THE CHALLENGE OF PROTEIN GROPS

AS A SUSTAINABLE SOURCE OF FOOD

AND FEED FOR THE FUTURE

EDITED BY: Antonio M. De Ron, Francesca Sparvoli, José J. Pueyo and Didier Bazile PUBLISHED IN: Frontiers in Plant Science

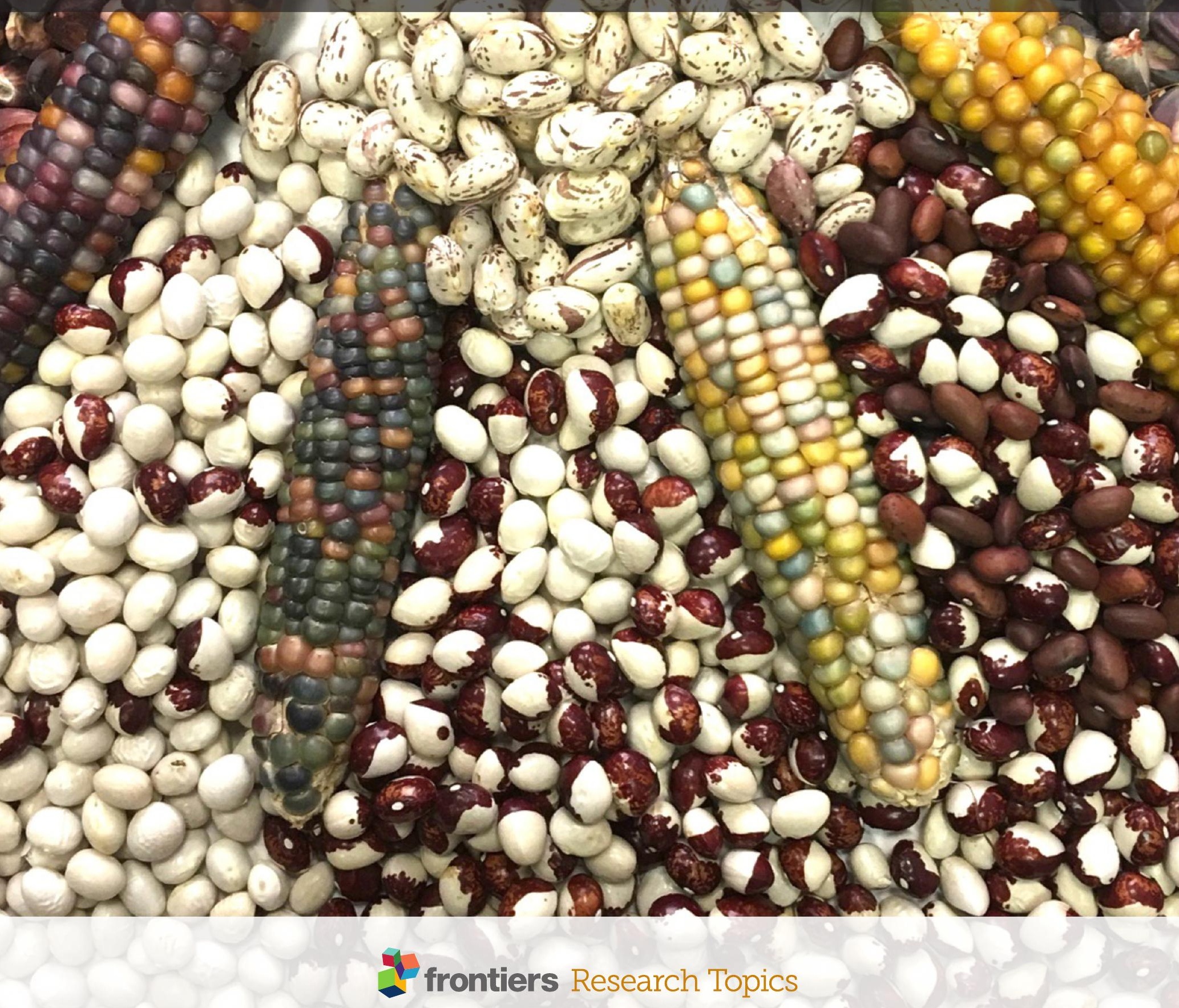




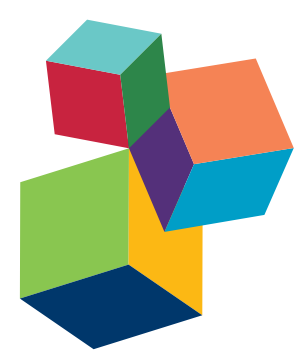

Frontiers Copyright Statement

(c) Copyright 2007-2017 Frontiers Media SA. All rights reserved.

All content included on this site, such as text, graphics, logos, button

icons, images, video/audio clips,

downloads, data compilations and

software, is the property of or is

licensed to Frontiers Media SA

("Frontiers") or its licensees and/or subcontractors. The copyright in the text of individual articles is the property of their respective authors, subject to a license granted to Frontiers.

The compilation of articles constituting

this e-book, wherever published,

as well as the compilation of all other

content on this site, is the exclusive property of Frontiers. For the conditions for downloading and copying of e-books from Frontiers' website, please see the Terms for Website Use. If purchasing Frontiers e-books from other websites or sources, the conditions of the website concerned apply.

Images and graphics not forming part of user-contributed materials may not be downloaded or copied without permission.

Individual articles may be downloaded and reproduced in accordance with the principles of the CC-BY licence subject to any copyright or other notices. They may not be re-sold as an e-book.

As author or other contributor you grant a CC-BY licence to others to reproduce your articles, including any graphics and third-party materials supplied by you, in accordance with the Conditions for Website Use and subject to any copyright notices which you include in connection with your articles and materials.

All copyright, and all rights therein, are protected by national and international copyright laws.

The above represents a summary only. For the full conditions see the Conditions for Authors and the Conditions for Website Use.

ISSN 1664-8714 ISBN 978-2-88945-162-3

DOI 10.3389/978-2-88945-162-3

\section{About Frontiers}

Frontiers is more than just an open-access publisher of scholarly articles: it is a pioneering approach to the world of academia, radically improving the way scholarly research is managed. The grand vision of Frontiers is a world where all people have an equal opportunity to seek, share and generate knowledge. Frontiers provides immediate and permanent online open access to all its publications, but this alone is not enough to realize our grand goals.

\section{Frontiers Journal Series}

The Frontiers Journal Series is a multi-tier and interdisciplinary set of open-access, online journals, promising a paradigm shift from the current review, selection and dissemination processes in academic publishing. All Frontiers journals are driven by researchers for researchers; therefore, they constitute a service to the scholarly community. At the same time, the Frontiers Journal Series operates on a revolutionary invention, the tiered publishing system, initially addressing specific communities of scholars, and gradually climbing up to broader public understanding, thus serving the interests of the lay society, too.

\section{Dedication to Quality}

Each Frontiers article is a landmark of the highest quality, thanks to genuinely collaborative interactions between authors and review editors, who include some of the world's best academicians. Research must be certified by peers before entering a stream of knowledge that may eventually reach the public - and shape society; therefore, Frontiers only applies the most rigorous and unbiased reviews.

Frontiers revolutionizes research publishing by freely delivering the most outstanding research, evaluated with no bias from both the academic and social point of view. By applying the most advanced information technologies, Frontiers is catapulting scholarly publishing into a new generation.

\section{What are Frontiers Research Topics?}

Frontiers Research Topics are very popular trademarks of the Frontiers Journals Series: they are collections of at least ten articles, all centered on a particular subject. With their unique mix of varied contributions from Original Research to Review Articles, Frontiers Research Topics unify the most influential researchers, the latest key findings and historical advances in a hot research area! Find out more on how to host your own Frontiers Research Topic or contribute to one as an author by contacting the Frontiers Editorial Office: researchtopics@frontiersin.org 


\section{THE CHALLENGE OF PROTEIN CROPS AS A SUSTAINABLE SOURCE OF FOOD AND FEED FOR THE FUTURE}

\section{Topic Editors:}

Antonio M. De Ron, Spanish National Research Council (CSIC), Spain Francesca Sparvoli, Consiglio Nazionale delle Ricerche (CNR), Italy José J. Pueyo, Spanish National Research Council (CSIC), Spain Didier Bazile, French Agricultural Research and International Cooperation Organization (CIRAD) \& Research Unit Management of Renewable Resources and the Environment (UPR GREEN) (CIRAD), France

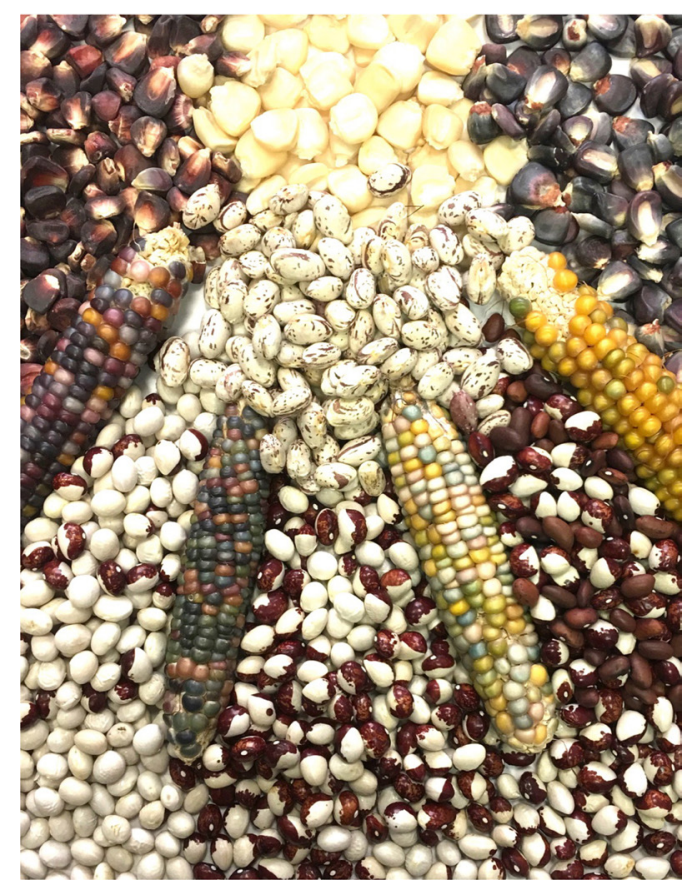

Seeds of common bean and maize. Seeds of legume and cereal crops are the main source of plant proteins for food and feed.

Photo by Francesca Sparvoli

Grain legumes, together with quinoa and amaranth (pseudocereals) and other crops are attractive candidates to satisfy the growing demand for plant protein production worldwide for food and feed. Despite their high value, many protein crops have not been adequately assessed and numerous species are underutilized. Special attention has to be paid to genetic diversity and 
landraces, and to the key limiting factors affecting yield, including water deficiency and other abiotic and biotic stresses, in order to obtain stable, reliable and sustainable crop production through the introduction and local adaptation of genetically improved varieties.

Legumes, the main protein crops worldwide, contribute to the sustainable improvement of the environment due to their ability to fix nitrogen and their beneficial effects on the soil. They play a key role in the crop diversification and sustainable intensification of agriculture, particularly in light of new and urgent challenges, such as climate change and food security. In addition, the role of legumes in nutrition has been recognized as a relevant source of plant protein, together with other benefits for health. Chapters dealing with common bean, lupine, soybean, lentil, cowpea and Medicago are included in this book. Most contributions deal with legumes, but the significant number of papers on different aspects of quinoa gives an idea of the increasing importance of this protein crop. Pseudocereals, such as quinoa and amaranth, are good sources of proteins. Quinoa and amaranth seeds contain lysine, an essential amino acid that is limited in other grains. Nutritional evaluations of quinoa indicate that it constitutes a source of complete protein with a good balance among all of the amino acids needed for human diet, and also important minerals, vitamins, high quality oils and flavonoids. Other protein crops also included in this book are hemp, cotton and cereals (maize, wheat and rice). Although cereals protein content is not high, their seeds are largely used for human consumption.

In this book are included articles dealing with all different aspects of protein crops, including nutritional value, breeding, genetic diversity, biotic and abiotic stress, cropping systems or omics, which may be considered crucial to help provide the plant proteins of the future. Overall, the participation of 169 authors in 29 chapters in this book indicates an active scientific community in the field, which appears to be an encouraging reflect of the global awareness of the need for sustainability and the promising future of proteins crops as a source of food and feed.

Citation: De Ron, A. M., Sparvoli, F., Pueyo, J. J., Bazile, D., eds. (2017). The Challenge of Protein Crops as a Sustainable Source of Food and Feed for the Future. Lausanne: Frontiers Media. doi: 10.3389/978-2-88945-162-3 


\section{Table of Contents}

07 Editorial: Protein Crops: Food and Feed for the Future

Antonio M. De Ron, Francesca Sparvoli, José J. Pueyo and Didier Bazile

\section{Chapter 1. Grain Legumes}

11 Trade-Offs between Economic and Environmental Impacts of Introducing Legumes into Cropping Systems

Moritz Reckling, Göran Bergkvist, Christine A. Watson, Frederick L. Stoddard, Peter M. Zander, Robin L. Walker, Aurelio Pristeri, Ion Toncea and Johann Bachinger

26 Proteomics and Metabolomics: Two Emerging Areas for Legume Improvement Abirami Ramalingam, Himabindu Kudapa, Lekha T. Pazhamala, Wolfram Weckwerth and Rajeev K. Varshney

47 Enhancing Legume Ecosystem Services through an Understanding of PlantPollinator Interplay

María J. Suso, Penelope J. Bebeli, Stefanie Christmann, Célia Mateus, Valeria Negri, Miguel A. A. Pinheiro de Carvalho, Renzo Torricelli and Maria M. Veloso

65 Influence of Rhizoctonia solani and Trichoderma spp. in growth of bean (Phaseolus vulgaris L.) and in the induction of plant defense-related genes Sara Mayo, Santiago Gutiérrez, Monica G. Malmierca, Alicia Lorenzana, M. Piedad Campelo, Rosa Hermosa and Pedro A. Casquero

76 Development of a qPCR Strategy to Select Bean Genes Involved in Plant Defense Response and Regulated by the Trichoderma velutinum - Rhizoctonia solani Interaction

Sara Mayo, Eleonora Cominelli, Francesca Sparvoli, Oscar González-López, Alvaro Rodríguez-González, Santiago Gutiérrez and Pedro A. Casquero

88 Seedling Emergence and Phenotypic Response of Common Bean Germplasm to Different Temperatures under Controlled Conditions and in Open Field Antonio M. De Ron, Ana P. Rodiño, Marta Santalla, Ana M. González, María J. Lema, Isaura Martín and Jaime Kigel

100 Exploitation of Common Bean Flours with Low Antinutrient Content for Making Nutritionally Enhanced Biscuits

Francesca Sparvoli, Monica Laureati, Roberto Pilu, Ella Pagliarini, Ivan Toschi, Gianluca Giuberti, Paola Fortunati, Maria G. Daminati, Eleonora Cominelli and Roberto Bollini

114 The future of lupin as a protein crop in Europe

M. Mercedes Lucas, Frederick L. Stoddard, Paolo Annicchiarico, Juana Frías, Cristina Martínez-Villaluenga, Daniela Sussmann, Marcello Duranti, Alice Seger, Peter M. Zander and José J. Pueyo 
Elena Bartkiene, Vadims Bartkevics, Vytaute Starkute, Daiva Zadeike

and Grazina Juodeikiene

124 The Potential for Engineering Enhanced Functional-Feed Soybeans for Sustainable Aquaculture Feed

Eliot M. Herman and Monica A. Schmidt

130 Agricultural practices altered soybean seed protein, oil, fatty acids, sugars, and minerals in the Midsouth USA

Nacer Bellaloui, H. Arnold Bruns, Hamed K. Abbas, Alemu Mengistu, Daniel K. Fisher and Krishna N. Reddy

144 Additive interactions of unrelated viruses in mixed infections of cowpea Nigna unguiculata $L$. Walp)

Imade Y. Nsa and Kehinde T. Kareem

157 Genetic Diversity of Cultivated Lentil (Lens culinaris Medik.) and Its Relation to the World's Agro-ecological Zones

Hamid Khazaei, Carolyn T. Caron, Michael Fedoruk, Marwan Diapari,

Albert Vandenberg, Clarice J. Coyne, Rebecca McGee and Kirstin E. Bett

164 Transcriptional profiling of Medicago truncatula during Erysiphe pisi infection Miguel Curto, Franziska Krajinski, Armin Schlereth and Diego Rubiales

Chapter 2. Quinoa and Amaranth

176 The Global Expansion of Quinoa: Trends and Limits

Didier Bazile, Sven-Erik Jacobsen and Alexis Verniau

182 Development of a Worldwide Consortium on Evolutionary Participatory Breeding in Quinoa

Kevin M. Murphy, Didier Bazile, Julianne Kellogg and Maryam Rahmanian

189 Worldwide Evaluations of Quinoa: Preliminary Results from Post International Year of Quinoa FAO Projects in Nine Countries

Didier Bazile, Cataldo Pulvento, Alexis Verniau, Mohammad S. Al-Nusairi, Djibi Ba, Joelle Breidy, Layth Hassan, Maarouf I. Mohammed, Omurbek Mambetov, Munira Otambekova, Niaz Ali Sepahvand, Amr Shams, Djamel Souici, Khaled Miri and Stefano Padulosi

207 Quinoa for Marginal Environments: Toward Future Food and Nutritional Security in MENA and Central Asia Regions

Redouane Choukr-Allah, Nanduri K. Rao, Abdelaziz Hirich, Mohammad Shahid, Abdullah Alshankiti, Kristina Toderich, Shagufta Gill and Khalil Ur Rahman Butt

218 Elevated Genetic Diversity in an $F_{2: 6}$ Population of Quinoa (Chenopodium quinoa) Developed through an Inter-ecotype Cross

Ouafae Benlhabib, Noura Boujartani, Peter J. Maughan, Sven E. Jacobsen

and Eric N. Jellen

227 Quinoa Seed Quality Response to Sodium Chloride and Sodium Sulfate Salinity Geyang Wu, Adam J. Peterson, Craig F. Morris and Kevin M. Murphy

235 New Insight into Quinoa Seed Quality under Salinity: Changes in Proteomic and Amino Acid Profiles, Phenolic Content, and Antioxidant Activity of Protein Extracts

Iris Aloisi, Luigi Parrotta, Karina B. Ruiz, Claudia Landi, Luca Bini, Giampiero Cai, Stefania Biondi and Stefano Del Duca 
256 Crossing Methods and Cultivation Conditions for Rapid Production of Segregating Populations in Three Grain Amaranth Species

Markus G. Stetter, Leo Zeitler, Adrian Steinhaus, Karoline Kroener, Michelle Biljecki

and Karl J. Schmid

\section{Chapter 3. Other Protein Crops}

264 How Integrated Management Strategies Promote Protein Quality of Cotton Embryos: High Levels of Soil Available N, N Assimilation and Protein Accumulation Rate

HongKun Yang, YaLi Meng, BingLin Chen, XingYue Zhang, YouHua Wang, WenQing Zhao and ZhiGuo Zhou

278 Cottonseed protein, oil, and mineral status in near-isogenic Gossypium hirsutum cotton lines expressing fuzzy/linted and fuzzless/linted seed phenotypes under field conditions

Nacer Bellaloui, Salliana R. Stetina and Rickie B. Turley

292 Where do Protein Bodies of Cereal Seeds Come From?

Emanuela Pedrazzini, Davide Mainieri, Claudia A. Marrano and Alessandro Vitale

299 Bacterial Communities in the Rhizosphere of Amilaceous Maize (Zea mays L.) as Assessed by Pyrosequencing

David Correa-Galeote, Eulogio J. Bedmar, Antonio J. Fernández-González, Manuel Fernández-López and Gregorio J. Arone

307 Variations in Protein Concentration and Nitrogen Sources in Different Positions of Grain in Wheat

Xiangnan Li, Longjing Zhou, Fulai Liu, Qin Zhou, Jian Cai, Xiao Wang, Tingbo Dai, Weixing Cao and Dong Jiang

317 Variability in Seed Traits in a Collection of Cannabis sativa L. Genotypes Incoronata Galasso, Roberto Russo, Sergio Mapelli, Elena Ponzoni, Ida M. Brambilla, Giovanna Battelli and Remo Reggiani 


\title{
Editorial: Protein Crops: Food and Feed for the Future
}

\author{
Antonio M. De Ron ${ }^{1 *}$, Francesca Sparvoli ${ }^{2}$, José J. Pueyo ${ }^{3}$ and Didier Bazile ${ }^{4,5}$ \\ ${ }^{1}$ Biology of Agrosystems, Misión Biológica de Galicia, Consejo Superior de Investigaciones Científicas (CSIC), Pontevedra, \\ Spain, ${ }^{2}$ Institute of Agricultural Biology and Biotechnology, Consiglio Nazionale delle Ricerche, Milan, Italy, ${ }^{3}$ Institute of \\ Agricultural Sciences (ICA), Consejo Superior de Investigaciones Cientificas (CSIC), Madrid, Spain, ${ }^{4}$ Directorate General in \\ Charge of Research and Strategy, French Agricultural Research and International Cooperation Organization (CIRAD), \\ Montpellier, France, ${ }^{5}$ Research Unit Management of Renewable Resources and the Environment (UPR GREEN) (CIRAD), \\ Montpellier, France
}

Keywords: cereals, legumes, plant proteins, quinoa

\section{Editorial on the Research Topic}

\section{Protein Crops: Food and Feed for the Future}

\section{OPEN ACCESS}

Edited and reviewed by: Diego Rubiales, Instituto de Agricultura Sostenible (CSIC), Spain

*Correspondence: Antonio M. De Ron amderon@mbg.csic.es

Specialty section:

This article was submitted to Crop Science and Horticulture, a section of the journal Frontiers in Plant Science

Received: 21 December 2016 Accepted: 18 January 2017 Published: 06 February 2017

Citation:

De Ron AM, Sparvoli F, Pueyo JJ and Bazile D (2017) Editorial: Protein Crops: Food and Feed for the Future.

Front. Plant Sci. 8:105. doi: 10.3389/fpls.2017.00105
Because of the protein content of their seeds, grain legumes, pseudocereals, and other crops are candidates to satisfy the growing demand for plant protein for food and feed. Crop production worldwide is highly specialized and currently relies on a very small number of species, raising questions about the sustainability of farming (Tilman et al., 2002). The role of legumes in nutrition has been recognized as a relevant source of plant protein together with other benefits for health. Soybean, peanut, common bean, pea, lupine, chickpea, faba bean, lentil, grass pea, cowpea, and pigeon pea are currently the most important legumes for human consumption and animal feed (De Ron, 2015). The integration of legumes into agriculture could reduce the current protein deficit and contribute to the transition to more sustainable agricultural systems. Legumes contribute to the sustainable improvement of the environment due to their ability to fix nitrogen and their beneficial effects on the soil (Drevon et al., 2015), having a tremendous potential in the reclamation of poor and marginal lands for agriculture (Coba de la Pe-a and Pueyo, 2012). Other protein crops include some minor crops, such as flax, hemp, or caraway, and some cereals have a certain potential, for they are quite abundant in food and feed. However, most importantly, chenopods called pseudocereals, such as amaranth and quinoa are recognized as excellent sources of protein and their seeds contain in particular the lysine, an essential amino acid that is limited in cereals. Nutritional evaluations of quinoa indicate that it constitutes a source of complete protein with a good balance of all of the amino acids needed for human diet, and also important minerals, vitamins, high quality oils, and flavonoids.

In this Research Topic (Protein crops: Food and feed for the future), were included papers dealing with different aspects of protein crops, such as breeding and selection of varieties for high yield or specific traits, biodiversity, sustainable cultivation, food and feed uses, nutritional value, health benefits, and socio-economic or environmental issues, which may be considered crucial to help provide the plant proteins of the future.

A novel assessment framework was developed by Reckling et al. and applied in five case study regions across Europe with the objective of evaluating trade-offs between economic and environmental effects of integrating legumes into cropping systems. Cropping systems with legumes significantly reduced nitrous oxide emissions and $\mathrm{N}$ fertilizer use in arable and forage systems. However, grain legumes reduced gross margins in 3 of 5 regions, while forage legumes 
increased gross margins in 3 of 3 regions. Legumes have been proved also beneficial in crop rotation and intercropping with cereals. Correa-Galeote et al. report on the variation of bacterial communities in the rhizosphere of maize as a result of intercropping with bur clover.

Therefore, increasing the cultivation of legumes could lead to economic competitive cropping systems and positive environmental impacts, but achieving this aim requires the development of novel management strategies informed by the involvement of advisors and farmers and it is often necessary to support farmers in their efforts to improve the biodiversity level on their farms. Since many legumes are bee-pollinated, the importance of the plant-pollinator interplay (PPI) in legumes lies in a combination of the importance of pollination for the production service and breeding strategies, plus the increasing urgency in mitigating the decline of pollinators through the development and implementation of conservation measures. The study by Suso et al. about PPI encompasses a range of reviews, opinions and perspectives and two basic approaches are proposed: (a) farming with alternative pollinators and (b) crop design system.

Lucas et al. focus on the future of lupin as a protein crop in Europe and conclude that lupin can be capable of promoting socio-economic growth and environmental benefits in Europe, provided that advanced breeding techniques deliver new productive lupin varieties, and novel processes are optimized to obtain high-quality protein ingredients for marketable foods to be offered to consumers. The need for optimizing lupin protein processing for increased nutritional value and food safety is also addressed by Bartkiene et al.

There is a growing awareness of the necessity to consider seed quality traits in breeding programs of protein crops. Bellaloui et al. discuss an agronomic study aimed to analyse the effects of management practices (planting date and seeding rate) on soybean seed composition. Seed storage proteins are a valuable source of essential amino acids for the diet; however in many legume seeds they often possess biological activities that might be either beneficial or detrimental to the consumer. Sparvoli et al. show how genetic removal, in common bean, of lectins and retention of $\alpha$-amylase inhibitor together with the reduction of phytate accumulation, may prove useful to produce bean composite flours for the preparation of novel foods, biscuits, with improved nutritional properties. Herman and Schmidt discuss on the potential for engineering soybeans for aquaculture feed by removing anti-nutritional proteins and increasing beneficial compounds.

Almost $50 \%$ of the global food protein supply comes from cereal seeds (faostat.fao.org). Most of these proteins (prolamins) accumulate in protein bodies (PB), large polymers formed in the endoplasmic reticulum (ER). On the contrary, the most common classes of seed storage proteins, the $2 \mathrm{~S}$ albumins and the $7 \mathrm{~S} / 11 \mathrm{~S}$ globulins accumulate in protein storage vacuoles (PSVs), which are the first subcellular compartment that evolved to store seed proteins. As the ER is mainly a compartment of transit, the permanent ER residence of proteins that do not play a role in typical ER functions seems a feature unique to prolamins. Based on the presence of specific domains in the different classes of storage proteins, together with phylogenetic studies of storage proteins evolution, Pedrazzini et al. formulate a hypothesis on how the PB evolved from the PSV as a simple solution to accommodate prolamin storage proteins. Li et al. analyse the variation of albumin, globulin, and glutenin contents in wheat grains as related to nitrogen application rates during different growth stages, which could be an effective approach to modulate the distribution of protein fractions for specific end-uses.

Ramalingam et al. review several proteomic and metabolomic studies on model and crop legumes, and discuss how integration of "omics" will contribute to the identification of accurate biomarkers in legume smart breeding programs. They discuss different methodological approaches, together with bioinformatics, and show how these could be used to tackle biological questions for understanding stress response mechanisms, cellular, and developmental processes and symbiosis.

Other protein crops also deserve attention. Hemp is an ancient crop that has been cultivated worldwide until the early twentieth century, after which its cultivation declined. Recently, interest in this multipurpose crop delivering fibers, shives, and seeds, has been renewed by an increasing demand not only for natural fibers but also for the high content and quality of seed protein and oil. Galasso et al. studied the composition of a collection of hemp genotypes of different geographical origins in order to identify potential genotypes to improve hemp cultivars. Their results reveal noticeable differences among hemp seed genotypes for antinutritional components, oil, and protein content. Collectively, this study suggests that the hemp seed is an interesting product in terms of protein, oil and antioxidant molecules, but a reduction of phytic acid would be desirable for both humans and monogastric animals. Bellaloui et al. and Yang et al. investigated the nutritional value of cotton, regarding protein content in seeds and embryos, respectively.

Crops growth is confronted by a variety of pathogens. Remaining healthy depends on their ability to recognize pathogens and to activate defense mechanisms against them. The plant defense responses are regulated by a broad number of signaling pathways. Transcription factors (TFs) control the transfer of genetic information from DNA to RNA by activation or repression of transcription, playing important roles in plant development and defense by regulating different signaling pathways (Singh et al., 2002; Udvardi et al., 2007).

Curto Rubio et al. used high-throughput quantitative realtime PCR (qPCR) technology to screen more than 1000 Medicago truncatula TFs in susceptible and resistant genotypes of $M$. truncatula after infection by the pathogen fungus Erysiphe pisi (powdery mildew). Seventy-nine TF genes, belonging to 33 families showed a significant transcriptional change in response to E. pisi infection. Forty eight TF genes were differentially expressed in the resistant genotypes compared to the susceptible one in response to E. pisi infection, including pathogenesisrelated transcriptional factors. The results suggest that these TF genes are among the E. pisi responsive genes in resistant M. truncatula that may constitute a regulatory network which controls the transcriptional changes in defense genes involved in resistance to E. pisi. 
The common bean is affected by a wide diversity of fungal pathogens, among them Rhizoctonia solani is one of the most important. Many Trichoderma species are well-known for their ability to promote plant growth and defense. Mayo et al. studied how the interaction of bean plants with $R$. solani and/or Trichoderma affect the plants growth and the level of expression of defense-related genes. Trichoderma isolates were evaluated in vitro for their potential to antagonize $R$. solani. The interaction of plants with $R$. solani and/or Trichoderma affects the level of expression of seven defense-related genes. Later on, Mayo et al. propose the use of biocontrol agents (BCAs) as a strategy to control bean diseases, mainly those caused by fungi. They combined in silico analysis and real time PCR to detect additional bean defense-related genes, regulated by the presence of Trichoderma velutinum and/or $R$. solani. Based in this strategy, from the 48 bean genes initially analyzed, 14 were selected, and only WRKY33, CH5b, and $h G S$ showed an up-regulatory response in the presence of T. velutinum. The strategy described in this work has shown to be effective to detect genes involved in plant defense, which respond to the presence of a BCA or to a pathogen and also to the presence of both.

Common bean is sensitive to low temperatures. Rapid and uniform seed germination and seedling emergence under diverse environmental conditions is a desirable characteristic for crops. Common bean genotypes differ in their low temperature tolerance regarding growth and yield. Cultivars tolerant to low temperature during the germination and emergence stages and carriers of the grain quality standards demanded by consumers are needed for the success of the bean crop. De Ron et al. studied the performance of 28 dry bean genotypes in open field and in growth chamber under low, moderate, and warm temperature. Screening of seedling emergence and phenotypic response of the bean germplasm under a range of temperatures in controlled growth chambers and under field conditions showed several genotypes with low temperature stress-tolerance at emergence and high yield potential that could be valuable genetic material for breeding programs.

Cowpea is a warm season legume that accounts for a huge portion of the dietary protein of the people in sub-Saharan Africa; however it is affected by many virus diseases that reduce yields. Nsa et al. studied the effects in three cowpea cultivars of single infections and co-infections of three unrelated viruses: Cowpea aphid-borne mosaic virus (CABMV), genus Potyvirus, cowpea mottle virus $(\mathrm{CMeV})$, genus Carmovirus, and Southern bean mosaic virus (SBMV), genus Sobemovirus. The treated plants were assessed for susceptibility to the viruses, growth, and yield. In all cases, early inoculation resulted in higher disease severity compared with late infection. Single, double and triple infections by CABMV, CMeV, and SBMV led to a complete loss of seeds in the three cowpea cultivars; only cultivar White produced some seeds at 30 DAP.

Lentil is the third most important cool-season grain legume in the world after chickpea and pea. Khazaei et al. studied the genetic diversity and population structure of lentil germplasm collection of 352 accessions from 54 countries to estimate genetic diversity and genetic structure using 1194 polymorphic single nucleotide polymorphism (SNP) markers which span the lentil genome. Using principal coordinate analysis, population structure analysis and UPGMA cluster analysis, the accessions were categorized into three major groups: (a) South Asia (sub-tropical savannah), (b) Mediterranean, and (c) northern temperate. Based on the results from this study, it is also clear that breeding programs still have considerable genetic diversity to mine within the cultivated lentil.

A number of articles focus on the importance of quinoa as a climate resilient grain option for climate change (Ruiz et al., 2014). This recent international recognition, for an underutilized crop from the Andean highlands, is largely due to its high nutritional value but underlines the importance of its high genetic diversity for adaptation to new environments (Bazile et al., 2015).

Bazile et al. give a critic of trends on the global expansion of quinoa. After centuries of neglect, the potential of quinoa was rediscovered during the second half of the twentieth century, and now more than 100 countries are testing or cultivating quinoa worldwide. Thanks to the high levels of genetic diversity, the crop is highly resilient to agro-ecological extremes and is tolerant to frost, drought, and salinity. The geographical increase in distribution of quinoa has highlighted the difficulty of access to quality seed, which is a key factor for testing the crop outside the Andes. In this context, research partnerships have allowed trials to be undertaken in non-traditional areas of cultivation.

Following the International Year of Quinoa in 2013, the Food and Agriculture Organization of the United Nations (FAO) initiated various trials at global level. Bazile et al. present field evaluations that were conducted in Asia and the Near East and North African countries. In each of the nine countries involved, the trials were carried out in different locations that globally represent the diversity of 19 agrarian systems for comparing 21 genotypes of quinoa using the same experimental protocol across the locations. Some genotypes showed higher yields for some specific locations when other genotypes were evaluated with stable and satisfactory levels of yield in each of the different trial sites. This production stability is of considerable importance especially under climate change uncertainty.

Choukr-Allah et al. consider the high tolerance and resistance of quinoa to many stresses and various cultivars have been screened for tolerance to salinity, water-use efficiency and nutritional quality. The authors summarized 15 years of studies on assessing the potential for introducing the crop in countries of the Middle East and North Africa and Central Asia and describe the key constraints for scaling-up the production under marginal growing conditions. Quinoa maintains productivity in poor soils and under water stress conditions and high salinity. Nevertheless, higher yields do not guarantee quinoa's success in the region and to be successful it must fit in the current cropping patterns, farming systems, marginal lands affected by salinity and alkalinity, and in the areas where the majority of the food crops could not be produced economically.

Also related to salt stress, Aloisi et al. reported the changes in proteomic and amino acid profiles, phenolic content and antioxidant activity in different quinoa cultivars when subjected to salinity stress. Wu et al. address the question of how the fertilization level influences salinity tolerance of quinoa and 
how soil salinity and fertility impact on seed quality traits, with particular attention to protein content and seed hardness and seed density.

Benlhabib et al. present an analysis of genetic diversity of $72 \mathrm{~F}_{2: 6}$ recombinant-inbred lines and parents developed through hybridization between highland and coastal germplasm groups of quinoa and evaluated for quantitative and qualitative traits. The study highlighted the extended diversity regenerated among the 72 lines and helped to identify potentially adapted quinoa genotypes for production in the Moroccan coastal environment, in particular with some lines resistant to downy mildew. Stetter et al. present a complementary research to Benlhabib et al. focused on amaranth. It appears of evidence that for the improvement of minor crops, efficient crossing methods are the basis of breeding programs. The authors developed three different crossing methods and compared their efficiency validated with genetic markers. The rapid production of segregating populations makes amaranth an attractive model for improvement by plant breeding.

\section{REFERENCES}

Bazile, D., Bertero, H. D., and Nieto, C. (Ed.) (2015). State of the Art Report on Quinoa Around the World in 2013. Rome: FAO and CIRAD, 603. Available online at: http://www.fao.org/3/a-i4042e.pdf

Coba de la Pe-a, T., and Pueyo, J. J. (2012). Legumes in the reclamation of marginal soils, from cultivar and inoculant selection to transgenic approaches. Agron. Sust. Dev. 32, 65-91. doi: 10.1007/s13593-0110024-2

De Ron, A. M. (Ed.) (2015). Grain Legumes. Series: Handbook on Plant Breeding. NewYork, NY; Dordrecht; Heidelberg; London: Springer.

Drevon, J. J., Alkama, N., Bargaz, A., Rodi-o, A. P., Sungthongwises, K., and Zaman-Allah, M. (2015). "The legume-rhizobia symbiosis," in Grain Legumes. Series: Handbook of Plant Breeding, ed A. M. De Ron (NewYork, NY; Dordrecht; Heidelberg; London: Springer), 267-290.

Ruiz, K. B., Biondi, S., Oses, R., Acu-a-Rodríguez, I. S., Antognoni, F., Martinez-Mosqueira, E. A., et al. (2014). Quinoa biodiversity and sustainability for food security under climate change. A review. Agron. Sustain. Dev. 34, 349-359. doi: 10.1007/s13593-0130195-0
Murphy et al. highlight the methods used for plant breeding and their impact on biodiversity in agricultural systems. Maintaining and increasing quinoa biodiversity is imperative, as the dynamics of the global expansion of quinoa may constitute a threat to farmers if the spread is generated with a narrow genetic base. Evolutionary participatory breeding (EPB) appears as a useful tool to develop new quinoa genetic material in cooperation with farmers. The global collaborative network on quinoa (GCNQuinoa) could be the baseline for participatory plant breeding programs to meet the needs of farmers across a diversity of agrosystems.

While these results on quinoa research suggest that this Andean crop is able to grow in many different environments, social, and cultural considerations remain crucial regarding its possible introduction in new cropping systems worldwide.

\section{AUTHOR CONTRIBUTIONS}

AD, FS, JP, and DB: drafting and revising the work and approval of the version to be published.

Singh, K. B., Foley, R. C., and Oñate-Sánchez, L. (2002). Transcription factors in plant defense and stress responses. Curr. Opin. Plant Biol. 5, 430-436. doi: 10.1016/S1369-5266(02)00289-3

Tilman, D., Cassman, K. G., Matson, P. A., Naylor, R., and Polasky, S. (2002). Agricultural sustainability and intensive production practices. Nature 418, 671-677. doi: 10.1038/nature01014

Udvardi, M. K., Kakar, K., Wandrey, M., Montanari, O., Murray, J., Andriankaja, A., et al. (2007). Legume transcription factors: global regulators of plant development and response to the environment. Plant Physiol. 144, 538-549. doi: 10.1104/pp.107.098061

Conflict of Interest Statement: The authors declare that the research was conducted in the absence of any commercial or financial relationships that could be construed as a potential conflict of interest.

Copyright (C) 2017 De Ron, Sparvoli, Pueyo and Bazile. This is an open-access article distributed under the terms of the Creative Commons Attribution License (CC BY). The use, distribution or reproduction in other forums is permitted, provided the original author(s) or licensor are credited and that the original publication in this journal is cited, in accordance with accepted academic practice. No use, distribution or reproduction is permitted which does not comply with these terms. 


\section{OPEN ACCESS}

Edited by:

Antonio M. De Ron,

Spanish National Research Council -

Misión Biológica de Galicia, Spain

Reviewed by:

Salvador Nadal Moyano,

Junta de Andalucía, Spain

Francisco Javier Ibarra Perez,

Instituto Nacional de Investigaciones

Forestales Agrícolas y Pecuarias,

Mexico

*Correspondence:

Moritz Reckling

moritz.reckling@zalf.de

${ }^{\dagger}$ Present Address:

Frederick L. Stoddard,

Department of Food and

Environmental Sciences, University of

Helsinki, Helsinki, Finland

Specialty section:

This article was submitted to Crop Science and Horticulture,

a section of the journal

Frontiers in Plant Science

Received: 31 March 2016

Accepted: 01 May 2016

Published: 23 May 2016

Citation:

Reckling $M$, Bergkvist $G$, Watson $C A$, Stoddard FL, Zander PM, Walker RL,

Pristeri A, Toncea I and Bachinger J (2016) Trade-Offs between Economic and Environmental Impacts of Introducing Legumes into Cropping

Systems. Front. Plant Sci. 7:669. doi: 10.3389/fpls.2016.00669

\section{Trade-Offs between Economic and Environmental Impacts of Introducing Legumes into Cropping Systems}

\author{
Moritz Reckling ${ }^{1,2 *}$, Göran Bergkvist ${ }^{2}$, Christine A. Watson ${ }^{2,3}$, Frederick L. Stoddard ${ }^{4 \dagger}$, \\ Peter M. Zander ${ }^{5}$, Robin L. Walker ${ }^{3}$, Aurelio Pristeri ${ }^{6}$, Ion Toncea ${ }^{7}$ and Johann Bachinger ${ }^{1}$ \\ ${ }^{1}$ Institute of Land Use Systems, Leibniz Centre for Agricultural Landscape Research, Müncheberg, Germany, ${ }^{2}$ Department of \\ Crop Production Ecology, Swedish University of Agricultural Sciences, Uppsala, Sweden, ${ }^{3}$ Crop and Soil Systems, \\ Scotland's Rural College, Aberdeen, UK, ${ }^{4}$ Department of Agricultural Sciences, University of Helsinki, Helsinki, Finland, \\ ${ }^{5}$ Institute of Socio-Economics, Leibniz Centre for Agricultural Landscape Research, Müncheberg, Germany, ${ }^{6}$ Department of \\ Agricultural Science, Mediterranean University of Reggio Calabria, Reggio Calabria, Italy, ${ }^{7}$ National Agricultural Research and \\ Development Institute, Fundulea, Romania
}

Europe's agriculture is highly specialized, dependent on external inputs and responsible for negative environmental impacts. Legume crops are grown on less than $2 \%$ of the arable land and more than $70 \%$ of the demand for protein feed supplement is imported from overseas. The integration of legumes into cropping systems has the potential to contribute to the transition to a more resource-efficient agriculture and reduce the current protein deficit. Legume crops influence the production of other crops in the rotation making it difficult to evaluate the overall agronomic effects of legumes in cropping systems. A novel assessment framework was developed and applied in five case study regions across Europe with the objective of evaluating trade-offs between economic and environmental effects of integrating legumes into cropping systems. Legumes resulted in positive and negative impacts when integrated into various cropping systems across the case studies. On average, cropping systems with legumes reduced nitrous oxide emissions by 18 and 33\% and $\mathrm{N}$ fertilizer use by 24 and 38\% in arable and forage systems, respectively, compared to systems without legumes. Nitrate leaching was similar with and without legumes in arable systems and reduced by $22 \%$ in forage systems. However, grain legumes reduced gross margins in 3 of 5 regions. Forage legumes increased gross margins in 3 of 3 regions. Among the cropping systems with legumes, systems could be identified that had both relatively high economic returns and positive environmental impacts. Thus, increasing the cultivation of legumes could lead to economic competitive cropping systems and positive environmental impacts, but achieving this aim requires the development of novel management strategies informed by the involvement of advisors and farmers.

Keywords: crop rotation, framework, land use and impacts, multi-criteria assessment, protein crops, resource-efficiency, rotation generator 


\section{INTRODUCTION}

Crop production in Europe is highly specialized and currently relies on a very small number of crop species, raising questions about the sustainability of farming (Tilman et al., 2002). Furthermore, less than $30 \%$ of the demand for protein feed supplement is produced within Europe (Bouxin, 2014). The reintegration of legumes into European agriculture could reduce the current protein deficit and contribute to the transition to more sustainable agricultural systems (Voisin et al., 2013).

The use of legumes also affects the performance of cropping systems and their environmental impacts, including (i) nitrogen supply via biological nitrogen fixation (BNF), reducing the demand for external nitrogen fertilizers, (ii) positive pre-crop benefits through a combination of residual nitrogen and breakcrop effects (Angus et al., 2015; Preissel et al., 2015), (iii) reduced fossil energy consumption in crop production (Jensen et al., 2011), and (iv) increased crop diversification and biodiversity (Köpke and Nemecek, 2010).

Despite these benefits, there are good reasons why European farmers grew grain legumes on only $1.6 \%$ of the arable land in 2014 (FAOstat, 2015). These include specialization in a few crops (Zander et al., 2016), low and unstable yields of grain legumes (Von Richthofen et al., 2006; Cernay et al., 2015; Reckling et al., 2015), low and unpredictable policy support (Bues et al., 2013), and lack of awareness of, and inability to evaluate the benefits of including legumes in the cropping system (Preissel et al., 2015). As a consequence, the area under grain legumes declined from $4.7 \%$ of arable land in 1961 to currently below $2 \%$ (FAOstat, 2015). Organic farms used $6.8 \%$ of their arable land for production of grain legumes in 2014 (Eurostat, 2015). Forage legume areas also declined because of a transformation of the ruminant feeding system toward maize silage and soybean oil cakes, and geographical separation between cereal and cattlerearing areas (Voisin et al., 2013).

By introducing legumes into the cropping system, farmers also introduce more complexity into their planning. To maximize the benefits of legumes while minimizing potential threats, a number of issues need to be taken into account. The preceding crop in a rotation should have a high ability to take up available nitrogen in order to reduce residual soil $\mathrm{N}$ levels and allow $\mathrm{BNF}$ of the legumes to be optimized. Legume crops need to be kept several years apart in the rotation because of their susceptibility to soil-borne diseases. The following crop should take up the residual $\mathrm{N}$ of the legume quickly, in order to minimize opportunities for nitrate leaching and nitrous oxide emission. Legume crops, their management and their effects on other crops in the rotation are specific to different climatic and edaphic conditions (Döring, 2015), e.g., narrow-leafed lupin (Lupinus angustifolius L.) is considered suitable for sandy soils with low soil $\mathrm{pH}$, whereas faba bean (Vicia faba L.) is typically grown on clay soils with sufficient rainfall, soybean (Glycine max (L.) Merr.) requires appropriate daylength to flower and sufficient growing degree-days to mature, and pea (Pisum sativum L.) is suitable for many different soil types. Therefore, the design of cropping systems needs to be site-specific and based on regional expert knowledge.
Although the positive effects of legumes on subsequent crops, including yield benefits and reductions in nitrogen fertilizer demand, soil tillage and biocide applications are well known (Angus et al., 2015; Preissel et al., 2015) from field experiments, these effects are difficult to formalize and are rarely considered in modeling (Bergez et al., 2010). Life cycle assessments comparing rotations with and without legumes have been carried out (e.g. Nemecek et al., 2008; Köpke and Nemecek, 2010) but these work with a limited set of rotations and do not include the detailed levels of field management required to evaluate all rotational effects.

In order to focus research and policy intervention, a method is needed to assess the economic and environmental effects of changes to cropping system design, such as the integration of legumes. To identify novel practices that achieve high economic and environmental benefits the method should provide a range of crop combinations and evaluate management options beyond current farming practices.

We earlier (Reckling et al., 2016) formulated an assessment framework that allows a systematic analysis of a large range of cropping options. It operates on the cropping system scale and takes rotational effects of crops such as legumes into account.

The objective of this study is to assess the economic and environmental effects of integrating legumes into cropping systems, in order to identify potentials and limitations of increasing legume cultivation in Europe. For the analysis we apply the cropping system framework by Reckling et al. (2016) in case studies in Germany, Italy, the United Kingdom, Romania, and Sweden. We hypothesize that the integration of legumes increases positive environmental impacts and at the same time reduces economic returns, thus leading to greater trade-offs between economic and environmental benefits.

\section{METHODS}

The static and rule-based framework developed by Reckling et al. (2016) was used to assess cropping systems in five case studies following three steps: (i) generate crop rotations (using a rule-based rotation generator), (ii) calculate the impact of crop production activities (using environmental and economic indicators), and (iii) assess and compare cropping systems with and without legumes. Following Bergez et al. (2010), we used a multi-criteria analysis for the identification of trade-offs.

\section{Case Studies}

Five contrasting regions were selected across Europe (Figure 1): Brandenburg (BB) in north-eastern Germany, Calabria (CB) in southern Italy, eastern Scotland (ES) in the United Kingdom, Sud-Muntenia (SM) in Romania, and Västra Götaland (VG) in western Sweden. The regions are characterized by contrasting climatic conditions and cropping systems, and were selected to represent a broad range of bio-physical and socio-economic conditions and possible roles of legume production. In all regions, legumes occupy less than $2.5 \%$ of the arable land. Within case studies, land capability types were defined by regional classifications and differences in soil texture (Table 1). 


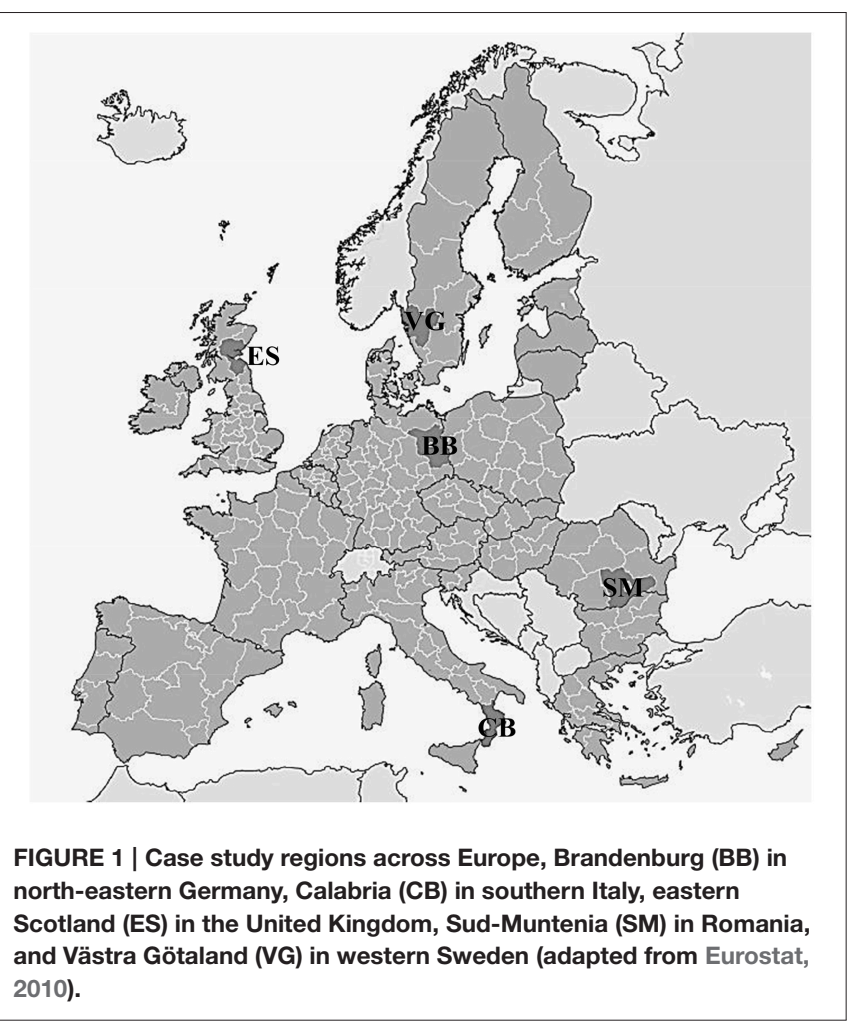

These determine the choice of the crops, their management and performance.

In Brandenburg, soil types are classified according to LELF (2010). Arable framing concentrates on Type 1-2 with higher yield potential and forage oriented farming on Type 3-4 with a lower yield potential. Soil Type 2 was selected for arable and Type 3 for forage systems. In Calabria, the assessment focused on arable rainfed systems in the lowlands, where pea and faba bean are the main annual legume crops. In Eastern Scotland, mixed cropping is common across soil Types $1-3$, and forage production on Types 3 and 4 (classified according to Bibby et al., 1982). For the assessment, Type 1 and 2 were selected for arable and Type 3 for forage systems. Cereals occupy $23 \%$ and grassland $73 \%$ of the agricultural land, while faba bean and pea together account for only $0.3 \%$ (Scottish-AgriculturalCensus, 2010). Arable farmers in the lowlands grow large shares of cereals and relatively few break crops, mainly winter and spring oilseed rape, potato, and swede. In the hilly areas, farms are dominated by grassland with low shares of clover. In SudMuntenia, Chernozem soils with high humus contents were selected, as they underlie the major cropping areas with mediumand large-scale farms. The study considered only arable farms, because information on forage grass production was limited. Legumes already grown include pea and soybean for feed and common bean (Phaseolus vulgaris L.) for the food market. In Västra Götaland, soils with high clay contents were selected for this study, because they represent the major cropping areas with both arable and mixed farms. Arable farmers use $80-100 \%$ of their land for cereals, and legumes and other break crops play minor roles (1.7 and $1.5 \%$ of the arable land). In forage-oriented farms, perennial grasses with a relatively low share of legumes $(<20 \%)$ are common.

Across the case studies, pea, faba bean and clover (Trifolium L.) (sole cropped or in mixture with grasses) were the most widely tested legumes. Wheat, barley and oilseed rape were the most common non-legume crops (Table 1).

\section{Generate Crop Rotations}

Crop rotations were generated with the previously described static and rule-based rotation generator (Reckling et al., 2016) that produces fixed and cyclical "agronomically sound rotations" for arable fields, following a fixed set of site-specific agronomic rules. In each region, agronomists defined suitable crops and then the restriction values for crop sequence, including suitability of crop-crop combinations, the minimum cultivation break and frequency restrictions (\% of a crop and crop type in a rotation) to allow the site-specific generation of rotations. In addition, a rotation of current farming ("business as usual") was based on the most common crops grown.

The rotation generator combined crops to produce all possible 3- to 6-year sequences following the criteria (i) crop sequence suitability (e.g., pea-rye), (ii) maximum frequency of a crop in the rotation (e.g., $20 \%$ for pea), (iii) minimum break between the same crop (4 years for pea), and (iv) maximum frequency of crops of the same crop type (e.g., $25 \%$ grain legumes). Duplicates and multiples were removed and rotations were filtered by soil type. Agronomists checked the plausibility of the results in comparison to the existing sequences.

\section{Calculate Impact Indicators}

The crop production activities (CPA) covered the crop, preceding crop and site-specific crop management. CPA were designed by collecting management data in a structured survey among 2-4 experienced agronomists in each case study in 2013. The data included inputs (seed, N-P-K fertilizer and pesticides), outputs (grain yield, forage yield, and straw yield) and management characteristics (fertilizer intensity, machinery use, and harvesting method) for each crop. In order to consider rotational effects, pre-crop types were defined according to their ability to influence the yield of the following crop through $\mathrm{N}$ from residue decomposition. Besides the effect on yield, differences in fertilization and agro-chemical applications were considered for each pre-crop type. The following types were separated, in the order of increasing $\mathrm{N}$ residue levels and positive effect on yield of subsequent crops: (i) spring cereals, winter cereals and maize; (ii) grain legumes, rapeseed and grass $(<10 \%$ legumes); (iii) forage legumes in pure stands and in mixtures with grass (>30\% legumes).

We selected five indicators to assess the economic and environmental performance of cropping systems. Nitrogen is not only important in crop production, it also contributes to environmental pollution of the atmosphere and water bodies, so nitrous oxide emission and nitrate leaching were selected as indicators. Nitrogen fertilizer use and nitrogen fertilizer efficiency were selected as indicators of resource use efficiency. Gross margins provided the standard indicator for economic 
TABLE 1 | Cropping characteristics of selected regions and land capability types.

\begin{tabular}{|c|c|c|c|c|c|c|}
\hline Region & Site & $\%$ sand & $\%$ clay & $\%$ of agric. area & Non-legume crops & Legume crops \\
\hline \multicolumn{7}{|c|}{ ARABLE CROPPING SYSTEMS } \\
\hline BB & Type 2 & 50 & 25 & 22 & WW, WR, SB, WB, WT, WOR, SM, SO & FP, FB \\
\hline CB & Type 3 & 47 & 23 & 36 & WB, DW, SO, WOR, WW, WT & FP, FB \\
\hline ES & Type 1/2 & 78 & 14 & 21 & SB, WB, SO, WO, SOR, WOR, SW, WW & FP, FB \\
\hline SM & Type 1 & 65 & 18 & 42 & WW, WOR, GM, SF, WB & FP, CB, SY \\
\hline VG & Type 1 & 17 & 33 & 50 & SB, SO, SW, WW, SOR, WOR, WR, WT & FP, FB \\
\hline \multicolumn{7}{|c|}{ FORAGE CROPPING SYSTEMS } \\
\hline BB & Type 3 & 70 & 20 & 37 & WW, WR, WOR, SM, SO, SB & FP, LU, GC, AF \\
\hline ES & Type 3 & 68 & 17 & 10 & SB, WB, SOR, WOR, SW, WW, SO, WO, GR & GC \\
\hline VG & Type 1 & 17 & 33 & 50 & SM, SB, SO, SW, WW, SOR, WOR, WR, WT, GR & $\mathrm{FP}, \mathrm{FB}, \mathrm{FP}-\mathrm{SO}, \mathrm{GC}$ \\
\hline
\end{tabular}

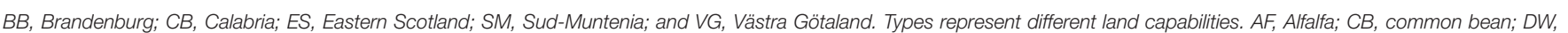

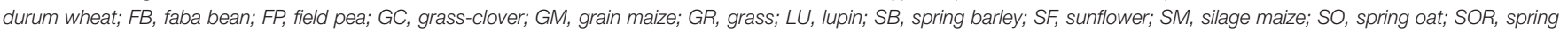
oilseed rape; SW, spring wheat; SY, soybean; WB, winter barley; WO, winter oat; WOR, winter oilseed rape; WR, winter rye; WT, winter triticale; WW, winter wheat.

performance of crops. All impacts were calculated on a perhectare and per-year basis.

\section{Calculation of Nitrate Leaching}

Nitrate leaching is calculated as nitrate- $\mathrm{N}$ based on the soil type, preceding crop and crop management as a function of the soil leaching probability and the nitrogen surplus. Adapted from Gäth and Wohlrab (1992) and Bachinger and Zander (2007), nitrate leaching is calculated as:

$$
N_{\text {leaching }}=N_{\text {surplus }} * L_{P}
$$

where $L_{P}$ is the leaching probability during the winter (mean winter precipitation divided by the water holding capacity at rooting depth and a crop-specific leaching coefficient) and the $\mathrm{N}_{\text {surplus }}$ is calculated as:

$$
N_{\text {surplus }}=\left(N_{\text {manureP }}+N_{\text {fertilizer }}+N_{\text {mineralization }}-N_{d f s}\right)
$$

where $N_{\text {manureP }}$ is the plant-available $\mathrm{N}$ content in solid and liquid manure and $N_{\text {mineralization }}$ is calculated as a function of the total organic $\mathrm{N}$ content (typical contents per soil type) and a region-specific mean annual mineralization rate modified by the pre-crop specific $\mathrm{N}$ supply level. $N_{d f s}$ is the nitrogen derived from soil and is calculated as:

$$
N_{d f s}=N_{\text {uptake }}-N_{\text {fixation }}
$$

Where $N_{\text {uptake }}$ is the nitrogen accumulated by the crop and $N_{\text {fixation }}$ is BNF of grain and forage legumes calculated as a function of the yield, the $\mathrm{N}$ content, the crop-specific ratio of above and below ground plant $\mathrm{N}$, the percentage of $\mathrm{N}$ derived from the atmosphere (\%Ndfa) depending on soil mineral $\mathrm{N}$ content using minimum and maximum \%Ndfa values (Peoples et al., 2009), the percentage of legumes in crop mixtures (grass-clover and cereal-legume intercropping), and the ratio of fixed $\mathrm{N}$ transferred to grass in grass-clover mixtures. The soil mineral $\mathrm{N}$ content is estimated taking into account possible $\mathrm{N}$ mineralization from preceding crop residues in spring, along with $\mathrm{N}$ inputs from plant-available $\mathrm{N}$ in manure and mineral $\mathrm{N}$ fertilizer (Reckling et al., 2016). Atmospheric nitrogen deposition and non-symbiotic nitrogen fixation were not considered because reliable data was not available.

\section{Calculation of Nitrogen Fertilizer Efficiency and Use}

Nitrogen fertilizer efficiency was calculated as the ratio of the $\mathrm{N}$ output in harvested grain or biomass to the $\mathrm{N}$ input from mineral and organic fertilizer and $\mathrm{N}$ in seed. $\mathrm{N}$ fertilizer use was calculated as the average $\mathrm{N}$ applied from organic and mineral $\mathrm{N}$ fertilizer.

\section{Calculation of Nitrous Oxide Emissions}

Soil-based nitrous oxide emission from crop cultivation was calculated with the IPCC 2006 Tier 1 methodology, including direct and indirect emission from applied fertilizer, manure and crop residues using standard factors and parameters, and assuming no emission from nitrogen fixation (IPCC, 2006).

\section{Calculation of Gross Margins}

Gross margins are calculated by subtracting variable costs (inputs, variable costs of machinery and services) from the revenues (yield multiplied by the product price). Labor costs, subsidies, interest and insurance were not taken into account. Data for the economic analysis were obtained from regional statistics (KTBL, 2010; LELF, 2010; Scottish-Agricultural-Census, 2010; SJV, 2011; ISTAT, 2013; MADR, 2013; SAC, 2013; Eurostat, 2015).

\section{Analysis of Cropping Systems}

Cropping systems were generated for each land capability and evaluated using the impact indicators. The evaluated systems were grouped as either arable, including only grain crops, or forage, including at least one forage crop, and the groups were further divided into those with legumes or without legumes. The dataset was analyzed by plotting environmental impacts against economic impacts of all single cropping systems and by calculating the mean impact and difference between arable and forage systems with and without legumes.

Trade-offs were analyzed between economic and environmental impacts using multi-criteria analysis based on five indicators: (i) Gross margin, (ii) nitrate leaching, 
(iii) nitrous oxide emission, (iv) $\mathrm{N}$ fertilizer use, and (v) $\mathrm{N}$ fertilizer efficiency. To allow relative comparisons, impacts were normalized for each land capability and arable and forage systems separately by dividing the impact of each single cropping system by the overall mean. Trade-offs were analyzed for selected cropping systems:

1) current farming without legumes

2) economic-environmental optimized systems without legumes

3) economic-environmental optimized systems with legumes

Current farming without legumes represents the most common cropping system per land capability based on the crop proportions and expert knowledge. Economic-environmental optimized cropping systems with and without legumes are selected from the large range of generated systems according to the criteria:

- Gross margin is equal to or $50 €$ lower than the system with the highest gross margin

- Nitrate leaching is equal to or lower than the system with the highest gross margin

- Nitrous oxide emission is equal to or lower than system with the highest gross margin

The selected cropping systems were compared in radar charts to identify trade-offs and to evaluate the performance from a systems perspective.

\section{Model Evaluation}

Generated rotations were compared by agronomists and advisors with current and plausible cropping patterns. Algorithms for nitrate leaching were already validated by Bachinger and Zander (2007) using HERMES, a dynamic model that simulates water and soil nitrogen dynamics (Kersebaum, 1995). Model outputs for each land capability type were compared against published data and by experts in nitrate leaching. The IPCC method to estimate nitrous oxide emissions is acknowledged to be simple and static, so is open to criticism (Philibert et al., 2012). Nevertheless, it is widely used in GHG inventories and emission models (Lokupitiya and Paustian, 2006; Berdanier and Conant, 2012) because it can be relatively easily applied with only a few input parameters. The model outputs were checked by a specialist in nitrous oxide emission measurements and models (B. Rees, pers. communication). Calculations of impacts at the cropping systems scale were evaluated by a panel of 12 agronomists who discussed the generated systems and compared the results with current management practices in their regions.

\section{RESULTS}

Integration of legumes affected the economic and environmental performance of cropping systems. The environmental performance was evaluated in terms of reduced leaching and nitrous oxide loss and lower fertilizer use, and economic performance was evaluated in terms of higher gross margin. On average across all regions, arable cropping systems with legumes had $18 \%$ less nitrous oxide emission, $24 \%$ less $\mathrm{N}$ fertilizer use, no increase in nitrate leaching and no decrease in gross margin (Table 2). In forage cropping systems, average effects were stronger, namely $33 \%$ less nitrous oxide emission, $38 \%$ less $\mathrm{N}$ fertilizer use, $22 \%$ less nitrate leaching and $21 \%$ higher gross margin (Table 2). Effects varied between the case studies due to the different crop management, bio-physical and socio-economic conditions, and between arable and forage systems.

TABLE 2 | Average economic and environmental impacts for cropping systems with and without legumes.

Region Site Legume Generated systems [no.] Gross margin [€/ha] $\mathrm{NO}_{3}-\mathrm{N}$ leaching [kg/ha] $\mathrm{N}$ fertilizer use [kg/ha] $\mathrm{N} 2 \mathrm{O}$ emissions [kg/ha]

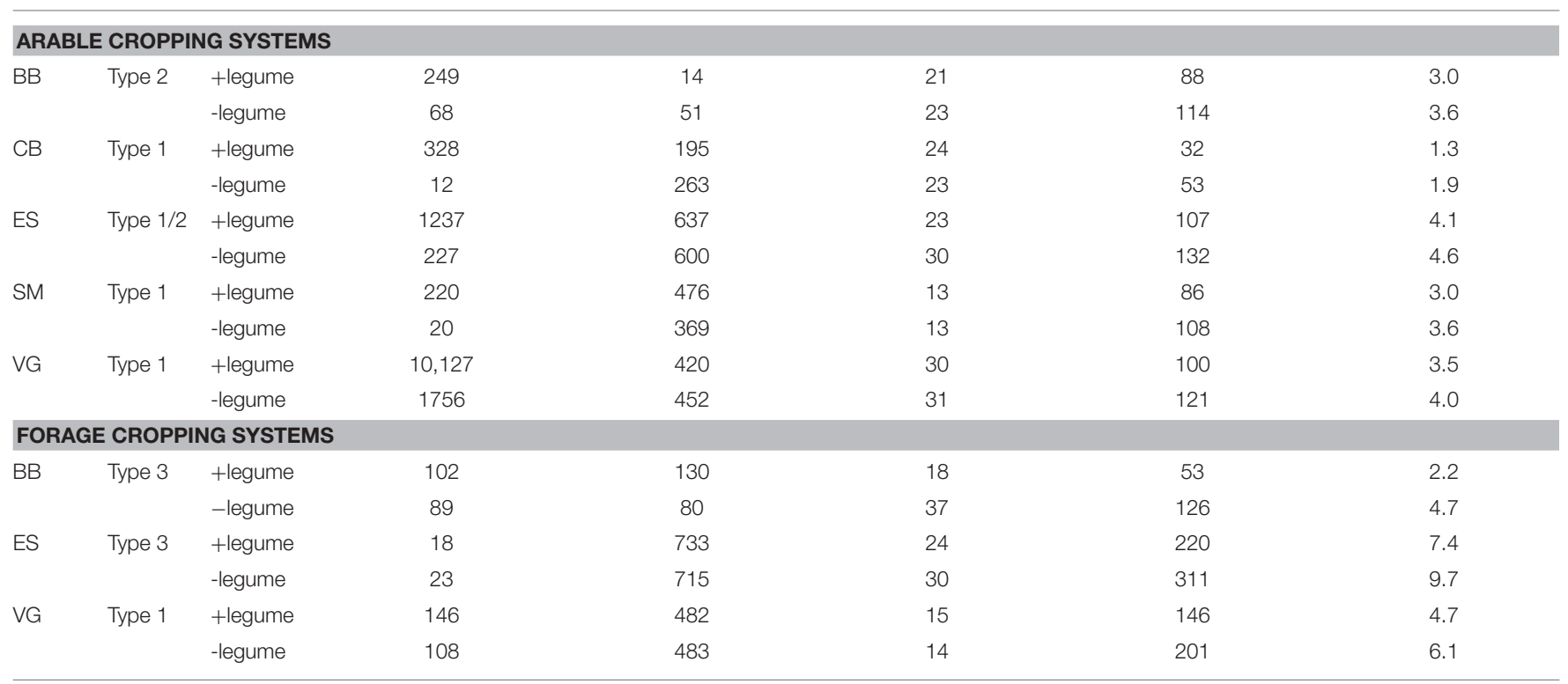

BB, Brandenburg; CB, Calabria; ES, Eastern Scotland; SM, Sud-Muntenia; and VG, Västra Götaland. Types represent different land capabilities. 


\section{Effects of Integrating Legumes into Arable Cropping Systems}

The inclusion of grain legumes, such as pea and faba bean, in arable cropping systems reduced $\mathrm{N}$ fertilizer use by $17-40 \%$ and nitrous oxide emission by $12-30 \%$ (Table 2). The smallest effects were found in VG for $\mathrm{N}$ fertilizer use and $\mathrm{ES}$ for nitrous oxide emission and the greatest in $\mathrm{CB}$ for $\mathrm{N}$ fertilizer use and nitrous oxide emission. Gross margins were 6 and 29\% higher in ES and $\mathrm{SM}$, and 7,26 , and $73 \%$ lower in $\mathrm{VG}, \mathrm{CB}$ and $\mathrm{BB}$, respectively (Table 2). Nitrate leaching was similar or slightly reduced in arable systems. Cropping systems with high gross margin tended to have higher nitrous oxide emissions than cropping systems with low gross margin. No relationship was observed between gross margin and nitrate leaching (Figure 2).

The current farming scenario without legumes achieved high economic returns in $\mathrm{BB}, \mathrm{CB}$ and $\mathrm{ES}$ and resulted in relatively high nitrate leaching and nitrous oxide emission in all regions (Figure 2). In VG and SM the current farming system achieved a low economic and environmental performance although it is practiced by a large share of farmers according to crop share statistics. The inclusion of oilseed rape improved gross margins in $\mathrm{BB}$ and VG and environmental benefits in cropping systems with and without legumes. The economic-environmental optimized system without legumes performed economically better than current farming in ES, SM and VG and environmentally better in all regions (Table 3).

The optimized system with legumes achieved 12 and 97\% higher gross margins in ES and SM than the optimized system without legumes, because of the high yield and high market value (for food use) of pea and common bean in the two regions (Table 3). Besides, adding a legume as another break crop to the cropping system led to an increased yield of the subsequent crop and hence to a higher gross margin. In VG, $\mathrm{CB}$, and $\mathrm{BB}$ the gross margin was $4-21 \%$ lower in the optimized system with legumes than in the optimized system without legumes, mainly due to low yields and low prices for pea and faba bean (Table 3). The optimized system with legumes had $12-25 \%$ lower $\mathrm{N}$ fertilizer use, 9-28\% lower nitrate leaching (except in CB) and 9-23\% lower nitrous-oxide emissions than that without legumes (Table 3). The major contributory factors to the improved environmental effects were that no $\mathrm{N}$ fertilizer was applied to the legume crop, the $\mathrm{N}$ fertilizer dose to the subsequent crop was reduced and substitution of winter wheat with crops that received smaller amounts of fertilizer. In CB, the optimized system with and without legumes reduced nitrate leaching by $72-$ $81 \%$ compared to common practice, because higher leaching was calculated for winter oat than for winter oilseed rape (Table 3). In $\mathrm{BB}$ and $\mathrm{CB}$, a proportion of legume-supported systems resulted in negative gross margins because they did not include a sufficient proportion of profitable crops such as oilseed rape (Figure 2).

\section{Effects of Integrating Legumes into Forage Cropping Systems}

The inclusion of forage legumes such as grass-clover and alfalfa and grain legumes in forage cropping systems reduced $\mathrm{N}$ fertilizer use by $27-58 \%$ and nitrous oxide emission by $23-52 \%$ (Table 2 ). The greatest reduction in $\mathrm{N}$ fertilizer use and nitrous oxide loss was found in $\mathrm{BB}$ where highly fertilized silage maize was replaced by unfertilized grass-clover. The effect was smaller in VG and ES where perennial grasses with low legume content were replaced by grass-clover including 30\% clover in the crop biomass. Gross margins were similar between systems with and without legumes in VG and ES and 62\% higher with legumes in BB (Table 2). Nitrate leaching was similar in both systems in VG and was reduced by the introduction of legumes in ES and BB. Similar to arable systems, forage cropping systems with high gross margins tended to have high nitrous oxide emissions, but no relationship was observed between gross margin and nitrate leaching (Figure 3).

The current cropping systems without legumes achieved high economic returns in $\mathrm{BB}$ and $\mathrm{VG}$ and resulted in relatively high nitrate leaching and nitrous oxide emission in all regions (Figure 3). In ES the current farming system with an additional cereal crop in the cropping system did not perform as well as the optimized systems economically or environmentally (Table 3 ), although it is practiced by a large proportion of farmers. Silage maize dominates current farming in $\mathrm{BB}$, and has the best economic performance. Maize yields relatively well on these soils and the economic value of silage maize for feed and biogas is high. The optimized system without legumes performed better economically than current farming in ES and VG and better environmentally in all regions (Table 3 ). In BB, the optimized system with a lower share of silage maize resulted in improved environmental performance than the current system, but with lower gross margin.

The optimized system with legumes achieved 3-4\% higher gross margins than without legumes in VG and ES because of the similar yield and price of grass-clover compared to grass, lower costs of fertilization, and a better pre-crop effect that led to a yield increase in the subsequent crop (Table 3). In BB, the integration of grass-clover resulted in $40 \%$ higher gross margin due to the high price of grass-clover forage, lower fertilizer costs and a greater pre-crop effect compared to silage maize, and despite the higher costs of harvesting 3 cuts of grass-clover rather than one crop of silage maize (Table 3). Across the regions, the optimized system with legumes had 23-54\% lower N fertilizer use, 21-58\% lower nitrous-oxide emissions, and, in $\mathrm{BB}, 70 \%$ lower nitrate leaching than that without legumes (Table 3). The major reasons for this difference include that no $\mathrm{N}$ was applied to the legume crop, the reduced $\mathrm{N}$ fertilizer doses to the subsequent crop and changes in the crop sequence.

\section{Trade-Offs between Economic and Environmental Impacts}

In all arable cropping systems, trade-offs were found between gross margin and environmental impacts (Figure 4). In BB, CB, and ES current farming had a high, above-average, economic and a low, below-average, environmental performance. The optimized system without legumes performed similarly in environmental terms to current farming in BB and ES. In CB, SM, and VG the optimized systems without legumes performed better on at least 

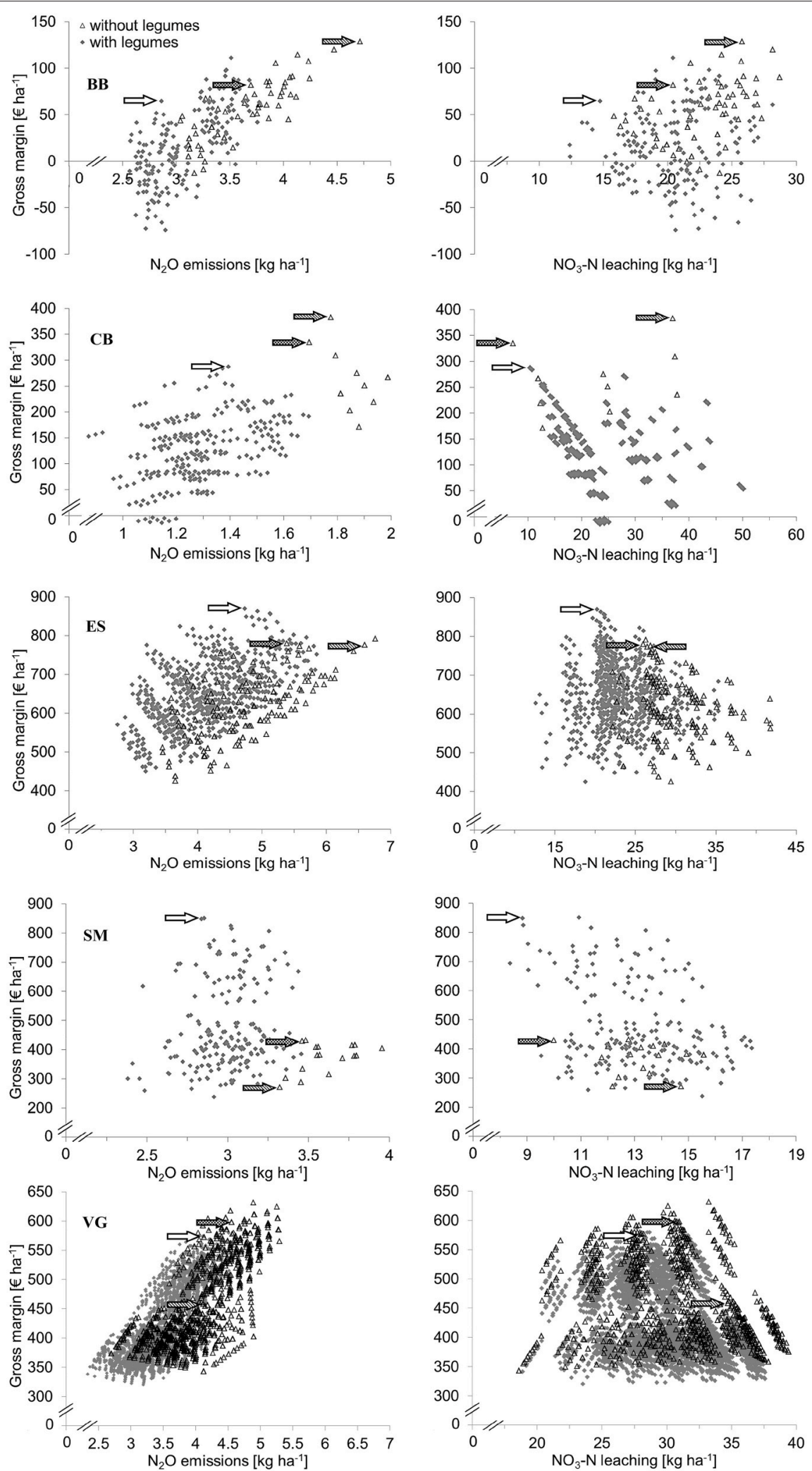

FIGURE 2 | Gross margin plotted against $\mathrm{N}_{2} \mathrm{O}$ emissions and $\mathrm{NO}_{3}-\mathrm{N}$ leaching for arable cropping systems with and without legumes in Brandenburg (BB), Calabria (CB), Eastern Scotland (ES), Sud-Muntenia (SM), and Västra Götaland (VG). Blank and dotted arrows indicate economic-environmental optimized systems with and without legumes and dashed arrows indicate current farming. 
TABLE 3 | Economic and environmental impacts for current and optimized cropping systems with and without legumes.

\begin{tabular}{|c|c|c|c|c|c|c|c|c|c|c|c|c|c|}
\hline Region & Site & System & Legume & Crop 1 & Crop 2 & Crop 3 & Crop 4 & Crop 5 & Crop 6 & $\begin{array}{l}\text { Gross } \\
\text { margin } \\
{[€ / h a]}\end{array}$ & $\begin{array}{c}\mathrm{NO}_{3}-\mathrm{N} \\
\text { leaching } \\
\text { [kg/ha] }\end{array}$ & $\begin{array}{c}\mathrm{N} \\
\text { fertilizer } \\
\text { use } \\
\text { [kg/ha] }\end{array}$ & $\begin{array}{c}\mathrm{N}_{2} \mathrm{O} \\
\text { emissions } \\
{[\mathrm{kg} / \mathrm{ha}]}\end{array}$ \\
\hline \multicolumn{14}{|c|}{ ARABLE CROPPING SYSTEMS } \\
\hline \multirow[t]{3}{*}{$\mathrm{BB}$} & Type 2 & Current & -legume & WW & WB & OR & & & & 128 & 26 & 139 & 4.7 \\
\hline & & Econ.-env. optimized & -legume & WR & WR & WR & SB & OR & & 82 & 20 & 125 & 3.7 \\
\hline & & Econ.-env. optimized & +legume & WW & WR & WR & WR & FP & & 64 & 15 & 94 & 2.9 \\
\hline \multirow[t]{3}{*}{$\mathrm{CB}$} & Type 1 & Current & -legume & WB & WO & WB & WO & & & 383 & 37 & 49 & 1.8 \\
\hline & & Econ.-env. optimized & -legume & WB & OR & WB & OR & & & 335 & 7 & 46 & 1.7 \\
\hline & & Econ.-env. optimized & +legume & WB & OR & WB & FB & OR & & 287 & 10 & 34 & 1.4 \\
\hline \multirow[t]{3}{*}{ ES } & Type 1/2 & Current & -legume & WB & OR & WW & WW & WB & & 776 & 27 & 192 & 6.6 \\
\hline & & Econ.-env. optimized & -legume & WB & OR & WB & WO & $\mathrm{SB}$ & & 780 & 26 & 156 & 5.4 \\
\hline & & Econ.-env. optimized & +legume & WB & OR & WB & WO & FP & & 869 & 20 & 130 & 4.7 \\
\hline \multirow[t]{3}{*}{ SM } & Type 1 & Current & -legume & GM & WW & SF & & & & 272 & 15 & 102 & 3.3 \\
\hline & & Econ.-env. optimized & -legume & GM & WB & OR & & & & 430 & 10 & 103 & 3.5 \\
\hline & & Econ.-env. optimized & +legume & GM & WB & OR & CB & & & 848 & 9 & 80 & 2.8 \\
\hline \multirow[t]{3}{*}{ VG } & Type 1 & Current & -legume & WW & WW & $\mathrm{SO}$ & & & & 459 & 35 & 124 & 4.1 \\
\hline & & Econ.-env. Optimized & -legume & WW & SB & OR & WR & SB & & 598 & 31 & 143 & 4.6 \\
\hline & & Econ.-env. optimized & +legume & WW & SB & OR & WR & FB & & 573 & 28 & 126 & 4.1 \\
\hline \multicolumn{14}{|c|}{ FORAGE CROPPING SYSTEMS } \\
\hline \multirow[t]{3}{*}{$\mathrm{BB}$} & Type 3 & Current & -legume & SM & $\mathrm{SM}$ & SM & WR & & & 262 & 40 & 166 & 6.9 \\
\hline & & Econ.-env. optimized & -legume & SM & WR & SM & SO & WR & & 131 & 34 & 131 & 4.8 \\
\hline & & Econ.-env. optimized & +legume & GC & GC & WR & WR & WR & SB & 184 & 10 & 60 & 2.0 \\
\hline \multirow[t]{3}{*}{ ES } & Type 3 & Current & -legume & GR & GR & GR & SB & $\mathrm{SO}$ & & 664 & 29 & 293 & 9.1 \\
\hline & & Econ.-env. optimized & -legume & GR & GR & GR & SB & & & 767 & 18 & 340 & 10.5 \\
\hline & & Econ.-env. optimized & +legume & GC & GC & GC & SB & & & 795 & 12 & 235 & 8.0 \\
\hline \multirow[t]{3}{*}{ VG } & Type 1 & Current & -legume & GR & GR & GR & WW & $\mathrm{SO}$ & & 535 & 12 & 222 & 6.6 \\
\hline & & Econ.-env. optimized & -legume & GR & GR & GR & OR & WT & SO & 551 & 12 & 212 & 6.3 \\
\hline & & Econ.-env. optimized & +legume & GC & GC & GC & OR & WR & SO & 567 & 13 & 163 & 5.0 \\
\hline
\end{tabular}

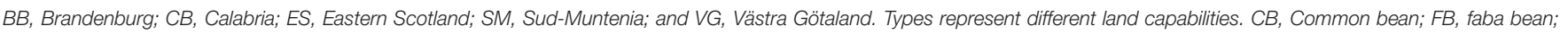
FP, field pea; GC, grass-clover; GM, grain maize; GR, grassland; OR, winter oilseed rape; SB, spring barley; SF, sunflower; SM, silage maize; SO, spring oat; WB, winter barley; WO, winter oat; WR, winter rye; WW, winter wheat; legumes are highlighted in bold.

one of the environmental measures, particularly nitrate leaching. The optimized systems with legumes performed economically well in all regions, except in $\mathrm{BB}$, and reached above-average scores for all environmental indicators (Figure 4).

Trade-offs between gross margin and environmental impacts were found for forage cropping systems without legumes (Figure 5). All systems without legumes performed below average, except regarding gross margin and nitrate leaching. The legume-supported systems reached above-average scores on all indicators, including gross margin. The environmental performance was generally good, especially considering nitrate leaching (Figure 5).

\section{DISCUSSION}

\section{Environmental and Economic Impacts}

The reduction in the use of $\mathrm{N}$ fertilizer when legumes were integrated into cropping systems, $17-40 \%$ in arable and $27-58 \%$ in forage systems (Table 2), is mainly attributable to the nitrogen added through the BNF of legume crops (Peoples et al., 2009).
The greatest savings were made in forage systems, because the perennial legumes included (clover and alfalfa) could fix more than $350 \mathrm{~kg} \mathrm{ha}^{-1}$ (Carlsson and Huss-Danell, 2003), which is much more than the corresponding figures for the annual legumes, pea and faba bean, that fix about $130 \mathrm{~kg} \mathrm{ha}^{-1}$ (Peoples et al., 2009). In BB, the $\mathrm{N}$ fertilizer savings were particularly large $(58 \%)$, because unfertilized grass-clover replaced silage maize that received high doses of $\mathrm{N}$ fertilizer. In VG and $\mathrm{ES}$, less $\mathrm{N}$ fertilizer was saved (27 and 29\%, respectively) (Table 2), because the grass-clover was fertilized, even if the doses were quite low. The different management of grass-clover represents common farming practice in the respective regions. The potential of BNF in grass-clover could be exploited more effectively than is common practice in VG and ES if $\mathrm{N}$ fertilizer doses to grassclover were reduced and/or distributed in time in a way that allows a sufficient share of legumes, as well as reducing losses (Eriksen et al., 2015). Nitrogen fertilizer savings in subsequent crops depend on the economic trade-off between securing maximum yields and maximizing $\mathrm{N}$ savings (Preissel et al., 2015). In our study, we used $\mathrm{N}$ fertilizer prices that were relatively 

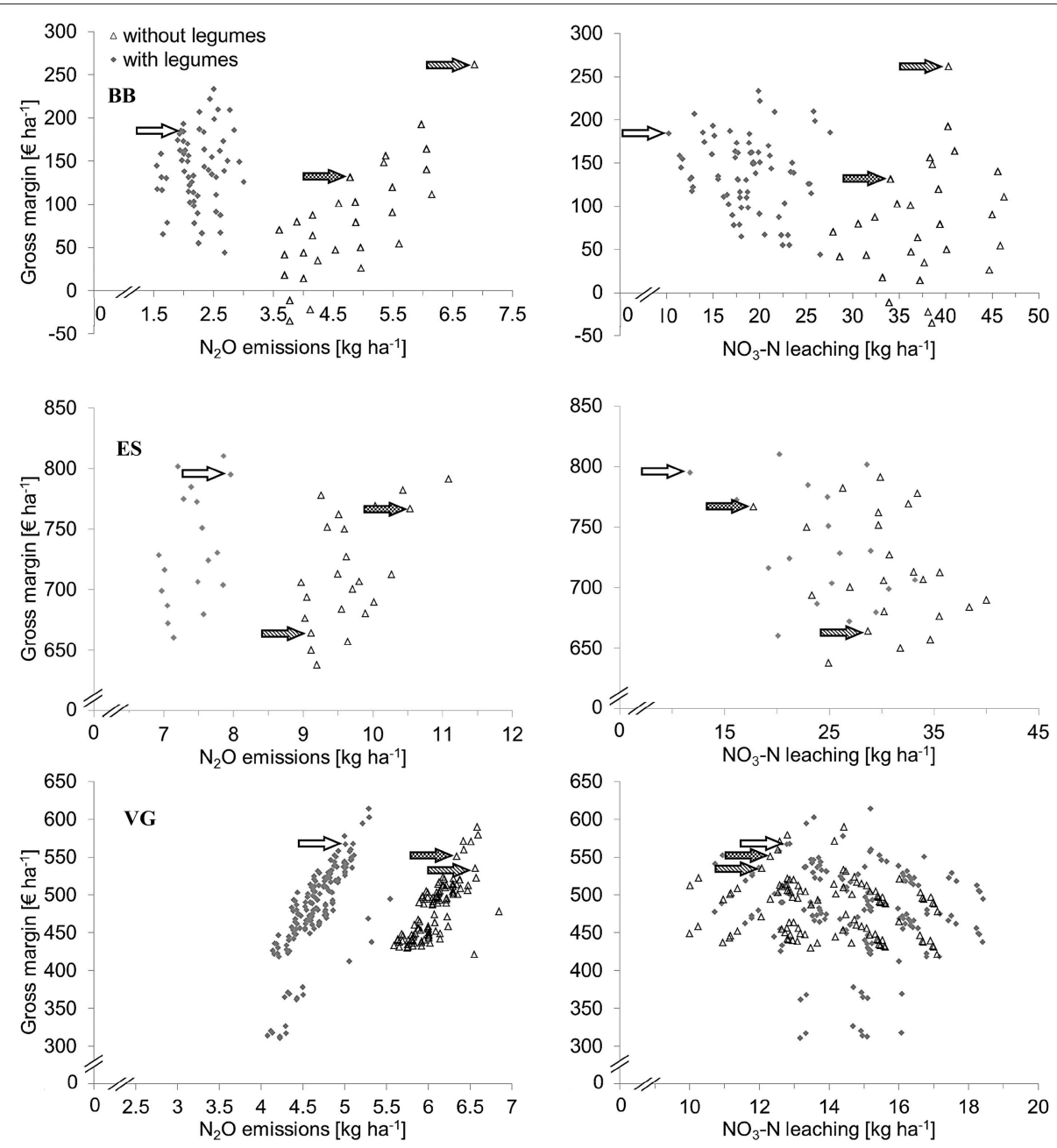

FIGURE 3 | Gross margin plotted against $\mathrm{N}_{2} \mathrm{O}$ emissions and $\mathrm{NO}_{3}-\mathrm{N}$ leaching for forage cropping systems with and without legumes in Brandenburg (BB), Eastern Scotland (ES), and Västra Götaland (VG). Blank and dotted arrows indicate economic-environmental optimized systems with and without legumes and dashed arrows indicate current farming.

low in relation to product prices. Therefore maintaining high yields came out as more important than saving $\mathrm{N}$ fertilizer, which means that we estimated small fertilizer savings, but high yield increases in subsequent crops. However, the framework allows new applications, such as to maximize $\mathrm{N}$ fertilizer savings, and the results of this analysis would have been different with this aim.

The average nitrous oxide emissions in cropping systems with legumes were 18 and 30\% lower in arable and forage systems, respectively (Table 2). The differences among regions were greater for forage systems than for arable systems. The difference was greatest in $\mathrm{BB}(52 \%)$ due to the lower amounts of $\mathrm{N}$ applied to grass-clover than to silage maize. There is a direct relationship between $\mathrm{N}$ fertilizer input and nitrous oxide emissions, as shown by several studies compiled by Buckingham et al. (2014). However, we have used the assumption that $1 \%$ of each $\mathrm{kg}$ of $\mathrm{N}$ fertilizer is released as field-based nitrous oxide emission (IPCC, 2006), and this is currently under consideration. Philibert et al. (2012) calculated lower emission factors (EF) when the amount of $\mathrm{N}$ applied was below $160 \mathrm{~kg} \mathrm{~N}^{-1}$, Hinton et al. (2015) estimated EF to be between 0.28 and $1.35 \%$ of applied $\mathrm{N}$ depending on the $\mathrm{N}$ input, and Rees et al. (2012) concluded from a meta-analysis using measured data from Europe that annual emissions from arable sites were significantly greater than predicted by IPCC. Besides $\mathrm{N}$ fertilizer inputs, field-based nitrous oxide emissions are influenced by soil, management and environmental conditions (Ball et al., 2014). It seems clear that the EF used in the present study is approximate, but there is no consensus on how to modify it to better represent actual emissions.

The risk of nitrous oxide emission is generally considered to be lower from legumes than from cereals, oilseed rape and grassland (Stehfest and Bouwman, 2006; Schwenke et al., 2015). In a meta-analysis, Jensen et al. (2011) calculated that emissions from legume crops were around $40 \%$ lower than those from $\mathrm{N}$ fertilized crops, and similar to unplanted soils or crops that were not $\mathrm{N}$ fertilized. The reason is that legume $\mathrm{N}$ inputs through $\mathrm{BNF}$ 


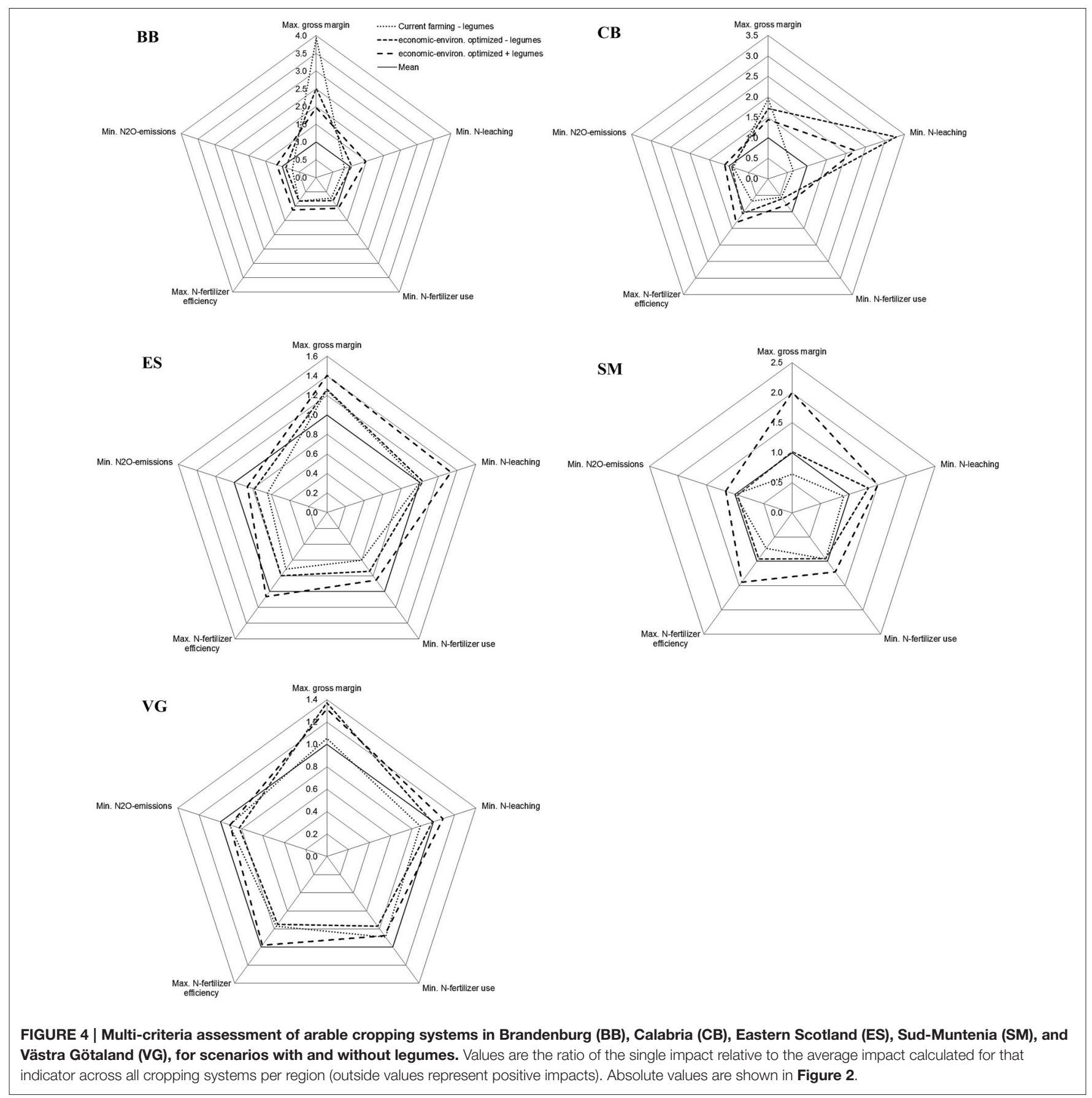

supplement the uptake of soil mineral $\mathrm{N}$ to meet crop $\mathrm{N}$ demand. The preferential use of soil mineral $\mathrm{N}$ leads to low availability of nitrate $\mathrm{N}$ for potential denitrification losses (Schwenke et al., 2015).

Nitrate leaching was similar with and without legumes in arable cropping systems (Table 2). Similarly to nitrous oxide emissions, nitrate leaching is soil-dependent and influenced by tillage, which did not differ between cropping systems with and without legumes. Tillage operations could in some circumstances be reduced before and after grain legumes (Luetke-Entrup et al.,
2003; López-Bellido et al., 2003, 2004), which could reduce losses of nitrogen and increase gross margins of legume-supported systems (Preissel et al., 2015). The lower leaching in CB in the optimized cropping systems than in the current system was a result of the substitution of winter oat by winter oilseed rape (Table 3). The latter can take up more $\mathrm{N}$ before winter and thus reduce the risk of nitrate leaching (Sieling and Kage, 2012).

In forage systems, management factors such as soil tillage, nitrogen fertilization and the management of legumes in grassclover mixtures influence nitrate leaching significantly (Eriksen 

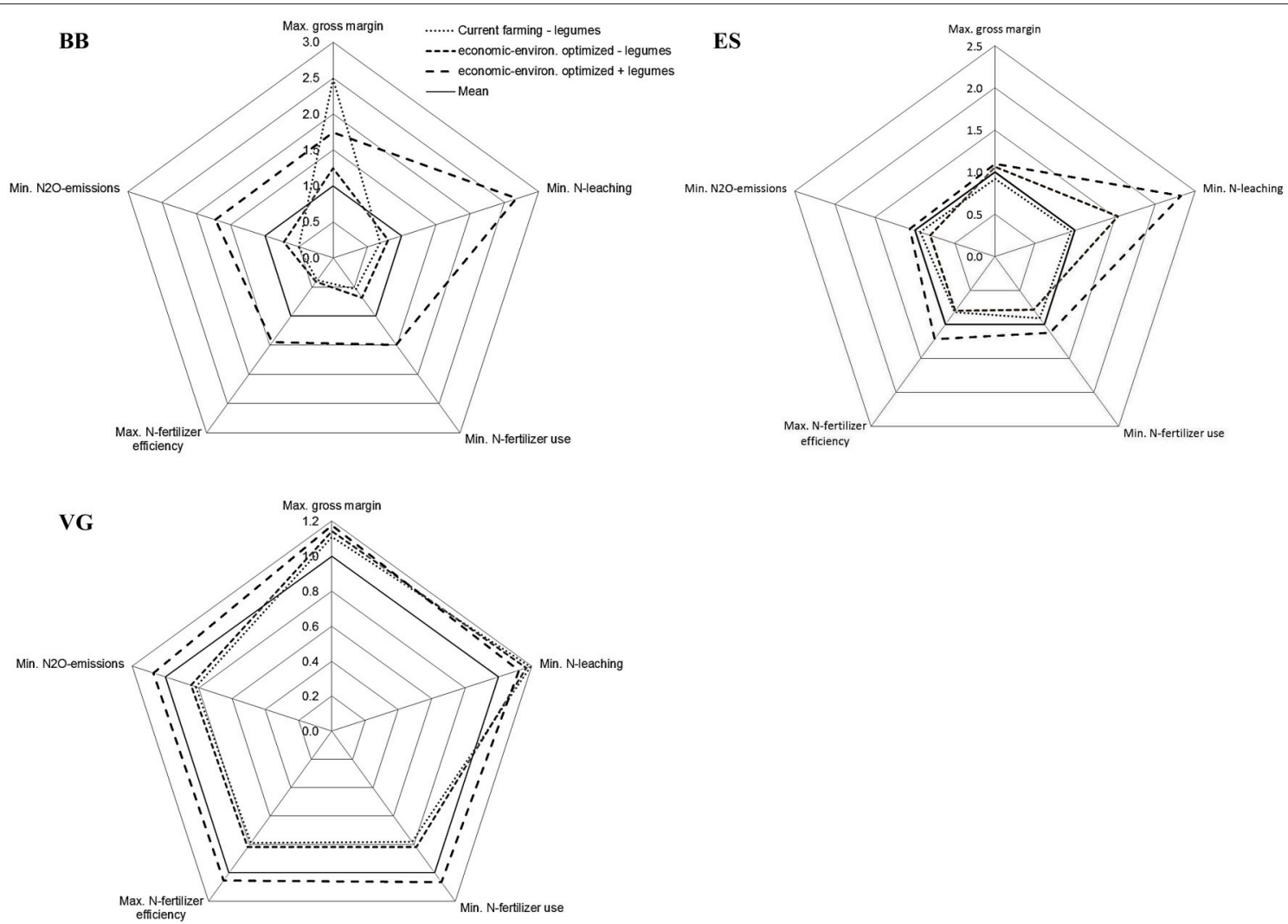

FIGURE 5 | Multi-criteria assessment of forage cropping systems in Brandenburg (BB), Eastern Scotland (ES), and Västra Götaland (VG), for scenarios with and without legumes. Values are the ratio of the single impact relative to the average impact calculated for that indicator across all cropping systems per region (outside values represent positive impacts). Absolute values are shown in Figure 3.

et al., 2015). In our study, nitrate leaching was similar in both optimized systems in VG, slightly reduced with legumes in ES and strongly reduced in BB (Table 3). Reduced leaching with legumes in $\mathrm{BB}$ can be attributed to continuous crop cover in grass-clover compared to winter fallow between maize crops and high $\mathrm{N}$ fertilization of the maize crop compared to no $\mathrm{N}$ fertilization of grass-clover, in combination with a humid winter climate that causes most of the leaching to occur from October to March. In ES, lower doses of $\mathrm{N}$ fertilizer to grass-clover than to pure grass explain the slightly lower leaching.

The so-called break-crop effect is another aspect that increases the efficiency of utilizing $\mathrm{N}$ after a legume. Roots of a given crop are healthier after an unrelated crop has been grown, because pathogen populations are reduced (Angus et al., 2015), allowing more $\mathrm{N}$ to be taken up by the crop, and reducing the availability of $\mathrm{N}$ for leaching. There is a risk of large leaching losses after incorporation of $\mathrm{N}$-rich crop residues, such as legume residues, so proper management is required to avoid losses (Eriksen et al., 2015). In our study, the risk of losses was reduced through the generation of optimized cropping systems where pre-crop effects were used effectively. Cover cropping is another option to reduce nitrate leaching after grain legumes (Plaza-Bonilla et al., 2015), but was not tested in this study.
Economic performance is regarded a key driver responsible for low adoption of legumes in cropping systems by farmers (Von Richthofen et al., 2006). As individual crops, legumes generally have lower gross margins than cereals and oilseed crops (Preissel et al., 2015). Our study revealed that the economic performance of legume crops was improved when assessed at the cropping system scale that allows consideration of breakcrop effects on fertilizer use, other inputs, and yields of the following crops. The rotational effects of legumes are generally not considered in the economic evaluations of cropping systems (Zander et al., 2016). When cropping system effects are included, the competitiveness of legumes improved and the difference in gross margin between systems with and without legumes ranged from -67 to $106 €$ in arable and from 0 to $50 €$ in forage systems across all regions (Figures 2, 3). The high economic performance of legume systems in SM depends on stable market prices for common bean.

\section{Trade-Offs between Economic and Environmental Impacts}

Cropping systems with a high economic performance tended to have high nitrous oxide emissions due to high input use. Such systems could be relevant for the intensification of agricultural systems, and concentrate the negative environmental impacts 
onto less arable land according to the land-sparing paradigm (Lamb et al., 2016).

The integration of grain legumes in arable systems had environmental benefits in all regions, but cropping systems with legumes also performed well economically in VG, SM, and ES (Figure 4). Thus the introduction of legumes in these regions added services without associated economic cost or losses in productivity, so they contributed to the ecological intensification of cropping systems (Doré et al., 2011). In CB and BB, the gross margin was lower in legume-supported systems than in those without legumes because of the low yields and prices of grain legumes.

Forage systems with legumes achieved both economic and environmental benefits in all regions because of relatively high yields, similar prices and better residual effects on subsequent crops considering yield and $\mathrm{N}$ fertilizer use (Figure 5).

Thus, adding a legume to cropping systems reduced tradeoffs compared to systems without legumes resulting either in (a) win-win situations where legume cultivation is economically attractive and increases environmental benefits, or (b) situations where legumes are not economically attractive but increase environmental benefits.

\section{Opportunities and Constraints for Integrating Legumes into Cropping Systems}

In the case of win-win situations, legumes are already economically attractive and provide environmental benefits. The opportunities for forage legumes are easier to utilize because of (i) their generally better economic and environmental performance compared to annual grain legumes, (ii) their high quality feed value for livestock (relatively high prices), and (iii) their relatively simple integration into existing temporary grassland. The perennial nature of forage legumes offers opportunities for climate change mitigation and adaptation (Lüscher et al., 2014), improved biodiversity (Stein-Bachinger and Fuchs, 2012) and soil organic carbon content (Jensen et al., 2011), and lowered risk of both soil erosion (Jensen et al., 2011) and weed infestation (Håkansson, 2003). The deep rooting of some species improves subsoil accessibility for subsequent crops and increases the water infiltration rate (Fischer et al., 2014). However, the adoption of forage legumes is restricted to mixed farms with crop and livestock production, farm collaborations with livestock farmers, and farms that deliver green biomass to biogas plants (Tidåker et al., 2014). In mixed farms, nutrient management through the integration of crops and livestock remains a challenge, because of the high risk of nitrogen losses in manure management (Watson et al., 2005). Important agronomic constraints in legume production include crop establishment and to maintain about one third of legumes in grass-clover mixtures to achieve the maximum benefits from the clover (Suter et al., 2015). When feeding clovers and alfalfa to livestock, there is also an increased risk that ruminants suffer problems with digestion, i.e., bloat (Majak et al., 1995; Dewhurst et al., 2009). The specialization of farming and associated spatial decoupling of livestock and crop production is the major reason for the low proportion of forage legumes in Europe (Lemaire et al., 2015).

There are situations in arable systems were the introduction of legumes has both economic and environmental advantages, especially were grain legumes achieve high prices as a human food product (SM and ES) and where grain yields are relatively high. However, in most situations, grain legumes are still not competitive and there are multiple reasons why European farmers do not grow these crops. The major drivers are economic forces favoring cost-effective production systems and gains from international trade over the economic benefits of diversified production systems (Zander et al., 2016). Agronomic risks include lower yields and yield stability of grain legumes compared to cereals (Cernay et al., 2015), especially under conditions with low water availability such as in BB (Reckling et al., 2015) and in CB. The magnitude and causes of yield variability requires further research (Döring, 2015) and is attributable partly to the much lower investment in legume breeding than in cereal breeding (Meynard et al., 2013). Management strategies to reduce yield stability beyond good farming practices are limited. Other limitations mentioned by experts from the case study regions were increased weed infestation, root rot caused by Aphanomyces euteiches in pea, susceptibility to soil compaction, and lack of adapted cultivars (Stoddard, 2013). These constraints require that the legume crop be carefully selected to fit the local conditions and that the crop rotation be well designed with sufficient breaks between legumes that are affected by similar pathogens.

Marketing constraints for grain legumes are mainly due to low prices and few marketing channels. Prices are currently often lower than the actual feed value, and EU-produced grain legumes have difficulties competing with other protein crops on the world market (Voisin et al., 2013). The value chains for EUproduced grain legumes for feed and food are poorly developed in many European countries (Meynard et al., 2013). Regional supply chains for the feed and food market could increase competitiveness of legumes in Europe (Voisin et al., 2013) e.g., a GMO-free protein supply. Furthermore, ecosystem services, including reduced nitrous oxide emissions and nitrate leaching, and increased biodiversity are currently not rewarded through payments, so they are not considered in farmers' economic calculations and could justify policy support (Zander et al., 2016).

Farmers and advisors seldom consider the long-term benefits, focusing instead on single years. This leads to an underestimation of the services provided by legumes. The valuation of such services requires an assessment at the cropping-system scale (Reckling et al., 2016). Appreciation of these services could lead to wider adoption of legumes by farmers, but would also require that practical information on their rotational effects and proper management is available (Stoddard, 2013).

\section{Framework Evaluation}

The framework was evaluated using (i) design validation, (ii) plausibility checking of outputs and (iii) end-user validation, 
as proposed by Bockstaller and Girardin (2003) and described in detail by Reckling et al. (2016). The plausibility checking, especially through experts, provided sufficient information to adapt the model for the specific application in each of the contrasting case studies. Several iterations were needed with agronomists to define the input data and to evaluate the outputs. Thus the quality of the model outputs was dependent to a large extent on the quality of the expert knowledge, as is usually the case for rule-based models (Bachinger and Zander, 2007; Naudin et al., 2015).

The major sensitivity of the framework was the quantification of pre-crop effects that were estimated by experts and supported by information from field experiments (Reckling et al., 2016). Data from field experiments and dynamic models alone do not provide all the required data. Field experiments show varying effects between years and sites, and dynamic models have difficulties in handling pre-crop effects (Lorenz et al., 2013; Kollas et al., 2015).

The static calculation of nitrate leaching and nitrous oxide emission quantifies effects that do not consider the variability of weather, yields and environmental impacts (as is the case in process-based models), and are used here to make only relative comparisons between cropping systems. Nevertheless, static approaches are often used for nitrous oxide emission (Lokupitiya and Paustian, 2006; Berdanier and Conant, 2012) and nitrate leaching (Bachinger and Zander, 2007; Stein-Bachinger et al., 2015) when numerous systems are compared and measurements are not sufficiently available. The equation for nitrate leaching is based on the $\mathrm{N}$ surplus at the end of the growing season of each crop and might not sufficiently take into account all losses from mineralizing crop residues. This is especially the case when the $\mathrm{N}$ surplus is low due to high $\mathrm{N}$ uptake, e.g., the combination of high yields and low $\mathrm{N}$ input. Potential $\mathrm{N}$ losses through mineralization of $\mathrm{N}$-rich residues from legumes after harvest until the end of the growing season might be underestimated. The indicators used for the evaluation were, however, found to be suitable to account for agronomic, environmental and economic aspects. Additional indicators such as erosion, soil organic matter, diseases and weed infestation, and the water foot print of crops could be added to the sustainability assessment in future.

The assessment framework generated and assessed large numbers of rotations that provide challenges to users, but the large number and diversity of systems provided the opportunity to explore cropping strategies outside existing system configurations and boundaries. This ability to explore a large number of options can also be used to complement information derived from life cycle assessments e.g., Nemecek et al. (2008). Through applying the selection criteria, we were able to identify novel economic-environmental optimized systems with legumes that could in the future be tested as prototypes by farmers (Vereijken, 1997).

A particular strength of this assessment framework is that it involves stakeholders from research and practical farming in the process of redesigning and assessing cropping systems that fulfill both economic and environmental aims, and that this can be done without having to test a wide range of systems on farms or experimental stations.

\section{Outlook}

To utilize the multiple services of legumes, their production needs to increase significantly. Where legumes already lead to win-win situations with high economic and environmental benefits, such effects need to be communicated to advisors and farmers, and regional supply chains need to be developed for the feed and human food market.

In situations where legumes are not (yet) competitive but their environmental impact is considered desirable, constraints of low yield stability, weed and disease infestation, require innovative solutions from on-farm and on-station experimentation, use of crop management tools and advances in plant breeding. In such situations, policy support could be justified.

\section{AUTHOR CONTRIBUTIONS}

All authors contributed to manuscript writing and read and approved the final manuscript. MR, GB, FS, CW, PZ, and JB designed the study. MR, PZ, and JB performed the modeling. RW, AP, IT, GB, MR, and JB provided data for the case studies and were involved in the validation of the results and interpretation.

\section{FUNDING}

The work was financed by the German Federal Ministry of Food and Agriculture; the Brandenburg Ministry of Sciences, Research and Cultural Affairs; the Scottish Government RESAS Strategic Research Programme; the EU FP7 project "Legume Futures" (Grant 245216 CP-FP), and the FACCE-ERA-NET+ project Climate-CAFE (Grant PTJ-031A544). The publication of this article was funded by the Open Access fund of the Leibniz Association.

\section{ACKNOWLEDGMENTS}

We thank Nicole Schläfke, Renate Wille, Kairsty Topp, Vera Eory, Juliette Maire, Bodil Frankow-Lindberg, Birgitta Båth, Marioara Rusu, and Michele Monti for providing and managing data, and for advice. 


\section{REFERENCES}

Angus, J. F., Kirkegaard, J. A., Hunt, J. R., Ryan, M. H., Ohlander, L., and Peoples, M. B. (2015). Break crops and rotations for wheat. Crop Pasture Sci. 66, 523-552. doi: 10.1071/CP14252

Bachinger, J., and Zander, P. (2007). ROTOR, a tool for generating and evaluating crop rotations for organic farming systems. Eur. J. Agr. 26, 130-143. doi: 10.1016/j.eja.2006.09.002

Ball, B. C., Griffiths, B. S., Topp, C. F. E., Wheatley, R., Walker, R. L., Rees, R. M., et al. (2014). Seasonal nitrous oxide emissions from field soils under reduced tillage, compost application or organic farming. Agric. Ecosyst. Environ. 189, 171-180. doi: 10.1016/j.agee.2014.03.038

Berdanier, A. B., and Conant, R. T. (2012). Regionally differentiated estimates of cropland $\mathrm{N}_{2} \mathrm{O}$ emissions reduce uncertainty in global calculations. Glob. Chang. Biol. 18, 928-935. doi: 10.1111/j.1365-2486.2011. 02554.x

Bergez, J. E., Colbach, N., Crespo, O., Garcia, F., Jeuffroy, M. H., Justes, E., et al. (2010). Designing crop management systems by simulation. Eur. J. Agr. 32, 3-9. doi: 10.1016/j.eja.2009.06.001

Bibby, J., Douglas, K. H., and Thomasson, A. J. S. R. (1982). Land Capability Classification for Agriculture. Soil Survey of Scotland Monograph. Aberdeen: The Macaulay Institute for Soil Research.

Bockstaller, C., and Girardin, P. (2003). How to validate environmental indicators. Agric. Syst. 76, 639-653. doi: 10.1016/S0308-521X(02)00053-7

Bouxin, A. (2014). Food and Feed Statistical Yearbook 2013. Brussels: FEFAC, European Feed Manufacturers' Association.

Buckingham, S., Anthony, S., Bellamy, P. H., Cardenas, L. M., Higgins, S., McGeough, K., et al. (2014). Review and analysis of global agricultural $\mathrm{N}_{2} \mathrm{O}$ emissions relevant to the UK. Sci. Total Environ. 487, 164-172. doi: 10.1016/j.scitotenv.2014.02.122

Bues, A., Preissel, S., Reckling, M., Zander, P., Kuhlmann, T., Topp, K., et al. (2013). The Environmental Role of Protein Crops in the New Common Agricultural Policy. European Parliament, Directorate General for Internal Policies, Policy Department B: Structural and Cohesion Policies, Agricultural and Rural Development IP/B/AGRI/IC/2012-067. 112. Available online at: www. europarl.europa.eu/studies [29.10.2015].

Carlsson, G., and Huss-Danell, K. (2003). Nitrogen fixation in perennial forage legumes in the field. Plant Soil 253, 353-372. doi: 10.1023/A:1024847017371

Cernay, C., Ben-Ari, T., Pelzer, E., Meynard, J.-M., and Makowski, D. (2015). Estimating variability in grain legume yields across Europe and the Americas. Sci. Rep. 5:11171. doi: 10.1038/srep11171

Dewhurst, R. J., Delaby, L., Moloney, A., Boland, T., and Lewis, E. (2009). Nutritive value of forage legumes used for grazing and silage. Irish J. Agri. Food Res. 48, 167-187. Available online at: http://www.jstor.org/stable/20720367

Doré, T., Makowski, D., Malézieux, E., Munier-Jolain, N., Tchamitchian, M., and Tittonell, P. (2011). Facing up to the paradigm of ecological intensification in agronomy: Revisiting methods, concepts and knowledge. Eur. J. Agr. 34, 197-210. doi: 10.1016/j.eja.2011.02.006

Döring, T. F. (2015). "Grain legume cropping systems in temperate climates," in Grain Legumes. Handbook of Plant Breeding, ed A. M. De Ron (New York, NY: Springer Science+Business Media), 401-434.

Eriksen, J., Askegaard, M., Rasmussen, J., and Søegaard, K. (2015). Nitrate leaching and residual effect in dairy crop rotations with grass-clover leys as influenced by sward age, grazing, cutting and fertilizer regimes. Agric. Ecosyst. Environ. 212, 75-84. doi: 10.1016/j.agee.2015.07.001

Eurostat (2010). Available online at: http://ec.europa.eu/eurostat/ [15.3.2012].

Eurostat (2015). Available online at: http://ec.europa.eu/eurostat/web/agriculture/ [29.10.2015]

FAOstat (2015). Production-Crops. Grain Legume Production Areas EU Countries and Switzerland. Available online at: http://faostat.fao.org [29.10.2015]

Fischer, C., Roscher, C., Jensen, B., Eisenhauer, N., Baade, J., Attinger, S., et al. (2014). How do earthworms, soil texture and plant composition affect infiltration along an experimental plant diversity gradient in grassland? PLoS ONE 9:e98987. doi: 10.1371/journal.pone.0098987

Gäth, S., and Wohlrab, B. (1992). Strategien zur Reduzierung Standort- und Nutzungsbedingter Belastungen des Grundwassers mit Nitrat. Oldenburg: Deutsche Bodenkundliche Gesellschaft-AG Bodennutzung in Wasserschutzund -schongebieten.
Håkansson, S. (2003). Weeds and Weed Management on Arable Land: An Ecological Approach. Wallingford: CABI Publishing. doi: 10.1079/9780851996516.0000

Hinton, N. J., Cloy, J. M., Bell, M. J., Chadwick, D. R., Topp, C. F. E., and Rees, R. M. (2015). Managing fertiliser nitrogen to reduce nitrous oxide emissions and emission intensities from a cultivated Cambisol in Scotland. Geod. Reg. 4, 55-65. doi: 10.1016/j.geodrs.2014.12.002

IPCC (2006). "2006 IPCC guidelines for national greenhouse gas inventories," in Prepared by the National Greenhouse Gas Inventories Programme, eds H. S. Eggleston, L. Buendia, K. Miwa, T. Ngara, and K. Tanabe (Institute for Global Environmental Strategies (IGES)).

ISTAT (2013). National Institute of Statistics (Italy). Available online at: www.istat. it [29.10.2015]

Jensen, E. S., Peoples, M. B., Boddey, R. M., Gresshoff, P. M., Hauggaard-Nielsen, H., Alves, B. J. R., et al. (2011). Legumes for mitigation of climate change and the provision of feedstock for biofuels and biorefineries. A review. Agron. Sust. Dev. 32, 329-364. doi: 10.1007/s13593-011-0056-7

Kersebaum, K. C. (1995). Application of a simple management model to simulate water and nitrogen dynamics. Ecol. Modell. 81, 145-156. doi: 10.1016/03043800(94)00167-G

Kollas, C., Kersebaum, K. C., Nendel, C., Manevski, K., Müller, C., Palosuo, T., et al. (2015). Crop rotation modelling - A European model intercomparison. Eur. J. Agron. 70, 98-111. doi: 10.1016/j.eja.2015.06.007

Köpke, U., and Nemecek, T. (2010). Ecological services of faba bean. Field Crops Res. 115, 217-233. doi: 10.1016/j.fcr.2009.10.012

KTBL (2010). Betriebsplanung Landwirtschaft, 22. Auflage. Darmstadt: Kuratorium für Technik und Bauwesen in der Landwirtschaft.

Lamb, A., Green, R., Bateman, I., Broadmeadow, M., Bruce, T., Burney, J., et al. (2016). The potential for land sparing to offset greenhouse gas emissions from agriculture. Nat. Clim. Chang. 6, 488-492. doi: 10.1038/nclimate2910

LELF (2010). Datensammlung für die Betriebsplanung und die Betriebswirtschaftliche Bewertung landwirtschaftlicher Produktionsverfahren im Land Brandenburg, 7. Auflage. Landesamt für Ländliche Entwicklung, Landwirtschaft und Flurneuordnung, Frankfurt (Oder). 131.

Lemaire, G., Gastal, F., Franzluebbers, A., and Chabbi, A. (2015). Grasslandcropping rotations: an avenue for agricultural diversification to reconcile high production with environmental quality. Environ. Manage. 56, 1065-1077. doi: 10.1007/s00267-015-0561-6

Lokupitiya, E., and Paustian, K. (2006). Agricultural soil greenhouse gas emissions. J. Environ. Qual. 35, 1413-1427. doi: 10.2134/jeq2005.0157

López-Bellido, L., López-Bellido, R. J., Castillo, J. E., and López-Bellido, F. J. (2004). Chickpea response to tillage and soil residual nitrogen in a continuous rotation with wheat: I. Biomass and seed yield. Field Crops Res. 88, 191-200. doi: $10.1016 /$ j.fcr.2004.01.011

López-Bellido, R. J., López-Bellido, L., López-Bellido, F. J., and Castillo, J. E. (2003). Faba bean (Vicia faba 1.) response to tillage and soil residual nitrogen in a continuous rotation with wheat (Triticum aestivum 1.) under rainfed Mediterranean conditions. Agron. J. 95, 1253-1261. doi: 10.2134/agronj2003.1253

Lorenz, M., Furst, C., and Thiel, E. (2013). A methodological approach for deriving regional crop rotations as basis for the assessment of the impact of agricultural strategies using soil erosion as example. J. Environ. Manage. 127, S37-S47. doi: 10.1016/j.jenvman.2013.04.050

Luetke-Entrup, N., Schneider, M., and Braun, J. (2003). Bearbeitungsgänge sparen, aber mit Augenmaß. Neue Land. Sond. Konserv. Bodenbearb. 9-14.

Lüscher, A., Mueller-Harvey, I., Soussana, J. F., Rees, R. M., and Peyraud, J. L. (2014). Potential of legume-based grassland-livestock systems in Europe: a review. Grass Forage Sci. 69, 206-228. doi: 10.1111/gfs.12124

MADR (2013). Project no. 6.2.3. "Technical-Economic Foundation of Production Technologies, Costs Determination and Estimation of the Profitability Level of Vegetal and Animal Products".

Majak, W., Hall, J. W., and McCaughey, W. P. (1995). Pasture management strategies for reducing the risk of legume bloat in cattle. J. Anim. Sci. 73, 1493-1498

Meynard, J. M., Messéan, A., Charlier, A., Charrier, F., Farès, M., Le Bail, M., et al. (2013). RE: Crop Diversification: Obstacles and Levers. Study of Farms and Supply Chains. Synopsis of the Study Report, INRA. Available online at: www6.paris.inra.fr/depe/content/download/3736/35824/version/1/ file/Version+Anglaise+Diversification-8pages.pdf [29.10.2015]. 
Naudin, K., Husson, O., Scopel, E., Auzoux, S., Giner, S., and Giller, K. E. (2015). PRACT (Prototyping Rotation and Association with Cover crop and no Till) a tool for designing conservation agriculture systems. Eur. J. Agron. 69, 21-31. doi: 10.1016/j.eja.2015.05.003

Nemecek, T., von Richthofen, J.-S., Dubois, G., Casta, P., Charles, R., and Pahl, H. (2008). Environmental impacts of introducing grain legumes into European crop rotations. Eur. J. Agron. 28, 380-393. doi: 10.1016/j.eja.2007.11.004

Peoples, M. B., Brockwell, J., Herridge, D. F., Rochester, I. J., Alves, B. J. R., Urquiaga, S., et al. (2009). The contributions of nitrogen-fixing crop legumes to the productivity of agricultural systems. Symbiosis 48, 1-17. doi: 10.1007/BF03179980

Philibert, A., Loyce, C., and Makowski, D. (2012). Quantifying uncertainties in $\mathrm{N}_{2} \mathrm{O}$ emission due to $\mathrm{N}$ fertilizer application in cultivated areas. PLoS ONE 7:e50950. doi: 10.1371/journal.pone.0050950

Plaza-Bonilla, D., Nolot, J.-M., Raffaillac, D., and Justes, E. (2015). Cover crops mitigate nitrate leaching in cropping systems including grain legumes: field evidence and model simulations. Agric. Ecosyst. Environ. 212, 1-12. doi: 10.1016/j.agee.2015.06.014

Preissel, S., Reckling, M., Schläfke, N., and Zander, P. (2015). Magnitude and farmeconomic value of grain legume pre-crop benefits in Europe: a review. Field Crops Res. 175, 64-79. doi: 10.1016/j.fcr.2015.01.012

Reckling, M., Döring, T., Stein-Bachinger, K., Bloch, R., and Bachinger, J. (2015). Yield stability of grain legumes in an organically managed monitoring experiment. Aspects Appl. Biol. 128, 57-62. doi: 10.13140/RG.2.1.1122.0966

Reckling, M., Hecker, J.-M., Bergkvist, G., Watson, C., Zander, P., Stoddard, F., et al. (2016). A cropping system assessment framework-evaluating effects of introducing legumes into crop rotations. Eur. J. Agron. 76, 186-197. doi: 10.1016/j.eja.2015.11.005

Rees, R. M., Augustin, J., Alberti, G., Ball, B. C., Boeckx, P., Cantarel, A., et al. (2012). Nitrous oxide emissions from European agriculture; an analysis of variability and drivers of emissions from field experiments. Biogeosci. Dis. 9, 9259-9288. doi: 10.5194/bgd-9-9259-2012

SAC (2013). Farm Management Handbook. Available online at: http://www.sruc. ac.uk/info/120376/farm_management_handbook [10.10.2014]

Schwenke, G. D., Herridge, D. F., Scheer, C., Rowlings, D. W., Haigh, B. M., and McMullen, K. G. (2015). Soil $\mathrm{N}_{2} \mathrm{O}$ emissions under $\mathrm{N}_{2}$-fixing legumes and $\mathrm{N}$ fertilised canola: a reappraisal of emissions factor calculations. Agric. Ecosyst. Environ. 202, 232-242. doi: 10.1016/j.agee.2015.01.017

Scottish-Agricultural-Census (2010). Scottish-Agricultural-Census. Available online at: http://www.gov.scot/Topics/Statistics/Browse/Agriculture-Fisheries/ PubFinalResultsJuneCensus [10.10.2014]

Sieling, K., and Kage, H. (2012). Efficient N management using winter oilseed rape. A review. Agron. Sust. Dev. 30, 271-279. doi: 10.1051/agro/2009036

SJV (2011). Yearbook of Agricultural Statistics 2011. Available online at: www. jordbruksverket.se/swedishboardofagriculture/statistics/agriculturalstatistics/ 2011.4.13af540213600c8df7a8000399.html [29.10.2015]

Stehfest, E., and Bouwman, L. (2006). $\mathrm{N}_{2} \mathrm{O}$ and NO emission from agricultural fields and soils under natural vegetation: summarizing available measurement data and modeling of global annual emissions. Nut. Cycl. Agroecosyst. 74, 207-228. doi: 10.1007/s10705-006-9000-7
Stein-Bachinger, K., and Fuchs, S. (2012). Protection strategies for farmland birds in legume-grass leys as trade-offs between nature conservation and farmers' needs. Organ. Agric. 2, 145-162. doi: 10.1007/s13165-012-0029-4

Stein-Bachinger, K., Reckling, M., Bachinger, J., Hufnagel, J., Koker, W., and Granstedt, A. (2015). Ecological recycling agriculture to enhance agroecosystem services in the baltic sea region: guidelines for implementation. Land 4, 737-753. doi: 10.3390/land4030737

Stoddard, F. L. (2013). Agronomic case studies in Legume Futures. Leg. Fut. Rep. 1.2., 83. Available online at: www.legumefutures.de/images/Legume_Futures_ Report_1.2.pdf

Suter, M., Finn, J. A., Connolly, J., Loges, R., and Lüscher, A. (2015). Gain in nitrogen yield from grass-legume mixtures is robust over a wide range of legume proportions and environmental conditions. Proc. Environm. Sci. 29, 187-188. doi: 10.1016/j.proenv.2015.07.253

Tidåker, P., Sundberg, C., Öborn, I., Kätterer, T., and Bergkvist, G. (2014). Rotational grass/clover for biogas integrated with grain production-A life cycle perspective. Agric. Syst. 129, 133-141. doi: 10.1016/j.agsy.2014.05.015

Tilman, D., Cassman, K. G., Matson, P. A., Naylor, R., and Polasky, S. (2002). Agricultural sustainability and intensive production practices. Nature 418, 671-677. doi: 10.1038/nature01014

Vereijken, P. (1997). A methodical way of prototyping integrated and ecological arable farming systems (I/EAFS) in interaction with pilot farms. Eur. J. Agron. 7, 235-250. doi: 10.1016/S1161-0301(97)00039-7

Voisin, A.-S., Guéguen, J., Huyghe, C., Jeuffroy, M.-H., Magrini, M.-B., Meynard, J.-M., et al. (2013). Legumes for feed, food, biomaterials and bioenergy in Europe: a review. Agron. Sust. Dev. 34, 361-380. doi: 10.1007/s13593-01 3-0189-y

Von Richthofen, J.-S., Pahl, H., Bouttet, D., Casta, P., Cartrysse, C., Charles, R., et al. (2006). What do European farmers think about grain legumes. Grain Legumes 45, 14-15. Available online at: www.ias.csic.es/grainlegumesmagazine/ Grain_Legumes_issue_45.pdf

Watson, C. A., Öborn, I., Eriksen, J., and Edwards, A. C. (2005). Perspectives on nutrient management in mixed farming systems. Soil Use Managem. 21, 132-140. doi: 10.1079/SUM2005304

Zander, P., Amjath-Babu, T. S., Preissel, S., Reckling, M., Bues, A., Schläfke, N., et al. (2016). Grain legume decline and potential recovery in European agriculture: a review. Agron. Sust. Dev. 36, 1-20. doi: 10.1007/s13593-016 $-0365-y$

Conflict of Interest Statement: The authors declare that the research was conducted in the absence of any commercial or financial relationships that could be construed as a potential conflict of interest.

Copyright (c) 2016 Reckling, Bergkvist, Watson, Stoddard, Zander, Walker, Pristeri, Toncea and Bachinger. This is an open-access article distributed under the terms of the Creative Commons Attribution License (CC BY). The use, distribution or reproduction in other forums is permitted, provided the original author(s) or licensor are credited and that the original publication in this journal is cited, in accordance with accepted academic practice. No use, distribution or reproduction is permitted which does not comply with these terms. 


\title{
Proteomics and Metabolomics: Two Emerging Areas for Legume Improvement
}

\author{
Abirami Ramalingam ${ }^{1}$, Himabindu Kudapa ${ }^{1}$, Lekha T. Pazhamala ${ }^{1}$, Wolfram Weckwerth ${ }^{2}$ \\ and Rajeev K. Varshney ${ }^{1,3 *}$ \\ ${ }^{1}$ International Crops Research Institute for the Semi-Arid Tropics (ICRISAT), Hyderabad, India, ${ }^{2}$ Department of Ecogenomics \\ and Systems Biology, University of Vienna, Vienna, Austria, ${ }^{3}$ School of Plant Biology and Institute of Agriculture, The \\ University of Western Australia, Crawley, WA, Australia
}

The crop legumes such as chickpea, common bean, cowpea, peanut, pigeonpea, soybean, etc. are important sources of nutrition and contribute to a significant amount of biological nitrogen fixation (>20 million tons of fixed nitrogen) in agriculture. However, the production of legumes is constrained due to abiotic and biotic stresses. It is therefore imperative to understand the molecular mechanisms of plant response to different stresses and identify key candidate genes regulating tolerance which can be deployed in breeding programs. The information obtained from transcriptomics

\section{OPEN ACCESS}

Edited by:

Francesca Sparvoli, Institute of Agricultural Biology and

Biotechnology, Italy

Reviewed by:

Hao Peng,

Washington State University, USA Luca Espen,

Università degli Studi di Milano, Italy

*Correspondence:

Rajeev K. Varshney r.k.varshney@cgiar.org

Specialty section:

This article was submitted to Crop Science and Horticulture, a section of the journal Frontiers in Plant Science

Received: 14 August 2015 Accepted: 25 November 2015 Published: 24 December 2015

Citation:

Ramalingam A, Kudapa $\mathrm{H}$, Pazhamala $L T$, Weckwerth $W$ and Varshney RK (2015) Proteomics and Metabolomics: Two Emerging Areas for Legume Improvement.

Front. Plant Sci. 6:1116. doi: 10.3389/fpls.2015.01116 has facilitated the identification of candidate genes for the given trait of interest and utilizing them in crop breeding programs to improve stress tolerance. However, the mechanisms of stress tolerance are complex due to the influence of multi-genes and post-transcriptional regulations. Furthermore, stress conditions greatly affect gene expression which in turn causes modifications in the composition of plant proteomes and metabolomes. Therefore, functional genomics involving various proteomics and metabolomics approaches have been obligatory for understanding plant stress tolerance. These approaches have also been found useful to unravel different pathways related to plant and seed development as well as symbiosis. Proteome and metabolome profiling using high-throughput based systems have been extensively applied in the model legume species, Medicago truncatula and Lotus japonicus, as well as in the model crop legume, soybean, to examine stress signaling pathways, cellular and developmental processes and nodule symbiosis. Moreover, the availability of protein reference maps as well as proteomics and metabolomics databases greatly support research and understanding of various biological processes in legumes. Protein-protein interaction techniques, particularly the yeast two-hybrid system have been advantageous for studying symbiosis and stress signaling in legumes. In this review, several studies on proteomics and metabolomics in model and crop legumes have been discussed. Additionally, applications of advanced proteomics and metabolomics approaches have also been included in this review for future applications in legume research. The integration of these "omics" approaches will greatly support the identification of accurate biomarkers in legume smart breeding programs.

Keywords: abiotic and biotic stresses, developmental process, functional genomics, signaling pathways, stress tolerance 


\section{INTRODUCTION}

The legume crops such as chickpea (Cicer arietinum), common bean (Phaseolus vulgaris), cowpea (Vigna unguiculata), faba bean (Vicia faba), lentil (Lens culinaris), lupin (Lupinus luteus), mungbean (Vigna radiata), pea (Pisum sativum), peanut (Arachis hypogaea), pigeonpea (Cajanus cajan), and soybean (Glycine max) have greatly contributed in providing nutrition, food security, and environmental sustainability (Graham and Vance, 2003; Varshney et al., 2013a). However, a majority of them are grown in marginal environments, subjected to abiotic (e.g., drought, heat, cold, salinity, waterlogging, heavy metal toxicities etc.) and biotic stresses (e.g., anthracnose, bean rust, bacterial blight, Fusarium wilt etc.), thus limiting their productivity (Dita et al., 2006; Varshney and Tuberosa, 2013; Rodziewicz et al., 2014). Moreover, these environmental conditions severely affect rhizobia-legume symbiosis, which contributes to $\sim 45 \%$ of nitrogen required for agriculture (Karmakar et al., 2015). Therefore, for the development of superior varieties with enhanced stress tolerance, it is extremely important to understand stress response mechanisms in legumes which include changes in gene expression, and the consequent variations in the transcriptome, proteome and metabolome.

The availability of high throughput and cost effective next generation sequencing (NGS) platforms as well as high throughput genotyping technologies, have facilitated the generation of massive genomic data for model as well as crop legumes. These platforms have been vital in producing the genome sequence assemblies for the following legumes: adzuki bean (Kang et al., 2015; Yang et al., 2015), chickpea (Varshney et al., 2013b), common bean (Schmutz et al., 2014), Lotus (Sato et al., 2008), Medicago (Young et al., 2011), mung bean (Kang et al., 2014), pigeonpea (Varshney et al., 2012), and soybean (Schmutz et al., 2010). Furthermore, whole genomeresequencing data are also becoming readily available for mining superior alleles (Varshney et al., 2009; Lam et al., 2010). Similarly, transcriptomics/gene expression studies, using a range of platforms, have been valuable for identifying candidate genes associated with tolerance/resistance to different stresses as well as several agronomic traits (Kudapa et al., 2013; Campbell et al., 2014; Brasileiro et al., 2015).

Although QTLs/candidate genes/ alleles have been used in breeding programmes, it has been observed that structural/ expression variation identified at the genetic level are not always translated into the "predicted" phenotype. Additionally, mechanisms involved in stress tolerance can be complicated, e.g., the involvement of metabolites, multigenes and posttranslational modifications (PTM) which cannot be investigated by genomics or transcriptomics approaches (Mazzucotelli et al., 2008; Weckwerth, 2011a). In this context, proteomics and metabolomics are promising approaches to enhance our understanding of functional molecules on specific aspects of multigene families and PTMs, instead of analyzing the genetic code (DNA) or transcript (RNA) abundance which may not correlate with their corresponding proteins (Weckwerth, 2011b; Hossain et al., 2012).
Proteomics, defined as the high-throughput study of proteins, has taken the lead in plant biological research and stress responses, especially due to the increasing number of plant genomes being sequenced and released (Pandey and Mann, 2000; Weckwerth, 2011b; Jorrín-Novo et al., 2015). In addition, advancements in mass spectrometry (MS), quantitative methods and bioinformatics approaches have allowed comprehensive identification, quantitation, validation and characterization for a wide range of proteins from specific organ/tissue/cells (Glinski and Weckwerth, 2006). The information obtained through these advanced approaches are valuable for deciphering protein structure and complex mechanisms such as enzymatic and regulatory functions of proteins coded by specific genes (Bachi and Bonaldi, 2008; Wienkoop et al., 2010a; Nanjo et al., 2011; Abdallah et al., 2012). Furthermore, proteomics approaches provide valuable information such as protein levels associated with stress tolerance, the modifications in proteomes under stress that link transcriptomics and metabolomics analyses as well as the role of expressed genes in the functionally translated regions of the genome linked to traits of interest (Kosová et al., 2011). Many proteomics based publications, especially related to plant development and other biological phenomenon such as symbiosis in legumes are available in the model legumes and Arabidopsis thaliana, as well as in some crop plants such as rice (Oryza sativa), wheat (Triticum aestivum), maize (Zea mays), soybean, tomato (Solanum lycopersicum) and tobacco (Nicotiana tabacum) (Jorrín-Novo et al., 2015).

In addition to proteomics, metabolomics is another important approach of functional genomics in which the identification and quantitation of metabolomes (collection of metabolites or small molecules) within a cell, tissue or organism produced through cellular metabolism, connects the cellular biochemical activity with the phenotype (Weckwerth, 2003). Major plant metabolomics approaches include metabolic fingerprinting, metabolite profiling and targeted analysis (Fiehn et al., 2000; Halket et al., 2005; Shulaev, 2006). Depending on the objective of study, different metabolomics approaches or a combination of approaches are applied. Furthermore, the use of MS, bioinformatics tools and softwares, allows metabolites to be measured quickly, simultaneously, in large numbers from a small amount of sample, which are spatially localized within the biological material (Bhalla et al., 2005; Patti et al., 2012). Since metabolites are closer to the phenotype, they reflect gene expressions and different regulatory processes more accurately and metabolomics is a powerful tool to study plant molecular phenotypes in response to stresses (Scherling et al., 2010; Arbona et al., 2013; Doerfler et al., 2013). For example, under abiotic stress conditions, the plant metabolism is affected due to factors such as inhibition of metabolic enzymes, shortage of substrate, extreme demand for specific compounds and a combination of these factors. Thus, the plant undergoes metabolic reprogramming to adapt to the predominant stress conditions through the production of anti-stress components such as compatible solutes, antioxidants and stress-responsive proteins (Wienkoop et al., 2008a; Obata and Fernie, 2012; Doerfler et al., 2014). In crop breeding programs, there has been great interest in using metabolites as selection 
biomarkers, since metabolites integrate the complex interaction between genotype and the environment (Fernie and Schauer, 2009).

With proteomics and metabolomics emerging as cutting edge functional biology disciplines for understanding plant adaptation mechanisms to stresses at cellular and developmental stages in different plant systems, there has been great interest in applying the knowledge to understand responses in different crop plants. These approaches, integrated with information obtained from genomics data, allow accurate identification of candidate genes and pathways involved in important agronomic traits that can be applied in crop breeding programs (Langridge and Fleury, 2011).

In this article different proteomics and metabolomics tools available and the use of these approaches in legumes especially to understand stress response mechanism, cellular and developmental processes and symbiosis, have been discussed. The assemblage of information and resources available for these aspects of legume proteomics and metabolomics will facilitate our understanding and utilization of these resources for legume crop improvement programs.

\section{PROTEOMICS APPROACHES AND CHALLENGES FOR LEGUME RESEARCH}

Proteomics approaches have been implemented according to the objective of the study and are based on certain criteria such as (1) descriptive proteomics for classification of proteins, (2) comparative proteomics for comparison of protein profiles (genotypes, cells, organs, developmental stages, stress response, etc., (3) PTMs that determine how proteins are modified, (4) Protein-protein interactions (PPI) for identifying protein complexes and interacting networks, (5) proteinomics for studying protein structure and functional groups, and (6) translational proteomics that involves transfer of methodology and knowledge for crop improvement (Jorrín-Novo et al., 2015). Data obtained through above mentioned approaches together with bioinformatics, provide significant information on biological processes and stress tolerance mechanisms that can be applied in crop breeding programs (Salekdeh and Komatsu, 2007; Nanjo et al., 2011; Hu et al., 2015).

Furthermore, the comprehensive analysis using differential proteomics in complex systems has demanded development of new technologies to study the cell proteome. Each approach has advantages and disadvantages from both the conceptual and the methodological perspectives. Several approaches are being used to study proteomics in plants and these include electrophoresis and/or chromatography combined with chemical or metabolic labeling and MS. In the following section, we have discussed the gel-based system which is significant for differential expression profiling for studying legume stress responses, and the advantages of the technologically more advanced gel-free, quantitative systems. The application of these approaches for protein profiling in model and crop legumes for understanding stress responses have been addressed with successful examples where proteomics approaches have been utilized for the advancement of crop legumes research. In the interest of channeling the usage of more advanced approaches for legume crop improvement research, the application of more advanced approaches applied in other plant systems, have also been addressed.

\section{Gel-Based System for Protein Differential Expression Profiling}

Gel-based systems have been widely applied for protein differential expression analysis which involves the use of twodimensional gel electrophoresis (2D-GE) or two-dimensional difference gel electrophoresis (2D-DIGE) for protein separation coupled with MS applications for the identification and quantitation of proteins (Kosová et al., 2011; Subramanian and Smith, 2013). The 2D-GE method has an advantage of resolving and visualizing thousands of spots corresponding to different molecular forms of same proteins. However, it has low sensitivity and is not suited for protein quantitation (Marouga et al., 2005; Abdallah et al., 2012). It was found that the use of fluorescent dyes in place of Coomassie can considerably increase the sensitivity and compatibility for MS analyses (Vanderschuren et al., 2013). These factors have led to the usage of a more refined gel electrophoresis method, called the 2D-DIGE which is based on pre-labeling of protein mixtures with fluorescence. Thus, 2DDIGE is a much more sensitive method, which allows protein detection at sub-picomolar levels for highly precise quantitative proteomic studies (Marouga et al., 2005). Few studies have been reported in legumes, which employed 2D-DIGE for monitoring early response to symbiosis and pathogenesis, such as in the root proteome in Medicago (Schenkluhn et al., 2010) and parasitic infections in pea (Castillejo et al., 2012). The advantages and disadvantages of 2D-GE and 2D-DIGE have been reviewed before (see Griffin and Aebersold, 2001; Marouga et al., 2005; Abdallah et al., 2012).

For identification of proteins separated by 2D-GE, MS based techniques were found to have high accuracy, resolution and sensitivity but the sample preparation seemed to be laborious (van Wijk, 2001; Subramanian and Smith, 2013). Earlier, the usage of techniques such as matrix-assisted laser desorption ionization time-of-flight (MALDI-TOF) MS and electrospray ionization (ESI) MS with tandem MS (MS/MS) overcame the slower and less sensitive methods (Griffin and Aebersold, 2001; Griffin et al., 2001; van Wijk, 2001). In particular, the application of tandem MS methods provided sequence information for more peptides accurately for the identification of proteins and PTMs (Pandey and Mann, 2000; Bachi and Bonaldi, 2008). The use of MALDI ionization with two TOF analysers, (MALDI TOF/TOF) and liquid chromatography tandem MS (LC-MS/MS) have been fairly new technologies for protein identification that enhanced the pipeline for de novo assembly. The availability of these tools, together with the genome sequences of more plants and accessibility of sequence databases, intensified the identification of protein spots in different plants (see Jorrín-Novo et al., 2015). The most commonly used pipeline for the identification of proteins involves comparing the MS/MS spectra to the reference databases (Romero-Rodríguez et al., 2014). Although SEQUEST, MASCOT and X!Tandem are the widely used database search 
programs for protein identification, MassMatrix is also suited for tandem MS data (Xu and Freitas, 2009; Senkler and Braun, 2012).

\section{Gel-Free Proteomics Tools and Approaches for Quantitation}

Shot-gun proteomics is a gel-free "bottom-up" strategy, in which complex peptide fractions produced after proteolytic digestion of proteins are analyzed by LC-MS/MS. The protein identification rates are enhanced using different peptide or protein fractionation strategies (Abdallah et al., 2012). This approach allows high throughput and comprehensive investigation, by providing an overview of the organelle or celltype proteomes (Glinski and Weckwerth, 2006). For large-scale proteomics, a multidimensional chromatography technique called multidimensional protein identification technology (MudPIT) has been used to separate complex protein samples prior to MS analyses to increase the proteome coverage and dynamic range (Washburn et al., 2001; Bachi and Bonaldi, 2008).

MS based protein quantitation strategies may include untargeted quantitation, (database dependent or database independent protein identification) or targeted absolute quantitation, which are important for biomarker discovery and protein stoichiometries in protein complexes (Wienkoop et al., 2008b; Weckwerth, 2011b; Schmidt and Urlaub, 2012). Relative quantitation is an unbiased, large-scale screening strategy, which is useful to detect proteins involved in unknown regulatory processes, protein modifications and mechanisms in systems biology. MS-based methods for protein quantitation can be label (stable-isotope in vivo or in vitro label) or non-label based (see Bachi and Bonaldi, 2008; Abdallah et al., 2012). Label based proteomics for relative quantitation consists of chemical labeling and metabolic labeling. The iTRAQ (isobaric tags for relative and absolute quantitation), is a chemical labeling approach and has been applied to study salinity stress tolerance in the phloem sap proteome of Cucumis sativus (Fan et al., 2015), leaf proteome of Vitis vinifera to understand heat stress response (Liu et al., 2014) and the root proteome of rice to investigate aluminum stress response (Wang et al., 2014). The platforms available for relative quantitation include Mass Accuracy Precursor Alignment (MAPA), ProtMax, MASS WESTERN, and PROMEX (Hoehenwarter et al., 2008; Weckwerth et al., 2014). Recently, a rapid shot-gun LC-MS approach for relative quantitation called full-scan (FS) selective peptide extraction (Selpex) was successfully used for generating a reference library of targeted peptides from leaf tissue of Medicago (Castillejo et al., 2014).

Selective reaction monitoring (SRM) or multiple reaction monitoring (MRM), which involves the use of triple-quadrupole (QQQ) MS is a quantitative, targeted and label-free approach, that complements untargeted shotgun methods due to its reliable quantitation of low abundance proteins in complex mixtures (Lange et al., 2008; Wienkoop et al., 2010b; Schmidt and Urlaub, 2012). This approach has been useful to measure a predetermined set of proteins that could constitute cellular networks or candidate biomarkers across samples consistent and reproducibly (Wienkoop et al., 2010b; Picotti and Aebersold, 2012). In Medicago, the MRM approach was applied for the absolute quantitation of sucrose synthase isoforms and $\mathrm{N}$ metabolism enzymes in symbiotic root nodules as well as in the analysis of nodule metabolism under drought stress (Wienkoop et al., 2008b; Larrainzar et al., 2009).

\section{Protein Profiling in Legumes}

Proteomic studies have been carried out predominantly in Medicago to investigate stress tolerance, seed physiology, plant growth, development and symbiosis which has immense significance in agricultural research (Colditz and Braun, 2010; Jorrín-Novo et al., 2015). A majority of studies have been carried out to investigate abiotic stress responses, e.g., drought tolerance in shoots, leaves and roots using gel-based as well as nongel based approaches (Table 1). For instance, 26 differentially expressed proteins were identified using 2D-GE and ESI-LCMS/MS approaches from leaf samples subjected to drought stress (Aranjuelo et al., 2011). The study revealed the regulation process involved in the synthesis of amino acids pertaining to osmoregulation. Using non-gel based LC-MS/MS approaches, root nodule proteome and symbiotic nitrogen fixation was studied under drought stress in Medicago (Larrainzar et al., 2007, 2009). The former study (Larrainzar et al., 2007) not only identified a large number of nodule proteins, but also grouped the drought responsive proteins from plant and bacteroid which was helpful in understanding the mechanism in which each symbiotic member responded to drought stress. The later study (Larrainzar et al., 2009) was important for the relative quantification of root nodule proteins and absolute quantification of a key enzyme in sucrose metabolism to understand the regulation of nitrogen fixation under drought stress. In order to understand the roles of diverse LEA proteins in developing seeds of Medicago, differential profiling was effectively used to identify proteins that may have a role in desiccation tolerance and seed longevity (Chatelain et al., 2012). The study of arbuscular mycorrhizal symbiosis in Medicago roots identified differential accumulation of 96 membrane proteins which could have a role during symbiosis when compared between the mycorrhizal and nonmycorrhizal roots. The study has also identified proteins that stimulated changes in membrane trafficking (Abdallah et al., 2014).

In addition, there is a significant contribution of proteomics for studying abiotic stress in soybean at subcellular, organ and whole plant levels. The methodologies applied and the major discoveries in these studies have been reviewed in a number of articles (see Hossain et al., 2013; Hossain and Komatsu, 2014a,b). A few important examples of proteomics approaches used for analyzing soybean abiotic stress are described here. For instance, osmotic stress in the plasma membrane of soybean seedling was studied using both gel-based and nanoLC MS/MS approaches (Nouri and Komatsu, 2010). While the former technique identified four up-regulated and eight down-regulated protein spots, the latter approach, identified 11 up-regulated and 75 down-regulated proteins, of which 7 were identified in both the studies. In dissecting the mechanism of Cadmium (Cd) uptake and distribution in soybean, 2D-GE and LC-MS/MS approaches were employed on contrasting soybean cultivars to identify 13 and 11 differentially expressed proteins, respectively. 
TABLE 1 | Key studies on protein differential expression analysis in response to various stress in some model and crop legumes.

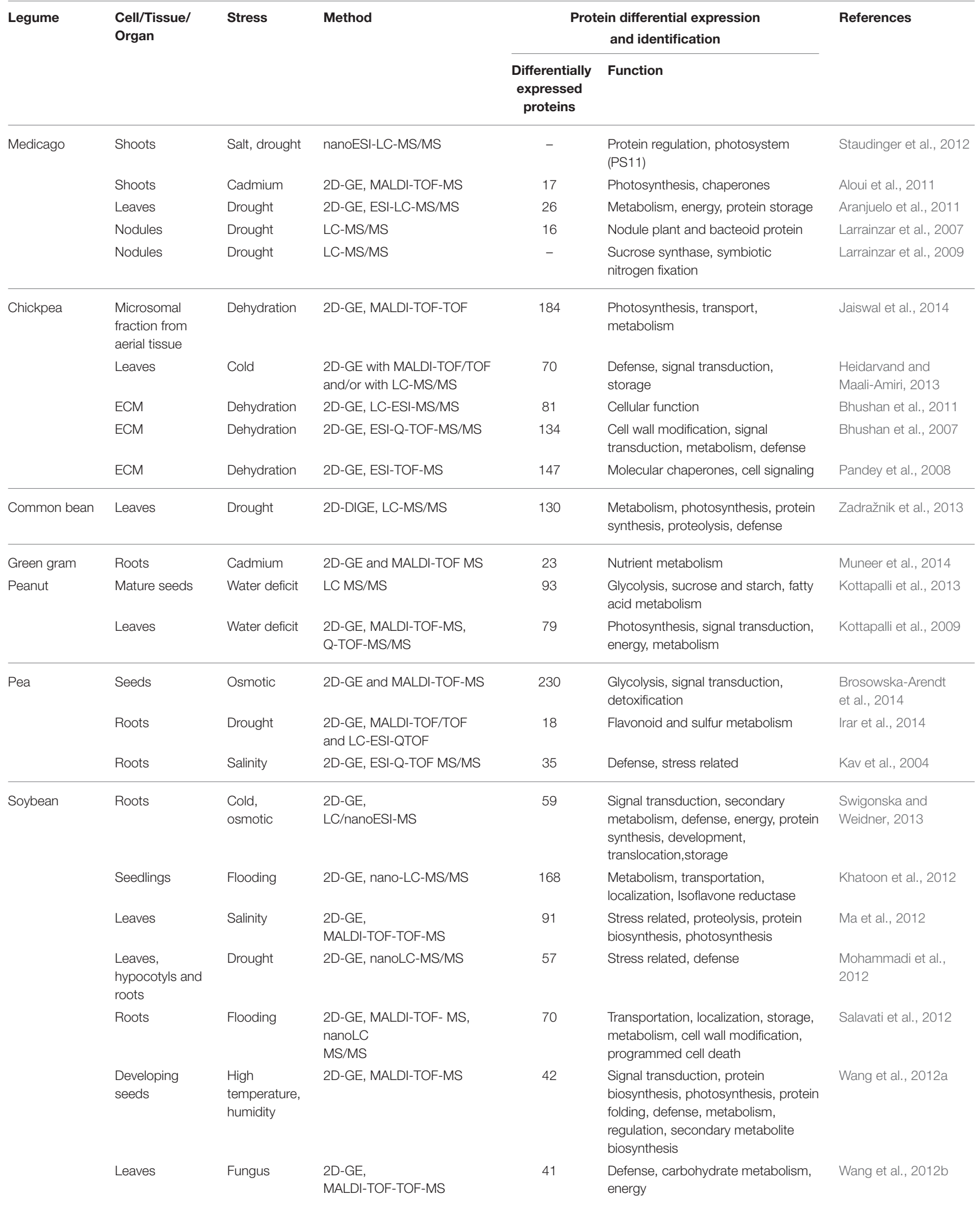


TABLE 1 | Continued

\begin{tabular}{|c|c|c|c|c|c|c|}
\hline \multirow[t]{2}{*}{ Legume } & \multirow[t]{2}{*}{$\begin{array}{l}\text { Cell/Tissue/ } \\
\text { Organ }\end{array}$} & \multirow[t]{2}{*}{ Stress } & \multirow[t]{2}{*}{ Method } & \multicolumn{2}{|c|}{$\begin{array}{c}\text { Protein differential expression } \\
\text { and identification }\end{array}$} & \multirow[t]{2}{*}{ References } \\
\hline & & & & $\begin{array}{l}\text { Differentially } \\
\text { expressed } \\
\text { proteins }\end{array}$ & Function & \\
\hline & $\begin{array}{l}\text { Plasma } \\
\text { membrane }\end{array}$ & Osmotic & 2D-GE, nano-LC-MS/MS & 96 & Transport & $\begin{array}{l}\text { Nouri and Komatsu, } \\
2010\end{array}$ \\
\hline & $\begin{array}{l}\text { Endoplasmic } \\
\text { reticulum }\end{array}$ & Flooding & $\begin{array}{l}\text { 2D-GE and BN-PAGE, } \\
\text { nano-LC-MS/MS }\end{array}$ & $\sim 50$ & Heat shock proteins, chaperonins & Komatsu et al., 2011 \\
\hline & $\begin{array}{l}\text { Microsomal } \\
\text { proteins }\end{array}$ & Cadmium & 2D-GE, nanoLC-MS/MS & 13 and 11 & Stress, protein biosynthesis & Ahsan et al., 2010a \\
\hline & $\begin{array}{l}\text { Leaves, stems } \\
\text { and roots }\end{array}$ & $\begin{array}{l}\text { High } \\
\text { temperature }\end{array}$ & $\begin{array}{l}\text { MALDI-TOF-MS, } \\
\text { nanoLC-MS/MS and protein } \\
\text { sequencing }\end{array}$ & $54,35,61$ & $\begin{array}{l}\text { Defense photosynthesis, secondary } \\
\text { metabolism, protein biosynthesis }\end{array}$ & Ahsan et al., 2010b \\
\hline & Roots & Waterlogging & $\begin{array}{l}\text { 2D-GE, MALDI-TOF- MS, } \\
\text { ESI-MS/MS }\end{array}$ & 24 & $\begin{array}{l}\text { Signal transduction, programmed } \\
\text { cell death, homeostasis and } \\
\text { metabolism }\end{array}$ & Alam et al., 2010 \\
\hline & $\begin{array}{l}\text { Hypocotyls, } \\
\text { roots }\end{array}$ & Salt & $\begin{array}{l}\text { 2D-GE } \\
\text { ESI-Q/TOF-MS/MS }\end{array}$ & $>20$ & LEA protein, protease inhibitor & Aghaei et al., 2009 \\
\hline & Seedlings & Aluminum & 2D-GE, MALDI-TOF-MS & 39 & $\begin{array}{l}\text { Defense, signal transduction, protein } \\
\text { folding, transport }\end{array}$ & Zhen et al., 2007 \\
\hline
\end{tabular}

In this study, highly up-regulated proteins associated with lignin biosynthesis indicated that xylem lignification may be preventing the translocation of Cd (Ahsan et al., 2010a). For deciphering the mechanism of heat tolerance in soybean seedlings, $2 \mathrm{D}$ GE, MALDI-TOF-MS, LC-MS/MS and protein sequencing were applied in which 54, 35 and 61 proteins were differentially expressed respectively in leaves, stems and roots, respectively (Ahsan et al., 2010b). The study showed that heat shock proteins (HSPs) and antioxidant defense related proteins were induced and identified different proteins involved in tissue specific and common defense mechanisms.

In other legume crops, there are reports of significant discoveries being made for understanding abiotic stress responses through protein profiling. In the case of chickpea, drought is one of the most important abiotic stresses that severely affect its productivity, for which efforts have been made to dissect the genetic basis of tolerance (Thudi et al., 2014; Varshney et al., 2014). Toward understanding proteomic response to dehydration stress in chickpea, a few protein profiling studies have been made available (Table 1). For instance, the investigation of a dehydration-responsive microsomal proteome with 2D-GE and MALDI-TOF/TOF identified 184 proteins that showed significant differential expression (Jaiswal et al., 2014). This study was significant in identifying a novel component involved in dehydration signaling called CaSUN1. In a different study, using 2D-GE with MALDI-TOF/TOF and LC-MS/MS, proteomic changes were identified at early stage of cold stress in chickpea leaves (Heidarvand and Maali-Amiri, 2013). The analysis indicated that energy resources and primary metabolites respond by recreating a new homeostasis in preparation for longterm cold stress adaptation. In an earlier study, 81 dehydration responsive proteins were identified from profiling analysis of the chickpea organellar proteome in two contrasting genotypes, with
2D-GE and LC-ESI-MS/MS (Bhushan et al., 2011). The study proposed that cell wall restructuring and the control of reactive oxygen species were mainly responsible for better adaptability to the stress.

A few studies have also been conducted in pea through protein profiling for understanding abiotic stress response and are being described here. For example, 2D-GE, MALDITOF/TOF, and LC-ESI-QTOF was employed to dissect the signaling pathway leading to inhibition of biological nitrogen fixation under drought stress (Irar et al., 2014). The study successfully identified 18 nodule proteins regulated by both pea and rhizobium genomes under drought stress and unraveling the SNF regulation machinery in nodules. In a different study, changes in protein accumulation in germinating seeds under different osmotic conditions were monitored with 2D-GE and MALDI-TOF-MS (Brosowska-Arendt et al., 2014). This work showed that under optimal conditions, proteins associated to glycolysis, fatty acids synthesis and detoxification under osmotic stress considerably decreased, while proteins involved in signal transduction and protection were accumulated. Similarly, investigation of salinity stress response in pea with 2D-GE and ESI-Q-TOF MS/MS approach revealed significant differential expression of 35 proteins, of which 10 pathogenesis-related (PR) proteins was reported for the first time to be involved in salinity stress response, possibly involving a new signal transduction pathway (Kav et al., 2004).

\section{Protein Reference Maps in Legumes}

Protein reference maps have been developed for model and crop legumes, which identify as many proteins as possible in a particular tissue or cell cultures at a given point of time. This provides useful insights into important plant processes, such as stress tolerance, nutrient uptake and symbiotic association 
with rhizobia (Brechenmacher et al., 2009). These resources will allow further studies for efficient proteomics applications for the crop legumes, especially through the classification and characterization of proteins related to development and stress tolerance important for unambiguous candidate gene identifications. Reference maps have been established in Medicago for cell suspension cultures, roots, leaves, stems, flowers and pods (Watson et al., 2003). A protein reference map generated for cell suspension culture of Medicago consisted of 1367 proteins with 907 unique accessions, which could identify a complete tricarboxylic acid cycle, a nearly complete glycolytic pathway, a partial ubiquitin pathway, enzymes involved in secondary metabolism through functional annotations (Lei et al., 2005). Other specialized proteome maps generated include Medicago mitochondria (Dubinin et al., 2011); cell wall (Gokulakannan and Niehaus, 2010); embryonic cell cultures generated from single protoplasts (Imin et al., 2004) and roots (Mathesius et al., 2011).

Lotus proteome reference maps for nodules and roots consist of 780 and 790 protein spots identified with 2D-GE, with $45 \%$ of the corresponding unique gene accessions common in both the tissues (Dam et al., 2014). The study showed that PTMs were more prominent in nodules rather than in roots. In addition to this, higher levels of proteins such as pathogen-related 10, heat shock, and redox processes related were found in the nodules prior to nitrogen fixation and nodulin related proteins were prevalent in mature nitrogen fixing nodules. In Lotus proteome reference maps were also generated for pod and seed development (Nautrup-Pedersen et al., 2010). Similar reference maps were also developed in crop legumes such as soybean and peanut. In soybean, a comprehensive proteome reference map was generated with 5702 proteins identified for a single root hair cell (Brechenmacher et al., 2012). A previous reference map in root hair cells identified 1492 proteins (Brechenmacher et al., 2009). Other proteome maps generated in soybean includes root apex and differentiated root zone (Mathesius et al., 2011); seed filling (Hajduch et al., 2005), leaf (Xu et al., 2006), and response to pathogen invasion (Mithöfer et al., 2002). Similarly, leaf proteome reference map was developed in peanut (Katam et al., 2010). In chickpea, reference maps were generated for understanding the complexity of plant nuclear proteins (Pandey et al., 2006) and membrane proteins (Jaiswal et al., 2012). In the case of pea, reference maps have been developed for vegetative tissues (Schiltz et al., 2004) and mature seeds (Bourgeois et al., 2009).

Proteome reference maps generated through gel-based approaches consist of gel images in which selected "spots" were linked with protein identity information with arrows and numbers (Senkler and Braun, 2012). However, webbased resources with interactive features of the reference maps have also been made available. Examples of these resources include the "Seed Proteome," the "Rice proteome database," and the "Arabidopsis seed proteome." Recently, for constructing proteome reference map, a software tool called "GelMap" was developed (Rode et al., 2011). This tool was used for generating a 3D GelMap of Arabidopsis complex 1 in which its unique proteins constituents has been defined (Peters et al., 2013).

\section{Legume Databases for Proteomics Analyses}

Various databases have been developed that store a large resource of plant proteins from the proteome reference maps of legumes and other plants, such as the plant proteomics database PROMEX (Hummel et al., 2007; Wienkoop et al., 2012). A continuously growing database, PROMEX consists of 116,364 tryptic peptide product ion spectra entries of 48,218 different peptide sequence entries from Lotus, Medicago, common bean and soybean as well as other plants such as Arabidopsis, rice, etc. The database could be searched for whole experiments with an experimental ID, meta-information and single proteins and their corresponding peptide reference spectra. Furthermore, new LC-MS/MS analyses can be searched against this spectral library (http://promex.pph.univie.ac.at/promex/). LegProt (http:// bioinfo.noble.org/manuscript-support/legumedb) is a legume specific protein database consisting of amino acid sequences translated from predicted gene models and 6-frame translations of tentative consensus sequences expressed sequence tags (ESTs) (Lei et al., 2011). The ProteomeXchange contains the dataset of seed phosphoproteins from Lotus (PXD000053, http:// proteomecentral.proteomexchange.org/cgi/GetDataset?ID=PXD 00053) and has been valuable for understanding the regulatory mechanisms of seed germination in legumes (Ino et al., 2014). This dataset contains a total of 721 phosphopeptides from 343 phosphoproteins in cotyledons and 931 phosphopeptides from 473 phosphoproteins in hypocotyls.

The Medicago PhosphoProtein Database (MPPD, http://phospho.Medicago.wisc.edu), contains 3457 unique phosphopeptides with 3404 non-redundant sites of phosphorylation on 829 proteins. This database represents the most comprehensive Medicago phosphorylation data, which allows browsing of identified proteins, searching proteins of interests, in addition to conducting BLAST searches of the database using peptide sequences and phosphorylation motifs as queries (Rose et al., 2012a). The Soybean Proteome Database (SPD, http://proteome.dc.affrc.go.jp/Soybean/) consists of a repository of functional analysis of abiotic stresses (flooding, drought, and salt). In total, it consists of 23 reference maps of soybean and proteins collected from several organs, tissues, and organelles of soybean (Ohyanagi et al., 2012). Recently, another database for storage, allergen, and anti-nutritional proteins from cultivated soybean called Soybean Protein Database (SoyProDB; http://bioinformatics.towson.edu/Soybean_Seed_Proteins_2D_ Gel_DB/Home.aspx) has also been developed (Tavakolan et al., 2013). In the case of yellow lupin (Lupinus luteus L.), a seedprotein catalog has been developed. In this study, 736 proteins corresponding to 152 unique proteins have been deposited in the WORLD-2DPAGE repository (http://world-2dpage.expasy.org/ repository/0066/; Ogura et al., 2014).

\section{Post-Translational Modifications (PTMs) in Legumes}

PTMs are required for the functionality of proteins that regulate processes and the subcellular localization that could be analyzed using proteomics approaches (Pandey and Mann, 
2000; Seo and Lee, 2004; Downes and Vierstra, 2005). Although mostly identified through MS approaches, PTM analyses are not straightforward as protein identifications, the reason being peptide analyses do not show the expected molecular mass and therefore more protein samples are required (Pandey and Mann, 2000). However, advancement in phosphoproteomics procedures, e.g., the enrichment of phosphopeptides using immobilized metal affinity chromatography (IMAC) or proteins with aluminum hydroxide or titanium dioxide called metal oxide affinity chromatography (MOAC) (Wolschin et al., 2005) have improved the identification efficiency (Ndassa et al., 2006; Chen et al., 2010; Hoehenwarter et al., 2013; Beckers et al., 2014). Among the PTMs, phosphorylation is the major posttranslational regulatory processes in all eukaryotes followed by ubiquitin and SUMO (Small Ubiquitin-like MOdifier) conjugations (Mazzucotelli et al., 2008).

The root proteome of the Medicago genotype, Jemalong A173457, revealed unique phosphopeptides that covers 3404 non-redundant sites of in vivo phosphorylation on 829 proteins (Grimsrud et al., 2010). The large scale phosphoproteomic study identified multiple sites of phosphorylation on a number of crucial proteins in rhizobial symbiosis initiation such as SICKLE, NUCLEOPORIN133 and INTERACTING PROTEIN OF DM13. Further, the rapid Nod-factors (NF) induced changes in the phosphorylation levels of 13,506 phosphosites in 7739 proteins was recently measured for rhizobia-legume symbiosis in Medicago which was found useful for quantifying phosphorylation, specifically associated with NF-signaling (Rose et al., 2012b).

In chickpea, a differential phosphoproteomic study in response to dehydration stress identified 91 phosphoproteins that are likely to be involved in cell defense, photosynthesis, photorespiration, molecular chaperones and ion transport. The study also identified multiple sites of phosphorylation in key regulatory and functional proteins (Subba et al., 2013). Recently, a nucleus-specific phosphoproteome map of 107 identified phosphoproteins was constructed in chickpea, which identified a collection of phosphoproteins involved in many cellular functions such as protein folding, signaling, gene regulation, DNA replication/repair/modification, metabolism, etc. (Kumar et al., 2014). In an earlier study, the PTMs of $\alpha \mathrm{AI}(\alpha-$ amylase inhibitor) were compared among transgenic pea and chickpea expressing $\alpha \mathrm{AI}$ from common bean, with the processed form of the protein from several bean varieties. The $\alpha \mathrm{AI}$ proteins displayed microheterogeneity due to differences in glycan addition frequency, variation in glycan processing and differences in C-terminal exopeptidase activity (Campbell et al., 2011). Thus, PTMs in common beans were also investigated on three seed defensive proteins $\alpha$ AI-1, $\alpha$ AI-2, and arcelin- 5 (Young et al., 1999). The data showed that the proteolytic cleavage is required for the activation of the proteins, which resulted in the loss of the terminal Asn residue in $\alpha \mathrm{AI}-1$, and a minimum of seven residues from the C-termini of all three proteins. Additionally, a significant difference in the glycosylation patterns of $\alpha \mathrm{AI}-1$ and $\alpha \mathrm{AI}-2$ has been reported, although the proteins showed high sequence homology. Similarly in pea, the presence of multiple trypsin inhibitors (TI) isoforms were attributed to PTMs and particularly post-translational processing at the C-terminus during the desiccation stage of seed development resulted in the appearance of TI isoforms in pea (Domoney et al., 1995).

Histone modifications and histone variants are known to be vital for various biological processes. In this context, variants of soybean histone, H3 and H4 and their PTMs were reported, which revealed several distinct variants of soybean histone and their modifications that were different from Arabidopsis ( $\mathrm{Wu}$ et al., 2009). The study thus, provides important biological information toward understanding histone modifications and their functional relevance in legumes.

This area of research possesses great promise for legume improvement as it is supported by advanced proteomics technologies, in particular, developments in the strategies for detection and selective isolation of proteins with known function. As discussed above functional properties of proteins are often regulated by PTMs of proteins and the numerous techniques developed can be applied to the global identification of PTMs and their processing sites in legumes.

\section{PPI Approaches and Application in Legume Research}

PPIs provide functional knowledge about proteins by analysing the interacting partners and interactomics, which is the largescale study of PPI networks using high throughput methods. This provides valuable insights for understanding cellular function, metabolism and signaling mechanisms (Braun et al., 2013; Stasi et al., 2015). Interactomes (maps of PPI) are constructed on the basis of experimental data and computational prediction of interactions (Stasi et al., 2015). Approaches used in PPI analyses and mapping can be genetic, biochemical or proteomics-based. The strategies involve direct interactions with binary methods such as yeast two-hybrid ( $\mathrm{Y} 2 \mathrm{H})$ or split ubiquitin, analyses of protein complexes with co-immunoprecipitation or affinity purification followed by MS (AP-MS), imaging and database analyses (Pandey and Mann, 2000; Fukao, 2012; Braun et al., 2013; Stasi et al., 2015). The identification of minor PPIs are also essential for studying transient interactions and proteins of lowabundance, which may be functionally relevant (Fukao, 2012). In recent years, many proteins have been identified with advances in MS technologies and large-scale proteomics, however, the consequence of this is the increased number of false positive protein identifications. Therefore, for the accurate appraisal of PPI identification, the inclusion of independent experimental validation is required (Fukao, 2012). Y2H system is sensitive in detecting transient and unstable interactions, and is suitable for PPI mapping and generating high-throughput data with a fine resolution to understand cellular process at the systemic level. However, it has limitations with incidences of false-positives and false-negative interactions. The PPIs are usually validated with techniques such as pull-down assays, co-immunoprecipitation, in-situ hybridization (von Mering et al., 2002; Parrish et al., 2006; Brückner et al., 2009).

Although limited PPI based studies have been undertaken in legumes, the studies involving stress signaling networks, nodule formation and symbiosis have provided valuable information 
in understanding these processes. The $\mathrm{Y} 2 \mathrm{H}$ system has been mostly used in model legumes to study symbiosis and nodulation mechanisms and in the crop legumes mainly to study stress responses (Table 2). For example in pea, using the $\mathrm{Y} 2 \mathrm{H}$ system and validation using in planta co-immunoprecipitation, the PPI observed among the heterotrimeric G proteins and GPCR protein were shown to be relevant in the salt and heat stress signaling pathways (Misra et al., 2007). In another study, the exposure to salt and cold simultaneously stimulated the expression of genes encoding a protein kinase $\left(P_{S} C I P K\right)$ and a calcineurin B-like protein ( $P s C B L$ ) in pea (Mahajan et al., 2006). Although immunofluorescence and confocal microscopy showed that the PsCBL was localized in the cytosol and PsCIPK in the outer membrane, $\mathrm{Y} 2 \mathrm{H}$ analysis indicated that both the protein products interacted and this was supported with Western blots. In chickpea, a few candidate interactors were identified for the 1R-MYB for drought tolerance with the Y2H system (Ramalingam et al., 2015).

Bimolecular fluorescence complementation (BiFC) uses the yellow fluorescent protein (YFP), split into two-non-overlapping $\mathrm{N}$-terminal (YN) and C-terminal (YC) fragments, where each fragment is cloned in-frame to the gene of interest, allowing the expression of fusion proteins. PPI detected in planta causes the detection of yellow fluorescence, which is not found for non-interacting pairs or non-fused YN/YC (Bracha-Drori et al., 2004). Although identification of PPI by screening a cDNA library is usually performed in yeast, BiFC technology was applied to screen an Arabidopsis cDNA library against a bait protein in planta since subcellular compartmentation and protein modifications differ between plant and yeast cells (Lee et al., 2012).

A challenging area in crops research would be the development of a proteome-wide PPI maps to understand the complex biological pathways and cellular networks. A high-throughput, Y2H system (HTP-YTH), suitable for mapping PPIs was described by Fang et al. (2002) to screen PPI in plants. This system involves a yeast gap-repair cloning and a selectivity that reduces false positives and negative clones with automation in laboratory procedures. This system has been used to study the defense signal transduction pathway in rice, where more than 100 genes were selected as "baits" for HTP-YTH screening in which many known and novel PPIs were identified. In Arabidopsis, the first binary PPI map for the interactome network of plants was developed (Arabidopsis Interactome

TABLE 2 | PPI analyses in some model and crop legume using Y2H and validated with other approaches.

\begin{tabular}{|c|c|c|c|c|}
\hline Legume & Stress/Condition & Interacting components & $\begin{array}{l}\text { Method of } \\
\text { confirmation }\end{array}$ & References \\
\hline \multirow[t]{3}{*}{ Lotus } & Nodule development & LjNSP2 homodimers & $\beta$-galactosidase assay & Murakami et al., 2013 \\
\hline & Nodule development & CASTOR homodimer, POLLUX homodimer & $\mathrm{BiFC}$ & Charpentier et al., 2008 \\
\hline & & SIP1 and SymRK & Pull down assay & Zhu et al., 2008 \\
\hline \multirow[t]{3}{*}{ Medicago } & Nodulation signaling & RAM1 and NSP2 & $\mathrm{BiFC}$ & Gobbato et al., 2012 \\
\hline & & NSP1 and NSP2 & $\mathrm{BiFC}$ & Hirsch et al., 2009 \\
\hline & & DMI3 and IPD3 & $\mathrm{BiFC}$ & Messinese et al., 2007 \\
\hline Chickpea & Salt & CaCIPK6 and NtCBL3 & - & Tripathi et al., 2009 \\
\hline \multirow[t]{2}{*}{ Pea } & Salt and heat & $\begin{array}{l}\text { G } \alpha \text { subunit with the } G_{\beta} \text { subunit and } \\
\text { phospholipase } C \text { at the calcium-binding } \\
\text { domain }\end{array}$ & Co-immunoprecipitation & Misra et al., 2007 \\
\hline & Salt and cold & PsCIPK and PsCBL & $\begin{array}{l}\beta \text {-galactosidase assay, } \\
\text { Far-western blotting }\end{array}$ & Mahajan et al., 2006 \\
\hline \multirow[t]{2}{*}{ Soybean } & Cold & SCOF-1 and SGBF-1 & $\beta$-galactosidase assay & Kim et al., 2001 \\
\hline & $\begin{array}{l}\text { ABA, drought, cold, } \\
\text { salt }\end{array}$ & $\begin{array}{l}\text { GmMYB76 homodimers, GmMYB76 and } \\
\text { GmMYB177, GmZIP46 homodimer, } \\
\text { GmZIP46 and GmZIP62, GmZIP46 and } \\
\text { GmMYB76. }\end{array}$ & $\beta$-galactosidase assay & Liao et al., 2008a \\
\hline
\end{tabular}

GmGAMYB1 in 
Mapping Consortium, 2011). The construction of these maps in legumes should facilitate system biology approaches and will greatly benefit legume crop improvement programs.

\section{METABOLOMICS APPROACHES IN LEGUMES}

Plants synthesize specialized metabolites that define the biochemical phenotype of a cell or tissue and can be viewed as the end products of gene expression (Sumner et al., 2003). Quantitative and qualitative measurements of cellular metabolites provide a broad view of the biochemical status of an organism that could be used to monitor and assess gene function (Fiehn et al., 2000). Furthermore, metabolomics contributes significantly to the study of stress biology by identifying different compounds such as by-products of stress metabolism, stress signal transduction molecules, molecules that are part of plant acclimation process etc. (Weckwerth, 2003; Larrainzar et al., 2009). The identified metabolic compounds could be further studied by direct measurement or correlating with the changes in transcriptome and proteome expression and confirmed by mutant analysis. In this section, recent applications of metabolomics approaches in the area of legume development, symbiosis and stress response are discussed.

Metabolomics is a rapidly developing technology and at present metabolic fingerprinting and metabolite profiling approaches are being used. For wide coverage of the vast range of metabolites present, several analytical techniques involving separation and detection are implemented (Scherling et al., 2010; Doerfler et al., 2013, 2014). The separation technique is selective for certain groups of metabolites which includes gas chromatography (GC) for volatile and primary metabolites such as sugars and amino acids (Weckwerth, 2011a), LC for mainly secondary metabolites (Scherling et al., 2010; Weckwerth, 2011a), capillary electrophoresis (CE) for ionic metabolites to be separated (Soga et al., 2003; Soga, 2007) and ultra-performance liquid chromatography (UPLC). UPLC is a powerful technique which has high resolution, sensitivity and throughput than conventional high performance liquid chromatography (HPLC). The MS analysers have been commonly used for metabolite profiling, particularly those that provide accurate mass measurements such as FTICR_MS, Orbitrap-MS or TOF-MS due to its fast scan times with improved deconvolution, run times and high mass accuracy (Scherling et al., 2010; Weckwerth, 2010, 2011a; Obata and Fernie, 2012; Arbona et al., 2013; Doerfler et al., 2013, 2014). GC-MS has been widely used in plant metabolomics research and electron impact (EI) supports strong interfacing of GC with MS which allows fragmentation patterns to be highly reproducible. Major metabolomics approaches and their applications in legumes are described below and are summarized in Table 3.

\section{Metabolite Profiling}

Metabolite profiling is the simultaneous measurement of all or a set of metabolites in a given sample. Several analytical techniques such as nuclear magnetic resonance (NMR), GCMS, LC-MS, capillary electrophoresis-MS (CE-MS) and Fourier transform infrared (FT-IR) spectroscopy have been reported for analysing the data from metabolite profiling (Sumner et al., 2003; Weckwerth, 2003; Shulaev, 2006). The advantages and disadvantages of each technique for metabolite profiling have been discussed previously (Fiehn et al., 2000; Roessner et al., 2000; Sumner et al., 2003; Weckwerth, 2003; Weckwerth et al., 2004a,b; Shulaev, 2006). The use of metabolic profiling has been limited in crop legumes but this approach has been successfully demonstrated in model legumes. For example, in Medicago, untargeted quantitative MS approach was used to profile metabolites treated with rhizobial Nod factors to study the metabolic changes between the symbionts (Zhang et al., 2013). The study showed decrease in concentration of (9)-HODE class of oxylipins upon Nod factor treatment in planta and together with jasmonic acid inhibited Nod factor signaling. This suggests an important role for the oxylipin pathway in Nod factor signaling in symbiosis. In a different study, under early salt and drought stress conditions, the involvement of certain metabolites in nutritional priming through symbiotic interaction of nodulated plants and $\mathrm{N}$-fertilized Medicago has been reported (Staudinger et al., 2012).

Similarly, to understand drought acclimation in model and forage legumes, a comprehensive and progressive reprogramming of metabolic pathways were suggested for increased water stress in Lotus (Sanchez et al., 2012). Using GC coupled to electron impact ionization (EI)-TOF-MS (GCEI-TOF-MS), this study reported gradual increase in most of the soluble molecules profiled. In addition, comparative metabolomics between the Lotus species showed the presence of metabolites that were conserved and unique in response to drought stress. Metabolite profiling using a combination of ionomic and GC-MS was conducted for the shoots of extremophile Lotus species, adapted to highly saline coastal regions and was compared with that of cultivated glycophytic grassland forage Lotus species, to understand salt tolerance mechanisms. The extremophile Lotus species was identified to have higher salt levels with a differential rearrangement of shoot nutrient levels upon salt exposure (Sanchez et al., 2011). In a similar study, the accumulation of alanine under anoxic conditions was examined in Lotus, which is highly tolerant to water logging (Rocha et al., 2010). High accumulation of succinate, alanine and the direct co-substrates for alanine synthesis, glutamate and gamma aminobutyric acid (GABA) in the roots of Lotus during water logging was reported. Whereas, majority of amino acids that are derived from TCA cycle intermediate were found to be decreased, which support earlier findings that the metabolic equilibriums are expected to drive the metabolic flux from glycolysis, via alanine synthesis and oxoglutarate to succinate, which prevents the accumulation of pyruvate activating fermentation leading to ATP production by succinyl- CoA ligase. In another study in Lotus, GC-TOF-MS (primary metabolism) and LC-FourierTransformation-MS (secondary metabolism) was applied to study plant/plant competition responses in a large biodiversity experiment (Scherling et al., 2010). Significant effects in Lotus were not associated with primary metabolism (sugars, amino acids, organic acids) but with the secondary metabolism. A 
TABLE 3 | Application of metabolomics approaches in stress, development and symbiotic processes in some legumes.

\begin{tabular}{|c|c|c|c|c|}
\hline Legume & Stress/Condition, Tissue & Methods & Metabolites & References \\
\hline \multirow[t]{2}{*}{ Lotus } & Drought, shoots & GC-EI-TOF-MS & Serine, proline, arabitol & Sanchez et al., 2012 \\
\hline & Salt, shoots & GC-MS & Citric acid, malic acid & Sanchez et al., 2011 \\
\hline \multirow[t]{6}{*}{ Medicago } & Metabolic reprogramming, roots & GC-MS, LC-MS & $\begin{array}{l}\text { Flavonoids, triterpenoid, starch hydrolysis } \\
\text { metabolites }\end{array}$ & Watson et al., 2015 \\
\hline & & LC-ESI-TOF-MS & Oxylipins & Zhang et al., 2012 \\
\hline & Flavonoid profiling, roots & LC-MS & Flavonoids & Staszków et al., 2011 \\
\hline & $\begin{array}{l}\text { Arbuscular mycorhizal symbiosis, } \\
\text { roots }\end{array}$ & GC-MS, HPLC, LC-MS & Amino acids, fatty acids, isoflavonoids & Schliemann et al., 2008 \\
\hline & Signaling pathway roots & $\begin{array}{l}\text { HPLC coupled to UV photodiode } \\
\text { array and ESI ion-trap } \\
\text { MS(HPLC-PDA-ESI-ITMS) }\end{array}$ & Phenylpropanoid, isoflavonoid & Farag et al., 2007 \\
\hline & Microbial, roots & $\begin{array}{l}\text { Reversed-phase } \\
\text { HPLC-PDA-ESI-MS } \\
\text { (HPLC-PDA-ESI-MS) }\end{array}$ & Saponins & Huhman and Sumner, 2002 \\
\hline Pea & Drought, leaves & NMR & Amino acids & Charlton et al., 2008 \\
\hline \multirow[t]{4}{*}{ Soybean } & Aphid infection, leaves & CE-TOF-MS & Flavonoids, alkaloids, amino acids & Sato et al., 2013 \\
\hline & Defense, cotyledon & LC-MS, NMR & Prenylated-isoflavones & Cheng et al., 2011 \\
\hline & Flooding, roots, hypocotyls & CE-MS & Succinate, citrate, pyruvate & Komatsu et al., 2011 \\
\hline & Symbiosis, root hairs & GC-MS, UPLC-QTOF-MS & Isoflavonoids, fatty acids,carboxylic acids & Brechenmacher et al., 2010 \\
\hline
\end{tabular}

significant gradient of several putative flavonoids structures showed a high correlation to increasing biodiversity in the close environment of the individual plant (Scherling et al., 2010).

As described above, plants show a variety of metabolic responses against varied abiotic stresses. With the availability of genomics platforms, scientists are now able to opt for metabolomics for studying metabolites involving non-biased approaches. However, it would be important to study if there are any common metabolic responses associated with all the abiotic stresses or if the responses are specific to the stress. In this context, Komatsu et al. (2011) identified 81 metabolites related to the mitochondria under flooding stress in roots and hypocotyls of soybean using CE-MS which showed that the TCAcycle-related metabolites, glycolysis related metabolites, GABA, pyruvate, NAD, NADH and amino acids increased, while ATP decreased. This kind of accumulation/increase in GABA and amino acids was also reported in Lotus unlike TCA cycle related metabolites were found to be low in Lotus and high in soybean. These studies support that integrative analysis is required on the metabolite accumulation within the context of understanding metabolic responses to stress.
Phosphorous is an essential component of energy metabolism, signaling molecules, and structural macromolecules. Therefore, studies have been conducted to understand the role of phosphorus in stress response metabolite profiling of common bean roots and nodules under $\mathrm{P}$ starvation (Hernández et al., 2007). Increase in the levels of most of the amino acids and several sugars was reported in P-stressed roots. It was suggested that the accumulation of sugars may be partitioned preferentially to P-stressed roots to support the expression of $\mathrm{P}$ stress-induced genes. On the other hand reduced amounts of organic acids are reported in P-starved roots that likely reflect exudation of these metabolites from the roots into the rhizosphere (Hernández et al., 2007). The metabolic response of P-starved nodules is in contrast to that of P-starved roots in common bean. It was reported that amino acids, $\mathrm{N}$-containing metabolites and sugars were decreased, while organic acids were accumulated in P-deficient nodules (Hernández et al., 2009). Such contrasting response may be due to the suppression of $\mathrm{N}$ supply from fixed $\mathrm{N}_{2}$ under environmental limitations such as P-starvation in nodules. Recently, metabolite profiling study in chickpea revealed 49 primary metabolites in contrasting salt stress responsive cultivars (Dias et al., 2015). 
One drawback with the metabolite profiling is that the data obtained is not sufficient to determine the regulation mechanisms of the pathways of interest. In order to overcome this issue, integrated analysis of metabolomics data together with that of proteomic and transcriptomic data need to be performed. For example in Medicago, an integrated metabolomics and transcriptomic approach was found useful to study metabolic reprogramming of the border cells in roots through cumulative and pathway specific datasets (Watson et al., 2015). This integrative approach showed that there were significant differences in the levels of phytohormone, supported by variation in lipoxygenases and auxin responsive transcripts in the border cells and root tips. Additionally, this approach identified metabolic resources for growth and development redirected to the border cells for the accumulation of specialized metabolites that were defense and symbiosis related. Similarly, Komatsu et al. (2011) used an integrative proteomic and metabolomics approach which was useful in identifying the expression and regulation of components linked to flooding stress in soybean seedlings. Larrainzar et al. (2009) integrated GC-TOF-MS metabolite profiling with untargeted and targeted proteomics to reveal nodule metabolic responses under drought stress and recovery by re-watering in Medicago. Metabolite profiling was able to show a highly pronounced reprogramming of metabolism during drought response and the ability of the nodules to recover completely after re-watering.

\section{Targeted and Untargeted Metabolite Analysis}

Metabolites are analyzed either using a targeted or untargeted method (Patti et al., 2012). The targeted method is used to analyse a specified set of metabolites which targets one or more pathways of interest and involves the setup of selected reaction monitoring methods of the standard compounds of the metabolite of interest, followed by the extraction of metabolites from the sample and analysis. The data obtained provides quantitation based on standardized methods for the metabolites of interest. This method has been widely used to follow the dynamics of a limited number of metabolites known to be involved in a particular stress and also for comparative metabolite profiling of a large number of known metabolites. For example, in a single chromatographic run, highly parallel targeted assays based on SRM can be used for sensitive simultaneous analysis of over 100 metabolites (see Bajad and Shulaev, 2007). Alternatively, quantitative profiling may provide in vivo enrichment of metabolites with stable isotopes like $\mathrm{C}-13$ and $\mathrm{N}-15$. This can be possible only by growing plants or plant cells in liquid media containing $\mathrm{N}$-15-labeled inorganic nitrogen sources or C-13-labeled carbon dioxide or glucose (Hegeman et al., 2007; Huege et al., 2007). Metabolic labeling combined with MS has been successfully used for quantitative metabolic profiling in microorganisms (Mashego et al., 2004; Lafaye et al., 2005; Wu et al., 2005).

On the other hand, the untargeted metabolite profiling is often used for global and broader applications, e.g., understanding cellular metabolism. In this approach, metabolites are isolated from samples followed by the LC-MS analysis. The data obtained is processed using bioinformatics and values for peaks of interest are searched against metabolite databases for possible identity. These metabolite identities are confirmed using tandem MS (MS/MS) data and retention time data compared with standard components. Untargeted metabolite profiling in Lotus demonstrated a major and reproducible change of the metabolic phenotype in the course of salt acclimatization, which was most evident for amino acid, sugars and organic acid metabolism (Sanchez et al., 2008). Accumulation of amino acids and other nitrogen-containing compounds is a remarkable biochemical feature of almost all plant stress responses reported so far. However, the main disadvantage of untargeted profiling is that it is a semi-quantitative method and provides relative concentration data based on the same "surrogate" internal standard. These semi-quantitative data have to be further validated using targeted quantitative assays.

\section{Metabolic Fingerprinting}

Metabolic fingerprinting is mainly used to identify metabolic signatures, for example, finding patterns associated with a particular stress response without precise quantitation/identification of different metabolites in the given sample. Features specific to a fingerprint can be identified using a variety of pattern recognition and multivariate statistical analysis (e.g., principal component analysis (PCA), self-organizing maps (SOMs) and hierarchical clustering, discriminant function analysis (DFA), ANOVA etc.) on the data (Sumner et al., 2003). Different analytical techniques, including NMR spectroscopy (Krishnan et al., 2005), MS (Goodacre et al., 2003), and FT-IR (Johnson et al., 2003) may be used to perform metabolic finger printing. Of these, MS is advantages over NMR spectroscopy because of the low sensitivity of NMR, which makes it difficult to detect low abundance cellular metabolites. On contrary, MS has high resolving power compared to NMR, providing higher sensitivity and lower detection limit but generates more complex spectrum because of its results in the form of discriminant ions which remains as a challenge for data validation. Moreover, a larger subset of metabolites associated with the phenotype can be identified using MS.

There have been few studies that reported the application of these approaches in legumes. Metabolic finger printing has been utilized to study drought in seven different model and forage species of the Lotus genus. Analysis using PCA of the metabolite features regardless metabolite identification status yielded sample "fingerprints," which classified primarily according to the genotype. Infrared (IR) spectroscopy has been used to obtain a snapshot of the sample metabolome (typically low-molecular-weight compounds) at a given time. This study identified metabolic compounds responsible for rapid fermentation for the efficient conservation of forage proteins (Johnson et al., 2004). In another study, NMR-based approach has been used for metabolic fingerprinting of 21 grass and legume cultivars. Applying PCA, variation between cultivars and the magnitude of changes in the metabolic fingerprint between the spring growth and the second regrowth was elucidated in the study. Furthermore, variation in metabolic compounds such as malic acid, choline, and glucose was reported due to seasonal change (Bertram et al., 2010). 


\section{Metabolomics Databases for Legumes}

As with transcriptomics and proteomics data, metabolomics approaches also generate huge datasets that require specialized data mining and bioinformatics tools. It is imperative to integrate functional genomics data to comprehensively study biological components, using a systemic approach, e.g., through the mathematical modeling of biological systems. Metabolomics data handling, mining and analysis etc. have been improved tremendously, due to advances in bioinformatics tools. In this scenario, several databases have been developed for plant metabolomics data analysis. For example, a metabolic pathway reconstruction was used to generate a pathway database for Medicago called MedicCyc (http://www.noble. org/MedicCyc/) which features more than 250 pathways with related genes, enzymes and metabolites (UrbanczykWochniak and Sumner, 2007). The database contains Medicago specific pathways including isoflavonoid, lignin and triterpene saponin biosynthesis which were added or modified based on literature and in-house expertise. MedicCyc is designed to visualize functional genomics datasets from Medicago within the biological context of metabolic pathways and has been believed that this is best achieved through the visualization of data within the biological context of metabolic pathways in legumes. The pathways were engineered to enable the correlated visualization of integrated functional genomics data. Another database, Soybean Knowledge Base (SoyKB) (http:// soykb.org) has been reported to be a comprehensive resource for soybean translational genomics and contains integrative information on soybean genomics, transcriptomics, proteomics and metabolomics (Joshi et al., 2014). This is a web resource that would not only be useful for soybean translational genomics, but also for legume crop improvement programs. Also, a database for plant metabolomics, PlantMetabolomics.org (PM) (http://www.plantmetabolomics.org) was developed (Bais et al., 2010). This database represents metabolomics data generated from Arabidopsis through an integration of experiments compiled from different platforms with visualization tools. PlantMetabolomics has been widely used for exploring, visualizing and downloading plant metabolomics data and well-annotated metabolomics datasets which is useful for establishing metabolomics as a functional genomics tool in legumes.

\section{Metabolomics for Crop Breeding}

There is growing interest in using metabolites as selection markers in crop breeding programs, because metabolite biomarkers have been linked with strong environmentallycontrolled traits (Steinfath et al., 2010). Mapping and metabolomics genome wide association studies (mGWAS) have been conducted to develop "metabotypes" using metabotype quantitative trait locus (mQTL) (association of genomic markers and metabolic markers) which enabled the associations between metabolic concentrations and genetic polymorphisms. Overall, plant metabolomics has benefited from a rich array of preexisting methodological approaches and bioanalytical knowledge for the characterization of the chemically diverse classes of metabolites. However, speedy progress in the application of these approaches in legumes will be quite useful for legume improvement.

\section{SUMMARY AND FUTURE OUTLOOK}

The development in the area of proteomics and metabolomics has enhanced the power of "omics" with the possibility of studying at different levels of plant regulations, namely transcriptome, proteome and the metabolome (Shulaev et al., 2008). Plant growth, development and stress responses are not straightforward to be able to understand by just looking into one or two level(s), which has been done traditionally. For instance, to understand the molecular basis of stress physiology and biochemistry, the quantitative correlation of different protein groups and classes of metabolites with stress levels are required for the identification of candidate bio-markers (Weckwerth, 2011b; Rodziewicz et al., 2014). Moreover, with the recent developments in the technologies, availability of legume genomics and protein databases, it would be possible to have efficient and high throughput identification of stress related proteins in crop legumes (Hiremath et al., 2011; Kosová et al., 2011; Varshney et al., 2013a; Hossain and Komatsu, 2014a,b).

Legume crops are widely cultivated in the semi-arid tropics where various abiotic and biotic stresses pose severe threats to the productivity. The crops are subjected to not only one stress, but also a combination of stresses at a given point of time under natural field conditions. To sustain legume cultivation under these environments, crop improvement programs requires resourceful methods such as integrated "omics" approaches to understand stress responses at the molecular level such as cellular mechanisms, signaling pathways, biochemical processes. Such studies have largely been initiated in model plants such as Arabidopsis. For instance, transcriptomics and the metabolomics data revealed a different pattern of defense response in Arabidopsis subjected to a combination of drought and heat stress. It was found that proline (Pro) was accumulated in response to drought stress, but during the combination of stresses, Pro was replaced by sucrose as osmoprotectants. Similar studies needs to be undertaken in legume crops such as chickpea, pigeonpea and peanut, which are of agronomic value especially in the semi-arid tropics.

In this review, the available proteomics and metabolomics resources for legume research and their applications for further our understanding of the stress biology in model as well as crop legumes have been provided (Figure 1). A large number of protein reference maps for various tissues are already available for model legume crops that will greatly support comparative proteomics approaches. There is a great need to generate proteome maps from crop legumes that would greatly help in precise understanding of various cellular processes and signaling pathways. These resources would improve the functional annotations of the gene models in identifying novel ORFs as in the case of Arabidopsis (Castellana et al., 2008) and also validates the existing annotation (Agrawal et al., 2012, 2013; Dash et al., 2015; Walley and Briggs, 2015). Moreover, it is now possible to uncover the regulation of 

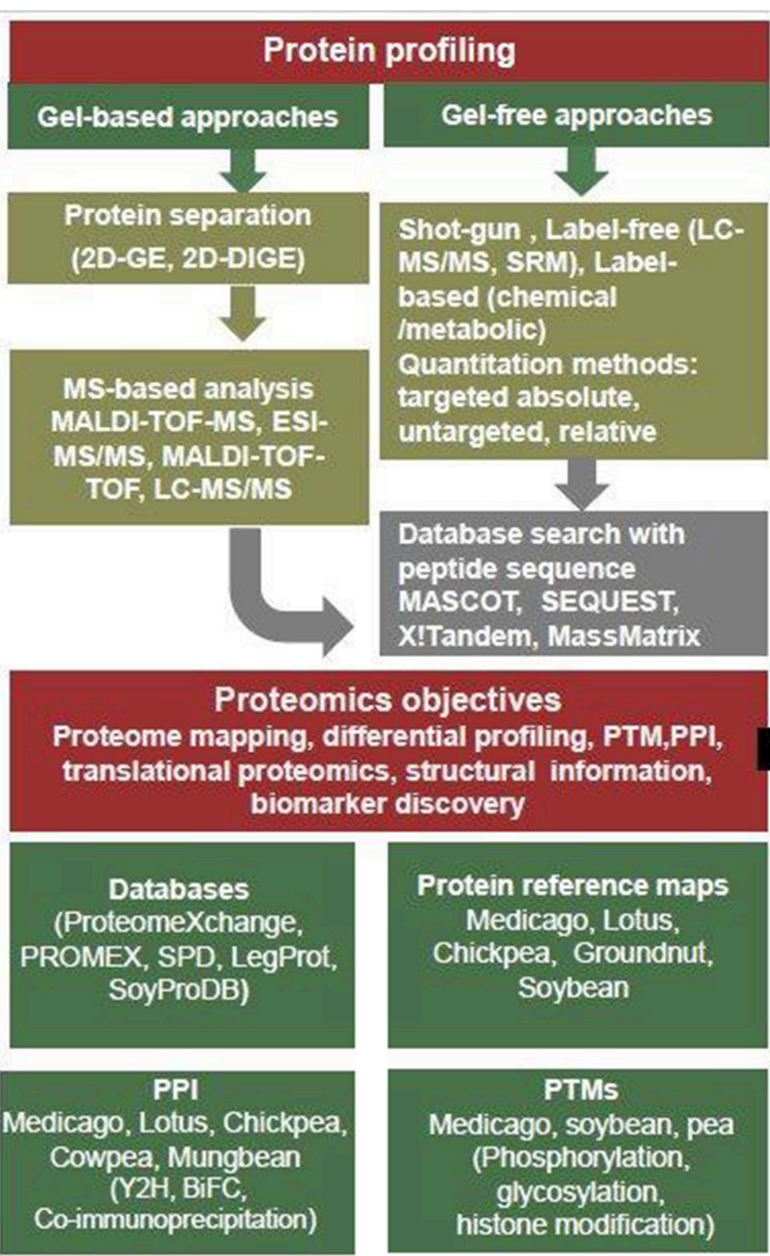

\section{Metabolomic profiling and fingerprinting}

\section{Profiling}

- Targeted approach (Metabolites in pathway(s) of interest)

- Untargeted approach (global)

Fingerprinting (Pattern of recognition)

-NMR, GC-MS, CE-MS, LC-MS, UPLC, HPLC, EI-TOF-MS, MS/MS

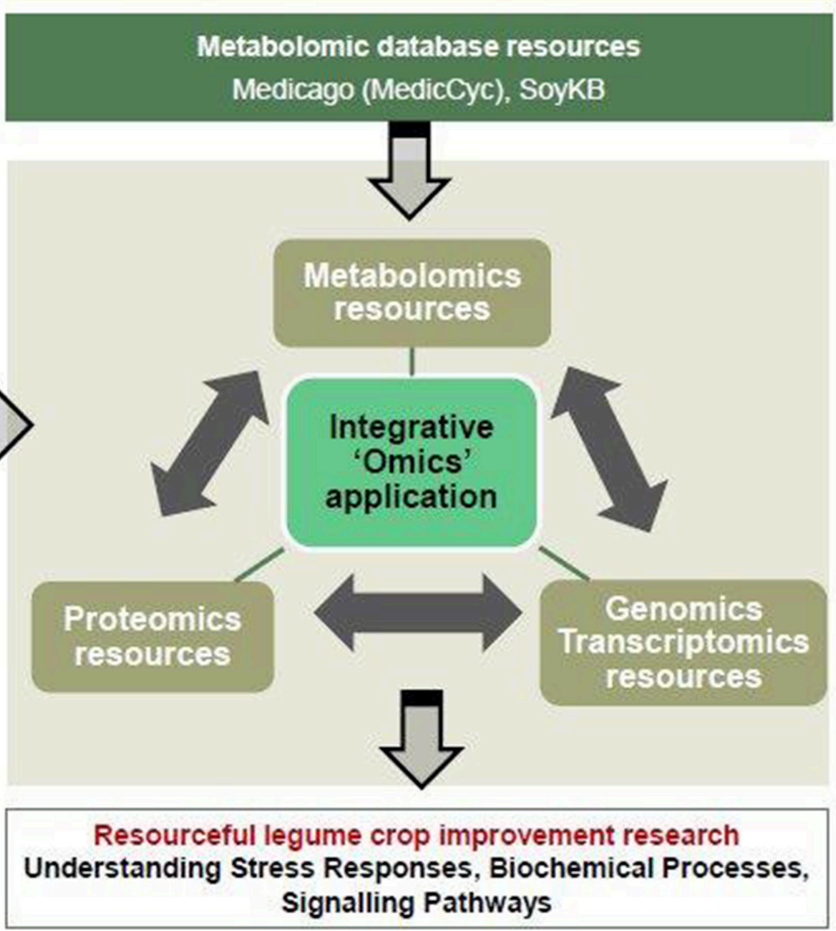

FIGURE 1 | Significant advances in proteomic and metabolomics for integrative "omics" approaches in legume crop research.

these processes by studying the post-translational modifications. Further, huge amount of information on metabolomics data related to stress responses in legumes have been generated which includes a large number of metabolic pathways under stress conditions. The integrative analyses of data through genomics, transcriptomics, proteomics and metabolomics will be important for a system biology approach and for efficient application in legume crop improvement. At this point, it would be worthwhile to caution that combining and integrating omics data together is challenging, because of the difficulty in correlating the data due to the differences in the time for quenching metabolism. In addition, some errors can be introduced due to the nature of the samples used and the lack of appropriate mathematical

\section{REFERENCES}

Abdallah, C., Dumas-Gaudot, E., Renaut, J., and Sergeant, K. (2012). Gel-based and gel-free quantitative proteomics approaches at a glance. Int. J. Plant Genomics 2012:494572. doi: 10.1155/2012/494572

Abdallah, C., Valot, B., Guillier, C., Mounier, A., Balliau, T., Zivy, M., et al. (2014). The membrane proteome of Medicago truncatula roots displays qualitative models that would allow identification of various biochemical and signaling pathways involved in stress response. Therefore, there is a need to advance at all the fronts, i.e., sampling strategy, data generation, data analysis, etc. in different disciplines so that precise system biology approach can be deployed to understand molecular basis of different traits related to plant biology as well as breeding applications.

\section{ACKNOWLEDGMENTS}

This work has been undertaken as part of the CGIAR Research Program on Grain Legumes. ICRISAT is a member of CGIAR Consortium.

and quantitative changes in response to arbuscular mycorrhizal symbiosis. J. Proteomics 108, 354-368. doi: 10.1016/j.jprot.2014.05.028

Aghaei, K., Ehsanpour, A. A., Shah, A. H., and Komatsu, S. (2009). Proteome analysis of soybean hypocotyl and root under salt stress. Amino Acids 36, 91-98. doi: 10.1007/s00726-008-0036-7

Agrawal, G. K., Pedreschi, R., Barkla, B. J., Bindschedler, L. V., Cramer, R., Sarkar, A., et al. (2012). Translational plant proteomics: A 
perspective. J. Proteomics 75, 4588-4601. doi: 10.1016/j.jprot.2012. 03.055

Agrawal, G. K., Sarkar, A., Righetti, P. G., Pedreschi, R., Carpentier, S., Wang, T., et al. (2013). A decade of plant proteomics and mass spectrometry: Translation of technical advancements to food security and safety issues. Mass Spectrom. Rev. 32, 335-365. doi: 10.1002/mas.21365

Ahsan, N., Donnart, T., Nouri, M. Z., and Komatsu, S. (2010b). Tissue-specific defense and thermo-adaptive mechanisms of soybean seedlings under heat stress revealed by proteomic approach. J. Proteome Res. 9, 4189-4204. doi: $10.1021 /$ pr100504j

Ahsan, N., Nanjo, Y., Sawada, H., Kohno, Y., and Komatsu, S. (2010a). Ozone stress-induced proteomic changes in leaf total soluble and chloroplast proteins of soybean reveal that carbon allocation is involved in adaptation in the early developmental stage. Proteomics 10, 2605-2619. doi: 10.1002/pmic.201000180

Alam, I., Lee, D. G., Kim, K. H., Park, C. H., Sharmin, S. A., Lee, H., et al. (2010). Proteome analysis of soybean roots under water logging stress at an early vegetative stage. J. Biosci. 35, 49-62. doi: 10.1007/s12038-010-0007-5

Aloui, A., Recorbet, G., Robert, F., Schoefs, B., Bertrand, M., Henry, C., et al. (2011). Arbuscular mycorrhizal symbiosis elicits shoot proteome changes that are modified during cadmium stress alleviation in Medicago truncatula. BMC Plant Biol. 11:75. doi: 10.1186/1471-2229-11-75

Arabidopsis Interactome Mapping Consortium (2011). Evidence for network evolution in an Arabidopsis interactome map. Science 333, 601-607. doi: 10.1126/science. 1203877

Aranjuelo, I., Molero, G., Erice, G., Avice, J. C., and Nogués, S. (2011). Plant physiology and proteomics reveals the leaf response to drought in alfalfa (Medicago sativa L.). J. Exp. Bot. 62, 111-123. doi: 10.1093/jxb/erq249

Arbona, V., Manzi, M., de Ollas, C., and Gómez-Cadenas, A. (2013). Metabolomics as a tool to investigate abiotic stress tolerance in plants. Int. J. Mol. Sci. 14, 4885-4911. doi: 10.3390/ijms14034885

Bachi, A., and Bonaldi, T. (2008). Quantitative proteomics as a new piece of the systems biology puzzle. J. Proteomics 71, 357-367. doi: 10.1016/j.jprot.2008.07.001

Bais, P., Moon, S. M., He, K., Leitao, R., Dreher, K., Walk, T., et al. (2010). PlantMetabolomics. org: A web portal for plant metabolomics experiments. Plant Physiol. 152, 1807-1816. doi: 10.1104/pp.109.151027

Bajad, S., and Shulaev, V. (2007). Highly-parallel metabolomics approaches using LC-MS for pharmaceutical and environmental analysis. Trends Analyt. Chem. 26, 625-636. doi: 10.1016/j.trac.2007.02.009

Beckers, G. J., Hoehenwarter, W., Röhrig, H., Conrath, U., and Weckwerth, W. (2014). Tandem metal-oxide affinity chromatography for enhanced depth of phosphoproteome analysis. Methods Mol. Biol. 1072, 621-632. doi: 10.1007/978-1-62703-631-3_42

Benkeblia, N., Shinano, T., and Osaki, M. (2007). Metabolite profiling and assessment of metabolome compartmentation of soybean leaves using nonaqueous fractionation and GC-MS analysis. Metabolomics 3, 297-305. doi: 10.1007/s11306-007-0078-y

Bertram, H. C., Weisbjerg, M. R., Jensen, C. S., Pedersen, M. G., Didion, T., Petersen, B. O., et al. (2010). Seasonal changes in the metabolic fingerprint of 21 grass and legume cultivars studied by nuclear magnetic resonance-based metabolomics. J. Agric. Food Chem. 58, 4336-4341. doi: 10.1021/jf904321p

Bhalla, R., Narasimhan, K., and Swarup, S. (2005). Metabolomics and its role in understanding cellular responses in plants. Plant Cell Rep. 24, 562-571. doi: 10.1007/s00299-005-0054-9

Bhushan, D., Jaiswal, D. K., Ray, D., Basu, D., Datta, A., Chakraborty, S., et al. (2011). Dehydration-responsive reversible and irreversible changes in the extracellular matrix: Comparative proteomics of chickpea genotypes with contrasting tolerance. J. Proteome Res. 10, 2027-2046. doi: 10.1021/pr200010f

Bhushan, D., Pandey, A., Choudhary, M. K., Datta, A., Chakraborty, S., and Chakraborty, N. (2007). Comparative proteomics analysis of differentially expressed proteins in chickpea extracellular matrix during dehydration stress. Mol. Cell. Proteomics 6, 1868-1884. doi: 10.1074/mcp.M700015-MCP200

Borg, S., Pødenphant, L., Jensen, T. J., and Poulsen, C. (1999). Plant cell growth and differentiation may involve GAP regulation of Rac activity. FEBS Lett. 453, 341-345. doi: 10.1016/S0014-5793(99)00750-4

Bourgeois, M., Jacquin, F., Savois, V., Sommerer, N., Labas, V., Henry, C., et al. (2009). Dissecting the proteome of pea mature seeds reveals the phenotypic plasticity of seed protein composition. Proteomics 9, 254-271. doi: 10.1002/pmic.200700903
Bracha-Drori, K., Shichrur, K., Katz, A., Oliva, M., Angelovici, R., Yalovsky, S., et al. (2004). Detection of protein-protein interactions in plants using bimolecular fluorescence complementation. Plant J. 40, 419-427. doi: 10.1111/j.1365313X.2004.02206.x

Brasileiro, A. C., Morgante, C. V., Araujo, A. C., Leal-Bertioli, S. C., Silva, A. K., Martins, A. C., et al. (2015). Transcriptome profiling of wild Arachis from water-limited environments uncovers drought tolerance candidate genes. Plant Mol. Biol. Rep. 1-17. doi: 10.1007/s11105-015-0882-x

Braun, P., Aubourg, S., Van Leene, J., De Jaeger, G., and Lurin, C. (2013). Plant protein interactomes. Annu. Rev. Plant Biol. 64, 161-187. doi: 10.1146/annurev-arplant-050312-120140

Brechenmacher, L., Lee, J., Sachdev, S., Song, Z., Nguyen, T. H. N., Joshi, T., et al. (2009). Establishment of a protein reference map for soybean root hair cells. Plant Physiol. 149, 670-682. doi: 10.1104/pp.108.131649

Brechenmacher, L., Lei, Z., Libault, M., Findley, S., Sugawara, M., Sadowsky, M. J., et al. (2010). Soybean metabolites regulated in root hairs in response to the symbiotic bacterium Bradyrhizobium japonicum. Plant Physiol. 153, 1808-1822. doi: 10.1104/pp.110.157800

Brechenmacher, L., Nguyen, T. H. N., Hixson, K., Libault, M., Aldrich, J., PasaTolic, L., et al. (2012). Identification of soybean proteins from a single cell type: The root hair. Proteomics 12, 3365-3373. doi: 10.1002/pmic.2012 00160

Broeckling, C. D., Huhman, D. V., Farag, M. A., Smith, J. T., May, G. D., Mendes, P., et al. (2005). Metabolic profiling of Medicago truncatula cell cultures reveals the effects of biotic and abiotic elicitors on metabolism. J. Exp. Bot. 56, 323-336. doi: $10.1093 / \mathrm{jxb} / \mathrm{eri058}$

Brosowska-Arendt, W., Gallardo, K., Sommerer, N., and Weidner, S. (2014). Changes in the proteome of pea (Pisum sativum L.) seeds germinating under optimal and osmotic stress conditions and subjected to post-stress recovery. Acta Physiol. Plant 36, 795-807. doi: 10.1007/s11738-013-1458-8

Brückner, A., Polge, C., Lentze, N., Auerbach, D., and Schlattner, U. (2009). Yeast two-hybrid, a powerful tool for systems biology. Int. J. Mol. Sci. 18, 2763-2788. doi: 10.3390/ijms10062763

Campbell, P. M., Reiner, D., Moore, A. E., Lee, R. Y., Epstein, M. M., and Higgins, T. J. V. (2011). Comparison of the $\alpha$-amylase inhibitor-1 from common bean (Phaseolus vulgaris) varieties and transgenic expression in other legumes posttranslational modifications and immunogenicity. J. Agric. Food Chem. 59, 6047-6054. doi: 10.1021/jf200456j

Campbell, R., Pont, S. D., Morris, J. A., McKenzie, G., Sharma, S. K., Hedley, P. E., et al. (2014). Genome-wide QTL and bulked transcriptomic analysis reveals new candidate genes for the control of tuber carotenoid content in potato (Solanum tuberosum L.). Theor. Appl. Genet. 127, 1917-1933. doi: 10.1007/s00122-014-2349-0

Castellana, N. E., Payne, S. H., Shen, Z., Stanke, M., Bafna, V., and Briggs, S. P. (2008). Discovery and revision of Arabidopsis genes by proteogenomics. Proc. Natl. Acad. Sci. U.S.A. 105, 21034-21038. doi: 10.1073/pnas. 0811066106

Castillejo, M. Á., Fernández-Aparicio, M., and Rubiales, D. (2012). Proteomic analysis by two-dimensional differential in gel electrophoresis (2D DIGE) of the early response of Pisum sativum to Orobanche crenata. J. Exp. Bot. 63, 107-119. doi: $10.1093 / \mathrm{jxb} / \mathrm{err} 246$

Castillejo, M. A., Staudinger, C., Egelhofer, V., and Wienkoop, S. (2014). Medicago truncatula proteomics for systems biology: Novel rapid shotgun LCMS approach for relative quantification based on full-scan selective peptide extraction (Selpex). Methods Mol. Biol. 1072, 303-313. doi: 10.1007/978-162703-631-3_22

Charlton, A. J., Donarski, J. A., Harrison, M., Jones, S. A., Godward, J., Oehlschlager, S., et al. (2008). Responses of the pea (Pisum sativum L.) leaf metabolome to drought stress assessed by nuclear magnetic resonance spectroscopy. Metabolomics 4, 312-327. doi: 10.1007/s11306-008-0128-0

Charpentier, M., Bredemeier, R., Wanner, G., Takeda, N., Schleiff, E., and Parniske, M. (2008). Lotus japonicus CASTOR and POLLUX are ion channels essential for perinuclear calcium spiking in legume root endosymbiosis. Plant Cell 20, 3467-3479. doi: 10.1105/tpc.108.063255

Chatelain, E., Hundertmark, M., Leprince, O., Gall, S. L., Satour, P., DelignyPenninck, S., et al. (2012). Temporal profiling of the heat-stable proteome during late maturation of Medicago truncatula seeds identifies a restricted subset of late embryogenesis abundant proteins associated with longevity. Plant Cell Environ. 35, 1440-1455. doi: 10.1111/j.1365-3040.2012.02501.x 
Chen, Y., Hoehenwarter, W., and Weckwerth, W. (2010). Comparative analysis of phytohormone-responsive phosphoproteins in Arabidopsis thaliana using TiO2-phosphopeptide enrichment and mass accuracy precursor alignment. Plant J. 63, 1-17. doi: 10.1111/j.1365-313x.2010.04218.x

Cheng, J., Yuan, C., and Graham, T. L. (2011). Potential defense-related prenylated isoflavones in lactofen-induced soybean. Phytochem. 72, 875-881. doi: 10.1016/j.phytochem.2011.03.010

Chung, E., Cho, C. W., So, H. A., Kang, J. S., Chung, Y. S., and Lee, J. H. (2013). Overexpression of $\mathrm{VrUBC} 1$, a mung bean E2 ubiquitin-conjugating enzyme, enhances osmotic stress tolerance in Arabidopsis. PLoS ONE 8:e66056. doi: 10.1371/journal.pone.0066056

Colditz, F., and Braun, H. P. (2010). Medicago truncatula proteomics. J. Proteomics 73, 1974-1985. doi: 10.1016/j.jprot.2010.07.004

Dam, S., Dyrlund, T. F., Ussatjuk, A., Jochimsen, B., Nielsen, K., Goffard, N., et al. (2014). Proteome reference maps of the Lotus japonicus nodule and root. Proteomics 14, 230-240. doi: 10.1002/pmic.201300353

Dash, S., Campbell, J. D., Cannon, E. K. S., Cleary, A. M., Huang, W., Kalberer, S. R., et al. (2015). Legume information system (LegumeInfo. org): A key component of a set of federated data resources for the legume family. Nucleic Acids Res. gkv1159. doi: 10.1093/nar/gkv1159

Den Herder, G., Yoshida, S., Antolín-Llovera, M., Ried, M. K., and Parniske, M. (2012). Lotus japonicus E3 ligase SEVEN IN ABSENTIA4 destabilizes the symbiosis receptor-like kinase SYMRK and negatively regulates rhizobial infection. Plant Cell 24, 1691-1707. doi: 10.1105/tpc.110.082248

Dias, D. A., Hill, C. B., Jayasinghe, N. S., Atieno, J., Sutton, T., and Roessner, U. (2015). Quantitative profiling of polar primary metabolites of two chickpea cultivars with contrasting responses to salinity. J. Chromatogr. B 1000, 1-13. doi: 10.1016/j.jchromb.2015.07.002

Dita, M. A., Rispail, N., Prats, E., Rubiales, D., and Singh, K. B. (2006). Biotechnology approaches to overcome biotic and abiotic stress constraints in legumes. Euphytica 147, 1-24. doi: 10.1007/s10681-006-6156-9

Doerfler, H., Lyon, D., Nägele, T., Sun, X., Fragner, L., Hadacek, F., et al. (2013). Granger causality in integrated GC-MS and LC-MS metabolomics data reveals the interface of primary and secondary metabolism. Metabolomics 9, 564-574. doi: 10.1007/s11306-012-0470-0

Doerfler, H., Sun, X., Wang, L., Engelmeier, D., Lyon, D., and Weckwerth, W. (2014). mzGroupAnalyzer-Predicting pathways and novel chemical structures from untargeted high-throughput metabolomics data. PLoS ONE 9:e96188. doi: 10.1371/journal.pone.0096188

Domoney, C., Welham, T., Sidebottom, C., and Firmin, J. L. (1995). Multiple isoforms of Pisum trypsin inhibitors result from modification of two primary gene products. FEBS Lett. 360, 15-20. doi: 10.1016/0014-5793(95)00070-P

Downes, B., and Vierstra, R. D. (2005). Post-translational regulation in plants employing a diverse set of polypeptide tags. Biochem. Soc. Trans. 33, 393-400. doi: 10.1042/BST0330393

Dubinin, J., Braun, H. P., Schmitz, U., and Colditz, F. (2011). The mitochondrial proteome of the model legume Medicago truncatula. Biochim. Biophys. Acta 1814, 1658-1668. doi: 10.1016/j.bbapap.2011.08.008

Fan, H., Xu, Y., Du, C., and Wu, X. (2015). Phloem sap proteome studied by iTRAQ provides integrated insight into salinity response mechanisms in cucumber plants. J. Proteomics 125, 54-67. doi: 10.1016/j.jprot.2015.05.001

Fang, Y., Macool, D. J., Xue, Z., Heppard, E. P., Hainey, C. F., Tingey, S. V., et al. (2002). Development of a high-throughput yeast two-hybrid screening system to study protein-protein interactions in plants. Mol. Genet. Genomics 267, 142-153. doi: 10.1007/s00438-002-0656-7

Farag, M. A., Huhman, D. V., Lei, Z., and Sumner, L. W. (2007). Metabolic profiling and systematic identification of flavonoids and isoflavonoids in roots and cell suspension cultures of Medicago truncatula using HPLC-UV-ESI-MS and GC-MS. Phytochemistry 68, 342-354. doi: 10.1016/j.phytochem.2006.10.023

Fernie, A. R., and Schauer, N. (2009). Metabolomics-assisted breeding: A viable option for crop improvement? Trends Genet. 25, 39-48. doi: 10.1016/j.tig.2008.10.010

Fiehn, O., Kopka, J., Dörmann, P., Altmann, T., Trethewey, R. N., and Willmitzer, L. (2000). Metabolite profiling for plant functional genomics. Nat. Biotechnol. 18, 1157-1161. doi: 10.1038/81137

Fukao, Y. (2012). Protein-protein interactions in plants. Plant Cell Physiol. 53, 617-625. doi: $10.1093 / p c p / p c s 026$
García-Villalba, R., León, C., Dinelli, G., Segura-Carretero, A., FernándezGutiérrez, A., Garcia-Cañas, V., et al. (2008). Comparative metabolomic study of transgenic versus conventional soybean using capillary electrophoresistime-of-flight mass spectrometry. J. Chromatogr. A 1195, 164-173. doi: 10.1016/j.chroma.2008.05.018

Glinski, M., and Weckwerth, W. (2006). The role of mass spectrometry in plant systems biology. Mass Spectrom. Rev. 25, 173-214. doi: 10.1002/mas.20063

Gobbato, E., Marsh, J. F., Vernié, T., Wang, E., Maillet, F., Kim, J., et al. (2012). A GRAS-type transcription factor with a specific function in mycorrhizal signaling. Curr. Biol. 22, 2236-2241. doi: 10.1016/j.cub.2012.09.044

Gokulakannan, G. G., and Niehaus, K. (2010). Characterization of the 'Medicago truncatula' cell wall proteome in cell suspension culture upon elicitation and suppression of plant defense. J. Plant Physiol. 167, 1533-1541. doi: 10.1016/j.jplph.2010.06.023

Goodacre, R., York, E. V., Heald, J. K., and Scott, I. M. (2003). Chemometric discrimination of unfractionated plant extracts analyzed by electrospray mass spectrometry. Phytochemistry 62, 859-863. doi: 10.1016/S0031-9422(02) 00718-5

Graham, P. H., and Vance, C. P. (2003). Legumes: Importance and constraints to greater use. Plant Physiol. 131, 872-877. doi: 10.1104/pp.017004

Griffin, T. J., and Aebersold, R. (2001). Advances in proteome analysis by mass spectrometry. J. Biol. Chem. 276, 45497-45500. doi: 10.1074/jbc.R100014200

Griffin, T. J., Gygi, S. P., Rist, B., Aebersold, R., Loboda, A., Jilkine, A., et al. (2001). Quantitative proteomic analysis using a MALDI quadrupole time-of-flight mass spectrometer. Anal. Chem. 73, 978-986. doi: 10.1021/ac001169y

Grimsrud, P. A., den Os, D., Wenger, C. D., Swaney, D. L., Schwartz, D., Sussman, M. R., et al. (2010). Large-scale phosphoprotein analysis in Medicago truncatula roots provides insight into in vivo kinase activity in legumes. Plant Physiol. 152, 19-28. doi: 10.1104/pp.109.149625

Hajduch, M., Ganapathy, A., Stein, J. W., and Thelen, J. J. (2005). A systematic proteomic study of seed-filling in soybean: Establishment of high resolution two-dimensional reference maps, expression profiles, and an interactive proteome database. Plant Physiol. 137, 1397-1419. doi: 10.1104/pp.104. 056614

Halket, J. M., Waterman, D., Przyborowska, A. M., Patel, R. K., Fraser, P. D., and Bramley, P. M. (2005). Chemical derivatization and mass spectral libraries in metabolic profiling by GC/MS and LC/ MS/MS. J. Exp. Bot. 56, 219-243. doi: $10.1093 /$ jxb/eri069

Hegeman, A. D., Schulte, C. F., Cui, Q., Lewis, I. A, Huttlin, E. L., Eghbalnia, H., et al. (2007). Stable isotope assisted assignment of elemental compositions for metabolomics. Anal. Chem. 79, 6912-6921. doi: 10.1021/ac070346t

Heidarvand, L., and Maali-Amiri, R. (2013). Physio-biochemical and proteome analysis of chickpea in early phases of cold stress. J. Plant Physiol. 170, 459-469. doi: 10.1016/j.jplph.2012.11.021

Hernández, G., Ramírez, M., Valdés-López, O., Goffard, N., Weiller, G., AparicioFabre, R., et al. (2009). Global changes in the transcript and metabolic profiles during symbiotic nitrogen fixation in phosphorus-stressed common bean plants. Plant Physiol. 151, 1221-1238. doi: 10.1104/pp.109.143842

Hernández, G., Ramírez, M., Valdés-López, O., Tesfaye, M., Graham, M. A., Czechowski, T., et al. (2007). Phosphorus stress in common bean: Root transcript and metabolic responses. Plant Physiol. 144, 752-767. doi: 10.1104/pp.107.096958

Hiremath, P. J., Farmer, A., Cannon, S. B., Woodward, J., Kudapa, H., Tuteja, R., et al. (2011). Large-scale transcriptome analysis in chickpea (Cicer arietinum L.), an orphan legume crop of the semi-arid tropics of Asia and Africa. Plant Biotechnol. J. 9, 922-931. doi: 10.1111/j.1467-7652.2011.00625.x

Hirsch, S., Kim, J., Muñoz, A., Heckmann, A. B., Downie, J. A., and Oldroyd, G. E. (2009). GRAS proteins form a DNA binding complex to induce gene expression during nodulation signaling in Medicago truncatula. Plant Cell 21, 545-557. doi: 10.1105/tpc.108.064501

Hoehenwarter, W., Thomas, M., Nukarinen, E., Egelhofer, V., Röhrig, H. Weckwerth, W., et al. (2013). Identification of novel in vivo MAP kinase substrates in Arabidopsis thaliana through use of tandem metal oxide affinity chromatography. Mol. Cell Proteomics 12, 369-380. doi: 10.1074/mcp.M112.020560

Hoehenwarter, W., van Dongen, J. T., Wienkoop, S., Steinfath, M., Hummel, J., Erban, A., et al. (2008). A rapid approach for phenotype-screening and database 
independent detection of cSNP/protein polymorphism using mass accuracy precursor alignment. Proteomics 8, 4214-4225. doi: 10.1002/pmic.200701047

Hossain, Z., Khatoon, A., and Komatsu, S. (2013). Soybean proteomics for unravelling abiotic stress response mechanism. J. Proteome Res. 12, 4670-4684. doi: $10.1021 / \mathrm{pr} 400604 \mathrm{~b}$

Hossain, Z., and Komatsu, S. (2014a). Potentiality of soybean proteomics in untying the mechanism of flood and drought stress tolerance. Proteomes 2, 107-127. doi: 10.3390/proteomes 2010107

Hossain, Z., and Komatsu, S. (2014b). Soybean proteomics. Methods Mol. Biol. 1072, 315-331. doi: 10.1007/978-1-62703-631-3_23

Hossain, Z., Nouri, M. Z., and Komatsu, S. (2012). Plant cell organelle proteomics in response to abiotic stress. J. Proteome Res. 11, 37-48. doi: 10.1021/pr200863r

Hu, J., Rampitsch, C., and Bykova, N. V. (2015). Advances in plant proteomics toward improvement of crop productivity and stress resistance. Front. Plant Sci. 6:209. doi: 10.3389/fpls.2015.00209

Huege, J., Sulpice, R., Gibon, Y., Lisec, J., Koehl, K., and Kopka, J. (2007). GCEI-TOF-MS analysis of in vivo carbon-partitioning into soluble metabolite pools of higher plants by monitoring isotope dilution after $13 \mathrm{CO} 2$ labelling. Phytochemistry 68, 2258-2272. doi: 10.1016/j.phytochem.2007.03.026

Huhman, D. V., and Sumner, L. W. (2002). Metabolic profiling of saponins in Medicago sativa and Medicago truncatula using HPLC coupled to an electrospray ion-trap mass spectrometer. Phytochemistry 59, 347-360. doi: 10.1016/S0031-9422(01)00432-0

Hummel, J., Niemann, M., Wienkoop, S., Schulze, W., Steinhauser, D., Selbig, J., et al. (2007). ProMEX: A mass spectral reference database for proteins and protein phosphorylation sites. BMC Bioinformatics 8:216. doi: 10.1186/14712105-8-216

Imin, N., De-Jong, F., Mathesius, U., Van Noorden, G., Saeed, N. A., Wang, X. D., et al. (2004). Proteome reference maps of Medicago truncatula embryogenic cell cultures generated from single protoplasts. Proteomics 4, 1883-1896. doi: 10.1002/pmic. 200300803

Ino, Y., Ishikawa, A., Nomura, A., Kajiwara, H., Harada, K., and Hirano, H. (2014). Phosphoproteome analysis of Lotus japonicus seeds. Proteomics 14, 116-120. doi: 10.1002/pmic.201300237

Irar, S., González, E. M., Arrese-Igor, C., and Marino, D. (2014). A proteomic approach reveals new actors of nodule response to drought in split-root grown pea plants. Physiol. Plant 152, 634-645. doi: 10.1111/ppl.12214

Jaiswal, D. K., Mishra, P., Subba, P., Rathi, D., Chakraborty, S., and Chakraborty, N. (2014). Membrane-associated proteomics of chickpea identifies Sad1/UNC84 protein (CaSUN1), a novel component of dehydration signaling. Sci. Rep. 4 , 4177. doi: 10.1038/srep04177

Jaiswal, D. K., Ray, D., Subba, P., Mishra, P., Gayali, S., Datta, A., et al. (2012). Proteomic analysis reveals the diversity and complexity of membrane proteins in chickpea (Cicer arietinum L.). Proteome Sci. 10, 59-5956. doi: 10.1186/14775956-10-59

Johnson, H. E., Broadhurst, D., Goodacre, R., and Smith, A. R. (2003). Metabolic fingerprinting of salt-stressed tomatoes. Phytochemistry 62, 919-928. doi: 10.1016/S0031-9422(02)00722-7

Johnson, H. E., Broadhurst, D., Kell, D. B., Theodorou, M. K., Merry, R. J., and Griffith, G. W. (2004). High-throughput metabolic fingerprinting of legume silage fermentations via Fourier transform infrared spectroscopy and chemometrics. Appl. Environ. Microbiol. 70, 1583-1592. doi: 10.1128/AEM.70.3.1583-1592.2004

Jorrín-Novo, J. V., Pascual, J., Sánchez-Lucas, R., Romero-Rodríguez, M. C., Rodríguez-Ortega, M. J., Lenz, C., et al. (2015). Fourteen years of plant proteomics reflected in Proteomics: Moving from model species and 2DE-based approaches to orphan species and gel-free platforms. Proteomics 15, 1089-1112. doi: 10.1002/pmic.201400349

Joshi, T., Fitzpatrick, M. R., Chen, S., Liu, Y., Zhang, H., Endacott, R. Z., et al. (2014). Soybean knowledge base (SoyKB): A web resource for integration of soybean translational genomics and molecular breeding. Nucleic Acids Res. 42, D1245-D1252. doi: 10.1093/nar/gkt905

Kang, Y. J., Kim, S. K., Kim, M. Y., Lestari, P., Kim, K. H., Ha, B. K., et al. (2014). Genome sequence of mungbean and insights into evolution within Vigna species. Nat. Commun. 5, 5443. doi: 10.1038/ncomms6443

Kang, Y. J., Satyawan, D., Shim, S., Lee, T., Lee, J., Hwang, W. J., et al. (2015). Draft genome sequence of adzuki bean, Vigna angularis. Sci. Rep. 5:8069. doi: $10.1038 /$ srep08069
Karmakar, K., Rana, A., Rajwar, A., Sahgal, M., and Johri, B. N. (2015). "Legumerhizobia symbiosis under stress," in Plant Microbes Symbiosis: Applied Facets, ed N. K. Arora (New Delhi; Heidelberg: Springer), 241-258.

Katam, R., Basha, S. M., Suravajhala, P., and Pechan, T. (2010). Analysis of peanut leaf proteome. J. Proteome Res. 9, 2236-2254. doi: 10.1021/pr901009n

Kav, N. N. V., Srivastava, S., Goonewardene, L., and Blade, S. F. (2004). Proteome-level changes in the roots of Pisum sativum in response to salinity. Ann. Appl. Biol. 145, 217-230. doi: 10.1111/j.1744-7348.2004.tb00378.x

Khatoon, A., Rehman, S., Hiraga, S., Makino, T., and Komatsu, S. (2012). Organ-specific proteomics analysis for identification of response mechanism in soybean seedlings under flooding stress. J. Proteomics 75, 5706-5723. doi: 10.1016/j.jprot.2012.07.031

Kim, J. C., Lee, S. H., Cheong, Y. H., Yoo, C. M., Lee, S. I., Chun, H. J., et al. (2001). A novel cold-inducible zinc finger protein from soybean, SCOF-1, enhances cold tolerance in transgenic plants. Plant J. 25, 247-259. doi: 10.1046/j.1365313x.2001.00947.x

Komatsu, S., Yamamoto, A., Nakamura, T., Nouri, M. Z., Nanjo, Y., Nishizawa, K., et al. (2011). Comprehensive analysis of mitochondria in roots and hypocotyls of soybean under flooding stress using proteomics and metabolomics techniques. J. Proteome Res. 10, 3993-4004. doi: 10.1021/pr2001918

Kosová, K., Vítámvás, P., Prášil, I. T., and Renaut, J. (2011). Plant proteome changes under abiotic stress-Contribution of proteomics studies to understanding plant stress response. J. Proteomics 74, 1301-1322. doi: 10.1016/j.jprot.2011.02.006

Kottapalli, K. R., Rakwal, R., Shibato, J., Burow, G., Tissue, D., Burke, J., et al. (2009). Physiology and proteomics of the water-deficit stress response in three contrasting peanut genotypes. Plant Cell Environ. 32, 380-407. doi: 10.1111/j.1365-3040.2009.01933.x

Kottapalli, K. R., Zabet-Moghaddam, M., Rowland, D., Faircloth, W., Mirzaei, M., Haynes, P. A., et al. (2013). Shotgun label-free quantitative proteomics of waterdeficit-stressed midmature peanut (Arachis hypogaea L.) seed. J. Proteome Res. 12, 5048-5057. doi: 10.1021/pr400936d

Krishnan, P., Kruger, N. J., and Ratcliffe, R. G. (2005). Metabolite fingerprinting and profiling in plants using NMR. J. Exp. Bot. 56, 255-265. doi: $10.1093 / \mathrm{jxb} /$ eri010

Kudapa, H., Ramalingam, A., Nayakoti, S., Chen, X., Zhuang, W. J., Liang, X., et al. (2013). Functional genomics to study stress responses in crop legumes: Progress and prospects. Funct. Plant Biol. 40, 1221-1233. doi: 10.1071/FP13191

Kumar, R., Kumar, A., Subba, P., Gayali, S., Barua, P., Chakraborty, S., et al. (2014). Nuclear phosphoproteome of developing chickpea seedlings (Cicer arietinum L.) and protein-kinase interaction network. J. Proteomics 105, 58-73. doi: 10.1016/j.jprot.2014.04.002

Lafaye, A., Labarre, J., Tabet, J. C., Ezan, E., and Junot, C. (2005). Liquid chromatography-mass spectrometry and $15 \mathrm{~N}$ metabolic labeling for quantitative metabolic profiling. Anal. Chem. 77, 2026-2033. doi: $10.1021 / \mathrm{ac} 048657 \mathrm{~g}$

Lam, H. M., Xu, X., Liu, X., Chen, W., Yang, G., Wong, F., et al. (2010). Resequencing of 31 wild and cultivated soybean genomes identifies patterns of genetic diversity and selection. Nat. Genet. 42, 1053-1059. doi: 10.1038/ng.715

Lange, V., Picotti, P., Domon, B., and Aebersold, R. (2008). Selected reaction monitoring for quantitative proteomics: A tutorial. Mol. Sys. Biol. 4, 222. doi: $10.1038 / \mathrm{msb} .2008 .61$

Langridge, P., and Fleury, D. (2011). Making the most of 'omics' for crop breeding. Trends Biotechnol. 29, 33-40. doi: 10.1016/j.tibtech.2010.09.006

Larrainzar, E., Wienkoop, S., Scherling, C., Kempa, S., Ladrera, R., Arrese-Igor, C., et al. (2009). Carbon metabolism and bacteroid functioning are involved in the regulation of nitrogen fixation in Medicago truncatula under drought and recovery. Mol. Plant Microbe Interact. 22, 1565-1576. doi: 10.1094/MPMI-22$12-1565$

Larrainzar, E., Wienkoop, S., Weckwerth, W., Ladrera, R., Arrese-Igor, C., and González, E. M. (2007). Medicago truncatula root nodule proteome analysis reveals differential plant and bacteroid responses to drought stress. Plant Physiol. 144, 1495-1507. doi: 10.1104/pp.107.101618

Lee, L. Y., Wu, F. H., Hsu, C. T., Shen, S. C., Yeh, H. Y., Liao, D. C., et al. (2012). Screening a cDNA library for protein-protein interactions directly in planta. Plant Cell 24, 1746-1759. doi: 10.1105/tpc.112.097998

Lei, Z., Dai, X., Watson, B. S., Zhao, P. X., and Sumner, L. W. (2011). A legume specific protein database (LegProt) improves the number of 
identified peptides, confidence scores and overall protein identification success rates for legume proteomics. Phytochemistry 72, 1020-1027. doi: 10.1016/j.phytochem.2011.01.026

Lei, Z., Elmer, A. M., Watson, B. S., Dixon, R. A., Mendes, P. J., and Sumner, L. W. (2005). A two-dimensional electrophoresis proteomic reference map and systematic identification of 1367 proteins from a cell suspension culture of the model legume Medicago truncatula. Mol. Cell Proteomics 4, 1812-1825. doi: 10.1074/mcp.D500005-MCP200

Liao, Y., Zou, H. F., Wang, H. W., Zhang, W. K., Ma, B., Zhang, J. S., et al. (2008a). Soybean GmMYB76, GmMYB92, and GmMYB177 genes confer stress tolerance in transgenic Arabidopsis plants. Cell Res. 18, 1047-1060. doi: $10.1038 / \mathrm{cr} .2008 .280$

Liao, Y., Zou, H. F., Wei, W., Hao, Y. J., Tian, A. G., Huang, J., et al. (2008b). Soybean GmbZIP44, GmbZIP62 and GmbZIP78 genes function as negative regulator of $A B A$ signaling and confer salt and freezing tolerance in transgenic Arabidopsis. Planta 228, 225-240. doi: 10.1007/s00425-008-0731-3

Liu, G. T., Ma, L., Duan, W., Wang, B. C., Li, J. H., Xu, H. G., et al. (2014). Differential proteomic analysis of grapevine leaves by iTRAQ reveals responses to heat stress and subsequent recovery. BMC Plant Biol. 14:110. doi: 10.1186/1471-2229-14-110

Ma, H., Song, L., Shu, Y., Wang, S., Niu, J., Wang, Z., et al. (2012). Comparative proteomic analysis of seedling leaves of different salt tolerant soybean genotypes. J. Proteomics 75, 1529-1546. doi: 10.1016/j.jprot.2011. 11.026

Mahajan, S., Sopory, S. K., and Tuteja, N. (2006). Cloning and characterization of CBL-CIPK signalling components from a legume (Pisum sativum). FEBS J. 273, 907-925. doi: 10.1111/j.1742-4658.2006.05111.x

Marouga, R., David, S., and Hawkins, E. (2005).The development of the DIGE system: 2D fluorescence difference gel analysis technology Anal. Bioanal. Chem. 382, 669-678. doi: 10.1007/s00216-005-3126-3

Mashego, M. R., Wu, L., Van Dam, J. C., Ras, C., Vinke, J. L., Van Winden, W. A., et al. (2004). MIRACLE: Mass isotopomer ratio analysis of U-13Clabeled extracts. A new method for accurate quantification of changes in concentrations of intracellular metabolites. Biotechnol. Bioeng. 85, 620-628. doi: 10.1002/bit.10907

Mathesius, U., Djordjevic, M. A., Oakes, M., Goffard, N., Haerizadeh, F., Weiller, G. F., et al. (2011). Comparative proteomic profiles of the soybean (Glycine max) root apex and differentiated root zone. Proteomics 11, 1707-1719. doi: 10.1002/pmic.201000619

Mazzucotelli, E., Mastrangelo, A. M., Crosatti, C., Guerra, D., Stanca, A. M., and Cattivelli, L. (2008). Abiotic stress response in plants: When posttranscriptional and post-translational regulations control transcription. Plant Sci. 174, 420-431. doi: 10.1016/j.plantsci.2008.02.005

Messinese, E., Mun, J. H., Yeun, L. H., Jayaraman, D. K., Rougé, P., Barre, A., et al. (2007). A novel nuclear protein interacts with the symbiotic DMI3 calciumand calmodulin-dependent protein kinase of Medicago truncatula. Mol. Plant Microbe Interact. 20, 912-921. doi: 10.1094/MPMI-20-8-0912

Misra, S., Wu, Y., Venkataraman, G., Sopory, S. K., and Tuteja, N. (2007). Heterotrimeric G-protein complex and G-protein-coupled receptor from a legume (Pisum sativum): Role in salinity and heat stress and cross-talk with phospholipase C. Plant J. 51, 656-669. doi: 10.1111/j.1365-313X.2007. 03169.x

Mithöfer, A., Müller, B., Wanner, G., and Eichacker, L. A. (2002). Identification of defence-related cell wall proteins in Phytophthora sojae-infected soybean roots by ESI-MS/MS. Mol. Plant Pathol. 3, 163-166. doi: 10.1046/j.13643703.2002.00109.x

Mohammadi, P., Moieni, A., Hiraga, S., and Komatsu, S. (2012). Organ-specific proteomic analysis of drought-stressed soybean seedlings. J. Proteomics 75, 1906-1923. doi: 10.1016/j.jprot.2011.12.041

Muneer, S., Hakeem, K. R., Mohamed, R., and Lee, J. H. (2014). Cadmium toxicity induced alterations in the root proteome of green gram in contrasting response towards iron supplement. Int. J. Mol. Sci. 15, 6343-6355. doi: 10.3390/ijms15046343

Murakami, Y., Imaizumi-Anraku, H., Kouchi, H., Kawagushi, M., and Kawasaki, S. (2013). The transcription activation and homodimerization of Lotus japonicus Nod factor Signaling Pathway2 protein. Plant Signal Behav. 8:e26457. doi: $10.4161 / \mathrm{psb} .26457$
Nanjo, Y., Skultety, L., Uváćková, L. U., Klubicová, K., Hajduch, M., and Komatsu, S. (2011). Mass spectrometry-based analysis of proteomic changes in the root tips of flooded soybean seedlings. J. Proteome Res. 11, 372-385. doi: $10.1021 / \mathrm{pr} 200701 \mathrm{y}$

Nautrup-Pedersen, G., Dam, S., Laursen, B. S., Siegumfeldt, A. L., Nielsen, K., Goffard, N., et al. (2010). Proteome analysis of pod and seed development in the model legume Lotus japonicus. J. Proteome Res. 9, 5715-5726. doi: $10.1021 / \mathrm{pr} 100511 \mathrm{u}$

Ndassa, Y. M., Orsi, C., Marto, J. A., Chen, S., and Ross, M. M. (2006). Improved immobilized metal affinity chromatography for large-scale phosphoproteomics applications. J. Proteome Res. 5, 2789-2799. doi: 10.1021/pr0602803

Nouri, M. Z., and Komatsu, S. (2010). Comparative analysis of soybean plasma membrane proteins under osmotic stress using gel-based and LC MS/MS-based proteomics approaches. Proteomics 10, 1930-1945. doi: 10.1002/pmic.200900632

Obata, T., and Fernie, A. R. (2012). The use of metabolomics to dissect plant responses to abiotic stresses. Cell. Mol. Life Sci. 69, 3225-3243. doi: 10.1007/s00018-012-1091-5

Ohyanagi, H., Sakata, K., and Komatsu, S. (2012). Soybean Proteome Database 2012: Update on the comprehensive data repository for soybean proteomics. Front. Plant Sci. 3:110. doi: 10.3389/fpls.2012.00110

Ogura, T., Ogihara, J., Sunairi, M., Takeishi, H., Aizawa, T., Olivos-Trujillo, M. R., et al. (2014). Proteomic characterization of seeds from yellow lupin (Lupinus luteus L.). Proteomics 14, 1543-1546. doi: 10.1002/pmic.201300511

Pandey, A., Chakraborty, S., Datta, A., and Chakraborty, N. (2008). Proteomics approach to identify dehydration responsive nuclear proteins from chickpea (Cicer arietinum L.). Mol. Cell. Proteomics 7, 88-107. doi: 10.1074/mcp.M700314-MCP200

Pandey, A., Choudhary, M. K., Bhushan, D., Chattopadhyay, A., Chakraborty, S., Datta, A., et al. (2006). The nuclear proteome of chickpea (Cicer arietinum L.) reveals predicted and unexpected proteins. J. Proteome Res. 5, 3301-3311. doi: 10.1021/pr060147a

Pandey, A., and Mann, M. (2000). Proteomics to study genes and genomes. Nature 405, 837-846. doi: 10.1038/35015709

Parrish, J. R., Gulyas, K. D., and Finley, R. L. (2006). Yeast two-hybrid contributions to interactome mapping. Curr. Opin. Plant Biol. 17, 387-393. doi: 10.1016/j.copbio.2006.06.006

Patti, G. J., Yanes, O., and Siuzdak, G. (2012). Innovation: Metabolomics: The apogee of the omics trilogy. Nat. Rev. Mol. Cell Biol. 13, 263-269. doi: $10.1038 / \mathrm{nrm} 3314$

Peters, K., Belt, K., and Braun, H. P. (2013). 3D gel map of Arabidopsis complex I. Front. Plant Sci. 4:153. doi: 10.3389/fpls.2013.00153

Picotti, P., and Aebersold, R. (2012). Selected reaction monitoring-based proteomics: Workflows, potential, pitfalls and future directions. Nat. Methods 9, 555-566. doi: 10.1038/nmeth.2015

Ramalingam, A., Kudapa, H., Pazhamala, L. T., Garg, V., and Varshney, R. K. (2015). Gene expression and Yeast two-hybrid studies of a 1RMYB transcription factor mediating drought stress response in root tissues of chickpea (Cicer arietinum L.). Front. Plant Sci. 6:1117. doi: 10.3389/fpls.2015.01117

Rocha, M., Licausi, F., Araújo, W. L., Nunes-Nesi, A., Sodek, L., Fernie, A. R., et al. (2010). Glycolysis and the tricarboxylic acid cycle are linked by alanine aminotransferase during hypoxia induced by waterlogging of Lotus japonicus. Plant Physiol. 152, 1501-1513. doi: 10.1104/pp.109.150045

Rode, C., Senkler, M., Klodmann, J., Winkelmann, T., and Braun, H. P. (2011). GelMap - a novel software tool for building and presenting proteome reference maps. J. Proteomics 74, 2214-2219. doi: 10.1016/j.jprot.2011.06.017

Rodziewicz, P., Swarcewicz, B., Chmielewska, K., Wojakowska, A., and Stobiecki, M. (2014). Influence of abiotic stresses on plant proteome and metabolome changes. Acta Physiol. Plant 36, 1-19. doi: 10.1007/s11738-013-1402-y

Roessner, U., Wagner, C., Kopka, J., Trethewey, R. N., and Willmitzer, L. (2000). Technical advance: Simultaneous analysis of metabolites in potato tuber by gas chromatography-mass spectrometry. Plant J. 23, 131-142. doi: 10.1046/j.1365313x.2000.00774.x

Romero-Rodríguez, M. C., Pascual, J., Valledor, L., and Jorrín-Novo, J. (2014). Improving the quality of protein identification in non-model species. Characterization of Quercus ilex seed and Pinus radiata needle proteomes 
by using SEQUEST and custom databases. J. Proteomics 105, 85-91. doi: 10.1016/j.jprot.2014.01.027

Rose, C. M., Venkateshwaran, M., Grimsrud, P. A., Westphall, M. S., Sussman, M. R., Coon, J. J., et al. (2012a). Medicago PhosphoProtein Database: A repository for Medicago truncatula phosphoprotein data. Front. Plant Sci. 3:122. doi: 10.3389/fpls.2012.00122

Rose, C. M., Venkateshwaran, M., Volkening, J. D., Grimsrud, P. A., Maeda, J., Bailey, D. J., et al. (2012b). Rapid phosphoproteomic and transcriptomic changes in the rhizobia-legume symbiosis. Mol. Cell. Proteomics 11, 724-744. doi: 10.1074/mcp.M112.019208

Sadhukhan, A., Panda, S. K., and Sahoo, L. (2014). The cowpea RING ubiquitin ligase VuDRIP interacts with transcription factor VuDREB2A for regulating abiotic stress responses. Plant Physiol. Biochem. 83, 51-56. doi: 10.1016/j.plaphy.2014.07.007

Salavati, A., Khatoon, A., Nanjo, Y., and Komatsu, S. (2012). Analysis of proteomic changes in roots of soybean seedlings during recovery after flooding. J. Proteomics 75, 878-893. doi: 10.1016/j.jprot.2011.10.002

Salekdeh, G. H., and Komatsu, S. (2007). Crop proteomics: Aim at sustainable agriculture of tomorrow. Proteomics 7, 2976-2996. doi: 10.1002/pmic.200700181

Sanchez, D. H., Lippold, F., Redestig, H., Hannah, M. A., Erban, A., Krämer, U., et al. (2008). Integrative functional genomics of salt acclimatization in the model legume Lotus japonicus. Plant J. 53, 973-987. doi: 10.1111/j.1365313X.2007.03381.x

Sanchez, D. H., Pieckenstain, F. L., Escaray, F., Erban, A., Kraemer, U. T. E., Udvardi, M. K., et al. (2011). Comparative ionomics and metabolomics in extremophile and glycophytic Lotus species under salt stress challenge the metabolic pre-adaptation hypothesis. Plant Cell Environ. 34, 605-617. doi: 10.1111/j.1365-3040.2010.02266.x

Sanchez, D. H., Schwabe, F., Erban, A., Udvardi, M. K., and Kopka, J. (2012). Comparative metabolomics of drought acclimation in model and forage legumes. Plant Cell Environ. 35, 136-149. doi: 10.1111/j.13653040.2011.02423.x

Sato, D., Akashi, H., Sugimoto, M., Tomita, M., and Soga, T. (2013). Metabolomic profiling of the response of susceptible and resistant soybean strains to foxglove aphid, Aulacorthum solani Kaltenbach. J. Chromatogr. B 925, 95-103. doi: 10.1016/j.jchromb.2013.02.036

Sato, S., Nakamura, Y., Kaneko, T., Asamizu, E., Kato, T., Nakao, M., et al. (2008). Genome structure of the legume, Lotus japonicus. DNA Res. 15, 227-239. doi: 10.1093/dnares/dsn008

Schenkluhn, L., Hohnjec, N., Niehaus, K., Schmitz, U., and Colditz, F. (2010). Differential gel electrophoresis (DIGE) to quantitatively monitor early symbiosis-and pathogenesis-induced changes of the Medicago truncatula root proteome. J. Proteomics 73, 753-768. doi: 10.1016/j.jprot.2009. 10.009

Scherling, C., Roscher, C., Giavalisco, P., Schulze, E. D., and Weckwerth, W. (2010). Metabolomics unravel contrasting effects of biodiversity on the performance of individual plant species. PLOS ONE 5:e12569. doi: 10.1371/journal.pone.0012569

Schiltz, S., Gallardo, K., Huart, M., Negroni, L., Sommerer, N., and Burstin, J. (2004). Proteome reference maps of vegetative tissues in pea. An investigation of nitrogen mobilization from leaves during seed filling. Plant Physiol. 135, 2241-2260. doi: 10.1104/pp.104.041947

Schliemann, W., Ammer, C., and Strack, D. (2008). Metabolite profiling of mycorrhizal roots of Medicago truncatula. Phytochemistry 69, 112-146. doi: 10.1016/j.phytochem.2007.06.032

Schmidt, C., and Urlaub, H. (2012). Absolute quantification of proteins using standard peptides and multiple reaction monitoring. Methods Mol. Biol. 893, 249-265. doi: 10.1007/978-1-61779-885-6_17

Schmutz, J., Cannon, S. B., Schlueter, J., Ma, J., Mitros, T., Nelson, W., et al. (2010). Genome sequence of the palaeopolyploid soybean. Nature 463, 178-183. doi: 10.1038 /nature 08670

Schmutz, J., McClean, P. E., Mamidi, S., Wu, G. A., Cannon, S. B., Grimwood, J., et al. (2014). A reference genome for common bean and genome-wide analysis of dual domestications. Nat. Genet. 46, 707-713. doi: 10.1038/ng.3008

Senkler, M., and Braun, H. P. (2012). Functional annotation of 2D protein maps: The GelMap portal. Front. Plant Sci. 14:87. doi: 10.3389/fpls.2012.00087
Seo, J., and Lee, K. J. (2004). Post-translational modifications and their biological functions: Proteomic analysis and systematic approaches. J. Biochem. Mol. Biol. 37, 35-44. doi: 10.5483/BMBRep.2004.37.1.035

Shulaev, V. (2006). Metabolomics technology and bioinformatics. Brief Bioinform. 7, 128-139. doi: 10.1093/bib/bbl012

Shulaev, V., Cortes, D., Miller, G., and Mittler, R. (2008). Metabolomics for plant stress response. Physiol. Plant. 132, 199-208. doi: 10.1111/j.13993054.2007.01025. $\mathrm{x}$

Soga, T. (2007). Capillary electrophoresis-mass spectrometry for metabolomics. Methods Mol. Biol. 358, 129-137. doi: 10.1007/978-1-59745244-1_8

Soga, T., Ohashi, Y., Ueno, Y., Naraoka, H., Tomita, M., and Nishioka, T. (2003). Quantitative metabolome analysis using capillary electrophoresis mass spectrometry. J. Proteome Res. 2, 488-494. doi: 10.1021/pr034020m

Stasi, M., De Luca, M., and Bucci, C. (2015). Two-hybrid-based systems: Powerful tools for investigation of membrane traffic machineries. J. Biotechnol. 202, 105-117. doi: 10.1016/j.jbiotec.2014.12.007

Staszków, A., Swarcewicz, B., Banasiak, J., Muth, D., Jasiñski, M., and Stobiecki, M. (2011). LC/MS profiling of flavonoid glycoconjugates isolated from hairy roots, suspension root cell cultures and seedling roots of Medicago truncatula. Metabolomics 7, 604-613. doi: 10.1007/s11306-011-0287-2

Staudinger, C., Mehmeti, V., Turetschek, R., Lyon, D., Egelhofer, V., and Wienkoop, S. (2012). Possible role of nutritional priming for early salt and drought stress responses in Medicago truncatula. Front. Plant Sci. 3:285. doi: 10.3389/fpls.2012.00285

Steinfath, M., Strehmel, N., Peters, R., Schauer, N., Groth, D., Hummel, J., et al. (2010). Discovering plant metabolic biomarkers for phenotype prediction using an untargeted approach. Plant Biotechnol. J. 8, 900-911. doi: 10.1111/j.14677652.2010.00516.x

Subba, P., Barua, P., Kumar, R., Datta, A., Soni, K. K., Chakraborty, S., et al. (2013). Phosphoproteomic dynamics of chickpea (Cicer arietinum L.) reveals shared and distinct components of dehydration response. J. Proteome Res. 12, 5025-5047. doi: 10.1021/pr400628j

Subramanian, S., and Smith, D. L. (2013). "A proteomics approach to study soybean and its symbiont Bradyrhizobium japonicum- a review," in A Comprehensive Survey of International Soybean Research-Genetics, Physiology, Agronomy and Nitrogen Relationships, ed J. Board (Rijeka: INTECH Open Access Publisher), 978-953.

Sumner, L.W., Mendes, P., and Dixon, R. A. (2003). Plant metabolomics: Largescale phytochemistry in the functional genomics era. Phytochemistry 62, 817-836. doi: 10.1016/S0031-9422(02)00708-2

Swigonska, S., and Weidner, S. (2013). Proteomic analysis of response to long-term continuous stress in roots of germinating soybean seeds. J. Plant Physiol. 170, 470-479. doi: 10.1016/j.jplph.2012.11.020

Tavakolan, M., Alkharouf, N. W., Khan, F. H., and Natarajan, S. (2013). SoyProDB: A database for the identification of soybean seed proteins. Bioinformation 9, 165-167. doi: 10.6026/97320630009165

Thudi, M., Upadhyaya, H. D., Rathore, A., Gaur, P. M., Krishnamurthy, L., Roorkiwal, M., et al. (2014). Genetic dissection of drought and heat tolerance in chickpea through genome-wide and candidate gene-based association mapping approaches. PLoS ONE 9:e96758. doi: 10.1371/journal.pone.0096758

Tripathi, V., Parasuraman, B., Laxmi, A., and Chattopadhyay, D. (2009). CIPK6, a CBL-interacting protein kinase is required for development and salt tolerance in plants. Plant J. 58, 778-790. doi: 10.1111/j.1365-313X.2009.03812.x

Urbanczyk-Wochniak, E., and Sumner, L. W. (2007). MedicCyc: A biochemical pathway database for Medicago truncatula. Bioinformatics 23, 1418-1423. doi: 10.1093/bioinformatics/btm040

Vanderschuren, H., Lentz, E., Zainuddin, I., and Gruissem, W. (2013). Proteomics of model and crop plant species: Status, current limitations and strategic advances for crop improvement. J. Proteomics. 93, 5-19. doi: 10.1016/j.jprot.2013.05.036

van Wijk, K. J. (2001). Challenges and prospects of plant proteomics. Plant Physiol. 126, 501-508. doi: 10.1104/pp.126.2.501

Varshney, R. K., Chen, W., Li, Y., Bharti, A. K., Saxena, R. K., Schlueter, J. A., et al. (2012). Draft genome sequence of pigeonpea (Cajanus cajan), an orphan legume crop of resource-poor farmers. Nat. Biotechnol. 30, 83-89. doi: $10.1038 /$ nbt.2022 
Varshney, R. K., Close, T. J., Singh, N. K., Hoisington, D. A., and Cook, D. R. (2009). Orphan legume crops enter the genomics era! Curr. Opin. Plant Biol. 12, 202-210. doi: 10.1016/j.pbi.2008.12.004

Varshney, R. K., Mohan, S. M., Gaur, P. M., Gangarao, N. V., Pandey, M. K., Bohra, A., et al. (2013a). Achievements and prospects of genomics-assisted breeding in three legume crops of the semi-arid tropics. Biotechnol. Adv. 31, 1120-1134. doi: 10.1016/j.biotechadv.2013.01.001

Varshney, R. K., Song, C., Saxena, R. K., Azam, S., Yu, S., Sharpe, A. G., et al. (2013b). Draft genome sequence of chickpea (Cicer arietinum) provides a resource for trait improvement. Nat. Biotechnol. 31, 240-246. doi: $10.1038 /$ nbt. 2491

Varshney, R. K., Thudi, M., Nayak, S. N., Gaur, P. M., Kashiwagi, J., Krishnamurthy, L., et al. (2014). Genetic dissection of drought tolerance in chickpea (Cicer arietinum L.). Theor. Appl. Genet. 127, 445-462. doi: 10.1007/s00122-013-2230-6

Varshney, R. K., and Tuberosa, R. (2013). “Translational genomics in crop breeding for biotic stress resistance: An introduction" in Translational Genomics for Crop Breeding: Biotic Stress, Vol. 1, eds R. K. Varshney and R. Tuberosa (Chichester, UK: John Wiley \& Sons), 1-9.

von Mering, C., Krause, R., Snel, B., Cornell, M., Oliver, S. G., Fields, S., et al. (2002). Comparative assessment of large-scale data sets of protein-protein interactions. Nature 417, 399-403. doi: 10.1038/nature750

Walley, J. W., and Briggs, S. P. (2015). Dual use of peptide mass spectra: Protein atlas and genome annotation. Curr. Plant Biol. 2, 21-24. doi: 10.1016/j.cpb.2015.02.001

Wang, L., Ma, H., Song, L., Shu, Y., and Gu, W. (2012a). Comparative proteomics analysis reveals the mechanism of pre-harvest seed deterioration of soybean under high temperature and humidity stress. J. Proteomics 75, 2109-2127. doi: 10.1016/j.jprot.2012.01.007

Wang, Y., Yuan, X., Hu, H., Liu, Y., Sun, W., Shan, Z., et al. (2012b). Proteomic analysis of differentially expressed proteins in resistant soybean leaves after Phakopsora pachyrhizi infection. J. Phytopathol. 160, 554-560. doi: 10.1111/j.1439-0434.2012.01949.x

Wang, Z. Q., Xu, X. Y., Gong, Q. Q., Xie, C., Fan, W., Yang, J. L., et al. (2014). Root proteome of rice studied by iTRAQ provides integrated insight into aluminum stress tolerance mechanisms in plants. J. Proteomics 98, 189-205. doi: 10.1016/j.jprot.2013.12.023

Washburn, M. P., Wolters, D., and Yates, J. R. (2001). Large-scale analysis of the yeast proteome by multidimensional protein identification technology. Nat. Biotechnol. 19, 242-247. doi: 10.1038/85686

Watson, B. S., Asirvatham, V. S., Wang, L., and Sumner, L. W. (2003). Mapping the proteome of barrel medic (Medicago truncatula). Plant Physiol. 131, 1104-1123. doi: $10.1104 /$ pp.102.019034

Watson, B. S., Bedair, M. F., Urbanczyk-Wochniak, E., Huhman, D. V., Yang, D. S., Allen, S. N., et al. (2015). Integrated metabolomics and transcriptomics reveal enhanced specialized metabolism in Medicago truncatula root Border Cells. Plant Physiol. 167, 1699-1716. doi: 10.1104/pp.114.253054

Weckwerth, W. (2003). Metabolomics in systems biology. Annu Rev Plant Biol. 54, 669-689. doi: 10.1146/annurev.arplant.54.031902.135014

Weckwerth, W. (2010). Metabolomics: An integral technique in systems biology. Bioanalysis 2, 829-836. doi: 10.4155/bio.09.192

Weckwerth, W. (2011a). Unpredictability of metabolism- the key role of metabolomics science in combination with next-generation genome sequencing. Anal. Bioanal. Chem. 400, 1967-1978. doi: 10.1007/s00216-011-4948-9

Weckwerth, W. (2011b). Green systems biology- From single genomes, proteomes and metabolomes to ecosystems research and biotechnology. J. Proteomics 75, 284-305. doi: 10.1016/j.jprot.2011.07.010

Weckwerth, W., Loureiro, M. E., Wenzel, K., and Fiehn, O. (2004a). Differential metabolic networks unravel the effects of silent plant phenotypes. Proc. Natl. Acad. Sci. U.S.A. 101, 7809-7814. doi: 10.1073/pnas.0303415101

Weckwerth, W., Wenzel, K., and Fiehn, O. (2004b). Process for the integrated extraction, identification and quantification of metabolites, proteins and RNA to reveal their co-regulation in biochemical networks. Proteomics 4, 78-83. doi: 10.1002/pmic.200200500

Weckwerth, W., Wienkoop, S., Hoehenwarter, W., Egelhofer, V., and Sun, X. (2014). "From proteomics to systems biology: MAPA, MASS WESTERN, PROMEX, and COVAIN as a user-oriented platform," in Plant Proteomics:
Methods and Protocols, eds J. V. Jorrin-Novo, S. Komatsu, W. Weckwerth, and S. Wienkoop (New York, NY: Springer, LLC), 15-27.

Wienkoop, S., Baginsky, S., and Weckwerth, W. (2010a). Arabidopsis thaliana as a model organism for plant proteome research. J. Proteomics 73, 2239-2248. doi: 10.1016/j.jprot.2010.07.012

Wienkoop, S., Larrainzar, E., Glinski, M., González, E. M., Arrese-Igor, C., and Weckwerth, W. (2008b). Absolute quantification of Medicago truncatula sucrose synthase isoforms and $\mathrm{N}$-metabolism enzymes in symbiotic root nodules and the detection of novel nodule phosphoproteins by mass spectrometry. J. Exp. Bot. 59, 3307-3315. doi: 10.1093/jxb/ern182

Wienkoop, S., Morgenthal, K., Wolschin, F., Scholz, M., Selbig, J., and Weckwerth, W. (2008a). Integration of metabolomic and proteomic phenotypes analysis of data covariance dissects starch and RFO metabolism from low and high temperature compensation response in Arabidopsis thaliana. Mol. Cell. Proteomics 7, 1725-1736. doi: 10.1074/mcp.M700273-MCP200

Wienkoop, S., Staudinger, C., Hoehenwarter, W., Weckwerth, W., and Egelhofer, V. (2012). ProMEX- a mass spectral reference database for plant proteomics. Front. Plant. Sci. 3:125. doi: 10.3389/fpls.2012.00125

Wienkoop, S., Weiß, J., May, P., Kempa, S., Irgang, S., Recuenco-Munoz, L., et al. (2010b). Targeted proteomics for Chlamydomonas reinhardtii combined with rapid subcellular protein fractionation, metabolomics and metabolic flux analyses. Mol Biosyst. 6, 1018-1031. doi: 10.1039/b920913a

Wolschin, F., Wienkoop, S., and Weckwerth, W. (2005). Enrichment of phosphorylated proteins and peptides from complex mixtures using metal oxide/hydroxide affinity chromatography (MOAC). Proteomics 5, 4389-4397. doi: 10.1002/pmic.200402049

Wu, L., Mashego, M. R., van Dam, J. C., Proell, A. M., Vinke, J. L., Ras, C., et al. (2005). Quantitative analysis of the microbial metabolome by isotope dilution mass spectrometry using uniformly 13C-labeled cell extracts as internal standards. Anal. Biochem. 336, 164-171. doi: 10.1016/j.ab.2004.09.001

Wu, T., Yuan, T., Tsai, S. N., Wang, C., Sun, S. M., Lam, H. M., et al. (2009). Mass spectrometry analysis of the variants of histone $\mathrm{H} 3$ and $\mathrm{H} 4$ of soybean and their post-translational modifications. BMC Plant Biol. 9:98. doi: 10.1186/14712229-9-98

Wu, W., Zhang, Q., Zhu, Y., Lam, H. M., Cai, Z., and Guo, D. (2008). Comparative metabolic profiling reveals secondary metabolites correlated with soybean salt tolerance. J. Agric. Food Chem. 56, 11132-11138. doi: 10.1021/jf8024024

Xu, C., Garrett, W. M., Sullivan, J., Caperna, T. J., and Natarajan, S. (2006). Separation and identification of soybean leaf proteins by two-dimensional gel electrophoresis and mass spectrometry. Phytochemistry 67, 2431-2440. doi: 10.1016/j.phytochem.2006.09.002

Xu, H., and Freitas, M. A. (2009). MassMatrix: A database search program for rapid characterization of proteins and peptides from tandem mass spectrometry data. Proteomics 9, 1548-1555. doi: 10.1002/pmic.200700322

Yang, K., Tian, Z., Chen, C., Luo, L., Zhao, B., Wang, Z. et al. (2015). Genome sequencing of adzuki bean (Vigna angularis) provides insight into high starch and low fat accumulation and domestication. Proc. Natl. Acad. Sci. U.S.A. 112, 13213-13218. doi: 10.1073/pnas.1420949112

Ye, H., Gemperline, E., Venkateshwaran, M., Chen, R., Delaux, P. M., HowesPodoll, M., et al. (2013). MALDI mass spectrometry-assisted molecular imaging of metabolites during nitrogen fixation in the Medicago truncatulaSinorhizobium meliloti symbiosis. Plant J. 75, 130-145. doi: 10.1111/tpj. 12191

Young, N. D., Debellé, F., Oldroyd, G. E., Geurts, R., Cannon, S. B., Udvardi, M. K., et al. (2011). The Medicago genome provides insight into the evolution of rhizobial symbioses. Nature 480, 520-524. doi: 10.1038/nature10625

Young, N. M., Thibault, P., Watson, D. C., and Chrispeels, M. J. (1999). Posttranslational processing of two K-amylase inhibitors and an arcelin from the common bean, Phaseolus vulgaris. FEBS Lett. 446, 203-206. doi: 10.1016/S00145793(99)00212-4

Zadražnik, T., Hollung, K., Egge-Jacobsen, W., Megliè, V., and Šuštar-Vozliè, J. (2013). Differential proteomic analysis of drought stress response in leaves of common bean (Phaseolus vulgaris L.). J. Proteomics 78, 254-272. doi: 10.1016/j.jprot.2012.09.021

Zhang, N., Venkateshwaran, M., Boersma, M., Harms, A., Howes-Podoll, M., den Os, D., et al. (2012). Metabolomic profiling reveals suppression of oxylipin biosynthesis during the early stages of legume-rhizobia symbiosis. FEBS Lett. 586, 3150-3158. doi: 10.1016/j.febslet.2012.06.046 
Zhang, Y., Zhao, L., Li, H., Gao, Y., Li, Y., Wu, X., et al. (2013). GmGBP1, a homolog of human ski interacting protein in soybean, regulates flowering and stress tolerance in Arabidopsis. BMC Plant Biol. 13:21. doi: 10.1186/1471-222913-21

Zhen, Y., Qi, J. L., Wang, S. S., Su, J., Xu, G. H., Zhang, M. S., et al. (2007). Comparative proteome analysis of differentially expressed proteins induced by Al toxicity in soybean. Physiol. Plant 131, 542-554. doi: 10.1111/j.13993054.2007.00979.x

Zhu, H., Chen, T., Zhu, M., Fang, Q., Kang, H., Hong, Z., et al. (2008). A novel ARID DNA-binding protein interacts with SymRK and is expressed during early nodule development in Lotus japonicus. Plant Physiol. 148, 337-347. doi: 10.1104/pp.108.119164
Conflict of Interest Statement: The authors declare that the research was conducted in the absence of any commercial or financial relationships that could be construed as a potential conflict of interest.

Copyright (c) 2015 Ramalingam, Kudapa, Pazhamala, Weckwerth and Varshney. This is an open-access article distributed under the terms of the Creative Commons Attribution License (CC BY). The use, distribution or reproduction in other forums is permitted, provided the original author(s) or licensor are credited and that the original publication in this journal is cited, in accordance with accepted academic practice. No use, distribution or reproduction is permitted which does not comply with these terms. 


\section{OPEN ACCESS}

Edited by:

Antonio M. De Ron, Spanish National Research Council Misión Biológica de Galicia, Spain

Reviewed by:

Fred Stoddard,

University of Helsinki, Finland

Pedro A. Casquero,

University of Leon, Spain

*Correspondence:

María J. Suso

ge1susom@uco.es

Specialty section:

This article was submitted to

Crop Science and Horticulture,

a section of the journal

Frontiers in Plant Science

Received: 05 December 2015 Accepted: 04 March 2016

Published: 18 March 2016

Citation:

Suso MJ, Bebeli PJ, Christmann S,

Mateus $C$, Negri V, Pinheiro

de Carvalho MAA, Torricelli $R$

and Veloso MM (2016) Enhancing Legume Ecosystem Services through

an Understanding of Plant-Pollinator

Interplay. Front. Plant Sci. 7:333.

doi: 10.3389/fpls.2016.00333

\section{Enhancing Legume Ecosystem Services through an Understanding of Plant-Pollinator Interplay}

\author{
María J. Suso ${ }^{*}$, Penelope J. Bebeli², Stefanie Christmann ${ }^{3}$, Célia Mateus ${ }^{4}$, \\ Valeria Negri5, Miguel A. A. Pinheiro de Carvalho ${ }^{6,7}$, Renzo Torricelli5 and \\ Maria M. Veloso ${ }^{8}$
}

\begin{abstract}
${ }^{1}$ Department of Plant Breeding, Institute for Sustainable Agriculture, Spanish National Research Council, Córdoba, Spain, ${ }^{2}$ Laboratory of Plant Breeding and Biometry, Department of Crop Science, Agricultural University of Athens, Athens, Greece, ${ }^{3}$ International Center for Agricultural Research in the Dry Areas, Rabat, Morocco, ${ }^{4}$ Agrarian and Forestry Systems and Plant Protection Unit, National Institute for Agrarian and Veterinarian Research/Linking Landscape, Environment, Agriculture and Food, Oeiras, Portugal, ${ }^{5}$ Department of Agricultural, Food and Environmental Sciences, University of Perugia, Perugia, Italy, ${ }^{6}$ ISOPlexis Genebank, University of Madeira, Funchal, Portugal, ${ }^{7}$ Mediterranean Institute of Environmental and Agricultural Sciences, University of Evora, Evora, Portugal, ${ }^{8}$ Biotechnology and Genetic Resources Unit, National Institute for Agrarian and Veterinarian Research/Linking Landscape, Environment, Agriculture and Food, Oeiras, Portugal
\end{abstract}

Legumes are bee-pollinated, but to a different extent. The importance of the plantpollinator interplay (PPI), in flowering crops such as legumes lies in a combination of the importance of pollination for the production service and breeding strategies, plus the increasing urgency in mitigating the decline of pollinators through the development and implementation of conservation measures. To realize the full potential of the PPI, a multidisciplinary approach is required. This article assembles an international team of genebank managers, geneticists, plant breeders, experts on environmental governance and agro-ecology, and comprises several sections. The contributions in these sections outline both the state of the art of knowledge in the field and the novel aspects under development, and encompass a range of reviews, opinions and perspectives. The first three sections explore the role of PPI in legume breeding strategies. PPI based approaches to crop improvement can make it possible to adapt and re-design breeding strategies to meet both goals of: (1) optimal productivity, based on an efficient use of pollinators, and (2) biodiversity conservation. The next section deals with entomological aspects and focuses on the protection of the "pest control service" and pollinators in legume crops. The final section addresses general approaches to encourage the synergy between food production and pollination services at farmer field level. Two basic approaches are proposed: (a) Farming with Alternative Pollinators and (b) Crop Design System.

Keywords: heterosis breeding, legume landraces, breeding for seed yield, pest control, Farming with Alternative Pollinators and Crop Design System

Agricultural land serves first and foremost to produce food and, to an increasing extent, it should contribute to the "green economy" by enhancing diversity in agro-ecosystems. It is therefore often necessary to support farmers in their efforts to improve the biodiversity level on their farms. Legumes are bee pollinated. To realize the full potential of the plant-pollinator interplay (PPI), 
a multidisciplinary approach is required. The article assembles an international team of genebank managers, geneticists, plant breeders, experts on environmental governance and agro-ecology and comprises several sections. Section "Basic Application-Oriented Questions in Heterosis Breeding: The Potential of Insect-Mediated Outcrossing in Grain Legumes" deals with heterosis breeding strategies. Regarding hybrid and heterotic population breeding, current strategies for using heterosis center on finding ways of reducing the cost and increasing the efficiency of producing hybrid seed. Optimum pollination should be focused on seed production technology based on insect-aided outcrossing. Section "Exploring the Role of Plant-Pollinator Interplay in Grain Legume Landrace Germplasm Conservation and Management" describes landrace pollination aspects from the perspective of ex situ and onfarm multiplication. The role played by pollinators can have positive or detrimental effects. While gene flow between different multiplication seed fields can be problematic, insufficient outcrossing within a single population can also create troubles. Data should be available to genebank curators, breeders and farmers to allow the selection of the multiplication strategy in the presence of pollinators and to predict or assess its impact on landrace genetic structure and seed production. Section "Developing Strategies for Seed Production and Seed Producers in Forage Legumes" focuses on strategies for seed production and seed producers in forage legumes. In forage legumes, the potential seed yield (PSY) is very high; many ovules are present per unit area at anthesis, but only a small part of them turns into mature seeds. Seed production is influenced by several factors including the presence of pollinators around seed crop. The section describes general strategies for seed production in forage legumes. Section "Protecting the Pollination Service from Pesticides" brings entomological aspects and focuses on the protection of the "pest control service" and pollinators in legume ecosystems. The use of pesticides to control pests not only affects negatively the pollinators but also the pests' natural enemies. Enhancing the pest control service of legume ecosystems, by avoiding pesticides and adopting adequate alternative control methods, and by promoting ecological infrastructures, has a positive impact on pollinators. Section "General Approaches to Encourage the Synergy between Food Production and Pollination Services" addresses general approaches to encourage the synergy between food production and pollination services. It is increasingly recognized that pollination has multiple aspects: farmers may use both pollinator-friendly crops and implement practices in the overall production scheme and management of their farms to increase the occurrence, health and visitation of pollinators, whether these are wild or managed. Designing the right crop and identifying the suite of practices, appropriate and effective in a particular site, is where management of pollination becomes key. Two basic approaches are proposed to manage pollination by farmers at field level: (a) Farming with Alternative Pollinators (FAP) and (b) taking into account that flowering crops could be effective determinants of bee diversity and density, the Crop Design System (CDS).

\section{BASIC APPLICATION-ORIENTED QUESTIONS IN HETEROSIS BREEDING: THE POTENTIAL OF INSECT-MEDIATED OUTCROSSING IN GRAIN LEGUMES}

\section{Background}

Heterosis (or hybrid vigor) is a natural phenomenon whereby hybrid offspring of genetically diverse individuals display improved physical and functional characteristics relative to their parents (Fu et al., 2014). Heterosis has been increasingly applied in breeding for nearly a century, with the aim of developing more vigorous, higher- yielding, and better-performing cultivars. Hybrid breeding is a success story in several allogamous species such as maize (Zea mays L.), sunflower (Helianthus annuus L.), and other crops, where, greater yield has been achieved (Coors and Pandey, 1999). Although it is technically more challenging, hybrid breeding is also being approached in partially outcrossing crops such as canola (Brassica napus L.) (Longin et al., 2012). Heterosis results in from $20 \%$ to over $50 \%$ yield increase in inbred species (Tester and Langridge, 2010). For many self-pollinating crop plants, hybrid breeding has been established only recently. The reason for this may lie in the limitations of methods available for controlling pollination and hybrid performance prediction, in particular when established heterotic groups are absent. Strategies for using heterosis more widely to increase yields in inbred crops center on finding ways of reducing the cost and increasing the efficiency of producing hybrid seed (Zhao et al., 2015). In grain legumes, very limited progress has been made in the use of heterosis. So far, hybrid pigeonpea [Cajanus cajan (L.) Millsp.] is the only success story to tell in legumes (Palmer et al., 2011).

\section{Goal and Key Constraints}

This section deals with the hybrid breeding technology for the exploitation of heterosis in legumes; it does not review the status quo of hybrid breeding, but highlights the main problems currently limiting our capacity to utilize heterotic potential by using the knowledge from the PPI.

The biological constraints of legumes (for more details, see Crop Design System) hamper the implementation of a costefficient hybrid seed production technology. The details have been discussed recently (for review, see Palmer et al., 2011; Suso et al., 2015). Briefly, Palmer et al. (2001), listed four components that are crucial for the successful development of hybrids in food legumes: parental combinations that produce heterotic progenies with yield higher than the best pure-line cultivars, a stable male-sterile and female-fertile system, an efficient pollen transfer mechanism from pollen parent to pod parent, and a profitable level of seed increase for the seedsman and grower. However, in legumes, most of the above mentioned components are lacking, making hybrid research an uphill task. Legume hybrid breeding has been less successful, than in other crops because of the lack of knowledge about high-yielding heterotic groups coupled with difficulties in implementing a cost-effective system for hybrid seed production as result of an efficient pollen transfer mechanism from pollen parent to pod parent (Palmer et al., 2011). 
Heterotic groups - genotypes or populations - that produce high-yielding progeny after crossing need to be identified. The determination and identification of heterotic combinations or associations require a large number of parental recombinations to be evaluated over a number of years, in many locations, and with an adequate number of replications per location. In legumes, a limiting factor in the study of heterosis is the lack of hybrid seed available for the agronomic performance tests. Most studies were based on a low number of environments with small-sized plots and low seed density (Ortiz-Perez et al., 2007; Palmer et al., 2012).

\section{Key Examples}

Despite these drawbacks, major attempts have been undertaken in the public and private domain to develop hybrid breeding programs in some legumes. Moreover, these attempts at crop improvement programs were recently stimulated by the demand for increased agricultural productivity per area in the face of dynamic environmental and biotic threats which the rapid global environmental changes will make increasingly challenging (Tester and Langridge, 2010). We summarized current knowledge on the extent of hybrid breeding technology through selected examples: a successful story, pigeonpea [Cajanus cajan (L.) Millsp], a predominantly selfing crop, soybean [Glycine max (L.) Merr.] and a partially allogamous crop, faba bean (Vicia faba L.).

\section{Pigeonpea}

The research by ICRISAT and its collaborators into sterility systems and agronomic performance studies was the catalyst that ensured the success of the pigeonpea hybrid. Saxena et al. (2013) reported that ICRISAT scientists, working with their Indian counterparts, developed the Hybrid ICPH 2671, which showed around $47 \%$ superiority for grain yield over the popular cv. control cultivar 'Maruti' in multilocation on-station testing and in the on-farm trials. The cytoplasmic-nuclear male sterility, combined with natural outcrossing of the crop (outcrossing was facilitated by a prolonged flowering period) was used to develop viable hybrid breeding technology. Saxena et al. (2013) reported that good amounts of seed set were obtained even with 25$30 \%$ outcrossing. The pollen vectors may visit the male-sterile plants several times, and on each visit, a certain proportion of the flowers are pollinated to set the pods, while the un-pollinated flowers should drop. This is followed by the emergence of new flowers on the same plant, some of which again set pods through open pollination. The outstanding performance of this hybrid has led to its release for cultivation in India by both a private seed company and a public university.

\section{Soybean}

A number of male-sterility systems with appropriate maintainers and restorers are available for soybean hybrid technology. The identification of cytoplasmic-nuclear male-sterile lines along with their maintainers and restorers has been achieved by intraspecific and interspecific hybridizations. Heterosis studies have shown that yield levels above the best parent are possible (Palmer et al., 2011).
Once a stable male-sterility system is obtained, it is necessary to transfer the pollen from the male parent to the female parent. For soybean, a major limitation is the movement of pollen from male parents to female parents. Most of cross-pollinations have been conducted with hand-emasculated plants. Manual cross-pollination to produce large quantities of hybrid seed is difficult and time-consuming. Ortiz-Perez et al. (2006a,b) and Palmer et al. (2009) recommended insect-aided technology for hybrid seed production and considered that the exploitation of the heterosis depends on basic information available on different aspects of the PPI. The role of pollen vectors in soybean production has been generally disregarded, with producers relying on self-pollination and pesticides to maintain yield levels (Milfont et al., 2013). However, growing evidence links improved soybean production to the activity of flower visitors. The studies by Martins (2013), Milfont et al. (2013), and Monasterolo et al. (2015) showed that pollinator activity is important for this crop, leading to increased yield.

Insect-aided crossing potential depends on the functional floral pollinator-related traits of the female and male parents. Studies in cultivated soybean revealed significant differences for floral discovery, attraction and reward traits of the seed parent (Ortiz-Perez et al., 2006a). Ortiz-Perez et al. (2008) indicated that the bees' preference for certain parental lines was the key factor in reducing hybrid seed production. Among various traits that influence outcrossing, volatiles are crucial. Differences in the emission of flower volatile compounds among soybean cultivars mediate the attractiveness of high and low seed set male-sterility lines. Suso et al. (2010) showed that higher seed setting of malesterile lines was associated with increases in the length of the standard and changes in its lobe shape. Wild soybean species have a greater ability for outcrossing. G. syndetika Pfeil and Craven is a perennial species noted for its relatively intense perfume. In this species, several volatile organic compounds were identified (Palmer et al., 2012; Pappas et al., 2012). Altogether, these results can be exploited to genetically enhance annual soybean cultivars for increased outcrossing.

\section{Faba bean}

Although good hybrid combinations have been found, none of the many published CMS (cytoplasmic male sterility) systems are employed in practical breeding, mostly due to instability and spontaneous reversion to pollen fertility (for an in-depth review, see Palmer et al., 2011). Hybrid cultivars are not available commercially yet, and are unlikely, due to the high cost of producing and growing very large seeds (Gnanasambandam et al., 2012).

Partially allogamous crops are bred as either self- or crossfertilizers. Breeding as a self-fertilized crop (line cultivar) leaves heterosis unexploited; moreover, there is a risk of lower yield stability (Maalouf et al., 1999). Genetic uniformity is now being questioned in sustainable agriculture, thus open-pollinated highly heterogeneous and heterozygous populations (mixture or synthetic cultivars) should be an objective in itself (Gasim and Link, 2007; Goldringer et al., 2010).

The heterozygosity level of a population is a function of its outcrossing rate. The expression of heterotic effects in synthetics 
is particularly dependent on the degree of outcrossing (Maalouf et al., 1999). A prerequisite of breeding synthetic cultivars is the breeding of lines with high cross-fertilization rate (Gasim and Link, 2007).

\section{Conclusions and Future Perspectives}

Hybrids and heterotic populations can contribute to food security through higher yield and resilience. The development of hybrid technology is severely limited, primarily because of its high seed cost due to low outcrossing. Optimization of the insect-aided outcrossing technology is required and should be coupled with the development of a clear understanding of the PPI.

In the context of pollinator decline (Klein et al., 2007), the efficient use of pollinators emerges as a key target (Christmann and Aw-Hassan, 2012; IPBES, 2013; Cost Action FA1307, 2014; Garibaldi et al., 2014). Besides, legume breeding for sustainable agriculture is linked to the development of non-food services such as environmental services (Helenius and Stoddard, 2007; Duc et al., 2014). As it has been the case in other problem-solving matters in plant breeding, the solution lies in the interdisciplinary domain rather than in unique expertise. Structured dialog, as suggested in the framework of the European Innovation Partnerships - Focus Group on Genetic Resources ${ }^{1}$, among pollination biologists, entomologists, as well as breeders, farmers and, beekeepers, offers promising possibilities to overcome the constraint. Significant breakthroughs can be made by mixing disciplines: combining expertise in the core disciplines of agroecology, apiculture/entomology, and breeding could result in the development of insect-mediated outcrossing technology.

\section{EXPLORING THE ROLE OF PLANT-POLLINATOR INTERPLAY IN GRAIN LEGUME LANDRACE GERMPLASM CONSERVATION AND MANAGEMENT}

\section{General Introduction to Grain Legume Pollination}

In modern agriculture, 19 grain legume crops provide food for mankind, worldwide, or locally (Broughton et al., 2003; Smýkal et al., 2015). Eleven of these crops have records of naturally occurring cross-pollination mediated by PPI (Purseglove, 1968; Klein et al., 2007; Suso et al., 2015). Among these crops are soybean (G. max), common bean (Phaseolus vulgaris L.), runner bean $(P$. coccineus $\mathrm{L}$.), lima bean ( $P$. lunatus Benth.), faba bean ( $V . f a b a$ L.), adzuki bean (Vigna angularis (Wild.) Ohwi \& H. Ohashi), mungo bean [V. radiata (L.) R. Wilczek], and cowpea [V. unguiculata (L.) Walp.], etc. Many legume crops are considered predominantly self-pollinated (Suso et al., 2011) and their domestication process has favored this pollination behavior (Allard, 1999) with the corresponding adaptation of flower structure and traits (further information is presented in

\footnotetext{
${ }^{1} \mathrm{http} / / /$ ec.europa.eu/eip/agriculture/en/content/genetic-resources-cooperation-
} models see Crop Design System). Self-pollination has contributed to crop segregation against wild crop relatives (McCouch et al., 2012) and other related legume crops. However, self-pollination can limit the crop diversity and adaptation to the environment, and many crops have kept alternative pollination and reproduction mechanisms to overcome these constrains. Thus, legume selfpollination cannot be seen as an unvarying process (Lloyd and Schoen, 1992), and can be compatible with a variable amount of pollen transfer within the inflorescence or between plants within populations or between populations.

\section{Assessing Grain Legume Landrace Diversity and Structure}

Many grain legumes are traditionally cultivated and maintained worldwide resulting in the evolution of wide genetic diversity represented by landraces (Negri et al., 2013). Genesys (2015) allow us to have an idea of the number of these landraces conserved ex situ. There are as many as 75,796 bean landraces, $69.11 \%$ of bean germplasm held in genebanks, 19,702 cowpea landraces (63.13\%), 31,880 chickpea landraces $(57.88 \%)$, and 5,754 faba bean landraces (27.95\%).

Crop landraces are locally adapted heterogeneous populations and a potential source of genotypes with functional traits needed for PPI and breeding improvement. Landraces have been the matter of widespread analysis but not always of consensual understanding (Zeven, 1998; Pinheiro de Carvalho et al., 2013). However, the importance of landraces, including legume landraces, for crop evolution, food production and agriculture sustainability (Upadhyaya et al., 2011) and eventually for the implementation of the IPM, FAP, and CDS approaches, is widely recognized (see Reducing Pesticide Application by an Integrated Pest Management Approach, Farming with Alternative Pollinators - An Approach to Gain a MassBasis for Pollinator Protection, and Crop Design System). Legume landraces are the result of evolutionary adaptation to agro-ecological conditions, and their structure includes the commitment to genetic singularity (assembly of genes and alleles defining the landrace), ecological complexity (defined by environmental adaptation) and socio-cultural value (Brush, 1999; Negri et al., 2009; Freitas et al., 2011).

Legume landraces are submitted to a general trend in modern agriculture characterized by its fast replacement by modern cultivars, with increasing danger of erosion and extinction (Almekinders and de Boef, 1999; Negri et al., 2009; Pinheiro de Carvalho et al., 2016). This trend determines an overall concern for their ex situ conservation in germplasm collections, while almost 150 thousands landraces of six major grain legumes are actually stored (see Genesys, 2015).

An important factor for understanding the role of legume landraces in PPI is the knowledge of landrace population structure, in order to comprehend their genetic diversity and the presence of genotypes with required functional traits, and to optimize their ex situ and in situ (on-farm) conservation and management and/or use in breeding programs. According to our knowledge, the research into this issue has been insufficiently developed. Legume landraces are, by nature, heterogeneous and 
have a complex structure, whereas traits and genes are evolving in dynamic equilibrium with environmental conditions. The heterogeneity of the landrace can be promoted by farmers in the field through seed exchange, natural or accidental selection and/or gene flow (including that mediated by pollinators through the PPI) within or between closely related landrace populations.

The sampling methodology of landrace material is aimed at gathering as much genetic diversity as possible, while keeping useful landraces traits. If the landrace is strictly selfpollinated, then the seeds harvested will change in terms of the increase of genotypes, leading to a greater fitness in population. In partially cross-pollinated landraces, which allow limited pollen exchange between flowers mediated by pollinators, even small natural outcrossing rates can create many population crosses, since they are composed of several genotypes cultivated in a proximity which allows PPI. In such conditions PPI pollen transfer and allele shuffling will increase the heterozygosity of the landrace. Recent data shows that natural outcrossing can contribute to increasing the genetic diversity of crop populations (Papa and Gepts, 2004; Barnaud et al., 2008).

\section{Impact of Pollinators on the Structure of Grain Legume Landraces: Risks and Opportunities}

The impact of pollinators on the heterogeneous structure of landraces is still not well understood. Pollinators can promote self-pollination or outcrossing within each landrace and among landraces or cultivars. Thus, landraces can be vulnerable to changes determined by crosses with other cultivars. Outcrossing with other homogenous cultivars can result in genetic loss of one or several landraces' useful genotypes and their functional traits. On the other hand, inbreeding depression appears to be related, with genetic erosion and reduction of heterozygosis, when the natural outcross or environmental adaptation are limited. The evolution of the landrace in the field and outcrossing among its genotypes should be facilitated by PPI to avoid inbreeding depression (Street et al., 2008c). Additionally, selfing was found to alter the performance of an open-pollinated population in a progressive way, leading to the expression change of those characters associated with pollinator discovery and attraction (Nadal et al., 2003; Suso and Río, 2014). Martin and Adams (1987), studying the extent of outcrossing in 15 bean landraces in Malawi, identified three different categories regarding their structure. One category was a group of four seed types without evidence of outcrossing among genotypes as result of recent landrace cultivation in the studied region, differences in their flowering times, absence of pollinators activity or pollen incompatibility. In the second category, intermediate outcrossing events between landraces were observed, while in the third category landrace structure had been largely determined by many outcrossing events (Martin and Adams, 1987). These observations point to a temporal change of landrace structure and diversity, promoted by PPI, and need to be taken into consideration, during landrace management.
The global strategy of ex situ conservation of legume landraces has resulted in the interruption of evolutionary processes (Brush, 1999). The process can restart periodically, during gene banks management actions, with regeneration or multiplication of accessions. Under cultivation or in on-farm conservation, landraces continue evolving and adapting to environmental conditions. However, this evolution can be threatened by genetic erosion resulting in the extinction of landraces when farmers abandon them or in their genetic pollution by bred cultivars. These cultivars are characterized by genetic gains in yield, but are homogeneous and may not hold the useful traits required for PPI improvement and hybrid seed production (Palmer et al., 2011; see also Key Examples). There are benefits derived from natural outcrossing within landraces for creating new variability and selecting highly adapted yielding cultivars (Saxena and Kumar, 2010). Cross-pollination can also be hazardous for the breeder wishing to maintain the genetic identity and purity of the crop cultivar (Gómez, 2004). The natural outcrossing occurring in several grain legume crops can affect the reliability of the breeding programs when improved cultivars outcross with unimproved plants in nearby fields (Rahman et al., 2001).

The gene flow occurring between landraces and related species depends on outcrossing rates, in addition to the socioeconomic conditions of their cultivation. Oaxacan farmers' practice multiple landrace cropping systems using common bean, runner bean and Phaseolus dumosus across an altitudinal gradient as a strategy to mitigate risks across a broad range of agronomic and household needs that cannot be addressed with single species or races (Worthington et al., 2012). De Mooy et al. (1990) showed that under traditional intercropping systems, outcrossing plays a positive role in maintaining genetic variability and stability of cowpea landraces. However, the outcrossing between landraces and bred cultivars increases the risks of genetic pollution (De Mooy et al., 1990). Additionally, the natural outcrossing in pigeon pea can be considered a constraint for the maintenance of genetic purity of the bred cultivars and genetic seed stocks. New cultivars have been contaminated in a short period of 3-4 years (Gupta et al., 1981). However, Singh et al. (2005) reviewed the long-term Indian pigeon pea breeding program and showed that cultivars were selected from landrace variations created by natural outcrossing, hybridization and segregation events (Singh et al., 1990 cited by Saxena, 2005; Singh et al., 2013).

\section{Ex situ and On-Farm Approaches and the Plant-Pollinator Interplay. A Complex Issue}

An important issue in genebank management is to maintain the necessary level of the heterozygosity and heterogeneity of landraces, which can be achieved through the management of pollinator-crop interplay (Suso et al., 2015). Trying to identify gaps in the management of grain legume landraces, Maggioni et al. (2002) analyzed the maintenance and regeneration protocols of 11 germplasm collections and realized that about $50 \%$ of them are multiplied under open-pollination conditions. An average contamination rate of $12 \%$ for these landraces was 
estimated, with a high variation between genotype frequency (Maggioni et al., 2002). These data point out the need to focus on collection management protocols, with particular attention to the regeneration procedures and conditions. Recently, recommendations have been drawn up for cowpea, chickpea, faba bean and lentil landrace management (Dumet et al., 2008; Street et al., 2008a,b,c) aiming at supporting the positive results of outcrossing within landraces and to avoid the detrimental effects of cross-pollination with different cultivars.

The Global Crop Diversity Trust has also published regeneration guidelines for many grain legumes, including cultivated chickpea landraces. Even though chickpea (Cicer arietinum L.) is mainly self-pollinated, an outcrossing rate of $5.6 \%$ has been detected (Rubio et al., 2010), mediated by insects (Purseglove, 1968). An isolation distance of $90 \mathrm{~cm}$ between plots has been recommended to avoid unwanted outcrosses (Street et al., 2008c). In pigeonpea, covering the flowering plants with muslin bags or insect-proof cages has been recommended to avoid cross-pollination (Upadhyaya et al., 2008). Regeneration of faba bean landrace germplasm (Street et al., 2008a) can take place in screen houses to avoid insect-facilitated cross-pollination with foreign landraces or cultivars, and it allows within-accession crossing to prevent inbreeding depression. The regeneration in open-pollinated conditions should keep isolation distances to facilitate pollination by natural out crossing within accession.

Self-pollinated Lens culinaris Medik. shows a degree of outcrossing, ranging from 0.06 to $5.12 \%$, depending on the cultivar, location, and year (Horneburg, 2006). Additionally, the outcrossing rate between individual flowers in the inflorescence can reach $22.2 \%$ within cultivars. Its regeneration will therefore require specific management aimed at controlling pollination, either by allowing insect pollination inside isolation cages or tunnels or in-field free open pollination, keeping the minimum distance between different landraces or cultivars to avoid undesirable outcrosses.

It is vital to maintain landrace integrity, because some genotypes have kept their floral structure, promoting natural outcrosses, which have almost disappeared through crop domestication. At the same time, the genetic mechanisms facilitating the maintenance of outcrossing are relevant in breeding (for both hybrids and populations). Therefore, these flower functional traits will be a useful tool for crop improvement as well as for in situ and on-farm conservation (see also Key Examples).

Thus, when regeneration actions are implemented, it is equally important to take notice of pollinators [particularly honey bees (A. mellifera), bumble bees (Bombus sp.) and hover flies (Syrphidae)] visiting crop flowers, and evaluate the accessions for additional phenological and morphological data. Street et al. (2008b) recommended: (1) coordinating periodic field inspections with entomologists during the growing season, to identify the most efficient visitor/pollinator and determine the relationship between pollinator and flower morphology; (2) evaluating the agronomic data in relation to phenology and flower morphology and structure. Besides, all off-type plants resulting from crossing should be kept and mixed to build a basis population to provide breeders with high-value material (for its outcrossing potential).

\section{DEVELOPING STRATEGIES FOR SEED PRODUCTION AND SEED PRODUCERS IN FORAGE LEGUMES}

\section{Seed Production in Forage Legumes Genetic and Environmental Factors in Seed Production}

Forage legumes show a wide variation in traits influencing breeding programs, cultivar maintenance and seed production. Perennial species are generally cross-pollinated by insects and, consequently, predominantly allogamous. Cultivars of these species (synthetic and improved populations from mass selection or recurrent selection) are populations of different, highly heterozygous genotypes assembled by the breeder in a delicate equilibrium, conditioned by the cross-mating system, that must be maintained during the lifetime of a cultivar, since eventual inbreeding has deleterious effects on forage and seed yields (Ceccarelli, 1986).

Annual medics and subterranean clover are generally selfpollinating species, with cleistogamous flowers and a selftripping mechanism (Katznelson and Morley, 1965; Lesins and Lesins, 1979). Because of their reproductive system (predominant autogamy) and the huge germplasm variation existing in nature, the breeding of these species has mostly been based on a pure-line selection from natural populations (Piano and Pecetti, 2010).

Seed yield, which is an important factor in determining the success of a cultivar, is determined by genetic and environmental (sensu lato) factors. The latter include the choice of the bestsuited environment (for soil and climate characteristics) and the choice of an appropriate agronomic technique, where seed sowing rate, care of the establishment (fertilization, irrigation, grazing, and/or cutting management, weeds and pest control) and number of pollinators should be carefully set up. Sub-optimal environmental conditions, cultural and harvesting practices and absence of pollinators negatively condition forage seed yield (Lorenzetti, 1981, 1993; Lorenzetti and Negri, 1995). While research has already clarified the best environmental conditions for seed production (for the main forage species at least), there is still a need to carry out further research on genetic factors affecting seed yield, on which we will focus the following section, with particular reference to minor legumes.

\section{Potential Seed Yield vs. Realized Seed Yield}

In most forage legumes, the realized seed yield (RSY) is very low compared with the potential seed yield (PSY). The PSY can be estimated multiplying the Potential Seed Number (PSN) per unit area by the average thousand seed weight (TSW) (Table 1). The former depends on inflorescence number per unit area, flowers per inflorescence, ovules-per flower (i.e., on the reproductive system size). It is noteworthy that each of these components interacts with the others. With the exception of ovule number 


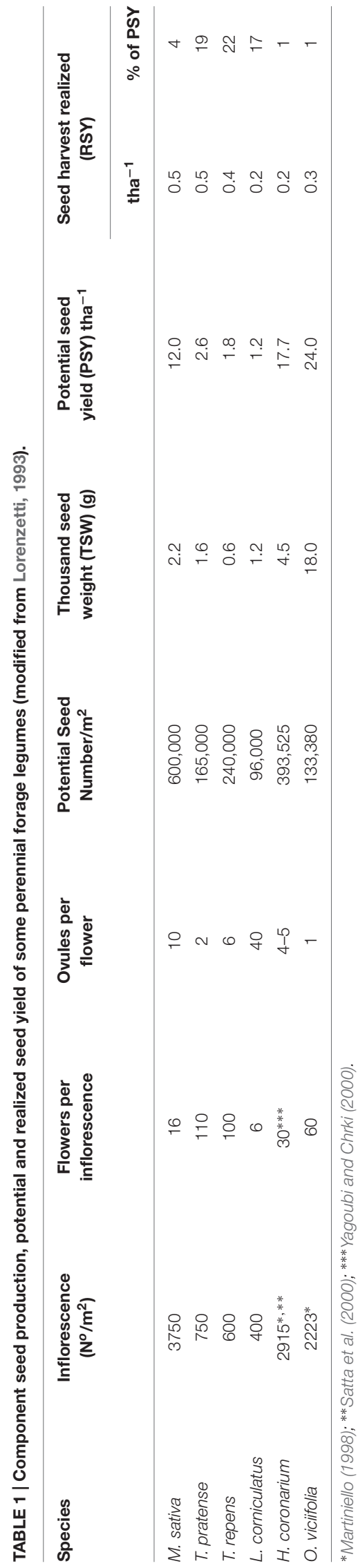

per flower, the less variable component, the other components show a high variability in all species (Lorenzetti, 1981; Rosellini et al., 1994), but few data on their hereditability exist. PSY is very high in forages since many ovules are present per unit area at anthesis; however, only a small part of these ovules turns into mature seed and only a part of this seed is collected (Table 1). RSY, in fact not only depends on the PSY, but also on the efficiency of the reproductive system (seed setting and maturation) and the percentage of harvested seed.

Pollination and fertilization are essential steps in the formation of mature seeds (Fairey and Hampton, 1998) and mostly depend on pollination. Pollinators like honey bees (A. mellifera), leafcutter bees (Megachile rotundata Fab.), alkali bees (Nomia melanderi Cockerell), and bumble bees (Bombus sp.), while foraging, operate flower tripping and fertilization and seed formation occur. Consequently, an adequate number of insects is a key factor in the production of forage seeds. Furthermore, crossing operated by pollinators allows the maintenance of a high level of heterozygosity and heterosis which, as mentioned above, condition important agronomic traits, such as forage and seed yields (Veronesi and Lorenzetti, 1983; Strickler, 1999; Strickler and Vinson, 2000; Pecetti and Proietti, 2003).

The environment strongly influences the success of pollination and fertilization through its effects on flowering pattern, flowering duration, pollen production and vitality, pollen tube growth, stigma receptivity, and ovule viability (Clifford and Scott, 1989; Hampton, 1990; Thomas and Pasumarty, 1996). However, the interplay plant-pollinator also plays a role. For example, in alfalfa, the bee foraging behavior is influenced by flower color (Steiner et al., 1992), flower aroma (Loper et al., 1974), and nectar availability (Kauffeld and Sorensen, 1971; Pecetti and Tava, 2000; Palmer et al., 2009). The latter are partially under genetic control (Loper and Waller, 1970; Loper and Lapioli, 1971; Loper and Berdel, 1978; Pecetti and Tava, 2000; Pecetti et al., 2002) while flower color is totally under genetic control (Barnes, 1966).

Moreover, fertilization may be reduced by genetically determined reproductive barriers such as incompatibility, plant sterility, or unreduced-gametes (Falcinelli, 1999). In addition, a high percentage (50\%) of embryo sac degeneration and/or fertilized egg abortion is observed in outcrossing forage legumes (Thomas, 1996) as a possible consequence of the genetic load resulting from deleterious recessive alleles (Wiens, 1984; Burbidge and James, 1991; Pasumarty et al., 1993; Rosellini et al., 1998). Breeding for traits like flower attractiveness to pollinators or low genetic load, factors that indeed condition reproductive efficiency, has been limited up to now.

Poor seed retention of forage legumes is another constraint in achieving PSY, since it causes considerable seed loss before and during harvesting. Forage legumes have not been fully domesticated yet and often show pod dispersal and/or dehiscence of pod prior to harvest. For example, seed shattering coupled with dehiscence of pod is high in Lotus corniculatus L., while pod dispersion is high in annual medics, Trifolium incarnatum L. and T. repens, where losses up to $40 \%$ of the total seed yield are reported (Clifford and McCartin, 1985). 


\section{Strategies for Increasing Seed Production in Forage Legumes}

Generally, the breeding programs of forage legumes are mainly designed to achieve a balance between persistence, quality, yield, and animal safety (Sewell et al., 2011), while they should also be addressed to increase seed production, since the commercial success of a forage cultivar also depends on its ability to produce seed. High seed yield facilitates the spread of the cultivar because low-cost seed can be easily placed on the market (Lorenzetti and Negri, 1995). In addition, several studies have shown that in forage legumes, seed yield is positively correlated with biomass production (Bolaños-Aguilar et al., 2002; Torricelli et al., 2007).

There are still ample margins for genetic improvement in seed production in forage legumes relying on existing genetic variation for traits that condition SYP (such as inflorescence number and seed number per plant, seed number per inflorescence and seed weight per inflorescence) and there is a high correlation with seed yield per plant (Bolaños-Aguilar et al., 2002; Sengul, 2006), attractiveness for pollinators and seed shattering.

Breeding in these crops is still at an early stage compared with other species. Both improved cultivars and populations are characterized by a wide genetic base, and thus, there is sufficient variation for seed yield to be substantially increased by selection and breeding. Improvements in seed yield will confer substantial benefits to breeding companies and farmers producing forages. It is noteworthy in the context of this paper that cultivars specifically bred for increased attractiveness to pollinators will also help in maintaining the wild pollinator fauna and increase honey production by honeybees (with a consequent increase in economic returns for farmers).

\section{PROTECTING THE POLLINATION SERVICE FROM PESTICIDES}

\section{The Pest Control Service in Legume Crops}

Legume crops are regularly attacked by several pests, pathogens and weeds, at different times of their growth cycles (Stevenson et al., 2007; El-Wakeil and El-Sebai, 2009; Pérez-De-Luque et al., 2010; Sillero et al., 2010; Stoddard et al., 2010; Kharrat and Ouchari, 2011; Stoddard, 2013; Arlauskiene et al., 2015), which strongly affect crop yields worldwide. This frequently compromises the choice of farmers for legume crops (Jensen et al., 2010). These attacks not only affect the crops' health but also limit the rotation and intercropping schemes that use legumes (Jensen et al., 2010).

Among the biotic constraints of legume crops, insect pests play a major role in yield reduction and several species are reported to be present in legume crops (Stevenson et al., 2007; Stoddard et al., 2010). More than 70 arthropod species have been reported attacking and damaging faba bean (Stoddard et al., 2010). However, not all these enemies are economically important (Nuessly et al., 2004; Stevenson et al., 2007).

The control of pests is crucial to optimizing production; however, frequently, natural enemies are not able to control pest outbreaks efficiently just by themselves in agricultural ecosystems. Presently, the use of pesticides still dominates the pest control programs of legume crops (Stevenson et al., 2007) and natural enemies or introduced (released) ones are chronically exposed to cocktails of pesticides. Predicted changes in global climate are likely to further exacerbate such problems in the future (Goulson et al., 2015).

Pesticides have a negative impact on the pests' enemies (and also on pollinators, as explained below) by direct mortality and by physiological and behavioral sub-lethal effects. Pesticide effects on their development, adult longevity, immunology, fecundity, sex ratio, mobility, navigation/orientation, feeding behavior, oviposition behavior and learning performance have a great influence in the abundance and pest control performance of those enemies (see details in Desneux et al., 2007). Pesticides disrupt/ weaken the natural pest control service in agricultural ecosystems.

\section{The Effect of Pesticides on the Pollination Service}

Pollination service is another ecological service essential in agricultural ecosystems, for both crops and wild plants. Many insect species contribute to pollination, including bees, hoverflies, butterflies, moths, and some beetles, by visiting a wide or narrow range of flower species to collect food (nectar and/or pollen), thus acting as generalist or specialist pollinators. Bees rely on either nectar or pollen at different stages of their life cycle with a total dependence on flowers, and are considered the most efficient pollinators. European bee species comprise the managed western honey bee (A. mellifera), 68 bumble bee species and over 1000 semi-social and solitary bee species (EASAC-The European Academies' Science Advisory Council, 2015).

There has been a steady decline in many pollinator groups, which has been attributed to several factors, many related to agricultural intensification, such as habitat loss and fragmentation, decreased resource diversity and pesticides application (Steffan-Dewenter et al., 2002; Kremen et al., 2007; Carré et al., 2009; Potts et al., 2010; Brown et al., 2012; Nieto et al., 2014).

Insecticides are the group of pesticides that pose the most direct risk to insect pollinators, especially the neonicotinoids (Woodcock, 2012; Sanchez-Bayo and Goka, 2014; Chagnon et al., 2015; Rundlöf et al., 2015). However, herbicide application also has the indirect effect of damaging the floral resources on which pollinators depend and delaying the flowering, so the timing between the period when food is most needed by pollinators and food availability is disrupted (Boutin et al., 2014; Nieto et al., 2014). Additionally, exposure to some fungicides can greatly increase the toxicity of insecticides, such as neonicotinoids and pyrethroids (Desneux et al., 2007; Goulson et al., 2015).

Pollinators can be exposed to insecticides during their application, by contact with residues, or from the ingestion of contaminated pollen, nectar, or guttation fluid. Exposure occurs in agricultural crops, flowering weedy crop boundaries and also in the neighboring semi-natural habitats where pollinators nest and forage, owing to pesticide drift (Potts et al., 2010). Numerous 
deaths of pollinators can result from insecticide application to a crop containing blooming weeds, even if the crop itself is not in bloom (Riedl et al., 2006; Gregorc and Ellis, 2011).

Exposure to insecticides may have a lethal effect (e.g., Gradish et al., 2010; Brittain and Potts, 2011; Tapparo et al., 2012; Pisa et al., 2015) or sub-lethal behavioral or physiological effects, such as a reduction in orientation abilities, reduction, or absence of foraging behavior, reduced feeding and life expectancy or compromised pollinators detoxification mechanisms and immune responses, rendering them more susceptible to diseases and parasites (Desneux et al., 2007; Mommaerts et al., 2010; Potts et al., 2010; Brittain and Potts, 2011; Henry et al., 2012; Pettis et al., 2012; Woodcock, 2012; Feltham et al., 2014; Godfray et al., 2014; Goulson et al., 2015).

The life-history traits of pollinator species affect their sensitivity to insecticides, such as body size (contact absorption rate and forage ranges), sociality (colonies essentially bioaccumulate pesticides and social pollinators experience exposure at greater doses for longer periods than solitary ones), flight season (overlapping with pesticide application periods), voltinism (univoltine species foraging when pesticides are applied are at a particular high risk), floral specialization (for polylectic pollinators, sources containing pesticides may be diluted by foraging in other plants or habitats), nesting behavior (ground or above ground nesting may be differently exposed to pesticides), and sex (different intrinsic susceptibility and body size) (see details in Williams et al., 2010; Brittain and Potts, 2011). Although synergistic interactions among traits remain to be explored, individual traits can be useful in predicting and understanding responses of related species to environmental disturbances (Williams et al., 2010).

It must be taken into account that the effects of pesticides on honey bees are poorly representative of the effects on other pollinators, including other bees. Indeed, bees (Apoidea) constitute a highly diverse group, and bees from different taxonomic groups differ widely in their vulnerability to pesticide exposure (Desneux et al., 2007).

In conclusion pollination service benefits from a healthy, robust pest control service in the respective agricultural ecosystem, which reduces the need for artificial pest control such as pesticide application.

\section{Reducing Pesticide Application by an Integrated Pest Management Approach}

The development of pests' resistance to pesticides, and the economic, health and environmental risks they pose have highlighted the need to reduce pesticide applications (see the European Union Sustainable Use of Pesticides Directive 2009/128/EC) and to adopt alternative pest management methods. To date, no single method provides efficient pest control, and an integrated approach (integrated pest management - IPM) is needed, in which methods are combined in order to maintain enemy populations below their respective economic threshold levels.

The use of pesticides is allowed in the IPM approach, but only when absolutely necessary and after a careful selection (Riedl et al., 2006; Stevenson et al., 2007; Macfadyen et al., 2015). Pest monitoring, together with the concept of economic threshold, allows the perception on when and where to apply pesticides and avoids unnecessary spraying. Removal and protection of bee colonies is also advised (Riedl et al., 2006).

Integrated pest management in legume crops has been widely discussed by several authors (e.g., Holt and Wratten, 1986; Redden et al., 2005; Sharma et al., 2007; Stevenson et al., 2007; Duc et al., 2010; Pérez-De-Luque et al., 2010; Rispail et al., 2010; Sillero et al., 2010; Stoddard, 2013). The wide range of regions where legumes are grown determines a high diversity of crop enemies (Stevenson et al., 2007), and their presence and abundance varies from region to region. Therefore, it is not feasible to define a generalized IPM strategy- it has to be adapted locally or at a regional level.

The biological control method may include releases of artificially reared beneficial organisms; however, a more sustainable way to implement this is to give the natural enemies occurring there the best conditions to increase their density and to enhance their impact on the pests - conservation biological control (CBC). This enhancement of the pest control service is achieved by managing the habitat and manipulating environmental factors (Rebek et al., 2005; Stevenson et al., 2007; Macfadyen et al., 2015).

The success of CBC, and also of the pollination service, is strongly linked to the temporal and spatial availability and quality of ecological infrastructures, inside and outside the farm limits (within a radius of approximately 100-200 m) (Boller et al., 2004), such as shrubs, herbaceous plants (ground covers, interrow strips, or crop boundaries), wooded field edges, stones, and bare undisturbed soil areas, inside the crop land or in the vicinity (Landis et al., 2000; Westerkamp and Gottsberger, 2000; Richards, 2001; Rebek et al., 2005; Schellhorn et al., 2008; Van Driesche et al., 2008; Köpke and Nemecek, 2010; Shackelford et al., 2013; Sigsgaard et al., 2013). Diverse floral resources (pollen and nectar), with phenological complementarity, apart from their evident importance for pollinators, are of great importance for both predators and parasitoids (Landis et al., 2000; Brown et al., 2012; Döring et al., 2012). When managing existing or introduced floral resources, implementing or preserving different types of shelters, it is essential to know a lot about the ecosystem being manipulated, including the morphology and bio-ecology of the target organisms present there (Landis et al., 2000).

Generally, faba bean offers a rich foraging habitat for several beneficial insects, with mass flowering and substantial amounts of nectar and pollen (Westphal et al., 2003; Nuessly et al., 2004; Köpke and Nemecek, 2010; Stoddard, 2013). For many natural enemies and pollinators, faba bean is considered a substitute for the loss of habitat diversity that results from agricultural intensification (Richards, 2001). Diverse legume leys provide a variety of flowers which bloom sequentially throughout the season to extend the availability of pollen and nectar for pollinators above that of standard leys (Brown et al., 2012).

When adopting IPM and selecting the most suitable pest management methods, it must be taken into account that not only pesticides may be dangerous to natural enemies and pollinators. 
Cultural practices such as removing weeds, vegetal debris and dead wood with insect galleries, as well as tillage procedures, may have a very negative impact on the beneficial fauna by the destruction of food and refuge sources (Fuchs and Hirnyck, 2000; Riedl et al., 2006; Stevenson et al., 2007; Williams et al., 2010), and so a trade-off of benefits- disadvantages must be considered to reach a correct decision (Saunders et al., 2015).

\section{GENERAL APPROACHES TO ENCOURAGE THE SYNERGY BETWEEN FOOD PRODUCTION AND POLLINATION SERVICES}

\section{Farming with Alternative Pollinators - An Approach to Gain a Mass-Basis for Pollinator Protection \\ The Urgent Need to Get a Mass-Basis for the Protection of Wild Pollinators}

The global decline of pollinator diversity is mainly a consequence of human mismanagement (agricultural chemicals, monocultures, landscape fragmentation, habitat loss) as referred to before, and increasingly of different climate change effects and the interaction of these stress factors (Potts et al., 2010). Climate change ultimately requires us to reflect on the PPI from a broader perspective to gain sufficient support for the protection of wild pollinators. Even in the case of agricultural crops, honey bees provide only $8-15 \%$ of services (Nabhan and Buchmann, 1997; see also MA, 2005, p. 759).

Pollinators have four key functions within ecosystems and for mankind, which make them indispensable and highlights the need for humans to enhance farming practices and the use of landscapes:

- 87 of the 115 most important food crops depend or benefit from pollination (Klein et al., 2007); this list of highvalue crops includes some food legumes such as faba bean, pigeon pea, common bean etc. The total yield, e.g., from faba bean with open access to pollinators is $185 \%$ higher compared to autonomous self-pollination (Nayak et al., 2015). This example demonstrates the importance of pollinators for food security and sustainable intensification of agricultural production. Some forage legumes such as alfalfa, clover and vetch depend mainly on wild pollinator species (alfalfa/Megachilidae; clover/Bombus);

- $85 \%$ of all terrestrial plants require pollinators (Ollerton et al., 2011), and so a loss of pollinators could cause a cascade of extinctions (Biesmeijer et al., 2006) and even pollinator population collapse if stress factors reached a tipping point (Lever et al., 2014). Pollinator protection by sustainable legume production can therefore contribute to the protection of the biodiversity of natural plants;

- Cross-pollination can enhance genetic diversity and thus adaptation to climate change (Christmann and Aw-Hassan, 2012), which is of importance for (1) legume-farmers, e.g., in remote mountainous areas with limited or no access to hybrids, and (2) the much higher number of natural plant species having no human breeders;

- Most other ecosystem services rely on pollination to a certain extent, and the partial, but simultaneous loss of these ecosystem services too could cause a spiral of poverty (Christmann et al., in review).

Cultivated legumes do not have exclusive mutualism between crop and pollinator as, e.g., fig, vanilla, or cocoa, but they are mainly pollinated by wild bees and also by honeybees. Legumes can play an important role in sustaining the diversity of wild bees and some wild bee species are indispensable to sustain some legumes, as the flower type of Fabaceae neither allows all insect pollinators to provide the service nor permits hand-pollination by humans. The extinction of wild bees (leaf cutting bees, solitary bees, or bumblebees) could significantly endanger legume production, in particular faba bean production in temperate or high altitude (above $2500 \mathrm{~m}$ ) regions and seed production of alfalfa. Faba bean flowers early and faces increasing risks of seasonal abnormalities in the course of climate change, which can prevent honeybees from providing service. Honeybees require $15^{\circ} \mathrm{C}$ and sunny days without rain and strong wind, whereas the availability of, e.g., bumblebees around the field can safeguard farmers' revenues despite unfavorable weather conditions.

Wild pollinators mostly work in a small radius $(50-2000 \mathrm{~m}$ ) around their nests (Kohler et al., 2008). Thus, they are more affected by monocultures, ploghing, chemicals, loss of diverse field edges and landscape fragmentation than honeybees, which fly up to $5 \mathrm{~km}$ and are supported by beekeepers (transport, food, hives). So the protection of wild pollinators requires a massbasis of local stakeholders ready to engage themselves in long term pollinator protection. Raising knowledge and awareness of these issues can increase the readiness of smallholders and some benevolent producers to engage in pollinator protection, but the number of commercial producers ready to reduce chemicals and to enhance habitats might be limited, if this action is based on good-will alone (Christmann et al., in review).

\section{The Methodology of FAP}

Hence, the leading research question for FAP (Christmann and Aw-Hassan, 2012) is: how to get a mass-basis of intrinsically motivated local actors? Such a high number of protagonists cannot be paid; even in industrialized countries, the incentive must be grounded on the economic value of ecosystem services (The Economics of Ecosystems and Biodiversity (TEEB)approach; TEEB, 2010).

Based on broad evidence that a high diversity of pollinators can significantly enhance the harvest of many crops in quantity and quality and that distance between nest and crop is the limiting factor (see literature in Ricketts et al., 2008; Christmann and Aw-Hassan, 2012; Garibaldi et al., 2014), the hypothesis is that by specific crop-based habitat enhancement the total net income from many horticulture crops can be significantly increased; the difference in income between FAP- and controlfields will be sufficient to motivate farmers in favor of pollinator protection by habitat enhancement. 
Farming with Alternative Pollinators compares the net revenue of FAP-fields and control fields: whereas control fields have the main crop in the entire field, FAP-fields use only $75 \%$ of the field for the main crop and $25 \%$ for habitat enhancement, consisting of three-season-forage buffets for pollinators and shelter (both based on further crops, spices, herbs, and medicinal plants), nesting support made out of local materials and a water source, e.g., a puddle or pond. Twelve or more different crops with different flower types and colors nourish pollinators for three seasons. The habitat enhancement in the field itself can optimally reduce the distance between nest and foraging crop. The different flower types and colors of petals attract a higher diversity of pollinators to FAP-fields during the flowering of the main crop. FAP uses a wide range of marketable plants instead of wildflower strips (e.g., (Garibaldi et al., 2014 recommend wildflower strips) to gain the highest possible income gain as an incentive. As the habitat zone and higher insect abundance also attract predators, FAP does not measure the economic value of pollination services, but of habitat enhancement. The methodology for fields and orchards is described in detail in Christmann et al., in review. The options of a purposeful combination of FAP and $\mathrm{CBC}$ should be explored and the potential of FAP for enhancing genetic diversity of crops and productivity of seeds should be analyzed. Such supplementary positive impacts on crop productivity by enhanced cross-pollination might additionally motivate farmers to enhance pollinator habitats.

Farming with Alternative Pollinators is a new approach with a limited number of field experiments in Uzbekistan and currently Morocco. The pilot project in Uzbekistan (main crops: cucumber and sour cherry; partly faba bean and partly sainfoin in the habitat zone; Christmann et al., 2014, in review) did on-farm trials mainly with smallholders, because in general they use less or no agricultural chemicals as they prefer low production costs and healthy food for their own families. Trials with smallholders can develop first data to convince commercial farmers too. Starting with large-scale producers can cause discussions about the use of chemicals. Also, due to their high number, smallholders can be important protagonists for pollinator protection.

\section{Results of First Field Experiments}

The FAP-pilot project confirmed: the FAP-fields attracted a clearly higher number of different pollinator and predator species, including effective wild bee species; for sour cherry, the harvest was double on FAP-sites, for cucumber even higher (Christmann et al., 2014, in review). Solitary bees (important pollinators for cucumber) used the nesting support. Due to the project duration (18 months), it was not possible to assess the adoption rate in the long term, but the readiness to protect pollinators and to take effective action based on data concerning yield increase was much higher in both project regions (Christmann et al., in review) than described in case studies recommending information campaigns (e.g., Kasina et al., 2009a).

\section{Potential of FAP with Special Regard to Legumes}

Pollinator-friendly legumes can significantly contribute to the habitat zone. Due to their specific flower type and the (for crops) unusual color of the petals, they can attract, e.g., wild bees, which are also effective for many other crops. According to the experience in Uzbekistan it is not difficult to convince farmers to seed faba bean or sainfoin in the habitat zone, as they know they also will benefit directly from these plants. They suggested using crops themselves, and would not consent to plants such as Laburnum anagyroides Medik or seed Indigofera tinctoria L., ornamental plants or wildflowers (weeds) to attract pollinators (Christmann et al., in review).

Pollinator-dependent legumes might benefit also substantially from FAP as a main crop. Current research on faba bean supports this assumption, since pollinators from nearby natural habitats can increase the harvest significantly (Musallam et al., 2004; Aouar-Sadli et al., 2008; Andersson et al., 2014). Also the production of string bean, soybean, kidney bean, and various peas etc. as main crops, as well as alfalfa-seed production, might benefit from FAP (Klein et al., 2007; referred also in the earlier paragraphs of this article).

As soon as the additional income gain by FAP-measures for these crops as a main crop has been assessed and planting instructions are spread, the intrinsic motivation of farmers for pollinator protection might rise significantly, as the approach does not require investment or long-term training. Including more marketable plants like other crops, spices and medicinal plants, rather than just pollinator-friendly legumes, can provide the necessary mass-basis for pollinator protection. Enhancing the environment might be a better option than the suggestion to breed for pollinator independent crops (Chaplin-Kramer et al., 2014), which has several negative effects: it does not take advantage of heterosis effect and bears the risk of lower yields (see "Basic Application-Oriented Questions in Heterosis Breeding: The Potential of InsectMediated Outcrossing in Grain Legumes"), creates the need for continuous breeding to adapt to the changing climate and runs the risk of local pollinator populations starving even more.

\section{Crop Design System}

In the CDS approach, breeders incorporate the potential benefits of pollen vectors into practices to increase the efficiency of hybrid seed technology and, in parallel, increase the occurrence, health and visitation of pollinators, whether these are wild, or managed by developing pollinator-friendly crops.

\section{Understanding the CDS Approach}

The rationale is the following:

(a) Animal-pollinated crops grown today are often suboptimal in attracting and sustaining their pollinator populations (Bailes et al., 2015). Cultivars are generally bred by the artificial selection of agronomic traits that are of commercial interest but with little regard to pollinator-related traits and preferences. Consequently, insect-pollinated cultivars may not be attractive to pollinators (Kobayashi et al., 2010). 
Legumes are known to have flowers with moveable parts that have to be actively handled by insects for pollination to take place. A flower has to be actively moved by a visitor to access the flower reward and, consequently, to perform pollination (Cordoba and Cocucci, 2011). Due to the morphological specialization in floral phenotype, effective pollen transfer requires several traits of the floral mechanism of plants to be adjusted to the morphology and behavior of animal pollen vectors (Garibaldi et al., 2015). Simple morphological mismatches appearing in the flowers are an obvious problem. For instance, hybrid seed production depends on the production and use of male-sterility lines. These lines are difficult to work with naturally in the fields, as the foraging behaviors of bees often change when confronted with such anomalies (Ortiz-Perez et al., 2006b). Information show that some traits may respond to pollinator-driven natural selection (Suso and Río, 2014). Suso and Río (2015) identified some architectural and floral patterns in faba bean that are the result of natural selection and reflect local adaptation to differences in pollination environments. Volatile compounds emitted by crop flowers mediate plant/pollinator interactions (Klatt et al., 2013).

In the CDS approach, breeders and farmers develop cultivars with enhanced heterozygosity and heterogeneity as result of appropriate functional floral traits (discovery, attraction, and reward) within the crop for supporting the bee pollinator populations used as agents of crossings. One idea proposed is to support native pollinators for insect-mediated outcrossing by designing a crop with appropriate functional flower traits (Suso et al., 2005; Palmer et al., 2009; Suso and Maalouf, 2010; Suso et al., 2015).

Legumes have great potential to be served by bee pollinators as crossing agents because they are visited by a great number of bees, such as bumble bees, honey bees, and wild bees (Free, 1993; Delaplane and Mayer, 2000). Non Apis bees, such as solitary bees, are an essential group of visitors and of incalculable value for pollination (e.g., Pierre et al., 1999; Kasina et al., 2009a,b; Suso et al., 2015), even in self-pollinated species such as soybean (Martins, 2013).

However, there is a gap in our knowledge about which particular insects visit and cross-pollinate a particular legume crop, and why. For many legumes, there is little specific data available and even if information on pollinators is available, the findings are often ambiguous (Suso et al., 2015). Besides, the pollinator species can vary from country to country, e.g., faba beans are mainly pollinated by wild bees in Spain and England, but by different species of Bombus in France (Bond and Kirby, 1999, 2001; Pierre et al., 1999).

(b) Declines in bee populations have increased interest in the ecological services of legumes (Köpke and Nemecek, 2010). Legume cultivation provides some key environmental benefits such as floral resources. Although the yield produced by legumes remains a major objective for breeders, other issues such as environmentally friendly, resilient production in the context of climate change, as well as new ecological services such as pollinator protection, are required (Duc et al., 2014). Improved value for the conservation of bee pollinators should be a key breeding objective.

\section{Concerns and Constrains}

Over many centuries, farmers have often selected many legume crops for their autogamous abilities or self-fertility. According to Hammer, (1984 cited by Smýkal et al., 2015), the "domestication syndrome" for legumes includes the transition from outcrossing to selfing. The shift from outcrossing or facultative selfing to strict inbreeding has been described as the single most common trend in legume domestication (Rick, 1988). One common view on the mating system of grain legumes, among germplasm curator and breeders, is that they display autogamous or mostly autogamous mating systems (Suso et al., 2011).

However, for the development of insect-aided outcrossing technology, selfing has to be bred out again and functional floral traits should be managed toward outcrossing. The transition from outbreeding ancestors to autogamously selfing lineages has been derived many times during plant evolution and, in most cases, it is accompanied by a characteristic set of morphological and functional changes to the flowers, together termed the selfing syndrome (Sicard and Lenhard, 2011). The ability of plants to evolve new mating behaviors is a notable feature of plant evolution (Chen et al., 2007).

Legumes possess flowers perfectly capable of outcrossing (Suso et al., 2015). According to Palmer et al. (2009), considering that pollinators respond to differences in discovery, attractiveness and reward, outcrossing could be increased through selection for floral characteristics that enhance its frequency and efficiency. A basic understanding of the variety of mechanisms by which flower morphology and structure influence pollinators' behavior and consequently pollen transfer, should allow plant breeders to manipulate the PPI in ways that increase insect-mediated outcrossing. Bailes et al. (2015), reviewing key topics in plant-pollinator research, also remark that floral traits are a promising and little-explored avenue for the improvement of crop yields.

Regarding the pollination crisis, breeders should confront the decline of pollinators. One tendency in plant breeding is to opt for plants that do not need pollinators (Partap and Ya, 2012; Chaplin-Kramer et al., 2014). However, yield barriers must be broken. Breaking yield barriers could be achieved by increasing potential yield by improvements in the efficiency of heterosis exploitation (Tester and Langridge, 2010). The clear message is that insect-aided cross-pollination is a key factor in the development of hybrid seed technology and pollinators are essential in providing this service. Changes from a crop that does not require cross-pollination, to crops that respond optimally to the presence of pollen vectors is the key. The proposed breeding strategy is to combine highly self-fertile genotypes which respond optimally to the presence of pollinators to produce high-yielding cultivars. Thus, insect-aided outcrossing allows the exploitation of heterosis potential but, in the absence of pollinators, a minimum yield 
is achieved (Suso et al., 1996). A delayed selfing mechanism provides reproductive assurance while allowing a high level of outcrossing when pollinators are not a limiting factor (Shirk and Hamrick, 2014).

\section{Making the Most of the PPI}

In order to maximize pollination success, the employment of new agronomic practices, and the maintenance of seminatural habitats in the agricultural landscape are essential (Andersson et al., 2014; Nayak et al., 2015; referred to also in section Farming with Alternative Pollinators - An Approach to Gain a Mass-Basis for Pollinator Protection) and should be coupled with the breeding procedure. The CDS approach, considering the management of natural resources, pollen vectors and improving the genetic characteristics of crops for functional floral traits, may prove faster, and more effective. The increasing demands for environmentally friendly agricultural practices and the decline in pollinators have established a favorable context for the development of pollinatorfriendly crops, while placing demands on plant breeders to develop cultivars that meet more complex objectives (Duc et al., 2014). But, unfortunately, the analysis of Duc et al. (2014), reflects a relatively small representation of scientific articles related to the hosting of pollinators. Additionally, in spite of the fact that hybrid breeding is considered a breeding technology to increase crop production in a changing world (Tester and Langridge, 2010), the option of considering hybrids and heterotic populations has received minor attention among the breeding approaches. Thus, substantial research is needed to understand better how plants with appropriate functional floral traits could enhance insectmediated outcrossing and the conservation of pollinator populations, which would help solve practical issues in the development of hybrids and heterotic populations and mitigate pollination declines.

\section{REFERENCES}

Allard, R. W. (1999). History of plant population genetics. Annu. Rev. Genet. 33, 1-27. doi: 10.1146/annurev.genet.33.1.1

Almekinders, C., and de Boef, W. (1999). The challenge of collaboration in the management of crop genetic diversity. ILEIA Newsl. 3-4, 5-7.

Andersson, G. K. S., Ekroos, J., Stjernman, M., Rundlöf, M., and Smith, H. G. (2014). Effects of farming intensity, crop rotation and landscape heterogeneity on field bean pollination. Agric. Ecosytst. Environ. 184, 145-148. doi: 10.1016/j.agee.2013.12.002

Aouar-Sadli, M., Louadi, K., and Doumandji, S. E. (2008). Pollination of the broad bean (Vicia faba L. var. major) (Fabaceae) by wild bees and honey bees (Hymenoptera: Apoidea) and its impact on the seed production in the Tizi-Ouzou area (Algeria). Afr. J. Agric. Res. 3, 266-272.

Arlauskiene, A., Kadziuliene, Z., Sarunaite, L., Deveikyte, I., and Semaskiene, R. (2015). "A contribution of legumes to diversity of crops in organic farming," in Proceedings of the Eucarpia International Symposium on Protein Crops, Pontevedra, 130.

Bailes, E. J., Ollerton, J., Pattrick, J. G., and Glover, B. J. (2015). How can an understanding of plant-pollinator interactions contribute to global food security? Curr. Opin. Plant Biol. 26, 72-79. doi: 10.1016/j.pbi.2015.06.002

Barnaud, A., Trigueros, G., McKey, D., and Joly, H. I. (2008). High outcrossing rates in fields with mixed sorghum landraces: how are landraces maintained? Heredity 101, 445-452. doi: 10.1038/hdy.2008.77

\section{AUTHOR CONTRIBUTIONS}

All authors contributed to manuscript writing and read and approved the final manuscript. However, regarding that the article include quite different contributions, aspects and disciplines, each author has led and participated mainly in one section, and is going to take responsibility of the particular section. Thus, the individual contributions of each author are being specified. MJS conceived and design the paper and carried out the Section "Basic Application-Oriented Questions in Heterosis Breeding: The Potential of InsectMediated Outcrossing in Grain Legumes". and Crop Design System. PB and MC carried out the Section "Exploring the Role of Plant-Pollinator Interplay in Grain Legume Landrace Germplasm Conservation and Management". VN and RT carried out the Section "Developing Strategies for Seed Production and Seed Producers in Forage Legumes". CM and MV carried out Section "Protecting the Pollination Service from Pesticides". SC carried out section Farming with Alternative Pollinators (FAP) An Approach to Gain a Mass-Basis for Pollinator Protection.

\section{ACKNOWLEDGMENTS}

MJS gratefully acknowledge the EU-FP7-KBBE-2009-3 project SOLIBAM (Strategies for Organic and Low-input Integrated Breeding and Management) and the H2020-SFS-2014-2 project DIVERSIFOOD (Embedding crop diversity and networking for local high quality food systems- GA number: 633571) for financial support. MJS, PB, MC, and RT wish to acknowledge the support from the COST Action FA1307 Super-B (www.superbproject.eu), supported by COST (European Cooperation in Science and Technology). SC wishes to acknowledge the support from BMUB, funding the pilot research in Uzbekistan within the International Climate Change Initiative.

Barnes, D. K. (1966). Flower color inheritance in diploid and tetraploid alfalfa: a reevaluation, in U.S. Dept. Agric. Tech. Bul. 1353, 3-28.

Biesmeijer, J. C., Roberts, S. P., M., Reemer, M., Ohlemüller, R., Edwards, M., et al. (2006). Parallel declines in pollinators and insect-pollinated plants in Britain and the Netherlands. Science 313, 351-354. doi: 10.1126/science. 112786

Bolaños-Aguilar, E. D., Huyghe, C., Ecalle, C., Hacquet, J., and Julier, B. (2002). Effect of cultivar and environment on seed yield in alfalfa. Crop Sci. 42, 45-50. doi: $10.2135 /$ cropsci2002.0045

Boller, E. F., Häni, F., and Poehling, H.-M. (2004). Ecological Infrastructures: Ideabook on Functional Biodiversity at the Farm Level - Temperate Zones of Europe. IOBCwprs Commision Integrated Production Guidelines Endorsment, Eschikon: Swiss Centre for Agricultural Extension and Rural Development, 212.

Bond, D., and Kirby, E. J. M. (1999). Anthophora plumipes (Hymenoptera: Anthophoridae) as a pollinator of broad bean (Vicia faba major). J. Apicul. Res. 38, 199-203. doi: 10.1080/00218839.1999.11101010

Bond, D. A., and Kirby, E. J. M. (2001). Further observations of Anthophora plumipes visiting autumn-sown broad bean (Vicia faba major) in the United Kingdom. J. Apicult. Res. 40, 113-114.

Boutin, C., Strandberg, B., Carpenter, D., Mathiassen, S. K., and Thomas, P. J. (2014). Herbicide impact on non-target plant reproduction: what are the toxicological and ecological implications? Environ. Pollut. 185, 295-306. doi: 10.1016/j.envpol.2013.10.009 
Brittain, C., and Potts, S. G. (2011). The potential impacts of insecticides on the life-history traits of bees and the consequences for pollination. Basic Appl. Ecol. 12, 321-331. doi: 10.1016/j.baae.2010.12.004

Brown, R. J., Döring, T. F., Storkey, J., Smith, J., Jones, H. E., and Potts, S. G. (2012). "Legume based plant mixtures for delivery of ecosystem services: pollinators," in Proceedings of the SAC and SEPA Biennial Conference, Agriculture and the Environment IX, Valuing Ecosystems: Policy, Economic and Management Interactions, ed. K. McCracken (Edinburgh: SAC Environmental), 156-162.

Broughton, W. J., Hernández, G., Blair, M., Beebe, S., Gepts, P., and Vanderleyden, J. (2003). Beans (Phaseolus spp.) - model food legumes. Plant Soil 252, 55-128. doi: 10.1023/A:1024146710611

Brush, S. B. (1999). Genes in the Field. On-Farm Conservation of Crop Diversity. Boca Raton, FL: Lewis Publishers. doi: 10.1201/9781420049824

Burbidge, A. H., and James, S. H. (1991). Post-zygotic seed abortion in the genetic system of Stylidium (Angiospermae, Stylidiaceae). J. Hered. 82, 319-328.

Carré, G., Roche, P., Chifflet, R., Morison, N., Bommarco, R., HarrisonCripps, J., et al. (2009). Landscape context and habitat type as drivers of bee diversity in European annual crops. Agric. Ecosyst. Environ. 133, 40-47. doi: 10.1016/j.agee.2009.05.001

Ceccarelli, S. (1986). "Problems of seed production in forage crops," in Seed Production Technologyed, eds J. P. Srivastava and L.T. Simarski (Aleppo: International Center for Agricultural Research in the Dry Areas).

Chagnon, M., Kreutzweiser, D., Mitchell, E. A. D., Morrissey, C. A., Noome, D. A., and Van der Sluijs, J. P. (2015). Risks of large-scale use of systemic insecticides to ecosystem functioning and services. Environ. Sci. Pollut. Res. 22, 119-134. doi: $10.1007 / \mathrm{s} 11356-014-3277-\mathrm{x}$

Chaplin-Kramer, R., Dombeck, E., Gerber, J., Knuth, K. A., Mueller, N. D., and Ziv, G., et al. (2014). Global malnutrition overlaps with pollinatordependent micronutrient production. Proc. R. Soc. B 281:20141799. doi: 10.1098/rspb.2014.1799

Chen, K. Y., Cong, B., Wing, R., Vrebalov, J., and Tanksley, S. D. (2007). Changes in regulation of a transcription factor lead to autogamy in cultivated tomatoes. Science 318, 643-645. doi: 10.1126/science.1148428

Christmann, S., and Aw-Hassan, A. A. (2012). Farming with alternative pollinators (FAP) -an overlooked win-win-strategy for climate change adaptation. Agric Ecosyt. Environ. 161, 161-164. doi: 10.1016/j.agee.2012.07.030

Christmann, S., Aw-Hassan, A., Rajabov, T., Khamraev, A. S., Symank, A., Ahmedov, A., et al. (2014). Farming with Alternative Pollinators (FAP) - A Practical Guide for Smallholders, Technical Report, Tashkent.

Clifford, P. T. P., and McCartin, S. J. M. (1985). Effects of pre-harvest treatment and mower and header types on seed loss and hard seed content at mowing, recovery and separation when harvesting a white clover seed crop. N. Z. J. Exp. Agric. 13, 307-316. doi: 10.1080/03015521.1985.10426099

Clifford, P. T. P., and Scott, D. (1989). Inflorescence, bumble bee, and climate interactions in seed crops of a tetraploid red clover (Trifolium pratense L.). J. Appl. Seed Prod. 7, 38-45.

Coors, J. G., and Pandey, S. (1999). The Genetics and Exploitation of Heterosis in Crops. Mexico: CIMMYT.

Cordoba, S. A., and Cocucci, A. A. (2011). Flower power: its association with bee power and floral functional morphology in papilionate legumes. Ann. Bot.108, 919-931. doi: 10.1093/aob/mcr196

Cost Action FA1307 (2014). Sustainable Pollination in Europe: Joint Research on Bees and Other Pollinators (SUPER-B). Available at: http://www.cost.eu/domains_actions/fa/Actions/FA1307 [Accessed August 11, 2015].

DeMooy, B. E., deMooy, C. J., and Burke, D. W. (1990). "Estimation of percentage natural outcrossing in cowpea (Vigna unguiculata L.) Walp in Botswana," in Proceedings of the Reports of Bean Improvement Cooperative and National Dry Bean Council Research Conference, Annual Report, Botswana, 122. Available at: http://nald c.nal.usda.gov/catalog/IND92013799

Delaplane, K. S., and Mayer, D. F. (2000). Crop Pollination By Bees. New York, NY: CABI Publishing. doi: 10.1079/9780851994482.0000

Desneux, N., Decourtye, A., and Delpuech, J. M. (2007). The sublethal effects of pesticides on beneficial arthropods. Annu. Rev. Entomol. 52, 81-106. doi: 10.1146/annurev.ento.52.110405.091440

Döring, T. F., Storkey, J., Baddeley, J. A., Crowley, O., Howlett, S. A., McCalman, H., et al. (2012). "Legume based plant mixtures for delivery of multiple ecosystem services: weed diversity and weed control," in Proceedings of the SAC and SEPA Biennial Conference, Agriculture and the Environment IX, Valuing Ecosystems: Policy, Economic and Management Interactions, ed. K. McCracken (Edinburgh: SAC Environmental), 163-168.

Duc, G., Agrama, H., Bao, S., Berger, J., Bourion, V., De Ron, A. M., et al. (2014). Breeding annual grain legumes for sustainable agriculture: new methods to approach complex traits and target new cultivar ideotypes. Crit. Rev. Plant Sci. 34, 381-411. doi: 10.1080/07352689.2014.898469

Duc, G., Bao, S., Baumc, M., Redden, B., Sadiki, M., Suso, M. J., et al. (2010). Diversity maintenance and use of Vicia faba L. genetic resources. Field Crop. Res. 115, 270-278. doi: 10.1016/j.fcr.2008.10.003

Dumet, D., Adeleke, R., and Faloye, B. (2008). "Regeneration guidelines: cowpea," in Crop specific Regeneration Guidelines [CDROM], eds M. E. Dulloo, I. Thormann, M. A. Jorge, and Hanson, J. (Rome: Hanson Rome in CGIAR System-wide Genetic Resource Programme), 8.

EASAC-The European Academies' Science Advisory Council (2015). Ecosystem Services, Agriculture and Neonicotinoids. EASAC policy report 26. Available at: www.easac.eu

El-Wakeil, N. E., and El-Sebai, T. N. E. (2009). Role of biofertilizer on faba bean growth, yield, and its effect on bean aphid and the associated predators. Arch. Phytopathol. Plant Protect. 42, 1144-1153. doi: 10.1080/032354007016 50882

Fairey, D. T., and Hampton, J. G. (1998). Forage Deed Production: Temperate Species, Vol. 1. New York, NY: CAB International.

Falcinelli, M. (1999). Temperate forage seed production: conventional and potential breeding strategies. J. New Seed 1, 37-66. doi: 10.1300/J153v01n01_04

Feltham, H., Park, K., and Goulson, D. (2014). Field realistic doses of pesticide imidacloprid reduce bumblebee pollen foraging efficiency. Ecotoxicology 23, 317-323. doi: 10.1007/s10646-014-1189-7

Free, J. B. (1993). Insect Pollination of Crops. New York, NY: Academic Press.

Freitas, G., Ganança, J. F. T., Nóbrega, H., Nunes, E., Costa, G., Slaski, J. J., et al. (2011). Morphological evaluation of common bean (Phaseolus vulgaris L.) diversity on the Island of Madeira. Genet. Resour. Crop Evol. 58, 861-874. doi: 10.1007/s10722-010-9624-y

Fu, D., Xiao, M., Hayward, A., Fu, Y., Liu, G., Jiang, G., et al. (2014). Utilization of crop heterosis: a review. Euphytica 197, 161-173. doi: 10.1007/s10681-0141103-7

Fuchs, S. J., and Hirnyck, R. E. (2000). Crop Profile for Lentil in Idaho State. Available at: http://www.ipmcenters.org/cropprofiles/docs/IDlentils.pdf [accessed May 2015].

Garibaldi, L. A., Bartomeus, I., Bommarco, R., Klein, A., Cunningham, S. A., and Aizen, M. A., et al. (2015). Trait matching of flower visitors and crops predicts fruit set better than trait diversity. J. Appl. Ecol. 52, 1436-1444. doi: $10.1111 / 1365-2664.12530$

Garibaldi, L. A., Carvalheiro, L. G., Leonhardt, S. D., Aizen, M. A., Blaauw, B. R., Isaacs, R., et al. (2014). From research to action: enhancing crop yield through wild pollinators. Front. Ecol. Environ. 12, 439-447. doi: 10.1890/ 130330

Gasim, S., and Link, W. (2007). Agronomic performance and the effect of selffertilization on German winter faba beans. J. Cent. Eur. Agric. 8, 121-128.

Genesys (2015). Gateway to Plant Genetic Resources. Available at: https://www.genesys-pgr.org/ (accessed July, 2015).

Gnanasambandam, A., Paull, J., Torres, A., Kaur, S., Leonforte, T., Li, H., et al. (2012). Impact of molecular technologies on faba bean (Vicia faba L.). Breed. Strategies Agron. 2, 132-166.

Godfray, H. C. J., Blacquière, T., Field, L. M., Hails, R. S., Petrokofsky, G., Potts, S. G., et al. (2014). A restatement of the natural science evidence base concerning neonicotinoid insecticides and insect pollinators. Proc. R. Soc. B 281:20140558. doi: 10.1098/rspb.2014.0558

Goldringer, I., Dawson, J., Rey, F., Vettoretti, A., Chable, V., Lammerts van Bueren, E., et al. (2010). "Breeding for resilience: a strategy for organic and lowinput farming systems?," in Proceedings of the EUCARPIA 2nd Conference of the Organic and Low- Input Agriculture Section, Paris.

Gómez, O. (2004). Evaluation of Nicaraguan Common Bean (Phaseolus vulgaris L.) landraces. Doctor's dissertation. Acta Universitatis agriculturae Sueciae, Agraria.

Goulson, D., Nicholls, E., Botías, C., and Rotheray, E. L. (2015). Bee declines driven by combined stress from parasites, pesticides, and Lack of flowers. Science 347:6229. doi: 10.1126/science.1255957 
Gradish, A. E., Scott-Dupree, C. D., Shipp, L., Harris, C. R., and Ferguson, G. (2010). Effect of reduced risk pesticides for use in greenhouse vegetable production on Bombus impatiens (Hymenoptera: Apidae). Pest Manag. Sci. 66, 142-146. doi: 10.1002/ps.1846

Gregorc, A., and Ellis, J. D. (2011). Cell death localization in situ in laboratory reared honey bee (Apis mellifera L.) larvae treated with pesticides. Pestic Biochem. Phys. 99, 200-207. doi: 10.1016/j.pestbp.2010.12.005

Gupta, S. C., Reddy, L. J., Sharma, D., Green, J. M., Murthi, A. N., and Saxena, K. B. (1981). "Maintenance of pigeonpea cultivars", in Proceedings of the International Workshop on Pigeonpeas, Vol. 1 (Patancheru: ICRISAT), 295-302.

Hammer, K. (1984). Das domestications syndrom. Kultupfanze 11, 11-34.

Hampton, J. G. (1990). Genetic variability and climatic factors affecting herbage legume seed production: an introduction. J. Appl. Seed Prod. 8, 45-51.

Helenius, J., and Stoddard, F. L. (2007). "Agro-ecosystem services from increased usage and novel applications of legumes. Integrating legume biology for sustainable agriculture," Sixth European Conference on Grain Legumes, Lisbon: Book of Abstracts.

Henry, M., Béguin, M., Requier, F., Rollin, O., Odoux, J. F., Aupinel, P., et al. (2012). A common pesticide decreases foraging success and survival in honey bees. Science 336, 348-350. doi: 10.1126/science.1215039

Holt, J., and Wratten, S. D. (1986). Components of resistance to Aphis fabae in faba bean. Entomol. Exp. Appl. 40, 35-40. doi: 10.1111/j.1570-7458.1986.tb0 2152.x

Horneburg, B. (2006). Outcrossing in lentil (Lens culinaris) depends on cultivar, location and year, and varies within cultivars. Plant Breed. 125, 638-640. doi: $10.1111 / \mathrm{j} .1439-0523.2006 .01290 . \mathrm{x}$

IPBES (2013). Report of the Second Session of the Plenary of the Intergovernmental Science-Policy Platform on Biodiversity and Ecosystem Services. Nairobi: United Nations Environment Program. Available at: http://www.ipbes.net [accessed August 11, 2015].

Jensen, E. S., Peoples, M. B., and Hauggaard-Nielsen, H. (2010). Faba bean in cropping systems. Field Crop. Res. 115, 203-216. doi: 10.1016/j.fcr.2009.10.008

Kasina, J. M., Hagen, M. L., Kraemer, M., Nderitu, J., Martius, C., and Wittmann, D. (2009a). Bee pollination enhances crop yield and fruit quality in Kakamega, western Kenya. E. Afr. Agric. For. J. 75, 1-11.

Kasina, J. M., Mburu, J., Kraemer, M., and Holm-Mueller, K. (2009b). Economic benefit of crop pollination by bees: a case of kakamega small-holder farming in western Kenya. J. Eco. Entom. 102, 467-473. doi: 10.1603/029.102.0201

Katznelson, J., and Morley, F. H. W. (1965). A taxonomic revison of sect. Calycomorphum of the genus Trifolium. I. The geocarpic species. Isr. J. Bot. 14, 112-134.

Kauffeld, N. M., and Sorensen, E. I. (1971). Interrelation of honey bee preference of alfalfa clones and flower color, aroma, nectar volume, and sugar concentration. Kansas Agric. Exp. Stn. Res. Publ. No 163, 1-14.

Kharrat, M., and Ouchari, H. (2011). Faba bean status and prospects in Tunisia. Grain Legumes 56, 11-12.

Klatt, B. K., Burmeister, C., Westphal, C., Tscharntke, T., and von Fragstein, M. (2013). Flower volatiles, crop varieties and bee responses. PLoS ONE 8:e72724. doi: 10.1371/journal.pone.0072724

Klein, A.-M., Vaissiere, B. E., Cane, J. H., Steffan-Dewenter, I., Cunningham, S. A., Kremen, C., et al. (2007). Importance of pollinators in changing landscapes for world crops. Proc. R. Soc. B. 274, 303-313. doi: 10.1098/rspb.2006.3721

Kobayashi, K., Tsukamoto, S., Tanaka, A., Nikura, S., and Ohsawa, R. (2010). Selective flower visitation behavior by pollinators in a radish $\mathrm{F} 1$ seed production field. Breed. Sci. 60, 203-211. doi: 10.1270/jsbbs.60.203

Kohler, F., Verhulst, J., Klink, R., and Kleijn, D. (2008). At what spatial scale do high-quality habitats enhance the diversity of forbs and pollinators in intensively farmed landscapes? J. Appl. Ecol 45, 753-762. doi: 10.1111/j.13652664.2007.01394.x

Köpke, U., and Nemecek, T. (2010). Ecological services of faba bean. Field Crop. Res. 115, 217-233. doi: 10.1016/j.fcr.2009.10.012

Kremen, C., Williams, N. M., Aizen, M. A., Gemmill-Herren, B., LeBuhn, G., and Minckley, R. et al. (2007). Pollination and other ecosystem services produced by mobile organisms: a conceptual framework for the effects of land-use-change. Ecol. Lett. 10, 299-314. doi: 10.1111/j.1461-0248.2007.01018.x

Landis, D. A., Wratten, S. D., and Gurr, G. M. (2000). Habitat management to conserve natural enemies of arthropod pests in agriculture. Annu. Rev. Entomol. 45, 175-201. doi: 10.1146/annurev.ento.45.1.175
Lesins, K. A., and Lesins, I. (1979). Genus Medicago (Leguminosae): A Taxogenetic Study. Dordrecht: Kluwer, 228. doi: 10.1007/978-94-009-9634-2

Lever, J. J., van Nees, E. H., Scheffer, M., and Bascompte, J. (2014). The sudden collapse of pollinator communities. Ecol. Lett. 17, 350-359. doi: $10.1111 /$ ele. 12236

Lloyd, D. G., and Schoen, D. J. (1992). Self- and cross-fertilization in plants. I. Functional dimensions. Int. J. Plant Sci. 153, 358-369. doi: 10.1086/ 297040

Longin, C. F. H., Mühleisen, J., Maurer, H. P., Zhang, H., Gowda, M., and Reif, J. C. (2012). Hybrid breeding in autogamous cereals. Theor. Appl. Genet. 125, 1087-1096. doi: 10.1007/s00122-012-1967-7

Loper, G. M., and Lapioli, A. M. (1971). Photoperiodic effects on the emanation of volatiles from alfalfa (Medicago sativa L.) florets. Plant Physiol. 49, 729-732. doi: $10.1104 /$ pp.49.5.729

Loper, G. M., and Waller, G. D. (1970). Alfalfa flower aroma and flower selection by honey bees. Crop Sci. 10, 66-68. doi: 10.2135/cropsci1970.0011183X001000010026x

Loper, G. M., Waller, G. D., and Berdel, R. L. (1974). Olfactory screening of alfalfa clones for uniform honey bee selection. Crop Sci. 14, 120-122. doi: 10. (2135)/cropsci1974.0011183X001400010037x

Loper, G. M., and Berdel, R. L. (1978). Seasonal emanation of ocimene from alfalfa flowers with three irrigation treatments. Crop Sci. 18, 447-452. doi: 10.2135/cropsci1978.0011183X001800030022x

Lorenzetti, F. (1981). "Relationship between dry matter and seed yield in Leguminous forage plants," in Proceedings of the Rep. Eucarpia Fodder Crops Section Meeting, ed. Van Bogaert (Merebelke: Rijksstation voor Plantenvereding), 57-74.

Lorenzetti, F. (1993). "Achieving potential herbage seed yields in species of temperate regions," in Proceedings of the XVII International Grassland Congress, eds M. J. Baker, J. R. Crush, and L. R. Humphreys (Palmerston North: Keeling \& Mundy LTD), 1621-1628.

Lorenzetti, F., and Negri V. (1995). Produzione potenziale e produzione reale delle colture da seme di leguminose foraggere. Riv. Agro. Anno 29, 242-259.

MA (2005). Ecosystems and Human Well-Being: Current State and Trends, Vol. 1, eds R. Hassan, R. Scholes, and N. Ash. Washington, DC: Island Press.

Maalouf, F. S., Suso, M. J., and Moreno, M. T. (1999). Choice of methods and indices for identifying the best parentals for synthetic varieties in faba bean. Agronomie 19, 705-712. doi: 10.1051/agro:19990805

Macfadyen, S., Davies, A. P., and Zalucki, M. P. (2015). Assessing the impact of arthropod natural enemies on crop pests at the field scale. Insect Sci. 22, 20-34. doi: 10.1111/1744-7917.12174

Maggioni, L., Ambrose, M., Schachl, R., Duc, G., and Lipman, E. (2002). Report of a Working Group on Grain Legumes. Rome: International Plant Genetic Resources Institute, 149.

Martin, B., and Adams M. W. (1987). Landraces of Phaseolus vulgaris (Fabaceae) in Northern Malawi a: legional variation. Econ. Bot. 41, 190-203. doi: 10.1007/BF02858965

Martiniello, P. (1998). Influence of agronomic factors on the relationship between forage production and seed yield in perennial forage grasses and legumes in a Mediterranean environment. Agronomie 18, 591-601. doi: 10.1051/agro:19980809

Martins, D. J. (2013). "People, plants and pollinators: uniting conservation, food security, and sustainable agriculture in east of Africa," in Conservation Biology: Voices from the Tropics, eds N. S. Sodhi, L. Gibson, and P. H. Raven (Hoboken, NJ: John Wiley \& Sons, Ltd.), 232-238.

McCouch, S. R., Kovach, M. J., Sweeney, M., Jiang, H., and Semon, M. (2012). "The dynamics of rice domestication: a balance between gene flow and genetic isolation," in Biodiversity in Agriculture: Domestication, Evolution, and Sustainability, eds P. Gepts, T. R. Famula, R. L. Bettinger, S. B. Brush, A. B. Damania, P. E. McGuire, et al. (Cambridge: Cambridge University Press), 311-329. doi: 10.1017/CBO97811390195 14.018

Milfont, M., Rocha, E. E. M., Lima, A. O. N., and Freitas, B. M. (2013). Higher soybean production using honeybee and wild pollinators, a sustainable alternative to pesticides and autopollination. Environ. Chem. Lett. 11, 335-341. doi: 10.1007/s10311-013-0412-8

Mommaerts, V., Reynders, S., Boulet, J., Besard, L., Sterk, G., and Smagghe, G. (2010). Risk assessment for side-effects of neonicotinoids against bumblebees 
with and without impairing foraging behavior. Ecotoxicology 19, 207-215. doi: 10.1007/s10646-009-0406-2

Monasterolo, M., Musicante, M. L., Valladares, G. R., and Salvo, A. (2015). Soybean crops may benefit from forest pollinators. Agric. Ecosyst. Environ. 202, 217-222. doi: 10.1016/j.agee.2015.01.012

Musallam, I. W., Haddad, N. J., Tawaha, A.-R. M., and Migdadi, O. S. (2004). The importance of bee-pollination in four genotypes of faba bean (Vicia faba L.). Int. J. Agricult. Biol. 6, 9-12.

Nabhan, G. P., and Buchmann, S. L. (1997). "Services provided by pollinators," in Nature's Services. Societal Dependence on Natural Ecosystems, ed. G. C. Daily (Washington, DC: Island Press), 133-150.

Nadal, S., Suso, M. J., and Moreno, M. (2003). Management of Vicia faba genetic resources: changes associated to the selfi ng process in the major, equina and minor groups. Gen. Resour. Crop Evol. 50, 183-192. doi: 10.1023/A:1022944017530

Nayak, G. K., Roberts, S. P. M., Garratt, M., Breeze, T. D., Tscheulin, T., HarrisonCripps, J., et al. (2015). Interactive effect of floral abundance and semi-natural habitats on pollinators in field beans (Vicia faba). Agric. Ecosyst. Environ. 199, 58-66. doi: 10.1016/j.agee.2014.08.016

Negri, V., Maxted, N., and Veterlainen, M. (2009). "European landrace conservation: an introduction," in 'European Landraces: On-farm Conservation, Management and Use. Bioversity Technical Bulletin No. 15, Bioversity International, eds M. Vetelainen, V. Negri, and N. Maxted (Italy: Bioversity International publisher), 1-22.

Negri, V., Pacicco, L., Bodesmo, M., and Torricelli, R. (2013). The First Italian Inventory of In Situ Maintained Landraces. Perugia: Morlacchi Editore.

Nieto, A., Roberts, S. P. M., Kemp, J., Rasmont, P., Kuhlmann, M., García Criado, M., et al. (2014). European Red List of bees. Luxembourg: Publication Office of the European Union.

Nuessly, G. S., Hentz, M. G., Beiriger, R., and Scully, B. T. (2004). Insects associated with Faba bean, Vicia faba (Fabales: Fabaceae), in southern Florida. Fla. Entomol. 87, 204-211. doi: 10.1653/00154040(2004)087[0204:IAWFBV]2.0.CO;2

Ollerton, J., Winfree, R., and Tarrant, S. (2011). How many flowering plants are pollinated by animals? Oikos 120, 321-326. doi: 10.1111/j. 1600$0706.2010 .18644 \mathrm{x}$

Ortiz-Perez, E., Horner, H. T., Hanlin, S. J., and Palmer, R. G. (2006a). Evaluation of insect-mediated seed set among soybean lines segregating for male sterility at the ms6 locus. Field Crop. Res. 97, 353-362. doi: 10.1016/j.fcr.2005.11.007

Ortiz-Perez, E., Horner, H. T., Hanlin, S. J., and Palmer, R. G. (2006b). Insectmediated seed-set evaluation of 21 soybean lines segregating for male sterility at 10 different loci. Euphytica 152, 351-360. doi: 10.1007/s10681-006-9222-4

Ortiz-Perez, E., Cianzio, S. R., Wiley, H., Horner, H. T., Davis, W. H., and Palmer, R. G. (2007). Insect-mediated cross-pollination in soybean [Glycine $\max ($ L.) Merrill] I. Agronomic performance. Field Crop. Res. 101, 259-268. doi: 10.1016/j.fcr.2006.12.003

Ortiz-Perez, E., Wiley, H., Horner, H. T., Davis, W. H., and Palmer, R. G. (2008). Insect-mediated cross-pollination in soybean [Glycine max (L.) Merrill]: II. Phenotypic recurrent selection. Euphytica 162, 269-280. doi: 10.1007/s10681007-9612-2

Palmer, R. G., Gai, J., Sun, H., and Burton, J. W. (2001). Production and evaluation of Hybrid Soybean. Plant Breed. Rev. 21, 263-307. doi: 10.1002/9780470650196.ch7

Palmer, R. G., Perez, P. T., Ortiz-Perez, E., Maalouf, F., and Suso, M. J. (2009). The role of crop-pollinator relationships in breeding for pollinator-friendly legumes: from a breeding perspective. Euphytica 170, 35-52. doi: 10.1007/s10681-0099953-0

Palmer, R. G., Gai, J., Dalvi, V. A., and Suso, M. J. (2011). "Male Sterility and Hybrid Production Technology," in Biology and Breeding of Food Legumes, eds A. Pratap and J. Kumar (Oxfordshire: CABI Publishers), 193-207. doi: 10.1079/9781845937669.0193

Palmer, R. G., Horner, H. T., Suso, M. J., Vangorp, M. L., and Pappas, A. L. (2012). "Soybean heterosis: the first steps," in Proceedings of the International Conference on Utilization of Heterosis in Crops (ICUHC). Toward a New Era of Crop Heterosis (Xi'an: Abstracts).

Papa, R., and Gepts, P. (2004). Gene Flow Between Crops and Their Wild Progenitors. Encyclopedia of Plant and Crop Science. New York, NY: Marcel Dekker, Inc. 488-491.
Pappas, A. L., Koziel, J. A., Healy, R. A., Horner, H. T., Bailey, T. B., Brown, A. H. D., et al. (2012). "Floral and aromatic analysis of the soybean perennial Glycine syndetika for future agronomic exploitation," in Proceedings of the XXII International Congress on Sexual Plant Reproduction, Melbourne, 13.

Partap, U., and Ya, T. (2012). The human pollinators of fruit crops in Maoxian County, Sichuan, China. Mt. Res. Dev. 32, 176-186. doi: 10.1659/MRDJOURNAL-D-11-00108.1

Pasumarty, S. V., Matsumura, T., Higuchi, S., and Yamada, T. (1993). Causes of low seed set in white clover (Trifolium repens L.). Grass Forage Sci. 48, 79-83. doi: 10.1111/j.1365-2494.1993.tb01839.x

Pecetti, L., and Tava, A. (2000). Effect of flower color and sampling time on volatile emanation in alfalfa flowers. Crop Sci. 40, 126-130. doi: $10.2135 /$ cropsci2000.401126x

Pecetti, L., Tava, A., Felicioni, A., Pinzauti, M., and Piano, E. (2002). Effect of three volatile compounds from lucerne flowers on their attractiveness towards pollinators. Bull. Insectol. 55, 21-27.

Pecetti, L., and Proietti, S. (2003). Estimation of cross-pollination by leafcutter bees in two lucerne genotypes with different flower colour. J. Genet. Breed. 57, 219-224.

Pérez-De-Luque, A., Eizenberg, H., Grenz, J. H., Sillero, J. C., Ávila, C., Sauerborn, J., et al. (2010). Broomrape management in faba bean. Field Crop. Res. 115, 319-328. doi: 10.1016/j.fcr.2009.02.013

Pettis, J. S., Engelsdorp, D., van, Johnson, J., and Dively, G. (2012). Pesticide exposure in honey bees results in increased levels of the gut pathogen Nosema. Naturwissenschaften 99, 153-158. doi: 10.1007/s00114-011-0881-1

Piano, E., and Pecetti, L. (2010). "Minor legume species," in Fodder Crops and Amenity Grasses, Handbook of Plant Breeding, Vol. 5, eds B. Boller, U. K. Posselt, and F. Veronesi (New York, NY: Springer), 477-500. doi: 10.1007/978-1-44190760-8_20

Pierre, J., Suso, M. J., Moreno, M. T., Esnault, R., and Le Guen, J. (1999). Diversite et efficacite de l'entomofaune pollinisatrice (Hymenoptera: Apidae) de la feverole (Vicia faba L.) sur deux sites, en France et en Espagne. Ann. Soc. Entomol.Fr.(N.S.) 35(suppl.), 312-318.

Pinheiro de Carvalho, M. A. A., Bebeli, P. J., Barata da Silva, A. M., Bettencourt, E., Slaski, J. J., and Dias, S. (2016). "Agro-biodiversity. the importance of inventories in the assessment of crop diversity and its time and spatial changes," in Genetic Erosion and Biodiversity, eds M. R. Ahuja and S. M. Jain (Berlin: Springer), $130-147$.

Pinheiro de Carvalho, M. A. A., Bebeli, P. J., Bettencourt, E., Costa, G., Dias, S., dos Santos, T. M. M., et al. (2013). Cereal landraces genetic resources in worldwide GeneBanks. Agron. Sustain. Dev. 33, 177-203. doi: 10.1007/s13593-0120090-0

Pisa, L. W., Amaral-Rogers, V., Belzunces, L. P., Bonmatin, J. M., Downs, C. A., Goulson, D., et al. (2015). Effects of neonicotinoids and fipronil on nontarget invertebrates. Environ. Sci. Pollut. Res. 22, 68-102. doi: 10.1007/s11356-0143471-x

Potts, S. G., Biesmeijer, J. C., Kremen, C., Neumann, P., Schweiger, O., and Kunin, W. E. (2010). Global pollinator declines: trends, impacts and drivers. Trends Ecol. Evol. 25, 345-353. doi: 10.1016/j.tree.2010.01.007

Purseglove (1968). Tropical Crops: Dicotyledons. London: Longman Group Limited, 246-250.

Rahman, M. A., Rahman, M. M., and Sarkar, M. A. (2001). Progress in isolation and purification of Lathyrus sativus breeding lines Lathyrus. Lathyrism Newsl. 2, 39-40.

Rebek, E. J., Sadof, C. S., and Hanks, L. M. (2005). Manipulating the abundance of natural enemies in ornamental landscapes with floral resource plants. Biol. Control 33, 203-216. doi: 10.1016/j.biocontrol.2005.02.011

Redden, B., Leonforte, T., Ford, R., Croser, J., and Slattery, J. (2005). "Pea (Pisum sativum)," in Genetic Resources, Chromosome Engineering, and Crop Improvement, eds R. Singh and P. Juahar (Boca Raton, FL: CRC Press), 49-83.

Richards, A. J. (2001). Does low biodiversity resulting from modern agricultural practice affect crop pollination and yield? Ann. Bot. 88, 165-172. doi: 10.1006/anbo.2001.1463

Rick, C. M. (1988). "Evolution of mating systems in cultivated plants," in Plant Evolutionary Biology, eds L. D. Gottlieb and S. Jain (London: Chapman \& Hall), 133-147.

Ricketts, T. H., Regetz, J., Steffan-Dewenter, I., Cunningham, S. A., Kremen, C., Bogdanski, A., et al. (2008). Landscape effects on crop pollination services: 
are there general patterns? Ecol. Lett. 11, 499-515. doi: 10.1111/j.14610248.2008.01157.x

Riedl, H., Johansen, E., Brewer, L., and Barbour, J. (2006). How to Reduce Bee Poisoning From Pesticides, PNW 591. Moscow, ID: University of Idaho, 24.

Rispail, N., Kalo, P., Kiss, G. B., Ellis, T. H. N., Gallardo, K., Richard, D., et al. (2010). Model legumes contribute to faba bean breeding. Field Crop. Res. 115, 253-269. doi: 10.1016/j.fcr.2009.03.014

Rosellini, D., Lorenzetti, F., and Bingham, E. T. (1998). Quantitative ovule sterility in Medicago sativa. Theor. Appl. Genet. 97, 1289-1295. doi: 10.1007/s001220051021

Rosellini, D., Veronesi, F., and Falcinelli, M. (1994). Recurrent selection of microgametophytic vigour in alfa-alfa and correlated response at the sporophytic level. Crop Sci. 34, 933-936. doi: 10.2135/cropsci1994.0011183X003400040017x

Rubio, J., Fernandez-Romero, M. D., Millán, T., Gil, J., and Suso, M. J. (2010). "Outcrossing rate and genetic structure on an open-flowering population of Cicer arietinum based on microsatellite markers," in Proceedings of the 5th Lnternaional Food Legumes Research Conference on and 7the European Conference on Grain Legumes for Global Health Legume Crops and Products for Food, Feed and Environmental Benefits (Antalyap: Book of Abstracts), 220.

Rundlöf, M., Andersson, G. K. S., Bommarco, R., Fries, I., Hederström, V., Herbertsson, L., et al. (2015). Seed coating with a neonicotinoid insecticide negatively affects wild bees. Nature 521, 77-80. doi: 10.1038/nature14420

Sanchez-Bayo, F., and Goka, K. (2014). Pesticide residues and bees-a risk assessment. PLOS ONE 9:e94482. doi: 10.1371/journal.pone.0094482

Satta, A., Acciaro, M., Floris, I., LentinI, A., and Sulas, L. (2000). "Insect pollination of sulla (Hedysarum coronarium L.) and its effect on seed production," in Legumes for Mediterranean Forage Crops, Pastures and Alternative Uses, ed. S. L. Zaragoza (Paris: CIHEAM), 373-377.

Saunders, M. E., Peisley, R. K., Rader, R., and Luck, G. W. (2015). Pollinators, pests, and predators: recognizing ecological trade-offs in agroecosystems. Ambio 45, 1-11. doi: 10.1007/s13280-015-0696-y

Saxena, K. B. (2005). "Pigeonpea [Cajanus cajan (L.) Millsp.]" in Genetic Resources, Chromosome Engineering, and Crop Improvement: Grain Legumes, Vol. 1, eds R. J. Singh and P. P. Jauhar (Boca Raton, CA: CRC Press), 85-110. doi: 10.1201/9780203489284.ch4

Saxena, K. B., and Kumar, R. V. (2010). Insect-aided natural out-crossing in four wild relativesof pigeonpea. Euphytica 173, 329-335. doi: 10.1007/s10681-0090088-0

Saxena, K. B., Kumar, R. V., Tikle, A. N., Saxena, M. K., Gautam, V. S., Rao, S. K., et al. (2013). ICPH 2671 - the world's first commercial food legume hybrid. Plant Breed. 132, 479-485. doi: 10.1111/pbr.12045

Schellhorn, N. A., Bellati, J., Paull, C. A., and Maratos, L. (2008). Parasitoid and moth movement from refuge to crop. Basic Appl. Ecol. 9, 691-700. doi: 10.1016/j.baae.2007.12.010

Sengul, S. (2006). Using path analysis to determine Lucerne (Medicago sativa L.) seed yield and its components. N. Z. J. Agric. Res. 49, 107-115. doi: 10.1080/00288233.2006.9513700

Sewell, J., Hill, R., and Reich, J. (2011). Persistence of Grazing Tolerant Lucernes under Australian, Conditions. Pasture Persistence. Grassland Res. Pract. Ser. 15, 187-190.

Shackelford, G., Steward, P. R., Benton, T. G., Kunin, W. E., Potts, S. G., Biesmeijer, J. C., et al. (2013). Comparison of pollinators and natural enemies: a metaanalysis of landscape and local effects on abundance and richness in crops. Biol. Rev. Camb. Philos. Soc. 88, 1002-1021. doi: 10.1111/brv.12040

Sharma, H. C., Gowda, C. L. L., Stevenson, P. C., Ridsill-Smith, T., Clement, S. L., Rao, R., et al. (2007). "Host plant resistance and insect pest management in chickpea," in Chickpea Breeding \& Management, eds S. S. Yadav, R. Redden, W. Chen, and B. Sharma (Oxfordshire: CABI International), 520-537.

Shirk, R. Y., and Hamrick, J. L. (2014). High but variable outcrossing rates in the invasive Geranium carolinianum (Geraniaceae). Am. J. Bot. 101, 1200-1206. doi: $10.3732 /$ ajb.1400224

Sicard, A., and Lenhard, M. (2011). The selfing syndrome: a model for studying the genetic and evolutionary basis of morphological adaptation in plants. Ann. Bot. 107, 1433-1443. doi: 10.1093/aob/mcr023

Sigsgaard, L., Betzer, C., Naulin, C., Eilenberg, J., Enkegaard, A., and Kristensen, K. (2013). The effect of floral resources on parasitoid and host longevity: prospects for conservation biological control in strawberries. J. Insect Sci. 13, 1-7. doi: 10.1673/031.013.10401

Sillero, J. C., Villegas-Fernández, A. M., Thomas, J., Rojas-Molina, M. M., Emerand, A. A., Fernández-Aparicio, M., et al. (2010). Faba bean breeding for disease resistance. Field Crop. Res. 115, 297-307. doi: 10.1016/j.fcr.2009.09.012

Singh, L., Gupta, S. C., and Farris, D. G. (1990). "Pigeonpea: breeding," in The Pigeonpea, ed. Y. L. Nene (Wallingford: CAB International), 375-399.

Singh, N. B., Singh, I. P., and Singh, B. B. (2005). "Pigeonpea breeding," in Advances in Pigeonpea Research, eds M. Ali and S. Kumar (Kanpur: Indian Institute of Pulses Research), 67-95.

Singh, N., Tyagi, R. K., and Pandey, C. (2013). Genetic Resources of Pigeonpea (Cajanus cajan): Conservation for Use. New Delhi: National Bureau of Plant Genetic Resources, 49p.

Smýkal, P., Coyne, C. J., Ambrose, M. J., Maxted, N., Schaefer, H., and Matthew, W., et al. (2015). Legume crops phylogeny and genetic diversity for science and breeding. Crit. Rev. Plant Sci. 34, 43-104. doi: 10.1080/07352689.2014.8 97904

Steffan-Dewenter, I., Munzenberg, U., Burger, C., Thies, C., and Tscharntke, T. (2002). Scale-dependent effects of landscape context on three pollinator guilds. Ecology 83, 1421-1432. doi: 10.1890/00129658(2002)083[1421:SDEOLC]2.0.CO;2

Steiner, J. J., Beuselinck, P. R., Peaden, R. N., Kojis, W. P., and Bingham, E. T. (1992). Pollinator effects on crossing and genetic shift in a three-flower-color alfalfa population. Crop Sci. 32, 73-77. doi: 10.2135/cropsci1992.0011183X003200010016x

Stevenson, P. C., Dhillon, M. K., Sharma, H. C., and El Bouhssini, M. (2007). "Insect pests of lentil and their management," in Lentil: An Ancient Crop for Modern Times, eds S. S. Yadav, D. L. McNeil, and P. C. Stevenson (Berlin: Springer), 331-348. doi: 10.1007/978-1-4020-6313-8_20

Stoddard, F. L., Nicholas, A. H., Rubiales, D., Thomas, J., and Villegas-Fernández, A. M. (2010). Integrated pest management in faba bean. Field Crop. Res. 115, 308-318. doi: 10.1016/j.fcr.2009.07.002

Stoddard, F. L. (2013). Agronomic case studies in Legume Futures. Legume Futures Report 1.2. Available at: http://www.legumefutures. de/images/Legume_Futures_Report_1.2.pdf

Street, K., Ismail, A., and Rukhkyan, N. (2008a). "Regeneration guidelines: faba bean," in Crop Specific Regeneration Guidelines [CD-ROM], eds M. E. Dulloo, I. Thormann, M. A. Jorge, and J. Hanson (Rome: CGIAR System-wide Genetic Resource Programme), 9.

Street, K., Ismail, A. and Rukhkyan, N. (2008b). "Regeneration guidelines: lentil," in Crop specific Regeneration Guidelines [CD-ROM], eds M. E. Dulloo, I. Thormann, M. A. Jorge, and J. Hanson (Rome: CGIAR System-wide Genetic Resource Programme), 10.

Street, K., Rukhkyan, N., and Ismail, A. (2008c). "Regeneration guidelines: chickpea," in Crop Specific Regeneration Guidelines [CD-ROM], eds M. E. Dulloo, I. Thormann, M. A. Jorge, and J. Hanson (Rome: CGIAR System-wide Genetic Resource Programme), 10.

Strickler, K. (1999). Impact of flower standing crop and pollinator movement on alfalfa seed yield. Environ. Entomol. 28, 178-187. doi: 10.1093/ee/28.2.178

Strickler, K., and Vinson, J. (2000). A simulation of the effect of pollinator movement on alfalfa seed production. Environ. Entomol. 29, 907-918. doi: 10.1603/0046-225X-29.5.907

Suso, M. J., Bebeli, P. J., and Palmer, R. (2015). "Reproductive biology of grain legumes," in Grain Legumes, Handbook of Plant Breeding, ed. A. de Ron (Berlin: Springer Science Business Media), 364-399.

Suso, M. J., Harder, L., Moreno, M. T., and Maalouf, F. (2005). New strategies for increasing heterozygosity in crops: Vicia faba mating system as a study case. Euphytica 143, 51-65. doi: 10.1007/s10681-005-2526-y

Suso, M. J., and Maalouf, F. (2010). Direct and correlated responses to upward and downward selection for outcrossing in Vicia faba. Field Crop. Res. 116, 116-126. doi: 10.1016/j.fcr.2009.12.001

Suso, M. J., Moreno, M. T., Mondragao Rodrigues, F., and Cubero, J. I. (1996). Reproductive biology of Vicia faba: role of pollination conditions. Field Crop. Res. 46, 81-91. doi: 10.1016/0378-4290(95)00089-5

Suso, M. J., Nadal, S., and Palmer, R. G. (2010). "Potential power of the plantpollinator relationship as a tool to enhance both environmental and production services of grain legumes in the context of low-input agriculture: what do we know?", in Proceedings of the Breeding for resilience: a strategy for organic and 
low-input farming systems. Eucarpia 2nd Conference of the Organic and LowInput Agriculture Section; 2010 Dec 1-3, eds I. Goldringer, J. Dawson, F. Rey, and A. Vettoretti (Paris: INRA), 23-26.

Suso, M. J., and Río, R. (2014). Faba bean gene-pools development for low-input agriculture: understanding early stages of natural selection. Euphytica 196, 77-93. doi: 10.1007/s10681-013-1015-y

Suso, M. J., and Río, R. (2015). A crop-pollinator inter-play approach to assessing seed production patterns in faba bean under two pollination environments. Euphytica 201, 231-251. doi: 10.1007/s10681-014-1200-7

Suso, M. J., Vishnyakova, M., Ramos, A., Duc, G., and Ambrose, M. (2011). An international survey on state of the art of grain legume management in gene banks. J. Agr. Sci. Tech. B 1, 975-981.

Tapparo, A., Marton, D., Giorio, C., Zanella, A., Soldà, L., Marzaro, M., et al. (2012). Assessment of the environmental exposure of honey bees to particulate matter containing neonicotinoid insecticides coming from corn coated seeds. Environ. Sci. Technol. 46, 2592-2599. doi: 10.1021/es2035152

TEEB (2010). The Economics of Ecosystems and Biodiversity. Ecological and Economic Foundations, ed. P. Kumar. London: Earthscan.

Tester, M., and Langridge, P. (2010). Breeding technologies to increase crop production in a changing world. Science 327, 818-822. doi: 10.1126/science. 1183700

Thomas, R. G. (1996). "Maximising seed production potential in white clover: factors influencing seed set per floret," in Proceedings of the White Clover: New Zealand's Competitive Edge, A Joint Symposium Between Agronomy Society of New Zealand and New Zealand Grassland Association Held at Lincoln University (Christchurch: Agronomy Society of New Zealand), 41-44.

Thomas, R. G., and Pasumarty, S. V. (1996). Limitations to seed set in white clover (Trifolium repens L.). I. Preliminary observations. J. App. Seed Prod. 14, 59-66.

Torricelli, R., Colesanti, N., and Falcinelli, M. (2007). "Improved seed production in a new Italian cultivar of lucerne (Medicago sativa L.)," in Proceedings of the Sixth International Herbage Seed Conference, Gjennestad, Norway - 18-20 June 2007, Bioforsk, 100-106.

Upadhyaya, H. D., Dwivedi, S. L., Ambrose, M., Ellis, N., Berger, J., Sm'ykal, P., et al. (2011). Legume genetic resources: management, diversity assessment, and utilization in crop improvement. Euphytica 180, 27-47. doi: 10.1007/s10681011-0449-3

Upadhyaya, H. D., Reddy, K. N., and Sastry, D. V. S. S. R. (2008). "Regeneration guidelines: pigeon pea," in Crop Specific Regeneration Guidelines [CD-ROM], eds M. EDulloo, I. Thormann, M. A. Jorge, and J. Hanson (Rome: CGIAR System-wide Genetic Resource Programme), 8.

Van Driesche, R. G., Lyon, S., Sanderson, J. P., Bennett, K. C., Stanek, E. J., III, and Zhang, R. (2008). Greenhouse trials of Aphidius colemani (Hymenoptera:
Braconidae) bankerplants for control of aphids (Hemiptera: Aphididae) in greenhouse spring floral crops. Fl. Entomol. 91, 583-591.

Veronesi, F., and Lorenzetti, F. (1983). Productivity and survival of alfalfa hybrid and inbred plants under competitive conditions. Crop Sci. 23, 557-580. doi: 10.2135/cropsci1983.0011183X002300030031x

Westerkamp, C., and Gottsberger, G. (2000). Diversity pays in crop pollination. Crop Sci. 40, 1209-1222. doi: 10.2135/cropsci2000.4051209x

Westphal, C., Steffan-Dewenter, I., and Tscharntke, T. (2003). Mass flowering crops enhance pollinator densities at a landscape scale. Ecol. Lett. 6, 961-965. doi: 10.1046/j.1461-0248.2003.00523.x

Wiens, D. (1984). Ovule survivorship, brood size, life history, breeding systems, and reproductive success in plants. Oecologia 64, 47-53. doi: 10.1007/BF00377542

Williams, N. M., Crone, E. E., Roulston, T. H., Minckley, R. L., Packer, L., and Potts, S. G. (2010). Ecological and life-history traits predict bee species responses toenvironmental disturbances. Biol. Conserv. 143, 2280-2291. doi: 10.1016/j.baae.2010.11.008.

Woodcock, T. S. (2012). Pollination in the Agricultural Landscape: Best Management Practices for Crop Pollination. Guelph, ON: University of Guelph.

Worthington, M., Soleri, D., Aragón-Cuevas, F., and Gepts, P. (2012). Genetic Composition and Spatial Distribution of Farmer-managed Phaseolus Bean Plantings: an Example from a Village in Oaxaca, Mexico. Crop Sci. 52, 17211735. doi: 10.2135/cropsci2011.09.0518

Yagoubi, N., and Chrki, A. (2000). Estimation of mating system parameters in Hedysarum coronarium L. (Leguminoseae, Fabaceae). Agronomie. EDP Sciences 20, 933-942. doi: 10.1051/agro:2000103

Zeven, A. C. (1998). Landraces: a review of definitions and classifications. Euphytica 104, 127-139. doi: 10.1023/A:1018683119237

Zhao, Y., Mette, M. F., and Reif, J. C. (2015). Genomic selection in hybrid breeding. Plant Breed. 134, 1-10. doi: 10.1111/pbr.12231

Conflict of Interest Statement: The authors declare that the research was conducted in the absence of any commercial or financial relationships that could be construed as a potential conflict of interest.

Copyright (c) 2016 Suso, Bebeli, Christmann, Mateus, Negri, Pinheiro de Carvalho, Torricelli and Veloso. This is an open-access article distributed under the terms of the Creative Commons Attribution License (CC BY). The use, distribution or reproduction in other forums is permitted, provided the original author(s) or licensor are credited and that the original publication in this journal is cited, in accordance with accepted academic practice. No use, distribution or reproduction is permitted which does not comply with these terms. 


\section{Influence of Rhizoctonia solani and Trichoderma spp. in growth of bean (Phaseolus vulgaris L.) and in the induction of plant defense-related genes}

\section{OPEN ACCESS}

Edited by:

Antonio M. De Ron,

Spanish National Research Council Misión Biológica de Galicia, Spain

Reviewed by:

Cristina Cabaleiro Sobrino, Universidade de Santiago de Compostela, Spain

Eleonora Cominelli, Istituto di Biologia e Biotecnologia Agraria, Consiglio Nazionale delle

Ricerche, Italy

*Correspondence:

Pedro A. Casquero,

Research Group of Engineering and Sustainable Agriculture, Natural Resources Institute, University of León, Av. Portugal 41 24071 León, Spain

pedro-casquero@unileon.es

Specialty section:

This article was submitted to Crop Science and Horticulture, a section of the journal

Frontiers in Plant Science

Received: 29 June 2015

Accepted: 18 August 2015

Published: 16 September 2015

Citation:

Mayo S, Gutiérrez S, Malmierca MG, Lorenzana A, Campelo MP, Hermosa $R$ and Casquero PA (2015) Influence of Rhizoctonia solani and Trichoderma spp. in growth of bean (Phaseolus vulgaris $L$.) and in the induction of plant defense-related genes.

Front. Plant Sci. 6:685. doi: 10.3389/fpls.2015.00685

\begin{abstract}
Sara Mayo ${ }^{1}$, Santiago Gutiérrez ${ }^{2}$, Monica G. Malmierca ${ }^{2}$, Alicia Lorenzana ${ }^{1}$, M. Piedad Campelo ${ }^{1}$, Rosa Hermosa ${ }^{3}$ and Pedro A. Casquero ${ }^{1 *}$
\end{abstract}

\begin{abstract}
${ }^{1}$ Research Group of Engineering and Sustainable Agriculture, Natural Resources Institute, University of León, León, Spain, ${ }^{2}$ Area of Microbiology, University School of Agricultural Engineers, University of León, Ponferrada, Spain, ${ }^{3}$ Department of Microbiology and Genetics, Spanish-Portuguese Centre for Agricultural Research, University of Salamanca, Salamanca, Spain
\end{abstract}

Many Trichoderma species are well-known for their ability to promote plant growth and defense. We study how the interaction of bean plants with $R$. solani and/or Trichoderma affect the plants growth and the level of expression of defense-related genes. Trichoderma isolates were evaluated in vitro for their potential to antagonize $R$. solani. Bioassays were performed in climatic chambers and development of the plants was evaluated. The effect of Trichoderma treatment and/or $R$. solani infection on the expression of bean defense-related genes was analyzed by real-time PCR and the production of ergosterol and squalene was quantified. In vitro growth inhibition of $R$. solani was between 86 and 58\%. In in vivo assays, the bean plants treated with Trichoderma harzianum T019 always had an increased size respect to control and the plants treated with this isolate did not decrease their size in presence of $R$. solani. The interaction of plants with $R$. solani and/or Trichoderma affects the level of expression of seven defense-related genes. Squalene and ergosterol production differences were found among the Trichoderma isolates, T019 showing the highest values for both compounds. T. harzianum T019 shows a positive effect on the level of resistance of bean plants to $R$. solani. This strain induces the expression of plant defense-related genes and produces a higher level of ergosterol, indicating its ability to grow at a higher rate in the soil, which would explain its positive effects on plant growth and defense in the presence of the pathogen.

Keywords: antifungal activity, defense-related genes, qPCR, ergosterol, squalene

\section{Introduction}

The common bean (Phaseolus vulgaris L.) is the third most important food legume crop worldwide, surpassed only by the soybean [Glycine $\max$ (L.) Merr.] and peanut (Arachis hypogea L.). Among the southern countries of the European Union, Spain together with Italy and Greece are the main common bean producers. León, a province located at the northwest of Spain, is 
the main producer province by quantity and quality, with almost $45 \%$ of Spanish production in 2014. Socio-economic conditions of León province enabled possible the maintenance of local varieties in traditional cropping systems, which are based in small-scale farms (Casquero et al., 2006). The high quality of this legume has been awarded with a Protected Geographic Indication (PGI) (EC Reg. n.256/2010 published on 26 March 2010, OJEU L880/17). In the last few years, however dry bean production has gone through difficulties due to relatively low yields (mainly caused by fungus, virus, and bacteria) and insufficient income for growers.

Root rots are the main diseases caused by soil fungi having their incidence on bean yield. Rhizoctonia solani JG Kühn [Teleomorph: Thanatephorus cucumeris (AB Frank) Donk] is the main root rot in León, being detected in $91.8 \%$ of affected plants in an evaluation of its occurrence in bean plants (Valenciano et al., 2006). Plant infection occurs through wounds or by a coating of an organ with mycelium, which tears the cuticle and penetrates the epidermis. This pathogen is more aggressive at temperatures between 15 and $18^{\circ} \mathrm{C}$ and in moist soils. It is a necrotrophic pathogen, distributed worldwide (Guerrero-González et al., 2011). R. solani is one of the root and hypocotyl pathogen that causes most economic losses worldwide.

Trichoderma (Teleomorph: Hypocrea) is a fungal genus that is found in the soil. It is a secondary fast growing opportunistic invasive, which produces large numbers of spores, enzymes able to degrade the fungal cell wall (chitinases, glucanases, and proteases) and compounds with antimicrobial activity. Many Trichoderma species are also well known as biocontrol agents (BCA) of important phytopathogenic fungi. The primary mechanisms of biocontrol used by Trichoderma in direct confrontation with pathogenic fungi are the mycoparasitism (Papavizas, 1985) antibiosis, and competition for nutrients with the pathogen (Harman and Kubicek, 1998).

Many Trichoderma species colonize the root surface and cause substantial changes in plant metabolism (Harman et al., 2004). The physical interaction between Trichoderma and plants is limited to the first cell layer of the epidermis and the root bark. This symbiotic relationship would thus protect plants against pathogens. Trichoderma induces the expression of genes involved in defense response and promotes plant growth, root development and nutrient availability (Hermosa et al., 2012). During the Trichoderma-plant interaction various classes of metabolites could induce resistance such as proteins with enzymatic activity, low molecular weight compounds, related to the fungal or the plant cell wall, originated by the enzymatic activity of Trichoderma (Woo et al., 2006; Woo and Lorito, 2007) and other secondary metabolites that trigger plant defense mechanisms against the pathogen (Hermosa et al., 2012; Malmierca et al., 2014), by inducing the expression of pathogenesis-related (PR) proteins that reduce the diseases symptoms. Thus, when the plant contacts with a pathogen it is activated a mechanism of systemic acquired resistance (SAR). However, when they interact with a non-pathogen organism the plants activated a mechanism for induced systemic resistance (ISR) (Hermosa et al., 2013; Mukherjee et al., 2013).
Squalene is a polyunsaturated terpene that is an intermediate in the ergosterol biosynthetic pathway, which has an essential function in the fungal cell structure. The levels of squalene will influence the level of ergosterol biosynthesis (Garaiová et al., 2013). In addition to its structural function, and as a result of its importance in fungal development, ergosterol is also able to activate the expression of a number of defense genes and could increase the resistance of plants against pathogens (Lochman and Mikeš, 2005).

In this work 23 Trichoderma isolates were collected from bean fields. These isolates were used to study their effect on the growth of bean plants, and also in the defense response of plants against the phytopathogen $R$. solani. Thus, parameters as plant growth in the presence of the pathogen and/or the different Trichoderma isolates were evaluated, also analyzing the level of expression of defense-related genes in plants treated with the selected Trichoderma isolate.

\section{Materials and Methods}

\section{Trichoderma and R. solani Isolates and Culture Collections}

The present study was conducted with twenty-three isolates of Trichoderma (Table 1) collected from the production area of the Protected Geographical Indication (PGI), called "Alubia La Bañeza-León,” without any genetic manipulation and three isolates from other collections. The Trichoderma isolates were stored in the collection "Pathogens and Antagonists of the Laboratory Diagnosis of Pests and Diseases" (PALDPD, University of León, León, Spain). R. solani R43 was also collected from plants of the same PGI and selected by its high virulence (Table 1).

\section{In vitro Antifungal Assays}

Trichoderma isolates were evaluated for their in vitro potential to antagonize the plant pathogenic fungus $R$. solani using two different tests. For all tests, plugs of $7 \mathrm{~mm}$ diameter collected from the edge of growing fungal colonies were used to inoculate potato dextrose agar medium (PDA) in sterile Petri dishes of $9 \mathrm{~cm}$ diameter. The dishes were incubated in the dark at $22^{\circ} \mathrm{C}$ for 7 days.

The aim of these tests was to study the percentage of $R$. solani growth inhibition caused by the different Trichoderma isolates.

The antifungal assay on membranes was used to quantify the ability of the Trichoderma isolates to produce metabolites and/or enzymes with inhibitory activity against $R$. solani. The surface of Petri dishes containing PDA medium was overlaid with a sterile cellophane membrane. Trichoderma plugs, extracted from PDA dishes grown for 7 days at $22^{\circ} \mathrm{C}$, were placed in the center of the dish with the cellophane sheet, containing PDA medium, and incubated for $48 \mathrm{~h}$ at $22^{\circ} \mathrm{C}$. Then, the cellophane membranes along with the mycelia of Trichoderma isolates were removed and $R$. solani plugs were placed in the same plates. Growth of $R$. solani was recorded after $72 \mathrm{~h}$ to calculate the percentage of pathogen growth inhibition (Figure 1A). Control PDA plates of $R$. solani, where Trichoderma spp. had not been previously grown, were also prepared in the same conditions as above. 
TABLE 1 | Trichoderma and Rhizoctonia strains used in this study.

\begin{tabular}{|c|c|c|c|}
\hline Lab. Code & Culture collection ${ }^{\star \star \star}$ & Localization/Received as & Identified as/References \\
\hline T001* $^{*}$ & PAULET20 & Bustillo del Páramo (León) & T. harzianum \\
\hline $\mathrm{TOO}^{*}$ & PAULET21 & Roperuelos del Páramo (León) & Trichoderma spp. \\
\hline T003* $^{*}$ & PAULET22 & Bercianos del Páramo (León) & Trichoderma spp. \\
\hline T004* $^{*}$ & PAULET23 & Riego de la Vega (León) & T. gamsii \\
\hline $\mathrm{TOO5}^{\star}$ & PAULET24 & Valderrey (León) & T. longibrachiatum \\
\hline T006* $^{*}$ & PAULET25 & San Esteban de Nogales (León) & Trichoderma spp. \\
\hline $\mathrm{T}_{007 *}^{*}$ & PAULET26 & Villamejil (León) & Trichoderma spp. \\
\hline T008* & PAULET27 & Fresno de la Vega (León) & T. citrinoviride \\
\hline T009 & PAULET28 & Bustillo del Páramo (León) & Trichoderma spp. \\
\hline T010 & PAULET29 & Bustillo del Páramo (León) & T. harzianum \\
\hline T011* & PAULET30 & San Pedro de Bercianos (León) & T. harzianum \\
\hline T012* & PAULET31 & Quintana del Castillo (León) & T. harzianum \\
\hline T013* & PAULET32 & Quintana del Castillo (León) & T. atroviride \\
\hline T014 & PAULET33 & Santa Marina del Rey (León) & Trichoderma spp. \\
\hline T015* & PAULET34 & San Cristobal de la Polantera (León) & T. harzianum \\
\hline T016 & PAULET35 & Bustillo del Páramo (León) & Trichoderma spp. \\
\hline T017 & PAULET36 & Bustillo del Páramo (León) & Trichoderma spp. \\
\hline T018 & PAULET37 & Urdiales del Páramo (León) & Trichoderma spp. \\
\hline T019* & PAULET38 & Carrizo de la Ribera (León) & T. harzianum \\
\hline T020 & PAULET39 & Soto de la Vega (León) & T. harzianum \\
\hline T021 & PAULET40 & Pozuelo del Páramo (León) & T. harzianum \\
\hline T022 & PAULET41 & Villaornate (León) & Trichoderma spp. \\
\hline T023 & PAULET42 & Cabreros del Río (León) & Trichoderma spp. \\
\hline T024* & IMI 352941 & T. atroviride & Hermosa et al., 2000 \\
\hline T025* & NBT 59 & T. virens & Hermosa et al., 2004 \\
\hline T34** & CECT 2413 & T. harzianum & Kullnig et al., 2001 \\
\hline $\mathrm{R} 43$ & PAULER006 & Santa María del Páramo (León) & Rhizoctonia solani \\
\hline
\end{tabular}

${ }^{*}$ Strains included in in vivo assays.

** Strain only used in ergosterol and squalene assays.

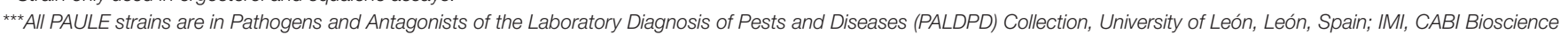
(Egham); NBT, Newbiotechnic S.A. (Seville); CECT, Spanish Type Culture Collection, Burjassot, Spain.

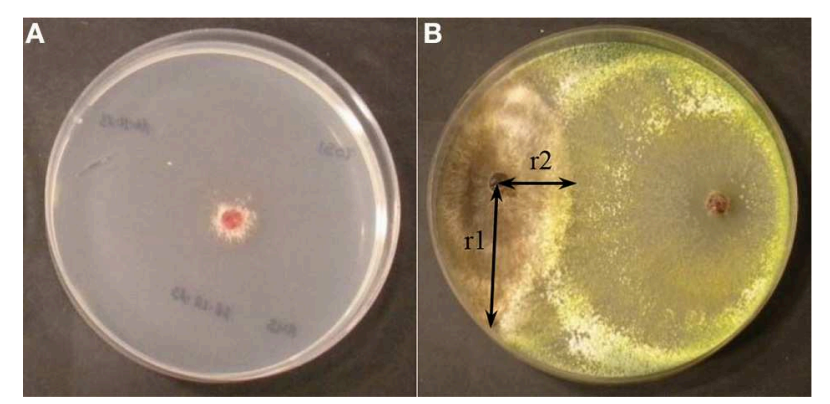

FIGURE 1 | (A) Inhibition of $R$. solani growth by metabolites of Trichoderma spp in assay on membranes (B) Growth of Trichoderma (right) and $R$. solani (left) in direct confrontation assays. Parameters to calculate the percentage of inhibition of growth in direct confrontation (ID) \% ID $=[(r 1-r 2) / r 1] \times 100$.

The percentage of inhibition (IM) was calculated after 3 days of growth of $R$. solani in this medium using the formula \% IM = $[(\mathrm{C}-\mathrm{T}) / \mathrm{C}] \mathrm{x} 100$ (C: diameter of the R. solani control; T: diameter of $R$. solani after being exposed to the metabolites of Trichoderma spp.). Experiments were performed with four replicates. The results were compared by analysis of variance (ANOVA) and Fisher least significant difference (LSD) tests using SAS (SAS Institute Inc., 2004, Cary, NC, USA).

The direct confrontation assays were used to verify the ability of Trichoderma spp. to overgrow the pathogen. Each Trichoderma isolate was grown in dual culture with $R$. solani $\mathrm{R} 43$. The isolates were placed $5.5 \mathrm{~cm}$ apart on the same plate and incubated at $22^{\circ} \mathrm{C}$ for 5 days. Experiments were performed with four replicates. The parameters were measured after 5 days: $r 1$ (distance between the pathogen sowing point and furthest point of the colony) and r2 (distance between the pathogen sowing point and the edge of the colony) from where $R$. solani and Trichoderma mycelia came into contact. Thus, the percentage of inhibition in the direct confrontation assay (ID) was calculated by the formula: \%ID $=[(\mathrm{r} 1-\mathrm{r} 2) / \mathrm{r} 1] \times 100$ (Figure 1B). Inhibition of $R$. solani growth was compared by analysis of variance (ANOVA) and Fisher least significant difference (LSD) tests using SAS. (SAS Institute Inc., 2004, Cary, NC, USA). 


\section{In vivo Assay of the Antifungal Activity}

These assays were only performed with those Trichoderma isolates that gave percentages of inhibition greater that $40 \%$ in membrane assays or $20 \%$ in direct confrontation assays, and that were able to sporulate on PDA medium.

The bioassays were performed in climatic chambers with 32 treatments as follow: 15 in vitro selected Trichoderma isolates against $R$. solani (R43) in order to test the antagonistic activity (RT0-number of Trichoderma isolate); 15 in vitro selected Trichoderma isolates in order to test their effect on plant (CT0number of Trichoderma isolate); one control with R. solani (RC); and one control without fungi (CC). Thirty pots were used per treatment, with polypropylene pots (1 liter capacity) with substrate ( $80 \%$ white peat, $20 \%$ black peat and $5.5 \mathrm{pH}$ ). Each pot was watered with $250 \mathrm{ml}$ of water prior inoculation. R. solani R43 was inoculated by surface irrigation with $50 \mathrm{ml}$ per pot of a suspension of triturated micromicete culture of this pathogen using five Petri dishes ( $18 \mathrm{ml}$ of PDA per dish) per liter of water. For control inoculation, only PDA medium was used without any pathogen. Pots were kept in a growth chamber for 8 days at $25^{\circ} \mathrm{C}(16 \mathrm{~h})$ and $16^{\circ} \mathrm{C}(8 \mathrm{~h}), 60 \%$ relative humidity $(\mathrm{RH})$ in the dark.

Trichoderma isolates were inoculated on PDA medium to grow in dark conditions $\left(25^{\circ} \mathrm{C}\right)$ for 1 week. After that, they were exposed to light in order to induce the formation of spores. Spores suspensions were prepared at a final concentration of $2 \times 10^{7}$ spores $/ \mathrm{ml}$. Bean seeds of "Canela" variety were surface sterilized (sodium hypochlorite $1 \%$ for $3 \mathrm{~min}$ and distilled water for $6 \mathrm{~min}$ ). Then, they were coated with a spore suspension of each Trichoderma isolate. The seeds were submerged in the spore suspension (45 seeds per $20 \mathrm{ml}$ spores suspension) and they were dried in a flow chamber for $12 \mathrm{~h}$. Coated seeds were sown after 8 days of the inoculation of $R$. solani R43. The culture was maintained for 45 days with a photoperiod of $16 \mathrm{~h}$ light, $25^{\circ} \mathrm{C} / 16^{\circ} \mathrm{C}$ (day/night), $60 \% \mathrm{RH}$ and brightness of 3500 lux. Irrigations were performed every 4 days with tap water (about $250 \mathrm{ml} /$ pot). On the $2 \mathrm{nd}-4$ th week a nutrient solution was added (Rigaud and Puppo, 1975). Plants were removed after 45 days from sowing, tissues with symptoms were placed in PDA medium, incubating the plates at $22^{\circ} \mathrm{C}$ for 5 days and identifying the fungus for fulfill Koch's postulates. The next parameters were evaluated in removed plants after 45 days from sowing: wet weight and dry weight $\left(72 \mathrm{~h}\right.$ in an oven, $82^{\circ} \mathrm{C}$ ) of the aerial part and root system.

The data were transformed by the formula $\sqrt{\mathrm{x}+0.5}$ and they were compared by analysis of variance (ANOVA) and Fisher least significant difference (LSD) tests using SAS (SAS Institute Inc., 2004, Cary, NC, USA).

\section{Nucleic Acid Extraction and Manipulation}

Genomic DNA from those isolates showing positive and negative phenotypic effects on bean plants, were extracted by growing the Trichoderma isolates in PDB medium (potato dextrose broth). Mycelia were then recovered by filtration, washed with $0.9 \% \mathrm{NaCl}$ and dried on absorbent filter paper. The procedure for fungal genomic DNA isolation was performed as previously described (Cardoza et al., 2006).

\section{PCR Amplification, Sequencing and DNA Analysis}

The amplification of the ITS regions of the nuclear rDNA gene cluster and an approximately $0.56 \mathrm{~kb}$ fragment of the tef 1 (translation elongation factor $1-\alpha$ ) gene were carried out with the primer pairs ITS1/ITS4 and EF1-728F/EF1-LLErev, respectively, as described previously (Hermosa et al., 2004). The PCR products were purified from agarose gels using the NucleoSpin Extract II Kit (Macherey-Nagel, Düren, Germany), according to the manufacturer's protocol. PCR fragments were sequenced in an ABI 377 Prism Sequencer (Applied Biosystems, Foster City, CA).

The Trichoderma ITS and tef1 sequences obtained in this work were analyzed using the online interactive key (available from http://www.isth.info/tools/blast/index.php) (Druzhinina et al., 2005).

\section{Analysis of Expression of Bean Defense-related Genes}

Three bean leaves from 45 day-old plants of each treatment were randomly collected and stored at $-80^{\circ} \mathrm{C}$ until use. Leaves were detached from plants inoculated with Trichoderma isolate showing positive phenotypic results in the in vivo test. Leaves were then reduced to a fine powder in a mortar under liquid nitrogen. Plant RNA isolation were performed as previously described (Malmierca et al., 2013).

cDNA were synthesized using $1 \mu \mathrm{g}$ total RNA and a Reverse Transcription System with an Oligo $(\mathrm{dT})_{15}$ as the primer (Promega, Madison, WT). cDNA were quantified using a Nanodrop 2000 (Thermo Scientific, Wilmington, DE) and used for further studies.

\section{Real Time-PCR Analysis}

In order to analyze the effect of Trichoderma treatment and/or R. solani infection of bean plants, oligonucleotides corresponding to seven defense-related genes were designed based on their available sequences (Table 2). PR1, PR2, PR3, and $P R 4$, which encode for pathogenesis related proteins related to the salicylate (SA) pathway; $\mathrm{CH} 5 b, \mathrm{CH} 1$ encoding for related to the jasmonate/ethylene pathway (JA/ET), and $P A L$ involved in the phenylpropanoid pathway, were selected to be analyzed in the present study. $\alpha$-actin (Upchurch and Ramirez, 2010; GuerreroGonzález et al., 2011) and PvEF1 $\alpha$ (this work) encoding genes were used as reference (housekeeping genes) for comparative analysis. The qPCR reactions were carried out using Step One Plus $^{\mathrm{TM}}$ (Applied Biosystems, Foster City, CA). The reactions were performed in a total volume of $20 \mu \mathrm{l}$ : $10 \mu \mathrm{l}$ Power SYBR ${ }^{\circledR}$ Green PCR Master Mix (Applied Biosystems, USA), $0.4 \mu \mathrm{l}$ Forward Primer $10 \mu \mathrm{M}, 0.4 \mu \mathrm{l}$ Reverse Primer $10 \mu \mathrm{M}, 5 \mu \mathrm{l}$ cDNA, and $\mathrm{H}_{2} \mathrm{O}$ to $20 \mu \mathrm{l}$. The REST $2009^{\odot}$ software (Pfaffl et al., 2002) was used to calculate the relative expression ratio and the significance of the differences between the gene expression levels. For each primer pair used in this work, we performed a standard curve with 320,160, 80, 40, 20, and $10 \mathrm{ng}$ cDNA to determine the PCR amplification efficiency (E value). Each measurement was made in triplicate (Malmierca et al., 2013). 
TABLE 2 | Oligonucleotides designed for Real-Time PCR analysis.

\begin{tabular}{|c|c|c|c|c|c|}
\hline Gene & Function gene & GenBank Accession number & Oligonucleotide name & Oligonucleotide sequence $\left(5^{\prime}-3^{\prime}\right)$ & References \\
\hline \multirow[t]{2}{*}{$P \vee E F 1 \alpha$} & Elongation Factor 1 & EF660340.1 & $E F 1 \alpha-F$ & CGGGTATGCTGGTGACTIT & This work \\
\hline & & & $\mathrm{EF} 1 \alpha-\mathrm{R}$ & CACGCTTGAGATCCTTGACA & \\
\hline \multirow[t]{2}{*}{$\alpha$-actin } & Actine & U60500.1 & $\alpha$-actin- $F$ & GAGCTATGAATTGCCTGATGG & This work \\
\hline & & & $\alpha$-actin-R & CGTITCATGAATTCCAGTAGC & \\
\hline \multirow[t]{2}{*}{$\mathrm{PVCH} 5 b$} & Chitinase & FE897014.1 & $\mathrm{CH} 5 \mathrm{~b}-\mathrm{F}$ & CAGCCAAAGGCTTCTACACC & This work \\
\hline & & & $\mathrm{CH} 5 \mathrm{~b}-\mathrm{R}$ & TTGTICGTGAGACGTTGC & \\
\hline \multirow[t]{2}{*}{ PVPR1 } & Pathogenesis related 1 & HO864272.1 & PR1-F & TGGTCCTAACGGAGGATCAC & This work \\
\hline & & & PR1-R & TGGCTITCCAGCTITGAGT & \\
\hline \multirow[t]{2}{*}{ PvPR2 } & Pathogenesis related 2 & HO864270.1 & PR2-F & GTGAAGGACGCCGATAACAT & This work \\
\hline & & & PR2-R & ACTGAGTTTGGGGTCGATTG & \\
\hline \multirow[t]{2}{*}{ PvPR4 } & Pathogenesis related 4 & HO864354.1 & PR4-F & CGCAGTGAGTGCATATTGCT & This work \\
\hline & & & PR4-R & TGTITGTCACCCTCAAGCAC & \\
\hline $\mathrm{PvCH} 1$ & Chitinase & & & & Pereira et al., 2014 \\
\hline PVPR3 & Pathogenesis related 3 & & & & Pereira et al., 2014 \\
\hline PVPAL & Phenylalanine ammonia-lyase & & & & Pereira et al., 2014 \\
\hline
\end{tabular}

\section{Quantification of Ergosterol and Squalene}

The Trichoderma selected strain was inoculated in $100 \mathrm{ml}$ of CM medium ( $0.5 \%$ malt extract, $0.5 \%$ yeast extract, and $0.5 \%$ glucose) with $10^{6}$ spores $/ \mathrm{ml}$, and incubated $24 \mathrm{~h}$ at $28^{\circ} \mathrm{C}$. Then $20 \mathrm{ml}$ from the previous cultures were inoculated on $100 \mathrm{ml}$ potato dextrose broth (PDB medium) and were incubated as before during 24$96 \mathrm{~h}$. The mycelia were filtered through nytal filters $(30 \mu \mathrm{m}$ diameter) and the liquid removed by drying between filter papers. The dry weight of the fungal pellet was calculated. Total intracellular sterols were extracted and ergosterol and squalene content were quantified as previously reported other authors (Cardoza et al., 2007; Ghimire et al., 2009). All measurements were made in duplicate in the Trichoderma selected isolate and a strain used as control of the same species of the selected isolate. The results were compared by analysis of variance (ANOVA) and Fisher least significant difference (LSD) tests using SAS (SAS Institute Inc., 2004, Cary, NC, USA).

\section{Results}

\section{Analysis of the in vitro Antagonistic Activity of Trichoderma Isolates with $R$. solani}

The first test to determine the in vitro antifungal ability of the different Trichoderma isolates was based on their ability to produce metabolites that may inhibit the growth of $R$. solani (Table 3, Figure 1A). Trichoderma isolates T003, T004, T006, T020, T022, T012, T013, T025, T016, T007, T024, T005, and T010 inhibited $R$. solani growth by more than $75 \%$, with the highest inhibition produced by T003, T004, T006, T020, and T022 (86.70\%). T019, T008, T002, T021, T001, T018, and T023 showed a remarkable inhibition (75-40\%). Finally, T015, T014, T017, T011, and T009 inhibited R. solani growth by less than $40 \%$, and T009 showed the lowest percentage (15.82\%).

T021 was the Trichoderma isolate showing the highest percentage of inhibition $(72.77 \%)$ in the direct confrontation assays (Table 3, Figure 1B), whereas T009 showed the lowest inhibition values (14.63\%). The inhibition percentages detected for the other Trichoderma isolates ranged from 47.13 to $30.55 \%$.

\section{Analysis of the in vivo Antagonistic Activity of Trichoderma Isolates with $R$. solani}

The results of the in vitro membrane assays and direct confrontation assays against $R$. solani, showed above, were used to select the isolates that would be used for the in vivo analysis. Thus, fifteen Trichoderma isolates (marked with asterisk in Tables 1, 3) were tested since they were able to sporulate in PDA medium and showed a percentage of inhibition of $R$. solani growth higher than $40 \%$ in the membrane assays, and/or $20 \%$ in the direct confrontation assays.

On plants removed after 45 days from sowing for Koch's postulates, $R$. solani was found and Trichoderma isolates were also present in the medium.

When dry aerial parts (Figure 2) were analyzed, plants treated with CT019 had the greatest weight of the aerial part, being significantly different from the control (CC). In the RT019 treatment, plants did not show significant differences in comparison with the control (CC), what is indicative of a biocontrol effect by T019 isolate.

In the case of the root system (Figure 3), the situation was similar to that observed in the aerial parts. CT019 treated plants were not significantly different in dry weight, although it was always greater than the weight of the control plants (CC). If the pathogen was present in the soil with same Trichoderma isolate, RT019 treatment, in the case of wet weight, there were no significant differences between control (CC) and control pathogen (RC) (data not shown).

Based on these results, the Trichoderma isolate T019 was selected for further studies since it showed the best positive effects on plant phenotype among all the analyzed isolates (Figure 4).

\section{Molecular Identification of Trichoderma Isolates}

Those Trichoderma isolates able to sporulate in PDA medium, showing a percentage of inhibition higher than 
TABLE 3 | In vitro antifungal activity of Trichoderma strains against R. solani R43.

\begin{tabular}{|c|c|c|c|c|c|}
\hline \multirow{2}{*}{$\begin{array}{l}\text { Strain } \\
\text { T003* }^{\star}\end{array}$} & \multicolumn{2}{|c|}{$\begin{array}{c}\text { Inhibition in growth assay } \\
\text { on membranes ( } \% \pm \\
\text { Standard error) (1) }\end{array}$} & \multirow{2}{*}{$\begin{array}{l}\text { Strain } \\
\text { T021 }\end{array}$} & \multicolumn{2}{|c|}{$\begin{array}{c}\text { Inhibition in growth assay } \\
\text { on direct confrontation } \\
\text { ( } \% \pm \text { Standard error) (2) }\end{array}$} \\
\hline & $86.70 \pm 0.15$ & $\mathrm{a}$ & & $72.77 \pm 4.49$ & $\mathrm{a}$ \\
\hline T004* & $86.70 \pm 0.15$ & $\mathrm{a}$ & T004* & $47.13 \pm 0.91$ & $b$ \\
\hline T006* & $86.70 \pm 0.15$ & a & T013* & $43.49 \pm 1.41$ & $\mathrm{bc}$ \\
\hline T020 & $86.70 \pm 0.15$ & a & T011* & $42.57 \pm 2.00$ & bcd \\
\hline T022 & $86.70 \pm 0.15$ & a & T014 & $41.99 \pm 1.33$ & cde \\
\hline T012* & $86.67 \pm 0.14$ & a & T023 & $41.57 \pm 1.40$ & cdef \\
\hline T013* & $85.04 \pm 1.04$ & a & T018 & $41.01 \pm 1.93$ & colefg \\
\hline T025* & $83.97 \pm 1.97$ & a & T020 & $39.21 \pm 1.41$ & cdefgh \\
\hline T016 & $82.29 \pm 2.33$ & a & T006* & $38.68 \pm 0.59$ & defghi \\
\hline T007* & $76.77 \pm 2.52$ & $\mathrm{~b}$ & T017 & $37.42 \pm 1.61$ & efghij \\
\hline T024* & $76.22 \pm 2.32$ & bc & T016 & $37.10 \pm 0.63$ & fghij \\
\hline T005* & $75.65 \pm 2.23$ & bc & T002* & $36.44 \pm 0.29$ & ghij \\
\hline T010 & $75.14 \pm 3.30$ & $\mathrm{bc}$ & T024* & $36.43 \pm 3.71$ & hij \\
\hline T019* & $72.36 \pm 2.01$ & bcd & T007* & $36.34 \pm 1.30$ & hij \\
\hline T008* & $70.61 \pm 1.82$ & cde & T005* & $36.16 \pm 0.67$ & hij \\
\hline T002* & $66.75 \pm 1.18$ & def & T019* & $35.09 \pm 1.01$ & hijk \\
\hline T021 & $65.98 \pm 0.77$ & ef & T022 & $35.05 \pm 0.34$ & hijk \\
\hline T001* & $65.51 \pm 1.75$ & $f$ & T008* & $35.04 \pm 0.89$ & hijk \\
\hline T018 & $54.12 \pm 2.35$ & $g$ & T012* & $34.59 \pm 0.98$ & ijk \\
\hline T023 & $48.26 \pm 3.10$ & $\mathrm{~h}$ & T025* & $33.28 \pm 4.24$ & kjl \\
\hline T015* & $39.99 \pm 0.82$ & i & T010 & $30.55 \pm 1.32$ & kl \\
\hline T014 & $38.82 \pm 2.96$ & $\mathrm{ij}$ & T015* & $28.84 \pm 0.85$ & $\operatorname{lm}$ \\
\hline T017 & $34.10 \pm 0.88$ & j & T001* & $24.66 \pm 0.74$ & $\mathrm{~m}$ \\
\hline T011* & $21.40 \pm 2.66$ & $\mathrm{k}$ & T003 $^{*}$ & $19.85 \pm 2.97$ & $\mathrm{n}$ \\
\hline T009 & $15.82 \pm 1.40$ & I & T009 & $14.63 \pm 0.83$ & 0 \\
\hline
\end{tabular}

Values in the same column followed by different letters are significantly different (Fisher's LSD. $p<0.05)$.

(1) Growth assay on membranes (effect of Trichoderma isolates on percent growth inhibition of R. solani. using cellophane membranes. (2) in direct confrontation assays (percentage of inhibition of R43 growth when grown confronted with Trichoderma strains during 5 days in PDA medium plates).

*Trichoderma isolates selected for in vivo experiments because they were able to sporulate in PDA medium and showed a percentage of inhibition higher than $40 \%$ in the membrane assays. and/or $20 \%$ in the direct confrontation assays.

$40 \%$ in the membrane assays, and/or $20 \%$ in the direct confrontation assays, were identified. ITS1 region of rDNA and a fragment of the translation elongation factor 1 (tef1) were amplified and sequenced for 12 isolates, and both nucleotide sequences were used for identification at species level. Eight out of these 12 isolates were identified as $T$. harzianum, and $T$. atroviride, T. gamsii, T. longibrachiatum and $T$. citrinoviride species were represented by one isolate (Table 1).

\section{Effect of Trichoderma Treatment and/or R. solani Infection in the Expression of Bean Defense Related Genes}

The amplification efficiencies of the oligo-pairs (Table 2) were: $\alpha$-actin 1.150, PvEF1 $\alpha$ 0.903, CH5b 0.883, CH1 1.098, PR1 1.094, PR2 1.048, PR3 0.947, PR4 0.922, and PAL 0.962. $\alpha$-actin and PvEF1 $\alpha$ were used as housekeeping genes to determine the relative expression level of the other genes analyzed in the present work. Trichoderma T019 strain was selected, based on its positive effects on bean phenotype with and without $R$. solani infection. The results included in Figure 5 showed that: (i) R. solani down-regulated the expression of all the $P$. vulgaris defense-related genes analyzed (Figure 5A), raising values of expression ranging between $0.099(p=0.029)$ for PR2 and 0.397 ( $p=0.045)$ for $\mathrm{CH} 1$; (ii) In plants treated with T019 compared with control plants (bean plants not treated with Trichoderma nor infected with $R$. solani) only the CH5b and PR2 were significantly up-regulated, raising comparative expression values of $1.495(p=0.000)$ and $24.492(p=0.000)$, respectively (Figure 5B). Finally, (iii) treatment with T019 and infection with $R$. solani significantly up-regulated the expression ratio of the analyzed genes, except $P A L$, in comparison with plants only infected with $R$. solani, with values ranging from 1.420 ( $p=$ $0.000)$ to $42.975(p=0.000)$ for $C H 1$ and $P R 4$ respectively (Figure 5C).

A non-remarkable significant up-regulation of the defenserelated gene expression was observed when expression ratios of the selected genes in plants treated with T. harzianum T019 and infected with $R$. solani were compared with those from plants untreated or uninfected (data not shown).

\section{Production of Ergosterol and Squalene by the Trichoderma Selected Strains}

Strain T019 (T. harzianum) was selected for this analysis because it was those producing the most remarkable positive phenotypic effect on beans (see above). T. harzianum T34 (Table 1) was used as control strain for comparative purposes because it is a well-known strain, widely characterized (Kullnig et al., 2001).

Production of ergosterol at $24 \mathrm{~h}$ of growth did not show significant differences between the strains T. harzianum (T34 and T019) (Table 4). When the production of ergosterol was analyzed at $96 \mathrm{~h}$, T019 produced significantly higher amounts than its respective control (T34).

Regarding the squalene production at $24 \mathrm{~h}, \mathrm{~T}$. harzianum (T34 and T019) strains did not show significant differences. However, at $96 \mathrm{~h}$ of growth, T019 produced amounts significantly higher than its control.

\section{Discussion}

Most of the Trichoderma isolates studied in the present work have been able to control the growth of $R$. solani. The percentage of growth inhibition in the direct confrontation assays raised values up to $72.77 \%$ for T021, but most of the isolates were between 47 and 24\%. By contrast, in the antifungal assays on membranes, those percentages ranged mostly between 86 and $58 \%$. In the assays conducted by Campelo et al. (2010) the percentage of inhibition in membrane assays using T. virens T59 (NBT59) and T. atroviride T11 (IMI352941) range between 100 and $84.7 \%$, respectively. The differences observed in the different in vitro assays might be due to the variability of genotypes, with differences in growth, sporulation and in their environmental 


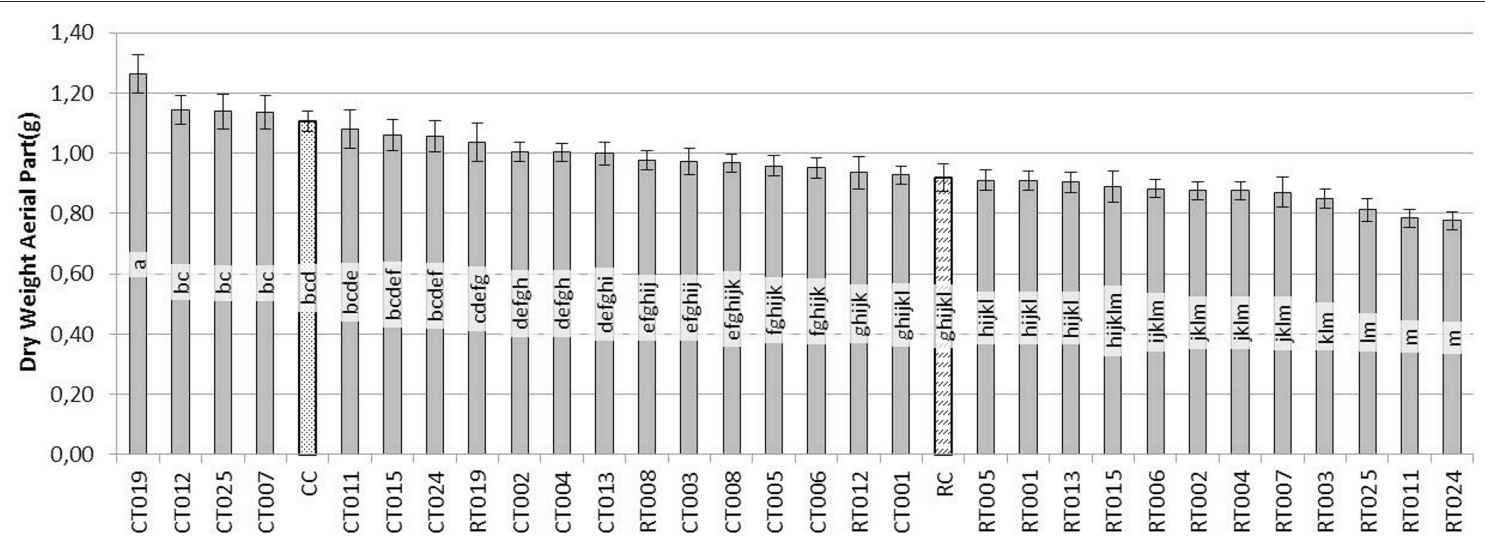

FIGURE 2 | Evaluation of the dry weight (g) of the aerial parts of bean plants grown during 45 days after sowing with 60 replicates. [Trichoderma isolates without pathogen (CTO-number of Trichoderma isolate), Trichoderma isolates with R. solani (RTO-number of Trichoderma isolate), R. solani control $(\mathrm{RC})$ and control without fungus $(\mathrm{CC})]$.

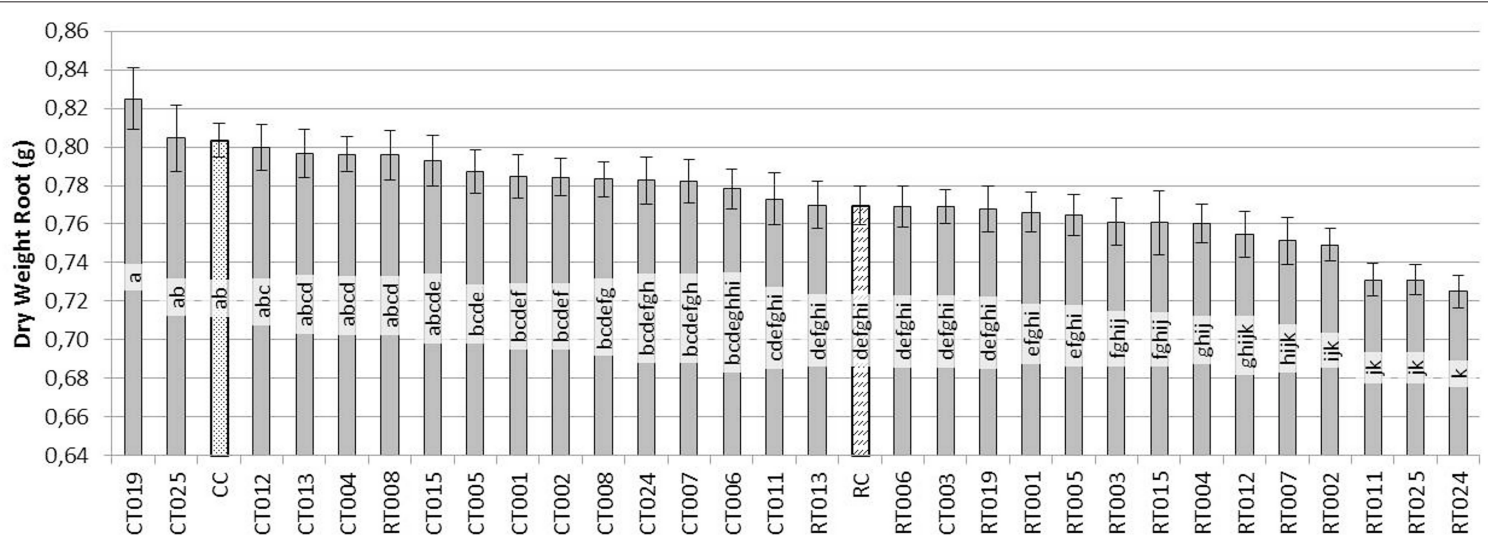

FIGURE 3 | Evaluation of the dry weight (g) of the root system of bean plants grown for 45 days after sowing with 60 replicates. [Trichoderma isolates without pathogen (CTO-number of Trichoderma isolate), Trichoderma isolates with R. solani (RTO-number of Trichoderma isolate), R. solani control $(\mathrm{RC})$ and control without fungus $(\mathrm{CC})]$.

biological interactions as a consequence of the prevalence of different modes of action on each of the analyzed strains (RuanoRosa et al., 2010).

The in vitro antagonistic activity of the Trichoderma isolates is an indication of their in vivo biological activity against phytopathogenic fungi. However, it has been found that the in vitro antifungal activity of Trichoderma does not correlate in a direct way with its in vivo ability to control diseases caused by phytopathogenic fungi, since many other factors influence that activity (Anees et al., 2010).

Regarding the in vivo assays, bean plants that had been in contact with some Trichoderma isolate had an increased size when the pathogen was not present. Pereira et al. (2014) also observed that $T$. harzianum was able to promote the growth of common bean plants in comparison to plants grown in its absence. However, in the present work only T019 treated plants did not decrease their size in the presence of $R$. solani. These data are in agreement with those from Yedidia and coworkers reporting a much stronger effect on cucumber plants treated with $T$. harzianum, which increased by $75 \%$ the length of the root, $95 \%$ aerial parts, $80 \%$ dry weight and $80 \%$ the size of the blade relative to the untreated control (Yedidia et al., 2001). T. harzianum was able to promote the growth of common bean plants in comparison to plants grown in its absence (Pereira et al., 2014).

However, in the present work opposite results were observed to those previously reported (Tello et al., 1985), indicating that infection with $R$. solani not always resulted in a reduction of the bean plant size, which was explained as a result of the activation of the natural plant-defense mechanisms (Cardoso and Echandi, 1987) that would lead to an enhanced development of the plants when this pathogen was present in the soil. In the present study we have observed that treatment with $R$. solani resulted in a down-regulation of all the bean defense-related genes analyzed, indicating a certain compensation effect between the intensity of plant defense response and plant growth (Hermosa et al., 2013). 


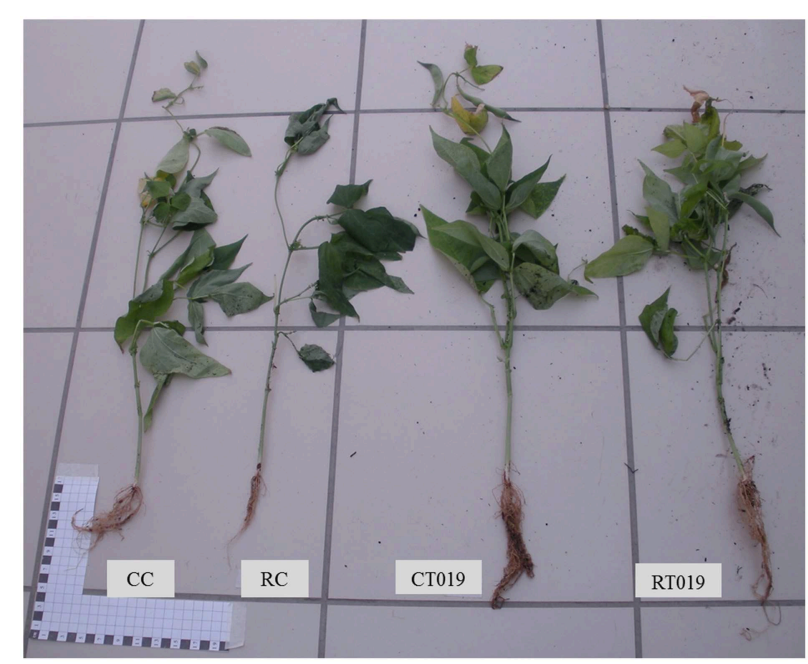

FIGURE 4 | Differences in growth of bean plants. CC plant control; RC plant with R. solani, CT019 plant with Trichoderma T019; RT019 plant with R. solani and Trichoderma T019.

Little is known about the effect of Trichoderma treatment and/or infection with $R$. solani in the expression of bean defenserelated gene expression. As indicated above, interaction of the plant with $R$. solani during 45 days of growth caused the repression of the seven defense-related genes studied ( $\mathrm{CH} 5 b$, CH1, PR1, PR2, PR3, PR4, and PAL1), as a mechanism to overcome the plant defense response and thus facilitating the progression of the infection process within the plant. Other authors have observed similar results in assays with tomato plants infected with Pseudomonas syringae pv. tomato, where a repression of PR1 and PR4 was shown, as an indication of the decrease in the plant self-defense mechanism, then facilitating disease progression (Zhao et al., 2003).

Expression of $P$. vulgaris defense-related genes was analyzed in leaves, even when the interaction with the pathogen $R$. solani is initially produced at the root level, to determine if the signals generated in roots as result of this interaction are able to systematically stimulate the bean defense along long distance from seed to leaf. When the interaction of plant with T019 was analyzed, $P R 2$ was up-regulated, $P R 4$ was slightly restrained but $P R 1$ and $P R 3$ were not affected. Pereira and coworkers observed that $T$. harzianum also seems to potentiate common bean response against the phytopathogenic fungus $R$. solani, as shown by the increase in the levels of glu1 and pod 3 in the double treatment in comparison to that obtained for plants in the presence of $R$. solani alone (Pereira et al., 2014). Other studies showed that there was an increase in expression $P R 1$ at $16 \mathrm{~h}$ of interaction (Guerrero-González et al., 2011). Rivière and coworkers suggested that $P R 1$ was down-regulated by the $\beta-1,3-$ glucanases (PR2 and PR3) (Rivière et al., 2008). In the present study, after 45 days of the inoculation of the biocontrol isolate we observed a higher expression of $P R 2$, which could be due to an alteration of plant defense responses at these longer times in comparison with shorter ones assayed in the previous reported works. This would result in a higher $\beta$-1,3-glucanase activity in the cell wall that would increase oligosaccharides released by its action, then acting as elicitors of the plant defense response and/or of the fungal secondary metabolism (Druzhinina et al., 2011).

In the case of plants inoculated with Trichoderma and seeded in soil infected with $R$. solani, when compared with plants only infected with $R$. solani, thus excluding the effect due exclusively to this pathogen, a significant up-regulation of all the analyzed genes was observed, except $P A L$, indicating that in presence of $R$. solani several mechanisms are induced in Trichoderma that potentiate its ability to elicit plant defense-responses. In previous works, during the interaction of Solanum tuberosum with $R$. solani it was observed that after $120 \mathrm{~h}$ from the initial infection and damage to the first floor, new outbreaks produce less symptoms, suggesting that the plant might be prepared to defend and avoid disease progression (Lehtonen et al., 2008). Other studies have shown that the PR4 was overexpressed in eggplants treated with BCAs suggesting that these agents promoted defensive reactions within the plant (Angelopoulou et al., 2014). In other assays with pepper plants an increase in the accumulation of PR1 and chitinases was also observed when these plants were previously inoculated with a strain of Fusarium oxysporum and subsequently exposed to Verticillium dahliae (Veloso and Díaz, 2012), emphasizing their importance in the response to pathogen infection and to abiotic stresses (Jung and Hwang, 2000). Thus, it could be inferred that the effect of Trichoderma would therefore modulate the response of the plant and prevent the suppression of defense genes caused by $R$. solani. However, the effect of the different Trichoderma strains in the different plants would indicate the existence of great differences between them.

In the case of $C H 5 b$, related to JA pathway, when $R$. solani was in contact with the plant for 45 days of development, the expression of this gene was down-regulated in comparison with non-infected plants. When bean plants were inoculated with Trichoderma, its expression was slightly induced. However, if the antagonist and the pathogen were present in the medium, the effect caused by $R$. solani was the overexpression of this gene. In previous works (Broglie et al., 1991) it was shown that $\mathrm{CH} 5 \mathrm{~b}$, responsible for chitinase production, was overexpressed in Nicotiana tabacum and Brassica napus infected with $R$. solani, and their disease symptoms were reduced. Benhamou et al. (1993) found that the expression of this gene caused a reduction of disease in B. napus infected with the same pathogen, which correlates with similar results observed in strawberry plants inoculated with Botrytis cinerea (Vellicce et al., 2006). In the present study we observed that the contact of the plant with the pathogen during a period of 45 days resulted in a significant repression of this gene, similarly to what it was observed for the expression of $\mathrm{CH} 1$, which thereby facilitates disease progression. However, in the presence of Trichoderma a similar effect to that previously described for SA-related genes was observed, so facilitating a prevention of disease progression.

In the case of the phenylalanine ammonia lyase (PAL), this is a key enzyme in the metabolic pathway of phenylpropanoid compounds by catalyzing the amino acid L-phenylalanine 

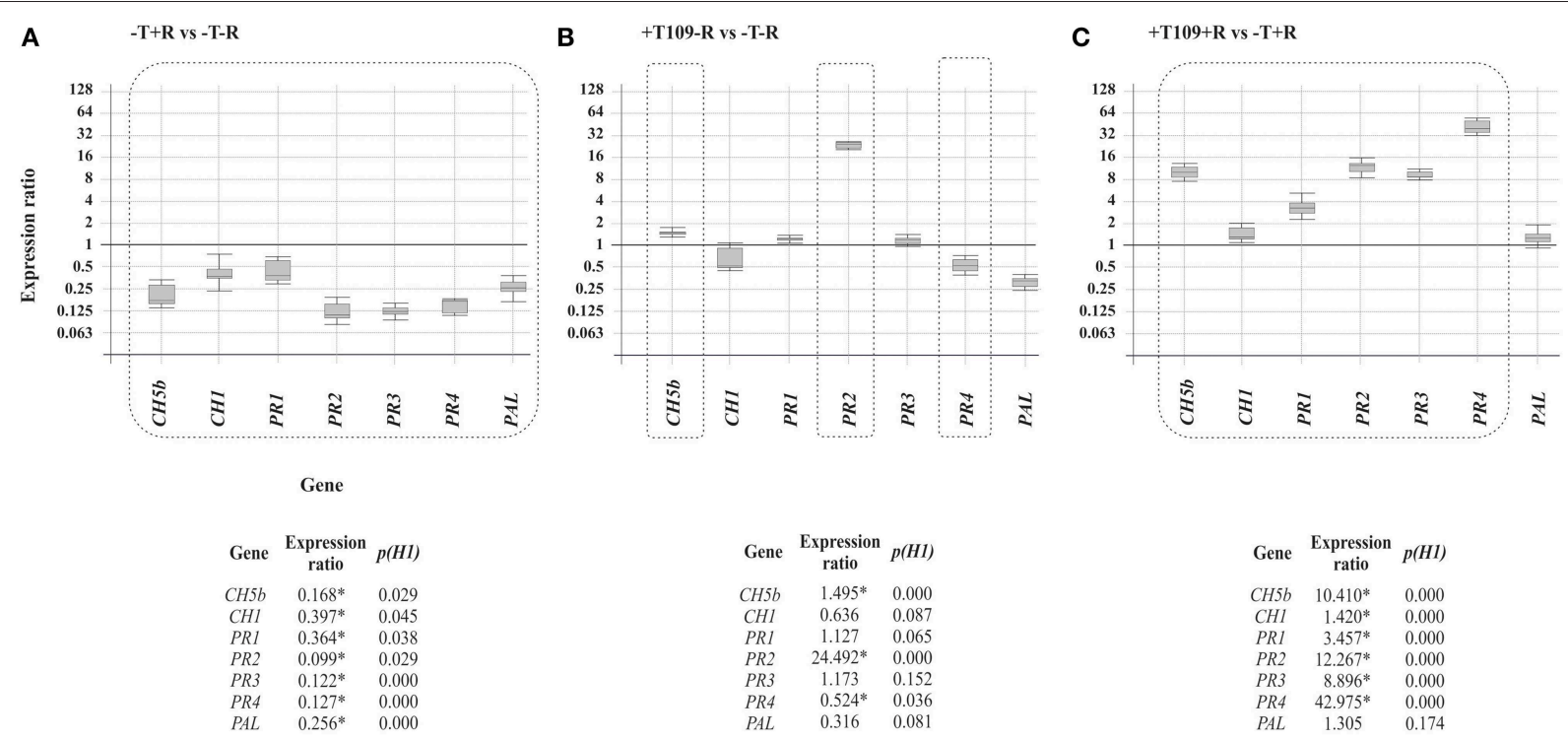

FIGURE 5 | Expression of $\mathbf{C H 5 b}, \mathbf{C H 1}, \mathbf{P R 1}, \mathbf{P R 2}, \mathbf{P R} 3, \mathbf{P R} 4$, and PAL genes in comparison with $\alpha$-actin and PvEF-1 $\alpha$ reference genes. The comparisons and statistical analysis were performed using the REST2009 ${ }^{\circ}$ program (Pfaffl et al., 2002). The numeric data are illustrated at the bottom of the figure and those showing differences statistically significant $(p<0.05)$ are indicated with an asterisk and they are boxed in the graphic representation. Comparison of the gene expression of the bean defense-related genes (A) in plants infected with $R$. solani R43 vs. control plants. (B) In plants treated with Trichoderma T019 vs. control plants (C) In plants infected with R. solani R43 and treated with Trichoderma T019 vs. plants infected with R. solani R43.

TABLE 4 | Ergosterol and squalene quantification from 24 and $96 \mathrm{~h}$ samples of the selected Trichoderma strains selected for this study.

\begin{tabular}{|c|c|c|c|c|}
\hline \multirow[t]{2}{*}{ Trichoderma spp. } & \multicolumn{2}{|c|}{ Ergosterol (mg E/g d wt) } & \multicolumn{2}{|c|}{ Squalene (mg S/g d wt) } \\
\hline & $24 \mathrm{~h}$ & $96 \mathrm{~h}$ & $24 \mathrm{~h}$ & $96 \mathrm{~h}$ \\
\hline T34 & $16.197 \pm 1.019 \mathrm{a}$ & $8.970 \pm 0.388 b$ & $0.317 \pm 0.020 a$ & $0.052 \pm 0.049 b$ \\
\hline T019 & $14.336 \pm 2.459 \mathrm{a}$ & $12.502 \pm 3.568 \mathrm{a}$ & $0.303 \pm 0.041 \mathrm{a}$ & $0.348 \pm 0.103 a$ \\
\hline
\end{tabular}

Values in the same column followed by different letters are significantly different (Fisher's LSD, $p<0.05$ ).

deamination, giving rise to trans-cinnamic acid and ammonia. The trans-cinnamic acid is used for the synthesis of various phenolic compounds, which are precursors in the synthesis of esters, coumarins, flavonoids, and lignin. The production of this enzyme is controlled during plant growth, but is also induced in cells neighboring the infection site and, besides infection, various environmental stimuli such as injury, heavy metal contamination, light and growth regulators (Rahman and Punja, 2005). In the present work, we observed that this gene was down-regulated or not affected in all the conditions assayed, indicating that in the analyzed strain, PAL activity would be more focused in the plant response against environmental stress than in the response against pathogens, which contrast with the role of this enzyme described in pepper, where it is involved in the positive regulation of SA-dependent defense signaling (Kim and Hwang, 2014).

Regarding squalene and ergosterol production, in this study it was found that there were differences among the isolates. Ergosterol is a sterol found in the fungal membrane, which although considered by the plant as a PAMP (pathogen associated molecular patterns) (Nürnberger et al., 2004) triggers a series of reactions (Cervone et al., 1997) which would cause an activation of the genes of plant defense (Rossard et al., 2010). Squalene is a precursor of the ergosterol biosynthetic pathway (Malmierca et al., 2013) located in the cellular membranes or accumulated as droplets in the cytoplasm whose physiological function, apart from acting as an ergosterol precursor, still remains unclear, but it might play some role in the elicitation of plant defense responses. Thus, in this assay, an increased production of these compounds by Trichoderma would result in the induction of defense genes in the bean, then the plant could grow better under a pathogen presence in the soil.

\section{Conclusions}

Trichoderma isolates inhibit the development of $R$. solani under in vitro conditions, when they grow in Petri dishes. In vivo conditions, the bean plants that had been in contact with the Trichoderma isolates always had an increased size when the 
pathogen was not present. When $R$. solani was present in the soil, the development of the bean plants was significantly reduced. However, bean plants treated with strain T019 did not decrease their size in the presence of $R$. solani.

The interaction of bean plants with $R$. solani caused, after 45 days of growth, the down-regulation of the seven defenserelated genes studied ( $C H 5 b, C H 1, P R 1, P R 2, P R 3, P R 4, P A L)$ as a mechanism to overcome the plant defense response and thus facilitating the progression of the infection process within the plant.

An increased production of ergosterol and squalene by Trichoderma resulted in the induction of defense genes in the bean plants. In this way, the plant would grow better under a pathogen presence in the soil.

T. harzianum T019 shows a positive effect on the level of resistance of bean plants to $R$. solani. This strain induces the expression of plant defense-related genes and produces a higher level of ergosterol, indicating its ability to grow at a higher rate in the soil, which would explain its positive

\section{References}

Anees, M., Tronsmo, A., Edel-Hermann, V., Hjeljord, L. G., Héraud, C., and Steinberg, C. (2010). Characterization of field isolates of Trichoderma antagonistic against Rhizoctonia solani. Fungal Biol. 114, 691-701. doi: 10.1016/j.funbio.2010.05.007

Angelopoulou, D. J., Naska, E. J., Paplomatas, E. J., and Tjamos, S. E. (2014). Biological control agents (BCAs) of verticillium wilt: influence of application rates and delivery method on plant protection, triggering of host defence mechanisms and rhizosphere populations of BCAs. Plant Pathol. 63, 1062-1069. doi: 10.1111/ppa.12198

Benhamou, N., Broglie, K., Chet, I., and Broglie, R. (1993). Cytology of infection of 35S-bean chitinase transgenic canola plants by Rhizoctonia solani: cytochemical aspects of chitin breakdown in vivo. Plant J. 4, 295-305. doi: 10.1046/j.1365313X.1993.04020295.x

Broglie, K., Chet, I., Holliday, M., Cressman, R., Biddle, P., Knowlton, S., et al. (1991). Transgenic plants with enhanced resistance to the fungal pathogen Rhizoctonia solani. Science 254, 1194-1197.

Campelo, P., Cardoza, R., Lorenzana, A., Hermosa, M., Monte, E., Reinoso, B., et al. (2010). Biological control of phytopathogenic fungi in bean (Phaseolus vulgaris L.) with Trichoderma atroviride and Trichoderma virens. [Abstract]. Bean Improv. Coop. Colo. 53, 114-115.

Cardoso, J., and Echandi, E. (1987). Biological control of Rhizoctonia root rot of snap bean with binucleate Rhizoctonia-like fungi. Plant Dis. 71, 167-170. doi: 10.1094/PD-71-0167

Cardoza, R. E., Hermosa, M. R., Vizcaíno, J. A., González, F., Llobell, A., Monte, E., et al. (2007). Partial silencing of a hydroxy-methylglutaryl-CoA reductaseencoding gene in Trichoderma harzianum CECT 2413 results in a lower level of resistance to lovastatin and lower antifungal activity. Fungal Genet. Biol. 44, 269-283. doi: 10.1016/j.fgb.2006.11.013

Cardoza, R. E., Vizcaíno, J. A., Hermosa, M. R., Sousa, S., González, F. J., Llobell, A., et al. (2006). Cloning and characterization of the erg1 gene of Trichoderma harzianum: effect of the erg1 silencing on ergosterol biosynthesis and resistance to terbinafine. Fungal Genet. Biol. 43, 164-178. doi: 10.1016/j.frb.2005.11.002

Casquero, P. A., Lema, M., Santalla, M., and De Ron, A. M. (2006). Performance of common bean (Phaseolus vulgaris L.) landraces from Spain in the Atlantic and Mediterranean environments. Genet. Res. Crop Evol. 53, 1021-1032. doi: 10.1007/s10722-004-7794-1

Cervone, F., Castoria, R., Leckie, F., and De Lorenzo, G. (1997). "Perception of fungal elicitors and signal transduction," in Signal Transduction in Plants, ed P. Aducci (Basel, Boston, Berlin: Birkhäuser), $153-177$. effects on plant growth and defense in the presence of the pathogen.

\section{Acknowledgments}

The grant awarded to Sara Mayo (FPU12/00677) by the Ministry of Education, Culture and Sport (Spain) according the Resolution of April 25, 2012 (BOE of 10 May), as amended by Resolution of May 18, 2012 (BOE of 22 May), and by Resolution of October 31,2012 (BOE of 12 November) and granted in the Resolution of December 20, 2012. Ministry of Economy and Competitiveness for National project "Farnesol as self-regulatory molecule in Trichoderma. Tyrosol and farnesol signaling in Trichodermabean interaction" (AGL2012-40041-C02-02). Junta de Castilla y León, Consejería de Educación for the project "Effect of terpenes and physiologically related compounds produced by Trichoderma parareesei in the development of common bean (Phaseolus vulgaris L.) and in defensive responses of this plant" (LE228U14). J. Alvarez for his technical support.

Druzhinina, I. S., Kopchinskiy, A. G., Komon, M., Bissett, J., Szakacs, G., and Kubicek, C. P. (2005). An oligonucleotide barcode for species identification in Trichoderma and Hypocrea. Fungal Genet. Biol. 42, 813-828. doi: 10.1016/j.fgb.2005.06.007

Druzhinina, I. S., Seidl-Seiboth, V., Herrera-Estrella, A., Horwitz, B. A., Kenerley, C. M., Monte, E., et al. (2011). Trichoderma: the genomics of opportunistic success. Nat. Rev. Microbiol. 9, 749-759. doi: 10.1038/nrmicro2637

Garaiová, M., Zambojová, V., Šimová, Z., Griac, P., and Hapala, I. (2013). Squalene epoxidase as a target for manipulation of squalene levels in the yeast Saccharomyces cerevisiae. FEMS Yeast Res. 14, 310-323. doi: 10.1111/15671364.12107

Ghimire, G. P., Hei, C. L., and Jae, K. S. (2009). Improved squalene production via modulation of the methylerythritol 4-phosphate pathway and heterologous expression of genes from Streptomyces peucetius ATCC 27952 in Escherichia coli. Appl. Environ. Microbiol. 75, 7291-7293. doi: 10.1128/AEM. 01402-09

Guerrero-González, M. L., Rodríguez-Kessler, M., Rodríguez-Guerra, R., González-Chavira, M., Simpson, J., Sanchez, F., et al. (2011). Differential expression of Phaseolus vulgaris genes induced during the interaction with Rhizoctonia solani. Plant Cell Rep. 30, 1465-1473. doi: 10.1007/s00299-011-1055-5

Harman, G. E., Howell, C. R., Viterbo, A., Chet, I., and Lorito, M. (2004). Trichoderma species-opportunistic, avirulent plant symbionts. Nat. Rev. Microbiol. 2, 43-56. doi: 10.1038/nrmicro797

Harman, G. E., and Kubicek, C. P. (1998). Trichoderma and Gliocladium: Enzymes, Biological Control and Commercial Applications. Vol. 2, London: CRC Press.

Hermosa, M. R., Grondona, I., Iturriaga, E. A., Díaz-Mínguez, J. M., Castro, C., Monte, E., et al. (2000). Molecular characterization and identification of biocontrol isolates of Trichoderma spp. Appl. Environ. Microbiol. 66, 1890-1898. doi: 10.1128/AEM.66.5.1890-1898.2000

Hermosa, M. R., Keck, E., Chamorro, I., Rubio, B., Sanz, L., Vizcaíno, J. A., et al. (2004). Genetic diversity shown in Trichoderma biocontrol isolates. Mycol. Res. 108, 897-906. doi: 10.1017/S0953756204000358

Hermosa, R., Belén Rubio, M., Cardoza, R. E., Nicolás, C., Monte, E., and Gutiérrez, S. (2013). The contribution of Trichoderma to balancing the costs of plant growth and defense. Int. Microbiol. 16, 69-80. doi: 10.2436/20.1501.01.181

Hermosa, R., Viterbo, A., Chet, I., and Monte, E. (2012). Plant-beneficial effects of Trichoderma and of its genes. Microbiol 158, 17-25. doi: 10.1099/mic.0. 052274-0

Jung, H. W., and Hwang, B. K. (2000). Pepper gene encoding a basic B-1,3glucanase is differentially expressed in pepper tissues upon pathogen infection 
and ethephon or methyl jasmonate treatment. Plant Sci. 159, 97-106. doi: 10.1016/S0168-9452(00)00334-4

Kim, D. S., and Hwang, B. K. (2014). An important role of the pepper phenylalanine ammonia-lyase gene (PAL1) in salicylic acid-dependent signalling of the defence response to microbial pathogens. J. Exp. Bot. 65, 2295-2306. doi: 10.1093/jxb/eru109

Kullnig, C., Krupica, T., Woo, S. L., Mach, R. L., Rey, M., Benítez, T., et al. (2001). Confusion abounds over identities of Trichoderma biocontrol isolates. Mycol. Res. 105, 770-772. doi: 10.1017/S0953756201229967

Lehtonen, M. J., Somervuo, P., and Valkonen, J. P. T. (2008). Infection with Rhizoctonia solani induces defense genes and systemic resistance in potato sprouts grown without light. Phytopathol 98, 1190-1198. doi: 10.1094/PHYTO98-11-1190

Lochman, J., and Mikeš, V. (2005). Activation of different defence-related genes expression by ergosterol. FEBS J. 272, 470. doi: 10.1111/j.17424658.2005.4739_12.x

Malmierca, M. G., Barua, J., Mccormick, S. P., Izquierdo-Bueno, I., Cardoza, R. E., Alexander, N. J., et al. (2014). Novel aspinolide production by Trichoderma arundinaceum with a potential role in Botrytis cinerea antagonistic activity and plant defence priming. Environ. Microbiol. 17, 1103-1118. doi: 10.1111/14622920.12514

Malmierca, M. G., Cardoza, R. E., Alexander, N. J., McCormick, S. P., Collado, I. G., Hermosa, R., et al. (2013). Relevance of trichothecenes in fungal physiology: disruption of tri5 in Trichoderma arundinaceum. Fungal Genet. Biol. 53, 22-33. doi: 10.1016/j.fgb.2013.02.001

Mukherjee, P. K., Horwitz, B. A., Herrera-Estrella, A., Schmoll, M., and Kenerley, C. M. (2013). Trichoderma research in the genome era. Annu. Rev. Phytopathol. 51, 105-129. doi: 10.1146/annurev-phyto-082712-102353

Nürnberger, T., Brunner, F., Kemmerling, B., and Piater, L. (2004). Innate immunity in plants and animals: Striking similarities and obvious differences. Immunol. Rev. 198, 249-266. doi: 10.1111/j.0105-2896.2004.0119.x

Papavizas, G. (1985). Trichoderma and Gliocladium: biology, ecology, and potential for biocontrol. Annu. Rev. Phytopathol. 23, 23-54. doi: 10.1146/annurev.py.23.090185.000323

Pereira, J. L., Queiroz, R. M. L., Charneaum, S. O., Felix, C. R., Ricart, C. A. O., Lopes Da Silva, F., et al. (2014). Analysis of Phaseolus vulgaris response to its association with Trichoderma harzianum (ALL-42) in the presence or absence of the phytopathogenic fungi Rhizoctonia solani and Fusarium solani. PLoS ONE 9:e98234. doi: 10.1371/journal.pone.0098234

Pfaffl, M. W., Horgan, G. W., and Dempfle, L. (2002). Relative expression software tool (REST) for group-wise comparison and statistical analysis of relative expression results in real-time PCR. Nucleic Acids Res. 30:e36. doi: 10.1093/nar/30.9.e36

Rahman, M., and Punja, Z. K. (2005). Biochemistry of ginseng root tissues affected by rusty root symptoms. Plant Physiol. Biochem. 43, 1103-1114. doi: 10.1016/j.plaphy.2005.09.004

Rigaud, J. R., and Puppo, A. (1975). Indole 3 acetic acid catabolism by soybean bacteroids. J. Gen. Microbiol. 88, 223-228. doi: 10.1099/00221287-88-2-223

Rivière, M., Marais, A., Ponchet, M., Willats, W., and Galiana, E. (2008). Silencing of acidic pathogenesis-related PR-1 genes increases extracellular $\beta-(1 \rightarrow 3)$ glucanase activity at the onset of tobacco defence reactions. J. Exp. Bot. 59, 1225-1239. doi: 10.1093/jxb/ern044
Rossard, S., Roblin, G., and Atanassova, R. (2010). Ergosterol triggers characteristic elicitation steps in Beta vulgaris leaf tissues. J. Exp. Bot. 61, 1807-1816. doi: $10.1093 / \mathrm{jxb} / \mathrm{erq} 047$

Ruano-Rosa, D., del Moral-Navarrete, L., and Lopez-Herrera, C. J. (2010). Selection of Trichoderma spp. isolates antagonistic to Rosellinia necatrix. Span. J. Agric. Res. 8, 1084-1097. doi: 10.5424/sjar/2010084-1403

Tello, J., Lacasa, A., and Molina, R. (1985). Una nota fitopatológica sobre el complejo parasitario del pie de la judía (Phaseolus vulgaris). ITEA 61, 57-69.

Upchurch, R. G., and Ramirez, M. E. (2010). Defense-related gene expression in soybean leaves and seeds inoculated with Cercospora kikuchii and Diaporthe phaseolorum var. meridionalis. Physiol. Mol. Plant Pathol. 75, 64-70. doi: 10.1016/j.pmpp.2010.08.007

Valenciano, J. B., Casquero, P. A., Boto, J. A., and Marcelo, V. (2006). Evaluation of the occurrence of root rots on bean plants (Phaseolus vulgaris) using different sowing methods and with different techniques of pesticide application. N.Z. J. Crop. Horticul. Sci. 34, 291-298. doi: 10.1080/01140671.2006. 9514419

Vellicce, G. R., Ricci, J. C. D., Hernández, L., and Castagnaro, A. P. (2006). Enhanced resistance to Botrytis cinerea mediated by the transgenic expression of the chitinase gene ch5B in strawberry. Transgenic Res. 15, 57-68. doi: 10.1007/s11248-005-2543-6

Veloso, J., and Díaz, J. (2012). Fusarium oxysporum Fo47 confers protection to pepper plants against Verticillium dahliae and Phytophthora capsici, and induces the expression of defence genes. Plant Pathol. 61, 281-288. doi: 10.1111/j.1365-3059.2011.02516.x

Woo, S. L., and Lorito, M. (2007). "Exploiting the interactions between fungal antagonists, pathogens and the plant for biocontrol," in Novel Biotechnologies for Biocontrol Agent Enhancement and Management, eds M. Vurro and J. Gressel (Dordrecht: Springer), 107-130.

Woo, S., Scala, F., Ruocco, M., and Lorito, M. (2006). The molecular biology of the interactions between Trichoderma spp., phytopathogenic fungi, and plants. Phytopathol 96, 181-185. doi: 10.1094/PHYTO-96-018

Yedidia, I., Srivastva, A. K., Kapulnik, Y., and Chet, I. (2001). Effect of Trichoderma harzianum on microelement concentrations and increased growth of cucumber plants. Plant Soil 235, 235-242. doi: 10.1023/A:1011990013955

Zhao, Y., Thilmony, R., Bender, C. L., Schaller, A., He, S. Y., and Howe, G. A. (2003). Virulence systems of Pseudomonas syringae pv. tomato promote bacterial speck disease in tomato by targeting the jasmonate signaling pathway. Plant J. 36, 485-499. doi: 10.1046/j.1365-313X.2003.01895.x

Conflict of Interest Statement: The authors declare that the research was conducted in the absence of any commercial or financial relationships that could be construed as a potential conflict of interest.

Copyright (C) 2015 Mayo, Gutiérrez, Malmierca, Lorenzana, Campelo, Hermosa and Casquero. This is an open-access article distributed under the terms of the Creative Commons Attribution License (CC BY). The use, distribution or reproduction in other forums is permitted, provided the original author(s) or licensor are credited and that the original publication in this journal is cited, in accordance with accepted academic practice. No use, distribution or reproduction is permitted which does not comply with these terms. 
OPEN ACCESS

Edited by:

Jaime Prohens,

Polytechnic University of Valencia,

Spain

Reviewed by:

Ana Paula Rodiño,

Misión Biológica de Galicia - CSIC,

Spain

Eriston Vieira Gomes,

University of São Paulo, Brazil

*Correspondence.

Pedro A. Casquero

pedro.casquero@unileon.es

Specialty section:

This article was submitted to

Crop Science and Horticulture,

a section of the journal

Frontiers in Plant Science

Received: 30 May 2016

Accepted: 12 May 2016

Published: 04 August 2016

Citation:

Mayo S, Cominelli E, Sparvoli F, González-López O,

Rodríguez-González A, Gutiérrez S

and Casquero PA (2016)

Development of a qPCR Strategy

to Select Bean Genes Involved

in Plant Defense Response

and Regulated by the Trichoderma

velutinum - Rhizoctonia solani

Interaction. Front. Plant Sci. 7:1109.

doi: 10.3389/fp/s.2016.01109

\section{Development of a qPCR Strategy to Select Bean Genes Involved in Plant Defense Response and Regulated by the Trichoderma velutinum - Rhizoctonia solani Interaction}

\author{
Sara Mayo', Eleonora Cominelli², Francesca Sparvoli², Oscar González-López', \\ Alvaro Rodríguez-González ${ }^{1}$, Santiago Gutiérrez ${ }^{3}$ and Pedro A. Casquero ${ }^{1 *}$
}

\begin{abstract}
'Research Group of Engineering and Sustainable Agriculture, Department of Agrarian Engineering and Sciences, Natural Resources Institute, University of León, León, Spain, ${ }^{2}$ Institute of Agricultural Biology and Biotechnology, Consiglio Nazionale delle Ricerche, Milan, Italy, ${ }^{3}$ Area of Microbiology, University School of Agricultural Engineers, University of León, Ponferrada,
\end{abstract} Spain

Bean production is affected by a wide diversity of fungal pathogens, among them Rhizoctonia solani is one of the most important. A strategy to control bean infectious diseases, mainly those caused by fungi, is based on the use of biocontrol agents (BCAs) that can reduce the negative effects of plant pathogens and also can promote positive responses in the plant. Trichoderma is a fungal genus that is able to induce the expression of genes involved in plant defense response and also to promote plant growth, root development and nutrient uptake. In this article, a strategy that combines in silico analysis and real time PCR to detect additional bean defense-related genes, regulated by the presence of Trichoderma velutinum and/or $R$. solani has been applied. Based in this strategy, from the 48 bean genes initially analyzed, 14 were selected, and only WRKY33, CH5b and hGS showed an up-regulatory response in the presence of T. velutinum. The other genes were or not affected (OSM34) or down-regulated by the presence of this fungus. $R$. solani infection resulted in a down-regulation of most of the genes analyzed, except PR1, OSM34 and CNGC2 that were not affected, and the presence of both, $T$. velutinum and $R$. solani, up-regulates $h G S$ and down-regulates all the other genes analyzed, except $\mathrm{CH} 5 b$ which was not significantly affected. As conclusion, the strategy described in the present work has been shown to be effective to detect genes involved in plant defense, which respond to the presence of a BCA or to a pathogen and also to the presence of both. The selected genes show significant homology with previously described plant defense genes and they are expressed in bean leaves of plants treated with T. velutinum and/or infected with $R$. solani.

Keywords: biotic stress, systemic acquired resistance, induced systemic resistance, hypersensitive response, defense genes, biocontrol agent, Phaseolus vulgaris 


\section{INTRODUCTION}

The common bean (Phaseolus vulgaris L.) is the most important food legume crop worldwide. Bean production is often affected by biotic and abiotic factors (Guerrero-González et al., 2011) by microorganisms, humidity, temperature... that are detected as signals for the activation of plant response mechanisms. This crop is affected by a wide diversity of fungal pathogens (Sclerotinia spp., Fusarium spp., Phytium spp., Botrytis spp.,...) among them Rhizoctonia solani JG Kühn [Teleomorph: Thanatephorus cucumeris ( $\mathrm{AB}$ Frank) Donk] has a remarkable importance as responsible of important economic losses in this crop (Valenciano et al., 2006). R. solani is a necrotrophic pathogen responsible for the root and hypocotyl diseases. Plant infection occurs through wounds or by the direct action of the fungal mycelium, which tears the cuticle and penetrates the epidermis (Guerrero-González et al., 2011).

As a strategy to control bean infectious diseases, mainly those caused by fungi, the use of biocontrol agents (BCA) can reduce the negative effects of plant pathogens and they also can promote positive responses in the plant (Shoresh et al., 2010). The genera Trichoderma, Gliocladium, Rhizobium, Pseudomonas, are beneficial organisms that have shown good efficiency as BCAs against pathogenic microorganisms. Trichoderma (Teleomorph: Hypocrea) is a fungal genus that is found in the soil, and it is a secondary fast growing opportunistic invasive. In addition, Trichoderma biocontrol strains are able to induce the expression of genes involved in defense response and also to promote plant growth, root development, and nutrient uptake (Hermosa et al., 2012).

The relationships established between plant and microorganisms are very diverse. When a plant is exposed to a pathogenic microorganism, the production of molecules associated to salicylic acid is increased, being this a systemic acquired resistance (SAR) response. The response of plants against non-pathogenic microorganisms is different, resulting in activation of signaling cascades that are dependent on jasmonic acid and ethylene, such as hydroperoxide lyase, peroxidase, and phenylalanine ammonia lyase, all of which belong to an induced systemic resistance (ISR) response (Druzhinina et al., 2011). Other responses result in a rapid cell death in infected tissues, then plants activate the hypersensitive response that involves the accumulation of salicylic acid, reactive oxygen species and an increased the influx of $\mathrm{Ca}^{2+}$ (Guerrero-González et al., 2011).

In the tripartite interaction of bean plants with the pathogen $R$. solani and a biocontrol Trichoderma species, several changes are produced in the plant, such as the increase in phenolic acid and lignin, accumulation of phytoalexins (Guerrero-González et al., 2011), and down- or up-regulation of defense-related genes expression (Mayo et al., 2015). Different categories of defenserelated genes whose expression is modulated by biotic stresses have been described in bean plant interacting with pathogen and non-pathogenic microorganisms (Mayo et al., 2015).

Our hypothesis is that the combination of real time PCR with "in silico" analysis is a valid strategy to identify bean defense-related genes regulated by BCAs and/or plant pathogens. The aim is develop a systematic strategy to detect bean defense-related genes regulated by the presence of Trichoderma velutinum and/or $R$. solani. Finally, the procedure has been validated by the analysis of expression of the selected genes in the presence or absence of these two fungi.

\section{MATERIALS AND METHODS}

\section{Trichoderma and Rhizoctonia solani Isolates and Culture Collections}

Trichoderma velutinum T028, was collected from the bean traditional production area (Protected Geographical Indication, PGI), called "Alubia La Bañeza - León” (EC Reg. n.256/2010 published on March 26th, 2010, OJEU L880/17), from a High Quality variety of beans (Figure 1) without any genetic manipulation. It was isolated from soil plot bean in the Astorga region (León, Spain). This isolate gave percentages of inhibition greater that $60 \%$ in membrane assays and $40 \%$ in direct confrontation assays with R. solani, and that was able to sporulate on potato-dextrose-agar (PDA) medium.

Rhizoctonia solani R43 was isolated from bean plants of the same PGI and selected based on its high virulence. The isolated strains were stored in the collection "Pathogens and Antagonists of the Laboratory Diagnosis of Pests and Diseases" (PALDPD, University of León, León, Spain).

Isolates were inoculated on PDA (Becton Dickinson, Germany) medium and grown at $25^{\circ} \mathrm{C}$ in the dark for 1 week. After this incubation time T. velutinum T028 was exposed to light in order to induce the spore's formation.

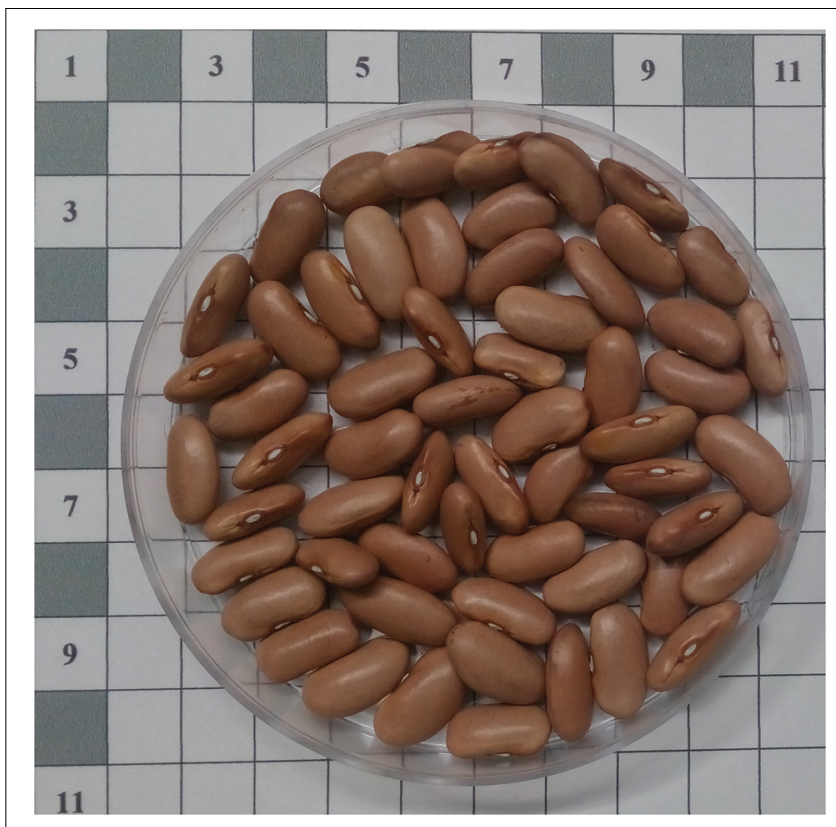

FIGURE 1 | Bean seeds of "Canela" variety of the Protected Geographical Indication "Alubia La Bañeza - León” (Spain). 


\section{Plant Materials and Growth Conditions}

Sixty bean seeds (Canela landrace, PGI "Alubia de la Bañeza León," Figure 1) per treatment were germinated and cultured in presence or absence of the fungi in four conditions according to the procedure previously described by Mayo et al. (2015): (i) T. velutinum (T028) isolate plus R. solani (R43) (RT028); (ii) T. velutinum isolate (T028) without pathogen $(\mathrm{C}=$ control) (CT028); (iii) control (without T. velutinum) with R. solani (RC) and (iv) control without fungi (CC). The culture was carried out in climatic chamber and growth conditions were performed as previously described (Mayo et al., 2015). Six bean leaves from 45 day-old plants of each treatment were randomly collected and stored at $-80^{\circ} \mathrm{C}$ until use.

\section{RNA Extraction and Purification}

The procedures for RNA extraction were performed as described previously (Reid et al., 2006). Bean leaves were lyophilized and were ground to a fine powder in liquid nitrogen using a mortar and pestle. The powder was mixed with $20 \mathrm{ml}$ of extraction buffer/g of sample (extraction buffer: 0.1\% SDS, $100 \mathrm{mM} \mathrm{LiCl}$, $10 \mathrm{mM}$ EDTA, $100 \mathrm{mM}$ Tris- $\mathrm{HCl}$, pH9) pre-warmed at $65^{\circ} \mathrm{C}$, and $20 \mathrm{ml} / \mathrm{g}$ of phenol-chloroform-isoamyl alcohol 25:24:1 (SigmaAldrich, St. Louis, MO, USA). Then, the mixtures, in eppendorf tubes, were centrifuged at $13,000 \mathrm{rpm}$ for $10 \mathrm{~min}$ at $4^{\circ} \mathrm{C}$. The aqueous layer was transferred to a new tube. This step was repeated twice. Nucleic acids were precipitated with 1 volume of $\mathrm{LiCl} 4 \mathrm{M}$, mixed and kept overnight at $4^{\circ} \mathrm{C}$. Tubes were then centrifuged at $13,000 \mathrm{rpm}$ for $30 \mathrm{~min}$ at $4^{\circ} \mathrm{C}$, and the resulting pellets were washed with ice cold ethanol 70\%-DEPC, centrifuged again at $13,000 \mathrm{rpm}$ for $10 \mathrm{~min}$ at $4^{\circ} \mathrm{C}$ and air dried. Finally, the pellets were dissolved in 50-200 $\mu \mathrm{H}_{2} \mathrm{O}$-DEPC and stored at $-20^{\circ} \mathrm{C}$ until use.

RNA concentrations and its purity were estimated from the A260/280 absorbance ratio with a NanoDrop (Thermo Scientific, Wilmington, DE, USA), considering the ideal absorbance ratio $(1.8 \leq \mathrm{A} 260 / 280 \leq 2.0)$ and $1 \%$ agarose gel was run to visualize the integrity of the RNA.

\section{cDNA Synthesis}

Approximately $5 \mu \mathrm{g}$ of RNA were treated with DNase using the TURBO DNAfree ${ }^{\mathrm{TM}}$ Kit (Applied Biosystems, Foster City, CA, USA), according to the manufacturer instructions. cDNA was synthesized using High-Capacity cDNA Reverse Transcription kit (Applied-Biosystems, Foster City, CA, USA) according to the manufacture's manual.

\section{qPCR Conditions and Analysis}

qPCR reactions were performed with 7300 System (Applied Biosystems, Foster City, CA, USA) using SYBR ${ }^{\circledR}$ Green. Each reaction was performed in $20 \mu \mathrm{l}$ containing $10 \mu \mathrm{l}$ of $2 \mathrm{X}$ Power SYBR Green PCR Master Mix (Life Technologies), 0.2-0.3 $\mu \mathrm{M}$ primers and cDNA samples diluted 1:20. Each qPCR reaction was performed in triplicate. Reactions were run using the cycling parameter described previously (Reid et al., 2006) and the qPCR data were analyzed by the $2^{-\Delta \Delta \mathrm{Ct}}$ method (Pfaffl, 2001). In order to analyze the qPCR data, Act11 gene was used as housekeeping to determine the relative expression level of the other genes analyzed in this work (Borges et al., 2012). T. velutinum T028 strain was selected as reference strain in this study based on its positive effects on bean phenotype with and without R. solani infection (data no published). For the determination of qPCR efficiency of each primer pairs, a standard curve was performed using the following cDNA dilutions: 1:4, 1:16, 1:64; 1:256 and 1:1024. Every measurement was made in triplicate. The corresponding qPCR efficiencies (E) were calculated for every primer pair with the software 7300 System SDS software (Applied Biosystems, Foster City, CA, USA) according to the equation $\mathrm{E}=\left(10^{-1 / \text { slope }}-1\right) \times 100$ (Rutledge and Stewart, 2008).

The significance of the differences between the gene expressions levels were compared by the Student's $t$-test using SAS (SAS Institute Inc., 2004, Cary, NC, USA).

\section{RESULTS}

\section{Selection of Putative Bean Defense-Related Genes}

Following an exhaustive and systematic analysis, summarized in the Figure 2, several bean genes were selected for their expression analysis in leaves from bean plants grown in interaction with T. velutinum and infected or not with Rhizoctonia solani. Thus, as result of the search in the literature, 48 genes were firstly found, showing stress and/or defense response (Table 1). Only those genes that resulted to be expressed in P. vulgaris leaves, based on transcriptomic data reported in the Phytozome database ${ }^{1}$, were considered for qPCR expression analysis in leaves. The genes for which we confirmed expression in leaves were considered for further analyses.

As result, from the 48 genes selected for their involvement in bean stress and/or defense responses, only 19 were selected which showed a detectable level of expression in bean leaves.

The selected genes can be included in nine different groups (Table 2): (i) involved in the regulation of the balance between necrotrophic and biotrophic pathogen responses: WRKY33 (WRKY transcription factor) (NM129404.3) (Bakshi and Oelmüller, 2014); (ii) pathogenesis related genes: PR1 (pathogenesis related 1) (HO864272) (Guerrero-González et al., 2011), PR2 ( $\beta$ 1-3 endoglucanase) (HO864270) (GuerreroGonzález et al., 2011), PR3 (chitinase class I) (TC18606) (Pereira et al., 2014), PR4 (pathogenesis related 4) (HO864354) (Guerrero-González et al., 2011), PR16a (germin.like protein 8) (CB540239) (Borges et al., 2012), IPER (basic peroxidase) (AF007211) (Upchurch and Ramirez, 2010), PPO (polyphenol oxidase) (EF158428) (Upchurch and Ramirez, 2010); (iii) related with the ethylene signaling pathway: ERF1 (ethylene-responsive transcription factor 1) (AF076277) (Lorenzo et al., 2003), ERF5 (ethylene-responsive transcription factor 5) (At5g47230) (Moffat et al., 2012), and CH5b (endochitinase precursor) (FE897014.1) (Vellicce et al., 2006); (iv) involved in phytoalexin

\footnotetext{
${ }^{1}$ http://phytozome.jgi.doe.gov/pz/portal.html
} 
biosynthesis: PAL1 (phenylalanine and histidine ammonialyase) (KF279696) (Kim and Hwang, 2014); (v) related in osmotin biosynthesis: OSM34 (osmitin-like protein) (At4g11650) (Sharma et al., 2013); (vi) involved in $\mathrm{Ca}^{2+}$ signaling: CNGC2 (cyclic nucleotide-gated ion channel 2) (CB542582) (Borges et al., 2012); (vii) needed for antimicrobials and oxylipins (defense signaling molecules): HPL (hydroperoxide lyase) (AW733791) (Upchurch and Ramirez, 2010), Lox2 (lipoxygenase 2) (D13949) (Upchurch and Ramirez, 2010), Lox7 (lipoxygenase 2) (Upchurch and Ramirez, 2010); (viii) GSTa (2,4-D inducible glutathione S-transferase) (HO864392) (Guerrero-González et al., 2011); and (ix) $h G S$ (homoglutathione synthetase) (HO864377) both related with oxidative stress (Guerrero-González et al., 2011).

However, only 14 genes were selected to the study of the expression genes because PR16a, IPER, PPO, Lox2, and Lox7, showing negative $\mathrm{qPCR}$ results, were finally discarded.

\section{Selection of a Trichoderma Strain to Validate the Gene Selection Strategy}

Trichoderma velutinum T028 was the selected isolate, based on its positive effect on bean growth. Thus, plants inoculated with this strain showed a significant increase in dry weight of both aerial parts and root system, including when $R$. solani was present in the substrate (Figure 3). Thus, when bean plants were treated with $T$. velutinum $\mathrm{T} 028$ they increased respect to control plants (CC) $4.75 \%$ their diameter of hypocotyl, 10.75\% their length of root system, 4.27 and 5.51\% in dry weight of aerial parts and root system, respectively. When plants were infected with $R$. solani, the action of $T$. velutinum T028 caused an increased respect to the control plant with the pathogen (RC) of the diameter of hypocotyl in $8.76,21.15 \%$ in the length of root system, and 11.05 and $3.43 \%$ in dry weight of aerial parts and root system respectively.

Based on these results, this isolate was used for further studies. In addition, this is the first report in which the effects of this strain on bean phenotype and plant gene regulation are studied.

\section{Effect of $R$. solani Infection on Expression of the Selected Genes. Validation of the Procedure Used to Select Bean Genes Involved in Defense Responses (Strategy Validation I)}

A significant down-regulation of expression of PR2, PR3, PR4, ERF1, ERF5, PAL1, HPL, and GTSa genes with ratios of expression ranging from 0.149 fold for $P A L 1$ and 0.763 fold for $P R 3$ was observed in bean plants grown in the presence of R. solani (RC) compared to control plants (CC). Conversely, expression of PR1, OSM34, CNGC2, and $h G S$ genes was upregulated, but with non-statistically significant differences with a ratios between 1.289 and 1.193 for $P R 1$ and $h G S$, respectively (Figure 4).

\section{Effect of Trichoderma on Expression of the Selected Genes (Strategy Validation II)}

Trichoderma treatment also down-regulates expression of most of the bean defense-related genes, but at a lower level than $R$. solani. Thus, when $T$. velutinum T028 was in the substrate (CT028), PR2, PR3, PR4, ERF1, ERF5, PAL1, CNGC2, HPL, and GSTa

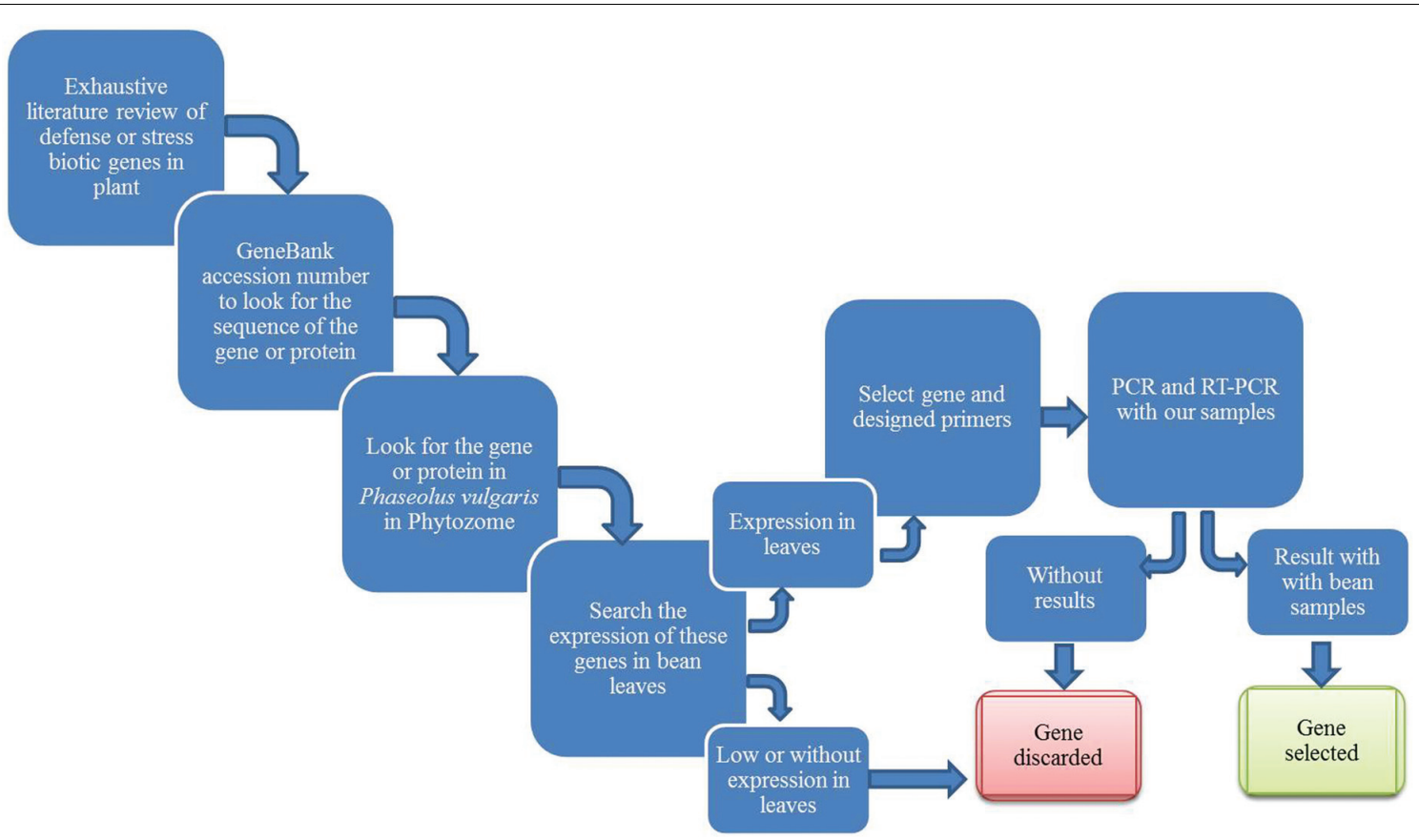

FIGURE 2 | Schematic representation of the work flow set up in the present work to select bean genes involved in plant defense. 
TABLE 1 | Genes selected for stress and/or defense response and their empirical expression in Phaseolus vulgaris leaves.

\begin{tabular}{|c|c|c|c|c|}
\hline Id & Gene & Accession number & Functional annotation & NCBI Phytozome \\
\hline 1 & Chit & AY357300.2 & Chitanase & \\
\hline 3 & Pod3 & AF485265.1 & Peroxidase & \\
\hline 4 & PR3 & TC18606 & Chitinase class I & Phvul.009G116600 \\
\hline 5 & Lox1 & U76687.2 & Lipoxygenase & \\
\hline 6 & PPO & EF158428 & Polyphenol oxidase & \\
\hline 7 & PR10 & AJ289155 & Stress-induced ribonuclease-like protein & \\
\hline 8 & PR12 & BU964598 & Defensin precursor & \\
\hline 9 & MMP2 & AY057902 & Matrix metalloproteinase 2 & \\
\hline 10 & $\mathrm{CHS}$ & X53958 & Chalcone synthase & \\
\hline 11 & AOS & DQ288260 & Allene oxide synthase & \\
\hline 15 & IPER & AF007211 & Basic peroxidase & Phvul.009G215000 \\
\hline \multicolumn{5}{|c|}{ Borges et al., 2012} \\
\hline 16 & PR16a & CB540239 & Germin-like protein 8 & Phvul.010G129900 \\
\hline 17 & PGla & CB542106 & Polygalacturonase-inhibitor-like protein & \\
\hline 18 & MAPKK & CB543156 & MEK map kinase kinase & \\
\hline 19 & PROF & CB543496 & Profilin & \\
\hline 20 & CNGC2 & CB542582 & Cyclin nucleotide-gated ion channel 2 & Phvul.008G036200 \\
\hline \multicolumn{5}{|c|}{ Guerrero-González et al., 2011} \\
\hline 21 & $P R 1$ & HO864272 & Pathogenesis related protein 1 & Phvul.003G109100 \\
\hline 22 & PR2 & HO864270 & Pathogenesis related protein 2 & Phvul.003G109200 \\
\hline 23 & PR4 & HO864354 & Pathogenesis related protein 4 & Phvul.006G102300 \\
\hline 31 & CPRD14 & HO864341 & CPRD14 protein & \\
\hline 32 & OPR5 & HO864304 & 12-oxophytodienoic acid 10, 11-reductase & \\
\hline 33 & GST22 & HO864275 & Glutathione S-transferase 22 & \\
\hline 34 & CPRD8 & HO864396 & CPRD8 protein & \\
\hline 35 & UDPGT & HO864301 & UDP-glucosyl transferase 72E1 & \\
\hline 36 & ERD15 & HO864375 & ERD15 protein & \\
\hline 37 & GTSa & HO864392 & 2,4-D inducible glutathione S-transferase & Phvul.002G241400 \\
\hline 38 & GST15 & HO864369 & Glutathione S-transferase 15 & \\
\hline \multicolumn{5}{|c|}{ Gallou et al., 2009} \\
\hline 39 & GST1 & J03679 & Gluthatione-S-transferase 1 & \\
\hline \multicolumn{5}{|c|}{ Lehtonen et al., 2008} \\
\hline 40 & TSI-1 & BQ121547 & TSI-1 protein & \\
\hline 41 & Lip & BQ112158 & Lipase-like protein & \\
\hline 42 & Amintransf2 & BQ517030 & Aminotransferase 2 & Phvul.006G029100 \\
\hline \multicolumn{5}{|c|}{ Bakshi and Oelmüller, 2014} \\
\hline 43 & WRKYЗ3 & NM129404.3 & WRKY transcription factors & Phvul.008G090300 \\
\hline \multicolumn{5}{|c|}{ Vellicce et al., 2006} \\
\hline 44 & $C H 5 b$ & FE897014.1 & Endochitinase precursor & Phvul.009G116500 \\
\hline Lor & t al., 2003 & & & \\
\hline
\end{tabular}


TABLE 1 | Continued

\begin{tabular}{|c|c|c|c|c|}
\hline Id & Gene & Accession number & Functional annotation & NCBI Phytozome \\
\hline \multicolumn{5}{|c|}{ Moffat et al., 2012} \\
\hline \multicolumn{5}{|c|}{ Kim and Hwang, 2014} \\
\hline 47 & PAL1 & KF279696 & Phenylalanine and histidine ammonia-lyase & Phvul.001G177800 \\
\hline \multicolumn{5}{|c|}{ Sharma et al., 2013} \\
\hline
\end{tabular}

were significantly down-regulated with expression ratios ranging from 0.168 for PR4 to 0.754 for ERF1. However, WRKY 33, $C H 5 b$, and $h G S$ were up-regulated when compared with the levels of expression in control plants, with relative expression levels between 2.462 for $C H 5 b$ and 1.576 for $h G S$ (Figure 5). OSM34 was slightly but not significantly up-regulated.

\section{Effect of Interaction of $T$. velutinum and $R$. solani on Expression of the Selected Genes (Strategy Validation III)}

When T. velutinum T028 and R. solani (RT028) were in the substrate, the genes WRKY33, PR2, PR3, PR4, ERF1, ERF5, PAL1, OSM34, HPL and GSTa were significantly down-regulated with values between 0.179 for PAL1 and 0.631 for WRKY33. In the case of PR1 and CNGC2, they were also down-regulated but not significantly respect to control plant (C). Conversely, $h G S$ was up-regulated with a significant ratio of 1.589 respect to control plants, while $\mathrm{CH} 5 \mathrm{~b}$ was not significantly up-regulated with a ratio of 1.613 (Figure 6).

\section{DISCUSSION}

Plants have developed some defensive strategies to perceive pathogen attack and to translate this perception into an appropriate adaptive response. During attack, plants are able to enhance their resistance (induced, acquired, hypersensitive) (Lodha and Basak, 2012). Contact with pathogenic and nonpathogenic microorganisms triggers two mechanisms: (i) SAR that is usually triggered by local infections, it provides longterm systemic resistance to pathogen attack and requires the involvement of the signal molecule salicylic acid (Durrant and Dong, 2004), and (ii) ISR that is known to result from colonization of roots by certain non-pathogenic microorganisms and is dependent on components of the jasmonic acid and ethylene signaling pathways (Shoresh et al., 2010). Then, the combination of both types of induced resistance response can protect the plant against pathogens and can even result in additive level of induced protection against pathogens through both the jasmonic acid/ethylene and salicylic acid pathways (Verhagen et al., 2006).

In the present work we developed a strategy to select genes involved in bean defense response, which would belong to those pathways, but also genes that can contribute to plant defense by other mechanisms. In this sense several previous works have described genes involved in bean defense response (GuerreroGonzález et al., 2011; Mayo et al., 2015). However, in the present work, by a systematic approach, 48 genes were initially considered, and 14 finally selected, which match with the criteria set up in this work: (i) they showed significant homology with previously described plant defense genes, and (ii) were expressed in bean leaves of plants treated with Trichoderma and/or infected with $R$. solani.

The expression of $P$. vulgaris defense-related genes was analyzed in leaves, although the interaction with Trichoderma and/or $R$. solani is initially produced at the root level, to determine if the signals generated in roots as result of this interaction are able to systematically stimulate the bean defense along long distance from roots to the leaves. The isolate T. velutinum T028 was selected following a similar strategy to that previously described (Mayo et al., 2015), and based on its positive effect on bean growth. In this work, to select a Trichoderma isolate, the results of the in vitro membrane assays and direct confrontation assays against $R$. solani were analyzed. Isolate Trichoderma T019 was then selected, showing a percentage of inhibition higher than $40 \%$ in the membrane assays, and/or $20 \%$ in the direct confrontation assays. This isolate also showed the best positive effects on plant phenotype among all the analyzed isolates.

WRKY transcription factors have been involved in the regulation of plant defense gene expression (Rushton and Somssich, 1998; Singh et al., 2002). Thus, WRKY33 has a role in biotic stress defense, where it regulates the balance between necrotrophic and biotrophic pathogen responses (Lippok et al., 2007; Pandey and Somssich, 2009; Birkenbihl et al., 2012). Previous studies have pointed out the involvement of Arabidopsis WRKY transcription factors in regulating the expression of PR genes by direct binding (Chen et al., 2002; Kim et al., 2006). A rapid pathogen-induced WKRY33 expression did not require salicylic acid signaling but a downregulation of this gene involved a direct activation of jasmonic acid (Bakshi and Oelmüller, 2014). In the present case, when bean plants were in contact T. velutinum T028 without pathogen, the WRKY33 gene expression was significantly up-regulated while the $P R$ genes expression (PR2, PR3 and PR4) was significantly down-regulated compared to expression levels in plants without Trichoderma treatment. In the present work, when $R$. solani was added to the substrate, expression of WRKY33 was significantly downregulated in plants with Trichoderma inoculation, while $P R 2, P R 3$ and PR4 were down-regulated. In the study by Mayo et al. (2015), 
TABLE 2 | Common bean sequences used for primer design for RT-PCR analysis.

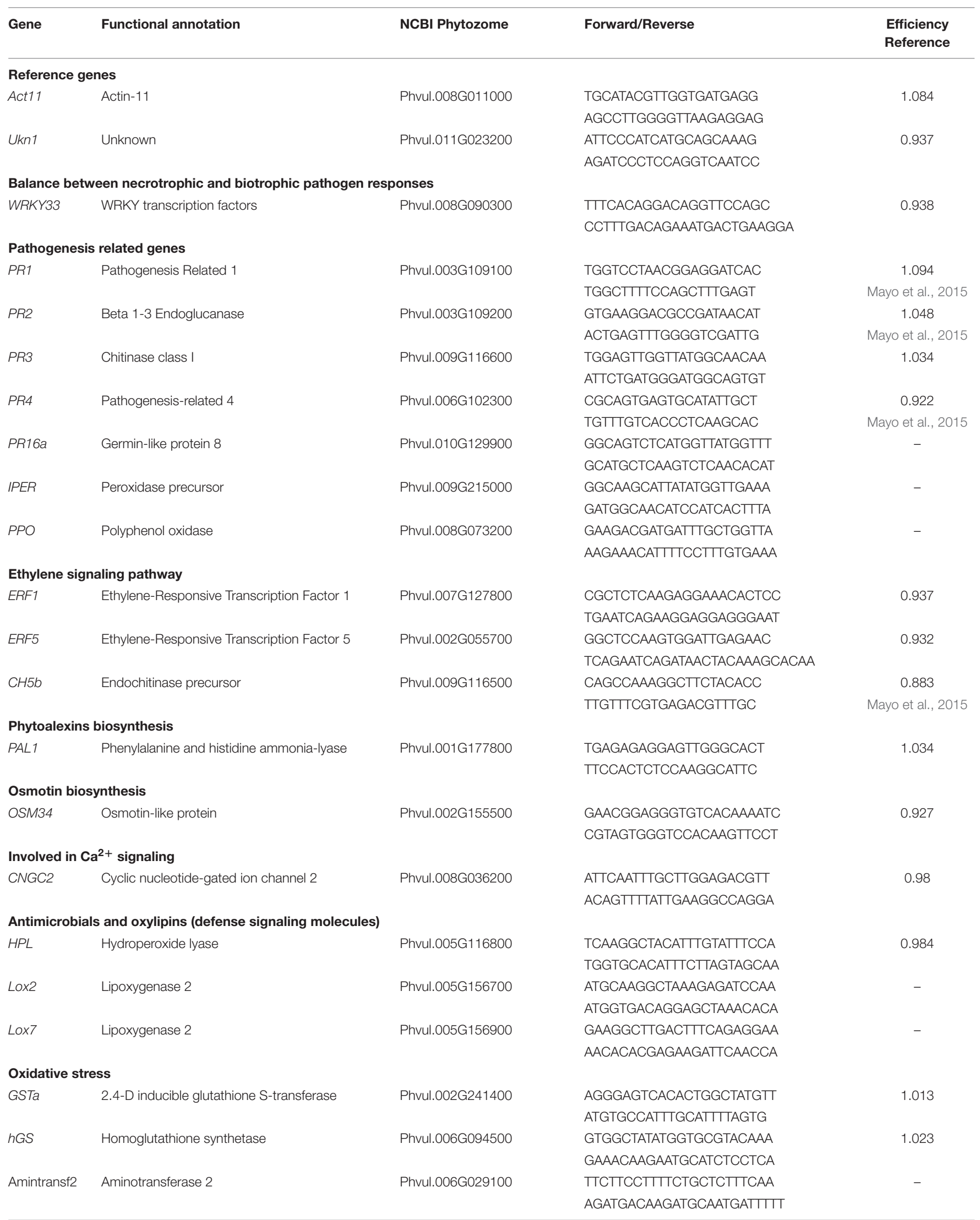

(-) Genes that empirically showing expression but showing negative qPCR results. 

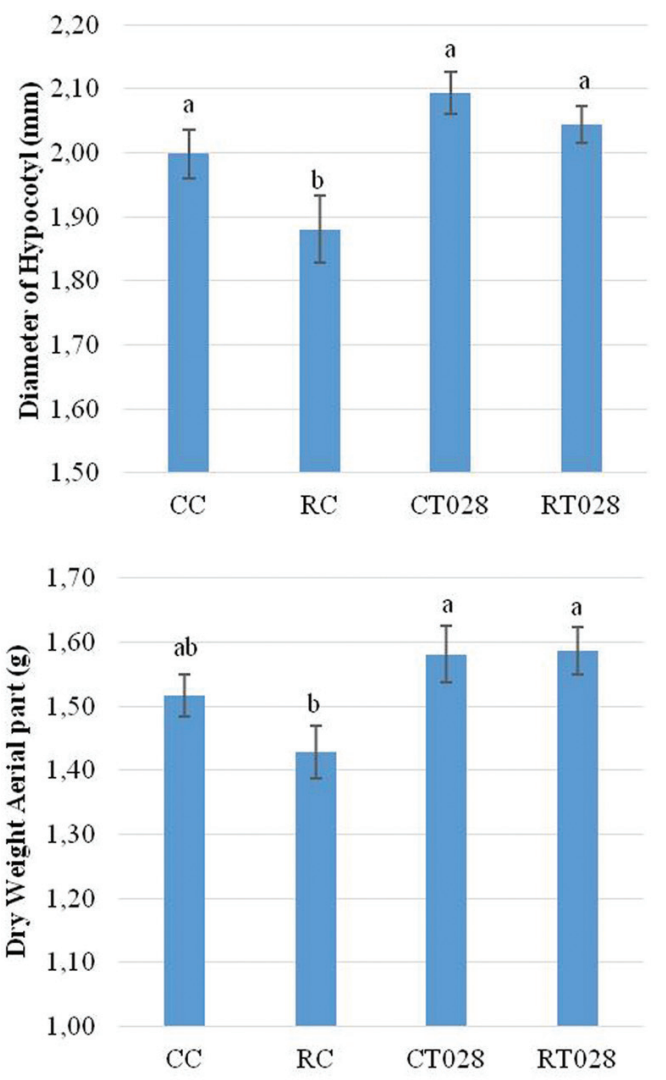

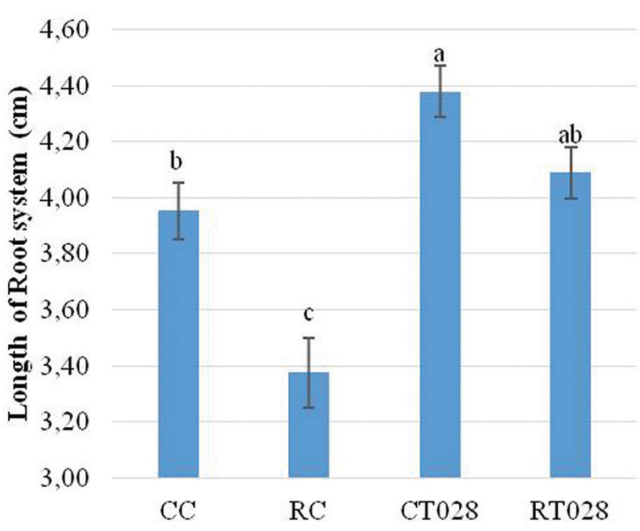

0,90

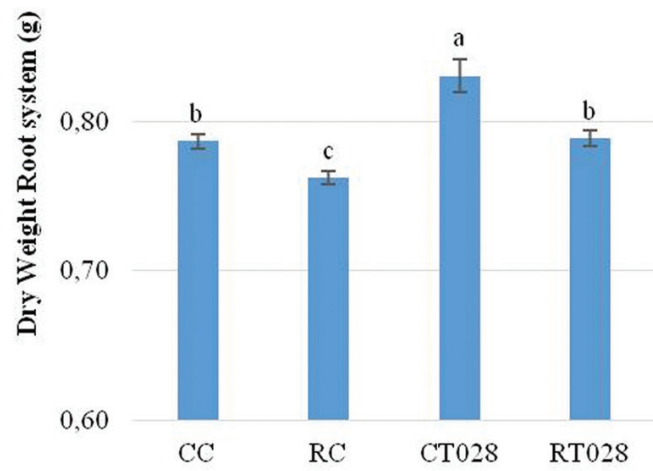

FIGURE 3 | Evaluation of the diameter of the hypocotyl (above left), length of root system (above right) dry weight of the aerial part (below left) and root system (below right) of bean plants grown during $\mathbf{4 5}$ days after sowing. [Trichoderma velutinum T028 without pathogen (CT028), T. velutinum T028 with Rhizoctonia solani (RT028), R. solani control (RC) and control without fungus (CC)]. Differences statistically significant respect to control plants ( $p<0.05)$ are indicated with different letters.

the expression of $P R 1, P R 2, P R 3$, and $P R 4$ was down-regulated when beans were inoculated with $R$. solani.

WRKY33 is also involved in the regulation of the expression of genes modulated by components of the ethylene signaling pathway. In this work, expression of the ERF1 and ERF5 reached similar significant values either with or without Trichoderma and or $R$. solani in the substrate. This result contrasts with previous reports showing that ERF5 was up-regulated and WRKY33 was down-regulated in Arabidopsis infected with Alternaria brassicicola (Son et al., 2012). WRKY33 would act as a represor of ERF1 and ERF5 expression. Thus, when the expression of WRKY33 is increased, expression of ERF1 and ERF5 is downregulated.

$\mathrm{CH} 5 \mathrm{~b}$ encodes an endochitinase precursor and it is also related with the ethylene signaling pathway. In previous works, it has been shown that, when this gene was over-expressed the $R$. solani symptoms were reduced in crops like Nicotiana tabacum and Brassica napus (Broglie et al., 1991). However, in this study, when bean plants were in contact with $R$. solani, the expression of this gene was down-regulated but not significantly, while treatment of these infected plants with $T$. velutinum resulted in its significant up-regulation. These results are in agreement with previous data, showing that the pathogen represses its expression, and the presence of Trichoderma induced it (Mayo et al., 2015).

$P A L$ plays an important role in plant defense; it is involved in the biosynthesis of salicylic acid, which is related to plant systemic resistance (Nugroho et al., 2002; Chaman et al., 2003). PAL gene expression is also regulated in response to pathogen infection. In this work, the presence of T. velutinum and R. solani in the soil resulted in a significant down-regulation of this gene compared with control plants.

Osmotins have plant protective effects against pathogen infection (Narasimhan et al., 2009). In this study, when T. velutinum or $R$. solani were present in the soil, the expression of OSM34 was not significantly up-regulated respect to control plants, but when both fungi were in the soil at the same time, OSM34 was slightly but significantly down-regulated.

The CNGC genes can be related to early plant defense responses due to changes in ion flux, including $\mathrm{H}^{+}$and $\mathrm{Ca}^{2+}$ influx and $\mathrm{K}^{+}$and $\mathrm{Cl}^{-}$efflux (Atkinson et al., 1996). The upregulation of $C N G C 2$ can confirm the importance of ion channels for the plant resistance response (Borges et al., 2012). In this work, this gene was up-regulated when $R$. solani was present in the soil not significant. Conversely, $C N G C 2$ was down-regulated 


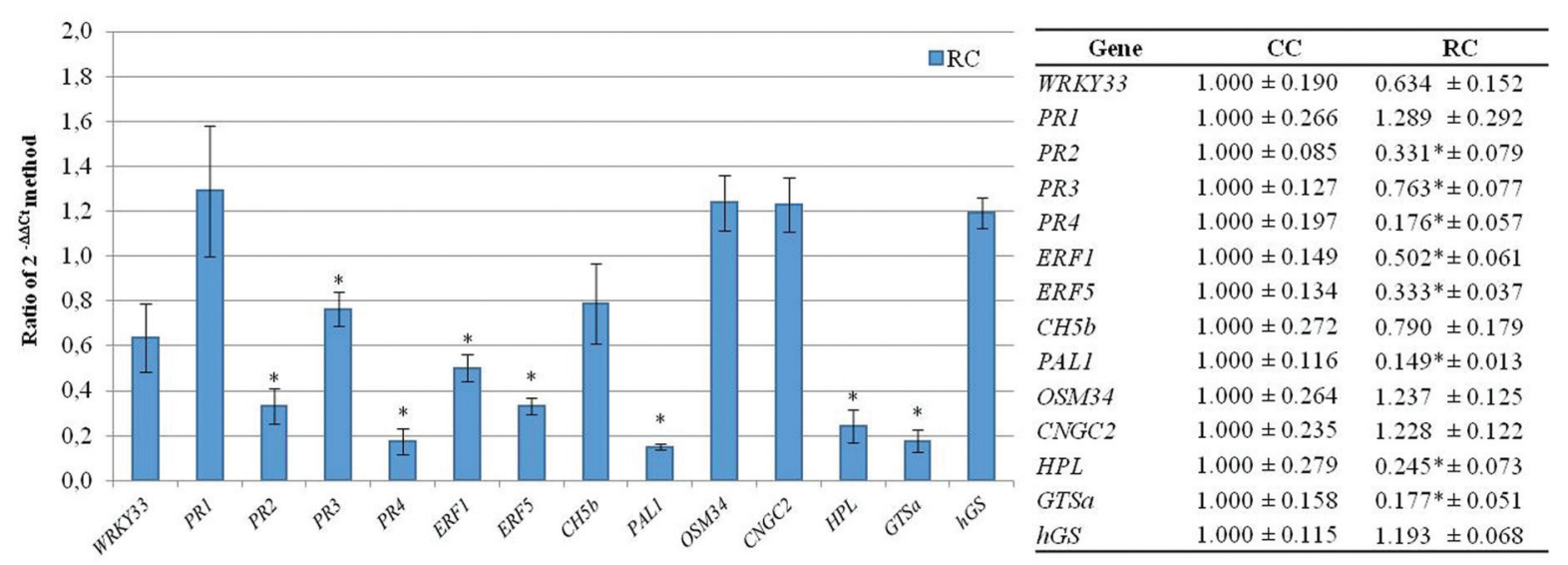

FIGURE 4 | Analysis of relative expression levels of the bean defense genes selected in the present work in bean plants infected with $R$. solani versus their levels of expression in control plants. The data were analyzed by the $2^{-\Delta \Delta \mathrm{Ct}}$ method. The differences statistically significant respect to control plants $(p<0.05)$ are indicated with an asterisk.

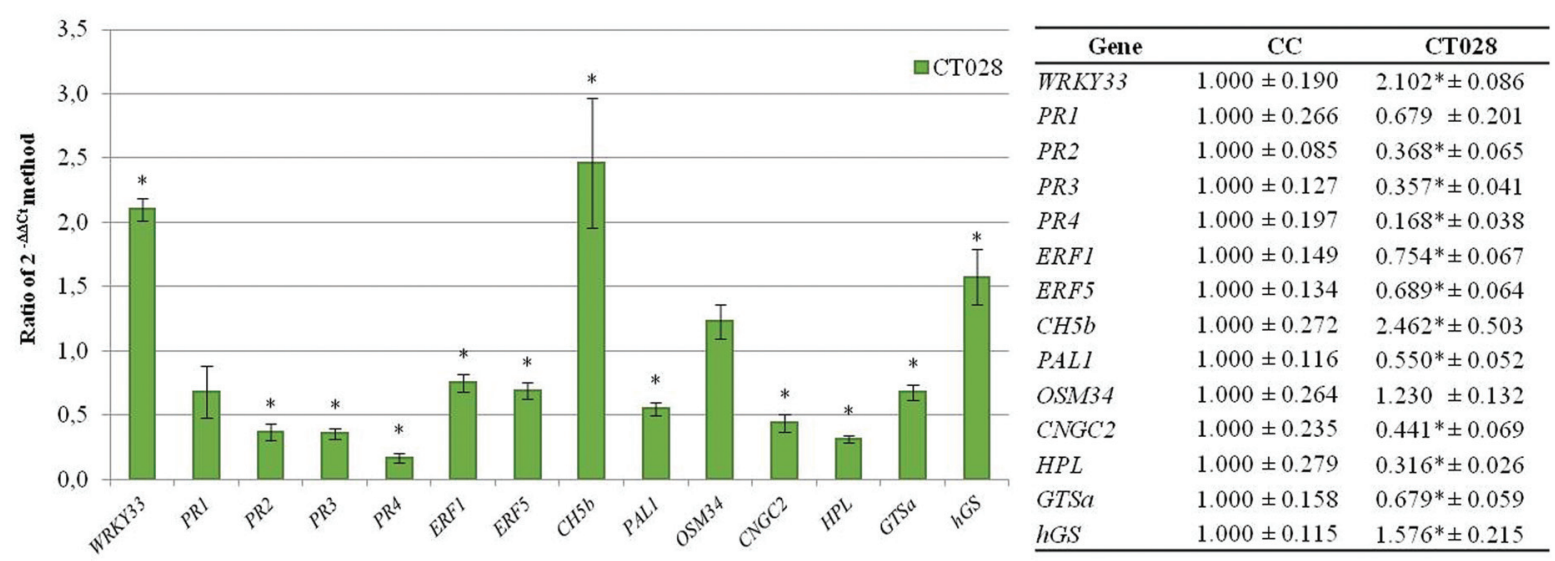

FIGURE 5 | Analysis of relative expression levels of the bean defense genes selected in the present work in bean plants treated with $T$. velutinum versus their levels of expression in control plants. The data were analyzed as indicated in the legend to the Figure 4.

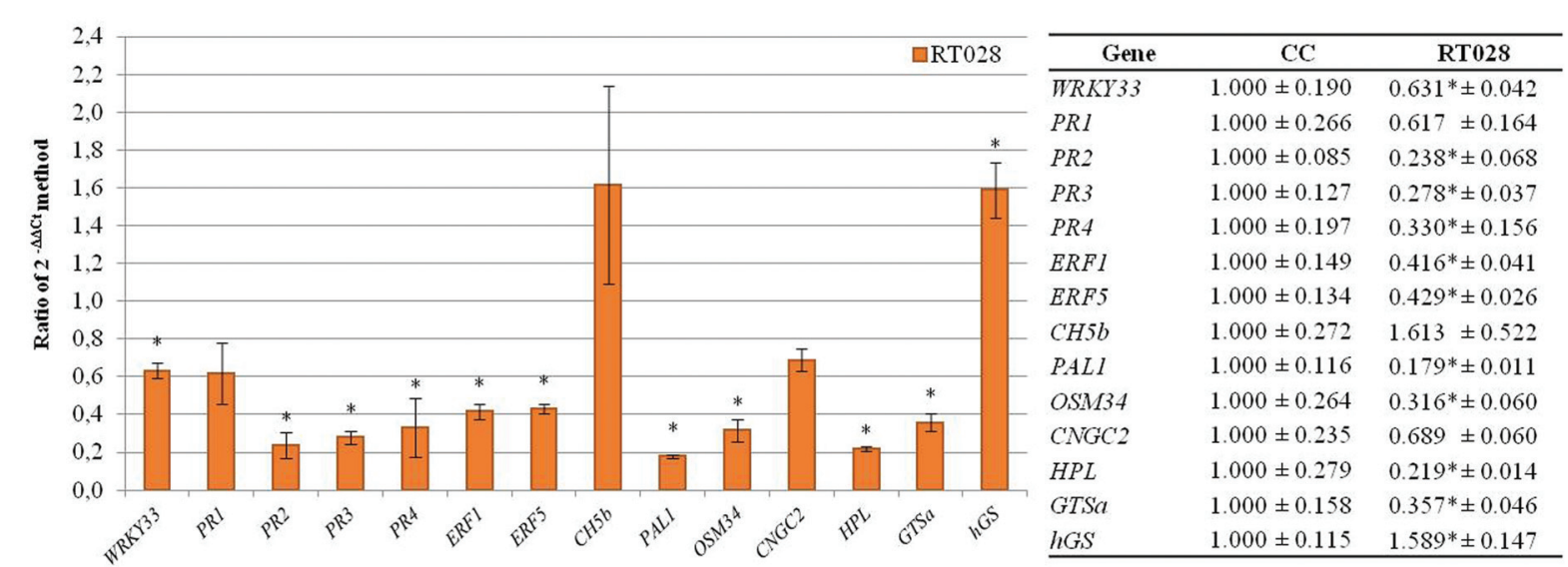

FIGURE 6 | Analysis of relative expression levels of the bean defense genes selected in the present work in bean plants infected with $R$. solani and treated with $\boldsymbol{T}$. velutinum versus their levels of expression in control plants. The data were analyzed as indicated in the legend to the Figure 4. 
in plants treated with T. velutinum. Then, the pathogen would induce an activation of hypersensitive defense mechanisms.

Hydroperoxide lyase $(H P L)$ is involved in the production of antimicrobial and defense signaling oxylipins (Noordermeer et al., 2001; Huang et al., 2010). In this study, the presence of T. velutinum and $R$. solani, resulted in a down-regulation of this gene expression respect to control plants. In previous works, when tomato plants were in contact with Botrytis cinerea, HPL expression increased $24 \mathrm{~h}$ after gray mold infection, but after that time the expression of this gene decreased gradually (Wan et al., 2013). In the present case, after 45 days in contact with the fungus T. velutinum and/or $R$. solani, its expression was down-regulated, indicating that the plant identifies Trichoderma and Rhizoctonia as two invader organisms, and some of the mechanisms activated against the presence of both are similar, independently of the final response specifically activated in the plant by each one.

GSTa (2,4-D inducible glutathione S-transferase) expression also responds to pathogen attack (Mauch and Dudler, 1993) and can be induced by molecules such as salicylic acid, methyl jasmonate, abscisic acid and $\mathrm{H}_{2} \mathrm{O}_{2}$ (Dixon et al., 2002; Moons, 2005). In Gossypium arboretum, GST provides resistance to fungal pathogens and oxidative stress (Barthelson et al., 2010). GST expression was up-regulated during fungal infection in barley, Arabidopsis, and cotton (Dowd et al., 2004; Durrant and Dong, 2004; Lu et al., 2005). However, in banana GST was down-regulated following Fusarium oxysporum f specialis (f. sp.) cubense infection (Wang et al., 2013), which is in agreement with the present case, where the expression of GSTa was downregulated when T. velutinum and/or R. solani were present in the soil.

$h G S$ encodes a homoglutathione synthetase that is involved in response to oxidative stress. There is not much information about the behavior of this gene in the plant. In the present study, when bean plants were in contact with $T$. velutinum and/or $R$. solani, expression of this gene was significantly up-regulated compared to control plants. In other studies, treatment of Medicago truncatula plants with compounds that release nitric oxide, a key signaling molecule in plants, induced expression of GST but not hGS in roots (Innocenti et al., 2007). Similarly, common bean plants treated with $\mathrm{H}_{2} \mathrm{O}_{2}$ showed upregulation of $h G S$ in nodules, whereas treatments with cadmium, sodium chloride, or jasmonic acid had no effect (Loscos et al., 2008).

\section{CONCLUSION}

From 48 genes initially analyzed, 14 bean genes were selected in the present work and only WRKY33, CH5b and $h G S$ showed

\section{REFERENCES}

Atkinson, M. M., Midland, S. L., Sims, J. J., and Keen, N. T. (1996). Syringolide 1 triggers $\mathrm{Ca} 2$ influx, $\mathrm{K}$ efflux, and extracellular alkalization in soybean cells carrying the disease-resistance gene Rpg4. Plant Physiol. 112, 297-302. doi: 10.1104/pp.112.1.297 an up-regulatory response in the presence of T. velutinum, the other genes were or not affected (OSM34) or down-regulated by the presence of this fungus. $R$. solani infection resulted in a down-regulation of most of the genes analyzed, except PR1, OSM34 and CNGC2 that were not affected, and the presence of both, T. velutinum and R. solani, up-regulates $h G S$ and downregulates all the other genes analyzed, except $C H 5 b$ which was not significantly affected.

As conclusion, the strategy described in the present work has been shown to be effective to detect genes involved in plant defense, which respond to the presence of a BCA or to a pathogen and also to the presence of both. The selected genes showed significant homology with described plant defense genes and they are expressed in bean leaves of plants treated with T. velutinum and/or infected with $R$. solani. The proposed strategy will be very useful in studies about the interaction of bean with pathogens and biocontrol fungi.

\section{AUTHOR CONTRIBUTIONS}

PC and SG conceived the research. SM, OG-L, and AR-G designed the research. SM, OG-L, and AR-G conducted the experiments. SM, PC, and SG analyzed data. SM, PC, SG, EC, and FS interpreted the data. SM, PC, SG, EC, and FS wrote the manuscript. All authors were agreed to be accountable for all aspects of the work in ensuring that questions related to the accuracy or integrity of any part of the work are appropriately investigated and resolved. All authors critically revised the manuscript. All authors approved the final version to be published.

\section{ACKNOWLEDGMENTS}

The grant awarded to Sara Mayo Prieto (FPU12/00677) by the Ministry of Education, Culture and Sport (Spain) according the Resolution of April 25, 2012 (BOE of 10 May), as amended by Resolution of May 18, 2012 (BOE of 22 May), and by Resolution of October 31, 2012 (BOE of 12 November) and granted in the Resolution of December 20, 2012. Ministry of Economy and Competitiveness for National project "Farnesol as self-regulatory molecule in Trichoderma. Tyrosol and farnesol signaling in Trichoderma-bean interaction" (AGL2012-40041-C02-02). Junta de Castilla y León, Consejería de Educación for the project "Effect of terpenes and physiologically related compounds produced by Trichoderma parareesei in the development of common bean (Phaseolus vulgaris L.) and in defensive responses of this plant" (LE228U14).

Bakshi, M., and Oelmüller, R. (2014). WRKY transcription factors. Plant Signal. Behav. 9:e27700. doi: 10.4161/psb.27700

Barthelson, R. A., Qaisar, U., and Galbraith, D. W. (2010). Functional analysis of the Gossypium arboreum genome. Plant Mol. Biol. Rep. 28, 334-343. doi: 10.1007/s11105-009-01 57-5 
Birkenbihl, R. P., Diezel, C., and Somssich, I. E. (2012). Arabidopsis WRKY33 is a key transcriptional regulator of hormonal and metabolic responses toward Botrytis cinerea infection. Plant Physiol. 159, 266-285. doi: 10.1104/pp.111.192641

Borges, A., Melotto, M., Tsai, S. M., and Caldas, D. G. G. (2012). Changes in spatial and temporal gene expression during incompatible interaction between common bean and Anthracnose pathogen. J. Plant Physiol. 169, 1216-1220. doi: 10.1016/j.jplph.2012.04.003

Broglie, K., Chet, I., Holliday, M., Cressman, R., Biddle, P., Knowlton, S., et al. (1991). Transgenic plants with enhanced resistance to the fungal pathogen Rhizoctonia solani. Science 254, 1194-1197. doi: 10.1126/science.254.5035. 1194

Chaman, M. E., Copaja, S. V., and Argandoña, V. H. (2003). Relationships between salicylic acid content, Phenylalanine Ammonia-Lyase (PAL) activity, and resistance of barley to aphid infestation. J. Agric. Food Chem. 51, 2227-2231. doi: $10.1021 / \mathrm{jf} 020953 \mathrm{~b}$

Chen, W., Provart, N. J., Glazebrook, J., Katagiri, F., Chang, H. S., Eulgem, T., et al. (2002). Expression profile matrix of Arabidopsis transcription factor genes suggests their putative functions in response to environmental stresses. Plant Cell 14, 559-574. doi: 10.1105/tpc.010410

Dixon, D. P., Lapthorn, A., and Edwards, R. (2002). Plant glutathione transferases. Genome Biol. 3, 3004.1-3004.10. doi: 10.1186/gb-2002-3-3-reviews3004

Dowd, C., Wilson, I. W., and McFadden, H. (2004). Gene expression profile changes in cotton root and hypocotyl tissues in response to infection with Fusarium oxysporum f. sp. vasinfectum. Mol. Plant-Microbe Interact. 17, 654667. doi: 10.1094/MPMI.2004.17.6.654

Druzhinina, I. S., Seidl-Seiboth, V., Herrera-Estrella, A., Horwitz, B. A., Kenerley, C. M., Monte, E., et al. (2011). Trichoderma: the genomics of opportunistic success. Nat. Rev. Microbiol. 9, 749-759. doi: 10.1038/nrmicro2637

Durrant, W. E., and Dong, X. (2004). Systemic acquired resistance. Annu. Rev. Phytopathol. 42, 185-209. doi: 10.1146/annurev.phyto.42.040803.140421

Gallou, A., Cranenbrouck, S., and Declerck, S. (2009). Trichoderma harzianum elicits defence response genes in roots of potato plantlets challenged by Rhizoctonia solani. Eur. J. Plant Pathol. 124, 219-230. doi: 10.1007/s10658-0089407-x

Guerrero-González, M. L., Rodríguez-Kessler, M., Rodríguez-Guerra, R., González-Chavira, M., Simpson, J., Sanchez, F., et al. (2011). Differential expression of Phaseolus vulgaris genes induced during the interaction with Rhizoctonia solani. Plant Cell Rep. 30, 1465-1473. doi: 10.1007/s00299-011-1055-5

Hermosa, R., Viterbo, A., Chet, I., and Monte, E. (2012). Plant-beneficial effects of Trichoderma and of its genes. Microbiology 158, 17-25. doi: 10.1099/mic.0.052274-0

Huang, F. C., Studart Witkowski, C., and Schwab, W. (2010). Overexpression of hydroperoxide lyase gene in Nicotiana benthamiana using a viral vector system. Plant Biotechnol. J. 8, 783-795. doi: 10.1111/j.1467-7652.2010.00508.x

Innocenti, G., Pucciariello, C., Le Gleuher, M., Hopkins, J., de Stefano, M., Delledonne, M., et al. (2007). Glutathione synthesis is regulated by nitric oxide in Medicago truncatula roots. Planta 225, 1597-1602. doi: 10.1007/s00425-0060461-3

Kim, D. S., and Hwang, B. K. (2014). An important role of the pepper phenylalanine ammonia-lyase gene (PAL1) in salicylic acid-dependent signalling of the defence response to microbial pathogens. J. Exp. Bot. 65, 2295-2306. doi: $10.1093 /$ jxb/eru109

Kim, K. C., Fan, B., and Chen, Z. (2006). Pathogen-induced Arabidopsis WRKY7 is a transcriptional repressor and enhances plant susceptibility to Pseudomonas syringae. Plant Physiol. 142, 1180-1192. doi: 10.1104/pp.106.082487

Lehtonen, M. J., Somervuo, P., and Valkonen, J. P. T. (2008). Infection with Rhizoctonia solani induces defense genes and systemic resistance in potato sprouts grown without light. Phytopathology 98, 1190-1198. doi: 10.1094/PHYTO-98-11-1190

Lippok, B., Birkenbihl, R. P., Rivory, G., Brümmer, J., Schmelzer, E., Logemann, E., et al. (2007). Expression of AtWRKY33 encoding a pathogen-or PAMPresponsive WRKY transcription factor is regulated by a composite DNA motif containing W box elements. Mol. Plant-Microbe Interact. 20, 420-429. doi: 10.1094/MPMI-20-4-0420

Lodha, T. D., and Basak, J. (2012). Plant-pathogen interactions: what microarray tells about it? Mol. Biotechnol. 50, 87-97. doi: 10.1007/s12033-011-9418-2
Lorenzo, O., Piqueras, R., Sánchez-Serrano, J. J., and Solano, R. (2003). Ethylene response factor 1 integrates signals from ethylene and jasmonate pathways in plant defense. Plant Cell 15, 165-178. doi: 10.1105/tpc.007468

Loscos, J., Matamoros, M. A., and Becana, M. (2008). Ascorbate and homoglutathione metabolism in common bean nodules under stress conditions and during natural senescence. Plant Physiol. 146, 1282-1292. doi: $10.1104 /$ pp.107.114066

Lu, Z.-X., Gaudet, D. A., Frick, M., Puchalski, B., Genswein, B., and Laroche, A. (2005). Identification and characterization of genes differentially expressed in the resistance reaction in wheat infected with Tilletia tritici, the common bunt pathogen. BMB Rep. 38, 420-431. doi: 10.5483/BMBRep.2005.38.4.420

Mauch, F., and Dudler, R. (1993). Differential induction of distinct glutathione-Stransferases of wheat by xenobiotics and by pathogen attack. Plant Physiol. 102, 1193-1201. doi: 10.1104/pp.102.4.1193

Mayo, S., Gutiérrez, S., Malmierca, M. G., Lorenzana, A., Campelo, M. P., Hermosa, R., et al. (2015). Influence of Rhizoctonia solani and Trichoderma spp. in growth of bean (Phaseolus vulgaris L.) and in the induction of plant defense-related genes. Front. Plant Sci. 6:685. doi: 10.3389/fpls.2015. 00685

Moffat, C. S., Ingle, R. A., Wathugala, D. L., Saunders, N. J., Knight, H., and Knight, M. R. (2012). ERF5 and ERF6 play redundant roles as positive regulators of JA/Et-mediated defense against Botrytis cinerea in Arabidopsis. PLoS ONE 7:e35995. doi: 10.1371/journal.pone.0035995

Moons, A. (2005). Regulatory and functional interactions of plant growth regulators and plant glutathione S-transferases (GSTs). Vitam. Horm. 72, 155202. doi: 10.1016/S0083-6729(05)72005-7

Narasimhan, M. L., Bressan, R. A., D’Urzo, M. P., Jenks, M. A., and Mengiste, T. (2009). Unexpected turns and twists in structure/function of PR-proteins that connect energy metabolism and immunity. Adv. Bot. Res. 51, 439-489. doi: 10.1016/S0065-2296(09)51011-7

Noordermeer, M. A., Veldink, G. A., and Vliegenthart, J. F. G. (2001). Fatty acid hydroperoxide lyase: a plant cytochrome P450 enzyme involved in wound healing and pest resistance. Chembiochem 2, 494-504. doi: 10.1002/14397633(20010803)2:7/8<494::AID-CBIC494>3.0.CO;2-1

Nugroho, L. H., Verberne, M. C., and Verpoorte, R. (2002). Activities of enzymes involved in the phenylpropanoid pathway in constitutively salicylic acid-producing tobacco plants. Plant Physiol. Biochem. 40, 755-760. doi: 10.1016/S0981-9428(02)01437-7

Pandey, S. P., and Somssich, I. E. (2009). The role of WRKY transcription factors in plant immunity. Plant Physiol. 150, 1648-1655. doi: 10.1104/pp.109.138990

Pereira, J. L., Queiroz, R. M. L., Charneau, S. O., Felix, C. R., Ricart, C. A. O., Lopes Da Silva, F., et al. (2014). Analysis of Phaseolus vulgaris response to its association with Trichoderma harzianum (ALL-42) in the presence or absence of the phytopathogenic fungi Rhizoctonia solani and Fusarium solani. PLoS ONE 9:e98234. doi: 10.1371/journal.pone.0098234

Pfaffl, M. W. (2001). A new mathematical model for relative quantification in real-time RT-PCR. Nucleic Acids Res. 29, e45-e45. doi: 10.1093/nar/29.9.e45

Reid, K. E., Olsson, N., Schlosser, J., Peng, F., and Lund, S. T. (2006). An optimized grapevine RNA isolation procedure and statistical determination of reference genes for real-time RT-PCR during berry development. BMC Plant Biol. 6:27. doi: 10.1186/1471-2229-6-27

Rushton, P. J., and Somssich, I. E. (1998). Transcriptional control of plant genes responsive to pathogens. Curr. Opin. Plant Biol. 1, 311-315. doi: 10.1016/13695266(88)80052-9

Rutledge, R. G., and Stewart, D. (2008). Critical evaluation of methods used to determine amplification efficiency refutes the exponential character of real-time PCR. BMC Mol. Biol. 9:96. doi: 10.1186/1471-2199-9-96

Sharma, S., Lin, W., Villamor, J. G., and Verslues, P. E. (2013). Divergent low water potential response in Arabidopsis thaliana accessions Landsberg erecta and Shahdara. Plant. Cell Environ. 36, 994-1008. doi: 10.1111/pce.12032

Shoresh, M., Harman, G. E., and Mastouri, F. (2010). Induced systemic resistance and plant responses to fungal biocontrol agents. Annu. Rev. Phytopathol. 48, 21-43. doi: 10.1146/annurev-phyto-073009-114450

Singh, K. B., Foley, R. C., and Oñate-Sánchez, L. (2002). Transcription factors in plant defense and stress responses. Curr. Opin. Plant Biol. 5, 430-436. doi: 10.1016/S1369-5266(02)00289-3

Son, G. H., Wan, J., Kim, H. J., Nguyen, X. C., Chung, W. S., Hong, J. C., et al. (2012). Ethylene-responsive element-binding factor 5, ERF5, is involved 
in chitin-induced innate immunity response. Mol. Plant-Microbe Interact. 25, 48-60. doi: 10.1094/MPMI-06-11-0165

Upchurch, R. G., and Ramirez, M. E. (2010). Defense-related gene expression in soybean leaves and seeds inoculated with Cercospora kikuchii and Diaporthe phaseolorum var. meridionalis. Physiol. Mol. Plant Pathol. 75, 64-70. doi: 10.1016/j.pmpp.2010.08.007

Valenciano, J. B., Casquero, P. A., Boto, J. A., and Marcelo, V. (2006). Evaluation of the occurrence of root rots on bean plants (Phaseolus vulgaris) using different sowing methods and with different techniques of pesticide application. New Zeal. J. Crop Hortic. Sci. 34, 291-298. doi: 10.1080/01140671.2006. 9514419

Vellicce, G. R., Ricci, J. C. D., Hernández, L., and Castagnaro, A. P. (2006). Enhanced resistance to Botrytis cinerea mediated by the transgenic expression of the chitinase gene CH5b in strawberry. Transgenic Res. 15, 57-68. doi: 10.1007/s11248-005-2543-6

Verhagen, B. W. M., Van Loon, L. C., and Pieterse, C. M. J. (2006). "Induced disease resistance signaling in plants," in Floriculture, Ornamental and Plant Biotechnology, ed. J. A. Teixeira da Silva (Utrecht: Global Science Books), 334-343.

Wan, X.-H., Chen, S.-X., Wang, C.-Y., Zhang, R.-R., Cheng, S.-Q., Meng, H.-W., et al. (2013). Isolation, expression, and characterization of a hydroperoxide lyase gene from cucumber. Int. J. Mol. Sci. 14, 22082-22101. doi: 10.3390/ijms141122082

Wang, Z., Huang, S., Jia, C., Liu, J., Zhang, J., Xu, B., et al. (2013). Molecular cloning and expression of five glutathione S-transferase (GST) genes from Banana (Musa acuminata L. AAA group, cv. cavendish). Plant Cell Rep. 32, 1373-1380. doi: 10.1007/s00299-013-1449-7

Conflict of Interest Statement: The authors declare that the research was conducted in the absence of any commercial or financial relationships that could be construed as a potential conflict of interest.

The reviewer EVG declared a past co-authorship with one of the authors SG to the handling Editor, who ensured that the process met the standards of a fair and objective review.

Copyright (C) 2016 Mayo, Cominelli, Sparvoli, González-López, Rodríguez-González, Gutiérrez and Casquero. This is an open-access article distributed under the terms of the Creative Commons Attribution License (CC BY). The use, distribution or reproduction in other forums is permitted, provided the original author(s) or licensor are credited and that the original publication in this journal is cited, in accordance with accepted academic practice. No use, distribution or reproduction is permitted which does not comply with these terms. 


\section{OPEN ACCESS}

Edited by:

Fred Stoddard,

University of Helsinki, Finland

Reviewed by:

Johann Vollmann,

University of Natural Resources and

Life Sciences, Vienna, Austria

Elisa Bellucci,

Marche Polytechnic University, Italy

Robert John French,

Department of Agriculture and Food,

Australia

*Correspondence:

Antonio M. De Ron

amderon@mbg.csic.es

Specialty section:

This article was submitted to Crop Science and Horticulture,

a section of the journal

Frontiers in Plant Science

Received: 26 April 2016

Accepted: 11 July 2016

Published: 02 August 2016

Citation:

De Ron AM, Rodiño AP, Santalla M,

González AM, Lema MJ, Martín I and

Kigel J (2016) Seedling Emergence and Phenotypic Response of Common Bean Germplasm to Different Temperatures under

Controlled Conditions and in Open Field. Front. Plant Sci. 7:1087. doi: 10.3389/fpls.2016.01087

\section{Seedling Emergence and Phenotypic Response of Common Bean Germplasm to Different Temperatures under Controlled Conditions and in Open Field}

\author{
Antonio M. De Ron ${ }^{1,2 *}$, Ana P. Rodiño ${ }^{1}$, Marta Santalla ${ }^{1}$, Ana M. González ${ }^{1}$, \\ María J. Lema ${ }^{2,3}$, Isaura Martín ${ }^{4}$ and Jaime Kigel ${ }^{5}$ \\ ${ }^{1}$ Biology of Agrosystems, Misión Biológica de Galicia, National Spanish Research Council, Pontevedra, Spain, ${ }^{2}$ Sistemas \\ Agroforestales, Unidad Asociada a la Misión Biológica de Galicia (CSIC), Pontevedra, Spain, ${ }^{3}$ Phytopathological Station do \\ Areeiro, Provincial Chamber, Pontevedra, Spain, ${ }^{4}$ National Center for Plant Genetic Resources, National Institute for \\ Agricultural and Food Research and Technology, Alcalá de Henares, Spain, ${ }^{5}$ The Robert H. Smith Faculty of Agriculture, \\ Food and Environment, Hebrew University of Jerusalem, Rehovot, Israel
}

Rapid and uniform seed germination and seedling emergence under diverse environmental conditions is a desirable characteristic for crops. Common bean genotypes (Phaseolus vulgaris L.) differ in their low temperature tolerance regarding growth and yield. Cultivars tolerant to low temperature during the germination and emergence stages and carriers of the grain quality standards demanded by consumers are needed for the success of the bean crop. The objectives of this study were (i) to screen the seedling emergence and the phenotypic response of bean germplasm under a range of temperatures in controlled chamber and field conditions to display stress-tolerant genotypes with good agronomic performances and yield potential, and (ii) to compare the emergence of bean seedlings under controlled environment and in open field conditions to assess the efficiency of genebanks standard germination tests for predicting the performance of the seeds in the field. Three trials were conducted with 28 dry bean genotypes in open field and in growth chamber under low, moderate, and warm temperature. Morpho-agronomic data were used to evaluate the phenotypic performance of the different genotypes. Cool temperatures resulted in a reduction of the rate of emergence in the bean genotypes, however, emergence and early growth of bean could be under different genetic control and these processes need further research to be suitably modeled. Nine groups arose from the Principal Component Analysis (PCA) representing variation in emergence time and proportion of emergence in the controlled chamber and in the open field indicating a trend to lower emergence in large and extra-large seeded genotypes. Screening of seedling emergence and phenotypic response of the bean germplasm under a range of temperatures in controlled growth chambers and under field conditions showed several genotypes, as landraces 272, 501, 593, and the cultivar Borlotto, with stress-tolerance at emergence, and high yield potential that could be valuable genetic material for breeding programs. Additionally, the 
potential genetic erosion in genebanks was assessed. Regarding bean commercial traits, under low temperature at sowing time seed reached larger size, and crop yield was higher compared to warmer temperatures at the sowing time. Therefore, early sowing of bean is strongly recommended.

Keywords: low temperature tolerance, Phaseolus vulgaris L., plant breeding, plant genetic resources, seedling emergence, yield

\section{INTRODUCTION}

The common bean (Phaseolus vulgaris L.) is native to the Americas where two major domestication centers and gene pools have been described, Andean and Mesoamerican, which differ in their adaptation to different climatic and eco-geographic conditions. Differences in the seed type and size are clear between both genetic pools (Singh et al., 1991; Santalla et al., 2001), having the Andean varieties larger seeds than the Mesoamerican ones. The diffusion of the common bean out of its American domestication centers appears to have been very complex, involving numerous introductions into different continents along a range of agrosystems. Several geographic regions have been proposed as secondary centers of diversification, such as Europe (Santalla et al., 2002; Angioi et al., 2010; Gioia et al., 2013), central-eastern and southern Africa, Brazil, and China (Bellucci et al., 2014). However, once out of the Americas, the spatial isolation between the Mesoamerican and Andean gene pools was not maintained, thus providing increased potential for their hybridization, and introgression. In Europe, this issue is highly relevant for breeding programs. Indeed, their hybridization has led to the recombination of the Mesoamerican and Andean traits resulting in novel and useful genotypes and phenotypes adapted to contrasting environmental conditions (i.e., resistance to biotic and abiotic stress; Rodiño et al., 2006; Angioi et al., 2010; Blair et al., 2010; Santalla et al., 2010). In contrast, various studies suggest that in other regions the introgression between these gene pools appears to be less relevant than in Europe (De Ron et al., 2015).

Early breeding efforts primarily focused on improved disease resistance and adaptation to local environments, with later efforts focused on improved seed quality, improved plant architecture, and breeding for yield (Duc et al., 2015). Yield per-se, tolerance to drought, adaptation to poor soils, and nutritional quality are priorities of bean breeders since the 1990s (De Ron et al., 2015). Seed germination and seedling emergence in the small seeded Mesoamerican genotypes is generally faster than that in the Andean ones, and this phenotypic trait has been used to distinguish between the two genetic pools (White and Montes, 1993). Faster emergence may reflect both genetic variation for adaptation to specific environments and effects of seed size in emergence. Seed size has been recognized as a factor affecting bean germination (Hanley et al., 2003; Kaya et al., 2008) and is probably related to water uptake, a key process in seedling emergence (Bewley, 1997). High seed vigor, good germination, and emergence are prerequisites for successful direct sowing in common bean. Thus, a better understanding of the genetics of the processes regulating germination and early growth under different conditions is important not only as a contribution to the knowledge of this species, but also has direct applications in plant breeding, and for germplasm conservation and regeneration.

The bean crop experienced a quick adaptive radiation throughout Europe in the Sixteenth Century (Zeven, 1997), where it was distributed through very different edapho-climatic environments. The microclimate of the cultivated areas, located at different latitudes, and altitudes, could have a strong influence on the recent evolution of this crop (Escribano et al., 1994; Santalla et al., 2002, 2010; Casquero et al., 2006; González et al., 2006; Papa et al., 2006; Rodiño et al., 2006). Several studies showed that a number of varieties with relevance for niche markets still survive on-farm in marginal areas of European countries (Zeven et al., 1999; Eichenberger et al., 2000; Rodiño et al., 2001, 2003, 2009; Negri and Tosti, 2002; Sicard et al., 2005) and in their areas or origin (De Ron et al., 2004; Galván et al., 2006). Common bean is adapted to relatively humid and cool climatic conditions with optimal average daily temperature for reproductive development ranging from 20 to $25^{\circ} \mathrm{C}$ (Wantanbe, 1953). Temperatures $>30^{\circ} \mathrm{C}$ during the day or $>20^{\circ} \mathrm{C}$ at night result in yield reduction (Hardwick and Andrews, 1980; Rainey and Griffiths, 2005), and seeds germinate poorly below $15^{\circ} \mathrm{C}$ (Kotowski, 1926; Kigel et al., 2015). Thus, it is necessary to restrict field sowing of beans to warm climates or to delay sowing until the soil is warm enough for satisfactory emergence in cool climates (Hardwick, 1972). Moreover, beans that are slow to germinate are also likely to be slow in growth (Kooistra, 1971). The physiological response of common bean to high temperature stress has been primarily studied through the use of controlled environmental testing in greenhouses and growth chambers (Porch, 2006). However, the long-term goal of breeding for stress tolerance is the development of germplasm with improved field adaptation to different temperatures. Therefore, in order to make maximum use of the available growing period, genotypes must be developed that are tolerant to low temperature during germination and early growth.

Seed germination is the process that commences with uptake of water by the dry seed-i.e., imbibition, and terminates with emergence of the seedling. Thus, the process involves two temporal stages, namely the germination stage and the emergence, or seedling-growth stage (Bewley and Black, 1985; Bewley, 1997). The emergence of the radicle marks the end of the first stage and the onset of the second. The sooner the radicle protrudes through the seed coat, the faster is the emergence. Fast seed germination is considered an important adaptive trait marking a quick transition to the growth phase in the life-cycle of a plant. The time taken for the germination 
process to be completed is one of the important parameters of seed quality (Copeland and McDonald, 1995; Dutt and Geneve, 2007). Vigorous, rapid, as well as uniform germination and emergence under diverse environmental conditions is a desirable attribute for seedling growth and, ultimately, grain yield in food legumes and cereal crops such as bean, rice, wheat, maize. Crop species vary widely in how fast their seeds germinate, the rate of emergence being the result of the interaction between the seed genotype and specific environmental, or ecological factors (Hernández-Nistal et al., 1989; Alonso-Blanco et al., 2003; Schmuths et al., 2006).

In past years, substantial progress was achieved by plant breeders in adapting crops such as maize, tomato, soybean, and common bean to suboptimal temperatures (Dickson, 1971; Holmberg, 1973; Skrdla and Mock, 1978; Patterson and Payne, 1983). Ideally, the best cold-tolerant genotypes should have successful water imbibition, germination, and emergence at low temperature (Kemp, 1978; Garcia-Huidobro et al., 1982; Dickson and Boettger, 1984a,b; Gummerson, 1986). The interval from seedling to maximum growth and blooming of bean plants should be shortened by selecting lines capable of rapid early growth during periods of low temperature following sowing.

The cultivation of dry bean in South Europe has traditionally taken place with sowings form April-June. But summer cultivation increases production costs by demanding greater irrigation and more tillage because of weed proliferation. Moreover, summer cultivation increases the likelihood of harvest coinciding with the onset of the rainy season, leading to crop failure, and yield losses. Cultivation in the spring period, however, is restricted because low temperatures at sowing delay germination, seedling emergence, and early growth. The alternative, therefore, is to use genotypes tolerant to low temperature at the germination, emergence, and early growth stages. Yet, little research has been done to breed this type of dry bean genotypes. Thus, further identification of cold tolerant genotypes already reported in a few instances (Dickson, 1971; Kooistra, 1971; Bannerot, 1979; Hardwick and Andrews, 1980; Dickson and Boettger, 1984a,b; Scully and Waines, 1987) is necessary.

The purpose of germination testing in genebanks and breeder collections is to provide information on the comparative and foreseeable field planting value of different seed samples. In the case that field testing seed emergence, which can be affected by the field conditions (Ellis et al., 1985), failed more than expected according the tests of germination, there may be a loss of genetic material of gene bank accessions that will imply a process of genetic erosion.

The objectives of this study were (i) to screen the seedling emergence and the phenotypic response of bean germplasm under a range of temperatures in controlled chamber and field conditions to display stress-tolerant genotypes with good agronomic performances and yield potential, and (ii) to compare the emergence of bean seedlings under controlled environment and in open field conditions to assess the efficiency of genebanks standard germination tests for predicting the performance of the seeds in the field.

\section{MATERIALS AND METHODS}

\section{Plant Material}

Twenty-eight accessions were used in this study-21 landraces, five breeding lines, and two cultivars. The seeds were maintained in the Misión Biológica de Galicia-Spanish National Research Council (MBG-CSIC, Pontevedra, Spain) germplasm facilities at $4^{\circ} \mathrm{C}$ and $40 \% \mathrm{RH}$ (relative humidity; Table 1 ).

\section{Controlled Climatic Chamber Experiments}

The experiments were carried out at the MBG-CSIC using a climatic chamber set at different day-lengths and temperatures that represent conditions of North-western Spain: $\mathrm{tc}_{1}: 14 / 10 \mathrm{~h}$ (day/night) at $14 / 10^{\circ} \mathrm{C}$ and $60 / 80 \% \mathrm{RH}$ representing field conditions in April (weighted average: $11.5^{\circ} \mathrm{C}, 68.3 \% \mathrm{RH}$ ); $\mathrm{tc}_{2}$ : $15 / 9 \mathrm{~h}$ at $17 / 12^{\circ} \mathrm{C}$ and $60 / 80 \% \mathrm{RH}$ representing conditions in May (weighted average: $15.1^{\circ} \mathrm{C}, 67.5 \% \mathrm{RH}$ ); and $\mathrm{tc}_{3}: 16 / 8 \mathrm{~h}$ at $22 / 15^{\circ} \mathrm{C}$ and $60 / 80 \% \mathrm{RH}$ representing conditions in June (weighted average: $19.7^{\circ} \mathrm{C}, 66.7 \% \mathrm{RH}$ ). Light was provided by seven very high output (VHO) fluorescent lamps with a photosynthetic photon flux (PPF) of $228 \mu \mathrm{mol} \mathrm{m}^{-2} \mathrm{~s}^{-1}$.

A randomized complete block design with three replications was used. Ten seeds of each genotype were sown in sterile peat in plastic containers $(30 \times 20 \times 12 \mathrm{~cm})$, with a plant to plant (seed to seed) distance of $2.5 \mathrm{~cm}$, and row to row distance of $5 \mathrm{~cm}$. Monitoring of emergence was carried out during ca. 30 days, starting 4 days after the beginning of the experiments. Seedlings with a hypocotyl-radicle axis $>3 \mathrm{~cm}$ were considered as emerged. Proportion of emergence (\% of sown seeds) and time to emergence (days from sowing to seedling emergence of all seeds sown) were measured.

\section{Field Experiments}

The open field experiments were conducted in the experimental farm of the MBG-CSIC at Pontevedra, Spain $\left(42^{\circ} 24^{\prime} \mathrm{N}\right.$ latitude, $8^{\circ} 38^{\prime} \mathrm{W}$ longitude, 40 masl $14^{\circ} \mathrm{C}$ average annual temperature, and $1600 \mathrm{~mm}$ annual rainfall). The soil, developed from granitic rocks, has a sandy loam texture, and a granular structure. It is classified as Humic Cambisol according to FAO criteria (FAOISRIC-ISSS, 1998).

To test temperature effects in the field, seeds were sown by hand at: (a) low temperature conditions $\left(\mathrm{tf}_{1}\right)$ on 10 April 2007; (b) moderate temperature conditions $\left(\mathrm{tf}_{2}\right)$ on 4 May 2007; and c) warm temperature conditions $\left(\mathrm{tf}_{3}\right)$ on 7 June 2007 (Table S1). Field trials were arranged according to a completely randomized block design with 30 plants per sowing treatment and two replications (crop density of 50000 plant ha $^{-1}$ ). Single row plots were $3.8 \mathrm{~m}$ in length and $0.8 \mathrm{~m}$ spaced. Plants were watered as needed, using drip irrigation. The following traits were measured: Emergence time (days) and emergence proportion (\%); expansion of the first trifoliate leaf (days); plant height at 10 and 20 days from emergence (centimeters); early plant vigor (from 1 to 9 scale); days from sowing to first open flower, beginning of flowering (50\% of plants with at least an open flower), end of flowering (days), and physiological mature pod (days); dry seed weight (g 100 seed $^{-1}$ ) and seed dimensions (millimeters). Seeds per pod and pods per plant were determined 
TABLE 1 | Origin, seed size, genetic pool, market class, and seed age of the common bean genotypes studied.

\begin{tabular}{|c|c|c|c|c|c|}
\hline Genotype $^{a}$ & Geographical origin & Seed size (g 100 seed $\left.^{-1}\right)$ & Genetic pool & Market class ${ }^{\mathbf{b}}$ & Seed age (years) \\
\hline \multicolumn{6}{|c|}{ LANDRACES } \\
\hline 200 & $43^{\circ} 07^{\prime} \mathrm{N}, 8^{\circ} 55^{\prime} \mathrm{W}, 247$ masl & 57 & Andean & White kidney & 1 \\
\hline 272 & $42^{\circ} 17^{\prime} \mathrm{N}, 8^{\circ} 12^{\prime} \mathrm{W}, 380$ masl & 36 & Andean & Purple caparron & 4 \\
\hline 391 & $42^{\circ} 13^{\prime} \mathrm{N}, 8^{\circ} 16^{\prime} \mathrm{W}, 545$ masl & 61 & Andean & Red pinto & 2 \\
\hline 399 & $43^{\circ} 29^{\prime} \mathrm{N}, 6^{\circ} 05^{\prime} \mathrm{W}, 37$ masl & 70 & Mesoamerican & Large great northern & 2 \\
\hline 413 & $43^{\circ} 26^{\prime} \mathrm{N}, 6^{\circ} 06^{\prime} \mathrm{W}, 420$ masl & 79 & Andean & Favada pinto & 4 \\
\hline 419 & $42^{\circ} 34^{\prime} \mathrm{N}, 8^{\circ} 53^{\prime} \mathrm{W}, 6$ masl & 73 & Mesoamerican & Large great northern & 1 \\
\hline 452 & $43^{\circ} 31^{\prime} \mathrm{N}, 7^{\circ} 06^{\prime} \mathrm{W}, 298$ masl & 79 & Andean & Favada & 1 \\
\hline 489 & $42^{\circ} 39^{\prime} \mathrm{N}, 2^{\circ} 32^{\prime} \mathrm{W}, 723$ masl & 40 & Andean & Guernikesa & 4 \\
\hline 501 & $43^{\circ} 14^{\prime} \mathrm{N}, 2^{\circ} 08^{\prime} \mathrm{W}, 312$ masl & 43 & Andean & Negro brillante & 2 \\
\hline 573 & $40^{\circ} 30^{\prime} \mathrm{N}, 5^{\circ} 45^{\prime} \mathrm{W}, 959$ masl & 35 & Mesoamerican & Common pinto & 1 \\
\hline 587 & $41^{\circ} 13^{\prime} \mathrm{N}, 5^{\circ} 29^{\prime} \mathrm{W}, 810$ masl & 37 & Mesoamerican & Great northern & 1 \\
\hline 593 & $41^{\circ} 23^{\prime} \mathrm{N}, 2^{\circ} 10^{\prime} \mathrm{E}, 8 \mathrm{masl}$ & 30 & Mesoamerican & Hook & 1 \\
\hline 623 & $41^{\circ} 23^{\prime} \mathrm{N}, 2^{\circ} 10^{\prime} \mathrm{E}, 8$ masl & 33 & Mesoamerican & Hook & 1 \\
\hline 837 & $43^{\circ} 11^{\prime} \mathrm{N}, 8^{\circ} 44^{\prime} \mathrm{W}, 135$ masl & 57 & Andean & Canario bola & 9 \\
\hline 838 & $43^{\circ} 15^{\prime} \mathrm{N}, 8^{\circ} 54^{\prime} \mathrm{W}, 50$ masl & 66 & Andean & White kidney & 1 \\
\hline 839 & $43^{\circ} 15^{\prime} \mathrm{N}, 8^{\circ} 53^{\prime} \mathrm{W}, 60$ masl & 81 & Andean & Favada & 1 \\
\hline 842 & $43^{\circ} 15^{\prime} \mathrm{N}, 8^{\circ} 53^{\prime} \mathrm{W}, 60$ masl & 66 & Andean & White kidney & 9 \\
\hline 917 & $43^{\circ} 41^{\prime} \mathrm{N}, 7^{\circ} 30^{\prime} \mathrm{W}, 40$ masl & 87 & Andean & Favada & 1 \\
\hline 921 & $43^{\circ} 41^{\prime} \mathrm{N}, 7^{\circ} 30^{\prime} \mathrm{W}, 40$ masl & 77 & Andean & Favada & 1 \\
\hline 924 & $43^{\circ} 41^{\prime} \mathrm{N}, 7^{\circ} 30^{\prime} \mathrm{W}, 40 \mathrm{masl}$ & 75 & Andean & Favada & 1 \\
\hline 1058 & $41^{\circ} 20^{\prime} \mathrm{N}, 7^{\circ} 45^{\prime} \mathrm{W}, 500$ masl & 47 & Mesoamerican & Great northern & 1 \\
\hline \multicolumn{6}{|c|}{ BREEDING LINES ${ }^{c}$} \\
\hline Andecha & $43^{\circ} 29^{\prime} \mathrm{N}, 5^{\circ} 26^{\prime} \mathrm{W}, 16$ masl & 76 & Andean & Favada & 4 \\
\hline Bonafema & $43^{\circ} 29^{\prime} \mathrm{N}, 5^{\circ} 26^{\prime} \mathrm{W}, 16$ masl & 76 & Andean & Favada & 1 \\
\hline Collacia & $43^{\circ} 29^{\prime} \mathrm{N}, 5^{\circ} 26^{\prime} \mathrm{W}, 16 \mathrm{masl}$ & 68 & Andean & Favada & 1 \\
\hline Montcau & $41^{\circ} 23^{\prime} \mathrm{N}, 2^{\circ} 07^{\prime} \mathrm{E}, 63$ masl & 32 & Mesoamerican & Hook & 1 \\
\hline Peregrina & $43^{\circ} 23^{\prime} \mathrm{N}, 8^{\circ} 08^{\prime} \mathrm{W}, 178$ masl & 46 & Mesoamerican & Great northern & 1 \\
\hline \multicolumn{6}{|l|}{ CULTIVARS } \\
\hline Borlotto & Vilmorin (France) & 54 & Andean & Cranberry & 1 \\
\hline Fukuryu & Agricultural Station (Japan) & 56 & Andean & Cranberry & 1 \\
\hline
\end{tabular}

${ }^{a}$ All the genotyes are from Spain, with the exception of the last two ones.

'Santalla et al. (2001).

${ }^{c}$ Originated from traditional landraces by individual selection.

on a plot average basis. Five plants were selected from the center of the plots for the estimation of grain yield (expressed in $\mathrm{kg}$ $\mathrm{ha}^{-1}$ ).

\section{Soil Properties}

Five soil samples were collected before sowing in the field. Samples were air dried and passed through a $2 \mathrm{~mm}$ sieve prior to analysis. Soil organic matter was estimated by weight loss after ignition (Schulte and Hopkins, 1996). Soil pH in $\mathrm{H}_{2} \mathrm{O}$ and $0.1 \mathrm{M}$ $\mathrm{KCl}$ were measured (solution ratio 1:2.5). Phosphorus availability was measured by a modification of the Olsen method (Olsen and Dean, 1965). Exchangeable cations were extracted with $1 \mathrm{M}$ $\mathrm{NH}_{4} \mathrm{Cl}$ (Peech et al., 1947) and determined by atomic absorption spectrophotometry. Effective cation exchange capacity (ECEC) was calculated as the sum of base cations plus aluminum. The soil had moderate levels of total organic matter (4.2\%) due to the long history of cultivation in the experimental plot and had an acidic reaction $(\mathrm{pH}=5.7)$ and significant levels of exchangeable aluminum, as expected in soil derived from granitic like rock. ECEC was relatively low, although the potassium levels were medium to high. High phosphorus availability was due to fertilization with organic manure for many years in the past. Overall, soil analyses showed that the experimental plot was representative of most agricultural soils intensively cultivated in the NW of Spain. The Table S2 summarizes the chemical properties of the soil of the experimental plot.

\section{Data Analyses}

Maximum, minimum, average temperatures and $\mathrm{RH}$ were daily measured in the controlled chamber and in the open field trials. The statistical analyses were conducted using the general linear model (GLM) procedure of the SAS 2000 statistical package. The least significant difference (LSD) $(P \leq 0.05)$ was used to evaluate differences among genotype means. Standard errors and coefficients of variation were also computed (Steel et al., 1997). 
Principal component analysis (PCA) was performed by NTSYS-pc v.2.10 (Rohlf, 2000) and the free software $\mathrm{R}^{\mathrm{mo}}$ modified by García-Pérez (2005) to display the ordination of the genotypes under both environments. The variables used in the PCA were emergence proportion and time to emergence at the three temperature levels $(\mathrm{t} 1, \mathrm{t} 2, \mathrm{t} 3)$ in the controlled chamber and in the open field. According to the scree diagram most of the variation was explained by the first (PC1), second (PC2), and third (PC3) principal components. PC1 and PC2 accounted for $56 \%$ of variation and were used for plotting the 28 genotypes.

\section{RESULTS}

\section{Screening in Controlled Chamber}

Marked differences in emergence proportion and time to emergence were found among genotypes at the different temperatures in the controlled chamber and in the open field sowings, respectively (Table 2). In the controlled chamber emergence proportion ranged from 30.0 to $96.7 \%$, with an average of $72.9 \%$ at low temperature $\left(\mathrm{tc} 1=14 / 8^{\circ} \mathrm{C}\right)$. Under $17 / 12^{\circ} \mathrm{C}(\mathrm{tc} 2)$ and $22 / 15^{\circ} \mathrm{C}$ (tc3), some genotypes reached $100 \%$ emergence, the averages were 88.2 and $94.4 \%$, respectively. Seedling emergence was delayed under the lower temperature in controlled conditions averaging $27.2 \mathrm{~d}$ at $14 / 8^{\circ} \mathrm{C}$ (tc1), but emergence time was drastically reduced to 7.8 and $4.6 \mathrm{~d}$ when temperature was increased to $17 / 12^{\circ} \mathrm{C}$ (tc2) and $22 / 15^{\circ} \mathrm{C}$ (tc3), respectively. Effects of genotype, temperature, and genotype $\times$ temperature interaction on emergence proportion and time to emergence were significant (Table 3), indicating differential and non-uniform responses of genotypes to temperature at the emergence stage.

\section{Screening in Open Field Trials}

In terms of average daily temperatures, the high temperature trials $\left(12.7-21.3^{\circ} \mathrm{C}\right)$, and the low temperature trials $\left(9.3-20.4^{\circ} \mathrm{C}\right)$ overlap. However, the low and high temperature trials were still

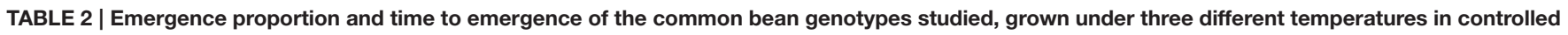
chamber, and open field.

\begin{tabular}{|c|c|c|c|c|c|c|c|c|c|c|c|c|}
\hline \multirow[t]{3}{*}{ Genotypes } & \multicolumn{6}{|c|}{ Emergence proportion (\%) } & \multicolumn{6}{|c|}{ Emergence time (d) } \\
\hline & \multicolumn{3}{|c|}{ Controlled chamber } & \multicolumn{3}{|c|}{ Open field } & \multicolumn{3}{|c|}{ Controlled chamber } & \multicolumn{3}{|c|}{ Open field } \\
\hline & tc1 & tc2 & tc3 & tf1 & $\mathrm{tf2}$ & tf3 & tc1 & tc2 & tc3 & tf1 & tf2 & tf3 \\
\hline 200 & 86.7 & 100.0 & 96.7 & 50.0 & 50.0 & 50.0 & 28.3 & 7.3 & 4.7 & 11.5 & 9.5 & 12.0 \\
\hline 272 & 96.7 & 86.7 & 96.7 & 58.3 & 58.3 & 50.0 & 23.0 & 8.0 & 3.7 & 15.5 & 9.5 & 12.0 \\
\hline 391 & 53.3 & 90.0 & 83.3 & 50.0 & 50.0 & 50.0 & 29.7 & 8.0 & 4.0 & 10.0 & 9.0 & 13.0 \\
\hline 399 & 63.3 & 73.3 & 86.7 & 75.0 & 50.0 & 50.0 & 30.3 & 10.0 & 9.3 & 13.5 & 8.5 & 12.5 \\
\hline 413 & 96.7 & 100.0 & 100.0 & 50.0 & 50.0 & 50.0 & 27.0 & 7.3 & 4.7 & 11.5 & 8.5 & 12.5 \\
\hline 419 & 73.3 & 90.0 & 90.0 & 75.0 & 50.0 & 40.0 & 29.0 & 8.0 & 4.7 & 13.5 & 8.0 & 13.0 \\
\hline 452 & 80.0 & 93.3 & 96.7 & 50.0 & 50.0 & 20.0 & 26.3 & 7.3 & 4.3 & 15.0 & 9.0 & 14.0 \\
\hline 489 & 70.0 & 70.0 & 100.0 & 50.0 & 50.0 & 41.7 & 26.3 & 7.7 & 4.0 & 13.0 & 10.0 & 13.0 \\
\hline 501 & 93.3 & 96.7 & 100.0 & 50.0 & 50.0 & 35.0 & 23.7 & 7.3 & 4.7 & 17.0 & 9.0 & 14.0 \\
\hline 573 & 33.3 & 80.0 & 83.3 & 50.0 & 45.0 & 43.3 & 29.3 & 8.0 & 3.7 & 12.5 & 13.5 & 13.5 \\
\hline 587 & 63.3 & 100.0 & 96.7 & 50.0 & 50.0 & 31.7 & 26.0 & 8.3 & 3.7 & 16.5 & 9.5 & 11.0 \\
\hline 593 & 90.0 & 96.7 & 100.0 & 50.0 & 40.0 & 50.0 & 24.3 & 7.3 & 4.0 & 14.5 & 14.0 & 13.0 \\
\hline 623 & 30.0 & 93.3 & 100.0 & 35.0 & 35.0 & 48.3 & 27.0 & 7.3 & 4.3 & 16.5 & 19.0 & 14.0 \\
\hline 837 & 63.3 & 80.0 & 96.7 & 50.0 & 50.0 & 50.0 & 30.7 & 8.0 & 5.0 & 11.5 & 8.5 & 12.5 \\
\hline 838 & 50.0 & 93.3 & 86.7 & 38.3 & 40.0 & 38.3 & 31.3 & 8.3 & 5.0 & 16.0 & 14.0 & 13.5 \\
\hline 839 & 33.3 & 90.0 & 76.7 & 31.7 & 56.7 & 50.0 & 30.3 & 7.3 & 4.3 & 17.5 & 14.5 & 13.0 \\
\hline 842 & 83.3 & 90.0 & 100.0 & 40.0 & 50.0 & 50.0 & 27.7 & 7.3 & 4.3 & 17.5 & 10.0 & 13.0 \\
\hline 917 & 63.3 & 80.0 & 86.7 & 40.0 & 50.0 & 20.0 & 24.7 & 7.3 & 4.7 & 18.0 & 8.0 & 14.0 \\
\hline 921 & 70.0 & 76.7 & 93.3 & 31.7 & 20.0 & 11.7 & 26.7 & 8.0 & 4.7 & 19.5 & 17.5 & 14.0 \\
\hline 924 & 76.7 & 76.7 & 93.3 & 36.7 & 21.7 & 21.7 & 27.0 & 7.3 & 4.3 & 19.0 & 23.5 & 14.0 \\
\hline 1058 & 86.7 & 90.0 & 100.0 & 58.3 & 58.3 & 50.0 & 26.0 & 7.3 & 4.7 & 12.5 & 10.0 & 12.5 \\
\hline Andecha & 96.7 & 96.7 & 100.0 & 45.0 & 50.0 & 31.7 & 27.3 & 7.3 & 4.7 & 16.0 & 8.5 & 13.5 \\
\hline Bonafema & 86.7 & 86.7 & 96.7 & 23.3 & 35.0 & 35.0 & 26.7 & 7.3 & 5.0 & 20.5 & 12.5 & 13.0 \\
\hline Collacia & 53.3 & 66.7 & 90.0 & 16.7 & 36.7 & 38.3 & 29.3 & 11.0 & 4.3 & 20.0 & 14.0 & 13.5 \\
\hline Montcau & 96.7 & 100.0 & 96.7 & 50.0 & 58.3 & 50.0 & 24.0 & 7.3 & 4.0 & 13.5 & 8.0 & 6.0 \\
\hline Peregrina & 83.3 & 93.3 & 96.7 & 23.3 & 33.3 & 26.7 & 26.7 & 8.3 & 4.0 & 20.0 & 13.0 & 14.0 \\
\hline Borlotto & 93.3 & 100.0 & 100.0 & 50.0 & 50.0 & 50.0 & 28.7 & 7.0 & 4.5 & 10.5 & 8.0 & 12.0 \\
\hline Fukuryu & 73.3 & 80.0 & 100.0 & 33.3 & 50.0 & 50.0 & 25.0 & 7.3 & 4.7 & 17.5 & 9.0 & 13.0 \\
\hline Average & 72.9 & 88.2 & 94.4 & 45.1 & 46.0 & 40.5 & 27.2 & 7.8 & 4.6 & 15.4 & 11.3 & 12.8 \\
\hline
\end{tabular}


TABLE 3 | Mean squares and coefficient of variation (CV) from the analysis of variance for the emergence proportion and time to emergence of the common bean genotypes studied, grown under three different temperatures in controlled chamber.

\begin{tabular}{|c|c|c|c|}
\hline Source of variation & $D f^{a}$ & $\begin{array}{c}\text { Emergence proportion } \\
(\%)\end{array}$ & $\begin{array}{c}\text { Emergence } \\
\text { time (d) }\end{array}$ \\
\hline Replications & 2 & 64.0 & 4.9 \\
\hline Genotype & 27 & $897.0^{\star \star}$ & $10.7^{\star \star}$ \\
\hline Temperature & 2 & $10278.4^{\star \star}$ & $12386.3^{\star \star}$ \\
\hline Genotype $\mathrm{x}$ temperature & 54 & $350.7^{\star \star}$ & $4.8^{\star}$ \\
\hline Error & 110 & 147.9 & 3.2 \\
\hline CV (\%) & & 14.3 & 13.4 \\
\hline
\end{tabular}

on the highest end of ideal common bean growing maximum and minimum temperatures $\left(20-25\right.$ and $15^{\circ} \mathrm{C}$ ) and thus probably experienced some temperature stress.

Analysis of variance of the 28 genotypes in the three open field experiments with different temperature conditions at sowing time is shown in Table 4. All the traits displayed significant differences among genotypes and among sowing times characterized by different temperatures, while significant genotype $\times$ sowing time interaction was only observed for dry seed weight, and length, pods per plant and yield. Only six genotypes showed emergence proportion higher than $50 \%$ in the earlier sowings at lower temperatures (tf1 and/or tf2), none under the warmer temperatures at the later sowing (tf3), averaging 45.1, 46.0 , and $40.5 \%$, respectively, at these sowing dates (Table 4). Differences in emergence time were smaller compared to the controlled chamber experiment. Emergence time was reduced from $15.4 \mathrm{~d}$ in the earlier, and colder conditions to 11.3 and 12.8 $\mathrm{d}$ in the later warmer conditions $(P=0.05)$. Early sowing in April associated with lower temperatures (tf1) significantly delayed by $4-5 \mathrm{~d}$ seedling emergence, first trifoliate leaf expansion, and beginning of flowering and by about $14-16 \mathrm{~d}$ the end of flowering and physiological maturity, compared to sowing in May (tf2). Sowing at warmer temperatures in June further reduce seed weight and plant yield. These results indicate that early sowing under lower temperatures produced taller plants with larger seeds and higher yield than later sowings under higher temperatures. In fact, the length of the vegetative and reproductive cycle stages was progressively reduced from the colder (tf1) to the warmer (tf2 and tf3) conditions. Thus, under lower temperatures plants had more time for growth, as well as for pod and seed set and maturation, resulting in larger seeds, and higher plant yield. The Table 5 displays the crop yield of the common bean genotypes studied, grown under three different temperatures in open field. The yield was clearly higher when sowing and growing under tf1 and tf2 than $\mathrm{tf} 3$ for all the genotypes, averaging 1188,849 , and $419 \mathrm{~kg}$ $\mathrm{ha}^{-1}$, respectively. Best performer was the landrace $419(3118 \mathrm{~kg}$ $\mathrm{ha}^{-1}$ at tf1) and the worst was the Japanese cultivar Fukuryu (149 kg ha-1 at tf1), the latter probably due to lack of adaptation to the growing area. Interestingly, the 10 top yielding genotypes corresponded to $\mathrm{tf} 1$, while the 10 worst genotypes were sown and grown at tf3, except the aforementioned cultivar Fukuryu (tf1). The performance of some genotypes was consistent through the different temperature conditions, such as $399,501,587$, and Borlotto.

\section{Comparison of Temperature Effect on Chamber and Field Trials}

The analysis of the emergence process was assessed in controlled chamber and in open field under three different temperature ranges by two variables: Time to emergence and proportion of emergence. Maximum, minimum, and the average values of these variables in chamber and field are shown in Table S3. Decrease in number of days to emergence and increase in emergence proportion when temperature increased occurred in the chamber trials but were less clear in the field (Table 4). Under favorable conditions (tc2 and/or tc3) 13 genotypes reached 100\% emergence in the growth chamber. In the open field, in contrast, only four genotypes showed emergence higher than $50 \%$ at tf2 and none at tf3, while emergence level was similar at tf1 and $\mathrm{tf} 2$, and higher than at tf3. Emergence time across genotypes was relatively stable at the lower temperatures (tc1) in the growth chamber, ranging from 23.0 to $31.3 \mathrm{~d}$, but in the field the range was 10.0 to $20.5 \mathrm{~d}$ in the first sowing (tf1).

Nine groups arose from the PCA ordination (Table 6, Figure 1). The $\mathrm{x}$-axis ( $\mathrm{PC1}$ ) represents variation in emergence time and proportion of emergence in the open field. Genotypes located at the left side have earlier emergence and higher emergence proportion than those on the right side. The $y$ axis represents variation in the same variables in the controlled chamber, with genotypes at the lower side showing earlier emergence, and higher emergence proportion than those at the upper side. Montcau is the only genotype in group 1, five genotypes are included in group 2 (200 272, 413 1058, Borlotto), eight in group $3(452,489,501,587,593,842$, Andecha, Fukuryu), four in group $4(623,917$, Bonafema, Peregrina), two in group $5(921,924)$, three in group $6(391,419,837)$, three in group $7(573,838,839)$, only Collacia in group 8 and 399 in group 9 .

\section{DISCUSSION}

In controlled environments and field conditions, studies have shown that the rate of germination and seedling emergence linearly increases with temperature in several crop species including legumes, such as cowpea, soybean, chickpea, and peanut (Covell et al., 1986; Ellis et al., 1986; Mohamed et al., 1988; Craufurd et al., 1996; Awal and Ikeda, 2002). In this work we found that the variation in emergence in a range of temperatures was greater in controlled chamber than in open field. It can be argued that environmental conditions in the chamber are strictly controlled and the weighted average of temperature and $\mathrm{RH}$ had a range of variation wider than in the field. However, seeds in the field are exposed to the natural not controlled environmental variation that could explain the fact that the proportion of emergence is approximately half of the value in the controlled chamber. In field experiments, several factors can affect seed germination, and therefore the proportion 
TABLE 4 | Mean squares, coefficient of variation (CV) and genotype means from the analysis of variance of agronomic traits of the common bean genotypes studied, grown under three different conditions in open field.

\begin{tabular}{|c|c|c|c|c|c|c|c|c|c|c|}
\hline \multirow[t]{2}{*}{ Trait } & \multicolumn{5}{|c|}{ Sources of variation } & \multirow[b]{2}{*}{ CV (\%) } & \multicolumn{4}{|c|}{ Mean } \\
\hline & Replications & Genotype (G) & Temperature $(\mathrm{T})$ & $\mathbf{G} \times \mathbf{T}$ & Error & & $\mathrm{tf1}$ & $\mathrm{tf2}$ & tf3 & LSD $^{b}$ \\
\hline Emergence time (d) & 20.02 & $31.02^{\star \star}$ & $236.74^{\star \star}$ & 11.14 & 7.50 & 20.81 & $15.36 a$ & $11.28 \mathrm{c}$ & $12.82 \mathrm{~b}$ & 1.26 \\
\hline Emergence proportion (\%) & 26.46 & $541.97^{\star \star}$ & $490.51^{*}$ & 146.86 & 151.19 & 28.04 & 45.06ab & $46.01 \mathrm{a}$ & $40.48 b$ & 4.59 \\
\hline Leaf expansion (d) & 3.72 & $15.55^{\star}$ & $327.25^{\star \star}$ & 10.14 & 7.88 & 14.32 & $22.36 \mathrm{a}$ & $17.84 b$ & 18.61b & 1.21 \\
\hline Plant height- 10 days (mm) & 5.28 & $58.86^{\star \star}$ & $3333.78^{\star \star}$ & 31.76 & 25.35 & 19.89 & $19.12 \mathrm{c}$ & $33.95 a$ & $22.85 b$ & 2.13 \\
\hline Plant height-20 days (mm) & 7.86 & $61.14^{\star \star}$ & $5004.26^{\star \star}$ & 18.87 & 29.61 & 12.85 & 53.30a & $37.68 \mathrm{~b}$ & $35.70 \mathrm{c}$ & 1.66 \\
\hline Early vigor (1-9) & 0.53 & $6.43^{\star *}$ & $60.06^{\star \star}$ & 2.22 & 2.54 & 28.52 & $6.46 a$ & $5.87 b$ & $4.42 \mathrm{c}$ & 0.57 \\
\hline First open flower (d) & 25.14 & $378.28^{\star \star}$ & $1139.59^{\star \star}$ & 22.74 & 24.69 & 8.66 & $61.96 \mathrm{a}$ & $57.21 b$ & $52.95 c$ & 1.80 \\
\hline Beginning of flowering (d) & 18.34 & $458.27^{\star \star}$ & $1267.97^{\star \star}$ & 33.01 & 23.89 & 7.87 & $66.79 a$ & $62.18 b$ & $57.23 c$ & 2.18 \\
\hline End of flowering (d) & 23.54 & $77.34^{\star \star}$ & $18036.40^{\star \star}$ & 12.63 & 9.02 & 2.77 & $124.98 a$ & $111.14 b$ & $88.26 \mathrm{c}$ & 1.35 \\
\hline Pod physiological maturity (d) & 7.04 & $90.77^{\star \star}$ & $16857.12^{\star \star}$ & 13.36 & 12.74 & 3.15 & $130.20 \mathrm{a}$ & $114.02 b$ & $94.72 \mathrm{c}$ & 1.39 \\
\hline Dry seed weight (g 100 seed $^{-1}$ ) & 78.38 & $1863.09^{\star \star}$ & $1825.58^{\star \star}$ & $89.76^{\star \star}$ & 43.11 & 11.15 & $63.75 a$ & $60.32 \mathrm{a}$ & $51.99 b$ & 3.66 \\
\hline Seed length (mm) & $4.74^{\star}$ & $65.45^{\star \star}$ & $12.98^{\star \star}$ & $1.78^{\star \star}$ & 0.72 & 5.14 & $16.96 \mathrm{a}$ & $16.62 \mathrm{a}$ & $16.09 b$ & 0.51 \\
\hline Seed width (mm) & 0.14 & $3.69^{\star \star}$ & $3.97^{\star \star}$ & 0.12 & 0.07 & 3.31 & $8.54 a$ & 8.33b & $7.99 c$ & 0.13 \\
\hline Seed thickness (mm) & 0.02 & $4.74^{\star \star}$ & $2.40^{\star \star}$ & 0.10 & 0.13 & 5.91 & $6.28 \mathrm{a}$ & $6.15 b$ & $5.80 c$ & 0.12 \\
\hline Seeds pod ${ }^{-1}$ & 1.92 & $4.94^{\star \star}$ & $4.13^{\star \star}$ & 0.61 & 0.55 & 17.64 & $4.35 a$ & $4.31 \mathrm{a}$ & $3.89 b$ & 0.30 \\
\hline Pods plant ${ }^{-1}$ & 119.76 & $2108.50^{\star \star}$ & $10732.38^{\star \star}$ & $804.45^{\star \star}$ & 321.42 & 55.00 & $31.23 b$ & $45.74 a$ & $20.14 c$ & 0.32 \\
\hline Yield (kg ha-1) & 67054.50 & $4086.89^{\star \star}$ & $55260.74^{\star \star}$ & $3145.16^{\star \star}$ & 1841.85 & 0.53 & $1188.20 \mathrm{~b}$ & $849.10 \mathrm{a}$ & 419.00c & 2.28 \\
\hline$D f^{a}$ & 1 & 27 & 2 & 54 & 56 & & & & & \\
\hline
\end{tabular}

*, ** significant at $P \leq 0.05, P \leq 0.01$, respectively.

${ }^{a} \mathrm{Df}$, degrees of freedom.

${ }^{b}$ Least Significant Difference. Means follow for the same letter are not significant different at $P \leq 0.05$.

of seedling emergence. One factor may be the presence of soilborne pathogens (Burke and Kraft, 1974) that affect seeds when the emergence is delayed and the seed remains more time on the soil. Days to emergence in the field varied slightly with increasing temperature in later sowings, but this variation could be due also to other environmental factors such as soil properties and hydrological conditions.

Clear genotypes $\times$ temperatures interactions were found for emergence proportion and time to emergence. For instance, genotypes 573 and 623 showed contrasting emergence proportions in response to $\mathrm{tf} 2$ and $\mathrm{tf} 3$. Furthermore, time to emergence in the field was advanced only by 1 day in 399 compared to 6 days in Collacia.

The bean germplasm from the Iberian Peninsula is characterized by large and extra-large seeds with this trait displaying a broad sense heritability of 0.70 (Escribano et al., 1994; Casquero et al., 2006; González et al., 2006). In our study

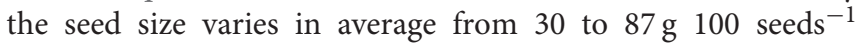
being $25 \%$ of the studied genotypes large and $43 \%$ extra-large seeded. The large seeds were the result of selection by farmers according to market and consumer preferences. Farmers use to germinate the large seeds in nurseries to ensure the process, then to transplant the seedlings to the open field. This practice could have favored selection for earlier emergence, but not for emergence proportion in the field. In fact, the PCA indicates a trend to lower emergence in large and extra-large seeded genotypes: the groups with good emergence (groups 1, 2, and 3) have seven large and extra-large seeded genotypes (50\%) and seven medium and small seeded ones (50\%). However, the groups with mediocre or bad emergence (groups 4, 5, 6, 7, 8, and 9) include 11 large and extra-large seeded genotypes (79\%) and only three medium and small ones (21\%).

With regard to market class and seed color, one of the factors that have been suggested as responsible for poor legume emergency in the field is imbibition damage caused by fast water uptake, resulting in cell death, solute leakage, and reduced emergence and growth. However, colored seeds which imbibed more slowly, suffer less damage than the white ones (Powell et al., 1986). Therefore, white seeded market classes are typically more susceptible to this damage. According to the PCA, the groups with good emergence (groups 1, 2, and 3) have eight white seeded genotypes, and six bicolor or colored, while the groups with bad emergence $(4,5,6,7,8$, and 9) included 11 white seeded genotypes and only three colored ones. These trends suggest that seed testa color could have some influence in seed germination and seedling emergence, in accordance with the results by other authors (Dickson, 1971; Powell et al., 1986).

Open field trials are the most reliable measure of low temperature tolerance since it is measured in the actual growth environment of the crop. In our research consistent comparable results were not observed between controlled chamber and field environments, in agreement with Kolasinska et al. (2000) and Khajeh-Hosseini and Rezazadeh (2011).

There is evidence that chilling tolerance at juvenile stages of development (germination, emergence, seedling growth) is under independent genetic control from chilling tolerance during 
TABLE 5 | Crop yield of the common bean genotypes studied, grown under three different temperatures (tf1, $\mathrm{tf2}$, and $\mathrm{tf} 3$ ) in open field.

\begin{tabular}{|c|c|c|c|}
\hline \multirow[t]{2}{*}{ Genotype } & \multicolumn{3}{|c|}{ Yield $\left(\mathrm{kg} \mathrm{ha}^{-1}\right)$} \\
\hline & tf1 & $\mathrm{tf2}$ & $\mathrm{tf3}$ \\
\hline 200 & 1025 & 946 & 373 \\
\hline 272 & 2236 & 811 & 448 \\
\hline 391 & 1463 & 955 & 404 \\
\hline 399 & 1594 & 1152 & 474 \\
\hline 413 & 468 & 477 & 465 \\
\hline 419 & 3118 & 1092 & 465 \\
\hline 452 & 818 & 1128 & 435 \\
\hline 489 & 367 & 431 & $\mathrm{~nm}^{*}$ \\
\hline 501 & 2738 & 1395 & 425 \\
\hline 573 & 777 & 907 & 355 \\
\hline 587 & 1643 & 1168 & 482 \\
\hline 593 & 1072 & 962 & 502 \\
\hline 623 & 825 & 734 & 382 \\
\hline 837 & 1084 & 862 & 393 \\
\hline 838 & 478 & 544 & 305 \\
\hline 839 & 1551 & 1567 & 445 \\
\hline 842 & 420 & 602 & 349 \\
\hline 917 & 1599 & 1234 & 356 \\
\hline 921 & 1199 & 443 & 339 \\
\hline 924 & 1055 & 706 & 526 \\
\hline 1058 & 2861 & 1006 & 432 \\
\hline Andecha & 1533 & 785 & 343 \\
\hline Bonafema & 370 & 461 & 379 \\
\hline Collacia & 884 & 921 & 319 \\
\hline Montcau & 626 & 496 & 428 \\
\hline Peregrina & 851 & 491 & 350 \\
\hline Borlotto & 471 & 1157 & 717 \\
\hline Fukuryu & 149 & 325 & 429 \\
\hline Average & 1188 & 849 & 419 \\
\hline
\end{tabular}

${ }^{*} n m$, not measured.

reproductive development (Kemp, 1973; Dickson and Petzoldt, 1987; Melo et al., 1997). Coincidence of tolerance to high and low temperature stress has been reported in snap beans (Dickson and Boettger, 1984a,b), oat (Mashiringi and Harahwa, 1985), and maize (Yacoob and Filion, 1986). Porch (2001) found that snap bean lines developed for cold tolerance had significant positive general combining ability (GCA) under high temperatures for traits related to high temperature tolerance.

It is generally accepted that germination rate is affected by seed shape or size and, therefore, the quantity of nutrients stored in the seed (Cui et al., 2002; Hanley et al., 2003; Nonogaki, 2006; Kaya et al., 2008). In the experiments here reported, large seeded genotypes needed more days to seedling emergence than small seeded ones, both in the growth chamber and in the open field experiments, and showed lower emergence in the field under real growing conditions. This fact may be related to the history of the bean crop in southwest Europe, since its introduction in the
TABLE 6 | Characteristics of the groups arising from the PCA of the common bean genotypes studied under different environments.

\begin{tabular}{|c|c|c|c|c|c|}
\hline Group & Genotype & $\begin{array}{c}\text { Genetic } \\
\text { pool }^{\mathrm{a}}\end{array}$ & $\begin{array}{l}\text { Seed } \\
\text { color }^{b}\end{array}$ & $\begin{array}{l}\text { Seed } \\
\text { size }^{c}\end{array}$ & $\begin{array}{c}\text { Emergence } \\
\text { score }^{d}\end{array}$ \\
\hline 1 & Montcau & $\mathrm{M}$ & w & $\mathrm{m}$ & $g$ \\
\hline \multirow[t]{5}{*}{2} & 200 & A & w & $\mathrm{m}$ & $g$ \\
\hline & 272 & A & $b$ & $\mathrm{~m}$ & $g$ \\
\hline & 413 & A & $\mathrm{b}$ & $\mathrm{x}$ & g \\
\hline & 1058 & $\mathrm{M}$ & w & 1 & $g$ \\
\hline & Borlotto & A & C & 1 & $g$ \\
\hline \multirow[t]{8}{*}{3} & 452 & A & w & $x$ & $g$ \\
\hline & 489 & A & c & $\mathrm{s}$ & $g$ \\
\hline & 501 & A & C & $\mathrm{m}$ & $g$ \\
\hline & 587 & $\mathrm{M}$ & w & $\mathrm{m}$ & $g$ \\
\hline & 593 & $\mathrm{M}$ & w & $\mathrm{m}$ & $g$ \\
\hline & 842 & A & w & $x$ & $g$ \\
\hline & Andecha & A & w & $x$ & $g$ \\
\hline & Fukuryu & A & $\mathrm{C}$ & I & $g$ \\
\hline \multirow[t]{4}{*}{4} & 623 & M & w & $\mathrm{m}$ & $\mathrm{m}$ \\
\hline & 917 & $A$ & w & $x$ & $\mathrm{~m}$ \\
\hline & Bonafema & A & w & $x$ & $m$ \\
\hline & Peregrina & $\mathrm{M}$ & w & 1 & $\mathrm{~m}$ \\
\hline \multirow[t]{2}{*}{5} & 921 & A & w & $x$ & $b$ \\
\hline & 924 & $A$ & w & $x$ & $b$ \\
\hline \multirow[t]{3}{*}{6} & 391 & A & C & 1 & $\mathrm{~m}$ \\
\hline & 419 & $\mathrm{M}$ & w & 1 & $\mathrm{~m}$ \\
\hline & 837 & A & C & 1 & $m$ \\
\hline \multirow[t]{3}{*}{7} & 573 & $\mathrm{M}$ & C & $\mathrm{m}$ & $\mathrm{m}$ \\
\hline & 838 & A & w & $x$ & $\mathrm{~m}$ \\
\hline & 839 & A & w & $x$ & $\mathrm{~m}$ \\
\hline 8 & Collacia & A & w & $x$ & $b$ \\
\hline 9 & 399 & M & w & $x$ & $\mathrm{~b}$ \\
\hline
\end{tabular}

${ }^{a} A$, Andean; M, Mesoamerican.

${ }^{b}$ w, white; $b$, bicoloured; $c$, colored.

${ }^{c} x$, extra-large (> $65 \mathrm{~g} 100$ seeds $\left.^{-1}\right)$; l, large (> $50 \mathrm{~g}^{100}$ seeds $^{-1}$ ); $\mathrm{m}$, medium (> $35 \mathrm{~g}$

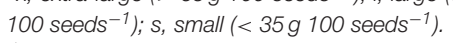

${ }^{d}$ According to the PCA ordination in Figure 1: g, good; $m$, mediocre; $b$, bad.

early Sixteenth Century. Farmers probably selected large seeded common bean genotypes that resembled the Old World faba bean (Vicia faba L.) that was cultivated at that time, thus introducing the new crop as a novelty, which explains the name "faba" or "haba" used often in Spain for the common bean. In spite of the pleiotropic effect that seed size probably had in delaying germination and reducing emergency, famers continued to select large seeded genotypes of common bean such as the currently grown in the northwest of the Iberian Peninsula (Escribano et al., 1994; Rodiño et al., 2001, 2009).

Nine groups arose from the PCA representing variation in emergence time and proportion of emergence in the controlled 


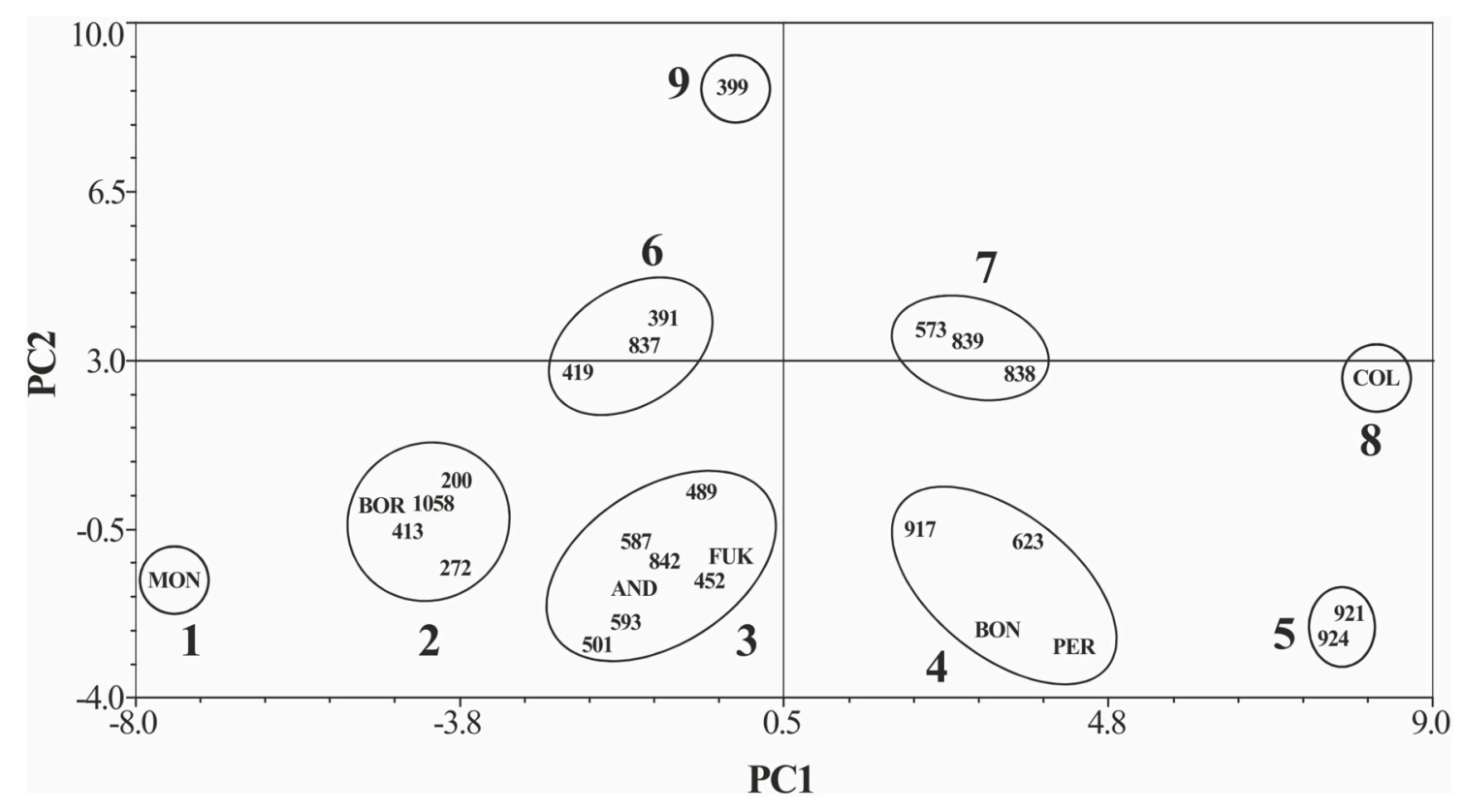

FIGURE 1 | Ordination the common bean genotypes studied according to the Principal Component Analysis (PCA). PC1, first principal component; PC2, second principal component.

chamber and in the open field. PCA indicates a trend to lower emergence in large and extra-large seeded genotypes. The genotypes 399 and 419 (large great northern market class), 921, 924, and Collacia (favada market class) are examples of white large seeded genotypes with poor germination in the field. In particular, according to the PCA, the favada market class genotypes (two in group 3 and six in groups 4, 5, 7, and 8), with very high market value, have lower proportion of emergence, probably due to the fact that farmers practice was to germinate the seed in the nursery, and afterwards transplant the seedling into the open field. Since then neither natural selection nor breeding had taken place to improve the germination and emergence of the favada market class genotypes or the large great northern ones whose seeds have similar market and uses by consumers. Nevertheless, in the present study some of these genotypes (e.g., 399, 419, 917, 921, and 924) showed high emergence $(>80 \%)$ when germinated at low temperature in a controlled environment chamber. This was probably due to the fact that these genotypes were selected in the past to be germinated in stable environments, not in unstable open field conditions.

In the experiments here reported, the emergence process was assessed by time to emergence and the proportion of seeds producing emerged seedlings, with relevance in the conservation of germplasm in gene banks. In order to conserve the genetic structure of the original accessions, regeneration of germplasm in the field should be performed only when the results of the germination tests display low germination. Emergence in controlled conditions in climatic chamber resembles the viability test used in gene banks, whereas sowing in the field is used to regenerate accessions. According to our results the viability tests should be complemented with vigor tests that can provide better estimation of field emergence (Hampton and Tekrony, 1995). For the same reason, in regeneration processes of bean germplasm it would be advisable to germinate seeds under favorable conditions (e.g., in nurseries) before transplanting the seedlings into the field, in order to prevent (or minimize) genetic erosion due to the mortality of viable seeds.

According to our results, temperature conditions had a relevant role in the crop yield, together with the environmental factors. The plant productivity, which is the major expression of the genotypes fitness, hade the higher values when sowing and growing the plants under low temperature, decreasing when the temperature was increased. It is relevant also to link the yield with the process of emergence of seedlings at different temperatures in the open field and in the controlled chamber. Taken into account these results, the genotypes 272, 501, 593, and 1058, and the cultivar Borlotto had assembled the best conditions for early sowing achieving good yield performance.

In conclusion, seed germination, seedling emergence, plant growth, and crop yield under different temperatures are of relevance for the selection of common bean genotypes with better performance under stress temperatures, but also may lead to potential genetic erosion in germplasm collections. As a result of the screen of seedling emergence and phenotypic response of bean germplasm under a range of temperatures in controlled chamber and field conditions, some genotypes such as landraces 272, 501, 593, and the cultivar Borlotto were identified as temperature stress-tolerant at sowing time and seedling emergence with good agronomic performance and yield potential and they could be a valuable genetic 
material for breeding programs. Additionally, the efficiency of bean genebanks standard germination tests for predicting the performance of the seeds in the field was assessed comparing the emergence of bean seedlings under controlled environment and in open field. Regarding bean commercial traits, under low temperature at sowing time bean seeds reached larger size, and the crop yield was higher compared to warmer temperatures at this stage. Therefore, early sowing of bean is strongly recommended.

\section{AUTHOR CONTRIBUTIONS}

$\mathrm{AD}$ : conception and experimental design of the work, including chamber, and field experiments; revising the work and approval of the version to be published. AR: experimental design of the work; acquisition and analysis of chamber and field data for the work; drafting and revising the work and approval of the version to be published. MS: interpretation of field and chamber data for the work; drafting and revising the work and approval of the version to be published. AG: statistical analysis of field and chamber experimental data; revising the work and approval of the version to be published. ML: interpretation of soil data for the work; revising the work and approval of the version to be published. IM: interpretation of germplasm data for the work; revising the work and approval of the version to be published. JK: analysis and interpretation of physiological data for the

\section{REFERENCES}

Alonso-Blanco, C., Bentsink, L., Hanhart, C. J., Blankestijn-De Vries, H., and Koornneef, M. (2003). Analysis of natural allelic variation at seed dormancy loci of Arabidopsis thaliana. Genetics 164, 711-729.

Angioi, S. A., Rau, D., Attene, G., Nanni, L., Bellucci, E., Logozzo, G., et al. (2010). Beans in Europe: origin and structure of the European landraces of Phaseolus vulgaris L. Theor. Appl. Genet. 121, 829-843. doi: 10.1007/s00122-010-1353-2

Awal, M. A., and Ikeda, T. (2002). Effects of changes in soil temperature on seedling emergence and phenological development in field-grown stands of peanut (Arachis hypogaea). Environ. Exp. Bot. 47, 101-113. doi: 10.1016/S00988472(01)00113-7

Bannerot, H. (1979). Cold tolerance in beans. Ann. Rep. Bean Improv. Coop. 22, 81-84.

Bellucci, E., Bitocchi, E., Rau, D., Rodriguez, M., Biagetti, E., Giardino, A., et al. (2014). "Genomics of origin, domestication and evolution of Phaseolus vulgaris," in Genomics of Plant Genetic Resources, eds R. Tuberosa, A. Graner, and E. Frison (Dordrecht: Springer), 483-507.

Bewley, J. D. (1997). Seed germination and plant dormancy. Plant Cell 9, 1055-1066. doi: 10.1105/tpc.9.7.1055

Bewley, J. D., and Black, M. (1985). Seeds: Physiology of Development and Germination. New York, NY: Plenum Press.

Blair, M. W., Knewston, S. J. B., Astudillo, C., Li, C. M., Fernandez, A. C., and Grusak, M. A. (2010). Variation and inheritance of iron reductase activity in the roots of common bean (Phaseolus vulgaris L.) and association with seed iron accumulation QTL. BMC Plant Biol. 10:215. doi: 10.1186/1471-222910-215

Burke, D. W., and Kraft, J. M. (1974). Responses of beans and peas to root pathogens accumulated during monoculture of each crop species. Phytopathology 64:4. doi: 10.1094/phyto-64-546

Casquero, P. A., Lema, M., Santalla, M., and De Ron, A. M. (2006). Performance of common bean landraces from Spain in the Atlantic and Mediterranean work; revising the work and approval of the version to be published.

\section{FUNDING}

Research was supported by the projects AGL2014-51809-R and RFP2013-00001 from the Spanish Government (Ministerio de Economía y Competitividad) and AGI/CSIC I+D+I 2014 OTR00114 from the Galician Government-CSIC (Spain).

\section{ACKNOWLEDGMENTS}

The authors are grateful to CRF-INIA (Alcalá de Henares, Spain), Polytechnic University of Cataluña (Barcelona, Spain), Agricultural Station (Hokkaido, Japan), and SERIDA (Villaviciosa, Spain) for supplying common bean germplasm. We thank to Albert Vandenberg and Pedro Revilla for reviewing the manuscript and for laboratory facilities, Josefina HernándezNistal for scientific assistance, Salvador Rodríguez for technical assistance, and Diputación de Pontevedra (Pontevedra, Spain) for farm facilities.

\section{SUPPLEMENTARY MATERIAL}

The Supplementary Material for this article can be found online at: http://journal.frontiersin.org/article/10.3389/fpls.2016. 01087 environments. Genet. Resour. Crop Evol. 53, 1021-1032. doi: 10.1007/s10722004-7794-1

Copeland, O. L., and McDonald, B. M. (1995). Principles of Seed Science and Technology. 3rd Edn. New York, NY: Chapman and Hall.

Covell, S., Ellis, R. H., Roberts, E. H., and Summerfield, R. J. (1986). The influence of temperature on seed germination rate in grain legumes. I. Acomparison of chickpea, lentil, soybean and cowpea at constant temperatures. J. Exp. Bot. 37, 705-715. doi: 10.1093/jxb/37.5.705

Craufurd, P. Q., Ellis, R. H., Summerfield, R. J., and Menin, L. (1996). Development in cowpea (Vigna unguiculata). 1. The influence of temperature on seed germination and seedling emergence. Exp. Agric. 32, 1-12. doi: 10.1017/S0014479700025801

Cui, K., Peng, S., Xing, Y., Xu, C., Yu, S., and Zhang, Q. (2002). Molecular dissection of seedling-vigor and associated physiological traits in rice. Theor. Appl. Genet. 105, 745-753. doi: 10.1007/s00122-002-0908-2

De Ron, A. M., Menéndez-Sevillano, M. C., and Santalla, M. (2004). Variation in primitive landraces of common bean (Phaseolus vulgaris L.) from Argentina. Genet. Resour. Crop Evol. 51, 883-894. doi: 10.1007/s10722-0051934-0

De Ron, A. M., Papa, R., Bitocchi, E., González, A. M., Debouck, D. G., Brick, M. A., et al. (2015). "Common bean," in Grain Legumes. Series: Handbook of Plant Breeding, ed A. M. De Ron (New York, NY; Dordrecht; Heidelberg; London: Springer), 1-36.

Dickson, M. H. (1971). Breeding beans, Phaseolus vulgaris L., for improved germination under unfavorable low temperature conditions. Crop Sci. 11, 848-850. doi: 10.2135/cropsci1971.0011183X001100060024x

Dickson, M. H., and Boettger, M. A. (1984a). Emergence, growth and blossoming of bean (Phaseolus vulgaris) at sub-optimal temperatures. J. Am. Soc. Hortic. Sci. 109, 257-260.

Dickson, M. H., and Boettger, M. A. (1984b). Effect of high and low temperatures on pollen germination and seed set in green beans. J. Am. Soc. Hortic. Sci. 109, 372-374. 
Dickson, M. H., and Petzoldt, R. (1987). Inheritance of low temperature tolerance in beans at several growth stages. Hortic. Sci. 22, 481-483.

Duc, G., Agrama, H., Bao, S., Berger, J., Bourion, V., Burstin, J., et al. (2015). Breeding annual legumes for adaptation to low input cropping systems and new areas: methods to approach more complex traits and target new variety ideotypes. Crit. Rev. Plant Sci. 34, 381-411. doi: 10.1080/07352689.2014.898469

Dutt, M., and Geneve, R. L. (2007). Time to radicle protrusion does not correlate with early seedling growth in individual seeds of impatiens and petunia. J. Am. Soc. Hortic. Sci. 132, 423-428.

Eichenberger, K., Gugerli, F., and Schneller, J. J. (2000). Morphological and molecular diversity of Swiss common bean cultivars (Phaseolus vulgaris L. Fabaceae) and their origin. Bot. Helv. 110, 61-77.

Ellis, R. H., Covell, S., Roberts, E. H., and Summerfield, R. J. (1986). The influence of temperature on seed germination rate in grain legumes. II. Interspecific variation in chickpea (Cicer arietinum L.) at constant temperature. J. Exp. Bot. 37, 1503-1515. doi: 10.1093/jxb/37.10.1503

Ellis, R. H., Hong, T. D., and Roberts, E. H. (1985). "Handbooks for Genebanks: no," in Handbook of Seed Technology for Genebanks, Vol. 2. Compendium of Specific Germination Information and Test Recommendations (Rome: International Board for Plant Genetic Resources (IBPGR)).

Escribano, M. R., De Ron, A. M., and Amurrio, J. M. (1994). Diversity in agronomical traits in common bean populations from Northwestern Spain. Euphytica 76, 1-6. doi: 10.1007/B. F.00024014

FAO-ISRIC-ISSS (1998). World Reference Base for Soil Resources. 84th World Soil Resources Reports, Food and Agriculture Organization of the United Nations, Rome.

Galván, M. Z., Menéndez-Sevillano, M. C., De Ron, A. M., Santalla, M., and Balatti, P. A. (2006). Genetic diversity among wild common beans from Northwestern Argentina based on morphoagronomic and RAPD data. Genet. Res. Crop Evol. 53, 891-900. doi: 10.1007/s10722-004-0981-20

Garcia-Huidobro, J., Monteith, J. L., and Squire, G. R. (1982). Time-temperature and germination of pearl millet (Pennisetum typhoides S \& H). J. Exper. Bot. 33, 288-296.

García-Pérez, A. (2005). Métodos Avanzados de Estadística Aplicada. Técnicas Avanzadas. Madrid: UNED.

Gioia, T., Logozzo, G., Attene, G., Bellucci, E., Benedettelli, S., Negri, V., et al. (2013). Evidence for introduction bottleneck and extensive inter-gene pool (Mesoamerica $\mathrm{x}$ Andes) hybridization in the European common bean (Phaseolus vulgaris L.) germplasm. PLoS ONE 8:e75974 doi: 10.1371/journal. pone. 0075974

González, A. M., Monteagudo, A. B., Casquero, P. A., De Ron, A. M., and Santalla, M. (2006). Genetic variation and environmental effects on agronomical and commercial quality traits in the main European market classes of dry bean. Field Crop Res. 95, 336-347. doi: 10.1016/j.fcr.2005.04.004

Gummerson, R. J. (1986). The effect of constant temperatures and osmotic potentials on the germination of sugar Beet. J. Exp. Bot. 37, 729-741. doi: 10.1093/jxb/37.6.729

Hampton, J. G., and Tekrony, D. M. (1995). Handbook of Vigour Test Methods. Zurich: International Seed Testing Association (ISTA).

Hanley, M. E., Unna, J. E., and Darvill, B. (2003). Seed size and germination response: a relationship for fire-following plant species exposed to thermal shock. Oecologia 134, 18-22. doi: 10.1007/s00442-002-1094-2

Hardwick, R. C. (1972). The emergence and early growth of french and runner beans (Phaseolus vulgaris L., and Phaseolus coccineus L.) sown at different dates. J. Hortic. Sci. 47, 395-410. doi: 10.1080/00221589.1972.11514483

Hardwick, R. C., and Andrews, D. J. (1980). Selection for cold tolerance in Phaseolus vulgaris, yield of selected lines grown in warm and cool environments. Ann. App. Biol. 95, 249-259. doi: 10.1111/j.1744-7348.1980. tb04744.x

Hernández-Nistal, J., Rodríguez, D., Nicolás, G., and Aldasoro, J. J. (1989). Abscisic acid and temperature modify the levels of calmodulin in embryonic axes of Cicer arietinum. Physiol. Plant. 75, 255-260 doi: 10.1111/j.13993054.1989.tb06177.x

Holmberg, S. A. (1973). Soybeans for cool temperature climates. Agric. Hortic. Gen. $31,1-20$.

Kaya, M., Kaya, G., Kaya, M. D., Atak, M., Saglam, S., Khawar, K. M., et al. (2008). Interaction between seed size and $\mathrm{NaCl}$ on germination and early seedling growth of some Torkish cultivars of chickpea (Cicer arietinum L.). J. Zhejiang Univ. Sci. B 9, 371-377. doi: 10.1631/jzus.B0720268

Kemp, G. A. (1973). Initiation and development of flowers in beans under suboptimal temperature conditions. Can. J. Plant Sci. 53, 623-627. doi: 10.4141/cjps73-122

Kemp, G. A. (1978). Growth of primary leaves of beans (Phaseolus vulgaris L.) under sub-optimal temperatures. Can. J. Plant Sci. 58, 169-174. doi: 10.4141/cjps78-025

Khajeh-Hosseini, M., and Rezazadeh. (2011). The electrical conductivity of soakwater of chickpea seeds provides a quick test indicative of field emergence. Seed Sci. Technol. 39, 692-696 doi: 10.15258/sst.2011.39.3.18

Kigel, J., Rosental, L., and Fait, A. (2015). "Seed physiology and germination of grain legumes," in Grain Legumes. Series: Handbook of Plant Breeding, ed A. M. De Ron (New York, NY; Dordrecht; Heidelberg; London: Springer), 327-363

Kolasinska, K., Szyrmer, J., and Dul, S. (2000). Relationship between laboratory seed quality tests and field emergence of common bean seed. Crop Sci. 40, 470-475. doi: 10.2135/cropsci2000.402470x

Kooistra, E. (1971). Germination of beans (Phaseolus vulgaris L.) at low temperatures. Euphytica 20, 208-213. doi: 10.1007/BF00056080

Kotowski, F. (1926). Temperature relation to germination of vegetable seed. Proc. Am. Soc. Hortic. Sci. 23, 176-184.

Mashiringi, N. A., and Harahwa, G. (1985). The response of twenty wheat (Triticum aestivum) varieties to growing conditions in Zimbabwe. Zimbabwe Agric. J. 82, 11-15.

Melo, L. C., Dos Santos, J. B., and Ramalho, M. A. P. (1997). Choice of parents to obtain common bean (Phaseolus vulgaris) cultivars tolerant to low temperatures at the adult stage. Braz. J. Genet. 20, 283-292.

Mohamed, H. A., Clark, J. A., and Ong, C. K. (1988). Genotypic differences in the temperature responses of tropical crops. I. Germination characteristics of groundnut (Arachis hypogaea L.) and pearl-millet (Pennisetum typhoides S \& L). J. Exp. Bot. 39, 1121-1128. doi: 10.1093/jxb/39.8.1121

Negri, V., and Tosti, N. (2002). Phaseolus genetic diversity maintained on-farm in central Italy. Genet. Res. Crop Evol. 49, 511-520. doi: 10.1023/A:1020902508166

Nonogaki, H. (2006). Seed germination - The biochemical and molecular mechanisms. Breed. Sci. 56, 93-105. doi: 10.1270/jsbbs.56.93

Olsen, S. R., and Dean, L. A. (1965). "Phosphorous" in Methods of Soil Science, ed C. A. Black (Madison, WI: American Society of Agronomy), 403-430.

Papa, R., Nanni, L., Sicard, D., Rau, D., and Attene, G. (2006). "The evolution of genetic diversity in Phaseolus vulgaris L.," in Darwin's Harvest: New Approaches to the Origins, Evolution and Conservation of Crops, eds T. J. Motley, N. Zerega, and H. Cross (New York, NY: Columbia University Press), 121-142.

Patterson, B. D., and Payne, L. A. (1983). Screening for chilling resistance in tomato seedlings. Hortic. Sci. 18, 340-341.

Peech, M., Alexander, L. T., Dean, L., and Reed, J. F. (1947). Methods of Soil Analysis for Soil Fertility Investigations. US Dept. of Agriculture Circ.

Porch, T. G. (2001). Genetics and Applications of Heat Tolerance in Common Bean. (Dissertation Ph.D.) thesis, Cornell University, Ithaca.

Porch, T. G. (2006). Application of stress indices for heat tolerance screening of common bean (Phaseolus vulgaris). J. Agron. Crop Sci. 192, 390-394. doi: 10.1111/j.1439-037X.2006.00229.X

Powell, A. A., Oliveira, D.e, M., A., and Matthews, S. (1986). The role of imbibition damage in determining the vigour of white and coloured seed lots of dwarf French beans. J. Exp. Bot. 37, 716-722. doi: 10.1093/jxb/37.5.716

Rainey, K. M., and Griffiths, P. D. (2005). Inheritance of heat tolerance during reproductive development in snap bean (Phaseolus vulgaris L.). J. Am. Soc. Hortic. Sci. 130, 700-706.

Rodiño, A. P., Gonzalez, A. M., Santalla, M., De Ron, A. M., and Singh, S. P. (2006). Novel genetic variation in common bean from the Iberian Peninsula. Crop Sci. 46, 2540-2546. doi: 10.2135/cropsci2006.02.0104

Rodiño, A. P., Monteagudo, A. B., De Ron, A. M., and Santalla, M. (2009). Ancestral landraces of common bean from the South of Europe and their agronomical value for breeding programmes. Crop Sci. 49, 2087-2099. doi: 10.2135/cropsci2008.07.0427

Rodiño, A. P., Santalla, M., De Ron, A. M., and Singh, S. P. (2003). A core collection of common bean from the Iberian Peninsula. Euphytica 131, 165-175. doi: 10.1023/A:1023973309788 
Rodiño, A. P., Santalla, M., Montero, I., Casquero, P., and De Ron, A. M. (2001). Diversity in common bean (Phaseolus vulgaris L.) germplasm from Portugal. Genet. Res. Crop Evol. 48, 409-417. doi: 10.1023/A:1012248002436

Rohlf, F. J. (2000). NTSYS-pc. Numerical Taxonomy and Multivariate Analysis. Exeter Software. New York, NY: Exeter Software.

Santalla, M., De Ron, A. M., and De La Fuente, M. (2010). Integration of genome and phenotypic scanning gives evidence of genetic structure in Mesoamerican common bean (Phaseolus vulgaris L.) landraces from the southwest of Europe. Theor. Appl. Genet. 120, 1635-1651. doi: 10.1007/s00122-010-1282-0

Santalla, M., De Ron, A. M., and Voysest, O. (2001). "European bean market classes," in Catalogue of Bean Genetic Resources, eds M. Amurrio, M. Santalla, and A. M De Ron (Pontevedra: PHASELIEU FAIR 3463-MBG-CSIC. Fundación Pedro Barrié de la Maza), 77-94.

Santalla, M., Rodiño, A. P., and De Ron, A. M. (2002). Allozyme evidence supporting South-western Europe as a secondary center of genetic diversity for common bean. Theor. App. Genet. 104, 934-944. doi: 10.1007/s00122-0010844-6

Schmuths, H., Bachmann, K., Weber, W. E., Horres, R., and Hoffmann, M. H. (2006). Effects of preconditioning and temperature during germination of 73 natural accessions of Arabidopsis thaliana. Ann. Bot. 97, 623-634. doi: $10.1093 / \mathrm{aob} / \mathrm{mcl} 012$

Schulte, E. E., and Hopkins, B. G. (1996). "Estimation of organic matter by weight loss- on ignition," in Soil Organic Matter Analysis and Interpretation, eds F. R. Magdoff, M. A.Tatabai, and E. A. Hanlon (Madison, WI: Soil Science Society of America), 21-31.

Scully, B., and Waines, J. G. (1987). Germination and emergence response of common and tepary beans to controlled temperature. Agron. J. 79, 287-291. doi: 10.2134/agronj1987.00021962007900020022x

Sicard, D., Nanni, L., Porfiri, O., Bulfon, D., and Papa, R. (2005). Genetic diversity of Phaseolus vulgaris L and P. coccineus L. landraces in central Italy. Plant Breed. 124, 464-472. doi: 10.1111/j.1439-0523.2005.01137.x

Singh, S. P., Gepts, P., and Debouck, D. G. (1991). Races of common bean (Phaseolus vulgaris Fabaceae). Econ. Bot. 45, 379-396. doi: 10.1007/BF02887079
Skrdla, W. H., and Mock, J. S. (1978). Evaluation of maize plant introductions for cold tolerance. Euphytica 27, 27-32. doi: 10.1007/BF00039116

Steel, R. G. D., Torrie, J. H., and Dickey, D. A. (1997). Principles and Procedures of Statistics. A Biometrical Approach. New York, NY: McGraw-Hill.

Wantanbe, H. (1953). Studies on the unfruitfulness of beans. 3. Influences of temperature on blooming and of relative humidity on the pollen activities of beans. J. Hortic. Ass. Jpn. 22, 172-176.

White, J. W., and Montes, C. (1993). The influence of temperature on seed germination in cultivars of common bean. J. Exp. Bot. 44, 1795-1800. doi: 10.1093/jxb/44.12.1795

Yacoob, R. K., and Filion, W. G. (1986). Temperature-stress response in maize: a comparison of several cultivars. Can. J. Genet. Cytol. 28, 1125-1131. doi: $10.1139 / g 86-156$

Zeven, A. C. (1997). The introduction of the common bean (Phaseolus vulgaris L.) into Western Europe and the phenotypic variation of dry bean collected in the Netherlands in 1946. Euphytica 94, 319-328. doi: 10.1023/A:10029402 20241

Zeven, A. C., Waninge, J., Van Hintum, T., and Singh, S. P. (1999). Phenotypic variation in a core collection of common bean (Phaseolus vulgaris L.) in the Netherlands. Euphytica 109, 93-106. doi: 10.1023/A:10036654 08567

Conflict of Interest Statement: The authors declare that the research was conducted in the absence of any commercial or financial relationships that could be construed as a potential conflict of interest.

Copyright (C) 2016 De Ron, Rodiño, Santalla, González, Lema, Martín and Kigel. This is an open-access article distributed under the terms of the Creative Commons Attribution License (CC BY). The use, distribution or reproduction in other forums is permitted, provided the original author(s) or licensor are credited and that the original publication in this journal is cited, in accordance with accepted academic practice. No use, distribution or reproduction is permitted which does not comply with these terms. 


\section{OPEN ACCESS}

Edited by:

Soren K. Rasmussen,

University of Copenhagen, Denmark

Reviewed by:

Michael A. Grusak,

USDA-ARS Children's Nutrition

Research Center, USA

Tom Warkentin

University of Saskatchewan, Canada

*Correspondence: Francesca Sparvoli sparvoli@ibba.cnr.it

Specialty section:

This article was submitted to Crop Science and Horticulture, a section of the journal

Frontiers in Plant Science

Received: 16 January 2016 Accepted: 10 June 2016

Published: 27 June 2016

Citation:

Sparvoli F, Laureati M, Pilu R, Pagliarini E, Toschi I, Giuberti G Fortunati P, Daminati MG, Cominelli E and Bollini R (2016) Exploitation of

Common Bean Flours with Low Antinutrient Content for Making Nutritionally Enhanced Biscuits.

Front. Plant Sci. 7:928.

doi: 10.3389/fpls.2016.00928

\section{Exploitation of Common Bean Flours with Low Antinutrient Content for Making Nutritionally Enhanced Biscuits}

\author{
Francesca Sparvoli ${ }^{1 *}$, Monica Laureati ${ }^{2}$, Roberto Pilu ${ }^{3}$, Ella Pagliarini ${ }^{2}$, Ivan Toschi ${ }^{3}$, \\ Gianluca Giuberti ${ }^{4}$, Paola Fortunati ${ }^{4}$, Maria G. Daminati ${ }^{1}$, Eleonora Cominelli ${ }^{1}$ and \\ Roberto Bollini ${ }^{1}$ \\ ${ }^{1}$ CNR, Institute of Agricultural Biology and Biotechnology, Milan, Italy, ${ }^{2}$ Department of Food, Environmental and Nutritional \\ Sciences, University of Milan, Milan, Italy, ${ }^{3}$ Department of Agricultural and Environmental Sciences - Production, Landscape, \\ Agroenergy, University of Milan, Milan, Italy, ${ }^{4}$ Alimentari e Ambientali, Facoltà di Scienze Agrarie, Istituto di Scienze degli \\ Alimenti e della Nutrizione, Università Cattolica del Sacro Cuore, Piacenza, Italy
}

Consumption of legumes is associated with a number of physiological and health benefits. Legume proteins complement very well those of cereals and are often used to produce gluten-free products. However, legume seeds often contain antinutritional compounds, such as phytate, galactooligosaccharides, phenolic compounds, lectins, enzyme inhibitors, whose presence could affect their nutritional value. Screening natural and induced biodiversity for useful traits, followed by breeding, is a way to remove undesirable components. We used the common bean cv. Lady Joy and the Ipa1 mutant line, having different seed composition for absence/presence of lectins, $\alpha$-amylase inhibitor, $(\alpha-\mathrm{Al})$ and phytic acid, to verify the advantage of their use to make biscuits with improved nutritional properties. We showed that use of unprocessed flour from normal beans (Taylor's Horticulture and Billò) must be avoided, since lectin activity is still present after baking, and demonstrated the advantage of using the cv. Lady Joy, lacking active lectins and having active $\alpha$-Al. To assess the contribution of bean flour to biscuit quality traits, different formulations of composite flours (B12, B14, B22, B24, B29) were used in combinations with wheat (B14), maize (gluten-free B22 and B29), or with both (B12 and B24). These biscuits were nutritionally better than the control, having a better amino acid score, higher fiber amount, lower predicted glycemic index (pGl) and starch content. Replacement of CV. Lady Joy bean flour with that of Ipa1, having a 90\% reduction of phytic acid and devoid of $\alpha$-Al, contributed to about a $50 \%$ reduction of phytic acid content. We also showed that baking did not fully inactivate $\alpha$-Al, further contributing to lowering the $\mathrm{pGl}$ of the biscuits. Finally, data from a blind taste test using consumers indicated that the B14 biscuit was accepted by consumers and comparable in terms of liking to the control biscuit, although the acceptability of these products decreased with the increase of bean content. The B22 gluten-free biscuits, although received liking scores that were just above the middle point of the hedonic scale, might represent a good compromise between health benefits (absence of gluten and lower pGl), expectations of celiac consumers and likeness.

Keywords: amino acid score, $\alpha$-amylase inhibitor, biscuits, consumers test, lectins, nutritional enhancement, phytic acid, predicted glycemic index 


\section{INTRODUCTION}

Legumes are well-recognized functional foods and their use as ingredients for food formulations is getting increasing attention (Vaz Patto et al., 2015). A number of studies has provided indications that consumption of legumes is associated with several physiological and health benefits, such as prevention of cardiovascular disease, obesity, diabetes mellitus, and cancer. These health benefits are attributed to high content in legume seeds of important soluble and insoluble fiber, to their slowly digestible starch properties as well as to prebiotic oligosaccharides, phenolic compounds and some proteins such as the lupin $\gamma$-conglutinin, and the soybean 7S globulin $\alpha^{\prime}$ chain (Duranti, 2006; Sparvoli et al., 2015). Some of these components regulate glycaemia and gastrointestinal function, whereas others provide antioxidant properties (Cardador-Martinez et al., 2002). In particular, due to their poor digestibility related to the inherent physical and structural properties of starch, legumes possess lower glycemic index (GI) when compared to cereal grains, a characteristic providing benefits for people with diabetes and/or cardiovascular disease (Hoover and Zhou, 2003; Sandhu and Lim, 2008). Moreover, being gluten-free, legumes could be used as ingredients to biofortify preparations for the celiac population.

Common bean (Phaseolus vulgaris L.) has significant cultural and historical importance as a staple food and is essential to human diets in many parts of the world. Bean seeds are a good source of energy, complex carbohydrates (dietary fibers, starch, and oligosaccharides), proteins, important minerals and vitamins (such as iron, zinc, B-vitamins, folates) as well as antioxidants and polyphenols required for human health. From a nutritional standpoint, bean seeds are higher in proteins than cereal grains (18-24 vs. $8-15 \%)$ and the amino acid profile of seed (storage) proteins well complements that of cereals, which are normally rich in sulfur amino acids and poor in lysine, tryptophan and threonine. However, seeds of common bean also contain a number of bioactive and/or antinutritional compounds, such as lectins, digestive enzyme inhibitors, phytate, galactooligosaccharides, phenolic compounds, whose presence could affect seed nutritional value (Sparvoli et al., 2015).

In bean seeds, major lectins are the erythroagglutinating and leucoagglutinating phytohemagglutinins (PHA-E and PHA-L, respectively). These PHAs, together with the evolutionary related $\alpha$-amylase inhibitor ( $\alpha$-AI), belong to the APA gene family of storage proteins and are inherited as a single Mendelian locus (Lioi et al., 2003). Bean $\alpha$-AI, also known as phaseolamin, is able to inhibit mammalian $\alpha$-amylases, thus inhibiting starch digestion by blocking access to a basic active site of the $\alpha$ amylase enzyme (Santimone et al., 2004). As $\alpha$-AI prevents the digestion of complex carbohydrates, it is widely used as basic active ingredient of commercial starch blocker preparations for the control of body weight (Barrett and Udani, 2011). However, $\alpha$-AI has also been shown to be effective in reducing postprandial plasma levels of glucose, insulin, C-peptide and gastric inhibitory polypeptide in healthy subjects as well as in individuals affected by diabetes mellitus (Layer et al., 1985, 1986).

Phytic acid (myo-inositol-1,2,3,4,5,6-hexakisphosphate) is highly negatively charged at physiological $\mathrm{pH}$ and easily precipitates in the form of phytate salts, binding important mineral cations such as iron, zinc, potassium, calcium, and magnesium. Monogastric animals, including humans, lack phytases in their digestive tract and fail to process the phytates present in seeds, thus phytic acid is poorly digested and decreases the nutritional value of the seeds (and derived foods) by limiting phosphorus and mineral bioavailability (Schlemmer et al., 2009).

The growing body of research on legumes health benefits has stimulated interest in increasing consumption of grain legumes and thus to expand their use in food products. In particular, biscuits are the largest category of snack items among baked food worldwide and they are one of the best ways to reach all segments of the population, due to their low manufacturing cost, convenience and long shelf life. For these reasons, biscuits represent a good way to propose composite flours (Chavan and Kadam, 1993).

Common beans have been regarded as useful ingredients for the production of snacks or baked products, like biscuits, bread or pasta, especially for the development of gluten-free products (Szafranski et al., 2005; Anton et al., 2009; Siddiq et al., 2013; Manonmani et al., 2014; Giuberti et al., 2015, 2016). Incorporation of common bean flour causes a positive impact on the levels of proteins, dietary fibers, resistant starch and predicted glycemic index, pGI (Anton et al., 2009; Giuberti et al., 2015, 2016). However, their use is limited by the presence of antinutritional factors. Consumption of raw or inadequately cooked beans is well known to cause poisoning, which clinical symptoms are extreme nausea, vomiting, diarrhea, severe acute gastroenteritis and intestinal malabsorption (Noah et al., 1980; Rodhouse et al., 1990, Petry et al., personal communication). The toxicity has been ascribed to the presence of active lectins (Kumar et al., 2013), that accumulate in substantial amounts in bean seeds. Thus, the use of bean flour needs typical processing involving cooking and grinding, or extrusion and grinding, both of which introduce a heating step that would inactivate enzyme inhibitors and lectins (Elkowicz and Sosulski, 1982; Alonso et al., 2000). Inactivation and/or removal of undesirable components are essential for improving bean overall nutritional quality and acceptability, aiming to favor their potentiality as human food and/or food ingredient.

Screening of natural and induced biodiversity for useful traits, followed by breeding, is a way for removal of undesirable components. Using this approach we identified genotypes showing differences in the content of PHA-E, PHA-L, $\alpha-\mathrm{AI}$ and phytic acid, and we used them as parents in breeding programs to produce lines with different sets of storage proteins (Confalonieri et al., 1992; Bollini et al., 1999; Campion et al., 2009; Figure 1).

Our purpose was to verify the advantage of using these genetic materials to make composite flours for the preparation of biscuits with improved nutritional properties. We based our work on the cv. Lady Joy and the lpa1 mutant line. The cv. Lady Joy has an active $\alpha$-AI and contains only low amounts of the so-called "pinto lectin," an almost inactive PHA whose presence in common beans has been reported with a frequency lower than 10\% (Pusztai et al., 1979, 1981; Voelker et al., 1986), consequently it has not erythroagglutinating activity (Figure 1C). The lpal line carries a mutation in the gene coding for the phytic acid transporter 


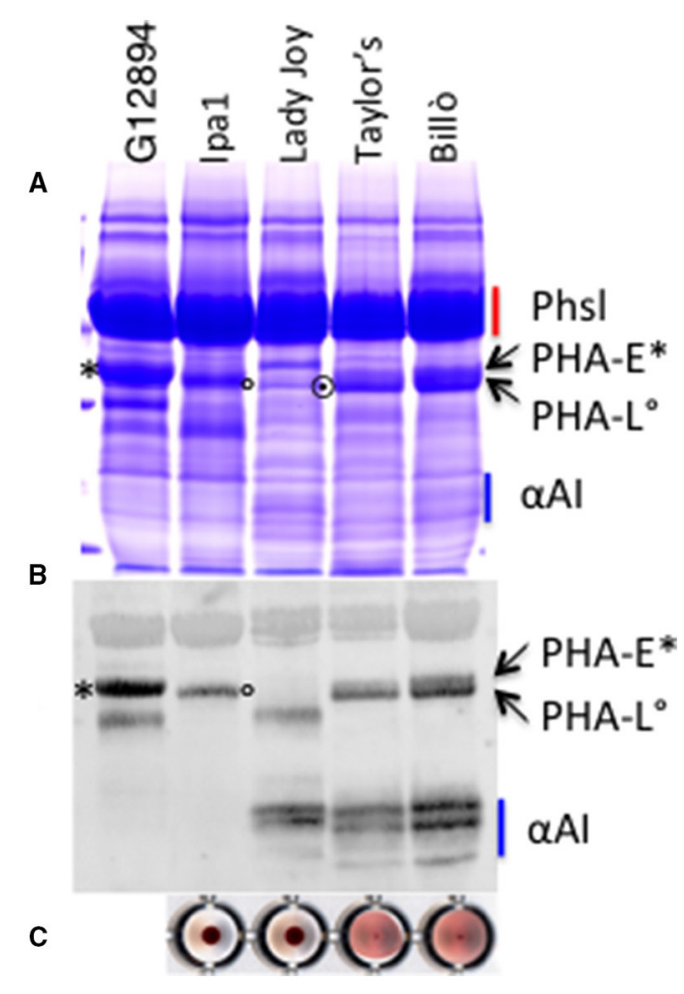

FIGURE 1 | SDS-PAGE analysis (A), immunoblot profiles (B), and hemagglutination test (C) of the seed extracts from the different genotypes used to prepare the bean containing biscuits. G12894, wild accession containing PHA-E. The major storage proteins are indicated. Red bar, Phsl, phaseolin polypeptides; blu bar, $\alpha$-Al; asterisk, PHA-E; circle, PHA-L; dotted circle, pinto lectin.

(Pvmrp1) and shows a reduction of seed phytic acid of about 90\% (Panzeri et al., 2011). Furthermore, this line is devoid of erythroagglutinating and $\alpha$-AI activities (lacks PHA-E and $\alpha$-AI), accumulating only low amounts of PHA-L (Figure 1B, compare lpa1 with G12984, a wild bean accession only containing PHAE, and the control cv. Taylor's Horticulture). We also used two wild type genotypes: the Italian landrace Billò, typically cultivated in Piedmont region (Piergiovanni and Lioi, 2010) and the cv. Taylor's Horticulture, that is the recurrent parent of the $\mathrm{cv}$. Lady Joy.

Here, we present data demonstrating the advantage of using the cv. Lady Joy, and the lpal line for the nutritional improvement of the biscuits. Having very low lectin activity or phytic acid content in their seeds, these bean flours do not need specific thermal treatments of the flour to inactivate PHA.

The resulting biscuits turned out to be nutritionally better than the control biscuit, having a better amino acid score, higher amount of fibers, lower starch content, and lower pGI. We also show that baking did not fully inactivate $\alpha$ AI, further contributing to lowering the pGI of the biscuits. Finally, we provide data from a consumer blind taste test using consumers showing that the best biscuit formulation (B14) had a good acceptability score, not significantly different from control biscuit.

\section{MATERIALS AND METHODS}

\section{Plant Materials}

Common bean whole flours were obtained by finely grinding the seeds with a Waring Commercial Blender (http://www.waringcommercialproducts.com/catalog.php?pcID =84_95\&products_id=296). Bean flours were used as such, as no processing involving cooking and grinding, or extrusion and grinding was applied prior their use. Seeds were obtained from genotypes differing for their PHA composition and/or presence or absence of phytic acid: (i) control genotypes: $\mathrm{cv}$. Taylor's Horticultural (Asgrow) and the Italian landrace Billò, a typical landrace of Cuneo, Piedmont region, both containing PHA-E, PHA-L, and $\alpha$-AI; (ii) cv. Lady Joy, lacking active PHA and containing $\alpha$-AI (Confalonieri et al., 1992); (iii) lpal mutant line, with $90 \%$ seed phytic acid reduction and lacking PHA-E and $\alpha$-AI (Campion et al., 2009). Commercial unprocessed flours of soft wheat ("00" type, http://www.molinochiavazza. it/page.php?pagina=7) and maize ("fioretto" type, http:// www.molinorossetto.com/it/farine-di-mais/1862-farina-fiorettogialla.html) were purchased from a local supplier.

\section{Preparation of Biscuits}

Biscuits were prepared by PRIMO PAN bakery (Battifollo, CN, Italy), based on the recipe of a typical Italian biscuit, from Cuneo region, called "Pasta di meliga" (http://www.primopan.com/ fogliemais.html). This typical biscuit is made with composite flour containing 67 and $33 \%$ of soft wheat and maize flours, respectively (Table 1, control). The ingredients for biscuits preparation included: $540 \mathrm{~g}$ of composite flour, $300 \mathrm{~g}$ raw cane sugar, $300 \mathrm{~g}$ butter, 2 medium eggs (ca. $110 \mathrm{~g}$ ), $2 \mathrm{~g}$ common salt, $14 \mathrm{~g}$ baking powder, natural vanilla flavor (Table 1). Biscuits were baked at $180^{\circ} \mathrm{C}$ for $12 \mathrm{~min}$. In addition to the control, five different types of biscuit samples were prepared (B12-B29) replacing maize and/or wheat with different amounts of common bean flours as described in Table 1.

\section{Hemagglutination Test}

Hemagglutinating activity in the extracts was estimated by a serial dilution procedure using human type A erythrocytes suspension (0.5\% in phosphate buffered saline buffer $\mathrm{pH} 7.0, \mathrm{PBS}$ ) adding $50 \mu \mathrm{l}$ of sample to the same volume of human erythrocytes. Agglutination was visually determined after $4 \mathrm{~h}$ incubation at room temperature. For each sample, serial dilutions in PBS, ranging from 1:10 to 1:1280, of seed and biscuit extracts were assayed. Results were recorded by monitoring how many $1: 1$ dilutions of the sample were necessary to prevent agglutination of red blood cells. Hemagglutinating unit (HAU) is defined as the reciprocal of the highest dilution still showing by eye agglutination of red cells, according to Trugo and von Baer (1998).

\section{Protein Extraction, SDS-PAGE and Immunoblot Analyses}

Total seed proteins extraction from dry bean seeds and biscuits, using 10 volumes of $20 \mathrm{mM}$ borate $\mathrm{pH}$ 9, and 
TABLE 1 | Main ingredients composition of biscuit samples.

\begin{tabular}{|c|c|c|c|c|c|c|c|c|c|c|c|}
\hline \multirow[t]{3}{*}{ Sample } & \multicolumn{6}{|c|}{ Flours composition } & \multirow[t]{3}{*}{ Total flours (g) } & & & & \multirow[t]{3}{*}{ Dough weight (g) } \\
\hline & \multicolumn{3}{|c|}{$\mathbf{g}$} & \multicolumn{3}{|c|}{$\%$ of dough weight } & & \multicolumn{3}{|c|}{ Other ingredients (g) } & \\
\hline & Wheat & Maize & Bean & Wheat & Maize & Bean & & Eggs & Butter & Sugar & \\
\hline Control & 360 & 180 & - & 29 & 14 & - & 540 & 110 & 300 & 300 & 1250 \\
\hline B12 & 240 & 150 & 150 & 19 & 12 & 12 & 540 & 110 & 300 & 300 & 1250 \\
\hline B14 & 360 & - & 180 & 29 & - & 14 & 540 & 110 & 300 & 300 & 1250 \\
\hline B22 & - & 270 & 270 & - & 22 & 22 & 540 & 110 & 300 & 300 & 1250 \\
\hline B24 & 120 & 120 & 300 & 10 & 10 & 24 & 540 & 110 & 300 & 300 & 1250 \\
\hline B29 & - & 180 & 360 & - & 14 & 29 & 540 & 110 & 300 & 300 & 1250 \\
\hline
\end{tabular}

separation by electrophoresis on an $15 \%$ sodium dodecylsulfatepolyacrylamide gel (SDS-PAGE) were as described by Bollini and Chrispeels (1978). Gels were stained with Coomassie Brilliant Blue R-250 or blotted on a supported nitrocellulose membrane (Hybond-C, GE-Healthcare). Immunoblot analysis was performed according to Burnette (1981) using rabbit antibodies against $P$. vulgaris recombinant $\alpha$-AI at 1:1000 dilution. These antibodies allow the detection of both PHA and $\alpha$-AI (Ceriotti et al., 1989). Peroxidase-linked anti-rabbit IgG was used as the secondary antibody.

\section{Assay of $\alpha$-Amylase Inhibitor Activity}

Inhibitory activity against human salivary $\alpha$-amylase (EC 3.2.1.1; Type IX-A) was measured by the increase of iodine staining after the action of the $\alpha$-amylase enzyme on soluble starch as described in Altabella and Chrispeels (1990). Briefly, different volumes (from 10 to $100 \mu \mathrm{l}$ ) of biscuit flour or seed extracts diluted 50 or 200 fold in $20 \mathrm{mM}$ borate buffer $\mathrm{pH} 9$, respectively, were preincubated with a fixed amount of $\alpha$-amylase $(0.15 \mathrm{U})$ for $30 \mathrm{~min}$ at room temperature in a final volume reaction of 300 $\mu l$. Then, $200 \mu \mathrm{l}$ of a $0.15 \%$ solution of potato starch was added and after $5 \mathrm{~min}$ at room temperature the reaction was stopped by adding $1 \mathrm{ml}$ of Iodine reagent (Varner and Mense, 1972) and absorbance was measured at $620 \mathrm{~nm}$. Results were expressed as units of $\alpha$-amylase inhibited per mg of flour, where one unit of inhibitor activity is the amount which will bring about $50 \%$ inhibition of the $\alpha$-amylase in 30 min under the above conditions according to Marshall and Lauda (1975).

\section{Determination of Phytic Acid Phosphate}

Phytic acid phosphate (PAP) fraction was determined by a ferric precipitation method (Pilu et al., 2003) and expressed as $\mu \mathrm{g} / \mathrm{mg}$ of sample. The amount of corresponding phytate level was then obtained by multiplying these values for the conversion factor 3.5484 .

\section{Bromatological Analysis and In Vitro Starch Digestion of Experimental Biscuits}

The chemical composition of biscuit samples was assayed according to AOAC standard methods (AOAC, 2000). Samples were milled and analyzed for dry matter (DM), ash, crude protein (CP), and ether extract (EE); starch content was measured enzymatically. Neutral detergent fiber (NDF), acid detergent fiber
(ADF), and acid detergent lignin (ADL) were determined using the filter bag system (ANKOM Technology, New York, USA). In particular, NDF was assessed according to the procedure of Mertens (Mertens et al., 2002), while ADF and ADL were determined according to Vansoest et al. (1991). Gross energy was measured using an adiabatic calorimeter (IKA 4000, Staufen, Germany). Total amino acid composition was evaluated on defatted flours according to Commission Regulation (EC) No $152 / 2009$ of 27 January 2009 laying down the methods of sampling and analysis for the official control of feed.

The multi-enzymatic protocol detailed by Giuberti et al. (2015) was used to evaluate the in vitro starch digestion of biscuits over time. The method, based on a 2-step enzymatic digestion incorporating a gastric $(\mathrm{pH}=2)$ and a pancreatic $(\mathrm{pH}=5.2)$ phase, has been shown to predict GI values with good accuracy for several cereal- and legume-based foods. Briefly, milled samples ( $800 \mathrm{mg}$ ) were carefully inserted into $50 \mathrm{ml}$ tubes containing glass balls and pre-treated with a $0.05 \mathrm{M} \mathrm{HCl}$ solution $(5 \mathrm{ml})$ containing pepsin $(5 \mathrm{mg} / \mathrm{ml}$; Sigma P-7000, Sigma Aldrich Co., Milan, Italy) for $30 \mathrm{~min}$ at $37^{\circ} \mathrm{C}$ under agitation. After the simulation of the gastric digestion, the $\mathrm{pH}$ of the solution was adjusted to 5.2 by adding $20 \mathrm{ml}$ of $0.1 \mathrm{M}$ acetate buffer. Then, $5 \mathrm{ml}$ of an enzyme mixture (amylase activity of about 7000 $\mathrm{U} / \mathrm{ml}$; Giuberti et al., 2015) given by pancreatin (Merck 7130; Merck KGaA, Darmstadt, Germany), amyloglucosidase (Sigma A-7095; Sigma Aldrich Co., Milan, Italy) and invertase (Sigma I-4504; Sigma Aldrich Co., Milan, Italy) enzymes was added. From each tube, aliquots were carefully taken at 0 (prior to the enzyme addition simulating the pancreatic phase) and at $30,60,120$, and $180 \mathrm{~min}$ after the enzyme addition. To each collected aliquot, absolute ethanol was added in order to stop the enzyme hydrolysis and the amount of released glucose was determined colorimetrically (glucose oxidase kit GODPOD 4058; Giesse Diagnostic snc, Rome, Italy). Commercial fresh white bread (starch content of $72.3 \% \mathrm{DM}$ ) was used as reference and a blank was also included to correct for the glucose in the amyloglucosidase solution. The percentage of digested starch at each time interval was calculated using a factor of 0.9 to convert mono to polysaccharides. For each treatment, samples were analyzed in duplicate. After the enzyme digestion, a hydrolysis index (HI) was derived from the ratio between the area under the hydrolysis curve (AUHC) of each biscuit samples and the corresponding AUHC of the reference fresh white bread as 
a percentage over the same period. From the obtained HI, a predicted GI value was then calculated with the formula pGI = $8.198+0.862 \times$ HI (Granfeldt, 1994).

\section{Consumer Test}

The acceptability of the six different types of biscuits was assessed involving 102 subjects (55 females and 47 males, age: mean $=24.0$; sd $=5.3$ ). Consumers were recruited according to their liking for and regular consumption of biscuits. These subjects were asked to taste one biscuit of each sample, and to express their liking according to a $100-\mathrm{mm}$ linear hedonic scale anchored at the extremes with "dislike extremely" (left of the scale, score $=0$ ) and "like extremely" (right of the scale, score $=100$ ). In order to balance the effects of serving order and carry-over, samples presentation order was systematically varied over participants (Macfie et al., 1989). Samples were served at room temperature (about $20^{\circ} \mathrm{C}$ ) in plastic plates coded with 3digit numbers and evaluated in individual booths in white light conditions. Participants were asked not to smoke, eat or drink anything, except water, for $1 \mathrm{~h}$ before the tasting sessions.

The study complied with the principles established by the Declaration of Helsinki and the protocol was approved by the Ethics Committee of the University of Milan. Written informed consent was obtained from each subject before the liking assessment was performed.

\section{RESULTS}

\section{Evaluation of Erythroagglutinating Activity in Bean Based Biscuits}

Usually, bean seeds accumulate substantial amounts of PHA (both L and E types) and $\alpha$-AI. Since consumption of raw or inadequately cooked beans may cause poisoning, to verify the role of baking on PHA activity we used different type of bean flours with or without PHAs. We compared different bean genotypes: (i) cv. Lady Joy flour, devoid of active lectin, (ii) its parental line, cv. Taylor's Horticulture, containing both PHA$\mathrm{E}$ and PHA-L, (iii) another commercial landrace, Billò, also containing both PHA-E and PHA-L, (iv) the lpal genotype, containing only PHA-L (not erythroagglutinating).

Biscuits were prepared according to the recipe of a typical Italian biscuit, from Cuneo region, called "Pasta di meliga," in which we replaced maize with bean (B14 formulation, Figure 2, Table 1).

The presence of active PHA was assessed using the erythroagglutination test (Figure 3). Results show that after baking, extracts of biscuits containing PHA (cv. Taylor's Horticulture and Billò) are still able to agglutinate erythrocytes after a serial dilution between 1:80 and 1:160 (Figure 3, black arrows). Three minutes of overbaking (baking from 12 to $15 \mathrm{~min}$ ) decreased the erythroagglutinating activity to $1: 40$ dilution (Figure 3, black arrowhead), confirming that proper heat treatment is needed for inactivation. As expected, no agglutination was detected on extracts of biscuits containing $\mathrm{cv}$. Lady Joy or lpal flours.

To quantify the residual PHA activity we compared the agglutination results with those made on corresponding amounts

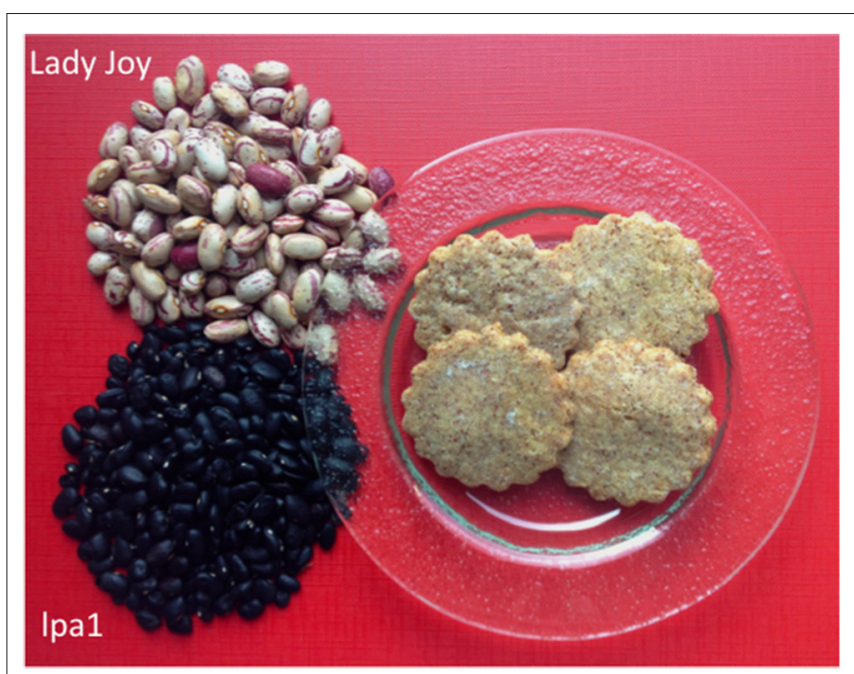

FIGURE 2 | Appearance of bean biscuits and bean seeds of the cv. Lady Joy and Ipa1 line.

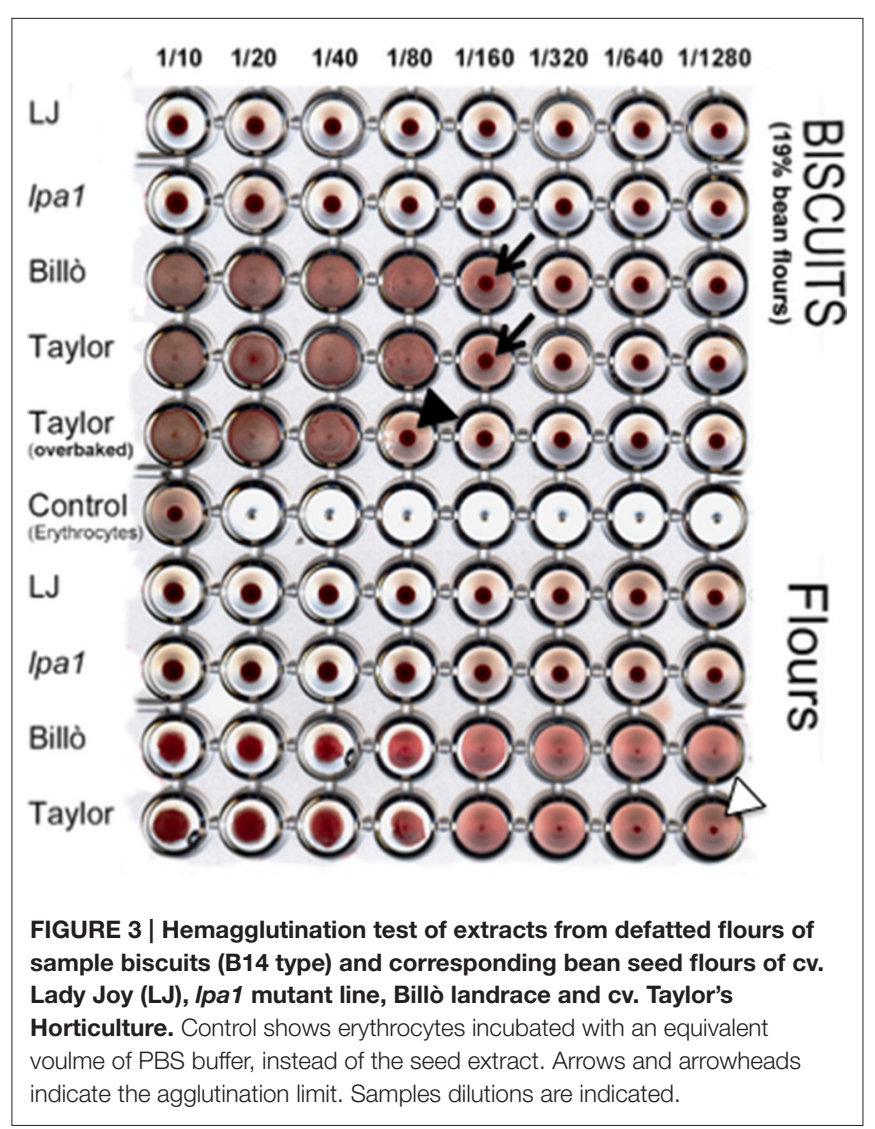

of unprocessed bean flours. We calculated that defatted extracts of B14 biscuits contain about 19\% of bean flour. Since at least three more 1:1 dilutions are needed to abolish agglutination in equivalent cv. Taylor's Horticulture and Billò extracts (Figure 3, white arrowhead), we estimated that the residual PHA activity in these biscuits was in the range of $5-10 \%$. 


\section{Bean Biscuits Formulation}

To assess the contribution of bean flour to biscuits quality traits, different formulations of composite flours were used in which wheat and/or maize flours were replaced by bean flour in the recipe of the biscuit "Pasta di meliga" (Table 1). To avoid any risk of poisoning due to residual active PHA after baking, we used the flour of cv. Lady Joy, which contains low amounts of the nontoxic "pinto lectin" and has an active $\alpha$-AI. Since celiac disease has been associated with high incidences of diabetes mellitus (Lamacchia et al., 2014) and there is considerable interest in lowering the GI of gluten free foods for the maintenance of a good glycemic control, two types of biscuits, in which bean and maize flours completely replaced that of wheat (B22 and B29, Table 1), were also prepared.

Composite flours contained different percentages of bean flour: 26.7, 32.1, 50.0, 53.6, and 64.3\% (B12, B14, B22, B24, $\mathrm{B} 29$, respectively), in combinations with wheat (B14), maize (B22 and B29), or with both wheat and maize flours (B12 and B24). Other ingredients included eggs, butter and sugar cane (Table 1). Biscuits were baked for $12 \mathrm{~min}$ at $180^{\circ} \mathrm{C}$.

In order to assess the effect of baking on bean proteins, biscuit protein extracts were analyzed by SDS-PAGE (Figure 4). Results showed that in all the formulations the protein profile was similar to that of cv. Lad Joy seed extract, with phaseolin being the most abundant protein, as in common bean flours (Figure 4, compare LJ with B12, B14, B22, B24, B29). This result indicates that in the biscuits bean proteins have undergone poor hydrolysis.

\section{Analysis of $\alpha$-Amylase Inhibitor Activity and Phytic Acid Content in Biscuits}

To verify the role of baking also on $\alpha$-AI, its activity was analyzed in defatted extracts of B12, B14, B22, B24, B29 and control ("Pasta di meliga") biscuits as well as in B14 biscuits made with flours of the cv. Taylor's Horticultural (Lady Joy recurrent parent) and the landrace Billò.

No relevant activity was present in control biscuits (not containing bean flour), while consistent $\alpha$-AI activity was measured in extracts of all biscuit types containing bean flours (Table 2). We observed that the level of $\alpha$-AI activity did not correlate with the amount of bean flour in the biscuits. In fact, the highest $\alpha$-AI activity was recorded in the B22 biscuit, which is not the one with the highest bean content. We then compared $\alpha$-AI activities of the different biscuits with those present in the corresponding raw bean flours. Data showed that samples B12, B24, and B29 retained a $\alpha-A I$ activity between 49.4 and $62.5 \%$, while higher activities were detected in samples B14 and B22, having 100 and $75.9 \%$ of the expected $\alpha$-AI activities, respectively (Table 2).

We assayed $\alpha$-AI activity also on extracts of B14 biscuits made with cv. Taylor's Horticulture and Billò flours. These biscuits retained about $34 \%$ of the starting $\alpha$-AI activity, while overbaking reduced $\alpha$-AI activity to about $10 \%$. As a control, we measured the $\alpha$-AI activity in the cv. Lady Joy B14 sample and confirmed that $100 \%$ of $\alpha$-AI activity was present (Table 2).
Phytic acid is a well-known antinutrient and is very stable to heat treatment. The use of flours almost devoid of phytic acid could therefore significantly improve the nutritional quality of baked products. To evaluate this point we quantified phytic acid in extracts of B14 biscuits made with flour of the lpal mutant, which is almost devoid of phytic acid, or with those of cv. Lady Joy, cv. Taylor's Horticulture, having normal phytic acid content (Table 3).

Results showed that biscuits made using lpal flour contained less phytic acid $(1.91 \mathrm{mg} / \mathrm{g})$ than biscuits made with flours of the other two genotypes ( 4.63 and $3.78 \mathrm{mg} / \mathrm{g}$ ). The reduction is $58.7 \%$ compared to phytic acid content of biscuits made with cv. Lady Joy, and $49.5 \%$ if compared to biscuits made with cv. Taylor's Horticulture (Table 3).

\section{Proximate Composition, In Vitro Predicted Glycemic Index Classification and Amino Acid Composition}

The different biscuits were also analyzed for their proximate composition, pGI, and total amino acid content (Tables 4, 5, respectively). Compared to control biscuits, the addition of bean flour increased protein content, from a minimum of 14\% (B12) up to $45.7 \%$ (B29), as well as ashes, neutral detergent fiber (NDF), acid detergent fiber (ADF) and, to a lower extent, lignin and crude fiber. The addition of bean flour to the recipe also affected starch content, which decreased from a minimum of 25.8 (B12) to $37.0 \%$ (B29) (Table 4). Lastly, pGI values (calculated using commercial white wheat bread as reference; pGI $=94.4$ ) decreased with the addition of bean flour in the composite, ranging from 88.9 to 61.9 for control and B29 biscuits, respectively.

As we found that B14 biscuits retain almost $100 \%$ of the activity of the $\alpha$-AI present in bean flour, we compared pGI values of B14 biscuits made with different bean flours. In order to estimate the contribution of the active $\alpha$-AI to the pGI, we used as a reference the pGI of biscuits made with the lpa 1 mutant, which is devoid of $\alpha$-AI (Table 2). Our results showed that B14 biscuits containing active $\alpha$-AI have lower pGI values than B14 biscuits made with flour of the lpal mutant, thus indicating that active $\alpha$-AI slightly contributes to the decrease of the pGI value (Table 4).

The presence of bean flour in the composite flours impacted also the amino acid composition of the biscuits: the highest effect was on the content of the essential amino acids Lys, Trp, and Arg, which compared to control biscuits raised one to two fold for Lys (118-275\%), 39-134\% for Trp, and 84-108\% for Arg. The average (B12-B29) increase for all the remaining amino acids was ranging from about $30 \%$ (Leu) up to $53 \%$ (Phe). The only slight decrease was observed for Glu, whose content decreased between 1.5 and $17.3 \%$ (B12, B22, B24, and B29) with the exception of B14 ( $16.7 \%$ increase), suggesting that wheat significantly contributes more than maize to the content of this amino acid (compare B14 with B29). The overall content of sulfur amino acids (Met and Cys) was not very much affected by bean flour addition and their average content increased between 7.6 and 13.3\% (B12-B29) with Met contributing more than Cys (average 30\% more and 6\% minus, respectively; see Table A1). 


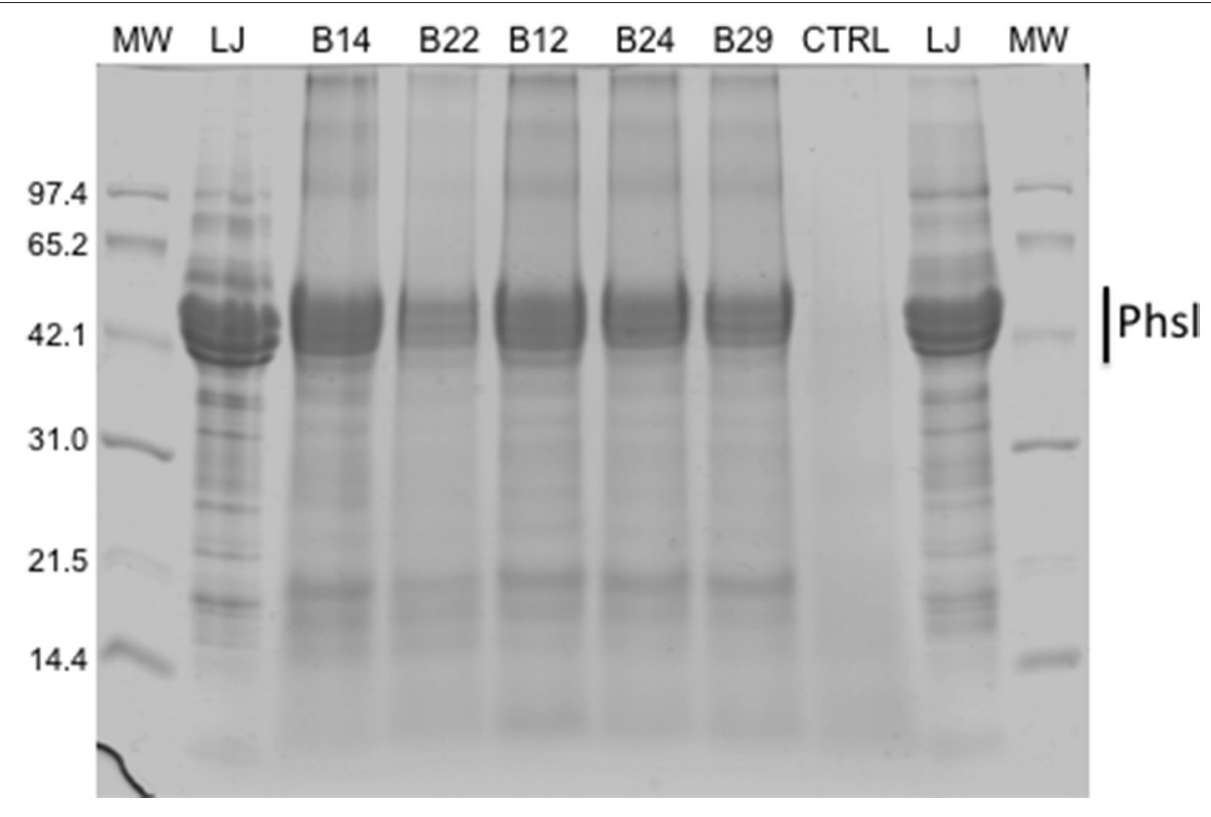

FIGURE 4 | SDS-PAGE analysis of extracts from defatted flours of sample biscuits (B14-B29, CTRL, control biscuit) and cv. Lady Joy seed extract (LJ). MW molecular weight markers. Bar indicates phaseolin polypeptides (Phsl).

TABLE 2 | $\alpha$-amylase inhibitory activity in extracts of defatted biscuit flours and bean flours of cv. Lady Joy (LJ), cv. Tayolor's Horticultural (TH), Billò (B), the Ipa1 mutant line, and over baked (OB).

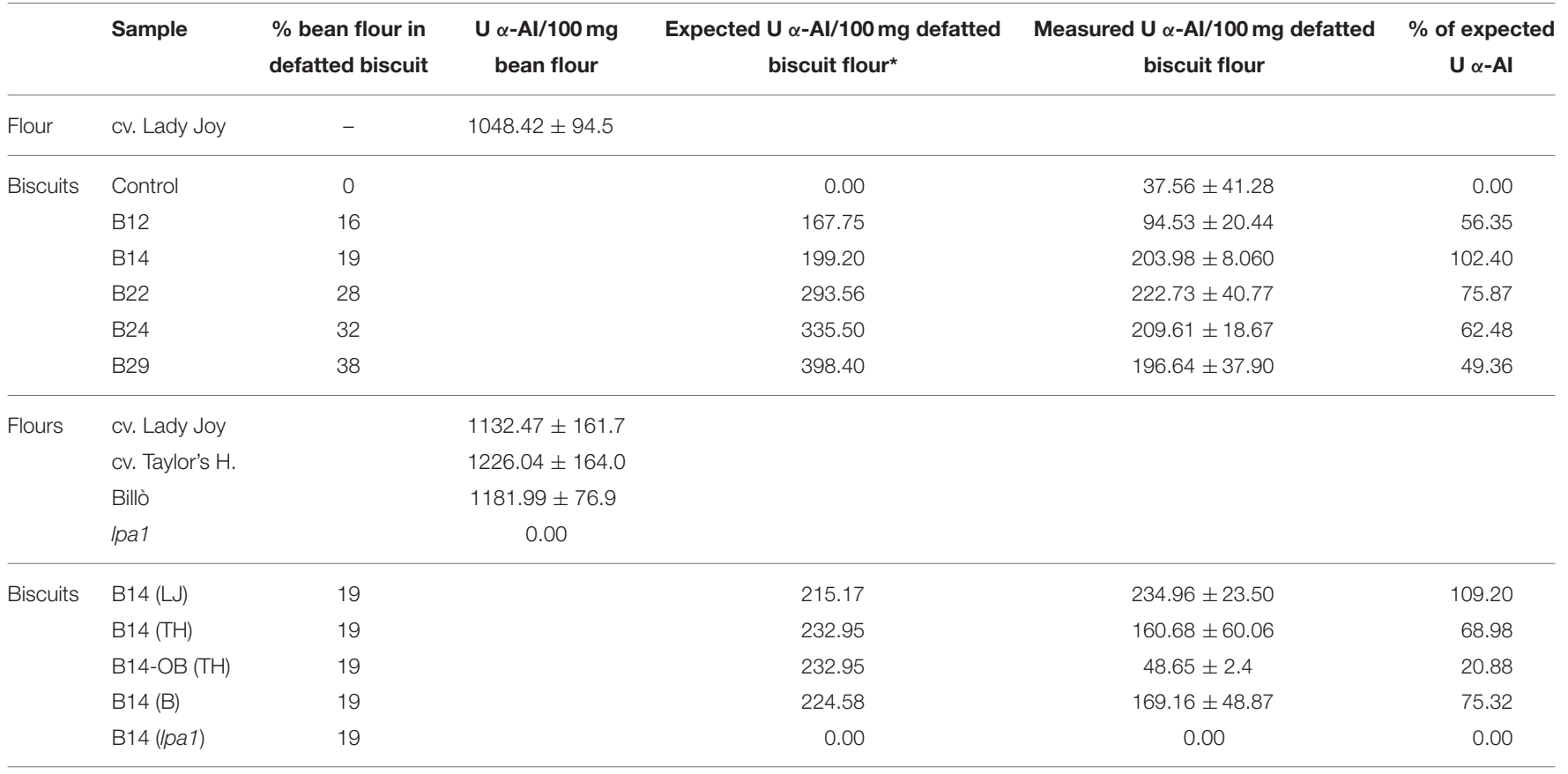

Units of inhibited $\alpha$-amylase are per $100 \mathrm{mg}$ of flours $(U \alpha$-Al/100 mg). *Values are the product of $\alpha$-Al activity in bean flour $x$ the percent of bean flour in defatted biscuit. B12-B29: see Table 1.

\section{Consumer Test}

The introduction of a new ingredient, the bean flour, to a conventional food, such as biscuits, could result in a product that might not fully meet the acceptability of the consumers. To verify the liking of the biscuits containing increasing amounts of cv. Lady Joy bean flour, their acceptability was tested in comparison with the traditional "Pasta di meliga" (control), in a blind taste test on a consumer sample of 102 subjects. Hedonic data were subjected to ANOVA considering samples $(n=6)$ as source of variation and liking scores as dependent variable. 
TABLE 3 | Phytic acid (PA) content in biscuit samples (defatted flours) and bean flours of the Ipa1 mutant line, cv. Lady Joy and cv. Tayolor's Horticultural.

\begin{tabular}{llr}
\hline & Sample & PA $\mathbf{~ g / / g}$ flour \\
\hline Biscuits & B14 (Ipa1) & $1.91 \pm 0.03$ \\
& B14 (LJ) & $4.63 \pm 0.81$ \\
& B14 (Taylor's H.) & $3.78 \pm 0.65$ \\
\hline \multirow{2}{*}{ Flours } & lpa1 & $2.38 \pm 0.05$ \\
& Lady Joy cV. & $9.32 \pm 0.23$ \\
& Taylor's H. cV. & $10.53 \pm 1.01$ \\
\hline
\end{tabular}

Mean hedonic values for the six biscuits (one control and five containing different amounts of bean flour) are reported in Table 6. ANOVA results showed a significant effect of the factor samples $\left[F_{(5,505)}=11.52, p<0.001\right]$ on liking ratings. LSD post-hoc comparison indicated that the B14 biscuits received an acceptability score that was statistically comparable to the control biscuit, and the acceptability of B12 biscuits, although statistically different from the control, was not statistically different from that of B14 biscuits, indicating that these two types were well liked by consumers. However, liking scores decreased with the increase of the bean flour content as the B22 (gluten-free) and B24 formulations were significantly less liked and received liking scores that were just above the middle point of the hedonic scale, although they were not significantly different from the B12 sample. The B29 biscuit, gluten-free but with the highest addition of bean flour, was the least liked sample.

\section{DISCUSSION}

The increasing awareness of the link between diet and health is the driving force to develop novel foods to meet consumer's needs, including the identification, development and improvement of new ingredients for food formulations.

In this work we assessed the advantage of using flours of nutritionally enhanced common bean genotypes for the production of bean based biscuits, with the aim to contribute to the diversification of healthier and more nutritional diet.

\section{Importance of Seed Nutrients Genetic Modulation for Biscuits Quality and Properties}

A number of studies reported improved nutritive value of high protein biscuits made using composite flours, including different amounts of flour from soybean, field pea, lentil, chickpea, lupin, and common bean (Shrestha and Noomhorm, 2002; Rababah et al., 2006; Zucco et al., 2011). In general, the consumption of common bean has not gone beyond the traditional processing and uses, due to the antinutritional factors present in the seeds. Extrusion, thermal treatments, such as roasting, and soaking at high temperature, followed by cooking and dehydration, are often used to eliminate lectin and enzyme inhibitors activities (Hoojjat and Zabik, 1984; Costa et al., 2006; Boye et al., 2010; Kelkar et al., 2012; Siddiq et al., 2013). However, these

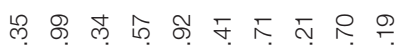

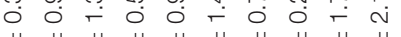

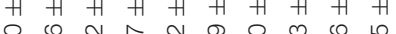

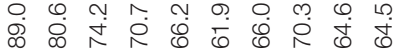

잉

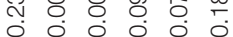

H H

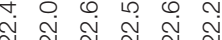

N N N N

\& مิ ชิ

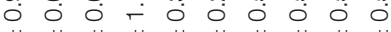

$\mathrm{H}+\mathrm{H}+\mathrm{H}+\mathrm{H}+\mathrm{H}+\mathrm{H}+\mathrm{H}+\mathrm{H}+\mathrm{H}$

字夜

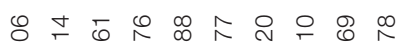

O

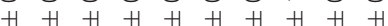

ஸ̣

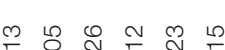

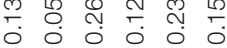

H H H H H H व व व व

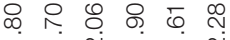

ᄃ. $8.0 \%$ ก

$\begin{array}{llllll}1 & 0 & 0 & 0 & 0 & 0\end{array}$

$H$ H H H H H o g व व

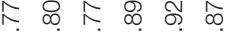

$\begin{array}{llllllll}0 & 0 & 0 & 0 & 0 & 0\end{array}$

ㄷ․

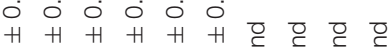

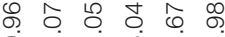

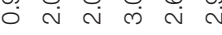

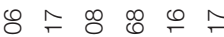

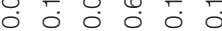

$\mathrm{H} H$ H H H H o व व व

ㅇ \&

$-\dot{N} \dot{\nabla} \dot{m}$

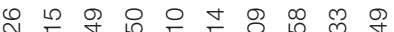

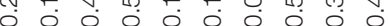

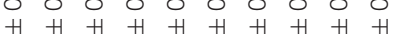
$\begin{array}{llllllllll}H & + & 0 & 0 & 0 & 0 & 0 & 0 & 0 & 0 \\ H & H & 0 & 0 & 0\end{array}$

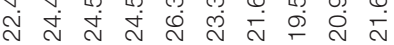

8 ก $\begin{array}{llllllllll}0 & 0 & 0 & 0 & 0 & 0 & 0 & 0 & 0 & 0\end{array}$ $\begin{array}{lllllllllllllll}H & H & H & H & H & H & H & H & H & H\end{array}$

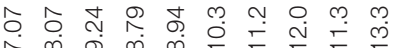

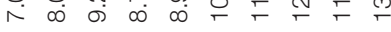

\& 8 8 \% $\begin{array}{llllllllll}0 & 0 & 0 & 0 & 0 & 0 & 0 & 0 & 0 & 0\end{array}$ స్ r a n a n a r a r

\section{ஜ}

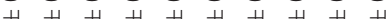
- 0 H 0 \%

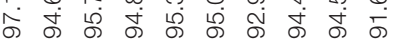

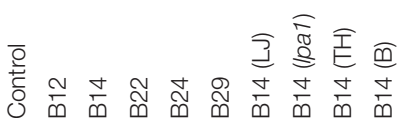




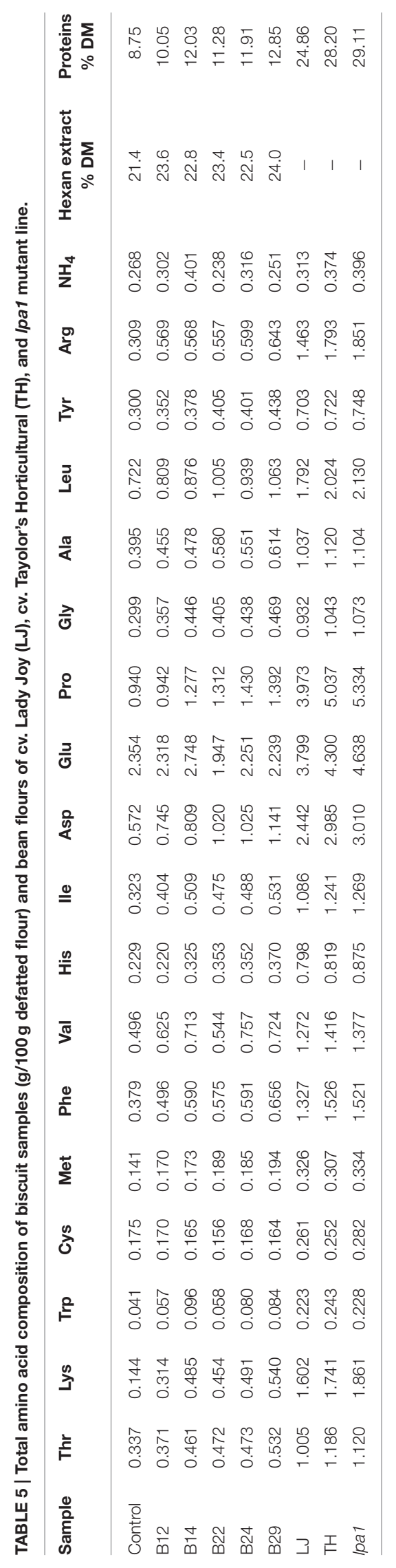

TABLE 6 | Results of the consumer test $(n=102)$ for evaluating the acceptability of the different bean biscuits compared to the control biscuit "Pasta di meliga."

\begin{tabular}{lll}
\hline Sample & Mean \pm SEM & LSD post-hoc groups \\
\hline Control & $69.8 \pm 2.3$ & $\mathrm{~d}$ \\
B12 & $60.7 \pm 2.5$ & $\mathrm{bc}$ \\
B14 & $64.6 \pm 2.5$ & $\mathrm{~cd}$ \\
B22 & $54.5 \pm 2.5$ & $\mathrm{~b}$ \\
B24 & $55.1 \pm 2.7$ & $\mathrm{~b}$ \\
B29 & $45.5 \pm 2.7$ & $\mathrm{a}$ \\
\hline
\end{tabular}

Identical letters indicate there is no statistically significant difference between the samples according to LSD post-hoc test. Consumers liking was expressed according to a 100-mm linear hedonic scale anchored at the extremes with "dislike extremely" (left of the scale, score $=0$ ) and "like extremely" (right of the scale, score $=100$ ).

types of processing are a source of additional costs for the producers and may lead to undesirable functional and sensory properties of the common bean flours (Batista et al., 2010). As a consequence, common bean is not a very common legume for flour fortification, despite its good protein and micronutrient content.

Here, we recommend the use of the cv. Lady Joy, containing an almost inactive and low abundant PHA (Pusztai et al., 1981; Confalonieri et al., 1992), to avoid any possible risk of lectin poisoning due to frequent and abundant consumption of these bean biscuits. In fact, we provide evidence that biscuits made with bean flours containing active PHA (Taylor's $\mathrm{H}$. and Billò) still retain residual lectin activity (about $5-10 \%$ of the lectin in the original flours), while no agglutination is detected in biscuits made using flour of the cv. Lady Joy (Figure 4).

Another advantage of avoiding preventive thermal treatments of the bean flours (possible if using the cv. Lady Joy) was the finding that substantial levels of $\alpha$-AI activity were retained in the bean biscuits, with the B14 (LJ) type showing the highest levels (Table 2). It is known that $\alpha$-AI and, in general, storage proteins belonging to the $P$. vulgaris lectin family, are stable proteins resistant to thermal degradation (Sparvoli et al., 1997). Nevertheless, the finding that cv. Lady Joy biscuits B14 retained such a high $\alpha$-AI activity was unexpected. A possible explanation could be that dry heating, as it occurs during baking, induces changes in the physicochemical properties of the dough and consequently of its components, causing enhancement of the $\alpha$ AI activity as has been shown for microwave treatment of beans (Oomah et al., 2014). The presence of active $\alpha$-AI was confirmed in cv. Taylor's Horticulture and Billò B14 biscuits, although to a lesser extent. This might be due to the fact that these two genotypes contain a different $\alpha$-AI isoform that might differently respond to heat treatments (Bollini et al., 1999).

\section{Biofortified Bean Biscuits: Improved Protein Content and Amino Acid Score, and Reduced Phytic Acid Content}

All biscuit formulas with composite bean flours showed a better nutritional composition, compared to the control, i.e., higher levels of protein and fiber, better amino acid score, and lower 
starch content along with a reduction in the pGI values. A similar behavior should be expected in food preparation containing different percentage of legume flour, as already reported for rice spaghetti, semolina spaghetti and teff tagliatelle prepared with increasing levels of bean flour (Gallegos-Infante et al., 2010; Giuberti et al., 2015, 2016). In addition, present findings are consistent with data obtained by other authors (Rababah et al., 2006; Zucco et al., 2011). For example, fortification with $12 \%$ of broad bean flour resulted in a protein increase from 16.6 to $20.2 \%$ (Rababah et al., 2006). Our results also indicate that the presence of maize in composite flours decreased biscuit protein value, as B14 biscuits, not containing maize, have higher protein content than B22-B29 samples (Tables 4, 5). Indeed, this is in accordance to the fact that wheat grains have average protein content higher than that of maize, traditionally an oil crop.

The nutritional enhancement of bean-containing biscuits was evident also for the amino acid composition, as the addition of bean flour increased the content of limiting essential amino acids (Lys, Thr, Trp) without lowering the content of sulfur amino acids. Furthermore, the replacement of cv. Lady Joy bean flour with that of $l_{p a 1}$ contributed to about a $50 \%$ reduction of phytic acid content in B14 biscuit formulations. Nutritional studies performed on volunteers indicated that iron absorption from lpal beans is significantly higher than from their parents with normal phytic acid levels (Petry et al., 2013, 2015). These results indicate that the $\mathrm{B} 14$ formulation containing lpal flour is suited to produce biofortified biscuits with an enhanced and more balanced protein content and with increased bioavailability of essential minerals.

One third of the global population is estimated to suffer from hidden hunger. Micronutrient deficiencies vary in severity and occur in every country, but they disproportionally affect women, children, and people living in developing countries (Von Grebmer et al., 2014). Thus, malnutrition and hidden hunger represent a big nutritional challenge. The bean biscuits we presented here could be further implemented and used as a biofortified food snack, especially for children, using the lpa1 bean flour in the B29 formulation, together with a flour from a biofortified maize lpa1 mutant (Cerino Badone et al., 2012; Landoni et al., 2013). In this case, we expect a novel food product having a very high mineral bioavailability and higher and balanced protein content (Tables 2, 3, A1).

\section{Biofortified Bean Biscuits: Predicted Glycemic Index and Consumer's Test}

The classification through the pGI can be useful to predict the likely in vivo glycemic response of food of interest and a reference material. Accordingly, foods can be categorized into low $(<55)$, medium (55-69), and high GI (>70) (Foster-Powell et al., 2002). With respect to the control, the pGI values of experimental biscuits decreased with the addition of bean flour, as already reported by Giuberti et al. (2015) where the presence of 20 and $40 \%$ of bean flour in gluten-free rice pasta reduced the pGI from 61 , in the control, to 37 and 31 , respectively. With respect to cereal flours, starch from bean is characterized by higher level of amylose and by larger size of starch granules, both starch structural properties that can contribute to impede overall starch degradability (Sandhu and Lim, 2008). Moreover, comparative studies reported that cereal starches are characterized by the A-type power diffraction pattern, which has an open structure rendering cereal starches highly digestible (Biliaderis, 1991). On the contrary, legume starches tend to exhibit the C-type crystallinity, which is more resistant to digestion due to a close-packed structure (Biliaderis, 1991). Lastly, the increasing amounts of fiber and protein found in bean-enriched biscuits, along with the possible formation of amylose-lipid complexes during cooking, could have contributed to further reduce the accessibility of amylase to hydrolyze the starch (Zhang et al., 2015).

The active $\alpha$-AI present in all bean biscuits (but the lpa1) represents an additional valuable feature, further contributing to the reduction of pGI (Table 5). Spadafranca et al. (2013) in a study performed on 12 volunteers to evaluate the effects of a standardized and purified $P$. vulgaris extract (PVE), containing $110.000 \mathrm{U}$ of $\alpha$-AI per $100 \mathrm{mg}$ tablet, showed lower increments in glucose, insulin and C-peptide $3 \mathrm{~h}$ after meal consumption associated to 1 PVE tablet. We calculate that five B14 biscuits are equivalent to $1 \mathrm{PVE}$ tablet, as they contain a comparable activity of $\alpha$-AI.

On the basis of the above results, favorable implications for human health with a particular regard to the control of glycemia, especially in diabetic individuals and in the celiac population, can be hypothesized. For instance, for the celiac population, the enrichment of gluten-free baked products with fiber component is beneficial, since a general low intake of this food component has been reported (Lamacchia et al., 2014). In addition, although there is an on-going debate, the reduction of the dietary glycemic response following the consumption of lower GI food products can favorably influence several physiological parameters implicated as markers for conditions including overweight and obesity, diabetes mellitus and risk of coronary heart disease (Livesey et al., 2008; Brand-Miller et al., 2009). This aspect is also of particular interest concerning the celiac population, because several gluten-free foods exhibit higher GI than their gluten containing counterparts, due to the lack of the gluten matrix (Pellegrini and Agostoni, 2015).

In this work we considered two different gluten-free biscuit formulations, B22 and B29, and showed that they had lower pGI compared to control biscuit, confirming an additive contribution of bean flour. The acceptability of these products decreased with the increase of bean content, however the B22 gluten-free biscuits might represent a good compromise between health benefits (absence of gluten and lower pGI), expectations of celiac consumers and likeness. It should be pointed out that the consumers' test was a blind one and was not directed to celiac subjects. Appearance, flavor and texture are reported to be the most critical properties for consumer's acceptance of glutenfree products (Pagliarini et al., 2010). It is well known that gluten-free food products have a lower acceptability compared to those containing wheat (Pagliarini et al., 2010; Laureati et al., 2012). Moreover, it has been reported that differences in liking between celiac and non-celiac consumers may arise when judging gluten-free products (Laureati et al., 2012), with celiac consumers 
providing higher scores than non-celiac subjects to products of lower quality. In future studies, it is advisable to involve regular consumers of gluten-free products, since they are probably more sensitive to the differences in terms of liking to verify if a better score could be gained for B22 and B29 gluten-free biscuits.

In this context, future perspectives of this study should address the identification of the properties of appearance, smell, taste and texture that characterize bean-flour enriched biscuit formulations in order to define the sensory attributes that drive consumer's preference. Actually, this piece of research is intended as a pilot study carried out in order to give insights on the potentiality of Lady Joy bean flour as gluten-free ingredient, a fundamental topic, which truly needs to be investigated systematically.

\section{CONCLUSIONS}

Food technology is important for the development of novel foods and food ingredients. Here we show the importance of the genetic and breeding approach to contribute and possibly facilitate the improvement of the primary product. In this work we show the feasibility of making nutritionally improved biscuits by the addition of bean flour of selected genotypes. We took advantage of a PHA inactive genotype (Lady Joy), which allows the direct use of flour without any thermal treatment. This resulted in biscuits containing fair amount of active $\alpha$ AI, which contributes to lowering the pGI. We also proved that the use of the lpal line could significantly contribute to the reduction of total phytates in the biscuits, a trait that is expected to increase minerals bioavailability. We are now working to combine the properties of $\mathrm{cv}$. Lady Joy (absence of active PHA and very active $\alpha-\mathrm{AI}$ ) with the low phytate content of the lpal mutant to produce a new common bean

\section{REFERENCES}

Alonso, R., Aguirre, A., and Marzo, F. (2000). Effects of extrusion and traditional processing methods on antinutrients and in vitro digestibility of protein and starch in faba and kidney beans. Food Chem. 68, 159-165. doi: 10.1016/S03088146(99)00169-7

Altabella, T., and Chrispeels, M. (1990). Tobacco plants transformed with the bean alpha-AI-gene express an inhibitor of insect alpha-amylase in their seeds. Plant Physiol. 93, 805-810. doi: 10.1104/pp.93.2.805

Anton, A. A., Fulcher, R. G., and Arntfield, S. D. (2009). Physical and nutritional impact of fortification of corn starch-based extruded snacks with common bean (Phaseolus vulgaris L.) flour: effects of bean addition and extrusion cooking. Food Chem. 113, 989-996. doi: 10.1016/j.foodchem.2008.08.050

AOAC (2000). Official Method of Analysis, 7th Edn. Washington, DC: Association of Official Analytical Chemists.

Azevedo, R. A., and Arruda, P. (2010). High-lysine maize: the key discoveries that have made it possible. Amino Acids 9, 979-989. doi: 10.1007/s00726-0100576-5

Barrett, M. L., and Udani, J. K. (2011). A proprietary alpha-amylase inhibitor from white bean (Phaseolus vulgaris): a review of clinical studies on weight loss and glycemic control. Nutr. J. 10:24. doi: 10.1186/1475-2891-10-24

Batista, K., Prudencio, S., and Fernandes, K. (2010). Changes in the functional properties and antinutritional factors of extruded hard-to-cook common beans (Phaseolus vulgaris, L.). J. Food Sci. 75, C286-C290. doi: 10.1111/j.17503841.2010.01557.x line that could be useful to address the health issue of diabetic individuals with micronutrient (iron, zinc, etc) deficiencies and that if combined with maize flours in the B22 formulation could meet the requirement of celiac disease affected patients, that are more exposed to the risk of developing type 1 diabetes. Finally these products could be further diversified and improved by using nutritionally enhanced maize flours from lpal mutants, anthocyanins enriched lines and "high quality protein" maize lines (Azevedo and Arruda, 2010; Landoni et al., 2013; Holding, 2014; Petroni et al., 2014).

\section{AUTHOR CONTRIBUTIONS}

FS conceptualized, designed, and supervised the project. MD, ML, RP, GG, PF carried out the experiments. FS, RB provided the bean genotypes and wrote the manuscript. FS, EC, ML, $\mathrm{RP}, \mathrm{EP}, \mathrm{GG}, \mathrm{RB}$ regularly discussed the experiments, analyzed the results, provided useful suggestions during the project and critically revised the manuscript. All authors read and approved the final manuscript.

\section{FUNDING}

This work was supported by the Programme FILAGRO "Strategie innovative e sostenibili per la filiera agroalimentare," as part of the activities defined within the Accordo Quadro Consiglio Nazionale delle Ricerche and Regione Lombardia.

\section{ACKNOWLEDGMENTS}

We thank Dr. Alessandra Benati for her support in providing contacts with "PrimoPan" bakery.

Biliaderis, C. G. (1991). The structure and interactions of starch with food constituents. Can. J Physiol. Pharmacol. 69, 60-78. doi: 10.1139/y91-011

Bollini, R., Carnovale, E., and Campion, B. (1999). Removal of anti nutritional factors from bean (Phaseolus vulgaris L.) seeds. Biotech. Agr. Soc. Environ. 3, 217-219.

Bollini, R., and Chrispeels, M. (1978). Characterization and subcellularlocalization of vicilin and phytohemagglutinin, 2 major reserve proteins of Phaseolus vulgaris L. Planta 142, 291-298. doi: 10.1007/BF00385080

Boye, J., Zare, F., and Pletch, A. (2010). Pulse proteins: processing, characterization, functional properties and applications in food and feed. Food Res. Int. 43, 414-431. doi: 10.1016/j.foodres.2009.09.003

Brand-Miller, J., McMillan-Price, J., Steinbeck, K., and Caterson, I. (2009). Dietary glycemic index: health implications. J. Am. Coll. Nutr. 28, 446-449. doi: 10.1080/07315724.2009.10718110

Burnette, W. (1981). Western blotting - electrophoretic transfer of proteins from sodium dodecyl sulfate-polyacrylamide gels to unmodified nitrocellulose and radiographic detection with antibody and radioiodinated protein-A. Anal Biochem. 112, 195-203. doi: 10.1016/0003-2697(81)90281-5

Campion, B., Sparvoli, F., Doria, E., Tagliabue, G., Galasso, I., Fileppi, M., et al. (2009). Isolation and characterisation of an lpa (low phytic acid) mutant in common bean (Phaseolus vulgaris L.). Theor. Appl. Genet. 118, 1211-1221. doi: 10.1007/s00122-009-0975-8

Cardador-Martinez, A., Loarca-Pina, G., and Oomah, B. (2002). Antioxidant activity in common beans (Phaseolus vulgaris L.). J. Agr. Food Chem. 50, 6975-6980. doi: 10.1021/jf020296n 
Cerino Badone, F., Amelotti, M., Cassani, E., and Pilu, R. (2012). Study of low Phytic Acid 1-7 (lpa1-7), a new ZmMRP4 Mutation in Maize. J. Hered. 103, 598-605. doi: 10.1093/jhered/ess014

Ceriotti, A., Vitale, A., and Bollini, R. (1989). Lectin-like proteins accumulate as fragmentation products in bean seed protein bodies. FEBS Lett. 250, 157-160. doi: 10.1016/0014-5793(89)80710-0

Chavan, J., and Kadam, S. (1993). Nutritional enrichment of bakery products by supplementation with nonwheat flours. Crit. Rev. Food Sci. Nutr. 33, 189-226. doi: 10.1080/10408399309527620

Confalonieri, M., Bollini, R., Berardo, N., Vitale, A., and Allavena, A. (1992). Influence of phytohemagglutinin on the agronomic performance of beans (Phaseolus vulgaris L.). Plant Breed. 109, 329-334. doi: 10.1111/j.14390523.1992.tb00192.x

Costa, G., Queiroz-Monici, K., Reis, S., and De Oliveira, A. (2006). Chemical composition, dietary fibre and resistant starch contents of raw and cooked pea, common bean, chickpea and lentil legumes. Food Chem. 94, 327-330. doi: 10.1016/j.foodchem.2004.11.020

Duranti, M. (2006). Grain legume proteins and nutraceutical properties. Fitoterapia 77, 67-82. doi: 10.1016/j.fitote.2005.11.008

Elkowicz, K., and Sosulski, F. W. (1982). Antinutritive factors in eleven legumes and their air-classified protein and starch fractions. J. Food Sci. 47, 1301-1304. doi: 10.1111/j.1365-2621.1982.tb07673.x

Foster-Powell, K., Holt, S. H. A., and Brand-Miller, J. C. (2002). International table of glycemic index and glycemic load values: 2002. Amer. J. Clin. Nutr. 76, 5-56.

Gallegos-Infante, J. S., Bello-Perez, L. A., Rocha-Guzman, N. E., Gonzales-Laredo, R. F., and Avial-Ontiveros, M. (2010). Effect of the addition of common bean (Phaseolus vulgaris L.) flour on the in vitro digestibility of starch and undigestible carbohydrates in spaghetti. J. Food Sci. 75, 151-156. doi: 10.1111/j.1750-3841.2010.01621.x

Giuberti, G., Gallo, A., Cerioli, C., Fortunati, P., and Masoero, F. (2015). Cooking quality and starch digestibility of gluten free pasta using new bean flour. Food Chem. 175, 43-49. doi: 10.1016/j.foodchem.2014.11.127

Giuberti, G., Gallo, A., Fiorentini, L., Fortunati, P., and Masoero, F. (2016). In vitro starch digestibility and quality attributes of gluten free 'tagliatelle' prepared with teff flour and increasing levels of a new developed bean cultivar. Starch 68, 374-378. doi: 10.1002/star.201500007

Granfeldt, Y. (1994). Foods Factors Affecting Metabolic Responses to Cereal Products. Ph.D. thesis, University of Lund.

Holding, D. R. (2014). Recent advances in the study of prolamin storage protein organization and function. Front Plant Sci. 20:276. doi: 10.3389/fpls.2014.00276

Hoojjat, P., and Zabik, M. (1984). Sugar-snap cookies prepared with wheat-navy bean-sesame seed flour blends. Cereal Chem. 61, 41-44.

Hoover, R., and Zhou, Y. (2003). In vitro and in vivo hydrolysis of legume starches by alpha-amylase and resistant starch formation in legumes a review. Carbohydr Polym. 54, 401-417. doi: 10.1016/S0144-8617(03) 00180-2

Kelkar, S., Siddiq, M., Harte, J., Dolan, K., Nyombaire, G., and Suniaga, H. (2012). Use of low-temperature extrusion for reducing phytohemagglutinin activity (PHA) and oligosaccharides in beans (Phaseolus vulgaris L.) cv. Navy and Pinto. Food Chem. 133, 1636-1639. doi: 10.1016/j.foodchem.2012.02.044

Kumar, S., Verma, A., Das, M., Jain, S., and Dwivedi, P. (2013). Clinical complications of kidney bean (Phaseolus vulgaris L.) consumption. Nutrition 29, 821-827. doi: 10.1016/j.nut.2012.11.010

Lamacchia, C., Camarca, A., Picascia, S., Di Luccia, A., and Ginafrani, C. (2014). Cereal-based gluten-free food: how to reconcile nutritional and technological properties of wheat proteins with safety for celiac disease patients. Nutrients 6 , 575-590. doi: 10.3390/nu6020575

Landoni, M., Badone, F., Haman, N., Schiraldi, A., Fessas, D., Cesari, V., et al. (2013). Low phytic acid 1 mutation in maize modifies density, starch properties, cations, and fiber contents in the seed. J. Agr. Food Chem. 61, 4622-4630. doi: $10.1021 / \mathrm{jf} 400259 \mathrm{~h}$

Laureati, M., Giussani, B., and Pagliarini, E. (2012). Sensory and hedonic perception of gluten-free bread: comparison between celiac and non-celiac subjects. Food Res. Int. 46, 326-333. doi: 10.1016/j.foodres.2011.12.020

Layer, P., Carlson, G., and Dimagno, E. (1985). Partially purified white bean amylase inhibitor reduces starch digestion in vitro and inactivates intraduodenal amylase in humans. Gastroenterology 88, 1895-1902. doi: $10.1016 / 0016-5085(85) 90016-2$
Layer, P., Zinsmeister, A., and Dimagno, E. (1986). Effects of decreasing intraluminal amylase activity on starch digestion and postprandial gastrointestinal function in humans. Gastroenterology 91, 41-48. doi: 10.1016/0016-5085(86)90436-1

Lioi, L., Sparvoli, F., Galasso, I., Lanave, C., and Bollini, R. (2003). Lectin-related resistance factors against bruchids evolved through a number of duplication events. Theor. Appl. Genet. 107, 814-822. doi: 10.1007/s00122-003-1343-8

Livesey, G., Taylor, R., Hulshof, T., and Howlett, J. (2008). Glycemic response and health - a systematic review and meta-analysis: relation between dietary glycemic properties and health outcomes. Am. J. Clin. Nutr. 87, 258-226.

Macfie, H. J. H., Bratchell, N., Greenhoff, K., and Vallis, L. V. (1989). Designs to balance the effect of order of presentation and first-order carry-over effects in hall tests. J. Sens. Stud. 4, 129-148. doi: 10.1111/j.1745-459X.1989.tb0 0463.x

Manonmani, D., Bhol, S., and Bosco, S. J. D. (2014). Effect of red kidney bean (Phaseolus vulgaris L.) flour on bread quality. Open Access Libr. J. 1, 1-6. doi: 10.4236/oalib.1100366

Marshall, J. J., and Lauda, C. M. (1975). Purification and properties of phaseolamin, an inhibitor of $\alpha$-amylase, from the kidney bean, Phaseolus vulgaris. J. Biol. Chem. 250, 8030-8037.

Mertens, D., Allen, M., Carmany, J., Clegg, J., Davidowicz, A., Drouches, M., et al. (2002). Gravimetric determination of amylase-treated neutral detergent fiber in feeds with refluxing in beakers or crucibles: collaborative study. J. AOAC Int. $85,1217-1240$

Noah, N., Bender, A., Reaidi, G., and Gilbert, R. (1980). Food poisoning from raw red kidney beans. Br. Med. J. 281, 236-237.

Oomah, B., Kotzeva, L., Allen, M., and Bassinello, P. (2014). Microwave and micronization treatments affect dehulling characteristics and bioactive contents of dry beans (Phaseolus vulgaris L.). J. Sci. Food Agr. 94, 1349-1358. doi: $10.1002 /$ jsfa.6418

Pagliarini, E., Laureati, M., and Lavelli, V. (2010). Sensory evaluation of gluten-free breads assessed by a trained panel of celiac assessors. Eur. Food Res. Technol. 231, 37-46. doi: 10.1007/s00217-010-1249-z

Panzeri, D., Cassani, E., Doria, E., Tagliabue, G., Forti, L., Campion, B., et al. (2011). A defective ABC transporter of the MRP family, responsible for the bean lpal mutation, affects the regulation of the phytic acid pathway, reduces seed myo-inositol and alters ABA sensitivity. New Phytol. 191, 70-83. doi: 10.1111/j.1469-8137.2011.03666.x

Pellegrini, N., and Agostoni, C. (2015). Nutritional aspects of gluten-free products. J. Sci. Food Agric. 95, 2380-2385. doi: 10.1002/jsfa.7101

Petroni, K., Pilu, R., and Tonelli, C. (2014). Anthocyanins in corn: a wealth of genes for human health. Planta 240, 901-911. doi: 10.1007/s00425-014-2131-1

Petry, N., Boy, E., Wirth, J. P., and Hurrell, R. F. (2015). Review: the potential of the common bean (Phaseolus vulgaris) as a vehicle for iron biofortification. Nutrients 11, 1144-1173. doi: 10.3390/nu7021144

Petry, N., Egli, I., Campion, B., Nielsen, E., and Hurrell, R. (2013). Genetic reduction of phytate in common bean (Phaseolus vulgaris L.) seeds increases iron absorption in young women. J. Nutr. 143, 1219-1224. doi: 10.3945/jn.113.175067

Piergiovanni, A. R., and Lioi, L. (2010). Italian common bean landraces: history, genetic diversity and seed quality. Diversity 2, 837-862. doi: 10.3390/d2060837

Pilu, R., Panzeri, D., Gavazzi, G., Rasmussen, S., Consonni, G., and Nielsen, E. (2003). Phenotypic, genetic and molecular characterization of a maize low phytic acid mutant (lpa241). Theor. Appl. Genet. 107, 980-987. doi: 10.1007/s00122-003-1316-y

Pusztai, A., Clarke, E., King, T., and Stewart, J. (1979). Nutritional-evaluation of kidney beans (Phaseolus vulgaris) - chemical composition, lectin content and nutritional-value of selected cultivars. J. Sci. Food Agr. 30, 843-848. doi: $10.1002 /$ jsfa. 2740300902

Pusztai, A., Grant, G., and Stewart, J. (1981). A new type of Phaseolus vulgaris (cv Pinto-III) seed lectin - isolation and characterization. Biochim. Biophys. Acta 671, 146-154. doi: 10.1016/0005-2795(81)90128-8

Rababah, T., Al-Mahasneh, M., and Ereifej, K. (2006). Effect of chickpea, broad bean, or isolated soy protein additions on the physicochemical and sensory properties of biscuits. J. Food Sci. 71, S438-S442. doi: 10.1111/j.17503841.2006.00077.x

Rodhouse, J., Haugh, C., Roberts, D., and Gilbert, R. (1990). Red kidney bean poisoning in the UK - an analysis of 50 suspected incidents between 
1976 and 1989. Epidemiol. Infect. 105, 485-491. doi: 10.1017/S0950268800 04810X

Sandhu, K., and Lim, S. (2008). Digestibility of legume starches as influenced by their physical and structural properties. Carbohyd. Polym. 71, 245-252. doi: 10.1016/j.carbpol.2007.05.036

Santimone, M., Koukiekolo, R., Moreau, Y., Le Berre, V., Rouge, P., MarchisMouren, G., et al. (2004). Porcine pancreatic alpha-amylase inhibition by the kidney bean (Phaseolus vulgaris) inhibitor (alpha-AI1) and structural changes in the alpha-amylase inhibitor complex. Biochim. Biophys. Acta 1696, 181-190. doi: 10.1016/j.bbapap.2003.11.001

Schlemmer, U., Frølich, W., Prieto, R. M., and Grases, F. (2009). Phytate in foods and significance for humans: food sources, intake, processing, bioavailability, protective role and analysis. Mol. Nutr. Food Res. 53(Suppl. 2), S330-S375. doi: 10.1002/mnfr.200900099

Shrestha, A., and Noomhorm, A. (2002). Comparison of physico-chemical properties of biscuits supplemented with soy and kinema flours. Int. J. Food Sci. Technol. 37, 361-368. doi: 10.1046/j.1365-2621.2002. 00574. $\mathrm{x}$

Siddiq, M., Kelkar, S., Harte, J., Dolan, K., and Nyombaire, G. (2013). Functional properties of flour from low-temperature extruded navy and pinto beans (Phaseolus vulgaris L.). Lwt-Food Sci. Technol. 50, 215-219. doi: 10.1016/j.lwt.2012.05.024

Spadafranca, A., Rinelli, S., Riva, A., Morazzoni, P., Magni, P., Bertoli, S., et al. (2013). Phaseolus vulgaris extract affects glycometabolic and appetite control in healthy human subjects. Br. J. Nutr. 109, 1789-1795. doi: 10.1017/S0007114512003741

Sparvoli, F., Bollini, R., and Cominelli, E. (2015). "Nutritional value," in Grain Legumes, ed A. De Ron (New York, NY: Springer Science+Business Media), 291-326.

Sparvoli, F., Daminati, M., Cantoni, R., and Bollini, R. (1997). "Possible role(s) of glycosylation on the biological properties of lectins and related proteins," in Biomedical and Therapeutical Applications of Dietary Lectins. COST Action 98, 7th Workshop, Vol. 6, eds S. Bardzoc and A. Pusztai (Lake Balaton: European Commission), 43-49.

Szafranski, M., Whittington, J. A., and Bessinger, C. (2005). Pureed cannellini beans can be substituted for shortening in brownies. J. Am. Diet. Assoc. 105, 1295-1298. doi: 10.1016/j.jada.2005.05.002
Trugo, L., and von Baer, D. (1998). Analytical Methods for the Analysis of Antinutritional Factors in Legume Seeds. Wageningen: Wageningen Press.

Vansoest, P., Robertson, J., and Lewis, B. (1991). Methods for dietary fiber, neutral detergent fiber, and nonstarch polysaccharides in relation to animal nutrition. J. Dairy Sci. 74, 3583-3597. doi: 10.3168/jds.S0022-0302(91)78551-2

Varner, J. E., and Mense, R. M. (1972). Characteristics of the process of enzyme release from secretory plant cells. Plant Physiol. 49, 187-189. doi: 10.1104/pp.49.2.187

Vaz Patto, M. C., Amarowicz, R., Aryee, A. N. A., Boye, J. I., Chung, H. J., MartínCabrejas, M. A., et al. (2015). Achievements and challenges in improving the nutritional quality of food legumes. Crit. Rev. Plant Sci. 34, 105-143. doi: 10.1080/07352689.2014.897907

Voelker, T., Staswick, P., and Chrispeels, M. (1986). Molecular analysis of 2 phytohemagglutinin genes and their expression in Phaseolus vulgaris cv Pinto, a lectin-deficient cultivar of the bean. EMBO J. 5, 3075-3082.

Von Grebmer, K., Saltzman, A., Birol, E., Wiesmann, D., Prasai, N., Yin, S., et al. (2014). 2014 Global hunger index: The Challenge of Hidden Hunger. Bonn; Washington, DC; Dublin: Welthungerhilfe; International Food Policy Research Institute; Concern Worldwide.

Zhang, B., Dhital, S., and Gidley, M. J. (2015). Densely packed matrices as rate determining features in starch hydrolysis. Trends Food Sci. Technol. 43, 18-31. doi: 10.1016/j.tifs.2015.01.004

Zucco, F., Borsuk, Y., and Arntfield, S. (2011). Physical and nutritional evaluation of wheat cookies supplemented with pulse flours of different particle sizes. Lwt Food Sci. Technol. 44, 2070-2076. doi: 10.1016/j.lwt.2011.06.007

Conflict of Interest Statement: The authors declare that the research was conducted in the absence of any commercial or financial relationships that could be construed as a potential conflict of interest.

Copyright (C) 2016 Sparvoli, Laureati, Pilu, Pagliarini, Toschi, Giuberti, Fortunati, Daminati, Cominelli and Bollini. This is an open-access article distributed under the terms of the Creative Commons Attribution License (CC BY). The use, distribution or reproduction in other forums is permitted, provided the original author(s) or licensor are credited and that the original publication in this journal is cited, in accordance with accepted academic practice. No use, distribution or reproduction is permitted which does not comply with these terms. 


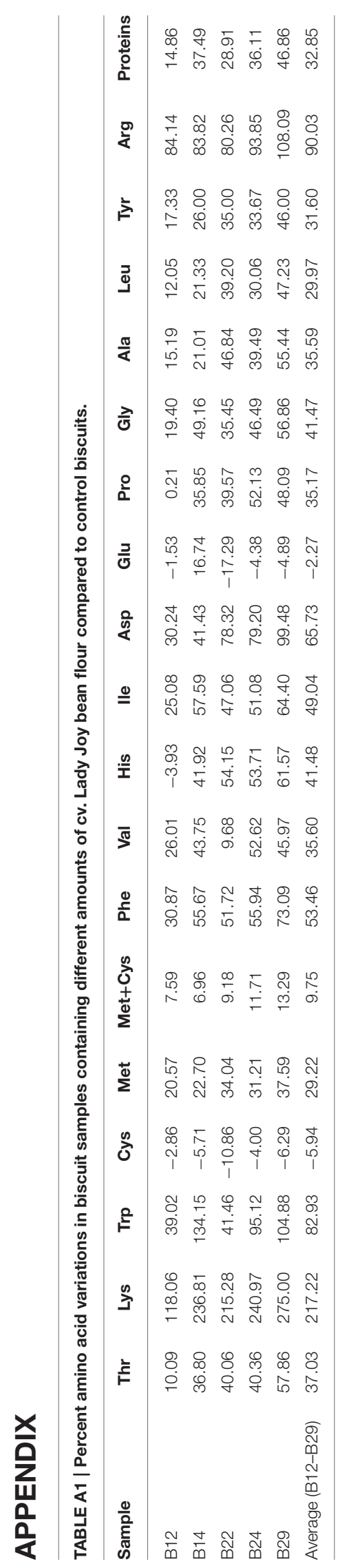




\title{
The future of lupin as a protein crop in Europe
}

\author{
M. Mercedes Lucas ${ }^{1}$, Frederick L. Stoddard ${ }^{2}$, Paolo Annicchiarico ${ }^{3}$, Juana Frías ${ }^{4}$, Cristina \\ Martínez-Villaluenga ${ }^{4}$, Daniela Sussmann ${ }^{5}$, Marcello Duranti $^{6}$, Alice Seger ${ }^{7}$, Peter M. \\ Zander $^{8}$ and José J. Pueyo ${ }^{1 *}$
}

${ }^{1}$ Institute of Agricultural Sciences, ICA-CSIC, Madrid, Spain, ${ }^{2}$ Department of Food and Environmental Sciences, University of Helsinki, Helsinki, Finland, ${ }^{3}$ Research Centre for Fodder Crops and Dairy Productions, CRA, Lodi, Italy, ${ }^{4}$ Institute of Food Science Technology and Nutrition, ICTAN-CSIC, Madrid, Spain, ${ }^{5}$ Fraunhofer-Institute for Process Engineering and Packaging, Freising, Germany, ${ }^{6}$ Department of Food Environment and Nutritional Sciences, Università degli Studi di Milano, Milan, Italy, ${ }^{7}$ Terrena Lup'Ingredients, Martigne-Ferchaud, France, ${ }^{8}$ Leibniz Centre for Agricultural Landscape Research, ZALF, Müncheberg, Germany

\section{OPEN ACCESS}

Edited by:

Soren K. Rasmussen, University of Copenhagen, Denmark

Reviewed by:

Ethalinda K. S. Cannon, lowa State University, USA Jens Stougaard,

Aarhus University, Denmark

*Correspondence: José J. Pueyo, Institute of Agricultural Sciences, ICA-CSIC,

Serrano, 115-bis, 28006 Madrid, Spain

jj.pueyo@csic.es

Specialty section:

This article was submitted to Crop Science and Horticulture, a section of the journal

Frontiers in Plant Science

Received: 09 July 2015 Accepted: 24 August 2015 Published: 08 September 2015

Citation

Lucas MM, Stoddard FL,

Annicchiarico P, Frías J, Martínez-Villaluenga $C$, Sussmann $D$, Duranti M, Seger A, Zander PM and Pueyo JJ (2015) The future of lupin as

a protein crop in Europe.

Front. Plant Sci. 6:705.

doi: $10.3389 /$ fp/s.2015.00705
Europe has become heavily dependent on soya bean imports, entailing trade agreements and quality standards that do not satisfy the European citizen's expectations. White, yellow, and narrow-leafed lupins are native European legumes that can become true alternatives to soya bean, given their elevated and high-quality protein content, potential health benefits, suitability for sustainable production, and acceptability to consumers. Nevertheless, lupin cultivation in Europe remains largely insufficient to guarantee a steady supply to the food industry, which in turn must innovate to produce attractive lupin-based protein-rich foods. Here, we address different aspects of the food supply chain that should be considered for lupin exploitation as a high-value protein source. Advanced breeding techniques are needed to provide new lupin varieties for socio-economically and environmentally sustainable cultivation. Novel processes should be optimized to obtain high-quality, safe lupin protein ingredients, and marketable foods need to be developed and offered to consumers. With such an integrated strategy, lupins can be established as an alternative protein crop, capable of promoting socio-economic growth and environmental benefits in Europe.

Keywords: lupin, protein crop, plant protein, breeding, protein ingredients, protein foods, food supply chain

The demand of the ever-growing world population for dietary protein is no longer sustainable through animal products alone. Soya bean has become the prevalent source of plant proteins for food and feed, and Europe depends on soya bean imports for $70 \%$ of its plant protein requirements. White lupin (Lupinus albus L.), yellow lupin (L. luteus L.) and narrow-leafed lupin (L. angustifolius L.), are native European legumes that represent a significant alternative to soya bean. Their seed protein content is high (up to 44\%) and its quality is good, they offer potential health benefits, and they contribute to the sustainability of cropping systems. Lupins are successful protein crops in Australia, where an important industry has developed to use lupin protein and other fractions, yet lupin production in Europe is insufficient to guarantee the stable and sufficient supply required for its use by the food and feed industry. Lupin is grown in several European countries, and although its grain yield is the world's highest in some parts of Europe, its cropping area remains modest and yields are highly variable. A slight rise of European cultivated area and production has occurred during the period 2000-2013 (Figure 1), representing 17.6\% of the world's production during that period (Figure 2). The Andean lupin (L. mutabilis Sweet) has been brought into cultivation in some parts of South America, but is not present in Europe on a commercial scale. 
$250 k$

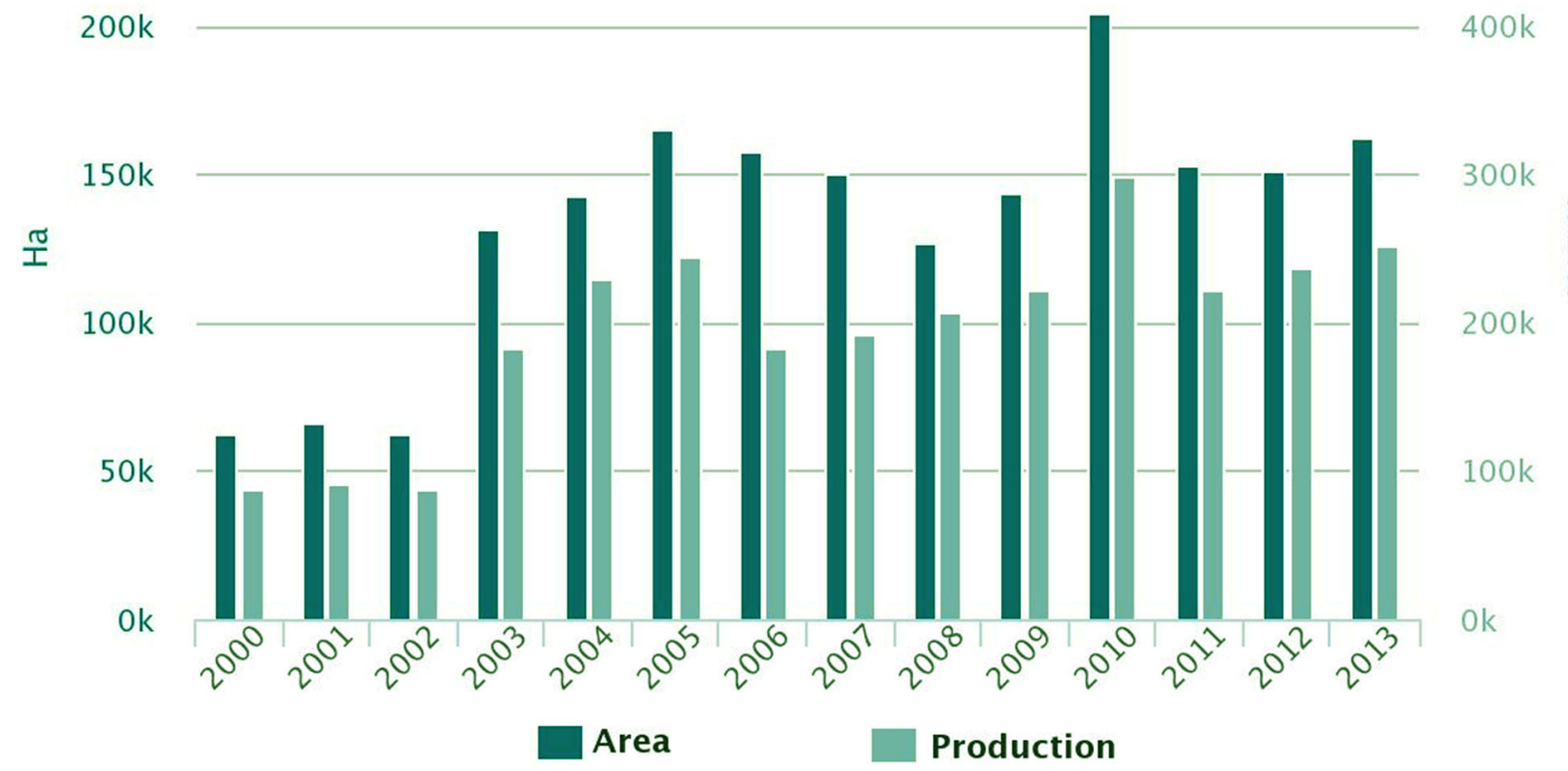

FIGURE 1 | Lupin cultivated areas and production in Europe. Source: FAOSTAT 2015.

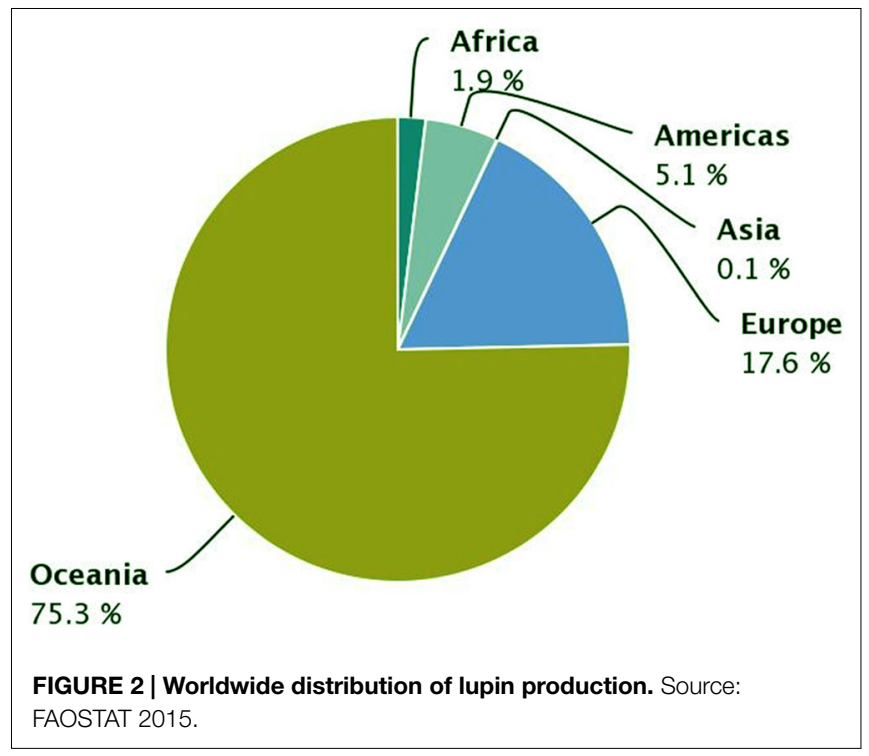

Insufficient grain yield in certain areas, largely due to limited breeding, constitutes a significant barrier to the expansion and economic sustainability of lupin cultivation in Europe. Lupin breeding has exploited little of the gene pool (Berger et al., 2012), partly due to the need to maintain a low alkaloid content (sweet seed). Landrace germplasm offers tremendous opportunities for increasing white lupin yield (Annicchiarico et al., 2010). Lupins are relatively more tolerant to several abiotic stresses than other legumes, and have a proven potential for the recovery of poor and contaminated soils (Fernández-Pascual et al., 2007; Coba de la Peña and Pueyo, 2012). Identifying germplasm with tolerance to a range of abiotic stresses may allow lupin cultivation to expand into a wider range of agro-climatic conditions across Europe. In areas subjected to frequent or occasional frosts, high vernalization requirement along with intrinsic cold tolerance is required, as sudden winter frost may lead to high mortality even in regions with relatively mild winters (Annicchiarico and Iannucci, 2007). Summer drought and progressive soil salinization are major climatic stresses in Mediterranean areas, yet information is lacking on the intrinsic genetic variation for drought tolerance, particularly in white lupin. Poor adaptation to calcareous soils is probably the main limit to the crop expansion, but white lupin landraces from Egypt or Italy that display tolerance to free calcium have been identified (Annicchiarico and Thami-Alami, 2012). The primary source of phosphate fertilizer (mined rock phosphate) is considered likely to be depleted within 40 years, emphasizing the importance of sustainable $\mathrm{P}$ management. When available $\mathrm{P}$ is limiting, lupins form specialized cluster root structures and/or release P-mobilizing carboxylates that free it from insoluble forms (Lambers et al., 2012), which could be exploited more widely to reduce the need for P supplementation.

To combine high protein quality, adaptation to environmental stresses and high yield, a suite of modern techniques is required. Extensive research in Australia has provided valuable information on the genetics of narrow-leafed lupin, and its genome sequence is nearly complete. Genomic selection is a particularly promising tool for improving complex, quantitatively inherited traits (Heffner et al., 2009). Combining phenotyping and genotyping 
data of the population into a model enables breeding values for each marker to be estimated. Genotyping-by-sequencing (GBS) can provide thousands of single nucleotide polymorphism (SNP) markers at much lower cost than earlier techniques (Elshire et al., 2011), but should be optimized for individual crops. GBS can help to identify and establish valuable genetic markers needed for genomic selection of each lupin species, and ultimately, to select cultivars adapted to the wide variety of European soils and climates, and to allow productive cultivation on poor and marginal lands.

Lupins form root nodules in which biological nitrogen fixation (BNF) takes place in symbiosis with compatible soil bacteria of the genus Bradyrhizobium. In general, European lupins that grow in acid soils can establish effective symbiosis with bradyrhizobia that are usually present in these soil, although they may be absent from neutral or alkaline soils. Where indigenous rhizobia are inadequate, inoculants boost BNF, improving grain yield and often its protein quality. BNF is strongly related to the physiological state of the host plant, so that environmental stresses not only have a detrimental effect on metabolism, growth and development of the plant, but also affect symbiosis. The Lupinus-Bradyrhizobium symbiosis has been described as being relatively tolerant to abiotic stresses (Fernández-Pascual et al., 2007). Rhizobial strain and lupin genotype interactions influence nodulation score, nitrogen fixation and plant growth. Specific Bradyrhizobium strains should be developed together with stresstolerant lupin genotypes, to promote lupin as a successful component of sustainable crop production systems.

To use lupin as a source of high quality and healthy proteins for food, several quality traits are worth extra attention: in particular, alkaloid content, which must be maintained as low as possible, and the conglutin- $\gamma$ protein fraction, which is of interest to control insulin resistance and diabetes (Terruzzi et al., 2011). The oil content of white lupin is $8-14 \%$ and its nutritional quality is excellent (Boschin et al., 2008). If this trait is further improved by breeding, it would increase the economic sustainability of the crop by making it dual-purpose for protein and oil, like soya bean. Iron deficiency is one of the most common and widespread nutritional disorder worldwide. Traditional iron supplementation methods can have negative effects on the consumer, and are unavailable to many people. The Fe-rich protein ferritin is abundant in some legume seeds, including lupin (Strozycki et al., 2007), and may represent a safe way to increase dietary iron intake. Lupin proteins influence lipid and glucose metabolism, as well as blood pressure levels (Duranti and Morazzoni, 2011). Amino acid sequences that may be related to hypotensive and lipid-lowering activities have been detected in lupin proteins (Bettzieche et al., 2009), although the components responsible for these effects are still undetermined and the underlying mechanisms remain illdefined. Another interesting aspect of lupin protein functionality is its possible effects on inflammatory processes and changes in the gut microbiome, which has a significant influence on several physiological parameters, including metabolism, nutrient absorption and immune function (Walsh et al., 2014).

Food processing affects structural features of proteins and peptides, exerting a strong impact on their techno-functional and bioactive properties. Some proteins display their activity through peptides that must be liberated upon digestion to achieve functionality, while other proteins, such as conglutin- $\gamma$, seem to be required in an intact form. The production of protein ingredients with improved nutritive, technological and health benefits presents the food industry with unique challenges. Technological approaches remain to be optimized to improve production, cost effectiveness, and sustainable and environmental feasibility of high-quality protein ingredients. The selection of pre-treatments, such as germination in mineral-fortified conditions (Frias et al., 2009), and the optimization of processing conditions to facilitate protein extraction and isolation, represent important technological targets, oriented toward sustainable and non-invasive techniques. Off-flavor production is an important constraint in legume flour milling, storage and use (Azarnia et al., 2011). Pre-treatment with electromagnetic microwave radiation provides potential advantages over conventional hydro-thermal pre-treatment, inactivating enzymes that cause off-flavor formation and enhancing seed brittleness, thereby assisting milling and fractionation with little effect on protein functionality, as has been demonstrated in oat (Keying et al., 2009). These techniques need to be properly adapted to lupin seeds. Different fractionation, isolation and purification techniques combined with pre-processing are used to obtain protein concentrates/isolates with targeted techno-functional properties. Wet fractionation techniques are conventionally used to obtain relatively pure protein isolates, although they are energy-intensive procedures requiring large amounts of water and they may affect protein functionality (Muranyi et al., 2013). Many processing conditions for protein isolation are well established, but they are amenable to improvement, as shown by the influence of various parameters on target properties of the protein preparation and on overall protein yield. Dry milling combined with air classification is an alternative strategy to wet extraction, and may provide ideal disentanglement with lower energy and water demands (Schutyser and van der Goot, 2011).

Protein isolates prepared by isoelectric precipitation exhibit excellent water absorption, emulsification and foam-forming activities (Rodríguez-Ambriz et al., 2005). In contrast, legume protein isolates prepared by salt-induced extraction and dilutionprecipitation with water have outstanding oil-binding capacity, emulsion stability and specific viscoelasticity, which affect their rheological properties, e.g., for bakery products (Marco and Rosell, 2008). Hence, the techno-functional properties of lupin proteins must be examined to optimize processing conditions and select suitable processing schemes, such as protein modification by microstructuring, to obtain ingredients for different food applications. Protein hydrolysates containing bioactive peptides are of great interest for the design of functional foods and nutraceuticals. Proteolysis of lupin storage proteins confers added value and targets functional properties as well as decreasing allergenicity (Clemente, 2000), and is commonly achieved by enzymatic treatments and fermentation. Recently developed high hydrostatic pressure and ultrasound-assisted methods increase the hydrolytic efficiency of enzymes (Zhang et al., 2012; GarciaMora et al., 2015). Sensory qualities and storage stability are key parameters to be taken into account when introducing lupin ingredients into the food industry. For example, neutral 
taste should be assured and maintained over a certain period in storage. Therefore, endogenous enzyme activities must be characterized to ensure the quality of the protein produced and to monitor storage stability. Lupin fiber could help to stabilize lupin protein based structures, as shown for other plant fiber materials (Laine et al., 2011). Accompanying components affect the technofunctional properties of the final protein products, so this has to be considered.

Technological challenges to optimize the production and processing of lupin protein relate to the integration of the process into a holistic concept that considers oil and fiber recovery, use of co-products for animal feed, bio-fuel (Pakarinen et al., 2011), or soil amendments, or even water recycling. After protein extraction, the large amount of dietary fiber (up to $40 \%$ of seed mass) has a potential role in functional foods. Innovative, costeffective and environmentally friendly technologies need to be explored for fiber purification and fractionation. Lupin oil is an attractive product because of its balanced fatty acid composition and its content of bioactive lipids (Kalogeropoulos et al., 2010). Within the context of co-product utilization, animal feed is one of the main contributors to overall profitability.

The safety aspects of lupin ingredients include the formation of biogenic amines and the presence of allergens. Lupin allergy is still quite rare, but cross-reactivity between peanut and lupin exists (Fiocchi et al., 2009), which has led to the inclusion of lupins in the allergen list of the EU directive on labeling (EU Directive $2006 / 142 / E C)$. Conglutins $-\alpha,-\beta,-\gamma$, and $-\delta$ are candidate allergens in lupin, of which conglutin- $\alpha$ has strong allergenicity (Holden et al., 2008). Considering the severity of allergic reaction to peanut, the cross-reactivity of new lupin derivatives needs to be carefully assessed, and commercially exploited lupin products properly labeled, to minimize the danger for potential allergic consumers.

Lupin ingredients are already used in foods in Europe but their use is much less common than that of soya bean or pea ingredients, despite their beneficial properties. Lupin ingredients (flour and protein isolates and concentrates) are mainly used in bakery and gluten-free products, albeit as minor components ( $<5 \%$ of the ingredients). Nevertheless, new products containing lupin ingredients enter the European market each year (Mintel GNPD, http://www.mintel.com/global-new-products-database). Given its high protein content, lupin flour is considered an excellent raw material to supplement different food products (Pollard et al., 2002) and can be used as an egg substitute in cakes, pancakes, biscuits, pasta, or bread (Dervas et al., 1999). The rich yellow color of lupin flour and some protein concentrates has considerable appeal and can be of value in pasta or noodle dishes (Doxastakis et al., 2002), yet it can be easily eliminated when necessary (Guémes-Vera et al., 2008). Lupin flour can be incorporated into wheat flour to improve the nutritional value of the final products with little or no detriment in product sensory quality, and lupin fiber can also be used as a source of dietary fiber (Clark and Johnson, 2002). In general, the addition of up to $10 \%$ lupin flour improves water binding, texture, shelf-life and aroma of bread, although the mixing time and dough stability decrease as the proportion of lupin flour is increased (Doxastakis et al., 2002; Pollard et al., 2002).
While European consumers have a positive opinion on plant protein consumption, many do not know that lupin is a plant protein source comparable to soya bean. Among the main trends in the current European food market are naturalness; environmentally friendly; vegetarian alternative to meat, milk or eggs; and health properties (Mintel GNPD). The development of novel lupin-based foods responding to these desiderata should probably focus first on replacement of animal products (meat alternative, vegetarian spreads, dessert creams, ice-cream, and vegetable drinks). Gluten-free products are often lower in protein than standard goods, and lupin-based goods (bakery products, pasta, breakfast cereals) would restore or even increase the protein content. Further targets include high-protein food products with excellent sensory properties (sausages, pasta, snacks, drinks, bread). Specific consumer groups can be addressed with suitable nutritional values, taste and texture. The major international nutraceutical markets (EU, USA, Japan) are expected to grow in the near future, with major areas of interest being chronic degenerative cardiovascular diseases, type-2 diabetes and neuroprotection. Despite their health-promoting effects (Duranti and Morazzoni, 2011; Terruzzi et al., 2011), lupin ingredients are scarcely used in probiotics and nutraceuticals, as only a few lupin-containing food supplements and products with health claims are present in Europe (Mintel GNPD). The European Food Safety Authority (EFSA) currently allows no health claim about lupin ingredients. If proper clinical trials demonstrate a preventive role of lupin-based foods toward ailments such diabetes, cardiovascular diseases, metabolic syndrome, and obesity, these health-promoting features may confer an important advantage over other protein crops.

Lupin production in Europe declined steadily during the second half of the 20th century (Preissel et al., 2015), mainly because of low productivity driven by seasonal variability (Cernay et al., 2015), the low price of lupin grain (de Visser et al., 2014), and EU policies favoring the importation of soya bean. Concern about the sustainability and dependence of non-European protein sources, as well as potential environmental advantages, have re-established interest in lupins and other legume crops, and production has increased since 2003 (Figure 1). To establish lupin-based products as serious alternatives, the supply chain of these products must be assessed in terms of production costs, yields and quality, as well as factors influencing the acceptance of lupin-based products by European consumers (de Visser et al., 2014). The complete supply chain has to be addressed, analyzing the factors contributing to low lupin production by European farmers, and the conditions necessary to increase supply and make lupin a viable alternative to existing crops. The potential benefits of lupins at the farm level include $\mathrm{N}$ fixation with positive direct and indirect effects for subsequent crop yields, lower phosphorus requirements, reduced soil loss, and biodiversity benefits (Nemecek et al., 2008). Value-chain characteristics to be considered comprise agricultural production in terms of cost factors and yield effects, agricultural policies driving farmers' decisions, production quantities and qualities demanded by processing companies, sustainability impacts, and factors influencing the acceptance of lupin products by European consumers. Life-cycle assessment methods (LCA) and 
participatory impact assessment techniques should be combined in a complementary manner. An in-depth investigation of the causes and driving forces leading to the current production and consumption situation of lupins in Europe would indicate which characteristics lupins have to fulfill and how framework situations need to be changed (e.g., agricultural production conditions, level of substitution in end-products) to make lupins into profitable protein crops.

The rising global demand for meat, dairy and fish products for human consumption is recognized as unsustainable, owing to the high environmental impact of animal production. Furthermore, diseases associated with excessive consumption of animal products are increasing in frequency. The world population continues to grow, so a trend toward diets containing more plant protein seems not just strongly recommended, but inevitable, and it would be wise to anticipate and direct this trend by adopting knowledge-based strategies. To best achieve this objective, advances in virtually all phases of the supply chain are needed. Lupins should be established as fundamental crops in the various agro-climatic zones and marginal lands of Europe, and their yields and adaptation genetically improved to ensure a continuous supply of quality grain. The combination of their low needs for fertilizer $\mathrm{N}$ and $\mathrm{P}$ with their exploitation of poor, degraded, stress-affected or contaminated soils to safely produce protein-rich crops could contribute to socio-economic development and environmental sustainability. Sustainable, innovative, and cost-efficient processing methods to produce

\section{References}

Annicchiarico, P., Harzic, N., and Carroni, A. M. (2010). Adaptation, diversity, and exploitation of global white lupin (Lupinus albus L.) landrace genetic resources. Field Crop Res. 119, 114-124. doi: 10.1016/j.fcr.2010.06.022

Annicchiarico, P., and Iannucci, A. (2007). Winter survival of pea, faba bean and white lupin cultivars in contrasting Italian locations and sowing times, and implications for selection. J. Agr. Sci. 145, 611-622. doi: 10.1017/S0021859607007289

Annicchiarico, P., and Thami-Alami, I. (2012). Enhancing white lupin (Lupinus albus L.) adaptation to calcareous soils through selection of lime-tolerant plant germplasm and Bradyrhizobium strains. Plant Soil 350, 131-144. doi: 10.1007/s11104-011-0889-5

Azarnia, S., Boye, J. I., Warkentin, T., and Malcolmson, L. (2011). Changes in volatile flavour compounds in field pea cultivars as affected by storage conditions. Int. J. Food Sci. Technol. 46, 2408-2419. doi: 10.1111/j.1365-2621.2011.02764.x

Berger, J. D., Buirchell, B. J., Luckett, D. J., and Nelson, M. N. (2012). Domestication bottlenecks limit genetic diversity and constrain adaptation in narrow-leafed lupin (Lupinus angustifolius L.). Theor. Appl. Genet. 124, 637-652. doi: 10.1007/s00122-011-1736-Z

Bettzieche, A., Brandsch, C., Eder, K., and Stangl, G. I. (2009). Lupin protein acts hypocholesterolemic and increases milk fat content in lactating rats by influencing the expression of genes involved in cholesterol homeostasis and triglyceride synthesis. Mol. Nutr. Food Res. 53, 1134-1142. doi: 10.1002/mnfr.200800393

Boschin, G., D’Agostina, A., Annicchiarico, P., and Arnoldi, A. (2008). Effect of genotype and environment on fatty acid composition of Lupinus albus L. seed. Food Chem. 108, 600-606. doi: 10.1016/j.foodchem.2007.11.016

Cernay, C., Ben-Ari, T., Pelzer, E., Meynard, J.-M., and Makowski, D. (2015). Estimating variability in grain legume yields across Europe and the Americas. Sci. Rep. 5, 11171. doi: 10.1038/srep11171

Clark, R., and Johnson, S. (2002). Sensory acceptability of foods with added lupin (Lupinus angustifolius) kernel fiber using pre-set criteria. J. Food Sci. 67, 356-361. doi: 10.1111/j.1365-2621.2002.tb11410.x high-protein ingredients should be devised to guarantee the socio-economic value of these crops. Reformulating traditional foods, rather than developing totally novel ones, may be a more effective strategy to capture the imagination of the European consumer, and lead to desirable changes in overall diet. There is huge potential market demand for lupin-based products, with niches in growing sectors, such as vegetarians, vegans, and people with intolerance or allergy to gluten, soya, milk, or egg. The incorporation of lupin ingredients as a source of protein for human consumption depends largely upon their nutritional quality, but also on their ability to be used as, or incorporated into, foods that will be readily consumed. There are European enterprises with a strong background and wealth of expertise on market analysis, consumer acceptance issues, legal issues and marketing tools, which are required to develop new and successful lupin products that constitute a serious European alternative to the prevailing soya bean-derived food monopoly.

\section{Funding}

This work was supported by a grant from MINECO (AGL201340758-R).

\section{Acknowledgments}

The authors wish to thank all participants in the LUSH Proteins Consortium for their valuable contribution.

Clemente, A. (2000). Enzymatic protein hydrolysates in human nutrition. Trends Food Sci. Tech. 11, 254-262. doi: 10.1016/S0924-2244(01)00007-3

Coba de la Peña, T., and Pueyo, J. J. (2012). Legumes in the reclamation of marginal soils, from cultivar and inoculant selection to transgenic approaches. Agron. Sustain. Dev. 32, 65-91. doi: 10.1007/s13593-011-0024-2

de Visser, C. L. M., Schreuder, R., and Stoddard, F. L. (2014). The EU's dependency on soya bean import for the animal feed industry and potential for EU produced alternatives. OCL 21, D407. doi: 10.1051/ocl/2014021

Dervas, G., Doxastakis, G., Hadjisavva-Zinoviadi, S., and Triantafillakos, N. (1999). Lupin flour addition to wheat flour doughs and effect on rheological properties. Food Chem. 66, 67-73. doi: 10.1016/S0308-8146(98)00234-9

Doxastakis, G., Zafiriadis, I., Irakli, M., Marlani, H., and Tananaki, C. (2002). Lupin, soya and triticale addition to wheat flour doughs and their effect on rheological properties. Food Chem. 77, 219-227. doi: 10.1016/S0308-8146(01) 00362-4

Duranti, M., and Morazzoni, P. (2011). Nutraceutical properties of lupin seed proteins. A great potential still waiting for full exploitation. Agro Food Ind. Hi Technol. 22, 20-23.

Elshire, R. J., Glaubitz, J. C., Sun, Q., Poland, J. A., Kawamoto, K., Buckler, E. S., et al. (2011). A robust, simple genotyping-by-sequencing (GBS) approach for high diversity species. PLoS ONE 6:e19379. doi: 10.1371/journal.pone.0019379

Fernández-Pascual, M., Pueyo, J. J., de Felipe, M. R., Golvano, M. P., and Lucas, M. M. (2007). Singular features of the Bradyrhizobium-Lupinus symbiosis. Dyn. Soil Dyn. Plant 1, 1-16.

Fiocchi, A., Sarratud, P., Terracciano, L., Vacca, E., Bernardini, R., Fuggetta, D., et al. (2009). Assessment of the tolerance to lupine-enriched pasta in peanut-allergic children. Clin. Exp. Allergy 39, 1045-1051. doi: 10.1111/j.13652222.2009.03199.x

Frias, J., Gulewicz, P., Martinez-Villaluenga, C., Pilarski, R., Blazquez, E., Jimenez, B., et al. (2009). Influence of germination with different selenium solutions on nutritional value and cytotoxicity of lupin seeds. J. Agric. Food Chem. 57, 1319-1325. doi: 10.1021/jf8028368

Garcia-Mora, P., Penas, E., Frias, J., Gomez, R., and Martinez-Villaluenga, C. (2015). High-pressure improves enzymatic proteolysis and the release of peptides with 
angiotensin I converting enzyme inhibitory and antioxidant activities from lentil proteins. Food Chem. 171, 224-232. doi: 10.1016/j.foodchem.2014.08.116

Guémes-Vera, N., Pena-Bautista, R. J., Jimenez-Martinez, C., Davila-Ortiz, G., and Calderon-Dominguez, G. (2008). Effective detoxification and decoloration of Lupinus mutabilis seed derivatives, and effect of these derivatives on bread quality and acceptance. J. Sci. Food Agric. 88, 1135-1143. doi: 10.1002/jsfa.3152

Heffner, E. L., Sorrells, M. E., and Jannink, J. L. (2009). Genomic selection for crop improvement. Crop Sci. 49, 1-12. doi: 10.2135/cropsci2008.08.0512

Holden, L., Sletten, G. B. G., Lindvik, H., Faeste, C. K., and Dooper, M. M. (2008). Characterization of IgE binding to lupin, peanut and almond with sera from lupin-allergic patients. Int. Arch. Allergy Immunol. 146, 267-276. doi: $10.1159 / 000121461$

Kalogeropoulos, N., Chiou, A., Ioannou, M., Karathanos, V. T., Hassapidou, M., and Andrikopoulos, N. K. (2010). Nutritional evaluation and bioactive microconstituents (phytosterols, tocopherols, polyphenols, triterpenic acids) in cooked dry legumes usually consumed in the Mediterranean countries. Food Chem. 121, 682-690. doi: 10.1016/j.foodchem.2010.01.005

Keying, Q., Changzhonga, R., and Zaigui, L. (2009). An investigation on pretreatments for inactivation of lipase in naked oat kernels using microwave heating. J. Food Eng. 95, 280-284. doi: 10.1016/j.jfoodeng.2009.05.002

Lambers, H., Bishop, J. G., Hopper, S. D., Etienne Laliberté, E., and Zúñiga-Feest, A. (2012). Phosphorus-mobilization ecosystem engineering: the roles of cluster roots and carboxylate exudation in young P-limited ecosystems. Ann. Bot. 110, 329-348. doi: 10.1093/aob/mcs130

Laine, P., Toppinen, E., Kivela, R., Taavitsainen, V. M., Knuutila, O., Sontag-Strohm, T., et al. (2011). Emulsion preparation with modified oat bran: optimization of the emulsification process for microencapsulation purposes. J. Food. Eng. 104, 538-547. doi: 10.1016/j.jfoodeng.2011.01.014

Marco, C., and Rosell, C. M. (2008). Functional and rheological properties of protein enriched gluten free composite flours. J. Food Eng. 88, 94-103. doi: 10.1016/j.jfoodeng.2008.01.018

Muranyi, S., Otto, C., Pickardt, C., Koehler, P., and Schweiggert-Weisz, U. (2013). Microscopic characterisation and composition of proteins from lupin seed (Lupinus angustifolius L.) as affected by the isolation procedure. Food Res. Int. 54, 1419-1429. doi: 10.1016/j.foodres.2013.10.004

Nemecek, T., von Richthofen, J. S., Dubois, G., Casta, P., Charles, R., and Pahl, H. (2008). Environmental impacts of introducing grain legumes into European crop rotation. Eur. J. Agron. 28, 380-393. doi: 10.1016/j.eja.2007.11.004

Pakarinen, A., Maijala, P., Stoddard, F. L., Santanen, A., Tuomainen, P., Kymalainen, M., et al. (2011). Evaluation of annual bioenergy crops in the boreal zone for biogas and ethanol production. Biomass Bioenerg. 35, 3071-3078. doi: 10.1016/j.biombioe.2011.04.022

Pollard, N. J., Stoddard, F. L., Popineau, Y., Wrigley, C. W., and MacRitchie, F. (2002). Lupin flours as additives: dough mixing, breadmaking, emulsifying, and foaming. Cereal Chem. 79, 662-669. doi: 10.1094/CCHEM.2002.79.5.662

Preissel, S., Reckling, M., Schläfke, N., and Zander, P. (2015). Magnitude and farmeconomic value of grain legume pre-crop benefits in Europe: a review. Field Crops Res. 175, 64-79. doi: 10.1016/j.fcr.2015.01.012

Rodríguez-Ambriz, S. L., Martinez-Ayala, A. L., Millan, F., and Davila-Ortiz, G. (2005). Composition and functional properties of Lupinus campestris protein isolates. Plant Food Hum. Nutr. 60, 99-107. doi: 10.1007/s11130-005-6835-z

Schutyser, M. A. I., and van der Goot, A. J. (2011). The potential of dry fractionation processes for sustainable plant protein production. Trends Food Sci. Technol. 22, 154-164. doi: 10.1016/j.tifs.2010.11.006

Strozycki, P. M., Szczurek, A., Lotocka, B., Figlerowicz, M., and Legocki, A. B. (2007). Ferritins and nodulation in Lupinus luteus: iron management in indeterminate type nodules. J. Exp. Bot. 58, 3145-3153. doi: 10.1093/jxb/erm152

Terruzzi, I., Senesi, P., Magni, C., Montesano, A., Scarafoni, A., Luzi, L., et al. (2011). Insulin-mimetic action of conglutin-gamma, a lupin seed protein, in mouse myoblasts. Nutr. Metab. Cardiovas. 21, 197-205. doi: 10.1016/j.numecd.2009.09.004

Walsh, C. J., Guinane, C. M., O’Toole, P. W., and Cotter, P. D. (2014). Beneficial modulation of the gut microbiota. FEBS Lett. 588, 4120-4130. doi: 10.1016/j.febslet.2014.03.035

Zhang, T., Jiang, B., Miao, M., Mu, W., and Li, Y. (2012). Combined effects of high-pressure and enzymatic treatments on the hydrolysis of chickpea protein isolates and antioxidant activity of the hydrolysates. Food Chem. 135, 904-912. doi: 10.1016/j.foodchem.2012.05.097

Conflict of Interest Statement: The authors declare that the research was conducted in the absence of any commercial or financial relationships that could be construed as a potential conflict of interest.

Copyright (ㅇ 2015 Lucas, Stoddard, Annicchiarico, Frías, Martínez-Villaluenga, Sussmann, Duranti, Seger, Zander and Pueyo. This is an open-access article distributed under the terms of the Creative Commons Attribution License (CC BY). The use, distribution or reproduction in other forums is permitted, provided the original author(s) or licensor are credited and that the original publication in this journal is cited, in accordance with accepted academic practice. No use, distribution or reproduction is permitted which does not comply with these terms. 


\title{
The Nutritional and Safety Challenges Associated with Lupin Lacto-Fermentation
}

\author{
Elena Bartkiene $^{1 *}$, Vadims Bartkevics ${ }^{2}$, Vytaute Starkute ${ }^{1}$, Daiva Zadeike $^{3}$ and \\ Grazina Juodeikiene ${ }^{3}$ \\ ${ }^{1}$ Food Safety and Quality, Lithuanian University of Health Sciences, Kaunas, Lithuania, ${ }^{2}$ Chemistry, BIOR, Ryga, Latvia, \\ ${ }^{3}$ Kaunas University of Technology, Kaunas, Lithuania
}

Keywords: lupins, fermentation, nutrition, safety, lactic acid bacteria

\section{INTRODUCTION}

OPEN ACCESS

Edited by:

José J. Pueyo,

Institute of Agricultural Sciences,

CSIC, Spain

Reviewed by:

Elena Peñas,

Institute of Food Technology and Nutrition (ICTAN-CSIC), Spain

Miroljub Bogdam Barac,

University of Belgrade, Serbia

*Correspondence:

Elena Bartkiene

elena.bartkiene@/smuni.It

Specialty section:

This article was submitted to Crop Science and Horticulture,

a section of the journal

Frontiers in Plant Science

Received: 26 April 2016

Accepted: 15 June 2016

Published: 28 June 2016

Citation:

Bartkiene E, Bartkevics V, Starkute V, Zadeike D and Juodeikiene G (2016) The Nutritional and Safety Challenges

Associated with Lupin Lacto-Fermentation.

Front. Plant Sci. 7:951.

doi: $10.3389 /$ fpls.2016.00951
A sustainable agriculture that can satisfy the ever-rising global demand for food and animal feeds is becoming a manifest necessity in the light of the growing population, and the security concerns in the increasing land-use for food/feed. There has been a recent growth of interest in the positive impact of the functional ingredients derived from plants on human health. The proposed beneficial effects of such ingredients span from a decrease in plasma glucose levels and in preventing hypertension, to controlling obesity, improving satiety and stabilizing plasma lipid homeostasis. Lupin is a sustainable crop that plays an important role in organic farming, thus, the interest in lupin as a protein source for human and animal nutrition has increased in recent years.

The use of legumes as a source of protein is somewhat limited by the low digestibility of most plant proteins (Neves et al., 2006). Previous digestibility studies of protein obtained from legumes have shown interactions between antinutritional compounds, such as trypsin inhibitors and tannins, and the decreased proteolytic susceptibility of protein complexes, thereby decreasing the food value of plant proteins (Agte et al., 1998). The digestibility of lupin protein could be improved by lactic acid fermentation (Bartkiene et al., 2015).

However, it should be noted that lactic acid fermentation is a traditional process for food and feed production that may also result in the formation of undesirable compounds (e.g., biogenic amines and D-lactate). Also, lupin exhibits useful techno functional properties allowing its use as an ingredient in the production of several palatable food products, such as biscuits, pasta, and bread (Lee et al., 2006; Guillamon et al., 2010; Jayasena and Nasar-Abbas, 2011, 2012; Bartkiene et al., 2013a, 2016b). For instance, the supplementation of wheat flour with the high-protein lupin flours can improve the nutritional quality of baked goods (Gomez et al., 2008).

In addition, lupine does not contain gluten (a mixture of proteins found in wheat and related grains), thus, it could be used as a functional ingredient in gluten-free foods (Scarafoni et al., 2009). However, the use of lupin flour with a high protein content for the production of cereal products may cause problems associated with the formation of acrylamide (Bartkiene et al., 2013b, 2016a). The tolerable daily intake (TDI) levels of acrylamide for neurotoxicity were estimated to be $40 \mu \mathrm{g} / \mathrm{kg}$ per day and for cancer-2.6 $\mu \mathrm{g} / \mathrm{kg}$ per day (Tardiff et al., 2010). Mitigation strategies propose modifying the product formulations or processing conditions, to minimize the pathways of acrylamide formation. For this reason, it is very important to assess the risk of new raw materials and technological procedures and to evaluate the safety parameters of the finished products. 


\section{THE APPLICATION OF LACTO-FERMENTATION TO IMPROVE THE NUTRITIONAL AND FUNCTIONAL VALUE OF LUPIN}

The in vitro digestibility of proteins depends on the type of fermentation (solid state or submerged fermentation), the type of microorganisms and the type of legume (Bartkiene et al., 2015). The low digestibility of plant proteins together with the limited content of essential amino acids result in a low nutritional value compared to animal proteins (Carbonaro et al., 2012). The fermentation of legumes enriches products with highvalue proteins of microbial origin, reduces the concentration of antinutritional factors, increases the antioxidant activity, and generally improve the nutritional characteristics (Bartkiene et al., 2011; Curiel et al., 2015).

Fermentation with lactobacilli has been reported to increase the concentrations of free amino acids in legumes (Curiel et al., 2014; Coda et al., 2015). The fermentation method (solid state or submerged fermentation), type of microorganisms applied for the fermentation (lactic acid bacteria), and the variety of lupin significantly affect the content of free essential and nonessential amino acids in fermented lupin wholemeal (Bartkiene et al., 2016b). By optimizing the fermentation technology it is possible to produce bioactive peptides, which are of great interest for the design of functional foods and nutraceuticals.

Our previous study demonstrated the potential contribution of fermented lupin to the human diet through improving the gut environment and eliminating pathogenic bacteria (Bartkiene et al., 2013b). The effect of diet supplemented with lupin flour that was fermented with a probiotic strain of Pediococcus acidilactici on the gastrointestinal tract (GIT) of Wistar rats resulted in the enhanced activities of $\alpha$-glucosidase, $\beta$-galactosidases, as well as high levels of lactic acid bacteria, bifidobacteria, and enterococci.

The lacto-fermentation of lupin flour had a significantly lowering effect on Escherichia coli compared to the control group. The dominant flora of the large intestine, like Bifidobacterium and anaerobic cocci, were found at high levels in diets containing fermented lupin.

\section{THE SAFETY PARAMETERS OF FERMENTED LUPINE-THE CONCENTRATIONS OF BIOGENIC AMINES AND D-LACTIC ACID}

Many strains of lactic acid bacteria have been referred to the European Food Safety Authority (EFSA) for safety assessment without raising any safety concerns. As a result, they have been included in the QPS (Qualified Presumption of Safety) list authorized for use in the food and feed chain within the European Union (EFSA, 2012). The same applies to the US, where they display the Generally Regarded as Safe (GRAS) status assigned by the U.S. Food and Drug Administration (FDA). However, some properties and enzymatic activity of the LAB can generate hazardous compounds such as biogenic amines (BAs), they should be avoided in food products (Linares et al., 2012).

Biogenic amines are formed by the enzymatic decarboxylation of amino acids and several factors as lupin variety, fermentation conditions, and the fermentative LAB strain have a significant effect on the free amino acid profile and content and, therefore, in the content of biogenic amines of lupins (Bartkiene et al., 2016b). Arginine is easily converted to agmatine and can be degraded to ornithine via bacterial activity, while ornithine undergoes decarboxylation to putrescine. Lysine can be converted by bacterial action into cadaverine. Histidine can be decarboxylated to histamine under certain conditions. Tyramine, tryptamine, and $\beta$-phenylethylamine arise in the same manner from tyrosine, tryptophan, and phenylalanine (Montet and Ray, 2016).

Microbially produced lactic acid is usually a mixture of the $\mathrm{L}(+)$ - and $\mathrm{D}(-)$-forms. As the latter cannot be metabolized by humans, excessive intake can result in acidosis, which is a disturbance in the acid-alkali balance in the blood. The potential toxicity of D-lactic acid is of particular concern for malnourished and sick people (Motarjemi, 2002). The increased levels of Dlactate in plasma and urine have been demonstrated in cases of intestinal ischaemia, short bowel, and appendicitis, and are considered as a marker of dysbiosis and/or increased intestinal permeability (Verbeke et al., 2015). Therefore, the desirable lactic acid isomer that should be produced in food and feed fermentation is L-lactate.

\section{THE APPLICATION OF LACTO-FERMENTATION TO REDUCE ACRYLAMIDE IN BAKERY PRODUCTS SUPPLEMENTED WITH LUPIN FLOUR}

Cereals are deficient in lysine but are rich in cysteine and methionine. Legumes, on the other hand, are rich in lysine but deficient in sulfur-containing amino acids. Hereby, the overall protein quality could be improved by combining cereals with legumes. However, the addition of protein-rich legume flours could increase the content of acrylamide in baked products. The peptides and amino acids formed by protein breakdown may serve as precursors for acrylamide formation during the heating of foods containing reducing sugars. The elimination of acrylamide precursors using biotechnological tools is regarded as a promising way of eliminating acrylamide.

The reduction of acrylamide concentration in cereal products can be achieved by the prolonged fermentation of dough as a consequence of extensive asparagine consumption by yeast (Claus et al., 2008; Sadd et al., 2008). Lacto-fermentation decreased the concentrations of asparagine and reduced saccharides in lupin and thus reduced the acrylamide content in wheat-lupin biscuits (Bartkiene et al., 2016a). The levels of acrylamide in wheat-lupin bread could be lowered to $49.1 \%$ by fermentation with $L$. sakei. The fermentation of lupin sourdoughs with Pedioccocus strains can decrease the levels of acrylamide in bread by $18.7 \%$ on average, compared to bread made with untreated lupin flour. 
For the purpose of enriching wheat bread with lupin as a highquality protein source, pure lactic acid bacteria cultures, such as L. sakei and $P$. pentosaceus, characterized by higher proteolytic activity, has been recommended for the fermentation of lupin flour in sourdough production (Bartkiene et al., 2013c). The most effective acrylamide reduction in biscuits (by 85\%) was reached using solid state fermentation of lupin with $P$. acidilactici. Fermented lupin supplementation offers a great potential in the development of bakery products with enhanced nutritional value and low acrylamide content.

\section{CONCLUSIONS}

Lupin is a good alternative source of protein, enabling affordable nutritional enrichment of food and feed and providing better access to protein for underserved populations. Most of the research toward this goal has been focused on improving the antioxidant activity, sensory properties, and the functional and nutritional value of the products. In our opinion, the influence of new ingredients included in the food/feed formulas should be subject to more complex analysis. The formation of undesirable compounds during the manufacturing process is unavoidable,

\section{REFERENCES}

Agte, V., Joshi, S., Khot, S., Parnikar, K., and Chiplonkar, S. (1998). Effect of processing on phytate degradation and mineral solubility in pulses. J. Food Technol. 35, 330-332.

Bartkiene, E., Bartkevics, V., Rusko, J., Starkute, V., Zadeike, D., and Juodeikiene, G. (2016b). Changes of the free amino acids and the biogenic amines in Lupinus luteus and Lupinus angustifolius during lactic acid fermentation. Int. J. Food Sci. Tech. doi: 10.1111/j.1365-2621.2011.02668.x

Bartkiene, E., Jakobsone, I., Juodeikiene, G., Vidmantiene, D., Pugajeva, I., and Bartkevics, V. (2013b). Effect of lactic acid fermentation of lupine wholemeal on acrylamide content and quality characteristics of wheat-lupine bread. Int. J. Food Sci. Nutr. 64, 890-896. doi: 10.3109/09637486.2013.805185

Bartkiene, E., Jakobsone, I., Pugajeva, I., Bartkevics, V., Zadeike, D., and Juodeikiene, G. (2016a). Reducing of acrylamide formation in wheat biscuits supplemented with flaxseed and lupine. LWT Food Sci. Technol. 65, 275-282. doi: 10.1016/j.lwt.2015.08.002

Bartkiene, E., Juodeikiene, G., Vidmantiene, D., Viskelis, P., and Urbonaviciene, D. (2011). Nutritional and quality aspects of wheat sourdough bread using $L$. luteus and L. angustifolius flours fermented by Pedioccocus acidilactici. Int. J. Food Sci. Tech. 46, 1724-1733. doi: 10.1111/j.1365-2621.2011.02668.x

Bartkiene, E., Juodeikiene, G., Vidmantiene, D., Zdunczyk, Z., Zdunczyk, P., Juśkiewicz, J., et al. (2013c). Influence of diets to Wistar rats supplemented with soya, flaxseed and lupine products treated by lactofermentation to improve their gut health. Int. J. Food Sci. Nutr. 64, 730-739. doi: 10.3109/09637486.2013.775230

Bartkiene, E., Krungleviciute, V., Juodeikiene, G., Vidmantiene, D., and Maknickiene, Z. (2015). Solid state fermentation with lactic acid bacteria to improve the nutritional quality of lupin and soya bean. J. Sci. Food Agric. 95, 1336-1342. doi: 10.1002/jsfa.6827

Bartkiene, E., Schleining, G., Rekstyte, T., Krungleviciute, V., Juodeikiene, G., Vaiciulyte-Funk, L., et al. (2013a). Influence of the addition of lupin sourdough with different lactobacilli on dough properties and bread quality. Int. J. Food Sci. Tech. 48, 2613-2620. doi: 10.1111/ijfs.12257

Carbonaro, M., Maselli, P., and Nucara, A. (2012). Relationship between digestibility and secondary structure of raw and thermally treated legume proteins: a Fourier transform infrared (FT-IR) spectroscopic study. Amino Acids 43, 911-921. doi: 10.1007/s00726-011-1151-4 and specific technological solutions may be encouraged to mitigate these issues. Lactic acid fermentation with selected bacterial strains could be used for increasing the functional value of lupin.

A complex analysis during all technological steps should be performed to avoid the formation of toxic compounds, in order to ensure the safety of the food products. In this case, the appropriate technological parameters should be optimized (the selection of starters for fermentation and the choice of lupin variety, appropriate design of fermentation process).

\section{AUTHOR CONTRIBUTIONS}

EB working on lupin proteins biotechnological treatment by using selected microorganisms, lupin proteins safety parameters and nutritional value evaluation. VB working on chemical methods development for toxic compounds in food produced with lupin seeds evaluation. VS working on lupin proteins isolation and safety parameters evaluation. DZ working on lupin proteins isolation and technological parameters evaluation. GJ working on lupin proteins hydrolysis and nutrition design.
Claus, A., Mongili, M., Weisz, G., Schieber, A., and Carle, R. (2008). Impact of formulation and technological factors on the acrylamide content of wheat bread and bread rolls. J. Cereal Sci. 7, 546-554. doi: 10.1016/j.jcs.2007.06.011

Coda, R., Melama, L., Rizzello, C. G., Curielb, J. A., Sibakov, J., Holopainena, U., et al. (2015). Effect of air classification and fermentation by Lactobacillus plantarum VTT E-133328 on faba bean (Vicia faba L.) flour nutritional properties. Int. J. Food Microbiol. 193, 34-42. doi: 10.1016/j.ijfoodmicro.2014.10.012

Curiel, J. A., Coda, R., Centomani, I., Summo, C., Gobbetti, M., and Rizzello, C. G. (2015). Exploitation of the nutritional and functional characteristics of traditional Italian legumes: the potential of sourdough fermentation. Int. J. Food Microbiol. 196, 51-61. doi: 10.1016/j.ijfoodmicro.2014. 11.032

Curiel, J. A., Coda, R., Limitone, A., Katina, K., Raulio, M., Giuliani, G., et al. (2014). Manufacture and characterization of pasta made with wheat flour rendered gluten-free using fungal proteases and selected sourdough lactic acid bacteria. J. Cereal Sci. 59, 79-87. doi: 10.1016/j.jcs.2013.09.011

EFSA (2012). Scientific Opinion on the maintenance of the list of QPS biological agents intentionally added to food and feed. EFSA J. 10, 3020.

Gomez, M., Oliete, B., Rosell, C. M., Pando, V., and Fernandez, E. (2008). Studies on cake quality made of wheat-chickpea flour blends. LWT Food Sci. Technol. 41, 1701-1709. doi: 10.1016/j.lwt.2007.11.024

Guillamon, E., Cuadrado, C., Pedrosa, M. M., Varela, A., Cabellos, B., Muzquiz, M., et al. (2010). Breadmaking properties of wheat flour supplemented with thermally processed hypoallergenic lupine flour. Span. J. Agric. Res. 8, 100-108. doi: 10.5424/sjar/2010081-1148

Jayasena, V., and Nasar-Abbas, S. M. (2011). Effect of lupin flour incorporation on the physical characteristics of dough and biscuits. Qual. Assur. Safety Crops Foods 3, 140-147. doi: 10.1111/j.1757-837X.2011.00100.x

Jayasena, V., and Nasar-Abbas, S. M. (2012). Development and quality evaluation of high-protein and high-dietary fiber pasta using lupin flour. J. Text. Stud. 43, 153-163. doi: 10.1111/j.1745-4603.2011.00326.x

Lee, Y. P., Mori, T. A., Sipsas, S., Barden, A., Puddey, I. B., Burke, V., et al. (2006). Lupin-enriched bread increase satiety and reduces energy intake acutely. Am. J. Clin. Nutr. 84, 975-980.

Linares, D. M., Del Rio, B., Ladero, V., Martinez, N., Fernandez, M., Martin, M. C., et al. (2012). Factors influencing biogenic amines accumulation in dairy products. Front. Microbiol. 3:180. doi: 10.3389/fmicb.2012.00180 
Montet, D., and Ray, R. C. (2016). Fermented Foods. Biochemistry and Biotechnology. New York, NY: CRC Press.

Motarjemi, Y. (2002). Impact of small scale fermentation technology on food safety in developing countries. Int. J. Food Microbiol. 75, 213-229. doi: 10.1016/S01681605(01)00709-7

Neves, V. A., Silva, S. I. Jr., and Silva, M. A. (2006). Isolamento da globulina majoritária, digestibilidade in vivo e in vitro das proteínas do tremoço-doce (Lupinus albus L.), var. Multolupa. Ciência e Tecnologia de Alimentos 26, 832-840. doi: 10.1590/S0101-20612006000400019

Sadd, P., Hamlet, C. G., and Liang, L. (2008). Effectiveness of methods for reducing acrylamide in bakery products. J. Agric. Food Chem. 56, 6154-6161. doi: 10.1021/jf7037482

Scarafoni, A., Ronchi, A., and Duranti, M. (2009). A realtime PCR method for the detection and quantification of lupin flour in wheat flour-based matrices. Food Chem. 115, 1088-1093. doi: 10.1016/j.foodchem.2008.12.087

Tardiff, G. T., Gargas, M. L., Kirman, C. R., Carson, M. L., and Sweeney, L. M. (2010). Estimation of safe dietary intake levels of acrylamide for humans. Food Chem. Toxicol. 48, 658-667. doi: 10.1016/j.fct.2009.11.048
Verbeke, K. A., Boobis, A. R., Chiodini, A., Edwards, C. A., Franck, A., Kleerebezem, M., et al. (2015). Towards microbial fermentation metabolites as markers for health benefits of prebiotics. Nutr. Res. Rev. 28, 42-66. doi: 10.1017/S09544224150 00037

Conflict of Interest Statement: The authors declare that the research was conducted in the absence of any commercial or financial relationships that could be construed as a potential conflict of interest.

Copyright (C) 2016 Bartkiene, Bartkevics, Starkute, Zadeike and Juodeikiene. This is an open-access article distributed under the terms of the Creative Commons Attribution License (CC BY). The use, distribution or reproduction in other forums is permitted, provided the original author(s) or licensor are credited and that the original publication in this journal is cited, in accordance with accepted academic practice. No use, distribution or reproduction is permitted which does not comply with these terms. 


\section{OPEN ACCESS}

Edited by:

José J. Pueyo,

Consejo Superior de Investigaciones

Cientificas, Spain

Reviewed by:

Abu Hena Mostafa Kamal,

University of Texas at Arlington, USA

Marty Riche,

Florida Atlantic University, USA Alfonso Clemente,

Spanish National Research Council,

Spain

${ }^{*}$ Correspondence:

Eliot M. Herman

emherman@email.arizona.edu

Specialty section:

This article was submitted to

Crop Science and Horticulture,

a section of the journal

Frontiers in Plant Science

Received: 27 December 2015

Accepted: 21 March 2016

Published: 5 April 2016

Citation:

Herman EM and Schmidt MA (2016)

The Potential for Engineering

Enhanced Functional-Feed Soybeans

for Sustainable Aquaculture Feed.

Front. Plant Sci. 7:440

doi: $10.3389 /$ fpls.2016.00440

\section{The Potential for Engineering Enhanced Functional-Feed Soybeans for Sustainable Aquaculture Feed}

\author{
Eliot M. Herman* and Monica A. Schmidt \\ School of Plant Sciences, University of Arizona, Tucson, AZ, USA
}

Aquaculture is the most rapidly growing segment of global animal production that now surpasses wild-capture fisheries production and is continuing to grow $10 \%$ annually. Sustainable aquaculture needs to diminish, and progressively eliminate, its dependence on fishmeal-sourced feed from over-harvested fisheries. Sustainable aquafeed sources will need to be primarily of plant-origin. Soybean is currently the primary global vegetable-origin protein source for aquaculture. Direct exchange of soybean meal for fishmeal in aquafeed has resulted in reduced growth rates due in part to soybean's anti-nutritional proteins. To produce soybeans for use in aquaculture feeds a new conventional line has been bred termed Triple Null by stacking null alleles for the feedrelevant proteins Kunitz Trypsin Inhibitor, lectin, and P34 allergen. Triple Null is now being further enhanced as a platform to build additional transgene traits for vaccines, altered protein composition, and to produce high levels of $\beta$-carotene an intrinsic orangecolored aquafeed marker to distinguish the seeds from commodity beans and as the metabolic feedstock precursor of highly valued astaxanthin.

Keywords: aquaculture, aquafeed, soybean, carotenoid, vaccines, Kunitz trypsin inhibitor, soybean agglutinin, P34 allergen

\section{AQUACULTURE IS THE FASTEST GROWING SEGMENT OF GLOBAL ANIMAL PRODUCTION THAT WILL REQUIRE NEW SUSTAINABLE SOURCES OF FEED}

The global human population is projected to grow to 9.6 billion individuals by 2050 and will continue to 12 billion individuals or more by 2100 (Gerland et al., 2014). This population growth will require an increase of $70 \%$ more animal production that translates to a need for $235 \%$ more animal feed. Soybean is the largest plant-source input of protein and reduced nitrogen for formulated animal feed that drives a global industry of production and consumption. Aquaculture is most rapidly growing sector of the animal food-production sectors, increasing at nearly $10 \%$ per year (Food and Agriculture Organization [FAO], 2012). Aquaculture-sourced fish surpasses wild capture fisheries in annual production and this differential is projected to rapidly increase due to overfishing and habitat destruction in parallel to increased production in the aquaculture sector. As aquaculture displaces wild capture fisheries providing feed sources for cultured animals presents an immense challenge. Many commercially raised fish are carnivorous (e.g., trout and salmon), while others are omnivores and herbivores (e.g., catfish, carp, and tilapia); therefore, different fish species vary in their capacity to effectively use different kinds of feed. Aquaculture currently over-relies on fishmeal to provide high-quality feed protein. Annual fishmeal production has been constant for 
the last 15 years at approximately 6.5 million metric tons. Current or projected increase of fishmeal production to support aquaculture growth cannot be sustained (Watson et al., 2015).

A sustainable feed solution for aquaculture would be for farmed fish to be fed renewable plant-sourced protein and oil products harvested from terrestrial farms (Gatlin et al., 2007; Naylor et al., 2009; Rust et al., 2011). While substituting plant protein for fishmeal is now routine at low levels, in most cases increasing the proportion of plant protein in fish feed limits fish growth rates and feed efficiency. There is an opportunity to engineer seed crops as functional feed ingredient that specifically address the challenges of increasing the proportion of plant-based protein in commercial fish aquafeeds. Soybean is currently used as a primary source of vegetable protein supplement for aquafeed (Tacon et al., 2012) and is a large US export (Hardy, 1996). Soybean, particularly at high inclusion levels, in aquaculture can result in reduced growth rate dues at least in part from soybean's anti-nutritional proteins (Krogdahl et al., 1994, 2010; Baeverfjord and Krogdahl, 1996; Burrells et al., 1999; BakkeMcKellep et al., 2000; Buttle et al., 2001; Lilleeng et al., 2007; Sahlmann et al., 2013). One approach is to use biotechnology to suppress anti-nutritional proteins or to alter seed protein composition (see Herman, 2003, for review). To some degree conventional breeding and stacking of traits derived from nonbiotech sources such as collections and mutation to enhance seed composition can also meet trait goals. Other enhancements that are not encoded within the soybean genome will require adding foreign genes to produce stacked trait soybeans optimized for high-performance feed-ingredient (Herman, 2009).

\section{BREEDING LOW ANTI-NUTRITIONAL BIOACTIVITY SOYBEANS AND ITS DEVELOPMENT AS A PLATFORM TO EXPRESS ADDITIONAL TRANSGENE TRAITS}

\section{Developing a Conventional Low Bioactivity Soybean Seed; the Creation of Triple Null}

Soybeans null for bioactive seed proteins that have been isolated from the USDA soybean collection include Kunitz trypsin inhibitor (KTI) null (Orf and Hymowitz, 1979; Hymowitz, 1986), soybean agglutinin (SBA) null (Orf et al., 1978; Goldberg et al., 1983) and immunodominant soybean allergen P34 protein nulls (Josephs et al., 2006). Each of these nulls has the potential to partially address concerns of soybean feed/food consumption and stacked together these traits can form a platform for engineering enhanced soybean varieties. A Triple Null stack of the recessive nulls of KTI, SBA, and P34 in the standard cultivar Williams (the archetype used to elucidate the soybean genome) has been produced (Schmidt et al., 2015a). The parental components of the Triple Null $(p 34, k t i, l e)$ stack include frame shift mutants of P34 (Josephs et al., 2006; Bilyeu et al., 2009) and KTI (Jofuku et al., 1989) that still accumulate a small amount of authentic protein due to a shift of the start methionine inward in the open reading frame Schmidt et al. (2015a). The Lectin (LE) null results from a large transposon and does not produce a functional transcript or lectin protein accumulation (Orf et al., 1978; Goldberg et al., 1983). Proteomic analysis of Triple Null shows that the line lacks this trio of bioactive proteins while retaining the full complement of other proteome constituents without any other collateral bioactive protein alterations (Schmidt et al., 2015a).

\section{Triple Null Soybeans Can Be Transformed to Stack Additional Traits}

To enable further enhancement of Triple Null its capacity for biolistic transformation was assessed. Somatic embryos were induced from Triple Null and used in test biolistic transformation studies using a hygromycin-selection cassette (see Schmidt and Herman, 2008; Schmidt et al., 2011, 2015b for methods). The resulting transformed lines were selected and regenerated into somatic embryos that were then germinated to produce a $T_{0}$ population of plants. The resulting $T_{1}$ seeds are a segregating population typically requiring two or three generations of recurrent selection to produce homozygote lines. Figure 1 shows PCR analysis of a segregating population assessed by the presence of the Hygromycin marker demonstrating that Triple Null that is bred into a Williams 82 background is capable of being transformed by the same protocols as cv Jack. This indicates that the traits of Triple Null can be used as a platform to stack additional transgene traits and can be exchanged for other standard transformation soybean lines.

\section{POTENTIAL BIOTECHNOLOGY TRAITS TO ENHANCE TRIPLE NULL'S PERFORMANCE AS AN INGREDIENT IN AQUAFEED}

The capacity to transform Triple Null is enabling for further biotechnological modifications using gene expression and silencing to further optimize performance of the seeds in aquafeeds. As an aquafeed source there are potential targets of expressing bioactive proteins such as vaccines and to further remodel the seed proteome such as by optimizing its protein content for digestibility. There are additional modifications that could be added to soybean that produces products that are not feasible to produce from soybean's genome. These traits could reproduce lipid-soluble carotenoids and omega fatty acids that would otherwise be obtained from fish oil with the same sustainability issues as fishmeal or from chemical synthesis and/or modification.

\section{Protein Enhancement}

The soybean seed proteome consists of two dominant seed storage proteins (SPs) 7S conglycinin and $11 \mathrm{~S}$ glycinin and a number of additional moderately abundant proteins that are often bioactive including Kunitz and Bowman Birk trypsin inhibitors, lectin, P34 allergen, urease and lipoxygenases. Triple 


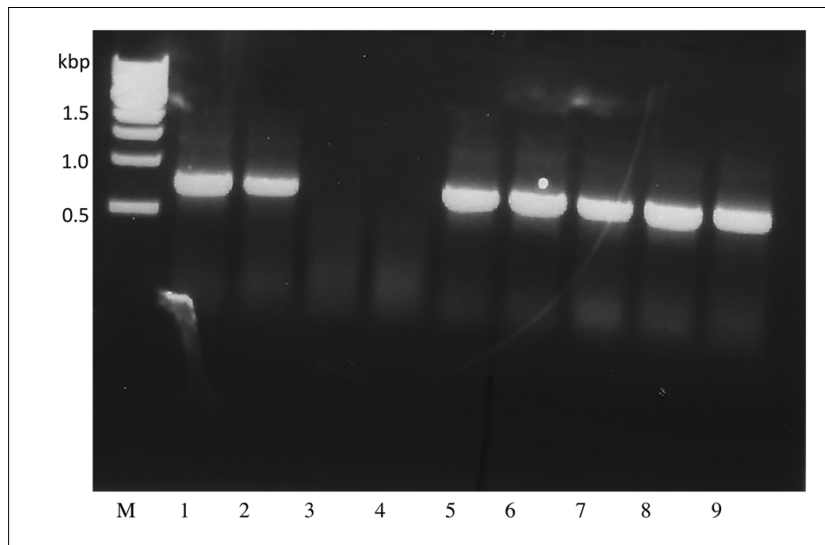

FIGURE 1 | Triple Null soybean is transformable via biolistic transformation of somatic embryos. Genomic PCR was performed on $T_{1}$ segregating Triple Null plants transformed with the hygromycin resistance marker gene expression cassette. Presence of the PCR amplicon $\sim 700$ bp in all samples except 3 and 4 indicate the presence of the hygromycin gene in those plants DNA, indicating a stable transformation event that is segregating in an expected Mendelian ratio.

Null stacking alleles from the USDA soybean collection addresses some of the most problematic bioactive proteins for aquafeed (Krogdahl et al., 1994; Buttle et al., 2001; Lilleeng et al., 2007). The SPs comprise about $65 \%$ of the seed's protein content and is regulated by genotype as a breeding trait. Most of the major soybean proteins are members of small gene families (for example Nielsen et al., 1989) making it difficult to identify conventional alleles that that silence all of the individual genes. Biotechnology approaches using co-suppression and RNAi has been used to knockout soybean genes and gene families of $\beta$-conglycinin (Kinney et al., 2001; Kim et al., 2014), P34 allergen (Herman et al., 2003), and both $\beta$-conglycinin and glycinin (Schmidt et al., 2011). Other lines have reduced Bowman Birk trypsin inhibitor (BBI) content by co-expressing an inactive variant (Livingstone et al., 2007) as not to further reduce the sulfur amino acid content of soybeans.

Silencing major soybean SPs results in compensatory remodeling of the proteome but not the seed protein content. This proteome compensation is termed protein rebalancing (Herman, 2014) that in soybean results when a major SP is silenced by either mutation or targeted genetic modification that should result in a significant shortfall of accumulated seed protein. The soybean remodels its proteome resulting in other intrinsic seed proteins increasing in accumulation to compensate for loss of major proteins that maintains both protein content (Schmidt et al., 2011) and total amino acid composition (Schmidt et al., 2011; Kim et al., 2014). From the biotechnologist's perspective proteome rebalancing can be exploited as a technology to replace major soybean SPs with other transgene products (Herman and Schmidt, 2014). Suppressing $\beta$-conglycinin that comprises $20 \%$ of the total seed protein results in enhanced accumulation of glycinin that maintains the standard protein content. Glycinin consists of a small five gene family to test the potential of exploiting the proteome rebalancing process a model GFP gene was constructed as a glycinin mimic and introgressed into $\beta$-conglycinin silenced seeds as an additional glycinin allele. This resulted in increasing GFP accumulation eight-fold from 1 to $8 \%$ of the total protein (Schmidt and Herman, 2008). Although this appears to be a potential engineering strategy to increase foreign protein content in seeds the proteome rebalancing processes by maintaining protein content and relative total amino acid this strategy does not appear to be effective to increase the sulfur amino acid content of soybean by expressing zein in a $\beta$-conglycinin suppressed background (Kim et al., 2014).

\section{Feed based Vaccines Are Economic Solution to Disease}

In animal production animal density is a key economic factor. High animal density is an economic necessity of production and this aggravates the problems of animal competition, waste management, and controlling the potential for disease. For fish production a number of bacterial and viral diseases have emerged that impact production. For many fish species especially the omnivorous and herbivorous species the low individual value of each fish limits the potential expenditure for antibiotic treatment or preventive vaccination. Fish can be immunized orally (Companjen et al., 2005; Plant and LaPatra, 2011; Tobar et al., 2011, 2015) or by bath exposure and if the vaccine antigens are inexpensive and effective this can enable disease prevention. There are problems to be resolved to make these protocols as effective as individual injection immunization particularly with primary sensitization (Munang'andu et al., 2015; Mutoloki et al., 2015). There is a large literature of prototype vaccines engineered by plant biotechnology for inclusion in food or feed especially for situations requiring mass immunization at minimal cost (Daniell et al., 2009). Plant biopharma is well matched to the needs and economic limitations of aquaculture (Clarke et al., 2013). Soybean feed-based vaccines can meet this need as a stacked trait. Soybean can be induced to produce up to $1 \%$ of its total protein shown as a prototype vaccine (Piller et al., 2005; Moravec et al., 2006). To produce greater levels of vaccine proteins technology has been developed to produce high-levels of heterologous proteins in soybean (Schmidt and Herman, 2008). An engineering strategy has been developed where transgenes encoding heterologous proteins can be produced at high levels, $>8 \%$ of the total protein, by mimicking the gene of a compensating glycinin. Using this type of technology it should be feasible to design feed vaccines that separately or simultaneously produce several antigens potentially stacked with a strong adjuvant such as the enterotoxin LTB (Moravec et al., 2006) that could be produced in different formulations to vary and stagger the sensitization. This approach may improve the performance of oral vaccines for aquaculture.

\section{Carotenoid Enhancement}

Astaxanthin $\left(3,3^{\prime}\right.$-dihydroxy $4,4^{\prime}$-diketo- $\beta$-carotene $)$ is the carotenoid responsible for giving many crustaceans and some bird species their signature pink/red color. Its principle commercial use is a flesh colorant for fish. Farm-raised salmonoid fish are deprived of phytoplankton and/or algae 
that would naturally provide this colorful compound, making it necessary to supplement fish diet with additive pigments in order to ensure that the resultant filets appeal to consumers. In the U.S. alone, astaxanthin sales per year are about \$200 million for just the salmon aquaculture industry (Lorenz and Cysewski, 2000). In order for plants to be an effective sustainable supply of this colorant it would have be both biosynthesized and accumulated at relevant levels and produced in a suitable delivery plant organ system. Proof-of-concept in the successful production of astaxanthin has been achieved in tobacco nectar (Mann et al., 2000; Ralley et al., 2004) and leaves (Hasunuma et al., 2008) and tubers of both potato (Gerjets and Sandmann, 2006) and carrot (Jayaraj et al., 2008). The highest level obtained was $64 \mu \mathrm{g}$ astaxantin/g of the nectar of transgenic tobacco plants. Production of this carotenoid in seeds has been shown to be possible but with marginal accumulation levels: transgenic canola (Brassica napus) seeds contained $0.2 \mu \mathrm{g}$ astaxanthin/g (Fujisawa et al., 2009) and transgenic soybean seeds $7 \mu \mathrm{g}$ astaxanthin/g dry seed (Pierce et al., 2015).

Variations in the amount of astaxanthin produced in different plant systems can be attributed to the inherent ability of certain plant organs to accumulate carotenoids, leaf and flower tissue more than seeds, and the catalytic attributes of the carotenoid biosynthetic enzymes. Enzymes in the carotenoid pathway have been reported to act upon an assortment of various substrates, often resulting in a mixture of carotenoids produced rather than a majority of a specific sought metabolite. Following on research showing that enhanced $\beta$-carotene levels could be achieved by the overexpression of a bacterial Pantoea phytoene synthase gene in soybean seeds (Figure 2) (Schmidt et al., 2015b), this construct was then combined individually with two ketolase genes from different sources: one from bacteria Brevundimonas spp and the other algae known for its high concentration of astaxanthin Haematococcus pluvialis. A mixture of carotenoids resulted with only one line using the bacterial ketolase producing detectable levels of astaxanthin (Pierce et al., 2015). Further emphasizing the importance of enzyme choice, three $\beta$-carotene ketolase enzymes were used from different sources in Arabidopsis leaves and found a wide variety of successful production of the desired astaxanthin carotenoid (Zhong et al., 2011). Up to $2 \mathrm{mg} / \mathrm{g}$ was produced when the ketolase gene from Chlamydomonas, compared to $0.24 \mathrm{mg} / \mathrm{g}$ when Chlorella was the gene source and non-detectable levels when Haematococcus ketolase gene was used. These findings emphasis the importance of enzyme selection in the biosynthesis of carotenoids giving promise to the future production of sustainable and commercially viable levels of this carotenoid pigment in a suitable plant delivery system.

\section{Feed-based Omega 3 Fatty Acids}

Desired oil modifications in seeds for aquaculture use would include the omega $3(\omega 3)$ fatty acids. These fatty acids have been shown to enhance fish growth rates (Tocher, 2015) and are one of the human health promoting constituents in fish (for review, Calder and Yaqoob, 2009). Many plant seed oils are abundant in the $\omega-3$ fatty acid $\alpha$ linolenic acid $\left(18: 3^{\Delta 9,12,15}\right)$ yet the health promoting long chain $\omega 3$ fatty

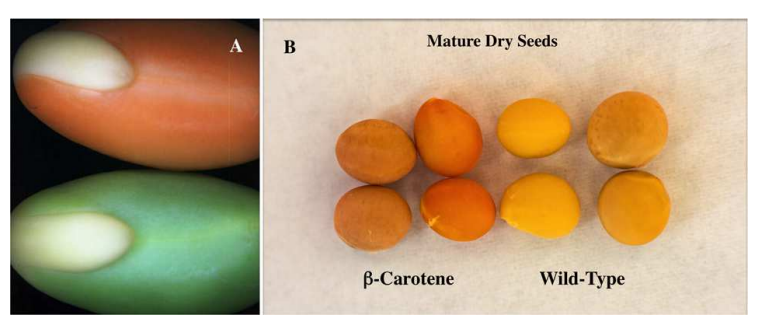

FIGURE 2 | $\beta$-carotene biofortified transgenic soybean seeds. (A) developing soybean cotyledons from (bottom) non-transgenic soybean compared to (top) chloroplast localized seed specific bacterial phytoene synthase transgenic soybean. (B; column 4) dry non-transgenic soybean seeds, (column 3) dry non-transgenic soybean seeds without seed coat, (column 2) dry transgenic soybean seeds without seed coat and (column 1) dry transgenic $\beta$-carotene soybean seeds. Enhanced $\beta$-carotene

accumulation in transgenic soybean seeds provides a visually obvious orange color in both developing cotyledons and dry seeds.

acids are more limiting in the human diet with their principle source being marine fish. Although a vital component to both human and marine life nutrition, long chain $\omega-3$ fatty acids eicosapentaenoic (EPA 20: $5^{\Delta 5,8,11,14,17}$ ) and docosahexaenoic acid (DHA 22:6 $6^{\Delta 4,7,10,13,16,19}$ ) must be consumed in diets due to human and fish being very limited in their ability to produce them (Williams and Burdge, 2006). The most successful research to produce DHA and EPA in oil seeds crops was reported in Brassica seeds and used nine introduced gene expression cassettes using fatty acid modifying enzymes from various marine sources to achieve $0.2 \%$ DHA and up to $15 \%$ EPA of total fatty acids (Wu et al., 2005). Research to produce these lipid compounds in plants has moved toward soon being able to make commercially viable levels for the aquaculture community.

\section{SOYBEANS IN FUNCTIONAL FEED WILL MEET AN URGENT NEED TO SUPPORT THE GROWING AQUACULTURE INDUSTRY}

Triple Null $(p 34, k t i, l e)$ is a potential platform in a path to stack additional transgene traits for functional aquafeed. The growing aquaculture industry and global demands for high quality animal protein will require the optimization of feed performance. Over the past two decades biotechnology has had a remarkable role in increasing yield and productivity. The next frontier is to enhance the performance of crops for specific end uses. The rapidly growing aquaculture industry over-dependent on unsustainable fishmeal sources is an ideal circumstance to explore the potential to design high performance functional feed ingredients.

\section{AUTHOR CONTRIBUTIONS}

This data contained and production of this manuscript is a collaborative effort of both MS and EH who contributed to all aspects of the manuscript's production. 


\section{REFERENCES}

Baeverfjord, G., and Krogdahl, A. (1996). Development and regression of soybean meal induced enteritis in Atlantic salmon, Salmo salar L., distal intestine: a comparison with the intestines of fasted fish. J. Fish Dis. 19, 375-387. doi: 10.1046/j.1365-2761.1996.d01-92.x

Bakke-McKellep, A. M., Press, C., Baeverfjord, G., Krogdahl, Å., and Landsverk, T. (2000). Changes in immune and enzyme histochemical phenotypes of cells in the intestinal mucosa of Atlantic salmon, Salmo salar L., with soybean mealinduced enteritis. J. Fish Dis. 23, 115-127. doi: 10.1111/j.1365-2095.2007.00519x

Bilyeu, K., Ren, C., Nguyen, H. T., Herman, E. M., and Sleper, D. A. (2009). Association of a four basepair insertion in the P34 gene with the low allergen trait in soybean. Plant Genome 2, 141-148. doi: 10.3835/plantgenome2009.01.0006

Burrells, C., Williams, P. D., Southgate, P. J., and Crampton, V. O. (1999). Immunological, physiological and pathological responses of rainbow trout (Oncorhynchus mykiss) to increasing dietary concentration of soybean proteins. Vet. Immunol. Immunopathol. 72, 277-288. doi: 10.1016/S01652427(99)00143-9

Buttle, L. G., Burrells, A. C., Good, J. E., Williams, P. D., Southgate, P. J., and Burrells, C. (2001). The binding of soybean agglutin (SBA) to the intestinal epithelium of Atlantic salmon, Salmo salar and rainbow trout, Oncorhynchus mykiss, fed high levels of soybean meal. Vet. Immunol. Immunopathol. 80, 237-244. doi: 10.1016/S0165-2427(01)00269-0

Calder, P. C., and Yaqoob, P. (2009). Omega 3 polyunsaturated fatty acids and human health outcomes. Biofactors 35, 266-272. doi: 10.1002/biof.42

Clarke, J. L., Waheed, M. T., Lossel, A. G., Martinussen, I., and Daniell, H. (2013). How can plant genetic engineering contribute to cost effective fish vaccine development for promoting sustainable aquaculture? Plant Mol. Biol. 83, 33-40. doi: 10.1007/s11103-013-0081-9

Companjen, A. R., Florack, D. E., Bastiaans, J. H., Matos, C. I., Bosch, D., and Rombout, J. H. (2005). Development of a cost-effective oral vaccination method against viral disease in fish. Dev. Biol. (Basel) 121, 143-150.

Daniell, H., Singh, N. D., Mason, H., and Streatfield, S. J. (2009). Plant-made vaccine antigens and biopharmaceuticals. Trends Plant Sci. 14, 669-679. doi: 10.1016/j.plants.2009.09.009

Food and Agriculture Organization [FAO] (2012) The State of World Fisheries and Aquaculture. Rome: FAO. Available at: http://www.fao.org/docrep/016/i2727e/ i2727e.pdf?

Fujisawa, M., Takita, E., Harada, H., Sakurai, N., Suzuki, H., Ohyama, K., et al. (2009). Pathway engineering of Brassica napus seeds using multiple key enzyme genes involved in ketocarotenoid formation. J. Exp. Bot. 60, 1319-1332. doi: 10.1093/jxb/erp006

Gatlin, D. M., Barrows, F. T., Brown, P., Dabrowski, K., Gaylord, T. G., Hardy, R. W., et al. (2007). Expanding the utilization of sustainable plant products in aquafeeds: a review. Aquacult. Res. 38, 551-579. doi: 10.1111/j.13652109.2007.01704.x

Gerjets, T., and Sandmann, G. (2006). Ketocartoenoid formation in transgenic potato. J. Exp. Bot. 57, 3639-3645. doi: 10.1093/jxb/erl103

Gerland, P., Raftery, A. E., Ševčíková, H., Li, N., Gu, D., Spoorenberg, T., et al. (2014). World population stabilization unlikely this century. Science 346, 234237. doi: 10.1126/science. 1257469

Goldberg, R. B., Hoschek, G., and Vodkin, L. O. (1983). An insertion sequence blocks the expression of a soybean lectin gene. Cell 33, 465-475. doi: 10.1016/0092-8674(83)90428-2

Hardy, R. W. (1996). Alternate protein sources for salmon and trout diets. Anim. Feed Sci. Technol. 59, 71-80. doi: 10.1016/0377-8401(95)00888-8

Hasunuma, T., Miyazawa, S., Yoshimura, S., Shinzaki, Y., Tomizawa, K., Shindo, K., et al. (2008). Biosynthesis of astaxanthin in tobacco leaves by transplastomic engineering. Plant J. 55, 857-868. doi: 10.1111/j.1365313X.2008.03559.x

Herman, E. M. (2003). Genetically modified soybeans and food allergies. J. Exp. Bot. 54, 1317-1319. doi: 10.1093/jxb/erg164

Herman, E. M. (2009). Biotechnology Could Combine Positive Traits in Seed-Based Fish Feed. St. Louis, MO: Global Aquaculture Advocate, 88-90.

Herman, E. M. (2014). Soybean seed proteome rebalancing. Front. Plant Sci. 5:437. doi: $10.3389 /$ fpls.2014.00437
Herman, E. M., Helm, R. M., Jung, R., and Kinney, A. J. (2003). Genetic modification removes an immunodominant allergen from soybean. Plant Physiol. 132, 36-43. doi: 10.1104/pp.103.021865

Herman, E. M., and Schmidt, M. A. (2014). "Towards using biotechnology to modify soybean seeds as protein biofactories," in Recent Advancements in Plant Expression in Crop Plants, eds K. Azhakanandam, A. Silverstone, H. Daniell, and M. R. Davey (Berlin: Springer), 193-212. doi: 10.1007/978-1-4939-2202-4_5

Hymowitz, T. (1986). Genetics and breeding of soybeans lacking the Kunitz trypsin inhibitor. Adv. Exp. Med. Biol. 199, 291-298. doi: 10.1007/978-1-4757-0022$0 \_18$

Jayaraj, J., Devlin, R., and Punja, Z. (2008). Metabolic engineering of novel ketocarotenoid production in carrot plants. Trans Res. 17, 489-501. doi: 10.1007/s11248-007-9120-0

Jofuku, K. D., Schipper, R. D., and Goldberg, R. B. (1989). A frameshift mutation prevents Kunitz trypsin inhibitor mRNA accumulation in soybean embryos. Plant Cell 1, 427-435. doi: 10.1105/tpc.1.4.427

Josephs, L. M., Hymowitz, T., Schmidt, M. A., and Herman, E. M. (2006). Evaluation of Glycine germplasm for nulls of the immunodominant allergen P34/Gly m Bd 30k. Crop Sci. 46, 1755-1763. doi: 10.2135/cropsci2005.12-0500

Kim, W.-S., Jez, J. J., and Krishnan, H. B. (2014). Effects of proteome rebalancing and sulfur nutrition on the accumulation of methionine rich $\delta$-zein in transgenic soybeans. Front. Plant Sci. 5:633. doi: 10.3389/fpls.2014.00633

Kinney, A. J., Jung, R., and Herman, E. M. (2001). Cosuppression of the $\alpha$-subunits of $\beta$-conglycinin in transgenic soybean seeds induces the formation of endoplasmic reticulum-derived protein bodies. Plant Cell 13, 1165-1178. doi: 10.1105/tpc.13.5.1165

Krogdahl, Å., Lea, T. B., and Olli, J. L. (1994). Soybean proteinase-inhibitors affect intestinal trypsin activities and amino acid digestibilities in rainbow trout (Oncorhynchus mykiss). Comp. Biochem. Physiol. 107, 215-219. doi: 10.1016/0300-9629(94)90296-8

Krogdahl, Å., Penn, M. H., Thorsen, J., Refstie, S., and Bakke, A. M. (2010). Important antinutrients in plant feedstuffs for aquaculture: an update on recent findings regarding responses in salmonids. Aquac. Res. 41, 333-344. doi: 10.1111/j.1365-2109.2009.02426.x

Lilleeng, E., Froystad, M. K., and Krogdahl, A. (2007). Effects of diets containing soybean meal on trypsin mRNA expression and activity in Atlantic salmon (Salmo salar L). Comp. Biochem. Physiol. A. Mol. Integr. Physiol. 147, 25-36. doi: 10.106/j.cbpa.2006.10.043

Livingstone, D., Beilinson, V., Kalyaeva, M., Schmidt, M. A., Herman, E. M., and Nielsen, N. C. (2007). Reduction of protease inhibitor activity by expression of a mutant Bowman-birk gene in soybean seed. Plant Mol. Biol. 64, 397-408. doi: 10.1007/s11103-007-9163-x.

Lorenz, R. T., and Cysewski, G. R. (2000). Commercial potential for Haematococcus microalgae as a neutral source of astaxantin. Trends Biotechnol. 18, 160-167. doi: 10.1016/S0167-7799(00)01433-5

Mann, V., Harker, M., Pecker, I., and Hirschberg, J. (2000). Metabolic engineering of astaxanthin production in tobacco flowers. Nat. Biotechnol. 18, 888-892. doi: $10.1038 / 78515$

Moravec, T., Schmidt, M. A., Herman, E. M., and Woodford-Thomas, T. (2006). Production of Escherichia coli heat labile toxin (LT) B subunit in soybean seed and its analysis of its immunogenicity as an oral vaccine. Vaccine 25, 1647-1657. doi: 10.1016/j.vaccine.2006.11.010

Munang'andu, H. M., Mutoloki, S., and Evensen, O. (2015). An overview of challenges limiting the design of protective mucosal vaccines for finfish. Front. Immunol. 6:542. doi: 10.3389/fimmu.2015.00542

Mutoloki, S., Munang'Andu, H. M., and Evensen, O. (2015). Oral vaccination of fish- antigen preparations, uptake, and immune induction. Front. Immunol. 6:519. doi: 10.3389/fimmu.2015.00519

Naylor, R. L., Hardy, R. W., Bureau, D. P., Chiu A., Elliott, M., Farrell, A. P., et al. (2009). Feeding aquaculture in an era of finite resources. Proc. Natl. Acad. Sci. U.S.A. 106, 15103-15110. doi: 10.1073/pnas.0905235106

Nielsen, N. C., Dickinson, C. D., Cho, T. J., Thanh, V. H., Scallon, B. J., Fischer, R. L., et al. (1989). Characterization of the glycinin gene family in soybean. Plant Cell 1, 313-328. doi: 10.1105/tpc.1.3.313

Orf, J. H., and Hymowitz, T. (1979). The inheritance of the absence of the Kunitz trypsin inhibitor in seed protein of soybeans. Crop Sci. 19, 107-109. doi: 10.2135/cropsci1979.0011183X001900010026x 
Orf, J. H., Hymowitz, T., Pull, S. P., and Pueppke, S. G. (1978). Inheritance of soybean seed lectin. Crop Sci. 18, 899-900. doi: 10.2135/cropsci1978.0011183X001800050058x

Pierce, E. C., LaFayette, P. R., Ortega, M. A., Joyce, B. L., Kopsell, D. A., and Parrott, W. A. (2015). Ketocarotenoid production in soybean seeds through metabolic engineering. PLoS ONE 10:e0138196. doi: 10.1371/journal.pone.0138196

Piller, K. J., Clemente, T. E., Jun, S. M., Petty, C. C., Sato, S., Pascual, D. W., et al. (2005). Expression and immunogenicity of an Escherichia coli K99 fimbriae subunit antigen in soybean. Planta 222, 6-18. doi: 10.1007/s00425-004-1445-9

Plant, K. P., and LaPatra, S. E. (2011). Advances in fish vaccine delivery. Dev. Comp. Immunol. 35, 1253-1259. doi: 10.1016/j.dci.2011.03.007

Ralley, L., Enfissi, E. M. A., Misawa, N., Schuch, W., Bramley, P. M., and Fraser, P. D. (2004). Metabolic engineering of ketocarotenoid formation in higher plants. Plant J. 39, 477-486. doi: 10.1111/j.1365-313X.2004.02151.x

Rust, M. B., Barrows F. T., Hardy, R. W., Lazur, A., Naughten, K., and Silverstein J. (2011). The Future of Aquafeeds. NOAA Technical Memorandum NMFS F/SPO-124. NOAA National Marine Fisheries Service. Available at: http://www. nmfs.noaa.gov/aquaculture/docs/feeds/the_future_of_aguafeeds_final.pdf

Sahlmann, C., Sutherland, B. J., Kortner, T. M., Koop, B. F., Krogdahl, A., and Bakke, A. M. (2013). Early response of gene expression in the distal intestine of Atlantic salmon (Salmo salar L.) during the development of soybean meal induced enteritis. Fish Shellfish Immunol. 34, 599-609. doi: 10.1016/j.fsi.2012.11.031

Schmidt, M. A., Barbazuk, W. B., Stanford, M., May, G., Song, Z., Hong, W., et al. (2011). Silencing of soybean seed storage proteins results in a rebalanced protein composition preserving seed protein content without major collateral changes in the metabolome and transcriptome. Plant Physiol. 156, 330-345. doi: 10.1104/pp.111.173807

Schmidt, M. A., and Herman, E. M. (2008). Proteome rebalancing in soybean seeds can be exploited to enhance foreign protein accumulation. Plant Biotechnol. J. 6, 832-842. doi: 10.1111/j.1467-7652.2008.00364.x

Schmidt, M. A., Hymowitz, T., and Herman, E. M. (2015a). Breeding and characterization of soybean Triple Null; a stack of recessive alleles of kunitz trypsin inhibitor, soybean agglutinin, and P34 allergen nulls. Plant Breed. 134, 310-315. doi: 10.1111/pbr.12265

Schmidt, M. A., Parrott, W. A., Hildebrand, D. F., Berg, R. H., Cooksey, A., Pendarvis, K., et al. (2015b). Transgenic soya bean seeds accumulating $\beta$-carotene exhibit the collateral enhancements of oleate and protein content traits. Plant Biotechnol. J. 13, 590-600. doi: 10.1111/pbi.12286

Tacon, A. G. J., Hasan, M. R., Allan, G., El-Sayed, A. F. M., Jackson, J., Kaushik, S. J., et al. (2012). "Aquaculture feeds : addressing the long-term sustainability of the sector," in Proceedings of the Global Conference on Aquaculture 2010. Farming the Waters for People and Food, eds R. P. Subasinghe, J. R. Arthur, D. M. Bartley, S. S. De Silva, M. Halwat, N. Hishamunda, et al. (Rome: FAO), 896.

Tobar, I., Aranciba, S., Torres, C., Vera, V., Soto, P., Carrasco, C., et al. (2015). Successive oral immunizations against Piscirickettsia salmonis and infectious salmon anemia virus are required to maintain a jlong-term protection in farmed salmonids. Front. Immunol. 6:244. doi: 10.3389/fimmu.2015. 00244

Tobar, J., Jerez, S. A., Caruffo, M., Bravo, C., Contreras, F., Bucarey, S., et al. (2011). Oral vaccination of Atlantic salmon (Salmo salar) against salmonid rickettsial septicaemia. Vaccine 29, 2336-2340. doi: 10.1016/j.vaccine.2010.12.107

Tocher, D. R. (2015). Omega 3 long chain polyunsaturated fatty acids and aquaculture in prespective. Aquaculture 449, 94-107. doi: 10.1016/j.aquaculture.2015.01.010

Watson, R. A., Nowara, G. B., Hartmann, K., Green, B. S., Tracey, S. R., and Carter, C. G. (2015). Marine foods sourced from farther as their use of global ocean primary production increases. Nat. Commun. 6:7365. doi: $10.1038 /$ ncomms 8365

Williams, C. M., and Burdge, G. (2006). Long chain n-3 PUFA: plant v marine sources. Proc. Nutr. Soc. 65, 42-50. doi: 10.1079/PNS2005473

Wu, G., Truksa, M., Datla, N., Vrinten, P., Bauer, J., Zank, T., et al. (2005). Stepwise engineering to produce high yields of very long-chain polyunsaturated fatty acids in plants. Nat. Biotechnol. 23, 1013-1017. doi: 10.1038/nbt1107

Zhong, J.-J., Huang, J.-C., Liu, J., Li, Y., Jiang, Y., Xu, Z.-F., et al. (2011) Functional characterization of various algal carotenoid ketolases reveals that ketolating zeaxanthin efficiently is essential for high production of astaxanthin in transgenic Arabidopsis. J. Exp. Bot. 62, 3659-3669. doi: 10.1093/jxb/err070

Conflict of Interest Statement: The authors declare that the research was conducted in the absence of any commercial or financial relationships that could be construed as a potential conflict of interest.

The reviewer AC and handling Editor declared their shared affiliation, and the handling Editor states that the process nevertheless met the standards of a fair and objective review.

Copyright (c) 2016 Herman and Schmidt. This is an open-access article distributed under the terms of the Creative Commons Attribution License (CC BY). The use, distribution or reproduction in other forums is permitted, provided the original author(s) or licensor are credited and that the original publication in this journal is cited, in accordance with accepted academic practice. No use, distribution or reproduction is permitted which does not comply with these terms. 


\title{
Agricultural practices altered soybean seed protein, oil, fatty acids, sugars, and minerals in the Midsouth USA
}

\author{
Nacer Bellaloui ${ }^{1}{ }^{*}$, H. Arnold Bruns ${ }^{2}$, Hamed K. Abbas ${ }^{3}$, Alemu Mengistu ${ }^{4}$, Daniel K. Fisher ${ }^{2}$ and \\ Krishna N. Reddy ${ }^{2}$ \\ ${ }^{1}$ Crop Genetics Research Unit, Plant Physiology, United States Department of Agriculture - Agricultural Research Service, Stoneville, MS, USA \\ ${ }^{2}$ Crop Production Systems Research Unit, Crop Production, United States Department of Agriculture - Agricultural Research Service, Stoneville, MS, USA \\ ${ }^{3}$ Biological Control of Pests Research Unit, Biological Control, United States Department of Agriculture - Agricultural Research Service, Stoneville, MS, USA \\ ${ }^{4}$ Crop Genetics Research Unit, Pathology, United States Department of Agriculture - Agricultural Research Service, Jackson, TN, USA
}

\section{Edited by:}

Antonio M. De Ron, National Spanish

Research Council, Spain

\section{Reviewed by:}

Leonardo Velasco, Institute for Sustainable Agriculture - National Spanish Research Council, Spain Rouxlene Van Der Merwe, University of the Free State, South Africa

\section{*Correspondence:}

Nacer Bellaloui, Crop Genetics Research Unit, Plant Physiology, United States Department of Agriculture - Agricultural Research Service, 141 Experiment Station Road, Stoneville, MS 38776, USA e-mail: nacer.bellaloui@ars.usda.gov
Information on the effects of management practices on soybean seed composition is scarce. Therefore, the objective of this research was to investigate the effects of planting date (PD) and seeding rate (SR) on seed composition (protein, oil, fatty acids, and sugars) and seed minerals ( $\mathrm{B}, \mathrm{P}$, and Fe) in soybean grown in two row-types (RTs) on the Mississippi Delta region of the Midsouth USA. Two field experiments were conducted in 2009 and 2010 on Sharkey clay and Beulah fine sandy loam soil at Stoneville, MS, USA, under irrigated conditions. Soybean were grown in $102 \mathrm{~cm}$ single-rows and $25 \mathrm{~cm}$ twin-rows in $102 \mathrm{~cm}$ centers at SRs of 20,30,40, and 50 seeds $\mathrm{m}^{-2}$. The results showed that in May and June planting, protein, glucose, $\mathrm{P}$, and $\mathrm{B}$ concentrations increased with increased SR, but at the highest SRs ( 40 and 50 seeds $\mathrm{m}^{-2}$ ), the concentrations remained constant or declined. Palmitic, stearic, and linoleic acid concentrations were the least responsive to SR increases. Early planting resulted in higher oil, oleic acid, sucrose, B, and P on both single and twin-rows. Late planting resulted in higher protein and linolenic acid, but lower oleic acid and oil concentrations. The changes in seed constituents could be due to changes in environmental factors (drought and temperature), and nutrient accumulation in seeds and leaves. The increase of stachyose sugar in $\mathbf{2 0 1 0}$ may be due to a drier year and high temperature in 2010 compared to 2009; suggesting the possible role of stachyose as an environmental stress compound. Our research demonstrated that PD, SR, and RT altered some seed constituents, but the level of alteration in each year dependent on environmental factors such as drought and temperature. This information benefits growers and breeders for considering agronomic practices to select for soybean seed nutritional qualities under drought and high heat conditions.

Keywords: seed protein, fatty acids, sugars, minerals, twin-rows, seeding rate, planting date

\section{INTRODUCTION}

Soybean is a major oil and protein crop globally. The seed quality is determined by its composition, including protein, oil, fatty acids, sugars, and minerals. Soybean seed contains on a dry weight basis about 380 to $420 \mathrm{~g} \mathrm{~kg}^{-1}$ protein, 190 to $230 \mathrm{~g} \mathrm{~kg}^{-1}$ oil, and based on the total oil, 120 to $130 \mathrm{~g} \mathrm{~kg}^{-1}$ palmitic acid, 30 to $40 \mathrm{~g} \mathrm{~kg}^{-1}$ oleic acid, 480 to $580 \mathrm{~g} \mathrm{~kg}^{-1}$ linoleic acid, and 50 to $80 \mathrm{~g} \mathrm{~kg}^{-1}$ linolenic acid. They also contain sugars such as monosaccharides (glucose and fructose), disaccharide (sucrose), and oligosaccharides (raffinose and stachyose). The mineral composition includes $\mathrm{P}, \mathrm{K}, \mathrm{Ca}, \mathrm{Mn}, \mathrm{Zn}, \mathrm{Fe}$, and $\mathrm{B}$ that are essential for human nutrition, and deficiency of these minerals in the diet can lead to human malnutrition and health problems (Samman et al., 1998; Bouis, 2003; Devirian and Volpe, 2003; Lu etal., 2008). It was reported that over 3 billion people are suffering from malnutrition of minerals, especially iron and zinc (Welch and Graham, 2004; Lu et al., 2008; White and Broadley, 2009). Higher oleic, and lower linoleic and linolenic acids are desirable because they contribute to oil stability. Lower raffinose and stachyose and higher sucrose, fructose, and glucose are desirable because mono- and disaccharides contribute to flavor and taste, but high raffinose and stachyose are indigestible and cause flatulence and diarrhea in non-ruminant animals (Liu, 1997). Seed composition constituents are genetically controlled; however, they are known to be influenced by biotic and abiotic factors such as genotype, maturity, growing season, geographic location, and agronomic practices (Harue and Hirokadzu, 1971; Chapman et al., 1976; Chy and Sheldon, 1979; Wilcox and Cavins, 1995).

Traditionally soybeans in the Midsouth are grown in singlerows, and between row-spacings of 88 to $102 \mathrm{~cm}$ are common for soybeans produced in the Mississippi Delta (Ebelhar, 2010). With the commercialization and availability of twin-row-planters (Mascagni et al., 2008; Bruns, 2011), more farmers in the Mississippi delta are becoming interested in twin-row production. Twin-row planters have the capability of planting twin-rows 7.5 to 10 inches (19.1 to $25.4 \mathrm{~cm}$ ) apart. Although yield responses of single-row vs. twin-row planting have been inconsistent across crops, years, and locations, soybean still has the largest 
positive response to yield increase in twin-rows vs. single-row (Mascagni et al., 2008). Also, in the Midsouth, including Mississippi, some growers plant soybean as a double-crop with wheat (Lehrsch et al., 1994; Minor and Wiebold, 1998; Heatherly, 2014), and in this production system, soybeans are usually planted in June. This is considered late planting, as opposed to early planting which usually occurs in April. Late planting exposes soybean to a new environment of drought, heat, photoperiod, and diseases, especially charcoal rot and phomopsis. In the Early Soybean Production System (ESPS) in the Midsouth, soybean cultivars of maturity group (MG) IV and $\mathrm{V}$ are planted in April-May and harvested in August-September (Heatherly, 1999; Ray etal., 2006) to avoid drought stress during late July through early September. In spite of the yield benefit of the ESPS (Heatherly et al., 1999; Ray et al., 2006), poor seed quality (Mengistu and Heatherly, 2006; Mengistu et al., 2007; Smith et al., 2008), and variability of seed constituents (Bellaloui et al., 2008, 2009b) remain a challenge. Therefore, optimizing the ESPS in the Midsouth for higher, sustainable seed quality is critical.

Although limited information is available on the effects of planting date (PD; Schnebly and Fehr, 1993; Jaureguy et al., 2013) and row-spacing (Boydak et al., 2002), and seeding rate (SR) and row-spacing (Bellaloui et al., 2014) on seed composition, to our knowledge there has been no research done on the combined effects of PD, row-type (RT), and SR on seed composition and mineral nutrition. Therefore, the objective of the current research was to evaluate the effects of single- and twin-row plantings (using 102 and $25 \mathrm{~cm}$ on $102 \mathrm{~cm}$ center row-spacing, respectively), early, intermediate, and late planting, and SRs of 20,30, 40, and 50 seeds $\mathrm{m}^{-2}$ on soybean seed composition in sandy and clay soils under the Midsouth agro-ecosystem. Our hypothesis was that PD combined with RT (single- vs. twin) and SR will subject the crop to a new growing environment, altering seed composition constituents.

\section{MATERIALS AND METHODS FIELD MANAGEMENT AND GROWTH CONDITIONS}

An experiment was performed under field conditions in 2009 and 2010 on Sharkey clay (very-fine, smectitic, thermic Chromic Epiaquerts) and sandy loam (Beulah fine sandy loam, coarse-loamy, mixed, active, thermic Typic Dystrudepts) soils in Stoneville, MS, USA. The current research focused on seed nutrition only, and the agronomic component, including yield, was previously published (Bruns, 2011). Field management and growth conditions were described in detail previously (Bruns, 2011). Briefly, single-row plots were planted using an Almaco cone plot planter (Allen Machine Company, Nevada, IA, USA), and twin-row plots were planted using a four unit Monosem NG-3 (Monosem, Edwardsville, KS, USA) twin-row planter set on $102-\mathrm{cm}$ centers and $25 \mathrm{~cm}$ between rows. In the Sharkey clay soil, soybean was planted in 2009 on 22 April, 20 May, and 17 June. In 2010, plantings occurred on 12 April, 11 May, and 2 June. In the sandy loam soil, PDs were 8 April, 11 May, and 8 June in 2009, and 14 April, 11 May, and 17 June in 2010. Cultivar Pioneer brand, 94B73, representative of most of the cultivars grown in the ESPS in the Midsouth was used. For weed control, a pre-plant application of trifluralin [2,6-dinitro- $N, N$-dipropyl-4(trifluoromethyl)aniline] at $0.7 \mathrm{~kg} \mathrm{ai} \mathrm{ha}^{-1}$ was applied, followed by two post-emergence applications of metolachlor [2-chloro- $\mathrm{N}$-(2ethyl-6-methylphenyl)-N-(2-methoxy-1-methylethyl) acetamide ] and glyphosate [2-[(phosphonomethyl)amino]acetic acid] at growth stage V2 to V3 (two to three trifoliolates) and at V5 to V6 (five to six trifoliolates). To control fungus, pyraclostrobin (carbamic acid, [[[[1-(4-cholrophenyl)-H-pyrazol3-yl] oxy]methyl]phenyl]methoxy-,methyl ester) was applied at factory label directions at V5 to V6. The experiments were furrow irrigated starting at R1 (beginning flowering) through R6 (full seed-fill), and an equivalent of $\sim 25 \mathrm{~mm} \mathrm{ha}^{-1}$ water was applied at 10 -days intervals. Soil analysis during the vegetative stage at both sites indicated that there were no nutrient deficiencies in soil in either year. Mature seeds at the R8 growth stage were collected, processed, and analyzed for seed composition constituent concentrations as described below.

\section{SOIL MINERALS, N, S, AND C ANALYSIS}

Soil nutrient analyses were performed at the University of Georgia's Soil, Plant, and Water Laboratory in Athens, GA. Concentrations of $\mathrm{K}$ were analyzed on a $5 \mathrm{~g}$ soil: $20 \mathrm{ml}$ Mehlich- 1 solution and the concentrations were determined using inductively coupled plasma spectrometry. Soil N, S, and C were determined by combusting samples using a $\mathrm{C} / \mathrm{N} / \mathrm{S}$ elemental analyzer having thermal conductivity cells (LECOCNS-2000 elemental analyzer, LECOCorporation, St. Joseph, MI, USA). A $0.25 \mathrm{~g}$ sample of soil was combusted in an oxygen atmosphere at $1350^{\circ} \mathrm{C}$, converting elemental $\mathrm{N}, \mathrm{S}$, and $\mathrm{C}$ into $\mathrm{N}_{2}, \mathrm{SO}_{2}$, and $\mathrm{CO}_{2}$. The gasses were then passed through infrared cells and $\mathrm{N}, \mathrm{S}$, and $\mathrm{C}$ were determined by the elemental analyzer.

\section{LEAF AND SEED MINERALS, N, S, AND C ANALYSIS}

Plant tissue samples were analyzed for different nutrients, including $\mathrm{N}, \mathrm{S}$, and $\mathrm{C}$ ratios. This was done by digesting $0.6 \mathrm{~g}$ of dried, ground plant materials in $\mathrm{HNO}_{3}$ in a microwave digestion system. Samples were ground using a Laboratory Mill 3600 (Perten, Springfield, IL, USA), and the concentration of $\mathrm{K}$ was determined using inductively coupled plasma spectrometry (Thermo Jarrell-Ash Model 61E ICP and Thermo JarrellAsh Autosampler 300; Bellaloui et al., 2011, 2014). For N, C, and $\mathrm{S}$ measurements, a $0.25 \mathrm{~g}$ ground-dried sample was combusted, and the percentages of $\mathrm{C}, \mathrm{N}$ and $\mathrm{S}$ were determined using the C/N/S elemental analyzer (Bellaloui et al., 2011, 2014). For B, P, and Fe, concentrations were determined as described below.

\section{SEED ANALYSIS FOR PROTEIN, OIL, FATTY ACIDS, AND SUGARS}

Mature seeds were analyzed for protein, oil, fatty acids, and sugars according to detailed methods as reported by Bellaloui et al. (2009b, 2010, 2014). Briefly, a 25 g sample of ground seed was analyzed for protein, oil, fatty acids, and sugars by near infrared reflectance (Wilcox and Shibles, 2001; Bellaloui et al., 2009b, 2010) using a diode array feed analyzer AD 7200 (Perten, Springfield, IL, USA). The calibration equation was initially developed by the University of Minnesota using Perten's Thermo Galactic Grams PLS IQ software using conventional chemical protocols with AOAC 
methods (AOAC, 1990a,b). Then, the calibration equation for protein, oil, fatty acids, and sugars has been updated from 6 months to 1 year to insure accuracy and validity of the equation. Seeds at maturity were harvested at $13 \%$ water moisture and seed concentrations for protein, oil, fatty acids, and sugars were immediately performed on $13 \%$ water moisture and expressed on dry weight basis (Wilcox and Shibles, 2001; Boydak et al., 2002; Bellaloui et al., 2010, 2014). The fatty acid concentrations (palmitic, stearic, oleic, linoleic, and linolenic acids) were determined relative to total oils (Bellaloui et al., 2009b, 2014).

\section{SEED GLUCOSE AND FRUCTOSE ANALYSIS}

The glucose level in seeds was measured by an enzymatic reaction using a Glucose (HK) Assay Kit, Product Code GAHK-20 (Sigma-Aldrich Co, St. Louis, MO, USA). A detailed description of the protocol and analysis was previously described by Bellaloui et al. (2014). Briefly, glucose and fructose were phosphorylated by adenosine triphosphate (ATP) and catalyzed by hexokinase, resulting in glucose-6-phosphate (G6P). The produced product (G6P) was then oxidized to 6-phosphogluconate by oxidized nicotinamide adenine dinucleotide (NAD) using glucose-6-phosphate dehydrogenase $(\mathrm{G} 6 \mathrm{PDH})$. The NAD was then reduced to $\mathrm{NADH}$, and the concentration of glucose was determined based on the increase in absorbance at $340 \mathrm{~nm}$. The seed samples involved were ground using the Laboratory Mill 3600, and a random sample of $0.1 \mathrm{mg}$ was extracted with deionized water. The sample solution was heated to aid extraction, and a sample of $100 \mu \mathrm{l}$ was added to $100 \mathrm{ml}$ of the Glucose (HK) Assay Reagent and then incubated at room temperature for $15 \mathrm{~min}$. A sample blank consisting of 100 and $1000 \mu \mathrm{l}$ deionized water, and a reagent blank with $1000 \mu \mathrm{l}$ of Glucose (HK) Assay Reagent and $100 \mu \mathrm{l}$ of deionized water were used. Samples were read at an absorbance of $340 \mathrm{~nm}$ using the Beckman Coulter DU 800 spectrophotometer in order to determine glucose levels, which were expressed as $\mathrm{mg} \mathrm{g}^{-1} \mathrm{dry}$ weight.

Levels of fructose were measured using the method reported by Bellaloui et al. (2014). Fructose was phosphorylated by ATP using hexokinase, producing fructose 6-phosphate (F6P). The F6P produced was then converted to G6P by phosphoglucose isomerase (PGI), and the P6G then oxidized to 6-phosphogluconate in the presence of NAD in a reaction catalyzed by G6PDH. An equimolar amount of NAD was then reduced to NADH, and the concentration of fructose was measured by the same method as described for glucose.

\section{BORON DETERMINATION}

Concentrations of boron in plant materials were measured using the azomethine-H method described by Lohse (1982) and Dordas et al. (2007), with a detailed description of the protocol reported by Bellaloui et al. (2014). Briefly, a ground sample of $1.0 \mathrm{~g}$ was ashed at $500^{\circ} \mathrm{C}$, extracted with $20 \mathrm{ml}$ of $2 \mathrm{M} \mathrm{HCl}$ at $90^{\circ} \mathrm{C}$ for $10 \mathrm{~min}$, and then a $2 \mathrm{ml}$ sample of the filtered mixture was added to $4 \mathrm{ml}$ of buffer solution (containing 25\% ammonium acetate, $1.5 \%$ EDTA, and $12.5 \%$ acetic acid). A volume of $4 \mathrm{ml}$ of fresh azomethine- $\mathrm{H}$ solution $(0.45 \%$ azomethine- $\mathrm{H}$ and $1 \%$ of ascorbic acid; John et al., 1975) was added. The concentrations of boron in leaves and seeds were determined at $420 \mathrm{~nm}$ using a Beckman
Coulter DU 800 spectrophotometer (Beckman Coulter Inc., Brea, CA, USA).

\section{IRON DETERMINATION}

Iron concentrations in leaves and seeds were determined according to Bandemer and Schaible (1944) and Loeppert and Inskeep (1996). The determination of the concentration was conducted by acid wet digestion, extraction, and reaction of the reduced ferrous Fe with 1,10-phenanthroline, as described by Bellaloui et al. (2011, 2014). Briefly, samples of $2 \mathrm{~g}$ of dried ground leaves and seeds were acid digested, and the soluble constituents were dissolved in $2 \mathrm{M}$ of $\mathrm{HCl}$. A volume of $4 \mathrm{ml}$ of an aliquot containing 1-20 $\mu \mathrm{g}$ of iron of the sample solution was transferred into a $25 \mathrm{ml}$ volumetric flask and diluted to $5 \mathrm{ml}$ using $0.4 \mathrm{M} \mathrm{HCl}$. A volume of $1 \mathrm{ml}$ of Quinol solution was added to the $5 \mathrm{ml}$ diluted sample solution and mixed. Three $\mathrm{ml}$ of the phenanthroline solution and $5 \mathrm{ml}$ of the tri-sodium citrate solution $(8 \% \mathrm{w} / \mathrm{v})$ were added. The solution was diluted to $25 \mathrm{ml}$ with distilled water and incubated at room temperature for $4 \mathrm{~h}$. Phenanthroline reagent solution of $0.25 \%$ $(\mathrm{w} / \mathrm{v})$ in $25 \%(\mathrm{v} / \mathrm{v})$ ethanol and quinol solution $(1 \% \mathrm{w} / \mathrm{v})$ was prepared. A standard curve was prepared using a concentration range from 0.0 to $4 \mu \mathrm{g} \mathrm{ml}^{-1}$ of $\mathrm{Fe}$ in $0.4 \mathrm{M} \mathrm{HCl}$, with concentrations measured at an absorbance of $510 \mathrm{~nm}$ using the Beckman Coulter DU 800 spectrophotometer.

\section{PHOSPHORUS DETERMINATION}

The yellow phosphor-vanado-molybdate complex method according to Cavell (1955) was used to determine phosphorus concentrations in leaves and seeds. The detailed description of the method was previously reported by Bellaloui et al. (2009b, 2014). Briefly, dried ground samples of $2 \mathrm{~g}$ of leaves and seed were ashed at $500^{\circ} \mathrm{C}$, and $10 \mathrm{ml}$ of $6 \mathrm{M} \mathrm{HCl}$ were added. The samples were placed in a water bath at $100^{\circ} \mathrm{C}$ to evaporate the solution to dryness. After the extraction of $\mathrm{P}$ using $2 \mathrm{ml}$ of $36 \% \mathrm{v} / \mathrm{v} \mathrm{HCl}$ under heat and filtration, $5 \mathrm{ml}$ of $5 \mathrm{M} \mathrm{HCl}$ and $5 \mathrm{ml}$ of ammonium molybdate-ammonium metavanadate reagent were added to $5 \mathrm{ml}$ of the filtrate. Ammonium molybdate-ammonium metavanadate was prepared by dissolving $25 \mathrm{~g}$ of ammonium molybdate and $1.25 \mathrm{~g}$ of ammonium metavanadate in $500 \mathrm{ml}$ of distilled water. The phosphorus standard curve was established by preparing standard solutions of phosphorus in a range of concentrations from 0 to $50 \mu \mathrm{g} \mathrm{ml}^{-1}$ using dihydrogen orthophosphates. The concentrations of phosphorus were measured at an absorbance of $400 \mathrm{~nm}$ using the Beckman Coulter DU 800 spectrophotometer.

\section{EXPERIMENTAL DESIGN AND STATISTICAL ANALYSIS}

The experimental design was a split-plot in a randomized complete block, with four replicates. A main plot was created for each PD, and subplots were a combination of either a single-row or twinrow planting with a SR of $20,30,40$, or 50 seeds $\mathrm{m}^{-2}$. Replicates within years [rep (year)] and planting date $\times$ rep (year) were considered as components of variance for random effects. Year, PD, and SR were modeled as fixed effects. Residuals of random effect factors as covariance parameters were shown in tables; the residual values refer to Restricted Maximum Residual Likelihood (REML), which reflects the total variance of the random parameters in the model. Analysis of variance of data was performed using PROC 
MIXED in SAS (Statistical Analysis System, Copyright 2002-2010, Cary, NC, USA). Means were separated by Fisher's protected LSD (0.05).

\section{RESULTS}

\section{ANALYSIS OF VARIANCE (ANOVA) OF PLANTING DATE, ROW-TYPE, AND SEEDING RATE IN CLAY SOIL}

In clay soil, ANOVA (Tables $\mathbf{1}$ and 2) showed that year (Y) and $\mathrm{PD}$, and their interactions $(\mathrm{Y} \times \mathrm{PD})$ had significant (ranged from $P \leq 0.05$ to $P \leq 0.0001$ ) effects on protein, oil, and fatty acids. RT had significant effects on protein, oil, stearic, and oleic acid, but not on palmitic, linoleic, and linolenic acids. However, interactions of $\mathrm{PD} \times \mathrm{RT}$ and $\mathrm{Y} \times \mathrm{RT}$ were significant for oil and oleic acid only, indicating that the response of oil and oleic acid to RT and PD were dependent on environmental factors in each year. Palmitic and stearic acids were the least affected by the interactions between Y, PD, and RT. Seeding rate had significant effects on protein, oil, and linoleic acid, while the interactions between SR, PD, and RT were significant for protein, oleic and linolenic acid. This indicated that the influence of SR on seed constituents was dependent on $\mathrm{PD}$ and RT. Based on this, the most sensitive constituents to agronomic practices and seasonal environmental factors were protein, oil, and oleic acid, and the least sensitive constituents to agronomic practices and environment were palmitic and stearic acids. Linoleic and linolenic acids were in between. The level of interactions between $\mathrm{Y}, \mathrm{PD}, \mathrm{RT}$, and $\mathrm{SR}$ for seed composition ranged from $P \leq 0.05$ to $P \leq 0.0001$, depending on the seed constituent, indicating the different sensitivity of seed composition constituents to environments and agricultural practices (Tables 1 and 2). Except for raffinose, sugars and mineral concentrations were significantly influenced by $\mathrm{Y}$ and $\mathrm{PD}$. However, $\mathrm{Y} \times \mathrm{PD}$ interactions were significant for B, P, and all sugars, except for stachyose. RT was significant for raffinose, glucose, fructose, $B$ and $P$, while $S R$ was significant for all minerals, and the sugars sucrose, raffinose, and glucose. Interactions between Y, PD, RT, and SR influenced some constituents. However, the least influenced constituent to agricultural practices was stachyose, while the most influenced constituents were the minerals (B, P, and Fe). Since Y interacted with other factors for some seed constituents, results were presented by year (Bellaloui et al., 2009b, 2010, 2011).

\section{ANALYSIS OF VARIANCE OF PLANTING DATE, ROW-TYPE, AND SEEDING RATE IN SANDY SOIL}

In sandy soil, $\mathrm{Y}, \mathrm{PD}$, and their interactions $(\mathrm{Y} \times \mathrm{PD})$ were significant (ranged from $P \leq 0.05$ to $P \leq 0.0001$ ) for protein, oil, and fatty acids, indicating that both Y and PD had different effects on these constituents, depending on the environmental factors in each year (Table 3). RT significantly interacted with $\mathrm{Y}$ and PD for the seed protein, oil, and palmitic acid, indicating that RT effects were dependent on Y and PD. However, RT on its own had no significant effects on these constituents. SR had significant effects on oil, oleic and linolenic acids, but its interaction $(\mathrm{Y} \times \mathrm{SR})$ had significant effects on protein, oil, oleic, and linolenic acid. It can be concluded that under sandy soil conditions the most responsive constituents to agronomic practices and environment were protein, oil, and oleic acid, while the least responsive constituents

Table 1 | Analysis of variance ( $F$ and $P$ values) of seed protein, oil, fatty acids $\left(\mathrm{g} \mathrm{kg}^{-1}\right)$ in soybean as influenced by year $(\mathrm{Y})$, seeding rate (SR), planting date (PD), and row-type (RT, single- or twin-row) in Sharkey clay soil in 2009 and 2010 at Stoneville, MS, USA.

\begin{tabular}{|c|c|c|c|c|c|c|c|c|c|c|c|c|c|c|c|}
\hline \multirow[b]{2}{*}{ Effect } & \multirow[b]{2}{*}{ DF } & \multicolumn{2}{|c|}{ Protein } & \multicolumn{2}{|c|}{ Oil } & \multicolumn{2}{|c|}{ Palmitic } & \multicolumn{2}{|c|}{ Stearic } & \multicolumn{2}{|c|}{ Oleic } & \multicolumn{2}{|c|}{ Linoleic } & \multicolumn{2}{|c|}{ Linolenic } \\
\hline & & $\boldsymbol{F}$ & $P$ & $\boldsymbol{F}$ & $P$ & $\boldsymbol{F}$ & $P$ & $\boldsymbol{F}$ & $\boldsymbol{P}$ & $\boldsymbol{F}$ & $P$ & $\boldsymbol{F}$ & $P$ & $\boldsymbol{F}$ & $\boldsymbol{P}$ \\
\hline PD & 2 & 194 & $* * *$ & 511 & $* * *$ & 10.5 & $* * *$ & 7.04 & $* *$ & 72 & $* * *$ & 7.89 & $* * *$ & 65 & $* * *$ \\
\hline$Y \times P D$ & 2 & 23.0 & $* * *$ & 119 & $* * *$ & 17.3 & $* * *$ & 4.7 & * & 87 & $* * *$ & 42.3 & $* * *$ & 27 & $* * *$ \\
\hline $\mathrm{Y} \times \mathrm{RT}$ & 1 & 23.3 & $* * *$ & 6.4 & * & 0.57 & NS & 3.18 & NS & 16.1 & $* * *$ & 0.13 & NS & 3.7 & NS \\
\hline $\mathrm{PD} \times \mathrm{RT}$ & 2 & 1.3 & NS & 5.6 & $* *$ & 0.75 & NS & 0.26 & NS & 10.6 & $* * *$ & 0.3 & NS & 1.6 & NS \\
\hline$Y \times P D \times R T$ & 2 & 13.6 & $* * *$ & 7.6 & $* * *$ & 0.65 & NS & 0.08 & NS & 3.1 & * & 0.61 & NS & 2.7 & NS \\
\hline $\mathrm{SR}$ & 3 & 6.4 & $* * *$ & 4.1 & $* *$ & 0.55 & NS & 0.87 & NS & 2.2 & NS & 2.05 & $*$ & 1.3 & NS \\
\hline $\mathrm{Y} \times \mathrm{SR}$ & 3 & 1.7 & NS & 0.69 & NS & 1.4 & NS & 0.48 & NS & 1.4 & NS & 0.07 & NS & 2.4 & NS \\
\hline $\mathrm{Y} \times \mathrm{RT} \times \mathrm{SR}$ & 3 & 0.26 & NS & 0.69 & NS & 1.8 & NS & 0.98 & NS & 1.6 & NS & 0.9 & NS & 0.61 & NS \\
\hline $\mathrm{PD} \times \mathrm{RT} \times \mathrm{SR}$ & 6 & 2.3 & * & 0.82 & NS & 0.85 & NS & 0.65 & NS & 3.6 & $* *$ & 1.8 & NS & 3.1 & $* *$ \\
\hline $\mathrm{Y} \times \mathrm{PD} \times \mathrm{RT} \times \mathrm{SR}$ & 6 & 2.2 & * & 1.0 & NS & 1.2 & NS & 1.0 & NS & 1.3 & NS & 1.3 & NS & 1.9 & NS \\
\hline Residual & & 35.8 & & 21 & & 41.3 & & 1.4 & & 109 & & 90 & & 32.1 & \\
\hline
\end{tabular}

${ }^{*} P \leq 0.05 ;{ }^{*} P \leq 0.01 ;{ }^{*}{ }^{*} P \leq 0.001 ; N S$, not significant. 
Table 2 | Analysis of variance ( $F$ and $P$ values) of sucrose, raffinose, stachyose, glucose, fructose $\left(\mathrm{mg} \mathrm{g}^{-1}\right)$, boron $\left(B, \mathrm{mg} \mathrm{kg}^{-1}\right)$, phosphorus $(P, \mathrm{~g}$ $\mathrm{kg}^{-1}$ ), and iron ( $\mathrm{Fe}, \mathrm{mg} \mathrm{kg}^{-1}$ ) in soybean as influenced by year (Y), seeding rate (SR), planting date (PD), and row-type (RT, single- or twin-row) in Sharkey clay soil in 2009 and 2010 at Stoneville, MS, USA.

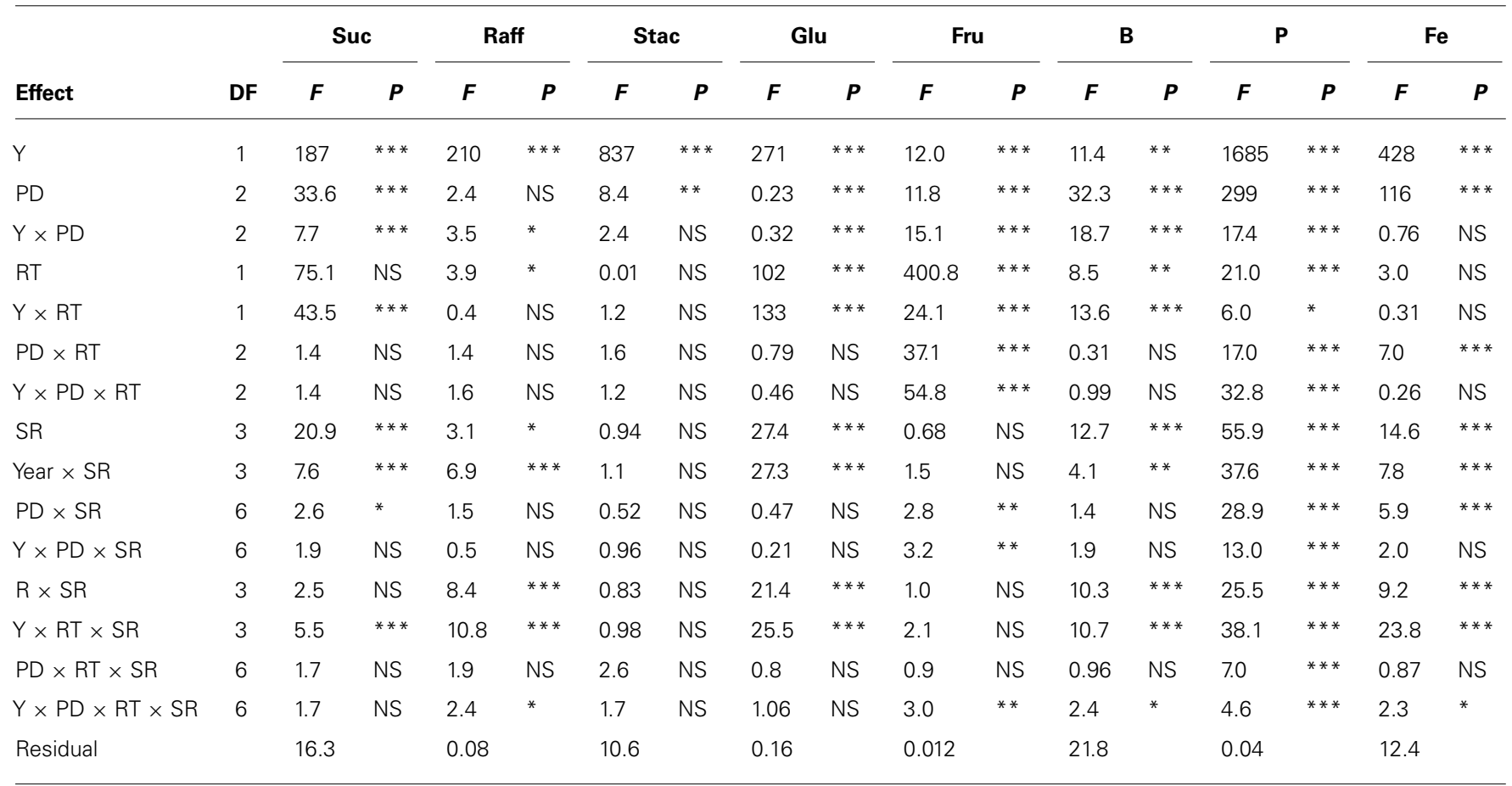

${ }^{*} P \leq 0.05 ;{ }^{*} P \leq 0.01 ;{ }^{*}{ }^{*} P \leq 0.001 ; N S$, not significant, suc, sucrose; raff, raffinose; stac, stachyose; glu, glucose; and fruc, fructose.

Table 3 | Analysis of variance ( $F$ and $P$ values) of seed protein, oil, fatty acids $\left(\mathrm{g} \mathrm{kg}^{-1}\right)$ in soybean as influenced by year (Y), seeding rate (SR), planting date (PD), and row-type (RT, single- or twin- row) in Beulah fine sandy loam soil in 2009 and 2010 at Stoneville, MS, USA.

\begin{tabular}{|c|c|c|c|c|c|c|c|c|c|c|c|c|c|c|c|}
\hline \multirow{2}{*}{$\begin{array}{l}\text { Effect } \\
Y\end{array}$} & \multirow{2}{*}{$\frac{\text { DF }}{1}$} & \multicolumn{2}{|c|}{ Protein } & \multicolumn{2}{|c|}{ Oil } & \multicolumn{2}{|c|}{ Palmitic } & \multicolumn{2}{|c|}{ Stearic } & \multicolumn{2}{|c|}{ Oleic } & \multicolumn{2}{|c|}{ Linoleic } & \multicolumn{2}{|c|}{ Linolenic } \\
\hline & & 240 & $* * *$ & 328 & $* * *$ & 14.4 & $* *$ & 33.1 & $* *$ & 300 & $* * *$ & 454 & $* * *$ & 226 & $* * *$ \\
\hline PD & 2 & 105 & $* * *$ & 123 & $* * *$ & 243 & $* * *$ & 37.9 & $* * *$ & 4.95 & * & 64.1 & $* * *$ & 0.59 & NS \\
\hline$Y \times P D$ & 2 & 82.1 & $* * *$ & 68.3 & $* * *$ & 54.1 & $* * *$ & 21.4 & $* * *$ & 23 & $* * *$ & 40.9 & $* * *$ & 3.3 & * \\
\hline $\mathrm{RT}$ & 1 & 0.01 & NS & 0.07 & NS & 0.26 & NS & 0.05 & NS & 2.3 & NS & 2.46 & NS & 0.04 & NS \\
\hline $\mathrm{Y} \times \mathrm{RT}$ & 1 & 10.1 & $* *$ & 0.14 & NS & 17.3 & $* * *$ & 0.18 & NS & 1.4 & NS & 0.24 & NS & 0.14 & NS \\
\hline$Y \times P D \times R T$ & 2 & 1.9 & NS & 10.3 & $* * *$ & 15.4 & $* * *$ & 0.52 & NS & 1.3 & NS & 0.7 & NS & 0.57 & NS \\
\hline SR & 3 & 0.24 & NS & 14.8 & $* * *$ & 1.3 & NS & 0.51 & NS & 28.9 & $* * *$ & 0.15 & NS & 27.7 & $* * *$ \\
\hline Year $\times$ SR & 3 & 3.7 & $* *$ & 6.3 & $* *$ & 0.79 & NS & 1.17 & NS & 30.1 & $* * *$ & 0.18 & NS & 31.9 & $* * *$ \\
\hline $\mathrm{PD} \times \mathrm{SR}$ & 6 & 2.4 & * & 12.2 & $* * *$ & 3.2 & $* *$ & 0.81 & NS & 2.1 & NS & 0.52 & NS & 1.3 & NS \\
\hline $\mathrm{Y} \times \mathrm{PD} \times \mathrm{SR}$ & 6 & 2.5 & * & 17.4 & $* * *$ & 4.4 & $* *$ & 1.01 & NS & 1.6 & NS & 0.48 & NS & 1.6 & NS \\
\hline $\mathrm{Y} \times \mathrm{PD} \times \mathrm{RT} \times \mathrm{SR}$ & 6 & 5.53 & $* * *$ & 3.4 & $* *$ & 1.94 & NS & 0.8 & NS & 3.23 & $* *$ & 0.43 & NS & 0.35 & NS \\
\hline Residual & & 53.6 & & 28.1 & & 29.6 & & 1.92 & & 155 & & 161 & & 38.1 & $* * *$ \\
\hline
\end{tabular}

${ }^{*} P \leq 0.05 ;{ }^{*} P \leq 0.01 ;{ }^{*}{ }^{*} P \leq 0.001 ; N S$, not significant. 
were stearic, linoleic, and linolenic acids. Sugars, except glucose, were significantly influenced by both Y and PD (Table 4); however, interaction with $Y$ did not affect sucrose, raffinose, or glucose. This showed that these sugars had the same response pattern in each year. Year, PD, and their interactions had significant effects on all minerals, indicating that these mineral levels were influenced by $\mathrm{PD}$, but this influence is also affected by yearly environmental factors. RT had significant effects on all sugars (except glucose) and minerals, and its interaction with Y or PD was mainly significant for minerals. Sucrose, fructose, $B$ and $P$ were the constituents most influenced by SR and its interactions with $\mathrm{Y}$ and $\mathrm{PD}$, reflecting the different response of seed constituents to agricultural practices such as PD, SR, and RT. It can be concluded that the least responsive constituents were stachyose, and glucose, and the most responsive were sucrose, fructose, and minerals ( $\mathrm{B}, \mathrm{P}$, and $\mathrm{Fe}$ ). Since $\mathrm{Y}$ interacted with other factors for some seed constituents, results were presented by year (Bellaloui et al., 2009b, 2010, 2011).

\section{EFFECTS OF PLANTING DATE, SEEDING RATE, AND ROW-TYPE ON SEED COMPOSITION IN CLAY SOIL}

Mean values in 2009 in clay soil and in April planting (Table 5) showed that protein concentrations decreased with increasing SR on single-rows, but increased on twin-rows. On the single-rows, the linolenic acid was reduced with SR, but remained constant or not consistent on the twin-rows. Oleic acid, sucrose, P, and B increased with increasing SR, but this increase continued only until a maximum concentration reached, after which the concentration deceased or remained constant. No consistent effects of SR increase were observed for the other seed constituents. A similar pattern was observed for $\mathrm{B}$ and $\mathrm{P}$ in May and June plantings on single- and twin-rows. However, for these PDs protein concentration increased with SR on single-row, but decreased on twin-row (Table 5). Linolenic acid increased with SR and then decreased at higher SR on single- and twin-rows. In May planting the concentrations of oil and minerals increased with SR increases on single- and twin-rows, but the pattern of oleic and protein varied, depending on RT. Generally, protein was higher in May and June plantings than in April planting, but oil concentration had the opposite trend, higher in April and lower in May and June plantings on single- or twin-rows. In 2010, except in May planting, protein, glucose, $\mathrm{B}$, and Fe concentrations increased with SR increase in April and June plantings (Table 6) on single- and twinrows. Protein was higher in May planting and oil was higher in April planting on single- and twin-rows, confirming the observation in 2009. Oleic acid was higher in 2010 than in 2009 for all PDs and on single- and twin-rows.

\section{EFFECTS OF PLANTING DATE, SEEDING RATE, AND ROW-TYPE ON SEED COMPOSITION IN SANDY SOIL}

Mean values in 2009 (Table 7) showed that in April planting on single-rows, oleic acid decreased and linolenic acid and minerals increased with increasing SR until the maximum concentration was achieved, after which the concentrations either became constant or declined. On twin-rows, protein, linolenic acid, sucrose, $\mathrm{B}$, and $\mathrm{P}$ concentrations increased with increasing SR, and oleic acid and stachyose decreased. In May planting, protein, oil,

Table 4 | Analysis of variance ( $F$ and $P$ values) of sucrose, raffinose, stachyose, glucose, fructose $\left(\mathrm{mg} \mathrm{g}^{-1}\right)$, and boron $\left(B, \mathrm{mg} \mathrm{kg}^{-1}\right)$, phosphorus $(P$, $\mathrm{g} \mathrm{kg}^{-1}$ ), and iron (Fe, $\mathrm{mg} \mathrm{kg}^{-1}$ ) in soybean as influenced by year (Y), seeding rate (SR), planting date (PD), and row-type (RT, single- or twin-row) in Beulah fine sandy loam soil in 2009 and 2010 at Stoneville, MS, USA.

\begin{tabular}{|c|c|c|c|c|c|c|c|c|c|c|c|c|c|c|c|c|c|}
\hline \multirow[b]{2}{*}{ Effect } & \multirow[b]{2}{*}{ DF } & \multicolumn{2}{|c|}{ Suc } & \multicolumn{2}{|c|}{ Raff } & \multicolumn{2}{|c|}{ Stac } & \multicolumn{2}{|c|}{ Glu } & \multicolumn{2}{|c|}{ Fru } & \multicolumn{2}{|c|}{ B } & \multicolumn{2}{|c|}{$\mathbf{P}$} & \multicolumn{2}{|c|}{ B } \\
\hline & & $F$ & $P$ & $\boldsymbol{F}$ & $\boldsymbol{P}$ & $\boldsymbol{F}$ & $P$ & $\boldsymbol{F}$ & $P$ & $\boldsymbol{F}$ & $P$ & $\boldsymbol{F}$ & $P$ & $\boldsymbol{F}$ & $P$ & $F$ & $\boldsymbol{P}$ \\
\hline Y & 1 & 500. & $* * *$ & 374 & $* * *$ & 153 & *** & 665 & $* * *$ & 5216 & *** & 442 & $* * *$ & 1044 & $* * *$ & 489 & $* * *$ \\
\hline PD & 2 & 13.8 & ** & 14.3 & $* *$ & 12.8 & $* *$ & 0.02 & NS & 22.41 & $* * *$ & 75.6 & *** & 79.9 & $* * *$ & 9.2 & ** \\
\hline$Y \times P D$ & 2 & 2.59 & NS & 2.3 & NS & 6.58 & $* *$ & 0.5 & NS & 2.71 & NS & 44.0 & $* * *$ & 79.75 & $* * *$ & 9.7 & $* *$ \\
\hline $\mathrm{RT}$ & 1 & 132 & $* * *$ & 23.6 & $* * *$ & 12.5 & $* *$ & 669 & NS & 407 & *** & 150 & $* * *$ & 162 & $* * *$ & 132 & $* * *$ \\
\hline$Y \times R T$ & 1 & 2.9 & NS & 57.6 & $* * *$ & 0.4 & NS & 2.26 & NS & 28.8 & *** & 7.17 & $* *$ & 28.6 & $* * *$ & 54.7 & $* * *$ \\
\hline$P D \times R T$ & 2 & 2.3 & NS & 1.47 & NS & 0.07 & NS & 0.56 & NS & 64.0 & $* * *$ & 2.79 & NS & 19.3 & $* * *$ & 17.8 & *** \\
\hline$Y \times P D \times R T$ & 2 & 1.3 & NS & 0.1 & NS & 1.86 & NS & 1.46 & NS & 26.8 & $* * *$ & 0.92 & NS & 17.8 & $* * *$ & 14.2 & $* * *$ \\
\hline SR & 3 & 8.6 & $* * *$ & 0.72 & NS & 0.61 & NS & 1.4 & NS & 5.41 & $* *$ & 54.7 & $* * *$ & 65.3 & $* * *$ & 1.06 & NS \\
\hline Year $\times$ SR & 3 & 2.6 & $*$ & 0.89 & NS & 0.86 & NS & 0.0 & NS & 0.0 & NS & 0.0 & NS & 0.0 & NS & 0.29 & NS \\
\hline $\mathrm{PD} \times \mathrm{SR}$ & 6 & 3.5 & * * & 5.5 & $* * *$ & 0.59 & NS & 0.38 & NS & 5.1 & *** & 22.91 & $* * *$ & 31.0 & $* * *$ & 2.8 & $* *$ \\
\hline$Y \times P D \times S R$ & 6 & 1.5 & NS & 2.29 & * & 0.45 & NS & 0.41 & NS & 6.9 & $* * *$ & 18.75 & $* * *$ & 61.4 & $* * *$ & 4.93 & *** \\
\hline$R \times S R$ & 3 & 3.6 & NS & 0.44 & NS & 0.88 & NS & 0.87 & NS & 5.1 & $* *$ & 12.48 & $* * *$ & 88.5 & $* * *$ & 6.8 & $* *$ \\
\hline $\mathrm{Y} \times \mathrm{RT} \times \mathrm{SR}$ & 3 & 0.7 & NS & 0.54 & NS & 1.27 & NS & 0.0 & NS & 0.0 & NS & 0.0 & NS & 0.03 & NS & 2.5 & NS \\
\hline $\mathrm{PD} \times \mathrm{RT} \times \mathrm{SR}$ & 6 & 3.87 & ** & 4.53 & $* *$ & 1.9 & NS & 0.86 & NS & 11.6 & $* * *$ & 0.93 & NS & 9.6 & $* * *$ & 17.5 & $* * *$ \\
\hline$Y \times P D \times R T \times S R$ & 6 & 5.1 & $* * *$ & 5.6 & $* * *$ & 0.27 & NS & 0.62 & NS & 17.4 & $* * *$ & 0.52 & NS & 12.2 & $* * *$ & 4.9 & $* *$ \\
\hline Residual & & 20 & & 0.25 & & 13.9 & & 0.14 & & 0.01 & & 9 & & 0.06 & & 12.8 & \\
\hline
\end{tabular}

${ }^{*} P \leq 0.05 ;{ }^{*} P \leq 0.01 ; * *{ }^{*} P \leq 0.001 ; N S$, not significant; suc, sucrose, raff, raffinose; stac, stachyose; glu, glucose; and fruc, fructose. 
Table 5 | Effects of row-type (RT, single, $\mathrm{S}$ or twin, $\mathrm{T})$, seeding rate (SR, seed $\left.\mathrm{m}^{-2}\right)$, and planting date on seed protein, oil, fatty acids (g kg $\left.\mathbf{~}^{-1}\right)$, sucrose (Suc), stachyose (Stac), glucose (Glu) $\left(\mathrm{mg} \mathrm{g}^{-1}\right)$, boron $\left(B, \mathrm{mg} \mathrm{kg}^{-1}\right)$, phosphorus $\left(\mathrm{P}, \mathrm{g} \mathrm{kg}^{-1}\right)$, and iron $\left(\mathrm{Fe}\right.$, $\left.\mathrm{mg} \mathrm{kg}^{-1}\right)$ in soybean in Sharkey clay soil in 2009 at Stoneville, MS, USA.

\begin{tabular}{|c|c|c|c|c|c|c|c|c|c|c|c|c|}
\hline Planting & RT & SR & Protein & Oil & Oleic & Linolenic & Suc & Stac & Glu & B & $\mathbf{P}$ & $\mathrm{Fe}$ \\
\hline \multirow[t]{9}{*}{ April } & & 20 & 420 & 242 & 231 & 67.6 & 46.5 & 26.7 & 2.8 & 38.3 & 4.5 & 66.0 \\
\hline & $\mathrm{S}$ & 30 & 413 & 242 & 237 & 66.4 & 47.2 & 26.3 & 2.8 & 44.7 & 4.5 & 66.0 \\
\hline & & 40 & 419 & 237 & 246 & 56.7 & 47.2 & 29.4 & 2.6 & 43.8 & 5.6 & 65.5 \\
\hline & & 50 & 417 & 242 & 256 & 56.3 & 45.9 & 26.3 & 2.8 & 36.4 & 4.5 & 63.8 \\
\hline & & LSD & 1.90 & 2.10 & 4.20 & 2.90 & 1.21 & 0.70 & 0.20 & 3.70 & 0.07 & 1.30 \\
\hline & $\mathrm{T}$ & 30 & 408 & 243 & 281 & 61.3 & 61.5 & 27.2 & 4.1 & 47.2 & 5.6 & 66.8 \\
\hline & & 40 & 408 & 244 & 275 & 61.5 & 62.5 & 26.8 & 4.1 & 48.2 & 5.6 & 73.3 \\
\hline & & 50 & 410 & 241 & 266 & 61.5 & 46.0 & 28.0 & 4.0 & 48.5 & 4.3 & 61.0 \\
\hline & & LSD & 3.50 & 2.70 & 5.00 & 2.30 & 1.10 & 1.10 & 0.21 & 1.30 & 0.08 & 1.40 \\
\hline \multirow{7}{*}{ May } & & 50 & 458 & 203 & 220 & 77.8 & 45.5 & 25.1 & 2.6 & 32.0 & 4.6 & 60.0 \\
\hline & & LSD & 2.30 & 2.00 & 3.30 & 3.50 & 0.81 & 1.00 & 0.16 & 1.90 & 0.09 & 1.90 \\
\hline & & 20 & 458 & 203 & 224 & 75.9 & 53.8 & 24.2 & 4.1 & 28.9 & 4.4 & 53.0 \\
\hline & $\mathrm{T}$ & 30 & 452 & 206 & 245 & 88.5 & 61.0 & 23.7 & 4.0 & 29.5 & 5.3 & 64.5 \\
\hline & & 40 & 447 & 201 & 230 & 73.9 & 62.3 & 21.8 & 4.3 & 38.9 & 4.6 & 64.0 \\
\hline & & 50 & 452 & 201 & 211 & 71.8 & 52.0 & 25.4 & 4.2 & 38.4 & 4.3 & 54.0 \\
\hline & & LSD & 4.40 & 2.90 & 4.70 & 2.90 & 0.91 & 1.20 & 0.19 & 4.50 & 0.18 & 1.20 \\
\hline \multirow[t]{4}{*}{ June } & & 20 & 439 & 223 & 204 & 72.7 & 55.8 & 24.2 & 2.7 & 26.6 & 3.4 & 52.5 \\
\hline & $S$ & 30 & 449 & 223 & 207 & 76.3 & 51.3 & 24.7 & 2.9 & 34.1 & 3.5 & 55.5 \\
\hline & & 50 & 422 & 224 & 209 & 83.7 & 54.8 & 27.3 & 4.1 & 39.2 & 3.7 & 57.8 \\
\hline & & LSD & 4.10 & 2.60 & 7.70 & 3.40 & 2.30 & 1.20 & 0.20 & 1.50 & 0.09 & 2.70 \\
\hline
\end{tabular}

Means were separated by Fisher's least significant difference LSD (0.05).

linolenic acid, B, and $\mathrm{P}$ increased with increasing SR on singlerows. On twin-rows, linolenic acid, sucrose, glucose, $\mathrm{B}$, and $\mathrm{P}$ concentrations increased, and protein and oleic acid concentrations decreased. In June planting on single-rows, linolenic acid, glucose, B, P, and Fe concentrations increased with increasing SR, but oleic acid decreased. On twin-rows, oleic acid decreased and linolenic acid, sucrose, and B concentrations increased. Protein was higher in June planting, but oil was higher in April planting. In 2010 (Table 8) in April planting, sucrose and Fe concentrations increased with increasing SR on single- and twinrows. In May planting, protein concentration decreased with SR, but oil concentration and B concentrations increased. In June planting, protein, $\mathrm{P}$, and $\mathrm{Fe}$ increased and oil and oleic acid decreased with SR on single- and twin-rows. Sucrose and B concentrations showed a pattern of increase with increasing SR on twin-rows.

\section{SOIL AND LEAF NUTRIENTS LEVELS}

Soil analyses showed that in clay soil, the average nutrient levels in 2009 and 2010, respectively, were: $\mathrm{C}=1.40$ and $1.10 \%$; $\mathrm{N}=0.13$ and $0.10 \% ; \mathrm{S}=31.2$ and $30.9 \mathrm{mg} \mathrm{kg}^{-1} ; \mathrm{K}=2340$ and $2570 \mathrm{mg} \mathrm{kg}^{-1} ; \mathrm{P}=355$ and $368 \mathrm{mg} \mathrm{kg}^{-1} ; \mathrm{B}=2.45$ and $2.92 \mathrm{mg} \mathrm{kg}^{-1}$; and $\mathrm{Fe}=20.13$ and $22.91 \mathrm{~g} \mathrm{~kg}^{-1}$. In sandy soil, the average nutrient levels, respectively, in 2009 and 2010 were: $\mathrm{C}=1.10$ and $1.30 \% ; \mathrm{N}=0.10$ and $0.11 \% ; \mathrm{S}=28.7$ and $30.1 \mathrm{mg} \mathrm{kg}^{-1} ; \mathrm{K}=2140$ and $2060 \mathrm{mg} \mathrm{kg}^{-1} ; \mathrm{P}=267$ and 
Table 6 | Effects of row-type (RT, single, $\mathrm{S}$ or twin, T), seeding rate (SR, seed $\left.\mathrm{m}^{-2}\right)$, and planting date on seed protein, oil, fatty acids $\left(\mathrm{g} \mathbf{k g}^{-1}\right)$, sucrose (Suc), stachyose (Stac), glucose (Glu) $\left(\mathrm{mg} \mathrm{g}^{-1}\right)$, boron $\left(B, \mathrm{mg} \mathrm{kg}^{-1}\right)$, phosphorus $\left(\mathrm{P}, \mathrm{g} \mathrm{kg}^{-1}\right)$, and iron $\left(\mathrm{Fe}, \mathrm{mg} \mathrm{kg}^{-1}\right)$ in soybean in Sharkey clay soil in $\mathbf{2 0 1 0}$ at Stoneville, MS, USA.

\begin{tabular}{|c|c|c|c|c|c|c|c|c|c|c|c|c|}
\hline Planting & RT & SR & Protein & Oil & Oleic & Linolenic & Suc & Stac & Glu & B & $\mathbf{P}$ & $\mathrm{Fe}$ \\
\hline \multirow[t]{9}{*}{ April } & & 20 & 419 & 231 & 279 & 66.3 & 23.8 & 38.3 & 1.6 & 28.1 & 3.6 & 47.8 \\
\hline & $S$ & 30 & 424 & 227 & 276 & 62.5 & 23.3 & 39.3 & 1.8 & 36.8 & 3.4 & 57.0 \\
\hline & & 40 & 421 & 230 & 282 & 60.7 & 23.8 & 42.3 & 2.6 & 33.9 & 3.5 & 52.8 \\
\hline & & 50 & 423 & 228 & 275 & 69.7 & 25.3 & 42.8 & 3.4 & 42.1 & 3.4 & 57.3 \\
\hline & & LSD & 2.80 & 1.90 & 4.70 & 2.40 & 3.40 & 2.20 & 0.21 & 1.30 & 0.10 & 1.40 \\
\hline & $\mathrm{T}$ & 30 & 423 & 226 & 289 & 64.2 & 21.5 & 44.3 & 2.0 & 27.0 & 3.4 & 53.0 \\
\hline & & 40 & 421 & 230 & 270 & 63.8 & 24.0 & 40.8 & 3.4 & 45.3 & 3.4 & 55.5 \\
\hline & & 50 & 421 & 228 & 284 & 66.1 & 22.0 & 40.5 & 2.3 & 30.5 & 3.3 & 55.0 \\
\hline & & LSD & 2.60 & 1.90 & 4.90 & 2.40 & 2.90 & 1.70 & 0.30 & 5.00 & 0.05 & 1.30 \\
\hline \multirow{7}{*}{ May } & & 50 & 443 & 212 & 289 & 70.7 & 27.5 & 40.3 & 3.8 & 34.3 & 3.4 & 52.5 \\
\hline & & LSD & 2.41 & 1.50 & 5.60 & 2.80 & 1.80 & 2.00 & 0.14 & 1.20 & 0.13 & 1.50 \\
\hline & & 20 & 437 & 217 & 278 & 68.5 & 33.3 & 37.5 & 1.9 & 36.8 & 3.4 & 56.0 \\
\hline & $\mathrm{T}$ & 30 & 438 & 215 & 287 & 66.8 & 24.5 & 37.8 & 1.9 & 26.3 & 2.3 & 44.0 \\
\hline & & 40 & 437 & 216 & 284 & 68.2 & 34.3 & 38.3 & 3.7 & 35.3 & 3.3 & 54.0 \\
\hline & & 50 & 440 & 216 & 282 & 65.5 & 33.5 & 42.0 & 1.7 & 35.5 & 3.4 & 43.5 \\
\hline & & LSD & 2.20 & 1.70 & 7.60 & 2.40 & 1.60 & 1.90 & 0.19 & & 0.07 & 1.50 \\
\hline \multirow[t]{4}{*}{ June } & & 20 & 419 & 210 & 286 & 72.4 & 35.3 & 42.3 & 1.8 & 29.6 & 2.4 & 41.5 \\
\hline & $S$ & 30 & 431 & 213 & 295 & 66.8 & 29.3 & 40.3 & 1.6 & 36.8 & 2.5 & 43.8 \\
\hline & & 50 & 437 & 203 & 278 & 67.5 & 24.8 & 38.8 & 1.7 & 39.3 & 3.5 & 47.0 \\
\hline & & LSD & 2.60 & 2.10 & 2.80 & 2.60 & 2.70 & 2.40 & 2.19 & 1.60 & 0.11 & 1.40 \\
\hline
\end{tabular}

Means were separated by Fisher's least significant difference LSD (0.05).

$284 \mathrm{mg} \mathrm{kg}^{-1} ; \mathrm{B}=1.20$ and $1.60 \mathrm{mg} \mathrm{kg}^{-1}$; and $\mathrm{Fe}=18.72$ and $18.89 \mathrm{~g} \mathrm{~kg}^{-1}$. Nutrient concentrations in leaf samples in clay soil, respectively in 2009 and 2010 were: $\mathrm{N}=5.20$ and $4.11 \%$; $\mathrm{S}=0.31$ and $0.27 \% ; \mathrm{K}=2000$ and $1570 \mathrm{mg} \mathrm{kg}^{-1} ; \mathrm{P}=390$ and $230 \mathrm{mg} \mathrm{kg}^{-1} ; \mathrm{B}=40.56$ and $33.76 \mathrm{mg} \mathrm{kg}^{-1}$; and $\mathrm{Fe}=209$ and $86 \mathrm{mg} \mathrm{kg}^{-1}$. Nutrient concentrations in leaves in sandy loam soil, respectively in 2009 and 2010, were: $\mathrm{N}=4.80$ and $4.76 \% ; \mathrm{S}=0.37$ and $0.23 \% ; \mathrm{K}=2.20$ and $1.43 \% ; \mathrm{P}=0.41$ and $0.29 \% ; \mathrm{B}=35.7$ and $28.70 \mathrm{mg} \mathrm{kg}^{-1}$; and $\mathrm{Fe}=157$ and $94.50 \mathrm{mg} \mathrm{kg}^{-1}$. The analysis of random samples of fully expanded leaves taken across the field at R5 to R6 showed adequate concentrations of nutrients in soybean grown in both sites. It was noticed that concentrations of nutrients, especially for K, B, P, and Fe, in 2009 were greater than in 2010 and this observation was attributed to heat and drier conditions in 2010.

\section{DISCUSSION}

EFFECTS OF PLANTING DATE, SEEDING RATE, AND ROW-TYPE ON SEED COMPOSITION

The current research showed that April planting (early planting) resulted in higher oil, oleic acid, sucrose, and minerals, especially $\mathrm{B}$ and $\mathrm{P}$, on both single and twin-rows, and June planting (late planting) resulted in higher protein and linolenic acid, but lower oleic acid and oil concentrations in 2009 and 2010. Previous research showed that the response of seed constituents to PD was mainly due to temperature differences (Dardanelli et al., 
Table 7 | Effects of row-type (RT, single, $S$ or twin, $T)$, seeding rate (SR, seed $\left.\mathbf{~}^{-2}\right)$, and planting date on seed protein, oil, fatty acids $\left(\mathrm{g} \mathrm{kg}^{-1}\right)$, sucrose (Suc), stachyose (Stac), glucose (Glu) ( $\left.\mathrm{mg} \mathrm{g}^{-1}\right)$, boron (B, $\left.\mathrm{mg} \mathrm{kg}^{-1}\right)$, phosphorus $\left(\mathrm{P}, \mathrm{g} \mathrm{kg}^{-1}\right)$, and iron (Fe, $\left.\mathrm{mg} \mathrm{kg}{ }^{-1}\right)$ in soybean in Beulah fine sandy loam soil in 2009 at Stoneville, MS, USA.

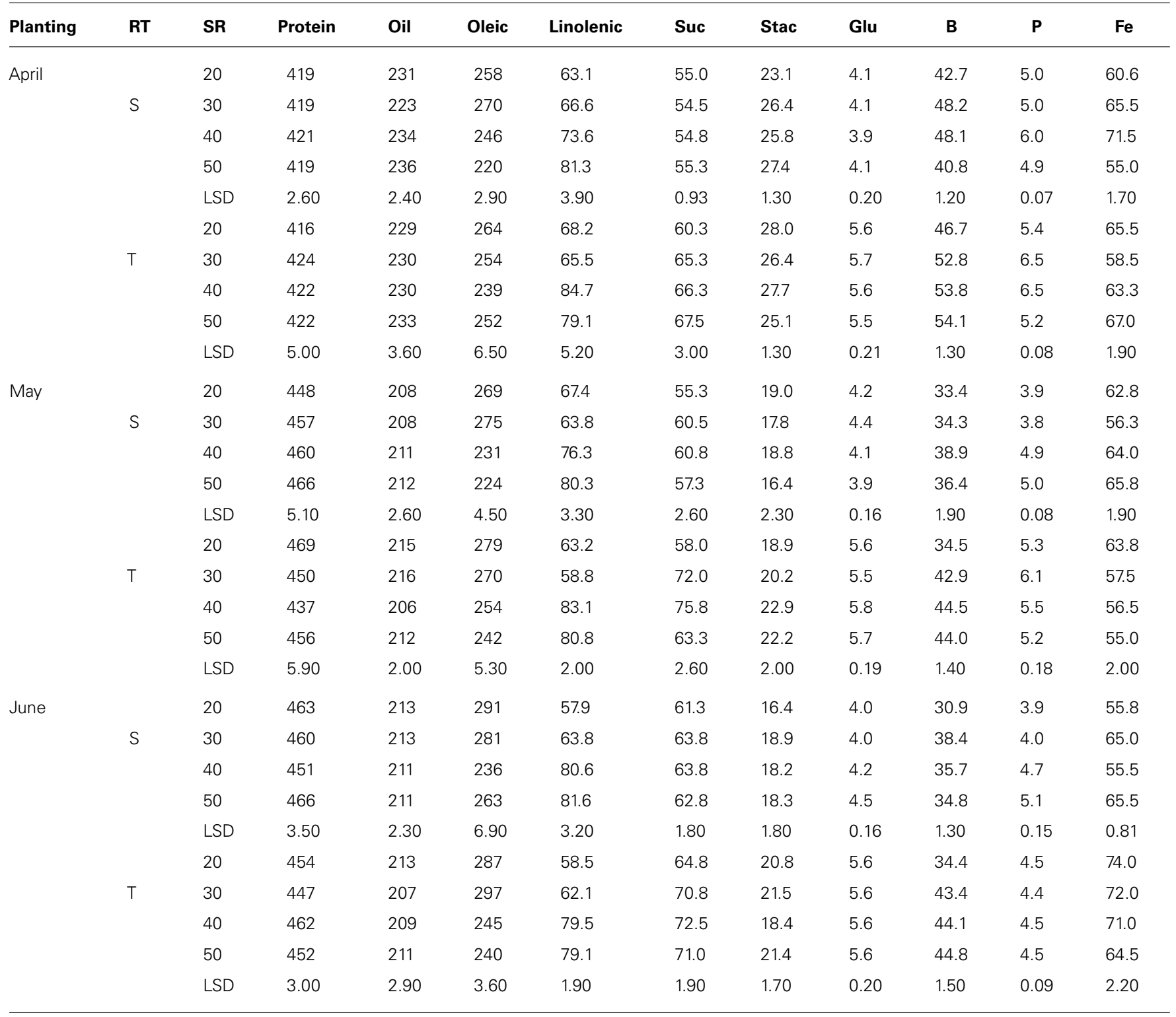

Means were separated by Fisher's least significant difference LSD (0.05). Suc, sucrose; stac, stachyose, and glu, glucose.

2006; Bellaloui et al., 2009b) or other environmental factors such as drought (Piper and Boote, 1999; Bellaloui et al., 2009b, 2014). Dardanelli et al. (2006) investigated the consistency of soybean MG effects and their interactions with the environment on protein and oil, and found that environment was mainly defined by PD and location, and that environment was the most important source of variation for protein and oil. Previous research on the effects of PD on seed composition is still inconsistent and inconclusive. For example, oil concentration increased with early planting, but this increase pattern was not consistent across locations (Helms et al., 1990; Kane et al., 1997; Pedersen and Lauer, 2004), indicating that the response of seed constituents to PDs depends on the environment under which soybeans are grown.
Our research showed that late planting (June planting) resulted in the increase of protein concentration and decrease in oil concentration, partially agreeing with those of Helms et al. (1990) and Pedersen and Lauer (2004). The high oleic acid and low linolenic acid concentrations in the early planting (April planting), and the low oleic acid and high linolenic acid in late planting, especially the June planting, could be due to the inverse relationship between these two constituents (Carver et al., 1986; Dornbos and Mullen, 1992; Bellaloui et al., 2009b) and temperature differences. Studying high oleic acid gemplasm lines, it was suggested that the instability of oleic acid across environments was due mainly to temperature effects on enzymes controlling biosynthesis of soybean seed fatty acids, especially during seed-fill (R5 to R6) stage 
Table 8 | Effects of row-type (RT, single, $\mathrm{S}$ or twin, $\mathrm{T})$, seeding rate (SR, seed $\left.\mathrm{m}^{-2}\right)$, and planting date on seed protein, oil, fatty acids $\left(\mathrm{g} \mathbf{k g}^{-1}\right)$, sucrose (Suc), stachyose (Stac), glucose (Glu) $\left(\mathrm{mg} \mathrm{g}^{-1}\right)$, boron $\left(B, \mathrm{mg} \mathrm{kg}^{-1}\right)$, phosphorus $\left(\mathrm{P}, \mathrm{g} \mathrm{kg}^{-1}\right)$, and iron $\left(\mathrm{Fe}\right.$, $\left.\mathrm{mg} \mathrm{kg}^{-1}\right)$ in soybean in Beulah fine sandy loam soil in $\mathbf{2 0 1 0}$ at Stoneville, MS, USA.

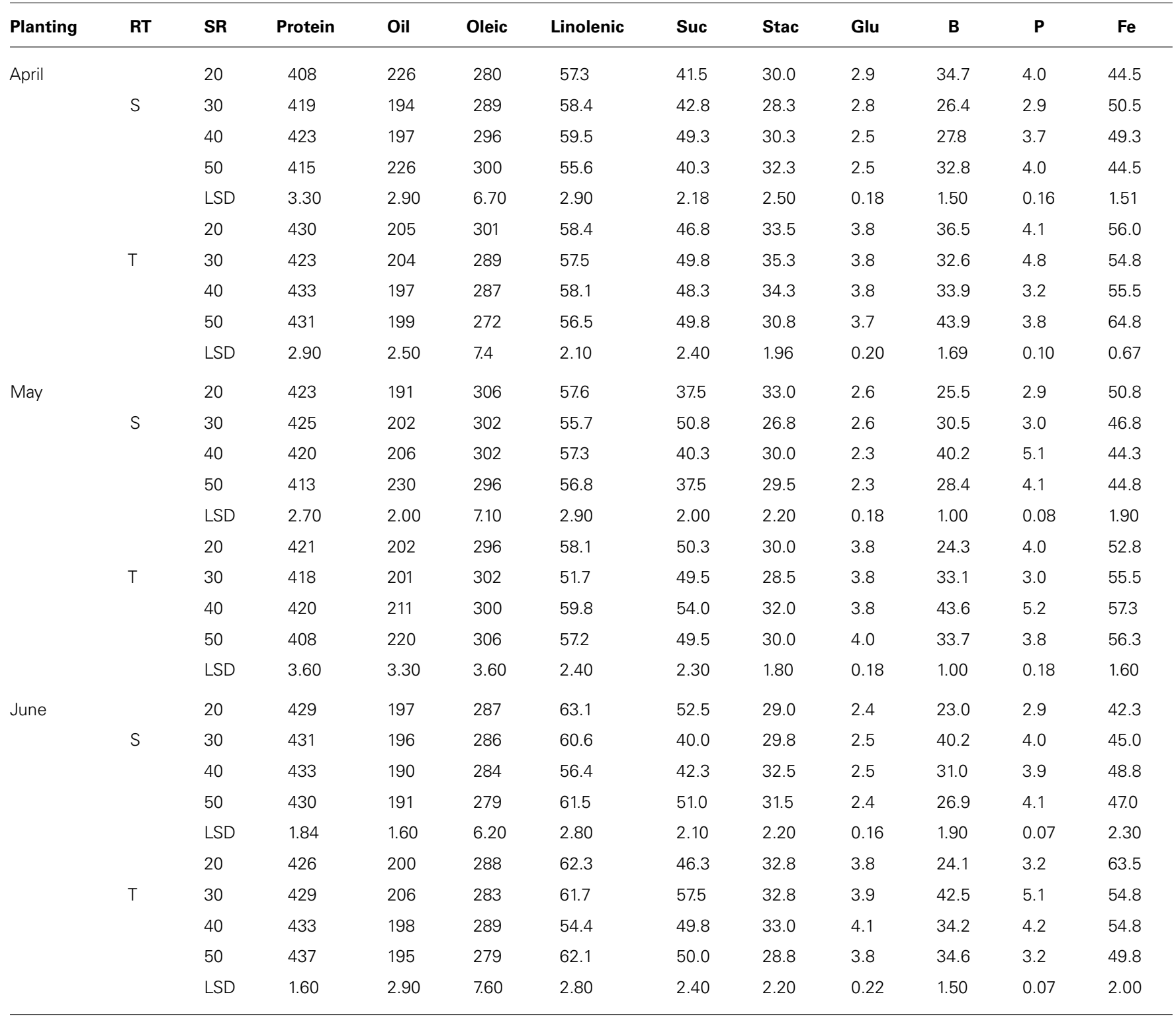

Means were separated by Fisher's least significant difference LSD (0.05). Suc, sucrose; stac, stachyose; and glu, glucose.

(Howell and Collins, 1957; Wilcox and Cavins, 1992; Bachlava and Cardinal, 2009). Bachlava and Cardinal (2009) suggested that the late planting may result in a decrease of linolenic and palmitic acids, but stearic acid may increase, and the changes in fatty acids with late planting may be due to temperature changes during seed maturation at later planting (Wilcox and Cavins, 1992). Other researchers showed that high oleic and low linolenic acids under warmer conditions (Carver et al., 1986) were explained as consequences of the effects of temperature on oleic acid and linoleic acid desaturases (Burton, 1991). These effects include a decrease in oleic- and linoleic-desaturase activities at $35^{\circ} \mathrm{C}$ (Cheesbrough, 1989), a decrease in $\omega-6$ desaturase enzyme (encoded by the FAD2-1A gene), and the degradation of desaturases at high growth temperatures of $30^{\circ} \mathrm{C}$ (Tang et al., 2005). Another possible explanation for high linolenic and low oleic acids in late planting is that late planting coincides with a cooler temperature that favors linolenic acid accumulation and lowering of oleic acid. This explanation could be supported by our findings that linolenic acid concentration increased with increased SR for all PDs in sandy soil in 2009. This may be due to shade effects and cooler temperatures in the lower plant canopy which resulted from higher plant densities at higher SR (40 and 50 seed $\mathrm{m}^{-2}$ ). Previous research investigated the effect of upper and lower plant parts on seed nutrients, including seed oil and protein (Collins and Cartter, 1956), protein (Escalante and Wilcox, 1993), protein, oil, oleic, and linolenic acids (Bellaloui and Gillen, 2010; 
Bellaloui et al., 2012). They found that seed located at the top central portion had lower seed oil and higher protein contents (Collins and Cartter, 1956), while Kochegura (1982) found that seeds from the higher plant canopy had higher seed protein than seeds at lower plant canopy. Other researchers found that there were no differences between the upper and lower canopy (Huskey et al., 1990). Bellaloui et al. (2012) found that the lower plant canopy had higher oil and linolenic acid, but lower protein and oleic acid. The differences in seed oil between the upper and lower plant canopy was explained to be due to environmental differences during seed oil synthesis and accumulation (Collins and Cartter, 1956). Others reported that the higher seed oil and linolenic acid and the lower protein and oleic acids in seeds in the upper plant canopy was due to cooler temperature and shade effects as cooler temperature enhances linolenic acid and oil (Bellaloui et al., 2012). Therefore, the higher linolenic acid concentration with increasing SR and late planting could be due to shade effects and cooler temperature. Since linolenic acid and oleic acid showed to have an inverse relationship (Bellaloui et al., 2009a,b), the lower oleic acid is a consequences of the higher linolenic acid.

Increasing protein and B with increasing SR in 2009 in sandy and clay soils, especially for May and June plantings, increasing linolenic acid in sandy soil in April planting, and increasing sucrose in 2009 in both clay and sandy soils indicated the positive response of these constituents to SR increases under these conditions. The higher concentrations of B and sucrose in twin-rows, compared to single-rows, for all PDs in 2009 can be explained in terms of light interception and row-spacing effects. Twin-rows resulted in narrow-plantings, while single-row plantings were wider. It was reported that narrow-row soybean had higher canopy radiation interception than wider rows (Shibles and Weber, 1966; Taylor et al., 1982), and differences in radiation interception was observed between narrow-row and wide-rows during the period from R6 (beginning seed-fill) to R7 (full seed-fill) stages (Fehr and Caviness, 1977). These differences were attributed to leaf area distribution and duration (Taylor et al., 1982). Other researchers reported that the reasons for higher yield in narrower rows (from 25 to $50 \mathrm{~cm}$ ) compared to wider rows (Bharati, 1977; Cooper, 1977) was usually attributed to the development of a full canopy (95\% light interception) before rapid seed development (Shibles and Weber, 1966), resulting in greater photosynthetic rate (Shibles and Weber, 1965), and nutrient uptake and translocation (Bellaloui et al., 2014). Therefore, the higher concentrations of sucrose and $\mathrm{B}$ in twin-rows could be due to the possible association between sucrose and B nutrition and light interception and photosynthesis. The higher concentrations of oleic acid in 2010, especially in clay soil, may be due to higher temperature and a drier year in 2010 (Figure 1). The higher concentrations of stachyose in 2010 may be due to a drier year and higher temperature in 2010 compared with 2009, suggesting a possible role of stachyose as an environmental stress compound under drought and high temperature. Although the biological functions of stachyose are still not clear (Ren et al., 2009), Obendorf (1997) reported that oligosaccharides, including stachyose, are required for the acquisition of desiccation tolerance during seed development and maturation, and could be involved in seed protection

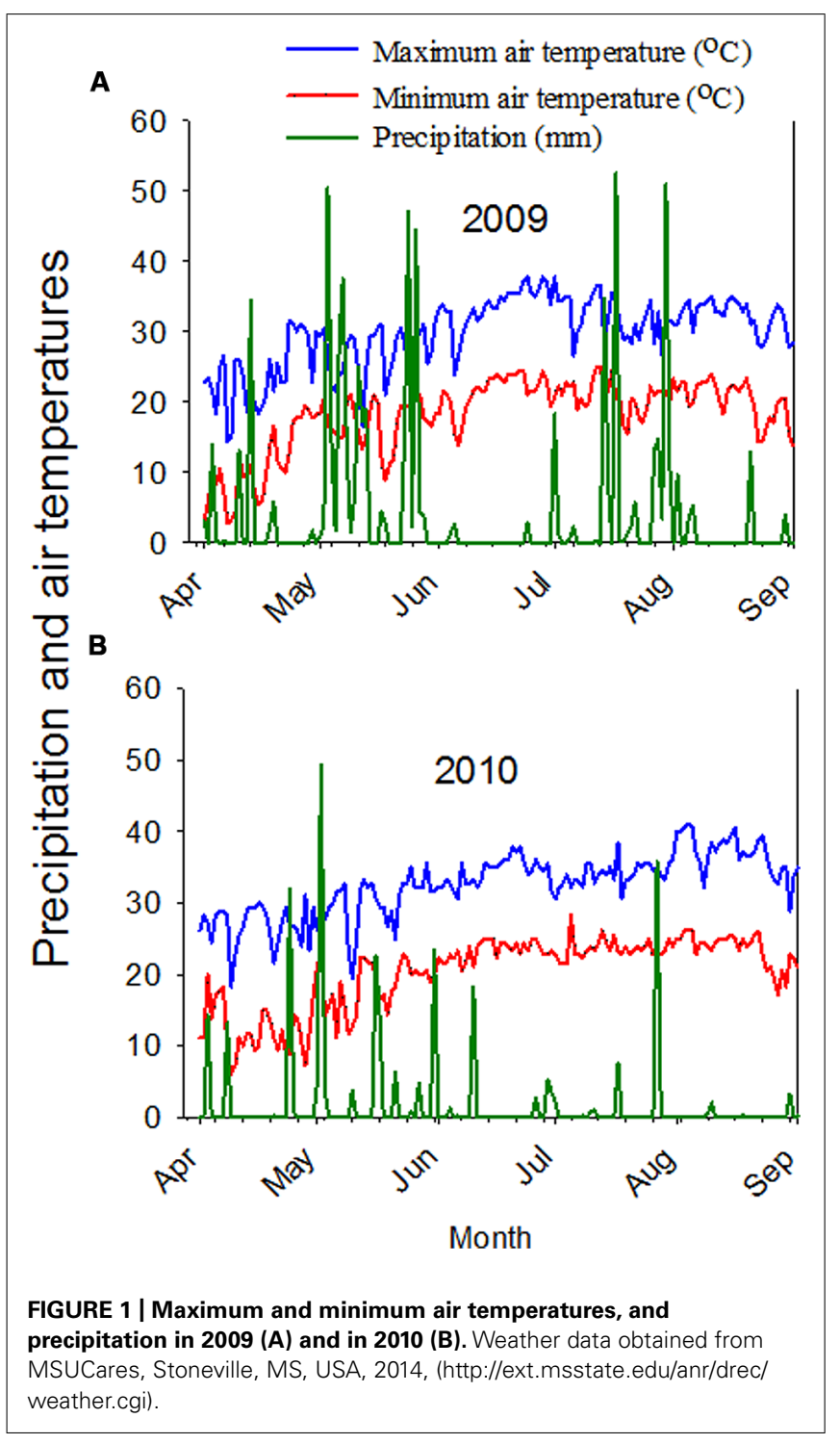

against damage during seed dehydration to ensure seed survival and storability.

Very limited information on the effects of SR and row-spacing on seed composition was reported, and what is available is on other species (Bellaloui et al., 2014). For example, effect of SR and row-spacing on seed protein and oil in rapeseed (Brassica napus L.) had no consistent effects (Kondra, 1975). Other researchers evaluated the effects of three row-spacing $(30,40$, and $50 \mathrm{~cm})$ on canola seed oil and found there was no relationship between rowspacing and oil, but the highest oil concentration was recorded at row-space of $30 \mathrm{~cm}$ (Mousavi et al., 2011). Studying the effects of SRs of $60,50,40$, and 30 plants $\mathrm{m}^{-2}$ on safflower (Carthamus tinctorius L.) oil under irrigated and rainfed conditions, Amoghein et al. (2012) found that the highest oil percentage was achieved by the lowest SR of 30 plants $\mathrm{m}^{-2}$, but no oil differences was observed between 40,50 , and 60 plant $\mathrm{m}^{-2}$. SR from 30,000 to 45,000 plants $\mathrm{ha}^{-1}$ resulted in higher oil in Sunflower (Helianthus annuus L.), while SR beyond 45,000 plants $\mathrm{ha}^{-1}$ resulted in a smaller increases 
(Gubbels and Dedio, 1986). Recently, Bellaloui et al. (2014) investigated the effects of SR and row-spacing on seed protein, oil, fatty acids, sugars, and minerals using four soybean cultivars (P 93M90, AG 3906, P 94B73, and V 52N3) in 2006 and 2007 in the Midsouth USA. They found that protein, oleic acid, sugars, $\mathrm{P}$, and $\mathrm{B}$ concentrations increased with the increase of SR in P 93M90 and AG 3906, but the concentrations of these constituents decreased after the maximum concentrations were reached, supporting our results. The pattern of increase was mainly observed in 2006 and depended on the row-spacing. In 2007, however, the opposite trend (protein and oleic decreased with SR) was noticed. In cultivars $\mathrm{P} 94 \mathrm{~B} 73$ and V 52N3 protein concentration increased with SR in 2006 and 2007 for both 38 and $76 \mathrm{~cm}$ row-spacing. An increase of oleic acid and a decrease of linolenic acid with row-spacing were observed in 2006. They concluded that SRs and row-spacing can alter some seed composition constituents, and the effect of SRs depended on the rate used, row-spacing, genotype, and the growing conditions of each year. They also reported that the different pattern (positive or negative) of seed constituents between years due to the environmental factors such as temperature and drought.

The response of some seed constituents to $\mathrm{PD}, \mathrm{SR}$, or RT was different between 2009 and 2010, and this may be due to high heat and drier year in 2010 . Weather data showed that the average maximum temperature reached $34^{\circ} \mathrm{C}$ in July and $37^{\circ} \mathrm{C}$ in August in 2010 compared with 32.2 and $34.4^{\circ} \mathrm{C}$ in July and August, respectively in 2009 (Figure 1; MSUCares, 2014). The precipitation in July was 46.0 and $6.1 \mathrm{~mm}$ in August in 2010, but was $203.7 \mathrm{~mm}$ in July and $36.1 \mathrm{~mm}$ in August in 2009 (Figure 1; MSUCares, 2014). Previous research showed that relationships between seed composition constituents differ between years due to heat and drought (Bellaloui et al., 2009b, 2014). Our soil analysis showed that both clay and sandy soils had adequate nutrient levels; however, leaf samples collected in 2009 and 2010 showed that leaf nutrient levels in 2010 were lower than in 2009, and this may be due to high heat and drier year in 2010, affecting the concentrations of seed composition and minerals.

We did not observe clear patterns for protein, oil, or fatty acids between single- and twin-rows, although other researchers found that row-spacing and irrigation significantly affected protein and oil contents, and that row-spacing (RS) of $70 \mathrm{~cm}$ had the highest protein content, followed by RS of 60,40 , and $50 \mathrm{~cm}$, respectively (Boydak et al., 2002). They also found that RS had a significant $(P<0.01)$ influence on oleic and linoleic acid content, and a rowspacing of $50 \mathrm{~cm}$ produced maximum oil value, but a row-spacing of $70 \mathrm{~cm}$ produced the highest protein value $(39.05 \%)$, and $50 \mathrm{~cm}$ produced the lowest value $(37.65 \%)$.

\section{CONCLUSION}

The current research showed that early planting resulted in higher soybean seed oil and oleic acid, but lower protein and linolenic acid concentrations. The late planting resulted in higher protein and linolenic acid. These changes in seed constituents were attributed mainly to temperature changes and drought, indicating that shifts in PDs create a new environment. Concentrations of protein, linolenic acid, sucrose and B increased with SR increases, possibly due to higher light interception and early canopy closure. The SRs that resulted in highest levels of seed constituents (for example, for protein, oil, oleic and linolenic acids, sucrose, and B) appear to be between 30 and 40 seed $\mathrm{m}^{-2}$. Rates beyond 40 seed $\mathrm{m}^{-2}$ may result in a negative effect on some seed constituents, possibly due to interplant competition for available nutrients and shade effects. The constituents most affected by management practices, and in our case PD, SR, and RT, appear to be protein, oleic and linolenic acids, sucrose, and B. Since the physiological and biochemical mechanisms of how management practices affect seed constituents are scarce, further research is needed to understand the mechanisms controlling these responses. The increase of stachyose in 2010 may be due to a drier year and high temperature in 2010 compared with 2009, suggesting a possible role of stachyose as an environmental stress compound, and further research is needed to confirm this observation. The current information benefits growers and breeders for considering environmental factors such as heat, drought, and agronomic practices in producing seed with higher quality, especially under double-cropping production systems of soybean and wheat in the Midsouth USA.

\section{ACKNOWLEDGMENTS}

Authors are thankful to Debbie Boykin for statistics assistance, Sandra Mosley for lab assistance, and Joseph Baugh for field management. This work was partially supported by the U.S. Department of Agriculture, Agricultural Research Service Project 6402-21220-012-00D. Mention of trade names or commercial products in this publication is solely for the purpose of providing specific information and does not imply recommendation or endorsement by the U.S. Department of Agriculture.

\section{REFERENCES}

Amoghein, R. S., Tobeh, A., and Jamaati-e-Somarin, S. (2012). Effect of plant density on phenology and oil yield of safflower herb under irrigated and rainfed planting systems. J. Med. Plant. Res. 6, 2493-2503.

AOAC. (1990a). “Method 988.05,” in Official Methods of Analysis, 15th Edn, ed. K. Helrich (Arlington, VA: Elsevier Science Publishing).

AOAC. (1990b). “Method 920.39," in Official Methods of Analysis, 15th Edn, ed. K. Helrich (Arlington, VA: Elsevier Science Publishing).

Bachlava, E., and Cardinal, A. J. (2009). Correlation between temperature and oleic acid seed content in three segregating soybean populations. Crop Sci. 49, 1328-1335. doi: 10.2135/cropsci2008.11.0660

Bandemer, S. L., and Schaible, P. J. (1944). Determination of iron. A study of the o-phenanthroline method. Ind. Eng. Chem. Anal. Ed. 16, 317-319. doi: 10.1021/i560129a013

Bellaloui, N., Abbas, H. K., Gillen, A. M., and Abel, C. A. (2009a). Effect of glyphosate-boron application on seed composition and nitrogen metabolism in glyphosate-resistant soybean. J. Agric. Food Chem. 57, 9050-9056. doi: 10.1021/jf901801z

Bellaloui, N., Smith, J. R., Ray, J. D., and Gillen, A. M. (2009b). Effect of maturity on seed composition in the early soybean production system as measured on near-isogenic soybean lines. Crop Sci. 49, 608-620. doi: 10.2135/cropsci2008.0 4.0192

Bellaloui, N., and Gillen, A. M. (2010). Soybean seed protein, oil, fatty acids, N, and S partitioning as affected by node position and cultivar differences. Agric. Sci. 1, 110-118.

Bellaloui, N., Mengistu, A., and Paris, D. R. (2008). Soybean seed composition in cultivars differing in resistance to charcoal rot (Macrophomina phaseolina). J. Agric. Sci. 146, 667-675. doi: 10.1017/S0021859608007971

Bellaloui, N., Mengistu, A., Walker, E. R., and Young, L. D. (2014). Soybean seed composition as affected by seeding rates and row spacing. Crop Sci. 54, 1782-1795. doi: 10.2135/cropsci2013.07.0463

Bellaloui, N., Smith, J. R., Gillen, A. M., Fisher, D. K., and Mengistu, A. (2012). Effect of shade on seed protein, oil, fatty acids, and minerals in soybean lines varying 
in seed germinability in the early soybean production system. Am. J. Plant Sci. 3, 84-95. doi: 10.4236/ajps.2012.31008

Bellaloui, N., Smith, J. R., Gillen, A. M., and Ray, J. D. (2010). Effect of maturity on seed sugars as measured on near-isogenic soybean (Glycine max) lines. Crop Sci. 50, 1978-1987. doi: 10.2135/cropsci2009.10.0596

Bellaloui, N., Smith, J. R., Gillen, A. M., and Ray, J. D. (2011). Effects of maturity, genotypic background, and temperature on seed mineral composition in nearisogenic soybean lines in the early soybean production system. Crop Sci. 51 1161-1171. doi: 10.2135/cropsci2010.04.0187

Bharati, M. P. (1977). Density and environment interaction in soybeans: a review Nepalese. J. Agric. 12, 239-247.

Bouis, H. E. (2003). Micronutrient fortification of plants through plant breeding: can it improve nutrition in man at low cost? Proc. Nutr. Soc. 62, 403-411. doi 10.1079/PNS2003262

Boydak, E., Alpaslan, M., Hayta, M., Gerçek, S., and Simsek, M. (2002). Seed composition of soybeans grown in the Harran region of Turkey as affected by row spacing and irrigation. J. Agric. Food Chem. 50, 4718-4720. doi: 10.1021/jf02 55331

Bruns, H. A. (2011). Planting date, rate, and twin-row vs. single-row soybean in the Mid-South. Agron. J. 103, 1308-1313. doi: 10.2134/agronj2011.0076

Burton, J. W. (1991). Recent developments in breeding soy-beans for improved oil quality. Fat Sci. Tech. 93, 121-128.

Carver, B. F., Burton, J. W., Carter, T. E., and Wilson, R. F. (1986). Response to environmental variation of soybean lines selected for altered unsaturated fatty acid composition. Crop Sci. 26, 1176-1180. doi: 10.2135/cropsci1986.0011183X002600060021x

Cavell, A. J. (1955). The colorimetric determination of phosphorus in plant materials. J. Sci. Food Agric. 6, 479-480. doi: 10.1002/jsfa.2740060814

Chapman, G. W., Robertson, J. A., and Budrick, D. (1976). Chemical composition and lipoxygenase activity in soybean affected by genotype and environment. J. Am Oil Chem. Soc. 53, 54-56. doi: 10.1007/BF02637391

Cheesbrough, T. M. (1989). Changes in the enzymes for fatty acid synthesis and desaturation during acclimation of developing soybean seeds to altered growth temperature. Plant Physiol. 90, 760-764. doi: 10.1104/pp.90 2.760

Chy, W. S., and Sheldon, V. L. (1979). Soybean oil quality as influenced by planting site and variety. J. Am. Oil Chem. Soc. 56, 71-75. doi: 10.1007/BF02914271

Collins, F. I., and Cartter, J. L. (1956). Variability in chemical composition of seed from different portions of the soybean plant. Agron. J. 48, 216-219. doi: 10.2134/agronj1956.00021962004800050006x

Cooper, R. L. (1977). Response of soybean cultivars to narrow rows and planting rates under weed free conditions. Agron. J. 69, 89-92. doi: 10.2134/agronj1977.00021962006900010023x

Dardanelli, J. L., Balzarini, M., Martinez, M. J., Cuniberti, M., Resnik, S., Ramunda, S. F., et al. (2006). Soybean maturity groups, environments, and their interaction define mega-environments for seed composition in Argentina. Crop Sci. 46, 1939 1947. doi: 10.2135/cropsci2005.12-0480

Devirian, T. A., and Volpe, S. L. (2003). The physiological effects of dietary boron. Crit. Rev. Food Sci. Nutr. 43, 219-231. doi: 10.1080/10408690390826491

Dordas, C., Apostolides, G., and Goundra, O. (2007). Boron application affects seed yield and seed quality of sugarbeets. J. Agric. Sci. 145, 377-384. doi $10.1017 /$ S0021859607006879

Dornbos, D. L., and Mullen, R. E. (1992). Soybean seed protein and oil contents and fatty-acid composition adjustments by drought and temperature. J. Am. Oil Chem. Soc. 69, 228-231. doi: 10.1007/BF02635891

Ebelhar, M. W. (2010). Twin-row corn boosts yields. Corn \& Soybean Digest. Available at: http://cornandsoybeandigest.com/corn/twin-row-cornboosts-yields [accessed June 21, 2011].

Escalante, E. E., and Wilcox, J. R. (1993). Variation in seed protein among nodes of normal- and high-protein soybean genotypes. Crop Sci. 33, 1164-1166. doi: 10.2135/cropsci1993.0011183X003300060011x

Fehr, W. R., and Caviness, C. E. (1977). Stages of soybean development. Iowa Coop. Ext. Serv. Iowa Agric. Home Econ. Exp. Stn. Spec. Rep. 80:11.

Gubbels, G. H., and Dedio, W. (1986). Effect of plant density and soil fertility on oilseed sunflower genotypes. Can. J. Plant Sci. 66, 521-527. doi: 10.4141/cjps 86-071

Harue, T., and Hirokadzu, T. (1971). Influence of location on the chemical composition of soybean seeds. Proc. Crop Sci. Soc. Jpn. 40, 530-534. doi: $10.1626 /$ jcs. 40.530
Heatherly, L. (2014). Doublecropping in the Midsouth. Mississippi Soybean Promotion Board (MSPB). Available at: http://mssoy.org/blog/doublecropping-in-themidsouth/ [accessed July 10, 2014].

Heatherly, L. G. (1999). "Early soybean production system (ESPS)," in Soybean Production in the Midsouth, eds L. G. Heatherly, and H. F. Hodges (Boca Raton, FL: CRC Press), 103-118.

Heatherly, L. G., Blaine, A., Hodges, H. F., Wesley, R. A., and Buehring, N. (1999). "Variety selection, planting dates, row spacing, and seeding rate," in Soybean Production in the Midsouth, eds L. G. Heatherly and H. F. Hodges (New York: CRC Press), 41-47.

Helms, T. C., Hurburgh, C. R. Jr., Lussenden, R. L., and Whited, D. A. (1990). Economic analysis of increased protein and decreased yield due to delayed planting of soybean. J. Prod. Agric. 3, 367-371. doi: 10.2134/jpa1990.0367

Howell, R. W., and Collins, F. I. (1957). Factors affecting linolenic and linoleic acid content of soybean oil. Agron. J. 49, 593-597. doi: 10.2134/agronj1957.00021962004900110007x

Huskey, L. L., Snyder, H. E., and Gbur, E. E. (1990). Analysis of single soybean seeds for oil and protein. J. Am. Oil Chem. Soc. 67, 686-688. doi: 10.1007/BF02540423

Jaureguy, L. M., Rodriguez, F. L., Zhang, L., Chen, P., Brye, K., Oosterhuis, D., et al. (2013). Planting date and delayed harvest effects on soybean seed composition. Crop Sci. 53, 2162-2175. doi: 10.2135/cropsci2012.12.0683

John, M. K., Chuah, H. H., and Neufeld, J. H. (1975). Application of improved azomethine-H method to the determination of boron in soils and plants. Anal. Lett. 8, 559-568. doi: 10.1080/00032717508058240

Kane, M. V., Steele, C. C., Grabau, L. J., MacKown, C. T., and Hildebrand, D. F. (1997). Early- maturing soybean cropping system: III. Protein and oil contents and oil composition. Agron. J. 89, 464-469. doi: 10.2134/agronj1997.00021962008900030016x

Kochegura, A. V. (1982). Differences in seed quality within the plant. Selektsiya I Semenovodstovo USSR 9, 24-25.

Kondra, Z. P. (1975). Effects of row spacing and seeding rate on rapeseed. Can. J. Plant Sci. 55, 339-341. doi: 10.4141/cjps75-052

Lehrsch, G. A., Whislerb, F. D., and Buehringc, N. W. (1994). Cropping system influences on extractable water for mono- and double-cropped soybean. Agric. Water Manage. 26, 13-25. doi: 10.1016/0378-3774(94)9 0021-3

Liu, K. (1997). Soybeans Chemistry, Technology, and Utilization. New York: Chapman \& Hall.

Loeppert, R. L., and Inskeep, W. P. (1996). "Colorimetric determination of ferrous iron and ferric iron by the 1,10-phenanthroline method," in Methods of soil analysis: Part 3, Chemical methods, ed. J. M. Bigham (Madison, WI: SSSA), 659-661.

Lohse, G. (1982). Microanalytical azomethine-H method for boron determination in plant tissue. Commun. Soil Sci. Plant Anal. 13, 127-134. doi: $10.1080 / 00103628209367251$

Lu, K., Li, L., Zheng, X., Zhang, Z., Mou, T., and Hu, Z. (2008). Quantitative trait loci controlling $\mathrm{Cu}, \mathrm{Ca}, \mathrm{Zn}, \mathrm{Mn}$, and Fe content in rice grains. J. Genet. 87, 305-310. doi: 10.1007/s12041-008-0049-8

Mascagni, H. J. R., Clawson, E., Lanclos, D., Boquet, D., and Ferguson, R. (2008). Comparing Single-Row, Twin-Row Configurations for Louisiana crop Production. LSU, Ag Center, Research \& Extension. Available at: http://text.lsuagcenter.com/en/communications/publications/agmag/Archive/ 2008/Summer/Comparing+SingleRow+TwinRow+Configurations+for+Louis iana+Crop+Production.htm [accessed July 13, 2014].

Mengistu, A., Castlebury, L. A., Smith, J. R., Rossman, A. Y., and Reddy, K. N. (2007). Identification and pathogenicity of Phomopsis isolates from weed hosts and their effect on soybean. Can. J. Plant Pathol. 29, 283-289. doi: 10.1080/07060660709507471

Mengistu, A., and Heatherly, L. G. (2006). Planting date, irrigation, maturity group, year, and environment effects on Phomopsis longicolla, seed germination, and seed health rating of soybean in the early soybean production system of the mid-southern USA. Crop Prot. 25, 310-317. doi: 10.1016/j.cropro.2005. 05.01

Minor, H. C., and Wiebold, W. J. (1998). Soybean-wheat double crop management in Missouri. MU Extension, University of Missouri-Columbia. Available at: http://extension.missouri.edu/p/G4953 [accessed July 10, 2014].

Mousavi, S. J., Sam-Daliri, M., and Bagheri, H. (2011). Study of planting density on some agronomic traits of rapeseed three cultivar (Brassica napus L.). Aust. J. Basic Appl. Sci. 5, 2625-2627. 
MSUCares. (2014). Mississippi State University, Extension service. Available at: http://ext.msstate.edu/anr/drec/weather.cgi [accessed August 20, 2014].

Obendorf, R. L. (1997). Oligosaccharides and galactosyl cyclitols in seed desiccation tolerance. Seed Sci. Res. 7, 63-74. doi: 10.1017/S09602585000 $0341 \mathrm{X}$

Pedersen, P., and Lauer, J. G. (2004). Response of soybean yield components to management system and planting date. Agron. J. 96, 1372-1381. doi: 10.2134/agronj2004.1372

Piper, E. L., and Boote, K. J. (1999). Temperature and cultivar effects on soybean seed oil and protein concentrations. J. Am. Oil Chem. Soc. 76, 1233-1242. doi: 10.1007/s11746-999-0099-y

Ray, J. D., Heatherly, L. G., and Fritschi, F. B. (2006). Influence of large amounts of nitrogen on non-irrigated and irrigated soybean. Crop Sci. 46, 52-60. doi: $10.2135 /$ cropsci2005.0043

Ren, C., Bilyeu, K. D., and Beuselinck, P. R. (2009). Composition, vigor, and proteome of mature soybean seeds developed under high temperature. Crop Sci. 49, 1010-1022. doi: 10.2135/cropsci2008.05.0247

Samman, S., Naghii, M. R., Lyons Wall, P. M., and Verus, A. P. (1998). The nutritional and metabolic effects of boron in humans and animals. Biol. Trace Elem. Res. 66, 227-235. doi: 10.1007/BF02783140

Schnebly, S. R., and Fehr, W. R. (1993). Effect of years and planting dates on fatty acid composition of soybean genotypes. Crop Sci. 33, 716-719. doi: 10.2135/cropsci1993.0011183X003300040016x

Shibles, R. M., and Weber, C. R. (1965). Leaf area, solar radiation interception and dry matter production by soybeans. Crop Sci. 5, 575-577. doi: 10.2135/cropsci1965.0011183X000500060027x

Shibles, R. M., and Weber, C. R. (1966). Interception of solar radiation and dry matter production by various soybean planting patterns. Crop Sci. 6, 55-59. doi: 10.2135/cropsci1966.0011183X000600010017x

Smith, J. R., Mengistu, A., Nelson, R. L., and Paris, R. L. (2008). Identification of soybean accessions with high germinability in high-temperature environments. Crop Sci. 48, 2279-2288. doi: 10.2135/cropsci2008.01.0026

Tang, G. O., Novitzky, W. P., Griffin, H. C., Huber, S. C., and Dewey, R. E. (2005). Oleate desaturase enzymes of soybean: evidence of regulation through differential stability and phosphorylation. Plant J. 44, 433-446. doi: 10.1111/j.1365-313X.2005.02535.x
Taylor, H. M., Mason, W. K., Bennie, A. T. P., and Rowse, H. R. (1982). Responses of soybeans to two row spacings and two soil water levels. I. An analysis of biomass accumulation, canopy development, solar radiation interception and components of seed yield. Field Crop Res. 5, 1-14. doi: 10.1016/0378-4290(82)90002-8

Welch, R. M., and Graham, R. D. (2004). Breeding for micronutrients in staple food crops from a human nutrition perspective. J. Exp. Bot. 55, 353-364. doi: 10.1093/jxb/erh064

White, P. J., and Broadley, M. R. (2009). Biofortification of crops with seven mineral elements often lacking in human diets - iron, zinc, copper, calcium, magnesium, selenium, and iodine. New Phytol. 182, 49-84. doi: 10.1111/j.1469-8137.2008.02738.x

Wilcox, J. R., and Cavins, J. F. (1992). Normal and low linolenic acid soybean strains: response to planting date. Crop Sci. 32, 1248-1251. doi: 10.2135/cropsci1992.0011183X003200050037x

Wilcox, J. R., and Cavins, J. F. (1995). Backcrossing high seed protein to a soybean cultivar. Crop Sci. 35, 1036-1041. doi: 10.2135/cropsci1995.0011183X003500040019x

Wilcox, J. R., and Shibles, R. M. (2001). Interrelationships among seed quality attributes in soybean. Crop Sci. 41, 11-14. doi: 10.2135/cropsci2001.41111x

Conflict of Interest Statement: The authors declare that the research was conducted in the absence of any commercial or financial relationships that could be construed as a potential conflict of interest.

Received: 21 November 2014; accepted: 13 January 2015; published online: 18 February 2015.

Citation: Bellaloui N, Bruns HA, Abbas HK, Mengistu A, Fisher DK and Reddy KN (2015) Agricultural practices altered soybean seed protein, oil, fatty acids, sugars, and minerals in the Midsouth USA. Front. Plant Sci. 6:31. doi: 10.3389/fpls.2015.00031

This article was submitted to Crop Science and Horticulture, a section of the journal Frontiers in Plant Science.

Copyright (C) 2015 Bellaloui, Bruns, Abbas, Mengistu, Fisher and Reddy. This is an open-access article distributed under the terms of the Creative Commons Attribution License (CC BY). The use, distribution or reproduction in other forums is permitted, provided the original author(s) or licensor are credited and that the original publication in this journal is cited, in accordance with accepted academic practice. No use, distribution or reproduction is permitted which does not comply with these terms. 


\section{OPEN ACCESS}

Edited by:

Antonio M. De Ron, Spanish National Research Council, Misión Biológica de Galicia - CSIC,

Spain

Reviewed by:

Carissa M. Soto

Naval Research Laboratory, USA

Ousmane Boukar,

International Institute of Tropical

Agriculture (IITA), Nigeria

Ana Campa,

Servicio Regional de Investigación y

Desarrollo Agroalimentario, Spain Deidre Fourie,

ARC-Grain Crops Institute,

South Africa

*Correspondence: Imade Y. Nsa,

Department of Microbiology, University of Lagos, Akoka, Yaba, Lagos, Nigeria imadee@hotmail.com: insa@unilag.edu.ng

Specialty section: This article was submitted to Crop Science and Horticulture, a section of the journal Frontiers in Plant Science

Received: 22 June 2015 Accepted: 17 September 2015

Published: 01 October 2015

Citation:

Nsa IY and Kareem KT (2015) Additive interactions of unrelated viruses in mixed infections of cowpea Nigna unguiculata L. Walp).

Front. Plant Sci. 6:812.

doi: 10.3389/fp/s.2015.00812

\section{Additive interactions of unrelated viruses in mixed infections of cowpea (Vigna unguiculata L. Walp)}

\author{
Imade $\mathrm{Y} . \mathrm{Nsa}^{1 *}$ and Kehinde T. Kareem ${ }^{2}$ \\ ${ }^{1}$ Department of Microbiology, University of Lagos, Lagos, Nigeria, ${ }^{2}$ Institute of Agricultural Research and Training, Moor \\ Plantation, Obafemi Awolowo University, Ibadan, Nigeria
}

This study was carried out to determine the effects of single infections and co-infections of three unrelated viruses on three cowpea cultivars (one commercial cowpea cultivar "White" and 2 IITA lines; IT81D-985 and TVu 76). The plants were inoculated with Cowpea aphid-borne mosaic virus (CABMV), genus Potyvirus, Cowpea mottle virus (CMeV), genus Carmovirus and Southern bean mosaic virus (SBMV), genus Sobemovirus singly and in mixture (double and triple) at 10, 20, and 30 days after planting (DAP). The treated plants were assessed for susceptibility to the viruses, growth, and yield. In all cases of infection, early inoculation resulted in higher disease severity compared with late infection. The virus treated cowpea plants were relatively shorter than buffer inoculated control plants except the IT81D-985 plants that were taller and produced more foliage. Single infections by CABMV, CMeV, and SBMV led to a complete loss of seeds in the three cowpea cultivars at 10 DAP; only cultivar White produced some seeds at 30 DAP. Double and triple virus infections led to a total loss of seeds in all three cowpea cultivars. None of the virus infected IITA lines produced any seeds except IT81D-985 plants co-infected with CABMV and SBMV at 30 DAP with a reduction of $80 \%$. Overall, the commercial cultivar "White" was the least susceptible to the virus treatments and produced the most yield (flowers, pods, and seeds). CABMV was the most aggressive of these viruses and early single inoculations with this virus resulted in the premature death of some of the seedlings. The presence of the Potyvirus, CABMV in the double virus infections did not appear to increase disease severity or yield loss. There was no strong evidence for synergistic interactions between the viruses in the double virus mixtures.

Keywords: cowpea, severity, virus, yield, interaction

\section{Introduction}

Cowpea (Vigna unguiculata (L). Walp), accounts for a huge portion of the dietary protein of the people in sub-Saharan Africa (Li et al., 2001). Nigeria ranks first in cowpea production worldwide, and is responsible for about $61 \%$ of total world production of cowpea (IITA, 2009). Historically, cowpeas are known to produce a more satisfactory yield than most leguminous plants under a variety of climatic, soil, and cultural conditions. However, they are very susceptible to insect pests (Lephale et al., 2012) and many virus diseases (Karungi et al., 2000) that reduce yields. More than 140 viruses have been identified as naturally infecting cowpea (Hughes and Shoyinka, 2003) but only nine have been reported in Nigeria (Taiwo, 2003), and these are Cowpea aphid-borne mosaic 
virus (CABMV), Cowpea golden mosaic virus (CPGMV), Southern bean mosaic virus (SBMV), Sunhemp mosaic virus (SHMV), Blackeye mosaic virus (BICMV), Cucumber mosaic virus (CMV), Cowpea mottle virus $(\mathrm{CMeV})$, Cowpea yellow mosaic virus (CPMV), and Cowpea mild mottle virus (CPMMV).

CABMV was first reported and described as a distinct Potyvirus infecting cowpea in Italy (Vidano and Conti, 1965; Lovisolo and Conti, 1966; Bashir et al., 2002), then in Nigeria in 1976 (Ladipo, 1976). It is of widespread in distribution in Nigeria and in other major cowpea growing regions of the world. It has also been reported from several African countries including, Kenya (Bock, 1973), Tanzania (Patel and Kuwite, 1982), Botswana (Burke et al., 1986), Uganda (Orawu et al., 2005). CABMV is the most important viral pathogen of cowpea in Nigeria, as far as pathogenic variation and yield losses are concerned (Raheja and Leleji, 1974; Thottappilly and Rossel, 1992; Shoyinka et al., 1997). It is readily transmissible by sap inoculation and by several aphids in a non-persistent manner (Vidano and Conti, 1965; Atiri, 1982; Atiri et al., 1984). It is also seed-borne in cowpea, but transmission is dependent upon cowpea cultivar and virus strain (Aboul-Ata et al., 1982; Gumedzoe, 1985). It has a wide experimental host range including many species in the Leguminosae, Chenopodiaceae, Curcubitaceae, Solanaceae, and Amaranthaceae (Lovisolo and Conti, 1966; Bos, 1970; Bock, 1973). Virus may induce local lesions or systemic infections depending on the host, infected plants show dark green vein-banding, leaf deformation, crinkling, blistering, and stunting (Bock and Conti, 1974). However, the severity of the symptoms depends on the interaction between the host cultivar and virus strain (Rossel and Thottappilly, 1985).

Field occurrence of SBMV on cowpea has also been reported from the world, U.S.A. (Kuhn, 1963), India (Singh and Singh, 1974), Ghana (Lamptey and Hamilton, 1974), Ivory Coast (Fauquet and Thouvenel, 1980), Senegal (Gaikward and Thottappilly, 1988), Togo (Gumedzoe et al., 1989), and Nigeria (Shoyinka et al., 1979). The systemic symptoms induced by SBMV include vein clearing, followed by a mild to severe mottling or coarse mosaic pattern (Shepherd and Fulton, 1962; Allen et al., 1981). It is transmissible by inoculation of sap, beetle, and is seed borne (Tremaine and Hamilton, 1983).

$\mathrm{CMeV}$ was first described from Nigeria, where it was isolated from Bambarra groundnut (Vigna subterranea) and cowpea, V. unguiculata (Robertson, 1963, 1966). In Nigeria, $\mathrm{CMeV}$ is commonly found in the southern rainforest and guinea savannah zones where most of the Bambara groundnut is grown (Rossel and Thottappilly, 1985). It has also been reported from other West African countries, Ivory Coast (Thouvenel, 1988), Togo (Gumedzoe et al., 1989), and the Republic of Benin (Thottappilly and Rossel, 1988). In tolerant cowpea varieties, the symptom of this virus consists basically of mottling; whereas in severe infections, $\mathrm{CMeV}$ may induce leaf distortion, reduction in leaf size and witches broom syndrome. $\mathrm{CMeV}$ is transmitted principally by a chrysomelid beetle vector, Ootheca mutabilis (Shoyinka et al., 1978) and by sap inoculation.

In Nigeria, there have been reports of single and multiple (double and triple) virus infections of cultivated cowpeas resulting in complete yield loss (Raheja and Leleji, 1974; Kareem and Taiwo, 2007; Taiwo et al., 2007). Mixed virus infection of plants often results in intensified symptom severity than those caused by each individual virus, and higher virus accumulation, a phenomenon referred to as synergy (Wang et al., 2002; Untiveros et al., 2007). Synergism occurs in mixed infections when a pair of the viruses is unrelated, with the overall effect of the viruses eliciting more severe disease symptoms in the host plant than those produced by each of the viruses separately (Cho et al., 2000; Murphy and Bowen, 2006). Examples of synergistic viral diseases have been recorded for many decades (Shi et al., 1996). The severity of the disease symptoms is also greatly increased if one of the infecting viruses is a member of the genus Potyvirus (Pio-Ribeiro et al., 1978). Nevertheless, not all combinations of unrelated viruses result in increased symptoms (Anjos et al., 1992). Shoyinka et al. (1978) reported that $\mathrm{CMeV}$ frequently occurred in mixed infections with Cowpea yellow mosaic virus (CYMV) or SBMV in cowpea fields located in southwestern Nigeria.

One method of detecting viruses is by biological properties (Naidu and Hughes, 2003). In this study, two International Institute of Tropical Agriculture (IITA) cowpea lines (IT81D985, TVu 76) and a local commercial cultivar (White) were evaluated for symptom severity, growth defects, and yield losses as a result of single and mixed infections of $\mathrm{CABMV}, \mathrm{CMeV}$, and SBMV. We also ascertained whether the qualitative observation of increased symptom severity, reductions in growth and yield presumably attributed to synergistic interactions of the viruses could be further validated by quantitative methods. Serological tests were done to determine relative virus concentration.

\section{Materials and Methods}

\section{Source and Cultivation of Cowpea Lines/Cultivars}

The cowpea lines, TVu 76 and IT 81D-985 used in this study were obtained from the Germplasm Resource Unit of the International Institute of Tropical Agriculture (IITA), Ibadan and the commercial "White" cultivar was purchased from Sandgrouse Market, Lagos. The IT81D-985 is a medium-late maturing semi spreading cowpea line (Singh, 2011) and line TVu 76 is known to be susceptible to leafhoppers (Raman et al., 1980). The cowpea lines/cultivars were cultivated as described by Kareem and Taiwo (2007).

\section{Source and Maintenance of Viruses}

The viruses used in this research were Cowpea aphid-borne mosaic virus genus, Potyvirus; Southern bean mosaic virus, genus Sobemovirus; and Cowpea mottle virus, genus Carmovirus. The viruses were obtained from infected cowpea plants or tissue stored over calcium chloride at the IITA Ibadan. These viruses were maintained on the Ife Brown variety in the Greenhouse of the Botanical Garden of University of Lagos (Kareem and Taiwo, 2007).

\section{Inoculation of Cowpea Cultivars with Viruses}

Seedlings were mechanically inoculated at 10, 20, and 30 days after planting. Each pot was labeled according to the type of virus treatment, including negative controls, name of cultivar and 
date of inoculation. Test plants were dusted with carborundum (180 mesh) to increase entry of the virus into the hosts. Single virus inoculum was prepared by grinding virus-infected leaves with a sterile mortar and pestle at the rate of $1 \mathrm{~g}$ of tissue to $2 \mathrm{ml}$ of buffer. However, mixed viral treatments were obtained by mixing saps from the relevant inocula at ratio $1: 1(\mathrm{~V} / \mathrm{V})$ just before inoculation. The pestle was used to rub sap over the upper leaf surface from base to the top of the leaf. After inoculation, the leaves were rinsed immediately with water to prevent the harmful effects of the sap. Inoculated plants were kept in the University of Lagos Greenhouse. The different treatments are listed below.

a. Single virus inoculation

Each of the cultivars: TVu 76, IT81D-985 and commercial cultivar "White" was inoculated singly with CABMV, CMeV, or SBMV.

b. Double virus inoculation

Each of the three cowpea lines/cultivar was inoculated with double virus mixtures i.e., CABMV + $\mathrm{CMeV}, \mathrm{CABMV}+$ SBMV, and $\mathrm{CMeV}+\mathrm{SBMV}$.

c. Triple virus inoculation

Each of the three cowpea lines was also inoculated with a combination of the three viruses, i.e., CABMV + SBMV + $\mathrm{CMeV}$.

d. Mock inoculation with buffer

Control plants of each of the cowpea lines/cultivars were inoculated with buffer only. The buffer was prepared by dissolving $17.4 \mathrm{~g}$ of di-potassium hydrogen orthophosphate $\left(\mathrm{K}_{2} \mathrm{HPO}_{4}\right)$ in one liter (1l) distilled water ( $0.1 \mathrm{M}$ solution); $0.1 \mathrm{M}$ potassium di-hydrogen orthophosphate $\left(\mathrm{KH}_{2} \mathrm{PO}_{4}\right)$ was added (3.4 $\mathrm{g}$ of $\mathrm{KH}_{2} \mathrm{PO}_{4}$ dissolved in $250 \mathrm{ml}$ ) to achieve a $\mathrm{pH}$ 7.5 (Walkey, 1985). The working concentration was $0.05 \mathrm{M}$.

\section{Inoculation Procedure}

\section{a. First stage inoculation}

The first inoculation was done 10 days after planting (DAP). The sap from the different virus treatments above or buffer was rubbed onto the plant surface of the fully expanded leaves of each of the three cultivars. Symptoms were observed at 10 , 20, and 30 days after inoculation (DAI).

b. Second stage inoculation

The second inoculation was done 20 DAP. The sap from the different virus treatments or buffer was rubbed on the leaves of each of the three cultivars. Symptoms were observed at 10, 20 , and 30 DAI.

\section{c. Third stage inoculation}

The second inoculation was carried out 30 days after planting. Symptoms were observed at 10, 20, and 30 DAI. After each inoculation, plants were rinsed with water to reduce the effect of caborundum on leaves that may interfere with photosynthesis. Plants were kept in the greenhouse under ambient temperatures ranging from 25 to $28^{\circ} \mathrm{C}$, watered every other day, and observed for symptom development.

\section{Experimental Design}

The experimental design used in the greenhouse was randomized complete block design (RCBD). There were three cowpea lines/cultivars, three replicates, three different stages of virus inoculation and eight virus treatments including the negative control $(3 \times 3 \times 3 \times 8)$ making a total of 216 pots.

\section{Effect of Virus Treatments on Disease Severity}

The reaction of the cultivars to mechanical inoculation with the viruses (single and multiple) and buffer was determined by rating inoculated cowpea plants based on symptom development at 10 , 20 , and $30 \mathrm{DAI}$. The rating was done on a scale of 1 to 5 , where 1, no symptoms; (resistant) 2, slight mosaic/mottle on leaves (susceptible); 3, moderate mosaic/mottle/blistering/curling of leaves (susceptible); 4, severe mosaic/mottle/blistering, leaf reduction (susceptible); 5, very severe symptoms leading to stunting, apical necrosis, and plant death.

\section{Effects of Virus Treatments on Growth Traits}

The effect of virus treatments and buffer on the plant height, number of leaves of White, IT81D-985 and TVu 76, was determined at 10 DAP 10 DAI and 30 DAP 10 DAI. The plant height was measured with a ruler in $\mathrm{cm}$. and the leaves were counted. The percentage reduction in plant height, and number of leaves in virus-infected plants was calculated as:

$\%$ reduction in plant height $=\left[\left(\mathrm{H}_{\mathrm{B}}-\mathrm{H}_{\mathrm{V}}\right) / \mathrm{H}_{\mathrm{B}}\right] \times 100$

Where:

$\mathrm{H}_{\mathrm{B}}=$ plant height of buffer inoculated plant

$\mathrm{H}_{\mathrm{V}}=$ plant height of virus inoculated plant

$\%$ reduction in leaf number $=\left[\left(\mathrm{L}_{\mathrm{B}}-\mathrm{L}_{\mathrm{V}}\right) / \mathrm{L}_{\mathrm{B}}\right] \times 100$

Where:

$\mathrm{L}_{\mathrm{B}}=$ number of leaves of buffer inoculated plant

$\mathrm{LV}_{\mathrm{V}}=$ number of leaves of virus inoculated plant

\section{Effect of Virus Treatments on Yield Parameters of the Cowpea Varieties}

The effect of virus treatments and buffer on the number of flowers, number of pods, pod length, seed number, and seed weight was determined. Pod length was measured with a ruler and the seeds were weighed on a Mettler -Toledo balance (Columbus, OH, U.S.A).

\section{Determination of the Type of Biological Response among the Viruses}

To determine the type of biological interactions existing between the viruses in double co-infections, we compared the growth traits of cowpea plants exposed to double virus treatments to the single virus inoculated plants. We calculated the degree of interactions of the viruses in the mixed virus treatments using the Abbott's formula (Abbott, 1925; Gisi, 1996).

$$
\mathrm{Cexp}=\mathrm{A}+\mathrm{B}-(\mathrm{AB} / 100)
$$

Where Cexp is the expected level of disease, and A and B are corresponding responses due to infection by virus $\mathrm{A}$ and $\mathrm{B}$, respectively, as observed in the experiment, $\mathrm{AB}$ is the response of the double virus infection (Murphy and Bowen, 2006). This was 
done for all the double infections, $\mathrm{CABMV}+\mathrm{CMeV}, \mathrm{CABMV}+$ $\mathrm{SBMV}$, and $\mathrm{CMeV}+\mathrm{SBMV}$ of the three cowpea varieties under investigation.

The means of the different growth traits were used to quantitatively determine the degree of the observed reduction. Plant height and leaf number were the two traits considered. Rather than using the strict mathematical definition of a synergistic interaction as the ratio of the observed response (Cobs) to the expected response (Cexp) if greater than 1.0, additive, if equal to 1 and antagonistic, if less than 1 . We adopted the cut offs by Gisi et al. (1985), where a biological response can be categorized as follows: (1) synergistic, if the interaction of the ratio of the observed response (Cobs) to the expected response (Cexp) is greater than $1.5,(2)$ additive, between 0.5 and 1.5 , and (3) antagonistic, less than 0.5 .

\section{Data Analysis}

All the data obtained were analyzed using Statistical Package for Social Scientists (SPSS) version 16.0 and Duncan Multiple Range Test was used to compare means obtained at 5\% level of probability (Little and Hills, 1972).

\section{Effect of Virus Treatment on Relative Virus Concentration}

Leaf samples from cultivar White, IT81D-985 and TVu 76 that received the different treatments were taken at 10 and 30 days after inoculation at the different stages of growth to determine virus concentration. The relative concentration of virus in single and mixed infections was determined serologically with antigen coated plate enzyme linked immunosorbent assay (ACP ELISA). The ELISA protocol was carried out as described as Taiwo et al. (2007).

\section{Results}

\section{Symptomatology}

The three cultivars/lines were susceptible to the three viruses used in this investigation. The inoculated leaves were observed for symptom development at different stages of growth after inoculation and recorded. The symptoms observed at 20 DAP were not intermediary or significantly different from those observed at 30 DAP (data not shown).

\section{Symptom Severity of Virus Treatments on Cowpea (White)}

The mixture of the triple viruses, $\mathrm{CABMV}+\mathrm{CMeV}+\mathrm{SBMV}$ induced the most symptoms. Its common symptoms were mosaic, mottling and especially apical necrosis that eventually led to plant death (Figure 1A). The triple virus treatments caused death at all stages of plant growth regardless of the time of inoculation. As for the double virus infections, only early inoculations (10 DAP) resulted in a severity score of 5 indicating that the plants died due to early infection (Table 1). Leaf deformation was observed in the mixture of CABMV and $\mathrm{CMeV}$ (Figure 1B). No symptoms were observed on the leaves of the buffer inoculated plants (Figure 1C).

For the single viruses, SBMV induced mostly mosaic and leaf curling symptoms but did not cause the death of any plants (Figure 1D). CABMV was the most aggressive of the viruses in this variety. Early inoculations with this virus resulted in premature death of some of the seedlings giving a severity score of 5 (Table 1). Its common symptoms included mosaic, vein banding, chlorosis, blistering (Figure 1E) and stunting. Whereas, single infections by $\mathrm{CMeV}$, induced mostly mottling and defoliation. The leaves of the buffer inoculated plants/controls
A

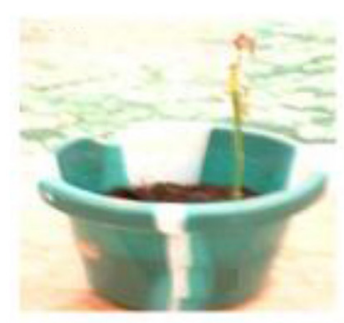

D

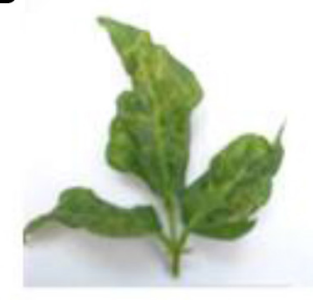

B

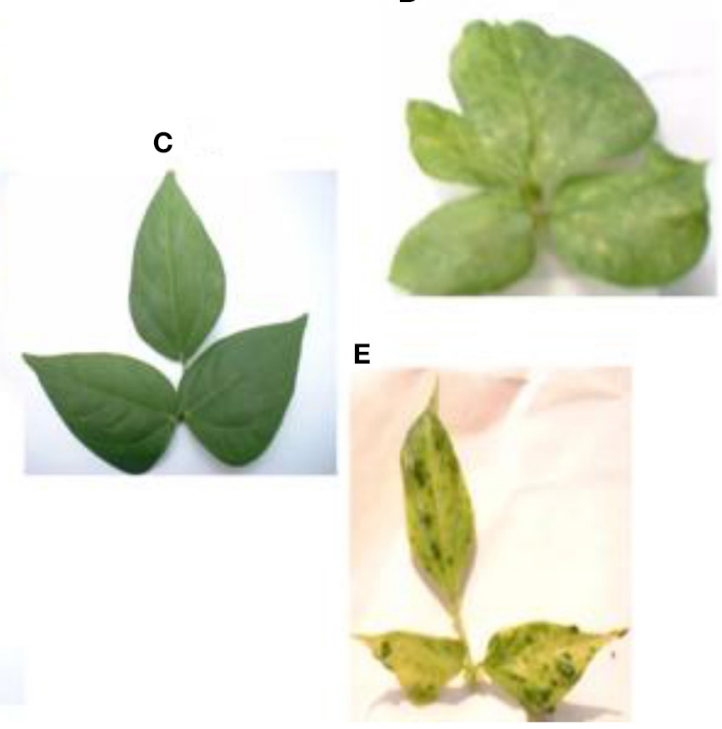

FIGURE 1 | Viral symptoms induced on the local commercial cultivar White. (A) Apical necrosis and defoliation induced by triple virus treatment, CABMV + $\mathrm{CMeV}+\mathrm{SBMV}$ that eventually led to plant death. (B) Leaf deformation and mosaic symptoms induced by double virus infection, CABMV + CMeV. (C) Buffer inoculated healthy fully expanded trifoliate leaves. (D) Mottling, leaf reduction and crinkling induced by SBMV. (E) Blistering and leaf chlorosis induced by CABMV. 
TABLE 1 | Severity of single and multiple virus treatments on cowpea varieties.

\begin{tabular}{|c|c|c|c|c|c|c|c|c|c|c|}
\hline \multirow[t]{2}{*}{ Virus treatment } & \multirow[t]{2}{*}{ DAP $^{x}$} & \multicolumn{3}{|c|}{$10^{y}$} & \multicolumn{3}{|c|}{$20^{y}$} & \multicolumn{3}{|c|}{$30^{y}$} \\
\hline & & White & IT81D-985 & TVu 76 & White & IT81D-985 & TVu 76 & White & IT81D-985 & TVu 76 \\
\hline \multirow[t]{2}{*}{ CABMV } & 10 & $3^{C}$ & $3^{c}$ & $4^{d}$ & $2.5^{\mathrm{bc}}$ & $5^{e}$ & $3^{c}$ & $5^{e}$ & $5^{e}$ & $4^{d}$ \\
\hline & 30 & $2^{b}$ & $2^{b}$ & $1^{\mathrm{a}}$ & $2^{b}$ & $3^{c}$ & $1^{a}$ & $2.5^{\mathrm{bc}}$ & $4^{d}$ & $2^{b}$ \\
\hline \multirow[t]{2}{*}{$\mathrm{CMeV}$} & 10 & $2^{b}$ & $2^{b}$ & $4.5^{\mathrm{de}}$ & $3.5^{\mathrm{cd}}$ & $2^{b}$ & $3.5^{\mathrm{cd}}$ & $3.5^{\mathrm{cd}}$ & $4^{d}$ & $4.5^{\mathrm{de}}$ \\
\hline & 30 & $1^{\mathrm{a}}$ & $2^{b}$ & $2^{b}$ & $2.5^{\mathrm{bc}}$ & $2^{b}$ & $2^{b}$ & $3.5^{\mathrm{cd}}$ & $3.5^{\mathrm{cd}}$ & $4.5^{\text {de }}$ \\
\hline \multirow[t]{2}{*}{ SBMV } & 10 & $2.5^{\mathrm{bc}}$ & $2^{b}$ & $2^{b}$ & $3^{c}$ & $2^{b}$ & $4^{d}$ & $3.5^{\mathrm{cd}}$ & $5^{e}$ & $4^{d}$ \\
\hline & 30 & $2^{b}$ & $2^{b}$ & $2^{b}$ & $2^{b}$ & $2^{b}$ & $2^{b}$ & $3^{c}$ & $2^{b}$ & $2^{b}$ \\
\hline \multirow[t]{2}{*}{$\mathrm{CABMV}+\mathrm{CMeV}$} & 10 & $2.5^{\mathrm{bc}}$ & $4^{d}$ & $4.5^{\mathrm{cd}}$ & $5^{e}$ & $2^{b}$ & $5^{e}$ & $5^{e}$ & $5^{e}$ & $5^{e}$ \\
\hline & 30 & $2^{b}$ & $2^{b}$ & $2^{b}$ & $2^{b}$ & $2^{b}$ & $5^{e}$ & $5^{\mathrm{e}}$ & $4^{d}$ & $5^{e}$ \\
\hline \multirow[t]{2}{*}{ CABMV + SBMV } & 10 & $2.5^{\mathrm{bc}}$ & $3.5^{\mathrm{cd}}$ & $4^{d}$ & $5^{e}$ & $3.5^{\mathrm{cd}}$ & $5^{e}$ & $5^{e}$ & $3.5^{\mathrm{cd}}$ & $5^{e}$ \\
\hline & 30 & $2^{b}$ & $3^{c}$ & $2^{b}$ & $2^{b}$ & $3^{c}$ & $4^{d}$ & $2.5^{\mathrm{bc}}$ & $3.5^{\mathrm{cd}}$ & $5^{e}$ \\
\hline \multirow[t]{2}{*}{$\mathrm{CMeV}+\mathrm{SBMV}$} & 10 & $2.5^{\mathrm{bc}}$ & $2^{b}$ & $3.5^{\mathrm{cd}}$ & $5^{e}$ & $3^{c}$ & $4.5^{\mathrm{de}}$ & $5^{e}$ & $5^{e}$ & $5^{e}$ \\
\hline & 30 & $2^{b}$ & $2^{b}$ & $2^{b}$ & $2^{b}$ & $2^{b}$ & $2^{b}$ & $2.5^{\mathrm{bc}}$ & $3^{c}$ & $3^{c}$ \\
\hline \multirow[t]{2}{*}{$\mathrm{CABMV}+\mathrm{CMeV}+\mathrm{SBMV}$} & 10 & $4.5^{\text {de }}$ & $4.5^{\text {de }}$ & $4^{d}$ & $4.5^{\mathrm{de}}$ & $3.5^{\mathrm{cd}}$ & $5^{e}$ & $5^{e}$ & $5^{\mathrm{e}}$ & $5^{\mathrm{e}}$ \\
\hline & 30 & $2^{b}$ & $2^{b}$ & $2^{b}$ & $2^{b}$ & $2^{b}$ & $3^{c}$ & $5^{e}$ & $5^{\mathrm{e}}$ & $3.5^{\mathrm{cd}}$ \\
\hline \multirow[t]{2}{*}{ BUFFER (negative control) } & 10 & $1^{\mathrm{a}}$ & $1^{\mathrm{a}}$ & $1^{\mathrm{a}}$ & $1^{\mathrm{a}}$ & $1^{\mathrm{a}}$ & $1^{a}$ & $1^{a}$ & $1^{\mathrm{a}}$ & $1^{a}$ \\
\hline & 30 & $1^{\mathrm{a}}$ & $1^{\mathrm{a}}$ & $1^{\mathrm{a}}$ & $1^{a}$ & $1^{a}$ & $1^{a}$ & $1^{a}$ & $1^{a}$ & $1^{a}$ \\
\hline
\end{tabular}

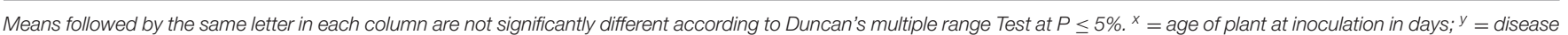

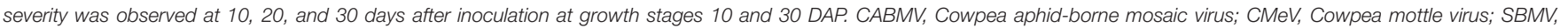
Southern bean mosaic virus.

were healthy, remained fully expanded and symptomless; the other plant parts also showed no disease symptoms.

\section{Symptom Severity of Virus Treatments on Line IT81D-985}

The most severe symptom induced by the triple virus infection of CABMV + CMeV + SBMV on IT81D-985 was apical necrosis. The symptoms induced on its primary leaves were reddish necrotic lesions. Double virus treatments involving CABMV + $\mathrm{CMeV}$ and $\mathrm{CMeV}+\mathrm{SBMV}$ led to the maximum severity score of 5 at 10 DAP, while early inoculation with CABMV + SBMV resulted in the highest severity score of 3.5 (Table 2).

In this variety, single inoculations with $\mathrm{CABMV}$ or $\mathrm{CMeV}$ caused death in some of the plants at 10 and 30 DAP, respectively. CABMV induced vein- banding, mosaic, internode shortening, chlorotic lesions and apical necrosis. In addition to the characteristic mottling of $\mathrm{CMeV}$ (Figure 2), reduction in size of leaves, defoliation, and apical necrosis were also observed. The general symptoms induced by SBMV on this line included mosaic, leaf reduction, and defoliation. Inoculations with each of the double virus mixtures caused the death of some of the plants. While early inoculation with $\mathrm{CMeV}+\mathrm{SBMV}$ at $10 \mathrm{DAP}$ resulted in the death of some plants, as well as severe mottling among other symptoms. Single inoculation with either CABMV or SBMV produced a severity score of 5 that caused death in the plants at 10 DAP 10 DAI and 10 DAP 30 DAI. The severity score of 2 was observed in the early- inoculated $\mathrm{CMeV}$ plants, severity worsened to a score of 4 at 10 DAP 30 DAI. Mottling was observed on the leaves of cowpea inoculated with $\mathrm{CMeV}$ (Figure 2A) but not death. The symptoms induced on its primary leaves were reddish necrotic lesions but like in the "White" variety, CABMV also induced chlorotic lesions.

\section{Symptom Severity of Virus Treatments on Cowpea (TVu 76)}

As observed in "White" and IT81D-985 varieties, triple infections resulted in chronic symptoms, most especially apical necrosis which eventually led to the death of some of the TVu 76 plants. Generally, all the virus treatments (whether single, double, or triple) induced defoliation, while the buffer inoculated plants remained healthy and symptomless.

Severity scores in plants with single virus treatments were significantly reduced at $30 \mathrm{DAP}$ compared with $10 \mathrm{DAP}$. TVu 76 plants inoculated with CABMV only at $30 \mathrm{DAP}$, were tolerant at the initial stage (10 DAI) of infection but later showed mild symptoms with a score of 2 at 30 DAI. The most common symptom caused by CABMV was internode shortening. Single infections by $\mathrm{CMeV}$ induced the most symptoms including mottling and apical necrosis, while SBMV induced basal necrosis and leaf curling among other symptoms. The severity scores of CABMV + CMeV and CABMV + SBMV at $10 \mathrm{DAP}$ were high (between 4 and 5) causing death of some plants (Table 1). At $30 \mathrm{DAP}, \mathrm{CMeV}+\mathrm{SBMV}$ induced less symptoms than CABMV + CMeV and CABMV + SBMV treatments and did not cause plant death. The buffer inoculated plants had a score of 1 implying that the plants remained healthy and symptomless.

\section{CABMV was the Most Aggressive of Single Virus Infections in the White Cultivar but not in Line TVu 76}

To determine the effects of single virus treatments to the different cowpea, we exposed each cowpea variety to single treatments of the 3 viruses-CABMV, CMeV, and SBMV. Generally, the entire virus treatments at 10 DAP caused a significant reduction in 
TABLE 2 | The effects of single virus, double infections of CABMV + CMeV treatments and buffer on plant height and number of leaves in the White cultivar, and TVu 76.

\begin{tabular}{|c|c|c|c|c|c|}
\hline \multirow[t]{2}{*}{ Growth Parameter } & & \multicolumn{2}{|c|}{ Plant Height (cm) } & \multicolumn{2}{|c|}{ Number of leaves } \\
\hline & & White & TVu 76 & White & TVu 76 \\
\hline TREATMENT & DAP $x$ & & & & \\
\hline \multirow[t]{2}{*}{ CABMV } & 10 & $19.19^{d}$ & $24.69^{c}$ & $3.44^{d}$ & $14.11^{b}$ \\
\hline & 30 & $92.99^{a b}$ & $46.84^{\mathrm{ab}}$ & $19.22^{\mathrm{ab}}$ & $16.67^{\mathrm{ab}}$ \\
\hline \multirow[t]{2}{*}{$\mathrm{CMeV}$} & 10 & $27.09^{d}$ & $16.3^{d}$ & $4.33^{d}$ & $5.25^{\mathrm{C}}$ \\
\hline & 30 & $52.78^{b c}$ & $36.12^{b}$ & $13.67^{b}$ & $12.22^{\mathrm{bc}}$ \\
\hline \multirow[t]{2}{*}{$\mathrm{CABMV}+\mathrm{CMeV}$} & 10 & $24.13^{d}$ & $16.08^{d}$ & $2.22^{d}$ & $6.33^{\mathrm{C}}$ \\
\hline & 30 & $98.64^{a b}$ & $34.14^{b}$ & $13.44^{b}$ & $12.33^{\mathrm{bc}}$ \\
\hline \multirow[t]{2}{*}{ BUFFER } & 10 & $50.09^{b c}$ & $34.43^{b}$ & $26.44^{\mathrm{a}}$ & $14.78^{b}$ \\
\hline & 30 & $102.44^{a}$ & $54.49^{a}$ & $27.11^{\mathrm{a}}$ & $18.33^{a}$ \\
\hline
\end{tabular}

Percent reduction in growth parameters of virus treated plants compared with mock inoculated plants

\begin{tabular}{|c|c|c|c|c|c|}
\hline \multirow[t]{2}{*}{ CABMV } & 10 & 61.69 & 28.29 & 86.99 & 4.53 \\
\hline & 30 & 9.22 & 14.04 & 29.10 & 9.06 \\
\hline \multirow[t]{2}{*}{$\mathrm{CMeV}$} & 10 & 45.92 & 52.66 & 83.62 & 64.48 \\
\hline & 30 & 48.48 & 33.71 & 49.58 & 33.33 \\
\hline \multirow[t]{2}{*}{ CABMV + CMeV (Cobs) } & 10 & 51.83 & 53.30 & 91.60 & 57.17 \\
\hline & 30 & 3.71 & 37.35 & 50.42 & 32.73 \\
\hline \multirow[t]{2}{*}{ Cexp } & 10 & 79.28 & 66.05 & 97.87 & 71.93 \\
\hline & 30 & 53.23 & 43.02 & 64.25 & 39.37 \\
\hline \multirow[t]{2}{*}{ Cobs: Cexp } & 10 & 0.65 & 0.81 & 0.94 & 0.79 \\
\hline & 30 & 0.07 & 0.87 & 0.78 & 0.83 \\
\hline
\end{tabular}

$D A P^{x}$, Growth parameters were measured 10 days after inoculation at 10 and 30 DAP. The age of the plant at 10 DAP was 20 days old, at 30 DAP, the age of the plant was 40 days old. CABMV, Cowpea aphid-borne mosaic virus; CMeV, Cowpea mottle virus. Each value is the mean of 3 replicates. In each column, means followed by the same letter are not significantly different $(P=0.05)$ according to Duncan's multiple range tests. Cexp $=A+B-A B / 100$, $A$ stands for CABMV alone and $B$ for CMeV alone. If Cobs: Cexp <0.5, interactions are antagonistic, Cobs: Cexp 0.5-1.5, interactions are additive, Cobs: Cexp >1.5, interactions are synergistic.

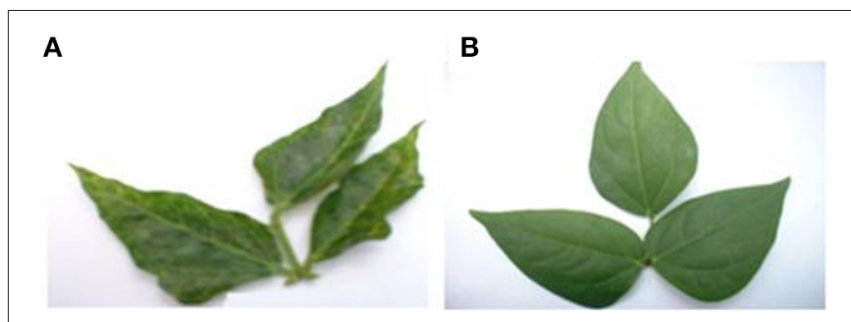

FIGURE 2 | (A) Mottling and leaf reduction induced on line IT81D-985 by $\mathrm{CMeV}$. (B) Symptomless buffer inoculated trifoliate leaf.

plant height and leaf number in comparison with the controls (Tables 2-4). We observed that in the White cultivar, CABMV was the most aggressive but caused the least reduction in vegetative growth in TVu 76 (Table 2). In the single infection of the cowpea cultivar "White," CABMV infected plants were the shortest with an average height of $19.19 \mathrm{~cm}$, while the average height of the healthy was $50.09 \mathrm{~cm}$. compared to the healthy's
TABLE 3 | The effects of single virus, double infections of CABMV + SBMV treatments and buffer on plant height and number of leaves in the White cultivar and TVu 76.

\begin{tabular}{|c|c|c|c|c|c|}
\hline \multirow[t]{2}{*}{ Growth Parameter } & & \multicolumn{2}{|c|}{ Plant Height (cm) } & \multicolumn{2}{|c|}{ Number of leaves } \\
\hline & & White & TVu 76 & White & TVu 76 \\
\hline TREATMENT & DAP $^{x}$ & & & & \\
\hline \multirow[t]{2}{*}{ CABMV } & 10 & $19.19^{d}$ & $24.69^{c}$ & $3.44^{d}$ & $14.11^{b}$ \\
\hline & 30 & $92.99^{a b}$ & $46.84^{b}$ & $19.22^{a b}$ & $16.67^{\mathrm{ab}}$ \\
\hline \multirow[t]{2}{*}{ SBMV } & 10 & $21.43^{d}$ & $16.07^{d}$ & $8.11^{\mathrm{C}}$ & $6.33^{\mathrm{C}}$ \\
\hline & 30 & $51.92^{\mathrm{bc}}$ & $34.13^{b}$ & $23.67^{a b}$ & $12.33^{b c}$ \\
\hline \multirow[t]{2}{*}{ CABMV + SBMV } & 10 & $26.24^{d}$ & $22.15^{\mathrm{C}}$ & $4.89^{d}$ & $2.67^{d}$ \\
\hline & 30 & $43.11^{\mathrm{C}}$ & $34.44^{b}$ & $15.67^{b}$ & $4.22^{\mathrm{cd}}$ \\
\hline \multirow[t]{2}{*}{ BUFFER } & 10 & $50.09 \mathrm{bc}$ & $34.43^{b}$ & $26.44^{\mathrm{a}}$ & $14.78^{b}$ \\
\hline & 30 & $102.44^{a}$ & $54.49^{a}$ & $27.11^{\mathrm{a}}$ & $18.33^{a}$ \\
\hline
\end{tabular}

Percent reduction in growth parameters of virus treated plants compared with mock inoculated plants

\begin{tabular}{llllll}
\hline CABMV & 10 & 61.69 & 28.29 & 86.99 & 4.53 \\
& 30 & 9.22 & 14.04 & 29.10 & 9.06 \\
SBMV & 10 & 57.22 & 53.33 & 69.33 & 57.17 \\
& 30 & 49.32 & 37.36 & 12.69 & 32.73 \\
CABMV + SBMV (Cobs) & 10 & 47.61 & 35.67 & 81.51 & 81.94 \\
& 30 & 57.92 & 36.80 & 42.20 & 76.98 \\
Cexp & 10 & 83.61 & 66.53 & 96.01 & 59.11 \\
& 30 & 53.99 & 46.16 & 38.10 & 38.83 \\
Cobs: Cexp & 10 & 0.57 & 0.54 & 0.85 & 1.39 \\
& 30 & 1.07 & 0.80 & 1.11 & 1.98
\end{tabular}

$D A P^{x}$,- Growth parameters were measured 10 days after inoculation at 10 and 30 DAP. The age of the plant at 10 DAP was 20 days old, at 30 DAP, the age of the plant was 40 days old. CABMV, Cowpea aphid borne mosaic virus; SBMV, Southern bean mosaic virus. Each value is the mean of 3 replicates. In each column, means followed by the same letters are not significantly different $(P=0.05)$ according to Duncan's multiple range tests. Cexp $=A+B-A B / 100$, A stands for CABMV alone and B for SBMV alone. If Cobs: Cexp < 0.5, interactions are antagonistic, Cobs: Cexp 0.5-1.5, interactions are additive, Cobs: Cexp > 1.5, interactions are synergistic.

average of 26 leaves. In TVu 76, single infections with $\mathrm{CMeV}$ and SBMV caused similar percentage reductions in plant height and leaf number at 10 and 30 DAP (Table 4).

\section{The Presence of a Potyvirus (CABMV) had no Significant Effect on Severity of Growth Reduction in the Double Infections}

On exposure to the different double virus combinations, $\mathrm{CABMV}+\mathrm{CMeV}, \mathrm{CABMV}+\mathrm{SBMV}, \mathrm{CMeV}+\mathrm{SBMV}$, there were no significant differences in the response of the White and $\mathrm{TVu} 76$ varieties to the 3 types of double infections (Tables 2-4).

In the White variety at $10 \mathrm{DAP}$, although the mixture of $\mathrm{CMeV}$ and SBMV caused the most stunting with an average height of $19.48 \mathrm{~cm}$, there was no significant difference in the average height and leaf number of the plants that received CABMV + $\mathrm{CMeV}$ or $\mathrm{CABMV}+\mathrm{SBMV}$ or $\mathrm{CMeV}+\mathrm{SBMV}$. However, there were significant differences in these parameters when the plants received the virus treatment at $30 \mathrm{DAP}$. 
All the TVu 76 plants that received virus treatments at 10 and 30 DAP were significantly shorter than the buffer inoculated plants. There was no significant differences in the effects of the 3 types of double virus infections on plant height at 10 and 30 DAP.

\section{Virus Treatments Promoted Increase in Foliage and Plant Height in the IITA Line IT81D-985}

Unexpectedly, some of the virus treated plants were taller and produced more leaves than the control plants. For the plants inoculated at $10 \mathrm{DAP}$, those that received the following treatments (i) $\mathrm{CMeV}$ (ii) CABMV + SBMV (iii) CABMV + $\mathrm{CMeV}$ were taller than the control plants and also produced more leaves. Considering those treated at $30 \mathrm{DAP}$, all the plants that received the virus treatments produced more leaves and were taller than the controls except those that were treated with SBMV alone (Table 5).

TABLE 4 | The effects of single virus, double infections of CMeV + SBMV treatments and buffer on plant height and number of leaves in the White cultivar and line TVu 76.

\begin{tabular}{|c|c|c|c|c|c|}
\hline \multirow[t]{2}{*}{ Growth Parameter } & & \multicolumn{2}{|c|}{ Plant Height (cm) } & \multicolumn{2}{|c|}{ Number of leaves } \\
\hline & & White & TVu 76 & White & TVu 76 \\
\hline TREATMENT & DAP $^{x}$ & & & & \\
\hline \multirow[t]{2}{*}{$\mathrm{CMeV}$} & 10 & $27.09^{d}$ & $16.3^{d}$ & $4.33^{d}$ & $5.25^{\mathrm{C}}$ \\
\hline & 30 & $52.78^{b c}$ & $36.12^{b}$ & $13.67^{b}$ & $12.22^{\mathrm{bc}}$ \\
\hline \multirow[t]{2}{*}{ SBMV } & 10 & $21.43^{d}$ & $16.07^{d}$ & $8.11^{\mathrm{C}}$ & $6.33^{\mathrm{C}}$ \\
\hline & 30 & $51.92^{\mathrm{bc}}$ & $34.13^{b}$ & $23.67^{a b}$ & $12.33^{b c}$ \\
\hline \multirow[t]{2}{*}{$\mathrm{CMeV}+\mathrm{SBMV}$} & 10 & $19.48^{d}$ & $18.2^{\mathrm{d}}$ & $4.33^{d}$ & $4.5^{\mathrm{cd}}$ \\
\hline & 30 & $87.48^{\mathrm{b}}$ & $34.00^{b}$ & $20.56^{a b}$ & $16.56^{\mathrm{ab}}$ \\
\hline \multirow[t]{2}{*}{ BUFFER } & 10 & $50.09^{b c}$ & $34.43^{b}$ & $26.44^{\mathrm{a}}$ & $14.78^{b}$ \\
\hline & 30 & $102.44^{\mathrm{a}}$ & $54.49^{a}$ & $27.11^{\mathrm{a}}$ & $18.33^{a}$ \\
\hline
\end{tabular}

Percent reduction in growth parameters of virus treated plants compared with mock inoculated plants

\begin{tabular}{lccccc}
\hline CMeV & 10 & 45.92 & 52.66 & 83.62 & 64.48 \\
& 30 & 48.48 & 33.71 & 49.58 & 33.33 \\
SBMV & 10 & 57.22 & 53.33 & 69.33 & 57.17 \\
& 30 & 49.32 & 37.36 & 12.69 & 32.73 \\
CMeV + SBMV (Cobs) & 10 & 61.11 & 47.14 & 83.62 & 69.55 \\
& 30 & 14.60 & 37.60 & 24.16 & 9.66 \\
Cexp & 10 & 76.86 & 77.90 & 94.98 & 84.79 \\
& 30 & 73.89 & 58.48 & 55.97 & 55.16 \\
Cobs: Cexp & 10 & 0.80 & 0.61 & 0.88 & 0.82 \\
& 30 & 0.20 & 0.64 & 0.43 & 0.18 \\
\hline
\end{tabular}

$D A P^{x}$, Growth parameters were measured 10 days after inoculation at 10 and 30 DAP. The age of the plant at 10 DAP was 20 days old, at 30 DAP, the age of the plant was 40 days old. CMeV, Cowpea mottle virus; SBMV, Southern bean mosaic virus. Each value is the mean of 3 replicates. In each column, means followed by the same letter are not significantly different $(P=0.05)$ according to Duncan's multiple range tests. If Cobs: Cexp <0.5, interactions are antagonistic, Cobs: Cexp 0.5-1.5, interactions are additive, obs: Cexp >1.5, interactions are synergistic.

\section{Co-infection with Triple Viruses did not have a Greater Impact than Double Virus Infections on Growth Traits}

To assess if the extent of damage caused by mixed infections could be greater if there were more than 2 viruses involved, we looked at the effects of the triple virus treatment CABMV + $\mathrm{CMeV}+\mathrm{SBMV}$ on the three cowpea varieties (Table 6). In all the cultivars/lines, the triple virus treated plants were significantly shorter than the control plants and produced less leaves. In White, there was no difference between the effects of the double and triple virus infections. However, the double infections were more aggressive than the triple virus infections in TVu 76 (Tables 2-4, 6).

\section{None of the Early Virus Inoculated Plants (10 DAP) Produced any Flowers, Pods, or Seeds}

In all the three varieties, none of the virus treated plants produced any flowers, pods or seeds at 10 DAP. However, the plants inoculated with buffer only, produced flowers and seeds. For those inoculated at $30 \mathrm{DAP}$, all the virus treated cultivar White plants produced an average of 1-4 flowers compared to the 7 flowers produced by the healthy. CABMV + SBMV, as well as $\mathrm{CABMV}+\mathrm{CMeV}+\mathrm{SBMV}$ treated ones produced the least-an average of 1 flower. All of the infected plants produced pods and seeds except those that received the CABMV $+\mathrm{CMeV}+\mathrm{SBMV}$ and CABMV + SBMV treatments.

The pods from the CABMV + CMEV treated plants were the shortest at $2.13 \mathrm{~cm}$ whereas the average length of the pods from the negative controls was $9.27 \mathrm{~cm}$. CMeV treated plants

TABLE 5 | The effect of single virus, double virus infections and buffer on plant height and leaf number in IT 81D-985.

\begin{tabular}{lccc}
\hline Treatment & DAP $^{\boldsymbol{x}}$ & Plant height (cm) & Leaf number \\
\hline CABMV & 10 & $8.47^{\mathrm{d}}$ & $4.33^{\mathrm{d}}$ \\
& 30 & $50.80^{\mathrm{ab}}$ & $13.89^{\mathrm{b}}$ \\
CMEV & 10 & $26.33^{\mathrm{c}}$ & $10.56^{\mathrm{b}}$ \\
& 30 & $61.24^{\mathrm{a}}$ & $19.33^{\mathrm{a}}$ \\
SBMV & 10 & $6.17^{\mathrm{d}}$ & $7.44^{\mathrm{c}}$ \\
& 30 & $21.22^{\mathrm{c}}$ & $17.89^{\mathrm{a}}$ \\
CABMV + CMeV & 10 & $21.87^{\mathrm{c}}$ & $8.78^{\mathrm{c}}$ \\
& 30 & $36.98^{\mathrm{b}}$ & $11.56^{\mathrm{b}}$ \\
CABMV + SBMV & 10 & $23.42^{\mathrm{c}}$ & $13.78^{\mathrm{b}}$ \\
& 30 & $50.94^{\mathrm{b}}$ & $15.56^{\mathrm{a}}$ \\
CMEV + SBMV & 10 & $13.41^{\mathrm{c}}$ & $3.78^{\mathrm{d}}$ \\
& 30 & $75.77^{\mathrm{ab}}$ & $18.33^{\mathrm{a}}$ \\
Buffer & 10 & $19.33^{\mathrm{c}}$ & $8.00^{\mathrm{d}}$ \\
& 30 & $34.71^{\mathrm{b}}$ & $8.56^{\mathrm{c}}$ \\
& & &
\end{tabular}

$D A P^{x}$, Growth parameters were measured 10 days after inoculation at 10 and 30 DAP. The age of the plant at 10 DAP was 20 days old, at 30 DAP, the age of the plant was 40 days old. CABMV, Cowpea aphid borne mosaic virus. CMeV, Cowpea mottle virus; SBMV, Southern bean mosaic virus. Each value is the mean of 3 replicates. In each column, means followed by the same letter are not significantly different $(P=0.05)$ according to Duncan's multiple range tests. 
TABLE 6 | The effect of co-infection with a mixture of the three viruses/buffer and age of plant at inoculation on growth parameters on commercial cultivar White and IITA lines IT81D-985 and TVu 76.

\begin{tabular}{|c|c|c|c|c|c|c|c|}
\hline \multirow[t]{2}{*}{ Virus Treatment } & \multirow[t]{2}{*}{ DAP $^{x}$} & \multicolumn{3}{|c|}{ PLANT HEIGHT } & \multicolumn{3}{|c|}{ LEAF NUMBER } \\
\hline & & White & IT81D-985 & TVu 76 & White & IT81D-985 & TVu 76 \\
\hline \multirow[t]{2}{*}{$\mathrm{CABMV}+\mathrm{CMeV}+\mathrm{SBMV}$} & 10 & $27.15^{d}$ & $13.82^{c}$ & $21.17^{\mathrm{C}}$ & $3.22^{\mathrm{d}}$ & $3.89^{d}$ & $10.111^{\mathrm{bc}}$ \\
\hline & 30 & $58^{b c}$ & $40.07^{a b}$ & $36.12^{\mathrm{b}}$ & $12^{b}$ & $6.89^{c}$ & $3.11^{d}$ \\
\hline \multirow[t]{2}{*}{ BUFFER } & 10 & $50.09^{b c}$ & $19.33^{C}$ & $34.43^{b}$ & $26.44^{\mathrm{a}}$ & $8^{C}$ & $14.78^{\mathrm{b}}$ \\
\hline & 30 & $102.44^{a}$ & $34.71^{\mathrm{b}}$ & $54.49^{a}$ & $27.11^{a}$ & $8.56^{\mathrm{C}}$ & $18.33^{a}$ \\
\hline
\end{tabular}

$D A P^{x}$, Growth parameters were measured 10 days after inoculation at 10 and 30 DAP. The age of the plant at 10 DAP was 20 days old, at 30 DAP, the age of the plant was 40 days old.

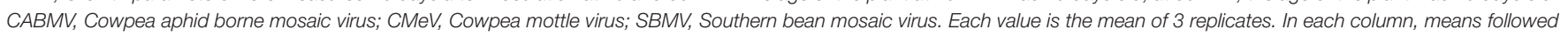
by the same letter are not significantly different $(P=0.05)$ according to Duncan's multiple range tests.

had the least yield of approximately 1 seed, while the CABMV treated plants as well as SBMV treated plants had the same average yield of 3 seeds. $\mathrm{CMeV}+\mathrm{SBMV}$ treated plants had the most yields for the double infections with an average seed number of 2 . Reduction in seed number ranged from 47 to $89 \%$. The average unit weight of the seeds produced from the virus treated plants was less than that produced by the control plants $(0.15 \mathrm{~g})$ except in those inoculated with $\mathrm{CMeV}+\mathrm{SBMV}$. CABMV treated plants produced the smallest seeds $(0.09 \mathrm{~g})$, while the average weight of seeds from $\mathrm{CMeV}+\mathrm{SBMV}$ treated ones was $0.21 \mathrm{~g}$.

For the IT81D-985 inoculated at 30 DAP, only those treated with CABMV singly, CABMV + $\mathrm{CMeV}$ and CABMV + SBMV and the healthy produced flowers. Of the virus treated plants that produced flowers, only those treated with CABMV + SBMV produced pods, which were one-third the length of those produced by the healthy. The average number of seeds they produced was 2 compared to about 5 produced by the control plants. The average unit weight of seeds from the healthy plants was at least 4 times the weight of the seeds from infected plant (Table 7).

Of the TVu 76 plants that received virus treatments at 30 DAP, only the plants infected singly with CABMV or $\mathrm{CMeV}$ produced flowers. All the others did not produce any flower or pod. None of the virus treated TVU plants produced any seeds. The control plants produced flowers, pods and seeds (Table 7).

\section{Co-infection with Triple Viruses Resulted Almost in a Total Loss in Yield Loss}

The triple virus infections resulted in a total loss of flowers, pods, and seeds except for the one flower produced by cultivar White. With regards to yield loss, the triple virus infections caused the most damage.

\section{Relative Concentration of the Viruses in the Cowpea Lines}

The ELISA test was also used to establish the virus concentration in the cowpea cultivar White, IITA lines- IT81D-985 and TVu 76 infected singly by CABMV, CMEV, and SBMV and in mixtures. The samples were obtained from the inoculated leaf samples at 10 and 30 DAP. The ELISA results showed that the cowpea cultivar White and TVu 76 were susceptible to the each of the viruses treatments (Taiwo et al., 2007) and the mock inoculated plants were virus free.

For IITA line, IT 81D-985, only SBMV was detected at reasonable concentrations in the applicable treatments. In the single virus treatments, the absorbance values of CABMV and $\mathrm{CMeV}$ were 0.1805 and 0.155 ; these values were not significantly different from their negative controls of 0.1355 and 0.113 , respectively. In the double infections and triple infections, the virus titres of $\mathrm{CABMV}$ and $\mathrm{CMeV}$ were also not significant to be considered positive. For the sample to be considered positive for virus presence, it must have at least twice the absorbance value of negative control. The optical density readings are shown in Figure 3.

\section{Lack of Strong Evidence for Synergistic Interactions among the Unrelated Viruses in the Mixed Infection}

Based on our preliminary statistical analysis, the effects on the growth parameters did not suggest synergistic interactions. To validate our observation on the absence of synergism, we quantified using the Abbott's formula and set the boundaries for the type of response according to Gisi et al. (1985) instead of using the strict mathematical cut offs of synergism as being defined as Cobs: Cexp > 1 (Gisi, 1996). From the calculations, the reductions in the vegetative traits are not indicative of synergism in White and TVu 76 except the reduction in leaf number by CABMV + SBMV in TVu 76 (Table 3). The dual infections in IT81D-985 resulted in an increase in plant height and number of leaves; therefore, we could not make any calculations for this line (Table 5). The data is more indicative of an additive relationship between these viruses in White and TVu 76 (Tables 2-4).

\section{Discussion}

Natural infections of cowpea grown in Nigeria by Cowpea aphidborne mosaic virus (CABMV), Southern bean mosaic virus (SBMV), and Cowpea mottle virus $(\mathrm{CMeV})$ and others have been reported by Shoyinka et al. (1997). Comparative studies of single and mixed unrelated virus infections of cowpea have been done (Pio-Ribeiro et al., 1978; Owolabi et al., 1988; Shoyinka et al., 1997; Martin et al., 2004; Orawu et al., 2005; Kareem and Taiwo, 2007; Taiwo et al., 2007; Akinjogunla et al., 2008). 


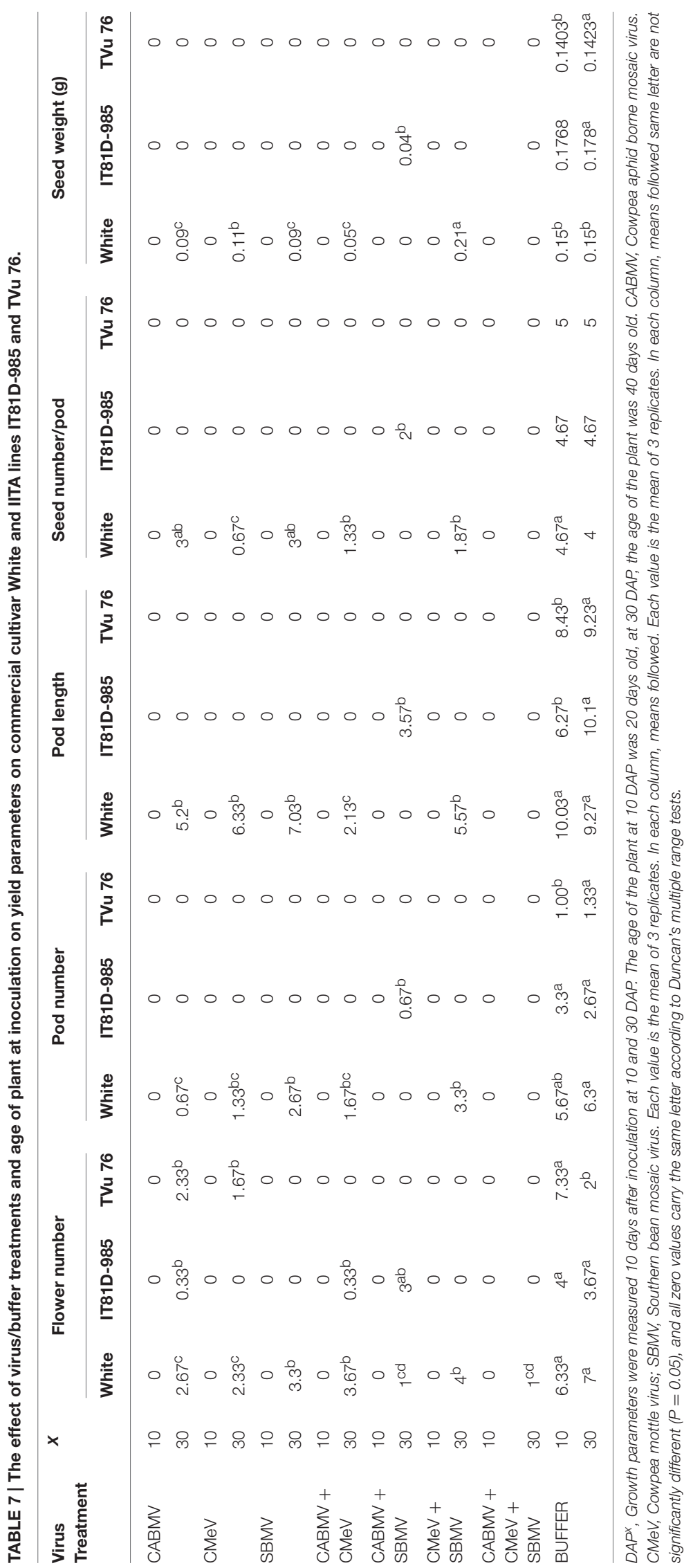




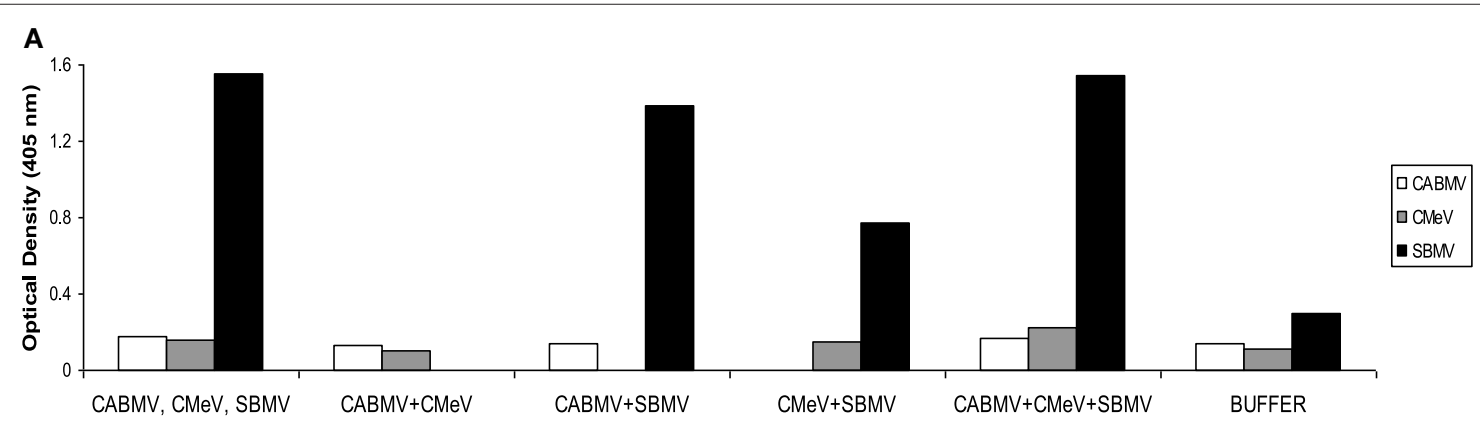

B

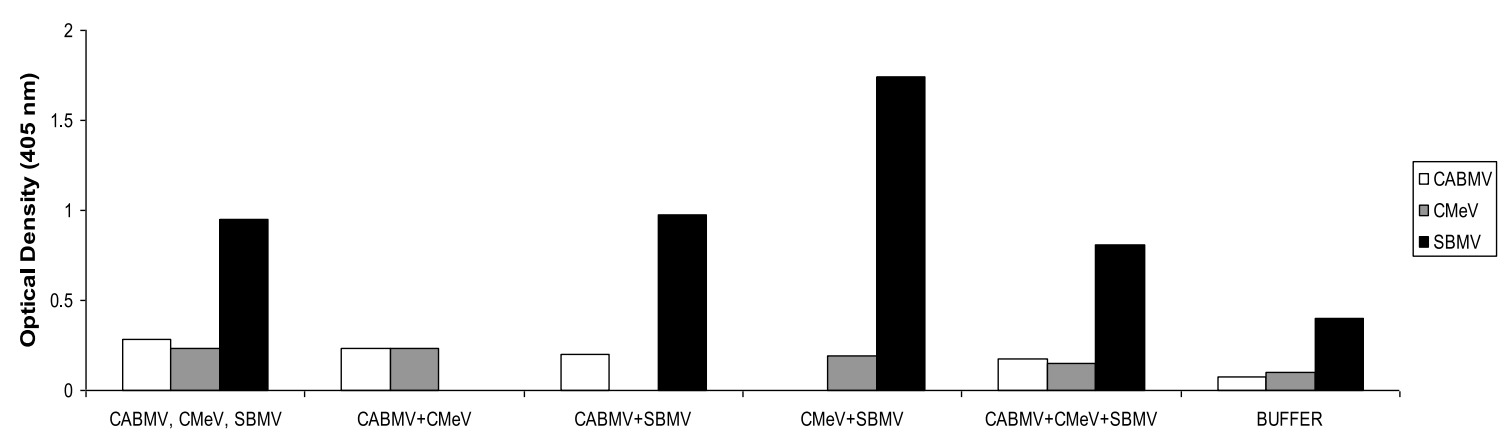

FIGURE 3 | Optical density readings (absorbance values) representing relative virus concentrations in leaf samples from IITA cowpea line IT81D-985 inoculated with single and mixed viruses at (A) 10 DAP (B) $\mathbf{3 0}$ DAP. The virus titres were determined by ACP ELISA. Values are averages of samples taken at 10 and 30 days after inoculation.

This study showed that the single and multiple virus infections have significant effects on the three Cowpea varieties investigated. The three Cowpea varieties- Commercial cultivar "White," IITA Lines IT 81D-985 and TVu 76 were susceptible to the single and mixed infections of Cowpea aphid-borne mosaic virus (CABMV), Southern bean mosaic virus (SBMV), and Cowpea mottle virus $(\mathrm{CMeV})$. The common symptoms induced by the virus infections were mosaic, mottling, vein banding, chlorosis, and stunting. The investigation confirmed that triple infections in the three varieties produced more severe visual symptoms than the double or single infections. Severity scores ranging from 2 to 5 were recorded for the cowpea cultivars infected with the viruses. The high scores might be due to the fact that cowpea is the primary host of these viruses. The severity of the virus diseases varied depending on the cowpea variety, the type of treatment and age at the onset of virus treatment. The severity of virus infection was higher at 10 DAP than at 30 DAP; the severity also increased from 10 DAI to 30 DAI. In line with this, Uyemoto et al. (1981) reported that early infection of plants by viruses results in a more drastic response than infections at an advanced stage. Kareem and Akinjogunla (2008) had also previously reported that the infection of cowpea with three unrelated viruses resulted in increased symptom severity at early stage of plant growth. The reason for this is that at an early stage of growth, cultivars might not have built up enough defense mechanism to combat diseases.

Generally, all the virus treatments caused some degree of reduction in vegetative growth and total yield. The virus-infected plants were shorter than buffer inoculated plants due to shorter internodes with fewer leaves. However, in IT81D-985, an anomaly was observed as some of the virus treated plants produced more leaves than the control and were taller. In spite of this, there was hardly any yield from this cultivar. This means that the increase in the number of leaves and plant height was due to the reaction to the virus presence. On the other hand, the most common symptom in TVu 76 was defoliation. There was also hardly any yield from the infected TVu 76 plants. The commercial cultivar, White was the least susceptible in terms of yield. The different responses could be due to the varying tolerance level of the cultivars. Our results show that virus diseases can damage an entire crop, leaves, stems, flowers, and seeds in these varieties under investigation.

The effect of CABMV and CMeV on "White" and TVu 76 led to at least $60 \%$ reduction in leaf number and ultimately caused the withering of all the leaves inoculated at 10 days after planting. Our results also did not show that the double virus infections had a more severe effect than the single virus infections. This supports the report of Anjos et al. (1992) that not all the combinations of unrelated viruses result in increased symptoms. In some cases, the single virus had a more devastating effect on the crop than double infections involving that same virus. For example, the IT81D-985 plants doubly infected with CABMV and SBMV were the only ones that produced seeds. Wells and Deba (1961) reported a similar occurrence of $100 \%$ loss in yield in cowpea due to the single infection of Cowpea mosaic virus (CpMV). 
Raheja and Leleji (1974) had also reported that CABMV caused a complete loss of yield of commercial cowpea.

Infections at 10 DAP with CABMV in "White," IT81D-985 and TVu 76 resulted in the death of some of the plants but not in those infected at 30 DAP. Of the 3 Cowpea varieties, the contrast in the impact of early and late infections on yield was most pronounced in the "White" variety. In the White variety, with the exception of plants that received the mixture of CABMV and SBMV, all the plants infected at 30 days after planting produced seeds unlike those infected at 10 DAP (Table 7). The results also correlate with previous information that the most severe effect on yield occurs as a result of early infection (Gay and Winstead, 1970; Kareem and Taiwo, 2007). This trend was also observed in the growth traits, as the heights of some "White" plants infected at $30 \mathrm{DAP}$ were not significantly different from the height of the control plants. This compares favorably with the results of investigation by Owolabi et al. (1988), who reported that Cultivar "Ife Brown" plants inoculated with CpMV alone and in combination with BlCMV at the initiation of flowering showed no significant difference in yield compared with the control. A similar trend was also observed in TVu 76. Despite the observation that the vegetative growth in IT81D-985 was an aberration from the other two, some of the IT81D-985 plants showed this trend.

It was also observed that the response of the cowpea varieties to virus infections differed from one variety to another. While the major symptom of virus treated $\mathrm{TVu} 76$ was defoliation, the response of the IT81D-985 plants to infection was increased leaf production. IT81D-985 is therefore sturdier than TVu 76 in response to virus infections. It is also significant that IT81D-985 was able to overcome infection by CABMV + SBMV and produced seeds, whereas, the White variety had no yield with the same treatment. The White variety shows similar characteristics to another local variety, Oloyin, reported by Kareem and Taiwo (2007) in that it was the most resistant of the three cultivars/varieties and produced seeds at 30 DAP.

Our results for growth and yield parameters for uninfected (buffer inoculated) IT81D-985 plants compare favorably with a previous report by Ekpo et al. (2012). They had evaluated plant height and leaf number of IT 81D-985 among other cowpea lines. In their report, at 21 days, plant height of IT81D-985 was $18.4 \pm 1.38 \mathrm{~cm}$ and at 14 days, the number of leaves was $8.42 \pm 0.2$, our average value at 20 days old for negative controls were $19.33 \mathrm{~cm}$ and 8 for plant height and number of leaves, respectively. However, at advanced stages of plant growth, our findings were different from Ekpo et al. (2012). At 42 days old, they recorded an average plant height of $89.8 \pm 13.72 \mathrm{~cm}$ and $18.25 \pm 0.32$ leaves, whereas at 40 days old, our healthy IT81D985 plants measured an average of $34.19 \mathrm{~cm}$ and produced an average of 9 leaves. For yield parameters, the IT81D-985 plants inoculated with buffer at $10 \mathrm{DAP}$ and $30 \mathrm{DAP}$ produced pods with an average length of 6.27 and $10.1 \mathrm{~cm}$, respectively, bearing similarity to the average pod length of $9.1 \pm 2.26 \mathrm{~cm}$ recorded by Ekpo et al. (2012). Although our IT81D-985 plants inoculated with the buffer at 30 DAP did not grow as tall as expected, we ruled out the possibility that these group of plants did not grow optimally because serological evaluation showed that they were virus free and they had a good yield.

Serological detection of the three viruses in all the treatments for White and TVu 76 validated that the symptoms and reductions in growth traits were due to virus presence. However, in line IT81D-985 virus treated plants, ELISA results showed substantial accumulation of SBMV but not significant concentrations of $\mathrm{CABMV}$ or $\mathrm{CMeV}$. Based on serological analysis, line IT81D-985 appeared resistant to CABMV and $\mathrm{CMeV}$. In spite of the symptomatic IT81D-985 leaf samples having low titres of these two viruses, and more vegetative growth, there was still a significant reduction in yield.

Although Shoyinka et al. (1978) reported that the response of plants most times to mixed infections is more of synergism, and Pio-Ribeiro et al. (1978) reported that the symptoms induced by mixed infection in California Black eye by cucumber mosaic virus and BICMV were more severe and distinct from the relatively mild symptoms caused by either of the viruses, it is clear from our results that the interaction of the viruses in the double virus infections could not be suggestive of synergism, because the overall effect of these mixed treatments was not stronger than the sum of the effects caused by each of the viruses separately in all the cowpea varieties. The quantitative analysis of the growth parameters provided evidence for an additive relationship in the double virus infections in cultivar White and line TVu 76. With the unexpected results of increase in plant height and number of leaves of virus treated IT81D-985, in comparison with the healthy plants, we could not assess this line for synergistic interactions of viruses as in cultivar White and TVu 76.

It has been well documented for decades that diseases caused by viruses have been responsible for great damage, causing serious losses in cowpea crop yield in several countries (Lima et al., 1979; Damiri et al., 2013). It is also evident that our observations here, agree with earlier findings of Lana and Adegbola (1977), who reported that economic injury to cowpea due to virus infection depends on three factors- virus isolate, tolerance/resistance of the infected Cowpea cultivar and most importantly the age of the host plant at the time of infection. In summary, the results obtained confirm that plant virus infections cause the decline of cowpea plants, lower product quality, and ultimately result in crop losses.

The basic approaches for the control of viruses have not been overly successful because of non-curable nature of viral infections, lack of resistant cultivars with good agronomic quality and diversity of viruses and their natural vectors (Lecoq, 1998). The implications of these results are that there is a need to intensify efforts in developing advanced cowpea breeding lines/cultivars with multiple resistance to economically important viruses. With few virus control strategies available, pathogen-derived resistance is a promising tool in viral disease management. Since its first demonstration by Powell-Abel et al. (1986), it has been used with success against several other virus groups (Hull and Davies, 1992; Bau et al., 2003). It offers great potential to develop in a short time plants with highly durable resistance and broad efficacy (Kaniewski and Lawson, 1998). IITA reported that one of its 2003 milestones was the initiation of field 
testing of transgenic cowpea plants. Cowpea lines resistant to CABMV have been identified (Dhanasekar and Reddy, 2015).

The benefits of increased cowpea production include improved nutrition for humans and livestock, improved soil properties and substantial opportunities for greater income. The control of these viruses therefore is crucial to sustainable cowpea production most especially in sub-Saharan Africa.

\section{References}

Abbott, W. S. (1925). A method of computing the effectiveness of an insecticide. J. Econ. Entomol. 18, 265-267. doi: 10.1093/jee/18.2.265a

Aboul-Ata, A. E., Allen, D. J., Thottappilly, G., and Rossel, H. W. (1982). Variation in rate of seed transmission of cowpea aphid-borne mosaic virus in cowpea. Trop. Grain Legume Bull. 25, 2-7.

Akinjogunla, O. J., Taiwo, M. A., and Kareem, K. T. (2008). Immunological and molecular diagnostic methods for detection of viruses infecting cowpea (Vigna unguiculata). Afr. J. Biotechnol. 7, 2099-2103. doi: 10.5897/AJB08.120

Allen, D. J., Anno-Nyako, F. O., Ochieng, R. S., and Ratinam, M. (1981). Beetle transmission of cowpea mottle and southern bean mosaic viruses in West Africa. Trop. Agric. (Trinidad) 58, 171-175.

Anjos, J. R., Jarlfors, U., and Ghabrial, S. A. (1992). Soybean mosaic virus, Potyvirus enhances the titre of two comoviruses in dually infected soybean plants. Phytopathology 82, 1022-1027. doi: 10.1094/Phyto-82-1022

Atiri, G. I. (1982). Virus-Vector-Host Relationship of Cowpea Aphid-Borne Mosaic Virus in Cowpea (Vigna Unguiculata (L). Walp). Ph.D. Thesis, University of Ibadan.

Atiri, G. I., Ekpo, E. J. A., and Thottappilly, G. (1984). The effect of aphidresistance in cowpea infestation and development of Aphis craccivora and the transmission of cowpea aphid-borne mosaic virus. Ann. Appl. Biol. 104, 339-346. doi: 10.1111/j.1744-7348.1984.tb05619.x

Bashir, M., Ahmad, Z., and Ghafoor, A. (2002). Cowpea aphid borne mosaic virusa review. Int. J. Pest Manage. 48, 155-168. doi: 10.1080/09670870110118722

Bau, H. J., Cheng, Y. H., Yang, J. S., and Yeh, S. D. (2003). Broadspectrum resistance to different geographic strains of Papaya ringspot virus in coat protein transgenic papaya. Phytopathology 93, 112-120. doi: 10.1094/PHYTO.2003.93.1.112

Bock, K. R. (1973). East African strains of cowpea aphid-borne mosaic virus. Ann. Appl. Biol. 74, 75-83.

Bock, K. R., and Conti, M. (1974). "Cowpea aphid-borne mosaic virus," in CMI/AAB Description of Plant Viruses, No. 134 (Surrey: Kew).

Bos, L. (1970). The identification of three new viruses isolated from Wisteria and Pisum in the Netherlands, and the problem of variation within the potato virus Y group. Neth. J. Plant Pathol. 76, 8-46. doi: 10.1007/BF01976763

Burke, D. W., Ditshipi, P., and DeMooy, C. J. (1986). Virus diseases of cowpeas in dry land and irrigated plots in Botswana. Plant Dis. 70, 801.

Cho, J. D., Kim, J. S., Choi, H. S., La, Y. J., and Kim, K. S. (2000). Ultrastructural aspects of mixed infections of watermelon mosaic Potyvirus isolated from pumpkin and cucumber green mottle mosaic tobamovirus from watermelon. Plant Pathol. J. 16, 216-221.

Damiri, D., Al-Shahwan, I. M., Al-Saleh, O. A., Abdalla, O. A., and Amer, M. A. (2013). Identification and Characterization of cowpea aphid-borne mosaic virus isolates in Saudi Arabia. J. Plant Pathol. 95, 79-85. doi: 10.4454/JPP.V95I1.019

Dhanasekar, P., and Reddy, K. (2015). Serological screening of cowpea genotypes for resistance against Cowpea Aphid Borne Mosaic virus using DAS-ELISA. Asian J. Plant Pathol. 9, 83-90. doi: 10.3923/ajppaj.2015.83.90

Ekpo, I. A., Agbor, R. B., Osuagwu, A. N., Okpako, E. C., and Ekanem, B. E. (2012). Evaluation of eight cowpea (Vigna unguiculata, L. Walp) spcies for yield and associated traits. Int. J. Pure Appl. Sci. Technol. 12, 1-7.

Fauquet, C., and Thouvenel, J. C. (1980). Plant Viral Diseases in the Ivory Coast, Documentation Techniques, No. 46. Reedition 1987. (Paris: ORSTOM).

Gaikward, D. G., and Thottappilly, D. (1988). Occurrence of southern bean mosaic virus in Senegal. J. Phytopathol. 121, 366-369.

\section{Acknowledgments}

This research was carried out as part of a Master's thesis by the first author under the supervision of Professor Monilola A. Taiwo at the University of Lagos. We thank Mr. Oye for excellent technical assistance in the greenhouse, and Chidiadi C. Nwachukwu for his assistance with the initial sorting of the data.

Gay, D. J., and Winstead, E. E. (1970). Seedborne viruses and fungi from southern pea grown in eight states. Plant Dis. Rep. 54, 243-245.

Gisi, U. (1996). Synergistic interactions of fungicides in mixture. Phytopathology $86,1273-1279$.

Gisi, U., Binder, H., and Rimbach, E. (1985). Synergistic interactions of fungicides with different modes of action. Trans. Br. Mycol. Soc. 85, 299-306. doi: $10.1016 /$ S0007-1536(85)80192-3

Gumedzoe, M. Y. (1985). Studies of Variability of the Cowpea Aphid-Borne Mosaic Virus (CAMBV) Complex in Nigeria. Ph.D. Thesis. University of Laval, Quebec, QC.

Gumedzoe, M. Y., Sunu, D. Y., and Thottappilly, G. (1989). "Inventaire des principaux viroses du niébé," in Papier Presente à la Conférence Biennale de l'Association Ouest Africaine, Cotonou le 10-15, Septembre.

Hughes, J. A., and Shoyinka, S.A. (2003). "Overview of viruses of legumes other than groundnut in Africa," in Plant Virology in Sub-Sahara Africa, eds J. A. Hughes and J. Odu (Proceedings of a conference organized by IITA Ibadan, Nigeria: International Institute of Tropical Agriculture), 553-68.

Hull, R., and Davies, J. W. (1992). Approaches to non-conventional control of plant virus disease. Crit. Rev. Plant Sci. 11, 17-33. doi: 10.1080/07352689209382328

International Institute of Tropical Agriculture (IITA) (2009). IITA Cereals and Legume Systems. Available online at: www.iita.org/cms/ details/cowpeaproject_details.asp

Kaniewski, W., and Lawson, C. (1998). "Coat protein and replicase-mediated resistance to plant viruses," in Plant Virus Disease Control, eds A. Hadidi, R. K. Khartepal, and K. Koagenezang (APS press), 65-78.

Kareem, K. T., and Akinjogunla, O. J. (2008). "Severity of infection of three unrelated viruses on cowpea cultivars," Proceedings of the Third Conference on Science and National Development, (Abeokuta), 102-107.

Kareem, K. T., and Taiwo, M. A. (2007). Interaction of viruses in cowpea: effect on growth and yield parameters. Virol. J. 4:15. doi: 10.1186/1743-422X-4-15

Karungi, J., Adipala, E., Ogenga-Latigo, M. W., Kyamanywa, S., and Oyobo, N. (2000). Pest management in cowpea. Part 1. Influence of planting time and planting density on cowpea field pests infestation in eastern Uganda. Crop Prot. 19, 231-236. doi: 10.1016/S0261-2194(00)00013-2

Kuhn, C. W. (1963). Field occurrence and properties of cowpea strain of southern bean mosaic virus. Phytopathology 53, 732-733.

Ladipo, J. L. (1976). A vein-banding strain of cowpea aphid-borne mosaic virus in Nigeria. Niger. J. Sci. 10, 77-78.

Lamptey, P. N., and Hamilton, R. I. (1974). A new cowpea strain of southern bean mosaic virus from Ghana. Phytopathology 64, 1100-1104. doi: 10.1094/Phyto64-1100

Lana, A. O., and Adegbola, M. O. K. (1977). Important virus diseases in West African crops. CMI Rev. Plant Pathol. 56, 854-855.

Lecoq, H. (1998). "Control of plant virus diseases by cross protection," in Plant Virus Disease Control, eds A. Hadidi, R. K. Khartepal, and K. Koagenezang (St. Paul, MN: APS press), 33-40.

Lephale, S., Addo-Bediako, A., and Ayodele, V. (2012). Susceptibility of seven cowpea cultivars (Vigna unguiculata) to cowpea beetle (Callosobruchus maculates). Agric. Sci. Res. J. 2, 65-69.

Li, C.-D., Fatokun, C. A., Ubi, B., Singh, B. B., and Scoles, G. J. (2001). Determining genetic similarities and relationships among cowpea breeding lines and cultivars by microsatellite markers. Crop Sci. 41, 189-197. doi: 10.2135/cropsci2001.411189x

Lima, J. A. A., Purcifull, D. E., and Hiebert, E. (1979). Purification, partial characterization and serology of blackeye cowpea mosaic virus. Phytopathology 69, 1252-1258. doi: 10.1094/Phyto-69-1252 
Little, T. M., and Hills, F. J. (1972). "Transformations," Statistical Methods in Agricultural Research, (Davis, CA: University of California), 103-120.

Lovisolo, O., and Conti, M. (1966). Identification of an aphid-transmitted cowpea mosaic virus. Neth. J. Plant Pathol. 72, 265-269. doi: 10.1007/BF02650216

Martin, E. M., Cho, J. D., Kim, S. C., Goeke, S. C., Kim, K. S., and Gergerich, R. C. (2004). Novel cytopathological structures induced by mixed infection of unrelated plant viruses. Phytopathology 94, 111-119. doi: 10.1094/PHYTO.2004.94.1.111

Murphy, J. F., and Bowen, K. L. (2006). Synergistic disease in pepper caused by the mixed infection of Cucumber mosaic virus and Pepper mottle virus. Phytopathology 96, 240-247. doi: 10.1094/PHYTO-96-0240

Naidu, R. A., and Hughes, J. A. (2003). "Methods for the detection of plant viral diseases," in Plant Virology in Sub-Sahara Africa, eds J. A. Hughes and J. Odu (Proceedings of a conference organized by IITA Ibadan, Nigeria: International Institute of Tropical Agriculture), 233-260.

Orawu, M., Melis, R., De Milliano, W., Laing, M., and Adipala, E. (2005). Occurrence and prevalence of cowpea virus diseases in Uganda. Afr. Crop Sci. Proc. 7, 1279-1283.

Owolabi, A. T., Taiwo, M. A., and Mabadeje, S. A. (1988). Effects of single and mixed inoculations with blackeye cowpea mosaic virus on two Nigerian cowpea cultivars. Niger. J. Basic Appl. Sci. 2, 25-33.

Patel, P. N., and Kuwite, C. (1982). Prevalence of cowpea aphid-borne mosaic virus and two strains of cowpea mosaic virus in Tanzania. Indian Phytopathol. 35, 467-472.

Pio-Ribeiro, G., Wyatt, S. D., and Kuhn, C. W. (1978). Cowpea stunt: a disease caused by the synergistic interaction of two viruses. Phytopathology 68, 1260-1265. doi: 10.1094/Phyto-68-1260

Powell-Abel, P., Nelson, R. S., De, B., Hoffmann, N., Rogers, S. G., Fraley, R. T., et al. (1986). Delay of disease development in transgenic plants that express the tobacco mosaic virus coat protein gene. Science 232, 738-742. doi: $10.1126 /$ science. 3457472

Raheja, A. K., and Leleji, O. I. (1974). An aphid-borne virus disease of irrigated cowpea in northern Nigeria. Plant Dis. Rep. 58, 1080-1084.

Raman, K. V., Singh, S. S., and van Emden, H. F. (1980). Mechanisms of resistance to leafhopper damage in cowpea. Entomol. Soc. Am. 73, 484-488. doi: $10.1093 /$ jee/73.4.484

Robertson, D. G. (1963). "Cowpea virus research in Nigeria," in Proceedings of the First Nigerian Grain Legume Conference. Samaru, Zaria. Institute of Agricultural Research, Ahmadu Bello University, Zaria, Nigeria.

Robertson, D. G. (1966). Seed-Borne Viruses of Cowpea in Nigeria. B.Sc. Thesis, University of Oxford, UK.

Rossel, H. W., and Thottappilly, G. (1985). Virus Diseases of Important Food Crops in Tropical Africa. (Ibadan: IITA Publication Series), 61.

Shepherd, R. J., and Fulton, R. W. (1962). Identity of a seed-borne virus of cowpea. Phytopathology 52, 489-493.

Shi, X. M., Pruss, G., Ge, X., Sriskanda, V., and Vance, V. B. (1996). Identification of Plant Viral Synergism Genes. Doctoral dissertation, University of South Carolina.

Shoyinka, S. A., Bozarth, R. F., Reese, J., and Okunsanya, B. O. (1979). Field occurrence and identification of southern bean mosaic virus (cowpea strain) in Nigeria. Turrialba 29, 111-116.

Shoyinka, S. A., Bozarth, R. F., Reese, J., and Rossel, H. W. (1978). Cowpea mottle virus: a seed-borne virus with distinctive properties infecting cowpea in Nigeria. Phytopathology 68, 693-699. doi: 10.1094/Phyto-68-693
Shoyinka, S. A., Thottappilly, G., Adebayo, G. G., and Anno-Nyako, F. O. (1997). Survey on cowpea virus incidence and distribution in Nigeria. Int. J. Pest Manage. 43, 127-132. doi: 10.1080/096708797 228816

Singh, B. B. (2011). Cowpea Genetics and Breeding - A Historical Perspective. Brasil: IV Reunião de Biofort Fiação.

Singh, R., and Singh, R. (1974). Natural infection of sickle senna (Cassia tora L.) and cowpea (Vigna sinensis savi) plants by some new strains of southern bean mosaic virus. Partugalia Data Biol. 13, 87-89.

Taiwo, M. A. (2003). "Viruses infecting legumes in Nigeria: case history," in Plant Virology in Sub-Saharan Africa, eds J. A. Hughes and B. Odu (Proceedings of Plant Virology Ibadan, Nigeria: IITA), 364-378.

Taiwo, M. A., Kareem, K. T., Nsa, I. Y., and Hughes, J. A. (2007). Cowpea viruses: effect of single and mixed infections on symptomatology and virus concentration. Virol. J. 4:95. doi: 10.1186/1743-422X-4-95

Thottappilly, G., and Rossel, H. W. (1988). Occurrence of cowpea mottle virus and other viruses (cowpea yellow mosaic virus, southern bean mosaic virus) in cowpea. FAO Plant Prot. Bull. 36, 184-185.

Thottappilly, G., and Rossel, H. W. (1992). Virus of cowpea in tropical Africa. Trop. Pest Manage. 38, 337-348. doi: 10.1080/09670879209 371724

Thouvenel, J. C. (1988). A serious disease caused by cowpea mottle virus in Ivory Coast. Plant Dis. 72, 363. doi: 10.1094/PD-72-0363F

Tremaine, J. H., and Hamilton, R. I. (1983). "Southern bean mosaic virus," in CMI/AAB Descriptions of Plant Viruses, No. 274 (Surrey: Kew).

Untiveros, M., Fuentes, S., and Salazar, L. F. (2007). Synergistic interaction of Sweet potato chlorotic stunt virus (Crinivirus) with carla-, cucumo-, ipomo-, and potyviruses infecting sweet potato. Plant Dis. 91, 669-676. doi: 10.1094/PDIS91-6-0669

Uyemoto, J. K., Claflin, L. E., Wilson, D. L., and Rayney, R. J. (1981). Maize chlorotic mottle and maize dwarf mosaic viruses: effect of single and double inoculations on symptomatology and yield. Plant Dis. 65, 9-41. doi: 10.1094/PD-65-39

Vidano, C., and Conti, M. (1965). Transmissione con afidi d'un 'cowpea mosaic virus' isolato da Vigna sinensis Endl. Ann. Accad. Sci. Torino (Italia), 99, 1041-1050.

Walkey, D. G. A. (1985). Applied Plant Virology. (New York, NY: John Wiley \& Sons), 329.

Wang, Y., Gaba, V., Yang, J., Palukaitis, P., and Gal-on, A. (2002). Characterization of synergy between Cucumber mosaic virus and potyviruses in cucurbit hosts. Phytopathology 92, 51-58. doi: 10.1094/PHYTO.2002.92.1.51

Wells, D. G., and Deba, R. (1961). Sources of resistance to cowpea yellow mosaic virus. Plant Dis. Rep. 45, 878-881.

Conflict of Interest Statement: The authors declare that the research was conducted in the absence of any commercial or financial relationships that could be construed as a potential conflict of interest.

Copyright (c) 2015 Nsa and Kareem. This is an open-access article distributed under the terms of the Creative Commons Attribution License (CC BY). The use, distribution or reproduction in other forums is permitted, provided the original author(s) or licensor are credited and that the original publication in this journal is cited, in accordance with accepted academic practice. No use, distribution or reproduction is permitted which does not comply with these terms. 


\title{
Genetic Diversity of Cultivated Lentil (Lens culinaris Medik.) and Its Relation to the World's Agro-ecological Zones
}

\author{
Hamid Khazaei ${ }^{1}$, Carolyn T. Caron ${ }^{1}$, Michael Fedoruk ${ }^{1}$, Marwan Diapari ${ }^{1,2}$, \\ Albert Vandenberg ${ }^{1}$, Clarice J. Coyne ${ }^{3}$, Rebecca McGee ${ }^{3}$ and Kirstin E. Bett ${ }^{1 *}$ \\ ${ }^{1}$ Department of Plant Sciences, University of Saskatchewan, Saskatoon, SK, Canada, ${ }^{2}$ London Research and Development \\ Centre, Agriculture and Agri-Food Canada, London, ON, Canada, ${ }^{3}$ USDA-ARS, Pullman, WA, USA
}

\section{OPEN ACCESS}

Edited by:

Antonio M. De Ron, Spanish National Research Council,

Spain

Reviewed by:

Matthew Nicholas Nelson, University of Western Australia,

Australia

Sripada M. Udupa,

ICARDA, Morocco

*Correspondence: Kirstin E. Bett

k.bett@usask.ca

Specialty section: This article was submitted to Crop Science and Horticulture, a section of the journal

Frontiers in Plant Science

Received: 24 May 2016

Accepted: 11 July 2016

Published: 26 July 2016

Citation:

Khazaei H, Caron CT, Fedoruk M, Diapari M, Vandenberg A, Coyne CJ, McGee R and Bett KE (2016) Genetic

Diversity of Cultivated Lentil (Lens culinaris Medik.) and Its Relation to the

World's Agro-ecological Zones.

Front. Plant Sci. 7:1093.

doi: 10.3389/fpls.2016.01093
Assessment of genetic diversity and population structure of germplasm collections plays a critical role in supporting conservation and crop genetic enhancement strategies. We used a cultivated lentil (Lens culinaris Medik.) collection consisting of 352 accessions originating from 54 diverse countries to estimate genetic diversity and genetic structure using 1194 polymorphic single nucleotide polymorphism (SNP) markers which span the lentil genome. Using principal coordinate analysis, population structure analysis and UPGMA cluster analysis, the accessions were categorized into three major groups that prominently reflected geographical origin (world's agro-ecological zones). The three clusters complemented the origins, pedigrees, and breeding histories of the germplasm. The three groups were (a) South Asia (sub-tropical savannah), (b) Mediterranean, and (c) northern temperate. Based on the results from this study, it is also clear that breeding programs still have considerable genetic diversity to mine within the cultivated lentil, as surveyed South Asian and Canadian germplasm revealed narrow genetic diversity.

Keywords: lentil, genetic diversity, population structure, germplasm, SNP markers, agro-ecological zones

\section{INTRODUCTION}

Cultivated lentil (Lens culinaris Medikus ssp. culinaris) is the third most important cool-season grain legume in the world after chickpea (Cicer arietinum L.) and pea (Pisum sativum L.) (FAO, 2015). Legumes are important components in farming systems, providing environmental and ecological benefits through crop rotation, especially by contributing to soil fertility and rhizosphere diversity through biological $\mathrm{N}_{2}$ fixation. Global annual lentil production was around 5 million metric tons ( $\mathrm{Tg}$ ) from nearly 4.3 million ha (Mha) in 2013. Canada was the largest producer, contributing $38 \%$ of the world's production, followed by India, Turkey, and Australia (FAO, 2015). Lentil was one of the first domesticated grain legumes, originating from the Near East center of origin (Zohary, 1999). Lentil subsequently spread to central Asia and the Mediterranean Basin (Cubero, 1981; Lev-Yadun et al., 2000). It is a relatively new crop in North America, first introduced into northwest USA in the 1930s and into the northern temperate prairies of North America in the late 1960s (Muehlbauer et al., 1995). Globally today, lentil is grown in three major distinct agro-ecological zones: Mediterranean, sub-tropical savannah, 
and northern temperate (Tullu et al., 2011). These zones each exhibit different day lengths and temperatures, which limits the exchange of germplasm between agro-ecological adaptation zones.

Success in crop breeding is a function of heritability, genetic diversity, and selection. Natural agro-biodiversity stored in genebanks can be used to expand the diversity in crops. These collections are a vital source for discovering useful genes/alleles, which serve as a cornerstone for any pre-breeding program. There are currently 58,405 Lens accessions held in various genebanks worldwide (FAO, 2010). International Center for Agricultural Research in the Dry Areas (ICARDA) hosts the largest collection (19\%) followed by the National Bureau of Plant Genetic Resources, India (17\%) and the Australian temperate field crops collection (9\%). Currently, the most accessible and accessed lentil collection is held by the USDAARS (United States Department of Agriculture-Agricultural Research Service; https://npgsweb.ars-grin.gov/).

Assessments of genetic diversity and relationships among preserved germplasm have important implications both for facilitating reliable documentation of genetic resources and for identifying material with possible utility for specific breeding purposes, particularly in cultivated lentil and other species with a narrow genetic base. Lentil is an autogamous diploid species with seven chromosome pairs and a relatively large genome of $\sim 4 \mathrm{Gbp}$ in the haploid complement (Arumuganathan and Earle, 1991). Considerable genetic diversity has been reported in Lens genetic resources for agro-morphological and phenological characteristics (e.g., Erskine and Choudhary, 1986; Erskine et al., 1989; Lazaro et al., 2001; Zaccardelli et al., 2012; Cristóbal et al., 2014). Molecular markers, as the more reliable and powerful of genetic tools, have been deployed to characterize lentil genetic resources. Various molecular marker techniques and types have been used for this purpose. These include restriction fragment length polymorphisms (RFLPs, Havey and Muehlbauer, 1989), amplified fragment length polymorphisms (AFLPs, Sharma et al., 1996; Alghamdi et al., 2014), random amplified polymorphic DNAs (RAPDs, Abo-Elwafa et al., 1995; Ford et al., 1997; Ferguson et al., 1998; Sonnante and Pignone, 2001), and inter simple sequence repeats (ISSRs, Scippa et al., 2008; Toklu et al., 2009; El-Nahas et al., 2011). Simple sequence repeats (SSRs) have the most widely used DNA markers for assessing genetic diversity in lentil (see Liu et al., 2008; Babayeva et al., 2009; Kaur et al., 2011; Zaccardelli et al., 2012; Dikshit et al., 2015; Idrissi et al., 2015).

In recent years, genome-wide nucleotide-level surveys from different individuals within or across species have received increasing emphasis (Yang et al., 2015). Development of gene-based single nucleotide polymorphisms (SNP) markers is effective for detecting genetic diversity in plant species (Frascaroli et al., 2013; Semagn et al., 2014). SNPs are the most abundant type of polymorphism in all genomes, which allows high-throughput genotyping that is low cost, locus specific, and co-dominant with simple documentation. So far, only a limited number of SNP markers have been used to study the genetic diversity in lentil (Lombardi et al., 2014; Basheer-Salimia et al., 2015). The population structure of global lentil accessions has not been extensively characterized based on their agro-ecological adaptation zones. The main aims of this study were to assess the population structure and genetic variation of a group of 352 lentil germplasm accessions of Canadian breeding lines (northern temperate adaptation) and ex situ germplasm collections of a diverse origin using a relatively large number of SNP markers spanning the genome.

\section{MATERIALS AND METHODS Plant Material and DNA Extraction}

A total of 352 lentil accessions originating from 54 countries were collected from various sources including breeding lines obtained from the Crop Development Centre (CDC) collection in Saskatoon, Canada, from ICARDA, and the USDA-ARS. The accession numbers and origins of the selected accessions are given in Supplementary Table 1. Accessions were assigned to different major agro-ecological zones: Mediterranean, subtropical savannah (particularly northeast India, Nepal's lowland, and western Bangladesh), and Northern temperate according to global agro-ecological zones v3.0 (IIASA/FAO, 2012). In the Mediterranean adaptation zone, sowing occurs after the autumn equinox following a hot dry summer, prior to the winter solstice. Similarly, in the sub-tropical savannah (South Asia) planting occurs after the autumn equinox to take advantage of declining day lengths and temperatures during the juvenile phase and increases during the reproductive phase. Temperate adaptation zones require planting to occur after the spring equinox following a cold winter, prior to the summer solstice. Germplasm originating from Iran and Turkey were not assigned to specific agro-ecological zones due to different agro-ecological climates within the country borders for lentil production areas and a lack of specific collection location that would facilitate this classification. Furthermore, ICARDA breeding lines and USDA lines designated W6 \# (hereafter referred to as USA breeding lines) were not assigned to an agro-ecological zones, since they were not specifically attributed a particular zone.

Canadian lines, along with ICARDA germplasm, were grown in Saskatoon, Canada and leaf tissues from at least five different plants were collected for genomic DNA extraction using a modified CTAB extraction method (Doyle and Doyle, 1990). DNA samples from leaf samples of greenhouse grown plants were provided by the USDA-ARS for the USA germplasm (PI and W6 lines; see Simon and Hannan, 1995).

\section{SNP Discovery and Genotyping}

The Lc1536 GoldenGate high-throughput assay (Illumina, San Diego, CA) described by Sharpe et al. (2013) was used to genotype the 352 lentil accessions. The SNP genotyping was performed on an Illumina BeadStation 500G (Illumina, San Diego, CA) at the National Research Council (NRC), in Saskatoon, Canada with the protocol supported by Illumina (Fan et al., 2003). A robust set of 1440 of the SNP markers was used for further analyses.

\section{Statistical Analysis}

The SNP marker data were analyzed using PowerMarker v. 3.25 (Liu and Muse, 2005) to calculate minor allele frequency (MAF), 
heterozygosity, gene diversity, and polymorphic information content (PIC).

\section{Population Structure and Genetic Diversity}

The program STRUCTURE v. 2.3.4 (Pritchard et al., 2000) was used to calculate the most probable number of sub-populations $(K)$ in the panel. Five independent runs were conducted for each $K$ ranging from 1 to 10 with both a burn-in time and Markov Chain Monte Carlo (MCMC) replication number of 500,000 using an admixture model. Selection of the best $K$ value was based on the procedure presented in Evanno et al. (2005) by submitting the results for each $K$ to the STRUCTURE HARVESTER website, which returned the $L(K)$ and $\Delta K$-value (Earl and vonHoldt, 2012), as well as a basic understanding of the nature of the germplasm. The results from STRUCTURE were presented at the country level, using the "rworldmap" package (South, 2011) in R (R Core Development Team, 2015).

Genetic structure of the lentil population was analyzed by performing principal coordinate analysis (PCoA) using GenAlEx v. 6.5 (Peakall and Smouse, 2012) based on standardized covariance of genetic distance for co-dominant markers. GenAlEx v. 6.5 was also used to calculate Analysis of Molecular Variance (AMOVA) among and within assigned groups. The genetic distance between genotypes and countries were computed using Nei's standard genetic distance (Nei, 1973) with PowerMarker software. A dendrogram was constructed from Nei's distance matrix using UPGMA and the resulting tree was visualized using iTOL v. 3.0 (Letunic and Bork, 2011).

The sequences of all the markers used in this study are described in Sharpe et al. (2013). All data are also available in KnowPulse (http://knowpulse.usask.ca) and Supplementary Table 2.

\section{RESULTS}

\section{SNP Markers Information}

Of 1400 SNP markers, $5.46 \%$ were monomorphic among the accessions, $2.14 \%$ generated ambiguous products, and $7.21 \%$ were rejected on the grounds of excess missing data points (>30\% missing data per marker). This filtering resulted in 1194 high quality polymorphic SNP markers for use in the clustering analyses. Around $40 \%$ of these SNP markers were previously mapped and shown to be evenly distributed throughout the genome (Sharpe et al., 2013). The overall PIC value was 0.3092 \pm 0.0789 . Measurements of the average observed heterozygosity over all loci and gene diversity were $0.0375 \pm 0.0755$ and 0.3932 \pm 0.1160 , respectively (Supplementary Table 3 ).

\section{Population Structure and Genetic Relationship}

We ran STRUCTURE for a range of $K$ (number of fixed subgroups or cluster) from 1 to 10 on the entire set of accessions. The estimated $\log$ probability of the data $[\operatorname{Ln} P(K)]$ for each $K$ plateaued at $K=3$. The maximum $\Delta K$-value was also reached at $K=3$ (Supplementary Figure 1) suggesting three distinct groups. Geographical distribution of the 352 lentil accessions along with their projected population structure are shown in Figure 1. These three clusters closely reflected the origins, pedigrees and breeding history of germplasm used in this study. Accessions collected from southern Asia and the Middle East were assigned to the same gene pool. Lentil accessions originating mainly from the Mediterranean, northeast Africa (along the Nile valley from Egypt to Ethiopia), and South America were assigned to the second group, whereas the third group consisted mostly of genotypes from northern latitudes (Canada and Russian). Results from principal coordinates analysis (Figure 2) were consistent with those of STRUCTURE and UPGMA cluster analysis by revealing three clusters (Supplementary Figure 2). The AMOVA based on PhiPT-values revealed that genetic variation mainly occurred within groups (86\%), while the variation between the groups was 14\% (Supplementary Table 4).

Based on the 1194 polymorphic SNP markers, three pairs of accessions were genetically indistinguishable despite having different accession numbers: PI 163589 and PI 320945, PI 431675 and PI 431731, and PI 297284 and PI 297285.

Based on Nei's genetic distance matrix, a close relationship exists between Indian material and germplasm from Nepal, Pakistan, and Afghanistan $(0.0575,0.0202$, and 0.1310, respectively). Generally, South Asian and Middle Eastern (i.e., Iran and Turkey) germplasm grouped together, however, some of the Iranian and Turkish germplasm skewed to those from Mediterranean and northern climates. Within the second group, germplasm from Chile exhibited the closest relationship to germplasm from Morocco (0.0828). Canadian cultivars and breeding lines, representative of group 3, were closely related to much of the "W6" numbered lines which used in USDA lentil breeding program as parents in recombinant inbred lines development (USA breeding materials; 0.0968) and Russian (0.0961) germplasm (Supplementary Table 5). Most of the Canadian breeding lines were clustered together with the exception of CDC Plato, CDC Imigreen and CDC Cherie. Conversely, USA breeding lines and ICARDA breeding lines were relatively well distributed among the three groups (Figure 2).

\section{DISCUSSION}

The deep population structure of cultivated lentil and its importance in explaining genetic diversity underscores the value of using global lentil genetic resources to broaden the genetic base in breeding programs and to improve our knowledge of adaptation in this species. Given the sample size (individuals and country of origins) and relatively high marker density, this study identified three major clusters of germplasm reflecting the geographical origins, pedigrees, and breeding history of accessions. We categorized these clusters as (a) subtropical, South Asian, (b) Mediterranean, including Southern Europe and North Africa, and (c) northern temperate climates. These three groups reflect the main climatic regions in which lentil is widely grown as described by Tullu et al. (2011).

Lentil accessions from South Asia (primarily Nepal, India, and Pakistan) have a narrow genetic base and are genetically more isolated relative to other origins included in our study. This is 


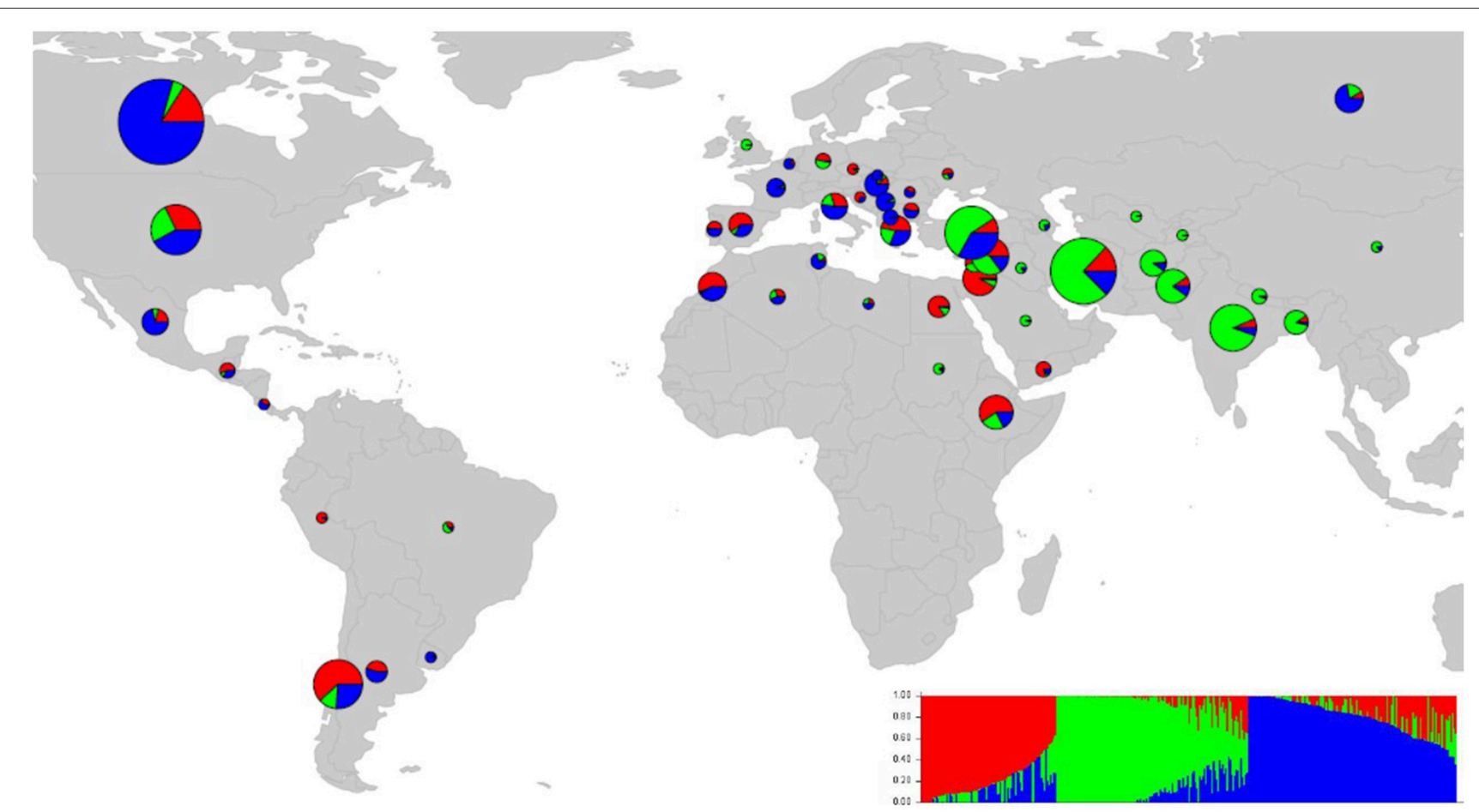

FIGURE 1 | Map of the world showing the country-specific distribution of 352 lentil accessions based on population structure $(\boldsymbol{K}=\mathbf{3})$. The size and color of pie chart is corresponding to sample size and the percentage of samples in each group, respectively.

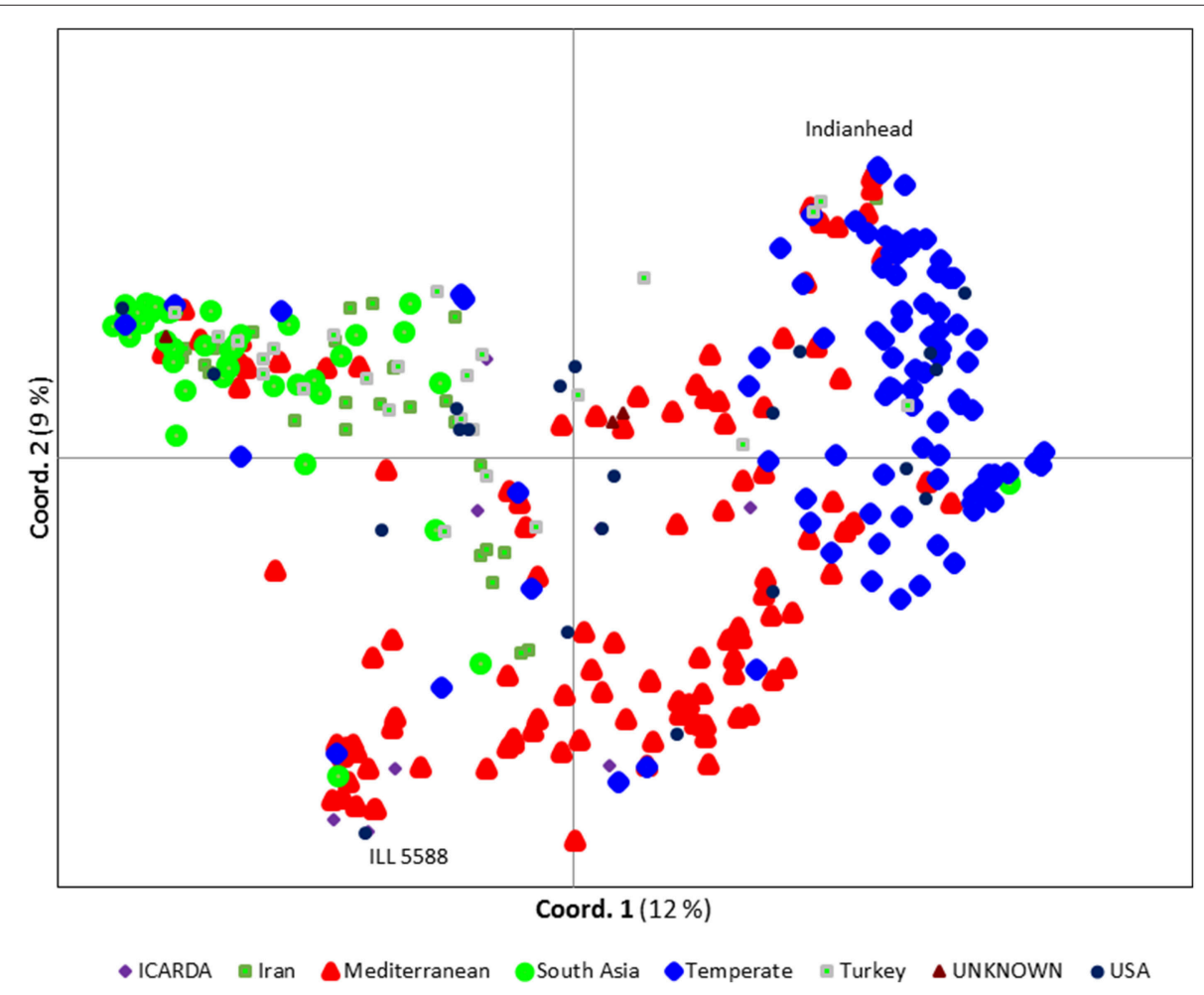

FIGURE 2 | Principal coordinate analysis (PCoA) of the 352 lentil accessions using 1194 polymorphic SNP markers. Each symbol/color combination represents different country of origin. The most two distinct accessions, Indianhead and ILL 5588, are labeled. 
likely a result of their specific phenological adaptation to the subtropical savannah environment and a potential genetic bottleneck during a time when lentil was introduced to South Asia around 2000 BC (Erskine et al., 1989). Most previous diversity studies of lentil diversity using molecular markers to date revealed two distinct groups: South Asian and all other origins (Ferguson et al., 1998; Alo et al., 2011; Kumar et al., 2014). In our study, however, germplasm from South Asia grouped with parts of material from Iran and Turkey, likely a stopping point on their move eastward on trade routes extending from the center of origin. A manual inspection of genetic distances between these regions shows that germplasm from Nepal, India, Afghanistan, and Pakistan are closely related, while relatively distant from Middle Eastern germplasm (Iran and Turkey). This implies there might be two distinct sub-groups within this particular cluster (South Asian and Middle Eastern). Germplasm from Afghanistan was found to be related to germplasm from South Asia as was previously reported using another set of molecular markers (e.g., Ferguson et al., 1998; Kumar et al., 2014).

Our results demonstrate that the Mediterranean, North African, and Chilean germplasm collections are similar with only a few deviations, following the classification of the Mediterranean agro-ecological zone. This is consistent with results from Ferguson et al. (1998) and Lombardi et al. (2014). Lentil was domesticated in the Eastern Mediterranean around 7-8 BC, after which it spread to Europe. Lentil was introduced to South America by the Spanish via Chile (after 1500 AD). Lentil is mainly grown as a winter crop in these regions, under conditions of declining day length followed by gradual increase in day length and temperature during the life cycle (6-7 month). This may explain the similarity among accessions of these regions.

Nearly $50 \%$ of the world's lentil production now originates from Canada (northern temperate climate) and Australia (Mediterranean climate) (FAO, 2015), regions in which lentil is a relatively new crop. Clearly, the breeding programs in these two regions have drawn from distinct global lentil genetic resources that originate in regions with climatic and growing conditions that match their local conditions. Most Canadian germplasm is related to Laird and Eston. Laird was the first Canadian lentil cultivar, released in 1978. It was a pure line selection derived from PI 343028, originally from Russia, selected for higher yield and larger seeds in the Canadian growing environment. Eston, the second cultivar released in Canada in 1980, was similarly selected for adaptation from a Turkish accession, PI 179307. The results from PCoA and genetic distance cluster analyses all demonstrate a narrower genetic variability among Canadian breeding lines. This may be attributable to relatively recent adaptation to long day northern temperate conditions in the prairies of Canada and selection pressure for improving yield and specific adaptations. A similar trend has been reported for Australian lentil breeding lines and cultivars (Lombardi et al., 2014). In contrast, breeding lines from the USA and ICARDA represent the most diverse material in this study and elsewhere (Alghamdi et al., 2014). ICARDA breeding strategies are more internationally-focused, covering a wide variety of regions and adaptive traits as part of CGIAR's policy. For example, ILL 7502 and ILL 7537 were bred for Western Asian climates, while ILL 8008 was targeted for South Asian climates (Shiv Kumar, personal communication).

Lentil domestication already has led to $\sim 40 \%$ loss in genetic diversity (Alo et al., 2011) and breeding in specific regions has narrowed this even more. Breeding new genotypes for traits of interest requires sampling broad genetic diversity. The various statistic methods we employed here support the presence of considerable genetic variability in global germplasm that is not being accessed in some regions. The results from population structure and PCoA in this study show, to some extent, a separation by origin of the accessions with closely related pedigrees typically group together. Phenotyping of available genetic diversity has demonstrated the importance of incorporating exotic germplasm into breeding programs focusing on biotic and abiotic stresses. For example, ILL 5588 (also known as PI 592998), an ICARDA accession originally collected from Jordan, is a known source of Ascochyta blight resistance in lentil (Erskine et al., 1996). It is clearly distinct from most of the temperate germplasm (Figure 2) but it has been used in the pedigrees of some Canadian lines, including CDC Plato and CDC Cherie. This may explain why they did not cluster with the other Canadian lines.

The grouping of some accessions outside of their geographic origin may be the result of outcrossing, migration, and adaptation during cultivation of the crop by local farmers. For example, Moroccan germplasm expresses slightly less variation compared to those from Turkey due to narrower environmental conditions (Idrissi et al., 2015). The major agro-morphological changes related to adaptation are mostly improvements to yield, increasing seed size, tolerance to biotic and abiotic stresses as well as improving market-dependent quality traits. Another source of division may be the growth habit of spring and winter types, which are most adapted to different climatic regions. It has also been noted that photoperiod plays a critical role to characterize lentil cultivation areas into the respective climatic regions (Tullu et al., 2011).

The availability of EST sequences and SNP discovery are strong tools for investigating polymorphism in different species, for quantifying biological factors that influence the patterns of genetic diversity and for investigating bottlenecks due to the domestication of crop species. An allele-specific Illumina Golden Gate 1536-SNP array was constructed using SNPs discovered in expressed sequence tag (EST) sequences from nine L. culinaris accessions. This study has confirmed that the sub-set of SNP markers previously reported by Sharpe et al. (2013) can provide good resolution at low cost for genetic characterization of cultivated lentil germplasm in relation to the world's agroecological zones.

\section{CONCLUSIONS}

Global cultivated lentil germplasm selected for this study clustered primarily based on eco-geographical origin into three basic groups: subtropical savannah, Mediterranean, and northern temperate. The narrow genetic base of some groups of germplasm (e.g., Canadian and South Asian) raises concern over the loss/penalty in yield due to biotic and abiotic stresses, particularly 
with the threat to global food security from climate change. This highlights the importance of harnessing the potential of lentil wild species in breeding programs by introgression of favorable genes from other regions. Based on the results from this study, it is also clear that breeding programs still have a lot of genetic diversity to mine within the cultivated species.

\section{AUTHOR CONTRIBUTIONS}

$\mathrm{KB}$ and $\mathrm{AV}$ designed the research; HK, and CTC analyzed data; MD and MF contributed to data generation; CJC and RM contributed germplasm and reagents; HK, CTC, KB, and AV contributed to writing of the manuscript.

\section{REFERENCES}

Abo-Elwafa, A., Muraik, K., and Shimada, T. (1995). Intra- and inter-specific variation in Lens species revealed by RAPD markers. Theor. Appl. Genet. 90, 335-340. doi: 10.1007/BF00221974

Alghamdi, S. S., Khan, A. M., Ammar, M. H., El-Harty, E. H., Migdadi, H. M., Abd El-Khalik, S. M., et al. (2014). Phenological, nutritional and molecular diversity assessment among 35 introduced lentil (Lens culinaris Medik.) genotypes grown in Saudi Arabia. Int. J. Mol. Sci. 15, 277-295. doi: 10.3390/ijms15010277

Alo, F., Furman, B. J., Akhunov, E., Dvorak, J., and Gepts, P. (2011). Leveraging genomic resources of model species for the assessment of diversity and phylogeny in wild and domesticated lentil. J. Hered. 102, 315-329. doi: 10.1093/jhered/esr015

Arumuganathan, K., and Earle, E. D. (1991). Nuclear DNA content of some important plant species. Plant Mol. Biol. 9, 208-218. doi: 10.1007/BF02672069

Babayeva, S., Akparov, Z., Abbasov, M., Mammadov, A., Zaifizadeh, M., and Street, K. (2009). Diversity analysis of Central Asia and Caucasian lentil (Lens culinaris Medik.) germplasm using SSR fingerprinting. Genet. Resour. Crop. Evol. 56, 293-298. doi: 10.1007/s10722-009-9414-6

Basheer-Salimia, R., Camilli, B., Scacchi, S., Noli, E., and Awad, M. (2015). Assessment of genetic diversity in lentils (Lens culinaris Medik.) based on SNPs. Genet. Mol. Res. 14, 5870-5878. doi: 10.4238/2015.June.1.4

Cristóbal, M. D., Pando, V., and Herrero, B. (2014). Morphological characterization of lentil (Lens culinaris Medik.) landraces from Castilla Y León, Spain. Pak. J. Bot. 46, 1373-1380.

Cubero, J. I. (1981). “Origin, taxonomy and domestication," in Lentils, eds C. Webb and G. Hawtin (London: Commonwealth Agricultural Bureaux), 15-38.

Dikshit, H. K., Singh, A., Singh, D., Aski, M. S., Prakash, P., Jain, N., et al. (2015). Genetic diversity in Lens species revealed by EST and genomic simple sequence repeat analysis. PLoS ONE 10:0138101. doi: 10.1371/journal.pone.0138101

Doyle, J. J., and Doyle, J. L. (1990). Isolation of plant DNA from fresh tissue. Focus $12,13-15$

Earl, D. A., and vonHoldt, B. M. (2012). STRUCTURE HARVESTER: a website and program for visualizing STRUCTURE output and implementing the Evanno method. Conserv. Genet. Resour. 4, 359-361. doi: 10.1007/s12686-0119548-7

El-Nahas, A., El-Shazly, H., Ahmed, S., and Omran, A. (2011). Molecular and biochemical markers in some lentil (Lens culinaris Medik.) genotypes. Ann. Agric. Sci. 56, 105-112. doi: 10.1016/j.aoas.2011.11.001

Erskine, W., Adham, Y., and Holly, L. (1989). Geographic distribution of variation in quantitative traits in a world lentil collection. Euphytica 43, 97-103. doi: 10.1007/BF00037901

Erskine, W., Bayaa, B., and Saxena, M. C. (1996). Registration of ILL 5588 lentil germplasm resistant to vascular wilt and ascochyta blight. Crop Sci. 36, 1080. doi: 10.2135/cropsci1996.0011183x0036000400055x

Erskine, W., and Choudhary, M. A. (1986). Variation between and within lentil landraces from Yemen republic. Euphytica 35, 695-700. doi: $10.1007 / \mathrm{BF} 00028577$

\section{ACKNOWLEDGMENTS}

This research was supported by Natural Sciences and Engineering Research Council of Canada (NSERC) and the Saskatchewan Pulse Growers. The authors would like to acknowledge Rob Stonehouse and Lacey-Anne Sanderson for their kind technical support during the experiments.

\section{SUPPLEMENTARY MATERIAL}

The Supplementary Material for this article can be found online at: http://journal.frontiersin.org/article/10.3389/fpls.2016. 01093

Evanno, G., Regnaut, S., and Goudet, J. (2005). Detecting the number of clusters of individuals using the software structure: a simulation study. Mol. Ecol. 14, 2611-2620. doi: 10.1111/j.1365-294X.2005.02553.x

Fan, J. B., Oliphant, A., Shen, R., Kermani, B. G., Garcia, F., Gunderson, K. L., et al. (2003). Highly parallel SNP genotyping. Cold Spring Harb. Symp. Quant. Biol. 68, 69-78. doi: 10.1101/sqb.2003.68.69

FAO (2010). The Second Report on the State of the World's Plant Genetic Resources for Food and Agriculture. Rome.

FAO (2015). FAOSTAT. Food and Agriculture Organization of the United Nations. Rome. Available online at: http://faostat.fao.org

Ferguson, M. E., Robertson, L. D., Ford-Lloyd, B. V., Newbury, H. J., and Maxted, N. (1998). Contrasting genetic variation amongst lentil landraces from different geographical origins. Euphytica 102, 265-273. doi: 10.1023/A:1018331432580

Ford, R., Pang, E. C. K., and Taylor, P. W. J. (1997). Diversity analysis and species identification in lens using PCR generated markers. Euphytica 96, 247-255. doi: 10.1023/A:1003097600701

Frascaroli, E., Schrag, T. A., and Melchinger, A. E. (2013). Genetic diversity analysis of elite European maize (Zea mays L.) inbred lines using AFLP, SSR, and SNP markers reveals ascertainment bias for a subset of SNPs. Theor. Appl. Genet. 126, 133-141. doi: 10.1007/s00122-012-1968-6

Havey, M. J., and Muehlbauer, F. J. (1989). Variability for restriction fragment lengths and phylogenies in lentil. Theor. Appl. Genet. 77, 839-843. doi: $10.1007 / \mathrm{BF} 00268336$

Idrissi, O., Udupa, S. M., Houasli, C., De Keyser, E., Van Damme, P., and De Riek, J. (2015). Genetic diversity analysis of Moroccan lentil (Lens culinaris Medik.) landraces using simple sequence repeat and amplified fragment length polymorphisms reveals functional adaptation towards agro-environmental origins. Plant Breed 134, 322-332. doi: 10.1111/pbr.12261

IIASA/FAO (2012). Global Agro-ecological Zones (GAEZv3.0). Rome; Laxenburg: IIASA: FAO.

Kaur, S., Cogan, N., Pembleton, L., Shinozuka, M., Savin, K., et al. (2011). Transcriptome sequencing of lentil based on second-generation technology permits large-scale unigene assembly and SSR marker discovery. BMC Genomics 12:265. doi: 10.1186/1471-2164-12-265

Kumar, S., Hamweih, A., Manickavelu, A., Kumar, J., Sharma, T. R., and Baum, M. (2014). "Advances in lentil genomics", in Legumes in Omics Era, eds S. Gupta, N. Nadarajan, and D. S. Gupta (New York, NY: Springer), 111-130.

Lazaro, A., Ruiz, M., De la Rosa, L., and Martin, I. (2001). Relationships between agro/morphological characters and climatic parameters in Spanish landraces of lentil (Lens culinaris Medik.). Genet. Resour. Crop Evol. 48, 239-249. doi: 10.1023/A:1011234126154

Letunic, I., and Bork, P. (2011). Interactive tree of life v2: online annotation and display of phylogenetic trees made easy. Nucleic Acids Res. 39, W475-W478. doi: 10.1093/nar/gkr201

Lev-Yadun, S., Gopher, A., and Abbo, S. (2000). The cradle of agriculture. Science 288, 1602-1603. doi: 10.1126/science.288.5471.1602

Liu, J., Guan, J. P., Xu, D. X., Zhang, X. Y., Gu, J., and Zong, X. X. (2008). Genetic diversity and population structure in lentil (Lens culinaris Medik.) 
germplasm detected by SSR markers. Acta Agron. Sin. 34, 1901-1909. doi: 10.1016/S1875-2780(09)60015-8

Liu, K., and Muse, S. V. (2005). PowerMarker: integrated analysis environment for genetic marker data. Bioinformatics 21, 2128-2129 doi: 10.1093/bioinformatics/bti282

Lombardi, M., Materne, M., Cogan, N. O., Rodda, M., Daetwyler, H. D., Slater, A. T., et al. (2014). Assessment of genetic variation within a global collection of lentil (Lens culinaris Medik) cultivars and landraces using SNP markers. BMC Genet. 15:150. doi: 10.1186/s12863-014-0150-3

Muehlbauer, F. J., Kaiser, W. J., Clement, S. L., and Summerfield, R. J. (1995). Production and breeding of lentil. Adv. Agron. 54, 283-332. doi: 10.1016/S00652113(08)60902-5

Nei, M. (1973). Analysis of gene diversity in subdivided populations. Proc. Natl. Acad. Sci. U.S.A. 70, 3321-3323. doi: 10.1073/pnas.70.12.3321

Peakall, R., and Smouse, P. E. (2012). GenAlEx 6.5: genetic analysis in Excel. Population genetic software for teaching and research-an update. Bioinformatics 28, 2537-2539. doi: 10.1093/bioinformatics/bts460

Pritchard, J. K., Stephens, M., and Donnelly, P. (2000). Inference of population structure using multilocus genotype data. Genetics, 155, 945-959.

R Core Development Team (2015). R: A Language and Environment for Statistical Computing. Vienna: R Foundation for Statistical Computing. Available online at: http://www.R-project.org

Scippa, G. S., Trupiano, D., Rocco, M., Viscosi, V., Di Michele, M., D’Andrea, A., et al. (2008). An integrated approach to the characterization of two autochthonous lentil (Lens culinaris) landraces of Molise (south-central Italy). Heredity 101, 136-144. doi: 10.1038/hdy.2008.39

Semagn, K., Babu, R., Hearne, S., and Olsen, M. (2014). Single nucleotide polymorphism genotyping using Kompetitive Allele Specific PCR (KASP): overview of the technology and its application in crop improvement. Mol. Breed. 33, 1-14. doi: 10.1007/s11032-013-9917-x

Sharma, S. K., Knox, M. R., and Ellis, T. H. N. (1996). AFLP analysis of the diversity and phylogeny of Lens and its comparison with RAPD analysis. Theor. Appl. Genet. 93, 751-758. doi: 10.1007/BF00224072

Sharpe, A., Ramsay, L., Sanderson, L.-A., Fedoruk, M. J., Clarke, W. E., Li, R., et al. (2013). Ancient orphan crop joins modern era: gene-based SNP discovery and mapping in lentil. BMC Genom 14:192. doi: 10.1186/1471-2164-14-192
Simon, C. J., and Hannan, R. M. (1995). Development and use of core subsets of cool-season food legume germplasm collections. HortScience 30, 907.

Sonnante, G., and Pignone, D. (2001). Assessment of genetic variation in a collection of lentil using molecular tools. Euphytica 120, 301-307. doi: 10.1023/A:1017568824786

South, A. (2011). rworldmap: a new R package for mapping global data. $R J .3$, 35-43.

Toklu, F., Karaköy, T., Hakle, I., Bicer, T., Brandolini, A., Kilian, B., et al. (2009). Genetic variation among lentil (Lens culinaris Medik.) landraces from Southeast Turkey. Plant Breed. 128, 178-186. doi: 10.1111/j.14390523.2008.01548.x

Tullu, A., Diederichsen, A., Suvorova, G., and Vandenberg, A. (2011). Genetic and genomic resources of lentil: status, use and prospects. Plant Genet. Resour. 9, 19-29. doi: 10.1017/S1479262110000353

Yang, H., Li, C., Lam, H. M., Clements, J., Yan, G., and Zhao, S. (2015). Sequencing consolidates molecular markers with plant breeding practice. Theor. Appl. Genet. 128, 779-795. doi: 10.1007/s00122-015-2499-8

Zaccardelli, M., Lupo, F., Piergiovanni, A. R., Laghetti, G., Sonnante, G., Daminati, M. G., et al. (2012). Characterization of Italian lentil (Lens culinaris Medik.) germplasm by agronomic traits, biochemical and molecular markers. Genet. Resour. Crop Evol. 59, 727-738. doi: 10.1007/s10722-011-9714-5

Zohary, D. (1999). Monophyletic vs. polyphyletic origin of the crops on which agriculture was founded in the Near East. Genet. Resour. Crop Evol. 46, 133-142. doi: 10.1023/A:1008692912820

Conflict of Interest Statement: The authors declare that the research was conducted in the absence of any commercial or financial relationships that could be construed as a potential conflict of interest.

Copyright (c) 2016 Khazaei, Caron, Fedoruk, Diapari, Vandenberg, Coyne, McGee and Bett. This is an open-access article distributed under the terms of the Creative Commons Attribution License (CC BY). The use, distribution or reproduction in other forums is permitted, provided the original author(s) or licensor are credited and that the original publication in this journal is cited, in accordance with accepted academic practice. No use, distribution or reproduction is permitted which does not comply with these terms. 


\section{OPEN ACCESS}

Edited by:

Antonio M. De Ron,

National Spanish Research Council,

Spain

Reviewed by:

Ana M. Gonzalez,

Misión Biológica de Galicia-Spanish

National Research Council, Spain

Tom Warkentin

University of Saskatchewan, Canada

*Correspondence:

Miguel Curto,

Department of Plant Breeding Institute for Sustainable Agriculture, Spanish National Research Council, Apdo. 4084, E-14080 Córdoba, Spain

b72curum@uco.es

Specialty section

This article was submitted to

Crop Science and Horticulture,

a section of the journal

Frontiers in Plant Science

Received: 05 May 2015

Accepted: 26 June 2015

Published: 09 July 2015

Citation:

Curto M, Krajinski F, Schlereth A and

Rubiales D (2015) Transcriptional profiling of Medicago truncatula during Erysiphe pisi infection.

Front. Plant Sci. 6:517. doi: 10.3389/fpls.2015.00517

\section{Transcriptional profiling of Medicago truncatula during Erysiphe pisi infection}

\author{
Miguel Curto $^{1 *}$, Franziska Krajinski ${ }^{2}$, Armin Schlereth ${ }^{2}$ and Diego Rubiales ${ }^{1}$ \\ 'Department of Plant Breeding, Institute for Sustainable Agriculture, Spanish National Research Council, Córdoba, Spain, \\ ${ }^{2}$ Department of Plant-Microbe Interactions, Max Planck Institute of Molecular Plant Physiology, Potsdam, Germany
}

Resistance to powdery mildew has been studied in a number of plant species, yet the molecular mechanisms remain largely unknown. Transcription factors (TFs) play a critical role in the plant defense response by regulating the transcriptional machinery which coordinates the expression of a large group of genes involved in plant defense. Using high-throughput quantitative real-time PCR (qPCR) technology more than 1000 Medicago truncatula TFs were screened in a pair of susceptible and resistant genotypes of $M$. truncatula after $4 \mathrm{~h}$ of Erysiphe pisi infection. Seventy nine TF genes, belonging to 33 families showed a significant transcriptional change in response to $E$. pisi infection. Forty eight TF genes were differentially expressed in the resistant genotypes compared to the susceptible one in response to $E$. pisi infection, including pathogenesis-related transcriptional factors, AP2/EREBP (APETALA2/ETHYLENE-RESPONSIVE ELEMENT BINDING FACTORS), WRKY (highly conserved WRKYGQK amino-acid sequence), MYB (Myeloblastoma), homeodomain (HD) and zinc finger $\mathrm{C}_{2} \mathrm{C}_{2}\left(\mathrm{CYS}_{2}-\mathrm{CYS}_{2}\right), \mathrm{C}_{2} \mathrm{H}_{2}$, $\left(\mathrm{CYS}_{2}-\mathrm{HIS}_{2}\right)$, LIM (Lin-11, Isl-1, Mec-3) gene families, which are involved in known defense responses. Our results suggest that these TF genes are among the E. pisi responsive genes in resistant $M$. truncatula that may constitute a regulatory network which controls the transcriptional changes in defense genes involved in resistance to E. pisi.

Keywords: Erysiphe pisi, legumes, Medicago truncatula, transcription factors, qPCR

\section{Introduction}

Plants grown in the natural environment are confronted by a variety of pathogens. Remaining healthy depends on their ability to recognize pathogens and to activate defense mechanisms against them. The plant defense responses are regulated by a broad number of signaling pathways. Transcription factors (TFs) control the transfer of genetic information from DNA to RNA by activation or repression of transcription, playing important roles in plant development and defense by regulating different signaling pathways (Singh et al., 2002; Udvardi et al., 2007). Data from several plant genome projects suggest that more than five percent of the plant genome encodes TF sequences (around 2000 TFs) (Riechmann and Ratcliffe, 2000). Therefore, many biologic processes, including responses to pathogens, are controlled by multiple genes managed by TFs (Singh et al., 2002). Several analyses have shown their differential expression in plants as responses to interactions with biotic and abiotic effectors (Udvardi et al., 2007). In spite of the importance 
of legumes as sources of protein and oil, and in the symbiotic nitrogen fixation, less than one percent of the transcript-specific regulation roles of TFs have been characterized in legumes (Udvardi et al., 2007). Among legumes, Medicago truncatula is a model with key attributes such as self-fertility, rapid generation time, and a small diploid genome (Singh et al., 2007) that have facilitated the use of molecular and genetic tools (Rose, 2008).

Powdery mildews are biotrophic plant pathogens that seriously constrain crop production worldwide (Bélanger et al., 2002). Erysiphe spp. cause considerable losses in various important legume crops (Sillero et al., 2006). This fungus has been classified into three physiologically specialized forms, f.sp. pisi specialized on Pisum, f.sp. medicaginis specialized on Medicago, and f.sp. vicia sativa, specialized on Vicia (Falloon and Viljanen-Rollinson, 2001). Breeding for powdery mildew resistance is the most desirable strategy to control this disease by means of resistant cultivars (Fondevilla and Rubiales, 2012; Rubiales et al., 2015). Consequently, several genes involved in resistance to powdery mildew have been reported in different plant species (Fondevilla et al., 2007; Yang et al., 2013; Barilli et al., 2014; Curto et al., 2015; Iglesias-García et al., 2015). High-throughput methods have resulted in identification of genes potentially associated with specific processes and characterization of the regulatory networks that control their expression (Czechowski et al., 2004; Caldana et al., 2007). Among them, DNA microarrays have been used successfully to characterize global gene expression patterns in M. truncatula (Foster-Hartnett et al., 2007; Samac et al., 2011; Zhang et al., 2014; Curto et al., 2015; Song et al., 2015) providing detailed information of metabolic pathways involved in the analyzed systems. Previous studies have analyzed the E. pisi/M. truncatula pathosystem (Foster-Hartnett et al., 2007; Samac et al., 2011; Curto et al., 2015) using different genotypes and microarray platforms, such as Mt16kOLI1, Mt16kOLI1plus (Küster et al., 2004, 2007), and Affymetrix GeneChip ${ }^{\circledR}$ (http://www.affymetrix. com). These studies have increased the knowledge of mechanisms involved in E. pisi resistance in $M$. truncatula, which are agreement that a wide variety of mechanisms and pathways are involved in E. pisi resistance including pathogenesis-related genes (i.e., PR10, Pprg2), as well as other genes involved in signal transduction, cell wall metabolism (i.e., Glucan endo-1,3-betaD-glucosidase, Pectinase) and abiotic stress, such as Heat shock protein 17.7, UVB-resistance protein BudCAR5, and Dehydrationresponsive protein $(R D 22)$. Although DNA microarrays have been shown to be five times less sensitive than qPCR (Czechowski et al., 2004), due to its high cost qPCR remains a technique used for low- to middle-scale studies. Several large-scale TF profiling approaches have employed the M. truncatula qPCRbased platform available (Kakar et al., 2008) in various studies (Verdier et al., 2008; Gao et al., 2010; Madrid et al., 2010; Villegas-Fernández et al., 2014). In this study, we screened the TF transcriptome of $M$. truncatula for altered expression during E. pisi infection using qPCR. Previous histological assessments showed that the resistance mechanisms carried out by the resistant genotype SA1306 is mainly related to hampering spore germination and further colony establishment by epidermal cell death as a hypersensitive response to E. pisi germlings that develop appressoria (Curto et al., 2015). Several mechanisms capable of monitoring changes in the plant cell wall are carried out by cellular signaling responses (Ringli, 2010; Cheung and $\mathrm{Wu}, 2011$ ). The present study has allowed us to identify the transcription factor-encoding genes involved in the $E$. pisi/M. truncatula pathosystem, which are candidates for further functional studies. In addition, this approach provides a model for the regulatory network controlling the expression of TF genes in this pathosystem.

\section{Materials and Methods}

\section{Plant Material, Growth Conditions, and Inoculation}

The study was performed through an analysis of two genotypes of M. truncatula, the commercial cultivar M. truncatula Gaertn. v. Parabinga and the accession SA1306, shown to be susceptible and resistant, respectively to E. pisi f.sp. Medicaginis (Curto et al., 2015).

The seeds of $M$. truncatula were pre-soaked in filter paper, kept in dark conditions at $4^{\circ} \mathrm{C}$ for $24 \mathrm{~h}$, and germinated in the dark for $48 \mathrm{~h}$ in a growth chamber at $65 \%$ relative humidity and $20^{\circ} \mathrm{C}$. The seedlings were placed in pots $(125 \mathrm{ml})$ containing a $1: 1$ mixture of perlite and sand substrate, fertilized with half-strength Hoagland's solution (Hoagland and Arnon, 1950) 3 times a week, and grown $\left(25^{\circ} \mathrm{C}, 12 \mathrm{~h}\right.$ photoperiod, $250 \mu \mathrm{mol} / \mathrm{m}^{2}$ light intensity, $80 \%$ relative humidity) for 4 weeks before pathogen inoculation.

As pathogen, we used a monosporic isolate of E. pisi f.sp. medicaginis strain $\mathrm{CO} 05$, derived from a mildew population collected on M. truncatula plants at Córdoba (Prats et al., 2007), which was maintained and propagated by infecting Parabinga plants. One day before inoculation the highly infected plants were shaken to remove old conidia in order to produce an inoculum with vigorous young spores. M. truncatula plants of both lines were inoculated when the fourth trifoliate leaf was completely expanded (4-week-old-plant). Inoculation was carried out using a setting tower to give an inoculum density of 5 conidia $\mathrm{mm}^{-2}$ (Prats et al., 2007). Five plants of each M. truncatula genotype were inoculated per triplicate, at the same time keeping five noninfected plants as a control, in total $60 \mathrm{M}$. truncatula plants. Thus, three independent biological replicates, five plants per condition (control and infected) and per genotype (Parabinga and SA1306), were performed with leaflets of control and E. pisi infected plants of both $M$. truncatula genotypes that were harvested $4 \mathrm{~h}$ after E. pisi inoculation. The samples were immediately washed with water, blot dried with filter paper, frozen in liquid nitrogen, and stored at $-80^{\circ} \mathrm{C}$ until RNA extraction.

\section{RNA Extraction, cDNA Synthesis, and qPCR Assays}

RNA was purified from collected samples using the Nucleospin RNA II kit (MACHEREY-NAGEL, Bethlehem, PA) following the manufacturer's procedure. The integrity of total RNA was assessed on 1\% agarose gels (samples were denatured in formaldehyde/formamide buffer), as well as for quantity and purity by using a NanoDrop ND-100 spectrophotometer (NanoDrop Technologies, Wilmington, DE) to measure the 
optical density. RNA samples were digested with RNasefree DNase1 (Ambion Inc., Houston, TX), according to the manufacturer's protocol. The absence of genomic DNA was checked by PCR analysis using primers designed on the M. truncatula ubiquitin gene intron sequence (Kakar et al., 2008).

Synthesis of first-strand CDNA was carried out with oligodT12-18 (Qiagen, Hilden, Germany) using SuperScript III reverse transcriptase (Invitrogen $\mathrm{GmbH}$, Karlsruhe, Germany). The efficiency of cDNA synthesis was evaluated by real-time quantitative PCR (qPCR) amplification of $5^{\prime}$ and $3^{\prime}$ regions of two reference genes, GAPDH (Glyceraldehyde 3 phosphate dehydrogenase) and Ubiquitin (Kakar et al., 2008). A single peak in the dissociation curve at the end of the PCR reaction allowed confirmation of the specificity of the amplified products.

A M. truncatula transcription factor platform composed of more than 1000 M. truncatula TFs gene-specific primers was used to carry out the qPCR experiments (Kakar et al., 2008). The qPCR reactions were carried out in triplicate in an optical 384-well plate with an ABI PRISM ${ }^{\circledR} 7900$ HT Sequence Detection System (Applied Biosystems, Foster City, CA) as described previously (Kakar et al., 2008).

\section{Normalization and Data Analysis}

SDS software ver. 2.3 (Applied Biosystems) was used to analyze fluorescent signals and calculate the quantification cycle $(\mathrm{Cq})$ (Bustin et al., 2009). The baseline data were collected from the fluorescence signal between cycles 3 and 15, and used to correct the fluorescence signal of the samples. The PCR efficiencies (E) and correlation coefficients $\left(R^{2}\right)$ from linear regression analysis were calculated for each performed PCR reaction by the software LinRegPCR ver. 7.5 as described previously (Kakar et al., 2008) (Table S1). The amplification reactions with $R^{2}<0.99$ that showed efficiencies lower than 1.8 were excluded for further analysis (24.4\% of reactions). TF genes were considered detected if they were expressed in at least two biological replicates with a $\mathrm{Cq}<40$.

Eight reference genes encoding Pentatricopeptide repeat protein (PPRrep; TC96273), Protein phosphatase 2A subunit A3 (PDF 2; TC107161), Polypyrimidine tract-binding protein homolog (PTB; TC111751), Helicase (CB892427), Ubiquitin (TC102473), Ubiquitin-protein ligase 7 (UPL7; TC111218), Ubiquitin-conjugating enzyme E2 (UBC; AW686873), and Ubiquitin-conjugating enzyme E2 9 (UBC9; TC106312) (Kakar et al., 2008) were studied in order to determine the best suited reference genes for transcript normalization. The expression stability of the eight reference genes was analyzed by the geNorm software (Vandesompele et al., 2002; Hellemans et al., 2007) for each cDNA sample under study. In addition, pair-wise comparison analysis allowed determining the optimal number of reference genes in this assay (Vandesompele et al., 2002).

Expression values were calculated from $\mathrm{E}^{\mathrm{Cq}}$ of each individual plot. To normalize the gene expression of each PCR reaction, ratios of the geometric mean of the selected reference genes to the different biological conditions were used using the Equation (1):

$$
\left(\frac{\left.\mathrm{E}_{\text {ref }} \text { (Cq ref }\right)}{\left.\mathrm{E}_{\text {gene }} \text { (Cq gene }\right)}\right)
$$

The relative induction/repression of TFs from E. pisi infected samples compared to untreated samples was calculated using the Equation (2):

$$
\left(\frac{\left(\frac{\mathrm{E}_{\text {ref }}(\mathrm{Cq} \text { ref })}{\mathrm{E}_{\text {gene }}(\mathrm{Cq} \text { gene })}\right) \text { infected condition }}{\left(\frac{\mathrm{E}_{\text {ref }}(\mathrm{Cq} \text { ref })}{\mathrm{E}_{\text {gene }}(\mathrm{Cq} \text { gene })}\right) \text { untreated condition }}\right)
$$

Non-parametric Levene's test and Spearman's correlation coefficient were used to verify the equality of variances in the samples and to study the similarity between TF gene expression profiles, respectively. TF genes showing statistically significant differences $(P<0.05)$ were clustered using a hierarchical cluster analysis by complete linkage. A model for the regulatory network controlling the expression of regulated genes induced by E. pisi in both M. truncatula genotypes studied was built using NodeXL (http://nodexl.codeplex.com).

\section{Results}

\section{Evaluation of Resistance in Medicago truncatula Genotypes}

Differences in the response to $E$. pisi between the two genotypes were not yet visible at the time the leaves were sampled for RNA extraction. Powdery mildew infection was macroscopically visible on remaining leaflets 2 weeks after inoculation, with profuse sporulation in the susceptible Parabinga genotype and absence of symptoms in the resistant SA1306 genotype (Figure S1). Thus, previous study described that colony formation was much higher in Parabinga than in SA1306, as well as the hypersensitive response associated with epidermal cell death was negligible in Parabinga, but marked in SA1306 (Curto et al., 2015). Former studies unveiled that at early E. pisi infection times, such as $4 \mathrm{~h}$ (Curto et al., 2015) and $12 \mathrm{~h}$ (Samac et al., 2011), M. truncatula plants induce a highly number of metabolic pathways in response to $E$. pisi infection. Hence, we choose to analyze the TF transcriptome of $M$. truncatula during E. pisi infection at $4 \mathrm{~h}$ after pathogen infection.

\section{Selection of Reference Genes}

Eight reference genes were studied to determine those best suited for transcript normalization. Transcripts levels of all reference genes were calculated, in each cDNA sample, using the average expression stability $(\mathrm{M})$ calculated by geNorm software (Figure 1A). All reference genes showed high average expression stability $(M<0.66)$ among them the UBC9, Helicase, PTB, and UPL7 reference genes showed the lower average expression stability (M) indicating a greater transcript stability (Figure 1A). Pair-wise variation (V) was also calculated as described by Vandesompele et al. (2002) allowing determining the optimal number of stable reference genes. The results indicated that the inclusion of a third gene $\left(\mathrm{V}_{3 / 4}\right)$ or more genes $\left(\mathrm{V}_{4 / 5}, \mathrm{~V}_{5 / 6}, \mathrm{~V}_{6 / 7}\right.$, and $\mathrm{V}_{7 / 8}$ ) has no significant effect (Figure 1B). Therefore, we selected UBC9 and Helicase as the best reference genes for this experiment, which were used for transcript normalization of the analyzed TF genes. 

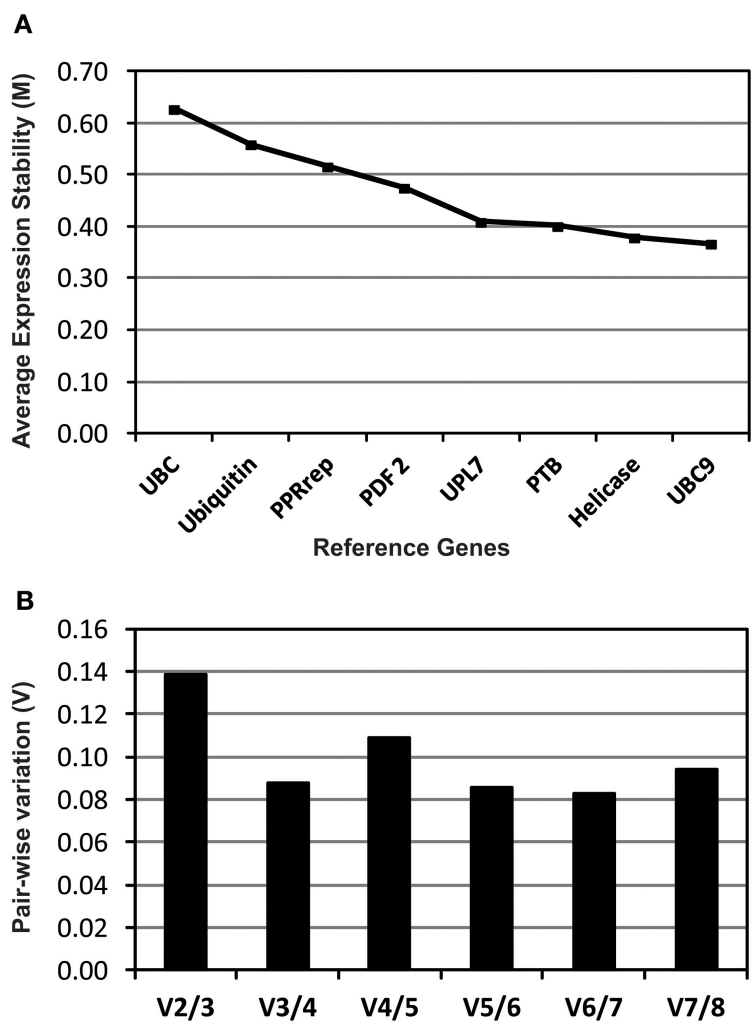

FIGURE 1 | Evaluation of candidate reference genes analyzed using geNorm software. Expression stability (A) and pair-wise variation (B) plots for the eight reference genes studied. A lower $M$ value indicates a more stable expression. The pair-wise variation $(\mathrm{V})$ values indicate the optimal number of reference genes.

\section{Expression Patterns of TF Genes in M. truncatula Following E. pisi Infection}

We analyzed and compared the expression patterns of TF genes in the susceptible cv. Parabinga and the resistant SA1306 genotypes at $4 \mathrm{~h}$ after $E$. pisi inoculation (Figure S1). A total of 623 genes of the qPCR TF platform (59.6\%) were considered detected $(C q<40 ; n \geq 2)$ and 95 showed statistically significant differences $(P<0.05)$ upon $E$. pisi infection in SA1306 and Parabinga genotypes. The relative gene expression ratios $(\mathrm{m}), \log _{2}$ expression ratios inoculated/control after E. pisi inoculation, were calculated for all TF genes. TF genes were considered to be differentially up- or down-regulated in response to E. pisi infection, if they met the prerequisites $p \leq 0.05$ and $m \geq 0.7$ or $m \leq-0.7$, respectively. We studied the expression pattern of these genes that showed statistically significant differences through a hierarchical clustering analysis based on gene expression profiles (Figure 2, Table 1).

\section{Transcription Factor Regulatory Network Induced by $E$. pisi Infection in $M$. truncatula}

The qPCR platform allowed identification of TF genes differentially expressed in the two $M$. truncatula genotypes in response to E. pisi infection. Genes regulated in response to E. pisi infection ( $p \leq 0.05$ and $m \geq 0.7$ or $m \leq-0.7$ ) were clustered into 10 groups with different expression patterns (Figure 3). The first group (GI) includes 15 genes that were down-regulated in SA1306. In Parabinga eight of the genes were differentially expressed, one was up-regulated and seven TFs were repressed. These genes belong to $12 \mathrm{TF}$ families, including $\mathrm{Zn}$-Finger members $\left(\mathrm{C}_{2} \mathrm{H}_{2}\left(\mathrm{CYS}_{2}\right.\right.$ - $\left.\mathrm{HIS}_{2}\right)$, TTF-type (THYROID TRANSCRIPTION FACTOR), LIM (Lin-11, Isl-1, Mec-3), AP2/EREBP (APETALA2/ETHYLENE-RESPONSIVE ELEMENT BINDING FACTORS), AUX/IAA (AUXIN/INDOLE ACETIC ACID), bHLH (BASIC-HELIX-LOOP-HELIX), BTB/POZ (BROAD COMPLEX, TRAMTRACK, BRICA-BRAC/POX VIRUS, AND ZINC FINGER), E2F (E2 FACTOR), HMG (HIGH-MOBILITY GROUP), MYB/HDlike (MYELOBLASTOSIS/ HOMEODOMAIN-LIKE), NAC (NAM/ATAF/CUC), and SBP (SQUAMOSA-PROMOTER BINDING PROTEIN) (Table 1). The second group (GII) includes 13 genes which were down-regulated in Parabinga, except for one gene that was not differentially expressed. In the resistant SA1306 genotype, three were up-regulated and other three repressed belong to $\mathrm{Zn}$-finger families, such as $\mathrm{C}_{2} \mathrm{C}_{2}\left(\mathrm{CYS}_{2}\right.$ $\left.\mathrm{CYS}_{2}\right)$ and $\mathrm{C}_{3} \mathrm{H}$-type I $\left(\mathrm{CYS}_{3}-\mathrm{HIS}_{1}\right)$ family. The remaining genes belong to AP2/EREBP, DDT (DNA BINDING HOMEOBOX, AND DIFFERENT TRANSCRIPTION FACTOR), HD, HD-like, HSF (HEAT STRESS TRANSCRIPTION FACTOR), NAC, RR (RESPONSE REGULATOR RECEIVER), and WRKY TF families.

Groups III, IV, and V include genes that were induced in both genotypes. Group III contains six genes that showed lower transcription levels in SA1306 than in Parabinga. These genes belong to AP2/EREBP, HD, HD-like, MYB, and MYB/HD-like TF families. Group IV includes nine genes that showed stronger transcription activation in the resistant SA1306 genotype. These genes belong to bHLH, $\mathrm{C}_{2} \mathrm{H}_{2}$ (Zn), HD, HD-like, MADS (MADS box), MYB, and PHD (PLANT HOMEODOMAIN MOTIF) TF families. Group V contains 10 genes that showed similar upregulation expression patterns in both susceptible and resistant genotypes. Genes of this fifth group encode proteins belonging to AP2/EREBP, ARF (AUXIN-RESPONSE FACTOR), bHLH, GRAS (GAI, RGA, SCR), HD, HTH (HELIX-TURN-HELIX), NAC, RR, and DHHC (ASP-HIS-HIS-CYS) (Zn) TF families.

Genes clustered in groups VI and VII were mainly not expressed differentially in either genotype. None of the genes included in group VI were regulated whereas seven of the 11 genes of group VII were regulated. Three genes were induced in both genotypes and they are members of HD-like, PHD, and bZIP (BASIC LEUCINE ZIPPER) TF families, respectively. The remaining four regulated genes of group VII were specifically upregulated in Parabinga and SA1306, which were included in HD, bZIP, and MYB/HD-like TF families.

Group VIII is comprised of six genes which were induced in Parabinga and not differentially expressed in SA1306. These genes belong to bHLH, FHA (FORKHEAD-ASSOCIATED), $\mathrm{MYB} / \mathrm{HD}$-like and three $\mathrm{Zn}$-finger TF families $\left(\mathrm{C}_{2} \mathrm{C}_{2}, \mathrm{CCHC}\right.$ (CYS-X8-CYS-X5-CYS-X3-HIS) and U1-type). The eight genes included in group IX were induced in SA1306, whereas in Parabinga half of them were up-regulated and the remaining genes were not differentially expressed. Genes of group IX are members of ARID (AT-RICH INTERACTION DOMAIN), $\mathrm{C}_{2} \mathrm{H}_{2}$ 


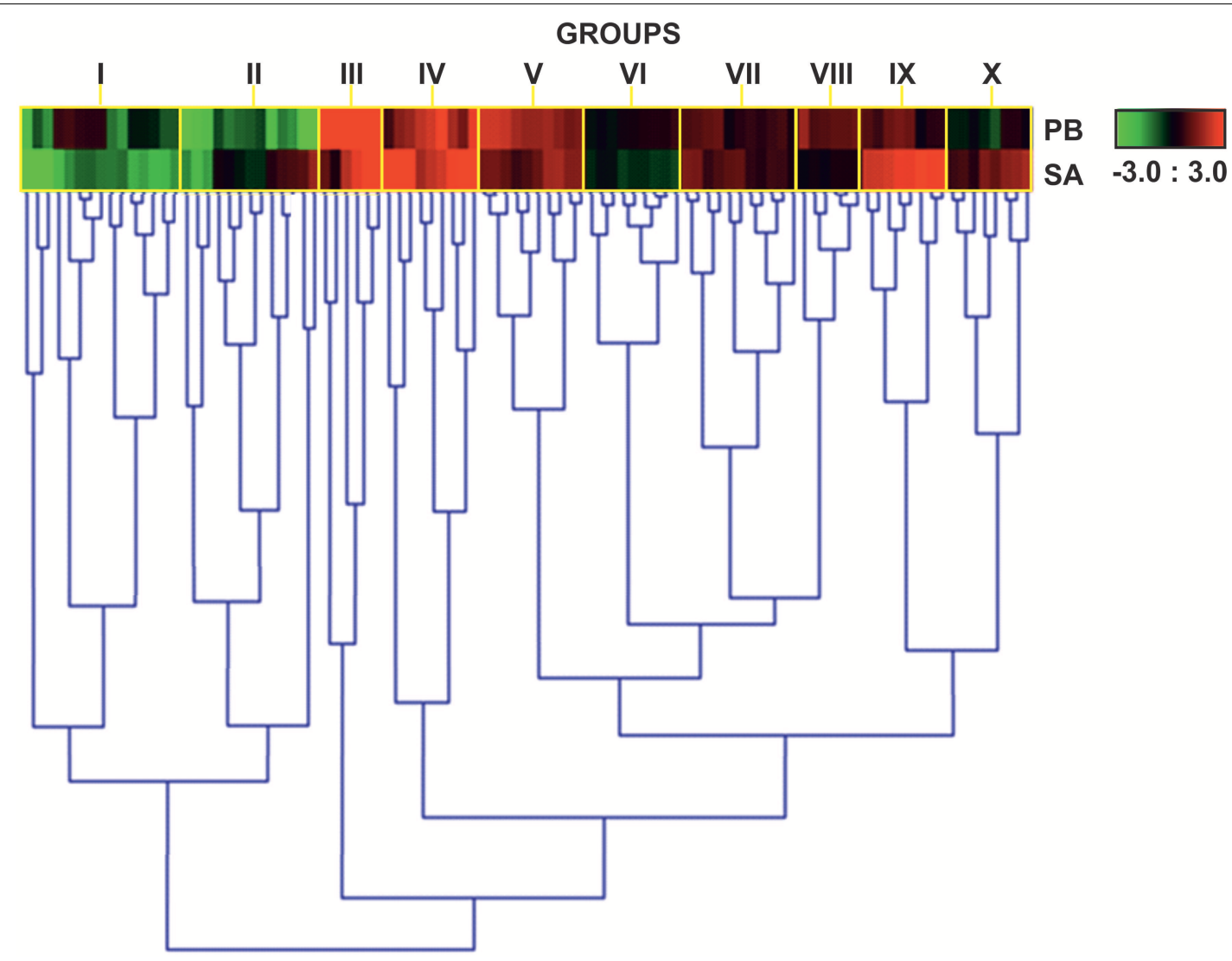

FIGURE 2 | Heat map expression profiles of TF genes. Heat map showing expression profiles of 95 genes that were differentially expressed in Parabinga (PB) and SA1306 (SA) M. truncatula genotypes in response to $E$. Pisi infection. Genes were considered differentially expressed if they met the prerequisites $p \leq 0.05$ and $m \leq-0.7$ or $m \geq 0.7$. Up-regulation $(m \geq 0.7)$ is indicated in red; down-regulation $(m \leq-0.7)$ in green; black indicates no differential expression $(-0.7 \leq m \leq 0.7)$. The heat map expression profiles are grouped by yellow rectangles $(I-X)$. Additional information is available in Table 1.
(Zn), HD, HD-like, NAC, and SBP TF families. Finally, group X includes eight genes which were mostly up-regulated in SA1306 and not differentially expressed in Parabinga. All genes belonging to this group were induced in the resistant genotype, except for two genes that were not differentially expressed. Only one repressed gene was detected in Parabinga. The genes of this last cluster belong to $\mathrm{Zn}$ finger families $\mathrm{C}_{2} \mathrm{C}_{2}, \mathrm{CCHC}, \mathrm{HD}$, HD-like, JUMONJI (JmjC domain), MYB/HD-like, and SBP TF families.

Around $80 \%$ of TF genes (79/95 genes) that showed statistically significant differences $(P<0.05)$ had at least a 1.6-fold change in transcript accumulation $(-0.7 \geq m \geq 0.7)$ (Table S2). To study the regulatory network controlling the expression and interactions of these 79 genes during E. pisi infection, we further analyzed their expression in the susceptible Parabinga and the resistant SA1306 genotypes (Figure 4).

Our analysis revealed that 16 and 18 of the $79 \mathrm{TF}$ genes were specifically regulated in Parabinga and in SA1306, respectively. The remaining 45 genes were regulated in both genotypes (Figure 4, Table S2). In the susceptible Parabinga genotype 10 of the 16 specifically regulated genes were induced and the remaining six genes were repressed. Most of the genes specifically induced in Parabinga are members of MYB/HD-like, $\mathrm{HD}, \mathrm{C}_{2} \mathrm{C}_{2}, \mathrm{CCHC}, \mathrm{RR}, \mathrm{FHA}, \mathrm{bHLH}$, and U1-type $\mathrm{Zn}$ finger families. Meanwhile, the six genes specifically down-regulated in Parabinga are included in RR, AP2/EREBP, NAC, HD-family, $\mathrm{C}_{3} \mathrm{H}$ - type $1(\mathrm{Zn})$, and DDT TF families. Moreover, the resistant SA1306 genotype showed 11 and seven genes specifically upand down-regulated, respectively. Genes specifically induced in SA1306 are members of HD/HD-like, MYB/HD-like, SBP, $\mathrm{C}_{2} \mathrm{C}_{2}$ (Zn), $\mathrm{C}_{2} \mathrm{H}_{2}(\mathrm{Zn})$, bZIP, ARID, and JUMONJI TF families. The genes down-regulated in SA1306 belong to LIM, $\mathrm{C}_{2} \mathrm{H}_{2}(\mathrm{Zn})$, TTF-type (Zn), MYB/HD-like, bHLH, E2F, and AUX/IAA TF families.

On the other hand, the genes regulated in both genotypes were mainly up-regulated; 30 induced and 10 repressed genes. Most of these 30 induced genes are in the HD-like, HD, and MYB TF families (Figure 4, Table S2). Moreover, the 10 repressed genes are included mainly in $\mathrm{Zn}$ finger families, $\mathrm{C}_{2} \mathrm{C}_{2}$ and $\mathrm{C}_{2} \mathrm{H}_{2}$. Interestingly, four of five common regulated genes were induced in SA1306 and repressed in Parabinga, and belong to the HD-like (TF626), CCHC (Zn) (TF660), WRKY (TF913), and AP2/EREBP 
TABLE 1 | Details of regulated TF genes clustered in groups among Medicago truncatula genotypes analyzed, SA1306 (SA), and Parabinga (PB), in response to Erysiphe pisi infection.

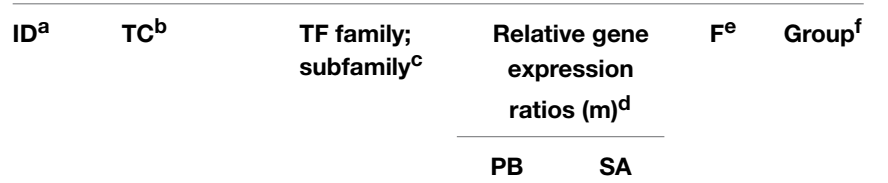

\begin{tabular}{|c|c|c|c|c|c|c|}
\hline TF700 & CX528154 & BTB/POZ & $-2.31^{\star}$ & $-3.55^{\star}$ & -1.24 & I \\
\hline TF479 & TC110178 & NAC & $-1.54^{\star}$ & $-1.12^{\star}$ & 0.42 & I \\
\hline TF823 & - & $\mathrm{C} 2 \mathrm{H} 2$ (Zn) & $-1.46^{\star}$ & $-3.36^{\star}$ & -1.89 & I \\
\hline TF436 & TC98230 & SBP & $-1.16^{\star}$ & $-1.24^{*}$ & -0.08 & I \\
\hline TF855 & - & AP2/EREBP & $-0.87^{\star}$ & $-1.49^{\star}$ & -0.62 & 1 \\
\hline TF303 & - & AP2/EREBP & $-0.86^{\star}$ & $-1.83^{*}$ & -0.97 & I \\
\hline TF485 & TC109384 & bHLH & $-0.83^{\star}$ & $-3.51^{\star}$ & -2.68 & I \\
\hline TF425 & - & $\mathrm{bHLH}$ & -0.36 & $-1.98^{\star}$ & -1.62 & I \\
\hline TF101 & TC103429 & $\mathrm{C} 2 \mathrm{H} 2(\mathrm{Zn})$ & -0.28 & $-1.62^{\star}$ & -1.34 & I \\
\hline TF8 & TC109476 & LIM & -0.28 & $-1.75^{\star}$ & -1.47 & I \\
\hline TF388 & BG448434 & TTF-type (Zn) & 0.61 & $-2.13^{*}$ & -2.74 & I \\
\hline TF244 & TC108091 & MYB/HD-like & 0.54 & $-1.04^{*}$ & -1.58 & I \\
\hline TF780 & - & AUX/IAA & 0.49 & $-1.21^{*}$ & -1.70 & 1 \\
\hline TF691 & BG450549 & E2F & 0.49 & $-1.00^{\star}$ & -1.49 & I \\
\hline TF233 & - & $\mathrm{HMG}$ & $0.72^{*}$ & $-1.43^{\star}$ & -2.15 & 1 \\
\hline TF962 & - & AP2/EREBP & $-5.08^{\star}$ & $1.56^{\star}$ & 6.64 & $\|$ \\
\hline TF626 & - & HD-like & $-3.62^{\star}$ & $0.91^{*}$ & 4.52 & ॥ \\
\hline TF913 & TC97332 & $\begin{array}{l}\text { WRKY family; } \\
\text { WRKY }\end{array}$ & $-1.48^{\star}$ & $0.77^{\star}$ & 2.25 & $\|$ \\
\hline TF393 & TC95605 & $\begin{array}{l}\text { C2C2 (Zn); } \\
\text { DOF }\end{array}$ & $-2.65^{\star}$ & $-2.19^{*}$ & 0.45 & $\|$ \\
\hline TF265 & - & $\begin{array}{l}\text { C2C2 (Zn); } \\
\text { DOF }\end{array}$ & $-4.31^{*}$ & $-1.42^{*}$ & 2.89 & $\|$ \\
\hline TF716 & AL382911 & $\begin{array}{l}\text { C2C2 (Zn); } \\
\text { GATA }\end{array}$ & $-2.46^{\star}$ & $-1.60^{*}$ & 0.86 & $\|$ \\
\hline TF767 & - & $\begin{array}{l}\text { C3H; } \\
\text { C3H-type } \\
1(\mathrm{Zn})\end{array}$ & $-2.42^{*}$ & 0.44 & 2.86 & ॥ \\
\hline TF822 & - & DDT & $-1.24^{\star}$ & 0.69 & 1.92 & $\|$ \\
\hline TF448 & TC97611 & $\mathrm{RR}$ & $-1.23^{\star}$ & 0.06 & 1.29 & $\|$ \\
\hline TF546 & AL366881 & AP2/EREBP & $-0.71^{\star}$ & 0.26 & 0.97 & $\|$ \\
\hline TF618 & - & HD family; HD & $-1.03^{\star}$ & -0.27 & 0.76 & $\|$ \\
\hline TF598 & TC95256 & NAC & $-0.92^{*}$ & -0.47 & 0.45 & $\|$ \\
\hline TF631 & TC96049 & HSF & -0.69 & -0.47 & 0.22 & $\|$ \\
\hline TF837 & - & MYB & $5.96^{\star}$ & $1.87^{\star}$ & -4.09 & III \\
\hline TF996 & - & HD-like & $4.24^{\star}$ & $3.23^{*}$ & -1.01 & III \\
\hline TF129 & TC102127 & MYB/HD-like & $4.45^{\star}$ & $2.37^{*}$ & -2.08 & III \\
\hline TF549 & TC107542 & $\begin{array}{l}\text { HD family; } \\
\text { HD-ZIP }\end{array}$ & $3.89^{\star}$ & $3.02^{*}$ & -0.87 & III \\
\hline TF3 & - & AP2/EREBP & $3.11^{*}$ & $1.02^{*}$ & -2.08 & III \\
\hline TF934 & TC109302 & MYB/HD-like & $4.22^{*}$ & 0.39 & -3.82 & III \\
\hline TF428 & - & $\mathrm{C} 2 \mathrm{H} 2(\mathrm{Zn})$ & $2.88^{\star}$ & $2.17^{\star}$ & -0.71 & IV \\
\hline TF879 & СB066652 & PHD & $2.42^{*}$ & $3.53^{*}$ & 1.11 & IV \\
\hline TF333 & - & bHLH & $2.18^{\star}$ & $1.99^{*}$ & -0.19 & IV \\
\hline TF322 & - & MADS & $1.98^{\star}$ & $1.70^{\star}$ & -0.28 & IV \\
\hline TF63 & - & MYB & $1.76^{\star}$ & $2.80^{*}$ & 1.04 & IV \\
\hline TF270 & - & $\mathrm{C} 2 \mathrm{H} 2$ (Zn) & $1.59^{\star}$ & $4.49^{*}$ & 2.90 & IV \\
\hline
\end{tabular}

TABLE 1 | Continued

\begin{tabular}{|c|c|c|c|c|c|c|}
\hline \multirow[t]{2}{*}{$I^{a}$} & \multirow[t]{2}{*}{$\mathrm{TC}^{\mathrm{b}}$} & \multirow[t]{2}{*}{$\begin{array}{l}\text { TF family; } \\
\text { subfamilyc }\end{array}$} & \multicolumn{2}{|c|}{$\begin{array}{l}\text { Relative gene } \\
\text { expression } \\
\text { ratios }(\mathrm{m})^{\mathrm{d}}\end{array}$} & \multirow[t]{2}{*}{$\mathrm{F}^{\mathrm{e}}$} & \multirow[t]{2}{*}{ Group ${ }^{f}$} \\
\hline & & & PB & SA & & \\
\hline TF258 & - & HD family; HD & $1.41^{*}$ & $5.26^{\star}$ & 3.85 & IV \\
\hline TF563 & - & MADS & $1.25^{*}$ & $3.13^{\star}$ & 1.88 & IV \\
\hline TF230 & TC109855 & HD-like & $0.71^{*}$ & $5.66^{\star}$ & 4.95 & IV \\
\hline TF600 & TC103296 & $\mathrm{bHLH}$ & $1.44^{\star}$ & $1.72^{\star}$ & 0.29 & V \\
\hline TF87 & TC96308 & ZF DHHC & $1.20^{*}$ & $1.40^{\star}$ & 0.20 & V \\
\hline TF639 & TC100932 & $\mathrm{bHLH}$ & $1.32^{*}$ & $1.40^{\star}$ & 0.08 & V \\
\hline TF296 & - & GRAS & $1.68^{*}$ & $1.66^{\star}$ & -0.02 & v \\
\hline TF565 & - & HTH; FIS & $1.57^{*}$ & $1.09^{*}$ & -0.48 & v \\
\hline TF386 & TC110943 & ARF & $1.55^{\star}$ & $0.79^{*}$ & -0.75 & v \\
\hline TF473 & TC107897 & AP2/EREBP & $2.15^{\star}$ & $1.22^{*}$ & -0.93 & V \\
\hline TF136 & TC96243 & NAC & $2.17^{\star}$ & $1.21^{\star}$ & -0.96 & v \\
\hline TF497 & BF636434 & HD family; HD & $2.08^{\star}$ & $1.01^{*}$ & -1.07 & v \\
\hline TF276 & BE249457 & $\mathrm{RR}$ & $1.71^{*}$ & 0.65 & -1.06 & V \\
\hline TF364 & TC96319 & $\begin{array}{l}\text { CCAAT; } \\
\text { CCAAT-HAP3 }\end{array}$ & -0.20 & -0.15 & 0.05 & $\mathrm{VI}$ \\
\hline TF196 & TC106782 & EIL & -0.05 & -0.12 & -0.07 & VI \\
\hline TF351 & TC103599 & $\mathrm{bHLH}$ & -0.21 & -0.42 & -0.21 & $\mathrm{VI}$ \\
\hline TF543 & TC101251 & $\mathrm{BD}$ & 0.25 & -0.44 & -0.70 & VI \\
\hline TF449 & - & $\mathrm{CCHC}(\mathrm{Zn})$ & 0.21 & -0.59 & -0.80 & $\mathrm{VI}$ \\
\hline TF438 & - & PHD & 0.38 & -0.43 & -0.81 & $\mathrm{VI}$ \\
\hline TF899 & - & $\mathrm{TCP}$ & 0.39 & -0.51 & -0.90 & $\mathrm{Vl}$ \\
\hline TF441 & - & JUMONJI & 0.41 & -0.54 & -0.95 & $\mathrm{VI}$ \\
\hline TF959 & - & C2H2 (Zn) & 0.60 & -0.67 & -1.28 & $\mathrm{VI}$ \\
\hline TF537 & - & bZIP & $1.09^{*}$ & $0.80^{\star}$ & -0.29 & VII \\
\hline TF429 & TC111833 & MYB/HD-like & $0.99^{*}$ & 0.59 & -0.40 & VII \\
\hline TF140 & TC107912 & PHD & $0.86^{*}$ & $1.22^{*}$ & 0.37 & VII \\
\hline TF97 & CX534602 & HD-like & $0.77^{\star}$ & $1.28^{\star}$ & 0.51 & VII \\
\hline TF588 & TC106806 & HD family; HD & $0.73^{*}$ & 0.63 & -0.10 & VII \\
\hline TF523 & TC102139 & MYB/HD-like & 0.63 & 0.67 & 0.04 & VII \\
\hline TF199 & - & NAC & 0.58 & 0.55 & -0.03 & VII \\
\hline TF464 & TC109097 & bZIP & 0.48 & $1.05^{\star}$ & 0.57 & VII \\
\hline TF437 & - & $\mathrm{CCHC}(\mathrm{Zn})$ & 0.47 & 0.44 & -0.03 & VII \\
\hline TF816 & TC96871 & MYB/HD-like & 0.25 & 0.29 & 0.04 & VII \\
\hline TF372 & TC96859 & HD family; HD & 0.15 & $0.97^{\star}$ & 0.82 & VII \\
\hline TF389 & TC104194 & $\begin{array}{l}\text { C2C2 (Zn); } \\
\text { DOF }\end{array}$ & $1.59^{*}$ & -0.06 & -1.66 & VIII \\
\hline TF1007 & - & $\mathrm{bHLH}$ & $1.11^{*}$ & 0.32 & -0.79 & VIII \\
\hline TF349 & TC98775 & $\mathrm{CCHC}(\mathrm{Zn})$ & $1.00^{*}$ & 0.30 & -0.70 & VIII \\
\hline TF179 & CX533076 & U1-type (Zn) & $0.99^{*}$ & 0.32 & -0.67 & VIII \\
\hline TF481 & TC112164 & $\mathrm{FHA}$ & $0.88^{*}$ & -0.03 & -0.91 & VIII \\
\hline TF143 & - & MYB/HD-like & $0.80^{*}$ & 0.21 & -0.59 & VIII \\
\hline TF797 & - & HD-like & $1.26^{\star}$ & $2.47^{\star}$ & 1.21 & IX \\
\hline TF200 & TC109833 & NAC & $1.10^{*}$ & $2.23^{\star}$ & 1.13 & IX \\
\hline TF814 & BG451025 & HD-like & $1.11^{*}$ & $2.45^{\star}$ & 1.34 & IX \\
\hline TF552 & TC107542 & $\begin{array}{l}\text { HD family; } \\
\text { HD-ZIP }\end{array}$ & $0.87^{*}$ & $2.09^{*}$ & 1.22 & IX \\
\hline TF666 & CX541503 & HD-like & 0.65 & $2.13^{\star}$ & 1.48 & IX \\
\hline TF982 & TC96831 & C2H2 (Zn) & 0.52 & $2.77^{\star}$ & 2.25 & IX \\
\hline
\end{tabular}

(Continued) 
TABLE 1 | Continued

\begin{tabular}{|c|c|c|c|c|c|c|}
\hline \multirow[t]{2}{*}{ ID $\mathrm{D}^{\mathrm{a}}$} & \multirow[t]{2}{*}{$T C^{b}$} & \multirow[t]{2}{*}{$\begin{array}{l}\text { TF family; } \\
\text { subfamilyc }\end{array}$} & \multicolumn{2}{|c|}{$\begin{array}{l}\text { Relative gene } \\
\text { expression } \\
\text { ratios }(m)^{d}\end{array}$} & \multirow[t]{2}{*}{$\mathbf{F}^{\mathrm{e}}$} & \multirow[t]{2}{*}{ Group ${ }^{f}$} \\
\hline & & & PB & SA & & \\
\hline TF901 & - & SBP & 0.48 & $2.71^{*}$ & 2.23 & IX \\
\hline TF27 & - & ARID & 0.05 & $3.00^{*}$ & 2.95 & IX \\
\hline TF1009 & - & JUMONJI & 0.08 & $1.73^{*}$ & 1.65 & $x$ \\
\hline TF726 & AL375449 & MYB/HD-like & 0.45 & $1.52^{*}$ & 1.07 & $x$ \\
\hline TF158 & - & HD-like & 0.44 & $1.43^{*}$ & 0.99 & $x$ \\
\hline TF540 & - & SBP & -0.38 & $1.31^{*}$ & 1.69 & $x$ \\
\hline TF660 & - & $\mathrm{CCHC}(\mathrm{Zn})$ & $-0.83^{\star}$ & $1.04^{*}$ & 1.88 & $x$ \\
\hline TF308 & - & $\begin{array}{l}\text { C2C2 (Zn); } \\
\text { DOF }\end{array}$ & -0.33 & $0.75^{*}$ & 1.08 & $x$ \\
\hline TF295 & - & HD family; HD & -0.35 & 0.65 & 1.00 & $x$ \\
\hline TF562 & TC98196 & HD family; HD & -0.08 & 0.48 & 0.55 & $x$ \\
\hline
\end{tabular}

Relative gene expression ratios $(m)$ are listed and sorted by cluster (Group).

a TF gene identification number. Additional information is given in Table S1.

${ }^{b}$ Identifier in the TIGR M. truncatula Gene index (MtGI 7).

cTF families; sub-families are showed as described (Kakar et al., 2008).

${ }^{d}$ Relative gene expression ratios values $(m)$ were calculated for Parabinga $(P B)$ and $S A 1306$ (SA) genotypes, using the following equation: $\log _{2}$ differential expression ratio ( $\left(\frac{\text { inoculated }}{\text { control }}\right)$.

${ }^{e}$ Fold change expression ratio (F) in SA1306 compared to Parabinga were calculated using the equation: $\log _{2}$ expression ratio ( $\left.\frac{S A 1306}{\text { Parabinga }}\right)$.

${ }^{f}$ Heat map expression profiles clustered. Additional information is given in Figures 2, 3.

* Genes were considered differentially expressed in response to $E$. pisi infection if to meet the prerequisites $p \leq 0.05$ and $m \leq-0.7$ or $m \geq 0.7$.

(TF962) TF families. The fifth gene, TF233 (HMG), was upregulated in Parabinga and repressed in SA1306.

Interestingly, 48 genes were differentially expressed in SA1306 compared to Parabinga $(P<0.05 ;-0.7>F>0.7)$ in response to E. pisi infection, and they belong to $25 \mathrm{TF}$ families (Figure 5, Table S3). Among them, the most represented TF families are HD-like, $\mathrm{C}_{2} \mathrm{H}_{2}(\mathrm{Zn})$, AP2/EREBP, MYB/HD-like, and HD/HD, which comprise approximately half of the differentially expressed genes. The bHLH, $\mathrm{C}_{2} \mathrm{C}_{2} / \mathrm{DOF}\left(\mathrm{CYS}_{2}-\mathrm{CYS}_{2} / \mathrm{DNA}-\mathrm{BINDING}\right.$ ONE ZINC FINGER) (Zn), HD-ZIP (HD-ZINC-REGULATED TRANSPORTER), MYB, NAC, and SBP TF families were also well represented $(\approx 24 \%)$ and the rest of TF families were poorly represented (Figure 5). Eleven of 25 families include genes with greater transcript accumulation $(F>0.7)$ in SA1306 compared to Parabinga (Figure 5). Among them, the genes belonging to the ARID, WRKY family/WRKY, $\mathrm{C}_{2} \mathrm{C}_{2}(\mathrm{Zn}) / \mathrm{DOF}, \mathrm{SBP}, \mathrm{HD}-$ like, MADS, and CCHC ( $\mathrm{Zn})$ families were up-regulated around two fold in SA1306 compared to Parabinga. Meanwhile only three families, TTF-type (Zn), bHLH, and HMG, include genes that were down-regulated $(F \leq-2)$ in SA1306 compared to Parabinga (Figure 5). Generally, families HD-ZIP and NAC include genes that were induced in both genotypes. However, the AP2/ERBP family was lightly up-regulated in SA1306 and almost not regulated in Parabinga (Table S3).

\section{Discussion}

Thanks to the high-throughput methods genes, potentially associated with specific processes and characterization of the regulatory networks that control their expression, have been identified (Czechowski et al., 2004; Caldana et al., 2007). DNA microarrays have been successfully applied to characterize global gene expression patterns in M. truncatula (FosterHartnett et al., 2007; Samac et al., 2011; Zhang et al., 2014; Curto et al., 2015; Song et al., 2015). Previous DNA microarray studies have analyzed the E. pisi/M. truncatula pathosystem (Foster-Hartnett et al., 2007; Samac et al., 2011; Curto et al., 2015) using different genotypes and microarray platforms, such as Mt16kOLI1, Mt16kOLI1plus, and Affymetrix GeneChip ${ }^{\circledR}$ (http://www.affymetrix.com), which have increased the knowledge of mechanisms involved in E. pisi resistance in $M$. truncatula. Several large-scale TF profiling approaches have employed the M. truncatula qPCR-based platform available (Kakar et al., 2008) in various studies (Verdier et al., 2008; Gao et al., 2010; Madrid et al., 2010; Villegas-Fernández et al., 2014; Noguero et al., 2015). In spite of the progress in characterizing TFs, those involved in the expression of stress-related genes in plants remain undiscovered (Singh et al., 2002). Particularly, the TFs involved in the defense mechanisms against E. pisi need to be clarified in order to completely understand the mechanisms involved in the plant's defense against this pathogen.

In our study we found that 95 of the TF genes analyzed (15\%) were expressed differentially. These results agree with similar approaches carried out in response to infection by Uromyces striatus $(\approx 13 \%$ ) (Madrid et al., 2010) and Botrytis spp. $(\approx 20 \%)$ (Villegas-Fernández et al., 2014). A subset of these genes belong to $25 \mathrm{TF}$ families (Figure 5, Table S3), including AUX/IAA, bHLH, E2F, HD, JUMONJI, MYB, SBP, and zinc finger families $\left(\mathrm{C}_{2} \mathrm{C}_{2}, \mathrm{C}_{2} \mathrm{H}_{2}, \mathrm{LIM}\right)$, were specifically regulated in the resistant SA1306 genotype suggesting that they act as major regulators of transcription throughout E. pisi defense responses.

Zinc finger and HD families represent the most of these genes specifically regulated in the resistant SA1306 genotype, and are members of the $\mathrm{C}_{2} \mathrm{H}_{2}(\mathrm{Zn}), \mathrm{C}_{2} \mathrm{C}_{2}$ /DOF, LIM, HD-like, HD/HD, and HD-ZIP TF families, which are agree with recent studies that used the same qPCR TF platform (Villegas-Fernández et al., 2014). The $\mathrm{C}_{2} \mathrm{H}_{2}(\mathrm{Zn})$ family playing a critical role as key transcriptional repressors involved in the defense response of plants to stress (Brayer and Segal, 2008; Ciftci-Yilmaz and Mittler, 2008; Kiełbowicz-Matuk, 2012) and M. truncatula to biotrophic and necrotrophic pathogens. Nevertheless, previous studies have reported the relationship between SBP genes and plant disease resistance, such as programmed cell death in Arabidopsis (Stone et al., 2005) and these studies are in agreement that programmed cell death, frequently associated to host cell death, is a common plant defense mechanisms against E. pisi (Curto et al., 2006; Barilli et al., 2014). Thus, a member of the SPB TF family ( $V p S B P 5)$ has been reported to be induced by powdery mildew (E. necator) (Hou et al., 2013), suggesting that this TF is involved in the resistance to powdery mildew by inducing salicylic acid and methyl jasmonate molecular signals. The $\mathrm{C}_{2} \mathrm{C}_{2}(\mathrm{Zn}) / \mathrm{DOF}$ family member have divergent physiological roles (Yanagisawa, 2002) including defense gene expression in response to salicylic acid and oxidative stress signals (Chen et al., 1996; Yanagisawa, 2002) and phytohormone-regulated expression (De Paolis et al., 1996; Yanagisawa, 2002). Recent studies have shown that $E$. pisi infection induces several enzymes, such as psCHS1 and 

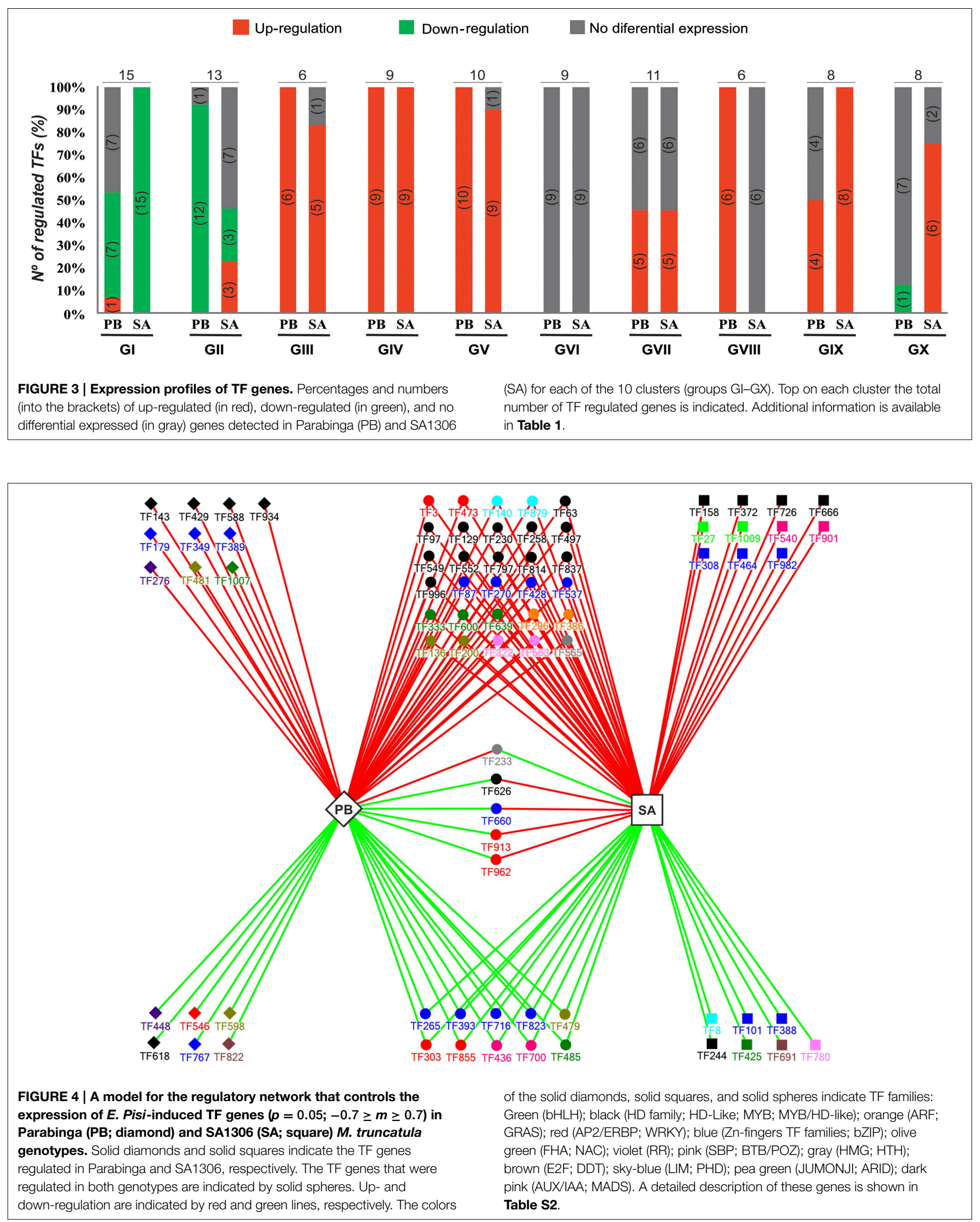

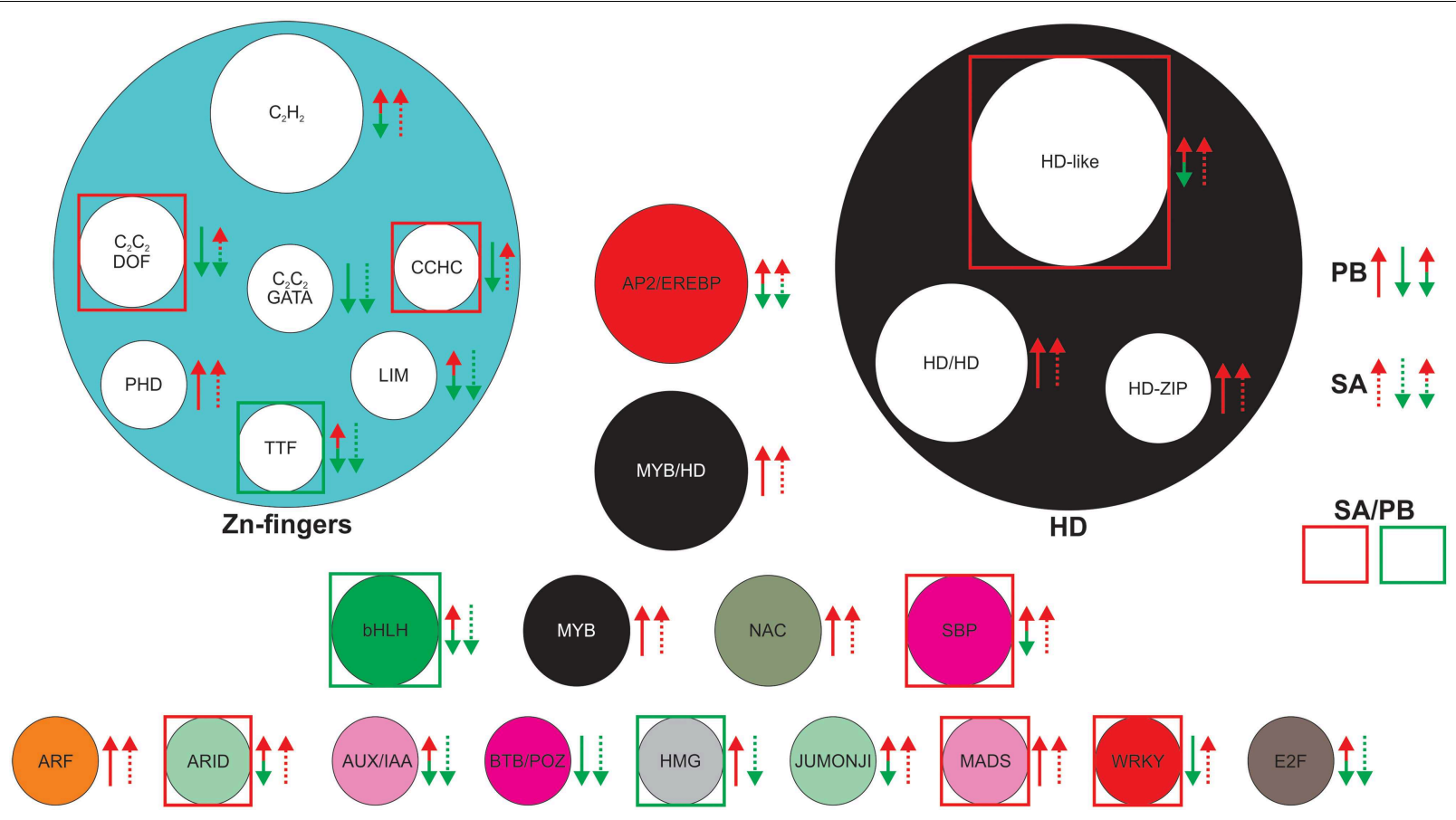

FIGURE 5 | Most differentially expressed TF families in SA1306 compared to Parabinga $(-0.7>F>0.7)$ in response to Erysiphe pisi infection. The TF families are represented by circles whose size is proportional to the number of genes in the family. Up-regulation $(m \geq 0.7)$ is indicated by red arrows; down-regulation ( $m \leq-0.7)$ by green arrows; no differential expression $(-0.7 \leq m \leq 0.7)$ are indicate by double red/green arrows; solid lines represent Parabinga (PB), and dotted lines SA1306 (SA). TF families highly up-regulated $(F \geq 1.8)$ and down-regulated $(F \leq-1.8)$ are indicated by red and green boxes, respectively. For expanded information, see Table S3.
PEAPAL2, involved in phenylpropanoid biosynthesis leading to biosynthesis of phytoalexin (Barilli et al., 2014), reinforcing the role of phenylpropanoid pathway in the elicited defense. Our findings are in agreement with these previous studies, suggesting the important role of these TF families in the resistance to E. pisi in M. truncatula.

Moreover, the LIM zinc TF family has also been found to be involved in the resistance mechanism against pathogens. Thus, several studies have revealed that this family has the capacity to regulate the expression of some lignin biosynthetic genes (Rogers and Campbell, 2004). In addition, previous studies have reported the role of lignification processes into the $M$. truncatula defense responses against to E. pisi infection (Prats et al., 2007). Our findings are in agreement with these previous studies and recent studies (Barilli et al., 2014), in which enzymes involved in ROIs stress, such as peroxidase $\operatorname{Pr} x 7$, are regulated in pea after E. pisi inoculation. Other studies have found that monolignol biosynthesis plays a critical role in cell wall apposition mediated defense against powdery mildew fungus penetration (Bhuiyan et al., 2009). Reactive oxygen intremediates (ROIs) are associated with the hypersensitive response (Torres et al., 2005), which has been related to programmed cell death (hypersensitive response) that plays a critical role in resistance to E. pisi (Barilli et al., 2014).

Our results also reveal a subset of TF genes that encode TFs with a HD (homeodomain) protein domain, which showed a different expression pattern in the resistant SA1306 compared to the susceptible Parabinga genotype in response to E. pisi infection. These genes belong to the HD-like and HD family/HD. Members of this family may play a role in the defense response against necrotrophic fungal pathogens regulated by jasmonic acid (Korfhage et al., 1994; Coego et al., 2005; Villegas-Fernández et al., 2014). In addition, a recent study has revealed that several homeodomain-like TF families are involved in the defense responses in $M$. truncatula when confronted with the necrotrophic pathogens, Botrytis fabae, and B. cinerea (VillegasFernández et al., 2014), supporting that these TF families may play an important role in the defensive mechanism of M. truncatula to fungal pathogens. Nevertheless, our results show that the Auxin/Indole-3-acetic acid (AUX/IAA) family was repressed in the resistant SA1306 genotype in response to $E$. pisi infection, which suggests that this family may play a role in the resistance mechanism against E. pisi. Earlier studies are agree with our results and have described that down regulation of auxin signaling contribute to plant induced immune responses in Arabidopsis (Navarro et al., 2006). The bHLH TFs were also induced in the resistant genotype in response to E. pisi infection, which are in agreement with the results obtained by Villegas-Fernández et al. (2014). The bHLH TFs up-regulated by E. pisi key TF regulating the expressions of jasmonic acid responsive genes (Fernández-Calvo et al., 2011), which mediate the transcriptional reprogramming associated with the plant immune response.

Our findings also indicate that E2F and JUMONJI TF families are involved in the defense response against E. pisi. 
The E2F transcription factor family has been found to induce transcription of genes required for cell cycle progression and DNA replication (Vandepoele et al., 2005). In addition, more than $100 \mathrm{E} 2 \mathrm{~F}$ target genes have been identified, including genes involved in several defense responses and signaling (RamirezParra et al., 2003). The members if the family JUMONJI play a role in the histone methylation process ( $\mathrm{Li}$ et al., 2013). Dimethylated or trimethylated histone H3 lysine 27 (H3K27me2/3) marks silent or repressed genes involved in stress responses in plants. Li et al. (2013) studied the jumonji $C$ protein gene JMJ705 that is induced by stress signals during pathogen infection, and is involved in methyl jasmonate-induced dynamic removal of $\mathrm{H} 3 \mathrm{~K} 27 \mathrm{me} 3$ and in gene activation increasing their basal and induced expression during pathogen infection (Balciunas and Ronne, 2000; Li et al., 2013). Our results are in agreement with the results obtained by Villegas-Fernández et al. (2014), which It is that have reported that the JUMONJI TF family may be involved in the defense response to a fungal pathogen, reinforcing the role of this TF family in defense responses against fungal pathogens.

Moreover, an important number of TF genes were regulated in both genotypes, which mainly belonged to AP2/EREBP (Singh et al., 2002; Gutterson and Reuber, 2004; Dietz et al., 2010; Villegas-Fernández et al., 2014), $\mathrm{C}_{2} \mathrm{H}_{2}$ (Zn) (Ciftci-Yilmaz and Mittler, 2008; Villegas-Fernández et al., 2014), MYB (Singh et al., 2002; Villegas-Fernández et al., 2014), HD (Coego et al., 2005; Villegas-Fernández et al., 2014), MYB/HD-like (Singh et al., 2002; Coego et al., 2005), NAC (Dangl and Jones, 2001; Villegas-Fernández et al., 2014), and PHD (Libault et al., 2007; Villegas-Fernández et al., 2014) TF families. Interestingly, the susceptible and resistant genotypes showed different expression patterns in five of these $\mathrm{TF}$ genes in response to E. pisi infection (Figure 4). Four of these five genes (TF626, TF660, TF913, and TF962) encode TFs belonging to known defense system pathways, which were induced in SA1306 and repressed in Parabinga, respectively. TF962 encodes a member of the AP2/EREBP family known to be linked to response to abiotic and biotic stresses (Dietz et al., 2010), as well as involved in response to a chitin elicitor and in metabolism of the plant hormone methyl jasmonate (McGrath et al., 2005; Libault et al., 2007). Results obtained were similar to those generated in of a study of $M$. truncatula of responses to Botrytis infection, in which AP2/EREBP was shown to be a key regulator of defense responses (Villegas-Fernández et al., 2014). TF913 encodes a WRKY protein, whose family members have been shown to be key components in the regulation of plant disease resistance (Eulgem and Somssich, 2007). Previous studies have shown that WRKY TF family members are involved in the regulation of $R$ gene-mediated disease resistance as well as in the regulation of transcriptional reprogramming associated with plant immune responses (Eulgem and Somssich, 2007; Buscaill and Rivas, 2014). Several genes encoding WRKY proteins (AtWRKY18, AtWRKY40) have been identified that confer resistance against powdery mildew (Shen et al., 2007). In addition, AtWRKY18 has been characterized to act as a positive regulator required for full SAR (Wang et al., 2006), whose transcriptional expression may be is linked to AtWRKY70, which modulates the cross-talk between signaling pathways regulating salicylic acid (SA)-dependent and jasmonic acid-dependent responses (Eulgem and Somssich, 2007). These results are in accord with results of recent studies in M. truncatula that WRKY TFs involved in the defensive reaction of M. truncatula to Uromyces and Botrytis (Madrid et al., 2010; Villegas-Fernández et al., 2014), supporting the critical role of this TF family in the plant defense responses against fungal pathogens.

The $\mathrm{Zn}$ finger family CCHC (TF660) was also detected as induced in SA1306 and repressed in Parabinga. Previous studies (Mangeon et al., 2010; Villegas-Fernández et al., 2014) are in agreement with our results, suggesting the important role of this TF family, A member of HD-like (TF626) was also induced in the resistant genotype and highly repressed in Parabinga. This family has been found to be involved in defense responses against fungal necrotroph pathogens regulated by jasmonic acid (Korfhage et al., 1994; Coego et al., 2005; Villegas-Fernández et al., 2014). We suggest that the 10 TFs that were repressed in both genotypes may play a role suppressing genes involved in photosynthetic metabolism leading to a reduction in the photosynthetic rate, as previously suggested (Swarbrick et al., 2006; Bolton, 2009).

\section{Conclusion}

We have screened more than 1000 TFs genes of M. truncatula for altered expression during E. pisi infection using qPCR platform. Forty eight TFs from them showed significant differences in the resistant SA1306 genotype compared to the susceptible Parabinga. These TF genes belong mainly to AP2/EREBP, WRKY, $\mathrm{MYB}, \mathrm{HD}$, and zinc finger families (C2C2, $\mathrm{C} 2 \mathrm{H} 2$, LIM) gene families, which are involved in known defense responses. In addition, we suggest a regulatory network that controls the expression in $M$. truncatula of genes involved in resistance to E. pisi. These results will help to systematically decipher the functional roles of TF genes and to develop new strategies against powdery mildew.

\section{Acknowledgments}

This work was supported by the European Commission FP6 Framework Programme Grain Legume Integrated Project (FOOD-CT-2004-506223) and by Spanish project AGL201452871-R co-financed by FEDER. We are grateful to Udvardi research Group (The Samuel Roberts Noble Foundation, Okla, USA) for advice and support and to Carolina Johnstone for grammatical review. This research work is lovingly dedicated to my mother, who has fought cancer two times.

\section{Supplementary Material}

The Supplementary Material for this article can be found online at: http://journal.frontiersin.org/article/10.3389/fpls.2015. 00517

Figure S1 | Macroscopic disease symptoms in the susceptible cultivar Parabinga (A) and in the resistant SA1306 accession (B). 
Table S1 | Complete list of TF genes. The accession numbers (AC), the TIGR Medicago gene accession numbers (TC) if available, as well as the transcription factor family and the subfamily names are shown as described Kakar et al. (Kakar et al., 2008). The quantification cycle (Cq), PCR efficiencies (PCReff) and correlation coefficients for the qPCR reactions performed on both Medicago truncatula genotypes, Parabinga and SA1306, in three biological replicates are indicated.

Table S2 | Details of regulated TF genes among Medicago truncatula genotypes analyzed, SA1306 (SA) and Parabinga (PB), in response to Erysiphe pisi infection ( $\mathbf{p} \leq \mathbf{0 . 0 5} ;-\mathbf{0 . 7} \geq \mathbf{m} \geq \mathbf{0 . 7}$ ). Relative gene expression ratios $(m)$ are listed and sorted by "Class" and "m" values. ID: Identification number of TF gene. TF family: Transcription factor family. TF subfamily: Subfamily names. m: Average $\log _{2}$ differential expression ratios (inoculated/control) for Parabinga (PB) and SA1306 (SA) genotypes. F: Fold change expression ratios of differentially expressed genes in SA1306 compared to Parabinga, $\log _{2}$ expression ratio SA1306/Parabinga. Class: Genes that were specifically regulated in SA1306 (SA), Parabinga (PB) and both genotypes (Common) (Figure 4).

Table S3 | Most differentially expressed TF families in SA1306 compared to Parabinga $(-0.7>F>0.7)$ in response to Erysiphe pisi infection.

\section{References}

Balciunas, D., and Ronne, H. (2000). Evidence of domain swapping within the jumonji family of transcription factors. Trends Biochem. Sci. 25, 274-276. doi: 10.1016/S0968-0004(00)01593-0

Barilli, E., Rubiales, D., Gjetting, T., and Lyngkjaer, M. F. (2014). Differential gene transcript accumulation in peas in response to powdery mildew (Erysiphe pisi) attack. Euphytica 195, 1-16. doi: 10.1007/s10681-014-1062-z

Bélanger, R. R., Bushnell, W. R., Dik, A. J., and Carver, T. L. W. (2002). The Powdery Mildews: A Comprehensive Treatise. St. Paul, MN: APS Press.

Bhuiyan, N. H., Selvaraj, G., Wei, Y., and King, J. (2009). Role of lignification in plant defense. Plant. Signal. Behav. 4, 158-159. doi: 10.4161/psb.4.2.7688

Bolton, M. D. (2009). Primary metabolism and plant defense-fuel for the fire. Mol. Plant Microbe Interact. 22, 487-497. doi: 10.1094/MPMI-22-5-0487

Brayer, K. J., and Segal, D. J. (2008). Keep your fingers off my DNA: protein-protein interactions mediated by $\mathrm{C} 2 \mathrm{H} 2$ zinc finger domains. Cell Biochem. Biophys. 50, 111-131. doi: 10.1007/s12013-008-9008-5

Buscaill, P., and Rivas, S. (2014). Transcriptional control of plant defence responses. Curr. Opin. Plant Biol. 20, 35-46. doi: 10.1016/j.pbi.2014.04.004

Bustin, S. A., Benes, V., Garson, J. A., Hellemans, J., Huggett, J., Kubista, M., et al. (2009). The MIQE guidelines: minimum information for publication of quantitative real-time PCR experiments. Clin. Chem. 55, 611-622. doi: 10.1373/clinchem.2008.112797

Caldana, C., Scheible, W.-R., Mueller-Roeber, B., and Ruzicic, S. (2007). A quantitative RT-PCR platform for high-throughput expression profiling of 2500 rice transcription factors. Plant Methods 3:7. doi: 10.1186/1746-4811-3-7

Chen, W., Chao, G., and Singh, K. B. (1996). The promoter of a $\mathrm{H}_{2} \mathrm{O}_{2}$ inducible, Arabidopsis glutathione S-transferase gene contains closely linked OBF- and OBP1-binding sites. Plant J. 10, 955-966. doi: 10.1046/j.1365313X.1996.10060955.x

Cheung, A. Y., and Wu, H.-M. (2011). THESEUS 1, FERONIA and relatives: a family of cell wall-sensing receptor kinases? Curr. Opin. Plant Biol. 14, 632-641. doi: 10.1016/j.pbi.2011.09.001

Ciftci-Yilmaz, S., and Mittler, R. (2008). The zinc finger network of plants. Cell Mol. Life Sci. 65, 1150-1160. doi: 10.1007/s00018-007-7473-4

Coego, A., Ramirez, V., Gil, M. J., Flors, V., Mauch-Mani, B., and Vera, P. (2005). An Arabidopsis homeodomain transcription factor, OVEREXPRESSOR OF CATIONIC PEROXIDASE 3, mediates resistance to infection by necrotrophic pathogens. Plant Cell 17, 2123-2137. doi: 10.1105/tpc.105.032375

Curto, M., Camafeita, E., Lopez, J. A., Maldonado, A. M., Rubiales, D., and Jorrín, J. V. (2006). A proteomic approach to study pea (Pisum sativum) responses to powdery mildew (Erysiphe pisi). Proteomics 6, S163-S174. doi: 10.1002/pmic.200500396

Curto, M., Krajinski, F., Küster, H., and Rubiales, D. (2015). Plant defense responses in Medicago truncatula unveiled by microarray analysis. Plant Mol. Biol. Rep. 33, 569-583. doi: 10.1007/s11105-014-0770-9

Czechowski, T., Bari, R. P., Stitt, M., Scheible, W.-R., and Udvardi, M. K. (2004). Real-time RT-PCR profiling of over 1400 Arabidopsis transcription factors: unprecedented sensitivity reveals novel root- and shoot-specific genes. Plant J. 38, 366-379. doi: 10.1111/j.1365-313X.2004.02051.x

Dangl, J. L., and Jones, J. D. G. (2001). Plant pathogens and integrated defence responses to infection. Nature 411, 826-833. doi: 10.1038/35081161

De Paolis, A., Sabatini, S., De Pascalis, L., Costantino, P., and Capone, I. (1996). A rolB regulatory factor belongs to a new class of single zinc finger plant proteins. Plant J. 10, 215-223. doi: 10.1046/j.1365-313X.1996.10020215.x
Dietz, K.-J., Vogel, M., and Viehhauser, A. (2010). AP2/EREBP transcription factors are part of gene regulatory networks and integrate metabolic, hormonal and environmental signals in stress acclimation and retrograde signalling. Protoplasma 245, 3-14. doi: 10.1007/s00709-0100142-8

Eulgem, T., and Somssich, I. E. (2007). Networks of WRKY transcription factors in defense signaling. Curr. Opin. Plant Biol. 10, 366-371. doi: 10.1016/j.pbi.2007.04.020

Falloon, R. E., and Viljanen-Rollinson, S. L. H. (2001). "Powdery mildew," in Compendium of Pea Diseases and Pests, 2nd Edn., eds J. M. Karaft and F. L. Pfleger (St. Paul, MN: APS Press), 28-29.

Fernández-Calvo, P., Chini, A., Fernández-Barbero, G., Chico, J.-M., Gimenez Ibanez, S., Geerinck, J., et al. (2011). The Arabidopsis bHLH transcription factors MYC3 and MYC4 are targets of JAZ repressors and act additively with MYC2 in the activation of jasmonate responses. Plant Cell 23, 701-715. doi: $10.1105 /$ tpc. 110.080788

Fondevilla, S., and Rubiales, D. (2012). Powdery mildew control in pea. A review. Agron. Sustain. Dev. 32, 401-409. doi: 10.1007/s13593-0110033-1

Fondevilla, S., Torres, A. M., Moreno, M. T., and Rubiales, D. (2007). Identification of a new gene for resistance to powdery mildew in Pisum fulvum, a wild relative of pea. Breed. Sci. 57, 181-184. doi: 10.1270/jsbbs.57.181

Foster-Hartnett, D., Danesh, D., Peñuela, S., Sharopova, N., Endre, G., Vandenbosch, K. A., et al. (2007). Molecular and cytological responses of Medicago truncatula to Erysiphe pisi. Mol. Plant Pathol. 8, 307-319. doi: 10.1111/j.1364-3703.2007.00395.x

Gao, L.-L., Kamphuis, L. G., Kakar, K., Edwards, O. R., Udvardi, M. K., and Singh, K. B. (2010). Identification of potential early regulators of aphid resistance in Medicago truncatula via transcription factor expression profiling. New Phytol. 186, 980-994. doi: 10.1111/j.1469-8137.2010. 03229.x

Gutterson, N., and Reuber, T. L. (2004). Regulation of disease resistance pathways by AP2/ERF transcription factors. Curr. Opin. Plant Biol. 7, 465-471. doi: 10.1016/j.pbi.2004.04.007

Hellemans, J., Mortier, G., De Paepe, A., Speleman, F., and Vandesompele, J. (2007). qBase relative quantification framework and software for management and automated analysis of real-time quantitative PCR data. Genome Biol. 8:R19. doi: $10.1186 / g b-2007-8-2-r 19$

Hoagland, D. R., and Arnon, D. I. (1950). The water-culture method of growing plants without soil. Calif. Agric. Exp. Stn. Circ. 347, 1-32.

Hou, H., Yan, Q., Wang, X., and Xu, H. (2013). A SBP-Box gene VpSBP5 from chinese wild Vitis Species Responds to Erysiphe necator and defense signaling molecules. Plant Mol. Biol. Rep. 31, 1261-1270. doi: 10.1007/s11105-01 3-0591-2

Iglesias-García, R., Rubiales, D., and Fondevilla, S. (2015). Penetration resistance to Erysiphe pisi in pea mediated by erl gene is associated with protein crosslinking but not with callose apposition or hypersensitive response. Euphytica 201, 381-387. doi: 10.1007/s10681-014-1221-2

Kakar, K., Wandrey, M., Czechowski, T., Gaertner, T., Scheible, W.-R., Stitt, M., et al. (2008). A community resource for high-throughput quantitative RT-PCR analysis of transcription factor gene expression in Medicago truncatula. Plant Methods 4:18. doi: 10.1186/1746-4811-4-18

Kiełbowicz-Matuk, A. (2012). Involvement of plant C2H2-type zinc finger transcription factors in stress responses. Plant Sci. 185-186, 78-85. doi: 10.1016/j.plantsci.2011.11.015 
Korfhage, U., Trezzini, G. F., Meier, I., Hahlbrock, K., and Somssich, I. E. (1994). Plant homeodomain protein involved in transcriptional regulation of a pathogen defense-related gene. Plant Cell 6, 695-708. doi: 10.1105/tpc.6.5.695

Küster, H., Becker, A., Firnhaber, C., Hohnjec, N., Manthey, K., Perlick, A. M., et al. (2007). Development of bioinformatic tools to support EST-sequencing, in silico- and microarray-based transcriptome profiling in mycorrhizal symbioses. Phytochemistry 68, 19-32. doi: 10.1016/j.phytochem.2006.09.026

Küster, H., Hohnjec, N., Krajinski, F., El Yahyaoui, F., Manthey, K., Gouzy, J., et al. (2004). Construction and validation of cDNA-based Mt6k-RIT macroand microarrays to explore root endosymbioses in the model legume Medicago truncatula. J. Biotechnol. 108, 95-113. doi: 10.1016/j.jbiotec.2003.11.011

Li, T., Chen, X., Zhong, X., Zhao, Y., Liu, X., Zhou, S., et al. (2013). Jumonji C domain protein JMJ705-mediated removal of histone H3 lysine 27 trimethylation is involved in defense-related gene activation in rice. Plant Cell 25, 4725-4736. doi: 10.1105/tpc.113.118802

Libault, M., Wan, J., Czechowski, T., Udvardi, M., and Stacey, G. (2007). Identification of 118 Arabidopsis transcription factor and 30 ubiquitin-ligase genes responding to chitin, a plant-defense elicitor. Mol. Plant Microbe Interact. 20, 900-911. doi: 10.1094/MPMI-20-8-0900

Madrid, E., Gil, J., Rubiales, D., Krajinski, F., Schlereth, A., and Millán, T. (2010). Transcription factor profiling leading to the identification of putative transcription factors involved in the Medicago truncatula-Uromyces striatus interaction. Theor. Appl. Genet. 121, 1311-1321. doi: 10.1007/s00122-01 $0-1390-x$

Mangeon, A., Junqueira, R. M., and Sachetto-Martins, G. (2010). Functional diversity of the plant glycine-rich proteins superfamily. Plant Signal. Behav. 5, 99-104. doi: 10.4161/psb.5.2.10336

McGrath, K. C., Dombrecht, B., Manners, J. M., Schenk, P. M., Edgar, C. I., Maclean, D. J., et al. (2005). Repressor- and activator-type ethylene response factors functioning in jasmonate signaling and disease resistance identified via a genome-wide screen of Arabidopsis transcription factor gene expression. Plant Physiol. 139, 949-959. doi: 10.1104/pp.105.068544

Navarro, L., Dunoyer, P., Jay, F., Arnold, B., Dharmasiri, N., Estelle, M., et al. (2006). A plant miRNA contributes to antibacterial resistance by repressing auxin signaling. Science 312, 436-439. doi: 10.1126/science. 1126088

Noguero, M., Le Signor, C., Vernoud, V., Bandyopadhyay, K., Sanchez, M., Fu, C., et al. (2015). DASH transcription factor impacts Medicago truncatula seed size by its action on embryo morphogenesis and auxin homeostasis. Plant J. 81, 453-466. doi: 10.1111/tpj.12742

Prats, E., Llamas, M. J., and Rubiales, D. (2007). Characterization of resistance mechanisms to Erysiphe pisi in Medicago truncatula. Phytopathology 97, 1049-1053. doi: 10.1094/PHYTO-97-9-1049

Ramirez-Parra, E., Fründt, C., and Gutierrez, C. (2003). A genome-wide identification of E2F-regulated genes in Arabidopsis. Plant J. 33, 801-811. doi: 10.1046/j.1365-313X.2003.01662.x

Riechmann, J. L., and Ratcliffe, O. J. (2000). A genomic perspective on plant transcription factors. Curr. Opin. Plant Biol. 3, 423-434. doi: 10.1016/S13695266(00)00107-2

Ringli, C. (2010). Monitoring the outside: cell wall-sensing mechanisms. Plant Physiol. 153, 1445-1452. doi: 10.1104/pp.110.154518

Rogers, L. A., and Campbell, M. M. (2004). The genetic control of lignin deposition during plant growth and development. New Phytol. 164, 17-30. doi: 10.1111/j.1469-8137.2004.01143.x

Rose, R. J. (2008). Medicago truncatula as a model for understanding plant interactions with other organisms, plant development and stress biology: past, present and future. Funct. Plant Biol. 35, 253-264. doi: 10.1071/FP07297

Rubiales, D., Fondevilla, S., Chen, W., Gentzbittel, L., Higgins, T. J. V., Castillejo, M. A., et al. (2015). Achievements and challenges in legume breeding for pest and disease resistance. Crit. Rev. Plant Sci. 34, 195-236. doi: 10.1080/07352689.2014.898445

Samac, D. A., Peñuela, S., Schnurr, J. A., Hunt, E. N., Foster-Hartnett, D., Vandenbosch, K. A., et al. (2011). Expression of coordinately regulated defence response genes and analysis of their role in disease resistance in Medicago truncatula. Mol. Plant Pathol. 12, 786-798. doi: 10.1111/j.13643703.2011.00712.x

Shen, Q.-H., Saijo, Y., Mauch, S., Biskup, C., Bieri, S., Keller, B., et al. (2007). Nuclear activity of MLA immune receptors links isolate-specific and basal disease-resistance responses. Science 315, 1098-1103. doi: $10.1126 /$ science. 1136372

Sillero, J., Fondevilla, S., Davidson, J., Patto, M., Warkentin, T., Thomas, J., et al. (2006). Screening techniques and sources of resistance to rusts and mildews in grain legumes. Euphytica 147, 255-272. doi: 10.1007/s10681-006-6544-1

Singh, K. B., Foley, R. C., and Oñate-Sánchez, L. (2002). Transcription factors in plant defense and stress responses. Curr. Opin. Plant Biol. 5, 430-436. doi: 10.1016/S1369-5266(02)00289-3

Singh, R. J., Chung, G. H., and Nelson, R. L. (2007). Landmark research in legumes. Genome 50, 525-537. doi: 10.1139/G07-037

Song, J., Wang, Y., Li, H., Li, B., Zhou, Z., Gao, S., et al. (2015). The F-box family genes as key elements in response to salt, heavy mental, and drought stresses in Medicago truncatula. Funct. Integr. Genomics 1-13. doi: 10.1007/s10142-0150438-Z

Stone, J. M., Liang, X., Nekl, E. R., and Stiers, J. J. (2005). Arabidopsis AtSPL14, a plant-specific SBP-domain transcription factor, participates in plant development and sensitivity to fumonisin B1. Plant J. 41, 744-754. doi: 10.1111/j.1365-313X.2005.02334.x

Swarbrick, P. J., Schulze-Lefert, P., and Scholes, J. D. (2006). Metabolic consequences of susceptibility and resistance (race-specific and broad-spectrum) in barley leaves challenged with powdery mildew. Plant Cell Environ. 29, 1061-1076. doi: 10.1111/j.1365-3040.2005.01472.x

Torres, M. A., Jones, J. D. G., and Dangl, J. L. (2005). Pathogen-induced, NADPH oxidase-derived reactive oxygen intermediates suppress spread of cell death in Arabidopsis thaliana. Nat. Genet. 37, 1130-1134. doi: 10.1038/ng1639

Udvardi, M. K., Kakar, K., Wandrey, M., Montanari, O., Murray, J., Andriankaja, A., et al. (2007). Legume transcription factors: global regulators of plant development and response to the environment. Plant Physiol. 144, 538-549. doi: 10.1104/pp.107.098061

Vandepoele, K., Vlieghe, K., Florquin, K., Hennig, L., Beemster, G. T. S., Gruissem, W., et al. (2005). Genome-wide identification of potential plant E2F target genes. Plant Physiol. 139, 316-328. doi: 10.1104/pp.105.066290

Vandesompele, J., De Preter, K., Pattyn, F., Poppe, B., Van Roy, N., De Paepe, A., et al. (2002). Accurate normalization of real-time quantitative RT-PCR data by geometric averaging of multiple internal control genes. Genome Biol. 3: research0034.0031. doi: 10.1186/gb-2002-3-7-research0034

Verdier, J., Kakar, K., Gallardo, K., Le Signor, C., Aubert, G., Schlereth, A., et al. (2008). Gene expression profiling of $M$. truncatula transcription factors identifies putative regulators of grain legume seed filling. Plant Mol. Biol. 67, 567-580. doi: 10.1007/s11103-008-9320-x

Villegas-Fernández, A. M., Krajinski, F., Schlereth, A., Madrid, E., and Rubiales, D. (2014). Characterization of transcription factor following expression profiling of Medicago truncatula - Botrytis spp interactions. Plant Mol. Biol. Rep. 32, 1030-1040. doi: 10.1007/s11105-014-0710-8

Wang, D., Amornsiripanitch, N., and Dong, X. (2006). A genomic approach to identify regulatory nodes in the transcriptional network of systemic acquired resistance in plants. PLoS Pathog. 2:e123. doi: 10.1371/journal.ppat.0020123

Yanagisawa, S. (2002). The Dof family of plant transcription factors. Trends Plant. Sci. 7, 555-560. doi: 10.1016/S1360-1385(02)02362-2

Yang, S., Tang, F., Caixetab, E. T., and Zhu, H. (2013). Epigenetic regulation of a powdery mildew resistance gene in Medicago truncatula. Mol. Plant. 6, 2000-2003. doi: 10.1093/mp/sst106

Zhang, J.-Y., Cruz De Carvalho, M. H., Torres-Jerez, I., Kang, Y. U. N., Allen, S. N., Huhman, D. V., et al. (2014). Global reprogramming of transcription and metabolism in Medicago truncatula during progressive drought and after rewatering. Plant Cell Environ. 37, 2553-2576. doi: 10.1111/pce.12328

Conflict of Interest Statement: The authors declare that the research was conducted in the absence of any commercial or financial relationships that could be construed as a potential conflict of interest.

Copyright (C) 2015 Curto, Krajinski, Schlereth and Rubiales. This is an open-access article distributed under the terms of the Creative Commons Attribution License (CC $B Y)$. The use, distribution or reproduction in other forums is permitted, provided the original author(s) or licensor are credited and that the original publication in this journal is cited, in accordance with accepted academic practice. No use, distribution or reproduction is permitted which does not comply with these terms. 


\section{OPEN ACCESS}

Edited by:

Paul Christiaan Struik,

Wageningen University, Netherlands

Reviewed by:

Colin Kahlil Khoury,

International Center for Tropical

Agriculture, Colombia

Eibertus Nicolaas van Loo,

Wageningen University and Research

Centre, Netherlands

${ }^{*}$ Correspondence:

Didier Bazile

didier.bazile@cirad.fr

Specialty section:

This article was submitted to

Crop Science and Horticulture,

a section of the journal

Frontiers in Plant Science

Received: 06 January 2016 Accepted: 22 April 2016

Published: 09 May 2016

Citation:

Bazile D, Jacobsen S-E and Verniau A (2016) The Global Expansion of Quinoa: Trends and Limits.

Front. Plant Sci. 7:622.

doi: 10.3389/fpls.2016.00622

\section{The Global Expansion of Quinoa: Trends and Limits}

\author{
Didier Bazile $^{1 *}$, Sven-Erik Jacobsen ${ }^{2}$ and Alexis Verniau ${ }^{3}$
}

${ }^{1}$ UPR Green, Department of Environment and Societies, French Agricultural Research and International Cooperation Organization, CIRAD, Montpellier, France, ${ }^{2}$ Department of Plant and Environmental Sciences, Faculty of Science, University of Copenhagen, Taastrup, Denmark, ${ }^{3}$ Ecole Supérieure d'Agricultures, Angers, France

Quinoa (Chenopodium quinoa Willd.) was first domesticated in Andean countries over 7000 years ago. Following the Spanish conquest, quinoa was rejected as "Indian food." After centuries of neglect, the potential of quinoa was rediscovered during the second half of the 20th century. Since then, the number of countries importing quinoa increased, with new producers appearing on the map and quinoa now being cultivated in areas outside the Andean countries. The geographical increase in distribution of quinoa has highlighted the difficulty of access to quality seed, which is a key factor for testing the crop outside the Andes. In this context, research partnerships have helped promote the exchange of quinoa germplasm and have allowed trials to be undertaken in nontraditional areas of cultivation. The number of countries growing the crop has increased rapidly from eight in 1980, to 40 in 2010, and to 75 in 2014. A further 20 countries have sown quinoa for the first time in 2015. In this paper, we analyze this trend and discuss the limits of quinoa's expansion. As commercial production of quinoa is expected to develop, changes in international regulatory frameworks on genetic resources are needed in order to facilitate plant breeding for the most adaptive varieties for each region.

Keywords: Chenopodium quinoa Willd., plant genetic resources, adaptation, climate change, benefit-sharing seed regulations, farmers' rights

\section{INTRODUCTION}

Quinoa (Chenopodium quinoa Willd.), is an annual species that originates from South America. Its domestication is thought to have begun in Andean region around 7000 years ago. Generations of farmers have been involved in quinoa selection, which explains the high levels of genetic diversity found today.

Quinoa has remained a staple food for indigenous people of the Andes over the centuries. Following the Spanish conquest, quinoa was rejected as "Indian food" but it has never disappeared despite the introduction of Old World species. After centuries of neglect, the nutritional status of quinoa was rediscovered during the second half of the 20th century, leading to a renaissance of its production (Repo-Carrasco et al., 2003).

Thanks to the high levels of genetic diversity, the crop is highly resilient to agro-ecological extremes (soils, rainfall, temperature, and altitude) and is tolerant to frost, drought, and salinity (Ruiz et al., 2014, 2015). Quinoa can be divided into different groups or ecotypes, reflecting its diffusion from the center of origin around Lake Titicaca. Each of these ecotypes is associated with sub-centers of diversity (Risi and Galwey, 1984), and highly adapted to specific environments. For 
example, the sea level ecotype from the central and southern part of Chile is the most adapted to temperate environments (Galwey, 1993; Jacobsen, 1997, 2003; Christiansen et al., 2010; Bendevis et al., 2014a,b), useful in developing new varieties for northern latitudes (Jacobsen, 2015). Quinoa diversity is divided into five main ecotypes (Bazile et al., 2013):

- Highlands in Peru and Bolivia;

- Inter-Andean valleys in Colombia, Ecuador, and Peru;

- Salares in Bolivia, Chile, and Argentina;

- Yungas in Bolivia;

- Sea level in Chile.

Research partnerships have often facilitated the exchange of germplasm and have had a powerful impact on this development by strengthened collaborations (Izquierdo et al., 2003). However, partnerships between research institutions for germplasm exchanges need to consider legal and ethical aspects related to the access to genetic resources for experimentation and fair commercial development (Bazile, 2015).

Today, quinoa is still considered a minor crop for global food and agriculture and often classified as a Neglected and Underutilized Species (NUS) with a high potential of development. However, its current distribution is now changing due to changes in consumption patterns. In this article, we analyze this trend, and present a case for the increase in production of quinoa around the world.

\section{SYNTHESIS OF EXISTING DATA}

A survey was conducted of resource persons who have been involved in quinoa research over the global level during the last 30 years. The survey identified those countries involved in quinoa research and cultivation. In addition, an analysis was conducted on the issues of access to quality seed of quinoa.

Quinoa project reports were studied to complement the information obtained through surveys. The genotypes used were described, together with their agromorphological characteristics and their origin was determined. The data generated was used to identify the key elements in the expansion of quinoa's cultivation.

\section{A NEGLECTED AND UNDERUTILIZED SPECIES (NUS) WITH A RAPID EXPANSION}

From at least 7000 years ago until the beginning of the 1980s, quinoa has only been connected to the Andes. However, when researchers in other countries understood the potential and benefits of quinoa, experimentation has not stopped growing. Studies have been performed in an increasing number of countries. The number of countries growing quinoa has risen rapidly from 8 in 1980 to 75 in 2014, with a further 20 countries which sowed quinoa for the first time in 2015 (Bazile and Baudron, 2015).
The first known experiment conducted outside the Andes took place in 1935 in Kenya (Elmer, 1942) using the creamcolored seed variety, obtained from the Royal Botanical Gardens, Kew, UK. Trials were conducted the response of quinoa to various nutrient deficiencies in 1948, on the tolerance of quinoa to salinity in 1950, and quinoa's growth response to temperature in 1968. Research was then undertaken using Chilean germplasm in the 1980s led by Colorado State University, USA. The same time period saw the beginning of commercial quinoa cultivation in Canada. Other countries followed and quinoa was introduced to UK (1983), Denmark (1984), Tibet (1984), India (1985), The Netherlands (1986), China (1988), Brazil and Cuba (1989) (Bazile et al., 2015).

The project "American and European Test of Quinoa" (Mujica et al., 2001) overseen by Angel Mujica and Sven-Erik Jacobsen together with Juan Izquierdo from the Food and Agriculture Organization of the United Nations (FAO) that began in 1996, was an important effort in the 1990s. The testing followed two other important activities, which were focused on cultivation in Europe: the EU project under the AIR ${ }^{1}$ program "Quinoa - a multiple crop for EC's Agriculture diversification, 1993-1997," coordinated by University of Cambridge, UK, and the Food and Agriculture COST Action 814 on "Crop Adaptation to Cool and Wet Climates, 19911996," coordinated by the Ministry of Agriculture, Belgium. Through international cooperation initiatives for promoting quinoa, field trials were set up in other countries such as Sweden, Poland, Czech Republic, Austria, Germany, Italy, and Greece.

The General Assembly of the United Nations declared 2013 as the International Year of Quinoa (IYQ). Quinoa was afforded a high profile as a crop with the potential to grow in importance in world agriculture (Jacobsen, 2003; Ruiz et al., 2015). The rapid expansion of the harvested area, with a doubling of countries from 2013, is rapidly changing the perception and representation of quinoa from a minor to a potential major crop (Figure 1).

The main quinoa producers in the world, however, are still Bolivia and Peru. In 2013, the area under quinoa cultivation in Bolivia was 75000 ha and in Peru 45000 ha. These two countries produce more than $80 \%$ of quinoa in the world, followed by Ecuador, USA, China, Chile, Argentina, France, and Canada, which together represent $15-20 \%$ of the world production (Bazile, 2015; Bazile et al., 2015). The area under quinoa cultivation in Europe has increased from 0 in 2008 to 5000 ha in 2015, mainly in France, Spain, and UK (Abbott, personal communication). China has first experimented with cultivating quinoa in Tibet in 1984, while today quinoa is cultivated in nine Chinese regions and over 2500 ha.

The activities carried out due to the IYQ have directly contributed to awareness raising for quinoa cultivation. FAO is actively involved in testing quinoa in 27 countries outside the

\footnotetext{
${ }^{1}$ FP3-AIR - Specific research and technological development and demonstration programme (EEC) in the field of agriculture and agro-industry, including fisheries, 1990-1994.
} 


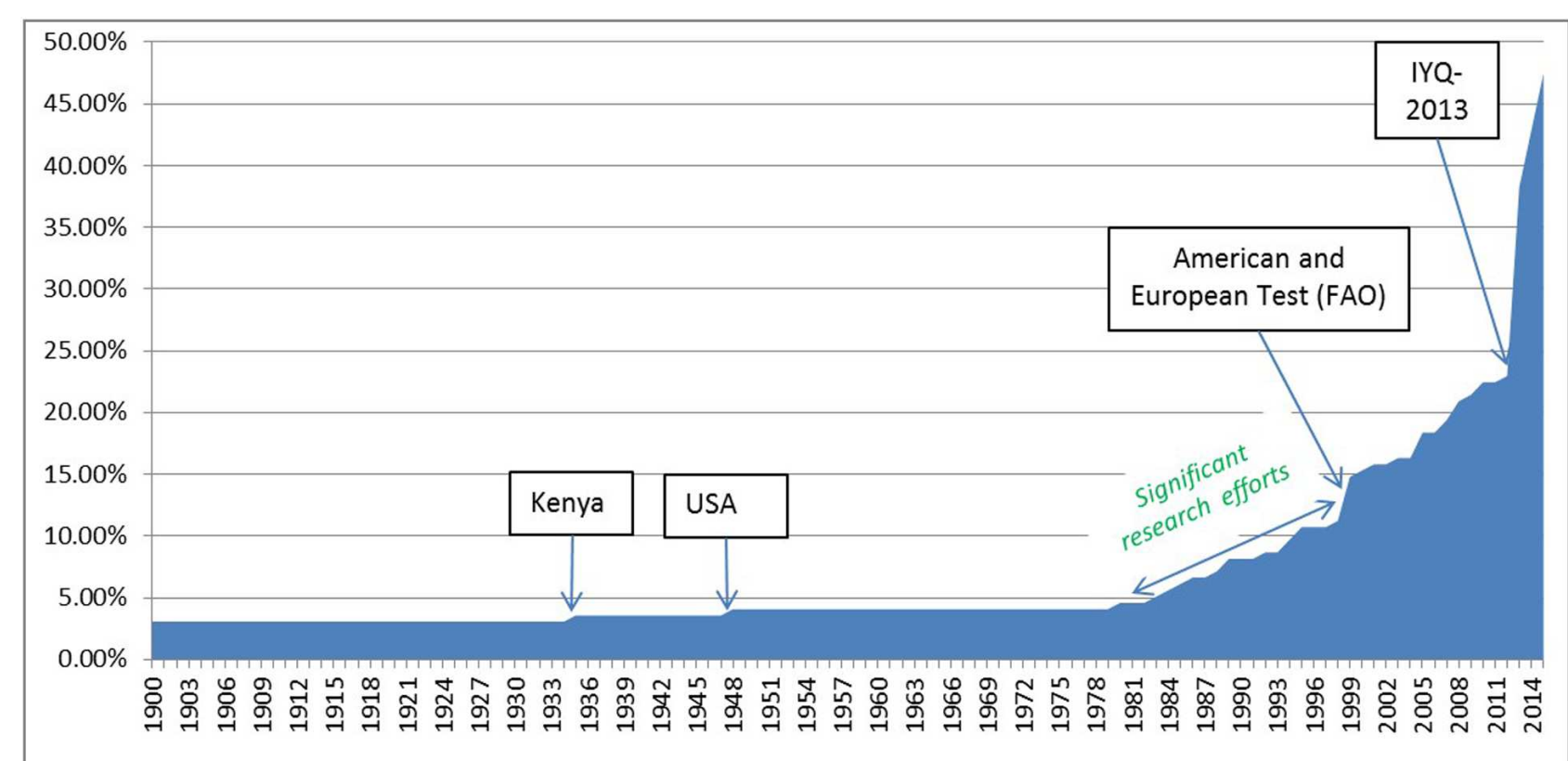

FIGURE 1 | Percentage of UN countries with quinoa experimentation or cultivation (1900-2015).

Andean region, with the aim to promoting food security, due to the nutritional qualities of quinoa and its resistance to abiotic stresses. During 2013-2015, evaluation of quinoa varieties were conducted in:

- Central and Southern Asia (Kyrgyzstan, Tajikistan, Sri Lanka, and Bhutan);

- Western Asia and North Africa (Algeria, Egypt, Iraq, Iran, Lebanon, Mauritania, Sudan, and Yemen);

- Africa (Djibouti, Kenya, Somalia, South Sudan, Ethiopia, Uganda, Zambia, Burkina Faso, Cameroon, Chad, Niger, Senegal, Togo, Ghana, and Guinea).

Size, shape, and compactness of the inflorescence are considered to be important for the rate of maturation. A large open inflorescence will dry quicker after rain and morning dew than a small, compact one, but it may also be prone to seed loss, as quinoa is relatively little domesticated, with few, modern varieties available (Jacobsen, 1997).

The breeding of quinoa, especially Europe and North America, concentrated on gains in time to maturity (around 150 days), high yield and uniformity (Zurita-Silva et al., 2014; Peterson et al., 2015). Another aim has been to reduce the content of saponins in the seed hull. Saponin is a bitter-tasting compound acting as a general defense against biotic stresses. For commercial production, however, the removal of saponins is an additional cost. As a result, sweet cultivars have been developed for use in Europe, especially in the Netherlands (Mastebroek et al., 2000, 2002) and in Denmark (Jacobsen, 2015).

Today quinoa is presently cultivated or tested in 95 countries of the world (Bazile, 2015). This global expansion of quinoa looks set to continue as increasing numbers of countries are testing quinoa.

\section{GLOBAL REGULATORY FRAMEWORKS FOR QUINOA CULTIVATION}

Considering the evolution of global regulations on seeds, two periods must be distinguished for their importance on biodiversity sovereignty: the signing of the Convention of Biological Biodiversity (CBD in Rio de Janeiro, Brazil in 1992) marks a particularly break and contrasts the before and the after for plant genetic resources' access and sharing. The CBD stipulates national sovereignty over biodiversity and obliges parties to form bilateral agreements for accessing their genetic resources (United Nations, 1992). In order to facilitate innovation in plant breeding, the International Treaty on Plant Genetic Resources for Food and Agriculture of 2004 provides a specific instrument for exchanging germplasm of the major food and forage crops (FAO, 2009). It established a global and Multilateral System (MLS) to allow farmers, plant breeders, and scientists to exchange plant genetic materials (Louafi et al., 2013).

Quinoa, unfortunately, is not one of the species pertaining to Annex 1 of the Treaty, which is a list of those species included the MLS of exchanges. The Declaration of Cordoba (2012) from the International Seminar "Crops for the XXI Century," the first international event celebrating the UN IYQ 2013, proposed the addition of minor crops to be included in the Annex I to the Treaty. To date, however, there has been no consensus reached.

The different regulations on plant genetic resources are usually applied at different levels (local, national, and international) and for different purposes (genetic resources, varieties and seeds, 


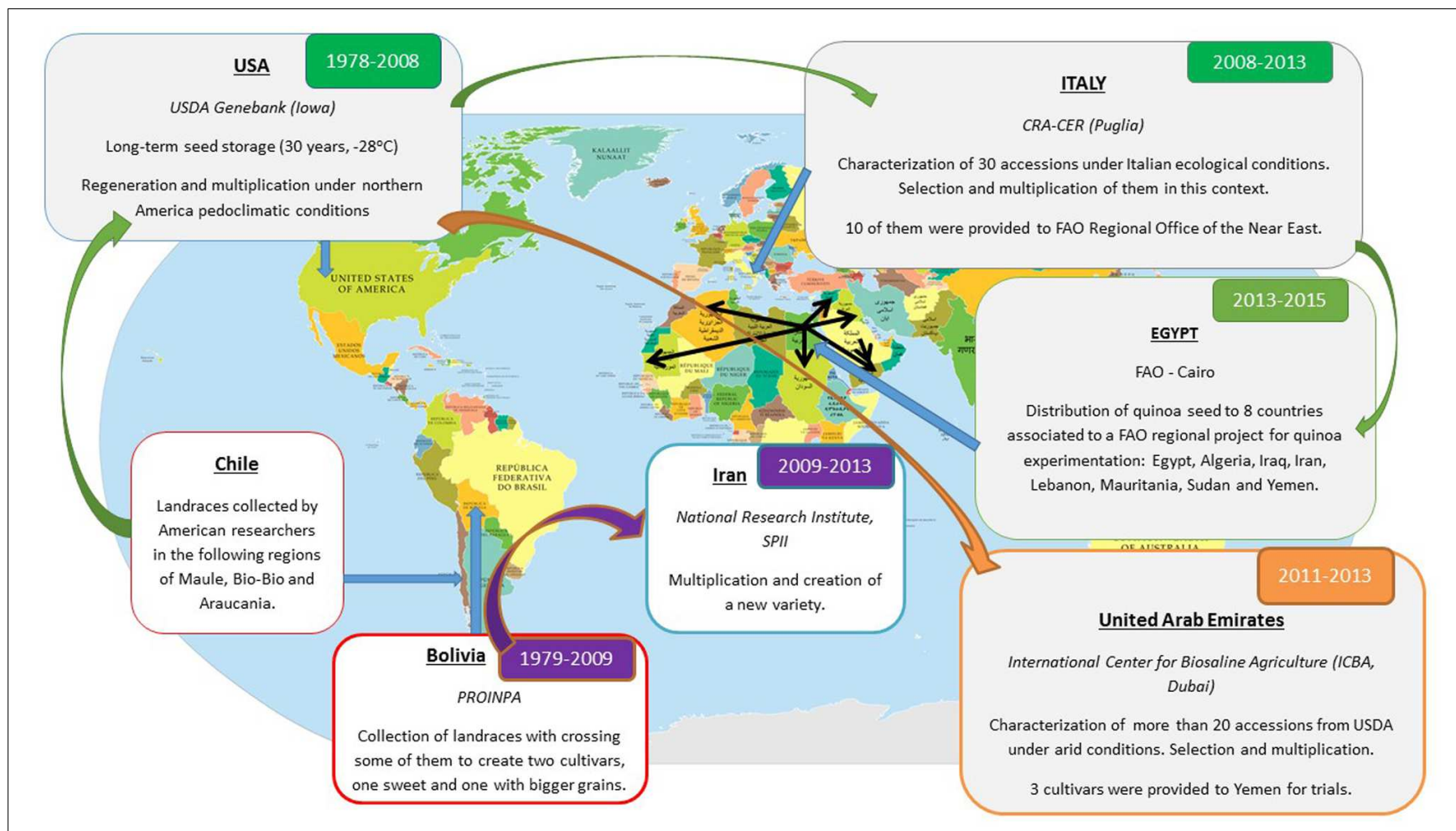

FIGURE 2 | An example of quinoa seed exchanges from Andean countries to new producers' countries.

agricultural by-products, etc.). For quinoa, however, there is no single existing legal framework providing a comprehensive coverage of all the issues related to the genetic resources and their sustainable management (Chevarria-Lazo et al., 2015). The CBD does regulate bilateral access and benefit sharing, but this is difficult to apply to quinoa as the crop is now planted internationally, not restricted to the Andean region, and this has been the case for decades.

Prior to 1992, 25 countries had developed ex situ collections of quinoa accessions, which are now distributed without legal necessity of prior agreement from Andeans countries (Rojas et al., 2015). As many countries had established collections prior to coming into force of the $\mathrm{CBD}$, this now means that these countries may develop new varieties from this germplasm without having to refer to the country of origin. Nevertheless, individuals and institutions do not have the right to share quinoa germplasm outside their country as per the terms of the $\mathrm{CBD}$, but this pertains only to those countries that have ratified the CBD. A country a signatory to the $\mathrm{CBD}$, however, has only a moral obligation not to go against it. To date, the $\mathrm{CBD}$ has been ratified by 196 countries (the Parties), and 168 have signed it before. However, there are a few countries, such as the USA, have not yet ratified this convention.

Overall, these legal regulations or restrictions at the global level to exchange germplasm across countries, only a small part of quinoa's genetic diversity is available to serve quinoa's adaptation in new environments for expansion of its cultivation.
Exchange of quinoa germplasm has been undertaken both formally via legal provisions (Standard Material Transfer Agreements - SMTAs), or informally via research networks (Figure 2). Only 25\% of genetic material exchanges correspond to individual exchanges, while $75 \%$ of the material has been exchanged among research networks. Considering personal links into existing partnerships, more than $85 \%$ of the respondents of the survey declared not signing MTAs.

\section{GENEFLOWS OF QUINOA}

Early stages of expansion highlighted the interest among importing countries and consumers for adapting quinoa to new environments, and it facilitated exchange of genetic resources. Now we are entering another phase of quinoa development, and a turning point with many countries interested in quinoa cultivation but with limitations for access to genetic resources.

Figure 2 illustrates the seed exchange among different partners involved in quinoa trials. Collections of quinoa accessions were created during the 1980s by Wilson (1988a,b, 1990) and taken back to the USA. The United States Department of Agriculture (USDA), Iowa, USA, maintains over 229 accessions, which are sent to those who request them. In Italy, the Cereal Research Centre (CER-CRA) obtained and evaluated 100 accessions for cultivation and development of varieties adapted to Mediterranean conditions. After undertaking germplasm characterization, low performing accessions were 
eliminated and some of the selected accessions were then distributed to Egypt (Figure 2). The FAO Regional Office for Near East in Cairo, Egypt, assisted in distributing quinoa accessions among National Research Institutions from eight countries (Algeria, Egypt, Iraq, Iran, Lebanon, Mauritania, Sudan, and Yemen) to conduct an evaluation of these genotypes in semi-arid and arid conditions. This example of seed flow demonstrates the importance of research networks in distributing quinoa seed at the global level. It also has limitations because of change of codes (unique identifier assigned to the accession in a genebank) from one genebank to another, and from Andean countries to other countries. Avoiding code change is one main challenge for data and germplasm sharing with transparency, but sometimes code change serves as a form of protection for plant breeders to mask the origin of the material they are developing.

The current situation provides a specific legal framework for access and exchange of genetic resources that have a strong impact on use and innovation. The Nagoya Protocol on Access and Benefit-sharing was adopted in Japan in 2010, entering in force in 2014 (70 Ratifications and 92 Signatures). It provides an international agreement, which aims at sharing the benefits from the utilization of genetic resources in a fair and equitable way, and contributing to the conservation of biological diversity and the sustainable use of its components. Considering the limits of the CBD for innovation in plant breeding, the Nagoya Protocol provides a new pathway for enhancing legal transparency on procedures for access and benefit-sharing, and for monitoring the utilization of genetic resources. Andean countries need to be active in this international agenda if they want to protect their interests on quinoa. Solutions need to be developed to better harmonize the different existing legal frameworks and to create new complementary ones. The rapid spread of quinoa at the global level provides an opportunity to consider more in depth the implications on seed exchange of the current regulatory instruments for genetic resources, so that they can be improved and implemented. In addition, it also offers an opportunity for plant breeders to reconsider models of varietal innovations in plant breeding to manage genetic resources for recombination without registration of intellectual property rights (IPR) for new varieties. In addition, adaptation of varieties to global climate changes needs to cope with an increasing unpredictability. Genetic structure of future varieties must be able to evolve to face those changes (Murphy et al., 2016). Landraces (crop populations with high level of intra-varietal diversity) from Central and Southern Chile, and in some cases from the highlands of Peru and Bolivia, are those most frequently often tested outside the Andes. In addition, researchers are mostly using only three varieties (Puno, Regalona, and Titicaca) registered through Plant Variety Protection (PVP; Bendevis et al.,

\section{REFERENCES}

Bazile, D. (2015). Le Quinoa: Les Enjeux d'une Conquête. Paris: Éditions Quæ, 112. Bazile, D., and Baudron, F. (2015). "The dynamics of the global expansion of quinoa growing in view of its high biodiversity," in State of the Art Report on Quinoa Around the World in 2013, eds D. Bazile, H. D. Bertero, and C. Nieto (Roma: FAO \& CIRAD), 42-55. 2014a,b; Jacobsen, 2015). The geographical expansion of quinoa cultivation has led to an increase in the number of PVP into action worldwide. Some countries (Netherlands, Chile, Canada, Denmark, Peru, UK, and Israel) have developed new varieties, and have set up a PVP certificate in the UPOV system. These commercial varieties facilitate access to seeds for quinoa testing. However, due to their physiological similarity being less day length sensitive than original material from Peru and Bolivia, the genetic diversity for quinoa experimentation and adaptation in new environments is narrow. Genebanks in the Andean region conserve more than $88 \%$ of the accessions available worldwide, which is considerable when taking into account the 16,422 accessions of quinoa and its wild relatives are conserved in 59 genebanks in 30 countries (Rojas et al., 2015). Despite the high levels of quinoa diversity conserved, this is currently not exchanged globally due to highly political issues of national sovereignty (Bazile, 2015).

Intellectual Property Rights should be addressed, recognizing the work of the Andean people in the selection and conservation of local quinoa landraces (Fuentes et al., 2012), and maintaining and adding value to quinoa's biodiversity for the benefit of world food security and poverty reduction. The future global seed system for quinoa cultivation needs to focus on increasing linkages among farmers, researchers, plant breeders, and politicians. This requires an iterative dialog at the national, regional, and international levels among all the stakeholders involved in managing and using quinoa diversity.

\section{AUTHOR CONTRIBUTIONS}

DB made the survey and networks analysis on quinoa global expansion and has written the first draft of the manuscript for revision by the co-authors. He is currently developing a Global Collaborative Network on Quinoa. S-EJ participated in many introduction of quinoa around the world. He shared his experience to integrate his global view on quinoa expansion to increase the quality of the mini-review. AV participated in various national projects on quinoa trials in the Near East and North Africa during his studies. He mapped the seed exchanges with the example of (Figure 2) in this manuscript.

\section{ACKNOWLEDGMENTS}

We would like to acknowledge the contributions of all colleagues, specifically from research institutions, that aided the authors in collecting the data for analyses and in understanding the complex relationships among them.

Bazile, D., Bertero, H. D., and Nieto, C. (eds). (2015). State of the Art Report on Quinoa Around the World in 2013. Roma: FAO \& CIRAD, 589.

Bazile, D., Fuentes, F., and Mujica, A. (2013). "Historical perspectives and domestication," in Quinoa: Botany, Production and Uses, eds A. Bhargava and S. Srivastava (Wallingford: CABI Publishing), 16-35.

Bendevis, M. A., Sun, Y., Rosenqvist, E., Shabala, S., Liu, F., and Jacobsen, S.-E. (2014a). Photoperiodic effects on short-pulse14C assimilation and 
overall carbon and nitrogen allocation patterns in contrasting quinoa cultivars. Environ. Exp. Bot. 104, 9-15. doi: 10.1016/j.envexpbot.2014. 03.002

Bendevis, M. A., Sun, Y., Shabala, S., Rosenqvist, E., Liu, F., and Jacobsen, S.-E. (2014b). Differentiation of photoperiod-induced ABA and soluble sugar responses of two quinoa (Chenopodium quinoa Willd.) Cultivars. J. Plant Growth Regul. 33, 562-570. doi: 10.1007/s00344-0139406-9

Chevarria-Lazo, M., Bazile, D., Dessauw, D., Louafi, S., Trommetter, M., and Hocdé, H. (2015). "Quinoa and the exchange of genetic resources: improving the regulation systems," in State of the Art Report on Quinoa Around the World in 2013, eds D. Bazile, H. D. Bertero, and C. Nieto (Roma: FAO \& CIRAD), 83-105.

Christiansen, J. L., Jacobsen, S.-E., and Jørgensen, S. T. (2010). Photoperiodic effect on flowering and seed development in quinoa (Chenopodium quinoa Willd.). Act Agric. Scand. 60, 539-544.

Elmer, L. A. (1942). Quinoa (Chenopodium quinoa). East Afr. Agric. J. 8, 21-23. doi: 10.1080/03670074.1942.11664212

FAO (2009). International Treaty on Plant Genetic Resources for Food and Agriculture. Rome: FAO, 68.

Fuentes, F., Bazile, D., Bhargava, A., and Martinez, E. A. (2012). Implications of farmers' seed exchanges for on-farm conservation of quinoa, as revealed by its genetic diversity in Chile. J. Agric. Sci. 150, 702-716. doi: $10.1017 /$ S0021859612000056

Galwey, N. W. (1993). The potential of quinoa as a multipurpose crop for agricultural diversification: a review. Ind. Crops Prod. 1, 101-106. doi: 10.1016/0926-6690(92)90006-H

Izquierdo, J., Mujica, A., Marathee, J. P., and Jacobsen, S.-E. (2003). Horizontal, technical cooperation in research on quinoa (Chenopodium quinoa Willd.). Food Rev. Int. 19, 25-29. doi: 10.1081/FRI-120018865

Jacobsen, S.-E. (1997). Adaptation of quinoa (Chenopodium quinoa) to Northern European agriculture: studies on developmental pattern. Euphytica 96, 41-48. doi: 10.1023/A:1002992718009

Jacobsen, S. E. (2003). The worldwide potential of quinoa (Chenopodium quinoa Willd.). Food Rev. Int. 19, 167-177. doi: 10.1081/FRI-120018883

Jacobsen, S. E. (2015). “Adaptation and scope for quinoa in northern latitudes of Europe," in State of the Art Report on Quinoa Around the World in 2013, eds D. Bazile, H. D. Bertero, and C. Nieto (Roma: FAO \& CIRAD), $436-446$.

Louafi, S., Bazile, D., and Noyer, J. L. (2013). "Conserving and cultivating agricultural genetic diversity: transcending established divides," in Cultivating Biodiversity to Transform Agriculture, ed. E. Hainzelin (Heidelberg: Springer), 181-230. doi: 10.1007/978-94-007-7984-6_6

Mastebroek, H. D., Limburg, H., Gilles, T., and Marvin, H. J. P. (2000). Occurrence of sapogenins in leaves and seeds of Quinoa (Chenopodium quinoa Willd.). J. Sci. Food Agric. 80, 152-156. doi: 10.1002/(SICI)10970010(20000101)80:1 < 152::AID-JSFA503> 3.0.CO;2-P

Mastebroek, H. D., Van Loo, E. N., and Dolstra, O. (2002). Combining ability for seed yield traits of Chenopodium quinoa breeding lines. Euphytica 125, 427-432. doi: 10.1023/A:1016030129541
Mujica, A., Jacobsen, S. E., Izquierdo, J., and Marathee, J. P. (2001). Resultados de la Prueba Americana y Europea de la Quinua. Puno: FAO, UNA \& CIP.

Murphy, K. M., Bazile, D., Kellogg, J., and Rahmanian, M. (2016). Seeds without Borders: development of a worldwide consortium on evolutionary participatory breeding in quinoa. Front. Plant Sci. 7:608. doi: 10.3389/fpls.2016.00608

Peterson, A., Jacobsen, S.-E., Bonifacio, A., and Murphy, K. (2015). A crossing method for quinoa. Sustainability 7, 3230-3243. doi: 10.3390/su7033230

Repo-Carrasco, R., Espinoza, C., and Jacobsen, S.-E. (2003). Nutritional value and use of the Andean crops quinoa (Chenopodium quinoa) and kañiwa (Chenopodium pallidicaule). Food Rev. Int. 19, 179-189. doi: 10.1081/FRI120018884

Risi, J. C., and Galwey, N. W. (1984). The Chenopodium grains of the Andes: inca crops for modern agriculture. Adv. Appl. Biol. 10, 145-216.

Rojas, W., Pinto, M., Alanoca, C., Gomez Pando, L., Leon-Lobos, P., Alercia, A., et al. (2015). "Quinoa genetic resources and ex situ conservation," in State of the Art Report on Quinoa Around the World in 2013, eds D. Bazile, H. D. Bertero, and C. Nieto (Roma: FAO \& CIRAD), 56-82.

Ruiz, K. B., Biondi, S., Martínez, E. A., Orsini, F., Antognoni, F., and Jacobsen, S.-E. (2015). Quinoa - a model crop for understanding salt tolerance mechanisms in halophytes. Plant Biosyst. 150, 357-371. doi: 10.1080/11263504.2015.1027317

Ruiz, K. B., Biondi, S., Oses, R., Acuña-Rodríguez, I. S., Antognoni, F., MartinezMosqueira, E. A., et al. (2014). Quinoa biodiversity and sustainability for food security under climate change. A review. Agron. Sustain. Dev. 34, 349-359. doi: 10.1007/s13593-013-0195-0

United Nations (1992). Convention on Biological Diversity. New York, NY: UNEP, 30.

Wilson, H. D. (1988a). Quinoa biosystematics I: domesticated populations. Econ. Bot. 42, 461-477. doi: 10.1007/BF02862791

Wilson, H. D. (1988b). Quinoa biosystematics II: free living populations. Econ. Bot. 42, 478-494. doi: 10.1007/BF02862791

Wilson, H. D. (1990). Quinua and relatives (Chenopodium sect. Chenopodium subsect. Cellulata). Econ. Bot. 44, 92-110. doi: 10.1007/BF02860478

Zurita-Silva, A., Fuentes, F., Zamora, P., Jacobsen, S. E., and Schwember, A. (2014). Breeding quinoa (Chenopodium quinoa Willd.): potential and perspectives. Mol. Breed. 34, 13-30. doi: 10.1007/s11032-014-0023-5

Conflict of Interest Statement: The authors declare that the research was conducted in the absence of any commercial or financial relationships that could be construed as a potential conflict of interest.

The reviewer EL and handling Editor declared their shared affiliation, and the handling Editor states that the process nevertheless met the standards of a fair and objective review.

Copyright (c) 2016 Bazile, Jacobsen and Verniau. This is an open-access article distributed under the terms of the Creative Commons Attribution License (CC BY). The use, distribution or reproduction in other forums is permitted, provided the original author(s) or licensor are credited and that the original publication in this journal is cited, in accordance with accepted academic practice. No use, distribution or reproduction is permitted which does not comply with these terms. 


\title{
Development of a Worldwide Consortium on Evolutionary Participatory Breeding in Quinoa
}

\author{
Kevin M. Murphy ${ }^{1 *}$, Didier Bazile², Julianne Kellogg ${ }^{1}$ and Maryam Rahmanian ${ }^{3}$ \\ 1 Sustainable Seed Systems Lab, Department of Crop and Soil Sciences, Washington State University, Pullman, WA, USA, \\ ${ }^{2}$ Unité Propre de Recherche Gestion des Ressources Renouvelables et Environnement, Department of Environment and \\ Societies, French Agricultural Research and International Cooperation Organization, Montpellier, France, ${ }^{3}$ Centre for \\ Sustainable Development, Tehran, Iran
}

\section{OPEN ACCESS}

Edited by:

Edmundo Acevedo,

University of Chile, Chile

Reviewed by:

Ricardo A. Pertuzé,

University of Chile, Chile

Stefania Grando,

International Crops Research Institute

for the Semi-Arid Tropics, India

Sven-Erik Jacobsen,

University of Copenhagen, Denmark

${ }^{*}$ Correspondence:

Kevin M. Murphy

kmurphy2@wsu.edu

Specialty section:

This article was submitted to Crop Science and Horticulture, a section of the journal

Frontiers in Plant Science

Received: 31 December 2015

Accepted: 20 April 2016

Published: 09 May 2016

Citation:

Murphy KM, Bazile D, Kellogg and Rahmanian M (2016)

Development of a Worldwide

Consortium on Evolutionary

Participatory Breeding in Quinoa.

Front. Plant Sci. 7:608.

do: $10.3389 /$ fpls.2016.00608
Chenopodium quinoa is gaining global importance due to its excellent protein quality and tolerance of abiotic stresses. The last 60 years have seen major strides in the expansion of quinoa crop production and experimentation. Quinoa's wide genetic diversity has led to its agronomic versatility and adaptation to different soil types, particularly saline soils, and environments with extremely variable conditions in terms of humidity, altitude, and temperature. The potential of quinoa to contribute to global food security was recognized in 2013 in the declaration of the International Year of Quinoa (IYQ). Promoting the use of improved homogeneous quinoa varieties standardized to comply with applicable norms on seeds or suited to intensified conventional agriculture farming systems may not generate the necessary resilience needed to respond to current and future global challenges. Maintaining and increasing quinoa biodiversity is imperative, as the dynamics of the global expansion of quinoa may constitute a threat to farmers if the spread is generated with a narrow genetic base. In this article, we propose that the method of evolutionary participatory breeding could be a useful tool to develop new quinoa genetic material in cooperation with farmers. We introduce preliminary results on quinoa population development with farmers in the Pacific Northwest region of the USA. We conclude that a global collaborative network on quinoa (GCN-Quinoa) could be the baseline for participatory plant breeding programs originating in developing or developed countries to meet the needs of farmers across a diversity of agronomic systems and a wide range of physical environments.

Keywords: evolutionary participatory breeding, Chenopodium quinoa, global network, population variety, agrobiodiversity

\section{QUINOA AS A KEYSTONE PROTEIN CROP FOR GLOBAL FOOD SECURITY}

Chenopodium quinoa Willd., a cultivated species of the Amaranthaceae family grown for its edible and highly nutritious achene, represents a neglected and underutilized species (NUS; Kahane et al., 2013). Quinoa is a gynomonoecious allotetraploid $(2 n=4 \times=36)$ and a facultative autogamous annual with outcrossing from 0.5 to $17.36 \%$ (Gandarillas, 1979; Silvestri and Gil, 2000). Protein content of quinoa typically ranges from 12 to $17 \%$, and is influenced by factors such as cultivar, 
soil fertility and environment (Rojas et al., 2015). Most notably, quinoa has a well-balanced complement of all essential amino acids, giving it a protein quality superior to that of other crop species (Repo-Carrasco et al., 2003). Quinoa also lacks gluten and therefore can be safely eaten by people with gluten allergies or celiac disease (Zevallos et al., 2015).

Andean farmers of South America took the first steps in domesticating quinoa from its wild forms more than 5,000 years ago (Planella et al., 2015). Seed exchanges and migrations of human populations played an important role in the early development of landraces with indehiscent, larger seeds, adaptations to drought and saline soils, and varietal differences in photoperiod, altitude, and diverse rainfall conditions (Bazile et al., 2013). Five ecotypes are globally recognized: Inter-Andean valleys (Colombia, Ecuador, and Peru); Altiplano (northern highlands in Peru and Bolivia); Yunga (Bolivia); Salares (salt flats or southern highlands in Bolivia, Chile and Argentina); and Coastal (coastal or sea level areas in central and southern Chile; Risi and Galwey, 1984; Fuentes et al., 2012; Bazile et al., 2013). Long-term selection in these sub-centers of quinoa biodiversity confers specific and often unique traits of adaptation to the landraces. Diversity is observed in a wide array of colors of plants and seeds, and through differences in branching patterns and panicle types, grain productivity, abiotic stress tolerances and disease resistance (Fuentes and Bhargava, 2011; Ruiz-Carrasco et al., 2011). Even if the potential adaptation to climate change inherent in quinoa confers probable utilization for agricultural development in other parts of the world, a better understanding of the diversity of the actual contexts of cultivation in its area of origin is crucial in order to predict where specific quinoa ecotypes would have the most agronomic impact (Jacobsen et al., 2015). Quinoa has higher drought and salinity tolerance than many other crops, and tremendous variation in these traits are evident among quinoa ecotypes and existing quinoa varieties and landraces (Bazile et al., 2015; Peterson and Murphy, 2015a). For example, the Salares ecotype is best suited to withstand extreme conditions of this cold high-altitude desert at 4.000 m.a.s.l. This ecotype is extremely drought resistant and adapted to saline and sandy soils surrounding the salt lakes. Alternatively, the quinoa of the Coastal ecotype grows from sea level up to 1500 m.a.s.l. and possesses characteristics specific to this unique environment; specifically, adaptation to high annual precipitation, resistant to pre-harvest sprouting, high evapotranspiration index, a comparatively high degree of heat tolerance, and salinity tolerance.

Due to quinoa's increasing popularity and recent expansion of cultivated area on a global scale, farmers and plant scientists are emphasizing morphological and physiological modifications that allow for mechanized harvesting, improved heat tolerance and downy mildew resistance, and reduced agricultural inputs (Bazile et al., 2015; Bonifacio et al., 2015). New sources of heat tolerance or disease resistance genes from wild relatives are being introgressed into commercial varieties by plant breeders; however, undesirable characteristics such as seed dormancy and shattering from the donor species can decelerate a quinoabreeding program (Zurita-Silva et al., 2014). The objective of this long-term participatory development project is to implement and successfully conduct a collaborative European-North American EPB program for quinoa with field sites in the host countries and in South America as well as across several countries in Africa and Asia.

\section{PARTICIPATORY PLANT BREEDING}

Participatory variety selection (PVS) and Participatory plant breeding (PPB) have been practiced in various regions and on various crops over the last 30 or more years. Much of the focus has been in developing countries, but there has also been some work in developed countries, often with a focus on low-input and/or organic agriculture (Chable et al., 2008; Ghaouti et al., 2008; Leroy et al., 2014; Campanelli et al., 2015). The primary difference between PPB and PVS is the extent of farmer participation in the various stages of the breeding program. PVS involves testing and selecting new varieties developed by the institutional system within farmers' fields and at local research stations in various environments and allowing farmers to compare these varieties with local farmer varieties. In a PPB program, farmers are routinely involved in decision-making throughout the entire breeding process and not just in the final testing of advanced breeding lines. Trials are conducted in farmers' fields and managed by farmers. Farmers score varieties according to criteria that they define. Data generated by breeders provides the basis for the selection of successful varieties by farmers, in dialog with breeders.

On marginal lands, and in relation to NUS and noncommercial crops, formal plant breeding has made less of an impact (Ceccarelli et al., 2009). To address this, plant breeders working on a diversity of crops in various regions began involving farmers in setting breeding objectives and in the subsequent selection and testing of breeding materials. This direct dialog benefits the breeding process with possible farmer participation in setting objectives, creating variability, selecting and testing breeding material, and producing and diffusing seed of new cultivars (Weltzien and Christinck, 2008). The objective of PPB research was to more appropriately respond to the specific needs of under-represented farmers and to optimize production, adoption, and food sovereignty through improved local adaptation of cultivars to relevant abiotic and biotic pressures, as well as to prevalent cultural norms (Ceccarelli et al., 2001; Sperling et al., 2001; Weltzien et al., 2003). Improvement of $\mathrm{PPB}$ continues as researchers identify different factors that affect breeding material performance including number of entries to be evaluated (Thiele et al., 1997; Ceccarelli et al., 2000), gender (Kamara et al., 1996; Sthapit et al., 1996; Weltzien et al., 1996), independent versus paired or group evaluations, and inclusion of on-station evaluations by farmers (McElhinny et al., 2007).

Among the important outcomes of PPB is the positive impact it has on biodiversity. PPB is a highly decentralized process where research occurs in a multitude of farmers' fields in different ecoregions and microclimates. PPB produces resilient varieties with high levels of phenotypic plasticity that often differ across locations depending on local climate and ecosystems, as well as social, cultural and economic factors influencing farmer 
preferences. With regard to quinoa, this diversity can be easily observed in situ in farmers' fields showing a wide array of colors in plants and seeds, different types of branching and panicles, as well as having variation in grain productivity, abiotic stress tolerance and disease resistance (Bazile et al., 2013). Varieties selected by farmers are often genetically variable, similar to traditional farmer landraces, in stark contrast to the majority of varieties produced by conventional breeding in which all the plants are genetically identical. The fact that farmers' varieties are not genetically uniform is precisely what makes them resilient to a variety of stresses that are made more unpredictable by climate change.

\section{EVOLUTIONARY PARTICIPATORY BREEDING (EPB)}

Due to the predominance of monocultures in global crop production, crop diversity occurs at a scale where individual genotype unit areas (GUAs; Mundt and Browning, 1985) are often many square kilometers (Newton et al., 2009). The genetic uniformity inherent in monocultures can restrict the crop's ability to tolerate diverse abiotic environmental stresses, pests and diseases, thereby leading to a potential decrease in the stability of the cropping system (Hooper et al., 2005; Hughes et al., 2008). Chakraborty and Newton (2011) suggest that strategies for establishing greater resilience and improved yield stability should focus on the introduction of increased genetic variability, both within and between cultivars, into agricultural systems. Such crop populations are better equipped to adapt to future unpredictable temporal climate shifts than are monocultures (Louafi et al., 2013). Genetically uniform cultivars have been shown to lack the ability to adjust and adapt to highly unpredictable environmental fluctuations, in direction and range, and novel stress factors (Verboom et al., 2010). Evolutionary breeding provides a framework with which to address current and predicted threats to agriculture as a result of climate change and agricultural intensification.

Evolutionary breeding involves four distinct stages: (i) creation of genetic diversity; (ii) multiplication of seeds from each cross followed by an equal mixing of populations if more than one cross will be used in a given population; (iii) repeated sowing and harvesting of the population in one or more agronomic environment without active selection of individual plants; and (iv) use of the seed in culturally relevant settings as food or feed, or use of the seed as a basis for further breeding (Döring et al., 2011). EPB merges the evolutionary breeding method described by Suneson (1956), Allard and Adams (1969), Allard (1988) and Phillips and Wolfe (2005) with farmer participatory breeding to develop high-yielding, disease-resistant cultivars of desired quality while maintaining a high degree of genetic variation to allow for adaptability to fluctuations in environmental conditions (Murphy et al., 2005; Döring et al., 2011; Murphy et al., 2013; Ruiz et al., 2014; Bazile, 2015).

\section{QUINOA POPULATIONS AND PRELIMINARY EPB METHODOLOGY}

Ten biparental quinoa populations were developed at Washington State University using seed from the USDA National Genetic Resources Program and from private seed companies in the US (Table 1) (Peterson et al., 2015). Parents were chosen based on one or more of the following characteristics from results of several years of preliminary testing: seed yield, day-neutral photoperiod, resistance to downy mildew, heat tolerance, drought tolerance, seed size, plant height, tolerance to lodging, mold and pre-harvest sprouting resistance, and plant and seed color. Seed was advanced to the $\mathrm{F}_{3}$ and populations were planted on two organic farms, located in Pullman and Quilcene, in 2014, each representing a distinct location and associated agronomic challenges (Washington State, USA). Pullman represents a very hot and dry climate in the major wheat growing region of the Pacific Northwest. Quilcene represents a cool, coastal climate with where late season rains caused significant yield loss due to pre-harvest sprouting and downy mildew (Peronospora variabilis) in 2010 and 2013.

No human selection was conducted on the $F_{3}$ quinoa populations in 2014, the hottest year on record in eastern Washington. Extremely low population yields in Pullman reflect

TABLE 1 | Pedigree and seed yield of 10 populations in two locations (Pullman: High-heat environment and Quilcene: Low-heat environment) in Washington State, USA in 2014.

\begin{tabular}{|c|c|c|c|c|}
\hline \multirow[t]{2}{*}{ Population designation } & \multirow[t]{2}{*}{ Female parent } & \multirow[t]{2}{*}{ Male parent } & \multicolumn{2}{|c|}{ Seed Yield $\left(\mathrm{g} / 400 \mathrm{~m}^{2}\right)$} \\
\hline & & & High-heat environment & Low-heat environment \\
\hline QUP11WA-101 & Biobio & Colorado 407D & 7.5 & 12579 \\
\hline QUP11WA-102 & Colorado 407D & QQ74 & 94.9 & 15215 \\
\hline QUP11WA-103 & Cherry Vanilla & Black & 1.3 & 10994 \\
\hline QUP11WA-104 & Kaslaea & QQ74 & 8.5 & 9371 \\
\hline QUP11WA-105 & QQ065 & QQ74 & 7.8 & 5132 \\
\hline QUP11WA-106 & QQ065 & Black & 20.8 & 6215 \\
\hline QUP11WA-107 & QQ74 & Black & 10.1 & 3864 \\
\hline QUP11WA-108 & QQ74 & Cherry Vanilla & no seed & 5009 \\
\hline QUP11WA-109 & Temuko & Biobio & no seed & 4113 \\
\hline QUP11WA-110 & Oro de Valle & Black & 7.3 & 4305 \\
\hline
\end{tabular}




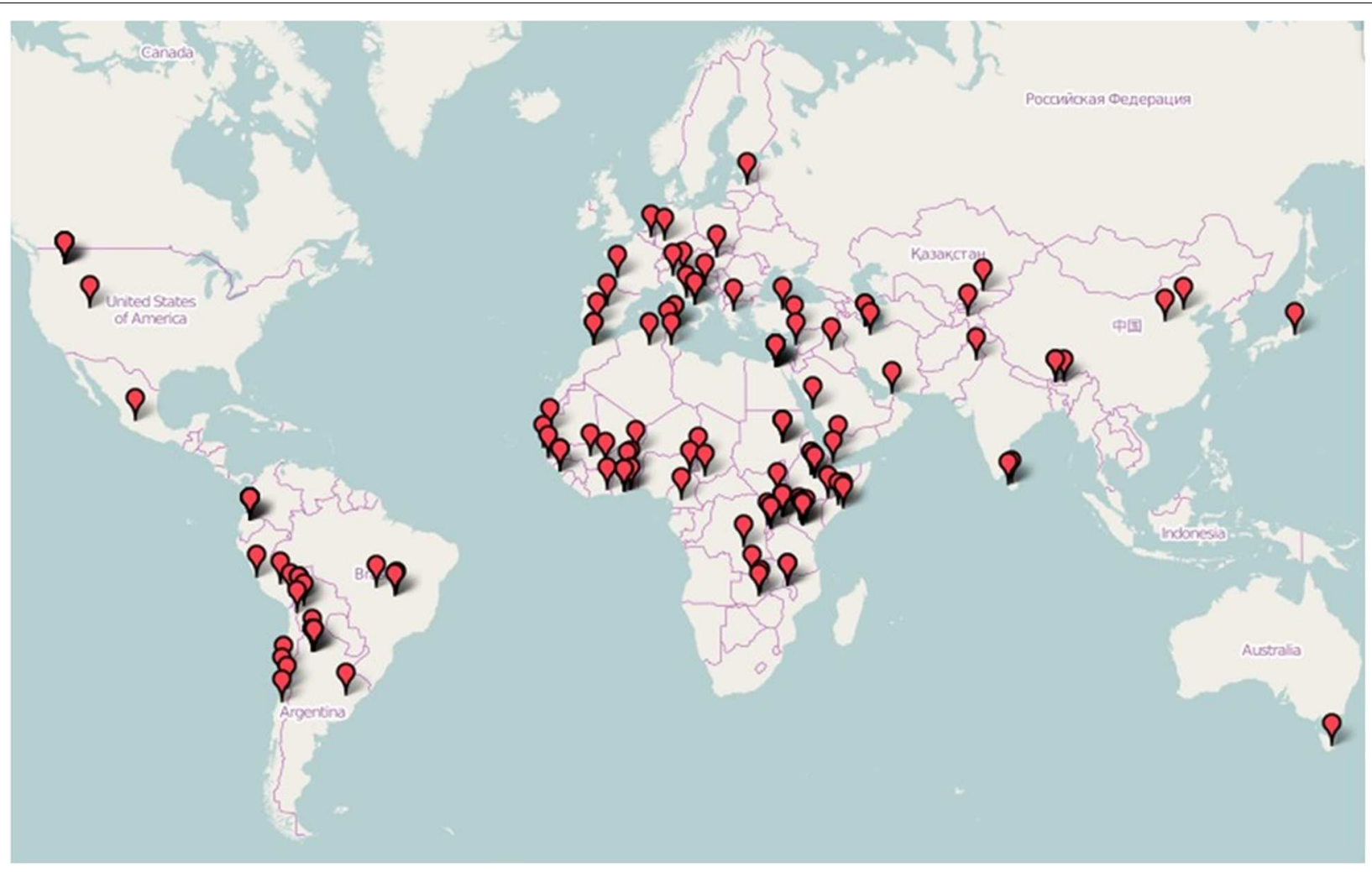

FIGURE 1 | Global distribution of the current members of the pilot Global Collaborative Network on Quinoa (GCN-Quinoa).

the effect of heat on pollen viability (Table 1). Variety trials, which included the majority the parents of the populations, adjacent to the population trials yielded no seed at all in any of the varieties. Population 102, derived from two relatively heat tolerant parents (Colorado 407D and QQ74) showed the highest seed yield (Table 1). The populations in Quilcene were not subject to heat stress, nor stress due to excessive rainfall, and subsequently seed yield was much higher. This evolutionary breeding method was repeated with $\mathrm{F}_{4}$ seed in 2015 in Quilcene, however, instead of a bulk harvest, 200 panicles from each population were harvested individually after a deliberate and prolonged exposure to rainy early autumn conditions to encourage pre-harvest sprouting in the populations. Additionally, farmers from a range of farms in the Olympic Peninsula conducted both negative and positive selection on six populations on a nearby organic farm in Chimacum, Washington. Some individual seed from each positively panicle will be saved and replanted in pedigree headrows in 2016, but the majority of the $\mathrm{F}_{5}$ seed will be bulked and the populations reconstituted; this seed will be distributed to collaborators in partner countries to begin the EPB process in 2016.

\section{PERSPECTIVES ON A GCN-QUINOA}

The pilot GCN-Quinoa is the first step in moving forward with $\mathrm{EPB}$ in quinoa. After just one year of existence, more than
150 researchers are part of this global network and represent a diversity of people from 60 countries who currently share a similar curiosity in adapting quinoa to their region (Figure 1). Nowadays, the number of research centers with experimental quinoa programs is increasing worldwide, even in regions that are not yet producing or consuming quinoa (Bazile et al., 2015; Peterson and Murphy, 2015b). However, the primary quinoa research centers are located in countries where quinoa has been grown traditionally for centuries. As a result, there exists a significant gap in varietal, agronomic, and cultural knowledge of quinoa production and consumption in Africa for example. In that context, the main objective of the GCN-Quinoa is to create a collaborative space to facilitate exchanges between producers, processors, distributors, politicians, and all persons involved in quinoa development for the promotion and sustainable use of quinoa genetic resources. Access and benefit sharing about quinoa seeds are of importance that is why a specific forum of discussion was generated online on this topic. Members of the GCN-quinoa interested in testing quinoa through EPB approach have joined, and can continue to join, the development of the Worldwide Consortium on Evolutionary Participatory Breeding in Quinoa and benefit from the expertise and support of the group.

Increasing consumer demand in quinoa and the usefulness of the crop in adapting to climate change means that the cultivation of quinoa is undergoing rapid expansion worldwide. It thus acknowledges the role that quinoa's biodiversity and high 
nutritional value could play in providing global food security. Since this minor crop could become a major crop, how can we continue the varietal improvement efforts that farmers in the Andean countries have been carrying out for generations? The achievement of this goal requires innovative approaches to plant breeding in order to avoid the well-known risks of genetic uniformity and vulnerability as well as to encourage sustainable agriculture with a decreased use of agrochemical inputs.

How does one breed for farmer's needs? Involving a large number of farmers in evaluation and selection throughout the selection process is an excellent way to take into account heterogeneity across environments (Kotschi, 2010). This form of decentralized selection is critical in involving and empowering farmers from the beginning of the breeding process. There is a need for a paradigm shift in agriculture which must enhance the role of agrobiodiversity and the need for innovation in plant breeding is particularly crucial (Ceccarelli, 2009). Increasing genetic diversity in quinoa populations may be a relevant method to adapt crops to environmental changes, in particular, to agricultural areas where environmental conditions are marginal for crop production. It will be important to consider market options, and the desire among end-users for a uniform product, when developing populations. Therefore, particular attention must be given to the selection toward uniformity of targeted quality traits, such as seed size and color, which can be identified visually post-harvest, and protein content and starch characteristics, which can be evaluated in a lab (Wu et al., 2014).

Evolutionary PPB with quinoa has the potential to contribute significantly to sustainable agriculture if a lasting connection can be made among all the relevant stakeholders. Linking researchers at the global level who are involved with quinoa trials in a collaborative network may be the most effective pathway. One of the challenges offered during the (IYQ, 2013) was to devise a means and methodology to increase the likelihood of quinoa's success in the introduction and development in non-traditional quinoa growing regions. Next-generation quinoa research will require a collaborative effort from a dedicated group of experts who possess the ability to evaluate the diverse global needs of quinoa research and to subsequently conduct this research in a cooperative manner (Bazile, 2013).

Given that quinoa cultivation and research is expanding to parts of world that are not familiar with the crop, researchers and farmers would benefit from being included in a network with other researchers facing similar challenges. They would also benefit from access to a wide range of germplasm which is currently concentrated in gene banks in Latin America, as noted above, and to which researchers in other parts of the world may not have ready access (Bazile et al., 2016). The International Treaty on Plant Genetic Resources for Food and Agriculture (the Treaty) proposes the implementation of a multilateral system (MLS) of exchange of genetic resources for both facilitate access to genetic resources and ensure benefit-sharing. The Treaty provides a monitoring of genetic resources with the harmonized material transfer agreements; certification of origin to prevent biopiracy and the disclosure of origin to share the benefits. With few notable exceptions (Argentina, Bolivia, and Colombia), Andean countries ${ }^{1}$ have ratified the Treaty. Under its MLS, countries agree to make available their genetic resources stored in collections that are under their control and in the public domain for 64 crops that are listed in Annex 1 of the Treaty. Although some discussions are under way to possibly enlarge this list, quinoa does not currently belong to this Annex 1 which means that, so far, the rules of the MLS do not apply for the exchanges of quinoa genetic material. The Convention on Biological Diversity (CBD) does only regulate bilateral access and benefit sharing, but this is difficult to apply to quinoa as the crop is now planted internationally, not restricted to the Andean region, and this has been the case for decades. For quinoa, there is no single existing legal framework providing a comprehensive coverage of all the issues related to the genetic resources and their sustainable management (Chevarria-Lazo et al., 2015). Considering that the status of quinoa is now rapidly changing from a NUS to a major crop for food and agriculture, the opportunity exists to question current dominant agricultural models and plant breeding methods and begin to move forward toward more participatory approaches in order to address the real needs of the end-users in a context of global changes (Anvar, 2008; Trommetter, 2010). Research and plant breeding in quinoa should be based on more flexible genetic material with the potential to maintain yield stability while continually evolving in response to changes in climate. Considering the GCN-Quinoa as a vector to disseminate evolutionary material, considered as open seed sources, with a high level of genetic diversity will certainly favor adaptation in new environments. Nevertheless, without direct dialog resulting from close collaborations between farmers and researchers, we may struggle to reach the next step for quinoa introduction to cropping systems for local diets.

\section{AUTHOR CONTRIBUTIONS}

KM wrote two sections of, and edited the entire manuscript. KM initiated the quinoa populations and along with JK set up and conducted the participatory breeding research. JK provided edits to the entire manuscript. DB and MR contributed significantly to the writing and editing of the manuscript. In addition, DB. initiated the Global Collaborative Network on Quinoa.

\section{ACKNOWLEDGMENT}

The authors thank all of the researchers, farmers and stakeholders who have already manifested their interest in joining the pilot GCN-Quinoa (gcn-quinoa.org) and who have requested quinoa crop populations to initiate trials.

\footnotetext{
${ }^{1}$ Contracting Parties: Chile (2016), Ecuador (2004), Peru (2003), Venezuela (2005); Only Signatorie: Argentina (2002), Colombia (2002); Non Contracting parties: Bolivia.
} 


\section{REFERENCES}

Allard, R. W. (1988). Genetic changes associated with the evolution of adaptedness in cultivated plants and their wild progenitors. J. Heredity 79, 225-238.

Allard, R. W., and Adams, J. (1969). Population studies in predominantly selfpollinating species. XIII. Intergenotypic competition and population structure in barley and wheat. Am. Nat. 103, 621-645. doi: 10.1086/282630

Anvar S. L. (2008). Semences et Droit. L'emprise d'un Modèle Economique Dominant sur une Réglementation Sectorielle, Thèse de Doctorat en droit, Université Paris 1 Panthéon-Sorbonne, Paris, 470.

Bazile, D. (2013). "The high genetic diversity of Chenopodium quinoa Willd and its global expansion. Invited speaker," in Proceeding of the International Quinoa Research Symposium 2013, Pullman: Washington State University. Available at: http://articles.extension.org/pages/68467/international-quinoa-research-sy mposium-broadcast-webinar [Accessed August 12-14, 2013].

Bazile, D. (2015). Le Quinoa : Les Enjeux d'une Conquête. (Paris: Editions Quae), 112.

Bazile, D., Bertero, H. D., and Nieto, C. (eds). (2015). State of the Art Report on Quinoa Around the World in 2013. (Rome: FAO and CIRAD), 603. Available at: http://www.fao.org/3/a-i4042e.pdf

Bazile, D., Fuentes, F., and Mujica, A. (2013). "Historical perspectives and domestication," in Quinoa: Botany, Production and Uses, eds A. Bhargava and S. Srivastava (Wallingford: CABI), 16-35.

Bazile, D., Jacobsen, S.-E., and Verniau, A. (2016). The global expansion of quinoa: trends and limits. Front. Plant Sci. Sect. Crop Sci. Horticult. 3:622.

Bonifacio, A., Gomez-Pando, L., and Rojas, W. (2015). "Quinoa breeding and modern variety development," in State of the Art Report on Quinoa Around the World in 2013, eds D. Bazile, H. D. Bertero, and C. Nieto (Rome: FAO/CIRAD), 172-191.

Campanelli, G., Acciarri, N., Campion, B., Delvecchio, S., Leteo, F., Fusari, F., et al. (2015). Participatory tomato breeding for organic conditions in Italy. Euphytica 204, 179-197. doi: 10.1007/s10681-015-1362-y

Ceccarelli, S. (2009). Evolution, plant breeding and biodiversity. J. Agric. Environ. Int. Dev. 103, 131-145.

Ceccarelli, S., Grando, S., Bailey, E., Amri, A., El-Felah, M., Nassif, F., et al. (2001). Farmer participation in barley breeding in Syria, Morocco, and Tunisia. Euphytica 122, 21-36.

Ceccarelli, S., Grando, S., Tutwiler, R., Baha, J., Martini, A. M., Salahieh, H., et al. (2000). A methodological study on participatory barley breeding I. Selection phase. Euphytica 111, 91-104. doi: 10.1023/A:1003717303869

Ceccarelli, S., Guimarães, E. P., and Weltzien, E. (eds). (2009). Plant Breeding and Farmer Participation. Rome: fao.

Chable, V., Conseil, M., Serpolay, E., and Le Lagadec, F. (2008). Organic varieties for cauliflowers and cabbages in Brittany: from genetic resources to participatory plant breeding. Euphytica 164, 521-529. doi: 10.1007/s10681-0089749-7

Chakraborty, S., and Newton, A. C. (2011). Climate change, plant diseases and food security: an overview. Plant Pathol. 60, 2-14. doi: 10.1016/j.jbiotec.2011.06.013

Chevarria-Lazo, M., Bazile, D., Dessauw, D., Louafi, S., Trommetter, M., and Hocdé, H. (2015). "Quinoa and the exchange of genetic resources: Improving the regulation systems," in State of the Art Report on Quinoa Around the World in 2013, eds D. Bazile, H. D. Bertero, and C. Nieto (Roma: FAO \& CIRAD), 83-105.

Döring, T. F., Knapp, S., Kovacs, G., Murphy, K. M., and Wolfe, M. S. (2011). Evolutionary plant breeding in cereals-Into a new era. Sustainability 3, 19441971. doi: 10.3390/su3101944

Fuentes, F., Bazile, D., Bhargava, A., and Martinez, E. A. (2012). Implications of farmers' seed exchanges for on-farm conservation of quinua, as revealed by its genetic diversity in Chile. J. Agric. Sci. 150, 702-716. doi: $10.1017 /$ S0021859612000056

Fuentes, F., and Bhargava, A. (2011). Morphological analysis of quinoa germplasm grown under lowland desert conditions. J. Agron. Crop Sci. 197, 124-134. doi: 10.1111/j.1439-037X.2010.00445.x

Gandarillas, H. (1979). “Mejoramiento genético," in Quinua y Kaniwa. Cultivos Andinos, Serie Libros y Materiales Educativos. Instituto Interamericano de Ciencias Agricolas, ed. M. E. Tapia (Bogota: Colombia), 65-82.

Ghaouti, L., Vogt-Kaute, W., and Link, W. (2008). Development of locally-adapted faba bean cultivars for organic conditions in Germany through a participatory breeding approach: participatory breeding of faba bean for organic conditions. Euphytica 162, 257-268. doi: 10.1007/s10681-007-9603-3

Hooper, D., Chapin, F., Ewel, J., Hector, A., Inchausti, P., Lavorel, S., et al. (2005). Effects of biodiversity on ecosystem functioning: a consensus of current knowledge. Ecol. Monogr. 75, 3-35. doi: 10.1111/brv.12110

Hughes, A., Inouye, B., Johnson, M., Underwood, N., and Vellend, M. (2008). Ecological consequences of genetic diversity. Ecol. Lett. 11, 609-623. doi: 10.1111/j.1461-0248.2008.01179.x

Jacobsen, S.-E., Sørensen, M., Pedersen, S. M., and Weiner, J. (2015). Using our agrobiodiversity: plant-based solutions to feed the world. Agron. Sustain. Dev. 35, 1217-1235. doi: 10.1007/s13593-015-0325-y

Kahane, R., Hodgkin, T., Jaenicke, H., Hoogendoorn, C., Hermann, M., Keatinge, J. D. H., et al. (2013). Agrobiodiversity for food security, health and income. Agron. Sustain. Dev. 33, 671-693. doi: 10.1007/s13593-013-0147-8

Kamara, A., Defoer, T., and de Groote, H. (1996). Selection of new varieties through participatory research, the case of corn in South Mali. Tropicultura 14, 100-105.

Kotschi, J. (2010). Reconciling agriculture with biodiversity and innovations in plant breeding. GAIA 1, 20-24.

Leroy, T., Coumaré, O., Kouressy, M., Trouche, G., Sidibé, A., Sissoko, S., et al. (2014). Inscription d'une variété de sorgho obtenue par sélection participative au Mali dans des projets multiacteurs. Agron. Environ. Soc. 4, 143-152.

Louafi, S., Bazile, D., and Noyer, J. L. (2013). "Conserving and cultivating agricultural genetic diversity: transcending established divides," in Cultivating Biodiversity to Transform Agriculture, ed. E. Hainzelin (Heidelberg: Springer), 181-230.

McElhinny, E., Peralta, E., Mazón, N., Danial, D. L., Thiele, G., and Lindhout, P. (2007). Aspects of participatory plant breeding for quinoa in marginal areas of Ecuador. Euphytica 153, 373-384. doi: 10.1007/s10681-006-9200-x

Mundt, C., and Browning, J. (1985). Development of crown rust epidemics in genetically diverse oat populations: effect of genotype unit area. Phytopathology 75, 607-610. doi: 10.1094/Phyto-75-1141

Murphy, K., Lammer, D., Lyon, S., Carter, B., and Jones, S. (2005). Breeding for organic and low-input farming systems: an evolutionary-participatory breeding method for inbred cereal grains. Renew. Agric. Food Syst. 20, 48-55. doi: 10.1079/RAF200486

Murphy, K. M., Carter, A. H., and Jones, S. S. (2013). "Evolutionary breeding and climate change," in Genomics and Breeding for Climate-Resilient Crops, ed. C. Kole (Berlin: Springer), 377-389.

Newton, A. C., Begg, G. S., and Swanston, J. S. (2009). Deployment of diversity for enhanced crop function. Ann. Appl. Biol. 154, 309-322. doi: 10.1371/journal.pone.0108179

Peterson, A. J., Jacobsen, S.-E., Bonifacio, A., and Murphy, K. M. (2015). A crossing method for quinoa. Sustainability 7, 3230-3243. doi: 10.3390/su7033230

Peterson, A. J., and Murphy, K. M. (2015a). Tolerance of lowland quinoa cultivars to sodium chloride and sodium sulfate salinity. Crop Sci. 55, 331-338. doi: 10.2135/cropsci2014.04.0271

Peterson, A. J., and Murphy, K. M. (2015b). "Quinoa cultivation for temperate north america: considerations and areas for investigation," in Quinoa: Improvement and Sustainable Production, eds K. M. Murphy and J. G. Matanguihan (Hoboken: Wiley-Blackwell).

Phillips, S. L., and Wolfe, M. S. (2005). Centenary review: evolutionary plant breeding for low input systems. J. Agric. Sci. 143, 245-254. doi: 10.1017/S0021859605005009

Planella, M. T., Lopez, M. L., and Bruno, M. C. (2015). "Domestication and prehistoric distribution," in State of the Art Report on Quinoa Around the World in 2013, eds D. Bazile, H. D. Bertero, and C. Nieto (Rome: FAO/CIRAD), 29-41.

Repo-Carrasco, R., Espinoza, C., and Jacobsen, S.-E. (2003). Nutritional value and use of the Andean crops quinoa (Chenopodium quinoa) and kañiwa (Chenopodium pallidicaule). Food Rev. Int. 19, 179-189. doi: 10.1081/FRI120018884

Risi, J., and Galwey, N. W. (1984). The Chenopodium grains of the Andes: inca crops for modern agriculture. Adv. Appl. Biol. 10, 145-216.

Rojas, W., Pinto, M., Alanoca, C., Gomez Pando, L., Leon-Lobos, P., Alercia, A., et al. (2015). "Quinoa genetic resources and ex situ conservation," in State of the Art Report on Quinoa Around the World in 2013, eds D. Bazile, H. D. Bertero, and C. Nieto (Rome: FAO/CIRAD), 56-82.

Ruiz, K. B., Biondi, S., Oses, R., Acuña-Rodríguez, I. S., Antognoni, F., MartinezMosqueira, E. A., et al. (2014). Quinoa biodiversity and sustainability for food 
security under climate change. A review. Agron. Sustain. Dev. 34, 349-359. doi: 10.1007/s13593-013-0195-0

Ruiz-Carrasco, K., Antognoni, F., Coulibaly, A. K., Lizardi, S., Covarrubias, A., Martinez, E. A., et al. (2011). Variation in salinity tolerance of four lowland genotypes of quinoa (Chenopodium quinoa Willd.) as assessed by growth, physiological traits, and sodium transporter gene expression. Plant Physiol. Biochem. 49, 1333-1341. doi: 10.1016/j.plaphy.2011.08.005

Silvestri, V., and Gil, F. (2000). Alogamia en quinua. Tasa en Mendoza (Argentina). Rev. Facult. Cienc. Agrarias Univ. Naci. Cuyo 32, 71-76.

Sperling, L., Ashby, J. A., Smith, M. E., Weltzien, E., and McGuire, S. (2001). A framework for analyzing participatory plant breeding approaches and results. Euphytica 122, 439-450. doi: 10.1023/A:10175053 23730

Sthapit, B. R., Joshi, K. D., and Witcombe, J. R. (1996). Farmer participatory crop improvement III. Participatory plant breeding, a case study for rice in Nepal. Exp. Agric. 32, 479-496. doi: 10.1017/S001447970000154X

Suneson, C. A. (1956). An evolutionary plant breeding method. Agron. J. 48, 188-191. doi: 10.2134/agronj1956.00021962004800040012x

Thiele, G., Gardner, G., Torrez, R., and Gabriel, J. (1997). Farmer involvement in selecting new varieties: potatoes in Bolivia. Exp. Agric. 33, 275-290. doi: $10.1017 /$ S0014479797003098

Trommetter, M. (2010). Flexibility in the implementation of intellectual property rights in agricultural biotechnology. Eur. J. Law Econ. 30, 223-245. doi: 10.1007/s10657-009-9133-7

Verboom, J., Schippers, P., Cormont, A., Sterk, M., Vos, C., and Opdam, P. (2010). Population dynamics under increasing environmental variability: implications of climate change for ecological network design criteria. Landsc. Ecol. 25, 1289-1298. doi: 10.1007/s10980-010-9497-7

Weltzien, E., and Christinck, A. (2008). "Participatory plant breeding: developing improved and relevant crop varieties with farmers," in Agricultural Systems: Agroecology and Rural Innovation for Development, eds S. S. Snapp and B. Pound (Amsterdam: Elsevier), 211-251.
Weltzien, E., Smith, M. E., Meitzner, L. S., and Sperling, L. (2003). Technical and Institutional Issues in Participatory Plant Breeding-from the Perspective of Formal Plant Breeding: A Global Analysis of Issues, Results, and Current Experience. PPB Monograph, PRGA Program No. 1. Colombia, 226.

Weltzien, E. W., Whitaker, M. L., and Anders, M. M. (1996). "Farmer participation in pearl millet breeding for marginal environments," in Participatory Plant Breeding: Proceedings of Workshop on Participatory Plant Breeding, eds P. Eyzaguirre and M. Iwanaga (Rome: IPGRI), 128-143.

Wu, G., Murphy, K. M., and Morris, C. (2014). Evaluation of texture differences among varieties of cooked quinoa. J. Food Sci. 79, S2337-S2345. doi: 10.1111/1750-3841.12672

Zevallos V. F., Herencia, L. I., and Ciclitira, P. J. (2015). "Quinoa, coeliac disease and gluten-free diet," in State of the Art Report of Quinoa in the World in 2013, eds D. Bazile, D. Bertero, and C. Nieto (Rome: FAO/CIRAD), 300-313.

Zurita-Silva, A., Fuentes, F., Zamora, P., Jacobsen, S.-E., and Schwember, A. R. (2014). Breeding quinoa (Chenopodium quinoa Willd.): potential and perspectives. Mol. Breed. 34, 13-30. doi: 10.1007/s11032-014-0023-5

Conflict of Interest Statement: The authors declare that the research was conducted in the absence of any commercial or financial relationships that could be construed as a potential conflict of interest.

The reviewer RP and handling Editor declared their shared affiliation, and the handling Editor states that the process nevertheless met the standards of a fair and objective review.

Copyright (c) 2016 Murphy, Bazile, Kellogg and Rahmanian. This is an open-access article distributed under the terms of the Creative Commons Attribution License (CC BY). The use, distribution or reproduction in other forums is permitted, provided the original author(s) or licensor are credited and that the original publication in this journal is cited, in accordance with accepted academic practice. No use, distribution or reproduction is permitted which does not comply with these terms. 


\section{OPEN ACCESS}

Edited by:

Soren K. Rasmussen,

University of Copenhagen, Denmark

Reviewed by:

Michael Benjamin Kantar,

University of Hawaii, USA

Redouane Choukallah,

International Center for Biosaline

Agriculture (ICBA), United Arab

Emirates

*Correspondence:

Didier Bazile

didier.bazile@cirad.fr

Specialty section:

This article was submitted to

Crop Science and Horticulture,

a section of the journal

Frontiers in Plant Science

Received: 24 March 2016 Accepted: 30 May 2016

Published: 21 June 2016

Citation:

Bazile D, Pulvento C, Verniau A,

Al-Nusairi MS, Ba D, Breidy J,

Hassan L, Mohammed MI,

Mambetov O, Otambekova M, Sepahvand NA, Shams A, Souici D,

Miri K and Padulosi S (2016) Worldwide Evaluations of Quinoa:

Preliminary Results from Post

International Year of Quinoa FAO

Projects in Nine Countries.

Front. Plant Sci. 7:850.

doi: 10.3389/fp/s.2016.00850

\section{Worldwide Evaluations of Quinoa: Preliminary Results from Post International Year of Quinoa FAO Projects in Nine Countries}

\author{
Didier Bazile ${ }^{1 *}$, Cataldo Pulvento ${ }^{2}$, Alexis Verniau ${ }^{3}$, Mohammad S. Al-Nusairi $^{4}$, Djibi Ba ${ }^{5}$, \\ Joelle Breidy ${ }^{6}$, Layth Hassan ${ }^{7}$, Maarouf I. Mohammed ${ }^{8}$, Omurbek Mambetov ${ }^{9}$, \\ Munira Otambekova ${ }^{10}$, Niaz Ali Sepahvand ${ }^{11}$, Amr Shams ${ }^{12}$, Djamel Souici ${ }^{13}$, Khaled Miri ${ }^{11}$ \\ and Stefano Padulosi ${ }^{14}$
}

\footnotetext{
${ }^{1}$ Unité Propre de Recherche Gestion des Ressources Renouvelables et Environnement, Department of Environments and Societies, French Agricultural Research and International Cooperation Organization, Montpellier, France, ${ }^{2}$ CNR-Institute for Agricultural and Forest Systems in the Mediterranean, Ercolano, Italy, ${ }^{3}$ ESA-Ecole Supérieur d'Agriculture, Angers, France, ${ }^{4}$ General Seed Multiplication Cooperation, Agricultural Research and Extension Authority, Dhamar, Yemen, ${ }^{5}$ Centre National de Recherche Agronomique et de Développement Agricole, Kaedi, Mauritania, ${ }^{6}$ Department of Plant Breeding, Lebanese Agricultural Research Institute, Zahlé, Lebanon, ${ }^{7}$ Plant Production Department, Ministry of Agriculture, Baghdad, Iraq, ${ }^{8}$ Genetics and Plant Breeding, Forage and Range Research Program, Agricultural Research Corporation, Khartoum North, Sudan, ${ }^{9}$ Seed Association of Kyrgyzstan, Bishkek, Kyrgyz, ${ }^{10}$ Seed Association of Tajikistan, Dushanbe, Tajikistan, ${ }^{11}$ Research Department, Seed and Plant Improvement Institute, Karaj, Iran, ${ }^{12}$ Crop Intensification Research Department, Field Crops Research Institute, Agricultural Research Center, Giza, Egypt, ${ }^{13}$ Département Recherche Expérimentation, Institut Technique de Développement de l'Agronomie Saharienne, Biskra, Algeria, ${ }^{14}$ Bioversity International, Maccarese, Italy
}

Chenopodium quinoa Willd., a high quality grain crop, is resistant to abiotic stresses (drought, cold, and salt) and offers an optimal source of protein. Quinoa represents a symbol of crop genetic diversity across the Andean region. In recent years, this crop has undergone a major expansion outside its countries of origin. The activities carried out within the framework of the International Year of Quinoa provided a great contribution to raise awareness on the multiple benefits of quinoa as well as to its wider cultivation at the global level. FAO is actively involved in promoting and evaluating the cultivation of quinoa in 26 countries outside the Andean region with the aim to strengthen food and nutrition security. The main goal of this research is to evaluate the adaptability of selected quinoa genotypes under different environments outside the Andean region. This paper presents the preliminary results from nine countries. Field evaluations were conducted during 2013/2014 and 2014/2015 in Asia (Kyrgyzstan and Tajikistan), and the Near East and North African countries (Algeria, Egypt, Iraq, Iran, Lebanon, Mauritania, and Yemen). In each country, the trials were carried out in different locations that globally represent the diversity of 19 agrarian systems under different agro-ecological conditions. Twenty-one genotypes of quinoa were tested using the same experimental protocol in all locations consisting in a randomized complete block design (RCBD) with three replicates. Some genotypes showed higher yields and the Q18 and Q12 landraces displayed greater adaptation than others to new environmental conditions. The Q21 and Q26 landraces were evaluated with stable and satisfactory levels of yield $\left(>1 \mathrm{t}^{\mathrm{h} \mathrm{h}^{-1}}\right)^{-}$in each of 
the different trial sites. This production stability is of considerable importance especially under climate change uncertainty. While these results suggest that this Andean crop is able to grow in many different environments, social, and cultural considerations remain crucial regarding its possible introduction as a staple food in new cropping systems around the world.

Keywords: Chenopodium quinoa Willd., plant genetic resources, seeds, adaptation, climate change, multi-local trials, agrobiodiversity, agroecology

\section{INTRODUCTION}

Chenopodium quinoa Willd. is a plant is a plant originated in the Andean Plateau, around Lake Titicaca, $3800 \mathrm{~m}$ above sea level (m.a.s.l.) on the Peruvian-Bolivian border (Heiser and Nelson, 1974; Jacobsen, 2003). Quinoa domestication began there about 7000 years ago (Bazile et al., 2013), and brought a significant increase in the genetic diversity of the species cultivated (Bhargava et al., 2007a,b). Such a high genetic diversity in quinoa is closely related to the vastness of its center of origin and the varied of human uses that have been influencing the selection process over time (Bazile and Negrete, 2009). This is the case with many domestication processes of numerous important crops and their wild relatives (Wilson, 1990). The selection exerted by local communities has led to many landraces, which can be still found grown especially in Bolivia and Peru (Risi and Galwey, 1984; Bazile et al., 2014; Bazile, 2015).

At global level, there are more than 6000 varieties of quinoa cultivated by farmers (Rojas et al., 2015). Those varieties can be classified into five main categories or ecotypes, according to their adaptation to specific agro-ecological conditions in major production areas (Bois et al., 2006; Rojas, 2003; Anabalón and Thomet-Isla, 2009; Fuentes et al., 2009a; Fuentes and Bhargava, 2011; Bazile et al., 2013, 2014). Quinoa of the interAndean valleys grows in areas between 2300 and 3500 m.a.s.l., characterized by annual rainfall between 700 and $1500 \mathrm{~mm}$. Quinoa grows in highlands (also called Altiplano of the Andes) between 3500 and 3900 m.a.s.l. in areas with an annual rainfall of 400-800 mm. Quinoa from the edges of deserts and high altitude salt lakes (Salares) grows in areas nearly 4000 m.a.s.l., characterized by a limited volume of annual rainfall (150-300 $\mathrm{mm}$ ) and with many days of frost. Quinoa found at sea level (Coastal) is adapted to the regions lying between sea level and 1000 m.a.s.l., where annual rainfall ranges from 500 to $1500 \mathrm{~mm}$ (Martínez et al., 2009). Quinoa from the Yungas grows under tropical moisture conditions and in areas with high levels of precipitations.

Considering the high genetic diversity of quinoa, the needs of the crop vary extensively by landrace or cultivar (Cleveland et al., 1994; Brookfield et al., 2002; Chevassus-au-Louis and Bazile, 2008). Due to the diverse characteristics of the five ecotypes, quinoa can be grown under very different climatic conditions (Jacobsen, 1997; Jacobsen et al., 2003; Christensen et al., 2007; Fuentes et al., 2009b, 2012). Sandy loam soils with good drainage and if possible, a high content of organic matter and nutrients are preferable for quinoa to better adapt to new environments. It is also advisable to work in neutral soils, although quinoa can tolerate different $\mathrm{pH}$ and grow in alkaline (to $\mathrm{pH} 9$ ) and acid soils as well (up to pH 4.5; Narea, 1976; Tapia, 1979). As previously described, quinoa cultivation can be pursued in many climatic conditions, including desert, hot, dry, cold and dry, temperate, and rainy or hot with high humidity (Bosque et al., 2003; Gesinski, 2008). Indeed, scientific evidence exist to confirm that quinoa tolerates very dry conditions and drought: quinoa uses water very effectively, even though it is a C3 plant, due to physiological mechanisms (Cocozza et al., 2013) that allow the plant to prevent moisture deficits, and tolerate and/or withstand lack of soil moisture. An ideal average temperature for quinoa would be around $15-20^{\circ} \mathrm{C}$, but some specific landraces can also withstand extreme temperatures from $-8^{\circ} \mathrm{C}$ to $+38^{\circ} \mathrm{C}$ (Bazile et al., 2015). Sensitivity periods to temperatures have been recorded mainly when seeds germination occurs in cold temperatures (frost) and when flowering takes place under high temperatures (FAO, 2011). Quinoa is able to endure extreme solar radiation, allowing it to store hours of heat necessary to carry out its vegetative and productive phases. Quinoa is cultivated in areas from $2^{\circ}$ North latitude to $47^{\circ}$ South latitude, from Colombia to Chile in South America.

There are varieties adapted to short days, long days and those photoperiod insensitive (Bertero et al., 1999; Bertero, 2001). There are two major phases in the development of quinoa plants: the vegetative phase is an active growth phase during which the plant acquires new properties to reach vegetative maturity. This is followed by the reproductive phase, which is the period when the plant will be able to produce flowers and seeds and reach physiological maturity. Depending on the photoperiod sensibility of each variety, each stage's duration can be modified depending on the length of days and on temperatures (Risi and Galwey, 1989, 1991; Jacobsen and Stølen, 1993). Photoperiod sensitivity is a key factor in the adaptation of this crop at new latitudes (Bertero, 2001).

For thousands of years, quinoa has been a staple food for Andean populations (Tagle and Planella, 2002; Planella et al., 2011; Martinez et al., 2015). Mainly used as a cereal grain (it is in many cases considered a pseudo-cereal), botanically speaking quinoa is an achene, a seed-like fruit with a hard coat (Cusack, 1984; NRC, 1989). Classified as a member of the Amaranthaceae (a large family of 160 genera and 2400 species- Sing, 2010) from the genus Chenopodium, the specie C. quinoa Willdenow is gaining importance particularly for its high and wellbalanced nutritional contents. Quinoa has exceptional nutritional properties, with high protein content in comparison to cereals, which is combined with a good balance of essential amino acids (Vega-Gálvez et al., 2010; Maureira and Martínez, 2012; Miranda 
et al., 2012; Lutz et al., 2013). The quinoa crop has recently undergone important developments around the world regarding its ability to withstand extreme conditions (Bazile et al., 2015). Its high genetic diversity provides opportunities for leveraging its hardiness and further its wide adaptation (Louafi et al., 2013). Many countries regularly face food insecurity problems, often combined with a difficult agricultural environment (FAO, 2015). Today, drought and soil salinization are major limiting factors in cultivation, a fact that is generating significant pressure on arable land availability. Considering these major challenges, quinoa hardiness is increasingly being appreciated by growers: today the crop is presently cultivated, or is under experimentation, in more than 95 countries and its cultivation continue to expand rapidly worldwide (Bazile, 2015; Bazile et al., 2016).

The main quinoa producers in the world are Bolivia, Peru, Ecuador, and the United States of America. In 2013, over 75,000 hectares of land were under quinoa cultivation in Bolivia and more than 45,000 hectares in Peru. These two countries are still the major producers in the Andes and in the world. Today the cultivation of quinoa has reached countries as far as Tibet, Morocco, France, India, China, the United Kingdom, Sweden, Denmark, Netherlands, and Italy, among others (Bhargava et al., 2006; Pulvento et al., 2010; Bazile, 2015; Bazile et al., 2015). From the 1950s to nowadays, trials for plant breeding and/or crop adaptation have been conducted in Andean countries but also in other parts of the world in order to better understand the domestication process of quinoa and to obtain quinoa germplasm adapted to new environmental conditions (Bonifacio et al., 2015; Jellen et al., 2015). Quinoa is a viable alternative for food insecure countries in a world facing increasingly climate challenges and set to feed a growing population in terms of both food and nutrition security (Galwey, 1992; Ruiz et al., 2014).

The FAO project "American and European Test of Quinoa" (1996-1998) was the first mechanism for the diffusion of quinoa worldwide and underlies the current global expansion of the crop (Mujica et al., 2001). Field trials were established in several countries to evaluate the performance of quinoa through multiple experiments at the international level. Since 1996, quinoa has been recognized as one of the most promising crops in terms of food security (Schlick and Bubenheim, 1996). While the major producers are still located in the Andean region, quinoa cultivation is a reality across all continents where germplasm originated from the Andes was successfully selected (Bazile et al., 2016).

The first objective of the International Year of Quinoa (IYQ) in 2013 was to increase the visibility of the great potential of quinoa biodiversity to contribute to global food security, especially in countries where the population has no access to other protein sources or where production conditions are limiting. In many of the countries in North Africa and the Near East, food security still remains a major problem for vulnerable population groups. Global agricultural production is facing problems that threaten its stability and sustainability, such as climate change, land salinization, limited water availability for agriculture (Gómez-Pando et al., 2010). Its high tolerance to extreme weather conditions and high nutritional value contributed to choosing quinoa as a potential crop to address these challenges (Delatorre-Herrera and Pinto, 2009; Orsini et al., 2011; Ruiz-Carrasco et al., 2011; Pulvento et al., 2012). Following the IYQ, the Food and Agriculture Organization of the United Nations (FAO) initiated a multi local test (through FAO-TCP for Technical Cooperation Programmes) in a number of countries of North Africa, the Near East and Asia viz. Algeria, Egypt, Iran, Iraq, Kyrgyzstan, Lebanon, Mauritania, Sudan, Tajikistan, and Yemen. During the implementation of these projects, FAO collaborated with many partners worldwide to access quinoa seeds and their expertise in the field of crop cultivation. Various research centers, universities and seed firms were mobilized to find quinoa seeds of different varieties to be tested. Twenty different genotypes were eventually made available to countries for the international trial programme.

The main goal of this research was to evaluate the adaptability of selected quinoa genotypes under different environments outside the Andean region. Two hypotheses have been put forward in this paper to assess the performance of quinoa genotypes in new environments viz: Hypothesis (1): "Within the high genetic diversity of the species, it is possible to identify the most suitable variety for each study site" and Hypothesis (2): "To meet the various uncertainties related to global change in each region, yield stability of one variety across all study sites represents an important indication of genotype suitability and decreased risks when cultivating it any other given area." Analysis of these two hypotheses will structure the discussion on two possible pathways: yield maximization for potential production and yield optimization for production stability (Tilman et al., 2002).

\section{MATERIALS AND METHODS}

\section{Study Sites}

The paper concerns an analysis of the results from nine countries with similar ecological conditions. In this set, countries are located in North Africa, the Near East, and Asia under quite similar semi-arid or arid climatic conditions, namely in Algeria, Egypt, Iran, Iraq, Kyrgyzstan, Lebanon, Mauritania, Tajikistan, and Yemen.

Within each of these countries, 2-5 sites were chosen to represent the diversity of agro-ecological zones at country level. The study sites are parts of the decentralized network of agronomic stations from each of the National Research Centers involved in the programme. We tested a set of 21 different quinoa genotypes and their responses in terms of agronomic performance cultivation in these contrasted environments were duly recorded. The locations of these sites are found in Figure 1. Table 1 also provides the principal descriptive data of each of 19 sites surveyed and used for this paper. The sowing dates reflect ecological and agronomic differences across study sites to adapt quinoa cycle into local cropping systems (Table 1).

\section{Genetic Resources Materials}

In Table 2, the different genotypes used were classified in three groups considering a gradient of the level of genetic diversity among them, from landraces (heterogeneous crop population varieties) to varieties under development (still conserving a degree of heterogeneity) and improved varieties (homogeneous 


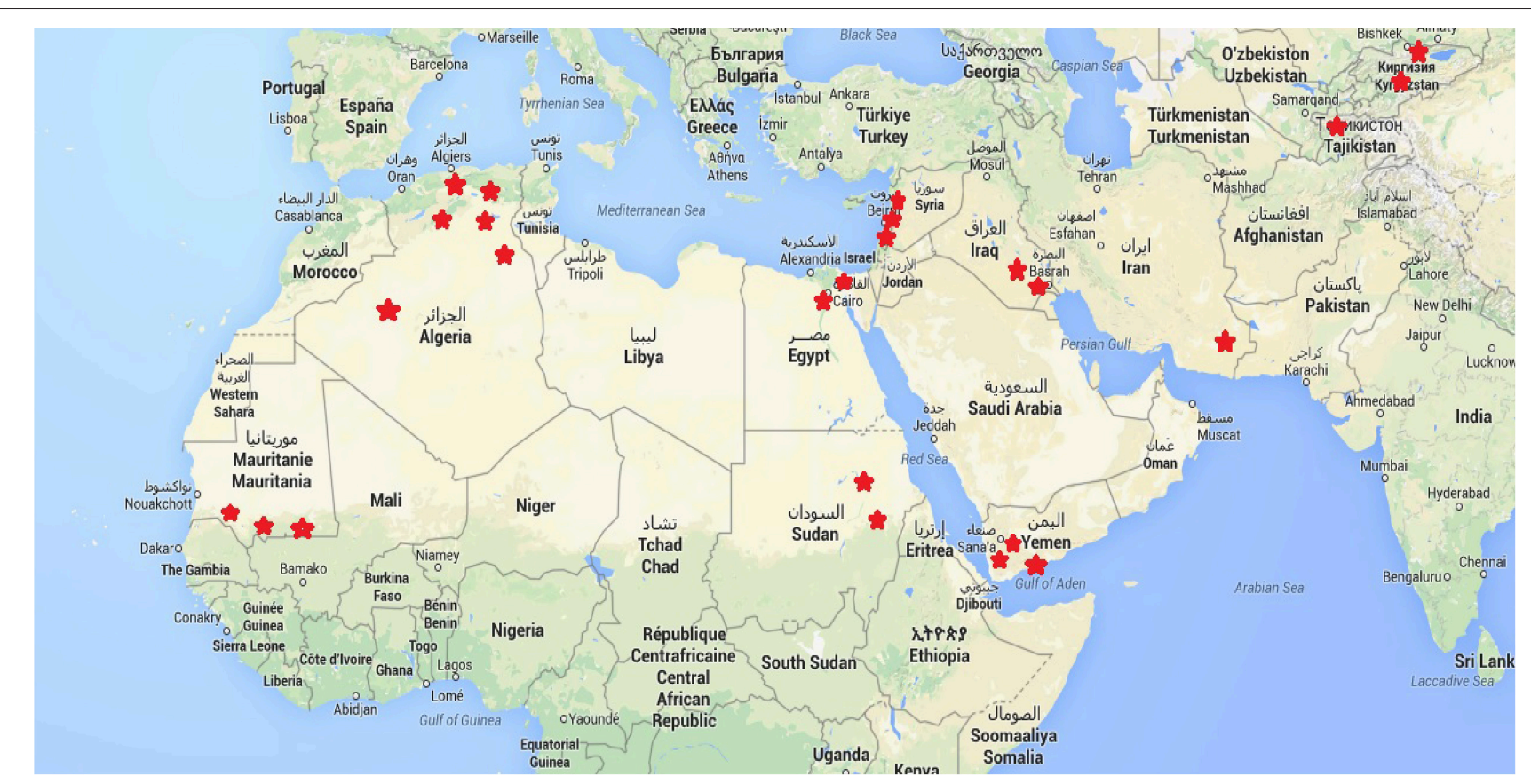

FIGURE 1 | Location of the study sites.

plants) protected by intellectual property rights (Plant Variety Protection, PVP; Bazile et al., 2016).

Due to the difficulties to access quinoa germplasm at global level, each country made specific requests through its networks. FAO has mobilized various partnerships to collect different quinoa accessions to carry out the study. First, through a collaboration with the International Center for Biosaline Agriculture (ICBA, a non-profit International Organization)a research center based in the United Arab Emirates (UAE) FAO could access specific varieties of quinoa. Seeds of the three varieties under development (Q1, Q2, and Q3) were obtained from ICBA to be made available for the trials in Yemen. FAO has also received seeds from the Centro di Ricerca per la Cerealicoltura (CRA-CER) in Italy which helped to expand the genotypes proposed in the tests. The seeds of nine accessions of landraces (Q12 to Q31) were obtained from CRA-CER who has been working and selecting these accessions in Italy after accessing them from the United States Department of Agriculture genebanks (USDA). These seeds had Chilean origin. Seeds were supplied to FAO-RNE which has distributed them to eight countries in the region. The two quinoa varieties (Sajama and Santamaria) were provided by PROINPA in Bolivia to the Seed and Plant Improvement Institute (SPII) in Iran. Selected seeds of early matured plants from the genotype "Sajama" have produced a new variety that was called "Iranshahr." Giza1 and Giza2 have been selected in Egypt from preliminary quinoa lines furnished by the University of Copenhagen in Denmark. Finally, Puno and Titicaca are two varieties of the Quinoa Quality Enterprise linked to the University of Copenhagen (Denmark). Regalona is the only quinoa variety with PVP developed by Von Baer Seeds for the Southern part of Chile (Von Baer et al., 2009). Finally, each country could choose from over 21 fairly differentiated genotypes.

\section{Experimental Protocol}

Given the specificities of the FAO TCP projects, each country independently developed its experiments with similar technical support provided at regional level. Due to the difficulties of accessing quinoa genetic resources for some countries and in order to avoid biases in the analysis, a senior statistician provided advice on how to overcome such gaps (not all the countries have tested the same genotypes) and how to define and conduct solid statistical analyses in order to obtain scientifically reliable results. To be able to do that, in each test, the experimental protocol was based on a common core curriculum. For each site, the parameters used were described in the Tables 1, 4 .

The experimental design, a randomized complete block design (RCBD) with three replicates, represents the core protocol. The distance between two blocks was more than $1 \mathrm{~m}$. Each of replicate plots is of the same area, i.e., $5 \mathrm{~m}$ long, $2 \mathrm{~m}$ wide, $10 \mathrm{~m}^{2}$. The replicate plot had four rows of plants to define an inter-rank of 50 $\mathrm{cm}$ and a $25 \mathrm{~cm}$ distance between plants. In addition, 3-5 seeds were sown per hole of about $1 \mathrm{~cm}$ depth that was covered later. In few cases, irrigation was to secure germination step. Fifteen days after sowing, considering the germination rate, two plants were kept per hole by eliminating the other and considered for the experiment.

It is however important to note that the different varietal types (landraces, varieties under development, and improved varieties) were not necessarily used in each trial. On some sites, 


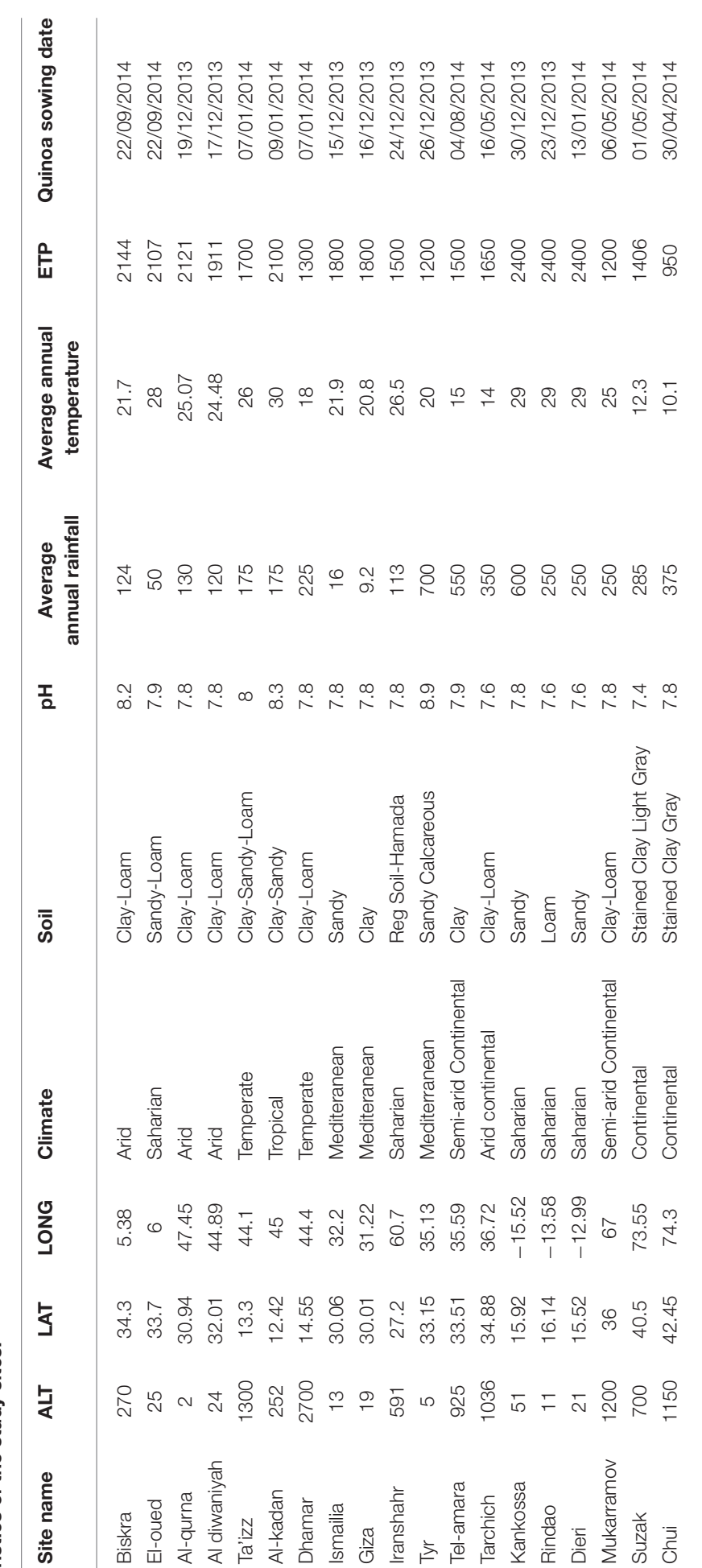

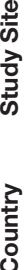

TABLE 2 | Classification of the 21 quinoa genotypes used for trials.

\begin{tabular}{lll}
\hline 10 Landraces & $\begin{array}{l}\text { 8 Varieties under } \\
\text { development }\end{array}$ & $\begin{array}{l}\text { 3 Improved varieties } \\
\text { (Registered with plant } \\
\text { variety protection) }\end{array}$ \\
\hline Q12 (Chile) & Sajama (Bolivia) & Regalona (Chile) \\
Q18 (Chile) & Santamaria (Bolivia) & Puno (Denmark) \\
Q19 (Chile) & Q1 (U.A.E) & Titicaca (Denmark) \\
Q21 (Chile) & Q2 (U.A.E) & \\
Q22 (Chile) & Q3 (U.A.E) & \\
Q26 (Chile) & GIZA 1 (Egypt) & \\
Q27 (Chile) & GIZA 2 (Egypt) & \\
Q29 (Chile) & SAJAMA Iranshar (Iran) & \\
Q31 (Chile) & & \\
Quinoa real (Bolivia) & & \\
\hline
\end{tabular}

only landraces were tested, and other tests were only conducted with improved varieties (see Tables 2, 3). These differences are explained by the difficulties to access germplasm and by the specific demands of each country. The resulting incompleteness of the experimental design has oriented our statistical analysis below.

Table 4 shows an example of a spreadsheet used to collect and measure data in the experimentation using the international descriptor list for quinoa (Bioversity International et al., 2013).

Pre-treating the seed with an insecticide and a fungicide was done before the test only, whenever such problems were found to exist in the area. In terms of soil preparation, plowing was performed by a weedier harrow to make the seedbed as thin as possible and facilitate soil-seed contact given the small diameter of the seeds.

\section{Statistical Analysis}

Estimates Yields were based on the whole plots $\left(10 \mathrm{~m}^{2}\right)$. As part of this study, two different averages were used to compare performances between genotypes and sites: the arithmetic average and the weighted average. Weighted average is important when you are dealing with frequencies or distributions. In our case, each site does not have the same number of genotypes studied, so the averages were weighted by the number of accessions used in the selected site to obtain actual weighted averages, reflecting more appropriately the results of the study for comparisons.

Considering the reality of our global experiment plan, the analysis of variance was only applied to an incomplete ANOVA table referring to some selected sites where similar genotypes were used extensively. A subset (16 sites $\times 11$ genotypes) was extracted from the whole data set to a posteriori generate an experiment plan that is almost complete (Table 3).

A two-way analysis of variance was performed with the $R$ Software to test for differences in yield across sites and genotypes. No site-genotype interaction was included in the model because of a lack of repetitions in some sites. Yield was square-rooted transformed prior to the analysis to stabilize the variance (See Supplementary Material). A one-way analysis of variance was also conducted to test for differences in square-rooted 
TABLE 3 | Distribution of the genotypes used for trials in the experiment plan.

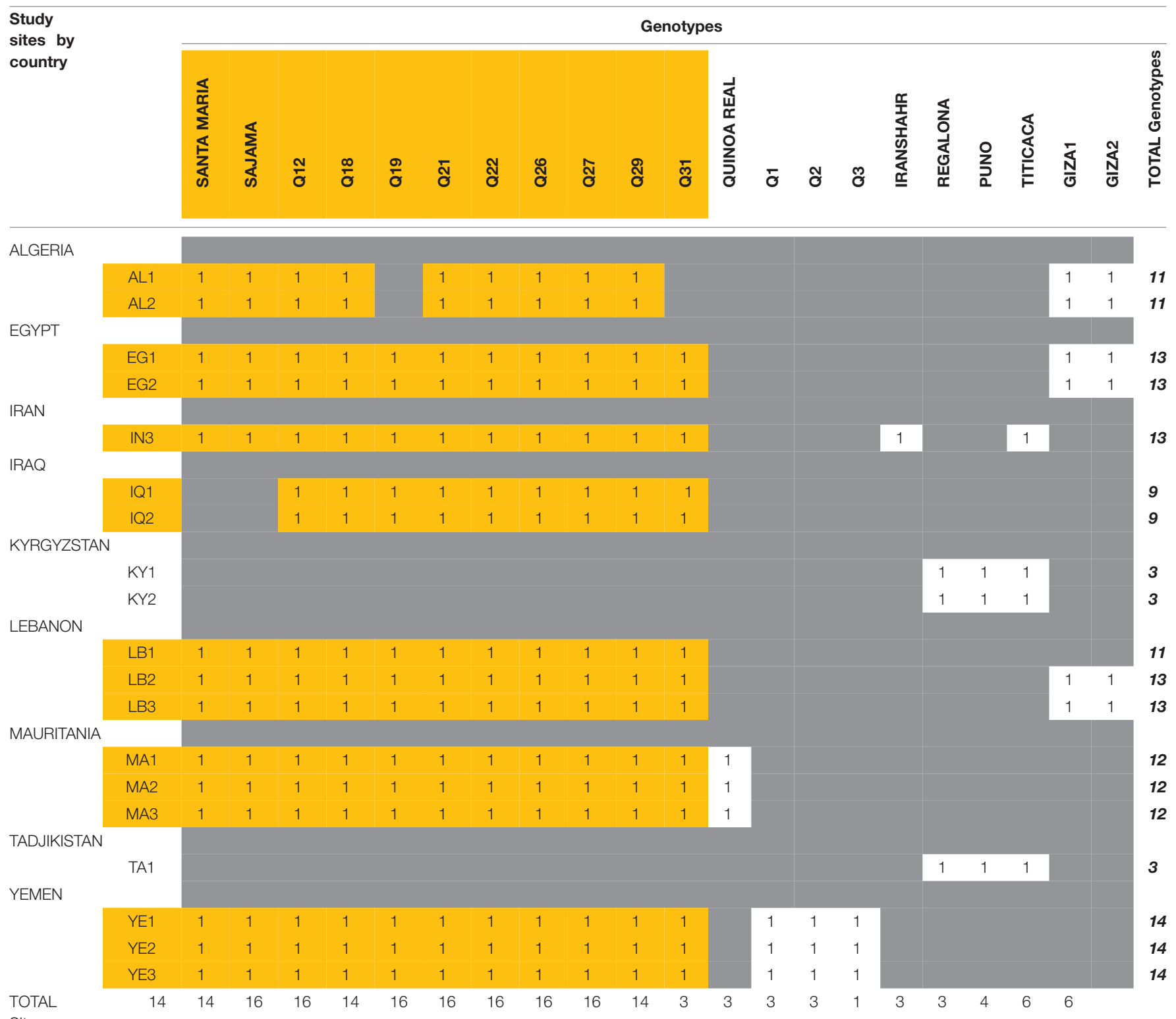

Sites

("1" = presence).

Subset (16 sites $\times 11$ genotypes) extracted from the whole data set to a posteriori generate an experiment plan that is almost complete for the ANOVA.

yield across sites, leaving the between-genotype variability in the residual variance. The analysis was complemented with a multiple comparison test using Tuckey's method. The same analysis was conducted using genotype instead of site as the predictor.

\section{RESULTS}

Yield was significantly different across sites and genotypes (twoway ANOVA: F-statistic for the site effect: 43.0, $p<0.001$, F-statistic for the genotype effect: $11.9, p<0.001$ ).

Even when leaving the genotype variability into the residual error, yield was significantly different across sites (one-way
ANOVA: F-statistic: 30.1, $p<0.001)$. Seven groups of sites out of sixteen were distinguished on the basis of yield (Figure 2).

Reciprocally, when leaving the site variability into the residual error, yield was significantly different across genotypes (one-way ANOVA: F-statistic: 3.3, $p<0.001$ ). Two groups of genotypes were distinguished on the basis of yield (Figure 3).

\section{Yield Performances per Site for All the Varieties Tested}

Table 3 and Figure 2 show that there are significant differences in yield among genotypes tested in different countries. A weighted average yield of $1.07 \mathrm{t}_{\mathrm{ha}} \mathrm{h}^{-1}$ was obtained for all varieties and all countries combined. The results demonstrate that quinoa can 
TABLE 4 | Example of a spreadsheet to collect data in the field.

\begin{tabular}{|c|c|c|c|c|}
\hline \multicolumn{5}{|l|}{$\begin{array}{l}\text { Country: } \\
\text { Study site: }\end{array}$} \\
\hline \multirow{2}{*}{$\begin{array}{l}\text { Varieties } \\
\text { Data observations/measures }\end{array}$} & \multicolumn{4}{|c|}{ Genotype 1} \\
\hline & R1 & $\mathbf{R} 2$ & R3 & Average \\
\hline \multicolumn{5}{|l|}{ Date of Sowing } \\
\hline \multicolumn{5}{|l|}{ Days to $2-8$ true leaves } \\
\hline \multicolumn{5}{|l|}{ Days to budding stage } \\
\hline \multicolumn{5}{|l|}{ Days to the beginning of flowering } \\
\hline \multicolumn{5}{|l|}{ Days to $50 \%$ flowering } \\
\hline \multicolumn{5}{|l|}{ Days to maturity } \\
\hline \multicolumn{5}{|l|}{ Date of harvesting } \\
\hline \multicolumn{5}{|l|}{ No. days from planting to harvest } \\
\hline \multicolumn{5}{|l|}{ Plant height (cm) } \\
\hline \multicolumn{5}{|l|}{ Number of branches/plant } \\
\hline \multicolumn{5}{|l|}{ 1000-seed weight (g) } \\
\hline \multicolumn{5}{|l|}{ Weight of the main head $(\mathrm{g})$} \\
\hline \multicolumn{5}{|l|}{ Width of panicle (cm) } \\
\hline \multicolumn{5}{|l|}{ Length of panicle (cm) } \\
\hline \multicolumn{5}{|l|}{ Panicle color } \\
\hline \multicolumn{5}{|l|}{ Germination Rate (\%) } \\
\hline \multicolumn{5}{|l|}{ Number of plants on the harvest area $\left(\mathrm{nb} / \mathrm{m}^{2}\right)$} \\
\hline \multicolumn{5}{|l|}{ Seed yield/plant (g) } \\
\hline Seed yield/ha (ton) & & & & \\
\hline
\end{tabular}

be adapted in many environments while having higher yields than those that can be obtained in Bolivia or Peru (about 1 t.ha ${ }^{-1}$ ). Considering the strong differences between varieties and countries, it is then necessary to investigate more in depth the results of each of the countries involved in the project. The Tuckey test highlights a large gradient of yields across the study sites from LB1 (group g) to MA3 (group a; Figure 2). The results by country are described below but we can underline here that the higher yields were obtained at LB1 (g), EG1 and EG2 (f, g), and AL1 and LB3 (e, f, g). In addition, MA3 (a), MA1 and MA2 and IQ1 $(\mathrm{a}, \mathrm{b})$ and, AL2 and YE3 (a, b, c) did not performed well and yields were always lower than in other locations.

\section{Algeria}

Two sites were analyzed and 13 genotypes were tested (Table 3). The yield average obtained was $1.65 \mathrm{t} \mathrm{ha}^{-1}$ at the first site (AL1) and $0.26 \mathrm{tha}^{-1}$ at the second site (AL2) which gives an arithmetic average of $0.96 \mathrm{t} \mathrm{ha}^{-1}$ for the country. Note that the lower values at the second site, never exceeding $0.78 \mathrm{t}^{-h^{-1}}{ }^{-1}$ contributed to the fact that the arithmetic average of the country remains below the value of the weighted average yield for all countries. The three genotypes Q26 (2.62 t.ha $\left.{ }^{-1}\right)$, Q18 (2.27 t.ha ${ }^{-1}$ ), and Q27 (2.17 t.ha ${ }^{-1}$ ) achieved the much higher yields at the first site.

\section{Egypt}

Two sites were analyzed and 13 genotypes were tested (Table 3). A higher value than the weighted average yield for all countries was obtained with $1.89{\mathrm{t} . h a^{-1}}$ at the first site (EG1) and 2.35 t.ha ${ }^{-1}$ at the second site (EG2). Very high yields were obtained with Q12 (3.87 t.ha ${ }^{-1}$ ), Q18 (3.17 t.ha ${ }^{-1}$ ), and Q29 (3.41 t.ha ${ }^{-1}$ ).

\section{Iran}

Only one site has been analyzed considering the lack of observations in the other sites and 11 genotypes were tested (Table 3). They produced an average yield of $0.85 \mathrm{t}^{-\mathrm{ha}^{-1}}$ for this site (IN3). Titicaca achieved the highest yield $\left(4.48 \mathrm{t}^{\mathrm{h}} \mathrm{ha}^{-1}\right)$ in Iran. Note however that some landraces like Q12 (1.03 t.ha $\left.{ }^{-1}\right)$ and Q21 (1.56 t.ha ${ }^{-1}$ ) have significantly higher values than the arithmetic average of the country.

\section{Iraq}

Two sites were analyzed for this study and nine genotypes were tested (Table 3) for an average yield of 0.10 t.ha ${ }^{-1}$ at the first site (IQ1) and $0.65{\mathrm{t} . h a^{-1}}^{\text {at }}$ the second site (IQ2). A value significantly lower than the weighted average yield for all countries was obtained at the two sites and an arithmetic mean for the country also much lower than all countries with only 0.36 t.ha ${ }^{-1}$.

\section{Kyrgyzstan}

Two sites were analyzed for the study and 3 genotypes were tested (Table 3) with an average yield of $0.90 \mathrm{t}^{\mathrm{ha}} \mathrm{a}^{-1}$ for the first site (KY1) and 1.03 t.ha $^{-1}$ for the second site (KY2) confirming a value significantly lower than the weighted average yields for all countries at the two sites. Any improved varieties achieved yields higher than 1.24 t.ha $^{-1}$.

\section{Lebanon}

Three sites were analyzed and 11 genotypes were tested (Table 3 ). An average yield of $2.7 \mathrm{t}^{-\mathrm{ha}^{-1}}$ was obtained at the first site (LB1), $1.15{\mathrm{t} . h a^{-1}}^{-1}$ at the second site (LB2) and $1.82{\mathrm{t} . h \mathrm{a}^{-1}}$ at the third site (LB3). A value higher than the weighted average yield for all countries was obtained at the three sites. In the first site, landraces Q12 (4.0 t.ha $\left.{ }^{-1}\right)$ and Q29 (4.5 t.ha ${ }^{-1}$ ) had very high yields which gave a high amplitude to this dataset. This is also the case at the second site where Q18 (2.8 t.ha $\left.{ }^{-1}\right)$ and GIZA1 (2.8 t.ha $\left.{ }^{-1}\right)$ had a high value. This phenomenon is also present at the third site where the two cultivars (GIZA1 and GIZA2) have zero yields as did the landrace Q31. But on the other hand, Q21 (4.3 t.ha $\left.{ }^{-1}\right)$ and Q27 (3.6 t.ha ${ }^{-1}$ ) achieved very high yields. These important differences between accessions explain a significant dispersion of yields in the three sites within the dataset.

\section{Mauritania}

Three sites were analyzed for this study and 9 genotypes were tested (Table 3) with an average yield of $0.08 \mathrm{t}^{\mathrm{h}} \mathrm{ha}^{-1}$ at the first site

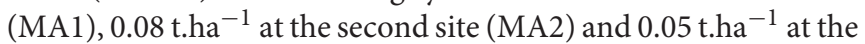
third site (MA3). The bad arithmetic average with $0.07 \mathrm{t} . \mathrm{ha}^{-1}$ was explained by low yields everywhere.

\section{Tajikistan}

One site was analyzed and only the three improved varieties were tested (Table 3 ). The study site (TA1) achieved an average yield of $2.13 \mathrm{t}^{\mathrm{h} \mathrm{h}^{-1}}$. PUNO and TITICACA achieved good performance with 2.2 t.ha $^{-1}$. 


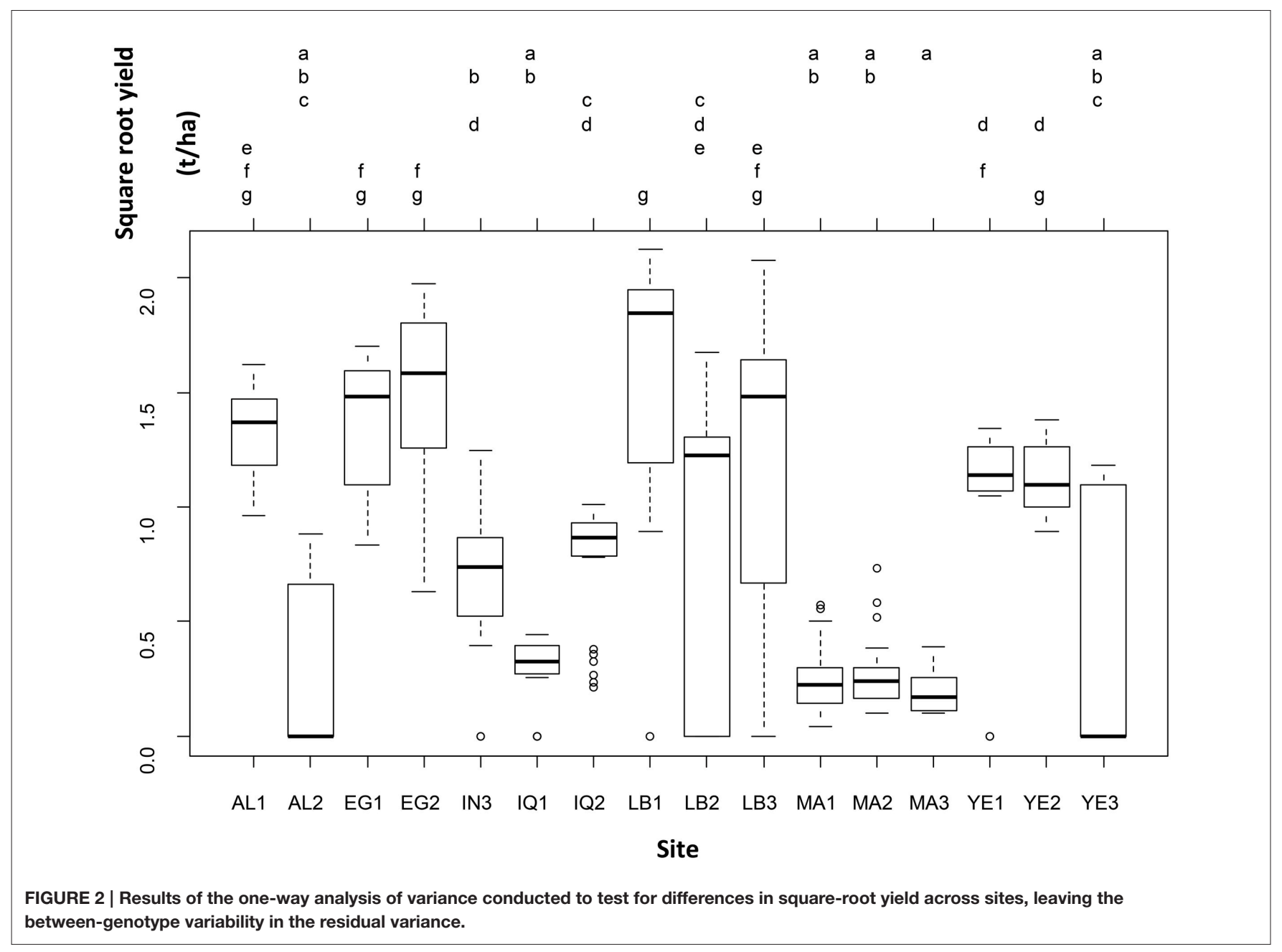

\section{Yemen}

Three sites were analyzed and 12 genotypes were tested (Table 3). The yield averages obtained were $1.21 \mathrm{t}^{-h^{-1}}$ at the first site

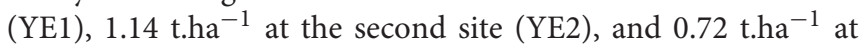
the third site (YE3). Thus, one can observe a value higher than the weighted average yields for all countries at the first two sites and an arithmetic average lower than that of all countries at the third site. Q27 achieved the higher yield with 1.9 t.ha ${ }^{-1}$ at YE2, following by Q26 with 1.8 t.ha $^{-1}$ at YE1 and Q1 with 1.7 t.ha ${ }^{-1}$ at YE3.

\section{Varieties Performances across Study Sites}

Figure 3 shows the performance for each genotype across the locations. Most of the genotypes presented higher yields than under Andean conditions in the farming systems examined. Most part of the accessions exhibited huge variations in seed yield data. Considering the large standard deviations observed for each genotype, it cannot be concluded that these accessions have a similar performance across the locations, to define the best one that can be sown with limited risks. The Tuckey test has generated two groups where Q12, Q18, Q21, and Q26 achieved the higher yields (Figure 3, group b) and where Q31 is represented as the lower one (Figure 3, group a); all the other genotypes are intermediaries.

\section{Analysis by Varietal Types \\ Landraces}

In this research project, nine landraces mainly from Chile were tested. When observing the results for each of the study sites (Figures 3, 4), a first dichotomy can be made within landraces. Indeed, we find that three landraces have yields averaging lower than the overall average of $1.07 \mathrm{t}^{-h a^{-1}}$ : Q19 (0.89 t.ha $\left.{ }^{-1}\right)$, Q22 (1.05 t.ha $\left.{ }^{-1}\right)$, and Q31 (0.24 t.ha $\left.{ }^{-1}\right)$. The other six landraces present above-average yields: Q12 (1.40 t.ha $\left.{ }^{-1}\right)$, Q18 (1.40 t.ha $\left.{ }^{-1}\right)$, Q21 (1.38 t.ha $\left.{ }^{-1}\right)$, Q26 (1.34 t.ha $\left.{ }^{-1}\right)$, Q27 (1.24 t.ha ${ }^{-1}$ ), and Q29 (1.20 t.ha $\left.{ }^{-1}\right)$. Nevertheless, the dispersion of data of such landraces is very high and standard deviations are ranging from 1.19 to 1.35 .

\section{Varieties under development}

As part of this study, eight varieties under development or without PVP (Table 2) were tested from different parts of the world (Bolivia, Egypt, Iran, and UAE). After reviewing the results of each of the study sites, they can be separated into two groups (Figures 3, 4). Four of these varieties have average 


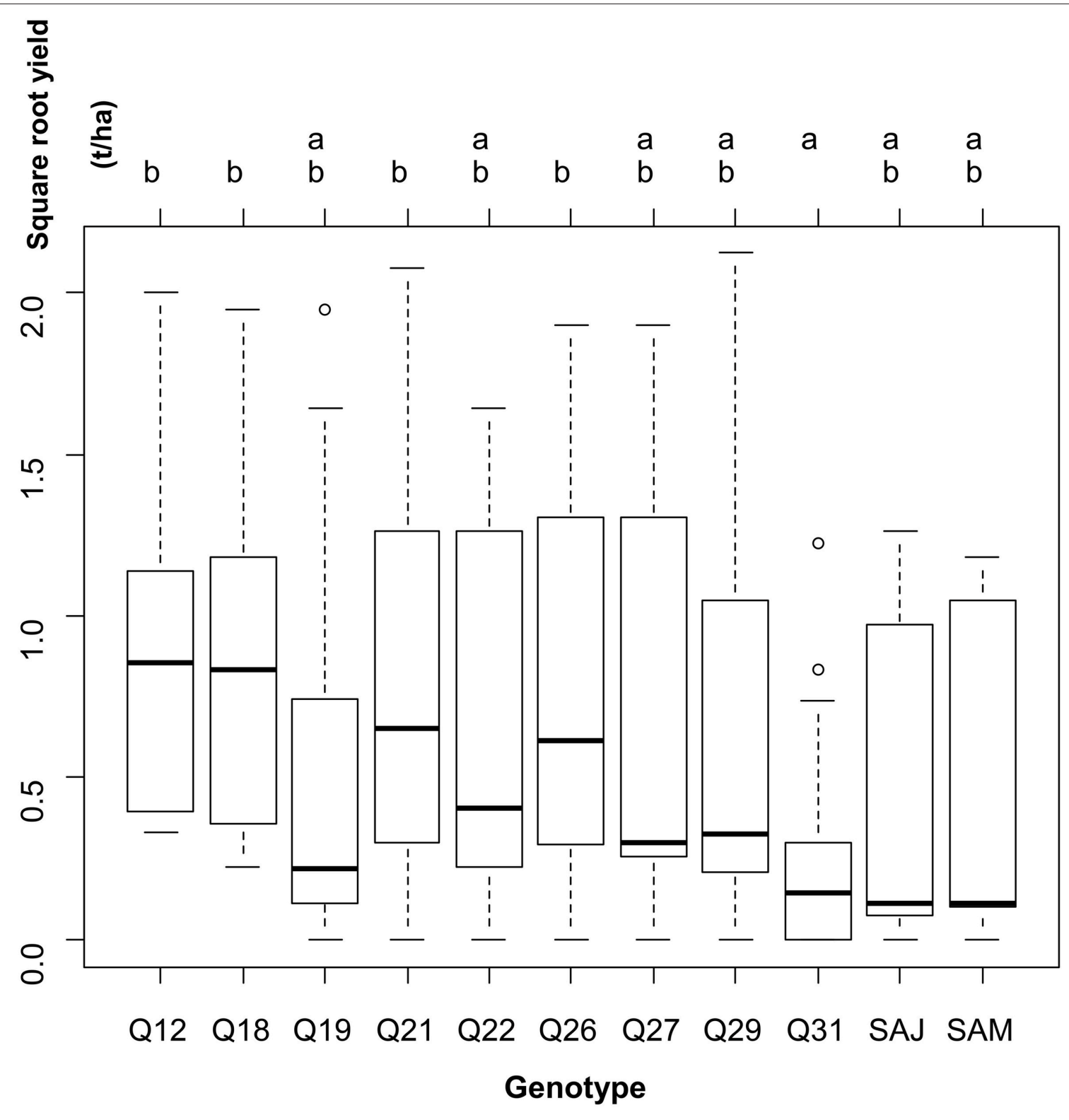

FIGURE 3 | Results of the one-way analysis of variance conducted to test for differences in square-root yield across genotypes, leaving the between-site variability in the residual variance.

yields below the global average of 1.07 t.ha $^{-1}$ : GIZA2 $(0.72$ t.ha $\left.{ }^{-1}\right)$, SANTAMARIA (0.59 t.ha $\left.{ }^{-1}\right)$, SAJAMA (0.58 t.ha $\left.{ }^{-1}\right)$, and SAJAMA IRANSHAR $\left(0.20 \mathrm{t}^{\mathrm{h} \mathrm{h}^{-1}}\right)$. The other four perform better: Q1 (1.30 t.ha $\left.{ }^{-1}\right)$, Q2 (1.13 t.ha $\left.{ }^{-1}\right)$, Q3 (1.13 t.ha $\left.{ }^{-1}\right)$, and GIZA1 $\left(1.88\right.$ t.ha $\left.^{-1}\right)$. Moreover, only GIZA1 achieved very promising performance for varieties under development.

\section{Improved varieties}

For this project, three improved varieties with PVP were also tested. These three varieties have very satisfactory performance levels (see Figure 4) in the few sites where there were tested. Indeed, it may be noted that these three varieties have yield averages much higher than the overall average from 1.37 t.ha ${ }^{-1}$ for REGALONA, to $1.45 \mathrm{t}^{\mathrm{h} \mathrm{h}^{-1}}$ for PUNO and to $2.05 \mathrm{t}^{\mathrm{h}} \mathrm{ha}^{-1}$ for TITICACA, which was the best variety among all the genotypes of panel. PUNO and REGALONA varieties have high uniformity. It is not the case with the variety TITICACA, which presents significant sources of heterogeneity.

\section{DISCUSSION}

\section{Germination Rates and Agronomic Practices}

A very low germination rate was noted for some genotypes tested. This might be due to stocking conditions where high humidity lowers germination quality between seasons (Coulibaly et al., 

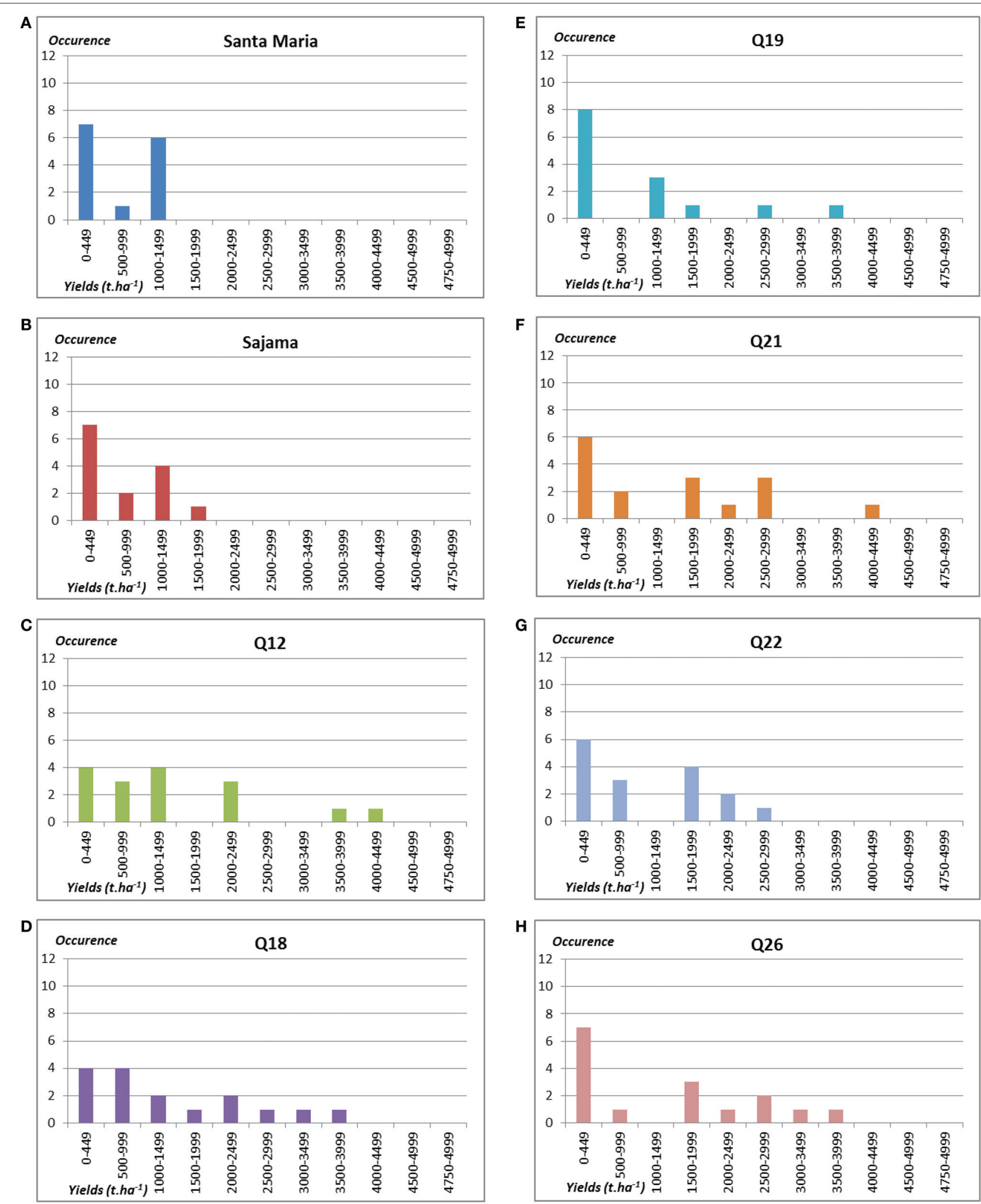

FIGURE 4 | Continued 

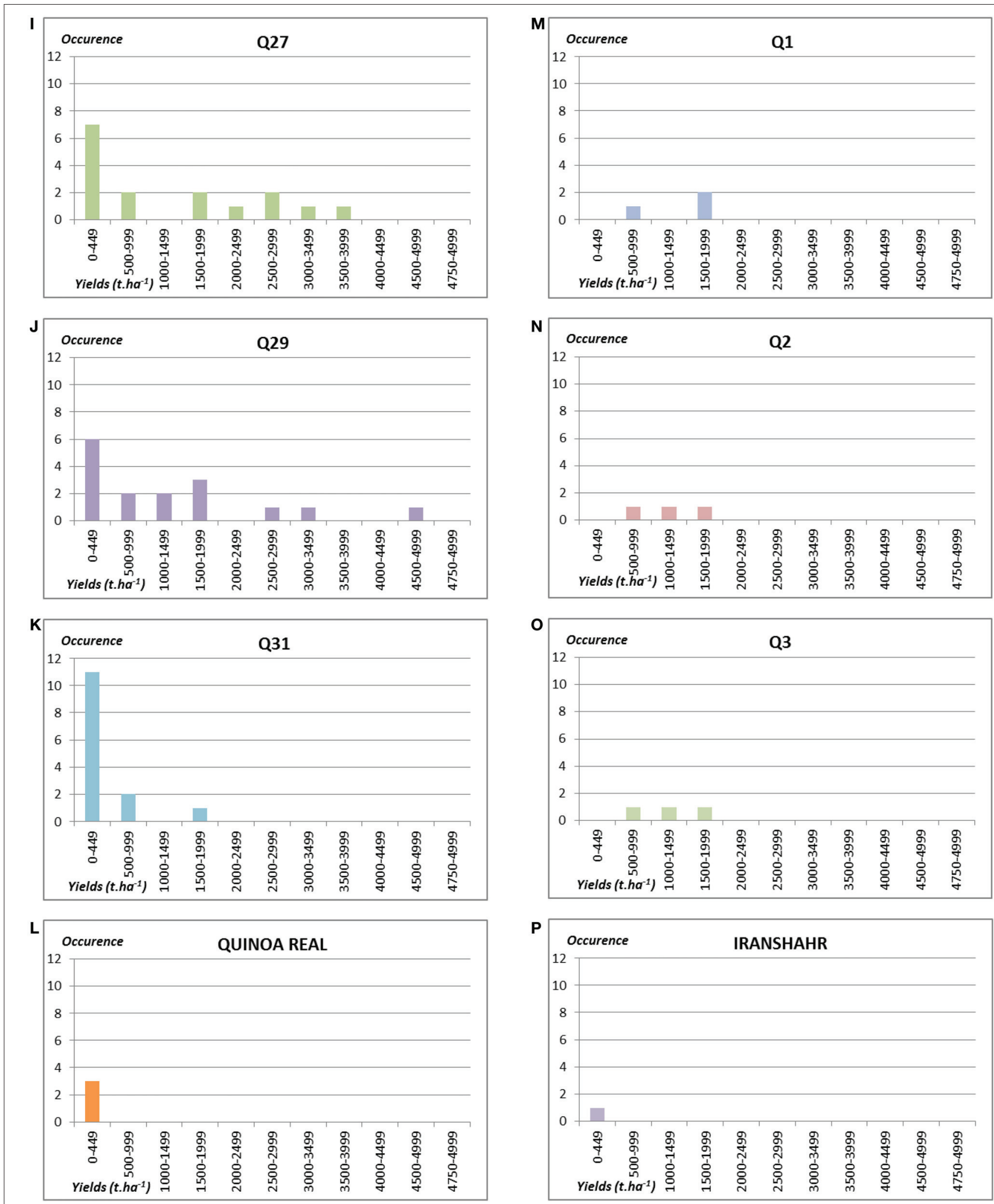

FIGURE 4 | Continued 

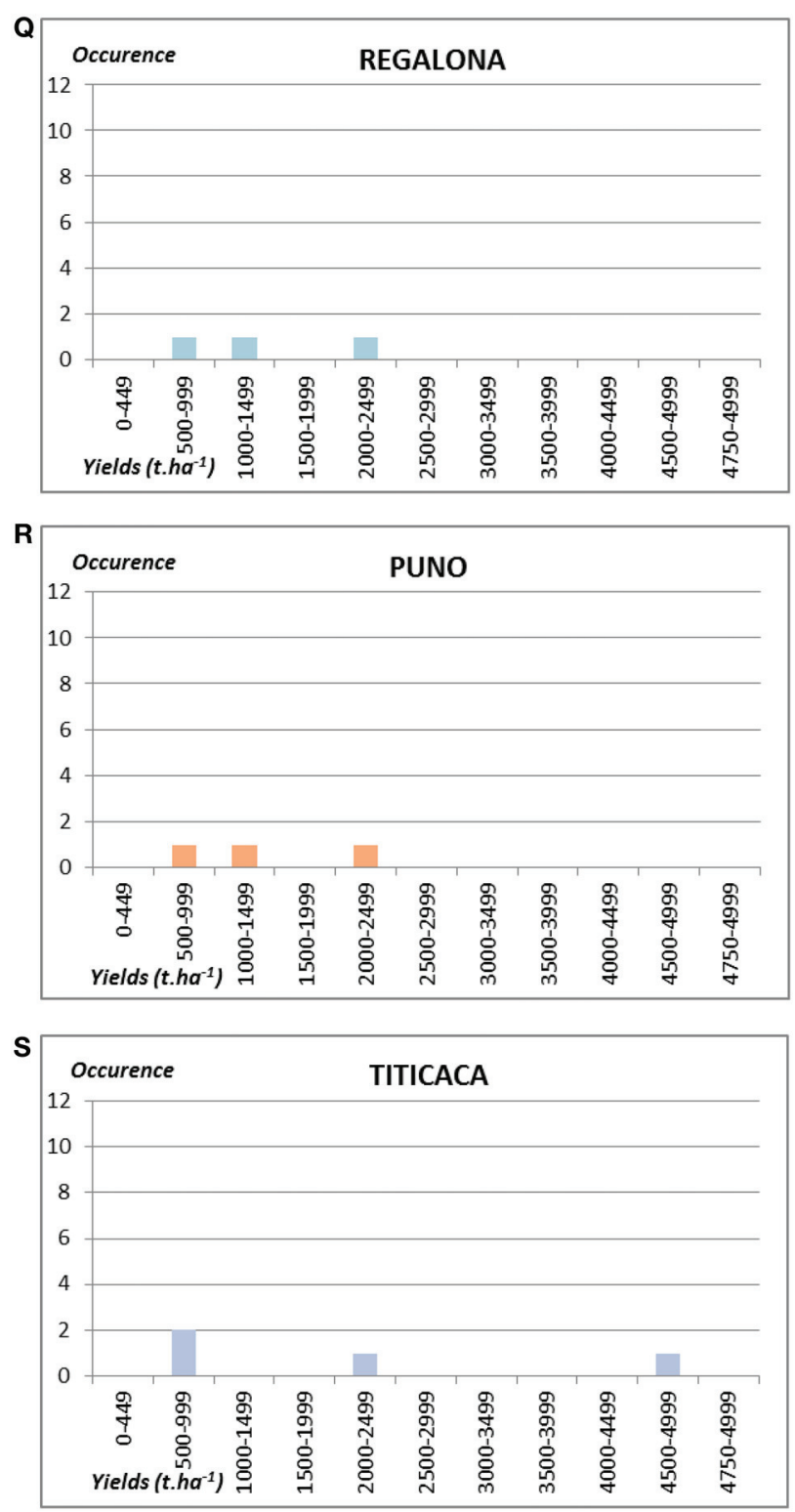

FIGURE 4 | Number of occurrences of plots by yields' classes in t.ha ${ }^{-1}$ for the 21 genotypes tested: (A) Santa Maria; (B) Sajama; (C) Q12; (D) Q18; (E) Q19; (F) Q21; (G) Q22; (H) Q26; (I) Q27; (J) Q29; (K) Q31; (L) Quinoa real; (M) Q1; (N) Q2; (O) Q3; (P) Iranshahr; (Q) Regalona; (R) Puno; (S) Titicaca; (T) Giza 1; (U) Giza 2.

2015). Large differences in yields were observed among the tested genotypes in the different countries. If global results show that quinoa could be adapted to many environments with higher yields than in traditional cropping systems of Peru or Bolivia, we can state that it is not the case everywhere.

Different reasons could explain the differences, in particular when the seeds of some accessions showed a low degree of germination. Seed quality is the first bottleneck for experimentation because many genotypes were conserved in genebanks for a long time before being sent by plane (submitted to high pressure). Soil preparation generally constitutes the second bottleneck. Before sowing, the soil should be properly
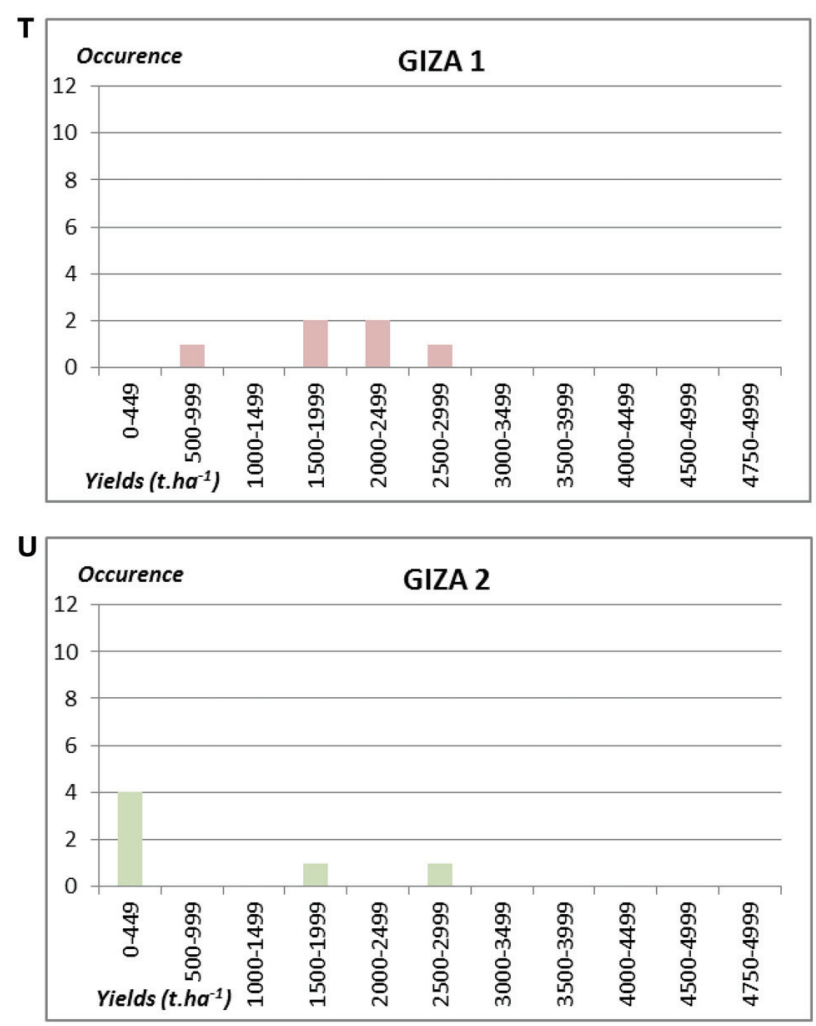

prepared to achieve a good seed-to-soil contact in the seedbed. The sowing date is another element of this second bottleneck. Preparing the soil under good weather conditions is crucial to obtain this ideal seedbed. All the conditions, including availability of seed accessions, are part of a good installation of the crop in the field avoiding too late sowing. Hot temperature is the third bottleneck and it is very important to plan the sowing date to avoid air temperatures higher than $32^{\circ} \mathrm{C}$ during the flowering stage, and taking into account that harvest must be done during the dry period. Therefore, the harvested seeds of quinoa need to be well stored at a temperature of less than $15^{\circ} \mathrm{C}$ under dry conditions to preserve their potential of germination. 


\section{Access to Genetic Resources}

Genebanks are mainly at the origin of development and distribution of our genotypes. However, genebanks should be distinguished as two different entities. We are interested first in genebanks from the Andean region, which can directly provide landraces from their ex situ conservation of national germplasm stocks, and secondly in genebanks outside the Andean region such as the USDA which has received seeds of that Andean area. It could also have received seeds from farmers' fields or through collaborations between genebanks. USDA collected landraces on its own account for plant breeding. In addition, USDA provides landraces from its collection to other breeders worldwide who request them in very small quantities (200 seeds) and for a limited number of accessions. While it seems easy to access this genetic material, it can be observed that the distribution is limited due to the limited access to quinoa germplasm and to the limited trade with the Andean countries because of the international regulatory context on seeds: farmers' rights, breeders' rights, phytosanitary rules, and protection of biodiversity against biopiracy. Farmers' varieties (landraces) should be recognized and protected by special agreements between research partner institutions for a particular exchange type, for example through a Standard Material Transfer Agreement (SMTA). The SMTA is a mandatory model for parties wishing to provide and receive material under the Multilateral System of the International Treaty on Plant Genetic Resources for Food and Agriculture but they are often difficult to implement. The Treaty recognizes the contribution that the local farmers of Andean regions, particularly in this case where they are located in centers of origin of crop diversity, have made for the conservation and development of plant genetic resources. Considering and using the SMTA, it gives governments the responsibility for implementing Farmers' Rights, clearly mentioned in its article 9.

Quinoa breeders can be two types: private institutes (Semillas Von Baer, Chile), public institutions (the University of Copenhagen or the CRA-CER Italy) or Non Profit International Organization such as the ICBA. If they have not developed their own collections, they could request access through genebanks, such as the USDA, in limited quantities. They multiply seeds before starting their breeding programs. After that they can benefited from results of this multiplication through the selection of landraces and assign new names. These cultivars or varieties are sold to other organizations, such as FAO that requests quinoa seeds to conduct international tests.

Following this process, there are different possible varietal types that were used in our tests: landraces, improved varieties and varieties under development. However, this genetic material could greatly change over the years and undergo several stages of selection and multiplication in different environmental conditions to those of its original environment. The characteristics of these accessions considered for our trials have probably changed significantly from their Andean nature of origin throughout all these steps.

It is important to note that this process has different pathways for seed diffusion but one can now see that they are related to each other. Being able to see them at separate stages in a continuum, we would see it more as a unique process of distribution and dissemination of genetic diversity of quinoa among different partners and institutions. The main consequence of these constraints on the access to genetic resources of quinoa is that a very low diversity is generally used to conduct trials (Bazile et al., 2016). This happens both, regarding the number of genotypes to be tested, and within the intrinsic genetic diversity of the genotypes for varieties under development and improved varieties that are developed for different environmental conditions (Murphy et al., 2016). These varieties have been selected so that they reduce their genetic heterogeneity which is synonymous to a lower adaptive capacity and contrary to high genetic diversity.

\section{Relationships with Original Ecotypes}

In order to determine if the characteristics of the sites could be compared to those of the original ecotypes of quinoa, a comparison was made based on three criteria: altitude, latitude and annual rainfall. We noticed that in crossing the variables two by two, we come to systematically identify a group of sites that comes close to the characteristics associated with an original ecotype. In all three cases, the data is always close to the coastal quinoa ecotype that grows at sea level altitude, and latitudes corresponding to more Mediterranean climates. Nevertheless, one cannot completely involve our study sites in this ecotype because their precipitation levels are too far apart. Most study sites have annual rainfall lower than $500 \mathrm{~mm}$, while in the region of origin for this coastal ecotype, from the Secano Costero in Chile's central region to the area of Araucanía in the southern Chile, rainfall ranges from 600 to $2000 \mathrm{~mm}$. Landraces from this ecotype, adapted to a level of such precipitation, will not grow easily in the more arid conditions of our study cases, where rainfall rarely exceeds $500 \mathrm{~mm}$, and where additionally $75 \%$ of our test sites receive less than $300 \mathrm{~mm}$ annually. If access to landraces was improved from the south-central area of Chile, it might be possible to make a screening of accessions to try to find this kind of highly drought resistant genotype. But as access conditions are limited, it is necessary to consider other solutions for quinoa adaptation in new environments.

No experimental site had the same ecological conditions that were observed where the original quinoa ecotypes grew. It is impossible to simultaneously align our three main experimental variables: latitude, altitude and rainfall. However, another solution is available: it is quite feasible to cross two genotypes from two ecotypes with different attributes. For example, using a variety from the ecotype of "Salares" capable of supporting arid conditions $(<200 \mathrm{~mm}$ rainfall per year) and another variety from the "coastal" ecotype that grows in areas with low altitudes $(0-1500 \mathrm{~m})$. A variety capable of growing at low altitude and resistant to low levels of rainfall would be interesting for our study since we find these conditions in most of our test sites. An application of this crossing method for quinoa plant breeding was already done in the past for other attributes, to obtain the variety Regalona, a hybrid between two sources, one close to the equator and the other at southern Chilean regions (Von Baer et al., 2009). 


\section{Discussion of the Two Strategic Objectives for Quinoa Research Related to Our Initial Hypothesis}

Plant adaptation in agriculture strategies is essential for building robust production systems that are resilient to the effects of climatic change while strengthening food production for the future (Araus et al., 2008). Choosing new sowing dates or switching the cultivation to a neglected or underutilized existing crop species or variety, may prove to be a very successful move to buffer the cultivation against the negative impact of climatic change. Leveraging the potential of genetic diversity along with the deployment of improved best practices, are relatively inexpensive measures affordable by farmers, especially when accompanied by adequate extension work. On the other end, conventional plant breeding and crop's improvements are more expensive and require more technology investment for pursuing the goal of greater productivity. For example, the increase in food production in recent years has been mostly achieved considering the expansion of irrigated lands. Water use efficiency appears now as a key factor to consider from another option of view the effects of climatic change (Seckler et al., 1999). In fact, assessment of new crops and varieties diffusion of traditional and improved crop varieties needs to take more into account agronomic practices suitable and sustainable for specific ecosystems.

In addition, during the twentieth century, plant breeding for major crops has contributed to the higher increase of crop productivity for the mentioned species. But at the same time, this successful story in crop yields was limited in more recent years with a stabilization of yields for wheat and rice (Conway and Toenniessen, 1999). The situation regarding crop yields nowadays is still more complex and local context of cultivation have to be more considered for agronomic practices but also for their social and cultural dimensions (Bazile, 2015).

\section{Maximizing Yields}

National breeding programs, which are primarily concerned on how to moderate food insecurity, may modify some aspects of the agricultural intensification derived from the Green Revolution strategy (Annicchiarico, 2002) in order to select and to produce improved germplasm, socio-economically convenient, capable of maximizing the agricultural potential of specific areas and farming systems in marginal areas, and of minimizing the occurrence of crop failures or very low yields in unfavorable years. The ultimate goal of this quinoa program is to achieve adaptation and yield stability targets, especially by using variety material with increased tolerance to prevailing biotic and abiotic stresses.

As part of our study, yield maximization strategies echoes our first hypothesis and it is important to remember: "Within the high genetic diversity of the species, it is possible to identify the most suitable variety for each study site." Based on the outcome of the studies, this first hypothesis was confirmed since there are always one or more genotypes of superior performance in one site when compared to other sites, reflecting their excellent adaptability for higher production under these particular ecological conditions.
However, we should ask whether this strategy to maximize productivity, considering the performance as yield per hectare, would not be somewhat inappropriate to the context of the study in terms of food security. In addition, the tested environments are all of them located in marginal areas under extremely dry conditions and sometime saline soils that cannot permit many choices of crops that support those. This intensive agricultural model would be relevant if we sought to obtain or to increase profits in the short term, for a major sale of production surplus, for example. In our context, we are not really focusing on production intensification but rather to contribute to the food security of local populations, mostly in a subsistence farming context, that is achieving sustainable productions also in difficult areas and climate conditions. Moreover, it is important to focus on the environmental risks linked to this agricultural conventional model more based on technology intensification than on ecological intensification. As part of a high production target, we often use a significant amount of inputs, such as pesticides or chemical fertilizers, which increase the level of pollution of the local environment and present health risks to populations (Caron et al., 2009). Given the limited investment capacity of small-scale farmers in these countries, the potential yield of these varieties of quinoa will never be achieved without an initial capital contribution, in terms of expenses related to inputs, to be deducted from the final performance available for sale or supply.

\section{Optimization for Stability of the Food Production}

From an ecological point of view, adaptation is considered as a process where the adaptedness is the level of adaptation of plant material to a given environment, and adaptability is the ability to present good adaptedness in a wide range of environments (Tigerstedt, 1994). For plant breeders, the two terms refer to a condition rather than a process. It shows the ability of plant material to be high-yielding with respect to a given environment or given conditions (Gallais, 1992). For agronomist, the accepted definitions need to consider more changes across time and space. Efforts may be developed for investigating crop genetic diversity in space and time under plant breeding programs, in relation with farmers and agronomists, for more sustainable crop production (Fu, 2015).

After demonstrating that a maximization strategy is not necessarily the most appropriate to our project, it is necessary to take a different look to find an alternative pathway for quinoa testing and introduction in new environments. We had already mentioned another strategy, raising the importance of a lasting and stable food system for the populations of the countries studied. This vision of stability, as an important dimension of food security, is embodied by our second hypothesis of work and concern directly the adaptability in a wide range of environments: "To meet the various uncertainties related to global change in each region, yield stability of one variety across all study sites represents an important indication of genotype suitability and decreased risks when cultivating it any other given area." We wanted to demonstrate, whether one or more varieties have a level of stable and efficient performance across all or most of our study sites. An average grain yield of all varieties in each 
location has been used to compare the performance of each variety. All varieties were listed with a higher yield than the average yield of the site. Finally, the varieties were classified based on the number of times each variety appeared higher than the average of the different study sites. Six genotypes (Q12, Q18, Q21, Q22, Q26, and Q27) have an occurrence of up to five and present good yield potentials with great stability across sites. This result was confirmed with a good ponderation by the number of sowing sites. This gives us another perspective for less considered varieties in the trials (Q22, Q27) considering the Tuckey's method used for comparisons. The improved varieties, Puno, Titicaca (and also Regalona) performed with more than $75 \%$ but they need to be tested under more various conditions considering their good yield production, with the first and third average yield among all the genotypes.

\section{Strategic Choices and Their Consequences}

As previously mentioned, the main objective of the FAO programme was primarily to explore ways to strengthen with quinoa cultivation the food and nutritional security of the countries involved. Our goal in fact was to achieve food security at different scales (local, national, and global). In addition, we have seen that the nutritional properties of quinoa are exceptional, especially due to its high protein levels correlated with a good balance of essential amino acids for humans. A slight amount of these grains in the daily diet would provide an interesting contribution in vegetable protein for local people which have no access to other sources of proteins.

Therefore, these two agricultural models, maximizing vs. optimization, do not tend toward the same goals and do not have the same impact on food security. They are incompatible, although they may coexist within a country in different geographical areas or for differentiated groups of farmers. As explained before, we would privilege more the food security of the countries through a yield optimization strategy that corresponds to the overall objective of the project supported by FAO for achieving adaptability and stability as requested by governments of these countries (Power, 1999). Therefore, the choice of a model that implies a specific kind of variety corresponds to the main objective. In our case, it should be preferable to choose genotypes that do not necessarily have higher yield levels. These genotypes must produce a higher average yield measured with a constant, regardless of its stability production and adaptability in marginal environments (Bazile and Weltzien, 2008; Choukr-Allah et al., 2016).

However, one should not completely ignore the model of maximizing returns (Fasoula, 2012). In fact, this strategy could be considered in those countries assuming a long term insertion of quinoa in the local agricultural landscapes and cropping systems. For example, some farmers in Morocco with larger areas are already able to take a greater risk with capital investment (equipment and inputs) to target the maximum potential of improved varieties of quinoa (Benlhabib et al., 2015).

\section{Conclusion: Looking for an Insertion in Local Cropping Systems}

In this research, the study tested two hypotheses in order to demonstrate the adaptability of quinoa outside the Andean area. One point of discussion through different perspectives must the sustainable establishment of an alien species in a new agricultural environment. The crop introduction has to move from the stage of agronomic testing to that of assessing in practice whether quinoa can take place easily and sustainably in local farming systems around the world. Focusing on major crops cultivated in project partner countries to better know their crop cycles, we analyzed the potential inclusion of quinoa in agricultural calendars. Three groups of countries differ significantly: first when quinoa is considered a winter crop (Lebanon, Egypt, and Yemen), second when it operates as a spring crop (Kyrgyzstan and Tajikistan) and finally where it is planted as a fall crop (Algeria, Iraq, Iran, and Mauritania). Such disparities are interesting to study more in depth, considering quinoa crop introduction in a geographical area in appearance with somewhat similar climatic conditions. This consideration underlines that climate data is the main lever of action to decide the sowing date and to understand the best position in the crop calendar for water use efficiency and to avoid high temperatures at the flowering period.

The main conclusion of this study is that we were able to measure and confirm the great adaptability of quinoa in new areas with very different climatic conditions than those of the area of origin of this crop. In each of the sites studied, we were able to identify one or more genotypes with a high performance level validating the hypothesis 1 of yield quality performance. High yield potentials in single locations were shown by some genotypes like Q29 (LB1), Titicaca (IN3), Q21 (LB3), Q12 (LB1), Q29 (EG2), Q27 (LB3), Puno (TA1), Q1 (YE3). Considering yield optimization within a long term vision, some genotypes maintain a good level of stable performance regardless of the specific site conditions in which they were tested. This result allows us to also validate our second hypothesis of yield stability across sowing sites. The genotypes Q12, Q18, Q21, and Q26, averaged the best yield across 16 sites. In an agroecological perspective, quinoa biodiversity could be interesting to diversify cropping systems in order to stabilize food production.

Quinoa should be considered for diversifying cropping systems for food security and it should not be incorporated as part of an already busy period of the crop calendar cycle, and then competing with other cultures that have already demonstrated their good performances in these areas. In addition, one must not focus solely on the agricultural side of the introduction of quinoa in a local agro-ecosystem. The social component remains an important part of the adaptation of exotic species and its appropriation by the new populations. The food system of the study area may be upset with the introduction of a new food. Local populations will have to integrate quinoa in their diets and they will need to be prepared and technically and culturally accompanied to handle the new grains. There is still doubt on this point because, although quinoa has exceptional 
nutritional properties, development of attractive and culturally acceptable food recipes will need to be developed to reach out to both rural and urban consumers. This work will be an important endeavor to take into account when considering the introduction of quinoa cultivations outside the Andean region.

\section{AUTHOR CONTRIBUTIONS}

MA, DB, JB, LH, IM, OM, MO, NS, AS, DS, and KM have established the multi-local trials across the different countries and have collected the data from the field. DB and CP have given a permanent technical assistance and some specific orientations for the experimental plan during the implementation of the trials and their monitoring. DB conducted the analysis on results from the multi-local trials and AV contributed to the standardization of the dataset and to the first step of statistical analysis. SP collaborated with $\mathrm{AV}$ and had a global overview of the writing process.

\section{REFERENCES}

Anabalón, R. L., and Thomet-Isla, M. (2009). Comparative analysis of genetic and morphologic diversity among quinoa accessions (Chenopodium quinoa Willd.) of the South of Chile and highland accessions. J. Plant Breed. Crop Sci. 1, 210-216. Available online at: http://www.academicjournals.org/journal/JPBCS/ article-abstract/43AB1941305

Annicchiarico, P. (2002). "Genotype x environment interactions - challenges and opportunities for plant breeding and cultivar recommendations," in Plant Production and Protection Paper $n$ 174. Rome: FAO.

Araus, J. L., Slafer, G. A., Royo, C., and Serret, M. D. (2008). Breeding for yield potential and stress adaptation in cereals. Crit. Rev. Plant Sci. 27, 377-412. doi: $10.1080 / 07352680802467736$

Bazile, D. (2015). Le Quinoa, Les Enjeux D’une Conquête. Versailles: Editions Quae.

Bazile, D., Fuentes, F., and Mujica, A. (2013). "Historical perspectives and domestication," in Quinoa: Botany, Production and Uses, eds A. Bhargava and S. Srivastava (Wallingford: CABI), 16-35.

Bazile, D., Jacobsen, S.-E., and Verniau, A. (2016). The global expansion of quinoa: trends and limits. Front. Plant Sci. 7:622. doi: 10.3389/fpls.2016.00622

Bazile, D., Martinez, E. A., and Fuentes, F. (2014). Diversity of quinoa in a biogeographical Island: a review of constraints and potential from arid to temperate regions of Chile. Not. Bot. Horti Agrobot. Cluj Napoca 42, 289-298. doi: $10.15835 /$ nbha.42.2.9733

Bazile, D., and Negrete, J. (2009). Quínoa y biodiversidad: Cuáles son los desafíos regionales? Rev. Geogr. Valparaíso 42, 1-141. Available online at: http:// geografiapucv.cl/revista-geografica-de-valparaiso/

Bazile, D., and Weltzien, E. (2008). Agrobiodiversités. Cah. Agric. 17, 73-256. Available online at: http://www.jle.com/fr/revues/agr/sommaire.phtml?cle parution $=2158$

Bazile, D., Bertero, H. D., and Nieto, C., (eds.). (2015). State of the Art Report on Quinoa Around the World in 2013. Rome: FAO; CIRAD. Available online at: http://www.fao.org/3/a-i4042e.pdf

Benlhabib, O., Jacobsen, S. E., Jellen, E. N., Maughan, P. J., and Choukr-Allah, R. (2015). "Status of quinoa production and research in Morocco," in State of the Art Report on Quinoa Around the World in 2013, eds D. Bazile, D. Bertero, and C. Nieto (Rome: Food and Agriculture Organization of the United Nations and CIRAD), 178-491.

Bertero, H. D., King, R. W., and Hall, A. J. (1999). Modelling photoperiod and temperature responses of flowering in quinoa (Chenopodium quinoa Willd.). Field Crops Res. 63, 19-34. doi: 10.1016/S0378-4290(99)00024-6

\section{ACKNOWLEDGMENTS}

The authors thank all the participants of the different TCPs for their collaboration during trials and in particular FAO Agricultural Officers from the Regional Offices and FAO Headquarters, Muhammad Dost (Regional coordinator for eight countries of FAO-RNE), Hafiz Muninjanov (Regional coordinator for KYR and TAJ), and Wilson Hugo (Technical assistance from FAO-HQ). In addition, the manuscript was edited by Diana Gutierrez Mendez and Benjamin Graub, the authors also thank them for their helpful comments. Nicolas Picard, senior statistician and modeler, has developed w us the statistical analysis. Funding was provided by FAO Technical Cooperation Programmes: TCP/KYR/3405; TCP/TAJ/3404 and $\mathrm{TCP} / \mathrm{RAB} / 3403$.

\section{SUPPLEMENTARY MATERIAL}

The Supplementary Material for this article can be found online at: http://journal.frontiersin.org/article/10.3389/fpls.2016. 00850

Bertero, H. D. (2001). Effects of photoperiod, temperature and radiation on the rate of leaf appearance in quinoa (Chenopodium quinoa Willd.) under field conditions. Ann. Bot. 87, 495-502. doi: 10.1006/anbo.2000.1362

Bhargava, A., Shukla, S., and Ohri, D. (2006). Chenopodium quinoa - an Indian perspective. Ind. Crops Prod. 23, 73-87. doi: 10.1016/j.indcrop.2005.04.002

Bhargava, A., Shukla, S., and Ohri, D. (2007a). Genetic variability and interrelationship among various morphological and quality traits in quinoa (Chenopodium quinoa Willd.). Field Crops Res. 101, 104-116. doi: 10.1016/j.fcr.2006.10.001

Bhargava, A., Shukla, S., and Ohri, D. (2007b). Genetic diversity for morphological and quality traits in quinoa (Chenopodium quinoa Willd.) germplasm. Genet. Resour. Crop Evol. 54, 167-173. doi: 10.1007/s10722-005-3011-0

Bioversity International, FAO, PROINPA, INIAF, and IFAD (2013). Descriptors for Quinoa (Chenopodium quinoa Willd.) and Wild Relatives. Rome; La Paz: Bioversity International; Fundación PROINPA; Instituto Nacional de Innovación Agropecuaria y Forestal; International Fund for Agricultural Development, Food and Agriculture Organization of the United Nations.

Bois, J. F., Winkel, T., Lhomme, J. P., Raffaillac, J. P., and Rocheteau, A. (2006). Response of some Andean cultivars of quinoa (Chenopodium quinoa Willd.) to temperature: effects on germination, phenology, growth and freezing. Eur. J. Agron. 25, 299-308. doi: 10.1016/j.eja.2006.06.007

Bonifacio, A., Gomez-Pando, L., and Rojas, W. (2015). "Quinoa breeding and modern variety development," in State of the Art Report on Quinoa Around the World 2013, eds D. Bazile, D. Bertero, and C. Nieto (Rome: FAO; CIRAD), 172-191.

Bosque, H., Lemeur, R., Van Damme, P., and Jacobsen, S. E. (2003). Ecophysiological analysis of drought and salinity stress of Quinoa (Chenopodium quinoa Willd.). Food Rev. Int. 19, 111-119. doi: 10.1081/FRI-120018874

Brookfield, H., Padoch, C., Parsons, H., and Stocking, M. (2002). Cultivating Biodiversity: Understanding, Analysing and Using Agricultural Diversity. London: ITDG Publishing and United Nations University.

Caron, P., Craufurd, P., Martin, A., Mc Donald, A., Abedini, W., Afiff, S., et al. (2009). "Impacts of AKST on development and sustainability goals," in Agriculture at a Crossroads. International Assessment of Agricultural Knowledge, Science and Technology for Development (IAASTD): global report, eds D. McIntyre Beverly, R. Herren Hans, J. Wakhungu, and T. Watson Robert (Washington, DC: Island Press), 145-253.

Chevassus-au-Louis, B., and Bazile, D. (2008). Cultiver la diversité. Cah. Agric. 17, 77-78. doi: 10.1684/agr.2008.0187 
Choukr-Allah, R., Rao, N. K., Hirich, A., Shahid, M., Alshankiti, A., Toderich, K., et al. (2016). Quinoa for marginal environments: toward future food and nutritional security in MENA and Central Asia Regions. Front. Plant Sci. 7:346. doi: 10.3389/fpls.2016.00346

Christensen, S. A., Pratt, D. B., Pratt, C., Nelson, P. T., Stevens, M. R., Jellen, E. N., et al. (2007). Assessment of genetic diversity in the USDA and CIP-FAO international nursery collections of quinoa (Chenopodium quinoa Willd.) using microsatellite markers. Plant Genet. Res. 5, 82-95. doi: $10.1017 /$ S1479262107672293

Cleveland, D. A., Soleri, D., and Smith, S. E. (1994). Do folk crop varieties have a role in sustainable agriculture? Bioscience 44, 740-751. doi: 10.2307/1312583

Cocozza, C., Pulvento, C., Lavini, A., Riccardi, M., d'Andria, R., and Tognetti, R. (2013). Effects of increasing salinity stress and decreasing water availability on ecophysiological traits of Quinoa (Chenopodium quinoa Willd.) grown in a Mediterranean-type agroecosystem. J. Agron. Crop Sci. 199, 229-240. doi: $10.1111 /$ jac. 12012

Conway, G., and Toenniessen, G. (1999). Feeding the world in the twenty-first century. Nature 402, C55-C58. doi: 10.1038/35011545

Coulibaly, A. K., Sangare, A., Konate, M., Traore, S., Ruiz, K. B., Martínez, E. A., et al. (2015). "Assessment and adaptation of quinoa (Chenopodium quinoa Willd) to the agroclimatic conditions in Mali, West Africa: an example of South-North-South cooperation," in State of the Art Report on Quinoa Around the World 2013, eds D. Bazile, D. Bertero, and C. Nieto (Rome: FAO; CIRAD), 524-533. Available online at: http://www.fao.org/3/a-i4042e/i4042e35.pdf

Cusack, D. F. (1984). Quinoa: grain of the Incas. Ecologist 14, 21-31.

Delatorre-Herrera, J., and Pinto, M. (2009). Importance of ionic and osmotic components of salt stress on the germination of four quinua (Chenopodium quinoa Willd.) selections. Chilean J. Agr. Res. 69, 477-485. doi: 10.4067/S071858392009000400001

FAO (2011). Quinoa: An Ancient Crop to Contribute to World Food Security. Santiago de Chile: FAO Regional Office for Latin America and the Caribbean.

FAO (2015). Panorama de la Seguridad Alimentaria en América Latina y el Caribe. Santiago de Chile: FAO Regional Office for Latin America and the Caribbean.

Fasoula, D. A. (2012). Nonstop selection for high and stable crop yield by two prognostic equations to reduce yield losses. Concept Paper. Agric. 2, 211-227. doi: 10.3390/agriculture2030211

Fu, Y.-B. (2015). Understanding crop genetic diversity under modern plant breeding. Theor. Appl. Genet. 128, 2131-2142. doi: 10.1007/s00122-015-2585-y

Fuentes, F., Bazile, D., Bhargava, A., and Martínez, E. A. (2012). Implications of farmers' seed exchanges for on-farm conservation of quinoa, as revealed by its genetic diversity in Chile. J. Agric. Sci. 150, 702-716. doi: 10.1017/S0021859612000056

Fuentes, F., Martínez, E. A., Hinrichsen, P. V., Jellen, E. N., and Maughan, P. J. (2009a). Assessment of genetic diversity patterns in Chilean quinoa (Chenopodium quinoa Willd.) germplasm using multiplex fluorescent microsatellite markers. Conserv. Genet. 10, 369-377. doi: 10.1007/s10592-008-9604-3

Fuentes, F., and Bhargava, A. (2011). Morphological analysis of quinoa germplasm grown under lowland desert conditions. J. Agron. Crop Sci. 197, 124-134. doi: 10.1111/j.1439-037X.2010.00445.x

Fuentes, F., Maughan, P. J., and Jellen, E. N. (2009b). Diversidad genética y recursos genéticos para el mejoramiento de la quínoa (Chenopodium quinoa Willd). Rev. Geogr. Valparaíso 42, 20-33. Available online at: http://geografiapucv.cl/wpcontent/uploads/2016/05/42-3.pdf

Gallais, A. (1992). Adaptation et adaptabilité en amélioration des plantes. Sel. Fr. $42,55-57$.

Galwey, N. W. (1992). The potential of quinoa as a multi-purpose crop for agricultural diversification: a review. Ind. Crops Prod. 1, 101-106. doi: 10.1016/0926-6690(92)90006-H

Gesinski, K. (2008). Evaluation of the development and yielding potential of Chenopodium quinoa Willd. under the climatic conditions of Europe. Acta Agrobot. 61, 185-189. doi: 10.5586/aa.2008.026

Gómez-Pando, L. R., lvarez-Castro, R. A., and Eguiluz-De La Barra, A. (2010). Effect of salt stress on Peruvian germplasm of Chenopodium quinoa Willd.: a promising crop. J. Agron. Crop Sci. 196, 391-396. doi: 10.1111/j.1439037X.2010.00429.x

Heiser, C. B., and Nelson, C. D. (1974). On the origin of cultivated Chenopods (Chenopodium). Genetics 78, 503-505.
Jacobsen, S.-E. (1997). Adaptation of quinoa (Chenopodium quinoa) to Northern European agriculture: studies on developmental pattern. Euphytica 96, 41-48. doi: 10.1023/A:1002992718009

Jacobsen, S. E. (2003). The worldwide potential of quinoa (Chenopodium quinoa Willd.). Food Rev. Int. 19, 167-177. doi: 10.1081/FRI-120018883

Jacobsen, S.-E., and Stølen, O. (1993). Quinoa-morphology and phenology and prospects for its production as a new crop in Europe. Eur. J. Agron. 2, 19-29. doi: 10.1016/S1161-0301(14)80148-2

Jacobsen, S. E., Mujica, A., and Jensen, C. R. (2003). The resistance of quinoa (Chenopodium quinoa Willd.) to adverse abiotic factors. Food Rev. Int. 19, 99-109. doi: 10.1081/FRI-120018872

Jellen, E. N., Maughan, P. J., Fuentes, F., and Kolano, B. A. (2015). "Botany, phylogeny and evolution," in State of the Art Report on Quinoa Around the World 2013, eds D. Bazile, D. Bertero, and C. Nieto (Rome: FAO; CIRAD), $12-23$.

Louafi, S., Bazile, D., and Noyer, J. L. (2013). "Conserving and cultivating agricultural genetic diversity: transcending established divides," in Cultivating Biodiversity to Transform Agriculture, ed E. Hainzelin (Heidelberg: Springer [Allemagne]), 181-230.

Lutz, M., Martínez, E. A., and Martínez, A. (2013). Daidzein and genistein contents in seeds of quinoa (Chenopodium quinoa Willd) from local ecotypes grown in arid Chile. Ind. Crops Prod. 49, 117-121. doi: 10.1016/j.indcrop.2013.04.023

Martinez, E. A., Fuentes, F., and Bazile, D. (2015). "History of Quinoa: its origin, domestication, diversification and cultivation with particular reference to the chilean context," in Quinoa: Improvement and Sustainable Production, eds K. Murphy and J. Matanguihan (Hoboken: Wiley-Blackwell). 19-24. doi: 10.1002/9781118628041.ch2

Martínez, E. A., Veas, E., Jorquera, C., San Martín, R., and Jara, P. (2009). Reintroduction of Chenopodium quinoa Willd. into arid Chile: cultivation of two lowland races under extremely low irrigation. J. Agron. Crop Sci. 195, 1-10. doi: 10.1111/j.1439-037X.2008.00332.x

Maureira, H., and Martínez, E. A. (2012). Nutritional aspects of six quinoa (Chenopodium quinoa Willd.) ecotypes from three geographical areas of Chile. Chilean J. Agric. Res. 72, 175-181. doi: 10.4067/S0718-58392012000200002

Miranda, M., Vega-Gálvez, A., Martinez, E. A., López, J., Rodríguez, M. J., Henríquez, K., et al. (2012). Genetic diversity and comparison of physicochemical and nutritional characteristics of six quinoa (Chenopodium quinoa Willd.) genotypes cultivated in Chile. Food Sci. Tech. 32, 835-843. doi: 10.1590/s0101-20612012005000114

Mujica, A., Jacobsen, S. E., Izquierdo, J., and Marathee, J. P. (2001). Resultados de la Prueba Americana y Europea de la Quinua. Puno: FAO; UNA; CIP.

Murphy, K. M., Bazile, D., Kellogg, J., and Rahmanian, M. (2016). Development of a worldwide consortium on evolutionary participatory breeding in Quinoa. Front. Plant Sci. 7:608. doi: 10.3389/fpls.2016.00608

Narea, A. (1976). "La producción de quinua en el Perú," in Segunda Convención Internacional de las Quenopodiáceas (Potosi: Universidad Boliviana Tomas Frias), 170-177.

National Research Council (NRC) (1989). Lost Crops of the Incas: Little-Known Plants of the Andes with Promise for Worldwide Cultivation. Washington, DC: National Academy Press.

Orsini, F., Accorsi, M., Gianquinto, G., Dinelli, G., Antognoni, F., Ruiz-Carrasco, K. B., et al. (2011). Beyond the ionic and osmotic response to salinity in Chenopodium quinoa: functional elements of successful halophytism. Funct. Plant Biol. 38, 818-831. doi: 10.1071/FP11088

Planella, M. T., Scherson, R., and McRostie, V. (2011). Sitio El Plomo y nuevos registros de cultígenos iniciales en cazadores del Arcaico IV en alto Maipo, Chile central. Chungara Rev. Antropol. Chil. 43, 189-202. doi: 10.4067/S071773562011000200003

Power, A. G. (1999). Linking ecological sustainability and world food needs. Dev. Sustain. 1, 185-196. doi: 10.1023/A:1010074805303

Pulvento, C., Riccardi, M., Lavini, A., D’Andria, R., Iafelice, G., and Marconi, E. (2010). Field trial evaluation of two Chenopodium quinoa genotypes grown under rainfed conditions in a typical Mediterranean environment in south Italy. J. Agron. Crop Sci. 196, 407-411. doi: 10.1111/j.1439-037X.2010.00431.x

Pulvento, C., Riccardi, M., Lavini, A., Iafelice, G., Marconi, E., and d'Andria, R. (2012). Yield and quality characteristics of quinoa grown in open field under different saline and non-saline irrigation regimes. J. Agron. Crop Sci. 198, 254-263. doi: 10.1111/j.1439-037X.2012.00509.x 
Risi, J. C., and Galwey, N. W. (1984). The Chenopodium grains of the Andes: Inca crops for modern agriculture. Adv. Appl. Biol. 10, 145-216.

Risi, J., and Galwey, N. W. (1989). "The Chenopodium grains of the Andes: a crop for temperate latitudes," in New Crops for Food and Industry, eds G. E. Wickens, N. Haq, and P. Day (London; New York, NY: Chapman \& Hall), 222-234.

Risi, J., and Galwey, N. W. (1991). Genotype x Environment interaction in the Andean grain crop quinoa (Chenopodium quinoa Willd.) in temperate environments. Plant Breed. 107, 141-147. doi: 10.1111/j.14390523.1991.tb00542.x

Rojas, W. (2003). Multivariate analysis of genetic diversity of Bolivian quinoa germplasm. Food Rev. Int. 19, 9-23. doi: 10.1081/FRI-120018864

Rojas, W., Pinto, M., Alanoca, C., Gómez-Pando, L., León-Lobos, P., Alercia, A., et al. (2015). "Quinoa genetic resources and ex situ conservation," in State of the Art Report on Quinoa Around the World 2013, eds D. Bazile, D. Bertero, and C. Nieto (Rome: FAO; CIRAD), 56-82.

Ruiz, K. B., Biondi, S., Oses, R., Acuña-Rodríguez, I. S., Antognoni, F., MartinezMosqueira, E. A., et al. (2014). Quinoa biodiversity and sustainability for food security under climate change: a review. Agron. Sustain. Dev. 34, 349-359. doi: 10.1007/s13593-013-0195-0

Ruiz-Carrasco, K. B., Antognoni, F., Coulibaly, A. K., Lizardi, S., Covarrubias, A., Martínez, E. A., et al. (2011). Variation in salinity tolerance of four lowland genotypes of quinoa (Chenopodium quinoa Willd.) as assessed by growth, physiological traits, and sodium transporter gene expression. Plant Physiol. Biochem. 49, 1333-1341. doi: 10.1016/j.plaphy.2011.08.005

Seckler, D., Barker, R., and Amarasinghe, U. (1999). Water scarcity in the twentyfirst century. Int. J. Water Resour. Dev. 15, 29-42. doi: 10.1080/07900629948916

Schlick, G., and Bubenheim, D. L. (1996). "Quinoa: candidate crop for NASA's controlled ecological life support systems," in Progress in New Crops, ed J. Janick (Arlington, TX: ASHS Press), 632-640.

Sing, S. (2010). Understanding the Weedy Chenopodium Complex in the North Central States. PhD thesis of Philosophy in Crop Sciences. University of Illinois at Urbana-Champaign, USA.

Tagle, M. B., and Planella, M. T. (2002). La Quínoa en la Zona Central de Chile, Supervivencia de una Tradición Prehispánica. Santiago: Editorial IKU.
Tapia, M. E. (1979). “Historia y distribución geográfica, in Quinua y Kañiwa. Cultivos Andinos," in Serie Libros y Materiales Educativos No 49, ed M. E. Tapia (Bogotá: Instituto Interamericano de Ciencias Agrícolas), 11-15.

Tigerstedt, P. M. A. (1994). Adaptation, variation and selection in marginal areas. Euphytica 77, 171-174. doi: 10.1007/BF02262628

Tilman, D., Cassman, K. G., Matson, P. A., Naylor, R., and Polasky, S. (2002). Agricultural sustainability and intensive production practices. Nature 418, 671-677. doi: 10.1038/nature01014

Vega-Gálvez, A., Miranda, M., Vergara, J., Uribe, E., Puente, L., and Martínez, E. A. (2010). Nutrition facts and functional potential of quinoa (Chenopodium quinoa Willd.), an ancient Andean grain: a review. J. Sci. Food Agric. 90, 2541-2547. doi: 10.1002/jsfa.4158

Von Baer, I., Bazile, D., and Martínez, E. A. (2009). Cuarenta años de mejoramiento de la quínoa (Chenopodium quinoa Willd.) en la Araucanía: origen de "La Regalona-B.” Rev. Geogr. Valparaíso 42, 34-44. Available online at: http:// geografiapucv.cl/wp-content/uploads/2016/05/42-4.pdf

Wilson, H. D. (1990). Quinua and relatives (Chenopodium sect. Chenopodium subsect. Cellulata). Econ. Bot. 44, 92-110. doi: 10.1007/BF02860478

Conflict of Interest Statement: The authors declare that the research was conducted in the absence of any commercial or financial relationships that could be construed as a potential conflict of interest.

The views expressed in this paper are those of the authors and do not necessarily reflect the views or policies of FAO.

Copyright (c) 2016 Bazile, Pulvento, Verniau, Al-Nusairi, Ba, Breidy, Hassan, Mohammed, Mambetov, Otambekova, Sepahvand, Shams, Souici, Miri and Padulosi. This is an open-access article distributed under the terms of the Creative Commons Attribution License (CC BY). The use, distribution or reproduction in other forums is permitted, provided the original author(s) or licensor are credited and that the original publication in this journal is cited, in accordance with accepted academic practice. No use, distribution or reproduction is permitted which does not comply with these terms. 


\section{Quinoa for Marginal Environments: Toward Future Food and Nutritional Security in MENA and Central Asia Regions}

\begin{abstract}
Redouane Choukr-Allah *, Nanduri K. Rao, Abdelaziz Hirich, Mohammad Shahid, Abdullah Alshankiti, Kristina Toderich, Shagufta Gill and Khalil Ur Rahman Butt
\end{abstract}

Research and Innovation Division, International Center for Biosaline Agriculture, Dubai, UAE

Quinoa is recognized as a crop of great value in terms of tolerance to abiotic stresses and there is growing interest to introduce it in marginal agricultural production systems worldwide. Also, quinoa is one of the most nutritious food crops currently known and the nutritive properties of the crop are seen as a mean to fight malnutrition globally. Various quinoa cultivars have been screened for tolerance to salinity, water-use efficiency and nutritional quality and the positive attributes found in them have created even wider global interest in its cultivation. This paper summarizes 15 years of studies on assessing the potential for introducing the crop in a few countries of the Middle East and North Africa (MENA) and Central Asia regions and describes the key constraints for scaling-up the production under marginal growing conditions in the newly introduced countries.

Keywords: irrigation, salinity, yield, sowing dates, food security

\section{INTRODUCTION}

Quinoa has been recognized as a climate resilient crop of great value and there is an increasing effort to introduce it in different marginal agriculture production systems of the world. Various quinoa cultivars have been screened for tolerance to abiotic stresses, especially salinity, drought, and frost and the positive attributes of the crop have created wider global interest in its cultivation (Jacobsen, 2003; Jacobsen et al., 2003). Also, quinoa is one of the most nutritious food crops currently known. The seeds contains high quality protein, which has all of the essential amino acids including lysine, methionine and threonine that are scarce in cereals and legumes (Repo-Carrasco et al., 2003). In view of its exceptional nutritional quality and ability to grow under marginal environments, the Food and Agriculture Organization of the United Nations (FAO) has identified quinoa as one of the crops that will play an important role in ensuring future food security and designated the year 2013 as the "Year of Quinoa" (Bazile et al., 2015). Worldwide, the demand for quinoa is growing, especially in the health food segment, but current supplies are unable to match it. Besides the use for human consumption, quinoa seed has other uses as livestock and poultry feed. The whole plant can be used as green fodder and harvest residues can be fed to the animals. In the context of the Middle East and North Africa (MENA) and Central Asia regions, where soil and water salinity is increasingly becoming a constraint to agricultural production, quinoa is seen as an alternative crop with significant potential to have a central role in sustaining farm productivity.

The International Center for Biosaline Agriculture (ICBA) in Dubai, United Arab Emirates (UAE) has been working since 2007 on quinoa in partnership with the Ministry of Environment 
and Water (MOEW) of the UAE, Abu Dhabi Farmer's Service Center (ADFSC) and its Peruvian partners [Instituto Nacional de Innovacion Agraria (INIA) and Universidad National Agraria La Molina (UNALM)] to evaluate the performance of several quinoa cultivars for their productivity when grown in marginal conditions. ICBA has identified and developed five high yielding salt- and heat-tolerant lines that are now ready to be tested in other agro-ecological zones. The FAO has also been in the forefront of quinoa research, keeping in view its unique nutritional characteristics and adaptability to a wide range of agro-ecological conditions, especially in the marginal areas and the organization has initiated a Regional Project TCP/RAB/3403 for the introduction, appropriation and institutionalization of its production in Algeria, Egypt, Iran, Iraq, Lebanon, Mauritania, Sudan, and Yemen. ICBA considers quinoa as an essential crop in its efforts to sustain agricultural productivity amidst growing threat of salinity and water scarcity, as well as to tackle hunger, malnutrition and poverty in the MENA and Central Asia regions. To fully exploit the potential of the crop for marginal environments, identification of new and high-yielding quinoa genotypes with good local adaptation and high nutritional quality are crucial, which requires intensified screening and adaption research.

This paper summarizes 15 years of studies in assessing the potential of quinoa for introducing it in some countries of the MENA and Central Asia Regions. In view of quinoa's potential as a stress-tolerant, climate resilient future-proof crop, ICBA is developing best practices for its production and management in marginal environments. The Center is also focusing on utilization and marketing of the produce and on fostering knowledge transfer, strengthening competencies and exchange of experiences among the various stakeholders within the MENA and Central Asia regions.

\section{SCREENING FOR LOCAL ADAPTATION AND YIELD POTENTIAL}

ICBA acquired 121 germplasm accessions from the United States Department of Agriculture (USDA) and evaluated them for growth performance and yield at its research station $\left(25^{\circ} 05^{\prime} 49^{\prime \prime}\right.$ $\mathrm{N}$ and $55^{\circ} 23^{\prime} 25^{\prime \prime} \mathrm{E}$ ) during the cropping season November 2006March 2007. The soil at the experimental site was fine sand and moderately alkaline ( $\mathrm{pH} 8.2$ ) with very low organic matter $(<0.5 \%)$. The seeds were sown in the first week of November 2006 and each accession was planted in three $3 \mathrm{~m}$-rows with spacing of $50 \mathrm{~cm}$ between the rows and one meter between two accessions. The distance between plants within each row was maintained at $25 \mathrm{~cm}$. Before sowing, the fertility of the soil was improved by incorporating organic fertilizer (compost) at the rate of $40 \mathrm{tha}^{-1}$ and during crop growth two split doses of NPK (20:20:20) at the rate of $50 \mathrm{~kg} \mathrm{ha}^{-1}$, were applied by banding alongside the rows. The amount of $\mathrm{N}$ added to the soil by the combination of compost and NPK was estimated to be $570 \mathrm{~kg} \mathrm{ha}^{-1}$. The plants were irrigated with low-salinity water with an electrical conductivity $\left(\mathrm{EC}_{w}\right)$ of $2-3 \mathrm{dS} \mathrm{m}{ }^{-1}$ using the drip system. Water was applied once every day for $20 \mathrm{~min}$ at a flow rate of $4 \mathrm{l} \mathrm{h}^{-1}$ per plant and in addition, about $60 \mathrm{~mm}$ of rainfall was received during the growing season. Of the 121 accessions planted, seeds were harvested from 73 accessions that survived through the growing season and based on the seed yield data, the top 20 performers were selected for subsequent evaluation, carried out during the cropping seasons 2007-08 and 2008-09 (Rao and Shahid, 2012). The agronomic practices such as irrigation and applications of fertilizers were the same as described previously. The data on grain yield of the 20 accessions from the two cropping seasons (2007-08 and 2008-09) are presented in Table 1. The seed yield among the accessions varied between 53.86 and $359.86 \mathrm{~g} \mathrm{~m}^{-2}$ in 2007-08, and between 3.32 and $258.42 \mathrm{~g} \mathrm{~m}^{-2}$ in 2008-09. Averaged over the two seasons, the yield ranged between $34.06 \mathrm{~g} \mathrm{~m}^{-2}$ and $238.99 \mathrm{~g} \mathrm{~m}^{-2}$ among the accessions. The studies showed that quinoa has good adaptation and can be successfully cultivated in the Arabian Peninsula. In a further study conducted at the ICBA Research Station in Dubai during 2009-10, the performance of five top-yielding accessions selected on the basis of average seed yield from previous studies were further evaluated using low-salinity irrigation water $\left(\mathrm{EC}_{\mathrm{w}} 2.8 \mathrm{dS} \mathrm{m}^{-1}\right.$ ) (Rao and Shahid, 2012). Analysis of the data revealed significant differences for plant height, number of primary branches and number of inflorescences per plant. However, differences among accessions for all other traits including inflorescence length, seed yield per plant, fresh and dry biomass were found to be marginal. The seed yield among the five accessions ranged between $374.4 \mathrm{~g} \mathrm{~m}^{-2}$ (Ames 13757) and $533.6 \mathrm{~g} \mathrm{~m}^{-2}$ (Ames 13761) with an average of $456.6 \mathrm{~g} \mathrm{~m}^{-2}$ over accessions. The dry matter yield, averaged over the accessions was $1464 \mathrm{~g} \mathrm{~m}^{-2}$, with accession Ames 13742 producing the maximum yield of $1624 \mathrm{~g} \mathrm{~m}^{-2}$ (Rao and Shahid, 2012). During course of the yield trials over the years, mass selection to eliminate inferior plant types and improve the yield potential of the selected accessions has resulted in five improved lines (Q1-Q5) which were used in further studies described below.

\section{AGRONOMIC EVALUATION AND PERFORMANCE OF SELECTED LINES}

The results from the preliminary trials undertaken at ICBA Research Station gave sufficient indication of the potential of quinoa as a salt-tolerant alternative crop. However, further investigations were needed to study the performance under a range of biophysical environments especially at various soil and water salinities to introduce the crop to the farmers. Hence field trials were conducted under a range of agro-ecological conditions-at Ghayathi in the Western Region of Abu Dhabi Emirate in 2012-13 with two of the five selected lines (Q3, Q5) and NSL 106399; and subsequently in five locations-three in the Northern Emirates (Dibba, Hamraniah, and Al Dhaid) and two in the Western Region of Abu Dhabi (Madinat Zayed and Ghayathi) in 2013-14, involving four of the five selected quinoa lines (Q1, Q2, Q3, and Q5) (Table 2). While the two locations planted in the Western Region were agricultural farms leased for the trials, those in the Northern Emirates were the federal government's regional research stations. Each set of lines was 
TABLE 1 | Grain yields in 20 selected quinoa accessions grown in winter 2007-08 and 2008-09 (source: Rao and Shahid, 2012).

\begin{tabular}{|c|c|c|c|c|}
\hline \multirow[t]{2}{*}{ Accession no. } & \multirow[t]{2}{*}{ Source } & \multicolumn{3}{|c|}{ Yield $\left(\mathrm{g} \mathrm{m}^{-2}\right)$} \\
\hline & & $2007-08$ & 2008-09 & Mean \\
\hline Ames 13220 & Bolivia & 84.57 & 100.95 & 92.76 \\
\hline Ames 13719 & USA & 74.61 & 87.05 & 80.83 \\
\hline Ames 13723 & USA & 80.09 & 134.42 & 107.25 \\
\hline Ames 13724 & USA & 132.32 & 62.42 & 97.37 \\
\hline Ames 13727 & USA & 111.39 & 133.02 & 122.2 \\
\hline Ames 13736 & USA & 57.50 & 109.07 & 83.29 \\
\hline Ames 13742 & USA & 359.86 & 118.12 & 238.99 \\
\hline Ames 13749 & USA & 211.06 & 79.25 & 145.16 \\
\hline Ames 13757 & USA & 187.26 & 120.32 & 153.79 \\
\hline Ames 13758 & USA & 64.81 & 3.32 & 34.06 \\
\hline Ames 13761 & USA & 60.50 & 258.42 & 159.46 \\
\hline Ames 21931 & Bolivia & 84.19 & 39.88 & 62.04 \\
\hline Ames 22154 & Chile & 50.85 & 45.13 & 47.99 \\
\hline Ames 22155 & Chile & 147.34 & 91.43 & 119.39 \\
\hline Ames 22157 & Chile & 118.58 & 133.33 & 125.95 \\
\hline NSL 106395 & USA & 105.77 & 119.25 & 112.51 \\
\hline NSL 106398 & USA & 54.00 & 193.92 & 123.96 \\
\hline NSL 106399 & USA & 167.10 & 148.65 & 157.87 \\
\hline NSL 86649 & USA & 53.86 & 143.1 & 98.48 \\
\hline PI 478410 & Bolivia & 58.06 & 13.27 & 35.67 \\
\hline Mean & & 113.19 & 106.72 & 109.95 \\
\hline SE & & 17.79 & 14.40 & 16.12 \\
\hline
\end{tabular}

sown in a randomized complete block design (RCBD) with three replications. In all cases, the distance between rows was $50 \mathrm{~cm}$ and between plants within the row was $25 \mathrm{~cm}$. Spacing between two adjacent plots was maintained at $1 \mathrm{~m}$. The amount of water and fertilizers applied were the same for all the five locations and as described in the previous section.

In 2012-13, at Ghayathi, despite high salinity of irrigation water $\left(\mathrm{EC}_{\mathrm{w}} 14-15 \mathrm{dS} \mathrm{m}^{-1}\right)$, the mean seed yield obtained of the three lines was $750 \mathrm{~g} \mathrm{~m}^{-2}$ which was on par with the highest yields reported from the non-saline traditional quinoa growing areas. The green biomass yield was also high, the mean of the three cultivars being $4.3 \mathrm{~kg} \mathrm{~m}^{-2}$, indicating the potential of quinoa as an alternative forage crop for saline areas (Rao et al., 2013). In 2013-14 trials, the salinity of irrigation water in the five locations varied between 2.3 and $18.9 \mathrm{dS} \mathrm{m}^{-1}$ (Table 2). Seed yield from trials in the Northern Emirates correlated with the salinity of irrigation water $\left(\mathrm{EC}_{w}\right)$, which was $6.1 \mathrm{dS} \mathrm{m}^{-1}$ in Dibba, $4.5 \mathrm{dS} \mathrm{m}^{-1}$ in Hamraniah and $2.3 \mathrm{dS} \mathrm{m}^{-1}$ in Al Dhaid. Thus, averaged over lines, the seed yield was highest in Al Dhaid $\left(541 \mathrm{~g} \mathrm{~m}^{2}\right)$, followed by Hamraniah $\left(398 \mathrm{~g} \mathrm{~m}^{-2}\right.$ ) and Dibba $\left(190 \mathrm{~g} \mathrm{~m}^{2}\right.$ ) (Rao, 2016; Figure 1). In the western region despite higher salinity of the irrigation water, significantly higher seed yields were recorded than those from the Northern Emirates. In Ghayathi, the mean seed yields of the four lines was $1050 \mathrm{~g} \mathrm{~m}^{-2}$, much higher than the yields recorded in the previous year $\left(750 \mathrm{~g} \mathrm{~m}^{-2}\right)$ from the same location. In Madinat Zayed the mean seed yield
TABLE 2 | Quinoa yield trails in the UAE: Soil and water characteristics at the locations and details of accessions evaluated (Rao, 2016).

\begin{tabular}{|c|c|c|c|c|}
\hline Location & $\begin{array}{l}\text { Growing } \\
\text { season }\end{array}$ & Soil texture & $\begin{array}{l}\text { Water salinity } \\
\left(\mathrm{dS} \mathrm{m}^{-1} \text { ) }\right.\end{array}$ & Identity \\
\hline $\begin{array}{l}\text { ICBA } \\
\text { Reserach } \\
\text { Station }\end{array}$ & $2009-10$ & Sand & 2.8 & $\begin{array}{l}\text { Ames 13742, Ames } \\
\text { 13749, Ames 13757, } \\
\text { Ames 13761, NSL } \\
106399\end{array}$ \\
\hline Ghayathi & $2012-13$ & Sand & 15.1 & $\begin{array}{l}\text { ICBA-Q3, ICBA-Q5, } \\
\text { NSL } 106399\end{array}$ \\
\hline Ghayathi & 2013-14 & Sand & 16.3 & ICBA-Q1, Q3, Q4, Q5 \\
\hline $\begin{array}{l}\text { Madinat } \\
\text { Zayed }\end{array}$ & $2013-14$ & Sandy loam & 18.9 & ICBA-Q1, Q3, Q4, Q5 \\
\hline Dibba & $2013-14$ & Sand & 6.1 & ICBA-Q1, Q3, Q4, Q5 \\
\hline Hamraniah & $2013-14$ & Sandy loam & 4.5 & ICBA-Q1, Q3, Q4, Q5 \\
\hline Al Dhaid & 2013-14 & Loamy sand & 2.3 & ICBA-Q1, Q3, Q4, Q5 \\
\hline
\end{tabular}

of the four lines was $700 \mathrm{~g} \mathrm{~m}^{-2}$, which was similar to the yield obtained from Ghayathi in the previous year (Rao et al., 2013; Rao, 2016; Figure 1). The results from yield trials showed high degree of variability in the performance of the different cultivars across locations (Supplementary Figure 1). While no major differences existed among the different locations in terms of radiation, mean maximum and minimum temperatures or precipitation during the growing period, the differences in yields could only be attributed to differences in physicochemical properties of the soils and water (other than the EC and $\mathrm{pH}$ which were measured), for which more detailed analyses are warranted. The two locations planted in the Western Region being agricultural soils, possibly had a more favorable microenvironment compared to the sandy soils with poorly developed profiles at the research stations in Northern Emirates. Nevertheless, the exceptionally higher yields obtained with highly saline irrigation water in the Western Region showed that quinoa, which is a facultative halophyte, not only endures salinity but also some of its cultivars prosper under saline conditions, therefore has huge potential as an alternative food and feed crop when growing traditional crops becomes uneconomical due to increased groundwater salinity. The results besides confirming quinoa's suitability to withstand high salinity in water and soils, also demonstrated that it has good adaptation to the hyperarid desert environments and is an excellent candidate for crop diversification in the UAE as well as other countries with similar climatic conditions.

In addition to the UAE, ICBA has recently undertook pilot trials in six other MENA and Central Asian countries and the selected quinoa lines proved to be very productive even under very poor soils (sandy soils) and under conditions of deficit irrigation and high salinity. For instance, in 2015, five quinoa lines (Q1-Q5) were evaluated for seed and forage yields in three countries of Central Asia, namely: Uzbekistan, Tajikistan and Kyrgyzstan under different eco-agroclimatic zones that significantly differ in soil characteristics and agricultural practices (ICBA, 2015). Results from Kyrgyzstan were variable due to late planning but in Uzbekistan the seed yields among the lines ranged between $294 \mathrm{~g} \mathrm{~m}^{-2}$ (Q3) and $557 \mathrm{~g} \mathrm{~m}^{-2}$ (Q5) with a mean 


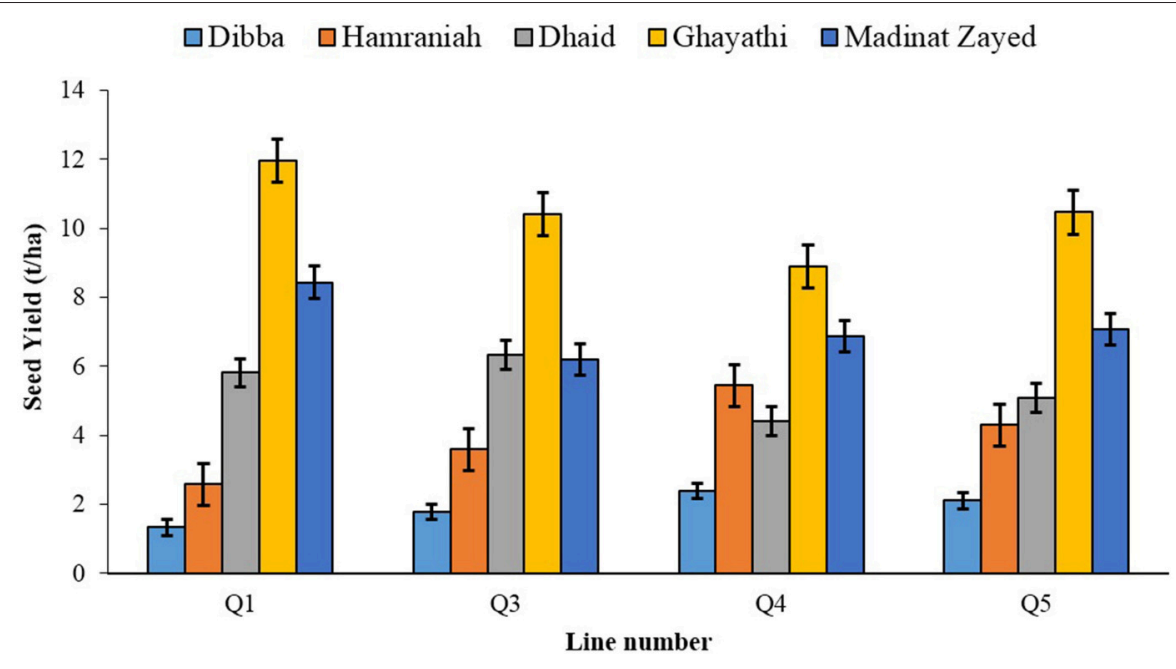

FIGURE 1 | The mean seed yields of four quinoa lines grown at five locations in the UAE during 2013-14. The error bars represent LSD of the means $(p<$ 0.05) (Rao, 2016).

of $436 \mathrm{~g} \mathrm{~m}^{-2}$ and in Tajikistan they ranged from $147 \mathrm{~g} \mathrm{~m}^{-2}$ (Q2) to $336 \mathrm{~g} \mathrm{~m}^{-2}$ (Q1) with a mean of $227 \mathrm{~g} \mathrm{~m}^{-2}$ (Figure 2).

The high yields obtained under marginal agro-climatic conditions in different locations are indicative of the potential and further investigations are needed to study the performance of a much wider range of genetically diverse accessions at various soil and water salinities to fully exploit the available genetic diversity within the crop. Quinoa is a predominantly self-pollinating species and considerable variation exists between cultivars for many of the desired characters. Therefore, it should be possible to select better adapted genotypes with high yields and nutritional quality combined with salt-and drought-tolerance. Identification of desirable genotypes needs to be followed by work on optimization of cultural practices to maximize productivity under the local conditions.

Quinoa was evaluated and introduced in Morocco for the first time in 2000-01 growing season in two locations, Khenifra (mountain region) and Rabat (coastal region). Obtained results are presented in Figure 3. Among the tested lines, G205-95DK performed the best under high altitude conditions due to its origin in the high altitudes of Altiplano (Andean Plateau), while most of the other lines showed adaptation to the coastal region probably because of provenance effect (Benlhabib et al., 2015).

In Morocco, in a field experiment conducted in Rhamna region, four selected quinoa lines (L11, L119, L123, L142, L143) along with two commercial varieties Titicaca and Puno were evaluated for phenological and agro-morphological characteristics (Filali, 2011). Titicaca and L143 recorded the highest seed yield $\left(1.5 \mathrm{t} \mathrm{ha}^{-1}\right)$, while L11 showed the lowest seed yield $\left(0.46 \mathrm{t} \mathrm{ha}^{-1}\right)$. The same lines when cultivated in the experimental farm of the Agronomic and Veterinary Medicine Hassan II Institute, Horticultural complex, Agadir (IAV-CHA) and tested under four different irrigation levels (100, 75,50 , and $25 \%$ of full irrigation), responded differently to water stress (Figure 4). The line L143 was most affected by water stress, L123 and L11 were less affected as the reduction in yield compared to the fully irrigated treatment was less (Supplementary Figures 5, 6).

\section{NUTRITIONAL QUALITY}

Quinoa seeds are an exceptionally nutritious food source, owing to their high protein content with all essential amino acids, lack of gluten, and high content of several minerals such as $\mathrm{Ca}, \mathrm{Mg}, \mathrm{Fe}$, and it is also rich in vitamins. Abiotic stress is known to induce considerable changes in the composition and quality of cereal grains such as wheat (Ashraf, 2014). Despite quinoa's growing popularity, the knowledge about the relationship between the growth conditions and the nutritional profile is still limited. Characterization of the nutritional and anti-nutritional properties of quinoa and evaluation of the effects of extreme growing conditions on the nutritional quality will provide strategic information for the introduction and promotion of quinoa in new environments besides aiding in the selection of nutritionally high and stable cultivars. Results from analysis of the chemical composition of the seeds harvested from three locations (Al Dahid, Ghayathi and Madinat Zayed) with varying levels of irrigation water salinity $(2.3,16.3$, and $18.9 \mathrm{dS} \mathrm{m}^{-1}$, respectively) showed that salt stress has marginal effect on the proximate composition (Rao, 2016). However, significant differences were found in the mineral content of the seeds. Especially, seeds harvested from Ghayathi and Madina zayed had higher $\mathrm{Na}$ content, but lower levels of $\mathrm{Ca}$ and $\mathrm{Fe}$ compared to those harvested from Al Dhaid (Rao, 2016). These findings have significant implications, especially for introduction of quinoa in highly saline areas, therefore detailed investigations are recommended for confirmation.

\section{AGRONOMIC TRIALS AND CULTIVATION}

In the FAO's Regional Project TCP/RAB/3403, 11 quinoa cultivars were evaluated for seed yield potential in seven of 


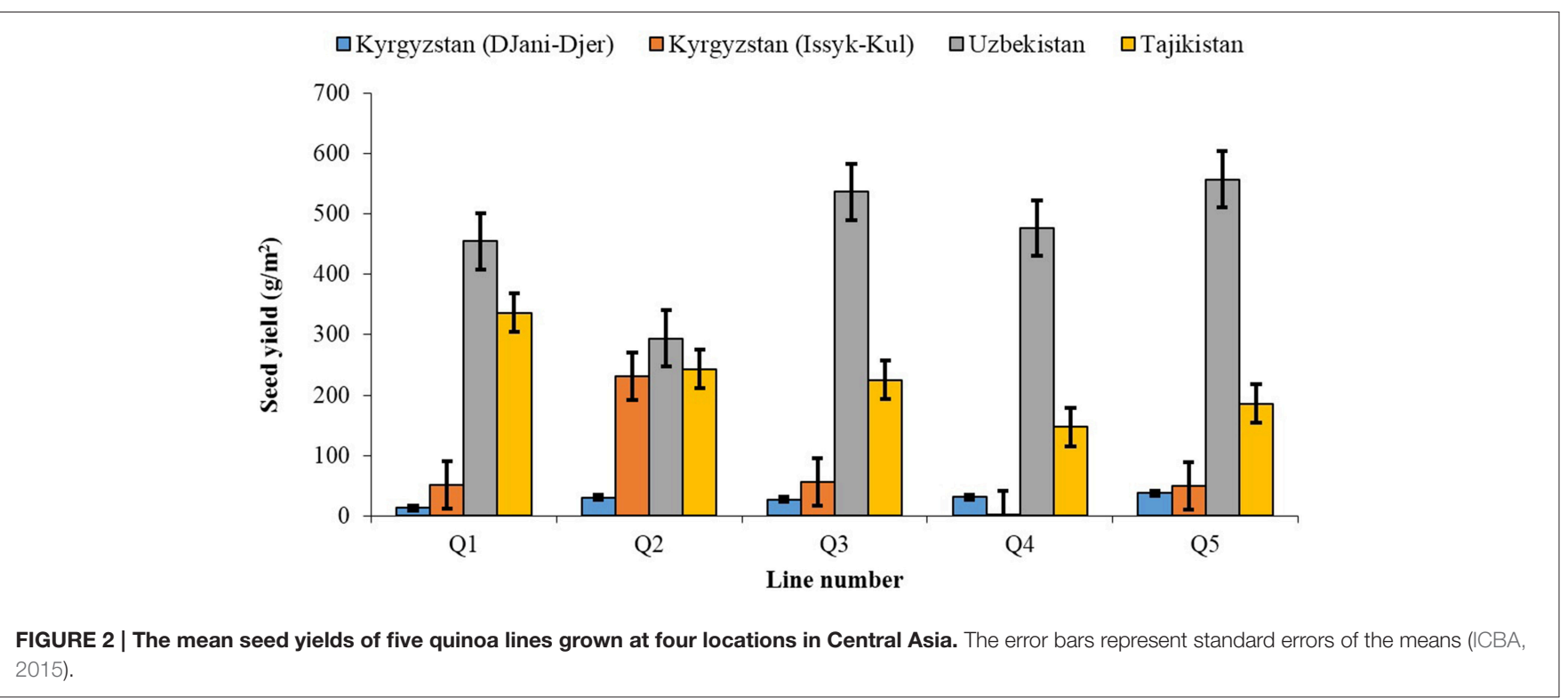

FIGURE 2 | The mean seed yields of five quinoa lines grown at four locations in Central Asia. The error bars represent standard errors of the means (ICBA, 2015).

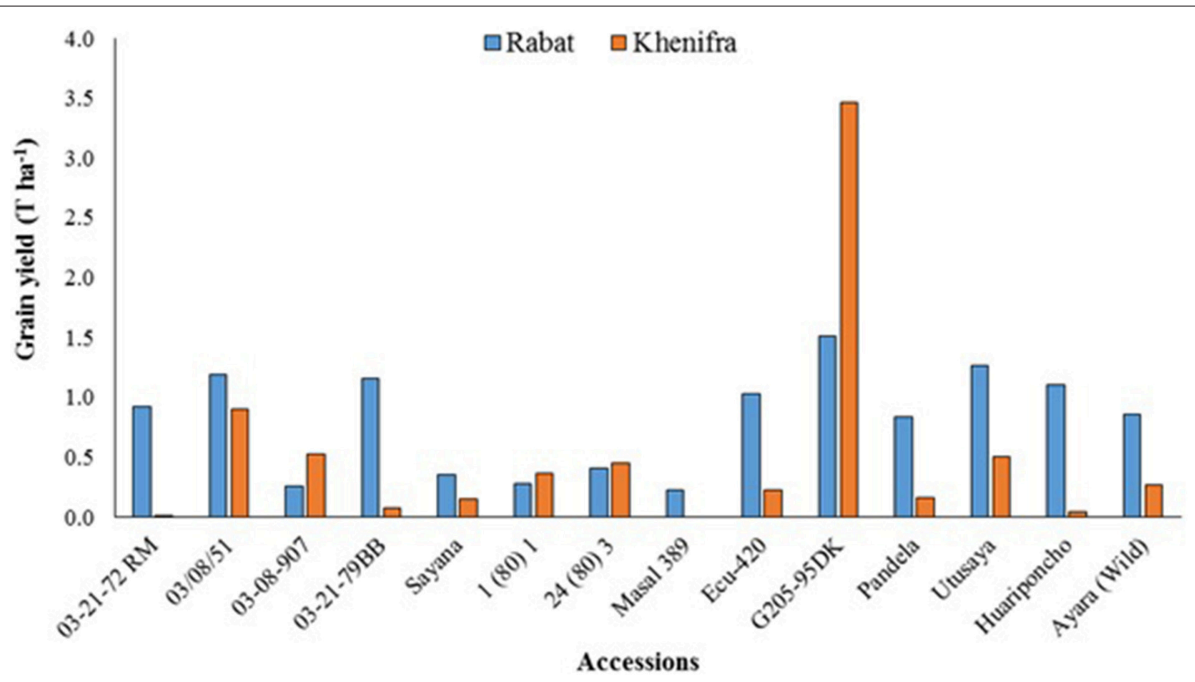

FIGURE 3 | Quinoa grain yields (T ha-1) under high altitude (Khenifra) and coastal (Rabat) conditions in 2000-01 season (Benlhabib et al., 2015).

the eight participating countries (Egypt, Iraq, Iran, Lebanon, Mauritania, Sudan and Yemen) during January-June 2014. Large yield variations per hectare were observed across locations within and between countries, the mean seed yields ranging from $0.11-0.96 \mathrm{t} \mathrm{ha}^{-1}$ in Iraq, $0.24-1.90 \mathrm{t} \mathrm{ha}^{-1}$ in Yemen, 0.41-3.87 $\mathrm{t} \mathrm{ha}^{-1}$ in Egypt, 1.50-7.50 $\mathrm{t} \mathrm{ha}^{-1}$ in Lebanon, 0.16$1.56 \mathrm{t} \mathrm{ha}^{-1}$ in Iran, and $0.03-0.23 \mathrm{t} \mathrm{ha}^{-1}$ in Mauritania. The highest yield $\left(7.50 \mathrm{t} \mathrm{ha}^{-1}\right)$ was recorded in Lebanon, followed by Egypt $\left(3.87 \mathrm{t} \mathrm{ha}^{-1}\right)$, while the lowest yield was recorded in Mauritania (0.23 $\mathrm{t} \mathrm{ha}^{-1}$ ) (Dost, 2015). A comparison of the cultivars across the countries/locations revealed very interesting and consistent patterns of performance that resulted in the identification of several potential/suitable varieties for the countries. The cultivars Q12 and Q21 exhibited outstanding performance in almost all the countries of evaluation, while Q29, Q18, Q19, Q22, Q26, and Q27 produced superior yields as compared to other cultivars at all locations/countries except Iran. Convinced by quinoa's performance, one private agriculture company in Egypt and a farmer in Lebanon planted quinoa on a large-scale (12 hectares) with a plan to further extend the production together with improvement in capacity of farmers and generating market demand. However, private sector involvement in quinoa production will mainly depend on the conducive policies of the governments, allocation of financial and human resources for research and development of new varieties along with the improvement in the production technologies, processing and utilization within the region. 


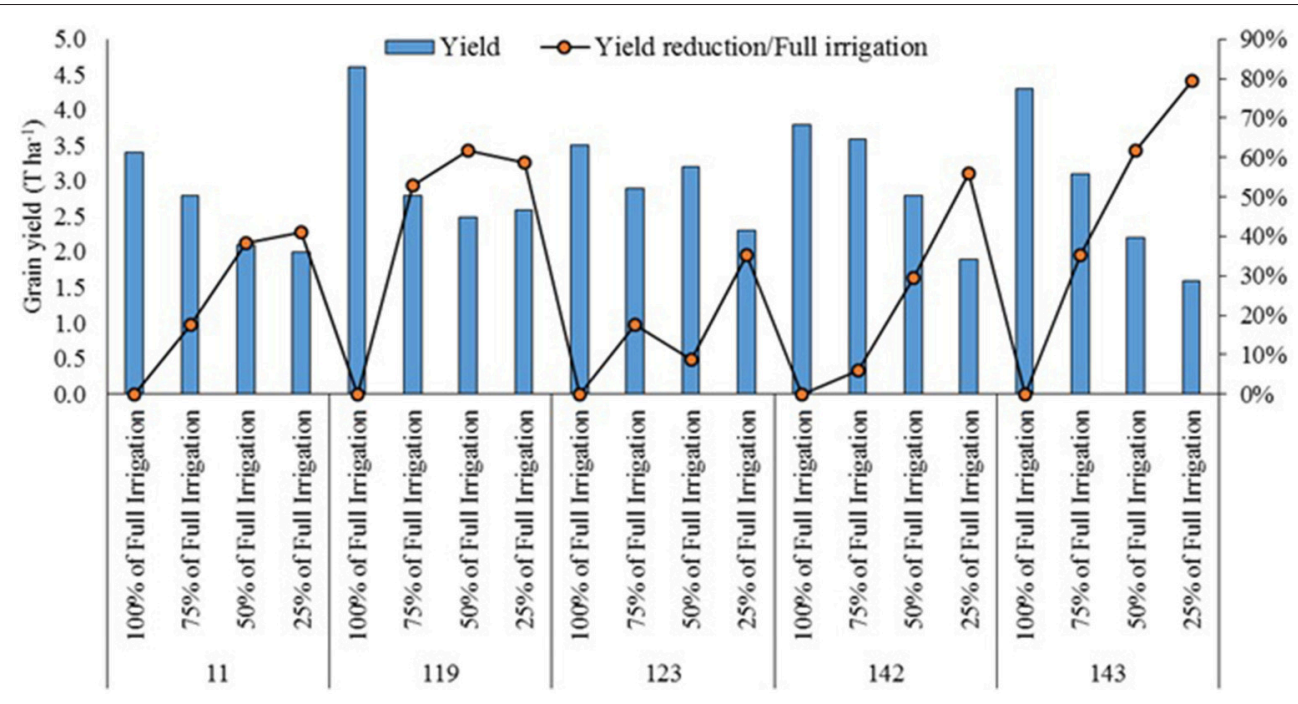

FIGURE 4 | Responses of 5 quinoa lines to different irrigation levels in terms of yield (Filali, 2011).

\section{CROPPING PRACTICES OF QUINOA PRODUCTION}

\section{Deficit Irrigation}

In quinoa, deficit irrigation (DI) strategy has been widely investigated as a valuable and sustainable production strategy in regions where intra-seasonal dry spells are of considerable importance (Garcia et al., 2003; Geerts et al., 2008). By limiting water applications to drought sensitive growth stages, the practice aims to maximize water productivity and to stabilize, rather than maximize yields (Geerts and Raes, 2009). Several experiments were conducted at IAV-CHA during two growing seasons 2010 and 2011 in order to evaluate the effect of deficit irrigation with treated wastewater (Hirich et al., 2012a,b,c, 2013). During the first season (2010), the response of the line D0708 with six deficit irrigation treatments (during vegetative growth, flowering, grain filling, during both vegetative growth and flowering stage, alternating with $100 \%$ of full irrigation as non-stress condition, and $50 \%$ of full irrigation as water deficit condition) were studied. During the second season (2011), two lines (D0708 and QM1113) were studied with six other irrigation treatments (rainfed, 0, 25, 50,75 , and $100 \%$ of full irrigation) only during the vegetative growth stage, while in the remaining stages, full irrigation was provided except for the rainfed treatment. The results clearly showed that the highest yields were obtained in the fully irrigated treatment followed by the treatment stressed during vegetative growth stage. When subjected to deficit irrigation during flowering, grain filling, during both vegetative growth and flowering, and during all crop stages, a yield reduction of $32,37,46$, and $50 \%$ was recorded respectively, in comparison with the control (full irrigation). The finding indicates that applying deficit irrigation during vegetative growth stage results in increased root to shoot ratio. Early water deficit induced root development and full irrigation applied during flowering and grain filling allowed the plants to produce more biomass due to the well-developed root system, possibly leading to higher levels of water and nutrient uptake. The increased yield observed in the treatment stressed during vegetative growth stage is probably due to maximized crop water productivity (CWP), which further allowed up to $19 \%$ water saving compared to full irrigation.

Statistical analysis carried out on grain yield of quinoa in response to several degrees of water stress applied during the vegetative growth stage revealed highly significant differences among the treatments $(p<0.001)$. The results clearly showed that line DO708 showed the highest grain yield compared to QM1113. While applying $50 \%$ of full irrigation during the vegetative growth stage resulted in 10 and $8 \%$ increase in seed yield in DO708 and QM1113 respectively compared to control (fully irrigated), applying $25 \%$ of full irrigation caused a yield reduction of 31 and 38\% (in DO708 and QM1113 respectively, compared to the control). It is obvious that CWP is maximized under 50\% deficit irrigation during the vegetative growth stage in both the lines.

Results from field trials conducted in sandy soil during two growing seasons (2011 and 2012) at the Experimental Station of Cadi Ayyad University located in $70 \mathrm{~km}$ south West Marrakech (Morocco) and also under rainfed condition in a farmer's field at Tnin Bouchan, about $250 \mathrm{~km}$ away from Agadir indicated that deficit irrigation (50 and 33\% of full irrigation) affected quinoa performance and resulted in seed yield reduction of $15.8,30.1 \%$ respectively in the first season (2011) and by 15.2 , $41.5 \%$ respectively, in the second season (2012) in comparison with full irrigation. Under rainfed conditions, seed yields were reduced by 62.1 and $59.3 \%$ in the 2011 and 2012 growing seasons, respectively compared to full irrigation. CWP was maximized in the treatment receiving 50\% of full irrigation (Fghire et al., 2013).

\section{Organic Amendment}

One of the most important factors which limits crop growth and production is water scarcity. As already discussed, studies show that deficit irrigation is a judicious strategy to increase water use efficiency and CWP in water-scarce environments. Organic 
amendments, on the other hand, improve the water holding capacity of soils and combing them with deficit irrigation can be a practical solution for sustainable yields as the negative effect of water scarcity can be compensated through the increased water holding capacity of the soils and there by improved availability of water and nutrients to the plants. In line with this, a study was conducted in at IAV-CHA to evaluate the combined effect of organic amendment (compost) and deficit irrigation using treated wastewater on productivity of the quinoa line (D0708) during October 2011 to March 2012 (Hirich et al., 2013, 2014a). Three levels of organic amendments $\left(0,5\right.$, and $\left.10 \mathrm{t} \mathrm{ha}^{-1}\right)$ combined with two levels of deficit irrigation (50 and 100\% of full irrigation) were studied. While the highest seed yield (66.3 $\mathrm{g} \mathrm{plant}^{-1}$ ) was recorded when quinoa was subjected to full irrigation and received $10 \mathrm{t} \mathrm{ha}^{-1}$ of compost, yield was low under water deficit conditions without organic amendment (Figure 5). The results indicated that organic amendment of 10 and $5 \mathrm{t} \mathrm{ha}^{-1}$ increased grain yield by 16 and $3 \%$ respectively, under full irrigation conditions, and by 18 and $13 \%$ under deficit irrigation (respectively). It can be concluded that organic amendment improve quinoa yields significantly under deficit irrigation conditions by improving the water holding capacity of the soils and the access to water and nutrients by the plants. Combining deficit irrigation and organic amendment also allowed maximizing the CWP.

\section{Quinoa Fertilization}

The combined effect of water stress and nitrogen application was studied on a quinoa line (D0708) in a field trial laid out in split-plot design at IAV-CHA, during the year 2013 (Supplementary Figures 2, 4). The treatments included four levels of water stress $(25,50,75$, and $100 \%$ of full irrigation) and seven different rates of nitrogen $(0,40,80,120,160,200$, and $240 \mathrm{~kg} \mathrm{ha}^{-1}$ ) application. The results suggested that seed yield increased with increasing nitrogen supply, though the response varied with the level of water stress (Figure 6). The yield was highest in the $50 \%$ of full irrigation treatment with $240 \mathrm{~kg} \mathrm{ha}^{-1}$ of nitrogen. CWP increased with higher supply of nitrogen and the degree of water stress, the value being highest in the most stressed treatment (25\% of full irrigation) and $240 \mathrm{~kg} \mathrm{ha}^{-1}$ of nitrogen and lowest with the full irrigation without nitrogen supply (Hirich, 2014).

In a field trial conducted in Wadi El-Natroon region, Beheira Governorate, Egypt during 2008/2009 and 2009/2010 winter seasons, Shams (2011) studied different rates of nitrogen fertilization $\left(0,90,180,270\right.$, and $\left.360 \mathrm{Kg} \mathrm{N} \mathrm{ha}{ }^{-1}\right)$ for improvement of growth and yield in sandy soils. High nitrogen fertilizer rate significantly increased yield during both the seasons. However, nitrogen use efficiency has reduced with increased rate of nitrogen application. In a pot experiment with two quinoa lines (Quinoa-52 and Quinoa-37) and two commercial varieties (Titicaca and Puno). Lavini et al. (2014) studied the effect of five rates of nitrogen application $(0,50,100$, 150 , and $200 \mathrm{mg} \mathrm{kg}^{-1}$ of soil). The results showed that both the lines responded similarly to the application of nitrogen and yield has improved significantly with increased nitrogen rate.

In studies conducted by Gomaa (2013) in Egypt, quinoa plants were fertilized with ammonium nitrate $(34 \% \mathrm{~N})$ at 0,120 ,
238, $357 \mathrm{~kg} \mathrm{ha}^{-1}$ in combination with nitrobin (biofertilizer) or calcium super phosphate $\left(15.5 \% \mathrm{P}_{2} \mathrm{O}_{5}\right)$ at $0,120,238,357 \mathrm{~kg}$ $\mathrm{ha}^{-1}$ in combination of phosphorin (biofertilizer). The plants performed the best in the treatment receiving $238 \mathrm{~kg} \mathrm{ha}^{-1}$ of ammonium nitrate in combination with nitrobin.

\section{Use of Saline Water}

A pot experiment was carried out at the IAV-CHA to evaluate the response of quinoa (line D0708) to different levels of irrigation

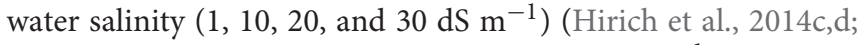
Lavini et al., 2014). Irrigation with water at $10 \mathrm{dS} \mathrm{m}^{-1}$ salinity has not affected seed yield significantly and yield reduction was only

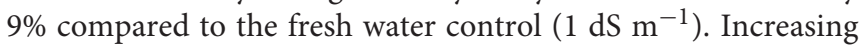
salinity to $20 \mathrm{dS} \mathrm{m}^{-1}$ and then to $30 \mathrm{dS} \mathrm{m}^{-1}$ resulted in a significant reduction in seed yield (24 and 34\%, respectively, compared to the control. Contrarily, saline water irrigation had positive effect on CWP-the most efficient treatment being the most salt-stressed treatment $\left(30 \mathrm{dS} \mathrm{m}{ }^{-1}\right)$, which produced $0.8 \mathrm{~kg}$ for $1 \mathrm{~m}^{3}$ of water and the lowest being the control $\left(0.6 \mathrm{~kg} \mathrm{~m}^{-1}\right)$ $(p<0.001)$. This difference in terms of CWP can be explained in terms of the difference in water uptake which was higher in the fresh water treatment and lower in the saline treatments.

Three lines of quinoa (QM1113, QS0938, D0708) cultivated in open field were irrigated with treated wastewater with different levels of salinity in order to assess the impact on yield (El Youssfi et al., 2012). Significant differences were found in the performance of the lines-QM1113 being the most productive with an average grain yield of $6.92 \mathrm{t} \mathrm{ha}^{-1}$ at $6 \mathrm{dS} \mathrm{m}^{-1}$ salinity followed by D0708 with an average yield of $5.65 \mathrm{t} \mathrm{ha}^{-1}$ at 3 $\mathrm{dS} \mathrm{m}^{-1}$. The findings indicated that quinoa yields increase with

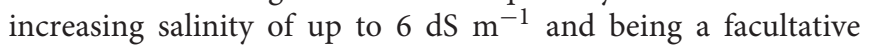
halophyte, salinity promotes growth but up to a certain threshold, beyond which growth and productivity start to be negatively affected. Results from these studies differ with those of Rao (2016) from on-farm trials where seed yields were maximum despite irrigation with highly saline water (15-18 $\left.\mathrm{dS} \mathrm{m}^{-1}\right)$; and those from field experiments in Turkey where salinity stress of up to $40 \mathrm{dS} \mathrm{m} \mathrm{m}^{-1}$ did not interfere with seed and biomass yields significantly (Yazar et al., 2015); or of Razzaghi et al. (2011) who showed an yield reduction of $50 \%$ with saline water of $40 \mathrm{dS} \mathrm{m}^{-1}$ compared with fresh water.

Simple seed germination tests were conducted at ICBA, Dubai to study the role of Bontera ${ }^{\mathrm{TM}}$ (Biofertilizer and humic acid) in enhancing seed germination and in mitigating the negative impact of salinity (Figure 7). Different levels of sodium chloride of up to $50 \%$ seawater salinity $(1.2 \% \mathrm{NaCl})$ were tested in combination with different dilutions of Bontera ${ }^{\mathrm{TM}}$ (ranging from 1:20 to 1:500). The results of these tests showed that salinity in the growth medium inhibited seed germination to the extent that at $1.6 \% \mathrm{NaCl}$ seed germination did not occur. At lower concentrations of Bontera $^{\mathrm{TM}}$ (dilutions of 1:500 and above) significantly enhanced seed germination and seedling growth, while higher concentrations (1:300) were inhibitory (Gill et al., 2016). Under field conditions, foliar application of Bontera ${ }^{\mathrm{TM}}$ consistently showed up to $28 \%$ improvement in grain yield. It was concluded that application of Bontera ${ }^{\mathrm{TM}}$ at $1-21 \mathrm{ha}^{-1}$ could reduce the chemical fertilizers application by $25 \%$. Foliar application of Bontera ${ }^{\mathrm{TM}}$ in combination with two irrigation 


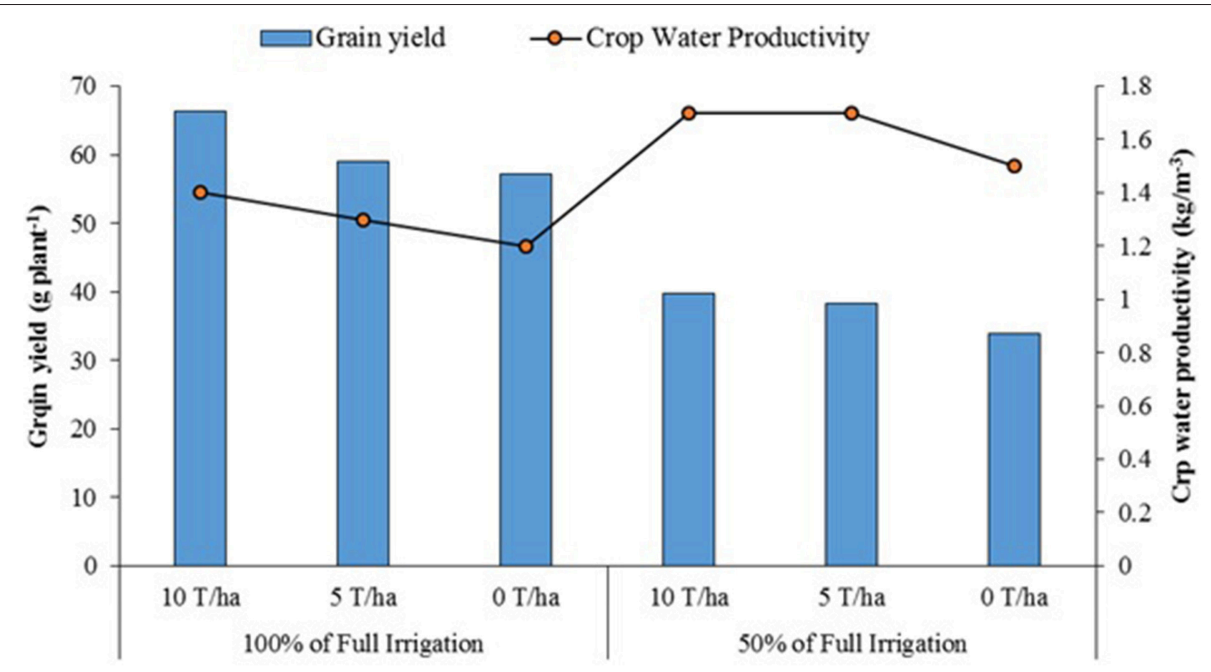

FIGURE 5 | Combined effect of organic amendment and deficit irrigation on quinoa yield and water productivity (Hirich et al., 2014a).

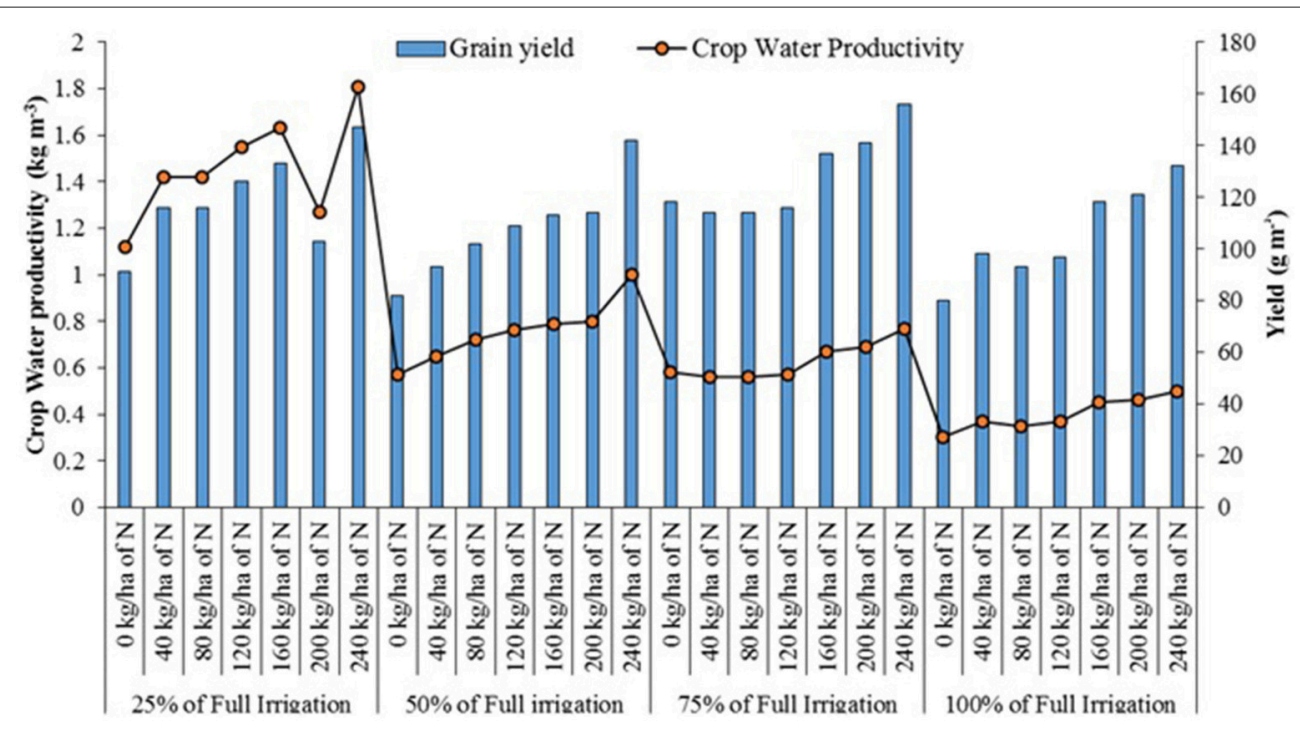

FIGURE 6 | Combined effect of water stress and N fertilization on quinoa yield and water productivity (Hirich, 2014).

regimes (100\% ET and 50\% ET) showed greater impact at 50\% ET suggesting a mitigating effect for stresses like water deficiency and drought (Gill et al., 2016).

\section{Sowing Dates}

Hirich et al. (2014b) carried out a series of field trials at IAV-CHA (Supplementary Figure 3), to investigate the effects of 10 sowing dates-from 1st November to 15th March with 15-day intervals, on quinoa performance. The study showed that sowing dates affected growth and productivity obviously due to differences in temperature, precipitation and radiation over time (Figure 8). Seed and dry matter yields were highest when quinoa was sown in November and early December. Reduced yield during the late sowing dates was explained by delay in germination due to prevailing low temperatures and occurrence of downy mildew during February and March triggered by high air humidity. The length of growing period increased from November to January and decreased from January to March. The longest growing period was when sown on 1st of January and the shortest was when sown on 15th of March.

\section{CONSTRAINTS FOR SCALING UP}

Poor germination and crop establishment are particular problems likely to be encountered with quinoa especially in saline or other marginal environments. In the trials conducted in the UAE and the FAO project countries, significantly high variability was observed in the yields of the lines/varieties. Therefore, understanding of the influence of edaphic and climatic factors on productivity in different locations is important. There 


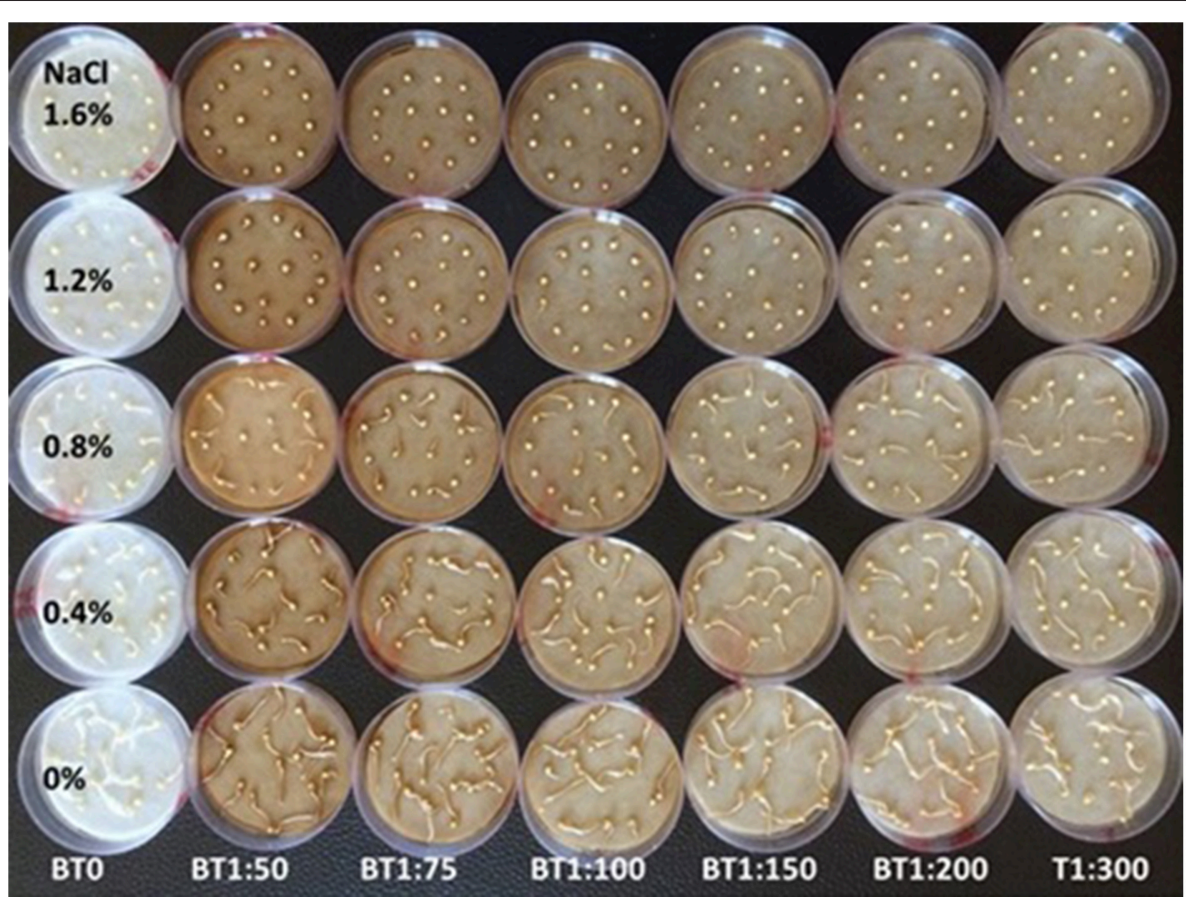

FIGURE 7 | Seed germination and seedling growth after 2 days- under different $\mathrm{NaCl}$ \& Bontera solution Concentrations (Gill et al., 2016).

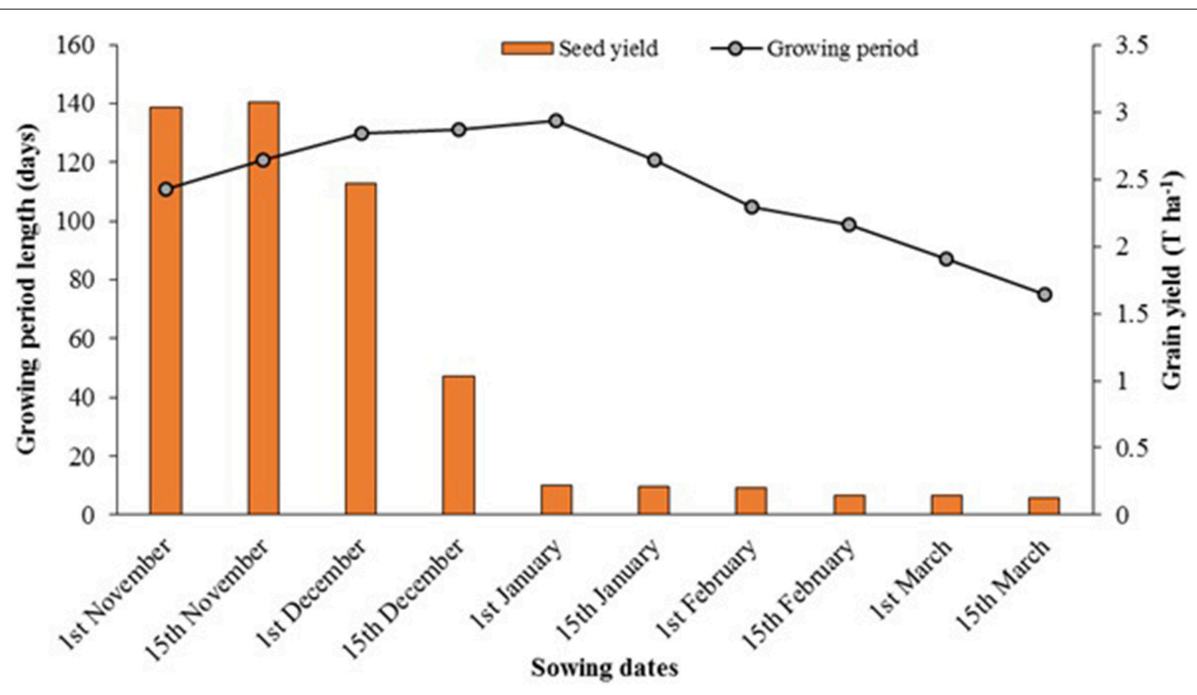

FIGURE 8 | Effect of sowing dates on quinoa yield and growing period length (Hirich et al., 2014b).

is also considerable knowledge gap regarding quinoa pests and diseases, particularly in areas outside its traditional growing regions. Some of the wild relatives such as Chonopodium murale and C. album are common weeds in several of the countries where quinoa is introduced and they possibly harbor a number of biotic stressors that can easily migrate onto cultivated species. Introduction and scaling-up of novel crops such as quinoa to non-traditional environments also requires the study and establishment of the entire chain and building capacity of the researchers and the farmers in the basics of production and harvesting, storage and processing technologies (Rao, 2016).

\section{CONCLUSIONS AND RECOMMENDATIONS}

In recent years, quinoa is receiving significant attention as a nutritively rich multi-purpose agro-industrial crop that can 
thrive in extreme soil and climatic conditions. Faced with the challenge of increasing the production of high-quality food to feed the growing population, quinoa offers an excellent alternative to ensure food and nutrition security in marginal environments in the MENA as well as other regions across the globe. In the MENA region, while many countries have recently initiated work on quinoa, Morocco and the UAE have made significant advances toward introducing the crop in the local production systems. In Morocco, IAV HASSAN II Institute has been studying quinoa since 2000 as a drought and salinitytolerant alternative crop to contribute to the food and nutrition security of people in the mountainous areas. As part of this program, quinoa has already been introduced to a dozen sites across the country and each year, the number of quinoa growers' is increasing all over the regions, especially Oujda, Benslimane, Fes, Boulmane, and Marrakech. However, quinoa yields over the years remained unpredictable and very low, averaging between 1.2 and $1.4 \mathrm{t} \mathrm{ha}^{-1}$, while the maximum attainable yield can be up to $8-10 \mathrm{t} \mathrm{ha}^{-1}$. A range of factors such as the choice of cultivars, optimal sowing date and nutrient management were suggested to affect the production. Constraints which still need to be overcome include crop stand establishment, sensitivity to high temperatures and salinity, weed control and saponin removal. Furthermore, there is also a need to design a product marketing strategy and raise awareness among farmers and the government agencies about quinoa's potential as a stress-tolerant alternative crop for marginal environments.

In the UAE, ICBA has been investigating quinoa since 2006 as an alternative crop for salt-affected areas. Significant progress has been made in identifying salt-tolerant lines combined with high yield potential. These lines are now being further evaluated for their yield potential in several countries including Uzbekistan, Tajikistan and Kyrgyzstan in Central Asia (through the project "Cross-regional partnerships for improving food and nutritional security in marginal environments of Central Asia" funded by the Islamic Development Bank); and Yemen, Jordan, and Egypt in the MENA region [as part of the collaborative project "Adaptation to climate change in West Asia and North Africa marginal environments through sustainable crop and livestock diversification" funded by the International Fund for Agricultural Development (IFAD), OPEC Fund for International Development (OFID)]. As with any other new crop, one of the key factors for successful introduction and establishment of quinoa under the novel climates will be the identification of appropriate planting material. It is therefore important to study the adaptation and yield potential of several quinoa genotypes from different provenances to select the most promising genotypes suitable for the local agroclimatic conditions. Information on these aspects as well as the economic assessment of the profitability of quinoa cultivation is essential, especially when planted under sub-optimal growth conditions with low-quality water in the targeted countries. ICBA's future research on quinoa focuses on developing best practices in relation to the production, management under diverse farming systems and agro-ecological regions, and on utilization and marketing of the produce, besides fostering knowledge transfer, strengthening competencies and exchange of experiences among quinoa researchers within the MENA and Central Asia regions.

It is well known that climate change is rapidly degrading the conditions of crop production. Salinization and aridity are forecasted to increase in most parts of the world, especially in the MENA region. As a consequence, new stress-tolerant genotypes of the existing crops or new and alternative crops or species must be identified and used for future food security. Stresstolerant crops such as quinoa offer major opportunities due to their comparative advantage over the staple food crops in terms of tolerance to harsh growing conditions. The results presented in this paper have indeed shown that quinoa maintains productivity in rather poor soils and under water stress conditions and high salinity. Moreover, quinoa seeds are an exceptionally nutritious food source, owing to their high protein content with all essential amino acids, lack of gluten, high mineral content (e.g., $\mathrm{Ca}$, $\mathrm{Mg}, \mathrm{Fe}$ ), and health-promoting compounds such as flavonoids. Higher yield do not guarantee quinoa's success in the region and to be successful it must fit in the current cropping patterns, farming systems and prove its worth in rigorous and properly designed on-farm trials in marginal lands affected by salinity and alkalinity and in the areas where the majority of the food crops could not be produced economically. For scaling up and large scale adoption by the farmers in the region, creating opportunities for marketing the produce are also vital. Quinoa being a new crop to the region, more efforts will be needed to create awareness for its incorporation in the social, cultural and dietary habits to increase the market demand besides strengthening the efforts to improve yields combined with nutritional quality, production, harvesting and post-harvesting practices along the value chain.

\section{AUTHOR CONTRIBUTIONS}

$\mathrm{RC}$ was the corresponding author collecting data and results as well as editing results related to North Africa region. NR was providing research results conducted by ICBA. He was reviewing the paper and editing the English writing. AH contributed to this paper by designing the figures, commenting the data and compiling all gathered results. He was also revising the formatting according to Journal Author guideline. KT provided results of research undertaken in Central Asia. Butt has been involved with Dr. NR in several experiment on quinoa. SG provided results related to seed treatment in order to improve crop tolerance to salinity. AA provided results related to seed treatment in order to improve crop tolerance to salinity.

\section{SUPPLEMENTARY MATERIAL}

The Supplementary Material for this article can be found online at: http://journal.frontiersin.org/article/10.3389/fpls.2016. 00346

Supplementary Figure 1 | Quinoa panicle variability in terms of color and panicle volume.

Supplementary Figure 2 | Trial on combined impacts of water stress and nitrogen application carried out in Agadir, Morocco. 
Supplementary Figure 3 | Sowing date trial carried out in Agadir, Morocco.

Supplementary Figure 4 | Quinoa harvest within field trial on combined impacts of water stress and nitrogen application carried out in Agadir, Morocco.

\section{REFERENCES}

Ashraf, M. (2014). Stress-induced changes in wheat grain composition and quality. Crc. Rev. Food. Sci. Nutr. 54, 1576-1583. doi: 10.1080/10408398.2011.644354

Bazile, D., Bertero, D., and Nieto, C. (2015). State of the Art Report on Quinoa around the World in 2013. Rome: Food and Agriculture Organization of the United Nations (FAO) \& CIRAD (Centre de coopération internationale en recherche agronomique pour le développement).

Benlhabib, O., Jacobsen, S. E., Jellen, E. N., Maughan, P. J., and Choukr-Allah, R. (2015). "Status of quinoa production and research in Morocco," in State of the Art Report on Quinoa around the World in 2013, eds D. Bazile, D. Bertero, and C. Nieto (Rome: Food and Agriculture Organization of the United Nations), 178-491.

Dost, M. (2015). "Field evaluation results across locations and identification of suitable QUINOA varieties," in Wrap up Workshop of Regional Quinoa Project (TCP/RAB/3403-FAO) (Rome: Food and Agriculture Organization of the United Nations).

El Youssfi, L., Choukr-Allah, R., Zaafrani, M., Mediouni, T., Ba Samba, M., and Hirich, A. (2012). Effect of domestic treated wastewater use on three varieties of Quinoa (Chenopodium quinoa) under semi arid conditions. World Acad. Sci. Eng. Technol. 68, 306-309.

Fghire, R., Oudou, I. A., Filali, K., Benlhabib, O., and Wahbi, S. (2013). "Deficit irrigation and fertilization impact on quinoa water and yield productions," in International Conference on Sustainable Water Use for Securing Food Production in the Mediterranean Region under Changing Climate, ed R. Choukr-Allah. (Agadir: SWUP-MED project), 409-416.

Filali, K. (2011). Caractérisation et Évaluation du Rendement de Lignées de Quinoa (Chenopodium quinoa) dans la Région de Rhamna. Rabat: Diplôme de Master en Biotechnologies et Amélioration Génétique des Productions Agricoles, Institut Agronomique et Vétérinaire Hassan II, Morocco.

Garcia, M., Raes, D., and Jacobsen, S.-E. (2003). Evapotranspiration analysis and irrigation requirements of quinoa (Chenopodium quinoa) in the Bolivian highlands. Agric. Water Manage. 60, 119-134. doi: 10.1016/S03783774(02)00162-2

Geerts, S., and Raes, D. (2009). Deficit irrigation as an on-farm strategy to maximize crop water productivity in dry areas. Agric. Water Manage. 96, 1275-1284. doi: 10.1016/j.agwat.2009.04.009

Geerts, S., Raes, D., Garcia, M., Vacher, J., Mamani, R., Mendoza, J., et al. (2008). Introducing deficit irrigation to stabilize yields of quinoa (Chenopodium quinoa Willd.). Eur. J. Agron. 28, 427-436. doi: 10.1016/j.eja.2007.11.008

Gill, S., Alshankiti, A., and Shahid, S. A. (2016). "Evaluation of BonteraTM microbial soil enhancer for improving crop production and water saving in sandy soils," in Joint Publication of ICBA and Flozyme, ed S. Gill (Dubai: International Center for Biosaline Agriculture).

Gomaa, E. F. (2013). Effect of Nitrogen, Phosphorus and biofertilizers on quinoa plant (Chenopodium quinoa). J. Appl. Sci. Res. 9, 5210-5222.

Hirich, A. (2013). Using deficit irrigation with treated wastewater to improve crop water productivity of sweet corn, chickpea, faba bean and quinoa. Moroccan Rev. Agron. Vet. Sci. 2, 15-22.

Hirich, A. (2014). Effects of Deficit Irrigation using Treated Wastewater and Irrigation with Saline Water on Legumes, Corn and Quinoa Crops. Ph.D., thesis, Hassan II Institue of Agronomy and Veterinary Medicine, Morocco.

Hirich, A., Choukr-Allah, R., and Jacobsen, S. E. (2014a). Deficit irrigation and organic compost improve growth and yield of quinoa and pea. J. Agron. Crop. Sci. 200, 390-398. doi: 10.1111/jac.12073

Hirich, A., Choukr-Allah, R., and Jacobsen, S. E. (2014b). Quinoa in Morocco - effect of sowing dates on development and yield. J. Agron. Crop. Sci. 200, 371-377. doi: 10.1111/jac.12071

Hirich, A., Choukr-Allah, R., and Jacobsen, S.-E. (2013). The combined effect of deficit irrigation by treated wastewater and organic amendment on quinoa (Chenopodium quinoa Willd.) productivity. Desal Water Treat 52, 2208-2213. doi: $10.1080 / 19443994.2013 .777944$
Supplementary Figure 5 | Field trial about responses of seven quinoa accessions to several irrigation levels.

Supplementary Figure 6 | Genetic and morphological variability of several quinoa accessions.

Hirich, A., Choukr-Allah, R., Jacobsen, S.-E., and Benlhabib, O. (2012a). Could quinoa be an alternative crop of wheat in the Mediterranean region: case of Morocco? Les Notes d'Alerte du CIHEAM 86, 1-8.

Hirich, A., Choukr-Allah, R., Jacobsen, S.-E., El Yousfi, L., and El Omari, H. (2012b). Using deficit irrigation with treated wastewater in the production of quinoa (Chenopodium quinoa Willd.) in Morocco. Rev. Científica UDO Agric. 12, 570-583.

Hirich, A., Choukr-Allah, R., Jelloul, A., and Jacobsen, S.-E. (2014c). Quinoa (Chenopodium quinoa Willd.) seedling, water uptake and yield responses to irrigation water salinity. Acta Hort. 1054, 145-152. doi: 10.17660/ActaHortic.2014.1054.16

Hirich, A., Choukr-Allah, R., Ragab, R., Jacobsen, S.-E., El Youssfi, L., and El Omari, H. (2012c). The SALTMED model calibration and validation using field data from Morocco. J. Mat. Environ. Sci. 3, 342-359.

Hirich, A., Jelloul, A., Choukr-Allah, R., and Jacobsen, S. E. (2014d). Saline water irrigation of quinoa and chickpea: seedling rate, stomatal conductance and yield responses. J. Agron. Crop. Sci. 200, 378-389. doi: 10.1111/jac.12072

ICBA (2015). ICBA Annual Report 2015. Dubai: International Center for Biosaline Agriculture.

Jacobsen, S. E. (2003). The worldwide potential for quinoa (Chenopodium quinoa Willd.). Food Rev. Int. 19, 167-177. doi: 10.1081/FRI-120018883

Jacobsen, S. E., Mujica, A., and Jensen, C. R. (2003). The resistance of Quinoa (Chenopodium quinoa Willd.) to adverse abiotic factors. Food Rev. Int. 19, 99-109. doi: 10.1081/FRI-120018872

Lavini, A., Pulvento, C., D’Andria, R., Riccardi, M., Choukr-Allah, R., Belhabib, O., et al. (2014). Quinoa's potential in the Mediterranean region. J. Agron. Crop Sci. 200, 344-360. doi: 10.1111/jac.12069

Rao, N. K. (2016). “Quinoa: a future-proof crop for climate smart agriculture," in Global Forum for Innovations in Agriculture-2016, ed R. Choukr-Allah (Abu Dhabi).

Rao, N. K., Rahman, K. U., and Ismail, S. (2013). "Quinoa: prospects as an alternative crop for salt-affected areas," in Book of Abstracts, 3rd International Conference on Neglected and Under-utilized Species (NUS) for Food Secure Africa, eds R. A. Hall, P. Rudebjer, and S. Padulosi (Rome: Bioversity International), 95.

Rao, N. K., and Shahid, M. (2012). Quinoa-a promising new crop for the Arabian Peninsula. American-Eurasian J. Agric. Environ. Sci. 12, 1350-1355.

Razzaghi, F., Ahmadi, S. H., Adolf, V. I., Jensen, C. R., Jacobsen, S. E., and Andersen, M. N. (2011). Water relations and transpiration of Quinoa (Chenopodium quinoa Willd.) under salinity and soil drying. J. Agron. Crop Sci. 197, 348-360. doi: 10.1111/j.1439-037X.2011.00473.x

Repo-Carrasco, R., Espinoza, C., and Jacobsen, S.-E. (2003). Nutritional value and use of the andean crops quinoa (Chenopodium quinoa) and Kañiwa (Chenopodium pallidicaule). Food Rev. Int. 19, 179-189. doi: 10.1081/FRI120018884

Shams, A. (2011). Response of quinoa to nitrogen fertilizer rates under sandy soil conditions. Int J. Water Resour. Arid Environ. 1, 318-325.

Yazar, A., Incekaya, Ç., Sezen, S. M., and Jacobsen, S.-E. (2015). Saline water irrigation of quinoa (Chenopodium quinoa) under Mediterranean conditions. Crop Pasture Sci. 66, 993-1002. doi: 10.1071/CP14243

Conflict of Interest Statement: The authors declare that the research was conducted in the absence of any commercial or financial relationships that could be construed as a potential conflict of interest.

Copyright (c) 2016 Choukr-Allah, Rao, Hirich, Shahid, Alshankiti, Toderich, Gill and Butt. This is an open-access article distributed under the terms of the Creative Commons Attribution License (CC BY). The use, distribution or reproduction in other forums is permitted, provided the original author(s) or licensor are credited and that the original publication in this journal is cited, in accordance with accepted academic practice. No use, distribution or reproduction is permitted which does not comply with these terms. 


\section{OPEN ACCESS}

Edited by:

Antonio M. De Ron,

Misión Biológica de Galicia-CSIC,

Spain

Reviewed by:

Pedro Revilla,

Misión Biológica de Galicia-CSIC

Spain

Svetla Sofkova-Bobcheva, Maritsa Vegetable Crops Research Institute, Bulgaria

${ }^{*}$ Correspondence: Eric N. Jellen jellen@byu.edu

Specialty section:

This article was submitted to Crop Science and Horticulture, a section of the journal

Frontiers in Plant Science

Received: 30 May 2016 Accepted: 02 August 2016 Published: 17 August 2016

Citation:

Benlhabib O, Boujartani N, Maughan PJ, Jacobsen SE and Jellen EN (2016) Elevated Genetic

Diversity in an $F_{2: 6}$ Population of Quinoa (Chenopodium quinoa)

Developed through an Inter-ecotype Cross. Front. Plant Sci. 7:1222. doi: 10.3389/fp/s.2016.01222

\section{Elevated Genetic Diversity in an $\mathrm{F}_{2: 6}$ Population of Quinoa (Chenopodium quinoa) Developed through an Inter-ecotype Cross}

\author{
Ouafae Benlhabib ${ }^{1}$, Noura Boujartani', Peter J. Maughan², Sven E. Jacobsen ${ }^{3}$ and \\ Eric N. Jellen ${ }^{2 *}$
}

\begin{abstract}
${ }^{1}$ Departement de Production, Protection et Biotechnologies Vegetales, Agronomic and Veterinary Institute Hassan II, Rabat, Morocco, ${ }^{2}$ Department of Plant and Wildlife Sciences, Brigham Young University, Provo, UT, USA, ${ }^{3}$ Department of Plant and Environmental Sciences, Faculty of Life Sciences, University of Copenhagen, Copenhagen, Denmark
\end{abstract}

Quinoa (Chenopodium quinoa) is a seed crop of the Andean highlands and Araucanian coastal regions of South America that has recently expanded in use and production beyond its native range. This is largely due to its superb nutritional value, consisting of protein that is rich in essential amino acids along with vitamins and minerals. Quinoa also presents a remarkable degree of tolerance to saline conditions, drought, and frost. The present study involved $72 \mathrm{~F}_{2: 6}$ recombinant-inbred lines and parents developed through hybridization between highland (0654) and coastal (NL-6) germplasm groups. The purpose was to characterize the quinoa germplasm developed, to assess the discriminating potential of 21 agro-morpho-phenological traits, and to evaluate the extent of genetic variability recovered through selfing. A vast amount of genetic variation was detected among the 72 lines evaluated for quantitative and qualitative traits. Impressive transgressive segregation was measured for seed yield (22.42 g/plant), while plant height and maturity had higher heritabilities (73 and 89\%, respectively). Other notable characters segregating in the population included panicle and stem color, panicle form, and resistance to downy mildew. In the Principal Component analysis, the first axis explained $74 \%$ of the total variation and was correlated to plant height, panicle size, stem diameter, biomass, mildew reaction, maturation, and seed yield; those traits are relevant discriminatory characters. Yield correlated positively with panicle length and biomass. Unweighted Pair Group Method with Arithmetic Mean-based cluster analysis identified three groups: one consisting of late, mildew-resistant, high-yielding lines; one having semi-late lines with intermediate yield and mildew susceptibility; and a third cluster consisting of early to semi-late accessions with low yield and mildew susceptibility. This study highlighted the extended diversity regenerated among the 72 accessions and helped to identify potentially adapted quinoa genotypes for production in the Moroccan coastal environment.

Keywords: quinoa, Chenopodium, downy mildew resistance, breeding, genetic diversity, Peronospora variabilis 


\section{INTRODUCTION}

Quinoa (Chenopodium quinoa) is an ancient Andean seed crop of exceptional nutritional, and in particular protein, quality (Koziol, 1992). Its cultivation has expanded within the past decade beyond its traditional range to more than 70 countries (Food Agriculture Organization of United Nations [FAO], 2012), although the Andean nations of Peru and Bolivia are still primary quinoa producers. Due to quinoa's exceptional nutritional value, its capacity for adaptation to diverse agro-ecological conditions and its high commercial value, quinoa is expected to play an essential role in the UN-FAO strategy to sustainably feed the world's growing population (Jacobsen et al., 2013).

Biologically, C. quinoa belongs to an allotetraploid $(2 n=4 x=36)$ complex found throughout the New World and whose root is the North American weed pitseed goosefoot (C. berlandieri). At least two other independent domestication events are presumed to have given rise to Mesoamerican vegetable and seed domesticates (C. berlandieri ssp. nuttaliae; Wilson and Heiser, 1979) as well as an extinct seed crop of the ancient cultures of eastern North America, C. berlandieri ssp. jonesianum (Smith and Yarnell, 2009; Kistler and Shapiro, 2011). After dispersing to the southeastern plains of South America, the weedy taxon of that region, avian goosefoot (C. hircinum), is presumed to have been brought into cultivation in the region of Lake Titicaca as C. quinoa and, at a later time, dispersed long-range to the Araucanian region on the Pacific slope in what is now Chile (Wilson, 1990; Bruno and Whitehead, 2003). Molecular genetic analyses have confirmed that the lowland Chilean coastal material is highly diverse (Fuentes et al., 2009), yet represents a branch that is separate from the even more variable quinoa germplasm of the High Andes (Christensen et al., 2007). The coastal Chilean germplasm includes genotypes that are highly resistant to heat and are day length insensitive - critical characters for quinoa breeders and agronomists seeking to expand quinoa's production into lowland subtropical and warm-season temperate environments around the world (Jacobsen et al., 2003).

The current work is part of a quinoa introduction and selection program initiated in 2000 in Morocco and involving Brigham Young University, the Institut Agronomique et Veterinaire Hassan II, and Copenhagen University of Denmark. Germplasm adapted to Moroccan production environments was to be identified in this long-term program through traditional breeding methodologies based upon the initial creation of highly diverse quinoa populations composed of the following: (1) in the first phase, introduction of internationally available cultivated quinoa varieties, landraces, and breeding lines; followed by, (2) introduction of breeding populations combining adaptive cultivated characteristics of lowland Chilean-origin $\times$ agronomically superior Andean Highland types; and then, (3) introduction of early-generation populations derived from disease-, pest-, and heat-resistant wild lowland $\times$ cultivated parent crosses. The primary objectives of this study, falling under phase 2 of the aforementioned project, were to (1) describe agro-morpho-phenological traits an $\mathrm{F}_{2: 6}$ population of recombinant-inbred lines (RIL) developed from an NL-6 (female, lowland) $\times 0654$ (male, highland) cross; (2) estimate the extent of genetic diversity present by the $\mathrm{F}_{6}$ generation in this population; and (3) estimate the discriminating potential and heritability of several of the evaluated traits.

\section{MATERIALS AND METHODS}

\section{Plant Material}

The quinoa population in this study was developed at BYU, Provo (USA), through a cross between the Dutch variety NL-6 (female) and Peruvian highland line 0654 (male) initially made by Alejandro Bonifacio, and was previously described in Maughan et al. (2012) as 'Pop39'. The NL-6 parent, which is derived from lowland Chilean germplasm, has yellow panicles, short stature, is early maturing, and is sufficiently heat-tolerant to allow for its cultivation at low elevations. Line 0654 is a highland Peruvian valley ecotype with red-purple panicles, late maturity, tall stature, and is heat susceptible. The population included in the current field evaluation consisted of $70 \mathrm{~F}_{2: 6}$ lines plus the two parents.

\section{Field Characterization and Statistical Analyses}

Characterization of Pop39 was performed on an experimental plot at the IAV Hassan II in Rabat, Morocco, near the laboratory to facilitate daily measurements of growth and phenology. The trial was sown indoors on January 29, transplanted to sandy loam soil in the field February 26-27, maintained through manual weeding, irrigated regularly as needed, and treated twice against aphids. No fungal control against mildew was applied. To protect against avian seed predation, an anti-sparrow net was used to cover the plots. The quinoa population was planted in two completely randomized blocks, with about 20 plants and five measurements per accession per block. Elementary plots were two rows of one-meter long and spaced $35 \mathrm{~cm}$ apart.

Sixteen quantitative and seven qualitative traits related to plant morphology, phenology and agronomic performance were measured. Quantitative measurements included seed diameter; plant height $($ at 60 days $=$ PH60, 75 days $=$ PH75, 90 days $=$ PH90, and at maturity $=$ PHM); panicle or inflorescence length (IL) and width (IW); sensitivity to downy mildew (SM); number of days to maturity (DM), and phenological stages and yield (GY) components, all of which were measured during the growing season and/or at harvest. Additional biomass data logged included main stem diameter (SD); root length (RL); aboveground fresh (FWA) and dry (DWA) weights; root fresh (FWR) and dry (DWR) weights; and overall biomass (BM). Qualitative traits used in the evaluation includes color of the seeds (ranged into a 1-10 scale by using classes of Photoshop color numerical codes), leaves (green/dark green) and stem (green/purple), and the panicle color (yellow/pink/orange/red/purple) and shape (globular versus amaranthiform). 
The evaluation of downy mildew (Peronospora variabilis) resistance (Choi et al., 2010) was performed in the laboratory on young leaves according to the inoculation method of Mhada et al. (2015). Pathogen development was evaluated every $48 \mathrm{~h}$ on three leaves per accession using a 0-5 notation scale where score 0 corresponds to no lesion, 1 to small and disperse lesions with less than $1 \mathrm{~mm}$ diameter and no sporulation on the lower side of the leaf, 2 to clearly individualized lesions increasing in number and size with a diameter between 0.5 and $1 \mathrm{~cm}$ without showing any sporulation on the lower side of the leaf, 3 to brown lesions, covering less than $50 \%$ of the leaf surface with a beginning of the sporulation at the lower side, 4 to lesions of larger size, covering more than $50 \%$ of the leaf area and 5 to lesions covering more than $91 \%$ of the leaf area, with a high sporulation rating on the lower and upper area.

Collected data were subjected to descriptive analyses, analysis of variance (ANCOVA) with two factors, the accessions as a fixed factor and the blocks as a random factor, and means comparisons using the Fisher test with XLSTAT software (XLSTAT-Pro 7.5). PAST software (v. 2.16, Hammer et al., 2001) was used to perform multivariate analyses to subtract the principal components which account for much of the variance, to compute the correlations matrix and to set up the dendrogram that gathers the $F_{2: 6}$ accessions into clusters using the Unweighted Pair Group Method with Arithmetic Mean (UPGMA) procedure (Sokal and Michener, 1958).

\section{RESULTS}

\section{Qualitative Traits}

The 72 studied lines represented a wide range of morphological variability that was reflected in seed and panicle coloration, plant and panicle size and shape, seed diameter, grain yield, maturity, and resistance to downy mildew (Figure 1). The population exhibited segregation for seed color, with $46 \%$ of the RIL's having white seeds and $22 \%$ having red seeds - the two main coloration patterns. The remaining lines were yellow (15\%), white-speckled red $(3 \%)$, yellow speckled with red $(7 \%)$, and light-yellow or orange ( $3 \%$ each).

Forty-six percent of the RIL's in the population had green stems and light green leaves (NL-6 parental type), 43\% had red stems and dark green leaves (0654-type), and the rest of the lines (8\%) were heterogeneous, having green stems streaked with red and light green leaves or fully red stems and light green leaves.

With respect to panicle variation, 35\% of the RIL's had yellow panicles like P1, 25\% were purple like P2, 19\% had red panicles, and $3 \%$ had pink panicles. Several RIL's exhibited panicle color segregation or heterogeneity, including yellow/red, yellow/purple, orange/ red, yellow/purple, and orange/purple. In addition to panicle color, the amaranth panicle shape (amaranthiform) was present in $60 \%$ of the RIL's, with the remaining 39\% exhibiting globular (glomerulate) panicles.

Branching pattern of the main stem was another discriminating trait. Sixty-nine percent of the RIL's did not

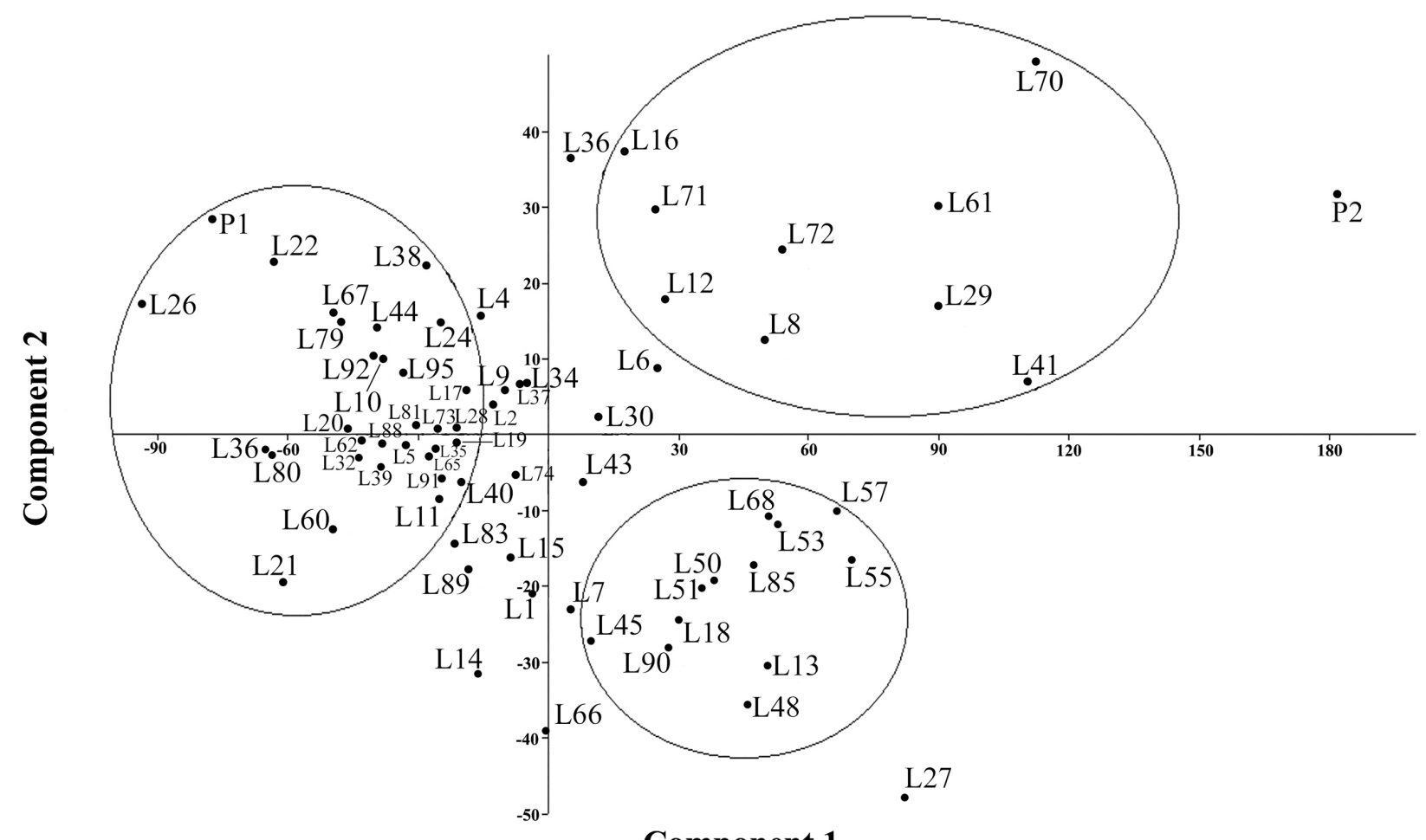

Component 1

FIGURE 1 | Principal Component Analysis (PCA) plot for the variables listed in Table 5. 
exhibit branching. Since branching is a wild-type trait and neither parent normally has a branched stem, the presence of significant stem branching in $\sim 30 \%$ of the lines indicates the two parents may have complementary genes suppressing stem branching.

\section{Quantitative Traits}

All 16 quantitative traits showed very highly significant differences among the RIL's for practically all the studied characters, providing strong evidence for the important diversity generated within the Pop39 and highlighting the relevant discriminating power of the variables used. Seed diameter, for example, varied from 1.71 to $2.11 \mathrm{~mm}$ and there were highly significant differences among lines, with $28 \%$ of the RIL's having larger seeds than the 0654 parent.

Sorting RIL's by plant height at maturity differentiated the population into five classes or groups, producing a distribution skewed slightly toward shorter plant height. The three intermediate classes - classes 2, 3, and 4 -encompassed more than $80 \%$ of the lines (Table 1 ). At maturity, the NL-6 parent was $63.90 \mathrm{~cm}$ tall and thus fell into in class 1; the 0654 parent at $214.88 \mathrm{~cm}$ height was at the upper end of class 5 . Consequently, transgressive segregants for plant height were not detected in the population.

Descriptive and variance analyses of biomass and its components highlighted the important diversity present among the $\mathrm{F}_{2: 6}$ lines. The main stem diameter varied between 0.54 and $1.89 \mathrm{~cm}$ (Table 2). The NL-6 parent had a diameter of $0.71 \mathrm{~cm}$ and for parent 0654 it was $1.15 \mathrm{~cm}$. The main root length of the whole population averaged $10.15 \mathrm{~cm}$ and varied among lines between 8.50 and $12.80 \mathrm{~cm}$. The panicle length also presented a wide variation among lines; it was three times greater in line L70 $(120.70 \mathrm{~cm})$ compared to L26 $(36.90 \mathrm{~cm})$. The panicle width also varied, ranging from 3.61 to $17.50 \mathrm{~cm}$ in lines L26 and L90, respectively (Table 2 ).

Lines could be classified into five biomass groups, with the NL-6 parent falling into class $2(13.16-20.10 \mathrm{~g})$ and 0654 grouping with class $5(50.52-79.39 \mathrm{~g})$. The aboveground and root biomasses had values for the 0654 parent of 196.85 and $12.94 \mathrm{~g}$, respectively (Table 2). The 0654 parent also presented the highest stem dry weight $(70.79 \mathrm{~g})$. Total plant dry biomass varied between 6.19 and $79.39 \mathrm{~g}$ for lines L26 and 0654, respectively. Standard deviations were variable among characters, being very high for the fresh weight of the aboveground plant and the length of the panicle. In contrast, standard deviations were minimal for stem diameter, plant size, and root dry weight (Table 2).

Five seed yield classes were identified, with $>80 \%$ of the lines falling into the three lowest classes and yielding less than $12 \mathrm{~g}$. The 0654 parent grouped in class 1 and yielded only $2.20 \mathrm{~g}$, while NL-6 yielded $12.18 \mathrm{~g}$ (class 4). Five of the RIL's in class 5 produced 17-23 g of seeds per plant, indicating the potential for positive transgressive segregation for yield in Pop39. The grain yield measured per individual ranged between 0.13 and $22.42 \mathrm{~g}$; the average yield of the whole $\mathrm{F}_{6}$ population was of $8.70 \mathrm{~g} /$ plant (Table 1). Thus, variation for seed production was highly significant in the population.
TABLE 1 | Main traits class's interval, average, and lines' percentage in quinoa $F_{2: 6}$ RIL Pop39.

\begin{tabular}{|c|c|c|c|c|c|}
\hline Traits & Class & Interval & Average & Number of lines & Lines' \% \\
\hline \multirow[t]{5}{*}{ PHM (cm) } & 1 & $61-90$ & 76.24 & 6 & 8.33 \\
\hline & 2 & $90-115$ & 105.13 & 23 & 31.94 \\
\hline & 3 & $115-140$ & 124.94 & 21 & 29.17 \\
\hline & 4 & $140-170$ & 159.31 & 15 & 20.83 \\
\hline & 5 & $170-215$ & 193.06 & 7 & 9.72 \\
\hline \multirow[t]{5}{*}{ GY (g/pl) } & 1 & $0.13-4.50$ & 2.54 & 15 & 20.83 \\
\hline & 2 & $4.5-8.0$ & 6.64 & 21 & 29.17 \\
\hline & 3 & $8.0-12.0$ & 9.62 & 22 & 30.56 \\
\hline & 4 & $12.0-17.0$ & 14.19 & 9 & 12.50 \\
\hline & 5 & $17.0-23.0$ & 20.48 & 5 & 6.94 \\
\hline \multirow[t]{5}{*}{ DM (days) } & 1 & $124-131$ & 127.15 & 10 & 13.89 \\
\hline & 2 & $131-140$ & 134.47 & 15 & 20.83 \\
\hline & 3 & $140-153$ & 147.2 & 24 & 33.33 \\
\hline & 4 & $153-70$ & 162.01 & 18 & 25.00 \\
\hline & 5 & $170-192$ & 187.31 & 5 & 6.94 \\
\hline \multirow[t]{6}{*}{ RPV (scores) } & 1 & $0.5-1.2$ & 1.0 & 8 & 11.11 \\
\hline & 2 & $1.4-1.7$ & 1.5 & 12 & 16.67 \\
\hline & 3 & $1.8-2.1$ & 2.0 & 13 & 18.06 \\
\hline & 4 & $2.2-2.3$ & 2.3 & 15 & 20.83 \\
\hline & 5 & $2.4-2.5$ & 2.5 & 7 & 9.72 \\
\hline & 6 & $2.6-3.0$ & 2.7 & 17 & 23.61 \\
\hline
\end{tabular}

PHM, Plant height at maturity; GY, grain yield; DM, days to maturity; YPV, resistance to peronospora variabilis. RPV scores: 1 = very resistance, $2=$ resistance, $3=$ slightly resistance, $4=$ slightly susceptible, $5=$ susceptible, $6=$ very susceptible.

TABLE 2 | Biomass and its components in $F_{2: 6}$ quinoa Pop39.

\begin{tabular}{lrrcccr}
\hline Character & Mean & Max & Highest line & Min & Lowest line & SD \\
\hline SD (cm) & 0.83 & 1.89 & L53 & 0.54 & $\mathrm{~L} 26$ & 0.42 \\
RL (cm) & 10.15 & 12.80 & $\mathrm{~L} 8$ & 8.50 & $\mathrm{~L} 39$ & 1.85 \\
IL (cm) & 66.85 & 120.70 & $\mathrm{~L} 70$ & 36.90 & $\mathrm{~L} 26$ & 26.18 \\
IW (cm) & 9.35 & 17.50 & $\mathrm{~L} 90$ & 3.61 & $\mathrm{~L} 26$ & 5.13 \\
FWA (g) & 51.52 & 196.85 & 0654 & 10.17 & $\mathrm{~L} 21$ & 43.21 \\
FWR (g) & 4.60 & 12.94 & 0654 & 0.83 & $\mathrm{~L} 21$ & 4.02 \\
DWA (g) & 18.14 & 70.79 & 0654 & 2.68 & $\mathrm{~L} 26$ & 16.39 \\
DWR (g) & 2.27 & 7.47 & $\mathrm{~L} 90$ & 0.47 & $\mathrm{~L} 26$ & 2.01 \\
BM (g/plt) & 29.11 & 79.39 & 0654 & 6.19 & $\mathrm{~L} 26$ & 21.91 \\
\hline
\end{tabular}

$\overline{S D}$, main stem diameter; $R L$, root length; IL, inflorescence length; $I W$, inflorescence width; FWA, above ground fresh weight; FWR, root fresh weight; DWA, above ground dry weight; DWR, root dry weight; BM, biomass. SD, standard deviation.

The $70 \mathrm{~F}_{6}$ quinoa lines grouped into five precocity classes when sorted by their number of days to maturity (Table 1), with a population DM average of 148 days and a standard deviation of 16.78 days. About $65 \%$ of the $\mathrm{F}_{2: 6}$ lines required less than 5 months to mature ( $<150$ days). The remaining $35 \%$ of lines required $>150$ days to mature and grouped in classes 4 and 5 . The ANOVA for DM, as with the other characters, had a highly significant $p$-value. The NL-6 parent was earlier than any of the RIL's, having reached maturity after only 124 days (Table 1). The 0654 parent, L48, and L90 were the latest-maturing genotypes at 192 days. 
TABLE 3 | ANOVA for resistance to mildew (Peronospora variabilis) infection in Pop39.

\begin{tabular}{lcccccc}
\hline & $\begin{array}{c}\text { Sum of } \\
\text { squares }\end{array}$ & $\begin{array}{c}\text { Mean } \\
\text { squares }\end{array}$ & Mean & SD & F Fisher & $\boldsymbol{P r}>\boldsymbol{F}$ \\
\hline 2 days & 30.388 & 0.428 & 0.488 & 0.554 & 1.732 & 0.003 \\
$\mathbf{4}$ days & 85.860 & 1.209 & 1.484 & 0.901 & 1.969 & $<0.0001$ \\
6 days & 133.950 & 1.887 & 2.223 & 1.040 & 2.772 & $<0.0001$ \\
8 days & 144.923 & 2.041 & 2.758 & 1.097 & 2.595 & $<0.0001$ \\
10 days & 181.966 & 2.563 & 3.428 & 1.095 & 4.908 & $<0.0001$ \\
12 days & 191.798 & 3.144 & 4.173 & 1.143 & 7.947 & $<0.0001$ \\
\hline
\end{tabular}

\section{Downy Mildew Resistance}

Analysis of variance for resistance to mildew showed significant differences among lines from the second day of the inoculation (Table 3). Perenospora variabilis symptoms did not show up right after the inoculation on all the RIL's - indicating that initial reaction to the pathogen depends on the genotype. The 70 F6 lines were classified in six groups (Table 1), with the most resistant ones in class 1 (L55, L7, L27, L41, and L89) and the most susceptible in class 6 (L37, L12, L18, L92, L44, and L95). The NL-6 parent was slightly susceptible to mildew while 0654 was among the most resistant. Fifty percent of the accessions (36/72) were classified between the two parents, indicating that transgressive segregation for resistance was substantial in Pop39. The resistant 0654 parent did not show any sporulation at day 12 from the inoculation; this behavior was also the case for other lines of the three first classes. Spores appeared on the 10th day on NL-6, which was also the case in lines falling into the other three susceptible classes. The most highly susceptible lines developed their first symptoms well before 10 days after inoculation.

Collected data showed that the pathogen infected every single RIL; however, the most susceptible genotypes rapidly developed symptoms and allowed for sporulation, whereas resistant lines delayed the progression and spread of disease, preventing spore growth and dissemination.

\section{Heritability}

Broad-sense heritability and variances for morphological characters are presented in Table 4. Phenotypic variances were greatest for PH (1154.7) and FWA (1066.97). The highest value of genotypic variance was also noted for $\mathrm{PH}$ (838.53); SD presented the lowest genotypic variance $(0.017)$. Highest heritability values were observed for DM (89\%) and PH (73\%). Heritabilities were intermediate for DWA (55\%), IL (46\%), and BM (46\%). Root length had the lowest heritability at $10 \%$. Grain yield heritability was measured at $42 \%$ in this population and environment.

\section{Principal Component Analysis}

Multivariate analysis was undertaken to highlight genotype groups with similar traits and significant correlations among characters. The principal components analysis (PCA) was computed to assess the contributions of individual variables to the global variance. Fifteen quantitative traits were used and data analysis showed that the four first components explained
$96 \%$ of the variance. These 15 traits' contributions are presented in Table 5 for each of the first four axes. The first principal component (PC1) explained $74 \%$ of the total variance. The variables positively correlated to $\mathrm{PC} 1$ were $\mathrm{PH}(0.92)$; IL and IW (0.85 and 0.64, respectively); SD (0.69); BM (0.94); and DM (0.65). Resistance to mildew was negatively correlated to PC1 $(-0.46)$. Grain yield was correlated to PC2 $(0.6)$, to which DM was negatively correlated $(-0.47)$.

According to the correlation matrix in Table 6, the most significant relationships among traits were GY with IL $(0.52)$ and BM (0.52); DM with PHM (0.71), DWA (0.72), DWR (0.64), and BM (0.54). Susceptibility to mildew (SM) was negatively correlated to PHM (0.54) and DM (0.47).

Figure 1 presents the PCA plot of Pop39 $\mathrm{F}_{2: 6}$ RIL's for PC1 and PC2. On the positive side of axis 1 are grouped 15 entries 0654 (P2), L70, L41, L61, L29, L27, L55, L57, L72, L53, L68, L13, L8, L85, L48 - that shared the characteristics of tall plant height, long and wide panicles, large stalk diameter, large biomass, and relatively long growth cycle. On the negative side of axis 1, RIL's L60, L67, L21, L22, L80, L31, NL-6 (P1), and L26 presented lower values of the aforementioned characters. Lines on the positive side of axis 2 (L70, L16, L36, and NL-6) are characterized by higher grain yield and earlier maturity; on the negative side of axis 2 are RIL's that matured later and had lower seed yield, notably lines L13, L14, L48, L27, and L66.

\section{Dendrogram}

A dendrogram was computed based on UPGMA using PAST v. 2.16 (Hammer et al., 2001). The F2:6 quinoa RIL Pop39 separated into three clusters (Figure 2). Group A included short-stature lines $(61-140 \mathrm{~cm})$ with small panicles $(36.9-69.4 \mathrm{~cm})$ and low biomass (6.19-29.56 $242 \mathrm{~g})$. With the exception of lines L9 and L89, they were also early maturing and susceptible to $P$. variabilis. Cluster A also included the NL-6 parent (P1).

Cluster B included six lines (0654 or P2, L29, L41, L72, L61, L70), all of tall size $(140-215 \mathrm{~cm})$ and having both long (90.2$120.7 \mathrm{~cm})$ and wide $(12.63-16.14 \mathrm{~cm})$ panicles. They were also of high biomass $(50.52-7.39 \mathrm{~g})$ and high grain yield (12-23 g). Parent 0654 was an exception because it yielded only $2.20 \mathrm{~g} / \mathrm{pl}$. However, 0654 and line L41 were very resistant to mildew, L70 and L29 had intermediate levels of resistance, while L61 was very susceptible.

Cluster C (Figure 2) consisted of 24 lines of somewhat large size $(115-170 \mathrm{~cm})$, long panicles $(57.8-120.7 \mathrm{~cm})$, high biomass (20.77-48.02 g), and late maturity (140-192 d). These RIL's were mostly resistant to downy mildew, except for highly susceptible lines L30, L12, and L18.

\section{DISCUSSION}

The presented cross operates between two contrasting lines, NL6 adapted to European lowlands and of short size $(63.90 \mathrm{~cm})$, and 0654 from the Peruvian valleys and taller $(214.88 \mathrm{~cm})$. There is a large genetic distance between $\mathrm{F}_{2: 6}$ accessions, and much variability within the population. Several authors reported that the valley ecotype is generally taller than highland ecotype 
TABLE 4 | Variance and broad-sense heritability components of 12 traits in quinoa $F_{2: 6}$ RIL Pop39.

\begin{tabular}{|c|c|c|c|c|}
\hline Character & Phenotypic variance & Genotypic variance & Environmental variance & Heritability \\
\hline Plant height at maturity $(\mathrm{cm})$ & 1154.70 & 838.53 & 316.17 & 0.73 \\
\hline Inflorescence length (cm) & 588.55 & 271.00 & 317.55 & 0.46 \\
\hline Inflorescence width (cm) & 19.67 & 7.99 & 11.68 & 0.40 \\
\hline Seed diameter (cm) & 0.047 & 0.017 & 0.03 & 0.36 \\
\hline Root length (cm) & 208.30 & 0.30 & 208.00 & 0.10 \\
\hline Above ground fresh weight (g) & 1066.97 & 423.33 & 643.64 & 0.39 \\
\hline Above ground dry weight (g) & 174.46 & 96.68 & 77.78 & 0.55 \\
\hline Root fresh weight $(\mathrm{g})$ & 7.36 & 2.81 & 4.55 & 0.38 \\
\hline Root dryweight (g) & 2.28 & 0.96 & 1.32 & 0.42 \\
\hline Biomass (g/pl) & 335.23 & 155.25 & 179.98 & 0.46 \\
\hline Grain yield (g/pl) & 40.38 & 17.08 & 23.30 & 0.42 \\
\hline Days to maturity (days) & 262.76 & 235.37 & 27.39 & 0.89 \\
\hline
\end{tabular}

TABLE 5 | Variables contribution to the four principal axes in quinoa $F_{2: 6}$ RIL Pop39.

\begin{tabular}{lrrrr}
\hline Character & Axis 1 & Axis 2 & Axis 3 & Axis 4 \\
\hline Plant high at day 60 & 0.0650 & -0.0968 & 0.6565 & 0.3918 \\
Plant high at day 75 & 0.1629 & -0.0308 & 0.8131 & 0.3110 \\
Plant high at day 90 & 0.4522 & -0.1115 & 0.8343 & 0.2193 \\
Plant high at day maturity & 0.9203 & -0.3676 & 0.0480 & 0.0026 \\
Inflorescence length (cm) & 0.8503 & 0.1523 & 0.2153 & -0.4385 \\
Inflorescence width (cm) & 0.6432 & 0.2206 & 0.0569 & -0.1345 \\
Seed diameter (cm) & 0.6985 & 0.1068 & 0.1202 & 0.0360 \\
Root length (cm) & 0.3010 & 0.1763 & 0.1327 & -0.0476 \\
Above ground fresh weight (g) & 0.9093 & 0.3786 & -0.1027 & 0.1235 \\
Above ground dry weight (g) & 0.9591 & 0.0183 & -0.1688 & 0.0238 \\
Root fresh weight (g) & 0.7962 & 0.2265 & -0.0886 & 0.0139 \\
Root dry weight (g) & 0.8589 & 0.0792 & -0.0944 & -0.0632 \\
Biomass (g/pl) & 0.9433 & 0.2053 & -0.0096 & -0.0542 \\
Grain yield (g/pl) & 0.3200 & 0.6045 & 0.4417 & -0.2231 \\
Days to maturity (days) & 0.6549 & -0.4757 & -0.4734 & 0.0613 \\
Mildew reaction & -0.4647 & 0.2946 & 0.0707 & -0.0330 \\
Variance & $74 \%$ & $11 \%$ & $9 \%$ & $3 \%$ \\
Sum of the variance & $74 \%$ & $85 \%$ & $93 \%$ & $96 \%$ \\
\hline
\end{tabular}

(Gandarillas, 1979; Cáceres, 1993; Mujica and Jacobsen, 1999; Carmen, 2008).

The $\mathrm{F}_{2: 6}$ quinoa RIL's presented a large genetic variability, which is translated to qualitative traits of stem, leaf, inflorescence and seed color diversity, and also by inflorescence shape and size variation. Seed color varies from black, brown, red, pink, yellow, orange to white. The inflorescence presents a very wide range of colors from white, yellow, pink, red to dark red, brown, and purple. Within the $\mathrm{F}_{2: 6}$ accessions, the leaf color was light green in $47 \%$ RIL's or dark green in $45 \%$; the stems were red $(43 \%)$ or green $(46 \%)$ and the inflorescence were mainly yellow (35\%) or red (25\%), but also orange, pink, purple.

Several authors have investigated the genetic control of a number of qualitative characters. A single gene with several alleles controls leaf color, red color is dominant over purple, dominating over green. The red color is dominant at leaves axils (Tapia et al., 1979; Izquierdo Fernández et al., 2001; Carmen, 2008).
Concerning the panicle shape, a 3:1 segregation of glomerulate upon amaranth forms was reported (Carmen et al., 2008). Male sterility identified in quinoa is nucleocytoplasmic, with three fertile plants for one sterile (Izquierdo Fernández et al., 2001; Carmen et al., 2008).

There is a wide range of seed colors being white, yellow, pink, dark red, brown, and purple (Cusack, 1984; Risi and Galwey, 1989). The genetic determination of this trait requires studying several cross descendants' between homozygote lines with contrasted seed colors. The tetraploid status of the species has to be taken into account while analyzing the offspring.

The UPGMA clustering and PCA showed that the highlandphenotype, relatively unadapted lines in Rabat's coastal climate formed a distinct group far apart from the other clusters. Highland-phenotype accessions- those most like the Peruvian 0654 parent - are of tall stature and have a more indeterminate growth pattern when cultivated in the mild and humid coastal environment. Moreover, since 0654 is susceptible to severe decreases in seed yield when exposed to elevated temperatures during flowering (personal observations), lowyielding lines may have inherited this characteristic from 0654 .

Maughan et al. (2012) reported that single-nucleotide polymorphism (SNP) markers identified in this same population (Pop39) displayed 95\% normal (non-distorted) segregation, with most of the skewed markers favoring the NL-6 alleles. They further speculated that this pattern of segregation distortion favoring SNP alleles from the NL-6 parent might be due to heat-induced sterility coming from the 0654 parent, even though the population was advanced to homozygosity each generation under relatively mild $\left(25^{\circ} \mathrm{C}\right)$ temperatures in the greenhouse. Consequently, it would be interesting in a future study to address the question of whether or not these NL- 6 skewed regions carry alleles for heat tolerance.

This highland RIL's are, however, highly resistant to downy mildew; they develop small lesions and have a long latent period when inoculated with oospores of $P$. variabilis under controlled conditions. The pathogen affected most genotypes at different intensities. 0654 parent was ranked among the most resistant $\mathrm{F}_{2: 6}$ RIL's group, probably carrying the resistance 
TABLE 6 | Correlation matrix among variables measured in $F_{2: 6}$ RIL quinoa Pop39.

\begin{tabular}{|c|c|c|c|c|c|c|c|c|c|}
\hline 0 & PHM & IL & FWA & DWA & FWR & DWR & BM & GY & DM \\
\hline IL & 0,72421 & & & & & & & & \\
\hline DWA & 0,85625 & 0,76911 & 0,88701 & & & & & & \\
\hline FWR & 0,62341 & 0,69904 & 0,8136 & 0,79851 & & & & & \\
\hline DWR & 0,72522 & 0,76126 & 0,7972 & 0,88355 & 0,93488 & & & & \\
\hline GY & 0,10011 & 0,52115 & 0,43149 & 0,23385 & 0,3125 & 0,29107 & 0,51739 & & \\
\hline DM & 0,71335 & 0,38958 & 0,46564 & 0,71753 & 0,51038 & 0,6402 & 0,53786 & $-0,31201$ & \\
\hline SM & $-0,53602$ & $-0,31489$ & $-0,32164$ & $-0,45151$ & $-0,40435$ & $-0,43732$ & $-0,38074$ & 0,06723 & $-0,468$ \\
\hline
\end{tabular}

A

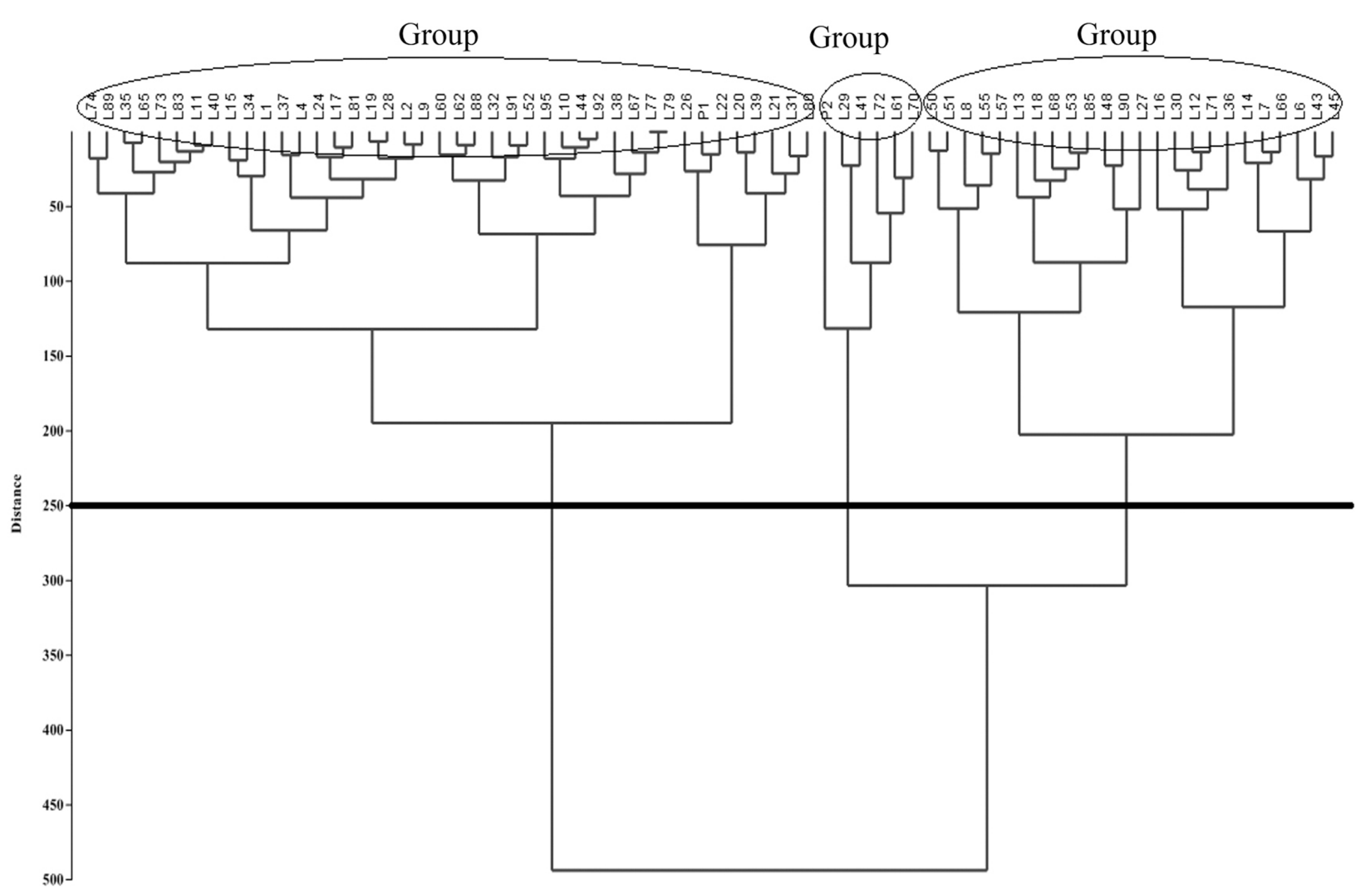

FIGURE 2 | Unweighted Pair Group Method with Arithmetic Mean (UPGMA)-based dendrogram showing three clusters (A-C) of RIL's in quinoa Pop39.

factor. This assumption is in agreement with Kitz (2008) from the same population. The highest resistance was that of RIL L55, which confirms our earlier greenhouse observations (unpublished).

According to Alfano and Collmer (1997), Campbell et al. (1999), and Eulgem et al. (2004), the valley ecotype is usually more resistant to downy mildew than early and short size lowland ecotype, corresponding to group A of the dendrogram. These results are in concordance with Bonifacio and Saravia (1999).
The data analyses confirmed the significant diversity of the quantitative traits. Seed yield was positively correlated to panicle length (0.52) and total biomass (0.52). Bhargava et al. (2007) state good correlation between grain yield and plant biomass while Carmen (2008) reports positive correlations with plant size, panicle width, total dry matter and crop earliness.

Mujica (1988) puts up two genetic indexes that are based on high inheritability traits that are highly correlated to grain 
yield; those are central glomeruli diameter (Index I1) and stem and panicle diameters (Index I2). The efficiency of the two indexes reached 144.48 and $148.32 \%$, respectively; they represent relevant parameters for germplasm evaluation and selection studies (Mujica, 1988).

Bertero et al. (2004) reported striking disparities for quinoa character correlations between cold/highland and lowland/tropical environments. For example, they reported grain yield versus biomass correlations of 0.92 and 0.48 in cold/highland versus warm/lowland test sites, respectively. Although Rabat is on the coastal plain and at mid-latitude, its proximity to the ocean moderates the temperature; nonetheless, the observed correlation of yield versus biomass of 0.52 in this study follows the heat-stress pattern detected by Bertero et al. (2004). Biomass was still a close second behind (IL, 0.521) for magnitude of correlation with grain yield in this study.

Bertero et al. (2004) also reported a disparity for quinoa grain yield-maturity correlations, having observed values of 0.87 and 0.37 in cold/highland and warm/lowland environments, respectively. In the present study, we found that grain yield and days to maturity were negatively correlated at -0.31 . This is most likely due to later-maturing lines encountering debilitating late spring-summer heat stress, especially for lines carrying alleles from the heat-susceptible parent 0654.

Bertero et al. (1999) reported strong effects on seed size and maturation from high temperatures and post-anthesis exposure to long days in Altiplano (variety 'Kanckolla') and Valley (variety 'Blanca de Junin') quinoa genotypes. Since our parental cultivar '0654' is of similar intermediate-late maturity and environmental adaptation as 'Blanca de Junin', it is not surprising that plant height, inflorescence size, biomass, seed diameter, and maturation all contributed heavily to Axis 1 of the PCA (Table 5). With a February sowing date at Rabat $\left(\sim 34^{\circ} \mathrm{N}\right.$ latitude), lines having greater allelic contributions for daylength sensitivity from '0654' would be expected to have delayed maturation, higher biomass, etc., whereas daylength-neutral alleles from the coastal genetic background of 'NL-6' would have contributed to markedly earlier maturation and relatively reduced biomass parameters under the same lengthening-day conditions - and without transgressive segregation for maturation from 'NL-6' contributing to a longer growth cycle.

Clearly, salient breeding objectives in the mild coastal climate of Rabat should include higher seed yield, shorter growing cycle and downy mildew resistance - though given the correlation

\section{REFERENCES}

Alfano, J. R., and Collmer, A. (1997). The type III (Hrp) secretion pathway of plant pathogenic bacteria: trafficking harpins, avr proteins, and death. J. Bacteriol. $179,5655-5662$.

Bertero, H. D., de la Vega, A. J., Correa, G., Jacobsen, S. E., and Mujica, A. (2004). Genotype and genotype-by-environment interaction effects for grain yield and grain size of quinoa (Chenopodium quinoa Willd.) as revealed by pattern analysis of international multi- environment trials. Field Crops Res. 89, 299-318.

Bertero, H. D., King, R. W., and Hall, A. J. (1999). Photoperiod-sensitive development phases in quinoa (Chenopodium quinoa Willd.). Field Crops Res. 60, 231-243. doi: 10.1016/S0378-4290(98)00128-2 between more lengthy growth cycle and higher mildew resistance, and in light of Rabat's lack of severe summer heat or winter freeze constraints, mildew resistance probably should not be sacrificed for earlier maturation. This is based on the presumption that the humid climate in Rabat is conducive to mildew spore exposure and growth year-round. Nevertheless, evaluations of larger numbers of segregating progeny from lowland $\times$ highland quinoa populations like Pop39 should facilitate uncoupling of traits like heat tolerance/early maturity + mildew susceptibility.

The extensive morphological variation present in just 70 F2:6 lines of the NL- $6 \times 0654$ RIL population, combined with the pattern of relatively moderate linkage distortion detected in this population using molecular markers (Maughan et al., 2012), bodes well for the continued use of lowland coastal $\times$ Andean highland quinoa breeding populations. The detection of positive transgressive segregants for seed size and yield, in particular, combined with an observed heritability for yield of approximately $40 \%$, bodes well for future selection to develop higher-yielding quinoa lines for the Moroccan coastal environment.

\section{AUTHOR CONTRIBUTIONS}

$\mathrm{OB}$ and $\mathrm{NB}$ designed the study. $\mathrm{PM}$ and EJ prepared the population. $\mathrm{NB}$ and $\mathrm{OB}$ collected the data. $\mathrm{NB}, \mathrm{OB}$, and $\mathrm{SJ}$ analyzed the data. OB, SJ, PM, and EJ obtained funding to support this project. $\mathrm{NB}, \mathrm{OB}$, and $\mathrm{EJ}$ took primary responsibility for writing the manuscript.

\section{FUNDING}

Funding for advancement of the population to the F2:6 generation was provided by Brigham Young University and the Holmes Family Foundation through grants in support of undergraduate research.

\section{ACKNOWLEDGMENTS}

The SWUP-Med project of the European Union provided principal funding for this study. The original cross for this population was made by Alejandro Bonifacio and we are grateful for his contribution of the $\mathrm{F}_{1}$ seed.

Bhargava, A., Shukla, S., and Ohri, D. (2007). Genetic variability and inter-relationship among various morphological and quality traits in quinoa (Chenopodium quinoa Willd.). Field Crops Res. 101, 104-116. doi: 10.1016/j.fcr.2006.10.001

Bonifacio, A., and Saravia, R. (1999). "Evaluación de la resistencia al mildiu en quinua," in Proceedings of the Tercer Taller de Preduza en Resistencia Duradera en Cultivos Altos en la Zona Andina, Cochabamba, 49-59.

Bruno, M., and Whitehead, W. T. (2003). Chenopodium cultivation and formative period agriculture at Chiripa, Bolivia. Lat. Am. Antiq. 14, 339-355. doi: $10.2307 / 3557564$

Cáceres, E. (1993). Cultivos Andinos. Bolivia: Instituto Nacional de Alimentacioìn y Nutrición, 126. 
Campbell, N. A., Reece, J. B., and Mitchell, L. G. (1999). Biology, 5th Edn. Menlo Park, CA: Benjamin/Cummings, 679-680.

Carmen, D. C. (2008). Diversité Génétique et Réponse aux Contraintes du Climat: Une Étude de cas à Partir de la Biologie des Populations de Quinoa (Chenopodium quinoa Willd.) de Bolivie. Doctoral thesis, Faculty of Agricultural Sciences in Gembloux, Gembloux.

Carmen, D. C., Mahy, G., and Winkel, T. (2008). La quinoa en Bolivie: une culture ancestrale devenue culture de rente "bio-équitable". Biotechnol. Agron. Soc. Environ. 12, 421-435.

Choi, Y. J., Danielsen, S., Lubeck, M., Hong, S. B., Delhey, R., and Shin, H. D. (2010). Morphological and molecular characterization of the causal agent of downy mildew on quinoa (Chenopodium quinoa). Mycopathologia 169, 403412. doi: 10.1007/s11046-010-9272-y

Christensen, S. A., Pratt, D. B., Pratt, C., Nelson, P. T., Stevens, M. R., Jellen, E. N., et al. (2007). Assessment of genetic diversity in the USDA and CIP-FAO international nursery collections of quinoa (Chenopodium quinoa Willd.) using microsatellite markers. Plant Genet. Resour. 5, 82-95. doi: $10.1017 /$ S1479262107672293

Cusack, D. (1984). Quinoa: grain of the Incas. Ecologist 14, 21-31.

Eulgem, T., Weigman, V. J., Chang, H., McDowell, J. M., Holub, E. B., Glazebrook, J., et al. (2004). Gene resistance signatures from three genetically separable resistance gene signaling pathways for downy mildew resistance. Plant Physiol. 135, 1129-1144. doi: 10.1104/pp.104.040444

Food Agriculture Organization of United Nations [FAO] (2012). International Year of Quinoa IYQ-2013. Available at: http://www.rlc.fao.org/en/about-fao/i yq-2012/

Fuentes, F., Martinez, E. A., Hinrichsen, P. V., Jellen, E. N., and Maughan, P. J. (2009). Assessment of genetic diversity patterns in Chilean quinoa (Chenopodium quinoa Willd.) germplasm using multiplex fluorescent microsatellite markers. Conserv. Genet. 10, 369-377. doi: 10.1007/s10592-008-9604-3

Gandarillas, H. (1979). "La quinua (Chenopodium quinoa Willd.), Genética y origen,” in La Quinua y la Kañiwa Cultivos Andinos, eds M. E. Tapia, H. Gandarillas, S. Alandia, A. Cardozo, and A. Mujica, (Bogota: CIID-IICA), 45-64.

Hammer, O., Harper, D. A. T., and Ryan, P. D. (2001). PAST: paleontological statistics software package for education and data analysis. Palaeontol. Electronica 4:9.

Izquierdo Fernández, J. I., Mujica, A., Jacobsen, S. E., Marathée, J. P., and Morón, C. (2001). Quinua (Chenopodium quinoa wild.) Ancestral cultivo andino, alimento del presente y futuro. Cultivos andinos. Versión 1.0. (CD-Rom). Santiago: Food and Agriculture Organization.

Jacobsen, S. E., Mujica, A., and Jensen, C. R. (2003). The resistance of quinoa (Chenopodium quinoa Willd.) to adverse abiotic factors. Food Rev. Int. 19, 99-109. doi: 10.1081/FRI-120018872

Jacobsen, S. E., Sorensen, M., Pedersen, S. M., and Weiner, J. (2013). Feeding the world: genetically modified crops versus agricultural biodiversity. Agron. Sustain. Dev. 33, 651-662. doi: 10.1007/s13593-013-0138-9

Kistler, L., and Shapiro, B. (2011). Ancient DNA confirms a local origin of domesticated chenopod in eastern North America. J. Archaeol. Sci. 38, 35493554. doi: 10.1016/j.jas.2011.08.023
Kitz, L. (2008). Evaluation of Downy Mildew (Peronospora farinosa F. Sp. CHENOPOD II) Resistance Among Quinoa Genotypes and Investigation of P. farinosa Growth Using Scanning Electron Microscopy. Ph.D. thesis, Brighan Young University, Provo, UT.

Koziol, M. J. (1992). Chemical composition and nutritional value of quinoa (Chenopodium quinoa Willd.). J. Food Composit. Anal. 5, 35-68. doi: 10.1016/0889-1575(92)90006-6

Maughan, P. J., Smith, S. M., Rojas-Beltran, J. A., Elzinga, D., Raney, J. A., Jellen, E. N., et al. (2012). Single nucleotide polymorphism identification, characterization, and linkage mapping in quinoa. Plant Genome 5, 114-125. doi: 10.3835/plantgenome2012.06.0011

Mhada, M., Ezzahiri, B., and Benlhabib, O. (2015). Assessment of downy mildew resistance (Peronospora farinosa) in a quinoa (Chenopodium quinoa Willd.) germplasm. Int. J. Biol. Med. Res. 6, 4748-4752.

Mujica, A. (1988). Genetic Parameters and Selection Indexes for Quinoa (Chenopodium quinoa Wild.). Doctoral thesis, Chapingo Autonomous University, Texcoco, 7.

Mujica, A., and Jacobsen, S. E. (1999). "Resistencia de la quinua a la sequía y otros factores abióticos adversos y su mejoramiento," in I Curso Internacional Sobre Fisiología de la Resistencia a Sequía en Quinua (Chenopodium quinoa Willd.) (Lima: CIP-DANIDA), 25-38.

Risi, I., and Galwey, N. M. (1989). "Chenopodium grains of the Andes: a crop for temperate latitudes," in New Crops For Food And Industry, eds G. E. Wickens, N. Haq, and P. Day (London: Chapman and Hall), 222-234.

Smith, B. D., and Yarnell, R. A. (2009). Initial formation of an indigenous crop complex in eastern North America at 3800 B.P. Proc. Natl. Acad. Sci. U.S.A. 106, 6561-6566. doi: 10.1073/pnas.0901846106

Sokal, R., and Michener, C. (1958). A statistical method for evaluating systematic relationships. Univ. Kansas Sci. Bull. 38, 1409-1438.

Tapia, M., Gandarillas, H., Alandia, S., Cardozo, A., Mujica, A., Ortiz, R., et al. (1979). La Quinua y la Kamwa. Cultivos andinos. Bogota: IICA, CIID, 228.

Wilson, H. D. (1990). Quinua and relatives (Chenopodium sect. Chenopodium subsect. Cellulata). Econ. Bot. 44, 92-110. doi: 10.1007/BF028 60478

Wilson, H. D., and Heiser, C. B. (1979). The origin and evolutionary relationships of 'Huauzontle' (Chenopodium nuttaliae Safford), domesticated chenopod of Mexico. Am. J. Bot. 66, 198-206. doi: 10.2307/2442525

Conflict of Interest Statement: The authors declare that the research was conducted in the absence of any commercial or financial relationships that could be construed as a potential conflict of interest.

Copyright (c) 2016 Benlhabib, Boujartani, Maughan, Jacobsen and Jellen. This is an open-access article distributed under the terms of the Creative Commons Attribution License (CC BY). The use, distribution or reproduction in other forums is permitted, provided the original author(s) or licensor are credited and that the original publication in this journal is cited, in accordance with accepted academic practice. No use, distribution or reproduction is permitted which does not comply with these terms. 
OPEN ACCESS

Edited by: Francesca Sparvoli, Consiglio Nazionale delle Ricerche,

Reviewed by: Albino Maggio,

University of Naples Federico II, Italy Antonio M. De Ron, Spanish National Research Council Misión Biológica de Galicia, Spain

*Correspondence: Kevin M. Murphy kmurphy2@wsu.edu

Specialty section: This article was submitted to Crop Science and Horticulture, a section of the journal Frontiers in Plant Science

Received: 16 December 2015

Accepted: 22 May 2016

Published: 03 June 2016

Citation:

Wu G, Peterson AJ, Morris CF and Murphy KM (2016) Quinoa Seed Quality Response to Sodium Chloride and Sodium Sulfate Salinity.

Front. Plant Sci. 7:790 doi: 10.3389/fpls.2016.00790

\section{Quinoa Seed Quality Response to Sodium Chloride and Sodium Sulfate Salinity}

\author{
Geyang Wu' ${ }^{1}$ Adam J. Peterson ${ }^{2}$, Craig F. Morris ${ }^{3}$ and Kevin M. Murphy2* \\ ${ }^{1}$ School of Food Science, Washington State University, Pullman, WA, USA, ${ }^{2}$ Department of Crop and Soil Sciences, \\ Washington State University, Pullman, WA, USA, ${ }^{3}$ United States Department of Agriculture/Agricultural Research \\ Service-Western Wheat Quality Laboratory, Washington State University, Pullman, WA, USA
}

Quinoa (Chenopodium quinoa Willd.) is an Andean crop with an edible seed that both contains high protein content and provides high quality protein with a balanced amino acid profile in embryonic tissues. Quinoa is a halophyte adapted to harsh environments with highly saline soil. In this study, four quinoa varieties were grown under six salinity treatments and two levels of fertilization, and then evaluated for quinoa seed quality characteristics, including protein content, seed hardness, and seed density. Concentrations of 8,16 , and $32 \mathrm{dS} \mathrm{m}{ }^{-1}$ of $\mathrm{NaCl}$ and $\mathrm{Na}_{2} \mathrm{SO}_{4}$, were applied to the soil medium across low (1 g N, $0.29 \mathrm{~g} \mathrm{P,} 0.29 \mathrm{~g} \mathrm{~K}$ per pot) and high ( $3 \mathrm{~g} \mathrm{~N}, 0.85 \mathrm{~g} \mathrm{P}$, $0.86 \mathrm{~g} \mathrm{~K}$ per pot) fertilizer treatments. Seed protein content differed across soil salinity treatments, varieties, and fertilization levels. Protein content of quinoa grown under salinized soil ranged from 13.0 to $16.7 \%$, comparable to that from non-saline conditions. $\mathrm{NaCl}$ and $\mathrm{Na}_{2} \mathrm{SO}_{4}$ exhibited different impacts on protein content. Whereas the different concentrations of $\mathrm{NaCl}$ did not show differential effects on protein content, the seed from $32 \mathrm{dS} \mathrm{m}^{-1} \mathrm{Na}_{2} \mathrm{SO}_{4}$ contained the highest protein content. Seed hardness differed among varieties, and was moderately influenced by salinity level $(P=0.09)$. Seed density was affected significantly by variety and $\mathrm{Na}_{2} \mathrm{SO}_{4}$ concentration, but was unaffected by $\mathrm{NaCl}$ concentration. The samples from $8 \mathrm{dS} \mathrm{m}^{-1} \mathrm{Na}_{2} \mathrm{SO}_{4}$ soil had lower density $\left(0.66 \mathrm{~g} / \mathrm{cm}^{3}\right)$ than those from $16 \mathrm{dS} \mathrm{m}^{-1}$ and $32 \mathrm{dS} \mathrm{m}^{-1} \mathrm{Na}_{2} \mathrm{SO}_{4}, 0.74$ and $0.72 \mathrm{~g} / \mathrm{cm}^{3}$, respectively. This paper identifies changes in critical seed quality traits of quinoa as influenced by soil salinity and fertility, and offers insights into variety response and choice across different abiotic stresses in the field environment.

Keywords: quinoa, soil salinity, protein content, hardness, density

\section{INTRODUCTION}

Quinoa (Chenopodium quinoa Willd.) has garnered much attention in recent years because it is an excellent source of plant-based protein and is highly tolerance of soil salinity. Because soil salinity affects between 20 and 50\% of irrigated arable land worldwide (Pitman and Läuchli, 2002), the question of how salinity affects seed quality in a halophytic crop like quinoa needs to be addressed. Protein content in most quinoa accessions has been reported to range from 12 to $17 \%$, depending on variety, environment, and inputs (Rojas et al., 2015). This range tends to 
be higher than the protein content of wheat, barley, and rice, which were reported to be $10.5-14 \%, 8-14 \%$, and $6-7 \%$, respectively (Orth and Shellenberger, 1988; Shih, 2006; Cai et al., 2013). Additionally, quinoa has a well-balanced complement of essential amino acids. Specifically, quinoa is rich in lysine, which is considered the first limiting essential amino acid in cereals (Taylor and Parker, 2002). Protein quality such as Protein Efficiency Ratio is similar to that of casein (Ranhotra et al., 1993). Furthermore, with a lack of gluten protein, quinoa can be safely consumed by gluten sensitive/intolerant population (Zevallos et al., 2014).

Quinoa shows exceptional adaptation to harsh environments such as drought and salinity (González et al., 2015). Soil salinity reduces crop yields and is a worldwide problem. In the United States, approximately 2.2 million hectares of cropland in 48 States were occupied by saline soils, while another 30.8 million hectares are at risk of becoming saline (United States Department of Agriculture [USDA], 2011). The salinity issue leads producers to grow more salt-tolerant crops, such as quinoa.

Many studies have focused on quinoa's tolerance to soil salinity, with a particular emphasis on plant physiology (RuizCarrasco et al., 2011; Adolf et al., 2012; Cocozza et al., 2013; Shabala et al., 2013) and agronomic characteristics such as germination rate, plant height, and yield (Prado et al., 2000; Chilo et al., 2009; Razzaghi et al., 2012; Peterson, 2013; Peterson and Murphy, 2015). For instance, Razzaghi et al. (2012) showed that the seed number per $\mathrm{m}^{2}$ and seed yield did not decrease as salinity increased from 20 to $40 \mathrm{dS} \mathrm{m}^{-1}$ in the variety Titicaca. Ruiz-Carrasco et al. (2011) reported that under $300 \mathrm{mM} \mathrm{NaCl}$, germination and shoot length were significantly reduced, whereas root length was inhibited in variety BO78; variety PRJ biomass was less affected and exhibited the greatest increase in proline concentration. Jacobsen et al. (2000) suggested that stomatal conductance, leaf area, and plant height were the characters in quinoa most sensitive to salinity. Wilson et al. (2002) examined salinity stress of salt mixtures of $\mathrm{MgSO}_{4}, \mathrm{Na}_{2} \mathrm{SO}_{4}, \mathrm{NaCl}$, and $\mathrm{CaCl}_{2}\left(3-19 \mathrm{dS} \mathrm{m}{ }^{-1}\right)$. No significant reduction in plant height and fresh weight were observed. In a comparison of the effects of $\mathrm{NaCl}$ and $\mathrm{Na}_{2} \mathrm{SO}_{4}$ on seed yield, quinoa exhibited greater tolerance to $\mathrm{Na}_{2} \mathrm{SO}_{4}$ than to $\mathrm{NaCl}$ (Peterson and Murphy, 2015).

Few studies have focused on the influence of salinity on seed quality in quinoa. Karyotis et al. (2003) conducted a field experiment in Greece ( $80 \mathrm{~m}$ above sea level, latitude: $39.7^{\circ} \mathrm{N}$ ). With the exception of Chilean variety 'No. 407', seven other varieties exhibited significant increases in protein (13-33\%) under saline-sodic soil, with electrical conductivity (EC) of 6.5 $\mathrm{dS} \mathrm{m}^{-1}$. Seed minerals contents of phosphorous, iron, copper, and boron did not decrease under saline conditions. Koyro and Eisa (2008) found a significant increase in protein and a decrease in total carbohydrates under high salinity $(500 \mathrm{mM})$. Increasing total soluble sugar, sucrose, and glucose were observed under salinity stress because of starch hydrolysis (Ruffino et al., 2010). The same study found a significant decrease in lipids and relative water content under salinity. Pulvento et al. (2012) indicated that fiber and saponin contents increased under saline conditions with well water/sea water ratio of 1:1 compared to those under non-saline soil.
Protein is one of the most important nutritional components of quinoa seed. The content and quality of protein contribute to the nutritional value of quinoa. Additionally, seed hardness is an important trait in crops such as wheat and soybeans. For instance, kernel hardness highly influences wheat end-use quality (Morris, 2002) and correlates with other seed quality parameters such as ash content, semolina yield, and flour protein content (Hrušková and Švec, 2009). Hardness of soybean influenced water absorption, seed coat permeability, cookability, and overall texture (Zhang et al., 2008). Quinoa seed hardness was correlated with the texture of cooked quinoa, influencing hardness, chewiness, and gumminess, and potentially consumer experience ( $\mathrm{Wu}$ et al., 2014). Furthermore, seed density is also a quality index and is negatively correlated with the texture of cooked quinoa such as hardness, cohesiveness, chewiness, and gumminess (Wu et al., 2014). Hence, protein content, seed hardness, and seed density were selected as indexes of quinoa seed quality in this study.

Chilean lowland varieties have been shown to be the most well-adapted to temperate latitudes (Bertero, 2003), and therefore they have been extensively utilized in quinoa breeding programs in both Colorado State University and Washington State University (Peterson and Murphy, 2015). The previous study found the varieties of CO407D, UDEC-1, Baer, and QQ065 exhibited extremely high tolerance to $\mathrm{Na}_{2} \mathrm{SO}_{4}$ and relatively high tolerance to $\mathrm{NaCl}$ in terms of agronomic performance such as yield, plant height, and leaf greenness (Peterson and Murphy, 2015). However, quinoa seed quality under salinity stress remains to be evaluated, since seed quality is critical to nutrition value as well as consumers' liking of the product. Hence, the objectives of this study were to (1) examine the effect of soil salinity on the protein content, seed hardness, and density of quinoa varieties, (2) determine the effect of different levels of two agronomically important soil salts, $\mathrm{NaCl}$ and $\mathrm{Na}_{2} \mathrm{SO}_{4}$, on seed quality, (3) determine the different influence of and $\mathrm{NaCl}$ and $\mathrm{Na}_{2} \mathrm{SO}_{4}$; and (4) test the influence of fertilization level on salinity tolerance of quinoa.

\section{MATERIALS AND METHODS}

\section{Genetic Material}

Quinoa germplasm was obtained from Dr. David Brenner at the USDA-ARS North Central Regional Plant Introduction Station in Ames, IA, USA. The four quinoa varieties, CO407D (PI 596293), UDEC-1 (PI 634923), Baer (PI 634918), and QQ065 (PI 614880 ), were originally sourced from lowland Chile. CO407D was released by Colorado State University in 1987. UDEC-1, Baer, and QQ065 were varieties from northern, central, and southern locations in Chile with latitudes of $34.63^{\circ} \mathrm{S}, 38.70^{\circ} \mathrm{S}$, and $42.50^{\circ}$ $\mathrm{S}$, respectively.

\section{Experimental Design}

A controlled environment greenhouse study was conducted using a split-split-plot randomized complete block design with three replicates per treatment. Factors included four quinoa varieties, two fertility levels, and seven salinity treatments 
(three concentration levels each of $\mathrm{NaCl}$ and $\mathrm{Na}_{2} \mathrm{SO}_{4}$ ). Three subsamples, each representing a single plant, were evaluated for each treatment combination. Quinoa variety was treated as the main plot, salinity level as the sub-plot, and fertilization as the sub-sub-plot. Salinity levels included 8, 16, and $32 \mathrm{dS} \mathrm{m}^{-1}$ of $\mathrm{NaCl}$ and $\mathrm{Na}_{2} \mathrm{SO}_{4}$. The details of controlling salinity levels were described by Peterson and Murphy (2015). In brief, fertilization was provided by a mixture of alfalfa meal, monoammonium phosphate, and feather meal. The low fertilization level included $1 \mathrm{~g}$ of $\mathrm{N}, 0.29 \mathrm{~g}$ of $\mathrm{P}$, and $0.29 \mathrm{~g}$ of $\mathrm{K}$ per pot; and the high fertilization level included $3 \mathrm{~g}$ of $\mathrm{N}, 0.86 \mathrm{~g}$ of $\mathrm{P}$, and $0.86 \mathrm{~g}$ of $\mathrm{K}$ per pot. Each pot contained about $1 \mathrm{~L}$ of Sunshine Mix \#1 (Sun Gro Horticulture, Bellevue, WA, USA) (dry density of $100 \mathrm{~g} / \mathrm{L}$, water holding capacity of ca. $480 \mathrm{~g} / \mathrm{L}$ potting mix). The entire experiment was conducted twice, with the planting dates of September 10th, 2011 and October 7th, 2011.

\section{Seed Quality Tests}

Quinoa was harvested at maturity. As described in the previous study (Peterson and Murphy, 2015), seeds were first stripped by hand from the inflorescences, and then threshed in a singlehead thresher (Precision Machine Company, Lincolin, NE, USA). The resulting material was cleaned using a Clipper Office Tester (Seedburo, Des Plaines, IL, USA) before the seed quality tests.

Protein content of quinoa was determined using the Dumas combustion nitrogen method (LECO Corp., Joseph, Mich., USA) (AACCI Method 46-30.01) (AACC International, 1995). A factor of 6.25 was used to convert nitrogen to protein. Seed hardness was determined using the Texture Analyzer (TA-XT2i) (Texture Technologies Corp., Scarsdale, NY, USA) and a modified rice kernel hardness method (Krishnamurthy and Giroux, 2001). A single quinoa kernel was compressed until the point of fracture using a $1 \mathrm{~cm}^{2}$ cylinder probe traveling at $5 \mathrm{~mm} / \mathrm{s}$. Repeat measurements were taken on nine random kernels. The seed hardness was recorded as the average peak force $(\mathrm{kg})$ of the repeated measures.

Seed density was determined using a pycnometer (Pentapyc 5200e, Quantachrome Instruments, Boynton Beach, FL, USA). Quinoa seed was placed in a closed micro container, and compressed nitrogen was suffused into the container. Pressure in the container was recorded both with and without nitrogen. The volume of the quinoa sample was calculated by comparing the standard pressure obtained with a stainless steel ball. Density was the seed weight divided by the displaced volume. Seed density was collected on only the second greenhouse experiment.

\section{Statistical Analysis}

Data were analyzed using the PROC GLM procedure in SAS (SAS Institute, Cary, NC, USA). Greenhouse experiment repetition was treated as a random factor in protein content and seed hardness analysis. Variety, salinity, and fertilization were treated as fixed factors. Fisher's LSD Test was used to access multiple comparisons. Pearson correlation coefficients between protein, hardness, and density were obtained via PROC CORR procedure in SAS, using the treatment means.

\section{RESULTS}

\section{Protein}

Variety, salinity, and fertilization all exhibited highly significant effects on protein content $(P<0.001)$ (Table 1). The greatest contribution to variation in seed protein was due to fertilization $(F=402.5)$. In contrast, salinity alone had a relatively minor effect, and the varieties responded similarly to salinity as evidenced by a non-significant interaction. Significant interactions, however, were found in variety $\times$ fertilization, as well as in salinity $\times$ fertilization, both of which are addressed in later paragraphs. It is worth noting that the two experiments produced different seed protein contents $(F=48.09$, $P<0.001)$ and an experiment $\times$ variety interaction was observed $(F=14.94, P<0.001)$ (data not shown). Upon closer examination, this interaction was caused by variety QQ065, which produced an overall mean protein content of $12.9 \%$ in Experiment 1 and $14.9 \%$ in Experiment 2. Protein contents of the other three varieties were essentially consistent across the two experiments.

Across all salinity and fertilization treatments, the variety protein means ranged from 13.0 to $16.7 \%$ (data not shown). As expected, high fertilization resulted in an increase in protein content across all varieties. The mean protein contents under high and low fertilization were 15.8 and 13.6\%, respectively (Table 2). The means of Baer and CO407D were the highest, 15.1 and $14.9 \%$, respectively. QQ065 contained 14.1\% protein, significantly lower than the other varieties.

Even though salinity effects were relatively smaller than fertilization and variety effects, salinity still had a significant effect on protein content (Table 1). The two types of salt exhibited different impacts on protein (Table 2). Protein content did not differ according to different concentrations of $\mathrm{NaCl}$ with means (across varieties and fertilization levels) from 14.7 to $14.9 \%$. Seed from $32 \mathrm{dS} \mathrm{m} \mathrm{m}^{-1} \mathrm{Na}_{2} \mathrm{SO}_{4}$, however, contained higher protein (15.2\%) than that from $8 \mathrm{dS} \mathrm{m}{ }^{-1}$ and $16 \mathrm{dS} \mathrm{m}{ }^{-1} \mathrm{Na}_{2} \mathrm{SO}_{4}(14.4$ and $14.2 \%$, respectively).

A significant interaction of salinity $\times$ fertilization was detected, indicating that salinity differentially impacted seed

TABLE 1 | Analysis of variance with $F$-values for protein content, hardness, and density of quinoa seed.

\begin{tabular}{lccc}
\hline Effect & \multicolumn{3}{c}{ F-values } \\
\cline { 2 - 4 } & Protein & Hardness & Density \\
\hline Model & $5.2^{* * *}$ & $3.6^{* * *}$ & $2.45^{* * *}$ \\
Variety & $24.6^{* * *}$ & $210.6^{* * *}$ & $22.82^{* * *}$ \\
Salinity & $9.8^{* * *}$ & $2.0^{\dagger}$ & $2.82^{*}$ \\
Fertilization & $402.5^{* * *}$ & 1.1 & 2.60 \\
Variety $\times$ Salinity & 1.0 & 1.0 & 0.36 \\
Variety $\times$ Fertilization & $20.6^{* * *}$ & $10.9^{* * *}$ & $4.60^{* *}$ \\
Salinity $\times$ Fertilization & $3.4^{* *}$ & 1.4 & 0.71 \\
Variety $\times$ Salinity $\times$ Fertilization & 0.8 & $1.6^{\dagger}$ & 1.55 \\
\hline
\end{tabular}

†Significant at the 0.10 probability level. *Significant at the $<0.05$ probability level. **Significant at the 0.01 probability level. *** Significant at the $<0.001$ probability level. 
TABLE 2 | Salinity level and composition, variety, and fertilization effects on quinoa seed protein content (\%).

\begin{tabular}{|c|c|c|c|c|c|}
\hline Salinity & Protein content (\%) & Variety & Protein content (\%) & Fertilization & Protein content $(\%)$ \\
\hline $8 \mathrm{dS} \mathrm{m}^{-1} \mathrm{NaCl}$ & $14.7 b c^{1}$ & CO407D & 14.9ab & High & $15.8 a$ \\
\hline $16 \mathrm{dS} \mathrm{m}^{-1} \mathrm{NaCl}$ & $14.8 \mathrm{ab}$ & UDEC-1 & $14.7 \mathrm{~b}$ & Low & $13.6 b$ \\
\hline $32 \mathrm{dS} \mathrm{m}^{-1} \mathrm{NaCl}$ & $14.9 a b$ & Baer & $15.1 \mathrm{a}$ & & \\
\hline $8 \mathrm{dS} \mathrm{m}^{-1} \mathrm{Na}_{2} \mathrm{SO}_{4}$ & $14.4 \mathrm{~cd}$ & QQ065 & $14.1 \mathrm{c}$ & & \\
\hline $16 \mathrm{dS} \mathrm{m} \mathrm{m}^{-1} \mathrm{Na}_{2} \mathrm{SO}_{4}$ & $14.2 d$ & & & & \\
\hline $32 \mathrm{dS} \mathrm{m}^{-1} \mathrm{Na}_{2} \mathrm{SO}_{4}$ & $15.2 \mathrm{a}$ & & & & \\
\hline
\end{tabular}

${ }^{1}$ Different letters in a given column indicate significant differences $(P<0.05)$.

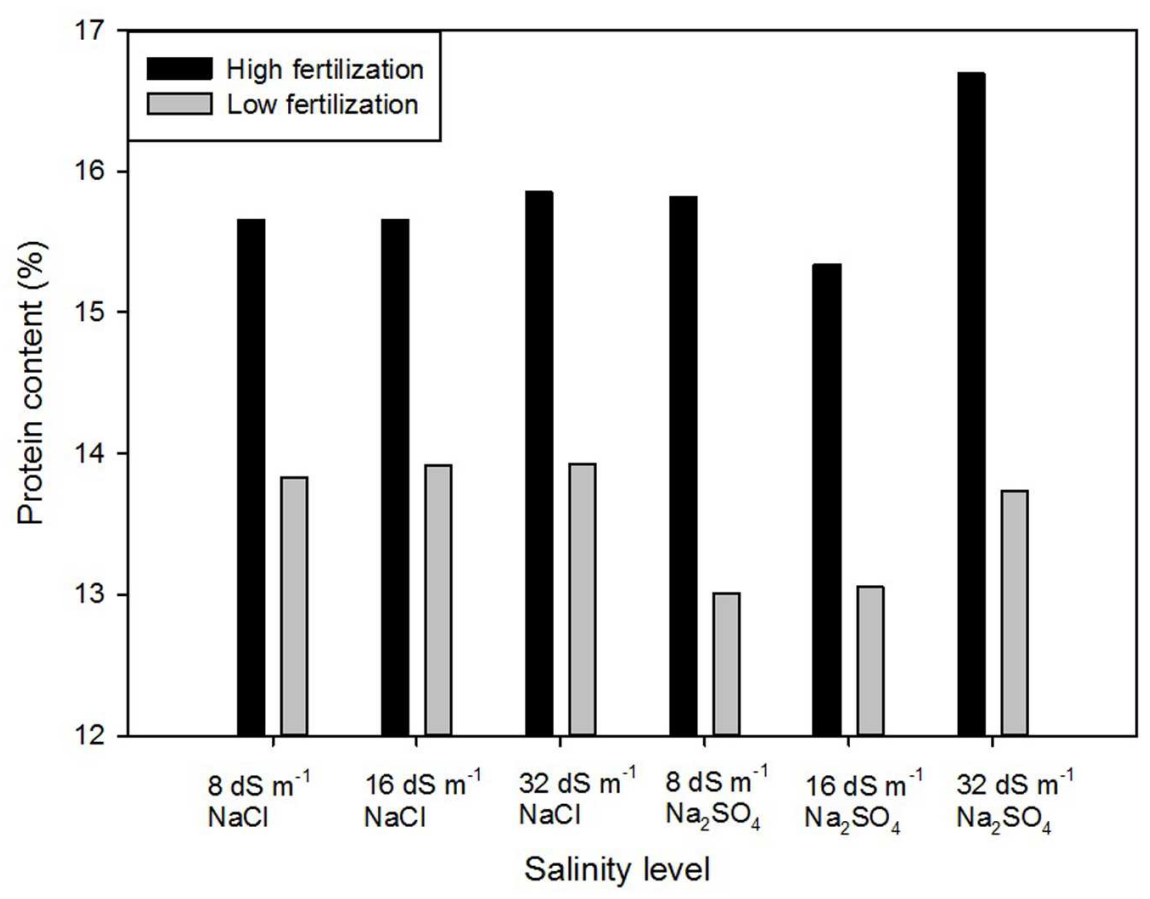

FIGURE 1 | Protein content (\%) of quinoa in response to combined fertility and salinity treatments.

protein content under high and low fertilization level (Figure 1). Within the high fertilizer treatment, seed protein content from $32 \mathrm{dS} \mathrm{m}^{-1} \mathrm{Na}_{2} \mathrm{SO}_{4}$ was significantly higher (16.7\%) than all other samples, which did not differ from each other $(\sim 13 \%)$. Within the low fertilizer treatment, seed protein content from $8 \mathrm{dS} \mathrm{m} \mathrm{m}^{-1}$ and $16 \mathrm{dS} \mathrm{m}^{-1} \mathrm{Na}_{2} \mathrm{SO}_{4}$ were significantly lower than those from the $\mathrm{NaCl}$ treatments and $32 \mathrm{dS} \mathrm{m}^{-1} \mathrm{Na}_{2} \mathrm{SO}_{4}$.

The significant interaction between variety and fertilization (Table 1) was due to the different response of QQ065. Protein mean of QQ065 from high fertilization was $14.4 \%$, lower than the other varieties. CO407D, UDEC-1, and Baer exhibited a decline of $16-18 \%$ in protein under low fertilization, while QQ065 dropped only $5 \%$.

\section{Hardness}

Variety exhibited the greatest influence on seed hardness ( $F=210.6, P<0.001)$, whereas fertilization did not show any significant effect (Table 1). Salinity exhibited a moderate effect $(F=2.0, P=0.09)$. Varieties responded consistently to salinity under various fertilization levels, since neither variety $\times$ salinity nor salinity $\times$ fertilization interaction was significant. However, a variety $\times$ fertilization interaction was observed, which will be discussed in a later paragraph. Similar to the situation in protein content, experiment repetition exhibited a significant influence on seed hardness. Whereas the hardness of CO407D was consistent across the two greenhouse experiments, the hardness of other three varieties all decreased by $8-9 \%$.

Mean hardness was significantly different among varieties. CO407D had the hardest seeds with hardness mean of $10.0 \mathrm{~kg}$ (Table 3). UDEC-1 was softer at $9.4 \mathrm{~kg}$, whereas Baer and QQ065 were the softest and with similar hardness means of 7.7 and $7.4 \mathrm{~kg}$, respectively.

Salinity exhibited a moderate impact on seed hardness $(P=0.09)$. The highest hardness mean was observed under 16 $\mathrm{dS} \mathrm{m} \mathrm{m}^{-1} \mathrm{Na}_{2} \mathrm{SO}_{4}$, whereas the lowest was under $8 \mathrm{dS} \mathrm{m}{ }^{-1} \mathrm{NaCl}$, with means of 8.9 and $8.3 \mathrm{~kg}$, respectively.

A significant fertilization $\times$ variety interaction was found for seed hardness. The hardness of UDEC-1 and Baer did not 
TABLE 3 | Salinity level and composition, variety, and fertilization effects on quinoa seed hardness (kg).

\begin{tabular}{lccc}
\hline Salinity & Hardness $\mathbf{( k g )})^{\mathbf{1}}$ & Variety & Hardness (kg) \\
\hline $8 \mathrm{dS} \mathrm{m}^{-1} \mathrm{NaCl}$ & 8.3 & CO407D & $10.0 \mathrm{a}^{2}$ \\
$16 \mathrm{dS} \mathrm{m}^{-1} \mathrm{NaCl}$ & 8.7 & UDEC-1 & $9.4 \mathrm{~b}$ \\
$32 \mathrm{dS} \mathrm{m}^{-1} \mathrm{NaCl}$ & 8.5 & Baer & $7.7 \mathrm{C}$ \\
$8 \mathrm{dS} \mathrm{m}^{-1} \mathrm{Na}_{2} \mathrm{SO}_{4}$ & 8.7 & QQ065 & $7.4 \mathrm{C}$ \\
$16 \mathrm{dS} \mathrm{m}^{-1} \mathrm{Na}_{2} \mathrm{SO}_{4}$ & 8.9 & & \\
$32 \mathrm{dS} \mathrm{m}^{-1} \mathrm{Na}_{2} \mathrm{SO}_{4}$ & 8.8 & &
\end{tabular}

${ }^{1}$ Hardness was significant at the 0.09 probability level. ${ }^{2}$ Different letters in a given column indicate significant differences $(P<0.05)$.

differ across fertilization level, whereas CO407D was harder under low fertilization, and QQ065 was harder under high fertilization.

\section{Seed Density}

Variety and salinity both significantly affected seed density, whereas fertilization did not show a significant influence (Table 1). The greatest contribution to variation in seed density was due to variety $(F=22.82)$. Salinity exhibited a relatively smaller effect, yet still significant $(F=2.82, P<0.05)$. Neither variety $\times$ salinity interaction nor salinity $\times$ fertilization interaction was observed, which indicated that varieties similarly responded to salinity under high and low fertilization levels. An interaction of variety $\times$ fertilization was found and the details are presented below.

Across all salinity and fertilization treatments, CO407D had the highest mean density, $0.80 \mathrm{~g} / \mathrm{cm}^{3}$, followed by Baer with $0.69 \mathrm{~g} / \mathrm{cm}^{3}$ (Table 4). UDEC-1 and QQ065 had the lowest and similar densities $\left(\sim 0.65 \mathrm{~g} / \mathrm{cm}^{3}\right)$.

With regard to salinity effect, the $\mathrm{Na}_{2} \mathrm{SO}_{4}$ treatments exhibited differential influence on seed density. Density means did not significantly change due to the increased concentration of $\mathrm{NaCl}$, which ranged from 0.68 to $0.71 \mathrm{~g} / \mathrm{cm}^{3}$ (Table 4). The samples from $8 \mathrm{dS} \mathrm{m}^{-1} \mathrm{Na}_{2} \mathrm{SO}_{4}$ soil had lower density $\left(0.66 \mathrm{~g} / \mathrm{cm}^{3}\right)$ than those from $16 \mathrm{dS} \mathrm{m}^{-1}$ and $32 \mathrm{dS} \mathrm{m}^{-1} \mathrm{Na}_{2} \mathrm{SO}_{4}, 0.74$ and $0.72 \mathrm{~g} / \mathrm{cm}^{3}$, respectively.

A significant variety $\times$ fertilization interaction was found. With closer examination, UDEC-1 and Baer yielded higher density seeds under high fertilization, whereas CO407D and QQ065 did not differ in density between fertilization treatments.

TABLE 4 | Salinity level and composition, variety, and fertilization effects on quinoa seed density $\left(\mathrm{g} / \mathrm{cm}^{3}\right)$.

\begin{tabular}{lccc}
\hline Salinity & Density $\left(\mathbf{g} / \mathbf{c m}^{\mathbf{3}}\right)$ & Variety & Density $\mathbf{( g / \mathbf { c m } ^ { \mathbf { 3 } } )}$ \\
\hline $8 \mathrm{dS} \mathrm{m}{ }^{-1} \mathrm{NaCl}$ & $0.69 \mathrm{bc}$ & CO407D & $0.80 \mathrm{a}$ \\
$16 \mathrm{dS} \mathrm{m}^{-1} \mathrm{NaCl}$ & $0.68 \mathrm{bc}$ & UDEC-1 & $0.66 \mathrm{bc}$ \\
$32 \mathrm{dS} \mathrm{m}^{-1} \mathrm{NaCl}$ & $0.71 \mathrm{abc}$ & Baer & $0.69 \mathrm{~b}$ \\
$8 \mathrm{dS} \mathrm{m}^{-1} \mathrm{Na}_{2} \mathrm{SO}_{4}$ & $0.66 \mathrm{c}$ & QQ065 & $0.65 \mathrm{c}$ \\
$16 \mathrm{dS} \mathrm{m}^{-1} \mathrm{Na}_{2} \mathrm{SO}_{4}$ & $0.74 \mathrm{a}$ & & \\
$32 \mathrm{dS} \mathrm{m}^{-1} \mathrm{Na}_{2} \mathrm{SO}_{4}$ & $0.72 \mathrm{ab}$ & & \\
\hline
\end{tabular}

${ }^{1}$ Different letters in a given column indicate significant differences $(P<0.05)$.

\section{Correlations of Protein, Hardness, and Density}

Correlation coefficients among seed protein content, hardness, and density are shown in Table 5. No significant correlation was detected between protein content and seed hardness. However, both protein content and hardness were correlated with seed density. The overall correlation coefficient was low $(r=0.19$, $P=0.03$ ) between density and protein. A marginally significant correlation was found between density and protein content of the seeds from $\mathrm{NaCl}$ salinized soil under low fertilization. No correlation was found between density and protein content of the seeds from $\mathrm{NaCl}$ salinized soil under high fertilization, or $\mathrm{Na}_{2} \mathrm{SO}_{4}$ salinized soil.

The overall correlation coefficient was $r=0.38(P<0.0001)$ between density and hardness. The low fertilization samples from both $\mathrm{NaCl}$ and $\mathrm{Na}_{2} \mathrm{SO}_{4}$ soil showed significant correlations between density and hardness, with coefficients of $r=0.51$ and 0.47 (both $P<0.005)$. The high fertility samples did not exhibit any correlation between density and hardness.

\section{Correlation with Yield, Leaf Greenness Index, Plant Height, and Seed Minerals Contents}

Correlation between seed quality and yield, leaf greenness index, plant height, and seed mineral concentration were obtained using data from Peterson and Murphy (2015) (Table 6). Seed hardness was significantly correlated with yield and plant height ( $r=0.35$ and 0.31 , respectively). Protein content and density, however, did not correlate with yield, leaf greenness, or plant height. Correlations were found between quality indices and the concentration of different minerals. Protein was negatively correlated with $\mathrm{Cu}$ and $\mathrm{Mg}(r=-0.52$ and -0.50 , respectively). Hardness was negatively correlated with $\mathrm{Cu}, \mathrm{P}$, and $\mathrm{Zn}(r=-0.37,-0.56,-0.29$, respectively), but was positively correlated with $\mathrm{Mn}(r=0.57)$. Density was negatively correlated with $\mathrm{Cu}(r=-0.35)$.

\section{DISCUSSION}

\section{Protein}

Although salinity exhibited a significant effect on seed protein content, the impact was relatively minor compared to fertilization and variety effects. In another words, over a wide range of saline soil, quinoa can grow and yield seeds with relatively stable protein content.

Protein content of quinoa growing under salinized soil ranged from 12.7 to $16.7 \%$ (data not shown), within the general range of protein content under non-saline conditions, which was 1217\% (Rojas et al., 2015). Saline soil did not cause a significant decrease in seed protein. It is interesting to notice that the samples from $32 \mathrm{dS} \mathrm{m}^{-1} \mathrm{Na}_{2} \mathrm{SO}_{4}$ tended to contain the highest protein, especially in variety QQ065. The studies of Karyotis et al. (2003) and Koyro and Eisa (2008) also indicated that protein content significantly increased under high salinity $(\mathrm{NaCl})$ whereas total carbohydrate decreased. In contrast, Ruffino et al. 
TABLE 5 | Correlation coefficients of protein, hardness, and density of quinoa seed.

\begin{tabular}{|c|c|c|c|c|c|}
\hline \multirow[t]{2}{*}{ Correlation } & \multirow[t]{2}{*}{ All } & \multicolumn{2}{|c|}{$\mathrm{NaCl}$} & \multicolumn{2}{|c|}{$\mathrm{Na}_{2} \mathrm{SO}_{4}$} \\
\hline & & High fertilization & Low fertilization & High fertilization & Low fertilization \\
\hline Protein - Density & $0.19^{*}$ & $0.13^{\text {ns }}$ & $0.29^{\dagger}$ & $0.26^{\text {ns }}$ & $0.19^{\text {ns }}$ \\
\hline Hardness - Density & $0.38^{* * *}$ & $0.27^{\mathrm{ns}}$ & $0.51^{* * *}$ & $0.22^{\mathrm{ns}}$ & $0.47^{* * *}$ \\
\hline
\end{tabular}

ns, Not significant. ${ }^{\dagger}$ Significant at the 0.10 probability level. * Significant at the $<0.05$ probability level. *** Significant at the $<0.001$ probability level.

TABLE 6 | Correlation coefficients of quinoa seed quality, and agronomic performance and seed mineral content ${ }^{\mathrm{a}}$.

\begin{tabular}{lccc}
\hline & Protein & Hardness & Density \\
\hline Yield & 0.04 & $0.35^{*}$ & 0.06 \\
Plant height & -0.04 & $0.31^{*}$ & 0.11 \\
$\mathrm{Cu}$ & $-0.52^{* * *}$ & $-0.37^{* *}$ & $-0.35^{*}$ \\
$\mathrm{Mg}$ & $-0.50^{* * *}$ & 0.04 & 0 \\
$\mathrm{Mn}$ & -0.06 & $0.57^{* * *}$ & $0.25^{\dagger}$ \\
$\mathrm{P}$ & -0.01 & $-0.56^{* * *}$ & -0.15 \\
$\mathrm{Zn}$ & -0.04 & $-0.29^{*}$ & $-0.28^{\dagger}$
\end{tabular}

aAgronomic and mineral contents taken from Peterson and Murphy (2015). †Significant at the 0.10 probability level. *Significant at the $<0.05$ probability level. ** Significant at the 0.01 probability level. *** Significant at the $<0.001$ probability level.

(2010) found that quinoa protein decreased under $250 \mathrm{mM} \mathrm{NaCl}$ salinity in a growth chamber experiment. It is reasonable to conclude that salinity exhibits contrasting effects on different quinoa genotypes.

$\mathrm{Na}_{2} \mathrm{SO}_{4}$ level exhibited a significant influence on protein content, whereas $\mathrm{NaCl}$ level did not. In the study of Koyro and Eisa (2008), however, seed protein content of the quinoa variety Hualhuas (origin Peru) increased under the highest salinity level of $500 \mathrm{mM} \mathrm{NaCl}$ compared to lower $\mathrm{NaCl}$ levels $(0-400 \mathrm{mM})$. This disagreement of $\mathrm{NaCl}$ influence may be due to diversity of genotypes. It is worth noting that quinoa protein contents in this paper were primarily above $13 \%$ based on a wet weight basis (asis-moisture of approximately $\sim 8-10 \%$ ) even under saline soil and low fertilization level. This protein content is generally equal to or higher than that of other crops such as barley and rice $(\mathrm{Wu}$, 2015). In conclusion, quinoa maintained high and stable protein content under salinity stress.

Besides seed quality, grain yield is another significant factor when quinoa is grown in marginal environments. The yield data were reported in the previous study (Peterson and Murphy, 2015). Under $32 \mathrm{dS} \mathrm{m}^{-1} \mathrm{Na}_{2} \mathrm{SO}_{4}$, the yield of CO407D, UDEC1 , and Baer decreased by $24.5,10.8$, and $11.6 \%$, respectively, and the yield decline was much lower than a barley variety Albacete (yield decline of $82.4 \%$ ). The yield decline of QQ065 was $54.5 \%$ under $32 \mathrm{dS} \mathrm{m}^{-1} \mathrm{Na}_{2} \mathrm{SO}_{4}$. In the same soil condition, both CO407D and QQ065 exhibited increased protein content. Hence, if only protein content is considered, QQ065 contained the highest protein under high $\mathrm{Na}_{2} \mathrm{SO}_{4}$; however, if both yield and protein content are considered, then CO407D is the variety more suitable for severe $\mathrm{Na}_{2} \mathrm{SO}_{4}$ affected areas since it exhibited a moderate yield decline and a significant increase in protein content. It also implies the importance to evaluate both agronomy performance and seed quality when studying crops' adaption to extreme environments.

\section{Hardness}

Quinoa seed hardness was only moderately affected by salinity $(P=0.09)$, indicating that quinoa primarily maintained seed texture when growing under a wide range of saline soil. CO407D exhibited the hardest seed $(10.0 \mathrm{~kg})$, whereas Baer and QQ065 were relatively soft $(7.4-7.7 \mathrm{~kg})$. A previous study indicated a hardness range of $5.8-10.9 \mathrm{~kg}$ among 11 quinoa varieties and 2 commercial samples (Wu et al., 2014). The commercial samples had hardness values of 6.2 and $7.1 \mathrm{~kg}$. Since commercial samples generally maintain stable quality and indicate an acceptable level for consumers, seed hardness around $7 \mathrm{~kg}$, as in Baer and QQ065, should be considered as acceptable quality. The hardness of CO407D was close to that of the colored variety 'Black' $(10.0 \mathrm{~kg})$, which had a thicker seed coat than that of the yellow seeded varieties. It was reported that a thicker seed coat is related to harder texture (Fraczek et al., 2005).

Even though the greenhouse is a highly controlled environment, and the two experiments were conducted in similar seasons (planted in September and October, respectively), seed protein and hardness were nevertheless different across the two experiments. However, ANOVA indicated modest-to-no significant interactions with salinity and fertilization such that responses to salinity and fertilization were consistent with little or no change in rank order. Even though the experiment $x$ variety interaction was significant, the $F$-values were relatively low compared to the major effects such as variety and fertilization, and neither of them exhibited cross-over interaction. This is a particularly noteworthy result for breeders, farmers, and processors.

\section{Density}

The range of seed density under salinity, $0.55-0.89 \mathrm{~g} / \mathrm{cm}^{3}$, was comparable to the density range of 13 quinoa samples $(0.58$ $0.76 \mathrm{~g} / \mathrm{cm}^{3}$ ) (Wu et al., 2014). Generally, CO407D had higher seed density $\left(0.71-0.89 \mathrm{~g} / \mathrm{cm}^{3}\right)$, which indicated that seed density of this variety was affected by salinity stress. In contrast, the density of QQ065 did not change according to salinity type or concentration, which indicated a stable quality under saline soil.

\section{Correlations}

The correlation between seed hardness and density was only significant under low fertilization, but not under high fertilization. The high fertilization level in the greenhouse experiment exceeded the amount of fertilizer that would 
normally be applied in field environments, whereas the low fertilization level was closer to the field situation. Therefore, correlation between hardness and density may still exist in field trials.

\section{CONCLUSION}

This study confirmed quinoa's tolerance to salinity stress in terms of seed quality. Under saline soil conditions, quinoa did not show any marked decrease in seed quality such as protein content, hardness, and density. Protein content even increased under high $\mathrm{Na}_{2} \mathrm{SO}_{4}$ concentration $\left(32 \mathrm{dS} \mathrm{m} \mathrm{m}^{-1}\right.$ ). Varieties exhibited great differential reactions to fertilization and salinity levels. QQ065 maintained a similar level of hardness and density, whereas seed of CO407D was both harder and higher density under salinity stress. If only seed quality is considered, then QQ065 is the most well-adapted variety in this study. Additionally, the influences of $\mathrm{NaCl}$ and $\mathrm{Na}_{2} \mathrm{SO}_{4}$ were different. The higher concentration of $\mathrm{Na}_{2} \mathrm{SO}_{4}$ tended to increase protein content and seed density, whereas $\mathrm{NaCl}$ concentration did not exhibit any significant difference on those quality indexes. In other words, quinoa can be grown in areas severely affected by $\mathrm{Na}_{2} \mathrm{SO}_{4}$ and still produce high protein seeds, which can be a good protein resource for vegetarians or malnourished populations.

In the present study, protein content, seed hardness, and density were selected as quinoa seed quality indexes due to their significance in nutritional value and end-use quality. Besides those factors, other seed components such as essential amino acids, starch, fiber, and minerals are also important to nutrition value. Their variation under different salt types should be investigated in the future. Hence, when quinoa is applied to

\section{REFERENCES}

AACC International (1995). Approved Methods of Analysis. Method 46-30. 01. Crude Protein - Combustion Method. St. Paul, MN: AACCI.

Adolf, V. I., Shabala, S., Andersen, M. N., Razzaghi, F., and Jacobsen, S. E. (2012). Varietal differences of quinoa's tolerance to saline conditions. Plant Soil 357, 117-129. doi: 10.1007/s11104-0121133-7

Bertero, H. D. (2003). Response of developmental processes to temperature and photoperiod in quinoa (Chenopodium quinoa Willd.). Food Rev. Int. 19, 87-97. doi: 10.1081/FRI-120018870

Cai, S., Yu, G., Chen, X., Huang, Y., Jiang, X., Zhang, G., et al. (2013). Grain protein content variation and its association analysis in barley. BMC Plant Boil. 13:35. doi: 10.1186/1471-2229-13-35

Chilo, G., Molina, M. V., Carabajal, R., and Ochoa, M. (2009). Temperature and salinity effects on germination and seedling growth on two varieties of Chenopodium quinoa. Agri-Scientia 26, 15-22.

Cocozza, C., Pulvento, C., Lavini, A., Riccardi, M., d'Andria, R., and Tognetti, R. (2013). Effects of increasing salinity stress and decreasing water availability on ecophysiological traits of quinoa (Chenopodium quinoa Willd.) grown in a mediterranean-type agroecosystem. J. Agron. Crop Sci. 199, 229-240. doi: $10.1111 /$ jac. 12012

Fraczek, J., Hebda, T., Slipek, Z., and Kurpaska, S. (2005). Effect of seed coat thickness on seed hardness. Can. Biosyst. Eng. 47, 41-45.

González, J. A., Eisa, S. S. S., Hussin, S. A. E. S., and Prado, F. E. (2015). “Quinoa: an Incan crop to face global changes in agriculture," in Quinoa: Improvement and Sustainable Production, eds K. M. Murphy and J. Matanguihan (Hoboken, NJ: John Wiley \& Sons), 7-11. a specific salinity area, the right varieties can be utilized not only with the consideration of yield but also the health benefits to local people. When addressing malnutrition in developed countries, the profile of essential amino acids, protein quality (such as protein efficiency ratio), and deficient minerals should be specifically evaluated. Furthermore, this study was based on greenhouse experiments. The soil conditions and environment in the field are much more complex than the soil medium used in the greenhouse. Based on the conclusion from this research, further field study should be conducted using the varieties best adapted to specific salt type and salinity level.

\section{AUTHOR CONTRIBUTIONS}

AP set up the experiment design in the greenhouse and grew, harvested, and processed quinoa samples. GW collected seed quality data such as protein content, seed hardness, and density. $\mathrm{AP}$ and GW together processed the data. GW also drafted the manuscript. KM and CM edited the manuscript.

\section{FUNDING}

The research was funded by USDA Organic Research and Extension Initiative, project number NIFA:GRANT11083982.

\section{ACKNOWLEDGMENT}

The authors acknowledge Alecia Kiszonas for assisting in the data analysis.

Hrušková, M., and Švec, I. (2009). Wheat hardness in relation to other quality factors. Czech J. Food Sci. 27, 240-248.

Jacobsen, S., Quispe, H., and Mujica, A. (2000). "Quinoa: an alternative crop for saline soils in the Andes," in Scientist and Farmer: Partners in Research for the 21st Century (Program Report 1999-2000), ed. W. Collins (Peru: International Potato Center), 403-408.

Karyotis, T., Iliadis, C., Noulas, C., and Mitsibonas, T. (2003). Preliminary research on seed production and nutrient content for certain quinoa varieties in a saline-sodic soil. J. Agron. Crop Sci. 189, 402-408. doi: 10.1046/j.09312250.2003.00063.x

Koyro, H.-W., and Eisa, S. (2008). Effect of salinity on composition, viability and germination of seeds of Chenopodium quinoa Willd. Plant Soil 302, 79-90. doi: 10.1007/s11104-007-9457-4

Krishnamurthy, K., and Giroux, M. J. (2001). Expression of wheat puroindoline genes in transgenic rice enhances grain softness. Nat. Biotechnol. 19, 162-166. doi: $10.1038 / 84435$

Morris, C. F. (2002). Puroindolines: the molecular genetic basis of wheat grain hardness. Plant Mol. Biol. 48, 633-647. doi: 10.1023/A:1014837431178

Orth, R. A., and Shellenberger, J. A. (1988). "Chapter 1: Origin, production, and utilization of wheat," in Wheat: Chemistry and Technology, 3th Edn, ed. Y. Pomeranz (St. Paul, MN: American Association of Cereal Chemists, Inc.), 11-12.

Peterson, A., and Murphy, K. (2015). Tolerance of lowland quinoa cultivars to sodium chloride and sodium sulfate salinity. Crop Sci. 55, 331-338. doi: $10.2135 /$ cropsci2014.04.0271

Peterson, A. J. (2013). Salinity Tolerance and Nitrogen Use Efficiency of Quinoa for Expanded Production in Temperate North America. Ph.D. thesis, Washington State University, Pullman. 
Pitman, M. G., and Läuchli, A., (2002). "Global impact of salinity and agricultural ecosystems," in Salinity: Environment-Plants-Molecules, eds A. Läuchli and U. Lüttge (Netherlands: Springer), 3-20.

Prado, F. E., Boero, C., Gallardo, M., and González, J. A. (2000). Effect of NaCl on germination, growth, and soluble sugar content in Chenopodium quinoa Willd. seeds. Bot. Bull. Acad. Sinica 41, 27-34.

Pulvento, C., Riccardi, M., Lavini, A., Iafelice, G., Marconi, E., and d'Andria, R. (2012). Yield and quality characteristics of quinoa grown in open field under different saline and non-saline irrigation regimes. J. Agron. Crop Sci. 198, 254-263. doi: 10.1111/j.1439-037X.2012. 00509.x

Ranhotra, G., Gelroth, J., Glaser, B., Lorenz, K., and Johnson, D. (1993). Composition and protein nutritional quality of quinoa. Cereal Chem. 70, 303-305.

Razzaghi, F., Ahmadi, S. H., Jacobsen, S. E., Jensen, C. R., and Andersen, M. N. (2012). Effects of salinity and soil-drying on radiation use efficiency, water productivity and yield of quinoa (Chenopodium quinoa Willd.). J. Agron. Crop Sci. 198, 173-184. doi: 10.1111/j.1439-037X.2011.00496.x

Rojas, W., Pinto, M., Alanoca, C., Pando, L. G., Leónlobos, P., Alercia, A., et al. (2015). “Chapter 1.5: Quinoa genetic resources and ex situ conservation," in FAO \& CIRAD. State of the Art Report on Quinoa in 2013, eds D. Bazile, D. Bertero, and C. Nieto (Rome: FAO), 56-82.

Ruffino, A., Rosa, M., Hilal, M., González, J., and Prado, F. (2010). The role of cotyledon metabolism in the establishment of quinoa (Chenopodium quinoa) seedlings growing under salinity. Plant Soil 326, 213-224. doi: 10.1007/s11104009-9999-8

Ruiz-Carrasco, K., Antognoni, F., Coulibaly, A. K., Lizardi, S., Covarrubias, A., Martínez, E. A., et al. (2011). Variation in salinity tolerance of four lowland genotypes of quinoa (Chenopodium quinoa Willd.) as assessed by growth, physiological traits, and sodium transporter gene expression. Plant Physiol. Biochem. 49, 1333-1341. doi: 10.1016/j.plaphy.2011.08.005

Shabala, S., Hariadi, Y., and Jacobsen, S.-E., et al. (2013). Genotypic difference in salinity tolerance in quinoa is determined by differential control of xylem $\mathrm{Na}+$ loading and stomatal density. J. Plant Physiol. 170, 906-914. doi: 10.1016/j.jplph.2013.01.014
Shih, F. F. (2006). "Chapter 6: Rice protein,” in Rice: Chemistry and Technology, 3th Edn, ed. E. T. Champagne (St. Paul, MN: American Association of Cereal Chemists, Inc.), 143-144.

Taylor, J. R. N., and Parker, M. L. (2002). “Chapter 3: Quinoa," in Pseudocereals and Less Common Cereals: Grain Properties and Utilization Potential, eds P. Belton and J. Taylor (Berlin: Springer Science and Business Media), 100-101.

United States Department of Agriculture [USDA] (2011). Soil and Water Resources Conservation Act (RCA). P 31. Available at: http://www.nrcs.usda.gov/Internet/FSE_DOCUMENTS/stelprdb1044939.pdf

Wilson, C., Read, J., and Abo-Kassem, E. (2002). Effect of mixed-salt salinity on growth and ion relations of a quinoa and a wheat variety. J. Plant Nutri. 25, 2689-2704. doi: 10.1081/PLN-120015532

Wu, G. (2015). "Nutritional properties of quinoa," in Quinoa: Improvement and Sustainable Production, eds K. M. Murphy and J. Matanguihan (Hoboken, NJ: John Wiley \& Sons), 193-194.

Wu, G., Morris, C. F., and Murphy, K. M. (2014). Evaluation of texture differences among varieties of cooked quinoa. J. Food Sci. 79, 2337-2345. doi: 10.1111/1750-3841.12672

Zevallos, V. F., Herencia, L. I., Chang, F., Donnelly, S., Ellis, H. J., and Ciclitira, P. J. (2014). Gastrointestinal effects of eating quinoa (Chenopodium quinoa Willd.) in celiac patients. Am. J. Gastroenterol. 109, 270-278. doi: 10.1038/ajg.2013.431

Zhang, B., Chen, P., Chen, C. Y., Wang, D., Shi, A., and Hou, A. (2008). Quantitative trait loci mapping of seed hardness in soybean. Crop Sci. 48, 1341-1349. doi: 10.2135/cropsci2007.10.0544

Conflict of Interest Statement: The authors declare that the research was conducted in the absence of any commercial or financial relationships that could be construed as a potential conflict of interest.

Copyright (C) 2016 Wu, Peterson, Morris and Murphy. This is an open-access article distributed under the terms of the Creative Commons Attribution License (CC BY). The use, distribution or reproduction in other forums is permitted, provided the original author(s) or licensor are credited and that the original publication in this journal is cited, in accordance with accepted academic practice. No use, distribution or reproduction is permitted which does not comply with these terms. 
OPEN ACCESS

Edited by: José J. Pueyo,

Consejo Superior de Investigaciones Científicas, Spain

Reviewed by:

Sara Maldonado,

University of Buenos Aires, Argentina

Sergey Shabala,

University of Tasmania, Australia

${ }^{*}$ Correspondence: Stefania Biondi stefania.biondi@unibo.it; Stefano Del Duca

stefano.delduca@unibo.it

${ }^{\dagger}$ These authors have contributed equally to this work.

Specialty section: This article was submitted to

Crop Science and Horticulture, a section of the journal

Frontiers in Plant Science

Received: 30 December 2015 Accepted: 28 April 2016 Published: 18 May 2016

Citation: Aloisi I, Parrotta L, Ruiz KB, Landi C, Bini L, Cai G, Biondi S and Del Duca S (2016) New Insight into Quinoa Seed

Quality under Salinity: Changes in Proteomic and Amino Acid Profiles,

Phenolic Content, and Antioxidant Activity of Protein Extracts.

Front. Plant Sci. 7:656. do: $10.3389 /$ fpls.2016.00656

\section{New Insight into Quinoa Seed Quality under Salinity: Changes in Proteomic and Amino Acid Profiles, Phenolic Content, and Antioxidant Activity of Protein Extracts}

\author{
Iris Aloisi ${ }^{1+}$, Luigi Parrotta ${ }^{1 \dagger}$, Karina B. Ruiz ${ }^{1,2}$, Claudia Landi ${ }^{3}$, Luca Bini ${ }^{3}$, \\ Giampiero Cai ${ }^{3}$, Stefania Biondi ${ }^{1 *}$ and Stefano Del Duca ${ }^{1 *}$
}

${ }^{1}$ Department of Biological, Geological and Environmental Sciences, University of Bologna, Bologna, Italy, ${ }^{2}$ Departamento de Producción Agrícola, Universidad de Chile, Santiago, Chile, ${ }^{3}$ Department of Life Sciences, University of Siena, Siena, Italy

Quinoa (Chenopodium quinoa Willd) is an ancient Andean seed-producing crop well known for its exceptional nutritional properties and resistance to adverse environmental conditions, such as salinity and drought. Seed storage proteins, amino acid composition, and bioactive compounds play a crucial role in determining the nutritional value of quinoa. Seeds harvested from three Chilean landraces of quinoa, one belonging to the salares ecotype (R49) and two to the coastal-lowlands ecotype, VI-1 and Villarrica (VR), exposed to two levels of salinity (100 and $300 \mathrm{mM} \mathrm{NaCl}$ ) were used to conduct a sequential extraction of storage proteins in order to obtain fractions enriched in albumins/globulins, $11 \mathrm{~S}$ globulin and in prolamin-like proteins. The composition of the resulting protein fractions was analyzed by one- and two-dimensional polyacrylamide gel electrophoresis. Results confirmed a high polymorphism in seed storage proteins; the two most representative genotype-specific bands of the albumin/globulin fraction were the 30- and 32-kDa bands, while the 11S globulin showed genotype-specific polymorphism for the 40- and 42-kDa bands. Spot analysis by mass spectrometry followed by in silico analyses were conducted to identify the proteins whose expression changed most significantly in response to salinity in VR. Proteins belonging to several functional categories (i.e., stress protein, metabolism, and storage) were affected by salinity. Other nutritional and functional properties, namely amino acid profiles, total polyphenol (TPC) and flavonoid (TFC) contents, and antioxidant activity (AA) of protein extracts were also analyzed. With the exception of Ala and Met in R49, all amino acids derived from protein hydrolysis were diminished in seeds from salt-treated plants, especially in landrace VI-1. By contrast, several free amino acids were unchanged or increased by salinity in R49 as compared with VR and Vl-1, suggesting a greater tolerance in the salares landrace. VR had the highest TPC and AA under non-saline conditions. Salinity increased TPC in all three landraces, with the strongest increase occurring in R49, and enhanced radical scavenging capacity in R49 and VR. Overall, results show that salinity deeply altered the seed proteome and amino acid profiles and, 
in general, increased the concentration of bioactive molecules and AA of protein extracts

in a genotype-dependent manner.

Keywords: antioxidant activity, Chenopodium quinoa, polyphenols, salt stress, seed storage proteins

\section{INTRODUCTION}

Quinoa (Chenopodium quinoa Willd., Amaranthaceae) is an Andean seed-producing crop cultivated since ca. 7000 years around Lake Titicaca in the Andean highlands (altiplano) from where it spread as far north as Ecuador and down to southern Chile, and from $3800 \mathrm{~m}$ above sea level to coastal and lowlands areas. This diversification in terms of native habitats and the wide genetic diversity has led to the identification of five ecotypes: salares (salt flats), highlands, inter-Andean valleys, yungas, and coastal-lowlands. The salares of the Andes are found principally in southern Bolivia, northern Chile, and Argentina. These highland deserts are extremely arid; temperatures often fall well below freezing and quinoa is the only crop that can grow under these edapho-climatic conditions (Fuentes et al., 2009). Other landraces (local varieties) are adapted to totally different environments. For example, in central and southern Chile, quinoa can grow at sea level; here annual rainfall, distributed throughout the year, ranges from 400 to $1500-2000 \mathrm{~mm}$ and soils have a high water retention capacity. Thus, quinoa has attracted the attention of scientists since over a decade mainly for its extreme tolerance and adaptability to unfavorable environmental conditions, such as salinity, drought, and frost (Jacobsen et al., 2003). However, different accessions, landraces, and cultivars of quinoa have been shown to exhibit different degrees of tolerance to abiotic, in particular salt stress (Gómez-Pando et al., 2010; Adolf et al., 2012; Peterson and Murphy, 2015).

Consumption of seeds is the most common use of quinoa, which is, therefore, considered a "cereal-like" crop. European and North American consumers are increasingly aware of the exceptional nutritional qualities of quinoa seeds that, together with sprouts, are now considered "functional foods" (VegaGálvez et al., 2010). This is because seeds have high protein content and contain all the essential amino acids (Abugoch et al., 2008). In quinoa seeds, protein and lipid reserves are accumulated in embryonic protein and lipid bodies, respectively, which occupy most of the cell (Prego et al., 1998). The classification of seed storage proteins dates from the turn of the century, when Osborne (1924) classified them into groups on the basis of their extraction and solubility, convention still used nowadays for storage proteins from plant seeds in general. These proteins are soluble in water (albumins), dilute saline solution (globulins), alcohol mixtures (prolamins), and dilute acid or alkali (glutelins). The major seed storage proteins include albumins, globulins, and prolamins (Shewry et al., 1995). The main protein fractions in quinoa seeds are globulins and albumins. Brinegar and Goundan (1993) characterized the 11S storage protein, chenopodin, and showed that it is composed of two subunit groups bridged by a disulfide bond, the acidic A subunit group (32-39 $\mathrm{kDa}$ ) and the basic B subunit group (22$23 \mathrm{kDa})$. Moreover, they reported that the $11 \mathrm{~S}$ and $2 \mathrm{~S}(8-9 \mathrm{kDa})$ polypeptides represented ca. 35 and $37 \%$, respectively of total proteins (Brinegar and Goundan, 1993; Brinegar et al., 1996). The genomic and amino acid sequences of the $11 \mathrm{~S}$ globulin of quinoa have been reported (Balzotti et al., 2008).

Quinoa seeds are also rich in bioactive compounds, such as vitamins (vitamin B2, vitamin E), carotene, tocopherols, and other molecules exerting antioxidant properties (e.g., phenolics) that scavenge harmful radicals (Paśko et al., 2009; Hirose et al., 2010; Repo-Carrasco-Valencia et al., 2010; Miranda et al., 2014). Diversity in these nutritional traits across genotypes has been reported (González et al., 2011; Vidueiros et al., 2015). In seeds of six quinoa genotypes from three geographical areas of Chile, significant differences in all the parameters analyzed were reported, with VR showing the highest content of protein, and vitamins E and C (Miranda et al., 2013, 2014). Seeds were also described as good sources of antioxidant compounds, although phenolic content, antioxidant and antimicrobial activities also varied among genotypes.

While many studies have been performed to investigate the tolerance of different quinoa genotypes to abiotic stress (mainly salinity) in terms of agronomic performance (growth, yield) and morpho/physiological mechanisms underlying salt tolerance (Adolf et al., 2013; Ruiz et al., 2016b), fewer have been devoted to the effects of high salinity on the nutritional quality of quinoa seeds. Increases or no effects in total protein content have been reported in most cases (Karyotis et al., 2003; Hariadi et al., 2011; Pulvento et al., 2012; Miranda et al., 2013). The effects of drought and salinity on seed phenolic content were investigated and only limited changes in these compounds under reduced irrigation (with or without salinity) were found (Gómez-Caravaca et al., 2012). Recently, increased total polyphenolics content (TPC) and antioxidant activity (AA) in methanol extracts of quinoa seeds harvested from plants grown under salinity has been observed, suggesting that stressful conditions may positively affect the seed's content of these important bioactive compounds (Ruiz et al., 2016a). Flour and the protein concentrate (PC) of seeds of amaranth, a close relative of quinoa, have been shown to contain polyphenols and to possess AA (Escudero et al., 2011). To date, these parameters have not been investigated in quinoa protein extracts.

The purpose of the present work was to investigate changes in the amino acid and protein profiles of seeds obtained from quinoa plants grown under saline conditions. In general, the capacity to accumulate more storage proteins in seeds plays an important role in the initial stages of the next generation, especially germination (Koyro and Eisa, 2008). Our purpose, however, was to conduct a more detailed investigation on the changes occurring under salinity in the relative amounts of different protein fractions (albumin/globulin fraction) and, more specifically, in spot patterns of individual proteins. The proteomic analysis reveals which proteins are involved in the 
salt-stress response in quinoa, thus contributing to a better understanding of the complex metabolic network involved in stress responses of halophytes (Koyro et al., 2013). In addition, the TPC and AA of protein extracts were evaluated in control and salinized seeds. These features strongly contribute to the nutraceutical properties of quinoa (Repo-CarrascoValencia et al., 2010; Abderrahim et al., 2015; Tang et al., 2015), may influence seed longevity (Sano et al., 2015), and can ultimately lead to the production of PCs to be used in the food industry and as dietary complements for their high protein level, functional properties, and low content of antinutritional factors (Cordero-De-Los-Santos et al., 2005; Escudero et al., 2011; Castel et al., 2014). To date, the phenolics content and AA of quinoa seed protein extracts have not yet been examined. Given the strong genotypic differences reported for all aspects of quinoa's responses to saline conditions (Adolf et al., 2012; Ruiz et al., 2016b), including nutritional aspects (Miranda et al., 2014; Abderrahim et al., 2015; Tang et al., 2015; Vidueiros et al., 2015), these parameters were comparatively analyzed in different genotypes. Thus, three Chilean landraces originating from contrasting habitats, namely R49, belonging to the salares ecotype, and two landraces belonging to the coastallowlands ecotype but from different latitudes and altitudes (VI1, Villarrica), were examined. Seeds were harvested from plants grown in a pot experiment under saline $(100$ or $300 \mathrm{mM} \mathrm{NaCl})$ and non-saline $(0 \mathrm{mM} \mathrm{NaCl})$ conditions.

\section{MATERIALS AND METHODS}

\section{Plant Material}

Seeds of three Chilean landraces of C. quinoa (Willd.), one belonging to the salares ecotype (R49) and two, VI-1 and Villarrica (VR), to the coastal-lowlands ecotype were collected along an altitudinal gradient from the arid northern highland with saline soils (R49, $3800 \mathrm{~m}$ a.s.l.) to sea level, and along a latitudinal gradient of ca. $2500 \mathrm{~km}$, from ca. $34^{\circ} \mathrm{S}$ (VI-1) down to the rainier southern region (ca. $39^{\circ} \mathrm{S}, \mathrm{VR}$ ) with higher precipitation and non-saline soils (Peterson and Murphy, 2015). All seeds were obtained from the National Seed Bank of Chile managed by INIA-Intihuasi (Vicuña, Chile).

Vernalized seeds were sown in 20-L plastic pots containing a garden soil:sand (1:1) mixture. When plants had four to six wellexpanded leaves (ca. 34 days after sowing) salt treatment was started by irrigating pots weekly with 0,100 , or $300 \mathrm{mM} \mathrm{NaCl}$ solutions; all the pots (control and salt-treated) were also watered weekly with 100-200 mL water supplemented with Phostrogen (N:P:K 10:10:27; 0.4 $\mathrm{g} \mathrm{L}^{-1}$; Bayer Garden, Cambridge, UK). Plants were grown (October to April) in a greenhouse under natural daylight conditions; the temperature was maintained at $23 \pm 3^{\circ} \mathrm{C}$. Seeds were collected at maturity starting from 91 days and up to 140 days after the first salt treatment, depending on the landrace, weighed, and stored in an air-tight container at $4^{\circ} \mathrm{C}$ until use.

\section{Chemicals}

All chemicals were obtained from Sigma-Aldrich (Milan, Italy) unless otherwise indicated.

\section{Seed Flour Preparation}

In order to remove saponins, quinoa seeds were washed repeatedly with cold water until there was no more foam in the wash water, and then dried at $50^{\circ} \mathrm{C}$ up to $15 \pm 3 \%$ moisture. The dried seeds were ground to a fine powder using a mortar and pestle. The resulting flour was defatted with hexane under continuous stirring overnight and then air-dried at room temperature. Flours were stored at $4^{\circ} \mathrm{C}$ until use.

\section{Preparation of Protein Fractions}

Protein fractions were prepared by a solvent-based sequential extraction following the methods of Ju et al. (2001), Bergamo et al. (2011), Džunková et al. (2011), and Zevallos et al. (2012), with slight modifications. Briefly, the albumin/globulin fraction was obtained by suspending $50 \mathrm{mg}$ of flour in $300 \mu \mathrm{l}$ of $5 \% \mathrm{NaCl}$; the suspension was then homogenized for $5 \mathrm{~min}$ and centrifuged at $5500 \mathrm{~g}$ for $10 \mathrm{~min}$. The procedure was repeated twice and the supernatants were collected. The flour was further extracted for prolamin-like proteins following the same procedure but using $250 \mu \mathrm{l}$ of $60 \%(\mathrm{v} / \mathrm{v})$ aqueous ethanol. The crude acid-soluble globulins and the 11S-enriched fraction were simultaneously isolated following Thanh et al. (1975). Briefly, defatted quinoa flour was extracted with $63 \mathrm{mM}$ Tris- $\mathrm{HCl}$ buffer containing 10 $\mathrm{mM} \beta$-mercaptoethanol, $\mathrm{pH} 7.8$, for $1 \mathrm{~h}$ and then centrifuged for $15 \mathrm{~min}$ at $9500 \times \mathrm{g}$. The extraction buffer was adjusted to $\mathrm{pH}$ 6.6, dialyzed against $63 \mathrm{mM}$ Tris- $\mathrm{HCl}$ containing $10 \mathrm{mM} \beta$ mercaptoethanol, $\mathrm{pH} 6.6$, at $4^{\circ} \mathrm{C}$ for at least $4 \mathrm{~h}$ and centrifuged for $20 \mathrm{~min}$ at $9500 \times \mathrm{g}$. The precipitate corresponded to the crude 11S fraction while the supernatant, which corresponded to the crude acid-soluble globulin fraction, was further purified by adjusting the $\mathrm{pH}$ to 4.8 . After centrifugation, the precipitate was dispersed in water and the $\mathrm{pH}$ was raised to 7.0. For assaying radical scavenging activity, extraction of the 11Senriched fraction was performed by omitting $\beta$-mercaptoethanol both in the extraction and dialyzing buffers (Thanh et al., 1975). Protein concentration was determined spectrophotometrically at $562 \mathrm{~nm}$ using the bicinchoninic acid kit and bovine serum albumin (BSA) as standard.

\section{SDS-PAGE and Gel Staining}

All one-dimensional electrophoretic runs were performed with the Mini Protean III apparatus (Bio-Rad Laboratories, Segrate, Italy). Proteins (40 $\mu \mathrm{g}$ lane $\mathrm{e}^{-1}$ ) were separated by SDS polyacrylamide gel electrophoresis (SDS-PAGE) according to the method of Laemmli (1970). For the glutenin-like enriched fraction, $2 \mathrm{M}$ urea was added to both stacking and resolving gels. The molecular mass standard was the Biomol (Hamburg, Germany) BLUEplus prestained Protein Ladder (10-180 kDa). Gels were fixed at room temperature in 50\% methanol: 5\% glacial acetic acid for $20 \mathrm{~min}$, then in 50\% methanol for $10 \mathrm{~min}$. After fixing, gels were washed twice in deionized water (10 min each) and stained with silver as previously described (Shevchenko et al., 1996) with minor modifications. Protein profiles were densitometrically analyzed using the AIDA software 4.14 (raytest Isotopenmessgeräte $\mathrm{GmbH}$, Straubenhardt, Germany). 


\section{Protein Precipitation}

For protein precipitation, 4 vol of $20 \%$ trichloroacetic acid (TCA) and $0.007 \% \beta$-mercaptoethanol in cold acetone were added to samples, mixed and kept at $-20^{\circ} \mathrm{C}$ for at least $45 \mathrm{~min}$. Proteins were pelleted by centrifugation at $15,000 \times \mathrm{g}$ for 15 min at $4^{\circ} \mathrm{C}$ and then washed with cold acetone containing $\beta$ mercaptoethanol. This step was repeated at least three times and residual acetone was finally removed by air-drying. The last pellet was resuspended with buffer for $2 \mathrm{DE}$ analysis (see below) and the protein concentration of samples was determined using a commercial kit (2-D Quant Kit, GE HealthCare, Milan, Italy), performed as described in the instruction manual and using BSA as reference.

\section{Two-Dimensional Electrophoresis (2DE)}

For 2DE analyses, 11-cm IPG Strips with a 3-10 pH gradient (Bio-Rad) were used in combination with $10 \%$ Criterion XT gels (Bio-Rad). Strips were rehydrated in the solubilization buffer (40 mM Tris, $8 \mathrm{M}$ urea, $2 \mathrm{M}$ thiourea, 2\% CHAPS, traces of bromophenol blue) to which $18 \mathrm{mM}$ DTT and $20 \mu \mathrm{l} \mathrm{ml} l^{-1}$ IPG buffer were added. Samples were dissolved to $1 \mathrm{mg} \mathrm{ml}^{-1}$ concentration in the solubilization buffer. Strips were rehydrated overnight in an Immobiline Dry Strip Reswelling Tray covered with a Dry Strip Cover PlusOne (GE HealthCare). Strips were run using a Protean IEF cell (Bio-Rad) through eight different steps:

- From 0 to $300 \mathrm{~V}$ for $30 \mathrm{~min}$.

- $300 \mathrm{~V}$ for $1 \mathrm{~h}$ and $30 \mathrm{~min}$.

- From 300 to $4000 \mathrm{~V}$ for $2 \mathrm{~h}$.

- $4000 \mathrm{~V}$ for $1 \mathrm{~h}$ and $30 \mathrm{~min}$.

- From 4000 to $8000 \mathrm{~V}$ for $1 \mathrm{~h}$ and $30 \mathrm{~min}$.

- $8000 \mathrm{~V}$ until a total of 20,000 Vhr (Volts $\mathrm{h}^{-1}$ ).

- From 8000 to $250 \mathrm{~V}$ for $10 \mathrm{~min}$.

- Hold step of $250 \mathrm{~V}$ until use of strips.

Strips were stored at $-80^{\circ} \mathrm{C}$ or used immediately. In both cases, they were equilibrated for $15 \mathrm{~min}$ in equilibration buffer $(50 \mathrm{mM}$ Tris- $\mathrm{HCl}, \mathrm{pH} 8.8$ containing $6 \mathrm{M}$ urea, 30\% glycerol, 2\% SDS, bromophenol blue, $10 \mathrm{mg} \mathrm{ml}^{-1}$ dithiothreitol). Proteins were then separated in the second dimension based on a Bis-Tris buffer system ( $\mathrm{pH}$ 6.4) that uses discontinuous chloride and MES or MOPS ion fronts to form moving boundaries to stack and then separate denatured proteins by size. Molecular weight standards of the Precision series (Bio-Rad) were run in parallel. Gels were stained with Bio-Safe Coomassie blue (Bio-Rad) as described in the instruction protocol.

\section{Spot Analysis}

Images of gels were captured using the Fluor-S Multi-Imager (Bio-Rad). The exposure time was $5-7 \mathrm{~s}$ for gels stained with Coomassie blue. Analysis of spots in 2DE gels was performed using the Spot Detection Wizard of PDQuest (Bio-Rad) by selecting the weakest protein spot and the larger protein clusters. Subsequently, spot analysis was improved manually by adding unidentified spots and by removing incorrect signals. After creating a Master (virtual) gel, spots were matched to determine qualitative and quantitative differences. Further analysis of spots was done using the Spot and Match set tools. The intensity of protein spots was normalized in relation to the total abundance of effective spots. After normalization and background subtraction, gels from control and treated samples were used to create a match set, which allowed the differential expression analysis between treated and control samples. Spots were considered as up- or down-regulated if their amount changed at least by a factor of 2 . All samples were analyzed in duplicate.

\section{Protein Identification by Mass Spectrometry}

Protein identification was performed as previously described (Hellman et al., 1995; Soskić et al., 1999). Spots of interest were manually excised, destained in $2.5 \mathrm{mM}$ ammonium bicarbonate and $50 \%(\mathrm{v} / \mathrm{v})$ acetonitrile and then dehydrated in acetonitrile. Gel pieces were rehydrated in trypsin solution and in-gel protein digestion was performed by an overnight incubation at $37^{\circ} \mathrm{C}$. For MALDI-TOF MS, $1.25 \mu \mathrm{l}$ of each protein digest was directly spotted onto the MALDI target and air-dried. After drying, $0.75 \mu \mathrm{l}$ of matrix solution $\left[5 \mathrm{mg} \mathrm{ml}^{-1} \alpha\right.$-cyano4-hydroxycynnamic acid in 50\% (v/v) acetonitrile and $0.5 \%$ $(\mathrm{v} / \mathrm{v})$ trifluoroacetic acid] was added to samples, which were allowed to dry again. Acquisition of mass spectra was performed using an Ultraflex III MALDI-TOF/TOF mass spectrometer (Bruker Daltonics, Billerica, MA, United States) in reflector positive mode. Spectra were analyzed by Flex Analysis software v. 3.0. Peptide mass fingerprinting (PMF) database searching was carried out in NCBInr and/or Swiss-Prot/TrEMBL databases set for Viridiplantae (Green Plants) using Mascot (Matrix Science Ltd., London, UK, http://www.matrixscience.com) online available software. The search settings were as follows: mass tolerance was set at $100 \mathrm{ppm}$, trypsin as the digestion enzyme with one allowed missed cleavage and oxidation of methionine as a variable modification. In order to accept identifications, the number of matched peptides, the extent of sequence coverage, and the probabilistic score were considered. Peptide digests that did not give unambiguous identifications were subjected to peptide sequencing by tandem mass spectrometry. MS/MS analysis was performed on the Ultraflex III MALDI-TOF/TOF instrument. Two to three PMF peaks showing a high intensity were CID (Collision Induced Dissociation) fragmented using Argon as collision gas, and MALDI-TOF/TOF tandem MS was performed in LIFT mode by software controlled data acquisition. Fragmented ions were analyzed using the Flex Analysis software v. 3.0. The MS/MS database search was carried out in NCBInr and/or Swiss-Prot/TrEMBL databases using the on-line MASCOT MS/MS ion search software. The following parameters were applied for the database search: taxonomy: Viridiplantae (Green Plants), trypsin specificity, one missed cleavage allowed, peptide precursor mass tolerance: $\pm 100 \mathrm{ppm}$, fragment mass tolerance $\pm 0.6 \mathrm{Da}$, peptide precursor charge state +1 , carbamidomethylation of cysteine as a fixed modification, oxidation of methionine as a possible modification. Protein identification was considered significant based on Mascot ion score, peptide coverage by " $b$ " and " $y$ " ions, and expected value. 


\section{Amino Acid Analysis}

Analysis of free amino acids and amino acids derived from complete hydrolysis of proteins in quinoa flour was performed in triplicate essentially as described by Silvanini et al. (2014) with some modifications. To analyze the content of free amino

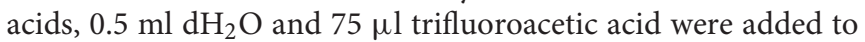
$20 \mathrm{mg}$ of quinoa flour. After mixing, samples were centrifuged at $12,000 \mathrm{~g}$ for $5 \mathrm{~min}$ at room temperature. Supernatants were dried by Speed-Vac and residues were dissolved in $20 \mu \mathrm{l}$ of 20 $\mathrm{mM} \mathrm{HCl}$. The content of protein-derived amino acids (PAAs) was determined by mixing each flour sample with $6 \mathrm{~N} \mathrm{HCl}$ and phenol crystal (around $1 \mathrm{mg}$ to avoid oxidation) followed by incubation for $24 \mathrm{~h}$ at $110^{\circ} \mathrm{C}$. After heating, samples were centrifuged at $4000 \mathrm{rpm}$ for $15 \mathrm{~min}$ at room temperature. The supernatant was dried under vacuum and the residue was dissolved in $20 \mu \mathrm{l}$ of $20 \mathrm{mM} \mathrm{HCl}$. Both free amino acids and those derived from hydrolysed proteins were derivatized according to the AccQ-Tag protocol (Waters, Milford, MA, USA). For HPLC analysis, a C18 AccQ-Tag column $(3.9 \times 150$ mm; Waters, Milford, MA, USA) was used. A gradient elution was performed using a phosphate buffer solution as eluent $A$ and acetonitrile:water 60:40 (v/v) as eluent $\mathrm{B}$. The temperature was set

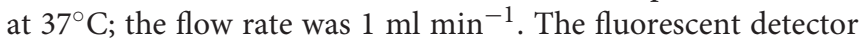
parameters were set as follows: $\lambda \mathrm{ex}=250 \mathrm{~nm}, \lambda \mathrm{em}=395 \mathrm{~nm}$, gain 1, eufs 100. All data are expressed as $\mathrm{mg} 100 \mathrm{~g}^{-1}$ flour.

\section{Extraction of Total Proteins}

Extraction of total proteins was performed essentially as described by Escudero et al. (2004). After adding $15 \mathrm{ml}$ of double distilled water to an aliquot $(50 \mathrm{mg})$ of the flour and continuous stirring for $30 \mathrm{~min}$ at room temperature (RT), the $\mathrm{pH}$ was taken to 9.0 with $0.1 \mathrm{~N} \mathrm{NaOH}$. After further stirring for $30 \mathrm{~min}$, the homogenate was centrifuged at $4500 \times \mathrm{g}$ for $20 \mathrm{~min}$ at RT. The supernatant was collected and taken to $\mathrm{pH} 5.0$ with 0.1 $\mathrm{N} \mathrm{HCl}$. After stirring for another $20 \mathrm{~min}$ and centrifugation at $4500 \times \mathrm{g}$ at $4^{\circ} \mathrm{C}$, the supernatant was discarded and the pellet re-suspended in $500 \mu \mathrm{l}$ of $63 \mathrm{mM}$ Tris- $\mathrm{HCl}, \mathrm{pH}$ 8.0. Protein concentration was determined spectrophotometrically at $562 \mathrm{~nm}$ using the bicinchoninic acid kit and BSA as standard.

\section{Total Phenolics and Flavonoid Contents}

The Folin-Ciocalteu (FC) assay was performed according to Singleton and Rossi (1965) with some modifications; $50 \mu \mathrm{l}$ of protein extract $\left(1 \mathrm{mg}\right.$ protein $\mathrm{ml}^{-1}$ ) were mixed with $250 \mu \mathrm{l}$ of FC reagent (previously diluted 10 -fold with distilled water) and $500 \mu \mathrm{l}$ distilled water. The reaction mixture was incubated for 1 min at room temperature and then $800 \mu \mathrm{l}$ of $20 \%$ (w/v) $\mathrm{Na}_{2} \mathrm{CO}_{3}$ was added. After incubation at $40^{\circ} \mathrm{C}$ for $30 \mathrm{~min}$, the absorbance was measured spectrophotometrically at $760 \mathrm{~nm}$ (Jasco V-530, Jasco Corporation, Tokyo, Japan). The total polyphenolic content (TPC) was evaluated from a gallic acid standard curve and was expressed as $\mathrm{mg}$ gallic acid equivalents (GAE) $\mathrm{g}^{-1}$ seed DW. The Prussian blue method (Hagerman and Butler, 1994) was also applied to determine total polyphenols. After adding $12 \mu \mathrm{l}$ of 0.1 $\mathrm{M} \mathrm{FeNH}_{4}\left(\mathrm{SO}_{4}\right)_{2}$ to $100 \mu \mathrm{l}$ protein extract $\left(1 \mathrm{mg}\right.$ protein $\mathrm{ml}^{-1}$ ), the mixture was incubated for $20 \mathrm{~min}$ at RT. Subsequently, 12 $\mu \mathrm{l}$ of $8 \mathrm{mM} \mathrm{K}_{3} \mathrm{Fe}(\mathrm{CN})_{6}$ were added, and after $5 \mathrm{~min}$ at room temperature, the optical density of the mixture was determined at $720 \mathrm{~nm}$. Gallic acid was used as standard to make a calibration curve and data were expressed as $\mathrm{mg} \mathrm{GAE} \mathrm{g}^{-1} \mathrm{DW}$.

The total flavonoid content (TFC) was determined with $\mathrm{AlCl}_{3}$ according to Liu et al. (2002) with slight modifications using rutin as standard. The seed protein extract $(50 \mu \mathrm{l})$ was added to $450 \mu \mathrm{l}$ of $100 \%$ methanol followed by $500 \mu \mathrm{l}$ of $2 \%(\mathrm{w} / \mathrm{v})$ $\mathrm{AlCl}_{3}$ in methanol. This reaction mixture was incubated for 15 min at room temperature. Finally, the absorbance of the reaction mixture was measured spectrophotometrically at $430 \mathrm{~nm}$. Data were expressed as $\mathrm{mg}$ rutin equivalents (RE) $\mathrm{g}^{-1} \mathrm{DW}$.

\section{Radical Scavenging Activity}

Free radical scavenging capacity of the protein extracts was determined by using the ABTS assay performed according to Arnao et al. (2001) with slight modifications. The $\mathrm{ABTS}^{+}$radical cation was generated by oxidizing a $2.0 \mathrm{mM}$ aqueous solution of ABTS with $70 \mathrm{mM} \mathrm{K}_{2} \mathrm{~S}_{2} \mathrm{O}_{8}$ and incubating in the dark for $24 \mathrm{~h}$ at room temperature. The reaction mixture contained $1.0 \mathrm{ml}$ of $\mathrm{ABTS}^{+}$(diluted with methanol in order to obtain an absorbance of 0.7 at $734 \mathrm{~nm})$ and different amounts $(12.5,25,50,100 \mu \mathrm{l})$ of sample solutions ( $1 \mathrm{mg} \mathrm{ml}^{-1}$ total protein or $11 \mathrm{~S}$ fraction) or Tris buffer for the blank. The absorbance at $734 \mathrm{~nm}$ was measured after a reaction time of $20 \mathrm{~min}$. Trolox equivalents per g DW of seeds were calculated using a standard curve prepared with a range of Trolox concentrations $(0-30 \mu \mathrm{M})$ in order to calculate the Total Antioxidant Activity (TAC) value for the samples.

\section{Statistical Analysis}

Two independent experiments were performed. Each experiment consisted of three pots per treatment $(0,100$, and $300 \mathrm{mM} \mathrm{NaCl})$, each containing one plant per landrace, and set up according to a randomized block design. Flour preparation, protein extractions, SDS-PAGE, and AIDA analyses were performed at least twice. TPC, TFC, and AA data were performed in triplicate from two separate protein extractions. To determine the overall significances, a two-way factorial analysis of variance (two-way ANOVA) was used with salt treatment and landrace as factors. Mean comparisons were made by applying Tukey's post-hoc test using InfoStat software (www.infostat.com.ar). Differences were considered significant at $P<0.05$.

\section{RESULTS}

\section{Seed Storage Protein Profiles and Spot Analysis}

Sequential extraction of the different protein fractions and isolation of the major storage proteins were performed on seeds of the three quinoa landraces (R49, VI-I, and VR) grown with/without 100 or $300 \mathrm{mM} \mathrm{NaCl}$. A combination of SDSPAGE and 2DE was used to, first of all, identify the fractions that were most affected by salt, and second the proteins that were most involved in the salt-induced response. The protein fraction analyzed was the one enriched in albumins/globulins. A general feature was the reduction of these storage proteins, expressed as $\mathrm{mg}$ protein $\mathrm{mg}^{-1}$ flour, after the $300 \mathrm{mM} \mathrm{NaCl}$ treatment. In 
particular, the albumin/globulin fraction declined by ca. 12, 7, and $15 \%$ in R49, VI-I, and VR, respectively (data not shown).

As shown in Figure 1, the proteins were resolved into distinct bands that spanned a broad range of apparent molecular weights from 15 to $>55 \mathrm{kDa}$. The image analysis software identified 10 major bands of 49 (1), 45 (2), 42 (3), 40 (4), 32 (5), 30 (6), 25 (7), $22(8), 19$ (9), and 16 (10) kDa. Some difference in the band patterns among genotypes was evident: in $\mathrm{R} 49$ the $32-\mathrm{kDa}(5)$ band was absent while VI-I seemed to lack the $30-\mathrm{kDa}$ band (6); VR had both the 32- (5); and the $30-\mathrm{kDa}$ (6) bands. Comparing the protein profiles in control and salt-treated seeds by SDSPAGE revealed that the $\mathrm{NaCl}$ treatment induced significant changes in the protein patterns and that the three landraces were differentially affected by salinity. In R49, the profile was very similar in seeds from control and $100 \mathrm{mM} \mathrm{NaCl}$-treated plants; in the $300 \mathrm{mM} \mathrm{NaCl}$ treatment, the high molecular weight bands (1-4) appeared to be slightly increased, while the low molecular weight bands (6-10) decreased significantly relative to controls (Figure 1B). Under saline conditions $(300 \mathrm{mM} \mathrm{NaCl})$, VI-I showed a decrease in different proteins; the decline was most evident in the low molecular-weight bands except for 8 and 9 (5-10; Figure 1C). Finally, in VR, both salt treatments decreased the intensity of bands 5,6 , and 7 in a concentration-dependent manner (Figure 1D).

Subsequently, a proteomic profile of the albumin/globulin fraction was performed by $2 \mathrm{DE}$ in control and salt-treated samples of landrace VR. This genotype was chosen because, in addition to changes in the SDS-PAGE band pattern, under salinity it also exhibited the highest TPC, TFC, and $\mathrm{AA}$ (see below) and the greatest percentage decrease in the albumin/globulin fraction. Two-DE gels revealed significant differences in protein composition between the two samples. Following the analysis of master gels obtained from albumin/globulin fractions, the nine spots whose abundance changed at least two-fold during treatments were selected and processed for identification by MALDI-TOF MS (Figure 2). Spots were numbered arbitrarily and correspond to numbers indicated in the identification list. As shown in Table 1, the spots analyzed by MS revealed specific correspondence to peptides in the protein database. They were clustered into seven functional categories: stress (chaperone/folding), transcription factors, respiration, photosynthesis, storage proteins, metabolism, and cell division. The spot intensities show that significant changes, either increases or decreases, occurred in response to salinity (Figure 3).

Three spots (1, 2, and 9) correspond to proteins involved in stress responses or as support in protein folding. In this category, spot 1 corresponds to a stromal chloroplast $70-\mathrm{kDa}$ heat shockrelated protein of Glycine soja, and to a putative heat shock protein of Ricinus communis. Analysis of the spot 2 sequence revealed a correspondence to several proteins with similar function: a GRP-78/luminal-binding protein of Spinacia oleracea, a luminal-binding protein of Beta vulgaris subsp. vulgaris, a luminal-binding protein 5 of Jatropha curcas, an endoplasmic reticulum chaperone binding protein of Lycium chinense, and a putative heat shock protein of $R$. communis. The intensity of
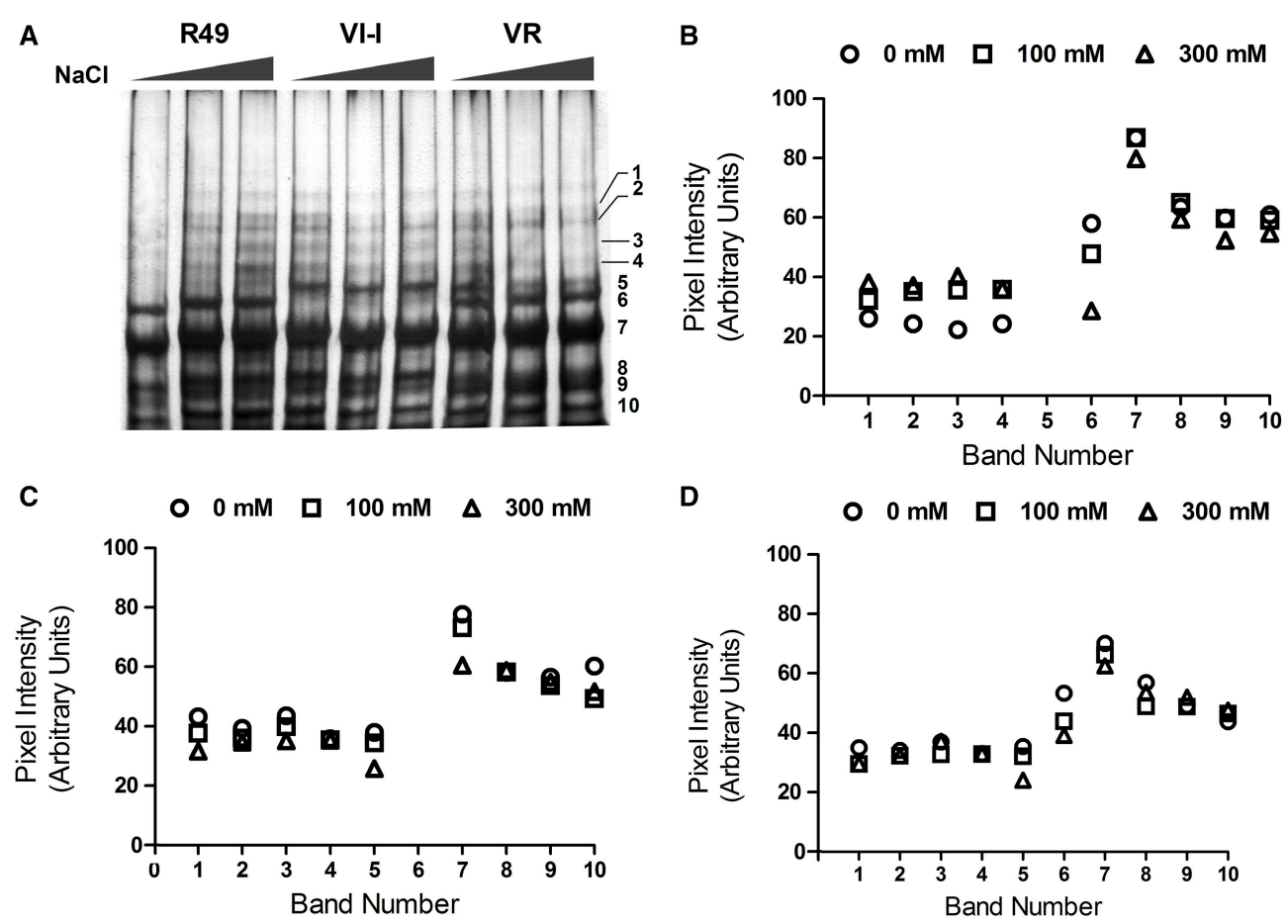

FIGURE 1 | Representative SDS-PAGE (A) of the albumin/globulin fraction of seeds from plants of three quinoa landraces (R49, VI-I, and VR) irrigated with 0, 100, or $\mathbf{3 0 0} \mathbf{~ m M ~ N a C l}$. The 10 major bands identified by the AIDA Image Analyzer software are numbered: 49 (1), 45 (2), 42 (3), 40 (4), 32 (5), 30 (6), 25 (7), 22 (8), 19 (9), and 16 (10) kDa. Intensity of the 10 major bands in control and salt-treated (100 or $300 \mathrm{mM} \mathrm{NaCl}$ ) samples of the three landraces: R49 (B), VI-I (C), and VR (D). 


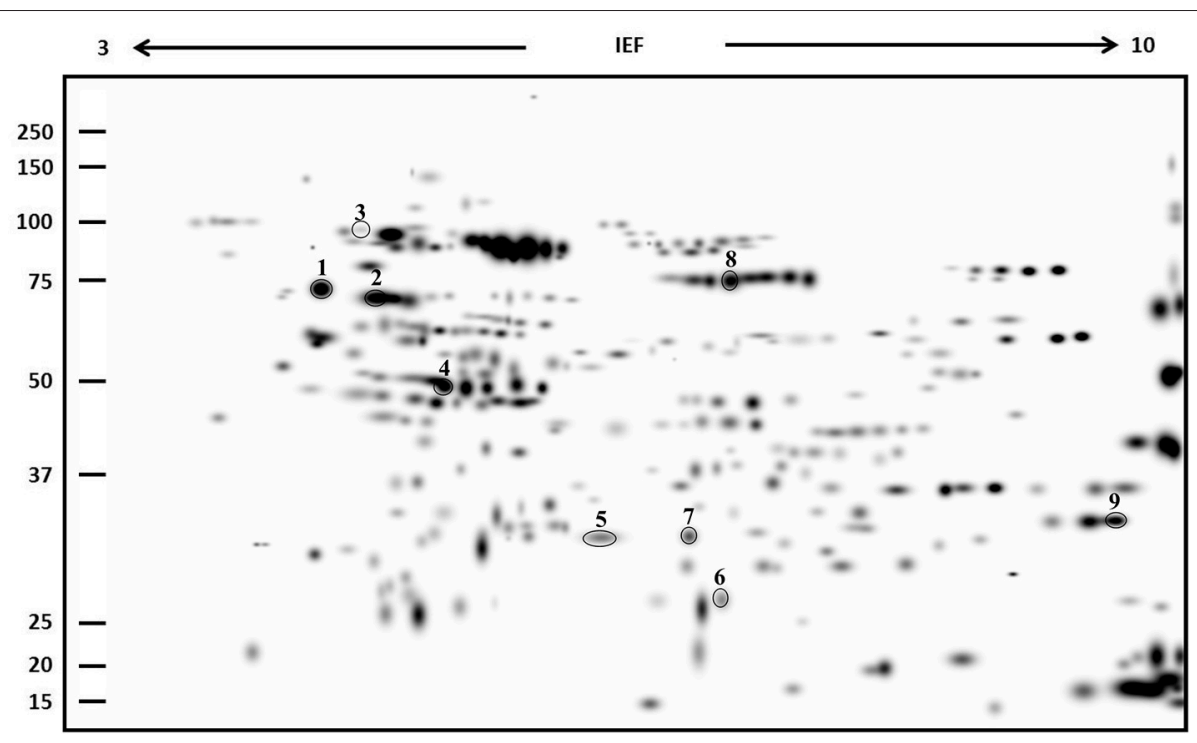

FIGURE 2 | Master gel obtained from the PDQuest-based comparison of 2DE gels of albumin/globulin fraction samples of VR (control and 300 mM $\mathrm{NaCl}$-treated). The virtual image of gel represents all spots as detected in all samples tested. Molecular mass standards are indicated on the left while the pH range is at the top. Equivalent protein contents $(200 \mu \mathrm{g})$ were loaded in each sample. Spots analyzed by MALDI-TOF are circled and numbered.

spots 1 and 2 increased after salt treatment. Spot 9 corresponded to peptidyl-prolyl cis-trans isomerase CYP20-1 of Amborella trichopoda, a protein involved in enhancing the correct folding of proteins; it decreased in seeds of salt-stressed plants as compared with controls. The second functional category is represented by spot 3, which corresponds to an Ethylene Insensitive3-like 1 protein of Phoenix dactylifera, a positive regulator in the ethylene response pathway. Its expression level decreased after treatment with $\mathrm{NaCl}$. A protein involved in respiration was also identified (although only in the NaCl-treated samples). Spot 4 was matched to a mitochondrial beta-subunit of ATP synthase in different organisms (Fragaria vesca subsp. vesca, Oryza sativa and Pyrus $\times$ bretschneideri). Spot 5 was identified as a plastid movement impaired protein of Medicago truncatula; the protein showed an increased level in the $\mathrm{NaCl}$-treated sample. Mass spectrometry analysis revealed that spot 6 corresponded to the $11 \mathrm{~S}$ seed storage globulin A of C. quinoa. The expression level of this protein showed a drastic decrease in samples treated with $\mathrm{NaCl}$. Spot 7 found homology with a pantothenate kinase of Micromonas $s p$. This protein converts pantothenate to phosphopantothenate, using ATP as phosphate donor and was significantly up-regulated under salinity. Finally, spot 8 matched to a helicase protein of Tetrahymena thermophile, a protein involved in the unwinding of DNA or RNA helix. The expression level of this protein was drastically increased in samples treated with $\mathrm{NaCl}$.

\section{Profiles and Spot Analysis of Globulin Fractions}

As the albumin/globulin fraction from seed proteins of quinoa was strongly affected by salinity, a further attempt was made to isolate and analyze the crude acid-soluble globulins and the 11S- (chenopodin) enriched fractions by SDS-PAGE. As shown in Figure 4, proteins of the crude acid-soluble globulin fraction were resolved into distinct bands that spanned a broad range of apparent molecular weights from 15 to $>90 \mathrm{kDa}$. Results showed that the protein profiles were represented by eight major and distinct bands, common to all three landraces, with molecular weights of $90,68,45,43,31,20,18$, and $15 \mathrm{kDa}$. Comparing the protein profiles in seeds from control and salt-treated plants revealed that the $\mathrm{NaCl}$ treatment did not induce significant changes in the protein composition; however, seeds of plants grown under $300 \mathrm{mM} \mathrm{NaCl}$ showed a general reduction in these storage proteins. In particular, the crude acid-soluble globulin fraction declined by $22.5,13.9$, and $25.3 \%$ in R49, VI-I, and VR, respectively.

As shown by $2 \mathrm{DE}$ followed by mass spectrometry of the albumin/globulin fraction, a general reduction of chenopodin, the major $11 \mathrm{~S}$ storage protein, was also evident (Figure 5). Proteins of the 11S-enriched fractions were resolved into distinct bands giving the typical electrophoretic pattern of chenopodin, in which subunits $\mathrm{A}(32-42 \mathrm{kDa})$ and $\mathrm{B}(22-23 \mathrm{kDa})$ were clearly evident (Figure 5A). By image analysis, five major bands of 42 (1), 40 (2), 32 (3), 24 (4), and 22 (5) kDa were identified. As already reported for the albumin/globulin fraction, protein band patterns showed some differences among genotypes: the $42-\mathrm{kDa}$ (1) band was absent in R49, VI-I did not present the $40-\mathrm{kDa}$ (2) band and VR had both. Comparing the protein profiles in control and salt-treated seeds by SDS-PAGE revealed that the $\mathrm{NaCl}$ treatment induced a significant reduction in the 11S-enriched fraction and that the three landraces were differentially affected by salinity (Figures 5B-D).

As previously done for the albumin/globulin fraction, a proteomic profile of the $11 \mathrm{~S}$ fraction was obtained by $2 \mathrm{DE}$. Only landrace VR was analyzed because, as outlined above, all of the five main bands were affected by salt treatment. The comparison between control and salt-treated samples revealed 


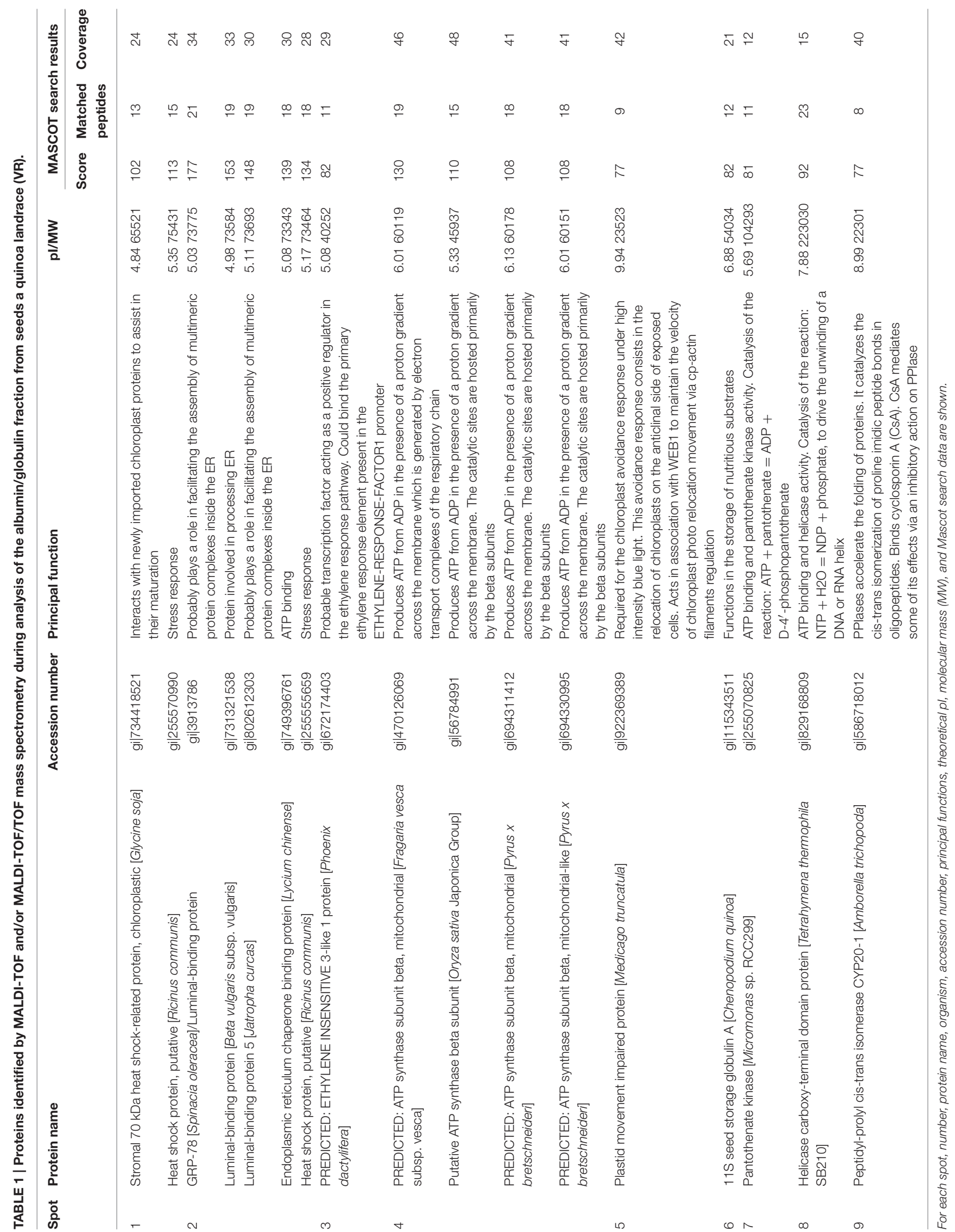




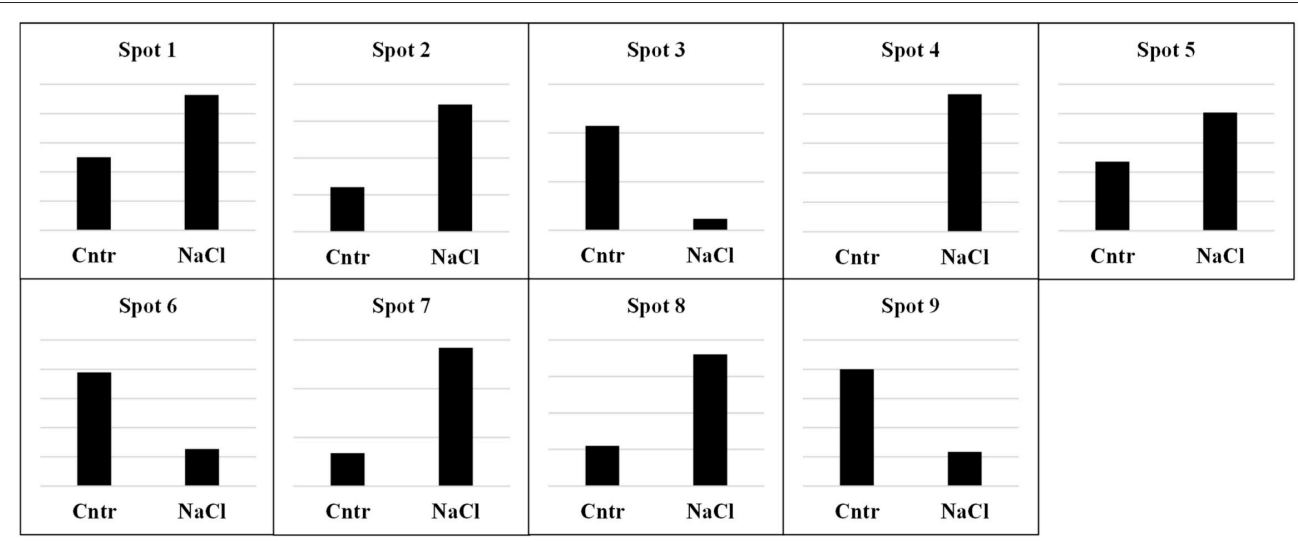

FIGURE 3 | Relative abundance of individual spots from the albumin/globulin fractions of quinoa seeds harvested from plants grown under control (Cntr) or saline ( $\mathbf{N a C l}$ ) conditions. Spots are numbered as shown in the master gel (Figure 2). Each spot is presented with the value of its relative quantification. The first column indicates the control sample, while the second column indicates the $300 \mathrm{mM} \mathrm{NaCl}$ treatment. Scales are different in the Y-axes of each graph because they were optimized to highlight the different intensity of spots between samples. In all cases, the most intense spot was used as reference for calibrating the scale of $Y$-axes.

remarkable differences in protein composition (Figure 6). Fifteen spots whose expression levels changed significantly were further analyzed by mass spectrometry. They revealed a specific correspondence to proteins found in databases (Table 2). As in the case of the albumin/globulin fraction, the proteins were clustered into five main functional categories: stress (chaperone/folding), storage proteins, photosynthesis, respiration, and ion transport. The spot intensities in seeds of plants grown under non-saline or saline $(300 \mathrm{mM} \mathrm{NaCl})$ conditions are represented in Figure 7. Three different proteins (spots 1, 2, and 4) are involved in stress (chaperone/folding). Two were identified as hypothetical and predicted proteins; however, subsequent BLAST analysis revealed that they corresponded to heat shock $70-\mathrm{kDa}$ protein 6, chloroplastic-like of Gossypium raimondii and heat shock cognate $70-\mathrm{kDa}$ protein 1 of Aegilops tauschii, respectively. The third protein was identified as a heat shock protein 90 of Nicotiana tabacum. In all cases, salt stress increased the accumulation of these proteins compared to controls.

For the storage category, nine spots corresponding to the 11S seed storage globulin of C. quinoa and a predicted-legumin Blike of $B$. vulgaris subsp. vulgaris were identified. The expression levels of these proteins changed significantly under salinity. In the case of $11 \mathrm{~S}$ globulin, the salt-treated sample showed a significant down-regulation or a complete disappearance of spots $6,9,10$, 11,12 , and 13. On the other hand, the expression level of spots 7 and 8 increased in salt-treated samples compared to controls. Spot 3 corresponds to a chloroplastic RuBisCO large subunit-binding protein, subunit alpha of Brassica napus. This photosynthetic protein increased after salt treatment. For the respiration category, spot 5 had a significant correspondence to mitochondrial ATP synthase subunit beta of Prunus mume and its intensity increased during stress. The ion transport category was represented by spot 14 , which corresponds to a predicted cation/ $\mathrm{H}^{(+)}$antiporter $6 \mathrm{~B}$ of Camelina sativa whose intensity increased during stress.

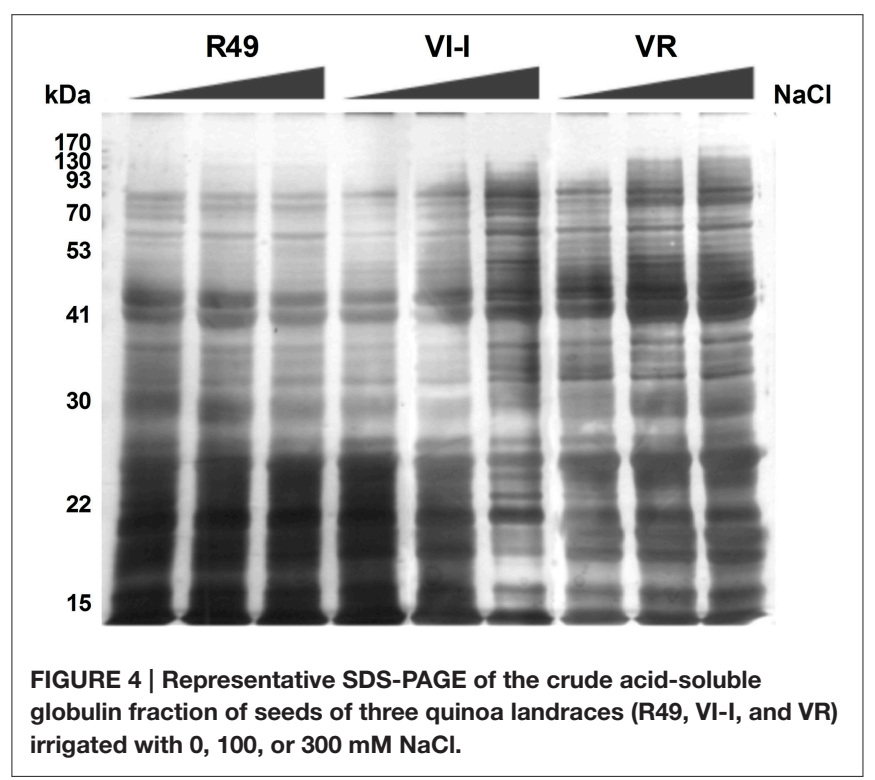

\section{Prolamin-Like Proteins}

Detecting and separating quinoa prolamin-like proteins was quite problematic because these polypeptides are scarcely soluble. The ethanol-soluble prolamin-like proteins were scarce and consisted of low-molecular weight polypeptides (Figure 8). Comparing the protein profiles in control and salt-treated seeds by SDS-PAGE revealed that the $\mathrm{NaCl}$ treatment did not induce changes in the pattern of proteins possibly related to gluten.

\section{Amino Acid Profiles}

The HPLC analysis of free amino acids and of amino acids derived from the complete hydrolysis of proteins (PAAs) from control and salt-treated seeds of quinoa revealed that some (Asp, Ser, Glu, Gly) were below the limit of detection in most samples 


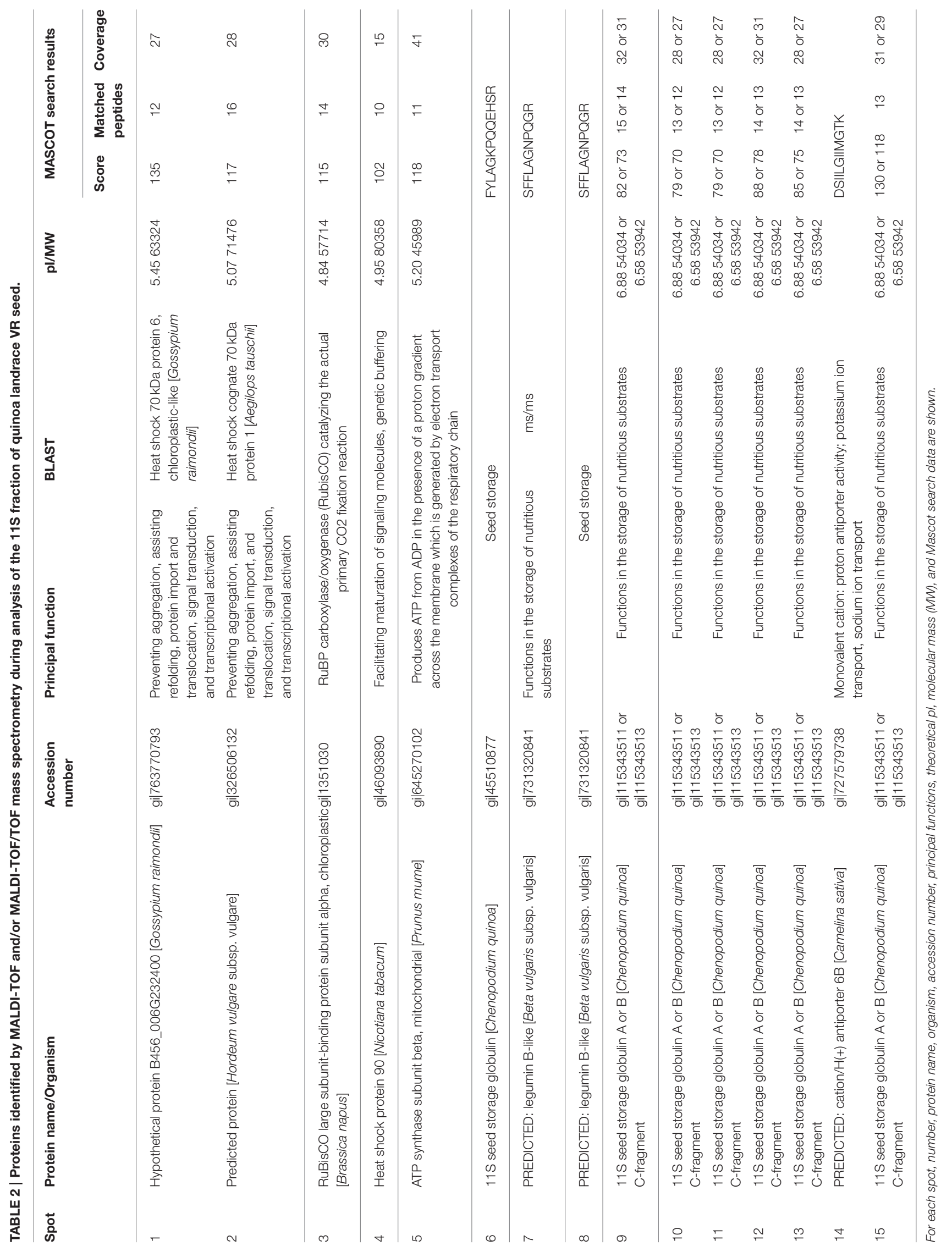



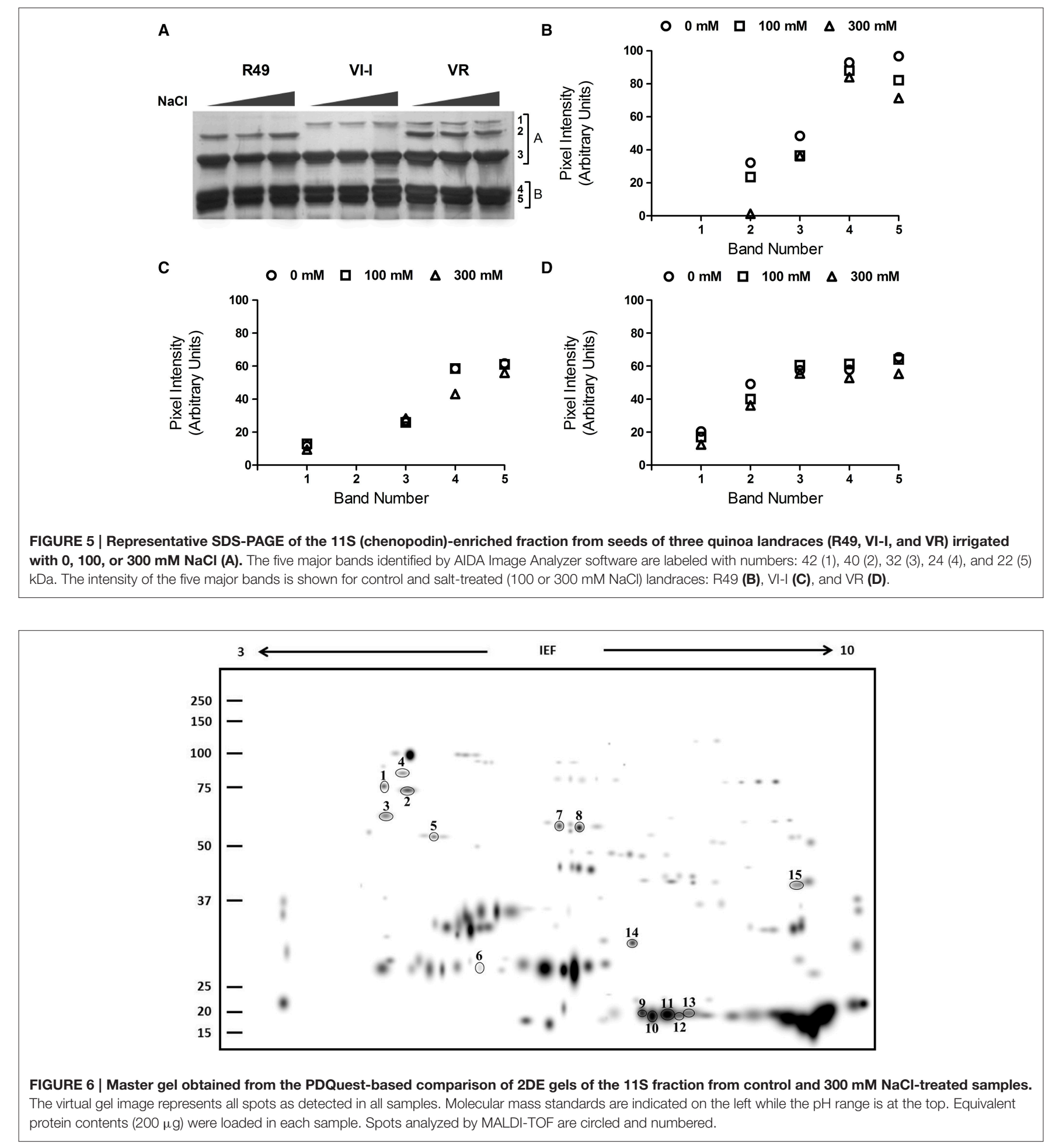

and were present only as free amino acids in VI-1 controls (Figure 9D, inset). Overall, the PAA profile was quite different from that of the free amino acids, both quantitatively (PAAs were much more abundant) and qualitatively (Figure 9).

In seeds of control plants, the profile derived from protein hydrolysis showed that Pro was the most abundant PAA in R49 and VR, followed by Cys and Tyr in R49, and by Ala, Cys, Tyr and Met in VR; in VI-1, Pro content was comparable to that of the other landraces, but the most abundant amino acid was Thr (several fold higher than the other amino acids, Figures 9A,C,E). In control plants of VI-1, by far the most abundant essential free amino acids were His and Thr, followed by essential and 


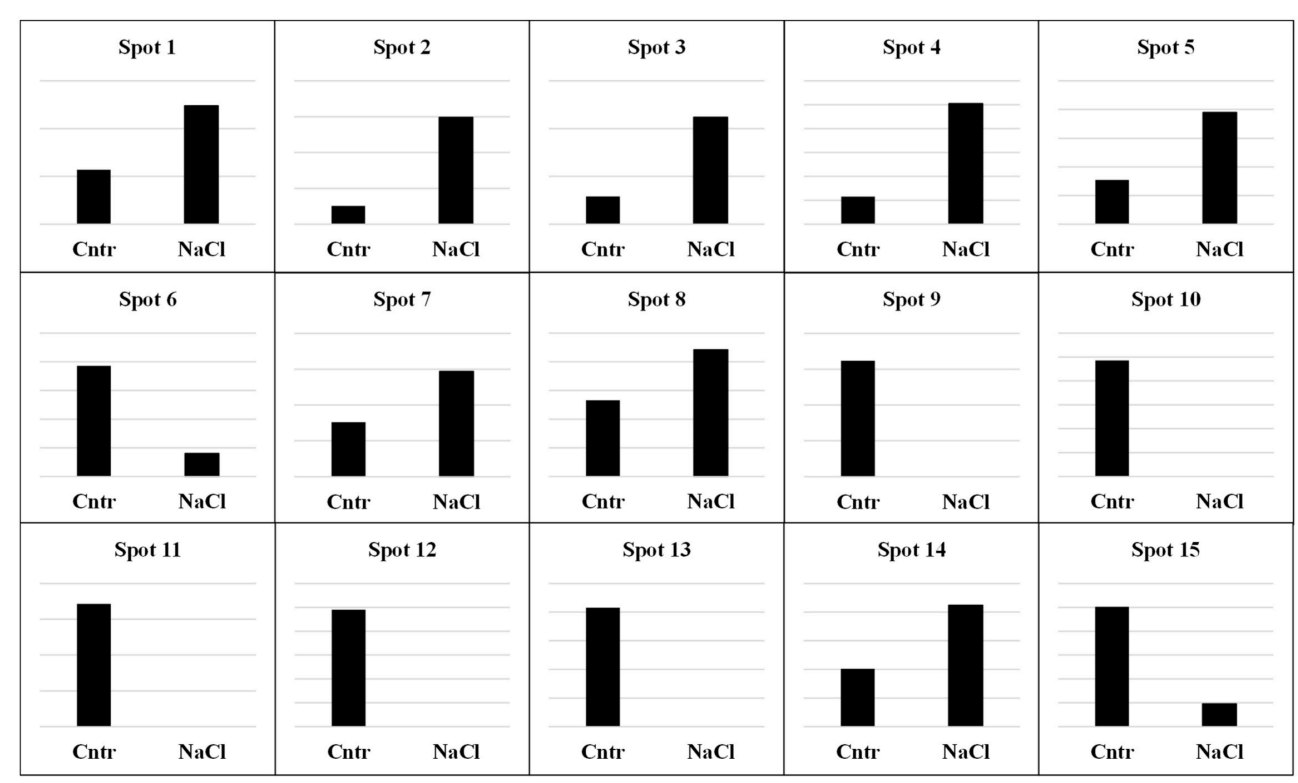

FIGURE 7 | Relative abundance of individual spots in the 11S fraction of quinoa seeds harvested from plants grown under control (Cntr) or saline (NaCl) conditions. Spots are numbered as shown in the master gel (Figure 6). Each spot is presented with the value of its relative quantification. Scales are different in the $Y$-axes of each graph because they were optimized to highlight the different intensity of spots between samples. In all cases, the most intense spot was used as reference for calibrating the scale of Y-axes.

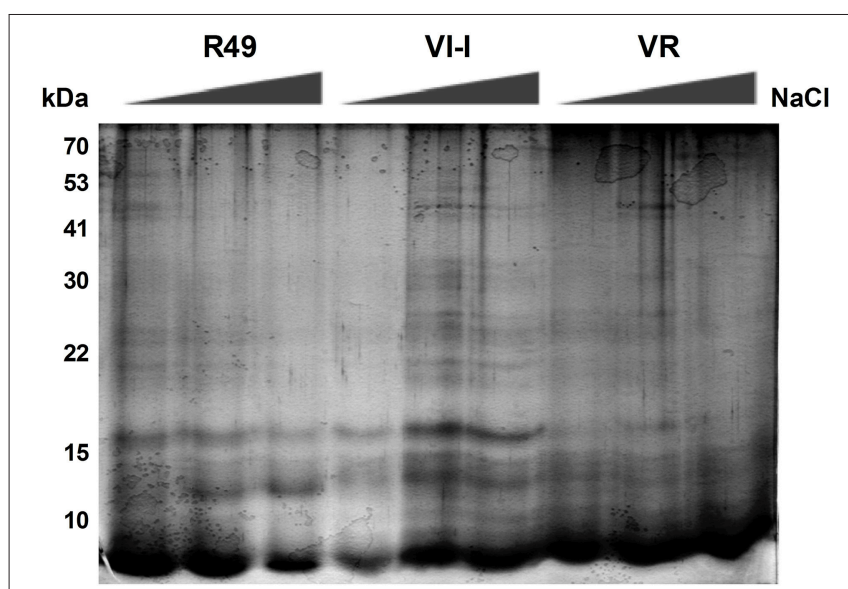

FIGURE 8 | Representative SDS-PAGE of the ethanol-soluble prolamin-like proteins.

non-essential amino acids Cys, Tyr, Leu, Glu; in R49 and VR these amino acids were much less abundant or not detectable (Figures 9B,D,F). Pro was the most represented free amino acid in these two landraces followed by Cys. Free Lys content was also ca. three-fold higher in VI-1 than in the other landraces.

Salt differentially affected the amino acid composition of the three landraces. Again, the PAA profile differed substantially from that of free amino acids also in salt-treated samples (Figures 9A,C,E). The non-essential PAAs Ala, Pro, and Cys decreased strongly in all landraces relative to controls, except for Ala in R49, which increased. In R49 there were no changes in some essential amino acids (Lys, Ile, Phe) while other essential amino acids (Thr, Val) declined. Met was the only essential PAA that increased in response to salinity, but only in R49. In VR, all essential PAAs either decreased (Met, Lys, Ile) or remained unchanged; only Leu increased slightly (30\% above control value). The strongest overall salt-induced reduction in amino acids was observed in VI-1.

It is noteworthy that in R49 free Thr was induced 60 -fold by salinity; other free essential amino acids, namely Val, Lys, and Ile were also significantly enhanced (ca. 2.6-, 2.1-, 3.6-fold) while Leu increased by 25\% (Figures 9B,D,E). By contrast, in VR and VI-1, all free essential amino acids were down-regulated, with the exception of Phe in VR, which increased by ca. 7.5-fold. Amongst the non-essential amino acids Ala, Pro, and Cys, there were no or very limited reductions $(20-40 \%)$ in all three landraces and Pro even increased by 30 and 60\% in R49 and VI-1, respectively. In VI-1, most free amino acids were undetectable in salt-treated samples showing a similar trend to the PAAs; in this landrace, Thr disappeared under salinity, whereas in R49 and VR the opposite occurred.

\section{Total Phenolic and Flavonoid Content, and Antioxidant Activity}

In the total proteins extracted from quinoa seeds, there was a genotype-dependent and salt-dependent variation in TPC as determined by the FC assay (Figure 10A). Under non-saline conditions, VR had the highest values followed by VI-1and R49. In all three landraces, seeds from plants grown with $300 \mathrm{mM}$ $\mathrm{NaCl}$ had higher TPC than those grown without $\mathrm{NaCl}$. The strongest increase (3.5-fold) was observed in R49 while in VR 

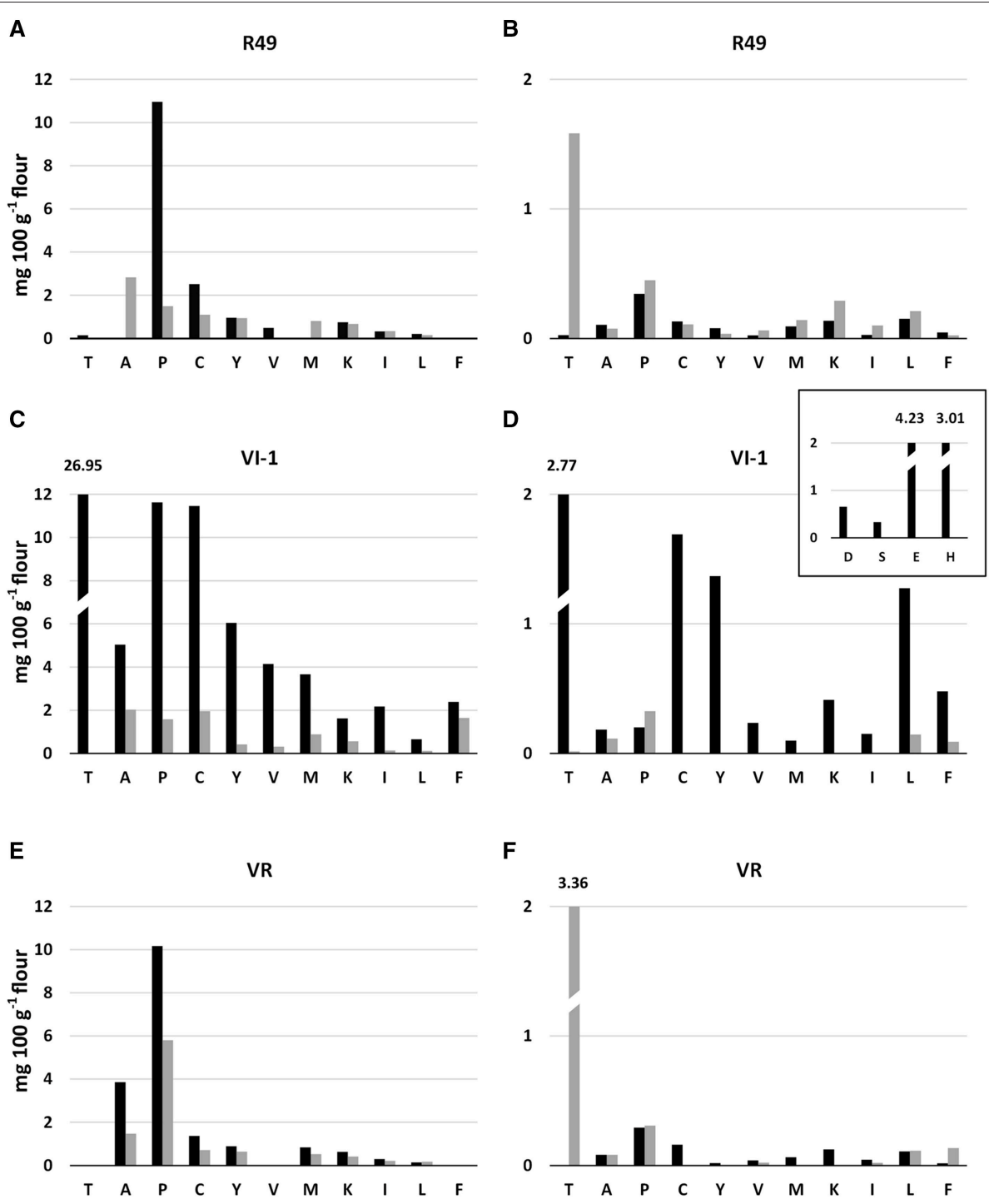

FIGURE 9 | Contents ( $\mathrm{mg}$ amino acid $100 \mathrm{~g}^{-1}$ flour) of protein-derived amino acids (A,C,E) and free amino acids (B,D,F) in seeds of control (black) and salt-treated (gray) landraces R49, Vl-1, and VR. D, Aspartic acid; S, Serine; E, Glutamic acid; G, Glycine; H, Histidine; R, Arginine; T, Threonine; A, Alanine; P, Proline; C, Cysteine; Y, Tyrosine; V, Valine; M, Methionine; K, Lysine; I, Isoleucine; L, Leucine; F, Phenylalanine.

and VI-1 the increase was lower (ca. 60-70\% above control levels). Using the Prussian blue method, polyphenols were below detection limit in R49 and VI-1 control and treated seeds; in VR controls, polyphenols were confirmed to be substantially more abundant than in the other two landraces $(0.17 \pm 0.007$ GAE $\mathrm{mg}^{-1} \mathrm{DW}$ ) and dramatically enhanced (five-fold) under salinity (data not shown). Flavonoid concentrations were similar in R49 and VI-1 but lower in VR; however, salinity enhanced TFC only in VR (Figure 10B). The radical scavenging capacity of the seed protein extracts assayed with the ABTS method indicated that TAC was significantly different between landraces, with VR exhibiting higher values as compared with R49 and VI-1. It was slightly but significantly higher in seeds from salt-treated plants of R49 and VR as compared with controls (Figure 10C). The 11S globulin-enriched fraction, extracted without using $\beta$-mercaptoethanol, also revealed some ABTS radical scavenging capacity. Values were much lower (0.005-0.006 mM Trolox equivalent $\mathrm{g}^{-1} \mathrm{DW}$ ) than those of the total protein extract and did not change significantly in salt-treated vs. control samples (data not shown).

\section{DISCUSSION}

Quinoa seeds are regarded as one of the most nutritionally well-balanced plant foods under cultivation, especially for their protein content and excellent balance between carbohydrates, lipids, and proteins. The increasing interest in quinoa as a crop is also centered on its remarkable adaptability to harsh environmental conditions such as low water content and high 

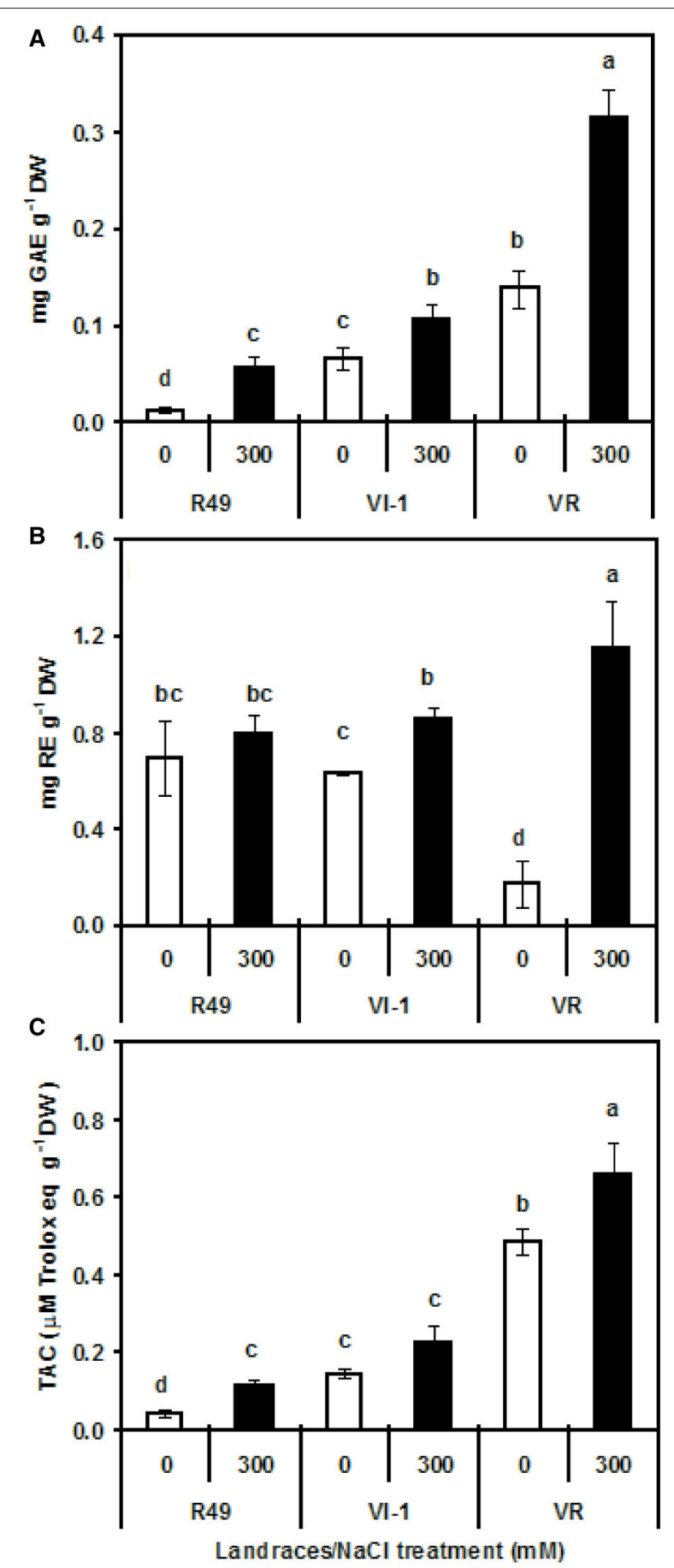

FIGURE 10 | Total polyphenols as assayed by the Folin-Ciocalteu method (A), total flavonoids (B), and total antioxidant activity (TAC, C) in protein extracts of quinoa seeds harvested from plants grown under control $(0)$ or saline $(300 \mathrm{mM} \mathrm{NaCl})$ conditions.

alkalinity of saline soils. Soil salinity is, in fact, among the major factors limiting crop yield and productivity worldwide and is expected to increase in the future. Several comparative studies have shown that the extent to which these and other parameters are affected by salinity in quinoa is strongly genotype-dependent (Gómez-Pando et al., 2010; Adolf et al., 2012).

In the framework of our current investigations on salinity tolerance in quinoa, we have measured growth, yield, germination capacity, leaf TPC, and seed quality in the three Chilean landraces R49, VI-I, and VR exposed to two levels of salinity (Ruiz et al., 2016a). In the present work, we aimed to delve further into the effects of salt treatment on the seed proteome and on the polyphenol content, and AA of seed extracts. The PC of Amaranthus cruentus seeds were reported to have improved nutritional quality as compared with flour through increased content of factors that directly or indirectly influence lipid metabolism and enhance antioxidant protection (Escudero et al., 2011). The AA of PCs obtained through different pilot-scale industrial processes from A. mantegazzianus seeds was recently evaluated in order to optimize production for the food industry (Castel et al., 2014).

\section{Proteomic Changes}

Preliminary analyses were conducted on the three different Chilean landraces in order to unveil genotype-specific responses. In fact, seed protein electrophoresis has been utilized in taxonomy for the explanation of the origin and evolution of a number of cultivated plants (Ahmad and Slinkard, 1992; Jha and Ohri, 1996; Nath et al., 1997; Ghafoor et al., 2002). Seed protein profiles of 40 wild and cultivated taxa of Chenopodium were congruent with taxonomic position, crossability relationships, and other biochemical characters, confirming SDS-PAGE as a powerful tool in solving taxonomic problems (Bhargava et al., 2005). More recently, Džunková and co-workers showed that both classical SDS-PAGE and chip electrophoresis analysis of seed storage proteins were useful methods for studying amaranth taxonomy in order to assess differences among different species and even among accessions (Džunková et al., 2011). They suggested that specific fractions, including albuminand glutelin-enriched fractions, instead of whole extracts, could better reveal differences among landraces. In the present study, the best candidates for SDS-PAGE-mediated polymorphism analysis were the most abundant seed storage proteins, i.e., the albumin/globulin fraction. In fact, results confirm a high polymorphism in seed storage proteins both in the position and in the intensity of some bands. The two most representative genotype-specific bands of this fraction were the $30-$ and $32-\mathrm{kDa}$ bands.

Because of their low concentrations, prolamins were not suitable for this type of analysis, both in quinoa, herein analyzed, and amaranth and Džunková et al. (2011). While the globulins of amaranth did not differ in band intensity and position (Džunková et al., 2011), we showed that chenopodin, the 11S globulin of quinoa, displayed a high polymorphism in the three landraces analyzed, with genotype-specific protein bands of 40and 42-kDa. Balzotti et al. (2008) reported the phylogenetic relationships between quinoa and 49 other species by using the coding DNA sequence for the well-conserved 11S basic subunit. On the basis of amino acid alignments, more than $74 \%$ sequence identity between amaranth and quinoa was revealed. 
To better understand the effects of salt stress on quinoa seeds, we further investigated the composition of storage proteins after irrigation with $300 \mathrm{mM} \mathrm{NaCl}$ as compared with untreated controls. Both quantitative and qualitative differences in protein expression as a result of salt treatment were found. The albumin/globulin fraction, and in particular the 11 S globulin, was the most affected fraction in terms of reduced percentage content as well as altered profile, both in SDS-PAGE and 2DE. Proteomic analysis revealed increases in the expression of proteins known to be involved directly in ER lumenal protein folding and in the assembly of proteins (Shewry et al., 1995), thus indirectly in the response to cellular stresses. Thus, despite a general decrease in protein content, up-regulation of some specific proteins, such as proteins involved in specific and fundamental cellular processes (photosynthesis and metabolism) as well as in stress adaptation, was observed by analyzing in detail the selected landrace (VR).

An interesting finding was that the expression level of heat shock-related protein (HSP70 and HSP90) and of a GRP-78/Luminal-binding protein (an endoplasmic reticulum chaperone involved in response to different types of stress) increased after salt treatment. Abiotic stresses, such as salinity, usually cause protein dysfunction. Maintaining proteins in their functional conformations thereby preventing the aggregation of non-native proteins is particularly important for cell survival under stress. Heat-shock proteins (HSPs)/chaperones are responsible for protein folding, assembly, translocation, and degradation in many normal cellular processes; they also stabilize proteins and membranes, and can assist in protein refolding under stress conditions (Wang et al., 2004). Data presented in this paper are supported by previous findings showing that at late developmental stages, when seeds undergo dehydration, LEA proteins, also regarded as chaperonins, are synthesized as part of the developmental program. Moreover, salinity triggers an abscisic acid (ABA)-mediated response that induces dehydrin accumulation in embryo cells (especially in the nucleus). This could imply a role for dehydrins as protective molecules for DNA when the cells are undergoing the normal dehydration process. Under salinity, however, dehydration could be drastically exacerbated, thereby favoring chaperonin accumulation (Burrieza et al., 2012). In Arabidopsis thaliana cell suspension cultures, the expression of HSP70 also increased under salt stress (Ndimba et al., 2005). Worthy of notice was the reduced content of a member of the peptidylprolyl cis-trans isomerase family, also called cyclophilins, known to assist protein folding by accelerating the isomerization of Xaa-Pro bonds, which is a rate-limiting step in the folding process of some proteins. This could be explained by the general decrease of proteins, including the substrates of cyclophilins.

Other proteins of quinoa seeds that responded to salinity are involved in the photosynthetic machinery, i.e., RuBisCO and plastid movement impaired protein. The RuBisCO enzyme is sensitive to salt stress as revealed by enhanced expression in leaves of salt-treated Triticum durum (Caruso et al., 2008). In developing embryos of $B$. napus L. (oilseed rape), RuBisCo acts without the Calvin cycle. This improves the carbon efficiency of developing green seeds in a metabolic context able to increase the efficiency of carbon use during the formation of oil (Schwender et al., 2004). Although photosynthesis is usually negatively affected by salinity, the enhanced expression of RuBisCO without the Calvin cycle could be involved in plant adaptation to salt stress at the seed level.

The Plastid Movement Impaired protein is required for regulation of chloroplast position in cells in order to reduce their avoidance. Unfortunately, it is difficult to assign a specific function in seed tissues to this protein directly involved in movement of plastids in response to light and normally expressed in leaves. We can only speculate that salt stress and, more generally, abiotic stress cause changes in the expression level of this protein. Salt stress also seemed to perturb proteins involved in respiration. In fact, the expression of mitochondrial ATP synthase was up-regulated in seeds of salt-treated quinoa plants. Mitochondrial ATP synthase is a key enzyme in plant metabolism and upregulation of its activity might be required in response to stressful conditions such salt stress. Increased production of ATP might fuel all the metabolic pathways generally involved in plant homeostasis. In addition, it has been proposed that increased activity of ATP synthase creates the driving force for $\mathrm{Na}^{+}$transport by the Salt Overly Sensitive1 (SOS1) membrane-localized proteins, whose activity is currently regarded as a strong basis for salt tolerance in plants (Zhu, 2003). Over-expression of an ATP synthase gene in Arabidopsis suspension-cultured cells increased resistance to salt, drought, oxidative, and cold stresses (Zhang et al., 2008). Upregulation of SOS1 gene expression was also reported for Chilean landraces of quinoa grown on saline media (Ruiz-Carrasco et al., 2011).

Salinity also affected three other main protein categories. Increased expression levels were observed for ion transport proteins (e.g., a cation $/ \mathrm{H}^{+}$antiporter), and for pantothenate kinase, and helicase carboxy-terminal domain protein involved in metabolism and cell division, respectively. The overexpression of these proteins during stress, and more specifically salt stress, has been reported in several studies. In particular, expression of the cation antiporter was found to increase in salt-treated tomato plants; the protein plays an important role in adaptation to salinity by improving $\mathrm{K}^{+}$accumulation (Rodriguez-Rosales et al., 2008). Pantothenate kinase activity plays a critical role in regulating intracellular coenzyme A (CoA) levels in bacteria and animals. In plants, pantothenate kinase activity was first reported in stroma of spinach chloroplasts, but little is known about the role of this enzyme (Tilton et al., 2006). Pantothenate, also known as vitamin B5 or "anti-stress vitamin," is part of the water-soluble B vitamin group. It is the key precursor of CoA and acyl carrier protein, which are essential co-factors for many metabolic enzymes (Raman and Rathinasabapathi, 2004). It was suggested that an increased level of CoA, mediated by an increased activity of pantothenate kinase, may be responsible for improved plant growth and stress resistance (Rubio et al., 2008). The main function of helicase is to bind ATP and to catalyze the reaction that drives the unwinding of DNA or RNA helix. Over-expression of this protein is one of the first cellular responses to stress conditions. Thus, pea DNA helicase was stimulated by salinity and cold stress in 
both shoots and roots (Vashisht et al., 2005). Moreover, DNA helicase 45 mRNA was induced in pea seedlings in response to high concentrations of salt and overexpression of this protein in transgenic tobacco plants conferred salinity tolerance, suggesting an important target pathway for manipulating stress tolerance in crop plants (Sanan-Mishra et al., 2005).

Ethylene Insensitive 3 -like 1 protein is probably a transcription factor acting as positive regulator in the ethylene signaling pathway. The expression of this protein decreased in salt-treated quinoa seeds. Ethylene plays important roles in multiple aspects of plant growth and development and its biosynthesis is induced by many stresses. However, its role in salt stress is uncertain. Cao et al. (2007) reported that alteration of ethylene signaling in Arabidopsis mutants affected salt-stress responses. El-Iklil and coworkers showed that lower ethylene production was associated with salt tolerance in tomato treated with high salt concentrations (El-Iklil et al., 2000), whereas higher ethylene production has been suggested as an indicator for salt tolerance in rice seeds (Khan et al., 1987). No information is available on the hormonal responses to abiotic stress in quinoa, except for one report on increased ABA levels under drought (Jacobsen et al., 2009).

The category exhibiting the greatest variation in expression levels under salinity was the functional category of seed storage proteins. Storage proteins accumulate in developing seeds as a source of nitrogen, carbon, sulfur, and amino acids for use in germination and growth of developing seedlings. Globulins generally fall into two major groups based on these coefficients: the 7-8S vicilin type and the 11-12S legumin type. Because legumin-type seed storage proteins vary in size, the 11-12S globulins are collectively referred to as legumins in other species (Balzotti et al., 2008). In our study, proteins belonging to this category were the $11 \mathrm{~S}$ globulin $\mathrm{A}$ or $\mathrm{B}$, and the legumin $\beta$ like. The 11S globulins are the major storage proteins in most legumes and in many other dicots (e.g., brassicas, composites, and cucurbits) and in some cereals (oats and rice; Shewry et al., 1995). Also known as chenopodin, the $11 \mathrm{~S}$ globulin is the major seed storage protein of quinoa. We found two different subunits of chenopodin; A and B subunit. The $11 \mathrm{~S}$ globulin is a hexamer consisting of six pairs of acidic $(34-36 \mathrm{kDa})$ and basic subunits $(22-24 \mathrm{kDa})$, with each subunit pair connected by a disulfide bond (Brinegar and Goundan, 1993). Recently, the quinoa $11 \mathrm{~S}$ globulin gene has been suggested to belong to a multigene family (Stevens et al., 2006), as in other species (Shewry et al., 1995). These proteins are related to each other based on their primary structures, with homologies reaching $63 \%$ between soybean proAlaB1b and pea prolegumin (Tandang-Silvas et al., 2010).

In the present paper, the 11S seed storage globulin displayed the strongest decrease in salt-treated samples as compared with controls; in fact, in all analyses we found a substantial downregulation of these proteins and even a complete absence of the corresponding spots in $2 \mathrm{DE}$ gels. The decrease in total protein content (Ruiz et al., 2016a) and in percentage content of the albumin/globulin fraction is thus ascribable to the severe reduction of the major seed storage protein, chenopodin. Decreased protein content with increasing salinity can also be attributed to disturbance in nitrogen metabolism or inhibition of nitrate absorption through reduced water uptake and decreased root permeability (Karyotis et al., 2003; Pulvento et al., 2012). The legumin $\beta$-like protein displayed an opposite trend insofar as the spot intensity increased in salt-treated samples. Legumin holoproteins are composed of six nearly identical subunits with molecular weights of 50-60 kDa. Each subunit is composed of two differently sized polypeptide chains. The larger more hydrophilic one $(30-40 \mathrm{kDa})$ is named $\alpha$-chain, whereas the smaller more hydrophobic one $(20 \mathrm{kDa})$ is named $\beta$-chain (Müntz, 1996). A relatively lower decrease in energy-rich storage compounds, such as storage proteins (legumin-like and 11S seed storage protein) was found in tolerant wheat genotypes than in less tolerant and sensitive genotypes upon cold treatment (Kosová et al., 2015). In soybean seeds, $\beta$-conglycinin and glycinin are the two major storage proteins; sulfur deficiency caused a substantial decrease in the level of glycinins and a concomitant increase in $\beta$-conglycinins; the subunit composition of these proteins was also affected (Gayler and Sykes, 1985). The crude acid-soluble globulin seemed not to be affected by growing quinoa under salinity, but since a crude extract and not a purified or highly enriched fraction (as was done for the 11S globulin) was analyzed, we cannot exclude possible changes in protein expression and content. Prolamin-related proteins likewise did not show evident changes in the presence of salt. This is probably due to the low amount of these peptides in gluten-free seeds such as those of quinoa. However, also in this case, we cannot exclude slight changes in this fraction, which represents a very interesting research topic with respect to gluten allergy also in so-called gluten-free crops.

\section{Amino Acid Profiles}

The amino acid profile of quinoa is one of its major nutritional attributes as it contains all the essential amino acids (Escuredo et al., 2014). The amino acid composition of quinoa seeds of cultivars from the Andean highlands (Bolivia/Argentina) and Argentinian Northwest were shown to vary depending on genotype and area of cultivation (González et al., 2011). Changes in the amino acid profile under drought and salinity, alone or combined, have been described in wild and cultivated barleys (Ahmed et al., 2013), but not in quinoa. In barley, the contents of all amino acids (except Met) were significantly increased relative to controls under drought alone or under combined stress in all genotypes, whereas they decreased or remained unchanged under salinity alone. Present results confirm the strongly genotype-dependent amino acid profile, both under control and salinized conditions, in the three Chilean landraces analyzed. Unexpectedly, the profiles of R49 (salares ecotype) and VR (coastal lowlands ecotype) were more similar than those of VR and VI-1, which belong to the same ecotype.

The non-essential amino acids Ala, Pro, and Cys were generally the most abundant PAAs in all three landraces. With the exception of VI-1, Pro was also the most abundant free amino acid; it was one of the few whose content was unaltered or enhanced by salinity in all three landraces, including VI1. Induction of free amino acids in response to abiotic stress 
is thought to play a role in plant stress tolerance because of their role as osmolytes. The accumulation of Gly, Pro, Ala, and $\mathrm{Val}$ is regarded as a general response to stress. In particular, the accumulation of branched-chain amino acids (BCAAs) Val, Leu, Ile-is induced by osmotic stress (Joshi et al., 2010). Ile is synthesized from Thr and Met; these three amino acids are synthesized in a highly regulated network depending on physiological and growth requirements. In the present study, free BCAAs (Val, Leu, Ile) were most strongly induced (twoto almost four-fold relative to controls) by salinity in R49, but either not affected or diminished in the other two landraces. It is noteworthy that Thr was also strongly upregulated by salinity in both R49 and VR, but not in VI-1. Unlike Pro, little is known about the function of the stress-induced response involving BCAAs, even though Pro may represent only $10 \%$ of the total free amino acids induced by drought (Shen et al., 1989). The increases in BCAAs of drought-stressed tomato leaves were also higher than that of Pro (Joshi et al., 2010). Moreover, it has been proposed that accumulation of free BCAAs may serve as a substrate for the synthesis of stress-induced proteins and that they may act as signaling molecules to regulate gene expression. Further research is needed in this area particularly for stresstolerant species such as quinoa.

As a general response to abiotic stress, all plants, even halophytes such as quinoa, accumulate amino acids, betaines, sugars, organic acids, and other osmoprotectants (Parida and Das, 2005; Ruiz-Carrasco et al., 2011). Thus, amino acid metabolism, including protease activation, may play an important role in plant tolerance, e.g., under drought stress (Joshi et al., 2010). As observed in the present study, increased contents of at least some amino acids could be the result of protein degradation, although de novo synthesis cannot be excluded. For example, Pro decreased in the protein fraction, but increased in the free form in all three landraces of quinoa under salinity. A better understanding of this process will also provide new insight into the negative vs. beneficial effects of abiotic stress on the nutritional qualities of quinoa.

In many cereals, two essential amino acids, Thr and Met, are less abundant than required for the human diet. In R49, free Thr was strongly induced by salinity; similarly, Met was induced by salt in both fractions (free and PAA) only in R49, suggesting that salt stress could improve the nutritional properties of this landrace. By contrast, Thr was very high in VI-1 under nonsaline conditions and fell below detection limit in seeds from salt-grown plants. In quinoa, the amount of the essential amino acid Lys is higher than that of cereals (Escuredo et al., 2014). In our study, Lys, representing about $10 \%$ of total free amino acids in control seeds, was also differentially affected by salinity in a genotype-dependent manner. Overall, this important amino acid was maintained or induced only in R49 in both fractions.

Based on these results, VI-1 seems to be the most sensitive to salinity in terms of amino acid profiles, and R49 the most tolerant, in accord with its origin from a highly stress-prone area. The differential response between the two coastal-lowlands landraces is in agreement with results for other parameters, namely AA, TPC, and TFC.

\section{Bioactive Molecules and Antioxidant Activity of Quinoa Seed Protein Extracts}

Plant-derived antioxidants (phenolic compounds, tocopherols, tocotrienols, ascorbic acid and carotenoids) are essential for counteracting oxidative stress and hence contrasting the insurgence of various diseases. In recent years, the quest for natural antioxidants for dietary as well as cosmetic and pharmaceutical uses is a major objective in plant research. AA is also a matter of great practical interest in food science since oxidation, mainly of lipids and proteins, can lead to the deterioration of quality attributes, such as flavor, aroma, and color. The health benefits of quinoa seeds derive from their nutritional properties (e.g., amino acid composition, minerals, vitamins) but also from their content in bioactive molecules, such as phenolic compounds, and their antioxidant activity. Not surprisingly, therefore, these aspects are increasingly attracting the attention of researchers (Abderrahim et al., 2015; Tang et al., 2015).

We have previously observed that TPC (measured with the FC method) in methanolic extracts of quinoa seed was genotypedependent and enhanced by salinity in landraces R49 and VR (Ruiz et al., 2016a). In R49, increased seed TPC could be related to better germination capacity on saline media. Improved antioxidant defense may also provide protection from salt stress during seedling establishment. Present results confirm this trend also for PCs obtained from the same seeds. Thus, in all three landraces, seed PCs from plants irrigated with $300 \mathrm{mM} \mathrm{NaCl}$ had higher phenolic contents than those grown without $\mathrm{NaCl}$. Spectrophotometric redox assays are among the most common methods for determining total phenolics in plant extracts as they detect the easily oxidized phenolic groups. While being the standard method for the determination of TPC, the FC assay is essentially a means of evaluating the redox potential of a plant extract and is, therefore, subject to interference by many compounds (e.g., sugars, proteins, organic acids; RodriguezAmaya, 2010). The Prussian blue method is less subject to interference from non-phenolic compounds and was, therefore, used in parallel in the present work to check for the presence of polyphenols in seed protein extracts. This method provided further proof that polyphenols were present. Although in most cases below detection limit or very low, they were dramatically enhanced in VR under salinity, thus confirming results of the FC assay.

Although protein extraction methods can also extract phenolic compounds, it is important to determine whether they maintain their bioactivity (Salgado et al., 2012). Based on the ABTS radical scavenging test, quinoa seed PC did exhibit AA. Values were also significantly different between landraces (highest in VR) and were slightly increased by salinity in R49 and VR, the two landraces in which TPC and flavonoids (the latter only in VR) also increased the most. These data point to some degree of correlation between phenolic content and AA. If, however, we consider the FC assay also as an indicator of redox potential, then it can be inferred that the antioxidant capacity of salt-treated samples was higher than that of controls in all three landraces. 
Phenolic compounds are mainly accumulated in the epidermis to protect photosynthetic tissues from excessive radiation. They also scavenge free radicals and other reactive species insofar as they possess many hydroxyl groups with a high capacity to scavenge ABTS and DPPH (2,2-diphenyl-1-picrylhydrazyl) radicals (Cai et al., 2006). To our knowledge, this is the first time that phenolic content and AA is determined for quinoa seed PCs. In seeds of A. cruentus, Escudero et al. (2011) reported

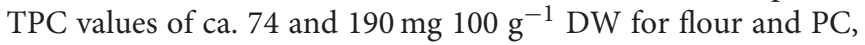

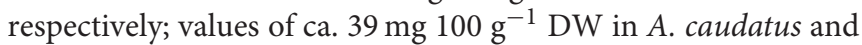
$56 \mathrm{mg} 100 \mathrm{~g}^{-1} \mathrm{DW}$ in A. paniculatus have also been reported (Klimczak et al., 2002). Based on the same FC assay, TPC for quinoa seeds $\left(10-70 \mathrm{mg} 100 \mathrm{~g} \mathrm{DW}^{-1}\right.$ ) were within the range of those reported above for amaranth flour extracts, but lower than those of the PC (prepared essentially as in the present work for quinoa seeds). Total flavonoids in A. cruentus (Escudero et al., 2011) were comparable to those of quinoa for seeds from plants grown under non-saline conditions, but were several fold higher in salt-treated quinoa seeds.

A strong correlation between TPC/TFC and AA has been reported in quinoa (Dini et al., 2010; Ismail et al., 2016). The concentration of phenolic compounds in quinoa protein extracts-as determined by the Prussian blue method-was too low to be regarded as the main component involved in AA, except possibly in VR from salt-treated plants. Their presence in proteins extracted by alkalinization followed by acidification may be attributed to a strong interaction between proteins and these secondary metabolites. Seeds, and plant material in general, are also rich in insoluble phenols, simple or highly polymerized (for example, tannins), which can be associated to carbohydrates and proteins (Ozdal et al., 2013). However, there is mounting evidence that proteins themselves can act as free radicals scavengers. The AA of proteins is due to complex interactions between their ability to inactivate ROS, scavenge free radicals, chelate pro-oxidative transition metals, reduce hydroperoxides, enzymatically eliminate specific oxidants, and alter the physical properties of food systems in a way that separates reactive species (Elias et al., 2008). This capacity depends on the amounts of hydrophobic and aromatic amino acids (Petchiammal and Hopper, 2014). The major seed storage proteins of legumes and other plant species have been reported to have radical scavenging potential. In hemp seed, edestin, a storage protein with all the typical features of the $11 \mathrm{~S}$ globulin storage proteins, was reported to possess antioxidant and antihypertensive activity (Girgih et al., 2014). Present data indicate that PCs from quinoa seeds also have this potential. Moreover, a low but detectable AA was also measured in the $11 \mathrm{~S}$ (chenopodin) fraction extracted without $\beta$-mercaptoethanol.

Establishing a link between salinity tolerance and accumulation of antioxidant molecules is not straightforward. Recently, foliar levels of antioxidant enzymes and molecules in two genotypes of quinoa (cvs Utusaya and Titicaca) with different levels of salinity tolerance were analyzed (Ismail et al., 2016). AA and TPC were slightly increased and rutin concentrations increased by ca. 25-fold in salt-exposed leaves of cv. Titicaca but not Utusaya. These results were interpreted as an indication that the more tolerant genotype Utusaya has a lesser requirement to trigger the accumulation of antioxidant molecules. In accord with this interpretation, foliar levels of polyphenols in control plants of R49 were lower than in VI-1 and VR (Ruiz et al., 2016a). Present results provide a similar picture: $\mathrm{VR}$, the landrace from the less stress-prone area (presumably the least tolerant of the three), exhibited the highest TPC and AA both under control and saline conditions and the highest TFC in the latter situation; by contrast, the tolerant salares landrace had the lowest values. Interestingly, the two coastal-lowlands landraces (VI-1 and VR) responded differently to salt treatment; PCs of VR seeds exhibited the stronger salt-induced increase in TPC, TFC, and AA. However, when comparing the salares landrace R49 with VR, no major differences could be observed in relative terms (treated vs. control), suggesting that the positive effects of salinity on the amount of bioactive molecules in quinoa PC could be regarded as a general effect and not an indicator of greater or lesser tolerance based on the habitat of origin. This confirms data from another study comparing two genotypes (Regalona and VR) grown under different environmental conditions (arid and cold-temperate), which indicated that nutritional and functional features were enhanced by cultivation in the more stressful arid region (Miranda et al., 2013).

\section{CONCLUSIONS}

In conclusion, the data presented here reveal that salinity induced deep changes in the amino acid composition and in protein profiles of the main seed storage proteins of quinoa as well as in the contents of bioactive molecules. The responses were differentially induced in the different landraces, providing evidence that breeding can further ameliorate the nutritional quality of quinoa. The AA of seed protein extracts can be explained by the presence of phenolics, but we cannot exclude that proteins themselves, including the $11 \mathrm{~S}$ fraction, possess this capacity. The proteomic analysis highlights some promising novel candidates with regard to salt-induced effects on seed quality. The most interesting ones should be further studied in terms of their structural and functional roles in order to enhance our understanding of the salt stress responses in a crop with such unique environmental adaptive characteristics and nutritional value. The strongly genotype-dependent responses to salinity confirm that quinoa landraces are a rich source of genetic variation with respect to stress tolerance and that they are useful for further improving adaptation of this species to diverse environments. Moreover, they confirm that in some cases (e.g., R49) abiotic stress may improve the nutritional properties of quinoa seeds.

\section{AUTHOR CONTRIBUTIONS}

KR designed the experiment and produced the plant material; IA, KR, CL, and LP performed the experiments, analyzed the data and prepared the figures; SB, SD, GC, and LB planned the research and interpreted the data; $\mathrm{IA}, \mathrm{SB}$, and LP wrote the article with contributions from all other authors. 


\section{ACKNOWLEDGMENTS}

Research was supported by funds (RFO) from the University of Bologna (Italy) to $\mathrm{SD}$ and $\mathrm{SB}$ and a CONICYT, FONDECYT/Postdoctoral grant (No. 3130624) to KR. The Departamento de Producción Agrícola (Universidad de Chile,

\section{REFERENCES}

Abderrahim, F., Huanatico, E., Segura, R., Arribas, S., Gonzalez, M. C., and Condezo-Hoyos, L. (2015). Physical features, phenolic compounds, betalains and total antioxidant capacity of coloured quinoa seeds (Chenopodium quinoa Willd.) from Peruvian Altiplano. Food Chem. 183, 83-90. doi: 10.1016/j.foodchem.2015.03.029

Abugoch, L. E., Romero, N., Tapia, C. A., Silva, J., and Rivera, M. (2008). Study of some physicochemical and functional properties of quinoa (Chenopodium quinoa Willd.) protein isolates. J. Agric. Food Chem. 56, 4745-4750. doi: $10.1021 / \mathrm{jf7} 703689 \mathrm{u}$

Adolf, V. I., Jacobsen, S.-E., and Shabala, S. (2013). Salt tolerance mechanisms in quinoa (Chenopodium quinoa Willd.). Environ. Exp. Bot. 92, 43-54. doi: 10.1016/j.envexpbot.2012.07.004

Adolf, V. I., Shabala, S., Andersen, M. N., Razzaghi, F., and Jacobsen, S.-E. (2012). Varietal differences of quinoa's tolerance to saline conditions. Plant Soil 357, 117-129. doi: 10.1007/s11104-012-1133-7

Ahmad, F., and Slinkard, A. E. (1992). Genetic relationships in the genus Cicer L. as revealed by polyacrylamide gel electrophoresis of seed storage proteins. Theor. Appl. Genet. 84, 688-692. doi: 10.1007/bf00224169

Ahmed, I. M., Cao, F., Han, Y., Nadira, U. A., Zhang, G., and Wu, F. (2013). Differential changes in grain ultrastructure, amylase, protein and amino acid profiles between Tibetan wild and cultivated barleys under drought and salinity alone and combined stress. Food Chem. 141, 2743-2750. doi: 10.1016/j.foodchem.2013.05.101

Arnao, M. B., Cano, A., Alcolea, J. F., and Acosta, M. (2001). Estimation of free radical-quenching activity of leaf pigment extracts. Phytochem. Anal. 12, 138-143. doi: 10.1002/pca.571

Balzotti, M. R. B., Thornton, J. N., Maughan, P. J., McClellan, D. A., Stevens, M. R., Jellen, E. N., et al. (2008). Expression and evolutionary relationships of the Chenopodium quinoa 11S seed storage protein gene. Int. J. Plant Sci. 169, 281-291. doi: 10.1086/523874

Bergamo, P., Maurano, F., Mazzarella, G., Iaquinto, G., Vocca, I., Rivelli, A. R., et al. (2011). Immunological evaluation of the alcohol-soluble protein fraction from gluten-free grains in relation to celiac disease. Mol. Nutr. Food Res. 55, 1266-1270. doi: 10.1002/mnfr.201100132

Bhargava, A., Rana, T. S., Shukla, S., and Ohri, D. (2005). Seed protein electrophoresis of some cultivated and wild species of Chenopodium. Biol. Plant. 49, 505-511. doi: 10.1007/s10535-005-0042-5

Brinegar, C., and Goundan, S. (1993). Isolation and characterization of chenopodin, the 11S seed storage protein of quinoa (Chenopodium quinoa). J. Agric. Food Chem. 41, 182-185. doi: 10.1021/jf00026a006

Brinegar, C., Sine, B., and Nwokocha, L. (1996). High-cysteine 2 S seed storage proteins from quinoa (Chenopodium quinoa). J. Agric. Food Chem. 44, 1621-1623. doi: 10.1021/jf950830+

Burrieza, H. P., Koyro, H.-W., Martínez-Tosar, L., Kobayashi, K., and Maldonado, S. (2012). High salinity induces dehydrin accumulation in Chenopodium quinoa Willd. cv. Hualhuas embryos. Plant Soil 354, 69-79. doi: 10.1007/s11104-0111045-y

Cai, Y. Z., Mei, S., Jie, X., Luo, Q., and Corke, H. (2006). Structure-radical scavenging activity relationships of phenolic compounds from traditional Chinese medicinal plants. Life Sci. 78, 2872-2888. doi: 10.1016/j.lfs.2005.11.004

Cao, W. H., Liu, J., He, X. J., Mu, R. L., Zhou, H. L., Chen, S. Y., et al. (2007). Modulation of ethylene responses affects plant salt-stress responses. Plant Physiol. 143, 707-719. doi: 10.1104/pp.106.094292

Caruso, G., Cavaliere, C., Guarino, C., Gubbiotti, R., Foglia, P., and Lagana, A. (2008). Identification of changes in Triticum durum L. leaf proteome in response to salt stress by two-dimensional electrophoresis and MALDI-TOF mass
Santiago) is gratefully acknowledged for providing the facilities for growing the plants. The authors are grateful to Dr. Pedro León (Seed Bank, INIA-Vicuña, Chile) for supplying the seeds and to Mr. Massimo Guarnieri (Department Life Sciences, University of Siena) for the technical assistance in amino acid analyses.

spectrometry. Anal. Bioanal. Chem. 391, 381-390. doi: 10.1007/s00216-0082008-X

Castel, V., Andrich, O., Netto, F. M., Santiago, L. G., and Carrara, C. R. (2014). Total phenolic content and antioxidant activity of different streams resulting from pilot-plant processes to obtain Amaranthus mantegazzianus protein concentrates. J. Food Eng. 122, 62-67. doi: 10.1016/j.jfoodeng.2013.08.032

Cordero-De-Los-Santos, J., Osuna-Castro, A., Borodanenko, A., and ParedesLópez, O. (2005). Physicochemical and functional characterization of Amaranth (Amaranthus hypochondriacus) protein isolates obtained by isoelectric precipitation and micellisation. Food Sci. Technol. Int. 11, 269-280. doi: 10.1177/1082013205056491

Dini, I., Tenore, G. C., and Dini, A. (2010). Antioxidant compound contents and antioxidant activity before and after cooking in sweet and bitter Chenopodium quinoa seeds. LWT Food Sci. Technol. 43, 447-451. doi: 10.1016/j.lwt.2009.09.010

Džunková, M., Janovská, D., Čepková, P. H., Prohasková, A., and Koláŕ, M. (2011). Glutelin protein fraction as a tool for clear identification of amaranth accessions. J. Cereal Sci. 53, 198-205. doi: 10.1016/j.jcs.2010.12.003

Elias, R. J., Kellerby, S. S., and Decker, E. A. (2008). Antioxidant activity of proteins and peptides. Crit. Rev. Food Sci. Nutr. 48, 430-441. doi: 10.1080/10408390701425615

El-Iklil, Y., Karrou, M., and Benichou, M. (2000). Salt stress effect on epinasty in relation to ethylene production and water relations in tomato. Agronomie 20, 399-406. doi: 10.1051/agro:2000136

Escudero, N. L., Albarracín, G. J., Lucero López, R. V., and Giménez, M. S. (2011). Antioxidant activity and phenolic content of flour and protein concentrate of Amaranthus cruentus seeds. J. Food Biochem. 35, 1327-1341. doi: 10.1111/j.1745-4514.2010.00454.x

Escudero, N. L., Arellano, M. L., Luco, J., Giménez, M. S., and Mucciarelli, S. (2004). Comparison of the chemical composition and nutritional value of Amaranthus cruentus flour and protein concentrate. Plant Foods Hum. Nutr. 59, 15-21. doi: 10.1007/s11130-004-0033-3

Escuredo, O., González Martín, M. I., Moncada, G. W., Fischer, S., and Hernández Hierro, J. M. (2014). Amino acid profile of the quinoa (Chenopodium quinoa Willd.) using near infrared spectroscopy and chemometric techniques. J. Cereal Sci. 60, 67-74. doi: 10.1016/j.jcs.2014.01.016

Fuentes, F. F., Martínez, E. A., Hinrichsen, P. V., Jellen, E. N., and Maughan, P. J. (2009). Assessment of genetic diversity patterns in Chilean quinoa (Chenopodium quinoa Willd.) germplasm using multiplex fluorescent microsatellite markers. Conserv. Genet. 10, 369-377. doi: 10.1007/s10592-0089604-3

Gayler, K. R., and Sykes, G. E. (1985). Effects of nutritional stress on the storage proteins of soybeans. Plant Physiol. 78, 582-585. doi: 10.1104/pp.78.3.582

Ghafoor, A., Ahmad, Z., Qureshi, A. S., and Bashir, M. (2002). Genetic relationship in Vigna mungo (L.) Hepper and V. radiata (L.) R. Wilczek based on morphological traits and SDS-PAGE. Euphytica 123, 367-378. doi: 10.1023/A:1015092502466

Girgih, A. T., He, R., Malomo, S., Offengenden, M., Wu, J., and Aluko, R. E. (2014). Structural and functional characterization of hemp seed (Cannabis sativa L.) protein derived antioxidant and antihypertensive peptides. J. Funct. Foods 6, 384-394. doi: 10.1016/j.jff.2013.11.005

Gómez-Caravaca, A., Iafelice, G., Lavini, A., Pulvento, C., Caboni, M., and Marconi, E. (2012). Phenolic compounds and saponins in quinoa samples (Chenopodium quinoa Willd.) grown under different saline and nonsaline irrigation regimes. J. Agric. Food Chem. 60, 4620-4627. doi: 10.1021/jf3002125

Gómez-Pando, L. R., Álvarez-Castro, R., and De La Barra, E. (2010). Effect of salt stress on Peruvian germplasm of Chenopodium quinoa Willd.: a promising crop. J. Agron. Crop Sci. 196, 391-396. doi: 10.1111/j.1439-037X.2010.00429.x 
González, J. A., Konishi, Y., Bruno, M., Valoy, M., and Prado, F. E. (2011). Interrelationships among seed yield, total protein and amino acid composition of ten quinoa (Chenopodium quinoa) cultivars from two different agroecological regions. J. Sci. Food Agr. 92, 1222-1229. doi: 10.1002/jsfa.4686

Hagerman, A. E., and Butler, L. G. (1994). Assay of condensed tannins or flavonoid oligomers and related flavonoids in plants. Methods Enzymol. 234, 429-437. doi: 10.1016/0076-6879(94)34113-3

Hariadi, Y., Marandon, K., Tian, Y., Jacobsen, S.-E., and Shabala, S. (2011). Ionic and osmotic relations in quinoa (Chenopodium quinoa Willd.) plants grown at various salinity levels. J. Exp. Bot. 62, 185-193. doi: 10.1093/jxb/erq257

Hellman, U., Wernstedt, C., Gonez, J., and Heldin, C. H. (1995). Improvement of an "In-Gel" digestion procedure for the micropreparation of internal protein fragments for amino acid sequencing. Anal. Biochem. 224, 451-455. doi: 10.1006/abio.1995.1070

Hirose, Y., Fujita, T., Ishii, T., and Ueno, N. (2010). Antioxidative properties and flavonoid composition of Chenopodium quinoa seeds cultivated in Japan. Food Chem. 119, 1300-1306. doi: 10.1016/j.foodchem.2009.09.008

Ismail, H., Dragiši Maksimović, H., Maksimović, V., Shabala, L., Živanović, B. D., Tian, Y., et al. (2016). Rutin, a flavonoid with antioxidant activity, improves plant salinity tolerance by regulating $\mathrm{K}^{+}$retention and $\mathrm{Na}^{+}$exclusion from leaf mesophyll in quinoa and broad beans. Funct. Plant Biol. 43, 75-86. doi: 10.1071/FP15312

Jacobsen, S. E., Liu, F., and Jensen, C. R. (2009). Does root-sourced ABA play a role for regulation of stomata under drought in quinoa (Chenopodium quinoa Willd.). Sci. Hortic. 122, 281-287. doi: 10.1016/j.scienta.2009.05.019

Jacobsen, S.-E., Mujica, A., and Jensen, C. R. (2003). The resistance of quinoa (Chenopodium quinoa Willd.) to adverse abiotic factors. Food Rev. Int. 19, 99-109. doi: 10.1081/FRI-120018872

Jha, S. S., and Ohri, D. (1996). Phylogenetic relationships of Cajanus cajan (L.) Millsp. (pigeon pea) and its wild relatives based on seed protein profiles. Genet. Res. Crop Evol. 43, 275-281. doi: 10.1007/BF00123279

Joshi, V., Joung, J.-G., Fei, Z., and Jander, G. (2010). Interdependence of threonine, methionine and isoleucine metabolism in plants: accumulation and transcriptional regulation under abiotic stress. Amino Acids 39, 933-947. doi: 10.1007/s00726-010-0505-7

Ju, Z. Y., Hettiarachchy, N. S., and Rath, N. (2001). Extraction, denaturation and hydrophobic properties of rice flour proteins. J. Food Sci. 66, 229-232. doi: 10.1111/j.1365-2621.2001.tb11322.x

Karyotis, T., Iliadis, C., Noulas, C., and Mitsibonas, T. (2003). Preliminary research on seed production and nutrient content for certain quinoa varieties in a saline-sodic soil. J. Agron. Crop Sci. 189, 402-408. doi: 10.1046/j.09312250.2003.00063.x

Khan, A. A., Akbar, M., and Seshu, D. V. (1987). Ethylene as an indicator of salt tolerance in rice. Crop Sci. 27, 1242-1248. doi: 10.2135/cropsci1987.0011183X002700060031x

Klimczak, I., Małecka, M., and Pachołek, B. (2002). Antioxidant activity of ethanolic extracts of amaranth seeds. Nahrung/Food 46, 184-186. doi: 10.1002/1521-3803(20020501)46:3<184::AID-FOOD184>3.0.CO;2-H

Kosová, K., Vítámvás, P., Urban, M. O., Klíma, M., Roy, A., and Prášil, I. T. (2015). Biological networks underlying abiotic stress tolerance in temperate crops-a proteomic perspective. Int. J. Mol. Sci. 16, 20913-20942. doi: 10.3390/ijms160920913

Koyro, H.-W., and Eisa, S. S. (2008). Effect of salinity on composition, viability and germination of seeds of Chenopodium quinoa Willd. Plant Soil 302, 79-90. doi: 10.1007/s11104-007-9457-4

Koyro, H.-W., Zörb, C., Debez, A., and Huchzermeyer, B. (2013). The effect of hyper-osmotic salinity on protein pattern and enzyme activities of halophytes. Funct. Plant Biol. 40, 787-804. doi: 10.1071/fp12387

Laemmli, U. K. (1970). Cleavage of structural proteins during assembly of head of bacteriophage-T4. Nature 227, 680-685. doi: 10.1038/227680a0

Liu, M., Li, X. Q., Weber, C., Lee, C. Y., Brown, J., and Liu, R. H. (2002). Antioxidant and antiproliferative activities of raspberries. J. Agric. Food Chem. 50, 2926-2930. doi: 10.1021/jf0111209

Miranda, M., Delatorre-Herrera, J., Vega-Gálvez, A., Jorquera, E., Quispe-Fuentes, I., and Martínez, E. A. (2014). Antimicrobial potential and phytochemical content of six diverse sources of quinoa seeds (Chenopodium quinoa Willd.). Agric. Sci. 5, 1015-1024. doi: 10.4236/as.2014.511110
Miranda, M., Vega-Gálvez, A., Martínez, E. A., López, J., Marín, R., Aranda, M., et al. (2013). Influence of contrasting environments on seed composition of two quinoa genotypes: nutritional and functional properties. Chil. J. Agric. Res. 73, 108-116. doi: 10.4067/S0718-58392013000200004

Müntz, K. (1996). Proteases and proteolytic cleavage of storage proteins in developing and germinating dicotyledonous seeds. J. Exp. Bot. 47, 605-622. doi: 10.1093/jxb/47.5.605

Nath, P., Ohri, D., Jha, S. S., and Pal, M. (1997). Seed protein electrophoresis of wild and cultivated species of Celosia (Amaranthaceae). Genet. Res. Crop Evol. 44, 241-245. doi: 10.1023/A:1008670407477

Ndimba, B. K., Chivasa, S., Simon, W. J., and Slabas, A. R. (2005). Identification of Arabidopsis salt and osmotic stress responsive proteins using two-dimensional difference gel electrophoresis and mass spectrometry. Proteomics 5, 4185-4196. doi: 10.1002/pmic.200401282

Osborne, T. B. (1924). The Vegetable Proteins. London: Longmans, Green.

Ozdal, T. C., Apanoglu, E., and Altay, F. (2013). A review on proteinphenolic interactions and associated changes. Food Res. Int. 51, 954-970. doi: 10.1016/j.foodres.2013.02.009

Parida, A. K., and Das, A. B. (2005). Salt tolerance and salinity effects on plants: a review. Ecotoxicol. Environ. Saf. 60, 324-349. doi: 10.1016/j.ecoenv.2004.06.010

Paśko, P., Bartoń, H., Zagrodzki, P., Gorinstein, S., Fołta, M., and Zachwieja, Z. (2009). Anthocyanins, total polyphenols and antioxidant activity in amaranth and quinoa seeds and sprouts during their growth. Food Chem. 115, 994-998. doi: 10.1016/j.foodchem.2009.01.037

Petchiammal, C., and Hopper, W. (2014). Antioxidant activity of proteins from fifteen varieties of legume seeds commonly consumed in India. Int. J. Pharm. Sci. 6, 476-479.

Peterson, A., and Murphy, K. (2015). Tolerance of lowland quinoa cultivars to sodium chloride and sodium sulfate salinity. Crop Sci. 55, 331-338. doi: $10.2135 /$ cropsci2014.04.0271

Prego, I., Maldonado, S., and Otegui, M. (1998). Seed structure and localization of reserves in Chenopodium quinoa. Ann. Bot. 82, 481-488. doi: 10.1006/anbo.1998.0704

Pulvento, C., Riccardi, M., Lavini, A., Iafelice, G., Marconi, E., and D’Andria, R. (2012). Yield and quality characteristics of Chenopodium quinoa Willd. grown in open field under different saline and not saline irrigation. J. Agron. Crop Sci. 198, 254-263. doi: 10.1111/j.1439-037X.2012.00509.x

Raman, S. B., and Rathinasabapathi, B. (2004). Pantothenate synthesis in plants Plant Sci. 167, 961-968. doi: 10.1016/j.plantsci.2004.06.019

Repo-Carrasco-Valencia, R., Hellström, J. K., Pihlava, J.-M., and Mattila, P. H. (2010). Flavonoids and other phenolic compounds in Andean indigenous grains: Quinoa (Chenopodium quinoa), kañiwa (Chenopodium pallidicaule) and kiwicha (Amaranthus caudatus). Food Chem. 120, 128-133. doi: 10.1016/j.foodchem.2009.09.087

Rodriguez-Amaya, D. B. (2010). Quantitative analysis, in vitro assessment of bioavailability and antioxidant activity of food carotenoids - A review. J. Food Comp. Anal. 23, 726-740. doi: 10.1016/j.jfca.2010.03.008

Rodriguez-Rosales, M. P., Jiang, X., Galvez, F. J., Aranda, M. N., Cubero, B., and Venema, K. (2008). Overexpression of the tomato $\mathrm{K}^{+} / \mathrm{H}^{+}$antiporter LeNHX2 confers salt tolerance by improving potassium compartmentalization. New Phytol. 179, 366-377. doi: 10.1111/j.1469-8137.2008.02461.x

Rubio, S., Whitehead, L., Larson, T. R., Graham, I. A., and Rodriguez, P. L. (2008), The coenzyme A biosynthetic enzyme phosphopantetheine adenylyltransferase plays a crucial role in plant growth, salt/osmotic stress resistance, and seed lipid storage. Plant Physiol. 148, 546-556. doi: 10.1104/pp.108.124057

Ruiz, K. B., Aloisi, I., Del Duca, S., Canelo, V., Torrigiani, P., Silva, H., et al. (2016a). Salares versus coastal ecotypes of quinoa: salinity responses in Chilean landraces from contrasting habitats. Plant Physiol. Biochem. 101, 1-13. doi: 10.1016/j.plaphy.2016.01.010

Ruiz, K. B., Biondi, S., Martínez, E. A., Orsini, F., Antognoni, F., and Jacobsen, S.-E. (2016b). Quinoa - a model crop for understanding salt tolerance mechanisms in halophytes. Plant Biosyst. 150, 357-371. doi: 10.1080/11263504.2015.1027317

Ruiz-Carrasco, K. B., Antognoni, F., Coulibaly, A. K., Lizardi, S., Covarrubias, A., Martínez, E. A., et al. (2011). Variation in salinity tolerance of four lowland genotypes of quinoa (Chenopodium quinoa Willd.) as assessed by growth, physiological traits, and sodium transporter gene expression. Plant Physiol. Biochem. 49, 1333-1341. doi: 10.1016/j.plaphy.2011.08.005 
Salgado, P. R., López-Caballero, M. E., Gómez-Guillén, M. C., Mauri, A. N., and Montero, M. P. (2012). Exploration of the antioxidant and antimicrobial capacity of two sunflower protein concentrate films with naturally present phenolic compounds. Food Hydrocoll. 29, 374-381. doi: 10.1016/j.foodhyd.2012.03.006

Sanan-Mishra, N., Pham, X. H., Sopory, S. K., and Tuteja, N. (2005). Pea DNA helicase 45 overexpression in tobacco confers high salinity tolerance without affecting yield. Proc. Natl. Acad. Sci. U.S.A. 102, 509-514. doi: 10.1073/pnas.0406485102

Sano, N., Rajjou, L., North, H. M., Debeaujon, I., Marion-Poll, A., and Seo, M. (2015). Staying alive: molecular aspects of seed longevity. Plant Cell Physiol. 57, 660-674. doi: 10.1093/pcp/pcv186

Schwender, J., Goffman, F., Ohlrogge, J. B., and Shachar-Hill, Y. (2004). Rubisco without the Calvin cycle improves the carbon efficiency of developing green seeds. Nature 432, 779-782. doi: 10.1038/nature03145

Shen, L., Foster, J. G., and Orcutt, D. M. (1989). Composition and distribution of free amino-acids in flatpea (Lathyrus sylvestris L.) as influenced by water deficit and plant-age. J. Exp. Bot. 40, 71-79. doi: 10.1093/jxb/40.1.71

Shevchenko, A., Wilm, M., Vorm, O., and Mann, M. (1996). Mass spectrometric sequencing of proteins silver-stained polyacrylamide gels. Anal. Chem. 68, 850-858. doi: 10.1021/ac950914h

Shewry, P. R., Napier, J. A., and Tatham, A. S. (1995). Seed storage proteins: structures and biosynthesis. Plant Cell 7, 945-956. doi: 10.1105/tpc.7.7.945

Silvanini, A., Dall'Asta, C., Morrone, L., Cirlini, M., Beghè, D., Fabbri, A., et al. (2014). Altitude effects on fruit morphology and flour composition of two chestnut cultivars. Sci. Hortic. 176, 311-318. doi: 10.1016/j.scienta.2014.07.008

Singleton, V. L., and Rossi, J. A. J. (1965). Colorimetry of total phenolics with phosphomolybdic-phosphotungstic acid reagent. Am. J. Enol. Vitic. 16, 144-158.

Soskić, V., Gorlach, M., Poznanović, S., Boehmer, F. D., and GodovacZimmermann, J. (1999). Functional proteomics analysis of signal transduction pathways of the platelet-derived growth factor beta receptor. Biochemistry 38, 1757-1764. doi: 10.1021/bi982093r

Stevens, M. R., Coleman, C. E., Parkinson, S. E., Maughan, P. J., Zhang, H. B., Balzotti, M. R., et al. (2006). Construction of a quinoa (Chenopodium quinoa Willd.) BAC library and its use in identifying genes encoding seed storage proteins. Theor. Appl. Genet. 112, 1593-1600. doi: 10.1007/s00122-0060266-6

Tandang-Silvas, M. R., Fukuda, T., Fukuda, C., Prak, K., Cabanos, C., Kimura, A., et al. (2010). Conservation and divergence on plant seed 11S globulins based on crystal structures. Biochim. Biophys. Acta 1804, 1432-1442. doi: 10.1016/j.bbapap.2010.02.016

Tang, Y., Li, X., Zhang, B., Chen, P. X., Liu, R., and Tsao, R. (2015). Characterisation of phenolics, betanins and antioxidant activities in seeds of three Chenopodium quinoa Willd. genotypes. Food Chem. 166, 380-388. doi: 10.1016/j.foodchem.2014.06.018
Thanh, V. H., Okubo, K., and Shibasaki, K. (1975). Isolation and characterization of the multiple 7 S globulins of soybean proteins. Plant Physiol. Biochem. 56, 19-22. doi: 10.1104/pp.56.1.19

Tilton, G. B., Wedemeyer, W. J., and Ohlrogge, J. (2006). Plant coenzyme A biosynthesis: characterization of two pantothenate kinases from Arabidopsis. Plant Mol. Biol. 61, 629-642. doi: 10.1007/s11103-00 6-0037-4

Vashisht, A. A., Pradhan, A., Tuteja, R., and Tuteja, N. (2005). Coldand salinity stress-induced bipolar pea DNA helicase 47 is involved in protein synthesis and stimulated by phosphorylation with protein kinase C. Plant J. 44, 76-87. doi: 10.1111/j.1365-313X.2005. 02511.x

Vega-Gálvez, A., Miranda, M., Vergara, J., Uribe, E., Puente, L., and Martínez, E. A. (2010). Nutrition facts and functional potential of quinoa (Chenopodium quinoa Willd.), an ancient Andean grain: a review. J. Sci. Food Agric. 90, 2541-2547. doi: 10.1002/jsfa.4158

Vidueiros, S. M., Curti, R. N., Dyner, L. M., Binaghi, M. J., Peterson, G., Bertero, H. D., et al. (2015). Diversity and interrelationships in nutritional traits in cultivated quinoa (Chenopodium quinoa Willd.) from Northwest Argentina. J. Cereal Sci. 62, 87-93. doi: 10.1016/j.jcs.2015. 01.001

Wang, W., Vinocur, B., Shoseyov, O., and Altman, A. (2004). Role of plant heatshock proteins and molecular chaperones in the abiotic stress response. Trends Plant Sci. 9, 244-252. doi: 10.1016/j.tplants.2004.03.006

Zevallos, V. F., Ellis, H. J., Suligoj, T., Herencia, L. I., and Ciclitira, P. J. (2012). Variable activation of immune response by quinoa (Chenopodium quinoa Willd.) prolamins in celiac disease. Am. J. Clin. Nutr. 96, 337-344. doi: 10.3945/ajcn.111.030684

Zhang, X., Liu, S., and Takano, T. (2008). Overexpression of a mitochondrial ATP synthase small subunit gene (AtMtATP6) confers tolerance to several abiotic stresses in Saccharomyces cerevisiae and Arabidopsis thaliana. Biotechnol. Lett. 30, 1289-1294. doi: 10.1007/s10529-008-9685-6

Zhu, J. K. (2003). Regulation of ion homeostasis under salt stress. Curr. Opin. Plant Biol. 6, 441-445. doi: 10.1016/S1369-5266(03)00085-2

Conflict of Interest Statement: The authors declare that the research was conducted in the absence of any commercial or financial relationships that could be construed as a potential conflict of interest.

Copyright (c) 2016 Aloisi, Parrotta, Ruiz, Landi, Bini, Cai, Biondi and Del Duca. This is an open-access article distributed under the terms of the Creative Commons Attribution License (CC BY). The use, distribution or reproduction in other forums is permitted, provided the original author(s) or licensor are credited and that the original publication in this journal is cited, in accordance with accepted academic practice. No use, distribution or reproduction is permitted which does not comply with these terms. 


\title{
Crossing Methods and Cultivation Conditions for Rapid Production of Segregating Populations in Three Grain Amaranth Species
}

\author{
Markus G. Stetter*, Leo Zeitler, Adrian Steinhaus, Karoline Kroener, Michelle Biljecki and \\ Karl J. Schmid
}

Institute of Plant Breeding, Seed Science and Population Genetics, University of Hohenheim, Stuttgart, Germany

\section{OPEN ACCESS}

Edited by:

Antonio M. De Ron, Spanish National Research Council (CSIC) - Misión Biológica de Galicia (MBG), Spain

Reviewed by: Johann Vollmann, University of Natural Resources and

Life Sciences, Austria

Peter Jeff Maughan, Brigham Young University, USA David M. Brenner,

lowa State University, USA

*Correspondence:

Markus G. Stetter

markus.stetter@uni-hohenheim.de

Specialty section: This article was submitted to Crop Science and Horticulture, a section of the journal Frontiers in Plant Science

Received: 20 April 2016 Accepted: 25 May 2016 Published: 07 June 2016

Citation:

Stetter MG, Zeitler L, Steinhaus A, Kroener K, Biljecki M and Schmid KJ (2016) Crossing Methods and

Cultivation Conditions for Rapid Production of Segregating Populations in Three Grain Amaranth

Species. Front. Plant Sci. 7:816 doi: $10.3389 / \mathrm{fp} / \mathrm{s} .2016 .00816$
Grain amaranths (Amaranthus spp.) have been cultivated for thousands of years in Central and South America. Their grains are of high nutritional value, but the low yield needs to be increased by selection of superior genotypes from genetically diverse breeding populations. Amaranths are adapted to harsh conditions and can be cultivated on marginal lands although little is known about their physiology. The development of controlled growing conditions and efficient crossing methods is important for research on and improvement of this ancient crop. Grain amaranth was domesticated in the Americas and is highly self-fertilizing with a large inflorescence consisting of thousands of very small flowers. We evaluated three different crossing methods (open pollination, hot water emasculation and hand emasculation) for their efficiency in amaranth and validated them with genetic markers. We identified cultivation conditions that allow an easy control of flowering time by day length manipulation and achieved flowering times of 4 weeks and generation times of 2 months. All three different crossing methods successfully produced hybrid $F_{1}$ offspring, but with different success rates. Open pollination had the lowest $(10 \%)$ and hand emasculation the highest success rate (74\%). Hot water emasculation showed an intermediate success rate (26\%) with a maximum of $94 \%$ success. It is simple to perform and suitable for a more large-scale production of hybrids. We further evaluated 11 single nucleotide polymorphism (SNP) markers and found that they were sufficient to validate all crosses of the genotypes used in this study for intra- and interspecific hybridizations. Despite its very small flowers, crosses in amaranth can be carried out efficiently and evaluated with inexpensive SNP markers. Suitable growth conditions strongly reduce the generation time and allow the control of plant height, flowering time, and seed production. In combination, this enables the rapid production of segregating populations which makes amaranth an attractive model for basic plant research but also facilitates further the improvement of this ancient crop by plant breeding.

Keywords: amaranth, hybridization, hot water emasculation, hand emasculation, genetic resources, marker assisted breeding 


\section{INTRODUCTION}

Ancient crops from the Americas such as quinoa (Chenopodium quinoa willd.) or amaranth (Amaranthus spp. L.) are a valuable addition to the human diet because of their high nutritional value. These pseudocereals have a high protein content and are rich in lysine and other essential amino acids that are limited in other grains (Vega-Gálvez et al., 2010; Rastogi and Shukla, 2013). In addition, these crops are well adapted to harsh environmental conditions and are therefore suitable for cultivation on marginal soils. Their yields are significantly lower than those of major crops due to a lack of plant breeding (Reta Alemayehu et al., 2015), but the presence of a high genetic and phenotypic diversity in these species indicates an excellent potential for breeding and variety development (Brenner et al., 2010).

Grain amaranth originated from Central and South America, where it was of great importance in pre-columbian agriculture until its cultivation strongly declined after the Spanish conquest (Sauer, 1967; Kauffman and Weber, 1990; Brenner et al., 2010). Three species of Amaranthus are cultivated for grain production: A. caudatus L., A. cruentus L., and A. hypochondriacus L. Amaranth expresses the $\mathrm{C}_{4}$ carbon cycle, which is more common in grasses but rare in dicots. Despite a high genetic diversity (Stetter et al., 2015), breeding efforts in amaranth so far were limited to the selection of suitable genotypes from landraces. Amaranth is mainly self-pollinating and has numerous intricate flowers, which make crosses more difficult than in other crops. The ability to efficiently carry out crosses is an important requirement for plant research to understand genetic basis of relevant traits (Moose and Mumm, 2008; Olsen and Wendel, 2013). Crosses are equally important for plant breeding and are used to generate new genetic variation and to introgress exotic material into breeding populations.

In many crops, hybrid varieties are characterized by strongly increased yields (Duvick, 2001). The application of hybrid breeding in amaranth is also very promising, because a midparent heterosis of up to $88 \%$ has been reported (Lehmann et al., 1991). The ability to conduct crosses on a large scale with little effort is of central importance for the development and production of hybrid crop varieties. To use this potential in minor crops, an improvement of crossing methods is essential (Veerappan et al., 2014).

Several approaches for hybrid production are available, but for all methods the key step is to prevent self-fertilization by the male parent. This is either by using appropriate genetic self-incompatibility systems or by mechanical and chemical treatments that lead to male sterility. In several species, cytoplasmatic male sterility (CMS) systems prevent selfing of the female crossing partner (Laser and Lersten, 1972). To use CMS systems for breeding male sterile female parent and male parents with restorer genes are needed to allow seed production in the hybrid progeny. Additionally, a maintainer line is needed that allows multiplying the male sterile line without loosing the CMS. Male sterility has been reported in A. hypochondriacus but is not yet developed sufficiently to be used for breeding (Peters and Jain, 1987). Mechanical emasculation methods are efficient if the male and female flower are well separated on the plant (e.g., as in maize) because then male flowers can be removed without interfering with the female inflorescence. In other crops like tomato and Medicago, anthers are removed before pollen shedding (Veerappan et al., 2014). Another physical method is the heat treatment of the flowers of the female parent to destroy the pollen, for example by a hot water treatment. Here, the temperature is crucial, as differences by few degrees can influence the efficiency of the emasculation (Mukasa et al., 2007; GarcíaYzaguirre and Carreres, 2008; Otsuka et al., 2010). Chemical gametocides are used in hermaphrodite crops for which no CMS systems are available or are too costly, for example in wheat (Dotlacil and Apltauerová, 1978). The grain amaranth species have male and female flowers on the same inflorescence where several female flowers are arranged circularly around a male flower (Figure 1). The flowers are less than $1 \mathrm{~mm}$ in diameter, which makes mechanical emasculation difficult. For this reason other emasculation methods such as a hot water treatment may be more efficient.

Frequently, crossing methods are not completely reliable and require the validation of progeny. Phenotypic traits with a dominant-recessive inheritance can be used to identify successful crosses. In amaranth, traits such as seed or leaf color differ between genotypes and are available for validation (Kulakow et al., 1985). For phenotypic traits to be useful, however, parents need to differ in at least one trait and the male parent needs to express the dominant allele. In contrast, molecular markers allow an efficient and early evaluation of crosses without restricting the combination of parents, and cost-efficient PCR-based marker systems are available for this purpose (Maughan et al., 2011).

For model plants it is important to take specific requirements of development into account. Amaranth shows a strong photoperiod sensitivity and starts to flower under short day conditions (Brenner et al., 2010). A single plant has the potential

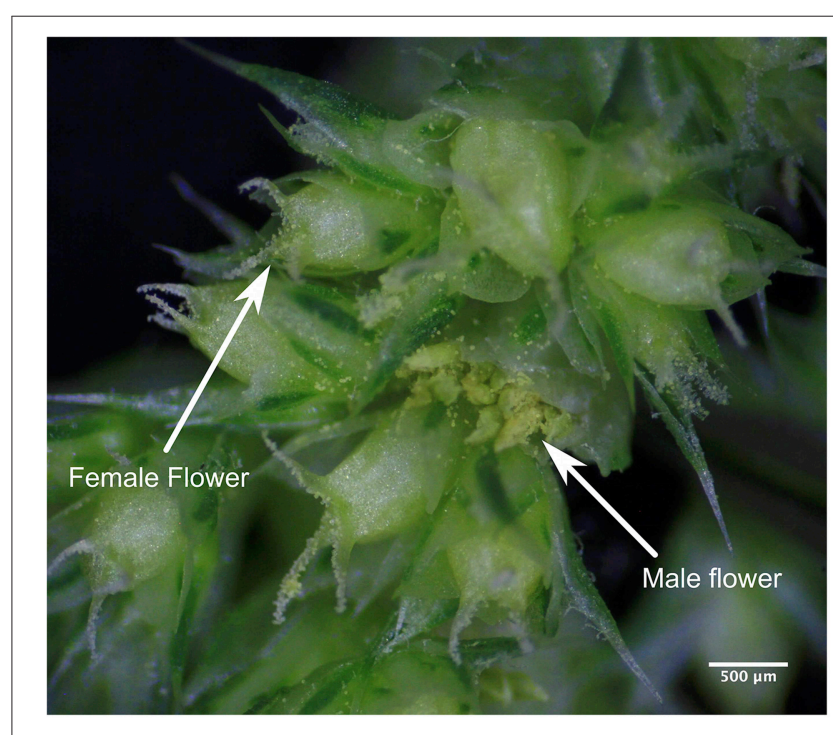

FIGURE 1 | Flower morphology. Inflorescence of $A$. caudatus consisting of flower clusters in which a male flower in the center is surrounded by several female flowers. 
to produce several thousands of seeds and can therefore produce large populations. However, under field conditions amaranth plants are usually tall and require a significant amount of space for cultivation. If flowering time, plant size and seed production can be controlled in climate chambers and greenhouses, an efficient propagation of the plant may be possible.

In the work presented here we study the efficiency of three different crossing methods and describe environmental conditions in a controlled environment (growth chamber) to achieve efficient and rapid generation of progeny for genetic studies. We suggest a method for hybrid identification with cost efficient PCR- based markers. Subsequently, we apply our method to three species of amaranth to evaluate its potential for the wider application to species within the genus Amaranthus.

\section{METHODS}

\subsection{Plant Material and Growth Conditions}

The amaranth accessions for testing the three crossing methods were selected to comprise accessions with green seedlings as female parent and accessions with red seedlings as male parent. Additionally, amaranth varieties were used to verify hybridization and the use of genetic markers (Table 1 and Table S2). Single seeds were planted in $7 \times 7 \mathrm{~cm}$ pots in standard gardening soil. Plants were grown for 2 weeks under long day conditions (Table 2) before transferring them step-wise in weekly intervals to short day conditions (Table 2). This helped to synchronize flowering of different genotypes and spread workload for performing the crosses.

\subsection{Crossing Methods}

We evaluated three different methods for crossing wild (A. hybr. and $A$. hybridus) and cultivated species of amaranth (Figure 2 and Table 1). The first method was open pollination by fixing the flowers of the female and male parent to each other and protecting them with a pollen proof bag (Sealed Air, Germany) from cross pollination by other plants. The second method was a warm water treatment of the inflorescence during flower initiation of the first emerging flowers (García-Yzaguirre and Carreres, 2008). Female flowers were dipped into a water bath of $45^{\circ} \mathrm{C}$ warm water for $10 \mathrm{~min}$ to emasculate the male flowers before proceeding as in the first method. The water treatment was repeated after 7 days. The third method was hand emasculation. For this approach, female flowers that were already open and all male flowers were removed from the inflorescence. The tip of the inflorescence was also removed to prevent the emergence of new flowers. The emasculation was repeated after 7 days and any flowers that developed later were removed. For all three methods plants were shaken daily to increase pollen dispersal and to assure cross-fertilization.

\subsection{Success Evaluation and Statistical Analysis}

Seeds of the female parent were harvested 4 weeks after crossing. For each cross 50 seeds were counted and planted in pots. Seedling color evaluation was performed 2 weeks after planting by counting green and red seedlings. The success rate was the

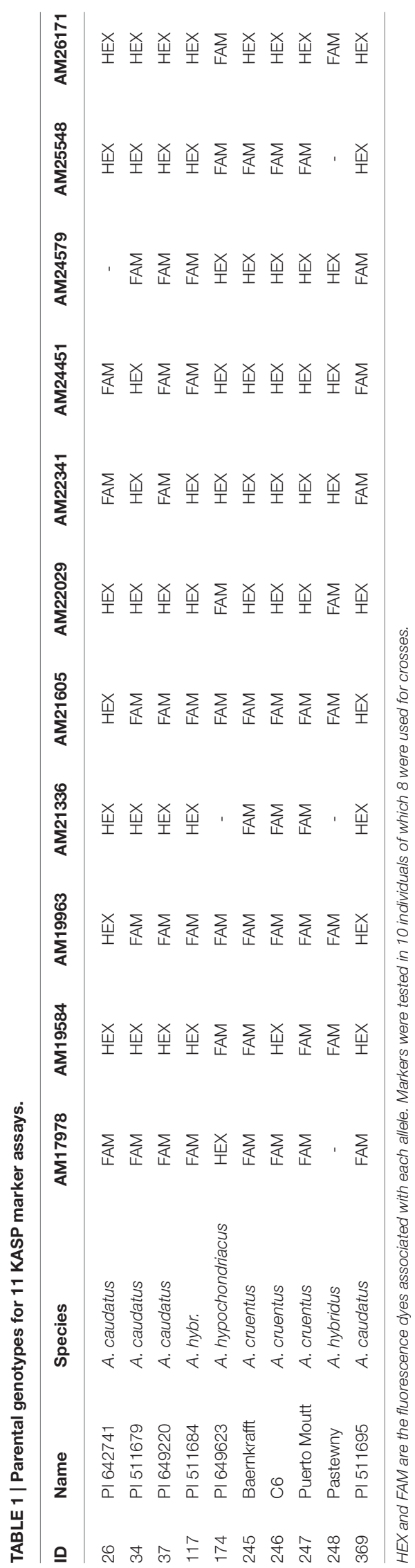


ratio between red and green offspring. Data analysis to test the differences between methods and between crossing types was conducted with a Generalized Linear Model (GLM) with binomial variance and a logit link function that included the crossing method $(\alpha)$, the crossing type ( $\beta$, Intra- and interspecific) and the interaction as factors:

$$
\log i t\left(\mu_{i j}\right)=\log \left(\frac{\mu_{i j}}{1-\mu_{i j}}\right)=\eta_{i j}=\mu+\alpha_{i}+\beta_{j}+(\alpha \beta)_{i j} .
$$

The calculation was done with the $\mathrm{R}$ statistical package version 3.2.0 using the stats library.

\subsection{DNA Extraction}

For genotyping the DNA was extracted with EconoSpin columns (Epoch Life Science Inc.) using 1\% CTAB extraction buffer (Saghai-Maroof et al., 1984). Dry leaf samples were homogenized and incubated for $2 \mathrm{~h}$ at $50^{\circ} \mathrm{C}$ in $400 \mu \mathrm{l} 1 \% \mathrm{CTAB}$ extraction buffer and $4 \mu \mathrm{l}$ Proteinase K. After addition of $300 \mu \mathrm{l}$ Ammonium acetate $(7.5 \mathrm{M})$ and $300 \mu \mathrm{l}$ Ethanol (96\%), the samples were centrifuged for $1 \mathrm{~min}$ at full speed. Then $800 \mu \mathrm{l}$ of the supernatant were transferred on a EconoSpinÂ placed in the collection tube and centrifuged for $1 \mathrm{~min}$. The flow through was discarded. The columns were washed twice with wash buffers from Saghai-Maroof et al. (1984) before eluting DNA twice with $50 \mu \mathrm{l}$ Tris- $\mathrm{HCl}(10 \mathrm{mM}, \mathrm{pH} 8)$.

\subsection{Evaluation of Genetic Markers}

Eleven KASP assays (LGC Berlin/Germany) were selected from Maughan et al. (2011) to validate crosses (Table S1). The assays were prepared with $5 \mu \mathrm{l}$ DNA $(10 \mathrm{ng} / \mu \mathrm{l})$ and $5 \mu$ l genotyping mix and run on the LightCycler 480 Instrument II (Roche Life Science) with standard settings as given by the KASP manual (LGC Berlin/Germany) and analyzed using the LightCycler 480

TABLE 2 | Growth conditions.

\begin{tabular}{lcccc}
\hline & Day length & Light intensity & Temp day & Temp night \\
\hline Long day & 16 & $150 \mathrm{mmol}$ & $35^{\circ} \mathrm{C}$ & $30^{\circ} \mathrm{C}$ \\
Short day & 8 & $150 \mathrm{mmol}$ & $30^{\circ} \mathrm{C}$ & $25^{\circ} \mathrm{C}$ \\
\hline
\end{tabular}

Parameters for amaranth in growth chamber for long and short day conditions.
Software. First, parental lines were evaluated to find polymorphic markers for each of the crosses. Later, these markers were used to validate the crosses. For a proof of concept we genotyped offspring that were evaluated before by their seedling color. Both offspring with green (selfed plants) and red (hybrids) were genotyped.

\subsection{Additional Hybrid Production}

The previously evaluated hand emasculation method was used to produce additional hybrids. Plants were grown as described above, but crossing partners were not restricted to different seedling colors. The success of the crosses was validated with SNP markers.

\section{RESULTS AND DISCUSSION}

\subsection{Cultivation and Life Cycle}

In the field the generation time of the three grain amaranth species is approximately 6 months and leads to very tall plants with thousands of flowers. To reduce the generation time, plant height and number of flowers, we cultivated the plants under short day conditions $(8 \mathrm{~h})$ and high temperature $\left(30^{\circ} \mathrm{C}\right)$ which both induced early flowering 4 weeks after planting. Additionally, we controlled the initiation of flowering by transferring plants from long day $\left(16 \mathrm{~h}, 35^{\circ} \mathrm{C}\right)$ conditions to short day conditions. Under long day conditions the plants displayed strong vegetative growth and did not flower within 10 weeks after planting, but started flowering approximately 14 days after a transfer to short day conditions. The step-wise transfer of plants from long to short-day conditions allows the production of plants in different flowering stages, which greatly facilitates synchronous flowering for crosses between genotypes that differ in their flowering time. This treatment is further useful to produce male parents that are able to shed large amounts of pollen when females parents start flowering. As soon as 4 weeks after flowering, mature seeds could be harvested. By employing these treatments, very short generation times can be achieved that allow up to six generations per year, which is comparable to the model plant Arabidopsis thaliana. In addition, plant height and seed number per plant can be controlled by adjusting growth conditions. Long day conditions lead to more vegetative growth, later flowering and more seeds, while short day conditions result in small early

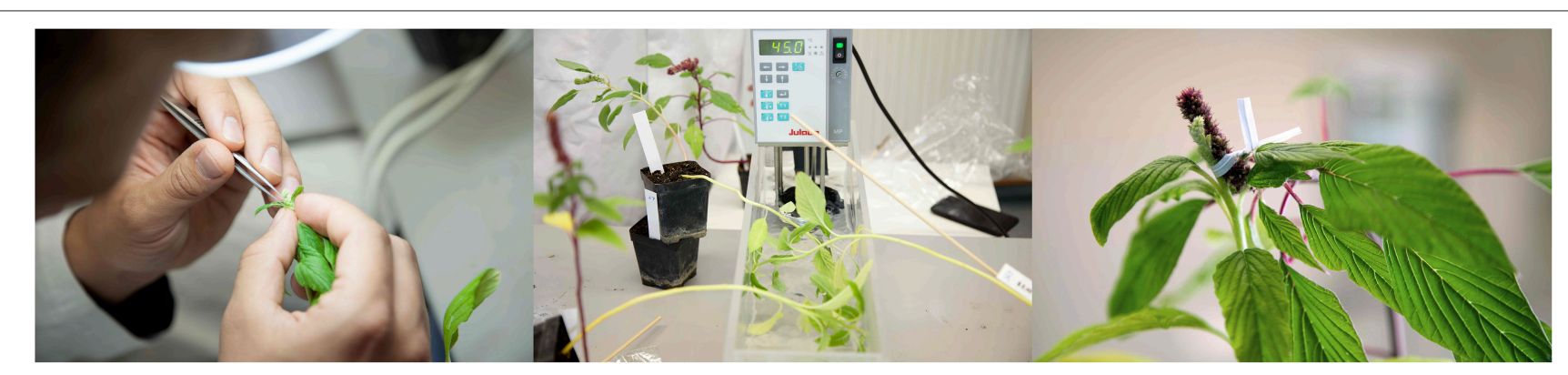

FIGURE 2 | Crossing methods. Three crossing methods: (A) Hand emasculation by removal of male flowers from female plant. (B) Hot water emasculation by 10 min treatment with $45^{\circ} \mathrm{C}$ water bath. (C) Fixing male and female flower to each other for better pollen transfer. 
TABLE 3 | Success rate of different crossing methods.

\begin{tabular}{|c|c|c|c|c|c|c|}
\hline & Type & $N$ & Mean (\%) & SD (\%) & Minimum (\%) & Maximum (\%) \\
\hline \multirow[t]{3}{*}{ Open pollination } & & 7 & $10^{c}$ & 5 & 4 & 18 \\
\hline & Intra-specific & 3 & 11 & 3 & 8 & 14 \\
\hline & Inter-specific & 4 & 10 & 6 & 4 & 18 \\
\hline \multirow[t]{3}{*}{ Heat treatment } & & 8 & $26^{b}$ & 35 & 0 & 94 \\
\hline & Intra-specific & 4 & 26 & 45 & 0 & 94 \\
\hline & Inter-specific & 4 & 27 & 27 & 0 & 57 \\
\hline \multirow[t]{3}{*}{ Hand emasculation } & & 11 & $74^{\mathrm{a}}$ & 29 & 17 & 100 \\
\hline & Intra-specific & 4 & 80 & 20 & 50 & 94 \\
\hline & Inter-specific & 7 & 71 & 34 & 17 & 100 \\
\hline
\end{tabular}

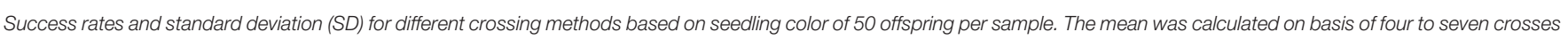
( $N$ ) per method and crossing type. Intra-specific crosses were performed with A. caudatus (PI $511679 \times$ PI 649220) and inter-specific crosses between A. caudatus (PI 511679$)$ and A.

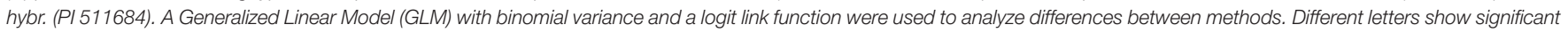
differences between methods. There was no significant difference between intra- and inter- specific crosses.

flowers. This is useful for different applications, because crosses require only few flowers, whereas the resulting $\mathrm{F}_{1}$ plants should produce larger amounts of seeds (e.g., for creating mapping populations).

\subsection{Crossing Methods}

We compared three crossing methods that included open pollination, hot water emasculation and hand emasculation. All three methods produced successful crosses, but the success rates and variances differed strongly between the methods (Table 3). The amount of seeds produced did not substantially differ between methods and the mother plants produced between 100 and 200 seeds. Open pollination between two plants under a single bag without emasculation of the female parent led to a mean success rate of $10 \%$ with a standard deviation (s.d.) of 0.05 . The hot water treatment of the female parent led to a significantly increased success rate of $26 \%$ but with a very high deviation (s.d. $=0.35$ ) and a minimal success rate of $0 \%$. However, the maximal success after hot water treatment was $94 \%$, which shows that the method has a high potential if the key conditions for a successful application can be identified. We sterilized flowers at $45^{\circ} \mathrm{C}$ and an adaptation of temperature may contribute to a higher rate of success. In other species (e.g., Acacia, buckwheat and rice), different temperatures change the efficiency of emasculation (Mukasa et al., 2007; García-Yzaguirre and Carreres, 2008; Otsuka et al., 2010). A temperature of $45^{\circ} \mathrm{C}$ for emasculation is rather high compared to other crops (GarcíaYzaguirre and Carreres, 2008), but not too high because the amaranth plants still set seeds after this treatment and a further optimization may be achieved by varying the length of the heat treatment. Overall, hot water emasculation works with amaranth and, if it can be further improved, is suitable for application in the field to large numbers of plants.

The most elaborated and time consuming method we evaluated was hand emasculation (Figure 3). The mean success rate of $74 \%$ was the highest of the three methods and the deviation (s.d. $=0.29$ ) was lower than of the heat treatment.

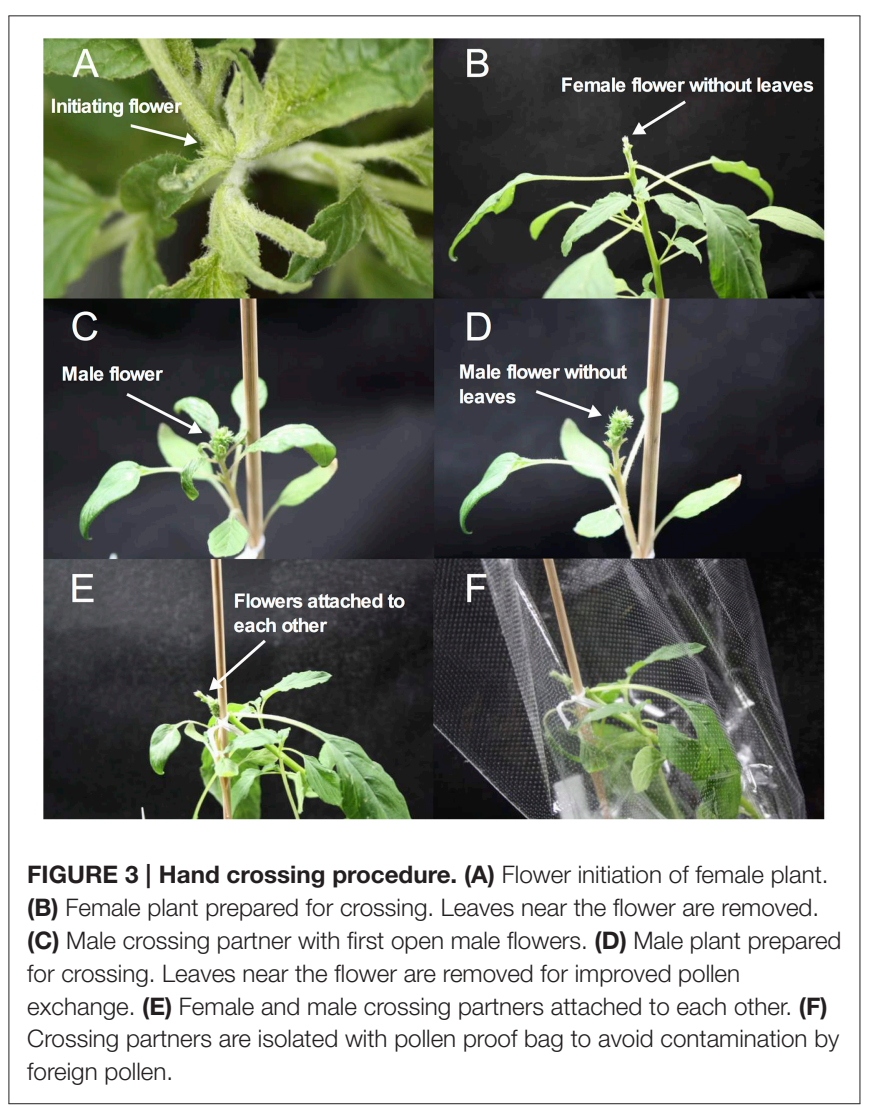

The minimum success was comparable to free pollination, but the maximum success was up to $100 \%$. Hand emasculation is difficult because amaranth has many small flowers and each male flower sheds enough pollen to pollinate a whole plant. Therefore it is critical to remove all male flowers from the female parent before flower dehiscence. The deviation can be decreased by keeping only few flower clusters per plant. We also tested whether intra- and inter-specific crosses are different in their 
efficiency, but there was no significant difference between intraand inter-specific crosses (Table 3). This shows that inter-specific hybridization is possible, but as the two species are closely related this might not be the case for distant member of the Amaranthus genus.

The comparison of the three methods shows that open pollination had low success rates, whereas heat treatment can be an effective and simple method for crosses if many seeds are required and simple morphological markers are available for the evaluation of offspring. Hand emasculation by well trained personnel shows the best performance. Since amaranth plants may produce thousands of seeds, a single successful cross can produce large $\mathrm{F}_{2}$ populations, and the number of hand crosses needed can be kept low, which decreases the work load of the method substantially and makes it suitable for large projects.

\subsection{Genetic Markers for Hybrid Identification}

Since no crossing method provides a $100 \%$ success rates, unsuccessful crosses have to be excluded in early stages. Furthermore, crossing partners should not be limited by phenotypic differences in certain traits (e.g., different seedling color), but all possible combinations parents should be available. We therefore evaluated all accessions used in this study with 11 PCR-based SNP markers. The markers were the most polymorphic from a set of 411 KASP markers from Maughan et al. (2011). Each marker was polymorphic between at least two lines and each cross segregated at least for one marker (Table 1). After evaluating the parental lines, we selected suitable markers to evaluate crosses.

First, we investigated progeny which had already been evaluated by their seedling color, because we expected green
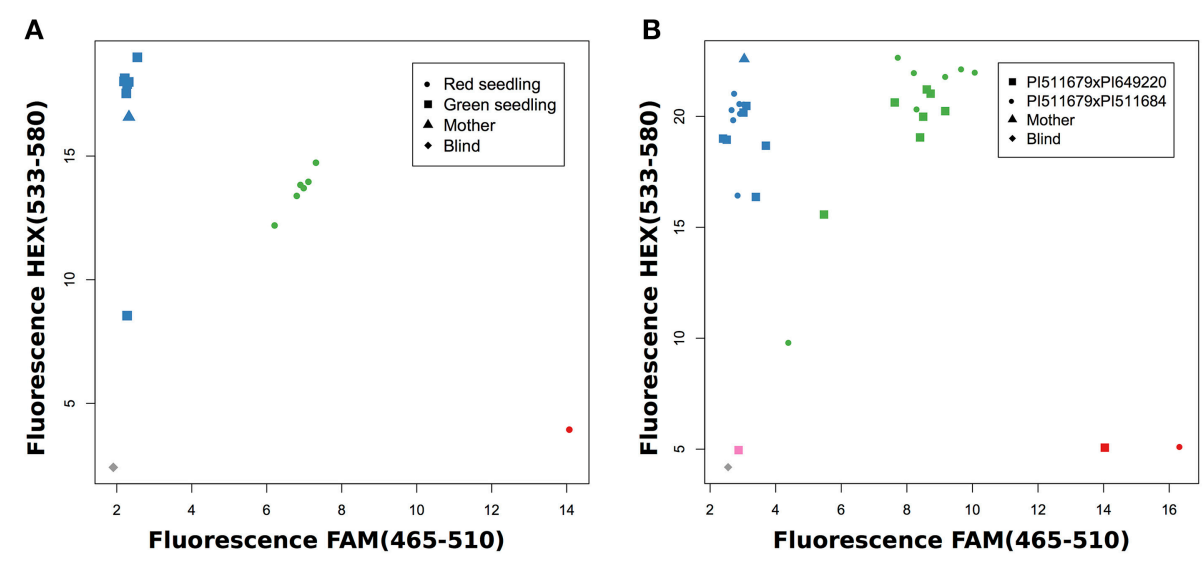

FIGURE 4 | SNP genotyping for known crosses. (A) Validation of PI511679xPI649220 with AM22341 and comparison with seedling color. (B) Validation of two crosses with AM24451.

TABLE 4 | Crosses of different amaranth varieties by hand emasculation and evaluation of success rates with SNP markers.

\begin{tabular}{|c|c|c|c|c|c|c|c|}
\hline & ID (Mother) & ID (Father) & Marker & Genotyped & Selfings & Successful crosses & Failed assay \\
\hline 1 & 34 & 245 & AM19584 & 7 & 0 & 7 & 0 \\
\hline 2 & 34 & 245 & AM19584 & 6 & 2 & 2 & 2 \\
\hline 3 & 34 & 245 & AM19584 & 7 & 2 & 4 & 1 \\
\hline 4 & 34 & 248 & AM19584 & 7 & 2 & 4 & 1 \\
\hline 5 & 34 & 248 & AM19584 & 7 & 0 & 6 & 1 \\
\hline 6 & 34 & 248 & AM19584 & 7 & 0 & 4 & 3 \\
\hline 7 & 245 & 26 & AM19584 & 6 & 4 & 2 & 0 \\
\hline 8 & 245 & 26 & AM19584 & 8 & 2 & 3 & 3 \\
\hline 9 & 247 & 248 & AM22029 & 10 & 1 & 9 & 0 \\
\hline 10 & 247 & 248 & AM22029 & 9 & 9 & 0 & 0 \\
\hline 11 & 248 & 245 & AM22029 & 6 & 6 & 0 & 0 \\
\hline 12 & 248 & 245 & AM22029 & 8 & 5 & 0 & 3 \\
\hline 13 & 248 & 245 & AM22029 & 7 & 4 & 0 & 3 \\
\hline
\end{tabular}

All crosses are interspecific crosses between the three grain amaranths and/or their putative ancestors. The ID of mothers and fathers corresponds to Table 1. 
seedlings to be homozygous for the maternal allele since the green allele is recessive, and red seedlings to be heterozygous. For example, the application of marker AM22341 in a cross of PI511679 $\times$ PI649220 showed that green seedlings were homozygous for the allele of parent PI511679 and red seedlings were heterozygous for both parental alleles (Figure 4A). Frequently, the same marker can be used in several crosses, which allows the evaluation of more than one cross simultaneously (Figure 4B). This strongly decreases the work load and the cost of the evaluation. When working with homozygous parental lines a single maker is sufficient to validate successful crosses.

To test the effectiveness of the hand crossing method and the validation with genetic markers, we produced hybrids between amaranth genotypes from different species and validated them with the marker system. Although not all crosses produced hybrids, for most crosses the number of hybrids produced was high and less than 10 offspring had to be evaluated per cross (Table 4).

\section{CONCLUSIONS}

Ancient and underutilized crops greatly benefit from the ongoing revolution in genomics. However, to utilize this information for the improvement of minor crops, efficient crossing methods which are the basis of breeding programs need to be established. We developed crossing methods and genetic markers for hybrid identification in amaranth and showed that these can be used for crosses within and between species. We further showed that the life cycle and plant size of amaranth can be reduced substantially when light and temperature conditions are adapted. For genetic and physiological studies a short generation time is advantageous, which is a common characteristic of model organisms for basic research. Under the conditions described here, generation times as short as those of $A$. thaliana are possible (Meyerowitz and Pruitt, 1985). Additionally, the amount of seeds can be controlled, which allows the production of large offspring

\section{REFERENCES}

Brenner, D. M., Baltensperger, D. D., Kulakow, P. A., Lehmann, J. W., Myers, R. L., Slabbert, M. M., et al. (2010). "Genetic resources and breeding of Amaranthus," in Plant Breeding Reviews, Vol. 19 (New York, NY: John Wiley \& Sons, Inc.), 227-285. doi: 10.1002/9780470650172.ch7

Clouse, J. W., Adhikary, D., Page, J. T., Ramaraj, T., Deyholos, M. K., Udall, J. A., et al. (2016). The amaranth genome: genome, transcriptome, and physical map assembly. Plant Gen. 9, 1-14. doi: 10.3835/plantgenome2015.07.0062

Dotlacil, L., and Apltauerová, M. (1978). Pollen sterility induced by ethrel and its utilization in hybridization of wheat. Euphytica 27, 353-360.

Duvick, D. N. (2001). Biotechnology in the 1930s: the development of hybrid maize. Nat. Rev. Genetics 2, 69-74. doi: 10.1038/35047587

García-Yzaguirre, A., and Carreres, R. (2008). Efficiency of different hybridization methods in single crosses of rice for pure line breeding. Spanish J. Agricult. Res. 6, 395-400. doi: 10.5424/sjar/2008063-332

Kauffman, C. S., and Weber, L. E. (1990). "Grain Amaranth," in Advances in New Crops, eds J. Janick and J. E. Simon (Portland: Timber Press), 127-139.

Kulakow, P., Hauptli, H., and Jain, S. (1985). Genetics of grain amaranths I. Mendelian analysis of six color characteristics. J. Heredity 76, 27-30. populations for genetic mapping. Furthermore, amaranth has a relatively small genome (500 $\mathrm{Mbp}$ ) with a reference sequence, and a large number of genotyped genebank accessions are available (Stetter et al., 2015; Clouse et al., 2016). Taken together, these resources and the possibility of interspecific crosses make the grain amaranth species a very suitable model organism for studying fundamental processes such as adaptation, speciation, heterosis, $\mathrm{C}_{4}$ photosynthetic metabolism, or domestication. The ability to conduct crosses from genetically diverse material facilitates the establishment of advanced breeding programs and the selection of improved genotypes using current breeding methods such as genomic selection will improve the value of this minor crop for agricultural production.

\section{AUTHOR CONTRIBUTIONS}

KS and MS designed the experiments. LZ, AS, KK, and MB performed the crosses. MS performed the genotyping and analyzed the data. KS and MS wrote the manuscript. All authors read and approved the final manuscript.

\section{ACKNOWLEDGMENTS}

We would like to thank the Institute of Crop Science of the University of Hohenheim for providing the amaranth varieties (Baernkrafft, C6, Puerto Moutt and Pastewny) and the USDAARS for providing germplasm. This work was funded by an endowment of the Stifterverband der Deutschen Wissenschaft grant to KS (Project H1705701502016296 ). MS, LZ, AS, KK, and $\mathrm{MB}$ were supported by the Humboldt Reloaded project at the University of Hohenheim, Germany (BMBF 01PL11003).

\section{SUPPLEMENTARY MATERIAL}

The Supplementary Material for this article can be found online at: http://journal.frontiersin.org/article/10.3389/fpls.2016. 00816

Laser, K., and Lersten, N. (1972). Anatomy and cytology of microsporogenesis in cytoplasmic male sterile angiosperms. Botan. Rev. 38, 425-454.

Lehmann, J., Clark, R., and Frey, K. (1991). Biomass heterosis and combining ability in interspecific and intraspecific matings of grain amaranths. Crop Sci. 31, 1111-1116.

Maughan, P. J., Smith, S. M., Fairbanks, D. J., and Jellen, E. N. (2011). Development, characterization, and linkage mapping of single nucleotide polymorphisms in the grain amaranths (Amaranthus sp.). Plant Gen. J. 4, 92-101. doi: 10.3835/plantgenome2010.12.0027

Meyerowitz, E. M., and Pruitt, R. E. (1985). Arabidopsis thaliana and plant molecular genetics. Science 229, 1214-1218.

Moose, S. P., and Mumm, R. H. (2008). Molecular plant breeding as the foundation for 21st century crop improvement. Plant Physiol. 147, 969-977. doi: $10.1104 /$ pp.108.118232

Mukasa, Y., Suzuki, T., and Honda, Y. (2007). Emasculation of Tartary buckwheat (Fagopyrum tataricum Gaertn.) using hot water. Euphytica 156, 319-326. doi: 10.1007/s10681-007-9377-7

Olsen, K. M., and Wendel, J. F. (2013). A bountiful harvest: genomic insights into crop domestication phenotypes. Ann. Rev. Plant Biol. 64, 47-70. doi: 10.1146/annurev-arplant-050312-120048 
Otsuka, J., Yamaguchi, S., Chigira, O., and Kato, K. (2010). Application of hot water emasculation to Acacia auriculiformis for controlled pollination. J. Forest Res. 15, 210-216. doi: 10.1007/s10310-009-0174-0

Peters, I., and Jain, S. (1987). Genetics of grain amaranths III. Gene-cytoplasmic male sterility. J. Heredity 78, 251-256.

Rastogi, A., and Shukla, S. (2013). Amaranth: a new millennium crop of nutraceutical values. Crit. Rev. Food Sci. Nutrit. 53, 109-125. doi: 10.1080/10408398.2010.517876

Reta Alemayehu, F., Bendevis, M. A., and Jacobsen, S.-E. (2015). The potential for utilizing the seed crop Amaranth (Amaranthus spp.) in east Africa as an alternative crop to support food security and climate change mitigation. J. Agron. Crop Sci. 201, 321-329. doi: 10.1111/jac.12108

Saghai-Maroof, M. A., Soliman, K. M., Jorgensen, R. A., and Allard, R. W. (1984). Ribosomal DNA spacer-length polymorphisms in barley: mendelian inheritance, chromosomal location, and population dynamics. Proc. Natl. Acad. Sci. U.S.A. 81, 8014-8018.

Sauer, J. (1967). The grain amaranths and their relatives: a revised taxonomic and geographic survey. Ann. Missouri Botan. Garden 54, 103-137.

Stetter, M. G., Müller, T., and Schmid, K. (2015). Incomplete domestication of South American grain amaranth (Amaranthus caudatus) from its wild relatives. bioRxiv.
Veerappan, V., Kadel, K., Alexis, N., Scott, A., Kryvoruchko, I., Sinharoy, S., et al. (2014). Keel petal incision: a simple and efficient method for genetic crossing in Medicago truncatula. Plant Methods 10:11. doi: 10.1186/17464811-10-11

Vega-Gálvez, A., Miranda, M., Vergara, J., Uribe, E., Puente, L., and Martínez, E. A. (2010). Nutrition facts and functional potential of quinoa (Chenopodium quinoa willd.), an ancient Andean grain: a review. J. Sci. Food Agricult. 90, 2541-2547. doi: 10.1002/jsfa. 4158

Conflict of Interest Statement: The authors declare that the research was conducted in the absence of any commercial or financial relationships that could be construed as a potential conflict of interest.

Copyright (C) 2016 Stetter, Zeitler, Steinhaus, Kroener, Biljecki and Schmid. This is an open-access article distributed under the terms of the Creative Commons Attribution License (CC BY). The use, distribution or reproduction in other forums is permitted, provided the original author(s) or licensor are credited and that the original publication in this journal is cited, in accordance with accepted academic practice. No use, distribution or reproduction is permitted which does not comply with these terms. 


\section{OPEN ACCESS}

Edited by:

Antonio M. De Ron,

Misión Biológica de Galicia - CSIC,

Spain

Reviewed by:

James Frelichowski,

United States Department

of Agriculture, USA

Mercedes Fernández-Pascual, Instituto de Ciencias Agrarias - CSIC,

Spain

${ }^{*}$ Correspondence: BingLin Chen

blchen@njau.edu.cn

Specialty section:

This article was submitted to Crop Science and Horticulture,

a section of the journal

Frontiers in Plant Science

Received: 25 April 2016

Accepted: 13 July 2016

Published: 02 August 2016

Citation:

Yang $H$, Meng $Y$, Chen $B$, Zhang $X$,

Wang Y, Zhao W and Zhou Z (2016)

How Integrated Management

Strategies Promote Protein Quality

of Cotton Embryos: High Levels

of Soil Available N, N Assimilation

and Protein Accumulation Rate.

Front. Plant Sci. 7:1118.

doi: 10.3389/fpls.2016.01118

\section{How Integrated Management} Strategies Promote Protein Quality of Cotton Embryos: High Levels of Soil Available N, N Assimilation and Protein Accumulation Rate

\author{
HongKun Yang, YaLi Meng, BingLin Chen*, XingYue Zhang, YouHua Wang, \\ WenQing Zhao and ZhiGuo Zhou
}

Key Laboratory of Crop Physiology and Ecology, Ministry of Agriculture, Nanjing Agricultural University and Jiangsu Collaborative Innovation Center for Modern Crop Production, Nanjing, China

Cottonseed is widely used as a source of ruminant feed and for industrial purposes. Therefore, there is a tremendous need to improve the nutritional value of cotton embryos. In this study, a conventional management (CM) and two integrated cotton management strategies $\left(\mathrm{IMS}_{1}, \mathrm{IMS}_{2}\right)$ were performed at two soil fertility levels to study the relationships among soil $\mathrm{N}, \mathrm{N}$ assimilation, embryonic protein accumulation and protein quality. The levels of proteins, essential amino acids, and semi-essential amino acids, especially those of glutamate, lysine, and methionine, were higher in IMS and $\mathrm{IMS}_{2}$ embryos than in $\mathrm{CM}$ embryos. These changes were significantly positively correlated with the soil-available $\mathrm{N}$ content, glutamine synthetase activity and peak value of protein accumulation rate and were negatively correlated with the free amino acid level. These results illustrated that integrated management strategies, especially the rates and timing of $\mathrm{N}$ application, raise the level of soil available $\mathrm{N}$, which is beneficial for $\mathrm{N}$ assimilation in developing cotton embryos. The protein content was limited by the rate of protein accumulation rather than by the free amino acid content. The combination of target yield fertilization, a growth-driven $\mathrm{N}$ application schedule, a high plant density and the seedling raising with bio-organic fertilizer can substantially improve protein quality in cotton embryos, especially at a soil with low soil organic matter and total nitrogen.

Keywords: cotton embryonic protein, integrated management strategies, soil available $\mathrm{N}, \mathrm{N}$ assimilation, proteinogenic amino acids

\section{INTRODUCTION}

Cotton embryos accumulate $40 \%-55 \%$ of their dry weight (DW) as storage protein, which has a substantial effect on their nutritional value (Alford et al., 1996; Mujahid et al., 2000). Therefore, evaluating the mechanisms controlling storage protein accumulation and protein quality in these embryos is crucial step for improving seed quality. Recently, considerable progress has been made

Abbreviations: AV-N, soil available nitrogen; CM, conventional management practices; Glu, glutamate; GS, glutamine synthetase; IMS, integrated management strategies; Lys, lysine; Met, methionine; NADH-GOGAT, NADH-dependent glutamate synthase. 
in elucidating the relationships among genotypes, environmental conditions and management practices, suggesting that protein content is a variety-specific quantitative characteristic (Yu et al., 2012) and that it is substantially influenced by environmental conditions (Li et al., 2009; Rotundo and Westgate, 2009) and crop management strategies (Sawan et al., 1993, 2001, 2006, 2009). Numerous studies show the many steps involved in protein accumulation in cottonseed embryos, involving seed biochemistry (Hodges, 2002; Hernandez-Sebastia et al., 2005; Zhang et al., 2014), N transport with the plant and from the soil (De Ruiter et al., 1986; Kullmann and Geisler, 1986; Crawford and Glass, 1998), soil N levels and the environment (Macduff and Hopper, 1986). Integration of the results of these studies into a complete management system targeting the final result of protein quality in cotton embryos is still needed.

Integrated management strategies are combinations of agronomic practices applied to achieve high yields, optimal quality, and long-term sustainability based on analyses of factors limiting the productivity and quality of crops under a given set of environmental conditions. The planting of high-yielding cultivars with optimal nutritional management (Sapkota et al., 2014) and water management strategies (Falkenberg et al., 2007), adequate plant populations (Dong et al., 2012), and strong seedlings (Mcgraw and Shaver, 2006) are major requirements for high yields and optimal quality. Integrated nutrient management improves the soil productivity, crop yield and quality of groundnuts (Prasad et al., 2002) and tomatoes (Javaria and Khan, 2011). Whether IMS can also improve protein quality in cotton embryos requires further elucidation.

A considerable amount of data is available regarding the "source-sink" relationships in the study of embryonic protein synthesis (Martre et al., 2003; Seong et al., 2004; Zhang et al., 2012), whereas the relationships between soil $\mathrm{N}$ and embryonic proteins are unclear. AV-N (i.e., the sum of $\mathrm{NH}_{4}^{+}-\mathrm{N}$ and $\mathrm{NO}_{3}^{-}$ $-\mathrm{N}$ ), as an indicator of soil $\mathrm{N}$ and fertility, can be directly utilized by crop roots (Crawford and Glass, 1998) and ultimately incorporated into storage protein (Kullmann and Geisler, 1986). Soil $\mathrm{N}$ increased with increasing fertilization rate (Sainju et al., 2006). Integrated nutritional management (Javaria and Khan, 2011; Singh et al., 2014) and soil temperature (Macduff and Hopper, 1986) can influence seasonal and regional variations in the soil available $\mathrm{N}$. Moreover, soil $\mathrm{N}$ also regulates $\mathrm{N}$ uptake and plant growth (Gastal and Lemaire, 2002). Therefore, it is necessary to explore the relationship between soil $\mathrm{N}$ and protein content.

Some of the crucial steps in initial $\mathrm{N}$ assimilation are the incorporation of $\mathrm{N}$ into carbon skeletons of storage proteins and its use for the biosynthesis of glutamine and then of Glu (Hodges, 2002). Glutamine synthetase (GS) and glutamate synthase (GOGAT) play a key role in $\mathrm{N}$ assimilation for its higher affinity to $\mathrm{NH}_{4}^{+}-\mathrm{N}$ than glutamate dehydrogenase (GDH) (Stewart and Rhodes, 1978; Hodges, 2002; Hodges et al., 2003; Muro-Pastor and Florencio, 2003; Muro-Pastor et al., 2005; Potel et al., 2009). N assimilation uses ferredoxin (Fd-GOGAT, EC 1.4.7.1) as an electron carrier in photorespiratory tissues (i.e., leaf samples) and $\mathrm{NADH}$ (NADH-GOGAT; EC 1.4.1.14) as an electron carrier in non-photorespiratory tissues (i.e., roots and seeds) (Canovas et al., 1998; Tamura et al., 2010; Konishi et al., 2014). The $\mathrm{NH}_{4}^{+}-\mathrm{N}$ and $\mathrm{NO}_{3}^{-}-\mathrm{N}$ concentrations in the soil tightly regulate the GS and GOGAT activities (Zhao and Shi, 2006). Although the functional roles of GS (EC 6.3.1.2) and GOGAT (EC 1.4.1.14) in plant roots and leaves have been well described (Ishiyama et al., 1998; Muro-Pastor and Florencio, 2003; Potel et al., 2009), the role of nitrogen assimilation in nonphotosynthetic organs is unclear, especially in protein-enriched cotton embryos.

Cotton embryos are characterized by high protein content and are rich in Lys and Glu, which serve as nutritional limiting factors for ruminants (Bertrand et al., 1998; Zhe et al., 2014). The globulin null soybean mutant accumulates high levels of free amino acids (Takahashi et al., 2003), suggesting a role of free amino acids in storage protein accumulation. Varietal and locational factors may influence the contents of free amino acids and storage protein in cottonseeds (Ikurior and Fetuga, 1987). A fundamental understanding of the conversion of free amino acids into storage proteins and the characterization of protein accumulation would clarify the factors limiting the protein accumulation rate.

The objective of the present study was to improve protein quality in cotton embryos by adopting IMS and to analyze how these strategies improve protein quality in terms of soil $\mathrm{N}, \mathrm{N}$ assimilation, the protein accumulation rate, proteinogenic amino acids, and the protein content. The initial hypothesis was that the combination of optimal $\mathrm{N}$ management practices, an adequate plant density and strong seedlings could be beneficial to protein quality in cotton embryos.

\section{MATERIALS AND METHODS}

\section{Growth Conditions and Integrated Management Strategies}

Field experiments were carried out at the Dafeng Experimental Station, Jiangsu, China $\left(120^{\circ} 45^{\prime} \mathrm{E}, 33^{\circ} 19^{\prime} \mathrm{N}\right)$, using a widely grown cotton cultivar, Siza-3 (Gossypium hirsutum L.), in 2012 and 2013. The soil at the experiment site was a typical sandy loam, and there were significant differences in the contents of organic matter and total nitrogen (Table 1), with low-fertility soil containing an average of $14.31 \pm 0.62 \mathrm{~g} \mathrm{~kg}^{-1}$ soil organic matter and $0.79 \pm 0.04 \mathrm{~g} \mathrm{~kg}^{-1}$ total nitrogen and high-fertility soil containing an average of $17.76 \pm 0.69 \mathrm{~g} \mathrm{~kg}^{-1}$ soil organic matter and $0.86 \pm 0.03 \mathrm{~g} \mathrm{~kg}^{-1}$ total nitrogen at a $0-20 \mathrm{~cm}$ depth of the soil profile.

Cotton plants were planted using a CM system and two integrated management strategies $\left(\mathrm{IMS}_{1}\right.$ and $\mathrm{IMS}_{2}$ ). A randomized complete block design with three replicates was used in a plot measuring $22 \mathrm{~m}$ long and $10 \mathrm{~m}$ wide. CM was a widely used practice in the Yangtze River cotton-producing region (i.e., $300 \mathrm{~kg} \mathrm{~N}$ ha $^{-1}+18,000$ plants ha ${ }^{-1}+$ seedling transplantation, with $40 \%$ of the $300 \mathrm{~kg} \mathrm{~N} \mathrm{ha}^{-1}$ applied as a basal fertilizer and $60 \%$ applied at the initial flowering stage). The two integrated management strategies $\left(\mathrm{IMS}_{1}\right.$ and $\mathrm{IMS}_{2}$ ) included different combinations of $\mathrm{N}$ rates, $\mathrm{N}$ application 
TABLE 1 | Soil nutrient status at the experimental site in 2012 and 2013.

\begin{tabular}{|c|c|c|c|c|c|c|c|c|c|c|}
\hline Year & $\begin{array}{l}\text { Soil } \\
\text { fertility }\end{array}$ & $\begin{array}{l}\text { Soil depth } \\
\text { (cm) }\end{array}$ & $\begin{array}{l}\text { Bulk density } \\
\quad\left(\mathrm{g} \mathrm{m}^{-3}\right)\end{array}$ & $\mathrm{pH}$ & $\begin{array}{l}\text { SOM } \\
\left(\mathrm{g} \mathrm{kg}^{-1}\right)\end{array}$ & $\begin{array}{l}\text { WSOC } \\
\left(\mathrm{mg} \mathrm{kg}^{-1}\right)\end{array}$ & $\begin{array}{c}\mathrm{TN} \\
\left(\mathrm{g} \mathrm{kg}^{-1}\right)\end{array}$ & $\begin{array}{c}\text { Av-N } \\
\left(\mathrm{mg} \mathrm{kg}^{-1}\right)\end{array}$ & $\begin{array}{c}\text { Av-P } \\
\left(\mathrm{mg} \mathrm{kg}^{-1}\right)\end{array}$ & $\begin{array}{c}\mathrm{Av}-\mathrm{K} \\
\left(\mathrm{mg} \mathrm{kg}^{-1}\right)\end{array}$ \\
\hline \multirow[t]{4}{*}{2012} & LF & $0-20$ & 1.20 & 8.47 & 14.80 & 46.70 & 0.79 & 23.90 & 19.00 & 364.50 \\
\hline & & $20-40$ & 1.51 & 8.55 & 12.60 & 42.80 & 0.67 & 18.00 & 14.20 & 273.40 \\
\hline & $\mathrm{HF}$ & $0-20$ & 1.16 & 8.26 & 18.30 & 51.20 & 0.86 & 28.60 & 23.50 & 384.00 \\
\hline & & $20-40$ & 1.47 & 8.34 & 15.70 & 46.50 & 0.73 & 21.40 & 17.60 & 288.10 \\
\hline \multirow[t]{4}{*}{2013} & LF & $0-20$ & 1.17 & 8.49 & 13.60 & 45.90 & 0.73 & 22.20 & 17.30 & 394.50 \\
\hline & & $20-40$ & 1.46 & 8.51 & 11.90 & 41.80 & 0.69 & 19.70 & 15.60 & 300.70 \\
\hline & $\mathrm{HF}$ & $0-20$ & 1.11 & 8.21 & 17.20 & 50.70 & 0.82 & 27.30 & 22.20 & 414.20 \\
\hline & & $20-40$ & 1.39 & 8.47 & 14.00 & 46.40 & 0.71 & 23.60 & 19.30 & 316.80 \\
\hline
\end{tabular}

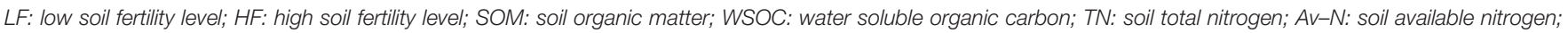
Av-P: soil available phosphor; Av-K: soil available potassium.

schedules, plant densities, and seedling-raising methods. $\mathrm{IMS}_{1}$ included an economic $\mathrm{N}$ rate, a high plant density and substrate seedling-raising method (i.e., $375 \mathrm{~kg} \mathrm{~N}^{-1}+30,000$ plants $\mathrm{ha}^{-1}+$ substrate seedling raising). Compared to the CM, in $\mathrm{IMS}_{2}$, the $\mathrm{N}$ rate was increased to $525 \mathrm{~kg} \mathrm{~N} \mathrm{ha}^{-1}$, the plant density was increased to 30,000 plants $\mathrm{ha}^{-1}$, and seedling transplantation was adopted (i.e., target yield fertilization + higher plant density + seedling transplantation).

Target yield fertilization and the economic $\mathrm{N}$ rate were calculated according to a previously described algorithm (Katayama et al., 1999; Baker and Young, 2004). The N application schedules of IMS 1 and IMS 2 were timed to coincide with growth-driven $\mathrm{N}$ demand of cotton plants, i.e., $20 \%$ applied as a basal fertilizer, $25 \%$ at the initial flowering stage, $40 \%$ at the full-bloom stage and $15 \%$ at the end of the flowering stage. $\mathrm{N}, \mathrm{P}$, and $\mathrm{K}$ were applied as urea $(46 \% \mathrm{~N})$, ordinary superphosphate $\left(12 \% \quad \mathrm{P}_{2} \mathrm{O}_{5}\right.$ and $12 \%$ sulfur) and potassium sulfate $\left(50 \% \mathrm{~K}_{2} \mathrm{O}\right.$ and $18 \%$ sulfur $)$ at a ratio of 1.0:0.6:1.1. For substrate seedling raising, seeds were planted with bio-organic fertilizer (20 million $\mathrm{g}^{-1}$ efficacious living-cell and $43.6 \%$ organic matter, purchased from Jiangsu Tianniang Ltd., China) at the rate of $10 \mathrm{~g}$ per seedling, and seedlings were transplanted in the field with a bio-organic fertilizer on May 15th (3-4 true leaves). For seedling transplantation, cottonseeds were planted in a nursery bed, and then seedlings with 3-4 true leaves were transplanted in the field without bio-organic fertilizer on May 15th. Integrated pest management and furrow irrigation were applied to avoid any biotic and abiotic stresses during the cotton growth period.

\section{Sampling}

White flowers from first fruiting nodes at the 7th-8th sympodial branches were tagged with the flowering date to ensure that boll samples in each treatment were collected at equivalent metabolic and developmental boll ages. Tagged boll samples were collected once every 7 days from 17 days after anthesis (DAA) until boll opening from 9:00 to 10:00 a.m. local time. Collected bolls were quickly separated into fibers, carpels, seed coats and embryos at $4^{\circ} \mathrm{C}$. About half of the embryo samples were placed in liquid nitrogen and stored at $-80^{\circ} \mathrm{C}$ for assays of GS and $\mathrm{NADH}$ dependent GOGAT. The other half of embryo samples were dried to a constant weight, and ground to pass through a $1 \mathrm{~mm}$ sieve, and then used to determine the free amino acid and protein contents, as well as the compositions of proteinogenic amino acids.

\section{Soil Available Nitrogen}

Fresh and uniformly mixed soil samples collected from 0-20 cm to 20-40 $\mathrm{cm}$ depths in each plot were assayed immediately. Soil samples $(5 \mathrm{~g})$ were extracted with $50 \mathrm{ml}$ of $0.01 \mathrm{M} \mathrm{CaCl}_{2}$ for $1 \mathrm{~h}$ using a shaking table. A continuous flow analyser (Bran and Luebbe TRAACS Model 2000 Analyzer) was used for determinations of the soil nitrate-nitrogen $\left(\mathrm{NO}_{3}^{-}-\mathrm{N}\right)$ and ammonium nitrogen $\left(\mathrm{NH}_{4}^{+}-\mathrm{N}\right)$ levels (Dahnke and Johnson, 1990). The soil-available nitrogen level was calculated as the sum of the nitrate-nitrogen $\left(\mathrm{NO}_{3}^{-}-\mathrm{N}\right)$ and ammonium nitrogen $\left(\mathrm{NH}_{4}^{+}-\mathrm{N}\right)$ levels.

\section{GS and NADH-Dependent GOGAT Assays}

The extraction and measurement of GS (EC 6.3.1.2) and NADHGOGAT (EC 1.4.1.14) were performed according to the method of Lea et al. (1990). Frozen embryo tissues (0.3 g) were homogenized in $5 \mathrm{ml}$ buffer containing $50 \mathrm{mM} \mathrm{KH}_{2} \mathrm{PO}_{4}(\mathrm{pH}$ 7.2), 2 mM EDTA, 2 mM DTT, 5\% (v/v) glycerol, and $1 \%(\mathrm{w} / \mathrm{v})$ insoluble PVPP. After centrifugation at $12,000 \times \mathrm{g}$ for $20 \mathrm{~min}$ at $4^{\circ} \mathrm{C}$, the supernatant homogenates were stored at $4^{\circ} \mathrm{C}$ for assays of GS, NADH-dependent GOGAT and soluble protein, which were conducted immediately.

\section{GS Assay}

The standard assay mixture $(1.6 \mathrm{ml})$ contained $0.15 \mathrm{mM}$ imidazole buffer ( $\mathrm{pH} 7.0$ ), $0.010 \mathrm{mM} \mathrm{MgSO}_{4}, 0.12 \mathrm{mM}$ Glu$\mathrm{Na}$, and $0.012 \mathrm{mM}$ ATP-Na. After adding $1.2 \mathrm{ml}$ crude enzyme and heating for $5 \mathrm{~min}$ at $25^{\circ} \mathrm{C}$, the reaction was initiated by $0.2 \mathrm{ml}$ of $1 \mathrm{M} \mathrm{NH} \mathrm{H}_{2} \mathrm{OH}$ for $15 \mathrm{~min}$ at $25^{\circ} \mathrm{C}$. The amount of $\gamma$-glutamyl hydroxamate $(\mathrm{GH})$ generated was determined by adding $0.8 \mathrm{ml}$ ferric chloride reagent $\left(0.37 \mathrm{mM} \mathrm{FeCl}_{2}, 0.67 \mathrm{mM}\right.$ $\mathrm{HCl}$ and $0.2 \mathrm{mM}$ trichloroacetic acid) and spectrophotometrically measuring absorbance at $540 \mathrm{~nm}$. The activity of GS is expressed as micromoles of $\gamma$-glutamyl hydroxamate formed per milligram of soluble protein per hour. 


\section{NADH-GOGAT Assay}

NADH-dependent GOGAT was assayed at $340 \mathrm{~nm}$ by coupling the reaction to $\mathrm{NADH}$ oxidation mediated by reductive amination of $\alpha$-ketoglutarate at saturating substrate concentrations (Muhitch, 2006). The reaction mixture contained $0.1 \mathrm{M}$ Tris- $\mathrm{HCl}(\mathrm{pH} 8.5), 0.2 \mathrm{M} \alpha$-ketoglutarate, $1.0 \mathrm{mM} \mathrm{CaCl}$, and $0.2 \mathrm{mM} \mathrm{NADPH}$. After heating to $25^{\circ} \mathrm{C}$, the reaction was initiated by adding monosodium Glu to a final volume $2.1 \mathrm{ml}$. After incubating the mixture in a water bath at $30^{\circ} \mathrm{C}$ for $30 \mathrm{~min}$, the rate of decline in the $\mathrm{NADH}$ concentration was determined using a spectrophotometer at $340 \mathrm{~nm}$. The activity of GOGAT is expressed as micromoles of $\mathrm{NADH}$ per milligram of soluble protein per hour.

\section{Soluble Protein Assay}

Soluble protein was quantified using the Bradford protocol (Bradford, 1976). The standard mixture contained $0.01 \%(\mathrm{w} / \mathrm{v})$ Coomassie brilliant blue (G-250), 4.7\% (w/v) ethanol and 8.5\% (w/v) phosphoric acid. After adding $50 \mu \mathrm{L}$ of extract to the standard mixture in a total volume of $5 \mathrm{ml}$, the contents were mixed thoroughly, and absorbance was spectrophotometrically measured at $595 \mathrm{~nm}$ against a blank reagent at the indicated time points, ranging from 2 to $30 \mathrm{~min}$. Soluble protein was quantified using a standard soluble protein (Bovine Serum Albumin).

\section{Free Amino Acid Content}

Free amino acids were extracted from dried and powdered cotton embryo tissues $(0.1 \mathrm{~g})$ with $5 \mathrm{ml}$ of $80 \%$ ethanol in a water bath at $80^{\circ} \mathrm{C}$ for $30 \mathrm{~min}$ (Hendrix, 1993). After three rounds of extraction, the samples were diluted to a final volume of $25 \mathrm{ml}$. Assays were performed in a 96-well polystyrene plate with a Benchmark microplate reader (Bio-Rad). The free amino acid content is expressed as $\mathrm{mg} \mathrm{g}^{-1} \mathrm{DW}$.

\section{Protein Content and Proteinogenic Amino Acid Profiles}

Protein content was calculated as the product of the 100-embryo weight (g), N concentration (\%) and 6.25. Proteinogenic amino acids were isolated from dried and powdered cotton embryos (30 mg) via acidolysis with $10 \mathrm{ml}$ of $6 \mathrm{M} \mathrm{HCl}$ for $24 \mathrm{~h}$ at $110^{\circ} \mathrm{C}$. The solution was diluted to a final volume of $50 \mathrm{ml}$ with 0.02 $\mathrm{M} \mathrm{HCl}$. Profiles of proteinogenic amino acids were determined using an automatic amino acid analyzer (L8900, Hitachi, Tokyo, Japan) (Hao et al., 2014).

A sigmoid growth curve was used to assess the accumulation of storage proteins in developing cotton embryos, where embryo protein is the protein content (g 100 embryos $^{-1}$ ) at developmental time (d), $P_{\max }$ is the protein content at maturity, $\mathrm{a}$ and $\mathrm{b}$ are constants, $\mathrm{DAA}_{1}$ is the start time of rapid embryo protein accumulation, and $\mathrm{DAA}_{2}$ is the termination time of rapid embryo protein accumulation. The duration is the difference (in days) between the two dates.

$$
\operatorname{Embryo}_{\operatorname{protein}_{(t)}}=\frac{P_{\max }}{1+a e^{b \times D A A}}
$$

$$
\begin{gathered}
\operatorname{Rate}_{(t)}=\frac{P_{\max } a b \times e^{b \times D A A}}{\left(1+a e^{b \times D A A}\right)^{2}} \\
\text { Duration }=D A A_{2}-D A A_{1}=\frac{1}{b} \ln \frac{2+\sqrt{3}}{a}-\frac{1}{b} \ln \frac{2-\sqrt{3}}{a}
\end{gathered}
$$

\section{Statistical Analysis}

Three-way analysis of variance was performed on at least three replicates to examine the effects of years, fertility levels, IMS and their interactions on the composition of proteinogenic amino acids. The measurements are expressed as the mean \pm SE. Fisher's least significant difference (LSD) test was used for statistical analyses. Statistical significance is indicated by $P<0.05$ or by $P<0.01$. SAS software was used for principal component analysis (PCA), and graphs were plotted using Origin software, version 9.0 .

\section{RESULTS}

\section{Protein Quality in Cotton Embryos}

When seeds were mature, the cotton embryos accumulated 42.7 $46.9 \%$ of their DW as storage protein. The protein contents ranged (g 100 embryos $^{-1}$ ) from $2.18-2.68 \mathrm{~g}$ in 2012 to 2.63$3.30 \mathrm{~g}$ in 2013 (Table 2). The protein contents in the $\mathrm{IMS}_{1}$ and $\mathrm{IMS}_{2}$ embryos were 14.5 and $24.3 \%$ higher at low soil fertility levels and 3.3 and $12.7 \%$ higher at high soil fertility levels, respectively, compared to those in the CM embryos $(P<0.05)$.

The protein content increased significantly with increases in the $\mathrm{N}$ rate and plant density (Table 2). The application of $20 \% \mathrm{~N}$ as basal fertilizer, $25 \% \mathrm{~N}$ at the initial flowering stage, $40 \% \mathrm{~N}$ at the full-bloom stage and $15 \% \mathrm{~N}$ at the end of the flowering stage showed advantages over the $\mathrm{N}$ application schedule of CM. The seedling-raising method also had beneficial effects on the protein content.

The acidolysis of proteinogenic amino acids from storage proteins indicated that the contents of total amino acids and each proteinogenic amino acid in the $\mathrm{IMS}_{1}$ and $\mathrm{IMS}_{2}$ embryos were increased compared to those in CM (Table 3). The contents of total proteinogenic, essential, semi-essential and non-essential amino acids in $\mathrm{IMS}_{2}$ increased by averages of 7.61, 8.04, 4.64, and $8.32 \%$, respectively, whereas they did not significantly differ between $\mathrm{IMS}_{1}$ and CM. Although, the soil fertility level did not affect the composition of proteinogenic amino acids, the interaction effect between fertility level and IMS was significant $(P<0.05$, Table 4), demonstrating IMS did not only affect proteinogenic amino acids profiles, but they also have a beneficial effect on soil fertility level.

Glu, Lys, and Met are nutritional limiting factors for ruminants fed cottonseed kernel protein flour. The contents of Glu, Lys, and Met in $\mathrm{IMS}_{2}$ were increased by 4.7, 2.9, and 3.4\% at a low fertility level and by $4.7,1.5$, and $4.0 \%$ at a high fertility level, respectively, compared to those embryos in CM (Figure 1). The ratio of each amino acid to the total proteinogenic amino acid content was relatively stable. These results indicate that the 
TABLE 2 | Mean protein content (g 100 embryos $^{-1}$ ) of recommended integrated crop management (ICM) as compared with conventional management practice (CM) for cottonseed embryos in 2012 and 2013.

\begin{tabular}{|c|c|c|c|c|c|c|c|c|c|}
\hline Year & $\begin{array}{c}\text { Soil } \\
\text { fertility }\end{array}$ & $\begin{array}{c}\text { Integrated } \\
\text { management } \\
\text { strategies }\end{array}$ & $\begin{array}{l}\text { N rates } \\
\left(\mathrm{kg} \mathrm{ha}^{-1}\right)\end{array}$ & $\begin{array}{l}\text { N application } \\
\text { schedule }\end{array}$ & $\begin{array}{l}\text { Plant density } \\
\text { (plant ha }^{-1} \text { ) }\end{array}$ & $\begin{array}{l}\text { Seedling raising } \\
\text { method }\end{array}$ & $\begin{array}{l}\text { Protein } \\
\text { content }\end{array}$ & $\begin{array}{c}\text { Increase } \\
\text { of IMS } \\
\text { over CM } \\
(\%)\end{array}$ & $P$ \\
\hline \multirow[t]{6}{*}{2012} & LF & $\mathrm{CM}$ & 300 & $120-180-0-0$ & 18,000 & ST & 2.01 & & \\
\hline & & $\mathrm{IMS}_{1}$ & 375 & $75-94-150-56$ & 30,000 & $\mathrm{SR}$ & 2.38 & 18.38 & $<0.05$ \\
\hline & & $\mathrm{IMS}_{2}$ & 525 & $105-131-210-79$ & 30,000 & ST & 2.65 & 32.16 & $<0.05$ \\
\hline & $\mathrm{HF}$ & $\mathrm{CM}$ & 300 & $120-180-0-0$ & 18,000 & ST & 2.35 & & \\
\hline & & $\mathrm{IMS}_{1}$ & 375 & $75-94-150-56$ & 30,000 & $\mathrm{SR}$ & 2.43 & 3.35 & ns \\
\hline & & $\mathrm{IMS}_{2}$ & 525 & $105-131-210-79$ & 30,000 & ST & 2.68 & 14.18 & $<0.05$ \\
\hline \multirow[t]{6}{*}{2013} & LF & $\mathrm{CM}$ & 300 & $120-180-0-0$ & 18,000 & ST & 2.63 & & \\
\hline & & $\mathrm{IMS}_{1}$ & 375 & $75-94-150-56$ & 30,000 & $\mathrm{SR}$ & 2.91 & 10.56 & $<0.05$ \\
\hline & & $\mathrm{IMS}_{2}$ & 525 & $105-131-210-79$ & 30,000 & ST & 3.06 & 16.47 & $<0.05$ \\
\hline & $\mathrm{HF}$ & $\mathrm{CM}$ & 300 & $120-180-0-0$ & 18,000 & ST & 2.97 & & \\
\hline & & $\mathrm{IMS}_{1}$ & 375 & $75-94-150-56$ & 30,000 & $\mathrm{SR}$ & 3.07 & 3.36 & ns \\
\hline & & $\mathrm{IMS}_{2}$ & 525 & $105-131-210-79$ & 30,000 & ST & 3.30 & 11.33 & $<0.01$ \\
\hline
\end{tabular}

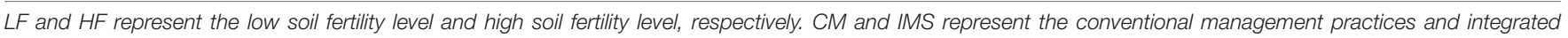

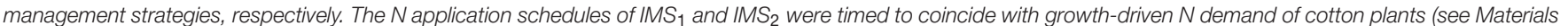

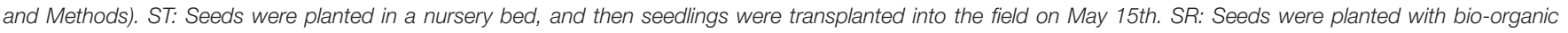

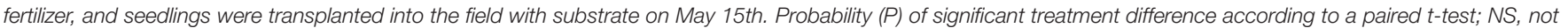
significantly different at $P=0.05$.

IMS increased the levels of proteinogenic amino acids but did not lead to an alteration in the level of any proteinogenic amino acid.

\section{Soil-Available Nitrogen Content}

Regardless of the soil fertility levels or soil depth, the contents of $\mathrm{NO}_{3}^{-}-\mathrm{N}$ and $\mathrm{NH}_{4}^{+}-\mathrm{N}$ were consistently significantly higher in $\mathrm{IMS}_{1}$ and $\mathrm{IMS}_{2}$ than in CM at soil depths of $0-20 \mathrm{~cm}$ and 20-40 cm (Figures 2A,B). Furthermore, the $\mathrm{NO}_{3}^{-}-\mathrm{N}$ and $\mathrm{NH}_{4}^{+}$ -N contents at the initial flowering stage were higher than those at the end of flowering and full-bloom stages. $\mathrm{IMS}_{2}$ exhibited the highest level of AV-N (sum of $\mathrm{NO}_{3}^{-}-\mathrm{N}$ and $\mathrm{NH}_{4}^{+}-\mathrm{N}$ ). Compared with CM, the contents of AV-N in $\mathrm{IMS}_{1}$ and $\mathrm{IMS}_{2}$ increased by averages of 6.37 and $16.86 \%$, respectively, at soil depths of $0-20 \mathrm{~cm}$ and by 9.72 and $21.52 \%$, respectively, at soil depths of $20-40 \mathrm{~cm}$. These results indicated that soil $\mathrm{N}$ availability was significantly increased in both $\mathrm{IMS}_{1}$ and $\mathrm{IMS}_{2}$ compared with CM.

\section{GS and NADH-Dependent GOGAT in Developing Cotton Embryos}

The enzymatic activities of GS and NADH-dependent GOGAT showed similar patterns of alterations, with their activities declining according to the duration of embryo growth in 2012 and 2013 (Figure 3). Compared with CM, GS activity increased during $\mathrm{IMS}_{1}$ and $\mathrm{IMS}_{2}$ embryo growth, with average increases of 4.5 and $13.6 \%$ at high fertility levels and of 11.2 and $16.5 \%$ at low fertility levels, respectively. Further, the NADH-dependent GOGAT activities in $\mathrm{IMS}_{1}$ and $\mathrm{IMS}_{2}$ increased by 13.9 and $24.1 \%$ at high fertility levels and by 9.1 and $20.6 \%$ at low fertility levels, respectively. These results indicated that the IMS enhanced the initial $\mathrm{N}$ assimilation in the developing cotton embryos.

\section{Free Amino Acid Supply and Protein Accumulation in Developing Cotton Embryos}

Free amino acids are precursors to storage proteins and are incorporated into proteins during the growth of cotton embryos. The free amino acid content increased sharply after 17 DAA, rising from a very low level to a maximum level at 24 DAA and then declining (Figure 4A). This content decreased dramatically, accompanied by rapid protein accumulation in the cotton embryos after 24 DAA. Moreover, at 24 DAA, the free amino acid content was significantly higher in CM than in $\mathrm{IMS}_{1}$ or $\mathrm{IMS}_{2}$.

Sigmoid growth curves, used to quantify the rate of protein accumulation (g 100 embryos $\mathrm{d}^{-1}$ ), may reveal the reasons and key stages associated with the differences in protein content among integrated management treatments (Figures 4B,C). Rapid embryo protein accumulation began at 17-22 DAA and terminated at 32-37 DAA, lasting from 11 to 15 days in 2012 and 2013 (Table 5). The peak rate of protein accumulation was positively correlated with the protein content at maturity. Change tendency of the protein content was opposite to that of the free amino acids. Accumulation of a large amount of protein coincided with a rapid decrease in the free amino acid content, and evaluation of the different management treatments revealed that the protein content was limited by the protein accumulation rate rather than by the free amino acid content.

\section{Protein Quality and Relationships}

The protein content was positively and significantly correlated with the content of soil available N, GS activity and peak rate of rapid protein accumulation, whereas it was negatively correlated with the free amino acid content (Table 6). Similar relationships 
TABLE 3 | Proteinogenic amino acid contents of cotton embryos under conventional management practices and two integrated management strategies at two fertility levels.

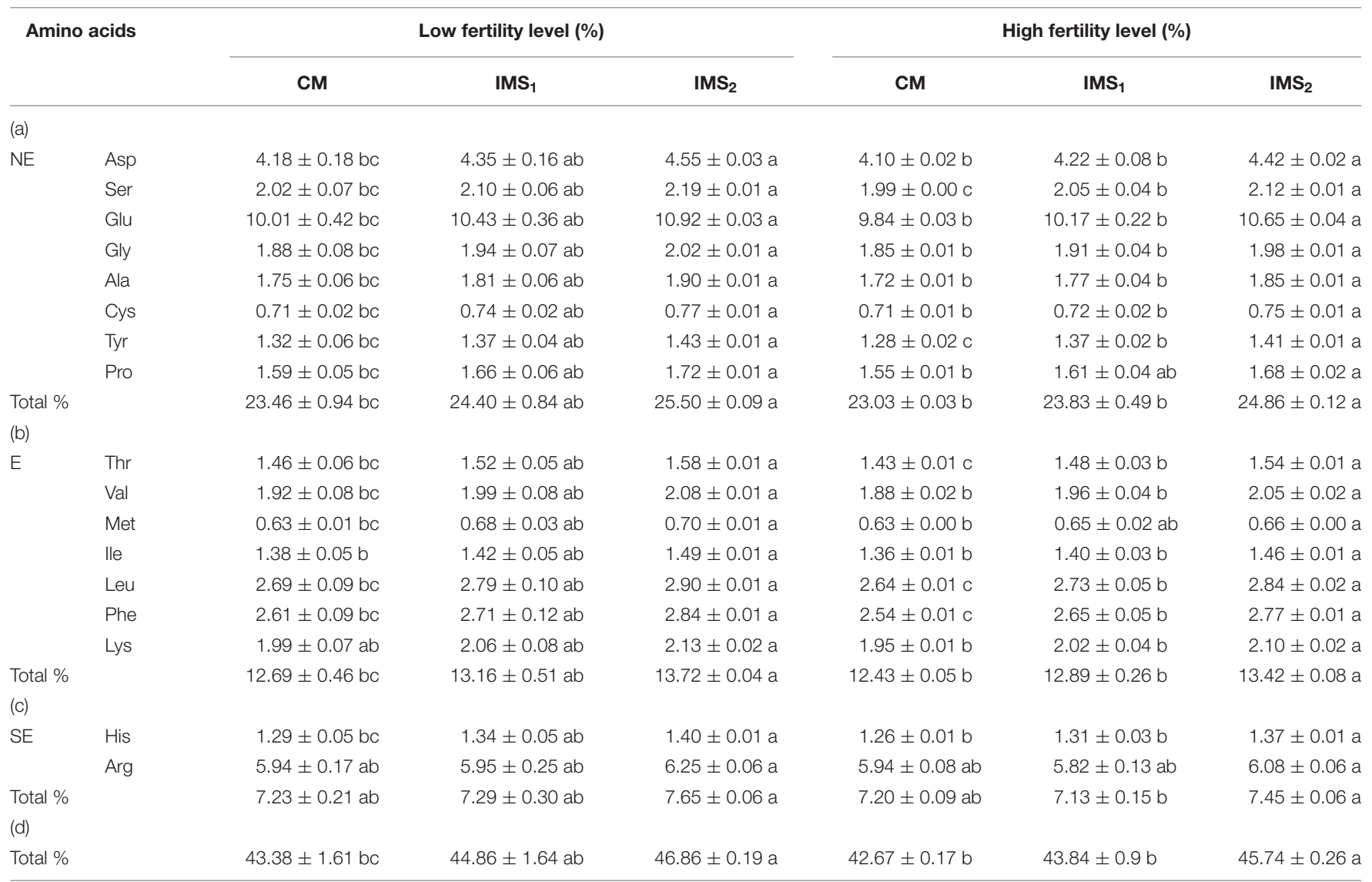

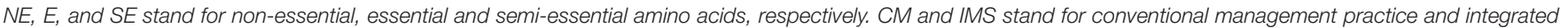

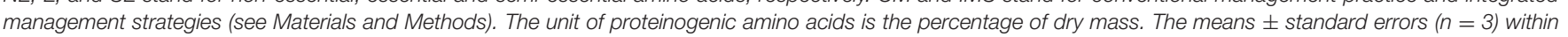
each column followed by the same letter are significantly different $(P<0.05)$ according to Fisher's least significant difference (LSD) test.

TABLE 4 | Results of ANOVA on the effects of year (Y), fertility levels (FL), integrated management strategies (M) and their interactions on protein content and proteinogenic amino acids in cotton embryos in 2012 and 2013.

\begin{tabular}{|c|c|c|c|c|c|c|c|c|c|}
\hline Effect & $d f$ & Protein content & TAA & NE & $\mathbf{E}$ & SE & Glu & Lys & Met \\
\hline Y & 1 & $235.71^{* *}$ & $0.64^{\mathrm{ns}}$ & $1.07^{\text {ns }}$ & $0.55^{\text {ns }}$ & $0.01^{n s}$ & $0.93^{\text {ns }}$ & $0.64^{\text {ns }}$ & $2.35^{\text {ns }}$ \\
\hline $\mathrm{FL}$ & 1 & $27.41^{* *}$ & $0.11^{\mathrm{ns}}$ & $0.07^{\text {ns }}$ & $0.46^{\text {ns }}$ & $0.01^{\mathrm{ns}}$ & $0.08^{\text {ns }}$ & $0.14^{\mathrm{ns}}$ & $2.54^{\mathrm{ns}}$ \\
\hline$M$ & 2 & $45.82^{* *}$ & $27.42^{* *}$ & $30.48^{* *}$ & $28.68^{* *}$ & $12.75^{* *}$ & $32.57^{* *}$ & $24.59^{* *}$ & $19.46^{* *}$ \\
\hline$Y \times F L$ & 1 & $2.03^{\text {ns }}$ & $6.35^{*}$ & $7.24^{*}$ & $4.38^{*}$ & $6.17^{*}$ & $7.17^{*}$ & $4.62^{*}$ & $5.71^{*}$ \\
\hline$Y \times M$ & 2 & $0.68^{\text {ns }}$ & $0.48^{\text {ns }}$ & $0.27^{\mathrm{ns}}$ & $0.31^{\mathrm{ns}}$ & $2.46^{\text {ns }}$ & $0.32^{\text {ns }}$ & $0.29^{\text {ns }}$ & $0.06^{n s}$ \\
\hline $\mathrm{FL} \times \mathrm{M}$ & 2 & $3.89^{*}$ & $5.00^{*}$ & $4.98^{*}$ & $4.42^{*}$ & $5.32^{*}$ & $4.95^{*}$ & $2.70^{\text {ns }}$ & $5.75^{*}$ \\
\hline $\mathrm{Y} \times \mathrm{FL} \times \mathrm{M}$ & 2 & $0.70^{\text {ns }}$ & $5.27^{*}$ & $5.22^{*}$ & $4.73^{*}$ & $5.81^{* *}$ & $5.20^{*}$ & $4.86^{*}$ & $3.18^{\text {ns }}$ \\
\hline
\end{tabular}

TAA, NE, E, and SE stand for total, non-essential, essential and semi-essential proteinogenic amino acids, respectively. $F$ values and significance levels $* * P<0.01$, ${ }^{*} P<0.05$ and $\left.{ }^{\mathrm{ns}} P \geq 0.05\right)$. df, degree of freedom.

were also observed for the levels of total proteinogenic amino acids, essential amino acids, semi-essential amino acids, Glu, Lys and Met.

In PCA, the first two principal components explained $61.4 \%$ and $18.5 \%$ of the total variance in the sample set, respectively, whereas the third principal component only accounted for $7.5 \%$ of the variance (Figure 5). Essential amino acids, semi-essential amino acids, Glu, Lys and Met were clustered as a group in the score plot. The content of soil available $\mathrm{N}$ and the peak rate of protein accumulation were most closely related to the proteinogenic amino acid profiles in the score plot. These results indicated that the improvements in the nutritional value of the cotton embryos observed with use of the IMS could be attributed to increases in soil $\mathrm{N}$ availability, the initial step of $\mathrm{N}$ assimilation and the conversion of free amino acids into storage proteins (Figure 6). 


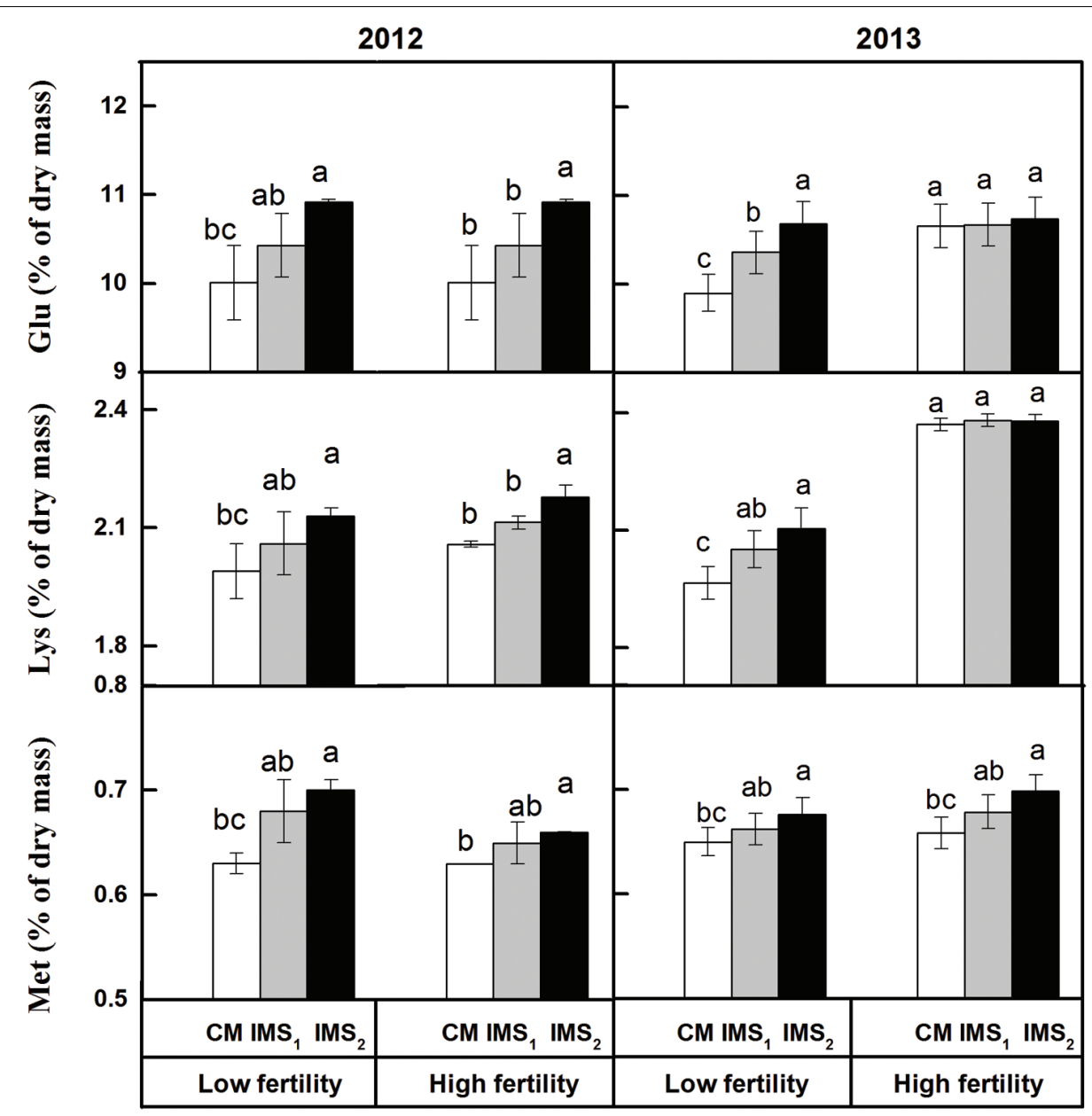

FIGURE 1 | Contents of Glu, Lys and Met in cotton embryos under conventional management practices and two integrated management strategies at two fertility levels in $\mathbf{2 0 1 2}$ and 2013. The data points represent the means, and the bars are the SEs. CM and IMS stand for conventional management practice and integrated management strategies (see Materials and Methods). Different letters indicate significant difference at the level of $P<0.05$.

\section{DISCUSSION}

The initial question that motivated us to perform this study was whether matching a high-yielding variety with IMS would prove useful in improving the nutritional value of cotton embryos. We were also particularly interested in the following questions: What improves their nutritional value? What is the physiological basis of high quality? What are the guidelines for increasing crop quality in other cotton-producing regions?

Most of the previous studies are focusing on the developing high fiber yield and optimal fiber propriety through crop breeding (Cai et al., 2014; Guan et al., 2014; Ning et al., 2014), stress-resistance cultivation (Kawakami et al., 2012; Liu et al., 2015; Kuai et al., 2016), and crop management (Kawakami et al., 2012). However, high-quality and nutritional value-added cottonseed by improving management practices can increase crop yields and improve farmers' livelihoods dramatically. In this study, the levels of proteins, essential amino acids, and semi-essential amino acids, especially those of Glu, Lys, and Met, were higher in $\mathrm{IMS}_{1}$ and $\mathrm{IMS}_{2}$ embryos than in CM embryos (Tables 2 and 3; Figure 1). In addition, fiber yield, seed yield, seed weight were also increased dramatically in $\mathrm{IMS}_{1}$ and $\mathrm{IMS}_{2}$ treatments (data not given). The impact of IMS on cotton embryo protein quality was more obvious at a low soil fertility level than at a high soil fertility level (Tables 2 and 3). Previously it has been reported that ruminants fed with cottonseed, like cows produce more milk and its protein content were also increased (Bertrand et al., 1998; Meyer et al., 2001; Taghizadeh et al., 2005). Therefore, an increase in the proteinogenic amino acid content achieved by adopting IMS resulted in enhanced nutritional value of the cotton embryos.

Nitrogen fertilization rate, plant density, and plant growth regulators have been shown to have a positive effect on cottonseed oil and protein contents (Sawan et al., 1993, 2006). In this study (using IMS), the $\mathrm{N}$ rate and fertility level contributed to the increase of protein content (Table 2), are in agreement with the results of the previous single-factor experiments (Chen et al., 2015), suggesting that protein content were increased with increasing $\mathrm{N}$ fertilization rate (Sawan et al., 1988; Egelkraut 

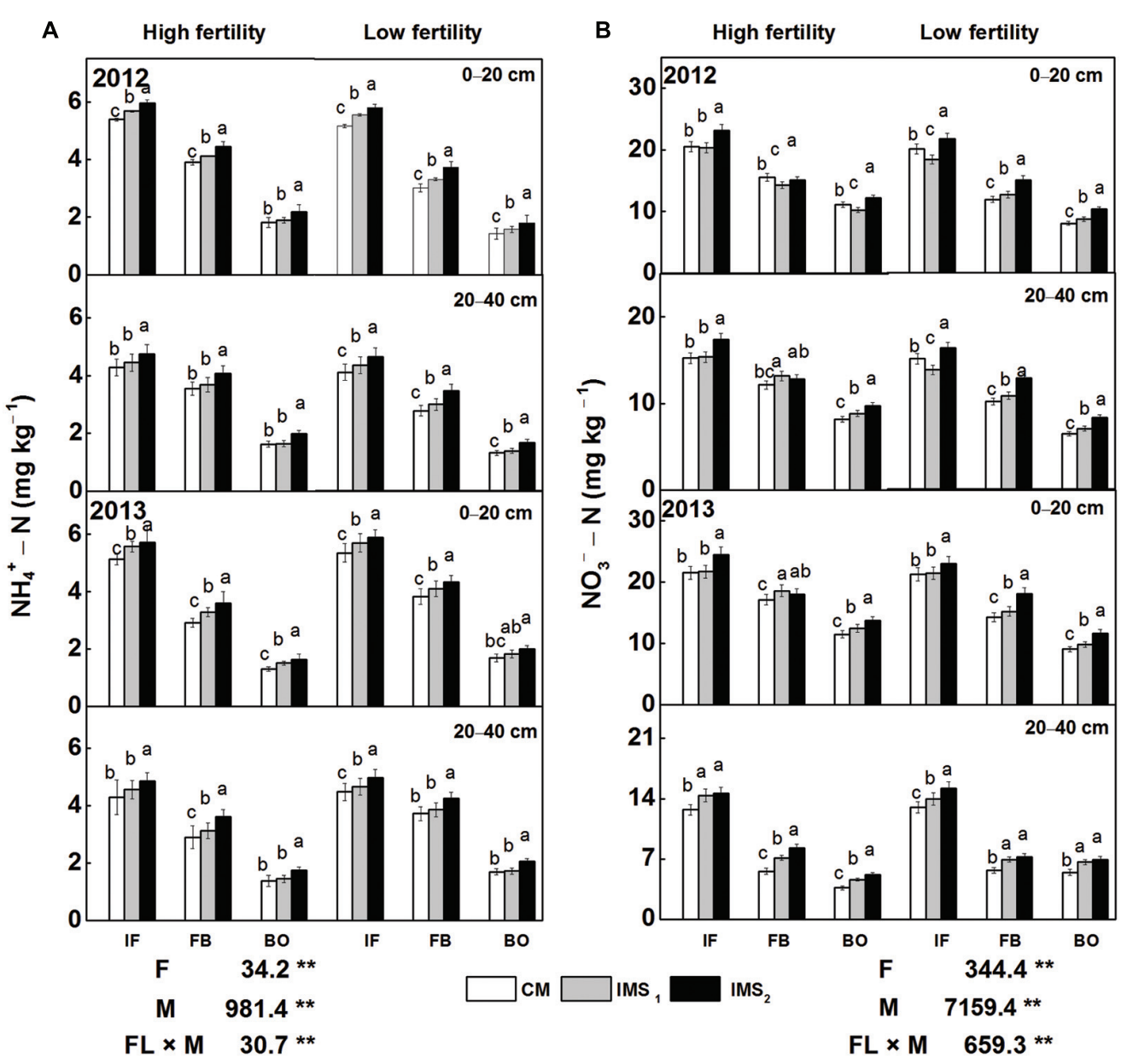

FIGURE 2 | Soil $\mathrm{NH}_{4}^{+}-\mathrm{N}(\mathrm{A})$ and $\mathrm{NO}_{3}^{-}-\mathrm{N}(\mathrm{B})$ levels under conventional management practices and two integrated management strategies at two fertility levels at soil depths of $\mathbf{0 - 2 0} \mathbf{c m}$ and $\mathbf{2 0 - 4 0 ~ c m ~ i n ~} \mathbf{2 0 1 2}$ and $\mathbf{2 0 1 3}$. CM and IMS stand for conventional management practices and integrated management strategies (see Materials and Methods). IF, BF, and BO stand for the initial flowering stage, full-bloom stage, and boll-opening stage, respectively. Different letters indicate significant difference at the level of $P<0.05$.

et al., 2004). As the plant density increased, the protein content were also increased (Rafiq et al., 2010), and it would not need an additional labor input because of mechanized transplanting. The $\mathrm{N}$ rate in $\mathrm{IMS}_{1}$ was an economic fertilization rate as reported previously (Chen et al., 2016) and it was higher compared with those reported at the other experimental stations (Rochester et al., 2001) might be attributed to the saline-alkali soil ( $\mathrm{pH}$ 8.26-8.47). Salt stress limits plant growth (Zhang et al., 2015) and therefore the high economic fertilization $\mathrm{N}$ rate $\left(375 \mathrm{~kg} \mathrm{ha}^{-1}\right)$. Further, increase in the application of $\mathrm{N}$ at flowering and boll-forming stage not only increased cotton yield and biomass (Yang et al., 2011), but also proved to be useful for increasing the protein content in cotton embryos (Table 2). Sufficient $\mathrm{N}$ supply from flowering to boll forming stage was the main reason for the increment of protein content. Additionally, this study also found that the substrate seedling-raising method contributed to increase in protein content. The probable reason behind this was that bio-organic fertilizer bring the efficacious living-cell into a soil and then affect the $\mathrm{N}$ availability in the soil (Inselsbacher et al., 2010). Due to unusual weather changes in recent years, temperature and photosynthetically active radiation in 2013 was higher than 2012, like cumulative photo-thermal product increased by $30.8 \%$ during the seed growth. Seed weight was increased dramatically; therefore, the protein content (g 100 embryos $^{-1}$ ) was higher than the year in 2012. Therefore, a set of the best management practices for cotton production was integrated that could increase protein quality in cotton embryos for feed purposes.

A study using a method based on isotope $\left({ }^{15} \mathrm{NH}_{4}^{+}\right.$and ${ }^{15} \mathrm{NO}_{3}^{-}$) labeling has demonstrated that soil $\mathrm{N}$ is ultimately translocated into seeds (Kullmann and Geisler, 1986), suggesting a potential contribution of soil $\mathrm{N}$ to the protein content of seeds. Embryos take up N, carbohydrates and free amino acids from seed coats (De Ruiter et al., 1986) and use them for embryo growth and the synthesis of storage proteins. Based on these studies, it appears that the soil $\mathrm{N}$ and seed protein contents are closely interrelated. In this study, as shown in 


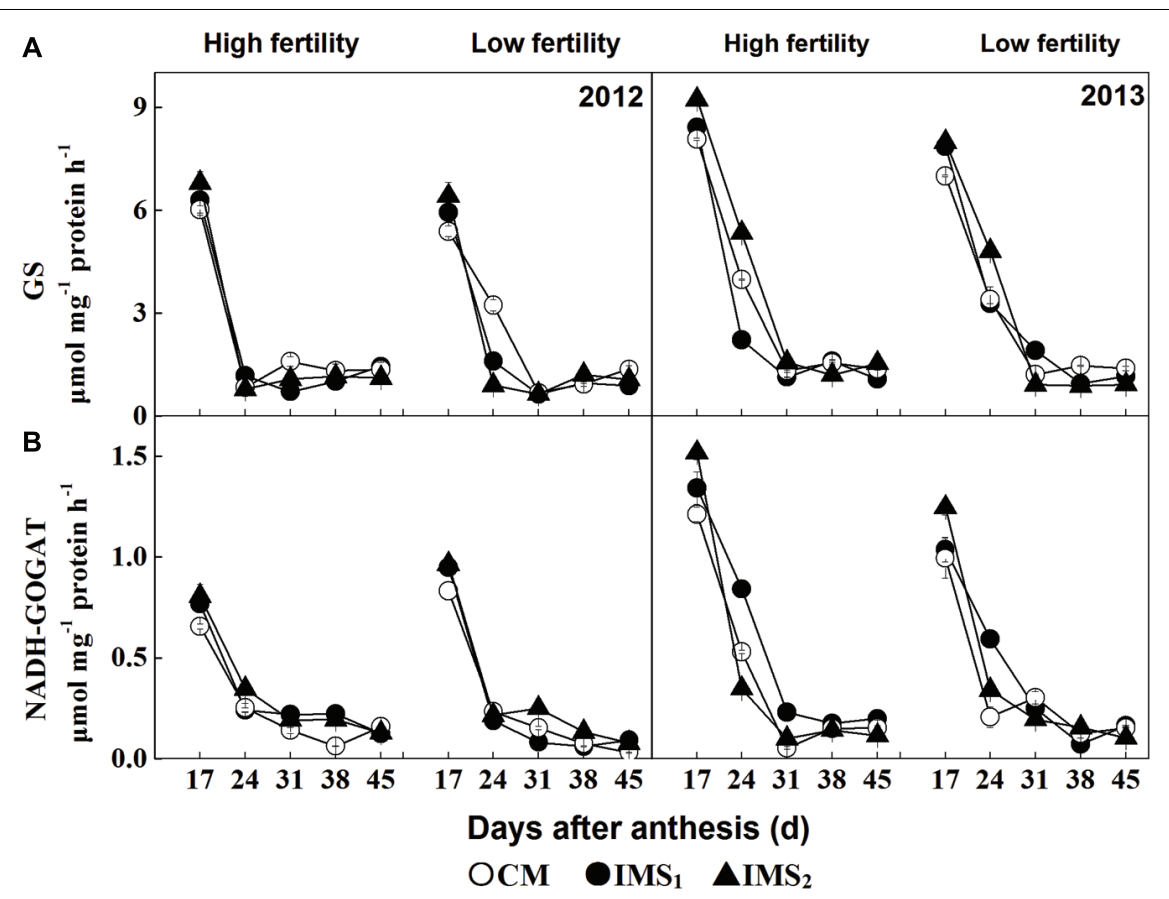

FIGURE 3 | Developmental profiles of glutamine synthetase (A) (GS, $\mu$ mol $\gamma$-glutamyl-hydroxamate $\mathrm{h}^{-1} \mathrm{~g}^{-1}$ soluble protein) and (B) NADH-dependent glutamate synthase (GOGAT, $\mu \mathrm{mol}$ NADH h $\mathrm{h}^{-1} \mathrm{~g}^{-1}$ soluble protein) for conventional management practices and two integrated management strategies at two fertility levels in 2012 and 2013. For each sample point, we tested at least three independent samples. The data points represent the means, and the bars are the SEs. CM and IMS stand for conventional management practices and integrated management strategies (see Materials and Methods).
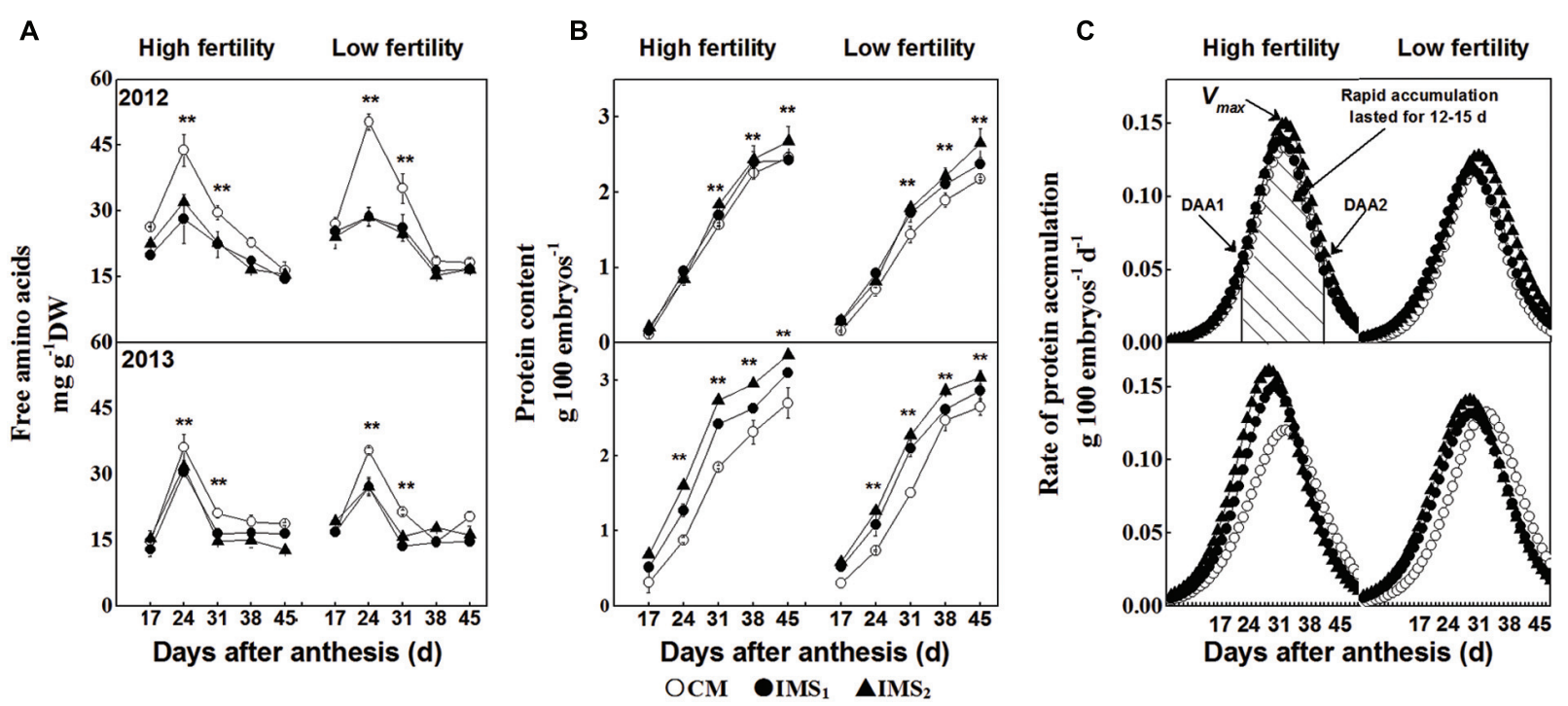

FIGURE 4 | The free amino acid contents (A) and protein accumulation $(B, C)$ in cotton embryos under conventional management practices and two integrated management strategies at two fertility levels in $\mathbf{2 0 1 2}$ and 2013. The data points represent the means, and the bars are the SEs. Characterization of proteins accumulated in the cotton embryos was calculated using sigmoid growth curves (see Materials and Methods). CM and IMS stand for conventional management practice and integrated management strategies (see Materials and Methods). ${ }^{*}$ and ${ }^{* *}$ denotes significant at the 0.05 level and 0.01 level, respectively.

Figure 2 and Table 2, the soil-available $\mathrm{N}$ and protein contents were higher in both $\mathrm{IMS}_{1}$ and $\mathrm{IMS}_{2}$ compared with that in CM. Similar results have been reported in tomato (Javaria and Khan, 2011) and potato (Zotarelli et al., 2015), suggesting that rate and timing of nitrogen fertilizer application increases the soil-available $\mathrm{N}$ content. Furthermore, principal component and Pearson's correlation analyses revealed that the soil-available nitrogen content was positively correlated with the nutritional 
TABLE 5 | Protein accumulation characteristics of cotton embryos under conventional management practices and two integrated management strategies at two fertility levels in 2012 and 2013.

\begin{tabular}{|c|c|c|c|c|c|c|c|c|c|c|c|}
\hline \multirow{3}{*}{$\begin{array}{l}\text { Fertility } \\
\text { levels }\end{array}$} & \multirow{3}{*}{$\begin{array}{l}\text { Integrated } \\
\text { management } \\
\text { strategies }\end{array}$} & \multicolumn{10}{|c|}{ Embryonic protein accumulation characteristics } \\
\hline & & \multicolumn{2}{|c|}{$\begin{array}{l}\mathrm{DAA}_{1} \\
\text { (d) }\end{array}$} & \multicolumn{2}{|c|}{$\begin{array}{l}\mathrm{DAA}_{2} \\
\text { (d) }\end{array}$} & \multicolumn{2}{|c|}{$\begin{array}{l}\text { Duration } \\
\text { (d) }\end{array}$} & \multicolumn{2}{|c|}{$\begin{array}{c}\text { Peak rate } \\
\left(\text { g } 100 \text { embryos }^{-1} \mathrm{~d}^{-1}\right)\end{array}$} & \multicolumn{2}{|c|}{$\begin{array}{l}\text { Protein content } \\
\left(\text { g } 100 \text { embryos }^{-1}\right)\end{array}$} \\
\hline & & 2012 & 2013 & 2012 & 2013 & 2012 & 2013 & 2012 & 2013 & 2012 & 2013 \\
\hline \multicolumn{12}{|l|}{ (a) } \\
\hline \multirow[t]{4}{*}{ High fertility } & $\mathrm{CM}$ & 21.6 & 20.5 & 33.5 & 35.4 & 11.9 & 15.0 & 0.134 & 0.120 & $2.35 \mathrm{~b}$ & $2.97 \mathrm{c}$ \\
\hline & $\mathrm{IMS}_{1}$ & 21.0 & 18.6 & 32.9 & 32.0 & 11.9 & 13.4 & 0.139 & 0.150 & $2.43 \mathrm{~b}$ & $3.07 \mathrm{~b}$ \\
\hline & $\mathrm{IMS}_{2}$ & 21.7 & 17.2 & 33.7 & 30.8 & 12.0 & 13.5 & 0.150 & 0.161 & $2.68 \mathrm{a}$ & $3.30 \mathrm{a}$ \\
\hline & CV\% & 2.49 & 9.37 & 3.49 & 6.82 & 5.71 & 5.12 & 7.77 & 13.81 & 7.00 & 5.55 \\
\hline \multicolumn{12}{|l|}{ (b) } \\
\hline \multirow[t]{4}{*}{ Low fertility } & $\mathrm{CM}$ & 21.2 & 22.7 & 32.8 & 37.0 & 11.5 & 14.3 & 0.116 & 0.132 & $2.01 \mathrm{bc}$ & 2.63 b \\
\hline & $\mathrm{IMS}_{1}$ & 19.8 & 19.1 & 33.2 & 34.0 & 13.4 & 14.9 & 0.118 & 0.132 & $2.38 \mathrm{~b}$ & $2.91 \mathrm{ab}$ \\
\hline & $\mathrm{IMS}_{2}$ & 21.1 & 18.5 & 35.0 & 33.3 & 13.9 & 14.8 & 0.127 & 0.141 & $2.65 \mathrm{a}$ & $3.06 \mathrm{a}$ \\
\hline & CV\% & 7.47 & 9.56 & 4.88 & 4.94 & 7.92 & 1.85 & 4.11 & 8.54 & 13.81 & 7.65 \\
\hline
\end{tabular}

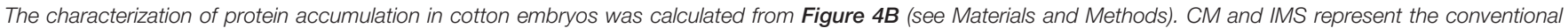

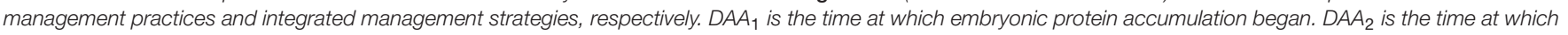

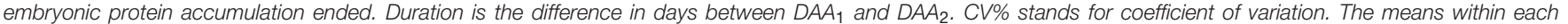
column followed by the same letter are not significantly different $(P<0.05)$ according to the least significant difference (LSD) test.

TABLE 6 | Correlations among protein traits, soil available $\mathbf{N}, \mathbf{N}$ assimilation and characterization of protein accumulation.

\begin{tabular}{|c|c|c|c|c|c|c|c|}
\hline \multirow[t]{2}{*}{ Quality traits } & \multicolumn{2}{|c|}{ Soil available $\mathbf{N}$} & \multirow[t]{2}{*}{ GS activity } & \multirow[t]{2}{*}{ NADH-GOGAT activity } & \multirow[t]{2}{*}{ Free amino acid content } & \multirow[t]{2}{*}{ Duration } & \multirow[t]{2}{*}{ Peak rate } \\
\hline & $0-20 \mathrm{~cm}$ & $20-40 \mathrm{~cm}$ & & & & & \\
\hline TAA & $0.805^{* *}$ & $0.708^{* *}$ & $0.552^{*}$ & $0.173^{\text {ns }}$ & $-0.621^{*}$ & $-0.049^{n s}$ & $0.702^{* *}$ \\
\hline$E$ & $0.772^{* *}$ & $0.737^{* *}$ & $0.471^{\mathrm{ns}}$ & $0.092^{n s}$ & $-0.614^{*}$ & $-0.130^{\mathrm{ns}}$ & $0.690^{* *}$ \\
\hline SE & $0.823^{* *}$ & $0.736^{* *}$ & $0.509^{*}$ & $0.131^{\mathrm{ns}}$ & $-0.434^{\mathrm{ns}}$ & $-0.152^{n s}$ & $0.601^{*}$ \\
\hline Glu & $0.703^{* *}$ & $0.639^{* *}$ & $0.503^{*}$ & $0.156^{\mathrm{ns}}$ & $-0.647^{* *}$ & $-0.062^{n s}$ & $0.718^{* *}$ \\
\hline Lys & $0.572^{*}$ & $0.508^{*}$ & $0.516^{*}$ & $0.123^{\text {ns }}$ & $-0.618^{*}$ & $0.013^{\text {ns }}$ & $0.623^{*}$ \\
\hline Met & $0.634^{* *}$ & $0.528^{*}$ & $0.493^{\text {ns }}$ & $0.125^{\mathrm{ns}}$ & $-0.575^{*}$ & $-0.108^{\text {ns }}$ & $0.752^{* *}$ \\
\hline
\end{tabular}

TAA, E, and SE stand for total, essential and semi-essential proteinogenic amino acids, respectively. Glu, Lys, and Met stand for glutamic acid, Iysine, and methionine,

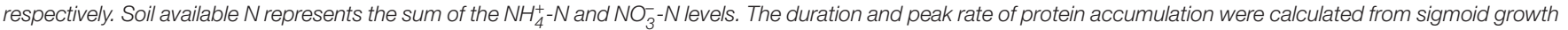
curves (see Materials and Methods). * and ** denotes significant at the 0.05 level and 0.01 level, respectively.

value of cotton embryos and that the soil-available nitrogen and proteinogenic amino acid profiles were closely matched in a score plot (Table 6; Figure 5). Therefore, high soil-available nitrogen content contributes to the nutritional value of cotton embryos.

A study using the isotope $\left({ }^{15} \mathrm{~N}\right)$ labeling also has shown that GS and NADH-GOGAT are correlated with $\mathrm{N}$ assimilation and re-distribution (Zhang et al., 2014). In this study, comparisons of management treatments at two fertility levels demonstrated that the protein content (g 100 embryos $^{-1}$ ) was positively correlated with the GS and NADH-dependent GOGAT activities (Table 6). Both soil N and GS activity increased protein content in cotton embryos (Figures 2 and 3; Table 2), that is consistent with previous research findings, suggesting that $\mathrm{NH}_{4}^{+}-\mathrm{N}$ and $\mathrm{NO}_{3}^{-}-\mathrm{N}$ concentrations in the soil tightly regulate the GS and GOGAT activities (Zhao and Shi, 2006), and that GS activity and actual accumulation of reduced $\mathrm{N}$ are closely interrelated in cotton (Radin et al., 1975) and wheat (Brunetti and Hageman, 1976). Therefore, a high level of soil available $N$ increases
$\mathrm{N}$ assimilation in developing cotton embryos (Figures 6A,B). Furthermore, GS plays a key role during the early stage of embryo growth.

A previous study reported that the synthesis of embryonic proteins is regulated by the supply of free amino acids (Takahashi et al., 2003; Hernandez-Sebastia et al., 2005). In this study, the free amino acid content decreased simultaneously with accumulating storage protein after 24 DAA (Figures 4A,B). The protein accumulation rate was higher in $\mathrm{IMS}_{1}$ and $\mathrm{IMS}_{2}$ embryos over CM, meanwhile the amino acids content showed the opposite trends, indicating that the protein content was limited by the protein accumulation rate rather than by the free amino acid content. Furthermore, the peak protein accumulation rate was positively associated with the protein content at maturity, exhibiting a period of rapid protein accumulation during 24-38 DAA (Figure 4C, Table 6), whereas the free amino acid content peaked at 24 DAA (Figure 4A). It seemed that embryos show an early period for free amino acids accumulation, which was characterized by large amount of free 


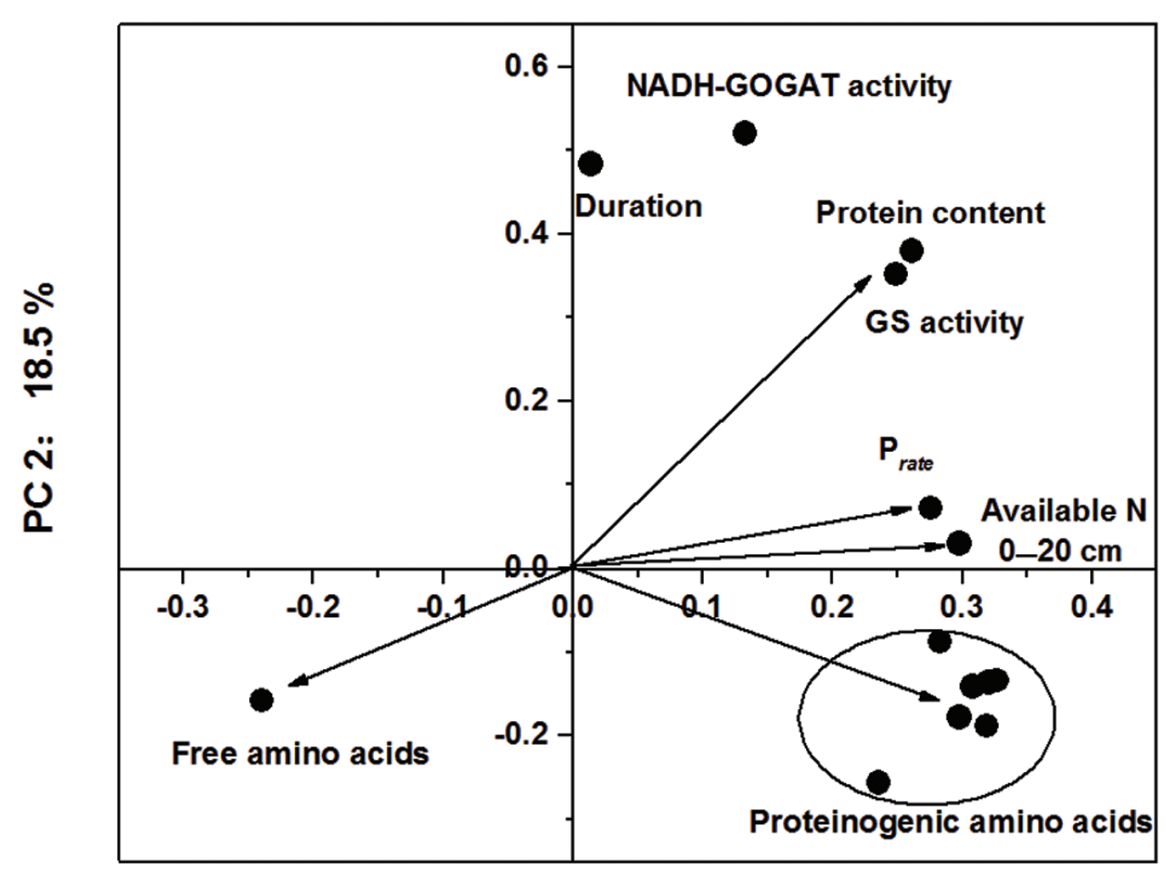

PC 1: $61.4 \%$

FIGURE 5 | Principal component analysis of the soil-available $\mathbf{N}$ content, $\mathbf{N}$ assimilation, rate of protein accumulation and proteinogenic amino acid profile based on the first two principal components (PC 1: principal component 1; and PC 2: principal component 2). The arrows represent the principal component loadings, and the ellipses represent the proteinogenic amino acid profiles.

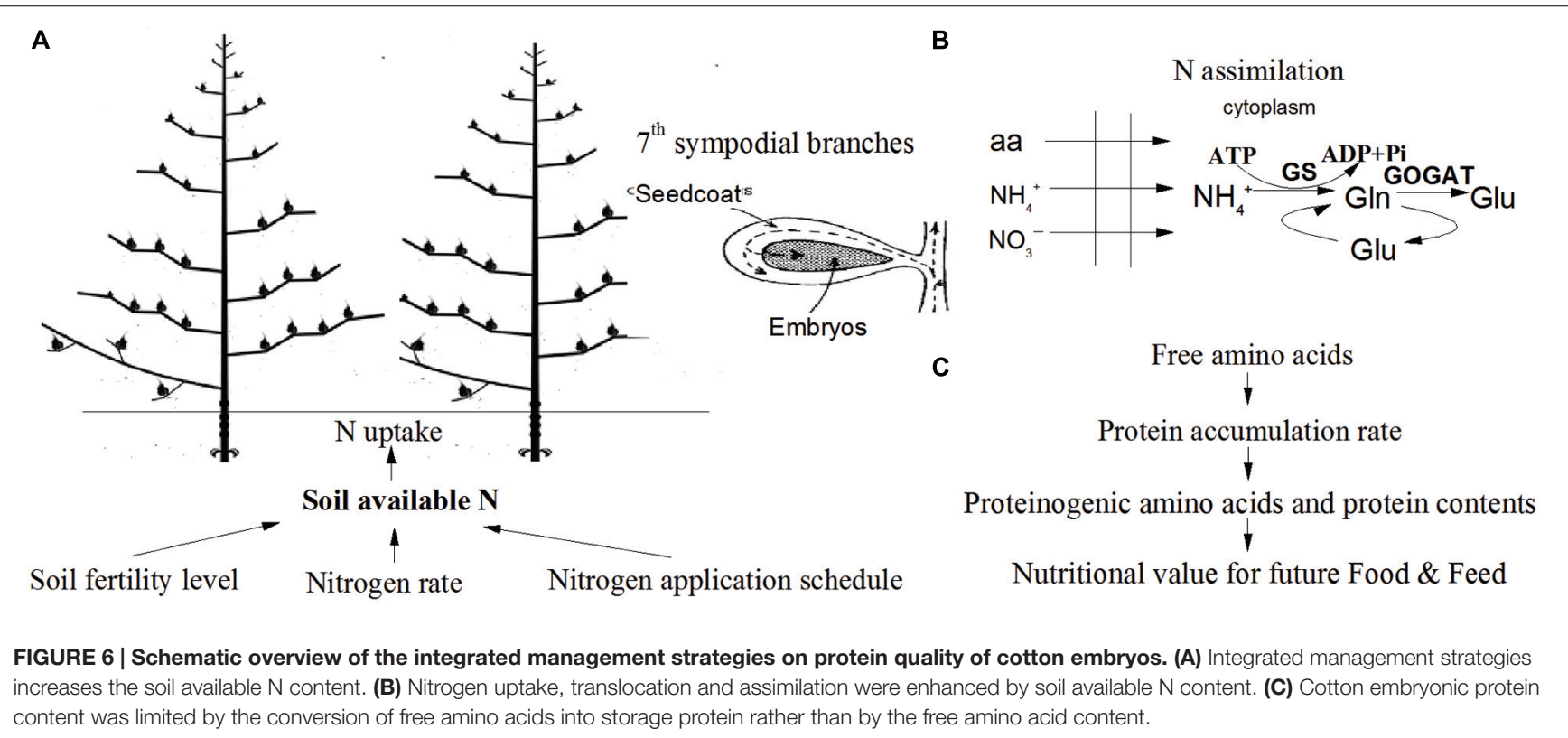

amino acid accumulation during 17-24DAA (Figure 4A). The embryos accumulate almost $75 \%$ of the total protein within rapid protein accumulation stage for a short period of time (lasted for 12-15 days) that was attributed to an early period of free amino acids accumulation. Similar study also observed in other researches, for instance in soybean (Rotundo et al.,
2011), suggesting protein content was determined by different combinations of rate and duration of content accumulation (Figure 6C).

The indeterminate growth habit of cotton results in the formation of bolls over a range of time as the plant grows and under different environmental and nutritional conditions 
(Bondada and Oosterhuis, 2001). In this study, the soil available $\mathrm{N}$ decreased with plant growth, so, late season boll are susceptible to low soil N. This could explain why protein content in upper fruiting branches was higher than middle and lower fruiting branches (Chen et al., 2015). Therefore, adding extra $\mathrm{N}$ and allocating $\mathrm{N}$ to flowering and boll forming stage are beneficial to soil available $\mathrm{N}$ for the formation of late season bolls and it might increase the uniformity of cottonseed. Previous results also suggest adding extra potash fertilizer or allocating it for the development of late season bolls might result in longer fibers in upper fruiting branches (Yang et al., 2016). To sum up, whether adding extra nitrogen and potash fertilizer, and allocating these fertilizer to the flowering and boll formatting stage could result in both longer fiber and high seed protein quality still further results.

\section{CONCLUSION}

A set of best management practices for cotton embryonic protein, collectively known as IMS, was assembled. The combined application of the economic $\mathrm{N}$ rate, growth-driven $\mathrm{N}$ application schedule, a high plant density, and seedling raising with bioorganic matter markedly improved protein quality, especially the levels of Glu, Lys, and Met. Increased protein quality was attributed to a high level of soil-available $\mathrm{N}$ content. Soil $\mathrm{NH}_{4}^{+}$ $-\mathrm{N}$ and $\mathrm{NO}_{3}^{-}-\mathrm{N}$ transported into seed and then utilized through GS and GOGAT pathway that are beneficial for $\mathrm{N}$ assimilation in developing cotton embryos. Reduced $\mathrm{N}$ and incorporated into carbon skeletons of storage protein was determined by the rate and duration of protein accumulation. The key factor limiting the protein content was the protein accumulation rate rather than the free amino acid content. Therefore, selection of a highyielding cultivar (SIZA-3) adapted to the cotton production

\section{REFERENCES}

Alford, B. B., Liepa, G. U., and Vanbeber, A. D. (1996). Cottonseed protein: what does the future hold? Plant Foods Hum. Nutr. 49, 1-11. doi: 10.1007/bf010 92517

Baker, D. A., and Young, D. L. (2004). Economically optimal nitrogen fertilization for yield and protein in hard red spring wheat. Agron. J. 96, 656-656. doi: 10.2134/agronj2004.1160

Bertrand, J. A., Pardue, F. E., and Jenkins, T. C. (1998). Effect of ruminally protected amino acids on milk yield and composition of Jersey cows fed whole cottonseed. J. Dairy Sci. 81, 2215-2220. doi: 10.3168/jds.S0022-0302(98) 75800-X

Bondada, B. R., and Oosterhuis, D. M. (2001). Canopy photosynthesis, specific leaf weight, and yield components of cotton under varying nitrogen supply. J. Plant Nutr. 24, 469-477. doi: 10.1081/pln-100104973

Bradford, M. M. (1976). A rapid and sensitive method for the quantitation of microgram quantities of protein utilizing the principle of protein-dye binding. Anal. Biochem. 72, 248-254. doi: 10.1006/abio.1976.9999

Brunetti, N., and Hageman, R. H. (1976). Comparison of in Vivo and in Vitro assays of nitrate reductase in wheat (Triticum aestivum L.) Seedlings. Plant Physiol. 58, 583-587. doi: 10.1104/pp.58.4.583

Cai, C., Ye, W., Zhang, T., and Guo, W. (2014). Association analysis of fiber quality traits and exploration of elite alleles in Upland cotton cultivars/accessions (Gossypium hirsutum L.). J. Integr. Plant Biol. 56, 51-62. doi: 10.1111/jipb. 12124

Canovas, F. M., Canton, F. R., Garcia-Gutierrez, A., Gallardo, F., and Crespillo, R. (1998). Molecular physiology of glutamine and glutamate biosynthesis region, transplantation of seedling with bio-organic fertilizer, a high plant density (30000 plant ha $\mathrm{h}^{-1}$ ), and an economic $\mathrm{N}$ fertilization rate $\left(375 \mathrm{~kg} \mathrm{ha}^{-1}, 20 \%\right.$ applied as a basal fertilizer, $25 \%$ at the initial flowering stage, $40 \%$ at the full-bloom stage and $15 \%$ at the end of the flowering stage) are highly recommended for cotton management to promote protein quality of cotton embryos for ruminants feed (Figure 6).

\section{AUTHOR CONTRIBUTIONS}

$\mathrm{ZZ}$, YM, and $\mathrm{BC}$ conceived the idea and led the study design. HY carried out the experiment, performed analysis and wrote the paper. XZ assisted in plant sampling and laboratory analysis. WZ and YW assisted manuscript writing and editing. All authors reviewed the manuscript.

\section{FUNDING}

This study was financially supported from the China Agriculture Research System (CARS-18-20) and the Special Fund of National Public Welfare Industry (Agriculture) R \& D Program (201203096, 201303002).

\section{ACKNOWLEDGMENTS}

We really appreciate Dr. Muhammad Abid and Dr. Rizwan Zahoor for their comments on this paper. We also thank all members in Dafeng Experimental Station, Nanjing Agricultural University for their helps on cotton management.

in developing seedlings of conifers. Physiol. Plant. 103, 287-294. doi: 10.1034/j.1399-3054.1998.1030217.x

Chen, B., Yang, H., Song, W., Liu, C., Xu, J., Zhao, W., et al. (2016). Effect of N fertilization rate on soil alkali-hydrolyzable $\mathrm{N}$, subtending leaf $\mathrm{N}$ concentration, fiber yield, and quality of cotton. Crop J. (in press). doi: 10.1016/j.cj.2016.03.006

Chen, M. L., Zhao, W. Q., Meng, Y. L., Chen, B. L., Wang, Y. H., Zhou, Z. G., et al. (2015). A model for simulating the cotton (Gossypium hirsutum L.) embryo oil and protein accumulation under varying environmental conditions. Field Crops Res. 183, 79-91. doi: 10.1016/j.fcr.2015.07.011

Crawford, N. M., and Glass, A. D. M. (1998). Molecular and physiological aspects of nitrate uptake in plants. Trends Plant Sci. 3, 389-395. doi: 10.1016/S13601385(98)01311-9

Dahnke, W. C., and Johnson, G. V. (1990). “Testing soils for available nitrogen," in Soil Testing and Plant Analysis, 3rd Edn. ed. R. L. Westerman (Madison, WI: SSSA), 127-139. doi: 10.2136/sssabookser3.3ed.c6

De Ruiter, H., Schuurmans, J., and Kollöffel, C. (1986). "Amino acid efflux from seed coats and from cotyledons of developing and germinating pea seeds," in Fundamental, Ecological and Agricultural Aspects of Nitrogen Metabolism in Higher Plants, eds H. Lambers, J. J. Neeteson, and I. Stulen (Rotterdam: Springer), 123-125. doi: 10.1007/978-94-009-4356-8_17

Dong, H. Z., Li, W. J., Eneji, A. E., and Zhang, D. M. (2012). Nitrogen rate and plant density effects on yield and late-season leaf senescence of cotton raised on a saline field. Field Crops Res. 126, 137-144. doi: 10.1016/j.fcr.2011. 10.005

Egelkraut, T. M., Kissel, D. E., Cabrera, M. L., Gascho, G. J., and Adkins, W. (2004). Nitrogen concentration in cottonseed as an indicator of $\mathrm{N}$ availability. Nutr Cycl. Agroecosys. 68, 235-242. doi: 10.1023/b:fres.0000019461.39144.29 
Falkenberg, N. R., Piccinni, G., Cothren, J. T., Leskovar, D. I., and Rush, C. M. (2007). Remote sensing of biotic and abiotic stress for irrigation management of cotton. Agric. Water Manag. 87, 23-31. doi: 10.1016/j.agwat.2006.05.021

Gastal, F., and Lemaire, G. (2002). N uptake and distribution in crops: an agronomical and ecophysiological perspective. J. Exp. Bot. 53, 789-799. doi: $10.1093 /$ jexbot/53.370.789

Guan, X., Song, Q., and Chen, Z. J. (2014). Polyploidy and small RNA regulation of cotton fiber development. Trends Plant Sci. 19, 516-528. doi: 10.1016/j.tplants.2014.04.007

Hao, X., Gao, J., Han, X., Ma, Z., Merchant, A., Ju, H., et al. (2014). Effects of open-air elevated atmospheric $\mathrm{CO} 2$ concentration on yield quality of soybean (Glycine $\max$ (L.) Merr). Agri. Ecosyst. Environ. 192, 80-84. doi: 10.1016/j.agee.2014.04.002

Hendrix, D. L. (1993). Rapid extraction and analysis of nonstructural carbohydrates in plant tissues. Crop Sci. 33, 1306-1311. doi: 10.2135/cropsci1993.0011183X003300060037x

Hernandez-Sebastia, C., Marsolais, F., Saravitz, C., Israel, D., Dewey, R. E., and Huber, S. C. (2005). Free amino acid profiles suggest a possible role for asparagine in the control of storage-product accumulation in developing seeds of low- and high-protein soybean lines. J. Exp. Bot. 56, 1951-1963. doi: $10.1093 /$ jxb/eri191

Hodges, M. (2002). Enzyme redundancy and the importance of 2oxoglutarate in plant ammonium assimilation. J. Exp. Bot. 53, 905-916. doi: $10.1093 /$ jexbot/53.370.905

Hodges, M., Flesch, V., Galvez, S., and Bismuth, E. (2003). Higher plant NADP $(+)$-dependent isocitrate dehydrogenases, ammonium assimilation and NADPH production. Plant Physiol. Biochem. 41, 577-585. doi: 10.1016/s09819428(03)00062-7

Ikurior, S. A., and Fetuga, B. L. A. (1987). Composition of some recommended Nigerian commercial cottonseed varieties. Food Chem. 26, 307-314. doi: 10.1016/0308-8146(87)90072-0

Inselsbacher, E., Umana, H. N., Stange, F. C., Gorfer, M., Schüller, E., Ripka, K., et al. (2010). Short-term competition between crop plants and soil microbes for inorganic N fertilizer. Soil Biol. Biochem. 42, 360-372. doi: 10.1016/j.soilbio.2009.11.019

Ishiyama, K., Hayakawa, T., and Yamaya, T. (1998). Expression of NADHdependent glutamate synthase protein in the epidermis and exodermis of rice roots in response to the supply of ammonium ions. Planta 204, 288-294. doi: $10.1007 / \mathrm{s} 004250050258$

Javaria, S., and Khan, M. Q. (2011). Impact of integrated nutrient management on tomato yield quality and soil environment. J. Plant Nutr. 34, 140-149. doi: 10.1080/01904167.2011.531605

Katayama, K., Ito, O., Adu-Gyamfi, J. J., Rao, T. P., Dacanay, E. V., and Yoneyama, T. (1999). Effects of NPK fertilizer combinations on yield and nitrogen balance in sorghum or pigeonpea on a vertisol in the semi. Soil Sci. Plant Nutr. 45, 143-150. doi: 10.1080/00380768.1999.10409330

Kawakami, E. M., Snider, J. L., Oosterhuis, D. M., and Mozaffari, M. (2012). Physiological and yield responses of field-grown cotton to application of urea with the urease inhibitor NBPT and the nitrification inhibitor DCD. Eur. J. Agron. 43, 147-154. doi: 10.1016/j.eja.2012.06.005

Konishi, N., Ishiyama, K., Matsuoka, K., Maru, I., Hayakawa, T., Yamaya, T., et al. (2014). NADH-dependent glutamate synthase plays a crucial role in assimilating ammonium in the Arabidopsis root. Physiol. Plant. 152, 138-151. doi: $10.1111 /$ ppl.12177

Kuai, J., Chen, Y., Wang, Y., Meng, Y., Chen, B., Zhao, W., et al. (2016). Effect of water logging on carbohydrate metabolism and the quality of fiber in cotton (Gossypium hirsutum L.). Front. Plant Sci. 7:877. doi: 10.3389/fpls.2016. 00877

Kullmann, A., and Geisler, G. (1986). "Translocation of labelled nitrogen in oilseed rape," in Fundamental, Ecological and Agricultural Aspects of Nitrogen Metabolism in Higher Plants, eds H. Lambers, J. J. Neeteson, and I. Stulen (Rotterdam: Springer), 127-129. doi: 10.1007/978-94-009-4356-8_18

Lea, P. J., Kwell, R. D., Chen, F. L., and Hecht, U. (1990). Enzymes of ammonia assimilation. Methods Plant Biochem. 3, 257-276. doi: 10.1016/B978-0-12461013-2.50022-8

Li, W., Meng, Y. L., Chen, B. L., and Wang, Y. H. (2009). Effects of climatic factors on fat and total protein contents in cottonseeds. Acta Ecol. Sin. 29, 1832-1839. doi: 10.3321/j.issn:1000-0933.2009.04.025
Liu, J. R., Meng, Y. L., Chen, B. L., Zhou, Z. G., Ma, Y. N., Lv, F. J., et al. (2015). Photosynthetic characteristics of the subtending leaf and the relationships with lint yield and fiber quality in the late-planted cotton. Acta Physiol. Plant. 37, 79. doi: $10.1007 /$ s1 1738-015-1824-9

Macduff, J. H., and Hopper, M. J. (1986). Effects of root temperature on uptake of nitrate and ammonium ions by barley grown in flowing-solution culture. Plant Soil 91, 303-306. doi: 10.1007/bf02198112

Martre, P., Porter, J. R., Jamieson, P. D., and Triboï, E. (2003). Modeling grain nitrogen accumulation and protein composition to understand the sink/source regulations of nitrogen remobilization for wheat. Plant Physiol. 133, 1959-1967. doi: $10.1104 /$ pp.103.030585

Mcgraw, J. B., and Shaver, G. R. (2006). Seedling density and seedling survival in Alaskan cotton grass tussock tundra. Ecography 5, 212-217. doi: 10.1111/j.16000587.1982.tb01039.x

Meyer, M., Shirley, J., Titgemeyer, E., Park, A., and VanBaale, M. (2001). Effect of mechanical processing and fat removal on the nutritive value of cottonseed for lactating dairy cows. J. Dairy Sci. 84, 2503-2514. doi: 10.3168/jds.s00220302(01)74701-7

Muhitch, M. J. (2006). Tissue distribution and developmental patterns of $\mathrm{NADH}$ - and ferredoxin-dependent glutamate synthase activities in maize (Zea mays) kernels. Physiol. Plant. 81, 481-488. doi: 10.1034/j.1399-3054.1991. 810406.x

Mujahid, A., Abdullah, M., Barque, A. R., and Gilani, A. H. (2000). Nutritional value of cottonseeds and it's derived products. II. Free gossypol, available lysine and in vitro protein digestibility. Asian-Australas. J. Anim. Sci. 13, 356-359. doi: 10.5713/ajas.2000.356

Muro-Pastor, M. I., and Florencio, F. J. (2003). Regulation of ammonium assimilation in cyanobacteria. Plant Physiol. Biochem. 41, 595-603. doi: 10.1016/s0981-9428(03)00066-4

Muro-Pastor, M. I., Reyes, J. C., and Florencio, F. J. (2005). Ammonium assimilation in cyanobacteria. Photosynthesis Res. 83, 135-150. doi: 10.1007/s11120-004-2082-7

Ning, Z., Chen, H., Mei, H., and Zhang, T. (2014). Molecular tagging of QTLs for fiber quality and yield in the upland cotton cultivar Acala-Prema. Euphytica 195, 143-156. doi: 10.1007/s10681-013-0990-3

Potel, F., Valadier, M. H., Ferrario-Méry, S., Grandjean, O., Morin, H., Gaufichon, L., et al. (2009). Assimilation of excess ammonium into amino acids and nitrogen translocation in Arabidopsis thaliana - roles of glutamate synthases and carbamoylphosphate synthetase in leaves. FEBS J. 276, 40614076. doi: 10.1111/j.1742-4658.2009.07114.x

Prasad, P. V. V., Satyanarayana, V., Murthy, V. R. K., and Boote, K. J. (2002). Maximizing yields in rice-groundnut cropping sequence through integrated nutrient management. Field Crops Res. 75, 9-21. doi: 10.1016/S03784290(01)00214-3

Radin, J. W., Sell, C. R., and Jordan, W. R. (1975). Physiological significance of the in vivo assay for nitrate reductase in cotton seedlings. Crop Sci. 15, 710-713. doi: 10.2135/cropsci1975.0011183x001500050029x

Rafiq, M. A., Ali, A., Malik, M. A., and Hussain, M. (2010). Effect of fertilizer levels and plant densities on yield and protein contents of autumn planted maize. Pak. J. Agric. Sci. 47, 201-208.

Rochester, I. J., Peoples, M. B., and Constable, G. A. (2001). Estimation of the N fertiliser requirement of cotton grown after legume crops. Field Crops Res. 70, 43-53. doi: 10.1016/s0378-4290(00)00150-7

Rotundo, J. L., Borrás, L., and Westgate, M. E. (2011). Linking assimilate supply and seed developmental processes that determine soybean seed composition. Eur. J Agron. 35, 184-191. doi: 10.1016/j.eja.2011.05.002

Rotundo, J. L., and Westgate, M. E. (2009). Meta-analysis of environmental effects on soybean seed composition. Field Crops Res. 110, 147-156. doi: 10.1016/j.fcr.2008.07.012

Sainju, U. M., Whitehead, W. F., Singh, B. P., and Wang, S. (2006). Tillage, cover crops, and nitrogen fertilization effects on soil nitrogen and cotton and sorghum yields. Eur. J. Agron. 25, 372-382. doi: 10.1016/j.eja.2006. 07.005

Sapkota, T. B., Majumdar, K., Jat, M. L., Kumar, A., Bishnoi, D. K., Mcdonald, A. J., et al. (2014). Precision nutrient management in conservation agriculture based wheat production of Northwest India: profitability, nutrient use efficiency and environmental footprint. Field Crops Res. 155, 233-244. doi: 10.1016/j.fcr.2013.09.001 
Sawan, Z. M., Basyony, A. E., McCuistion, W. L., and El Farra, A. H. A. (1993). Effect of plant population densities and application of growth retardants on cottonseed yield and quality. J. Am. Oil Chem. Soc. 70, 313-317. doi: 10.1007/BF02545314

Sawan, Z. M., Elfarra, A. A., and Ellatif, S. A. (1988). Cottonseed, protein and oil yields, and oil properties as affected by nitrogen and phosphorus fertilization and growth regulators. J. Agron. Crop Sci. 161, 50-56. doi: 10.1111/j.1439037X.1988.tb00643.x

Sawan, Z. M., Fahmy, A. H., and Yousef, S. E. (2009). Direct and residual effects of nitrogen fertilization, foliar application of potassium and plant growth retardant on Egyptian cotton growth, seed yield, seed viability and seedling vigor. Acta Ecol. Sin. 29, 116-123. doi: 10.1016/j.chnaes.2009. 05.008

Sawan, Z. M., Hafez, S. A., and Basyony, A. E. (2001). Effect of nitrogen fertilization and foliar application of plant growth retardants and zinc on cottonseed, protein and oil yields and oil properties of cotton. J. Agron. Crop Sci. 186, 183-191. doi: 10.1046/j.1439-037X.2001.00473.x

Sawan, Z. M., Hafez, S. A., Basyony, A. E., and Alkassas, A. (2006). Cottonseed, protein, oil yields and oil properties as affected by nitrogen fertilization and foliar application of potassium and a plant growth retardant. World J. Agric. Sci. 2, 56-65. doi: 10.3989/gya.2007.v58.i1.7

Seong, R. C., Ji, H. P., Park, S. J., and Cho, J. Y. (2004). Effects of sourcesine alteration on dry matter accumulation and protein content in soybean. J. Bacteriol. 186, 3621-3630.

Singh, K., Chand, S., and Yaseen, M. (2014). Integrated nutrient management in Indian basil (Ocimum basilicum). Ind. Crop Prod. 55, 225-229. doi: 10.1016/j.indcrop.2014.02.009

Stewart, G., and Rhodes, D. (1978). Nitrogen metabolism of halophytes III. Enzymes of ammonia assimilation. New Phytol. 80, 307-316. doi: 10.1111/j.1469-8137.1978.tb01563.x

Taghizadeh, A., Mesgaran, M. D., Valizadeh, R., Shahroodi, F. E., and Stanford, K. (2005). Digestion of feed amino acids in the rumen and intestine of steers measured using a mobile nylon bag technique. J. Dairy Sci. 88, 1807-1814. doi: 10.3168/jds.s0022-0302(05)72855-1

Takahashi, M., Uematsu, Y., Kashiwaba, K., Yagasaki, K., Hajika, M., Matsunaga, R., et al. (2003). Accumulation of high levels of free amino acids in soybean seeds through integration of mutations conferring seed protein deficiency. Planta 217, 577-586. doi: 10.1007/s00425-003-1026-3

Tamura, W., Hidaka, Y., Tabuchi, M., Kojima, S., Hayakawa, T., Sato, T., et al. (2010). Reverse genetics approach to characterize a function of NADH-glutamate synthase 1 in rice plants. Amino Acids 39, 1003-1012. doi: $10.1007 /$ s00726-010-0531-5
Yang, G., Tang, H., Tong, J., Nie, Y., and Zhang, X. (2011). Effect of fertilization frequency on cotton yield and biomass accumulation. Fuel Energy Abstr. 125, 161-166. doi: 10.1016/j.fcr.2011.08.008

Yang, J., Hu, W., Zhao, W., Chen, B., Wang, Y., Zhou, Z., et al. (2016). Fruiting branch $\mathrm{K}+$ level affects cotton fiber elongation through Osmoregulation. Front. Plant Sci. 7:13. doi: 10.3389/fpls.2016.00013

Yu, J. W., Yu, S. X., Fan, S. L., Song, M. Z., Zhai, H. H., Li, X. L., et al. (2012). Mapping quantitative trait loci for cottonseed oil, protein and gossypol content in a Gossypium hirsutum x Gossypium barbadense backcross inbred line population. Euphytica 187, 191-201. doi: 10.1007/s10681-012-0630-3

Zhang, S., Wang, T., Liu, Q., Gao, X., Zhu, X., Zhang, T., et al. (2015). Quantitative trait locus analysis of boll-related traits in an intraspecific population of Gossypium hirsutum. Euphytica 203, 121-144. doi: 10.1007/s10681-014-1281-3

Zhang, Y., Zhang, Y., Liu, N., Su, D., Xue, Q., Stewart, B. A., et al. (2012). Effect of source-sink manipulation on accumulation of micronutrients and protein in wheat grains. J. Plant Nutr. Soil Sci. 60, 1026-1036. doi: 10.1002/jpln.201100224

Zhang, Z. H., Wang, W. M., Guan, C. Y., Liu, Q., Rong, X. M., Han, Y. L., et al. (2014). Mechanisms of nitrogen re-disrtibution in response to enzyme activities and the effects on nitrogen use efficiency in brassica napus during later growing stage. Pak. J. Bot. 46, 1789-1795.

Zhao, X. Q., and Shi, W. M. (2006). Expression analysis of the glutamine synthetase and glutamate synthase gene families in young rice (Oryza sativa) seedlings. Plant Sci. 170, 748-754. doi: 10.1016/j.plantsci.2005.11.006

Zhe, S., Ying, L., Hong-bao, P., and Xue-jun, G. (2014). Application of protein feed processed by microbial fermentation to dairy cow. J. Northeast Agric. Univ. 21, 39-44. doi: 10.1016/S1006-8104(14)60020-9

Zotarelli, L., Rens, L. R., Cantliffe, D. J., Stoffella, P. J., Gergela, D., and Burhans, D. (2015). Rate and timing of nitrogen fertilizer application on potato 'FL1867'. Part I: plant nitrogen uptake and soil nitrogen availability. Field Crops Res. 183 246-256. doi: 10.2134/agronj14.0193

Conflict of Interest Statement: The authors declare that the research was conducted in the absence of any commercial or financial relationships that could be construed as a potential conflict of interest.

Copyright (c) 2016 Yang, Meng, Chen, Zhang, Wang, Zhao and Zhou. This is an open-access article distributed under the terms of the Creative Commons Attribution License (CC BY). The use, distribution or reproduction in other forums is permitted, provided the original author(s) or licensor are credited and that the original publication in this journal is cited, in accordance with accepted academic practice. No use, distribution or reproduction is permitted which does not comply with these terms. 


\section{Cottonseed protein, oil, and mineral status in near-isogenic Gossypium hirsutum cotton lines expressing fuzzy/linted and fuzzless/linted seed phenotypes under field conditions}

\section{OPEN ACCESS}

Edited by:

Antonio M. De Ron, National Spanish Research Council

(CS/C), Spain

Reviewed by: Josefina Hernández Nistal, Universidad de Santiago de

Compostela, Spain Miroslav Griga,

AGRITEC Plant Research Ltd., Czech

Republic

*Correspondence:

Nacer Bellaloui,

Crop Genetics Research Unit, Research Plant Physiologist, United

States Department of Agriculture,

Agricultural Research Service, 141 Experiment Station Road, PO Box 345

Stoneville, MS 38776, USA

nacer.bellaloui@ars.usda.gov

Specialty section:

This article was submitted to Crop Science and Horticulture, a section of the journal Frontiers in Plant Science

Received: 12 January 2015

Accepted: 20 February 2015

Published: 19 March 2015

Citation:

Bellaloui N, Stetina SR and Turley RB

(2015) Cottonseed protein, oil, and mineral status in near-isogenic

Gossypium hirsutum cotton lines expressing fuzzy/linted and fuzzless/linted seed phenotypes under field conditions.

Front. Plant Sci. 6:137. doi: 10.3389/fp/s.2015.00137

\author{
Nacer Bellaloui*, Salliana R. Stetina and Rickie B. Turley \\ Crop Genetics Research Unit, Plant Physiology, United States Department of Agriculture, Agricultural Research Service, \\ Stoneville, MS, USA
}

Cotton is an important crop in the world and is a major source of oil for human consumption and cotton meal for livestock. Cottonseed nutrition (seed composition: protein, oil, and minerals) determines the quality of seeds. Therefore, maintaining optimum levels of cottonseed nutrition is critical. Physiological and genetic mechanisms controlling the levels of these constituents in cottonseed are still largely unknown. Our previous research conducted under greenhouse conditions showed that seed and leaf nutrition differed between fuzzless and fuzzy seed isolines. Therefore, the objective of this research was to investigate the seed fuzz phenotype (trait) effects on seed protein, oil, N, C, S, and minerals in five sets of near-isogenic mutant cotton lines for seed fuzz in a 2-year experiment under field condition to evaluate the stability of the effect of the trait on seed nutrition. The isolines (genotypes) in each set differ for the seed fuzz trait (fuzzless/linted seed line, $N$ lines, and fuzzy/linted seed line, $F$ lines). Results showed that seed protein was higher in the fuzzy genotype in all sets, but seed oil was higher in fuzzless genotype in all sets. The concentrations of seed $\mathrm{Ca}$ and $\mathrm{C}$ were higher in all fuzzless genotypes, but N, S, B, Fe, and Zn were higher in most of the fuzzy genotypes. Generally, minerals were higher in leaves of $F$ lines, suggesting the translocation of minerals from leaves to seeds was limited. The research demonstrated that fiber development could be involved in cottonseed composition. This may be due to the involvement of fiber development in carbon and nitrogen metabolism, and the mobility of nutrients from leaves (source) to seed (sink). This information is beneficial to breeders to consider fuzzless cottonseed for potential protein and oil use and select for higher oil or higher protein content, and to physiologists to further understand the mobility of minerals to increase the quality of cottonseed nutrition for food and feed.

Keywords: cottonseed protein, cottonseed oil, cottonseed composition, minerals, near-isogenic cotton, cotton mutants 


\section{Introduction}

Cotton is a major crop in the world (Yu et al., 2012). Cotton fiber is a source of natural textile, and cottonseed is a source of oil for human consumption, cotton meal and minerals for livestock feed (Yu et al., 2012; He et al., 2013). Therefore, maintaining high quality fiber and cottonseed nutritional value is critical. There are four cultivated cotton species, Gossypium hirsutum (about $95 \%$ of the cultivated cotton), G. barbadense, G. arboreum, and G. herbaceum together (the last three represent about 5\%) (Turley et al., 2007; Padmalatha et al., 2012; Bellaloui and Turley, 2013; Stetina et al., 2014). Cotton fibers are developed from the ovule epidermis and are single-celled seed trichomes, and about $30 \%$ of the seed epidermal cells differentiate into spinable fibers (Arpat et al., 2004; Wilkins and Arpat, 2005). The development of cotton fibers comprises four stages named fiber cell initiation, fiber cell elongation/primary cell wall (occurs up to 20 days postanthesis), fiber cell synthesis/secondary cell wall (occurs between 5 and 15 days post-anthesis, and the secondary cell wall synthesis begins at about 20 days post-anthesis and reaches up to 45 days post-anthesis), and maturation (occurs from 45 to 50 days postanthesis) where fibers dehydrate and produce mature cotton lint (Ji et al., 2003; Arpat et al., 2004; Wilkins and Arpat, 2005).

Upland cotton (Gossypium hirsutum) is the predominant species commercially cultivated in the United States and produces two types of fiber on cottonseed, lint and fuzz. Lint fiber is economically valuable, and it is longer and develops faster than the fuzz (Stewart, 1975; Seagull and Giavalis, 2004; Stetina et al., 2014). During ginning process, lint fiber is removed and fuzz fiber left behind (Bechere et al., 2009). There are two naturally occurring mutations in cotton allowing the development of lint fiber, but preventing development of fuzz fiber, and these are referred to as the fuzzless seed alleles and designated as genotypes $N_{1-}$ (dominant fuzzless allele) or $n_{2} n_{2}$ (recessive fuzzless allele) (Turley and Kloth, 2002; Turley et al., 2007; Stetina et al., 2014).

It is reported that the fuzzless trait in cottonseed is a novel tool to use to understand the biology, genetics, and biochemical and metabolic processes (Turley et al., 2007; Padmalatha et al., 2012). In spite of the few reports available on the effects of fuzzless trait on the biology, genetics, and molecular biology (Padmalatha et al., 2012), very limited information is available on the effects of fuzzless trait on cottonseed composition (Yu et al., 2012; Bellaloui and Turley, 2013; He et al., 2013). For example, the effect of fuzzless trait was investigated on genetic products (Turley and Kloth, 2002; Gou et al., 2007; Turley et al., 2007; Zhao et al., 2009; Pang et al., 2010a,b; Liu et al., 2012), physiological (Ruan et al., 2003; Shi et al., 2006; Yang et al., 2006; Wang et al., 2010; Wang and Ruan, 2010; Zhang et al., 2011), and molecular (Machado et al., 2009; Guan et al., 2011; Walford et al., 2011). It was also reported that carbohydrate and energy metabolisms are involved in fiber development and carbon skeletons for the synthesis of cell wall polysaccharides and fatty acids (Gou et al., 2007; Yang et al., 2008; Pang et al., 2010a,b), and actin cytoskeleton to trigger the secondary cell wall synthesis (Li et al., 2002, 2005; Wang et al., 2010; Wang and Ruan, 2010).

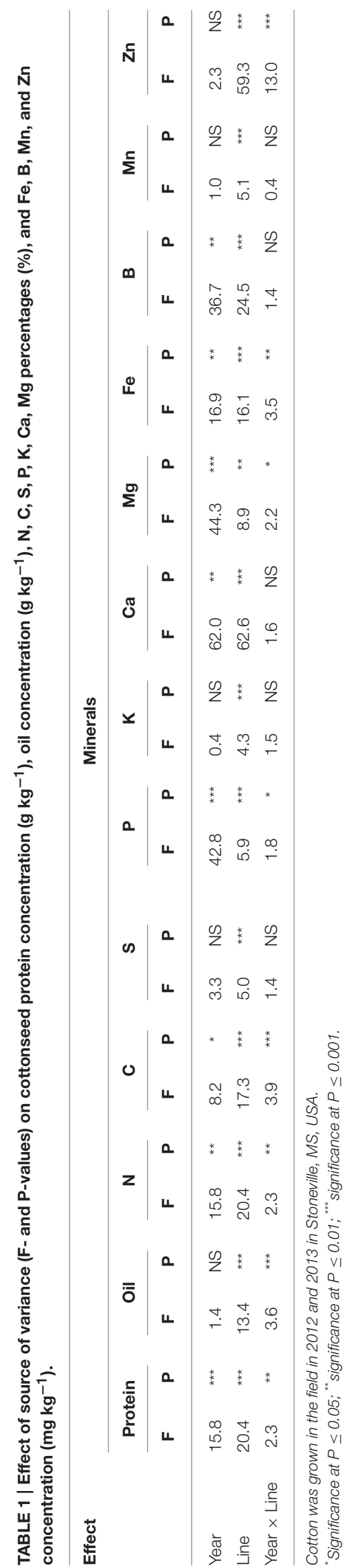




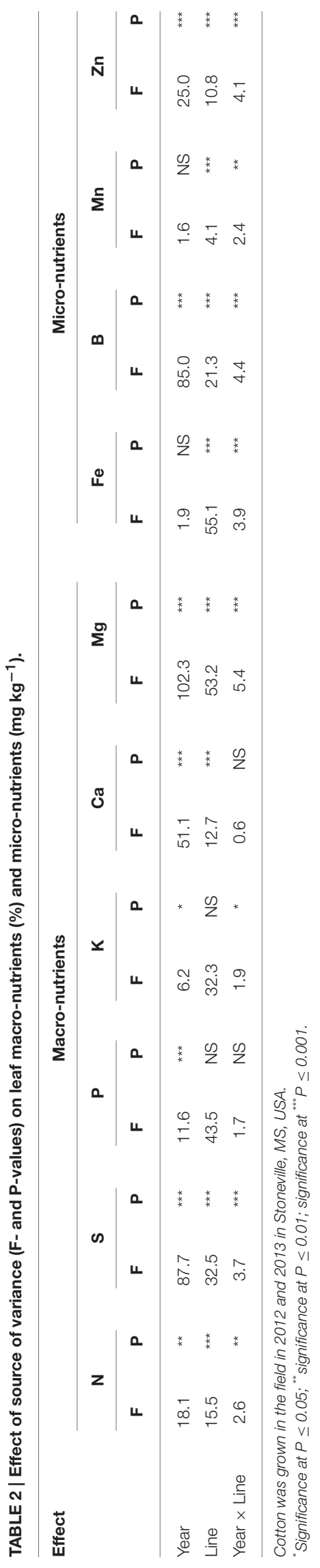

Cottonseed quality is also determined by its content of mineral and non-mineral nutrients such as $\mathrm{N}, \mathrm{C}, \mathrm{S}, \mathrm{K}, \mathrm{Ca}, \mathrm{Zn}$, and $\mathrm{Fe}$ because of their direct or indirect contribution to: protein synthesis (such as $\mathrm{N}, \mathrm{K}, \mathrm{S}$ ); oil (such as $\mathrm{C}$ and $\mathrm{N}$ ); carbohydrates (such as $\mathrm{C}, \mathrm{K}, \mathrm{B}$ ); metabolite synthesis $(\mathrm{Cu}, \mathrm{Zn}$, $\mathrm{Fe}, \mathrm{Mg}$ ); integrity of cell membrane and cell wall structure (Ca and B); cell membrane, lipid synthesis, energy transfer and phosphorylation reactions, carbohydrate metabolism, and nutrient active uptake processes (P); and osmoregulation, stomatal closure, carbohydrate movement, and nutrient mobility (K). The physiological and biochemical roles of these nutrients in plant growth and development were previously reported (Mengel and Kirkby, 1982; Marschner, 2012). The benefit of minerals to human health was also previously reported. For example, an unbalanced diet of micronutrients such as Fe, Zn, leads to human malnutrition (Samman et al., 1998; Fletcher et al., 2004; Lu et al., 2008). Therefore, maintaining an optimum level of Fe, Zn, Cu, Mo, Mn (Zhang et al., 2004; Heinemann et al., 2005) in seeds of major crops that are used for protein and oil sources is critical. It was reported that nutrient uptake, translocation, redistribution, and accumulation are processes controlling the concentrations of minerals in seeds (Grusak and DellaPenna, 1999; White and Broadley, 2005), and most of the genetic basis of these process are not known (Ding et al., 2010).

To our knowledge this is the first comprehensive report to use near-isogenic cotton mutant genotypes for the fuzzless trait to evaluate its effects on cottonseed nutrition (protein, oil, $\mathrm{N}, \mathrm{S}, \mathrm{C}$, and minerals) under field conditions. Our hypothesis was that because several metabolic processes including carbon metabolism and polysaccharide cell wall synthesis are involved in fiber development (Li et al., 2002, 2005; Gou et al., 2007; Yang et al., 2008; Wang et al., 2010; Wang and Ruan, 2010; Pang et al., 2010a,b), cottonseed nutrition will be influenced as protein and oil are results of carbon, nitrogen, and carbohydrate metabolism. Since we observed differences in leaf and seed nutrients between lines, we hypothesized that nutrients in lint fiber may be impacted as well. Therefore, we investigated the effect of fuzz trait on nutrient content in lint for 1 year only to obtain preliminary results for future research planning.

\section{Materials and Methods}

\section{Germplasm Development}

The development of near-isogenic lines was described in detail elsewhere (Stetina et al., 2014). The near-isogenic lines (NILs), one expressing the fuzzy/linted and one expressing fuzzless/linted phenotype were developed in five upland cotton backgrounds (Stetina et al., 2014). The following are five sets of $F$ and $N$ lines/genotypes: Sure-Grow (SG) $747 \mathrm{~F}$ vs. SG $747 \mathrm{~N}$; MD51ne $F$ vs. MD51ne N; STV 7Agl F vs. STV 7 A $g l N$; DP $5690 F$ vs. DP 5690 N; DES 119 F vs. DES 119 N. Genotype SA 243 ("Ballard naked seed," PI 528610) was the fuzzless parent with the dominant fuzzless seed allele, $N_{1} N_{1}$ (Turley et al., 2007), and DP 444 BG/RR (Monsanto Company, St. Louis, MO, USA) was used as the commercial check. 
TABLE 3 | Effect of fuzzless/linted $(N)$ and fuzzy/linted $(F)$ cottonseed phenotypes on cottonseed protein concentration ( $\mathrm{g} \mathrm{kg}^{-1}$ ), oil concentration ( $\mathrm{g}$ $\mathrm{kg}^{-1}$ ), N, C, S, P, K, Ca, Mg percentages (\%), and Fe, B, Mn, and Zn concentration ( $\mathrm{mg} \mathrm{kg}^{-1}$ ).

\begin{tabular}{|c|c|c|c|c|c|c|c|c|c|c|c|c|c|}
\hline Line/genotype & Protein & Oil & C & $\mathbf{N}$ & $\mathbf{S}$ & Ca & $\mathbf{K}$ & Mg & $\mathbf{P}$ & B & $\mathrm{Fe}$ & Mn & $\mathrm{Zn}$ \\
\hline SA 243 & 226 ef & 270 ef & $51.3 \mathrm{~d}$ & $3.27 \mathrm{~g}$ & $0.26 \mathrm{e}$ & $0.13 f$ & 0.94 e & $0.34 \mathrm{~g}$ & $0.56 \mathrm{~g}$ & $10.0 \mathrm{f}$ & $44.3 \mathrm{e}$ & $13.4 \mathrm{c}$ & $29.9 \mathrm{e}$ \\
\hline DP 444 BG/RR & $287 a$ & $282 \mathrm{~cd}$ & $51.3 \mathrm{~d}$ & $4.10 \mathrm{bc}$ & $0.34 \mathrm{c}$ & 0.14 e & 1.03 bc & $0.40 \mathrm{~b}$ & $0.64 \mathrm{bc}$ & $12.8 \mathrm{a}$ & $52.8 \mathrm{~b}$ & $13.3 \mathrm{~cd}$ & $56.9 \mathrm{c}$ \\
\hline DES $119 N$ & $240 d$ & $293 b$ & $52.8 \mathrm{~b}$ & 3.48 ef & $0.30 \mathrm{~d}$ & $0.18 \mathrm{a}$ & $0.95 \mathrm{e}$ & $0.41 b$ & $0.65 \mathrm{~b}$ & $11.7 \mathrm{~b}$ & $49.0 \mathrm{c}$ & $14.0 \mathrm{~b}$ & $33.9 \mathrm{e}$ \\
\hline DES $119 F$ & $276 b$ & $260 \mathrm{fg}$ & $50.8 \mathrm{e}$ & $4.32 \mathrm{a}$ & $0.40 \mathrm{a}$ & $0.12 \mathrm{~g}$ & $1.05 b$ & $0.43 \mathrm{a}$ & $0.72 \mathrm{a}$ & $12.8 \mathrm{a}$ & $62.4 \mathrm{a}$ & $14.6 \mathrm{a}$ & $81.7 \mathrm{a}$ \\
\hline DP $5690 N$ & $217 \mathrm{~g}$ & $323 a$ & $53.3 \mathrm{a}$ & $3.25 \mathrm{~g}$ & $0.27 \mathrm{e}$ & $0.15 \mathrm{c}$ & $0.91 \mathrm{f}$ & $0.35 \mathrm{f}$ & $0.55 \mathrm{~g}$ & $9.3 \mathrm{~g}$ & $39.9 \mathrm{~g}$ & $12.2 \mathrm{f}$ & $25.9 f$ \\
\hline DP $5690 F$ & $252 c$ & $275 \mathrm{fg}$ & $50.9 e$ & $3.95 \mathrm{c}$ & $0.37 \mathrm{~b}$ & $0.11 \mathrm{~h}$ & $0.99 d$ & $0.38 \mathrm{~cd}$ & $0.59 \mathrm{f}$ & $11.6 \mathrm{~b}$ & $48.7 \mathrm{~cd}$ & $11.5 \mathrm{~g}$ & $76.8 \mathrm{~b}$ \\
\hline MD 51ne N & $221 \mathrm{fg}$ & $274 \mathrm{fg}$ & 52.2 c & $3.27 \mathrm{~g}$ & $0.26 \mathrm{e}$ & $0.18 \mathrm{a}$ & 0.95 e & $0.37 \mathrm{e}$ & 0.59 ef & $10.7 \mathrm{~d}$ & $44.6 \mathrm{e}$ & $13.6 \mathrm{c}$ & $30.8 \mathrm{e}$ \\
\hline MD 51ne $F$ & $274 \mathrm{~b}$ & $257 \mathrm{gh}$ & 50.9 e & $4.15 a b$ & $0.35 \mathrm{bc}$ & $0.14 d$ & $1.05 \mathrm{bc}$ & $0.39 c$ & $0.62 \mathrm{~cd}$ & $11.7 \mathrm{~b}$ & $50.6 \mathrm{c}$ & $12.2 \mathrm{f}$ & $75.6 \mathrm{~b}$ \\
\hline SG $747 N$ & 229 e & 288 bc & $52.1 \mathrm{c}$ & $3.31 \mathrm{fg}$ & $0.26 \mathrm{e}$ & $0.17 b$ & 0.94 e & 0.37 de & $0.61 \mathrm{de}$ & $11.0 \mathrm{c}$ & $42.2 \mathrm{f}$ & $12.9 \mathrm{de}$ & $31.2 \mathrm{e}$ \\
\hline SG $747 F$ & $285 a$ & $250 \mathrm{~h}$ & $50.3 \mathrm{~g}$ & $4.11 \mathrm{bc}$ & $0.31 d$ & $0.12 \mathrm{fg}$ & 1.02 c & $0.38 \mathrm{c}$ & $0.65 \mathrm{bc}$ & $12.9 \mathrm{a}$ & $50.1 \mathrm{c}$ & $12.7 \mathrm{e}$ & $50.7 \mathrm{~d}$ \\
\hline STV $7 A g / N$ & $197 \mathrm{~h}$ & 264 e & $52.7 \mathrm{~b}$ & 3.57 de & $0.26 \mathrm{e}$ & $0.15 \mathrm{c}$ & 0.95 e & $0.38 \mathrm{~cd}$ & 0.59 ef & 10.4 e & $39.3 \mathrm{~g}$ & $11.3 \mathrm{~g}$ & 30.0 ef \\
\hline STV $7 \mathrm{Ag} / \mathrm{F}$ & $255 c$ & $234 \mathrm{i}$ & $50.5 f$ & $3.72 \mathrm{~d}$ & $0.30 \mathrm{~d}$ & $0.11 \mathrm{~h}$ & $1.10 \mathrm{a}$ & $0.40 \mathrm{~b}$ & $0.65 \mathrm{~b}$ & $11.8 \mathrm{~b}$ & $47.0 \mathrm{~d}$ & $11.4 \mathrm{~g}$ & $55.9 \mathrm{c}$ \\
\hline
\end{tabular}

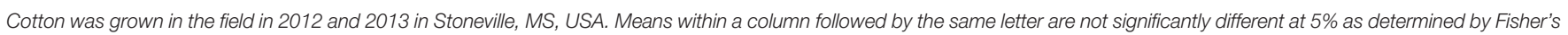
LSD test.

TABLE 4 | Effect of fuzzless/linted $(N)$ and fuzzy/linted $(F)$ cottonseed phenotypes on leaf $\mathrm{Ca}, \mathrm{K}, \mathrm{Mg}, \mathrm{P}, \mathrm{N}$, and $\mathrm{S}$ percentages (\%), and $\mathrm{B}, \mathrm{Fe} \mathrm{Mn}$, and Zn concentration $\left(\mathrm{mg} \mathrm{kg}^{-1}\right)$.

\begin{tabular}{|c|c|c|c|c|c|c|c|c|c|c|}
\hline Line/genotype & $\mathrm{Ca}$ & $\mathbf{K}$ & Mg & $\mathbf{P}$ & $\mathbf{N}$ & $\mathbf{S}$ & B & $\mathrm{Fe}$ & Mn & $\mathrm{Zn}$ \\
\hline SA 243 & 3.39 de & $2.01 \mathrm{f}$ & $1.09 \mathrm{c}$ & $0.42 \mathrm{~g}$ & $4.23 \mathrm{~d}$ & $0.96 \mathrm{e}$ & 40.9 e & $78.6 \mathrm{~g}$ & $166.6 \mathrm{~cd}$ & $37.4 \mathrm{~d}$ \\
\hline DP 444 BG/RR & $3.36 \mathrm{e}$ & $1.88 \mathrm{~g}$ & $0.95 d$ & $0.39 \mathrm{~h}$ & $3.84 \mathrm{f}$ & $0.84 \mathrm{f}$ & $40.2 \mathrm{e}$ & $77.6 \mathrm{~g}$ & $158.3 f$ & $37.4 \mathrm{~cd}$ \\
\hline DES $119 \mathrm{~N}$ & $4.31 \mathrm{~b}$ & $3.48 \mathrm{~b}$ & $1.22 \mathrm{~b}$ & $0.75 \mathrm{a}$ & $4.41 \mathrm{C}$ & $1.61 \mathrm{~b}$ & $51.9 \mathrm{~b}$ & $120.2 \mathrm{~b}$ & $170.8 \mathrm{~b}$ & $42.4 \mathrm{a}$ \\
\hline DES $119 F$ & $3.09 \mathrm{f}$ & $2.38 \mathrm{e}$ & 0.78 e & $0.58 d$ & $3.40 \mathrm{~g}$ & $0.96 \mathrm{e}$ & 40.2 e & $90.1 \mathrm{de}$ & $172.8 \mathrm{ab}$ & 30.8 e \\
\hline DP $5690 N$ & $3.58 \mathrm{~cd}$ & $2.78 \mathrm{c}$ & $1.00 \mathrm{~d}$ & 0.65 c & $4.04 \mathrm{c}$ & $1.70 \mathrm{a}$ & $48.0 \mathrm{c}$ & $91.3 \mathrm{~d}$ & $173.3 \mathrm{a}$ & 39.0 b \\
\hline DP $5690 F$ & $3.68 \mathrm{c}$ & $1.70 \mathrm{~h}$ & $0.67 \mathrm{f}$ & $0.65 \mathrm{c}$ & $3.80 \mathrm{c}$ & $1.31 \mathrm{~d}$ & $41.3 \mathrm{e}$ & $87.2 \mathrm{e}$ & $167.8 \mathrm{c}$ & $37.4 \mathrm{~d}$ \\
\hline MD 51ne N & $4.70 \mathrm{a}$ & $2.84 \mathrm{c}$ & $1.68 \mathrm{a}$ & $0.70 \mathrm{~b}$ & $4.90 \mathrm{~b}$ & $1.54 \mathrm{~b}$ & $55.1 \mathrm{a}$ & $141.0 \mathrm{a}$ & $162.3 \mathrm{e}$ & $42.3 \mathrm{a}$ \\
\hline MD 51ne $F$ & 3.66 c & $1.55 \mathrm{i}$ & $0.98 d$ & $0.51 \mathrm{f}$ & $3.80 \mathrm{f}$ & $0.97 \mathrm{e}$ & $43.3 \mathrm{~d}$ & $117.2 \mathrm{~b}$ & 162.0 e & $31.6 \mathrm{e}$ \\
\hline SG $747 N$ & $4.45 b$ & $2.61 d$ & $1.61 \mathrm{a}$ & $0.64 \mathrm{c}$ & $4.60 \mathrm{~b}$ & $1.42 \mathrm{c}$ & $50.7 \mathrm{~b}$ & 119.7 b & $165.1 \mathrm{~d}$ & $43.5 \mathrm{a}$ \\
\hline SG $747 F$ & 3.38 e & 1.60 hi & $0.93 d$ & $0.43 \mathrm{~g}$ & $3.81 \mathrm{f}$ & 0.89 ef & 40.3 e & $82.0 \mathrm{f}$ & $167.9 \mathrm{c}$ & $36.7 \mathrm{~d}$ \\
\hline STV $7 A g / N$ & $4.88 \mathrm{a}$ & $3.81 \mathrm{a}$ & 1.22 b & $0.75 \mathrm{a}$ & $4.81 \mathrm{a}$ & $1.38 \mathrm{~cd}$ & $50.3 \mathrm{~b}$ & $139.4 \mathrm{a}$ & $162.5 \mathrm{e}$ & $37.2 \mathrm{~d}$ \\
\hline STV $7 A g / F$ & 3.40 de & $2.48 \mathrm{e}$ & $0.75 \mathrm{e}$ & $0.55 \mathrm{e}$ & $2.98 \mathrm{~h}$ & $0.85 f$ & $40.2 \mathrm{f}$ & 107.2 c & $162.5 \mathrm{e}$ & $38.8 \mathrm{bc}$ \\
\hline
\end{tabular}

Cotton was grown in the field in 2012 and 2013 in Stoneville, MS, USA.

Means within a column followed by the same letter are not significantly different at $5 \%$ as determined by Fisher's LSD test.

\section{Field Management and Growth Conditions}

A field experiment was conducted in 2012 and 2013 at Stoneville, MS, USA. Field management and growth conditions were previously detailed in Stetina et al. (2014). Briefly, cottonseeds were planted on four-row plots spaced $1.02 \mathrm{~m}$ apart and each plot was $9.14 \mathrm{~m}$ long with a $3.04 \mathrm{~m}$ alley between plots. Planting dates were 25 April 2012 and 13 May 2013. Insecticides and fungicides were applied to control pest and diseases. Field management was conducted according to the standard agronomic practices for cotton production in the Mississippi Delta region (http://msucares.com/crops/cotton/index.html). Cotton bolls were harvested from 10 adjacent plants at three (2012) and four (2013) intervals to prevent the loss of fuzzless cottonseed content due to rain and wind. The harvest intervals were on 28 August, 6 September, and 27 September in 2012, and on 5 September, 12 September, 17 September, and 27 September in
2013. A defoliant was used prior to the last harvest date each year. Cottonseed samples were processed at the USDA ARS Cotton Ginning Laboratory at Stoneville, MS, USA and saw-ginned on 20 November 2012 and 12 November 2013. Cottonseed were collected and acid-delinted for seed composition analyses. Soil samples were taken across the field by dividing the field into four main sections and taking 20-25 samples from each section at depth of $30.5 \mathrm{~cm}$. Therefore, one composite sample (combined 20-25 samples) represented each section. Analysis of the four samples, representing the four sections, showed uniformity in main soil nutrients. Therefore, the soil nutrients shown in here are average of the four sections representing the entire field. For leaf sampling, the most recent fully expanded leaves were taken from each plot. Fourteen leaves were taken from the middle two rows (7 leaves were taken along each one middle row to ensure equal distribution of leaves along each row). Leaves were 
oven-dried at $65^{\circ} \mathrm{C}$ and ground into fine particle with Laboratory Mill 3600 (Perten, Springfield, IL). Seed and leaf C, N, K, Ca, Mn, and $\mathrm{Zn}$ were analyzed at the University of Georgia's Soil, Plant, and Water Laboratory, Athens, GA. Seed protein, oil, B, Fe, P, and leaf $\mathrm{B}, \mathrm{Fe}$, and $\mathrm{P}$ were analyzed as described below.

\section{Boron Analysis}

Boron concentrations in the most recently fully expanded leaves at boll stage and mature seeds were determined according to Lohse (1982) using the azomethine- $\mathrm{H}$ method, and samples were prepared according to John et al. (1975). Boron concentration was determined spectrophotometrically by reading the samples at $420 \mathrm{~nm}$ using a Beckman Coulter DU 800 spectrophotometer (Fullerton, CA). The concentration of B was measured after color development, and B concentration was expressed as $\mathrm{mg} \mathrm{B} \mathrm{kg}^{-1} \mathrm{dwt}$.

\section{Iron Analysis}

Iron concentrations in the most recently fully expanded leaves at boll stage and mature seeds were measured according to Bandemer and Schaible (1944) and Loeppert and Inskeep (1996). The concentration was determined by acid wet digestion (Analytical Methods Committee, 1959), extraction, and reaction of the

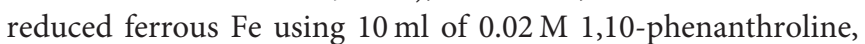
and the samples were prepared for measurement using a quinol solution of $1 \%(\mathrm{w} / \mathrm{v})$ reagent. Concentrations of $\mathrm{Fe}$ in leaves and seeds were measured spectrophotometrically at $510 \mathrm{~nm}$ using a Beckman Coulter DU 800 spectrophotometer (Fullerton, $\mathrm{CA}$ ).iron was expressed as $\mathrm{mg} \mathrm{Fe} \mathrm{kg}^{-1} \mathrm{dwt}$.

\section{Phosphorus Analysis}

Phosphorus concentrations in leaves and mature seeds were determined according to Cavell (1955) using the yellow phosphor-vanado-molybdate complex. Briefly, $2 \mathrm{~g}$ of dried ground samples were ashed and $10 \mathrm{ml}$ of $6 \mathrm{M} \mathrm{HCl}$ was added. The samples, then were placed in a water bath to evaporate the solution to dryness. Then, a $2 \mathrm{ml}$ of $36 \% \mathrm{v} / \mathrm{v} \mathrm{HCl}$ was added and the samples were boiled. Ten milliliters of distilled water was added to the samples and the solution was brought to boil for a few seconds, and then diluted to $50 \mathrm{ml}$ with distilled water, and then filtered. To measure $\mathrm{P}$ concentration, a reagent of $5 \mathrm{ml}$ of $5 \mathrm{M} \mathrm{HCl}$ and $5 \mathrm{ml}$ of ammonium molybdate-ammonium metavanadate was freshly prepared and added to the filtrate. The concentration of $\mathrm{P}$ was measured spectrophotometrically using a Beckman Coulter DU 800 spectrophotometer at $400 \mathrm{~nm}$. The concentration of $\mathrm{P}$ in the sample was expressed as a percentage.

\section{Analyses of N, S, C in Leaves and Seed}

The most recently fully expanded leaves during boll stage and mature seeds were analyzed for minerals, N, S, and C concentrations by digesting $0.6 \mathrm{~g}$ of dried, ground seed in $\mathrm{HNO}_{3}$ in a microwave digestion system. The concentrations of minerals in the samples were determined using inductively coupled plasma spectrometry (ICP) (Bellaloui and Turley, 2013; Bellaloui et al., 2014). For N, S, and C measurements, a 0.25 g ground-dried sample was combusted in an oxygen atmosphere at $1350^{\circ} \mathrm{C}$ to convert elemental N, S, and N, S, and C were measured by an elemental

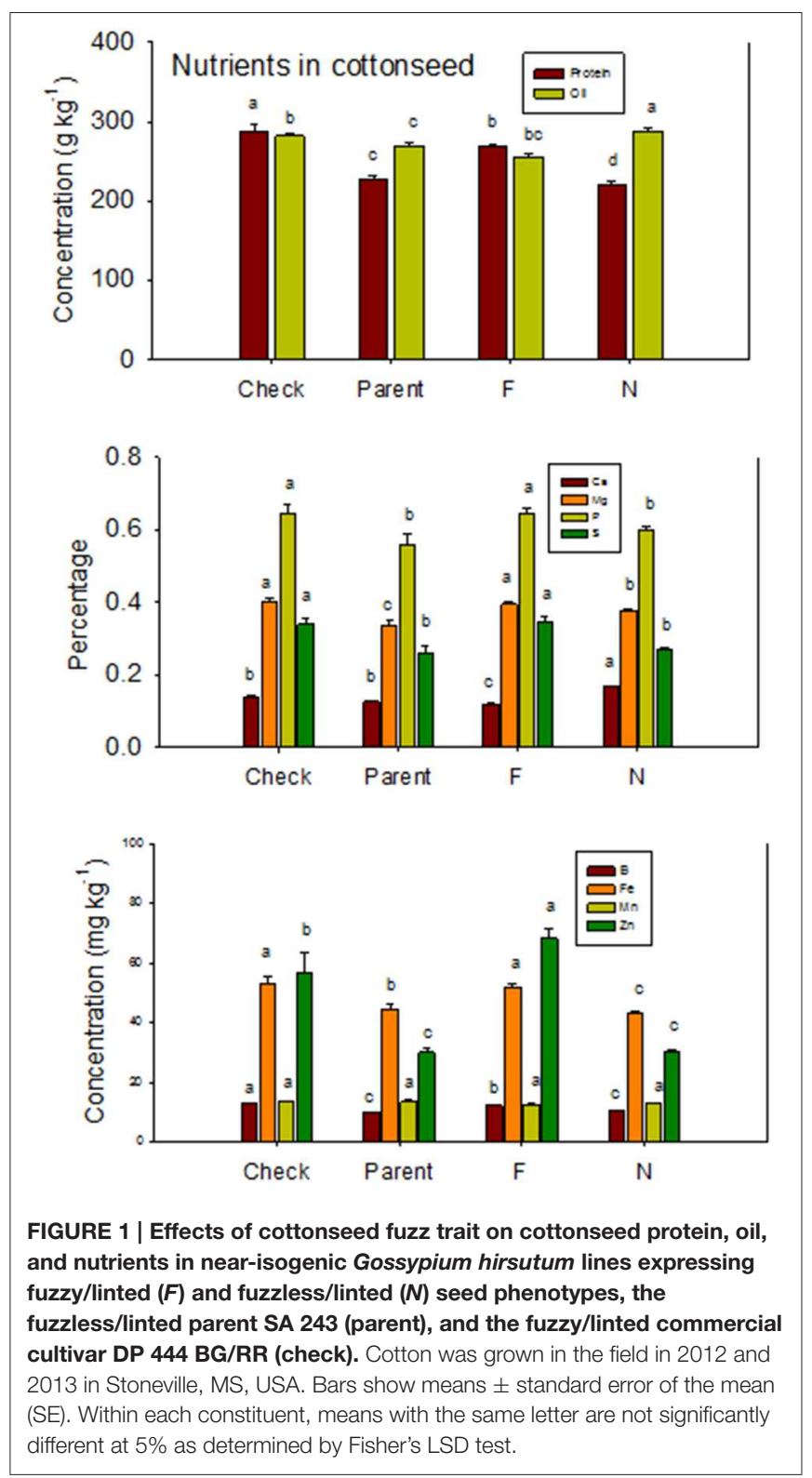

analyzer using thermal conductivity cells (LECOCNS-2000 Elemental Analyzer, LECO Corporation, St. Joseph, MI) (Bellaloui et al., 2011, 2014).

\section{Analyses of Minerals, N, S, and C in Soil}

Mineral concentrations in soil, N, S, and C were analyzed at The University of Georgia's Soil, Plant, and Water Laboratory, Athens, GA. The concentrations of the minerals $\mathrm{K}$ and Mn were determined using a 5-g soil: $20 \mathrm{ml}$ Mehlich-1 solution and analyzed using inductively coupled plasma (ICP) spectrometry (Bellaloui et al., 2009). Percentages of $\mathrm{N}, \mathrm{S}$, and $\mathrm{C}$ were determined in a 0.25 -g sample of soil by combusting samples in an oxygen atmosphere at $1350^{\circ} \mathrm{C}$, and converting elemental $\mathrm{N}, \mathrm{S}$, and $\mathrm{C}$ into $\mathrm{N}_{2}, \mathrm{SO}_{2}$, and $\mathrm{CO}_{2}$ gasses, and $\mathrm{N}, \mathrm{S}$, and $\mathrm{C}$ were determined by elemental analyzer using thermal conductivity cells (LECOCNS2000 Elemental Analyzer LECO Corporation, St. Joseph, MI, 

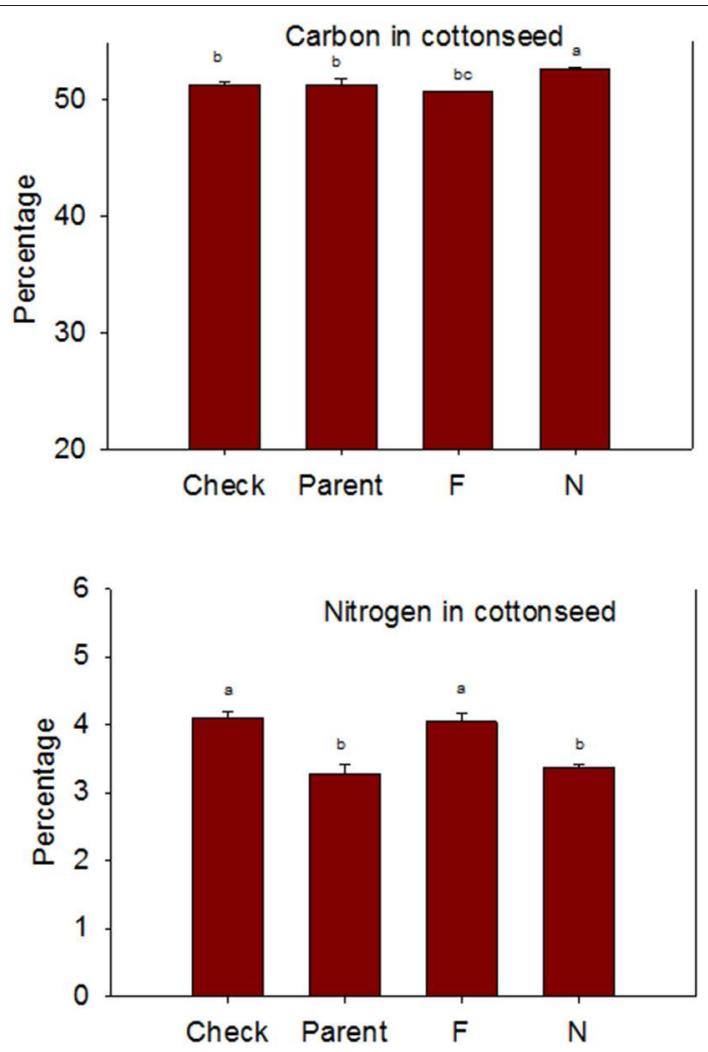

FIGURE 2 | Effects of cottonseed fuzz trait on cottonseed carbon (C) and nitrogen $(\mathrm{N})$ in near-isogenic Gossypium hirsutum lines expressing fuzzy/linted $(F)$ and fuzzless/linted $(N)$ seed phenotypes, the

fuzzless/linted parent SA 243 (parent), and the fuzzy/linted commercial cultivar DP 444 BG/RR (check). Cotton was grown in the field in 2012 and 2013 in Stoneville, MS, USA. Bars show means \pm standard error of the mean (SE). Within each constituent, means with the same letter are not significantly different at $5 \%$ as determined by Fisher's LSD test.

USA) (Bellaloui et al., 2009). Concentrations of N, S, and C were expressed as percentages.

\section{Cottonseed Protein and Oil Analysis}

Mature cottonseeds were collected from each plot and analyzed for protein and oil. Briefly, approximately $25 \mathrm{~g}$ of seed was ground using a Laboratory Mill 3600 (Perten, Springfield, IL). Protein and oil in cottonseed were analyzed by near infrared reflectance according to Wilcox and Shibles (2001) and Bellaloui and Turley (2013) using a diode array feed analyzer AD 7200 (Perten, Springfield, IL). Calibrations were developed using Perten's Thermo Galactic Grams PLS IQ software, and the calibration equation was established according to AOAC methods (Association of Official Analytical Chemists (AOAC), 1990a,b). Cottonseed protein and oil were expressed on a seed dry matter basis (Wilcox and Shibles, 2001; Boydak et al., 2002; Bellaloui and Turley, 2013).

\section{Experimental Design and Data Analysis}

This experiment was a part of a large experiment that was designed in a split-plot. The main plot was line (genotype),
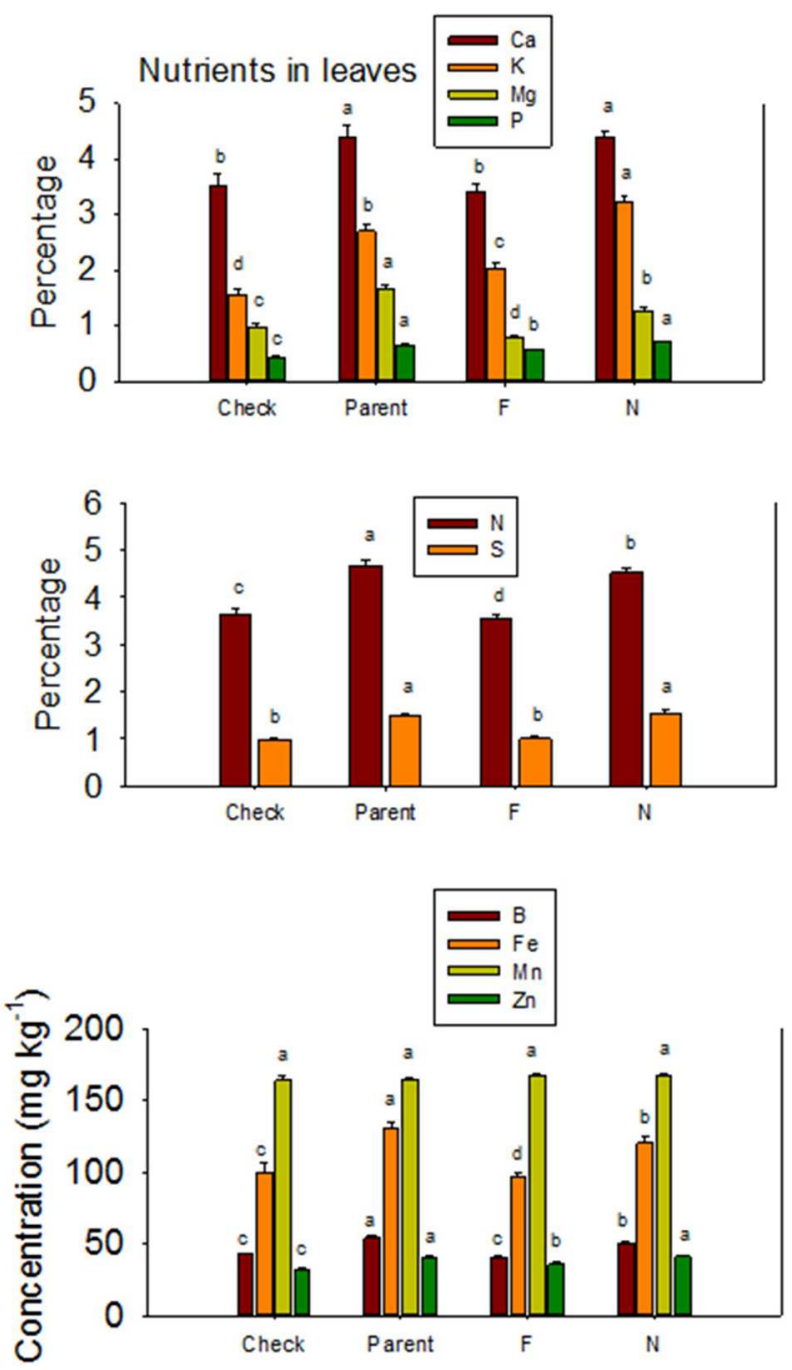

FIGURE 3 | Effects of cottonseed fuzz trait on cotton leaf nutrients in near-isogenic Gossypium hirsutum lines expressing fuzzy/linted $(F)$ and fuzzless/linted $(N)$ seed phenotypes, the fuzzless/linted parent SA 243 (parent), and the fuzzy/linted commercial cultivar DP 444 BG/RR (check). Cotton was grown in the field in 2012 and 2013 in Stoneville, MS, USA. Bars show means \pm standard error of the mean (SE). Within each constituent, means with the same letter are not significantly different at $5 \%$ as determined by Fisher's LSD test.

and plots were arranged in a randomized complete block design with 4 replications. The subplot was ginning method (Stetina et al., 2014). In the current study, we were interested in the conventional saw ginning method only. Therefore, the only factors under the study were line, year, and their interactions. Analysis of variance was conducted using Proc Mixed model in SAS (Statistical Analysis System; SAS Institute, Inc., Cary, NC, 2002-2012). Year (Y), Line, and Y $\times$ Line interactions were modeled as fixed effects, and replicates and their interactions were considered random effects. Means were separated by Fisher's protected least significant difference test at the 5\% level of significance using SAS (2002-2012). 
Correlation was performed using PROC CORR in SAS (2002-2012).

\section{Results and Discussion}

\section{Analysis of Variance}

ANOVA showed that year and line were the major sources of variability for seed composition constituents (Table 1). Based on Fvalue, year is more important for some constituents such as $\mathrm{P}, \mathrm{Mg}$, and $\mathrm{B}$ than line, suggesting that growing season may have also an influence on these constituents. For others constituents such as protein, $\mathrm{N}, \mathrm{C}$, and $\mathrm{Zn}$, genotypic effects were more important than year effects, suggesting the significant effects of genetic factors in controlling the accumulation of these constituents. There were no significant effects of year on oil, $\mathrm{S}, \mathrm{K}$, and $\mathrm{Mn}$, suggesting that the response of these constituents was similar in each year. All constituents were influenced by line, but the magnitude of the accumulation in seeds differed, depending on the line. For leaf $\mathrm{N}$, S, and minerals (Table 2), year was significant for all nutrients, except for Fe and Mn. Line had significant effects for all nutrients, except for $\mathrm{P}$ and $\mathrm{K}$, suggesting that the levels of nutrients in leaves were significantly influenced by the line. Since the $F$-value of year $\times$ line interactions was smaller (less significant: its contribution to the model was smaller) compared with that of the main effect of year or line, the data were combined across the 2 years.

\section{Effects of Fuzz Trait on Seed and Leaf Composition}

Lines with fuzzy seeds $(F)$ had higher concentrations of seed protein, $\mathrm{N}$ and $\mathrm{S}$ than lines with fuzzless seeds $(N)$ (with exception of STV 7Agl N), but $N$ lines had higher oil and C than in seeds of $F$ lines (Table 3). Calcium levels in seed were higher in $N$ lines than in $F$ lines. Minerals $\mathrm{K}, \mathrm{Mg}, \mathrm{P}$, and $\mathrm{Zn}$ in seeds were higher in $F$ lines than in $N$ lines. Nitrogen (for STV 7Agl) and Mn (for DP 5690, SG 747, and STV 7Agl) did not follow the general trend of other nutrients of higher nutrient accumulation in $F$ lines. Instead $N$ did not differ between $N$ and $F$ lines in STV 7Agl, and Mn was higher in the $N$ line for DP 5690, but did not differ between $N$ and $F$ lines in SG 747 and STV 7Agl. Except for Mn in DES 119, MD 51ne, and STV 7Agl, and Ca, P, and N for DP 5690, $\mathrm{N}$ and $\mathrm{S}$ and all minerals in leaves were higher in $N$ lines than in $F$ lines (Table 4). When the data were expressed across all lines for the fuzz trait ( $F$ vs. $N$ ) (Figures 1, 2), oil, C, and Ca, were higher in cottonseed in $N$ lines than in $F$ lines, but protein and other minerals, except $\mathrm{Mn}$, were higher in $F$ lines than in $N$ lines, supporting data expressed on line set basis. Fuzzless and fuzzy lines had similar concentrations of $\mathrm{Mn}$.

The higher accumulation of nutrients of $N$ lines than $F$ lines in leaves was also observed when all $F$ lines were compared with all $N$ lines, except for Mn (Figure 3). Each line set accumulated different levels of protein, oil, or other nutrients, and the level ranged from about $197 \mathrm{~g} \mathrm{~kg}^{-1}$ to $287 \mathrm{~g} \mathrm{~kg}^{-1}$ for protein, and $234 \mathrm{~g} \mathrm{~kg}^{-1}$ to $293 \mathrm{~g} \mathrm{~kg}^{-1}$ for oil. Similar wider ranges of other nutrients were observed between $F$ and $N$ lines. Cotton leaves accumulated higher concentrations of nutrients in $N$ lines than in $F$ lines, except for $\mathrm{Ca}$ and $\mathrm{P}$ in DP 5690, and $\mathrm{Mn}$ in DES 119, MD 51ne, and STV 7Agl (Table 4). Generally, both the fuzzless parent (SA 243) and the commercial cultivar DP 444 BG/RR accumulated comparable levels of nutrients to the isogenic lines sets.

Nutrient accumulation in lint differed between $N$ and $F$ lines (Table 5). Some $N$ lines accumulated more nutrients in the lint than their equivalent $F$ lines, and other $N$ lines accumulated less nutrients than their equivalent $F$ lines, reflecting genotypic differences in nutrient accumulations in lint. For example, Ca, K, S, B, $\mathrm{Fe}$, and $\mathrm{Na}$ contents were higher in $N$ lines than in $F$ lines, except in MD 51ne and SG 747 for K, DP 5690 and DES 119 for S, SG 747 and DP 5690 for B, DP 5690 for Fe, and MD 51ne for Na. All lines had at least one nutrient where $F$ line was higher than $N$ line, with the exception of MD 51ne where the $N$ line was always higher or equal to the $F$ line. Generally, a similar trend was also found when data were expressed on fuzz trait basis (all $F$ lines vs. all $N$ lines) (Figure 4).

The higher protein and lower oil concentrations in cottonseed may suggest there is a potential commercial use for fuzzless seed as a source for food (oil) and feed (cottonseed meal). Our results

TABLE 5 | Effect of fuzzless/linted $(N)$ and fuzzy/linted $(F)$ cottonseed phenotypes on cotton lint nutrients $\mathrm{Ca}, \mathrm{K}, \mathrm{Mg}, \mathrm{P}, \mathrm{C}, \mathrm{N}, \mathrm{S}$ percentages (\%), and Fe, B, $\mathrm{Mn}, \mathrm{Na}$, and $\mathrm{Zn}$ concentration $\left(\mathrm{mg} \mathrm{kg}^{-1}\right)$.

\begin{tabular}{|c|c|c|c|c|c|c|c|c|c|c|c|c|}
\hline Line & $\mathrm{Ca}$ & $\mathbf{K}$ & Mg & $\mathbf{P}$ & C & $\mathbf{N}$ & $\mathbf{S}$ & B & $\mathrm{Fe}$ & Mn & $\mathrm{Na}$ & $\mathrm{Zn}$ \\
\hline SA 243 & $0.07 \mathrm{f}$ & $0.48 \mathrm{a}$ & 0.06 de & $0.03 \mathrm{bc}$ & $44.3 \mathrm{a}$ & $0.74 \mathrm{~g}$ & 0.030 e & $3.1 \mathrm{de}$ & 7.1 ef & $3.2 \mathrm{e}$ & $40.5 \mathrm{c}$ & 3.8 ef \\
\hline DP 444 BG/RR & 0.09 e & $0.51 \mathrm{~b}$ & 0.07 bc & $0.03 \mathrm{bc}$ & $44.0 \mathrm{bc}$ & $0.97 \mathrm{bcd}$ & 0.033 de & $3.0 \mathrm{e}$ & $6.4 f$ & $3.7 \mathrm{ed}$ & 31.6 ef & $5.3 \mathrm{ab}$ \\
\hline DES $119 N$ & $0.15 \mathrm{c}$ & $0.53 \mathrm{a}$ & 0.07 bc & $0.02 \mathrm{~d}$ & $44.1 \mathrm{bc}$ & 0.88 def & $0.038 \mathrm{bc}$ & $4.1 \mathrm{bc}$ & $9.6 \mathrm{c}$ & 5.0 bc & $52.4 \mathrm{a}$ & $4.1 \mathrm{de}$ \\
\hline DES $119 F$ & $0.06 \mathrm{f}$ & $0.46 \mathrm{e}$ & $0.06 \mathrm{c}$ & $0.03 \mathrm{a}$ & $43.8 \mathrm{de}$ & $1.05 a b$ & $0.035 \mathrm{~cd}$ & $2.7 \mathrm{e}$ & 7.0 ef & $4.1 \mathrm{~d}$ & $41.2 \mathrm{bc}$ & $5.5 \mathrm{a}$ \\
\hline DP $5690 N$ & $0.11 \mathrm{~d}$ & $0.50 \mathrm{~b}$ & 0.07 b & $0.02 \mathrm{~cd}$ & 43.9 cde & $0.79 \mathrm{fg}$ & 0.030 e & $3.3 \mathrm{de}$ & 6.8 ef & $4.6 \mathrm{C}$ & $53.0 \mathrm{a}$ & $3.3 f$ \\
\hline DP $5690 F$ & 0.07 ef & $0.46 \mathrm{e}$ & $0.07 \mathrm{~b}$ & $0.02 \mathrm{~d}$ & $44.1 \mathrm{bc}$ & 0.96 bcde & 0.030 e & $2.9 \mathrm{e}$ & $8.5 \mathrm{~d}$ & $4.8 \mathrm{c}$ & $45.9 \mathrm{~b}$ & $4.1 \mathrm{de}$ \\
\hline MD 51neN & $0.19 \mathrm{a}$ & 0.47 de & $0.06 \mathrm{c}$ & $0.03 \mathrm{~b}$ & 44.0 cde & $0.84 f$ & $0.053 \mathrm{a}$ & $4.5 \mathrm{~b}$ & $11.1 \mathrm{~b}$ & $6.6 \mathrm{a}$ & 33.2 ef & $5.2 \mathrm{ab}$ \\
\hline MD 51ne $F$ & 0.09 e & 0.47 cde & 0.05 e & $0.02 \mathrm{~cd}$ & $44.1 \mathrm{bc}$ & 0.87 ef & $0.038 \mathrm{bc}$ & $3.0 \mathrm{e}$ & 6.7 ef & $4.7 \mathrm{C}$ & 34.1 de & $4.5 \mathrm{~cd}$ \\
\hline SG $747 N$ & $0.11 d$ & $0.43 \mathrm{f}$ & $0.06 \mathrm{c}$ & $0.02 \mathrm{~d}$ & 44.0 cde & 0.95 cde & $0.038 \mathrm{bc}$ & $3.0 \mathrm{e}$ & $9.1 \mathrm{~cd}$ & $3.7 \mathrm{ed}$ & $39.2 \mathrm{~cd}$ & $5.2 \mathrm{ab}$ \\
\hline SG $747 F$ & 0.09 e & $0.43 f$ & $0.06 \mathrm{~d}$ & $0.03 \mathrm{~b}$ & $44.0 \mathrm{cb}$ & $0.99 \mathrm{abc}$ & 0.033 de & $3.6 \mathrm{~cd}$ & $7.4 \mathrm{e}$ & $4.1 \mathrm{~d}$ & $26.3 \mathrm{fg}$ & $4.3 \mathrm{cde}$ \\
\hline STV 7Agl N & 0.17 b & $0.54 \mathrm{a}$ & 0.08 a & $0.02 \mathrm{~cd}$ & 43.8 e & $1.09 \mathrm{a}$ & 0.040 b & $5.9 \mathrm{a}$ & $14.0 \mathrm{a}$ & $5.4 \mathrm{~b}$ & 33.7 ef & $4.5 \mathrm{~cd}$ \\
\hline STV 7Agl F & 0.07 ef & 0.48 cde & 0.07 bc & $0.03 \mathrm{~b}$ & $44.1 \mathrm{bc}$ & $0.79 \mathrm{fg}$ & $0.030 \mathrm{e}$ & $2.8 \mathrm{e}$ & 6.7 ef & $3.4 \mathrm{e}$ & $23.6 \mathrm{~g}$ & $4.7 \mathrm{bc}$ \\
\hline
\end{tabular}



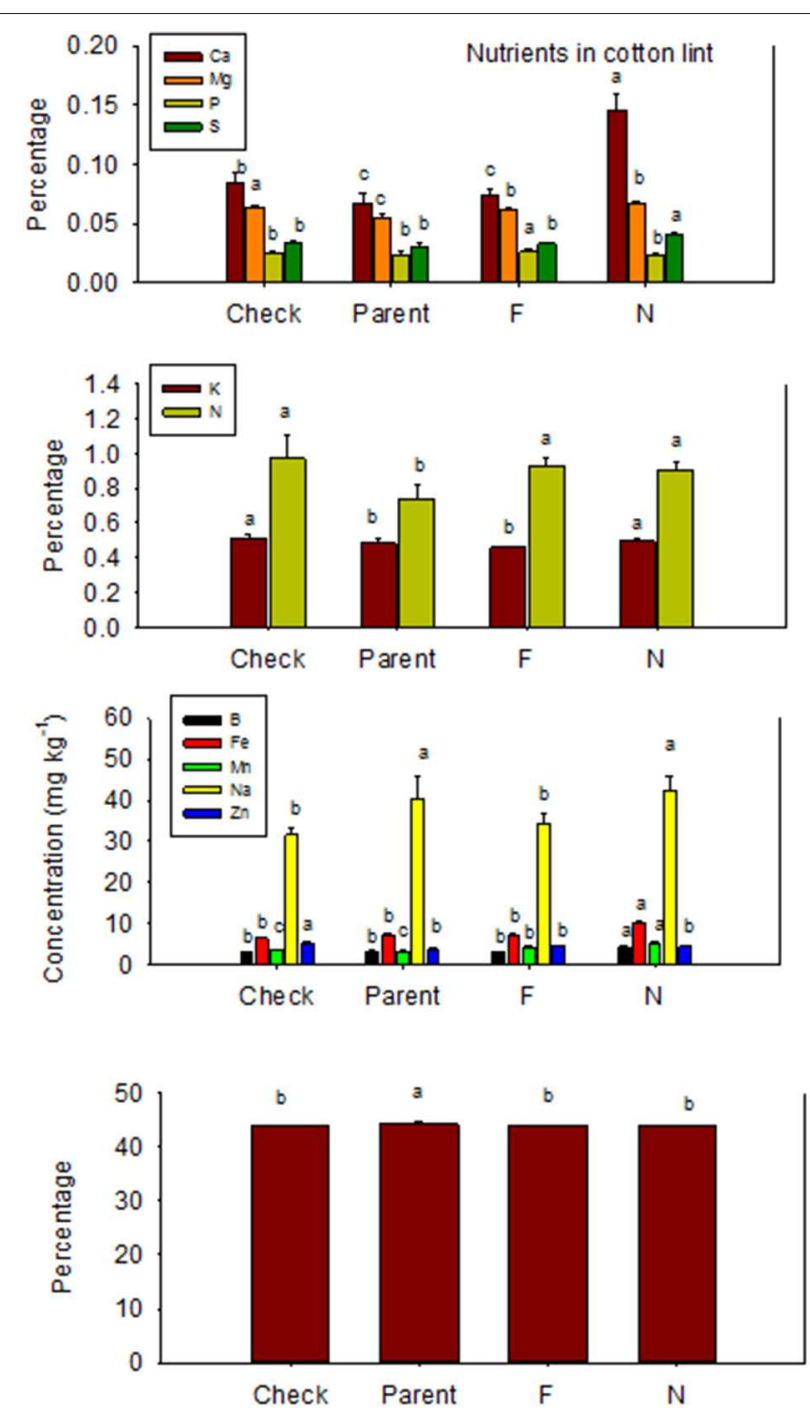

FIGURE 4 | Effects of cottonseed fuzz trait on cotton lint nutrients in near-isogenic Gossypium hirsutum lines expressing fuzzy/linted $(F)$ and fuzzless/linted $(N)$ seed phenotypes, the fuzzless/linted parent SA 243 (parent), and the fuzzy/linted commercial cultivar DP 444 BG/RR (check). Cotton was grown in the field in 2013 only in Stoneville, MS, USA. Bars show means \pm standard error of the mean (SE). Within each constituent, means with the same letter are not significantly different at $5 \%$ as determined by Fisher's LSD test.

are in agreement with previous reports (Bechere et al., 2009). They developed homozygous naked-tufted $\mathrm{M}_{8}$ mutant lines and evaluated the developed lines for lint yield, fiber quality, seed oil content, ginning efficiency, and yarn spinning. They found that lint yield in the naked-tufted seed mutants was lower when compared with their original fuzzy parents. Also, they found that seed oil content in naked-tufted seed mutants was higher than fuzzy parents. Bellaloui and Turley (2013), working on similar material with the same trait, but under greenhouse conditions, and found, generally, that fuzzless cotton seeds accumulated higher oil and lower protein compared with their equivalent fuzzy seeds, showing an inverse relationship between protein and oil. The inverse relationship between protein and oil was previously reported in other species such as corn (Kebede et al., 2013), soybean (Burton, 1985; Ray et al., 2006; Bellaloui et al., 2013a,b), and cottonseed (Pettigrew and Dowd, 2011). Pettigrew and Dowd (2011) investigated the effect of irrigation and planting dates on seed oil and protein in six conventional cotton (Gossypium hirsutum) cultivars (DP 445BR, DP 555BR, FM 800BR, FM 960BR, ST 4892BR, and ST 5599BR), planted at late April and late May, and under irrigated and non-irrigated (dryland) field conditions in Stoneville, MS, from 2005 to 2008. They found irrigation increased the concentrations of seed oil, but decreased seed protein. However, under dryland conditions, protein was higher than oil at late April and late May planting. Their results showed opposite trend between protein and oil, agreeing with the current results.

The higher accumulation of some nutrients in seeds of $F$ lines than in $N$ lines, and the higher accumulation of some other nutrients in $N$ lines than in $F$ lines was also previously reported (Bellaloui and Turley, 2013), and this could be due to genotypic differences. The consistent higher accumulation of nutrients in leaves of $N$ lines than in $F$ lines across line sets suggests limited translocation of nutrients from leaves to seed, and this may explain the lower accumulation of minerals in seeds of some fuzzless isolines. For example, B showed higher accumulation in leaves and lower accumulation in seed of $N$ lines than in the $F$ lines. It is known that cotton has higher B requirement for growth, development, and seed quality. Although B is mobile in sugar-alcohol containing species such as rice, pears, almond, and celery, it looks like B in cotton has limited mobility (Brown and Shelp, 1997; Brown et al., 2002; Dordas, 2006) from leaves to seed. Although high concentrations of B occurred in leaves (ranged from 40 to $55 \mathrm{mg} \mathrm{B} \mathrm{kg}-1$ ), cottonseed contained limited concentrations of $\mathrm{B}\left(<20 \mathrm{mg} \mathrm{B} \mathrm{kg}^{-1}\right)$. Soil used in our experiment had adequate $B$ concentration (ranged from 1.5 to $2.0 \mathrm{mg} \mathrm{kg}^{-1}$ ) to support the crop. The limited mobility of B in cotton may be one reason of why cotton sometimes responds to foliar B application. Dordas (2006) investigated the foliar application of B on cotton and found there was an increase in cotton lint, and concluded that it is possible that the critical levels of B in cotton have been assessed by visual symptoms and not by yield of comparative field studies. It must be noted also that some other studies did not find a positive response to foliar B applications (Heitholt, 1994).

The other mineral that showed an interesting pattern was $\mathrm{Ca}$. The accumulation of $\mathrm{Ca}$ in leaves, seeds, and lint was higher in $N$ lines than in $F$ lines, and this could be explained by the fact that Ca taken up was not used for further physiological and biochemical processing involved in lint fiber development and structure such as the synthesis of cell wall polysaccharides and fatty acids (Gou et al., 2007; Yang et al., 2008; Pang et al., 2010a,b) and the secondary cell wall synthesis (Li et al., 2002, 2005; Wang et al., 2010; Wang and Ruan, 2010). Also, the down-regulation of calcium and phytohormone mediated signals observed at fiber initiation stage in the fuzzless mutants (Padmalatha et al., 2012) could explain the higher levels of $\mathrm{Ca}$ in leaves, seeds, and lint due to lower requirements of $\mathrm{Ca}$ by fuzzless seed lines. The significant role of $\mathrm{Ca}$ in cotton was previously reported and involved 
in pollen germination (Brewbaker and Kwack, 1963), pollen tube growth (Zhang et al., 1997), stimulation of fertilization (Faure et al., 1994; Tian and Russell, 1997), and egg activation (Digonnet et al., 1997), and this is because $\mathrm{Ca}$ is required for vesicle fusion at the tip of the elongating tube (Pierson et al., 1996). It was also found that $\mathrm{Ca}$ enhances the antioxidant enzyme activity and protects the plant under oxidative stress conditions through reactive oxygen species (ROS) scavenging (Jiang and Huang, 2001). The apparent explanation that seed $\mathrm{Ca}$ in $N$ lines was higher than in $F$ lines could be due to higher requirements of $\mathrm{Ca}$ by $N$ lines for physiological and structural functions, may be, for seed protection from severe environmental conditions such as drought and high heat as seeds in $N$ lines are covered with little lint compared with $F$ lines. If this is the case, then germination rate between $N$ and $F$ lines should be different as seed of $N$ lines may be more hard seeded because of the higher $\mathrm{Ca}$ accumulation. The higher Ca content in leaves and lint may support the higher
Ca requirement by $N$ lines compared with $\mathrm{F}$ lines. Because the current results showed higher $\mathrm{Ca}$ in leaves and seeds in $N$ lines than in $F$ lines, it would be worthwhile to further investigate the effects of Ca supply on Ca partitioning in different plant tissues, including seeds and relate that to the rate of ROS enzymes and germination rates.

\section{Correlations Between Nutrients in Fuzzless and Fuzzy Near-Isogenic Lines}

In $N$ lines, protein was positively correlated with $\mathrm{Ca}, \mathrm{B}, \mathrm{Fe}, \mathrm{Mn}$, and $\mathrm{Zn}$, and oil was positively correlated with $\mathrm{C}$ (Table 6). There were positive correlations between $\mathrm{Ca}$ and $\mathrm{K}, \mathrm{Mg}, \mathrm{P}, \mathrm{B}, \mathrm{Fe}, \mathrm{Mn}$, and $\mathrm{Zn}$, and positive correlations between $\mathrm{Mg}$ and $\mathrm{P}, \mathrm{B}, \mathrm{Fe}, \mathrm{Mn}$, and $\mathrm{Zn}$. Positive correlations were observed between $\mathrm{P}$ and $\mathrm{B}, \mathrm{Fe}$, $\mathrm{Mn}$, and $\mathrm{Zn}$, and between $\mathrm{S}$ and $\mathrm{Mn}$. Boron was positively correlated with $\mathrm{Fe}, \mathrm{Mn}$, and $\mathrm{Zn}$, and $\mathrm{Fe}$ had a positive correlation with $\mathrm{Mn}$ and $\mathrm{Zn}$. A positive correlation between $\mathrm{Mn}$ and $\mathrm{Zn}$ was also

TABLE 6 | Pearson correlation coefficient ( $R$ - and $P$-values) between nutrients in cottonseed of near-isogenic Gossypium hirsutum lines expressing the fuzzless/linted seed phenotype across 2012 and 2013.

\begin{tabular}{|c|c|c|c|c|c|c|c|c|c|c|c|c|c|c|}
\hline & & Protein & Oil & $\mathrm{Ca}$ & $\mathbf{K}$ & $\mathbf{M g}$ & $\mathbf{P}$ & C & $\mathbf{N}$ & $\mathbf{S}$ & B & $\mathrm{Fe}$ & Mn & $\mathrm{Zn}$ \\
\hline \multirow[t]{2}{*}{ Protein } & $R$ & 1 & & & & & & & & & & & & \\
\hline & $P$ & & & & & & & & & & & & & \\
\hline \multirow[t]{2}{*}{ Oil } & $R$ & NS & 1 & & & & & & & & & & & \\
\hline & $P$ & & & & & & & & & & & & & \\
\hline \multirow[t]{2}{*}{$\mathrm{Ca}$} & $R$ & 0.63 & NS & 1 & & & & & & & & & & \\
\hline & $P$ & $\star \star \star *$ & & & & & & & & & & & & \\
\hline \multirow[t]{2}{*}{ K } & $R$ & NS & NS & 0.36 & 1 & & & & & & & & & \\
\hline & $P$ & & & * & & & & & & & & & & \\
\hline \multirow[t]{2}{*}{$\mathrm{Mg}$} & $R$ & NS & NS & 0.52 & 0.54 & 1 & & & & & & & & \\
\hline & $P$ & & & $* \star \star$ & $* * *$ & & & & & & & & & \\
\hline \multirow[t]{2}{*}{$P$} & $R$ & NS & NS & 0.58 & 0.61 & 0.92 & 1 & & & & & & & \\
\hline & $P$ & & & $* \star *$ & $* \star *$ & $* * \star$ & & & & & & & & \\
\hline \multirow[t]{2}{*}{ C } & $R$ & NS & 0.41 & NS & NS & NS & NS & 1 & & & & & & \\
\hline & $P$ & & ** & & NS & & NS & & & & & & & \\
\hline \multirow[t]{2}{*}{$\mathrm{N}$} & $R$ & NS & NS & NS & NS & NS & NS & NS & 1 & & & & & \\
\hline & $P$ & & & & & & NS & & & & & & & \\
\hline \multirow[t]{2}{*}{ S } & $R$ & NS & NS & NS & NS & NS & NS & NS & NS & 1 & & & & \\
\hline & $P$ & & & & & & NS & & & & & & & \\
\hline \multirow[t]{2}{*}{$B$} & $R$ & 0.49 & NS & 0.70 & NS & 0.77 & 0.73 & NS & NS & NS & 1 & & & \\
\hline & $P$ & $\star *$ & & $\star \star \star *$ & & $* * *$ & $* * *$ & & & & & & & \\
\hline \multirow[t]{2}{*}{$\mathrm{Fe}$} & $R$ & 0.51 & NS & 0.65 & 0.40 & 0.75 & 0.71 & NS & NS & NS & 0.74 & 1 & & \\
\hline & $P$ & $* * *$ & & $\star * \star$ & * & $* * \star$ & $* * *$ & & & & $* * *$ & & & \\
\hline \multirow[t]{2}{*}{$\mathrm{Mn}$} & $R$ & 0.40 & NS & 0.49 & NS & 0.38 & 0.35 & NS & NS & 0.30 & 0.51 & 0.45 & 1 & \\
\hline & $P$ & * & & $\star \star$ & & * & * & & & * & $* * *$ & ** & & \\
\hline \multirow[t]{2}{*}{$\mathrm{Zn}$} & $R$ & 0.31 & NS & 0.65 & NS & 0.59 & 0.50 & NS & NS & NS & 0.75 & 0.62 & 0.41 & 1 \\
\hline & $P$ & * & & 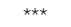 & & 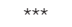 & $\star *$ & & & & 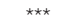 & 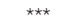 & $\star \star$ & \\
\hline
\end{tabular}

$N=40 ;{ }^{*} p \leq 0.5 ;{ }^{* *} p \leq 0.01 ;{ }^{* * *} p \leq 0.001$ 
TABLE 7 | Table 6 Pearson correlation coefficient ( $R$ - and $P$-values) between nutrients in cottonseed of near-isogenic Gossypium hirsutum lines expressing the fuzzy/linted seed phenotype across 2012 and 2013.

\begin{tabular}{|c|c|c|c|c|c|c|c|c|c|c|c|c|}
\hline Protein & Oil & $\mathrm{Ca}$ & $\mathbf{K}$ & $\mathbf{M g}$ & $\mathbf{P}$ & C & $\mathbf{N}$ & $\mathrm{s}$ & B & $\mathrm{Fe}$ & Mn & $\mathrm{Zn}$ \\
\hline
\end{tabular}

Protein

1

\begin{tabular}{|c|c|c|c|c|c|c|c|c|c|c|c|c|c|c|}
\hline Oil & $\begin{array}{l}R \\
P\end{array}$ & NS & 1 & & & & & & & & & & & \\
\hline \multirow[t]{2}{*}{$\mathrm{Ca}$} & $R$ & 0.41 & & 1 & & & & & & & & & & \\
\hline & $P$ & ** & & & & & & & & & & & & \\
\hline \multirow[t]{2}{*}{ K } & $R$ & NS & NS & NS & 1 & & & & & & & & & \\
\hline & $P$ & & & & & & & & & & & & & \\
\hline \multirow[t]{2}{*}{$\mathrm{Mg}$} & $R$ & NS & NS & NS & NS & 1 & & & & & & & & \\
\hline & $P$ & & & & & & & & & & & & & \\
\hline \multirow[t]{2}{*}{ P } & $R$ & NS & NS & NS & NS & 0.95 & 1 & & & & & & & \\
\hline & $P$ & & & & & 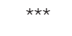 & & & & & & & & \\
\hline \multirow[t]{2}{*}{ C } & $R$ & NS & NS & NS & NS & NS & NS & 1 & & & & & & \\
\hline & $P$ & & & & & & & & & & & & & \\
\hline \multirow[t]{2}{*}{$\mathrm{N}$} & $R$ & NS & NS & NS & NS & NS & NS & NS & 1 & & & & & \\
\hline & $P$ & & & & & & & & & & & & & \\
\hline \multirow[t]{2}{*}{$S$} & $R$ & NS & NS & NS & NS & NS & NS & NS & NS & 1 & & & & \\
\hline & $P$ & & & & & & & & & & & & & \\
\hline \multirow[t]{2}{*}{$B$} & $R$ & 0.50 & NS & 0.41 & NS & 0.46 & 0.44 & NS & NS & NS & 1 & & & \\
\hline & $P$ & $* \star *$ & & * & & $* *$ & ** & & & & & & & \\
\hline \multirow[t]{2}{*}{$\mathrm{Fe}$} & $R$ & NS & NS & NS & NS & 0.62 & 0.58 & NS & NS & NS & 0.54 & 1 & & \\
\hline & $P$ & & & & & $\star * \star$ & $* \star *$ & & & & $* * *$ & & & \\
\hline \multirow[t]{2}{*}{$\mathrm{Mn}$} & $R$ & NS & NS & 0.36 & NS & 0.54 & 0.51 & NS & NS & NS & 0.61 & 0.67 & 1 & \\
\hline & $P$ & & & * & & $\star \star \star *$ & 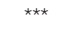 & & & & $* \star \star$ & 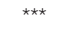 & & \\
\hline \multirow[t]{2}{*}{$\mathrm{Zn}$} & $R$ & NS & 0.37 & NS & NS & NS & NS & NS & NS & NS & NS & 0.31 & & 1 \\
\hline & $P$ & & * & & & & & & & & & * & & \\
\hline
\end{tabular}

$N=40 ;{ }^{*} p \leq 0.5 ;{ }^{* *} p \leq 0.01 ;{ }^{* \star *} p \leq 0.001$.

observed. In $F$ lines, positive correlations between protein and $\mathrm{B}$, and between protein and $\mathrm{Ca}$ were observed (Table 7). Oil was positively correlated with $\mathrm{Zn}$, and $\mathrm{Ca}$ had a positive correlation with $\mathrm{B}$ and $\mathrm{Mn}$. Both $\mathrm{Mg}$ and $\mathrm{P}$ had positive correlation with $\mathrm{B}$, $\mathrm{Fe}$, and $\mathrm{Mn}$. Boron had positive correlations with $\mathrm{Fe}$ and $\mathrm{Mn}$, and $\mathrm{Fe}$ had positive correlations with $\mathrm{Mn}$ and $\mathrm{Zn}$.

Correlation between nutrients in $N$ lines and $F$ lines showed significant positive correlation between some nutrients in both $N$ and $F$ lines, but some nutrients did not correlate (Tables 6, 7). For example, in $N$ lines protein was positively correlated with $\mathrm{Ca}, \mathrm{B}, \mathrm{Fe}, \mathrm{Mn}$, and $\mathrm{Zn}$, suggesting the indirect involvement of these minerals with protein synthesis. Also, the positive correlations between $\mathrm{Ca}$ and oil, and between $\mathrm{C}$ and oil support the observation of similar trends of these nutrients in $N$ lines, and the involvement of carbon in oil metabolism. Nutrients such as
P, Ca, and B; Ca, B, and Mn; Mg, B, Fe, and Mn; P, B, and Mn; $\mathrm{Fe}, \mathrm{Mn}$, and $\mathrm{Zn}$ showed consistency of positive correlation in both $N$ and $F$ lines. Other nutrients were not consistent between $N$ and $F$ lines, and this is due to genotypic effects. The positive and negative correlation between cation and anion nutrients were previously reported, although this correlation depends on growth conditions, genotype, and nutrient supply (Mengel and Kirkby, 1982; Marschner, 2012).

Nutrient uptake, translocation, redistribution, and accumulation are processes controlling the accumulation of minerals in seeds (Grusak and DellaPenna, 1999; White and Broadley, 2005), and most of the genetic basis of these process are still not known (Ding et al., 2010). Previous research on fuzzless cottonseed showed that fiber development involves physiological, biochemical, and molecular processes (Turley et al., 2007; 

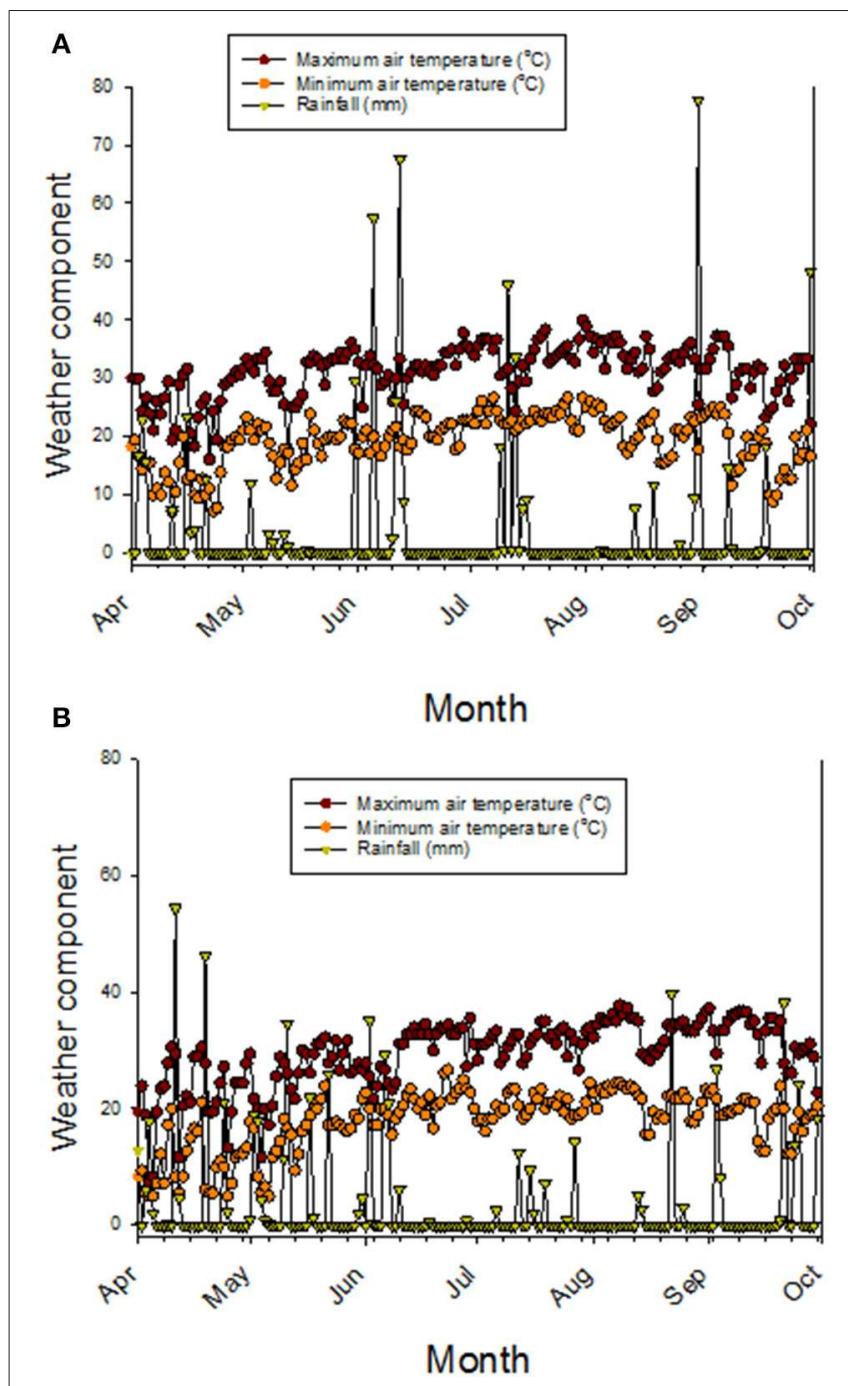

FIGURE 5 | Maximum and minimum air temperatures, and precipitation (rainfall) in 2012 (A) and in 2013 (B). Weather data obtained from MSUCares, Stoneville (2015).

Padmalatha et al., 2012). For example, it was found that the fiber development involved processes related to genetics (Turley and Kloth, 2002; Gou et al., 2007; Turley et al., 2007; Zhao et al., 2009; Liu et al., 2012), phytohormones (Yang et al., 2006; Zhang et al., 2011), transcription factors such as MYB25 (Machado et al., 2009), turgor mechanism (Ruan et al., 2003; Wang et al., 2010; Wang and Ruan, 2010), soluble sugars, potassium, and organic acids, ion-transporters ( $\mathrm{H}+$-ATPases and $\mathrm{K}+$-transporter) (Wang et al., 2010; Wang and Ruan, 2010); upregulation of potassium and sugar transporters to maintain the turgor pressure (Ruan et al., 2004), carbohydrate and energy metabolism and carbon skeletons for the synthesis of cell wall polysaccharides and fatty acids (Gou et al., 2007; Yang et al., 2008; Pang et al., 2010a,b), and the secondary cell wall synthesis (Li et al., 2002, 2005; Wang et al., 2010; Wang and Ruan, 2010). Because these processes were involved in fiber development, then it is expected to have differences in phenotypic traits such as protein, oil, and minerals between fuzzy and fuzzless cottonseed. This is because of the involvement of carbon skeleton and energy metabolism (Gou et al., 2007; Yang et al., 2008; Pang et al., 2010a,b), K (Ruan et al., 2004; Wang et al., 2010), and Ca (Padmalatha et al., 2012) in fiber development. The only difference between each line set investigated here is cottonseed fuzz trait, so differences in cottonseed composition constituents observed in our study could be explained by the trait as each isoline set has the same genetic background (Bellaloui and Turley, 2013). It must be noted here that information explaining nutrient dynamics in fuzzy and fuzzless cottonseed is almost non-existent (Bellaloui and Turley, 2013), and most of research on fuzzless cottonseed has been on cell biology, genetic, and molecular biology (Padmalatha et al., 2012; Bellaloui and Turley, 2013). However, it is possible that cottonseed differences could be explained by stored energy metabolism differences such as sugars, hormones signaling, and lower lint yields (Turley et al., 2007; Stetina et al., 2014) in fuzzless mutants. These major down regulations, mentioned above, and lower lint yield may have led to a nutrient imbalance, resulting in differences in nutrient uptake, transport, mobility from leaves (source) to seed (sink), and nutrient accumulation in leaves and cottonseed between $N$ and $F$ isogenic lines during boll development. The weather data (Figures 5A,B) showed that during May-August (reproduction stage period for cotton), the maximum and minimum temperatures in 2012 were higher than in 2013. For example, maximum temperatures in 2012 in May, June, July, August, and September were, respectively, $30.9,31.7,33.9$, and $33.8^{\circ} \mathrm{C}$ vs. $26.2,30.4,31.5$, and $33.8^{\circ} \mathrm{C}$ in 2013. Rainfall (mm) was higher in April and May in 2013 than in 2012, but in June, July, August, generally the rainfall was higher in 2012 than in 2013. Bearing in mind the rainfall in 1 day can reach upto $77 \mathrm{~mm}$ (for example in 2012 in August) or upto $40 \mathrm{~mm}$ (for example, in August in 2013). It is clear that the different patterns in temperature and rainfall between years (Figures 5A,B) will impact soil moisture, and consequently nutrient uptake, transport, and accumulation of nutrients in seeds. The effect of rainfall and temperatures in each year could be minimum since the experiment was irrigated and all genotypes were exposed to the same growing conditions. However, we still do not know the response of $F$ and $N$ genotypes or genotypes in each isoline set (fuzzy or fuzzless) to soil moisture resulted from rainfall or higher temperatures and their effects on nutrients uptake and transport. Therefore, we cannot exclude the effect of weather conditions on seed nutrients accumulation in $F$ and $N$ genotypes or on genotypes in the two sets of isolines. Further research is needed to determine the response of $F$ and $N$ genotypes to growing conditions such as drought and heat under field conditions.

It appears that the higher accumulation of nutrients of most $N$ lines in their leaves and lower accumulation of nutrients $(\mathrm{N}$, $\mathrm{S}$, and minerals) in seeds of some of the $F$ lines could be due to limited mobility of the nutrients from leaves to seed and lower nutrients demand by seeds of these $N$ line, resulting in higher accumulation of nutrients in leaves. Some $N$ lines did not follow this pattern, and this could be due to genotypic differences and fuzz trait. The real mechanism of how the fuzz development is involved in differential nutrients between $N$ and $F$ lines are still 
not understood (Bellaloui and Turley, 2013), and further research on the effects of nutrient supply on nutrient mobility is needed. Also, research is underway, and more biochemical and cellular methods including gene expression profiling based on RNA sequencing will be used to identify the gene responsible for this trait.

\section{Conclusions}

The current research demonstrated that seed protein was higher in fuzzy genotype in all near-isogenic sets, but seed oil was higher in fuzzless genotype in all near-isogenic sets, suggesting possible commercial use for fuzzless seed as source of both protein or oil as the level of protein and oil was competitive with the commercial check. The higher carbon in all fuzzless cottonseed may indicate that higher energy-storage occurred in fuzzless cottonseed. The higher accumulation of most of nutrients in leaves of fuzzless lines and lower accumulation of these nutrients in cottonseed suggested limited mobility and translocation of these nutrients in fuzzless lines compared with fuzzy lines. The research demonstrated that fuzz fiber development altered cottonseed composition, and this may be due to the

\section{References}

Analytical Methods Committee (1959). Analysts. Her majesty's stationery office, London. 84, 214.

Arpat, A. B., Waugh, M., Sullivan, J. P., Gonzales, M., Frisch, D., Main, D., et al. (2004). Functional genomics of cell elongation in developing cotton fibers. Plant Mol. Biol. 54, 911-929. doi: 10.1007/s11103-004-0392-y

Association of Official Analytical Chemists (AOAC), (1990a). "Method 988.05," in Official Methods of Analysis, 15th Edn., ed K. Helrich (Arlington, VA: AOAC), 70.

Association of Official Analytical Chemists (AOAC), (1990b). "Method 920.39," in Official Methods of Analysis, 15th Edn., ed K. Helrich (Arlington, VA: AOAC), 79.

Bandemer, S. L., and Schaible, P. J. (1944). Determination of iron. A study of the o-phenanthroline method. Ind. Eng. Chem. Anal. Ed. 16, 317-319. doi: 10.1021/i560129a013

Bechere, E., Auld, D. L., and Hequet, E. (2009). Development of 'naked-tufted' seed coat mutants for potential use in cotton production. Euphytica 167, 333-339. doi: 10.1007/s10681-009-9890-y

Bellaloui, N., Gillen, A. M., Mengistu, A., Kebede, H., Fisher, D. K., Smith, J. R., et al. (2013a). Responses of nitrogen metabolism and seed nutrition to drought stress in soybean genotypes differing in slow-wilting phenotype. Front. Plant Sci. 4:498. doi: 10.3389/fpls.2013.00498

Bellaloui, N., Hanks, J. E., Fisher, D. K. and Mengistub, A. (2009). Soybean seed composition is influenced by within-field variability in soil nutrients. Plant Manag. Netw. 8. doi: 10.1094/CM-2009-1203-01-RS

Bellaloui, N., Hu, Y., Mengistu, A., Kassem, M. A., and Abel, C. A. (2013b). Effects of foliar boron application on seed composition, cell wall boron, and seed $\delta 15 \mathrm{~N}$ and $\delta 13 \mathrm{C}$ isotopes in water-stressed soybean plants. Front. Plant Sci. 4:270. doi: $10.3389 /$ fpls.2013.00270

Bellaloui, N., Mengistu, A., Walker, R. R., and Young, L. D. (2014). Soybean seed composition affected by seeding rates and row spacing in the Midsouth USA. Crop Sci. 54, 1782-1795. doi: 10.2135/cropsci2013.07.0463

Bellaloui, N., Smith, J. R., Gillen, A. M., and Ray, J. D. (2011). Effects of maturity, genotypic background, and temperature on seed mineral composition in near-isogenic soybean lines in the early soybean production system. Crop Sci. 51, 1161-1171. doi: 10.2135/cropsci2010.04.0187 involvement of fuzz fiber development in carbon and nitrogen metabolism, and the mobility of nutrients from leaves (source) to seed (sink). This information is beneficial to breeders for selection for higher oil or higher protein content, and to physiologist to further understand the mechanisms of mineral movement within plants. Understanding the mechanisms of nutrient mobility from leaves to seed would help for selecting for cottonseed with higher nutritional quality for human consumption and animal feed.

\section{Acknowledgments}

Technical support for this project provided by S. Mosley, K. Jordan, and R. Manning is appreciated. Funding for this research was provided by USDA, Agricultural Research Service projects 6066-21220-012-00D, 6066-21000-051-00D, and 6066-22000-074-00D. Mention of trade names or commercial products in this publication is solely for the purpose of providing specific information and does not imply recommendation or endorsement by the Unites States Department of Agriculture (USDA). USDA is an equal opportunity provider and employer.
Bellaloui, N., and Turley, R. B. (2013). Effects of fuzzless cottonseed phenotype on cottonseed nutrient composition in near isogenic cotton (Gossypium hirsutum L.) mutant lines under well-watered and water stress conditions. Front. Plant Sci. 4:516. doi: 10.3389/fpls.2013.00516

Boydak, E., Alpaslan, M., Hayta, M., Gercek, S., and Simsek, M. (2002). Seed composition of soybeans grown in the Harran region of Turkey as affected by row spacing and irrigation. J. Agric. Food Chem. 50, 4718-4720. doi: $10.1021 /$ jf0255331

Brewbaker, J. L., and Kwack, B. H. (1963). The essential role of calcium ion in pollen germination and pollen tube growth. Am. J. Bot. 50, 859-865. doi: $10.2307 / 2439772$

Brown, P. H., Bellaloui, N., Wimmer, M. A., Bassil, E. S., Ruiz, J., Hu, H., et al. (2002). Boron in plant biology. Plant Biol. 4, 205-223. doi: 10.1055/s-200225740

Brown, P. H., and Shelp, B. J. (1997). Boron mobility in plants. Plant Soil 193, 85-101. doi: 10.1023/A:1004211925160

Burton, J. W. (1985). "Breeding soybean for improved protein quantity and quality," in World Soybean Research Conference III: Proceedings, ed R. Shibles (Ames; Boulder: Westview Press), August 12-17. 361-367.

Cavell, A. J. (1955). The colorimetric determination of phosphorus in plant materials. J. Sci. Food Agric. 6, 479-480. doi: 10.1002/jsfa.2740060814

Digonnet, C., Aldon, D., Leduc, N., Dumas, C., and Rougier, M. (1997). First evidence of a calcium transient in flowering plants at fertilization. Dev. Camb. Engl. 124, 2867-2874.

Ding, G., Yang, M., Hu, Y., Liao, Y., Shi, L., and Xu, F. (2010). Quantitative trait loci affecting seed mineral concentrations in Brassica napus grown with contrasting phosphorus supplies. Ann. Bot. 105, 1221-1234. doi: 10.1093/aob/ mcq050

Dordas, C. (2006). Foliar boron application affects lint and seed yield and improves seed quality of cotton grown on calcareous soils. Nutr. Cycl. Agroecosyst. 76, 19-28. doi: 10.1007/s10705-006-9037-7

Faure, J. E., Digonnet, C., and Dumas, C. (1994). An in vitro system for adhesion and fusion of maize gametes. Science 263, 1598-1600. doi: 10.1126/science.263.5153.1598

Fletcher, R. J., Bell, I. P., and Lambert, J. P. (2004). Public health aspects of food fortification: a question of balance. Proc. Nutr. Soc. 63, 605-614. doi: $10.1079 /$ PNS2004391 
Gou, J. Y., Wang, L. J., Chen, S. P., Hu, W. L., and Chen, X. Y. (2007). Gene expression and metabolite profiles of cotton fiber during cell elongation and secondary cell wall synthesis. Cell Res. 17, 422-434. doi: 10.1038/sj.cr. 7310150

Grusak, M. A., and DellaPenna, D. (1999). Improving the nutrient composition of plants to enhance human nutrition and health. Ann. Rev. Plant Physiol. Plant Mol. Biol. 50, 133-161. doi: 10.1146/annurev.arplant.50.1.133

Guan, X., Lee, J. J., Pang, M., Shi, X., Stelly, D. M., and Chen, Z. J. (2011). Activation of Arabidopsis seed hair development by cotton fiber-related genes. PLoS ONE 6:e21301. doi: 10.1371/journal.pone.0021301

He, Z., Shankle, M., Zhang, H., Way, T. R., Tewolde, H., and Uchimiya, M. (2013). Mineral composition of cottonseed is affected by fertilization management practices. Agron. J. 105, 341-350. doi: 10.2134/agronj2012.0351

Heinemann, R. J. B., Fagundes, P. L., Pinto, E. A., Penteado, M. V. C., and LanferMarquez, U. M. (2005). Comparative study of nutrient composition of commercial brown, parboiled and milled rice from Brazil. J. Food Comp. Anal. 18, 287-296. doi: 10.1016/j.jfca.2004.07.005

Heitholt, J. J. (1994). Supplemental boron, boll retention percentage, ovary carbohydrates and lint yield in modern cotton genotypes. Agron. J. 86, 492-497. doi: 10.2134/agronj1994.00021962008600030007x

Ji, S. J., Lu, Y. C., Feng, J. X., Wei, G., Li, J., Shi, Y. H., et al. (2003). Isolation and analyses of genes preferentially expressed during early cotton fiber development by subtractive PCR and cDNA array. Nucleic Acids Res. 31, 2534-2543. doi: $10.1093 /$ nar/gkg358

Jiang, Y., and Huang, B. (2001). Effects of calcium on antioxidant activities and water relations associated with heat tolerance in two cool-season grasses. J. Exp. Bot. 52, 341-349. doi: 10.1093/jexbot/52.355.341

John, M. K., Chuah, H. H., and Neufeld, J. H. (1975). Application of improved azomethine-H method to the determination of boron in soils and plants. Anal. Lett. 8:559-568. doi: 10.1080/00032717508058240

Kebede, H., Abbas, H. K., Fisher, D. K., and Bellaloui, N. (2013). Relationship between aflatoxin contamination and physiological responses of corn plants under drought and heat stress. Toxins 4, 1385-1403. doi: 10.3390/toxins4111385

Li, X. B., Cai, L., Cheng, N. H., and Liu, J. W. (2002). Molecular characterization of the cotton GhTUB1 gene that is preferentially expressed in fiber. Plant Physiol. 130, 666-674. doi: 10.1104/pp.005538

Li, X. B., Fan, X. P., Wang, X. L., Cai, L., and Yang, W. C. (2005). The cotton ACTIN1 gene is functionally expressed in fibers and participates in fiber elongation. Plant Cell 17, 859-875. doi: 10.1105/tpc.104.029629

Liu, K., Han, M., Zhang, C., Yao, L., Sun, J., and Zhang, T. (2012). Comparative proteomic analysis reveals the mechanisms governing cotton fiber differentiation and initiation. J. Proteomics 75, 845-856. doi: 10.1016/j.jprot.2011.09.025

Loeppert, R. L., and Inskeep, W. P. (1996). "Colorimetric determination of ferrous iron and ferric iron by the 1,10-phenanthroline method," in Methods of Soil Analysis: Part 3, Chemical Methods, ed J. M. Bigham (Madison, WI: SSSA), 659-661.

Lohse, G. (1982). Microanalytical azomethine-H method for boron determination in plant tissue. Commun. Soil Sci. Plant Anal. 13, 127-134. doi: 10.1080/00103628209367251

Lu, K., Li, L., Zheng, X., Zhang, Z., Mou, T., and Hu, Z. (2008). Quantitative trait loci controlling $\mathrm{Cu}, \mathrm{Ca}, \mathrm{Zn}, \mathrm{Mn}$ and $\mathrm{Fe}$ content in rice grains. J. Genet. 87, 305-310. doi: 10.1007/s12041-008-0049-8

Machado, A., Wu, Y., Yang, Y., Llewellyn, D. J., and Dennis, E. S. (2009). The MYB transcription factor GhMYB25 regulates early fiber and trichome development. Plant J. 59, 52-62. doi: 10.1111/j.1365-313X.2009.03847.x

Marschner, P. (2012). Marschner's Mineral Nutrition of Higher Plants. 3rd Edn. San Diego, CA: Academic Press.

Mengel, K., and Kirkby, E. A. (1982). Principles of Plant Nutrition, 3rd Edn. Worblaufen; Bern: International Potash Institute.

MSUCares, Stoneville, M. S. (2015). (http://ext.msstate.edu/anr/drec/weather.cgi). Verified on February 10, 2015.

Padmalatha, K. V., Patil, D. P., Kumar, K., Dhandapani, G., Kanakachari, M., Phanindra, M. L. V., et al. (2012). Functional genomics of fuzzless-lintless mutant of Gossypium hirsutum L. cv. MCU5 reveal key genes and pathways involved in cotton fiber initiation and elongation. BMC Genomics 13:624-638. doi: 10.1186/1471-2164-13-624
Pang, C. Y., Wang, H., Pang, Y., Xu, C., Jiao, Y., Qin, Y. M. X., et al. (2010a). Comparative proteomics indicates that biosynthesis of pectic precursors is important for cotton fiber and Arabidopsis root hair elongation. Mol. Cell. Proteomics 9, 2019-2033. doi: 10.1074/mcp.M110.000349

Pang, C. Y., Wang, H., Song, W. Q., and Zhu, Y. X. (2010b). The cotton ATP synthase $\delta 1$ subunit is required to maintain a higher ATP/ADP ratio that facilitates rapid fiber cell elongation. Plant Biol. 12, 903-909. doi: 10.1111/j.14388677.2009.00313.x

Pettigrew, W. T., and Dowd, M. K. (2011). Varying planting dates or irrigation regimes alters cottonseed composition. Crop Sci. 51, 2155-2164. doi: 10.2135/cropsci2011.02.0085

Pierson, E. S., Miller, D. D., Callaham, D. A., van Aken, J., Hackett, G., and Hepler, P. K. (1996). Tip-localized calcium entry fluctuates during pollen tube growth. Dev. Biol. 174, 160-173. doi: 10.1006/dbio.1996.0060

Ray, J. D., Fritschi, F. B., and Heatherly, L. G. (2006). Large application of fertilizer $\mathrm{N}$ at planting affects seed protein and oil concentrations in the early soybean production system. Field Crops Res. 99, 67-74. doi: 10.1016/j.fcr.2006. 03.006

Ruan, Y. L., Llewellyn, D. J., and Furbank, R. T. (2003). Suppression of sucrose synthase gene expression represses cotton fiber cell initiation, elongation, and seed development. Plant Cell 15, 952-964. doi: 10.1105/tpc.010108

Ruan, Y. L., Xu, S. M., White, R., and Furbank, R. T. (2004). Genotypic and developmental evidence for the role of plasmodesmatal regulation in cotton fiber elongation mediated by callose turnover. Plant Physiol. 136, 4104-4113. doi: 10.1104/pp.104.051540

Samman, S., Naghii, M. R., Lyons Wall, P. M., and Verus, A. P. (1998). The nutritional and metabolic effects of boron in humans and animals. Biol. Trace Elem. Res. 66, 227-235. doi: 10.1007/BF02783140

SAS (2002-2012). Statistical Analysis System. (Cary, NC: SAS Institute Inc).

Seagull, R. W., and Giavalis, S. (2004). Pre- and post- anthesis application of exogenous hormones alters fiber production in Gossypium hirsutum L. cultivar Maxxa GTO. J. Cotton Sci. 8, 105-111. Available online at: https://www.cotton.org/journal/2004-08/2/upload/jcs08-105.pdf

Shi, Y. H., Zhu, S. W., Mao, X. Z., Feng, J. X., Qin, Y. M., Zhang, L., et al. (2006) Transcriptome profiling, molecular biological, and physiological studies reveal a major role for ethylene in cotton fiber cell elongation. Plant Cell. 18, 651-664. doi: 10.1105/tpc.105.040303

Stetina, S. R., Turley, R. B., Bellaloui, N., and Boykin, J. C. (2014). Yield and fiber quality of five pairs of near-isogenic cotton (Gossypium hirsutum L.) lines expressing fuzzless/linted and fuzzy/linted seed phenotypes. J. Crop Improv. 28, 680-699. doi: 10.1080/15427528.2014.931901

Stewart, J. M. (1975). Fiber initiation on the cotton ovule (Gossypium hirsutum). Am. J. Bot. 62, 723-730. doi: 10.2307/2442061

Tian, H. Q., and Russell, S. D. (1997). Micromanipulation of male and female gametes of Nicotiana tabacum: II. Preliminary attempts for in vitro fertilization and egg cell culture. Plant Cell Rep. 16, 657-661. doi: 10.1007/BF01275510

Turley, R. B., and Kloth, R. H. (2002). Identification of a third fuzzless seed locus in upland cotton (Gossypium hirsutum L.). J. Hered. 93, 359-364. doi: 10.1093/jhered/93.5.359

Turley, R. B., Vaughn, K. C., and Scheffler, J. A. (2007). Lint development and properties of fifteen fuzzless seed lines of Upland cotton (Gossypium hirsutum L.). Euphytica 156, 57-65. doi: 10.1007/s10681-006-9351-9

Walford, S. A., Wu, Y., Llewellyn, D. J., and Dennis, E. S. (2011). GhMYB25like: a key factor in early cotton fiber development. Plant J. 65, 785-797. doi: 10.1111/j.1365-313X.2010.04464.x

Wang, J., Wang, H. Y., Zhao, P. M., Han, L. B., Jiao, G. L., Zheng, Y. Y., et al. (2010). Overexpression of a profilin (GhPFN2) promotes the progression of developmental phases in cotton fibers. Plant Cell Physiol. 51, 1276-1290. doi: $10.1093 / \mathrm{pcp} / \mathrm{pcq} 086$

Wang, L., and Ruan, Y. L. (2010). Unraveling mechanisms of cell expansion linking solute transport, metabolism, plasmodesmtal gating and cell wall dynamics. Plant Signal. Behav. 5, 1561-1564. doi: 10.4161/psb.5.12.13568

White, P. J., and Broadley, M. R. (2005). Biofortifying crops with essential mineral elements. Trends Plant Sci. 10, 586-593. doi: 10.1016/j.tplants.2005. 10.001

Wilcox, J. R., and Shibles, R. M. (2001). Interrelationships among seed quality attributes in soybean. Crop Sci. 41, 11-14. doi: 10.2135/cropsci2001.41111x 
Wilkins, T. A., and Arpat, A. B. (2005). The cotton fiber transcriptome. Physiol. Plant. 124, 295-300. doi: 10.1111/j.1399-3054.2005.00514.x

Yang, S. S., Cheung, F., Lee, J. J., Ha, M., Wei, N. E., Sze, S. H., et al. (2006). Accumulation of genome-specific transcripts, transcription factors and phytohormonal regulators during early stages of fiber cell development in allotetraploid cotton. Plant J. 47, 761-775. doi: 10.1111/j.1365-313X.2006.02829.x

Yang, Y. W., Bian, S. M., Yao, Y., and Liu, J. Y. (2008). Comparative proteomic analysis provides new insights into the fiber elongating process in cotton. J. Proteome Res. 7, 4623-4637. doi: 10.1021/pr800550q

Yu, J., Yu, S., Fan, S., Song, M., Zhai, H., Li, X., et al. (2012). Mapping quantitative trait loci for cottonseed oil, protein and gossypol content in a Gossypium hirsutum $\times$ Gossypium barbadense backcross inbred line population. Euphytica 187, 191-201. doi: 10.1007/s10681-012-0630-3

Zhang, J. S., Yang, H. Y., Zhu, L., and Tong, H. (1997). Ultracytochemical localization of calcium in the pollen tube track of cotton gynoecium. Acta Bot. Sin. 39, 121-125.

Zhang, M. W., Guo, B. J., and Peng, Z. M. (2004). Genetic effects on $\mathrm{Fe}, \mathrm{Zn}, \mathrm{Mn}$ and $\mathrm{P}$ contents in indica black pericarp rice and their genetic correlations with grain characteristics. Euphytica 135, 315-323. doi: 10.1023/B:EUPH.0000013340.98344.60
Zhang, M., Zheng, X., Song, S., Zeng, Q., Hou, L., Li, D., et al. (2011). Spatiotemporal manipulation of auxin biosynthesis in cotton ovule epidermal cells enhances fiber yield and quality. Nat. Biotechnol. 29, 453-458. doi: 10.1038/ nbt. 1843

Zhao, P. M., Wang, L. L., Han, L. B., Wang, J., Yao, Y., Wang, H. Y., et al. (2009). Proteomic identification of differentially expressed proteins in the ligon lintless mutant of upland cotton (Gossypium hirsutum L.). J. Proteome Res. 9, 1076-1087. doi: 10.1021/pr900975t

Conflict of Interest Statement: The authors declare that the research was conducted in the absence of any commercial or financial relationships that could be construed as a potential conflict of interest.

Copyright (c) 2015 Bellaloui, Stetina and Turley. This is an open-access article distributed under the terms of the Creative Commons Attribution License (CC $B Y)$. The use, distribution or reproduction in other forums is permitted, provided the original author(s) or licensor are credited and that the original publication in this journal is cited, in accordance with accepted academic practice. No use, distribution or reproduction is permitted which does not comply with these terms. 


\section{OPEN ACCESS}

Edited by:

Antonio M. De Ron,

Spanish National Research Council,

Spain

Reviewed by:

Hao Peng

Washington State University, USA

Carmen Cuadrado,

Instituto Nacional de Investigación y

Tecnología Agraria y Alimentaria,

Spain

*Correspondence: Alessandro Vitale vitale@ibba.cnr.it

Specialty section:

This article was submitted to

Crop Science and Horticulture,

a section of the journal

Frontiers in Plant Science

Received: 30 May 2016

Accepted: 18 July 2016

Published: 04 August 2016

Citation:

Pedrazzini E, Mainieri D, Marrano CA and Vitale $A$ (2016) Where do Protein Bodies of Cereal Seeds Come From?

Front. Plant Sci. 7:1139.

doi: 10.3389/fpls.2016.01139

\section{Where do Protein Bodies of Cereal Seeds Come From?}

\author{
Emanuela Pedrazzini, Davide Mainieri, Claudia A. Marrano and Alessandro Vitale*
}

Istituto di Biologia e Biotecnologia Agraria, Consiglio Nazionale delle Ricerche, Milano, Italy

Protein bodies of cereal seeds consist of ordered, largely insoluble heteropolymers formed by prolamin storage proteins within the endoplasmic reticulum (ER) of developing endosperm cells. Often these structures are permanently unable to traffic along the secretory pathway, thus representing a unique example for the use of the ER as a protein storage compartment. In recent years, marked progress has been made in understanding what is needed to make a protein body and in formulating hypotheses on how protein body formation might have evolved as an efficient mechanism to store large amounts of protein during seed development, as opposed to the much more common system of seed storage protein accumulation in vacuoles. The major key evolutionary events that have generated prolamins appear to have been insertions or deletions that have disrupted the conformation of the eight-cysteine motif, a protein folding motif common to many proteins with different functions and locations along the secretory pathway, and, alternatively, the fusion between the eight-cysteine motif and domains containing additional cysteine residues.

Keywords: cereals, evolution of subcellular compartments, prolamins, protein bodies, protein sorting, seeds

\section{INTRODUCTION}

Almost 50\% of the global food protein supply comes from cereal seeds (faostat.fao.org). Most of these proteins accumulate in protein bodies (PB), large polymers formed in the endoplasmic reticulum (ER). First discovered in the endosperm cells of maize developing seeds (Duvick, 1961), PBs are the most striking example for an alternative, perhaps surprising, use of the ER.

The ER is the port of entry of the secretory pathway, which leads to the cell surface or the inner hydrolytic compartments using the Golgi apparatus and various endosomes as intermediate sorting stations. The ER takes care of promoting efficient, correct structural maturation of proteins that enter the secretory pathway and selects for disposal those with incurable structural defects (Anelli and Sitia, 2008). In this respect, it is therefore mainly a compartment of transit. Its use to temporarily store proteins is very uncommon and almost exclusive to plants (Herman and Schmidt, 2004). More strikingly, the permanent ER residence of proteins that do not play a role in typical ER functions seems a feature unique to the major storage proteins of Poaceae seeds, the most studied being those of cereals (Shewry and Halford, 2002).

\section{ER-LOCATED PROTEIN BODIES EVOLVED WITH GRASSES}

The Poaceae family (commonly termed grasses) originated less than 80 million years ago (Gaut, 2002), a long time after the most common classes of seed storage proteins, the $2 S$ albumins and the 7S/11S globulins universally present in seeds and even in fern spores, had appeared 
(Rödin and Rask, 1990; Shutov et al., 1998). 2S albumins and $7 \mathrm{~S} / 11 \mathrm{~S}$ globulins accumulate in protein storage vacuoles (PSVs, Vitale and Hinz, 2005), which are therefore the first subcellular compartment that evolved to store seed proteins. PSV proteins start their life in the ER and reach their destination mainly by Golgi- and endosome-mediated traffic, even if direct traffic from the ER to PSVs also occurs (Vitale and Hinz, 2005). PSVs have higher luminal $\mathrm{pH}$ and lower hydrolytic activity than vegetative, lytic vacuoles and they are ready to receive hydrolytic enzymes during germination, when they are transformed into lytic vacuoles for the rapid degradation of storage proteins.

Prolamins evolved with grasses, where they are in most cases the major, or almost exclusive, storage proteins (Shewry and Halford, 2002). Unlike the soluble 2S albumins and 7S/11S globulins, most prolamins rapidly form very large polymers that become insoluble to various extents because of inter-chain disulfide bonds or hydrophobic interactions. These electron dense, round-shaped structures with diameters between 0.5 and $2 \mu \mathrm{m}$ were originally termed protein granules (Duvick, 1961; Khoo and Wolf, 1970) and then renamed PB (Graham et al., 1962). PB often detach from the ER cisternae, but retain ribosomes on their cytosolic face and do not fuse with other subcellular structures (Burr and Burr, 1976; Yamagata and Tanaka, 1986). Rice, maize, and sorghum prolamins exclusively form $\mathrm{PB}$, whereas other prolamins, such as those of wheat, can either form $\mathrm{PB}$ or traffic through the Golgi complex to PSVs, or undergo autophagy (Rubin et al., 1992). The destiny depends on the individual prolamin, but also on the seed developmental stage: in wheat, the ability to traffic is more prominent at early stages and the formation of PB in the ER lumen favored at later stages (Tosi et al., 2009).

\section{A WIDE SUPERFAMILY OF SMALL CYS-RICH PROTEINS}

Common features of many, but not all, prolamins (Table 1) are a repeated domain rich in Pro or Gln residues (hence the name) and a domain derived from the eight-cysteine motif (8CM, José-Estanyol et al., 2004), characterized by eight Cys residues arranged in a specific order, forming four intra-chain disulfide bridges necessary to maintain a scaffold of alphahelical segments connected by variable loops. The 8CM motif is common to a wide group of proteins termed the prolamin superfamily, with different functions and localizations along the secretory pathway. Besides many prolamins, the superfamily includes cereal $\alpha$-globulins, trypsin inhibitors and $\alpha$-amylase inhibitors, as well as hybrid proline-rich proteins (HyPRPs), nonspecific lipid transfer proteins (nsLTPs) and $2 \mathrm{~S}$ albumins, the latter three classes being widespread in plants. HyPRPs are cell wall proteins. nsLTPs are also secreted, often remaining anchored to the plasma membrane via a GPI anchor. $2 \mathrm{~S}$ albumins, cereal $\alpha$-globulins, trypsin inhibitors and $\alpha$-amylase inhibitors are seed proteins accumulated in PSV. In prolamins and in the PSVlocated members of the group three conserved regions, termed $\mathrm{A}, \mathrm{B}$, and $\mathrm{C}$, can be identified within the 8CM motif, which in this case is therefore also termed ABC domain (Kreis et al., 1985).

Based on the presence or not of the $8 \mathrm{CM} / \mathrm{ABC}$ domain and additional Cys residues available for inter-chain disulfide bonds, prolamins have been divided into high molecular weight (HMW), cysteine rich (S-rich), cysteine poor (S-poor), and $\alpha$-prolamins, the latter being the only group that does not seem related to 8CM proteins (Shewry and Halford, 2002). Based on amino acid sequence similarity and phylogenetic analysis, prolamins have also been divided into three groups named I, II, and III (Xu and Messing, 2009). The two criteria indeed largely overlap: $\alpha$-prolamins are in group I, S-rich and S-poor in group II and HMW in group III. Group II members are present in all cereals. Group III is present in Pooideae (wheat, barley, Brachypodium) but not in Panicoideae (maize, sorghum, millet) and rice, whereas group $\mathrm{I}$ is only present in Panicoideae and perhaps Oryzeae.

Group III is considered the most ancient, followed by II and then I. Sequence similarity indicates that an ancestral group III prolamin may have originated by duplication of the $\alpha$-globulin gene before the divergence of the different Poaceae subfamilies (Xu and Messing, 2009; Gu et al., 2010). Sequence alignments of orthologous regions of chromosomes suggest that the absence of group III prolamins in non-Pooideae families is due to gene loss after duplication (Xu and Messing, 2009).

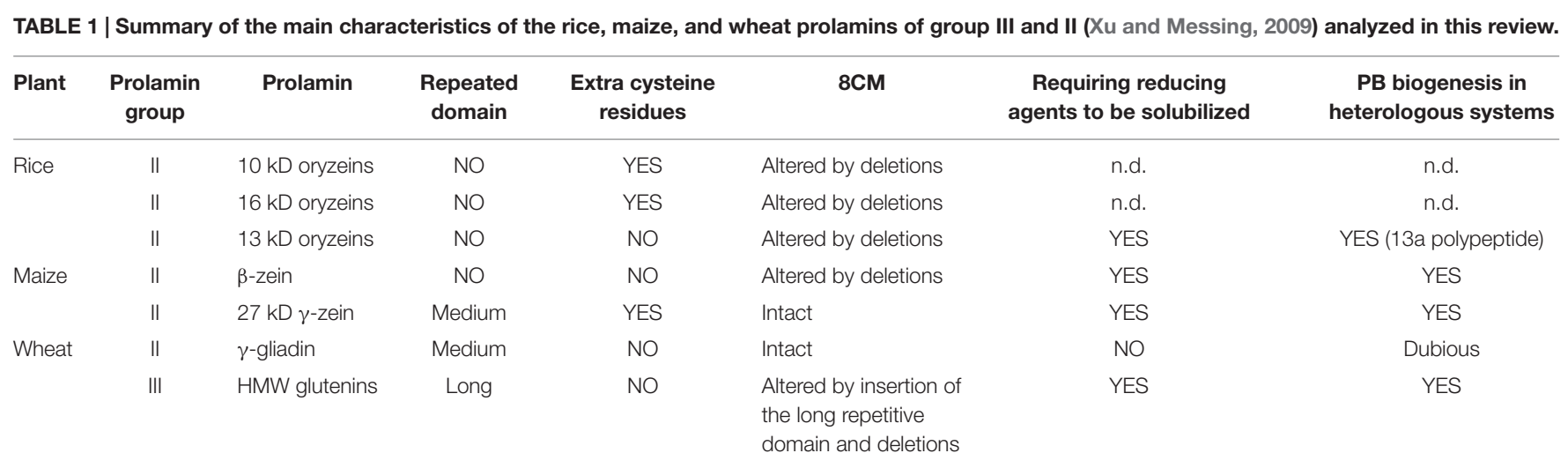

n.d.: not determined. 


\section{THE EVOLUTIONARY SHIFT FROM PSV TO THE ER}

Phylogenetic studies thus indicate that early prolamins may have evolved by insertions/mutations in a gene encoding a PSV protein, causing a tendency to shift accumulation from PSV to the ER itself. Retention in the port of entry of the secretory pathway is clearly more parsimonious than traffic to vacuoles and can therefore increase cellular fitness in tissues that spend considerable energy to accumulate storage material, but it raises two new requirements: (i) minimizing deleterious effects on ER functions, (ii) finding a new strategy for the degradation of storage proteins during germination. The rapid formation of large polymers seems to have satisfied the first requirement. For the second, a key event has probably been the change in the tissue accumulation from cotyledons to the endosperm: the latter undergoes programmed cell death at the end of maturation. The loss of membrane integrity allows access of hydrolytic enzymes synthesized and secreted by the surrounding aleurone during germination. Roles in the efficient formation of PBs are played by protein machineries specific of the endosperm (Vitale and Boston, 2008; Holding, 2014) and by elements in the coding mRNAs that target the transcripts to specific ER regions (Tian and Okita, 2014). However, a number of prolamins can efficiently form $\mathrm{PBs}$ also when expressed individually in vegetative tissues of transgenic plants (Table 1; Geli et al., 1994; Shani et al., 1994; Bagga et al., 1995; Bellucci et al., 2000; Saito et al., 2009), indicating that PB formation exploits the general folding machinery of the ER present in all tissues and that the key features to form a PB reside in prolamins themselves, as we discuss in the next two paragraphs.

\section{RICE AND WHEAT}

Rice prolamins, encoded by about twenty genes, are small polypeptides $(10,13$, or $16 \mathrm{kD})$ assigned to group II prolamins (Xu and Messing, 2009) and lacking any Pro- or Gln- rich repeated domain, indicating that the repetitions found in many other prolamins are not strictly necessary to form PBs (Onda and Kawagoe, 2011; Saito et al., 2012). A rice $\alpha$-globulin-GFP fusion that is correctly sorted to PSV instead accumulates into ER-located PBs when the formation of the disulfide bond between Cys79 in the B region and Cys171 in the $\mathrm{C}$ region is inhibited by mutagenesis (Kawagoe et al., 2005). The hypervariable loop between regions $\mathrm{B}$ and $\mathrm{C}$ is much shorter in rice prolamins than in $\alpha$-globulin, whereas in wheat HMW prolamins it is extensively elongated by the insertion of the very large repetitive domain rich in Pro and Gln (Kawagoe et al., 2005; Onda and Kawagoe, 2011). The authors suggested that these changes in length inhibit the formation of a critical intra-chain disulfide bond of the $8 \mathrm{CM}$ motif, making two Cys residues available for inter-chain disulfide bonds that allow the formation of PBs (Kawagoe et al., 2005). These insertions/deletions in $\alpha$-globulin may thus have been the oldest events leading to group III formation in Pooideae and group II formation in Pooideae and rice (Table 1). It should also be underlined that only two $13 \mathrm{kD}$ rice prolamins have the complete set of Cys of the 8CM motif and no other Cys residue, the other rice polypeptides having from 0 to 13 Cys residues in total (Onda and Kawagoe, 2011). Moreover, the two HMW prolamins of wheat ( $\mathrm{x}$-type and y-type HMW glutenin) have four to seven Cys residues, depending on the alleles. When expressed individually in transgenic tobacco these wheat prolamins form large polymers that are soluble only when reduced (Shani et al., 1994). Therefore, the loss of key Cys residues of the 8CM motif or the acquisition of new Cys residues can also be crucial for $\mathrm{PB}$ formation. Furthermore, at least once the initial rice $\mathrm{PB}$ core is formed, the rice prolamins without Cys residues can join it through interactions that are most probably of hydrophobic nature (Onda and Kawagoe, 2011).

Rice 13a prolamin has four Cys residues and is located in mid regions of the mature PB. When expressed in yeast as a GFP fusion, it forms insoluble PBs, whereas rice $\alpha$-globulin similarly tagged with GFP is delivered to the yeast vacuole (Masumura et al., 2015). Deletions studies made on GFP fusions indicated the 13a portions corresponding to the $\mathrm{B}$ or $\mathrm{C}$, but not A, regions form structures that resemble $\mathrm{PBs}$ by fluorescence microscopy, consistently with the view that the Cys residues in the $\mathrm{B}$ and $\mathrm{C}$ domains are important for $\mathrm{PB}$ formation. Unlike the full-length protein, these deletion mutants are soluble also in the absence of reducing agent, suggesting that there is not a direct causal relationship between insolubility and PB assembly. The polymerization state was not investigated, but the authors concluded that hydrophobic interactions may be important for the assembly of 13a prolamin into PBs (Masumura et al., 2015).

\section{MAIZE}

Maize prolamins (zeins) are grouped into four classes (Holding, 2014). $\alpha$-zeins, polypeptides between 19 and $22 \mathrm{kD}$ encoded by about twenty genes, are the most abundant. Three genes encode the $\gamma$-zeins $(16 \mathrm{kD}, 27 \mathrm{kD}$, which is another very abundant zein, and $50 \mathrm{kD})$, whereas $\beta$-zein $(15 \mathrm{kD})$ and $\delta$-zein $(10 \mathrm{kD}, 18 \mathrm{kD})$ are the products of one and two genes, respectively. During seed development, $\gamma$ - and $\beta$ - zeins, which belong to group II prolamins, are synthesized first. The $27 \mathrm{kD} \gamma$-zein and $\beta$-zein form $\mathrm{PBs}$ when expressed individually in transgenic vegetative tissues (Table 1; Geli et al., 1994; Bagga et al., 1995).

Treatments of isolated maize $\mathrm{PBs}$ with reducing agents solubilize the 27 and $50 \mathrm{kD} \gamma$-zeins but not the other zeins (Vitale et al., 1982). The $27 \mathrm{kD} \gamma$-zein is perhaps the best studied prolamin and plays a fundamental role in the correct formation of maize PBs. A natural duplication of its locus generates Quality Protein Maize (Wu et al., 2010; Liu et al., 2016), whereas a deletion encompassing the 27 and $50 \mathrm{kD} \gamma$-zein genes reduces PB number to $12 \%$ and alters PB morphology (Yuan et al., 2014). The hypothesis that the more recent group I prolamins exploited the PB forming ability of the more ancient ones is also supported by the finding that co-expression of $27 \mathrm{kD} \gamma$-zein enhances the accumulation of a $19 \mathrm{kD}$ a-zein polypeptide expressed in transgenic tobacco (Coleman et al., 1996). $27 \mathrm{kD} \gamma$-zein is composed of an $\mathrm{N}$-terminal domain characterized by eight repeats of the amphipathic sequence ValHisLeuProProPro and 


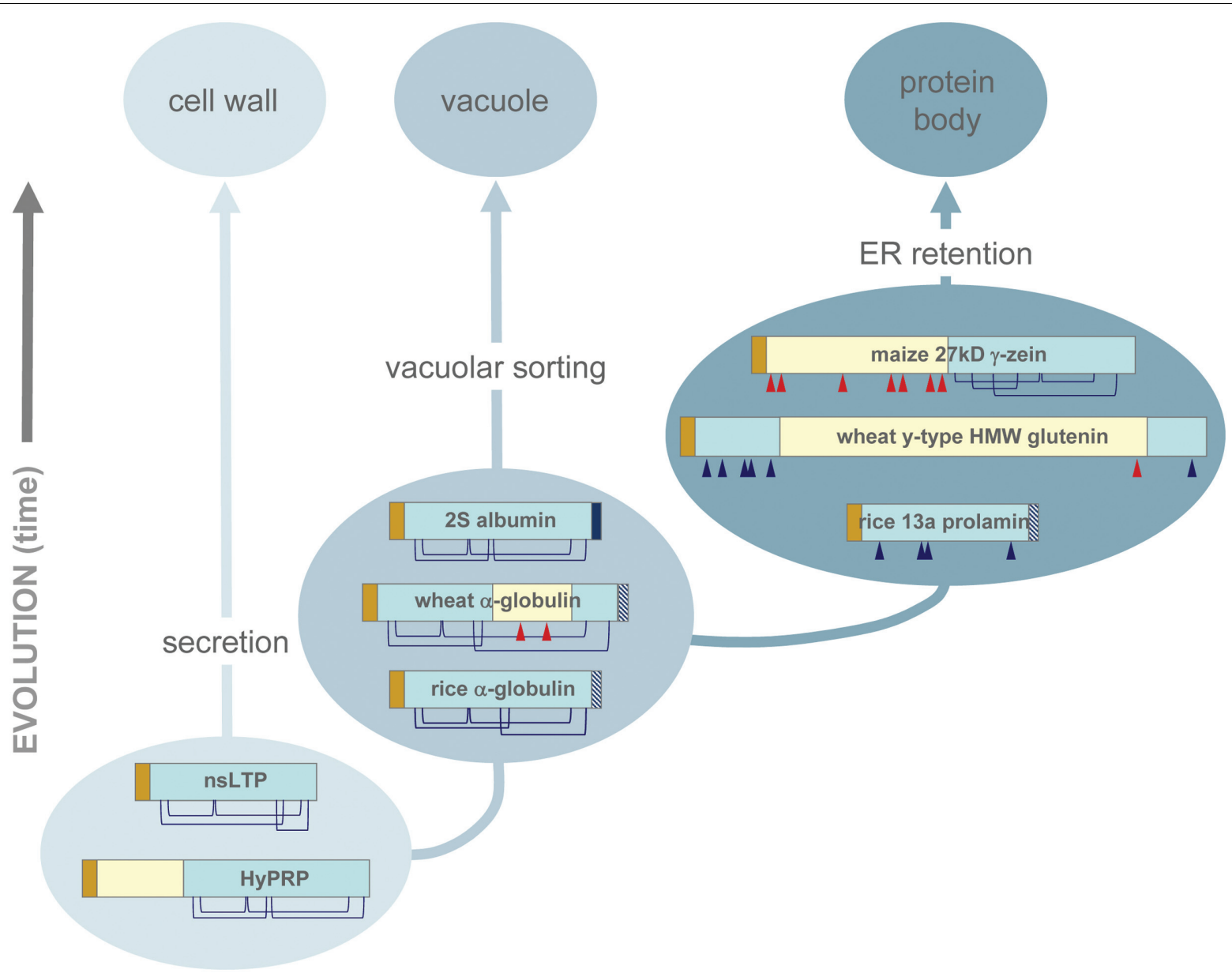

FIGURE 1 | A model of the evolutionary events at the origin of protein bodies. The model describes the origin of prolamins that are self-sufficient for PB formation and probably have a dominant effect on those that evolved later. Proteins with $8 \mathrm{CM}$ motifs are secreted or sorted to the vacuole, depending on the presence or not of vacuolar sorting signals. Insertions that do not alter the disulphide bonds of the 8CM motif do not affect protein destiny. Extensive polymerization that leads to PB formation can result from insertions that inhibit the correct formation of the four intra-chain bonds, deletions that involve the loss of critical Cys residues, or additions of domains containing new Cys residues. The repetitive domains may also mediate interactions with the ER membrane. Vacuolar sorting signals may be present or absent in PB proteins, but PB formation has anyway a dominant effect on intracellular traffic. Phylogenetic analysis and the results of protein engineering and expression of individual genes indicate that the PB forming prolamins evolved from superfamily members that are sorted to the vacuole, not from secreted members. The schematic protein structures are based on the following GenBank accessions: EU968356.1 (maize nsLTP), EU964401.1 (maize HyPRP), CAA40015.1 (2S albumin), ABG68034.1 (wheat $\alpha$-globulin), BAA09308.1 (rice $\alpha$-globulin), 27 kD zein, ABG68035.1 (wheat y-type HMW glutenin), and BAA36697.1 (rice 13a prolamin). Brown: signal peptide; light blue: conserved 8CM motif, or portions of it; yellow: Pro-rich or Glu-rich domain; blue: vacuolar sorting sequence; striped blue: putative vacuolar sorting sequence; blue lines: intra-chain disulphide bonds of the $8 \mathrm{CM}$ motif; blue triangles: Cys residues originating from the $8 \mathrm{CM}$ motif; red triangle: Cys residues that do not originate from the $8 \mathrm{CM}$ motif.

seven Cys residues, followed by a C-terminal 8CM/ABC domain. The N-terminal domain promotes PB formation when fused to other proteins that are otherwise available for intracellular traffic (Mainieri et al., 2004; Llop-Tous et al., 2010), although this dominant effect is not universal (de Virgilio et al., 2008; Ceresoli et al., 2016). Deletion of the N-terminal domain from $\gamma$-zein causes secretion of the remaining $A B C$ domain, and the reciprocal deletion causes ER retention of the $\mathrm{N}$-terminal domain, although in this case the typical round-shaped PBs are not formed (Geli et al., 1994). Zeolin, a fusion of the N-terminal domain with entire sequence of the $7 \mathrm{~S}$ storage protein phaseolin (which does not contain Cys residues), forms PBs that, like those formed by the wild type $\gamma$-zein, are insoluble unless reduced, and it traffics along the secretory pathway when cells are treated in vivo with reducing agents (Mainieri et al., 2004; Pompa and Vitale, 2006). The importance of inter-chain disulfide bonds was confirmed by mutagenesis of the full-length $\gamma$-zein: when the seven Cys residues of the $\mathrm{N}$-terminal domain were mutated to Ser, $\gamma$-zein was efficiently secreted (Mainieri et al., 2014). Progressive mutagenesis of these Cys residues gives rise to intermediate phenotypes, both in the full-length protein and a fusion between the N-terminal-domain and GFP: PBs become progressively smaller and ER retention is negatively affected (Llop-Tous et al., 2010; Mainieri et al., 2014). Deletion of the amphipathic repeats indicates that these are also important for $\mathrm{PB}$ formation (Llop-Tous et al., 2010). Altogether, these data indicate 
that the addition of an amphipathic sequence rich in Cys residues to an $8 \mathrm{CM}$ sequence has been crucial for this prolamin.

If six out of seven Cys residues of the N-terminal domain are mutated, the $27 \mathrm{kD} \gamma$-zein is in large part secreted, but a detectable proportion is sorted to the vacuole via a pathway that is sensitive to brefeldin A and wortmannin, two inhibitors of Golgi-mediated, vacuolar sorting (Mainieri et al., 2014). This indicates that limited multimerization avoids secretion and favors PSV sorting, whereas extensive polymerization promotes PB biogenesis. Protein sorting to PSVs requires sorting motifs recognized by specific receptors, but key recognition events could occur in the ER lumen and transient polymerization could contribute to sorting efficiency (Robinson et al., 2005; Robinson and Pimpl, 2014). Remarkably, the natural vacuolar sorting signal of phaseolin can be in part replaced by the insertion of a novel Cys residue that leads to polymerization of trimers (Pompa et al., 2010), underlining the evolutionary relationships between traffic to the vacuole and PB formation.

\section{A WORKING MODEL FOR THE ORIGIN OF PROTEIN BODIES}

The use of the ER to store proteins has evolved only recently and constitutes an interesting example of a simpler solution for protein accumulation (accumulation in the ER itself) evolving from more complex ones (sorting to vacuoles or secretion). Figure 1 presents its possible evolutionary paths. All the evidence obtained studying prolamins that are self-sufficient to form PBs indicates that these structures have emerged by both exploiting and remodeling the 8CM scaffold. New availability of Cys residues involved in inter-chain disulfide bonds has been critical in this process and has resulted from either of two probably independent events: extensive insertions in the hypervariable region between the $\mathrm{B}$ and $\mathrm{C}$ domains characterize group III prolamins, whereas the addition of a new Cys-containing domain to an $8 \mathrm{CM} / \mathrm{ABC}$ domain that can be virtually intact or mutated to variable extents characterizes many group II prolamins. The changes that have generated prolamins must thus have occurred more than once in evolution. Their success in being tolerated, without causing misfolding that would have led to degradation by ER quality control may be due to the rather simple folds of the small A, B, and C domains. The full explanation, however, must be more complex, since for example $7 \mathrm{~S}$ globulins have a threedimensional structure that does not tolerate great alterations (Hoffman et al., 1988; Pedrazzini et al., 1997; Lasserre et al., 2015), but zeolin is a very stable PB-forming protein fusion

\section{REFERENCES}

Anelli, T., and Sitia, R. (2008). Protein quality control in the early secretory pathway. EMBO J. 27, 315-327. doi: 10.1038/sj.emboj.7601974

Bagga, S., Hank Adams, H., John, D., Kemp, J. D., and Sengupta-Gopalan, C. (1995). Accumulation of 15-kilodalton zein in nove1 protein bodies in transgenic tobacco. Plant Physiol. 107, 13-23.

Banc, A., Desbat, B., Renard, D., Popineau, Y., Mangavel, C., and Navailles, L. (2009). Exploring the interactions of gliadins with model membranes:
(Mainieri et al., 2014) and, conversely, a proportion of at least one rice $13 \mathrm{kD}$ prolamin is detected in a ubiquitinated form in rice endosperm in which proteasome activity was inhibited, suggesting that normally this prolamin fails in part to fold properly and is degraded by ER quality control (Ohta and Taikawa, 2015).

It should be underlined that the formation of very large polymers is not per se sufficient to avoid traffic from the ER, at least in animal cells, a typical example being the secretion of procollagen fibrils (Bonfanti et al., 1998; Saito and Katada, 2015). Assembled PBs are heteropolymeric ordered structures in which certain polypeptides, such as $\gamma$ - and $\beta$-zeins and rice $13-16 \mathrm{kD}$ prolamins, are preferentially located at the PB surface (Lending and Larkins, 1989; Saito et al., 2012). Persistent interactions with chaperones and direct interactions with the luminal face of the ER membrane could thus play a major role in ER retention. Indeed, purified gliadins or a synthetic version of the Pro-rich repeat of $27 \mathrm{kD} \gamma$-zein directly interact in vitro with systems that mimic the inner face of the ER membrane (Kogan et al., 2004; Banc et al., 2009). It was suggested that also in gliadins the interactions could be mediated by the repeated region (Banc et al., 2009), even if the gliadin repeats are hydrophilic, unlike the amphipathic repeat of $\gamma$-zein, and the ability of $\gamma$-gliadin to form $\mathrm{PB}$ in the absence of other wheat prolamins is dubious (Napier et al., 1997; Tosi et al., 2009). Cell wall HyPRP proteins also have a hydrophilic, proline-rich repetitive $\mathrm{N}$-terminal domain and an 8CM C-terminal domain (Dvorakova et al., 2007), but they are clearly unable to form $\mathrm{PB}$. More detailed analysis and in vivo testing of the prolamin repetitive domains is therefore needed to determine their role in $\mathrm{PB}$ formation.

\section{AUTHOR CONTRIBUTIONS}

EP and AV wrote the manuscript. DM and CM contributed to the critical selection of the works discussed in the manuscript.

\section{FUNDING}

Supported by "Filagro" project of CNR-Regione Lombardia.

\section{ACKNOWLEDGMENT}

We thank Aldo Ceriotti, Massimilano Lauria, Angelo Viotti for the useful suggestions.

effect of confined geometry and interfaces. Biopolymers 91, 610-622. doi: 10.1002/bip.21188

Bellucci, M., Alpini, A., Paolocci, F., Cong, L., and Arcioni, S. (2000). Accumulation of maize $\gamma$-zein and $\gamma$-zein:KDEL to high levels in tobacco leaves and differential increase of BiP synthesis in transformants. Theor. Appl. Genet. 101, 796-804. doi: 10.1007/s001220051546

Bonfanti, L., Mironov, A. A. Jr., Martinez-Menarguez, J. A., Martella, O., Fusella, A., Baldassarre, M., et al. (1998). Procollagen traverses the Golgi stack without leaving the lumen of cisternae: evidence for 
cisternal maturation. Cell 95, 993-1003. doi: 10.1016/S0092-8674(00) 81723-7

Burr, B., and Burr, F. A. (1976). Zein synthesis in maize endosperm by polyribosomes attached to protein bodies. Proc. Natl. Acad. Sci. U.S.A. 73, 515-519. doi: 10.1073/pnas.73.2.515

Ceresoli, V., Mainieri, D., Del Fabbro, M., Weinstein, R., and Pedrazzini, E. (2016). A fusion between domains of the human bone morphogenetic protein-2 and maize $27 \mathrm{kD} \gamma$-Zein accumulates to high levels in the endoplasmic reticulum without forming protein bodies in transgenic tobacco. Front. Plant Sci. 7:358. doi: 10.3389/fpls.2016.00358

Coleman, C. E., Herman, E. M., Takasaki, K., and Larkins, B. A. (1996). The maize $\gamma$-zein sequesters $\alpha$-zein and stabilizes its accumulation in protein bodies of transgenic tobacco endosperm. Plant Cell 8, 2335-2345. doi: $10.1105 /$ tpc.8.12.2335

de Virgilio, M., De Marchis, F., Bellucci, M., Mainieri, D., Rossi, M., Benvenuto, E., et al. (2008). The human immunodeficiency virus antigen Nef forms protein bodies in leaves of transgenic tobacco when fused to zeolin. J. Exp. Bot. 59, 2815-2829. doi: 10.1093/jxb/ern143

Duvick, D. N. (1961). Protein granules of maize endosperm cells. Cereal Chem. 38, 374-385.

Dvorakova, L., Cvrckova, F., and Fischer, L. (2007). Analysis of the hybrid prolinerich protein families from seven plant species suggests rapid diversification of their sequences and expression patterns. BMC Genomics 8:412. doi: 10.1186/1471-2164-8-412

Gaut, B. S. (2002). Evolutionary dynamics of grass genomes. New Phytol. 154, 15-28. doi: 10.1046/j.1469-8137.2002.00352.x

Geli, M. I., Torrent, M., and Ludevid, D. (1994). Two structural domains mediate two sequential events in $\gamma$-zein targeting: protein endoplasmic reticulum retention and protein body formation. Plant Cell 6, 1911-1922. doi: 10.1105/tpc.6.12.1911

Graham, J. S. D., Jennings, A. C., Morton, R. K., Palk, B. A., and Raison, J. K. (1962). Protein bodies and protein synthesis in developing wheat endosperm. Nature 196, 967-969. doi: 10.1038/196967a0

Gu, Y. Q., Wanjugi, H., Coleman-Derr, D., Kong, X., and Anderson, O. A. (2010). Conserved globulin gene across eight grass genomes identify fundamental units of the loci encoding seed storage proteins. Funct. Integr. Genomics 10, 111-122. doi: 10.1007/s10142-009-0135-x

Herman, E. M., and Schmidt, M. A. (2004). Endoplasmic reticulum to vacuole trafficking of endoplasmic reticulum bodies provides an alternate pathway for protein transfer to the vacuole. Plant Physiol. 136, 3440-3446. doi: 10.1104/pp.104.051722

Hoffman, L. M., Donaldson, D. D., and Herman, E. M. (1988). A modified storage protein is synthesized, processed, and degraded in the seeds of transgenic plants. Plant Mol. Biol. 11, 717-729. doi: 10.1007/BF00019513

Holding, D. R. (2014). Recent advances in the study of prolamin storage protein organization and function. Front. Plant Sci. 5:276. doi: 10.3389/fpls.2014.00276

José-Estanyol, M., Gomis-Rüth, F., and Puigdomènech, P. (2004). The eightcysteine motif, a versatile structure in plant proteins. Plant Physiol. Biochem. 42, 355-365. doi: 10.1016/j.plaphy.2004.03.009

Kawagoe, Y., Suzuki, K., Tasaki, M., Yasuda, H., Akagi, K., Katoh, E., et al. (2005). The critical role of disulfide bond formation in protein sorting in the endosperm of rice. Plant Cell 17, 1141-1153. doi: 10.1105/tpc.105.030668

Khoo, U., and Wolf, M. J. (1970). Origin and development of protein granules in maize endosperm. Am. J. Bot. 57, 1042-1050. doi: 10.2307/2441269

Kogan, M. J., Lopez, O., Cocera, M., Lopez-Iglesias, C., De La Maza, A., and Giralt, E. (2004). Exploring the interaction of the surfactant N-terminal domain of gamma-Zein with soybean phosphatidylcholine liposomes. Biopolymers 73 , 258-268. doi: 10.1002/bip.10578

Kreis, M., Forde, B. G., Rahman, S., Miflin, B. J., and Shewry, P. R. (1985). Molecular evolution of the seed storage proteins of barley, rye and wheat. J. Mol. Biol. 183, 499-502. doi: 10.1016/0022-2836(85)90017-8

Lasserre, E., Ko, T., Dyer, J., and Murai, N. (2015). Modified bean seed protein phaseolin did not accumulate stably in transgenic tobacco seeds after methionine enhancement mutations. Am. J. Plant Sci. 6, 640-650. doi: 10.4236/ajps.2015.65069

Lending, C. R., and Larkins, B. A. (1989). Changes in the zein composition of protein bodies during maize endosperm development. Plant Cell 1, 1011-1023. doi: $10.2307 / 3869002$
Liu, H., Shi, J., Sun, C., Gong, H., Fan, X., Qiu, F., et al. (2016). Gene duplication confers enhanced expression of $27-\mathrm{kDa} \gamma$-zein for endosperm modification in quality protein maize. Proc. Natl. Acad. Sci. U.S.A. 113, 4964-4969. doi: $10.1073 /$ pnas. 1601352113

Llop-Tous, I., Madurga, S., Giralt, E., Marzabal, P., Torrent, M., and Ludevid, M. D. (2010). Relevant elements of a maize $\gamma$-zein domain involved in protein body biogenesis. J. Biol. Chem. 285, 35633-35644. doi: 10.1074/jbc.M110. 116285

Mainieri, D., Morandini, F., Maîtrejean, M., Saccani, A., Pedrazzini, E., and Vitale, A. (2014). Protein body formation in the endoplasmic reticulum as an evolution of storage protein sorting to vacuoles: insights from maize $\gamma$-zein. Front. Plant Sci. 5:331. doi: 10.3389/fpls.2014.00331

Mainieri, D., Rossi, M., Archinti, M., Bellucci, M., De Marchis, F., Vavassori, S., et al. (2004). Zeolin: a new recombinant storage protein constructed using maize $\gamma$-zein and bean phaseolin. Plant Physiol. 136, 3447-3456. doi: 10.1104/pp.104.046409

Masumura, T., Shigemitsu, T., Morita, S., and Satoh, S. (2015). Identification of the region of rice $13 \mathrm{kDa}$ prolamin essential for the formation of ER-derived protein bodies using a heterologous expression system. Biosci. Biotechnol. Biochem. 79, 566-573. doi: 10.1080/09168451.2014.991684

Napier, J. A., Richard, G., Turner, M. F. P., and Shewry, P. R. (1997). Trafficking of wheat gluten proteins in transgenic tobacco plants: $\gamma$-gliadin does not contain an endoplasmic reticulum retention signal. Planta 203, 488-494. doi: $10.1007 / \mathrm{s} 004250050218$

Ohta, M., and Taikawa, F. (2015). OsHrd3 is necessary for maintaining the quality of endoplasmic reticulum-derived protein bodies in rice endosperm. J. Exp. Bot. 66, 4585-4593. doi: 10.1093/jxb/erv229

Onda, Y., and Kawagoe, Y. (2011). Oxidative protein folding: selective pressure for prolamin evolution in rice. Plant Signal. Behav. 6, 1966-1972. doi: 10.4161/psb.6.12.17967

Pedrazzini, E., Giovinazzo, G., Bielli, A., de Virgilio, M., Frigerio, L., Pesca, M., et al. (1997). Protein quality control along the route to the plant vacuole. Plant Cell 9, 1869-1880. doi: 10.2307/3870531

Pompa, A., De Marchis, F., Vitale, A., Arcioni, S., and Bellucci, M. (2010). An engineered C-terminal disulfide bond can partially replace the phaseolin vacuolar sorting signal. Plant J. 61, 782-791. doi: 10.1111/j.1365-313X.2009.04113.x

Pompa, A., and Vitale, A. (2006). Retention of a bean phaseolin/maize $\gamma$-zein fusion in the endoplasmic reticulum depends on disulfide bond formation. Plant Cell 18, 2608-2621. doi: 10.1105/tpc.106.042226

Robinson, D. G., Oliviusson, P., and Hinz, G. (2005). Protein sorting to the storage vacuoles of plants: a critical appraisal. Traffic 6, 615-625. doi: 10.1111/j.16000854.2005.00303.x

Robinson, D. G., and Pimpl, P. (2014). Receptor-mediated transport of vacuolar proteins: a critical analysis and a new model. Protoplasma 251, 247-264. doi: 10.1007/s00709-013-0542-7

Rödin, J., and Rask, L. (1990). Characterization of matteuccin, the 2.2S storage protein of the ostrich fern. Evolutionary relationship to angiosperm seed storage proteins. FEBS J. 192, 101-107. doi: 10.1111/j.1432-1033.1990.tb19201.x

Rubin, R., Levanony, H., and Galili, G. (1992). Evidence for the presence of 2 different types of protein bodies in wheat endosperm. Plant Physiol. 99, 718-724. doi: 10.1104/pp.99.2.718

Saito, K., and Katada, T. (2015). Mechanisms for exporting large-sized cargoes from the endoplasmic reticulum. Cell. Mol. Life Sci. 72, 3709-3720. doi: 10.1007/s00018-015-1952-9

Saito, Y., Kishida, K., Takata, K., Takahashi, H., Shimada, T., Tanaka, K., et al. (2009). A green fluorescent protein fused to rice prolamin forms protein body-like structures in transgenic rice. J. Exp. Bot. 60, 615-627. doi: 10.1093/jxb/ern311

Saito, Y., Shigemitsu, T., Yamasaki, R., Sasou, A., Goto, F., Kishida, K., et al. (2012). Formation mechanism of the internal structure of type I protein bodies in rice endosperm: relationship between the localization of prolamin species and the expression of individual genes. Plant J. 70, 1043-1055. doi: 10.1111/j.1365313X.2012.04947.x

Shani, N., Rosenberg, N., Kasarda, D. D., and Galili, G. (1994). Mechanisms of assembly of wheat high molecular weight glutenins inferred from expression of wild-type and mutant subunits in transgenic tobacco. J. Biol. Chem. 269, 8924-8930. 
Shewry, P. R., and Halford, N. G. (2002). Cereal seed storage proteins: structures, properties and role in grain utilization. J. Exp. Bot. 53, 947-958. doi: $10.1093 /$ jexbot/53.370.947

Shutov, A. D., Braun, H., Chesnokov, Y. V., and Bäumlein, H. (1998). A gene encoding a vicilin-like protein is specifically expressed in fern spores. Eur. J. Biochem. 252, 79-89. doi: 10.1046/j.1432-1327.1998.25 20079.x

Tian, L., and Okita, T. W. (2014). mRNA-based protein targeting to the endoplasmic reticulum and chloroplasts in plant cells. Curr. Opin. Plant Biol. 22, 77-85. doi: 10.1016/j.pbi.2014. 09.007

Tosi, P., Parker, M., Gritsch, C. S., Carzaniga, R., Martin, B., and Shewry, P. R. (2009). Trafficking of storage proteins in developing grain of wheat. J. Exp. Bot. 60, 979-991. doi: 10.1093/jxb/ern346

Vitale, A., and Boston, R. S. (2008). Endoplasmic reticulum quality control and the unfolded protein response: insights from plants. Traffic 9, 1581-1588. doi: 10.1111/j.1600-0854.2008.00780.x

Vitale, A., and Hinz, G. (2005). Sorting of proteins to storage vacuoles: how many mechanisms? Trends Plant Sci. 10, 316-323. doi: 10.1016/j.tplants.2005. 05.001

Vitale, A., Smaniotto, E., Longhi, R., and Galante, E. (1982). Reduced soluble proteins associated with maize endosperm protein bodies. J. Exp. Bot. 33, 439-448. doi: 10.1093/jxb/33.3.439
Wu, Y., Holding, D. R., and Messing, J. (2010). Gamma-zein is essential for endosperm modification in quality protein maize. Proc. Natl. Acad. Sci. U.S.A. 107, 12810-12815. doi: 10.1073/pnas.1004721107

Xu, J.-H., and Messing, J. (2009). Amplification of prolamin storage protein genes in different subfamilies of the Poaceae. Theor. Appl. Genet. 119, 1397-1412. doi: 10.1007/s00122-009-1143-x

Yamagata, H., and Tanaka, K. (1986). The site of synthesis and accumulation of rice storage proteins. Plant Cell Physiol. 27, 135-145.

Yuan, L., Dou, Y., Kianian, S. F., Zhang, C., and Holding, D. R. (2014). Deletion mutagenesis identifies a haploinsufficient role for $\gamma$-zein in opaque2 endosperm modification. Plant Physiol. 164, 119-130. doi: 10.1104/pp.113. 230961

Conflict of Interest Statement: The authors declare that the research was conducted in the absence of any commercial or financial relationships that could be construed as a potential conflict of interest.

Copyright (C) 2016 Pedrazzini, Mainieri, Marrano and Vitale. This is an open-access article distributed under the terms of the Creative Commons Attribution License (CC BY). The use, distribution or reproduction in other forums is permitted, provided the original author(s) or licensor are credited and that the original publication in this journal is cited, in accordance with accepted academic practice. No use, distribution or reproduction is permitted which does not comply with these terms. 


\title{
Bacterial Communities in the Rhizosphere of Amilaceous Maize (Zea mays L.) as Assessed by Pyrosequencing
}

\author{
David Correa-Galeote ${ }^{1 *}$, Eulogio J. Bedmar ${ }^{*}$, Antonio J. Fernández-González", \\ Manuel Fernández-López ${ }^{1}$ and Gregorio J. Arone ${ }^{2}$
}

1 Department of Soil Microbiology and Symbiotic Systems, Estación Experimental del Zaidín, Agencia Estatal Consejo Superior de Investigaciones Cientificas, Granada, Spain, ${ }^{2}$ Department of Agricultural Sciences, National University of Huancavelica, Huancavelica, Peru

\section{OPEN ACCESS}

Edited by:

Antonio M. De Ron,

Spanish National Research Council,

Spain

Reviewed by:

Birgit Mitter,

Austrian Institute of Technology,

Austria

Christel Baum,

University of Rostock, Germany

Diego Flores,

Colegio de Postgraduados, Mexico

*Correspondence:

David Correa-Galeote david.correa@eez.csic.es

Eulogio J. Bedmar

eulogio.bedmar@eez.csic.es

Specialty section:

This article was submitted to Crop Science and Horticulture,

a section of the journal

Frontiers in Plant Science

Received: 29 April 2016

Accepted: 27 June 2016

Published: 29 July 2016

Citation:

Correa-Galeote D, Bedmar EJ,

Fernández-González AJ,

Fernández-López M and Arone Gu

(2016) Bacterial Communities

in the Rhizosphere of Amilaceous

Maize (Zea mays L.) as Assessed by

Pyrosequencing.

Front. Plant Sci. 7:1016

doi: 10.3389/fpls.2016.01016
Maize (Zea mays L.) is the staple diet of the native peasants in the Quechua region of the Peruvian Andes who continue growing it in small plots called chacras following ancestral traditions. The abundance and structure of bacterial communities associated with the roots of amilaceous maize has not been studied in Andean chacras. Accordingly, the main objective of this study was to describe the rhizospheric bacterial diversity of amilaceous maize grown either in the presence or the absence of bur clover cultivated in soils from the Quechua maize belt. Three 16S rRNA gene libraries, one corresponding to sequences of bacteria from bulk soil of a chacra maintained under fallow conditions, the second from the rhizosphere of maize-cultivated soils, and the third prepared from rhizospheric soil of maize cultivated in intercropping with bur clover were examined using pyrosequencing tags spanning the V4 and V5 hypervariable regions of the gene. A total of 26031 sequences were found that grouped into 5955 distinct operational taxonomic units which distributed in 309 genera. The numbers of OTUs in the libraries from the maize-cultivated soils were significantly higher than those found in the libraries from bulk soil. One hundred ninety seven genera were found in the bulk soil library and 234 and 203 were in those from the maize and maize/bur clover-cultivated soils. Sixteen out of the 309 genera had a relative abundance higher than $0.5 \%$ and the were (in decreasing order of abundance) Gp4, Gp6, Flavobacterium, Subdivision3 genera incertae sedis of the Verrucomicrobia phylum, Gemmatimonas, Dechloromonas, Ohtaekwangia, Rhodoferax, Gaiella, Opitutus, Gp7, Spartobacteria genera incertae sedis, Terrimonas, Gp5, Steroidobacter and Parcubacteria genera incertae sedis. Genera Gp4 and Gp6 of the Acidobacteria, Gemmatimonas and Rhodoferax were the most abundant in bulk soil, whereas Flavobacterium, Dechloromonas and Ohtaekwangia were the main genera in the rhizosphere of maize intercropped with bur clover, and Gp4, Subdivision3 genera incertae sedis of phylum Verrucomicrobia, Gp6 and Rhodoferax were the main genera in the rhizosphere of maize plants. Taken together, our results suggest that bur clover produces specific changes in rhizospheric bacterial diversity of amilaceous maize plants.

Keywords: maize rhizospheric soil, bacterial community, pyrosequncing, 16S rRNA gene, Andean chacras 


\section{INTRODUCTION}

Maize (Zea mays L.) reached the southern Andean highlands after its domestication in Mexico some 8700 years before the present (Piperno et al., 2009; van Heerwaarden et al., 2011; Grobman et al., 2012), and is since then the staple diet of the Quechua natives. Despite 500 years of Western colonization and national modernization efforts, native peasants continue growing amilaceous maize as did their ancestors, mostly without chemical fertilization and no irrigation. Most farmers (94.3\%) do not use certified seeds because the fields are planted with their own community seeds (Instituto Nacional de Estadística e Informática [INEI], 2012), which include more than 1600 entries grouped in 55 races (Manrique, 1997).

It is well established that, in addition to mineral fertilizers, which are not usually employed in the Quechua agricultural practices, $\mathrm{N}$ inputs to agriculture can be obtained from symbiotic nitrogen fixation by rhizobial bacteria inside root nodules of leguminous plants (Sprent, 2009), and that rhizobia associated with feed/fodder legumes contribute a substantial proportion of this fixed nitrogen (Herridge et al., 2008). In the traditional Quechua farming, amilaceous maize seeds are used for sowing at each growing season and plants grow associated with innately emerged bur clover (Medicago hispida, Gaernt. syn. M. polymorpha L.), an annual shrub of the Fabaceae family within the tribe Trifolieae which, though native to the Mediterranean basin, is distributed worldwide. In the Peruvian Andes bur clover grows up to almost $4000 \mathrm{~m}$ above sea level where it is also widely used as a pasture and as a cover crop, greatly contributing to the fodder units required for feeding of the national cattle. The microsymbiont from nodules of bur clover has been isolated and identified as Ensifer medicae, and the rhizobial-M. hispida symbiotic association has been suggested to be the main $\mathrm{N}$ source for growth of maize plants in the Andean chacras (Arone et al., 2014).

The plant rhizosphere is a complex environment where soil microbial communities play a key role in ecosystems functions and are among the most complex, diverse and important assemblages in the biosphere. Accordingly, the study of plant-associated microorganisms is of great interest for their contribution to plant nutrition, hormonal control of plant growth, disease suppression, etc. (Bastida et al., 2009). Many studies on the composition and community structure of plant rhizosphere have been performed by using culturedependent techniques and low-resolution molecular methods such as DGGE, TGGE, PLFA and SSCP profiles of microbial communities. Although isolation of culturable bacteria is appropriate for functional analysis, this approach often shows a rather limited diversity because a high percentage of naturally occurring bacteria remains in a non-culturable state (Oliver, 2000; Torsvik et al., 2002). Culture-independent molecular methods provide additional information on the diversity of bacterial communities by analyzing and comparing a very large number of samples. Among them, the bar-coded pyrosequencing technology introduced by 454 Life Science (Margulies et al., 2005; Rothberg and Leamon, 2008) describes microbial community composition in complex habitats such as the deep sea (Sogin et al., 2006; Huse et al., 2008; Polymenakou et al., 2015), human microbiome (Sundquist et al., 2007; Dethlefsen et al., 2008; Claesson et al., 2009; Hang et al., 2014), coastal microbial mats (Bolhuis and Stal, 2011; Tytgat et al., 2014) and dental implants (Kumar et al., 2012; Dabdoub et al., 2013), and soil (Uroz et al., 2010; Mao et al., 2011; Barberán et al., 2012; Sun et al., 2014; Sengupta and Dick, 2015).

Previous studies have analyzed bacterial taxa associated with maize. Some of these studies have been focused on the culturable fraction (Rai et al., 2007; Rijavec et al., 2007; Pereira et al., 2009) and others have assessed bacterial diversity independently of culture (Schmalenberger and Tebbe, 2003; Herschkovitz et al., 2005a,b; Sanguin et al., 2006a,b; Pereira et al., 2011; Ikeda et al., 2013). New generation sequencing has also been used to analyze the diversity and heritability of the maize rhizosphere microbiome under field conditions (Peiffer et al., 2013) and the importance of rare taxa for bacterial diversity in the rizosphere of Bt- and conventional maize varieties (Dohrmann et al., 2013). Because bacterial diversity in roots of amilaceous maize grown in Andean chacras has not been studied using high throughput sequencing, the main objective of this work was to describe the abundance and structure of bacterial communities associated with the roots of amilaceous maize by pyrosequencing. Given that amilaceous maize grows together with bur clover, we hypothesized that plant cultivation type could affect abundance and composition of maize rhizospheric bacteria. To test this hypothesis, we have analyzed changes on rhizospheric bacterial diversity in the absence and the presence of bur clover.

\section{MATERIALS AND METHODS}

\section{Site Description and Soil Sampling}

Chacras are small parcels of soil $\left(200-10000 \mathrm{~m}^{2}\right)$ used by Quechua peasants to grow maize, quinoa, wheat, potatoes, and others cereals and vegetables. Soils were taken from three chacras within the same soil plot located near Allpas ( $12^{\circ} 50^{\prime} 27^{\prime \prime} \mathrm{S}$, $74^{\circ} 34^{\prime} 14^{\prime \prime} \mathrm{W}$, at $3537 \mathrm{~m}$ above sea level), a village in the province of Acobamba (Huancavelica, Peru). At sampling time, one chacra had been cultivated with amilaceous maize (M soil) for 3 years, other with maize and bur clover (MT soil) for 5 years, and the third was bulk soil from a chacra under fallow conditions (B soil) for at least 3 years. Seeds of $Z$. mays $\mathrm{L}$. morphotype Qarway were planted by mid October 2012 and soil samples collected 120 days later, when the plants were at the grain filling stage. At the second hilling stage, 75-90 days after planting, bur clover looms up without previous sowing and has to be removed by hand to maintain the chacras free of the legume. Lateral roots ( $\sim 2 \mathrm{~mm}$ diameter, $2-3 \mathrm{~cm}$ long; $5-10 \mathrm{~cm}$ depth) were taken from maize plants grown at four different sites within each chacra and four replicates were sampled for each site. After cleaning of the soil attached to the roots, the remaining adhering rhizospheric soil was carefully removed and pooled together to obtain $2 \mathrm{~g}$. Bulk soil samples (four sites, four replicates/site; $0-5 \mathrm{~cm}$ depth) were taken from the chacra under fallow conditions. Soil samples were kept at $-20^{\circ} \mathrm{C}$ until further processing. The three chacras belong to the same soil plot, which 
has a sandy-loam texture (62.5\% sand, $30.0 \%$ silt, $7.5 \%$ clay); the average $\mathrm{pH}$ was 6.3 and that of the soil organic matter was $28.7 \mathrm{~g} / \mathrm{soil} \mathrm{kg}$.

\section{Extraction of DNA from Soil}

DNA was extracted from $250 \mathrm{mg}$ of unfrozen soil as previously indicated (Correa-Galeote et al., 2013). Essentially, samples were homogenized in $1 \mathrm{ml}$ of extraction buffer containing 100 mM Tris (pH 8.0), 100 mM EDTA, $100 \mathrm{mM} \mathrm{NaCl}, 1 \%$ $(\mathrm{w} / \mathrm{v})$ polyvinylpyrrolidone and $2 \%(\mathrm{w} / \mathrm{v})$ sodium dodecyl sulfate using a $2-\mathrm{ml}$ mini-bead-beater tube containing $0.5 \mathrm{~g}$ and $0.1 \mathrm{~g}$ of $106-\mu \mathrm{m}$ - and 2-mm-diameter glass beads, respectively, for $60 \mathrm{~s}$ at $1600 \mathrm{rpm}$. Cell debris was eliminated by centrifugation (14000 rpm for $5 \mathrm{~min}$ at $4^{\circ} \mathrm{C}$ ). Proteins were removed by treatment with $5 \mathrm{M}$ sodium acetate. After treatment for $12 \mathrm{~h}$ with ice-cold isopropanol, nucleic acids were precipitated by centrifugation ( $14000 \mathrm{rpm}$ for $30 \mathrm{~min}$ at $4^{\circ} \mathrm{C}$ ), washed with $70 \%$ ice-cold ethanol, recentrifruged (14000 rpm for $15 \mathrm{~min}$ at $4^{\circ} \mathrm{C}$ ) and air-dried for $30 \mathrm{~min}$. Finally, DNA was purified using GeneClean columns (Qiagen). Quality and size of DNA were checked by electrophoresis on $1 \%$ agarose and quantified by spectrophotometry at $260 \mathrm{~nm}$ using a Nanodrop spectrophotometer (NanoDrop ND1000).

\section{Amplification and Pyrosequencing of DNA from Maize Roots}

Polymerase chain reaction (PCR) amplification of the hypervariable V4-V5 regions of the 16S rRNA gene was performed over each individual DNA extraction from soils using universal primers U519F and U926R (Baker et al., 2003) joined to a multiplex identifier sequence (Binladen et al., 2007; Parameswaran et al., 2007). For each sample, amplicons were generated in several replicate PCRs using mixtures $(25 \mu \mathrm{l})$ that contained 25 pmol of each primer, $1.8 \mathrm{mM} \mathrm{MgCl}_{2}, 0.2 \mathrm{mM}$ dNTPs, $1 \times$ the corresponding Taq buffer, $1 \mathrm{U}$ of Taq Master (5 Prime, USA) and $10 \mathrm{ng}$ of the DNA template. The PCR program consisted of an initial denaturation step at $94^{\circ} \mathrm{C}$ for $4 \mathrm{~min}, 25$ cycles of denaturation at $94^{\circ} \mathrm{C}$ for $15 \mathrm{~s}$, primer annealing at $55^{\circ} \mathrm{C}$ for $45 \mathrm{~s}$ and extension at $72^{\circ} \mathrm{C}$ for $1 \mathrm{~min}$, followed by a final step of heating at $72^{\circ} \mathrm{C}$ for $10 \mathrm{~min}$. Amplicons of the same treatment were pooled to reduce per-PCR variability and purified using the ultracentrifugal filters Ultracel-100 K membranes (Amicon) according to the manufacturer's instructions. After quantification by Nanodrop ND1000 and visualization of the DNA by agarose electrophoresis, the samples were combined in equimolar amounts and pyrosequenced in a Roche Genome Sequencer FLX system using 454 Titanium chemistry at LifeSequencing S.L. (Valencia, Spain).

\section{Taxonomic Assignment of Sequence Reads and Diversity Indexes}

Raw sequences were processed through the Ribosomal Database Project (RDP) pyrosequencing pipeline ${ }^{1}$ release 11 (Cole et al., 2014). Sequences were trimmed for primers, filtered and assigned

${ }^{1}$ http://pyro.cme.msu.edu to three libraries (BG, MG and MTG containing 16S rRNA gene sequences from $\mathrm{B}, \mathrm{M}$ and $\mathrm{MT}$ soils, respectively) according to their tags. Sequences shorter than 150 base pair, with quality scores $<20$ or containing any unresolved nucleotides were removed from the dataset. Chimeras were identified using the Uchime tool from FunGene database (Edgar et al., 2011) and removed from the dataset. Sequences were aligned using the Infernal alignment tool in RDP (Nawrocki et al., 2009). Aligned sequences were clustered into operational taxonomic units (OTUs) defined at 97\% similarity cutoff using Complete Linkage Clustering RDP tool and their relative abundances calculated. The number of sequences in each OTU was employed to calculate the Good's coverage index, which is considered a relative measure of how well the sequences obtained represent the entire populations (Hughes and Bohannan, 2004). The RDP Classifier, a Bayesian rRNA classifying algorithm (Wang et al., 2007), was used to assign phylogenetic groups based on sequence similarity. Matches with an RDP confidence estimate below $60 \%$ were designated as unclassified bacteria. Shannon $\left(\mathrm{H}^{\prime}\right)$ and Simpson $\left(\mathrm{S}^{\prime}\right)$ diversity indexes and Jaccard indexes $\left(J_{\text {class }}\right.$ and $J_{\text {abund }}$ ) were used to analyze the alpha- and beta-diversity, respectively (Chao et al., 2005).

\section{Statistical Analyses}

Relative abundances of the main genera were compared using the $G$-test (w/Yates') + Fischer's test in the STAMP software (Parks et al., 2014). Comparisons among the total number of sequences in the genomic libraries were done using the Newcombe-Wilson method (Newcombe, 1998; Parks and Beiko, 2010).

\section{Accession Numbers}

Pyrosequencing reads have been sent to GenBank to obtain their under accession numbers. They are available as KX479121KX485313, KX559454-KX570596 and KX470813-KX479120 for the genomic libraries MTG, MG and BG, respectively.

\section{RESULTS}

\section{Pyrosequencing and Sequence Analysis}

A total of 38786 sequences were obtained from the three 16S rDNA samples sent to pyrosequencing, of which 26031 were retained after filtering and removing chimeras. The mean number of total retained sequences per library was 8677 , ranging from 6286 to 11387 . Average length of retained sequences was

TABLE 1 | Number of OTUs, values of Good's coverage index, and Shannon and Simpson biodiversity indexes in genomic libraries from bulk soil (BG) and rhizospheric soil of maize (MG) and maize intercropped with clover (MTG) grown in Andean chacras.

\begin{tabular}{lccc}
\hline Diversity index & MTG & MG & BG \\
\hline Number of OTUs & 1846 & 3287 & 2318 \\
Good's coverage & 85.58 & 84.92 & 86.44 \\
Shannon & 6.55 & 7.21 & 7.06 \\
Simpson & 0.0056 & 0.0031 & 0.0016
\end{tabular}


TABLE 2 | Number of taxa and of 16S rRNA sequences in genomic libraries from bulk soil (BG) and rhizospheric soil of maize (MG) and maize intercropped with clover (MTG) grown in Andean chacras.

\begin{tabular}{|c|c|c|c|c|c|c|}
\hline & \multicolumn{6}{|c|}{ Genomic Libraries } \\
\hline & \multicolumn{2}{|c|}{ MTG } & \multicolumn{2}{|c|}{ MG } & \multicolumn{2}{|c|}{ BG } \\
\hline & Number of taxa & $\begin{array}{c}\text { Number of } \\
\text { sequences (\%) }\end{array}$ & Number of taxa & $\begin{array}{c}\text { Number of } \\
\text { sequences (\%) }\end{array}$ & Number of taxa & $\begin{array}{c}\text { Number of } \\
\text { sequences (\%) }\end{array}$ \\
\hline Phylum & 21 & $5711(90.85)$ & 23 & $9594(84.26)$ & 21 & $6898(82.53)$ \\
\hline Class & 41 & $5066(80.59)$ & 44 & $8390(73.68)$ & 42 & $8358(75.93)$ \\
\hline Order & 43 & 3933 (62.57) & 51 & $5136(45.10)$ & 45 & 3391 (39.97) \\
\hline Family & 43 & 3607 (57.38) & 51 & $4141(36.37)$ & 45 & $2620(31.35)$ \\
\hline Genus & 203 & $3131(49.81)$ & 234 & $5650(49.62)$ & 197 & $4003(47.89)$ \\
\hline Unclassified sequences & & $575(9.15)$ & & $1793(15.75)$ & & $1460(17.47)$ \\
\hline
\end{tabular}

The percentage of sequences in relation with the total number of sequences is shown in brackets.

$374 \pm 5$ base pair (mean \pm SD). All the sequences aligned correctly in the expected position of the 16S rDNA sequence of Escherichia coli and were grouped at 97\% similarity in 5955 distinct OTUs, of which 1846, 3287 and 2318 were found in MTG, MG and BG libraries, respectively (Table 1), which also contained 575 (9.15\%), 1793 (15.75\%), and 1460 (17.47\%) unclassified sequences, respectively (Table 2; Figure 1). The remaining valid sequences distributed into 21,23 , and 21 phyla, respectively (Table 2 ), of which phyla Proteobacteria, Bacteroidetes, Acidobacteria, Actinobacteria, Firmicutes, Verrucomicrobia, Gemmatimonadetes, Planctomycetes, Chloroflexi, Armatimonadetes, Parcubacteria, Nitrospirae, Candidatus Saccharibacteria, candidate division WPS2, candidate division WPS-1, Latescibacteria, BRC1 and Microgenomates (in decreasing abundance) were found in all the three libraries (Figure 1). Phyla Spirochaetes and Chlamydiae were found in MTG and MG and so were Ignavibacteriae and Cyanobacteria in libraries MG and BG, respectively (Figure 1). Hydrogenedentes was detected only in MTG, DeinococcusThermus in MG and Elusimicrobia in BG (Figure 1). Numbers of classes, orders, families and genera are also shown in Table 2.

\section{Coverage and Diversity Indexes}

With values of the Good's coverage index higher than $80 \%$ at 90\% confidence interval (Table 1), the Shannon index of 5.13 for OTUs in the MTG clone library was lower than those of 7.06 and 7.21 for the FG and MG libraries, respectively. The Simpson index, however, showed clear differences among the OTUs in each library, with values of $0.0016,0.0031$ and 0.0056 for the MTG, MG and BG clone libraries, respectively (Table 1).

Sequences in library BG distributed in 197 genera, a value lower than those of 203 and 234 found in libraries MTG and MG, respectively (Table 2). Of the total number of genera, the BG library shared 132 and 155 with the MTG and MG libraries, respectively, and libraries MTG and MG had 159 genera in common (Table 3). Values of the $J_{\text {class }}$ index were similar between the BG and MG libraries (0.56) and the MTR and MG libraries (0.57), values that were higher than that $(0.49)$ between the MTG and BG libraries (Table 3). The $J_{\text {abund }}$ indexes for the multiple comparisons among genomic libraries were similar (Table 3 ).

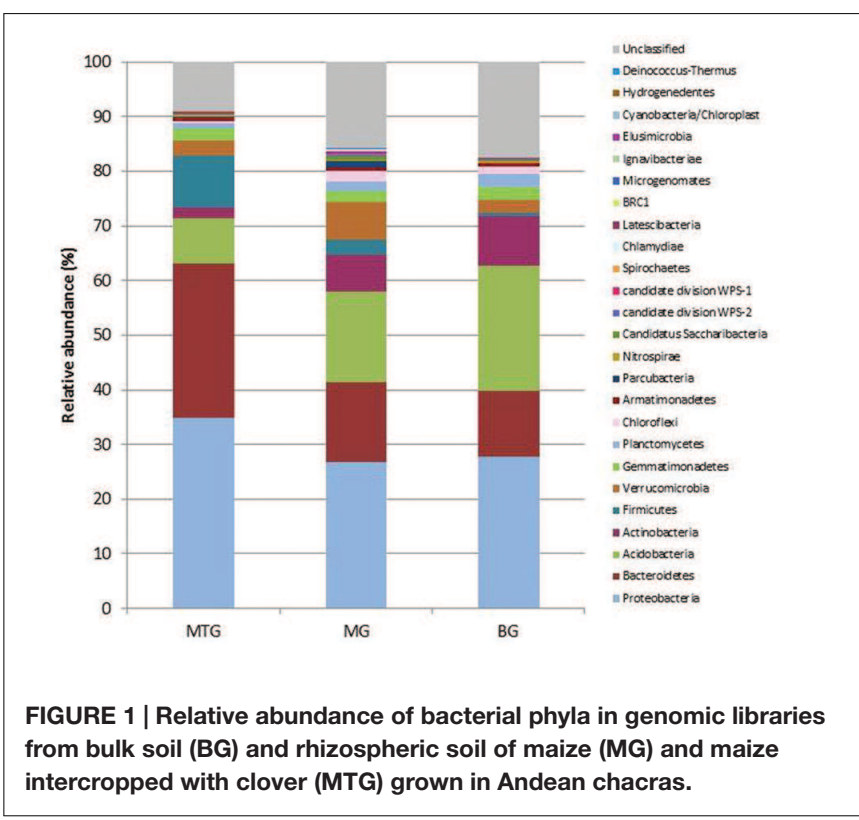

TABLE 3 | Number of shared genera among genomic libraries, and Jaccard similarity indexes using presence/absence $\left(J_{\text {class }}\right)$ and relative abundances $\left(J_{\text {abund }}\right.$ ) of the genera in libraries from bulk soil (BG) and rhizospheric soil of maize (MG) and maize intercropped with clover (MTG) grown in Andean chacras.

\begin{tabular}{lccc}
\hline & Number of shared genera & $\boldsymbol{J}_{\text {class }}$ & $\boldsymbol{J}_{\text {abund }}$ \\
\hline MTG-BG & 132 & 0.49 & 0.94 \\
MG-BG & 155 & 0.56 & 0.92 \\
MTG-MG & 159 & 0.57 & 0.98 \\
\hline
\end{tabular}

\section{Taxonomic Composition and Statistical Analyses}

From the total 26031 sequences, only 12784 could be assigned to 309 different genera (Supplementary Table S1). Sixteen (16) out of the 309 genera had a relative abundance higher than $0.5 \%$, and represented the $64.23 \%$ of the total number of sequences classified at the genus level. Altogether, these genera were (in 


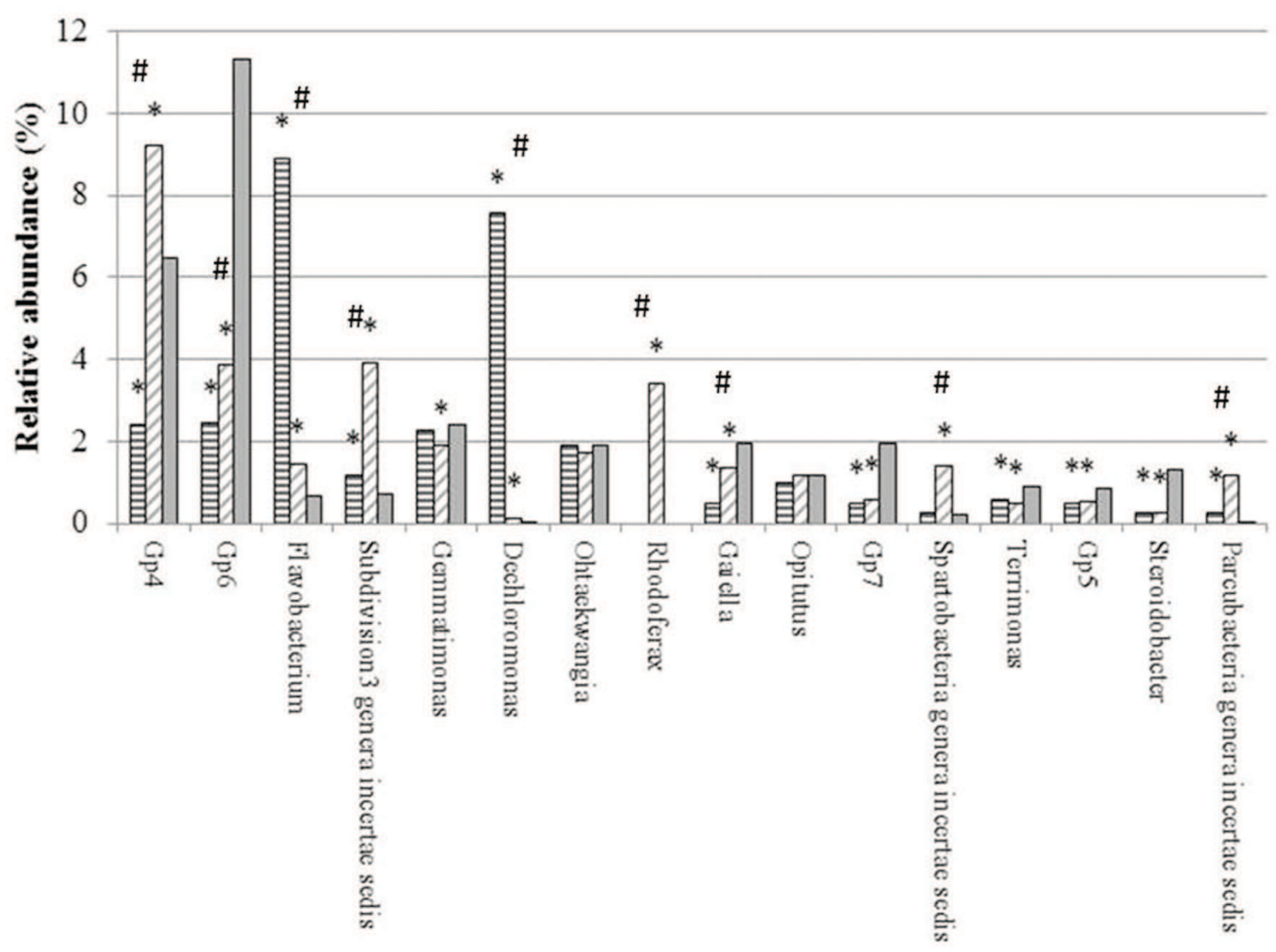

FIGURE 2 | Relative abundance (\%) of main genera in genomic libraries from bulk soil (BG, solid gray) and rhizospheric soil of maize (MG, horizontal gray lines) and maize intercropped with clover (MTG, diagonal gray lines) grown in Andean chacras. Relative abundances were compared using the combined $\mathrm{G}+$ Fischer tests $(\alpha \leq 0.05)$. *indicates statistically differences between the sequences in the MTG and MG libraries as compared to the BG library; \# indicates statistically differences between the sequences in the MTG and MG libraries.

decreasing order of abundance) Gp4, Gp6, Flavobacterium, Subdivision3 genera incertae sedis of the Verrucomicrobia phylum, Gemmatimonas, Dechloromonas, Ohtaekwangia, Rhodoferax, Gaiella, Opitutus, Gp7, Spartobacteria genera incertae sedis, Terrimonas, Gp5, Steroidobacter and Parcubacteria genera incertae sedis (Supplementary Table S1) (Figure 2).

Relative abundances of genera Flavobacterium, Subdivision3 genera incertae sedis of the Verrucomicrobia phylum, Dechloromonas and Parcubacteria in each the MTG and MG libraries were statistically higher than those in the BG library. In contrast, the number of sequences of genera Gp6, Gaiella, Gp7, Terrimonas, Gp5 and Steroidobacter in the MTG and MG libraries were lower than those in the BG library.

Numbers of sequences of genera Flavobacterium and Dechloromonas in the MTG library were statistically higher than those in the MG library (Figure 2). On the other hand, the relative abundance of genera Gp4, Gp6, Subdivision3 genera incertae sedis of phylum Verrucomicrobia, Rhodoferax, Gaiella and Spartobacteria in MTG were significantly lower than those in the MG library (Figure 2). Differences in the relative abundance of sequences corresponding to genera Gemmatimonas, Ohtaekwangia, Opitutus, Gp7, Terrimonas, Gp5,
Steroidobacter and Parcubacteria were not found between the MTG and MG libraries (Figure 2).

\section{DISCUSSION}

Using 454 next generation sequencing we assessed the composition and abundance of bacterial rhizospheric communities of amilaceous maize plants grown in Andean chacras following Quechua traditional practices. The total number of genera found in our genomic libraries were higher than those obtained in previous studies based on culturedependent and culture-independent methods (Gomes et al., 2001; Schmalenberger and Tebbe, 2002; Baumgarte and Tebbe, 2005; Brusetti et al., 2005; Aira et al., 2010; Miethling-Graff et al., 2010; Pereira et al., 2011; García-Salamanca et al., 2013; Li et al., 2014), and similar to those found in the rhizosphere of different maize varieties after pyrosequencing (Dohrmann et al., 2013).

Altogether, a $12.71 \%$ of the total sequences found inside roots corresponded to unclassified bacteria, which indicates the presence of hitherto uncultured bacterial groups. A high percentage of unclassified sequences have also been reported in the rhizosphere of maize plants when pyrosequencing was used to study bacterial biodiversity (Dohrmann et al., 2013). 
A variety of bacteria have been reported to be rhizospheric, among them mostly Proteobacteria, Firmicutes, Actinobacteria, Acidobacteria and Bacteroidetes (Sharma et al., 2005; DeAngelis et al., 2009; Peiffer et al., 2013; Uroz et al., 2013). In our study, regardless of soil provenance, members of phylum Proteobacteria were the most abundant (29.10\%) followed by those of Bacteroidetes (17.03\%), Acidobacteria (16.62\%), Actinobacteria (6.40\%), Verrucomicrobia (4.48\%) and Firmicutes (3.55\%) (Figure 1). Although the amount of bacterial phyla found in the three genomic libraries was similar (Table 2), the number of OTUs and values of the Shannon and Simpson indexes for the MTG library were lower than those for the BG library (Table 1), which suggests that intercropping reduced richness of the community. These results agree with those previously published that show that microbial diversity in maize rhizospheric soils was lower than in bulk soils (GarcíaSalamanca et al., 2013; Peiffer et al., 2013). This reduction can be explained if one considers that maize plants intercropped with bur clover somehow select from the bulk soil those bacteria able to promote plant growth and development; e.g., due to the symbiosis with E. medicae (Arone et al., 2014) free-living $N_{2}$ bacteria would not be required for plant growth. Our results also show that diversity in the MG library was higher than that in the BG library (Table 1). It is possible that the absence of bur clover promts the plants to increase bacterial diversity around the roots to improve their nutrition. Alternatively, it is also possible that more time is required for the maize crop to become well established in soils, which, in turn, could alter the soil microbiota. On the other hand, clear dominance of specific rhizospheric bacteria was not found, and multiple comparisons analyses of the values of the $\beta$-diversity indexes indicated the three genomic libraries have a similar bacterial community core. The absence of specific phylogenetic groups between bulk and rhizospheric soil from an oak forest after pyrosequencing of the 16S rRNA gene has been reported (Uroz et al., 2010).

Plants influence the abundance and composition of the bacterial rhizospheric community by secreting a variety of compounds through their roots into the surrounding soil to feed and manipulate the microbes that live there (Johnston-Monje et al., 2016). If one considers the bulk soil of the chacras as the reservoir of microbial communities, whereas maize increased relative abundance of genera Gp4, Flavobacterium, Subdivision3 genera incertae sedis of the Verrucomicrobia phylum, Dechloromonas, Rhodoferax, Spartobacteria and Parcubacteria

\section{REFERENCES}

Aira, M., Gómez-Brandón, M., Lazcano, C., Bååth, E., and Domínguez, J. (2010). Plant genotype strongly modifies the structure and growth of maize rhizosphere microbial communities. Soil Biol. Biochem. 42, 2276-2281. doi: 10.1016/j.soilbio.2010. 08.029

Arone, G., Calderón, C., Moreno, S., and Bedmar, E. J. (2014). Identification of Ensifer strains isolated from root nodules of Medicago hispida grown in association with Zea mays in the Quechua region of the Peruvian Andes. Biol. Fertil. Soils 50, 185-190. doi: 10.1007/s00374-0130825-3 incertae sedis, the maize-bur clover consortium increased relative abundance of Flavobacterium, Subdivision3 genera incertae sedis of the Verrucomicrobia phylum, Dechloromonas and Parcubacteria incertae sedis.

It is well established that plant genotype plays a significant role in shaping plant-associated microbial communities and in determining the biological outcome of such associations (Smith and Goodman, 1999; Ding et al., 2013). In this sense, 16S rRNA-based pyrosequencing studies on microbial diversity from rhizosphere of different maize genotypes have shown that variation in microbial diversity could be attributed to host genetics (Bouffaud et al., 2012; Peiffer et al., 2013). Because seeds of amilaceous maize used for planting were the same and the environmental conditions (including soil type and physicochemical properties), were, also, the same for the three chacras, our results suggest that the plant could be a main factor controlling bacterial diversity in their rhizosphere, whether alone (maize) or in consortium with other plants (maize and bur clover). Since richness of bacteria colonizing maize roots was based on pyrosequencing, it is not known whether the detection of bacteria based on DNA signature alone represent active microbes that are interacting with the host plant.

\section{AUTHOR CONTRIBUTIONS}

Conceived and designed the experiments: DC-G, EJB, GJA. Performed the experiments: DC-G, GJA. Analyzed the data: DC-G, EJB. Contributed reagents/materials/analysis tools: AJF-G, MF-L. Improve the paper: AJF-G, MF-L, GJA. Wrote the paper: DC-G, EJB.

\section{ACKNOWLEDGMENTS}

This study was supported by ERDF-cofinanced grant PE2012AGR1968 from Consejería de Economía, Innovación y Ciencia (Junta de Andalucía, Spain) and the CSIC-sponsored I-COOP Agrofood project 2014CD0013.

\section{SUPPLEMENTARY MATERIAL}

The Supplementary Material for this article can be found online at: http://journal.frontiersin.org/article/10.3389/fpls.2016.01016

Baker, G. C., Smith, J. J., and Cowan, D. A. (2003). Review and re-analysis of domain-specific $16 \mathrm{~S}$ primers. J. Microbiol. Methods 55, 541-555. doi: 10.1016/j.mimet.2003.08.009

Barberán, A., Bates, S. T., Casamayor, E. O., and Fierer, N. (2012). Using network analysis to explore co-occurrence patterns in soil microbial communities. ISME J. 6, 343-351. doi: 10.1038/ismej.2011.119

Bastida, F., Moreno, J. L., Nicolás, C., Hernández, T., and García, C. (2009). Soil metaproteomics: a review of an emerging environmental science. Significance, methodology and perspectives. Eur. J. Soil Sci. 60, 845-859. doi: 10.1111/j.15746941.2011.01284.x

Baumgarte, S., and Tebbe, C. C. (2005). Field studies on the environmental fate of the Cry1 Ab Bt-toxin produced by transgenic maize (MON810) and its effect on 
bacterial communities in the maize rhizosphere. Mol. Ecol. 14, 2539-2551. doi: 10.1111/j.1365-294X.2005.02592.x

Binladen, J., Gilbert, M. T., Bollback, J. P., Panitz, F., Bendixen, C., Nielsen, R., et al. (2007). The use of coded PCR primers enables high-throughput sequencing of multiple homolog amplification products by 454 parallel sequencing. PLoS ONE 2:e197. doi: 10.1371/journal.pone.0000197

Bolhuis, H., and Stal, L. J. (2011). Analysis of bacterial and archaeal diversity in coastal microbial mats using massive parallel 16S rRNA gene tag sequencing. ISME J. 5, 1701-1712. doi: 10.1038/ismej.2011.52

Bouffaud, M.-L., Kyselková, M., Gouesnard, B., Grundmann, G., Muller, D., and Moënne-Loccoz, Y. (2012). Is diversification history of maize influencing selection of soil bacteria by roots? Mol. Ecol. 21, 195-206. doi: 10.1111/j.1365294X.2011.05359.x

Brusetti, L., Francia, P., Bertolini, C., Pagliuca, A., Borin, S., Sorlini, C., et al. (2005). Bacterial communities associated with the rhizosphere of transgenic Bt 176 maize (Zea mays) and its non transgenic counterpart. Plant Soil 226, 11-21. doi: 10.1007/s11104-005-5399-x

Chao, A., Chazdon, R. L., Colwell, R. K., and Shen, T. J. (2005). A new statistical approach for assessing similarity of species composition with incidence and abundance data. Ecol. Lett. 8, 148-159. doi: 10.1111/j.1461-0248.2004.00707.x

Claesson, M. J., O'Sullivan, O., Wang, Q., Nikkilä, J., Marchesi, J. R., Smidt, H., et al. (2009). Comparative analysis of pyrosequencing and a phylogenetic microarray for exploring microbial community structures in the human distal intestine. PLoS ONE 4:e6669. doi: 10.1371/journal.pone.0006669

Cole, J. R., Wang, Q., Fish, J. A., Chai, B., McGarrell, D. M., Sun, Y., et al. (2014). Ribosomal database project: data and tools for high throughput rRNA analysis. Nucleic Acids Res. 42, D633-D642. doi: 10.1093/nar/gkt1244

Correa-Galeote, D., Tortosa, G., and Bedmar, E. J. (2013). Determination of denitrification genes abundance in environmental samples. Metagenomics 2, 235702. doi: $10.4303 / \mathrm{mg} / 235702$

Dabdoub, S., Tsigarida, A. A., and Kumar, P. S. (2013). Patient-specific analysis of periodontal and peri-implant microbiomes. J. Dent. Res. 92, 168S-175S. doi: $10.1177 / 0022034513504950$

DeAngelis, K. M., Brodie, E. L., DeSantis, T. Z., Andersen, G. L., Lindow, S. E., and Firestone, M. K. (2009). Selective progressive response of soil microbial community to wild oat roots. ISME J. 3, 168-178. doi: 10.1038/ismej.2008.103

Dethlefsen, L., Huse, S., Sogin, M. L., and Relman, D. A. (2008). The pervasive effects of an antibiotic on the human gut microbiota, as revealed by deep 16S rRNA sequencing. PLoS Biol. 6:e280. doi: 10.1371/journal.pbio.00 60280

Ding, T., Palmer, M. W., and Melcher, U. (2013). Community terminal restriction fragment length polymorphisms reveal insights into the diversity and dynamics of leaf endophytic bacteria. BMC Microbiol. 13:1. doi: 10.1186/1471-2180-13-1

Dohrmann, A. B., Küting, M., Jünemann, S., Jaenicke, S., Schlüter, A., and Tebbe, C. C. (2013). Importance of rare taxa for bacterial diversity in the rhizosphere of Bt-and conventional maize varieties. ISME J. 7, 37-49. doi: 10.1038/ismej.2012.77

Edgar, R. C., Haas, B. J., Clemente, J. C., Quince, C., and Knight, R. (2011). UCHIME improves sensitivity and speed of chimera detection. Bioinformatics 27, 2194-2200. doi: 10.1093/bioinformatics/btr381

García-Salamanca, A., Molina-Henares, M. A., Dillewijn, P., Solano, J., PizarroTobías, P., Roca, A., et al. (2013). Bacterial diversity in the rhizosphere of maize and the surrounding carbonate-rich bulk soil. Microb. Biotechnol. 6, 36-44. doi: 10.1111/j.1751-7915.2012.00358.x

Gomes, N. C. M., Heuer, H., Schönfeld, J., Costa, R., Mendonca-Hagler, L., and Smalla, K. (2001). Bacterial diversity of the rhizosphere of maize (Zea mays) grown in tropical soil studied by temperature gradient gel electrophoresis. Plant Soil 232, 167-180. doi: 10.1023/A:1010350406708

Grobman, A., Bonavia, D., Dillehay, T. D., Piperno, D. R., Iriarte, J., and Holst, I. (2012). Preceramic maize from Paredones and Huaca Prieta, Peru. Proc. Natl. Acad. Sci. U.S.A. 109, 1755-1759. doi: 10.1073/pnas.1120270109

Hang, J., Desai, V., Zavaljevski, N., Yang, Y., Lin, X., Satya, R. V., et al. (2014). 16S rRNA gene pyrosequencing of reference and clinical samples and investigation of the temperature stability of microbiome profiles. Microbiome 2, 31. doi: 10.1186/2049-2618-2-31

Herridge, D. F., Peoples, M. B., and Boddey, R. M. (2008). Global inputs of biological nitrogen fixation in agricultural systems. Plant Soil 311, 1-18. doi: 10.1007/s11104-008-9668-3
Herschkovitz, Y., Lerner, A., Davidov, Y., Okon, Y., and Jurkevitch, E. (2005a). Azospirillum brasilense does not affect population structure of specific rhizobacterial communities of inoculated maize (Zea mays). Environ. Microbiol. 7, 1847-1852. doi: 10.1111/j.1462-2920.2005.00926.x

Herschkovitz, Y., Lerner, A., Davidov, Y., Rothballer, M., Hartmann, A., Okon, Y., et al. (2005b). Inoculation with the plant-growth-promoting rhizobacterium Azospirillum brasilense causes little disturbance in the rhizosphere and rhizoplane of maize (Zea mays). Microb. Ecol. 50, 277-288. doi: 10.1007/s00248004-0148-x

Hughes, J. B., and Bohannan, B. J. M. (2004). "Application of ecological diversity statistics in microbial ecology," in Molecular Microbial Ecology Manual, Section 7, eds G. A. Kowalchuk, F. J. de Bruijn, I. M. Head, A. D. Akkermans, and J. D. van Elsas (Berlin: Springer), 3223-3246.

Huse, S. M., Dethlefsen, L., Huber, J. A., Welch, D. M., Relman, D. A., and Sogin, M. L. (2008). Exploring microbial diversity and taxonomy using SSU rRNA hypervariable tag sequencing. PLoS Genet. 4:e1000255. doi: 10.1371/journal.pgen.1000255

Ikeda, A. C., Bassani, L. L., Adamoski, D., Stringari, D., Cordeiro, V. K., Glienke, C. et al. (2013). Morphological and genetic characterization of endophytic bacteria isolated from roots of different maize genotypes. Microb. Ecol. 65, 154-160. doi: 10.1007/s00248-012-0104-0

Instituto Nacional de Estadística e Informática [INEI] (2012). IV Censo Nacional Agropecuario. Perú: Instituto Nacional de Estadística e Informática.

Johnston-Monje, D., Lundberg, D. S., Lazarovits, G., Reis, V. M., and Raizada, M. N. (2016). Bacterial populations in juvenile maize rhizospheres originate from both seed and soil. Plant Soil 1-19. doi: 10.1007/s11104-016-2826-0

Kumar, P. S., Mason, M. R., Brooker, M. R., and O'Brien, K. (2012). Pyrosequencing reveals unique microbial signatures associated with healthy and failing dental implants. J. Clin. Periodontol. 39, 425-433. doi: 10.1111/j.1600051X.2012.01856.X

Li, X., Rui, J., Mao, Y., Yannarell, A., and Mackie, R. (2014). Dynamics of the bacterial community structure in the rhizosphere of a maize cultivar. Soil Biol. Biochem. 68, 392-401. doi: 10.1016/j.soilbio.2013.10.017

Manrique, A. (1997). El maíz en el Perú. $2^{\text {a }}$ Edn. Peru: CONCYTEC.

Mao, Y., Yannarell, A. C., and Mackie, R. I. (2011). Changes in N-transforming archaea and bacteria in soil during the establishment of bioenergy crops. PLoS ONE 6:e24750. doi: 10.1371/journal.pone.0024750

Margulies, M., Egholm, M., Altman, W. E., Attiya, S., Bader, J. S., Bemben, L. A., et al. (2005). Genome sequencing in microfabricated high-density picolitre reactors. Nature $15,376-380$.

Miethling-Graff, R., Dockhorn, S., and Tebbe, C. C. (2010). Release of the recombinant Cry3Bbl protein of Bt maize MON88017 into field soil and detection of effects on the diversity of rhizosphere bacteria. Eur. J. Soil Biol. 46, 41-48. doi: 10.1016/j.ejsobi.2009.10.003

Nawrocki, E. P., Kolbe, D. L., and Eddy, S. R. (2009). Infernal 1.0: inference of RNA alignments. Bioinformatics 25, 1335-1337. doi: 10.1093/bioinformatics/btp157

Newcombe, R. G. (1998). Two-sided confidence intervals for the single proportion: comparison of seven methods. Stat. Med. 17, 857-872. doi: 10.1002/(SICI)10970258(19980430)17:8<857::AID-SIM777> 3.0.CO;2-E

Oliver, J. D. (2000). "The public health significance of viable but nonculturable bacteria," in Nonculturable Microorganisms in the Environment, eds R. R. Colwell and D. J. Grimes (Washignton, DC: ASM Press), 277-300.

Parameswaran, P., Jalili, R., Tao, L., Shokralla, S., Gharizadeh, B., Ronaghi, M., et al. (2007). A pyrosequencing-tailored nucleotide barcode design unveils opportunities for large-scale sample multiplexing. Nucleic Acids Res. 3, e130. doi: 10.1093/nar/gkm760

Parks, D. H., and Beiko, R. G. (2010). Identifying biologically relevant differences between metagenomic communities. Bioinformatics 26, 715-721. doi: 10.1093/bioinformatics/btq041

Parks, D. H., Tyson, G. W., Hugenholtz, P., and Beiko, R. G. (2014). STAMP: statistical analysis of taxonomic and functional profiles. Bioinformatics 30, 3123-3124. doi: 10.1093/bioinformatics/btu494

Peiffer, J. A., Spor, A., Koren, O., Jin, Z., Tringe, S. G., Dangl, J. L., et al. (2013). Diversity and heritability of the maize rhizosphere microbiome under field conditions. Proc. Natl. Acad. Sci. U.S.A. 110, 6548-6553. doi: $10.1073 /$ pnas. 1302837110

Pereira, C. J., Pereira, E. J. G., Cordeiro, E. M. G., Della Lucia, T. M. C., Tótola, M. R., and Guedes, R. N. C. (2009). Organophosphate resistance in the maize 
weevil Sitophilus zeamais: magnitude and behavior. Crop Protect. 28, 168-173. doi: 10.1016/j.cropro.2008.10.001

Pereira, P., Ibáñez, F., Rosenblueth, M., Etcheverry, M., and Martínez-Romero, E. (2011). Analysis of the bacterial diversity associated with the roots of maize (Zea mays L.) through culture-dependent and culture-independent methods. ISRN Ecol. 2011:938546. doi: 10.5402/2011/938546.

Piperno, D. R., Ranere, A. J., Holst, I., Iriarte, J., and Dickau, R. (2009). Starch grain and phytolith evidence for early ninth millennium BP maize from the Central Balsas River Valley, Mexico. Proc. Natl. Acad. Sci. U.S.A. 106, 5019-5024. doi: 10.1073/pnas. 0812525106

Polymenakou, P. N., Christakis, C. A., Mandalakis, M., and Oulas, A. (2015). Pyrosequencing analysis of microbial communities reveals dominant cosmopolitan phylotypes in deep-sea sediments of the eastern Mediterranean Sea. Res. Microbiol. 166, 448-445. doi: 10.1016/j.resmic.2015. 03.005

Rai, R., Dash, P. K., Prasanna, B. M., and Singh, A. (2007). Endophytic bacterial flora in the stem tissue of a tropical maize (Zea mays L.) genotype: isolation, identification and enumeration. World J. Microbiol. Biotechnol. 23, 853-858. doi: $10.1007 / \mathrm{s} 11274-006-9309-\mathrm{z}$

Rijavec, T., Lapanje, A., Dermastia, M., and Rupnik, M. (2007). Isolation of bacterial endophytes from germinated maize kernels. Can. J. Microbiol. 53, 802-808. doi: 10.1139/W07-048

Rothberg, J. M., and Leamon, J. H. (2008). The development and impact of 454 sequencing. Nat. Biotechnol. 26, 1117-1124. doi: 10.1038/nbt 1485

Sanguin, H., Herrera, A., Oger-Desfeux, C., Dechesne, A., Simonet, P., Navarro, E., et al. (2006a). Development and validation of a prototype 16S rRNA-based taxonomic microarray for Alphaproteobacteria. Environ. Microbiol. 8, 289-307. doi: 10.1111/j.1462-2920.2005.00895.x

Sanguin, H., Remenant, B., Dechesne, A., Thioulouse, J., Vogel, T. M., Nesme, X., et al. (2006b). Potential of a 16S rRNA-based taxonomic microarray for analyzing the rhizosphere effects of maize on Agrobacterium spp. and bacterial communities. Appl. Environ. Microbiol. 72, 4302-4312. doi: 10.1128/AEM.02686-05

Schmalenberger, A., and Tebbe, C. C. (2002). Bacterial community composition in the rhizosphere of a transgenic, herbicide-resistant maize (Zea mays) and comparison to its non-transgenic cultivar Bosphore. FEMS Microbiol. Ecol. 40, 29-37. doi: 10.1111/j.1574-6941.2002.tb00933.x

Schmalenberger, A., and Tebbe, C. C. (2003). Bacterial diversity in maize rhizospheres: conclusions on the use of genetic profiles based on PCR-amplified partial small subunit rRNA genes in ecological studies. Mol. Ecol. 12, 251-262. doi: 10.1046/j.1365-294X.2003.01716.x

Sengupta, A., and Dick, W. A. (2015). Bacterial community diversity in soil under two tillage practices as determined by pyrosequencing. Microb. Ecol. 70, 853-859. doi: 10.1007/s00248-015-0609-4

Sharma, S., Aneja, M. K., Mayer, J., Munch, J. C., and Schloter, M. (2005). Characterization of bacterial community structure in rhizosphere soil of grain legumes. Microb. Ecol. 49, 407-415. doi: 10.1007/s00248-004$0041-7$
Smith, K. P., and Goodman, R. M. (1999). Host variation for interactions with beneficial plant-associated microbes. Annu. Rev. Phytopathol. 37, 473-491. doi: 10.1146/annurev.phyto.37.1.473

Sogin, M. L., Morrison, H. G., Huber, J. A., Welch, D. M., Huse, S. M., Neal, P. R., et al. (2006). Microbial diversity in the deep sea and the underexplored "rare biosphere". Proc. Natl. Acad. Sci. U.S.A. 103, 12115-12120. doi: $10.1073 /$ pnas. 0605127103

Sprent, J. I. (2009). Legume Nodulation: A Global Perspective. Oxford: WileyBlackwell.

Sun, J., Zhang, Q., Zhou, J., and Wei, Q. (2014). Pyrosequencing technology reveals the impact of different manure doses on the bacterial community in apple rhizosphere soil. Appl. Soil Ecol. 78, 28-36. doi: 10.1016/j.apsoil.2014.02.004

Sundquist, A., Bigdeli, S., Jalili, R., Druzin, M. L., Waller, S., Pullen, K. M., et al. (2007). Bacterial flora-typing with targeted, chip-based pyrosequencing. BMC Microbiol. 7:108. doi: 10.1186/1471-2180-7-108

Torsvik, V., Øvreås, L., and Thingstad, T. F. (2002). Prokaryotic diversitymagnitude, dynamics, and controlling factors. Science 296, 1064-1066. doi: $10.1126 /$ science. 1071698

Tytgat, B., Verleyen, E., Obbels, D., Peeters, K., De Wever, A., D’hondt, S., et al. (2014). Bacterial diversity assessment in Antarctic terrestrial and aquatic microbial mats: a comparison between bidirectional pyrosequencing and cultivation. PLoS ONE 9:e97564. doi: 10.1371/journal.pone.0097564

Uroz, S., Buée, M., Murat, C., Frey-Klett, P., and Martin, F. (2010). Pyrosequencing reveals a contrasted bacterial diversity between oak rhizosphere and surrounding soil. Environ. Microbiol. Rep. 2, 281-288. doi: 10.1111/j.17582229.2009.00117.x

Uroz, S., Ioannidis, P., Lengelle, J., Cébron, A., Morin, E., Buée, M., et al. (2013). Functional assays and metagenomic analyses reveals differences between the microbial communities inhabiting the soil horizons of a Norway spruce plantation. PLOS ONE 8:e55929. doi: 10.1371/journal.pone.0055929

van Heerwaarden, J., Doebley, J., Briggs, W. H., Glaubitz, J. C., Goodman, M. M., Gonzalez, J. D. J. S., et al. (2011). Genetic signals of origin, spread, and introgression in a large sample of maize landraces. Proc. Natl. Acad. Sci. U.S.A. 108, 1088-1092. doi: 10.1073/pnas.1013011108

Wang, Q., Garrity, G. M., Tiedje, J. M., and Cole, J. R. (2007). Naive Bayesian classifier for rapid assignment of rRNA sequences into the new bacterial taxonomy. Appl. Environ. Microb. 73, 5261-5267. doi: 10.1128/AEM.00062-07

Conflict of Interest Statement: The authors declare that the research was conducted in the absence of any commercial or financial relationships that could be construed as a potential conflict of interest.

Copyright ( 2016 Correa-Galeote, Bedmar, Fernández-González, Fernández-López and Arone. This is an open-access article distributed under the terms of the Creative Commons Attribution License (CC BY). The use, distribution or reproduction in other forums is permitted, provided the original author(s) or licensor are credited and that the original publication in this journal is cited, in accordance with accepted academic practice. No use, distribution or reproduction is permitted which does not comply with these terms. 


\section{OPEN ACCESS}

Edited by:

Antonio M. De Ron,

Spanish National Research Council (CSIC) - Misión Biológica de Galicia,

Spain

Reviewed by:

Francisco Perez-Alfocea,

Consejo Superior de Investigaciones

Cientificas, Spain

Wanda Waaterworth

University of Leeds, UK

${ }^{*}$ Correspondence:

Qin Zhou

qinzhou@njau.edu.cn;

Dong Jiang

jiangd@njau.edu.cn

Specialty section: This article was submitted to Crop Science and Horticulture, a section of the journal

Frontiers in Plant Science

Received: 26 April 2016

Accepted: 13 June 2016

Published: 28 June 2016

Citation:

Li X, Zhou L, Liu F, Zhou Q, Cai J, Wang $X$, Dai T, Cao W and Jiang $D$

(2016) Variations in Protein

Concentration and Nitrogen Sources in Different Positions of Grain in Wheat. Front. Plant Sci. 7:942. doi: 10.3389/fpls.2016.00942

\section{Variations in Protein Concentration and Nitrogen Sources in Different Positions of Grain in Wheat}

\author{
Xiangnan $\mathrm{Li}^{1,2}$, Longjing Zhou ${ }^{1}$, Fulai Liu ${ }^{2}$, Qin Zhou ${ }^{1 *}$, Jian Cai ${ }^{1}$, Xiao Wang ${ }^{1}$, \\ Tingbo Dai ${ }^{1}$, Weixing $\mathrm{Cao}^{1}$ and Dong Jiang ${ }^{1 *}$
}

' National Engineering and Technology Center for Information Agriculture/Key Laboratory of Crop Physiology and Ecology in Southern China, Ministry of Agriculture, Nanjing Agricultural University, Nanjing, China, ${ }^{2}$ Department of Plant and

Environmental Sciences, Faculty of Science, University of Copenhagen, Taastrup, Denmark

The distribution patterns of total protein and protein components in different layers of wheat grain were investigated using the pearling technique, and the sources of different protein components and pearling fractions were identified using ${ }^{15} \mathrm{~N}$ isotope tracing methods. It was found that $\mathrm{N}$ absorbed from jointing to anthesis (JA) and remobilized to the grain after anthesis was the principal source of grain $\mathrm{N}$, especially in the outer layer. For albumin and globulin, the amount of $\mathrm{N}$ absorbed during different stages all showed a decreasing trend from the surface layer to the center part. Whereas, for globulin and glutenin, the $\mathrm{N}$ absorbed after anthesis accounted for the main part indicating that for storage protein, the utilization of $\mathrm{N}$ assimilated after anthesis is greater than that of the stored $\mathrm{N}$ assimilated before anthesis. It is concluded that manipulation of the $\mathrm{N}$ application rate during different growth stages could be an effective approach to modulate the distribution of protein fractions in pearled grains for specific end-uses.

Keywords: protein, pearling, wheat, nitrogen, isotope

\section{INTRODUCTION}

The grain protein concentration, which to a great extent determines the quality of pasta and bread, is one of the major pricing factors for wheat trading, and is an important nutritional factor for human health (Masclaux-Daubresse et al., 2008). The protein concentration is closely related to the nitrogen $(\mathrm{N})$ content and dry mass in wheat grains and can be separated into the fractions of albumin, gliadin, globulin, and glutenin according to its solubility in different solvents (Malik et al., 2013). Grain $N$ is derived from $N$ that is absorbed after anthesis and from remobilized $N$ that is stored in vegetative organs assimilated before anthesis. Both $\mathrm{N}$ sources support the synthesis of the storage proteins in grain (Dupont and Altenbach, 2003), and the former contributes approximately $60-95 \%$ to the grain N (Palta and Fillery, 1995). On the contrary, approximately $90 \%$ of carbon in grain is acquired by the concurrent photo-assimilates after anthesis (Zhang et al., 2011). In an extreme case, when plants are grown without $\mathrm{N}$ supply after the emergence of flag leaf, the remobilization of $\mathrm{N}$ from leaves, glumes, stems, and roots contributed $40,23,23$, and $16 \%$ to grain N, respectively (Simpson et al., 1983).

In many studies, the contribution of the pre-anthesis assimilated $\mathrm{N}$ to grain $\mathrm{N}$ was calculated by the difference in the $\mathrm{N}$ stored in vegetative organs at anthesis and maturity (Arduini et al., 2006; Ercoli et al., 2008). Similarly, the contribution of post-anthesis absorbed $\mathrm{N}$ to grain $\mathrm{N}$ was calculated by the difference between the $\mathrm{N}$ in the mature grain and the remobilized $\mathrm{N}$ assimilated pre-anthesis 
(Shi et al., 2012a). However, errors might exist using this method, due to the loss of senescence organs (especially for leaves, which present the highest $\mathrm{N}$ concentration and account for a large share of the remobilized N), nutrient leaches, and the fact that the post-anthesis absorbed $\mathrm{N}$ might be retained in vegetative organs such as sheathes and stems, which usually depends on the $\mathrm{N}$ supply level of the soil and the $\mathrm{N}$ fertilization level (Shi et al., 2012a). To overcome these problems, the ${ }^{15} \mathrm{~N}$ isotope tracing technique is used to discriminate the $\mathrm{N}$ that is absorbed at different growth stages and between the concurrent absorbed $\mathrm{N}$ and the remobilized $\mathrm{N}$ (Kichey et al., 2007). It has been shown that the contribution of $\mathrm{N}$ assimilated before anthesis to grain $\mathrm{N}$ is largely dependent on the genotypes, soil $\mathrm{N}$ status, field management practices such as fertilization and irrigation, and environmental conditions (Ferrise et al., 2010). However, the contributions of $\mathrm{N}$ that is absorbed during different growing periods and the $\mathrm{N}$ that is remobilized from different vegetative organs to the mature grains have not been well-quantified.

Pearling (debranning) is a new milling technique in the milling and baking industry, where the bran layers are removed sequentially by friction and abrasion operations with a type of device that is a modified rice polisher (Beta et al., 2005). The advantages of pearling before roller milling include improving the semolina yield and the quality of the durum wheat (Dexter and Wood, 1996), enhancing the bread-baking quality (Mousia et al., 2004), depressing the xylanase activity in both whole meal and flour (Gys et al., 2004), and decreasing the $\alpha$-amylase activity in the flour of pre-harvest sprouted grains of wheat (Hareland, 2003). At the same time, pearling provides us an effective way to investigate the distribution patterns of both the chemical components and quality of flour that are located at different positions in wheat grain, which is essential for separating the milling fractions for specific products (Liu et al., 2007). The distribution patterns of ash, iron, and zinc and the phenolic compounds, with antioxidant activity, have been illustrated in different pearling fractions (Fares et al., 1996; Beta et al., 2005; Liu et al., 2007, 2008). It has also been reported that the flour obtained from pearled wheat was significantly different from the unpearled wheat in terms of flour particle size distribution, percentage of damaged starch granules, starch gelatinization temperature, moisture, and concentrations in the chemical compositions, such as ash and protein (Mousia et al., 2004). In addition, a clear gradient in the protein concentration has been found across the starchy endosperm by microscopy, which was higher in the sub-aleurone cells and lower in the central endosperm cells (Tosi et al., 2011). Moreover, the concentrations of the protein components, such as the gluten proteins, were reported to be expressed in gradient along the different parts of the endosperm (Tosi et al., 2009). However, little is known about the concentration gradient of proteins and protein fractions in different layers of wheat grain and the $\mathrm{N}$ sources of those proteins. In this study, the distribution patterns and concentration gradient of the protein and protein components in wheat grain were investigated using the pearling technique, and the ${ }^{15} \mathrm{~N}$ isotope tracing technique was used to discriminate the $\mathrm{N}$ sources of different protein components in different pearling fractions of grain. The results will be essential in understanding the mechanisms of grain quality formation and exploring better $\mathrm{N}$ fertilization protocols for super quality wheat production.

\section{MATERIALS AND METHODS}

\section{Experimental Design and Materials}

Seeds of winter wheat (Triticum aestivum L., cv. Yangmai 16) were sown into $40-\mathrm{L}$ pots $(45 \mathrm{~cm}$ in length, $30 \mathrm{~cm}$ in both width and height, with a valve near the bottom) filled with $16 \mathrm{~kg}$ deionized quartz sand. Eight seeds were sown in each pot. A Hoagland nutrient solution that contained $3.0 \mathrm{mM} \mathrm{NH}_{4} \mathrm{NO}_{3}$ $\left({ }^{15} \mathrm{NH}_{4}{ }^{15} \mathrm{NO}_{3}, 5.0 \%\right.$ in ${ }^{15} \mathrm{~N}$ abundance), $1.0 \mathrm{mM} \mathrm{KH} \mathrm{KO}_{2} \mathrm{PO}_{4}$, $2.0 \mathrm{mM} \mathrm{KCl}, 2.0 \mathrm{mM} \mathrm{CaSO}_{4}, 1.0 \mathrm{mM} \mathrm{MgSO}, 1.0 \mathrm{mM} \mathrm{NaCl}$, $2.3 \mu \mathrm{M} \mathrm{H}_{3} \mathrm{BO}_{3}, 0.46 \mu \mathrm{M} \mathrm{MnSO}_{4}, 0.038 \mu \mathrm{M} \mathrm{ZnSO}_{4}, 0.016 \mu \mathrm{M}$ $\mathrm{CuSO}_{4}, 0.006 \mu \mathrm{M} \mathrm{H} \mathrm{H}_{2} \mathrm{MoO}_{4}, 89.3 \mu \mathrm{M} \mathrm{FeSO}$, and $43.1 \mu \mathrm{M}$ $\mathrm{Na}_{2}$ EDTA was used to provide nutrients to the plants. Depending on the winter wheat growth stages, four treatments were established: (1) $\mathrm{NH}_{4} \mathrm{NO}_{3}$ was replaced with ${ }^{15} \mathrm{NH}_{4}^{15} \mathrm{NO}_{3}$ during the period of emergence (November 14 ${ }^{\text {th }} 2011$ ) to jointing (March 12 ${ }^{\text {th }}$ 2012; EJ); (2) $\mathrm{NH}_{4} \mathrm{NO}_{3}$ was replaced with ${ }^{15} \mathrm{NH}_{4}$ ${ }^{15} \mathrm{NO}_{3}$ during the period of jointing-anthesis (April 12 ${ }^{\text {th }} 2012$; JA); (3) $\mathrm{NH}_{4} \mathrm{NO}_{3}$ was replaced with ${ }^{15} \mathrm{NH}_{4}{ }^{15} \mathrm{NO}_{3}$ during the period of anthesis-maturity ( $2^{\text {nd }}$ June 2012; AM); and (4) $\mathrm{NH}_{4} \mathrm{NO}_{3}$ was applied during the whole growth season as the control (NN). The wheat plants were grown in a greenhouse to prevent any rainfall. Nine liters of nutrient solution with or without ${ }^{15} \mathrm{NH}_{4}{ }^{15} \mathrm{NO}_{3}$ were applied to each pot every 7 days. The experiment was a completely randomized block design, with three replicates per treatment. Each replicate included six pots. Uniform tillers that flowered on the same day were tagged. All plants in one pot were harvested and separated into the root, stem, leaf, and spike (chaff and grain at maturity) for each replicate at anthesis and maturity. The samples were then dried at $80^{\circ} \mathrm{C}$ until a constant weight was reached. The pots left were harvested at maturity to record the grain yield.

Approximately $10 \mathrm{~g}$ of wheat grain was used for analysis of the whole grain $\mathrm{N}$ concentration and the concentrations of total protein and protein components. Following the methods of Liu et al. (2007), the wheat grain was pearled into eight fractions $\left(\mathrm{P}_{1}-\mathrm{P}_{8}\right)$ from the surface layer to the center part, with two rice polishers (JNMJ7 and JNMJ6, Taizhou Grain Industry Instrument Corp, China). The pearling fractions were classified as $\mathrm{P}_{1}(0-10 \%), \mathrm{P}_{2}(10-20 \%), \mathrm{P}_{3}(20-30 \%), \mathrm{P}_{4}$ (30$40 \%), \mathrm{P}_{5}(40-50 \%), \mathrm{P}_{6}(50-60 \%), \mathrm{P}_{7}(60-70 \%)$, and $\mathrm{P}_{8}(70-$ $80 \%)$. The pearling residue (20\% of the whole grain) of each sample was ground in a stainless grinder (DJ-04B, Shanghai Dianjiu Machinery Manufactory, China) and designated as $\mathrm{P}_{9}$ (80-100\%).

\section{Chemical Analysis}

The protein concentration in the whole wheat grain and the albumin, globulin, glutenin, and gliadin fractions were determined by the micro-Kjeldhl distillation method of AACC 46-13.01 with some modifications (Li et al., 2012).

The natural abundance and atom $\%$ of ${ }^{15} \mathrm{~N}$ in the plant samples were detected using a pattern ZHT-O2 mass spectrometer 
following the method by Shi et al. (2012b) at the Agroforestry Academy, Chemical Institute of Hebei Province, China.

\section{Calculation Methods}

The percentage of $\mathrm{N}$ that was derived from fertilizer- $\mathrm{N}$ (Ndff, $\%$ ) was calculated by the following Equation (1) (Cookson et al., 2001; Shi et al., 2012b):

$$
\operatorname{Ndff}(\%)=\frac{c-b}{a-b} \times 100
$$

where $a$ is the atom $\%$ of ${ }^{15} \mathrm{~N}$ in the fertilizer, $b$ is the atom $\%$ of ${ }^{15} \mathrm{~N}$ in the unfertilized plants, and $c$ is the atom $\%$ of ${ }^{15} \mathrm{~N}$ in the fertilized plants.

The accumulation of $\mathrm{N}$ that was assimilated by the plants from fertilizer- $\mathrm{N}$ was calculated following Equations (2) and (3):

$$
\begin{aligned}
& \text { Plant total } \mathrm{N} \text { accumulation }(\mathrm{mg} / \text { plant }) \\
& \qquad=\frac{\text { plant dry weight } \times \mathrm{N} \text { concentration }}{100} \times 1000 \\
& \mathrm{~N} \text { from fertilizer } \mathrm{N}(\mathrm{mg} / \text { plant })=\frac{(2) \times \mathrm{Ndff}_{\text {plant }}}{100}
\end{aligned}
$$

The $\mathrm{N}$ distribution was calculated as the ratio of the ${ }^{15} \mathrm{~N}$ accumulation amount (mg) in a given organ to the total ${ }^{15} \mathrm{~N}$ accumulation in the whole plant ( $\mathrm{mg}$ ).

The remobilization of stored $\mathrm{N}$ from a given organ to grain (RAN) was calculated by subtracting the $\mathrm{N}$ at maturity from the $\mathrm{N}$ at anthesis in this organ. The translocation efficiency of the stored $\mathrm{N}$ in a given organ (TEN) was calculated following Equation (4):

$$
\operatorname{TEN}(\%)=\frac{\text { RAN }}{\mathrm{N} \text { amonut at anthesis }} \times 100
$$

The contribution of RAN to grain N (CRAN) was calculated following Equation (5):

$$
\operatorname{CRAN}(\%)=\frac{\mathrm{RAN}}{\mathrm{N} \text { amount in grain at maturity }} \times 100
$$

\section{Statistical Analysis}

All of the data were subjected to one-way ANOVA using SPSS version 20.0 for windows (IBM SPSS Statistics, Chicago, IL, USA). The Duncan's multiple range test was used to check the significance of the difference between treatments.

\section{RESULTS}

\section{Distribution of Assimilated $\mathrm{N}$ before Anthesis in the Organs at Anthesis}

The percentages of $\mathrm{N}$ derived from fertilizer-N (Ndff, \%) in all organs (root, stem, leaf, and spike) were different between during EJ (Emergence-Jointing) and JA (Jointing-Anthesis; Figure 1A). The values of Ndff in the root and leaf during EJ were significantly higher than those during JA, while an opposite trend was found in the Ndff in the stem and spike. The accumulation of $\mathrm{N}$ during EJ in the root and leaf was 11.1 and $10.9 \%$ higher than that

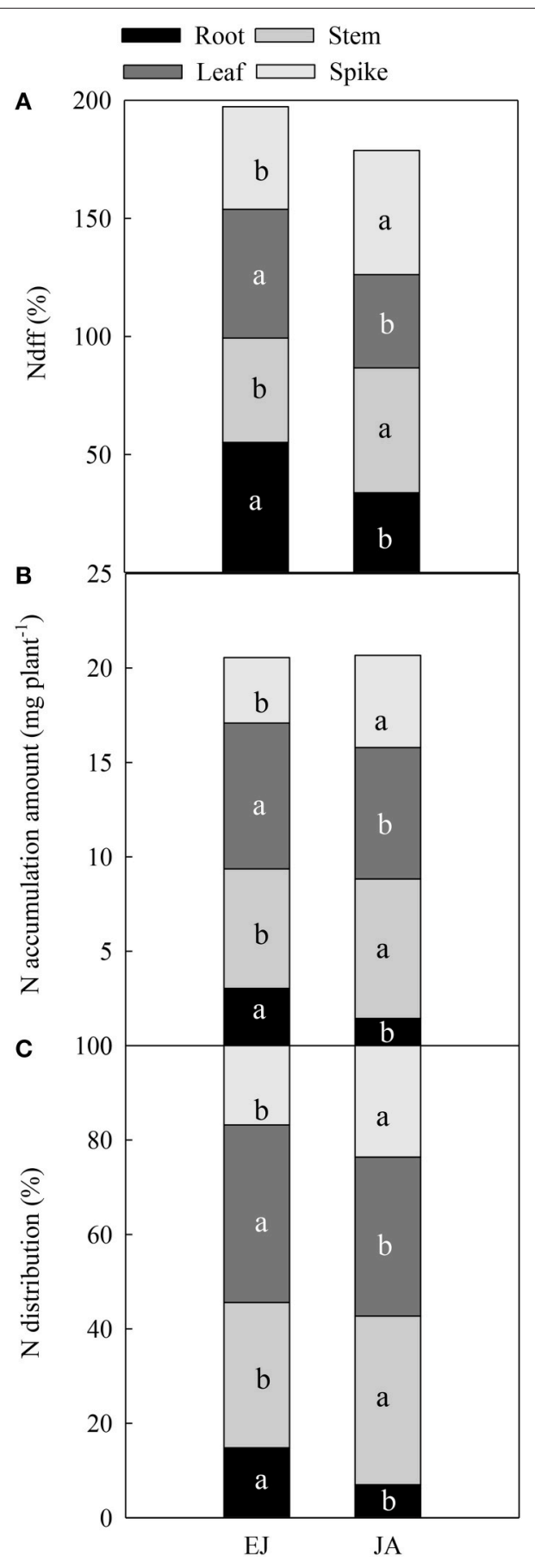

FIGURE 1 | Percentage of N derived from fertilizer-N (Ndff, \%) (A), accumulation amount (B), and contribution of absorbed $\mathbf{N}(C)$ during periods from emergence to jointing (EJ), and from jointing to anthesis (JA). Different small letters for the same organ (root, stem, leaf, and spike) indicate significant difference at 0.05 level between different growth periods. Sheath was included in stem.

during JA, while the $\mathrm{N}$ accumulation during EJ in the stem and spike was 14.3 and $29.1 \%$ lower than that during JA (Figure 1B). The distribution of $\mathrm{N}$ in the root and leaf that was absorbed during EJ was significantly higher than that during JA $(P<0.05$; 


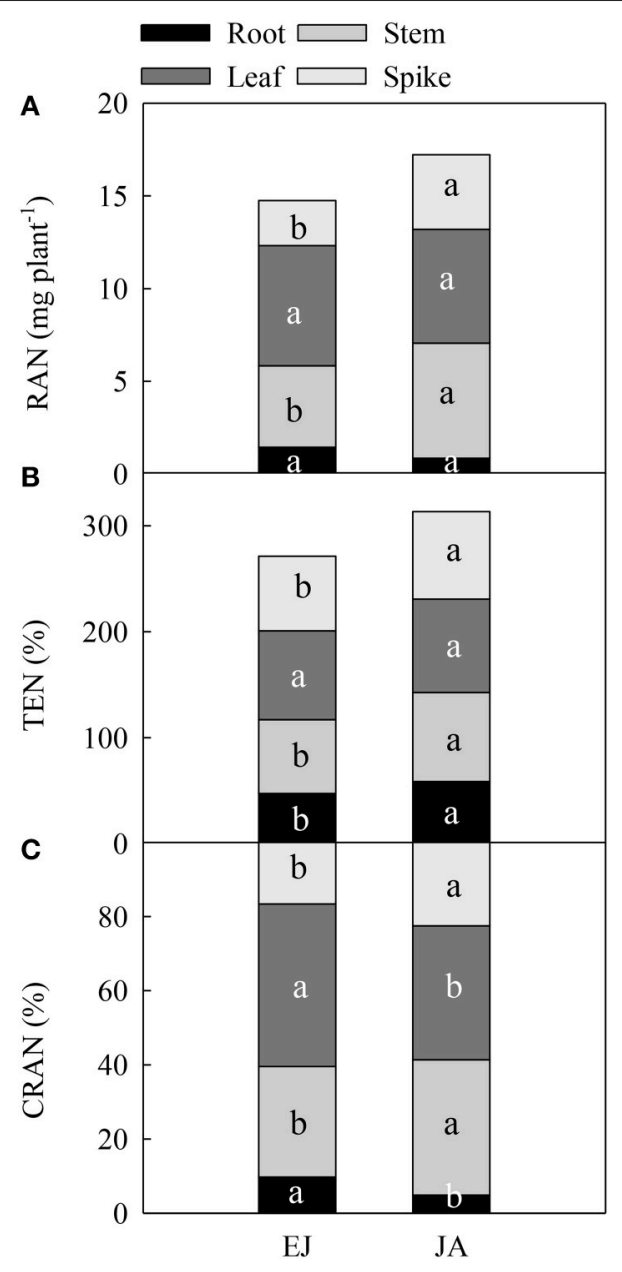

FIGURE 2 | Redistribution amount of $\mathbf{N}$ absorbed during periods from emergence to jointing (EJ) and from jointing to anthesis (JA) into grains (RAN) (A), translocation efficiency of the stored $\mathbf{N}$ in a given organ (TEN) (B), and contribution of RAN to grain N (CRAN) (C). Different small letters for the same organ (root, stem, leaf and spike) indicate significant difference at 0.05 level between different growth periods. Sheath was included in stem.

Figure 1C). On the contrary, the distribution of $\mathrm{N}$ in the stem and spike that was absorbed during EJ was significantly lower than that during JA.

\section{Remobilization of the Stored $\mathrm{N}$ before Anthesis to the Grain}

The remobilization of $\mathrm{N}$ (RAN) stored during EJ in the stem and spike to the grain was significantly lower than the RAN stored during JA (Figure 2A). However, for the leaf and root, no significant difference was found in RAN stored during EJ and JA. The translocation efficiency of $\mathrm{N}$ stored during EJ in the stem, spike and root (TEN) was significantly lower than TEN stored during JA (Figure 2B). The contribution of RAN (CRAN) stored in the leaf and root during EJ to grain was higher than the CRAN stored during JA (Figure 2C).

\section{Distribution of Assimilated $\mathbf{N}$ at Different Periods in Organs at Maturity}

The Ndff during EJ was the highest among all of the growth periods (Figure 3A). In the chaff and grain, the Ndff during EJ and JA was significantly higher than that during AM $(P<0.05)$. In the stem and leaf, the Ndff during JA was significantly higher than those during AM, but it was lower than that during EJ $(P<0.001)$. In the root, The Ndff during JA was significantly lower than that during AM (Anthesis-Maturity; $P<0.001$ ).

There was no difference in dry weight of plants among these treatments at maturity (Figure 3B). The $\mathrm{N}$ accumulation in the chaff (including the spike axis and kernel husks) during EJ and JA were higher than that during AM (Figure 3C). The highest amount of $\mathrm{N}$ accumulation in the stem and leaf was found during EJ, followed by during JA, and the lowest was during AM. However, the highest $\mathrm{N}$ accumulation in the root was observed during EJ, followed by during AM, and the lowest was during JA. In addition, the distribution of $\mathrm{N}$ absorbed during $\mathrm{AM}$ was higher than that absorbed during EJ and JA (Figure 3D).

\section{Distribution of Assimilated $\mathrm{N}$ at Different Periods in the Pearling Fractions of the Grain}

Across the pearling fractions in the grain, the highest Ndff during EJ was found in the $\mathrm{P}_{2}$ fraction, whereas the lowest Ndff was in the fractions $\mathrm{P}_{6}$ and $\mathrm{P}_{9}$ (Table 1). The Ndff during JA showed a decreasing trend from the surface layer to the center part, where the highest $\mathrm{Ndff}$ were found in the fractions $\mathrm{P}_{1}$, $\mathrm{P}_{2}$, and $\mathrm{P}_{3}$. However, the Ndff during $\mathrm{AM}$ showed an opposite trend: the highest Ndff-values were in $\mathrm{P}_{7}$ and $\mathrm{P}_{8}$. The highest accumulation of $\mathrm{N}$ was found in the $\mathrm{P}_{9}$ fraction in all these treatments. In addition, the average amount of $\mathrm{N}$ accumulation in the $\mathrm{P}_{1}-\mathrm{P}_{4}$ fractions was higher than in the $\mathrm{P}_{5}-\mathrm{P}_{9}$ fractions. In each pearling fraction the $\mathrm{N}$ was mostly accumulated during JA. The distribution of $\mathrm{N}$ assimilated during EJ, JA, and AM increased from the surface layer to the center part (except for $\mathrm{P}_{1}$ and $\left.\mathrm{P}_{9}\right)$.

\section{Distribution of the Assimilated $\mathrm{N}$ in the Protein Fractions of Pearled Grains}

The contribution of assimilated $\mathrm{N}$ during JA to the protein and protein fractions in each layer was the highest, except for the glutenin concentration in the $\mathrm{P}_{1}$ fraction (Figure 4). For the total protein, the contribution of $\mathrm{N}$ assimilated during EJ was higher than that during $\mathrm{AM}$ in the fractions $\mathrm{P}_{1}-\mathrm{P}_{6}$; however, it was opposite in the fractions $\mathrm{P}_{7}-\mathrm{P}_{9}$. For albumin, the contribution of $\mathrm{N}$ assimilated during EJ was significantly higher than during $\mathrm{AM}$ in the fractions $\mathrm{P}_{1}-\mathrm{P}_{6}$ and $\mathrm{P}_{9}$, and the contribution of $\mathrm{N}$ assimilated during EJ to the globulin was significantly higher than that during AM in all of the fractions. For gliadin, the $\mathrm{N}$ assimilated during EJ contributed more than that during AM in the fractions $\mathrm{P}_{1}-\mathrm{P}_{3}$, whereas the opposite was true in the fractions $\mathrm{P}_{4}-\mathrm{P}_{9}$. In the fractions of $\mathrm{P}_{2}-\mathrm{P}_{8}, \mathrm{~N}$ assimilated during $\mathrm{AM}$ contributed more to glutenin than that during EJ; however, 


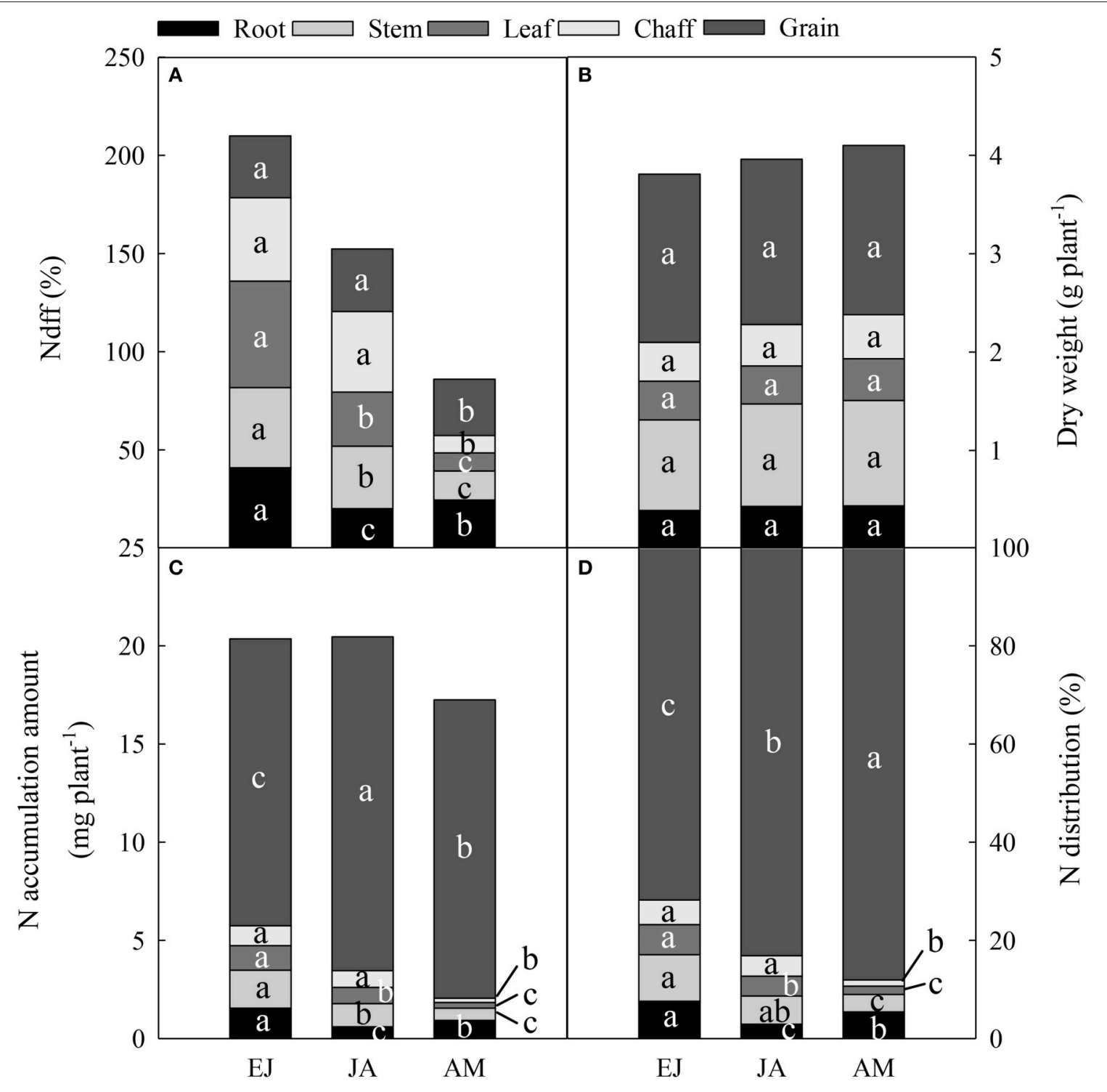

FIGURE 3 | Percentage of $\mathbf{N}$ derived from fertilizer-N (Ndff, \%) (A), dry weight in different vegetative organs at maturity (B), accumulation amount (C), and contribution (D) of absorbed $\mathbf{N}$ during periods from emergence to jointing (EJ), from jointing to anthesis (JA) and from anthesis to maturity (AM). Different small letters for the same organ (root, stem, leaf, chaff, and grain) indicate significant difference at 0.05 level between different growth periods. The chaff includes spike axis and kernel husks. Sheath was included in stem.

in fraction $\mathrm{P}_{1}$, the $\mathrm{N}$ contribution was found to be in the order: during $\mathrm{AM}>$ during $\mathrm{JA}>$ during $\mathrm{EJ}$.

Across the pearling fractions, the distribution of $\mathrm{N}$ assimilated during EJ, JA, and AM in albumin and globulin was decreased from the surface layer to the center part (Figure 5). In albumin, the distribution of $\mathrm{N}$ assimilated during JA was higher than that during the other two periods in $\mathrm{P}_{1-4}$. Also, a decreased trend from $\mathrm{P}_{2}$ to $\mathrm{P}_{8}$ was found in both gliadin and glutenin. For gliadin, the distribution of $\mathrm{N}$ assimilated during JA was higher than those during the other two periods in $\mathrm{P}_{1-4}$, and in $\mathrm{P}_{5-9}$ the distribution of $\mathrm{N}$ assimilated during AM was the highest among these three periods. For glutenin, in $\mathrm{P}_{1-4}$ the distribution of $\mathrm{N}$ assimilated during $\mathrm{AM}$ was the highest among the three periods, and in $\mathrm{P}_{5-9}$ the distribution of $\mathrm{N}$ assimilated during JA and AM was higher than that during EJ.

\section{DISCUSSION}

\section{Distribution and Remobilization of $\mathbf{N}$ Stored at Different Periods}

The $\mathrm{N}$ utilization of plants involves several processes, such as uptake, assimilation, translocation, and remobilization (Masclaux-Daubresse et al., 2008). Our study illustrated the spatial and temporal $\mathrm{N}$ distribution in the grains, using ${ }^{15} \mathrm{~N}$ isotope tracing technique. During vegetative growth stages, 


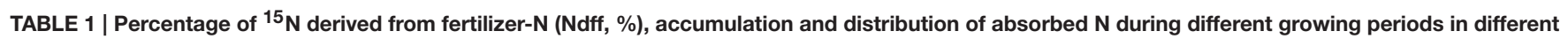
pearling fractions of wheat grain.

\begin{tabular}{|c|c|c|c|c|c|c|c|c|c|c|c|c|}
\hline \multirow[t]{2}{*}{ Pearling fractions } & \multicolumn{3}{|c|}{ Ndff (\%) } & \multicolumn{3}{|c|}{$\begin{array}{c}\mathrm{N} \text { accumulation } \\
\text { amount (mg plant } \\
-1\end{array}$} & \multicolumn{3}{|c|}{ N contribution $(\%)$} & \multicolumn{3}{|c|}{$\mathrm{N}$ distribution (\%) } \\
\hline & EJ & JA & AM & EJ & JA & AM & EJ & JA & AM & EJ & JA & AM \\
\hline$P_{1}$ & $31.14 \mathrm{c}$ & $31.59 \mathrm{a}$ & $25.10 f$ & $1.82 b$ & $2.16 c$ & $1.67 d$ & $8.87 b$ & $10.43 c$ & $9.66 d$ & $12.35 c$ & $12.67 c$ & $11.68 d$ \\
\hline$P_{2}$ & $31.70 a$ & $31.70 \mathrm{a}$ & $26.65 \mathrm{e}$ & $2.10 a$ & $2.31 b$ & $1.86 b$ & $10.23 a$ & 11.16b & $10.81 b$ & $14.25 b$ & $13.56 b$ & $13.06 \mathrm{~b}$ \\
\hline$P_{3}$ & $31.40 b$ & $31.58 a$ & $27.08 d$ & $1.84 b$ & $2.13 c$ & $1.76 c$ & $8.97 b$ & 10.30c & $10.19 c$ & $12.50 \mathrm{c}$ & $12.51 c$ & $12.31 \mathrm{c}$ \\
\hline$P_{4}$ & $31.05 c$ & $31.31 b$ & $27.36 c$ & $1.62 \mathrm{c}$ & $1.83 d$ & $1.54 \mathrm{e}$ & $7.89 c$ & $8.84 d$ & $8.92 \mathrm{e}$ & $10.98 d$ & $10.74 d$ & $10.78 \mathrm{e}$ \\
\hline$P_{5}$ & $31.00 \mathrm{c}$ & $31.37 \mathrm{~b}$ & $27.80 \mathrm{~b}$ & $1.46 \mathrm{~d}$ & $1.69 \mathrm{e}$ & $1.45 f$ & $7.13 d$ & $8.19 e$ & $8.43 f$ & $9.93 e$ & $9.95 e$ & $10.19 f$ \\
\hline$P_{6}$ & $30.23 e$ & $31.01 \mathrm{c}$ & $27.87 b$ & $1.43 d$ & $1.62 \mathrm{e}$ & $1.41 f$ & $6.96 d$ & $7.85 e$ & $8.17 f$ & $9.70 e$ & $9.53 e$ & $9.87 f$ \\
\hline$P_{7}$ & $30.40 d$ & $31.04 c$ & $28.32 a$ & $1.20 \mathrm{e}$ & $1.42 f$ & $1.23 \mathrm{~g}$ & $5.83 e$ & $6.85 f$ & $7.15 \mathrm{~g}$ & $8.12 f$ & $8.32 f$ & $8.64 \mathrm{~g}$ \\
\hline$P_{8}$ & 30.38de & $31.09 c$ & $28.28 a$ & $1.10 f$ & $1.25 \mathrm{~g}$ & $1.14 \mathrm{~h}$ & $5.34 f$ & $6.03 \mathrm{~g}$ & $6.62 \mathrm{~h}$ & $7.43 \mathrm{~g}$ & $7.32 \mathrm{~g}$ & $7.99 \mathrm{~h}$ \\
\hline$P_{9}$ & $30.23 e$ & $31.10 \mathrm{c}$ & $27.27 \mathrm{c}$ & $2.18 a$ & $2.62 a$ & $2.21 \mathrm{a}$ & $10.59 a$ & $12.68 a$ & $12.81 \mathrm{a}$ & $14.74 a$ & $15.40 \mathrm{a}$ & $15.48 \mathrm{a}$ \\
\hline$P_{1-4}$ & 31.32 & 31.55 & 26.55 & 1.85 & 2.11 & 1.71 & 8.99 & 10.18 & 9.90 & 12.52 & 12.37 & 11.96 \\
\hline$P_{5-9}$ & 30.45 & 31.12 & 27.91 & 1.43 & 1.24 & 5.98 & 7.20 & 8.32 & 8.42 & 30.45 & 31.12 & 27.91 \\
\hline$P_{5-9} / P_{1-4}$ & 0.97 & 0.99 & 1.05 & 0.68 & 0.73 & 0.66 & 0.73 & 0.66 & 0.68 & 0.97 & 0.99 & 1.05 \\
\hline
\end{tabular}

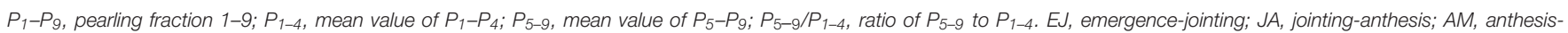

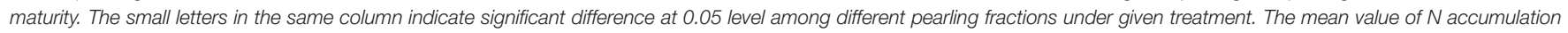
amount, $N$ contribution and $N$ distribution in fractions of $P_{5-9}$ were the total values divided by 6 , respectively, since the $P_{5-9}$ fractions accounted for $60 \%$ of the total grain mass.

the leaves have the highest $\mathrm{N}$ accumulation of any component because the photosynthetic apparatus is the greatest sink of $\mathrm{N}$ in cereal plants (Flood and Martin, 2001). In this study, the highest $\mathrm{N}$ accumulation was found in the leaves at anthesis, which mostly accumulated before jointing (Figure 1). However, the $\mathrm{N}$ that accumulated in the stem during JA was higher than that accumulated during EJ because of the rapid stem extension after jointing (Figure 1). During the initial phase of the grain growth, $\mathrm{N}$ accumulation continues in the stem and leaf, while the grain turn to be the major sink of $\mathrm{N}$ (Ferrise et al., 2010). In agreement with the study of Bertheloot et al. (2008) with bread wheat, here the $\mathrm{N}$ concentration in all of the organs started to decrease after anthesis with a rate that was proportional to their $\mathrm{N}$ concentration (Figure 2). The leaf and stem have nearly equal contributions to the grain $\mathrm{N}$, but the main part of remobilized $\mathrm{N}$, e.g., amino acids (lysine and proline) and small peptides, from these two organs was accumulated at different stages. For example, in leaf the remobilization of $\mathrm{N}$ stored during EJ was higher than the $\mathrm{N}$ stored during JA, whereas in the stems the remobilization of $\mathrm{N}$ stored during JA was higher than the $\mathrm{N}$ stored during EJ.

Rapid accumulation of $\mathrm{N}$ in grain after anthesis resulted in more than $70 \%$ of the total $\mathrm{N}$ being in the grains at maturity, which was mainly attributed to the remobilization of $\mathrm{N}$ assimilated during JA (Figure 3). In addition, the main part of the $\mathrm{N}$ was stored temporarily in the stems among all of the vegetative tissues after anthesis (Figure 3). Simpson et al. (1983) reported that the leaves, glumes, stems, and roots contribute 40, 23, 23, and $16 \%$, respectively, to the daily rate of grain $\mathrm{N}$ accumulation in wheat at mid-grain filling. Here, the contribution of $\mathrm{N}$ stored during EJ was $43.8,29.8,9.5$, and $16.5 \%$ from the leaves, stem, roots, and spike, respectively, to the grain. The $\mathrm{N}$ stored during JA in the leaves, stem, roots and spike contributed 36.1,36.5, 5.0, and
23.7\%, respectively, to the grain (Figure 2). This finding indicates that the contribution of $\mathrm{N}$ stored in the stems to the grain $\mathrm{N}$ increased during later vegetative growth stages before anthesis.

The Ndff during EJ was significantly higher than that during JA and AM in all of the organs, except for in the chaff and grain (Figure 3), suggesting that $\mathrm{N}$ assimilated during EJ is significantly involved in the functioning of meristematic tissue. It has been reported that up to $75 \%$ of the reduced $\mathrm{N}$ in cereal leaves is located in mesophyll cells, mostly as Rubisco (Bertheloot et al., 2008). On the other hand, the $\mathrm{N}$ absorbed and stored during JA and AM was mainly involved in the $\mathrm{N}$ metabolism as mobile constituents, which is remobilized to the growing grain during the reproductive stage (Bertheloot et al., 2008). Consistent with this, here higher accumulations and distributions of $\mathrm{N}$ stored during JA and AM than during EJ in mature grain were found.

\section{Distribution of N Stored at Different Periods in the Protein Fractions of Pearled Grains}

Wheat grain consists of the embryo, starchy endosperm, and bran. Among these three distinct parts, the bran comprises the pericarp (fruit coat), testa (seed coat), and outer endosperm (aleurone layer), which was included in fraction $\mathrm{P}_{1}$ in the present study (Mousia et al., 2004). The starchy endosperm consists of an outer aleurone layer and inner columns of starchy endosperm cells, which are packed with most of the starch and protein, accounting for approximately $80 \%$ of the grain mass (Tosi et al., 2011). During milling, the germ and bran are removed, leaving the starchy endosperm as the principal contributor to white flour (Dupont and Altenbach, 2003). Typically, the protein concentration is relatively low in the cells near the endosperm cavity and increases in an outward radial direction 


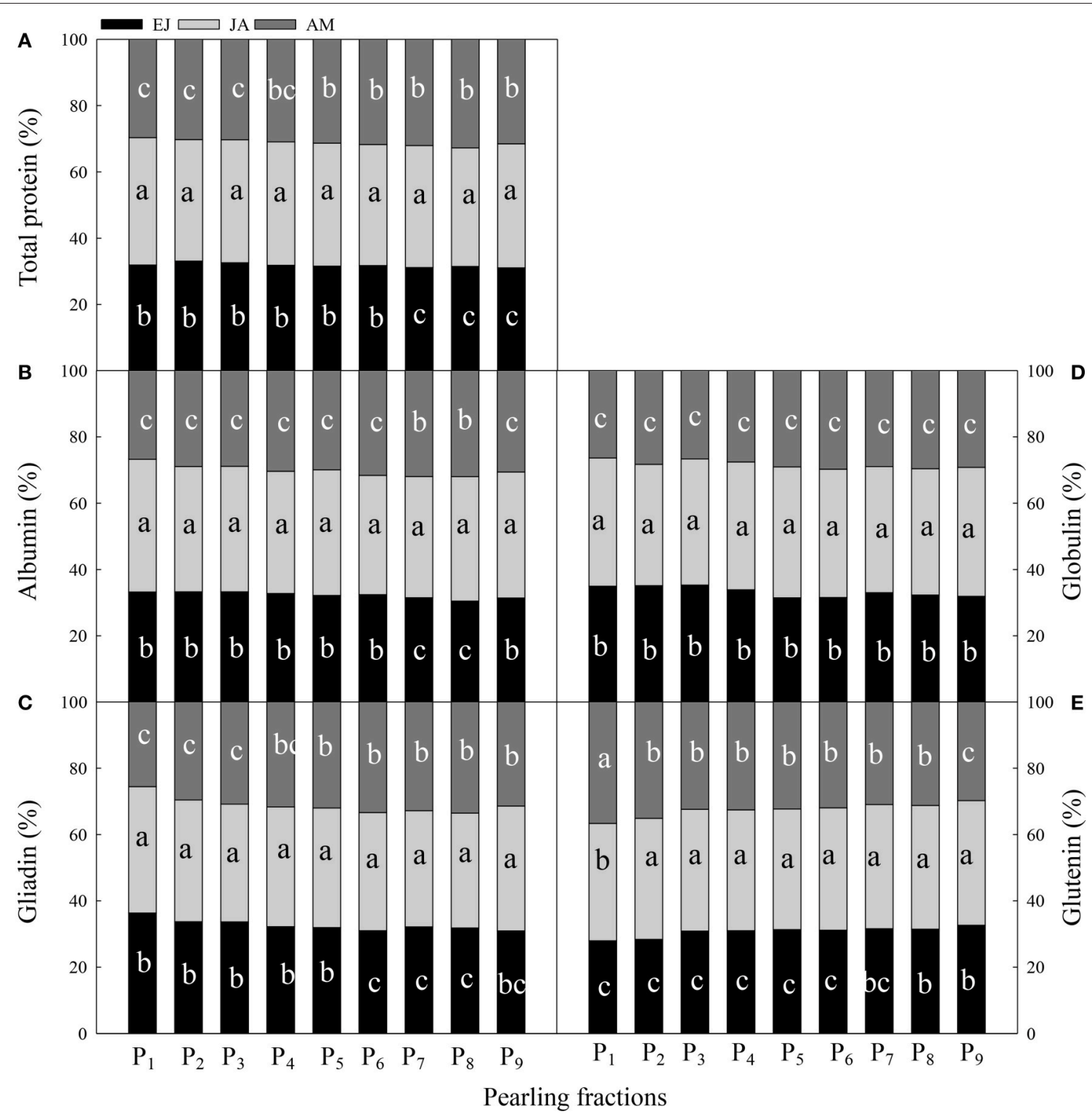

FIGURE 4 | Contribution of absorbed N druing periods from emergence to jointing (EJ), from jointing to anthesis (JA) and from anthesis to maturity (AM) to the total protein (A) and protein components (albumin, B; globulin, C; gliadin, D; glutenin, E) of different pearling fractions of wheat grain. P1-P9, pearling fraction 1-9. Different small letters for the same period (EJ, from emergence stage to jointing stage; JA, from jointing to anthesis stage; AM, from anthesis to maturity stage) indicate significant difference at 0.05 level between pearling fractions.

in a mature endosperm (Farrand and Hinton, 1974; Tosi et al., 2011). In the present study, the $\mathrm{N}$ accumulation also showed an increased trend from $\mathrm{P}_{8}$ to $\mathrm{P}_{2}$, where the endosperm is located (Table 1). Although it has been well-known that the gradients in the protein distribution are present in cereal grains, the underlying mechanisms by which the gradient was established during grain filling is still unclear. Tosi et al. (2011) hypothesized that the gradients have a developmental basis because both the quantitative and qualitative gradients of the protein distribution appear to follow the radial pattern of cell development in the endosperm. They concluded that the gradients in the gluten protein composition are related to the origin of the subaleurone cells, which are different from other starchy endosperm cells that derived from the re-differentiation of aleurone cells, but it could also be related to the signals produced by the maternal tissue that affect specific domains of the gluten protein gene promoters (Tosi et al., 2011).

It should be noted that the Ndff during EJ and JA increased from the inner starchy endosperm to the outer layer with a peak value in $\mathrm{P}_{2}$, while it was decreased in $\mathrm{P}_{1}$ (which corresponds to the outer layers, aleurone, and subaleurone; Table 1). However, the Ndff during $\mathrm{AM}$ decreased from $\mathrm{P}_{8}$ (inner layer) to $\mathrm{P}_{1}$. The 


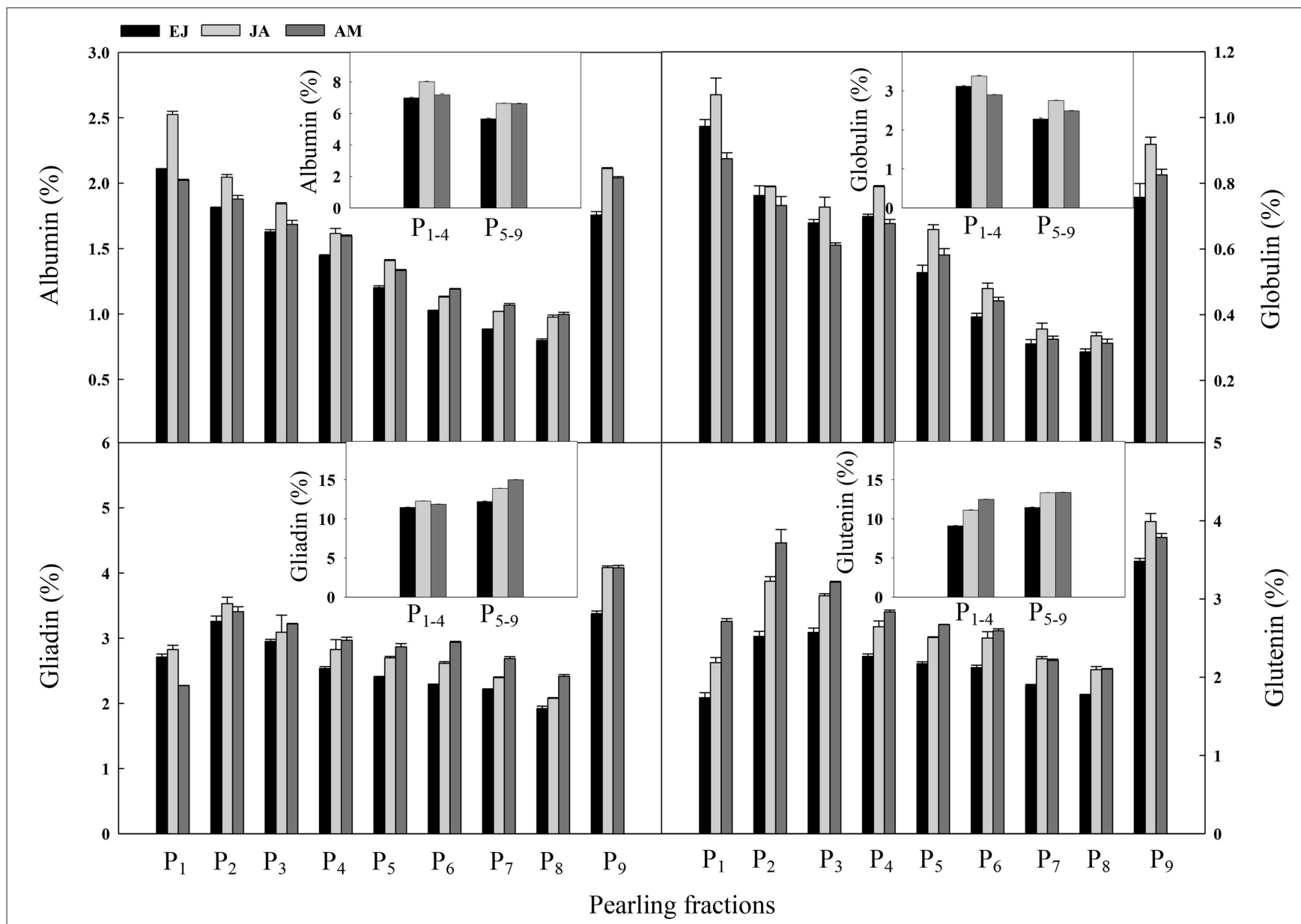

FIGURE 5 | Distribution of absorbed N druing periods of emergence-jointing (EJ), jointing-anthesis (JA), and anthesis-maturity (AM) in the protein components of different pearling fractions of wheat grain. $P_{1}-P_{9}$, pearling fraction $1-9 ; P_{1-4}$, mean value of $P_{1}-P_{4} ; P_{5-9}$, mean value of $P_{5}-P_{9}$.

varied trends in Ndff during the different growing stages could be explained by the fact that two separate pathways may operate in the developing wheat grain (Tosi et al., 2009). It could be hypothesized that the remobilization of $\mathrm{N}$ that stored before anthesis depends on the apoplast transport through the aleurone layer to the endosperm cells, and the $\mathrm{N}$ assimilated after anthesis is transferred following the radial direction in the endosperm cells. Nevertheless, the establishment of these gradients should be further studied considering the biological process of the grain development.

The contributions of $\mathrm{N}$ assimilated during different periods to the grain protein fractions varied across all of the layers, although the largest contribution was found from $\mathrm{N}$ assimilated during JA in each layer (Figure 4). For the albumin and gliadin, the contribution of $\mathrm{N}$ assimilated during EJ and JA declined from the outer layer to the inner layer, whereas the $\mathrm{N}$ contribution assimilated after anthesis showed a reversed trend. For globulin, the contribution of $\mathrm{N}$ assimilated during EJ decreased, whereas that during JA and AM increased from the outer layer to the inner layer. For glutenin, the contribution of $\mathrm{N}$ assimilated before anthesis increased, whereas the $\mathrm{N}$ contribution assimilated after anthesis decreased from the outer layer to the inner layer. The different contributions of $\mathrm{N}$ after anthesis to the protein fractions could be related to the synthesis process and transport pathway of each protein fraction during grain development (Zhu et al., 2005). The main grain structural proteins are synthesized during early grain growth, and later, the storage proteins, including gliadin and glutenin, are accumulated (Stone and Nicolas, 1996). The gliadin is a compound accumulated relatively late but fast, and part of the structural proteins can be converted into storage proteins during the latter grain-filling stage (Stone and Nicolas, 1996).

The distributions of $\mathrm{N}$ in grain assimilated during different periods across all of the layers showed different trends (Figure 5). For example, for albumin and gliadin, the $\mathrm{N}$ assimilated during JA had a high distribution ratio in the outer layer, whereas the contribution of $\mathrm{N}$ assimilated after anthesis increased faster than that before anthesis. This finding indicates that the utilization efficiency of $\mathrm{N}$ assimilated during JA is higher for structural proteins and greater amount of structural proteins were converted to storage proteins in the inner layers. However, for globulin and glutenin, the $\mathrm{N}$ assimilated after anthesis had 
the highest distribution ratio in most of the layers indicating that the utilization efficiency of those $\mathrm{N}$ is higher than that assimilated before anthesis for storage proteins, especially in the inner layers. This result is in accordance with the finding that the increased $\mathrm{N}$ application rate after anthesis can enhance the protein concentration in the endosperm of wheat grain (Johansson et al., 2004).

In conclusion, our results indicated that the remobilization of $\mathrm{N}$ stored from jointing to anthesis was the major contributor to the grain $\mathrm{N}$, especially in the outer layer. The distributions of $\mathrm{N}$ assimilated during EJ, JA, and AM in albumin and globulin showed a decreased trend from the surface layer to the center part. For globulin and glutenin, the $\mathrm{N}$ stored after anthesis had the highest distribution ratio in most of the layers, which indicates that the utilization efficiency of $\mathrm{N}$ assimilated after anthesis is higher than that before anthesis for the storage proteins. Therefore, modification in the $\mathrm{N}$ application rate during the different growth stages should be an effective approach to regulating the distribution of the protein fractions in the pearled grains for specific end-use. However, the

\section{REFERENCES}

Arduini, I., Masoni, A., Ercoli, L., and Mariotti, M. (2006). Grain yield, and dry matter and nitrogen accumulation and remobilization in durum wheat as affected by variety and seeding rate. Eur. J. Agron. 25, 309-318. doi: 10.1016/j.eja.2006.06.009

Bertheloot, J., Martre, P., and Andrieu, B. (2008). Dynamics of light and nitrogen distribution during grain filling within wheat canopy. Plant Physiol. 148, 1707-1720. doi: 10.1104/pp.108.124156

Beta, T., Nam, S., Dexter, J. E., and Sapirstein, H. D. (2005). Phenolic content and antioxidant activity of pearled wheat and roller-milled fractions. Cereal Chem. 82, 390-393. doi: 10.1094/CC-82-0390

Cookson, W., Rowarth, J., and Cameron, K. (2001). The fate of autumn-, late winter-and spring-applied nitrogen fertilizer in a perennial ryegrass (Lolium perenne L.) seed crop on a silt loam soil in Canterbury, New Zealand. Agric. Ecosyst. Environ. 84, 67-77. doi: 10.1016/S0167-8809(00)0 0196-1

Dexter, J., and Wood, P. (1996). Recent applications of debranning of wheat before milling. Trends Food Sci. Technol. 7, 35-41. doi: 10.1016/0924-2244(96)81326-4

Dupont, F. M., and Altenbach, S. B. (2003). Molecular and biochemical impacts of environmental factors on wheat grain development and protein synthesis. J. Cereal Sci. 38, 133-146. doi: 10.1016/S0733-5210(03)00 $030-4$

Ercoli, L., Lulli, L., Mariotti, M., Masoni, A., and Arduini, I. (2008). Postanthesis dry matter and nitrogen dynamics in durum wheat as affected by nitrogen supply and soil water availability. Eur. J. Agron. 28, 138-147. doi: 10.1016/j.eja.2007.06.002

Fares, C., Troccoli, A., and Di Fonzo, N. (1996). Use of friction debranning to evaluate ash distribution in Italian durum wheat cultivars. Cereal Chem. 73, 232-234.

Farrand, E., and Hinton, J. (1974). Study of relationships between wheat protein contents of two UK varieties and derived flour protein contents at varying extraction rates. II. Studies by hand-dissection of individual grains. Cereal Chem. 51, 56-65.

Ferrise, R., Triossi, A., Stratonovitch, P., Bindi, M., and Martre, P. (2010). Sowing date and nitrogen fertilization effects on dry matter and nitrogen dynamics for durum wheat: an experimental and simulation study. Field Crops Res. 117, 245-257. doi: 10.1016/j.fcr.2010.03.010

Flood, R. G., and Martin, P. J. (2001). Nitrogen accumulation and distribution at anthesis and maturity in ten wheats grown at three sites in north-western Victoria. Aust. J. Exp. Agric. 41, 533-540. doi: 10.1071/EA98025 establishment of these gradients should be further studied while considering the biological processes that are involved in the grain development.

\section{AUTHOR CONTRIBUTIONS}

DJ and QZ designed research; XL and LZ performed the experiment; XW, JC, XL, and LZ analyzed the data; LF, TD, WC, and $\mathrm{DJ}$ revised the manuscript.

\section{ACKNOWLEDGMENTS}

This study is supported by projects of the National Natural Science Foundation for Distinguished Young Scientists (31325020), the National Natural Science Foundation of China (31471445, 31171484, and 31401326), the China Agriculture Research System (CARS-03), Jiangsu Collaborative Innovation Center for Modern Crop Production (JCIC-MCP), Collaborative Innovation Center of Gene Resources, and the National Non-profit Program by Ministry of Agriculture (2014039).

Gys, W., Courtin, C. M., and Delcour, J. A. (2004). Reduction of xylanase activity in flour by debranning retards syruping in refrigerated doughs. J. Cereal Sci. 39, 371-377. doi: 10.1016/j.jcs.2004.01.003

Hareland, G. A. (2003). Effects of pearling on falling number and $\alpha$-amylase activity of preharvest sprouted spring wheat. Cereal Chem. 80, 232-237. doi: 10.1094/CCHEM.2003.80.2.232

Johansson, E., Prieto-Linde, M. L., and Svensson, G. (2004). Influence of nitrogen application rate and timing on grain protein composition and gluten strength in Swedish wheat cultivars. J. Plant Nutr. Soil Sci. 167, 345-350. doi: 10.1002/jpln.200320332

Kichey, T., Hirel, B., Heumez, E., Dubois, F., and Le Gouis, J. (2007). In winter wheat (Triticum aestivum L.), post-anthesis nitrogen uptake and remobilisation to the grain correlates with agronomic traits and nitrogen physiological markers. Field Crops Res. 102, 22-32. doi: 10.1016/j.fcr.2007. 01.002

Li, X., Cai, J., Li, H., Bo, Y., Liu, F., Jiang, D., et al. (2012). Effect of Shading from jointing to maturity on high molecular weight glutenin subunit accumulation and glutenin macropolymer concentration in grain of winter wheat. J. Agron. Crop Sci. 198, 68-79. doi: 10.1111/j.1439-037X.2011.0 0484.x

Liu, Z., Wang, H., Wang, X., Zhang, G., Chen, P., and Liu, D. (2007). Phytase activity, phytate, iron, and zinc contents in wheat pearling fractions and their variation across production locations. J. Cereal Sci. 45, 319-326. doi: 10.1016/j.jcs.2006.10.004

Liu, Z., Wang, H., Wang, X.-E., Xu, H., Gao, D., Zhang, G., et al. (2008). Effect of wheat pearling on flour phytase activity, phytic acid, iron, and zinc content. LWT Food Sci. Technol. 41, 521-527. doi: 10.1016/j.lwt.2007. 04.001

Malik, A. H., Kuktaite, R., and Johansson, E. (2013). Combined effect of genetic and environmental factors on the accumulation ofproteins in the wheat grain and their relationship to bread-making quality. J. Cereal Sci. 57, 170-174. doi: 10.1016/j.jcs.2012.09.017

Masclaux-Daubresse, C., Reisdorf-Cren, M., and Orsel, M. (2008). Leaf nitrogen remobilisation for plant development and grain filling. Plant Biol. 10, 23-36. doi: 10.1111/j.1438-8677.2008.00097.x

Mousia, Z., Edherly, S., Pandiella, S. S., and Webb, C. (2004). Effect of wheat pearling on flour quality. Food Res. Int. 37, 449-459. doi: 10.1016/j.foodres.2004.02.012

Palta, J., and Fillery, I. (1995). N application increases pre-anthesis contribution of dry matter to grain yield in wheat grown on a duplex soil. Crop Pasture Sci. 46, 507-518. doi: 10.1071/AR9950507 
Shi, Z., Jing, Q., Cai, J., Jiang, D., Cao, W., and Dai, T. (2012b). The fates of ${ }^{15} \mathrm{~N}$ fertilizer in relation to root distributions of winter wheat under different $\mathrm{N}$ splits. Eur. J. Agron. 40, 86-93. doi: 10.1016/j.eja.2012.01.006

Shi, Z., Li, D., Jing, Q., Cai, J., Jiang, D., Cao, W., et al. (2012a). Effects of nitrogen applications on soil nitrogen balance and nitrogen utilization of winter wheat in a rice-wheat rotation. Field Crops Res. 127, 241-247. doi: 10.1016/j.fcr.2011.11.025

Simpson, R. J., Lambers, H., and Dalling, M. J. (1983). Nitrogen redistribution during grain growth in wheat (Triticum aestivum L.). Plant Physiol. 71, 7-14. doi: $10.1104 /$ pp.71.1.7

Stone, P., and Nicolas, M. (1996). Varietal differences in mature protein composition of wheat resulted from different rates of polymer accumulation during grain filling. Func. Plant Biol. 23, 727-737. doi: 10.1071/pp9960727

Tosi, P., Gritsch, C. S., He, J., and Shewry, P. R. (2011). Distribution of gluten proteins in bread wheat (Triticum aestivum) grain. Ann. Bot. 108, 23-35. doi: 10.1093/aob/mcr098

Tosi, P., Parker, M., Gritsch, C. S., Carzaniga, R., Martin, B., and Shewry, P. R. (2009). Trafficking of storage proteins in developing grain of wheat. J. Exp. Bot. 60, 979-991. doi: 10.1093/jxb/ern346
Zhang, X., Jiang, D., Zheng, C., Dai, T., and Cao, W. (2011). Post-anthesis salt and combination of salt and waterlogging affect distributions of sugars, amino acids, $\mathrm{Na}^{+}$and $\mathrm{K}^{+}$in wheat. J. Agron. Crop Sci. 197, 31-39. doi: 10.1111/j.1439037X.2010.00438.x

Zhu, X., Zhou, J., Feng, C., Guo, W., and Peng, Y. (2005). Differences of protein and its component accumulation in wheat for different end uses. Acta Agron. Sin. 31, 342-347.

Conflict of Interest Statement: The authors declare that the research was conducted in the absence of any commercial or financial relationships that could be construed as a potential conflict of interest.

Copyright (c) 2016 Li, Zhou, Liu, Zhou, Cai, Wang, Dai, Cao and Jiang. This is an open-access article distributed under the terms of the Creative Commons Attribution License (CC BY). The use, distribution or reproduction in other forums is permitted, provided the original author(s) or licensor are credited and that the original publication in this journal is cited, in accordance with accepted academic practice. No use, distribution or reproduction is permitted which does not comply with these terms. 


\section{OPEN ACCESS}

Edited by:

Antonio M. De Ron,

Spanish National Research Council -

Misión Biológica de Galicia, Spain

Reviewed by:

Miroslav Griga

AGRITEC Plant Research Ltd.,

Czech Republic

Albert Vandenberg,

University of Saskatchewan, Canada

*Correspondence:

Remo Reggiani

reggiani@ibba.cnr.it

Specialty section:

This article was submitted to Crop Science and Horticulture,

a section of the journal

Frontiers in Plant Science

Received: 15 February 2016 Accepted: 05 May 2016 Published: 20 May 2016

Citation:

Galasso I, Russo R, Mapelli S, Ponzoni E, Brambilla IM, Battelli G and Reggiani $R$ (2016) Variability in Seed

Traits in a Collection of Cannabis

sativa L. Genotypes.

Front. Plant Sci. 7:688

doi: 10.3389/fpls.2016.00688

\section{Variability in Seed Traits in a Collection of Cannabis sativa L. Genotypes}

\author{
Incoronata Galasso ${ }^{1}$, Roberto Russo ${ }^{1}$, Sergio Mapelli ${ }^{1}$, Elena Ponzoni ${ }^{1}$, Ida M. Brambilla ${ }^{1}$, \\ Giovanna Battelli ${ }^{2}$ and Remo Reggiani ${ }^{1 *}$ \\ ${ }^{1}$ Institute of Agricultural Biology and Biotechnology, CNR, Milano, Italy, ${ }^{2}$ Institute of Science of Food Production, CNR, \\ Milano, Italy
}

The seed of Cannabis sativa $\mathrm{L}$. is an expanding source of proteins and oil for both humans and animals. In this study, the proximate composition of a collection of hemp cultivars and accessions of different geographical origins grown under the same conditions for 1 year was analyzed in order to identify potential accessions to improve hemp cultivars. Fatty acids, tocopherols, and antinutritional components, as well as concentrations of crude protein and oil were quantified. The seed oil concentrations varied between 285 and $360 \mathrm{~g} \mathrm{~kg}^{-1}$ dry seed (DS), while crude protein ranged between 316 and $356 \mathrm{~g} \mathrm{~kg}^{-1}$ dry matter (DM). The seed oil was mainly composed of unsaturated fatty acids and, as expected, the dominant fatty acids were linoleic and $\alpha$-linolenic acid. A high variability among the cultivars and accessions was also detected for polyphenolic content which ranged from 5.88 to $10.63 \mathrm{~g} \mathrm{~kg}^{-1} \mathrm{DM}, \mathrm{cv}$. Felina was the richest, whereas $\mathrm{cv}$. Finola had the lowest polyphenolic content. Regarding antinutritional compounds in seed, a high variability was detected among all genotypes analyzed and phytic acid was particularly abundant (ranging between 43 and $75 \mathrm{~g} \mathrm{~kg}^{-1} \mathrm{DM}$ ). In conclusion, our results reveal noticeable differences among hemp seed genotypes for antinutritional components, oil and protein content. Collectively, this study suggests that the hemp seed is an interesting product in terms of protein, oil and antioxidant molecules but a reduction of phytic acid would be desirable for both humans and monogastric animals. The high variability detected among the different genotypes indicates that an improvement of hemp seed might be possible by conventional and/or molecular breeding.

\footnotetext{
Keywords: protein, antinutritional compounds, fatty acids, tocopherols, polyphenols, antioxidant activity,
} genotype diversity

\section{INTRODUCTION}

Cannabis sativa L. (hemp) is a wind-pollinated annual plant that originated in central Asia (Li, 1973, 1974; Mukherjee et al., 2008). Hemp, naturally, is a dioecious crop, but some monoecious cultivars have been obtained as a result of earlier breeding efforts. Hemp is an ancient crop that has been cultivated worldwide until the early twentieth century, after which its cultivation declined. Recently, interest in this multipurpose crop delivering fibers, shives, and seeds, has been renewed by an increasing demand not only for natural fibers but also for the high content and quality of seed protein and oil. Hemp seed contains $25-35 \%$ oil, $20-25 \%$ protein, $20-30 \%$ carbohydrates, $10-15 \%$ 
insoluble fiber, vitamins, and minerals such as phosphorus, potassium, magnesium, sulfur, and calcium (Deferne and Pate, 1996; Callaway, 2004; House et al., 2010). The increasing demand for vegetable oils and proteins, along with current awareness about their nutritional and functional role in human diet, has made indispensable to characterize new plant sources. In this regard, hemp seed contains all the essential amino acids and fatty acids necessary to maintain healthy human life, and it might be a new good source of nutrients for both humans and livestock (Osburn, 1992; Deferne and Pate, 1996; Callaway, 2004; Russo and Reggiani, 2015a).

The principal value of hemp seed oil resides in its fatty acid composition. It contains the two dietary essential fatty acids:

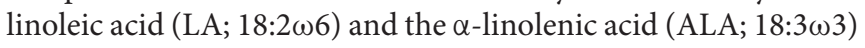
in the ratio of 2.5-3:1, which has been claimed as ideal for human nutrition (Simopoulos, 2008). In addition, the biological metabolites of LA and ALA, the $\gamma$-linolenic acid (GLA; 18:3 $\omega 6$ ) and stearidonic acid (SDA; 18:4 33 ) are also present in hemp seed oil, which makes the nutritional value of hemp seed superior to other seed oils (Leizer et al., 2000). Both GLA and SDA act as precursors for the rapid synthesis of longer chain fatty acids which are involved in many important biological processes (Guil-Guerrero et al., 2010).

Furthermore, hemp seeds are also an excellent nutritional source of high quality proteins (Russo and Reggiani, 2015a), as they are easily digested, absorbed, and utilized (House et al., 2010). The two main proteins in hemp seeds are the globulin edestin and albumin (Callaway, 2004; Tang et al., 2006). Edestin, which accounts for about $60-80 \%$ of the total protein content (Odani and Odani, 1998; Tang et al., 2006), has been the subject of intensive investigation for a long time. Recently, an accurate characterization of edestin at biochemical and molecular level has been done by Kim and Lee (2011) and Docimo et al. (2014). This storage protein, which contains exceptionally high levels of arginine and glutamic acids (Russo and Reggiani, 2015a), is easily digested and rich in all essential amino acids (Callaway, 2004; House et al., 2010; Docimo et al., 2014).

However, the nutritional quality of plant proteins, as measured by their amino acid composition and digestibility, is influenced by numerous factors. The amino acid composition may be influenced by genotypic variability or agronomic conditions such as soil fertility and postharvest processing that alters the ratio of seed components (e.g., shelling). The digestibility of proteins may be affected by protein structure, presence of antinutritional compounds and high temperature processing (Sarwar, 1997). Recently, it has been demonstrated that hemp meal (a by-product obtained from mechanical or solvent extraction of the seed oil) contains some antinutritional compounds that need to be considered carefully in feeding applications (Russo and Reggiani, 2013). In hemp seed, the content of phytic acid (inositol hexaphosphate, $\mathrm{IP}_{6}$ ) and trypsin inhibitors appeared fairly high compared to a number of other antinutritional compounds analyzed (Russo and Reggiani, 2015b). Phytic acid is the main organic form of phosphorus present in plant seeds. Its presence reduces protein digestibility and increases the excretion of endogenous nitrogen, amino acids and minerals, in particular bivalent cations (Cowieson et al., 2004). The trypsin inhibitors are present in many species of Leguminosae, Brassicaceae, and Gramineae, and reduce the biological activity of trypsin enzyme which is involved in the breakdown of many different proteins (Guillamón et al., 2008).

Therefore, in this work several hemp cultivars and accessions of different origins, grow in the same environment for 1 year, have been characterized for quantity and quality of seed oil, protein content, antinutritional compounds, polyphenols, tocopherols, and antioxidant activity. The objective of this study was the evaluation of these biochemical traits in order to identify potential accessions to improve hemp cultivars. To achieve this goal, we analyzed the seeds of 20 genotypes composed by the most used non-drug hemp cultivars that are allowed to be cultivated in Europe and a collection of hemp accessions obtained from genebanks.

\section{MATERIALS AND METHODS}

\section{Plant Material}

Seeds of hemp cultivars (cvs) and accessions were kindly provided by the genebank of IPK Gatersleben (Leibniz Institute of Plant Genetics and Crop Plant Research) (http://gbis.ipk-gatersleben.de/), the Centro di Ricerca per le Colture Industriali (CRA-CIN, Italy) and by seed companies (Table 1). All genotypes were sown at the beginning of spring (30 March 2014) in pots of about $1 \mathrm{~m}^{2}$ with three replications. The pots were fertilized with $80 \mathrm{~kg} \mathrm{ha}^{-1} \mathrm{~N}$ after the seedlings had emerged. The average air temperature was $19.4^{\circ} \mathrm{C}$ (30 March31 October 2014). During summer the plants were irrigated when necessary. In order to avoid cross-pollination among the different cvs and accessions, all pots were covered with two layers of cloth $(\sim 200$ mesh; Virexgomma, Somma Lombardo, Varese, Italy) during flowering time. Seeds were harvested at maturity from August to October. The harvesting date depended on the genotype. After harvesting, seeds were forced-air-dried $\left(30^{\circ} \mathrm{C}\right)$ in a ventilated chamber until $8 \%$ seed moisture was reached. Thousand seed weight (TSW) was established on five replicates of 100 seeds each. Seeds were manually counted and weighed. During the flowering stage, a pool of inflorescences (flowers and leaves) were collected from each pot. The $\Delta^{9}$ tetrahydrocannabinol (THC) concentration was measured in each genotype by the HPTLC densitometry method according to Fischedick et al. (2009).

\section{Total Protein and Antinutritional Compound Analysis}

Samples of whole seeds were ground in a mortar and defatted by extracting with hexane $(1: 10, \mathrm{w} / \mathrm{v})$. Protein was extracted from defatted flours using a Plant Total Protein Extraction Kit (Sigma-Aldrich, St. Louis, USA). The Plant Total Protein Extraction Kit includes two reagents, a plant specific protease inhibitor cocktail and a chaotropic reagent with increased solubilizing power to extract more hydrophobic proteins. Protein content was determined via a Quantum Protein Kit (Euroclone, Milan, Italy), using Bovine Serum Albumin (BSA) as standard. 
TABLE 1 | List of hemp accessions (from CAN19 to CAN58) and cultivars (from cv. Finola to cv. Kc Dora) analyzed in this study.

\begin{tabular}{|c|c|c|c|c|}
\hline Hemp genotypes & Sex type & Origin & Flowering time & Supply source \\
\hline C. sativa CAN19 & $D^{*}$ & Italy & End July & $\mathrm{IPK}^{\mathrm{a}}$ \\
\hline C. sativa CAN20 & $\mathrm{D}$ & Korea & End July & IPK \\
\hline C. sativa CAN24 & $\mathrm{D}$ & Italy & End July & IPK \\
\hline C. sativa CAN26 & $\mathrm{D}$ & Turkey & End July & IPK \\
\hline C. sativa CAN39 & $\mathrm{D}$ & China & End July & IPK \\
\hline C. sativa CAN40 & $\mathrm{D}$ & Italy & End July & IPK \\
\hline C. sativa CAN48 & $\mathrm{D}$ & Italy & End July & IPK \\
\hline C. sativa CAN51 & $\mathrm{D}$ & Argentina & End July & IPK \\
\hline C. sativa CAN58 & $\mathrm{D}$ & Spain & End August & IPK \\
\hline C. sativa cv. Finola & $\mathrm{D}$ & Finland & End June & Seed company ${ }^{b}$ \\
\hline C. sativa cv. Carmagnola & $\mathrm{D}$ & Italy & Beginning August & Seed companyc \\
\hline C. sativa cv. Carmagnola Selezionata (CS) & $\mathrm{D}$ & Italy & Beginning August & CRA-CIN ${ }^{d}$ \\
\hline C. sativa cv. Fibranova & $\mathrm{D}$ & Italy & Beginning August & CRA-CIN ${ }^{d}$ \\
\hline C. sativa cv. Fedora & $M^{\star \star}$ & France & Middle July & Seed company ${ }^{e}$ \\
\hline C. sativa cv. Futura 75 & M & France & Middle July & Seed company ${ }^{e}$ \\
\hline C. sativa cv. Felina 32 & M & France & Middle July & Seed company ${ }^{e}$ \\
\hline C. sativa cv. Ferimon & $M$ & France & Middle July & Seed company ${ }^{e}$ \\
\hline C. sativa cv. Codimono & $\mathrm{M}$ & Italy & Middle July & CRA-CIN ${ }^{f}$ \\
\hline C. sativa cv. Carmaleonte & $M$ & Italy & Middle July & CRA-CIN ${ }^{f}$ \\
\hline C. sativa cv. Kc Dora & M & Hungary & End July & Seed company ${ }^{g}$ \\
\hline
\end{tabular}

a IPK, Leibniz Institute of Plant Genetics and Crop Plant Research, Gatersleben, Germany.

${ }^{b}$ Exchange with other scientists. Seed company: Finola Ltd, http://www.finola.fi/.

${ }^{c}$ Assocanapa srl, Carmagnola (Torino, Italy) http://www.assocanapa.org/.

${ }^{d}$ CRA-CIN, Centro di ricerca per le colture industriali, Rovigo (IT) from dr. G. Grassi.

${ }^{e}$ Cooperative Centrale des Producteurs de Semences de Chanvre (Le Mans, France).

${ }^{f}$ CRA-CIN, Centro di ricerca per le colture industriali (CRA-CIN), Bologna (IT) from dr. A. Di Candilo.

${ }^{g}$ Agromag Kft, Hungary, http://www.agromag.hu/en.

$D^{\star}$, Dioecious plant; $M^{\star \star}$, Monoecious plant.

Phytic acid was extracted from defatted flour as described by De Boland et al. (1975) with minor modifications. Briefly, the phytic acid was extracted with $0.4 \mathrm{~N} \mathrm{HCl}$ plus $0.7 \mathrm{M} \mathrm{Na}_{2} \mathrm{SO}_{4}$ and then precipitated by $15 \mathrm{mM} \mathrm{FeCl}_{3}$ in $0.2 \mathrm{~N} \mathrm{HCl}$. The phosphorus content of the precipitate was colorimetrically determined, after acid digestion with sulfuric acid, according to Chen et al. (1956). Phytic acid content was determined by multiplying the phytate phosphorus content by a constant factor of 3.55 (Raboy and Dickinson, 1984).

Trypsin inhibitor (TI) was extracted from defatted flour with $2 \mathrm{mM}$ glycine buffer $\mathrm{pH} 11$ containing $2 \mathrm{mM} \mathrm{NaCl}$, $10 \mathrm{mM}$ urea, and $25 \mathrm{mM}$ EDTA. Trypsin inhibitor activity was measured as described by Russo and Reggiani (2013). To $200 \mu \mathrm{L}$ of extract were added $200 \mu \mathrm{L}\left(40 \mu \mathrm{g} \cdot \mathrm{mL}^{-1}\right)$ of trypsin and then pre-incubated at $37^{\circ} \mathrm{C}$ for $3 \mathrm{~min}$. After this, $500 \mu \mathrm{L}\left(0.4 \mathrm{mg} \cdot \mathrm{mL}^{-1}\right)$ of N-benzoyl-DL-arginine-pnitroanilide (pre-warmed to $37^{\circ} \mathrm{C}$ ) were added to start the reaction. After incubation at $37^{\circ} \mathrm{C}$ for $10 \mathrm{~min}, 100 \mu \mathrm{L} 30 \%$ $(\mathrm{v} / \mathrm{v})$ acetic acid was added to terminate the reaction and the mix centrifuged. Activity of trypsin was measured by the absorbance at $410 \mathrm{~nm}$ due to p-nitroaniline released. One unit of TI was defined as 0.01 decreases in absorbance at $410 \mathrm{~nm}$ under assay conditions compared with the control (without inhibitor).

\section{Total Phenolic Content, Antioxidant Activity and Tocopherols}

Phenols were extracted from defatted flours with $80 \%$ ethanol at $70^{\circ} \mathrm{C}$ (Russo and Reggiani, 2015c). The total phenolic (TP) content was determined using the Folin-Ciocalteu method according to Velioglu et al. (2006). One hundred microliters of the ethanolic extract or caffeic acid standard (50, 100, 200, 400 $\mu \mathrm{g})$ was combined with $500 \mu \mathrm{L}$ of Folin-Ciocalteu reagent and allowed to stand at room temperature for $5 \mathrm{~min}$. Then, $400 \mu \mathrm{L}$ of 60 g.L $\mathrm{L}^{-1}$ sodium carbonate solution were added to the mix and the tubes were heated at $45^{\circ} \mathrm{C}$ for $15 \mathrm{~min}$. The absorbance was measured at $765 \mathrm{~nm}$ after sitting for $30 \mathrm{~min}$ in the dark. The results were expressed as caffeic acid equivalents per $\mathrm{g}$ of dry weight ( $\left.\mathrm{g} \mathrm{CAE} \mathrm{kg}^{-1} \mathrm{DW}\right)$.

The antioxidant activity of the ethanolic extracts was determined by the Antioxidant Assay Kit (Sigma-Aldrich, Milan, Italy) according to the manufacturer's protocol. In the kit, trolox (a water-soluble vitamin $\mathrm{E}$ analog) was present as a standard for antioxidant control. Trolox has been broadly applied in assaying food samples (Re et al., 1999; Huang et al., 2005; Russo and Reggiani, 2015c). The results were expressed as mol trolox equivalents per $\mathrm{kg}$ of dry weight ( $\left.\mathrm{mol} \mathrm{TE} \mathrm{kg}^{-1} \mathrm{DW}\right)$.

Tocopherols were analyzed in HPLC by direct injection of oil (Gimeno et al., 2000). The tocopherol isomers were separated 
using an HPLC Jasco Tritotar III and Jasco MD910 Diode Array Detector (DAD). An amount of 5-10 $\mu \mathrm{l}$ of pure oil was loaded into a Kinetex $2.6 \mu \mathrm{m} \mathrm{C18} \mathrm{100A} \mathrm{(Phenomenex,} \mathrm{Castel} \mathrm{Maggiore,}$ Italy) column $(100 \times 4.6 \mathrm{~mm})$ and eluted with $1.5 \mathrm{ml} . \mathrm{min}^{-1}$ $\mathrm{MeOH} 95 \%$. The DAD spectra gave information on the purity of tocopherols. Absorbance at $280 \mathrm{~nm}$ was elaborated by the Borwin software system to determine tocopherol amounts in comparison with standards (Sigma-Aldrich, St. Louis, USA).

\section{Oil Content and Fatty Acid Composition}

Lipid extraction was performed according to Hara and Radin (1978). The fatty acid composition was determined in triplicate by gas chromatography (GC) of the oils after transesterification according to Christie (1982) with the modifications described by Chouinard et al. (1999). Gas chromatography analyses were carried out on an Agilent 6890 GC system (Paolo alto, CA) equipped with autosampler, on column injector, and a FID detector. The separation was performed on a $100 \%$ dimethylpolysiloxane column (CP-Sil 88 for FAME, $100 \mathrm{~m} \times 0.25$ $\mathrm{mm} \times 25 \mu \mathrm{m})$ adopting the following conditions. The carrier gas was $\mathrm{H}_{2}$ at a constant pressure of 28 psi. Column temperature was set at $190^{\circ} \mathrm{C}$ for $22 \mathrm{~min}$, and then increased to $240^{\circ} \mathrm{C}$ at a rate of $10^{\circ} \mathrm{C} \mathrm{min}^{-1}$ and maintained for $13 \mathrm{~min}$. Fatty acid methyl esters (FAMEs) were identified by comparison of their retention times with known standards (37-component FAME mix, Supelco 47885-U) and expressed as relative percentage of total fatty acids.

\section{Statistical Analyses}

All analyses were carried out in triplicate. Analysis of Variance (ANOVA) was applied to establish significant differences $(P \leq$ 0.01 ) between hemp genotypes in the levels of seed traits using SPSS version 16.0 software. Mean separation was performed using Duncan's test and referring to $P \leq 0.05$ probability level. Pearson's correlations between seed traits were also calculated.

\section{RESULTS}

All hemp plants considered in this study were analyzed for THC content (Figure 1). They showed THC concentrations between 0.07 and $0.19 \%$ in their inflorescences, which are below the authorized limit of $0.20 \%$ on a DM basis set in Italy.

\section{Protein and Antinutritional Compounds}

Hemp seed meal was found to be a rich protein source with a mean protein content of $337 \mathrm{~g} . \mathrm{kg}^{-1}$ of DW (Table 2). ANOVA analysis revealed significant differences in protein content among cvs and accessions. The CAN20 and CAN40 accessions showed the highest protein concentration in the meal (356 and 354 g.kg-1 DW, respectively). Pearson analysis demonstrated that the total protein content was highly correlated with TSW indicating that protein is an important component of seed weight (Table 3). A positive correlation is also evident among protein content and antioxidant activity (Table 3 ).

Phytate content was very high in all tested meals (mean $5.84 \%)$. The lowest phytate content was detected in Kc Dora $4.37 \%$ and CAM40 4.38\%, whereas cv Felina meal showed almost twice that amount (7.55\%; Table 2). Regarding the antinutritional

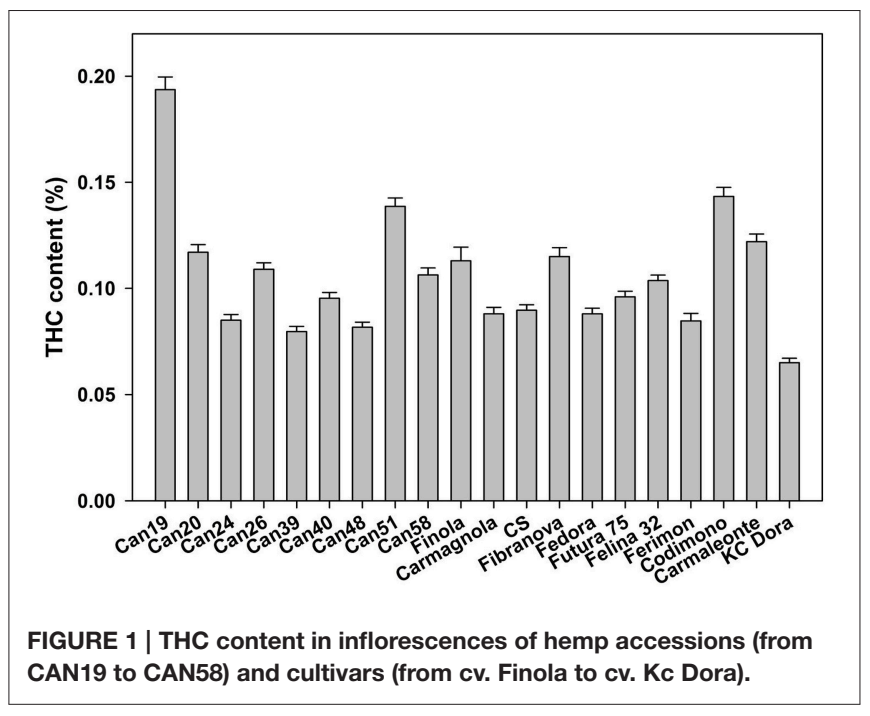

TI, Table 2 shows that its activity varied from 10.8 to 27.8 unit.mg ${ }^{-1}$ of defatted flour. Among hemp genotypes, Fibranova exhibited the lowest TI activity (Table 2). ANOVA analysis also evidenced significant differences in TI activity between cvs and accessions (cvs are on average richer in TI). Pearson analysis indicated that TI activity is highly correlated to phytate content (Table 3).

\section{Tocopherols, Total Phenolic Content, and Antioxidant Activity}

All hemp seeds analyzed in this study contained primarily $\gamma$ tocopherol and lesser quantities of $\alpha$ - and $\delta$-tocopherols (data not shown). Table 2 reports the total content of tocopherols including all isomers identified. The average of tocopherol concentration for hemp genotypes was $0.88 \mathrm{~g} \cdot \mathrm{Kg}^{-1}$ of oil. ANOVA analysis showed that the differences in tocopherols among all genotypes and groups (cvs vs. accessions) are significant at 0.01 level. A similar result was observed for TP content (Table 2). The highest TP content was detected in some monoecious cultivars (see those with "a" letters in parenthesis by the Duncan's range test). According to Table 2, TP varied from 5.88 to $10.63 \mathrm{mg} \mathrm{CAE} \mathrm{g}^{-1} \mathrm{DW}$ of defatted flour. Pearson analysis showed that TP content was positively correlated to antioxidant activity $(r=0.775$, Table 3$)$ and tocopherols $(r=0.275)$ at the 0.01 and 0.05 levels, respectively. These data support the notion that phenols and tocopherols are effective scavengers of free radicals. As can be seen in Table 2, antioxidant activity ranged from 0.92 to $1.70 \mathrm{~mol} \mathrm{TE} \mathrm{kg} \mathrm{kg}^{-1} \mathrm{DW}$ of defatted flour. The $\mathrm{cv}$ Felina exhibited the highest antioxidant activity (Table 2).

\section{Oil Content and Composition}

The oil content of the hemp seed genotypes ranged from 28.5 to $36.0 \%$ (Table 2) with an average of $31.9 \%$. The highest oil content was measured in CAN58 (36\%) and the lowest in Carmaleonte $(28.5 \%)$. ANOVA revealed significant differences among genotypes for oil content at the 0.01 level. 
TABLE 2 | Seed traits in hemp accessions (from CAM19 to CAN58) and cultivars (from Finola to Kc Dora).

\begin{tabular}{|c|c|c|c|c|c|c|c|c|c|c|}
\hline & TSW ${ }^{a}$ & Oilb & $\operatorname{ALA}(\omega 3)^{\mathrm{c}}$ & LA $(\omega 6)^{c}$ & Toc $^{d}$ & Protein ${ }^{d}$ & Phytate $^{d}$ & $\mathrm{TI}^{\mathrm{e}}$ & $T P^{f}$ & Antiox 9 \\
\hline CAN19 & 17.5 (efgh) & 32.5 (defg) & $15.9(n)$ & 60.2 (b) & 0.76 (gh) & 345 (cd) & $53.0(f)$ & $18.4(f)$ & 7.34 (gh) & $1.66(a b c)$ \\
\hline CAN20 & $19.1(\mathrm{~cd})$ & 29.5 (hij) & $18.5(g)$ & 59.4 (de) & 0.82 (efgh) & 356 (a) & 60.1 (de) & $13.8(k)$ & $9.01(\mathrm{bc})$ & $1.67(a b c)$ \\
\hline CAN24 & 13.9 (i) & 32.7 (cdef) & $28.6(a)$ & $46.6(\mathrm{~m})$ & 0.85 (defg) & 331 (ef) & 60.3 (de) & $17.1(\mathrm{~g})$ & $6.93(h)$ & $1.22(\mathrm{~g})$ \\
\hline CAN26 & 21.7 (b) & $34.9(a b)$ & $19.3(f)$ & $57.1(\mathrm{~g})$ & 0.85 (defg) & 345 (c) & 44.6 (h) & 15.8 (hij) & $8.65(\mathrm{~cd})$ & 1.63 (bcd) \\
\hline CAN39 & $9.4(k)$ & 30.4 (ghij) & 15.8 (o) & $55.2(j)$ & 0.60 (i) & 320 (gh) & 53.7 (f) & $11.3(\mathrm{l})$ & 7.90 (ef) & 1.44 (f) \\
\hline CAN40 & $19.3(\mathrm{~cd})$ & 34.8 (abc) & 20.5 (c) & $54.0(I)$ & 0.69 (hi) & 354 (ab) & 43.8 (h) & 16.3 (ghi) & 7.83 (fg) & $1.46(f)$ \\
\hline CAN48 & $22.8(\mathrm{a})$ & 34.0 (abcd) & $12.8(q)$ & 58.7 (f) & 0.75 (gh) & 339 (cde) & 73.3 (a) & 18.9 (ef) & 8.39 (de) & 1.59 (de) \\
\hline CAN51 & 21.6 (b) & $35.4(a b)$ & $20.0(\mathrm{e})$ & 56.1 (i) & 0.79 (fgh) & 336 (de) & 65.5 (b) & 20.2 (cd) & 7.86 (fg) & $1.46(f)$ \\
\hline CAN58 & 16.7 (h) & 36.0 (a) & 20.2 (d) & $54.4(k)$ & 0.86 (defg) & 346 (bc) & 61.7 (cd) & 16.2 (ghij) & 9.03 (bc) & 1.66 (abc) \\
\hline Finola & $11.6(j)$ & 32.7 (cdef) & 21.1 (b) & $59.6(\mathrm{~cd})$ & 0.90 (defg) & 317 (gh) & $63.0(\mathrm{bcd})$ & 16.2 (ghij) & 5.88 (i) & 0.92 (h) \\
\hline Carmagnola & $21.4(b)$ & 31.0 (fghi) & $18.4(\mathrm{~g})$ & 56.4 (h) & $1.10(\mathrm{bc})$ & 334 (ef) & 52.7 (f) & 19.4 (de) & $9.03(\mathrm{bc})$ & $1.62(\mathrm{bcd})$ \\
\hline CS & $19.3(\mathrm{~cd})$ & 31.6 (efgh) & 17.8 (i) & 59.7 (cd) & 1.10 (b) & 316 (h) & $58.5(\mathrm{e})$ & 21.1 (c) & 9.22 (b) & 1.60 (de) \\
\hline Fibranova & 18.3 (de) & 30.7 (fghij) & 15.7 (o) & 58.6 (f) & 1.35 (a) & 325 (fg) & 53.1 (f) & $10.8(I)$ & 9.40 (b) & $1.62(\mathrm{bcd})$ \\
\hline Fedora & 17.8 (efg) & 29.2 (ij) & $16.3(l)$ & 59.7 (c) & 0.93 (def) & 339 (cde) & $64.4(\mathrm{bc})$ & 21.0 (c) & 7.51 (fg) & $1.56(\mathrm{e})$ \\
\hline Futura 75 & 17.9 (ef) & 29.8 (hij) & $17.5(j)$ & 60.1 (b) & 0.96 (cde) & 337 (cde) & 75.0 (a) & $27.8(a)$ & 10.29 (a) & $1.68(a b)$ \\
\hline Felina 32 & 16.8 (gh) & 29.6 (hii) & $16.0(n)$ & $60.2(b)$ & 0.98 (bcd) & 343 (cd) & 75.5 (a) & 26.6 (b) & 10.63 (a) & 1.70 (a) \\
\hline Ferimon & 17.1 (fgh) & 30.1 (hij) & $16.2(\mathrm{~m})$ & $62.0(\mathrm{a})$ & 0.95 (de) & 344 (cd) & 72.7 (a) & 26.4 (b) & 10.11 (a) & $1.68(a b)$ \\
\hline Codimono & 17.3 (efgh) & 33.8 (bcde) & $18.2(h)$ & 56.4 (hi) & 0.97 (bcde) & 336 (de) & 48.1 (g) & 15.4 (ij) & 9.09 (bc) & $1.67(a b c)$ \\
\hline Carmaleonte & $19.3(\mathrm{~cd})$ & 28.5 (j) & $13.1(p)$ & $59.1(\mathrm{e})$ & 0.61 (i) & 345 (cd) & 44.3 (h) & 15.2 (j) & $10.11(\mathrm{a})$ & 1.67 (abc) \\
\hline Kc Dora & 19.5 (c) & 31.5 (fgh) & $16.8(k)$ & 56.2 (hi) & 0.88 (defg) & 332 (ef) & 43.7 (h) & 16.9 (gh) & 9.00 (bc) & 1.66 (abc) \\
\hline Mean \pm SEM & $17.9 \pm 0.4$ & $31.9 \pm 0.3$ & $17.9 \pm 0.4$ & $57.5 \pm 0.4$ & $0.88 \pm 0.04$ & $337 \pm 2$ & $58.4 \pm 1.4$ & $18.2 \pm 0.6$ & $8.66 \pm 0.16$ & $1.56 \pm 0.02$ \\
\hline P genotype & $<0.01$ & $<0.01$ & $<0.01$ & $<0.01$ & $<0.01$ & $<0.01$ & $<0.01$ & $<0.01$ & $<0.01$ & $<0.01$ \\
\hline P group* & ns & $<0.01$ & $<0.05$ & $<0.01$ & $<0.01$ & $<0.01$ & ns & $<0.01$ & $<0.01$ & ns \\
\hline
\end{tabular}

a Data expressed as g.1000 seeds.

${ }^{b}$ Data expressed as \% of seed weight.

${ }^{c}$ Data expressed as \% of total fatty acids.

${ }^{d}$ Data expressed as $\mathrm{g} \cdot \mathrm{kg}^{-1}$ of oil.

${ }^{e}$ Data expressed as unit. $\mathrm{mg}^{-1}$.

${ }^{f}$ Data expressed as $\mathrm{g}$ CAE $\mathrm{kg}^{-1}$.

${ }^{g}$ Data expressed as $\mathrm{mol}$ TE $\mathrm{kg}^{-1}$.

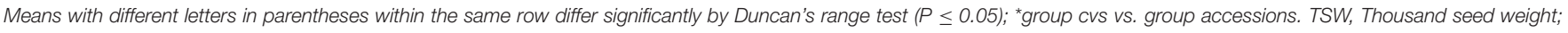

Toc, tocopherols; TI, Trypsin inhibitor activity; TP, Total phenolic content; Antiox, Antioxidant activity.

TABLE 3 | Pearson correlation coefficient (r) among seed traits in twenty hemp genotypes.

\begin{tabular}{|c|c|c|c|c|c|c|c|c|c|}
\hline & TSW & Oil & $\operatorname{ALA}(\omega 3)$ & $\operatorname{LA}(\omega 6)$ & Toc & Protein & Phytate & TI & TP \\
\hline Oil & 0.192 & 1 & & & & & & & \\
\hline $\mathrm{ALA}(\omega 3)$ & $-0.280^{\star}$ & $0.386^{\star \star}$ & 1 & & & & & & \\
\hline Toc & 0.132 & -0.106 & 0.034 & 0.189 & 1 & & & & \\
\hline Protein & $0.402^{\star \star}$ & 0.074 & -0.080 & 0.065 & $-0.314^{*}$ & 1 & & & \\
\hline TP & $0.346^{\star \star}$ & $-0.367^{\star \star}$ & $-0.486^{\star \star}$ & $0.389^{\star *}$ & $0.272^{*}$ & $0.259^{*}$ & 0.140 & $0.379^{* *}$ & 1 \\
\hline Antiox & $0.540^{\star \star}$ & -0.198 & $-0.580^{\star \star}$ & $0.372^{* *}$ & 0.152 & $0.463^{\star \star}$ & -0.035 & 0.237 & $0.775^{\star \star}$ \\
\hline
\end{tabular}

${ }^{* *}$ Correlation is significant at the 0.01 level; ${ }^{*}$ Correlation is significant at the 0.05 level.

TSW, Thousand seed weight; Toc, tocopherols; TI, Trypsin inhibitor activity; TP, Total phenolic content; Antiox, Antioxidant activity.

The fatty acid composition of hemp seed oils is shown in Table 4. The principal saturated fatty acid was the palmitic acid (PA; 16:0) in all genotypes ranging between 5.98 and $8.60 \%$, followed by stearic acid (SA; 18:0), varying from 2.26 to $4.61 \%$. The saturated fatty acid fraction represents on average $10.8 \%$ of the total fatty acids (Table 5). The major monounsaturated fatty acid was oleic acid (OA; 18:1cis9, average of 13.4\%) (Table 4) and CAN39 seeds showed the highest OA (16.8\%) content. With respect to the essential fatty acids, the seeds of the analyzed genotypes contained on average $55.7 \%$ of LA and $17.4 \%$ of 


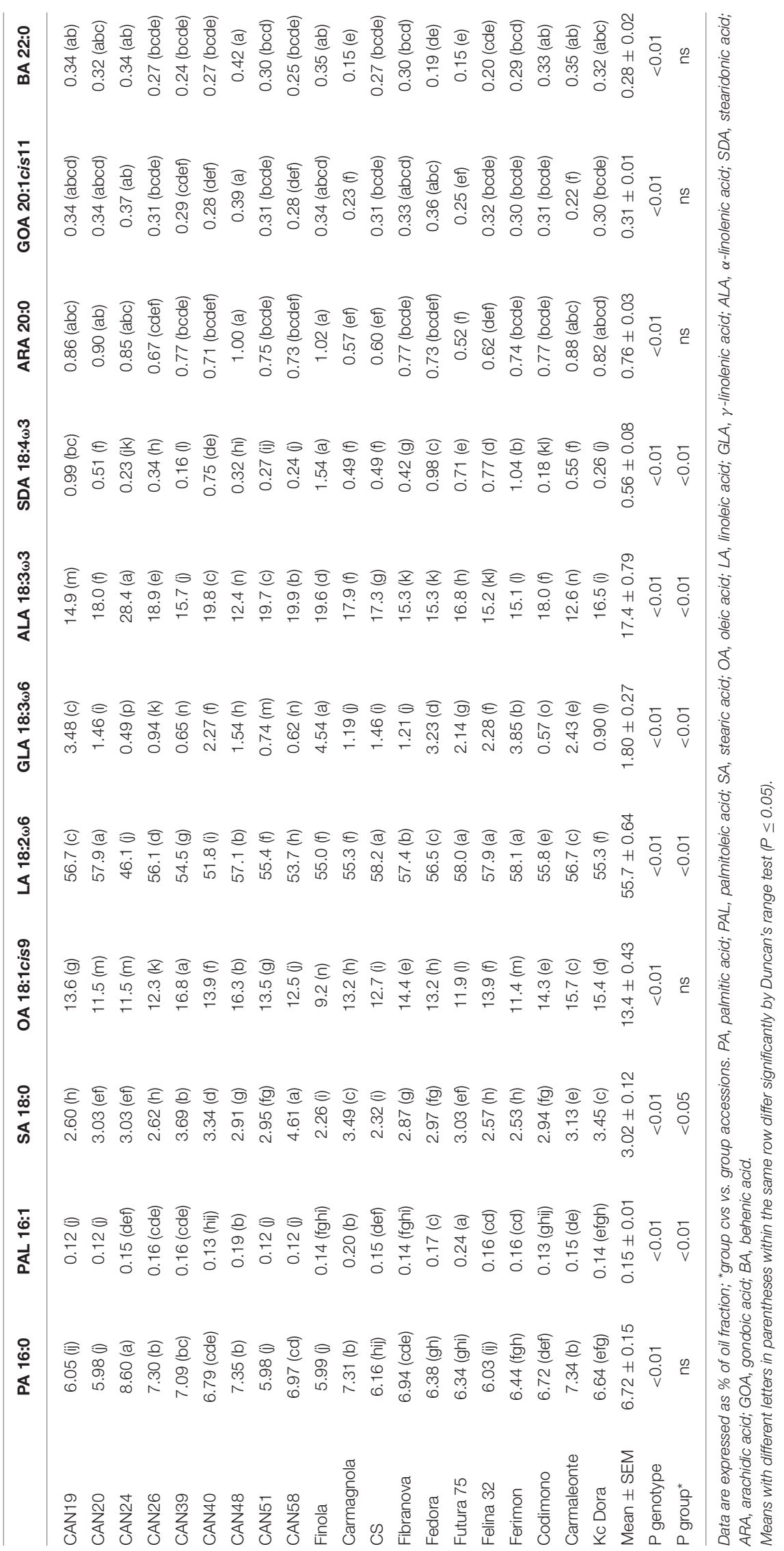


TABLE 5 | Saturated fatty acid (SFA), monounsaturated fatty acid (MUFA), polyunsaturated fatty acid (PUFA), and LA:ALA $(\omega 6: \omega 3)$ ratio in twenty hemp accessions (from CAN19 to CAN58) and cultivars (from cv. Finola to cv. Kc Dora).

\begin{tabular}{lcccc}
\hline & SFA & MUFA & PUFA & LA:ALA ratio \\
\hline CAN19 & 9.8 & 14.1 & 76.1 & 3.78 \\
CAN20 & 10.2 & 11.9 & 77.8 & 3.22 \\
CAN24 & 12.8 & 12.0 & 75.2 & 1.63 \\
CAN26 & 10.9 & 12.8 & 76.4 & 2.96 \\
CAN39 & 11.8 & 14.2 & 71.0 & 3.49 \\
CAN40 & 11.1 & 14.3 & 74.6 & 2.63 \\
CAN48 & 11.7 & 16.9 & 71.4 & 4.60 \\
CAN51 & 10.0 & 13.9 & 76.1 & 2.80 \\
CAN58 & 12.6 & 12.9 & 74.6 & 2.69 \\
Finola & 9.6 & 9.7 & 80.7 & 2.82 \\
Carmagnola & 11.5 & 13.6 & 74.9 & 3.06 \\
CS & 9.4 & 13.2 & 77.5 & 3.36 \\
Fibranova & 10.9 & 14.8 & 74.3 & 3.72 \\
Fedora & 10.3 & 13.7 & 76.0 & 3.67 \\
Futura 75 & 10.0 & 12.4 & 77.6 & 3.44 \\
Felina 32 & 9.4 & 14.4 & 76.2 & 3.77 \\
Ferimon & 10.0 & 11.9 & 78.1 & 3.84 \\
Codimono & 10.8 & 14.7 & 74.5 & 3.11 \\
Carmaleonte & 11.7 & 16.1 & 72.2 & 4.52 \\
Kc Dora & 11.2 & 15.9 & 72.9 & 3.35 \\
Mean \pm SEM & $10.8 \pm 0.2$ & $13.7 \pm 0.4$ & $75.4 \pm 0.5$ & $3.32 \pm 0.15$ \\
\hline Data & 13.9 &
\end{tabular}

Data are expressed as \% of total fatty acids.

ALA, resulting in a $\omega 6: \omega 3$ ratio $=3.3: 1$ (Table 5). Significant differences among the genotypes analyzed were observed for LA (from 46.1 to $58.1 \%$ ), the fatty acid present in hemp seed in the greatest proportion. Furthermore, noticeable differences in ALA concentration were observed, with the greatest amount measured in CAM24 (28.4\%) and the lowest quantity in CAN48 and Carmaleonte $(\sim 12 \%)$.

The other fatty acids: palmitoleic (POA; 16:1), GLA, SDA, arachidic (ARA; 20:0), gondoic (GOA; 20:1cis11), and behenic acid (BA; 22:0) were found in small amounts. However, the percentage of GLA was the highest among the other minor unsaturated fatty acids and varied from 0.49 to $4.54 \%$. The highest content of GLA was detected in Finola (4.54\%) which also contained the highest quantity of SDA (1.54\%), followed by Ferimon, CAN19 and Fedora with a content in GLA of $3.85,3.48,3.23 \%$, respectively and of $1.04,0.99,0.98 \%$ of SDA, respectively (Table 4). ANOVA revealed significant variability among genotypes for all the fatty acids at the 0.01 level. Pearson correlation analysis showed that ALA is positively correlated with oil content and inversely correlated with LA, TP content and antioxidant activity (Table 3), whereas LA was positively correlated with antinutritional compounds (phytate and TI), TP content and antioxidant activity.

\section{DISCUSSION}

The possibility of using industrial hemp cvs with low levels of THC $(<0.20 \%$ THC on a DM basis as established by the European Union and also by Italian law) is leading to a re-introduction of this plant into the Italian production systems. In the 2015 about 2,000 hectares (ha) have been cultivated with hemp in Italy and in all countries of European Union its cultivation has reached 25,224 ha (European Industrial Hemp Association, http://eiha.org/downloads/). The interest on hemp is due to the huge number of products that can be obtained from this plant (Small and Marcus, 2002), and recently it is also largely focused on seeds, which are rich in healthy nutritional fats and proteins for both humans and animals (Mustafà et al., 1999; Rodriguez-Leyva and Pierce, 2010; Girgih et al., 2014).

In this work we have compared the seed composition of several cvs that are allowed to be cultivated in Europe with a collection of hemp accessions obtained from the IPK genebank and grown for 1 year in the same environment. Although hemp tend to perform better in its area of development (Dempsey, 1975), in our trial, except CAM58 which richest the flowering stage (see Table 1) and, consequently, seed maturation very late and $\mathrm{cv}$ Finola which was able to produce a discrete quantity of seeds but the plant remained very short, all the remaining hemp genotypes showed a good environmental adaptation.

In general, a high variability $(P<0.01)$ for all biochemical traits among the genotypes analyzed was observed (Table 2). Some of them, such as TSW, total protein, oil and ALA content appeared higher in hemp seed accessions than in cvs. In fact, protein content was $>31 \%$ in all meals analyzed but CAN20 and CAN40 had the highest protein content $\sim 35 \%$ and the latter also had an oil content of up to $34.8 \%$.

As reported by others (Kriese et al., 2004; Chen et al., 2010; Vonapartis et al., 2015), the most abundant fatty acids in our hemp seed collection proved to be LA (average 55.7\%), ALA $(17.4 \%)$, and OA (13.4\%), which together represent $86.5 \%$ of total fatty acids (Tables 4, 5). However, a high variability for LA and ALA content was detected among all hemp genotypes (Table 4) and the most different was CAN24 which showed 46.1 and 28.4\% of LA and ALA respectively, giving a ratio $\omega 6: \omega 3$ (LA:ALA) of 1.63:1, which has never been reported before. In fact, a significant number of previous studies on hemp oil composition (Oomah et al., 2002; Callaway, 2004; Vonapartis et al., 2015) as well as most of the hemp genotypes analyzed in this study showed a $\omega 6: \omega 3$ ratio of roughly $3: 1$, reported as optimal for human nutrition (Erasmus, 1993; Leizer et al., 2000).

Simopoulos (2008) reported a $\omega 6: \omega 3$ ratio of 15-16.7:1 in Western European and American food supplies. This very high ratio, in today's diets, promotes the pathogenesis of many diseases, including cancer, cardiovascular and inflammatory and autoimmune diseases, whereas increased levels of $\omega 3$ polyunsaturated fatty acids (PUFAs) exert suppressive effects (Simopoulos, 2008; Gómez Candela et al., 2011). Therefore, the oil of CAN24 might be used, particularly in Western diets characterized by high LA and low ALA fatty acid intake, which is considered detrimental to health (Simopoulos, 2008; Gómez Candela et al., 2011). 
In addition to LA and ALA, hemp seed oil also contains their direct metabolites GLA and SDA, which serve as intermediaries in the formation of longer-chain fatty acids and vital hormonelike prostaglandins in the body (Guil-Guerrero et al., 2010). The results of this study confirm that hemp seed oil is a good source of PUFA, especially GLA and SDA, but the content detected among the hemp seed genotypes varies greatly, even more than that detected for LA and ALA. As expected, the cv Finola showed the highest content of GLA (4.54\%) and SDA (1.54\%; Table 4) which is in agreement with results from other authors (Callaway, 2004; Vonapartis et al., 2015). However, an interesting level of GLA and SDA was also detected in CAN19, Fedora and Ferimon showing a content of GLA $>3.0 \%$ and of SDA $\sim 1 \%$.

Hemp seeds showed a substantial content of tocopherols and total phenols (Table 2). In particular, TP content was higher in hemp seed than in flax, which is a species extremely rich in total phenols (Russo and Reggiani, 2015c). The high antioxidant activity detected in almost all hemp seeds, due to tocopherols and TP content, can ensure high oxidative stability that would makes hemp oil suitable for food and industrial applications, as well as improving the nutritional quality of human diets.

However, despite the functional and nutraceutical properties of this crop, the seeds are not totally free of antinutritional compounds, such as phytic acid, condensed tannins, cyanogenic glycosides, saponin and TI (Russo and Reggiani, 2013; Pojić et al., 2014). The quite high content (mean 5.84\%) of phytate in our hemp collection confirmed the findings of Russo and Reggiani (2013) and the variability between the different hemp genotypes was statistically significant (Table 2). In some genotypes, the phytate content was more than $7 \%$ (those with letter "a" by Duncan's range test). Therefore, the use of such meal appears rather limited for monogastric animals and in human nutrition since a high level of phytic acid will affect protein digestibility, organoleptic properties, and bioavailability of macro- and microelements (Guillamón et al., 2008). Furthermore the high phytate content found, particularly in French hemp seed cultivars, will greatly limit the use of this protein source in novel food or feed formulations.

In addition, TI is responsible for the reduced digestibility of seed proteins and, for this reason, it constitutes one of the main antinutritional factor in plant seeds (Peric et al., 2013). As shown in Table 2, considerable variability in TI activity among all hemp meals was evident. However, the levels of TI in hemp seeds were

\section{REFERENCES}

Callaway, J. C. (2004). Hemp seed as a nutritional resource: an overview. Euphytica 140, 65-72. doi: 10.1007/s10681-004-4811-6

Chen, T., He, J., Zhang, J., Zhang, H., Qian, P., Hao, J., et al. (2010). Analytical characterization of hemp seed (seed of Cannabis sativa L.) oil from eight regions in China. J. Diet Suppl. 7, 117-129. doi: 10.3109/19390211003781669

Chen, P. S., Toribara, T. Y., and Warner, H. (1956). Microdetermination of phosphorus. Anal. Chem. 28, 1756-1758. doi: 10.1021/ac60119a033

Christie, W. W. (1982). A simple procedure for rapid transmethylation of glycerolipids and cholesteryl esters. J. Lipid Res. 23, 1072-1075.

Chouinard, P. Y., Corneau, L., Barbano, D. M., Metzger, L. E., and Bauman, D. E. (1999). Conjugated linoleic acids alter milk fatty acid lower in comparison with those observed in some cereals and soybean (Sosulki et al., 1988; Tsukamoto et al., 1995). Pearson coefficient $(r=0.679)$ indicated a strong positive correlation between TI activity and phytate content (Table 3 ) and this is in agreement with previous results reported by Russo and Reggiani (2015b) who analyzed six hemp cultivars grown in two different experimental fields for two consecutive years.

In conclusion our analysis confirmed that hemp seed is an excellent product in terms of protein content, oil composition and antioxidant molecules for food and feed formulations, but the content of some antinutritional compounds, particularly phytate will influence negatively its potential nutritional value. Therefore, an improvement for this trait would be necessary. Based on our data, some hemp accessions present in our germplasm collection, appear to contain more interesting traits than the cultivated hemp cvs. In fact, hemp accessions appeared more interesting then hemp cvs with regard to protein and oil content. In particular the Italian CAN40 showed a high content of both, therefore, might be used to improve oil and protein content in hemp cvs through hybridization and selection. Furthermore, the Italian accession CAM40 might be also a good candidate to reduce the content of phytate. In general, we observed that, among the genotypes analyzed, all Italian dioic and monoic cvs showed less phytate content than the monoic French cvs. This trend, except for CAN48, is also observed in the other Italian accessions. Therefore, our analysis evidenced that, although we analyzed a small segment of hemp germplasm collection and for only 1 year, probably an improvement of the cultivated hemp cvs for some traits might be possible using the large genetic variability present in the germplasm of hemp.

\section{AUTHOR CONTRIBUTIONS}

This work was carried out in collaboration among authors. The authors of this manuscript worked together to design, conduct, analyze and interpret the findings of these experiments. All the authors read and approved the final manuscript.

\section{ACKNOWLEDGMENTS}

This study was partially supported by the Research Project FilAgro-Strategie innovative e sostenibili per la filiera agroalimentare-Accordo Quadro N. 18093/RCC del 5/8/2013. composition and inhibit milk fat secretion in dairy cows. J. Nutr. 129, 1579-1584.

Cowieson, A. J., Acamovic, T., and Bedford, M. R. (2004). The effects of phytase and phytic acid on the loss of endogenous amino acids and minerals from chickens. Brit. Poultry Sci. 45, 101-108. doi: 10.1080/00071660410001 668923

De Boland, A. R., Garner, G. B., and O’Dell, B. L. (1975). Identification and properties of phytate in cereal grains and oilseed products. J. Agric. Food Chem. 23, 1186-1189. doi: 10.1021/jf60202a038

Deferne, J. L., and Pate, D. W. (1996). Hemp seed oil: a source of valuable essential fatty acids, J. Int. Hemp Assn. 3, 4-7.

Dempsey, J. M. (ed.). (1975). “Hemp," in Fibre Crops (Gainesville, FL: University of Florida Press), 46-89. 
Docimo, T., Caruso, I., Ponzoni, E., Mattana, M., and Galasso, I. (2014). Molecular characterization of edestin gene family in Cannabis sativa L. Plant Physiol. Bioch. 84, 142-148. doi: 10.1016/j.plaphy.2014.09.011

Erasmus, U. (1993). Fats that Heal, Fats that Kill. Books Alive, 7436 Fraser Drive, V5J 5B9 (Burnaby, B.C).

Fischedick, J. T., Glas, R., Hazekamp, A., and Verpoorte, R. (2009). A qualitative and quantitative HPTLC densitometry method for the analysis of cannabinoids in Cannabis sativa L. Phytochem. Analysis 20, 421-426. doi: 10.1002/pca.1143

Gimeno, E., Calero, E., Castellote, A. I., Lamuela-Raventós, R. M., de la Torre, M. C., and López-Sabater, M. C. (2000). Simultaneous determination of $\alpha$ tocopherol and $\beta$-carotene in olive oil by reversed-phase high-performance liquid chromatography. J. Chromatogr. A 881, 255-259. doi: 10.1016/S00219673(00)00272-7

Girgih, A. T., He, R., Malomo, S., Offengenden, M., Wu, J., and Aluko, R. E. (2014). Structural and functional characterization of hemp seed (Cannabis sativa L.) protein-derived antioxidant and antihypertensive peptides. J. Funct. Foods 6: 384-394. doi: 10.1016/j.jff.2013.11.005

Gómez Candela, C., Bermejo López, L. M., and Loria Kohen, V. (2011). Importance of a balanced omega 6/omega 3 ratio for the maintenance of health: nutritional recommendations. Nutr. Hosp. 26, 323-329. doi: 10.1590/S021216112011000200013

Guil-Guerrero, J. L., Rincon-Cervera, M. A., and Venegas-Venegas, E. (2010). Gamma-linolenic and stearidonic acids: purification and upgrading of C18-PUFA oils. Eur. J. Lipid Sci. Technol. 112, 1068-1081. doi: 10.1002/ejlt.200900294

Guillamón, E., Pedrosa, M. M., Burbano, C., Cuadrado, C., and Muzquiz, M. (2008). The trypsin inhibitors present in seed of different grain legume species and cultivar. Food Chem. 107, 68-74. doi: 10.1016/j.foodchem.2007. 07.029

Hara, A., and Radin, N. S. (1978). Lipid extraction of tissues with a low-toxicity solvent. Anal. Biochem. 90, 420-426. doi: 10.1016/0003-2697(78)90046-5

House, J. D., Neufeld, J., and Leson, G. (2010). Evaluating the quality of protein from hemp seed (Cannabis sativa L.) products through the use of the protein digestibility corrected amino acid score method. J. Agric. Food Chem. 58, 11801-11807. doi: 10.1021/jf102636b

Huang, D., Ou, B., and Prior, R. L. (2005). The chemistry behind antioxidant capacity assays. J. Agr. Food Chem. 53, 1841-1856. doi: 10.1021/jf030723c

Kim, J. J., and Lee, M. J. (2011). Isolation and characterization of edestin from Cheungsam hempseed. J. Appl. Biol. Chem. 54, 84-88. doi: 10.3839/jabc.2011.015

Kriese, U., Schumann, E., Weber, W. E., Beyer, M., Bruhl, L., and Matthaus, B. (2004). Oil content, tocopherol composition and fatty acid patterns of the seeds of 51 Cannabis sativa L. genotypes. Euphytica 137, 339-351. doi: 10.1023/B:EUPH.0000040473.23941.76

Leizer, C., Ribnicky, D., Poulev, A., Dushenkov, S., and Raskin, I. (2000). The composition of hemp seed oil and its potential as an important source of nutrition. J. Nutraceut. Funct. Med. Foods 2, 35-53. doi: 10.1300/J133v02n04_04

Li, H. L. (1973). An archaeological and historical account of cannabis in China. Econ. Botany 28, 437-444.

Li, H. L. (1974). The origin and use of Cannabis in eastern Asia linguistic-cultural implications. Econ. Botany 28, 293-301.

Odani, S., and Odani, S. (1998). Isolation and primary structure of a methionine and cystine-rich seed protein of Cannabis sativa L. Biosci. Biotechnol. Biochem. $62,650-654$.

Oomah, B. D., Busson, M., Godfrey, D. V., and Drover, J. C. (2002). Characteristics of hemp (Cannabis sativa L.) seed oil. Food Chem. 76, 33-43. doi: 10.1016/S0308-8146(01)00245-X

Osburn, L. (1992). Hemp seed: the most nutritionally complete food source in the world. Part two: hemp seed oils and the flow of live force. Hemp Line J. 1, $12-13$.

Mukherjee, A., Roy, S. C., De Bera, S., Jiang, H. E., Li, X., Li, C. S., et al. (2008). Results of molecular analysis of an archaeological hemp (Cannabis sativa L.) DNA sample from North West China. Genet. Resour. Crop Evol. 55, 481-485. doi: 10.1007/s10722-008-9343-9

Mustafà, A. F., McKinnon, J. J., and Christensen, D. A. (1999). The Nutritive Value of Hemp Meal for Ruminants. Canadian. J. Anim. Sci. 79, 91-95. doi: 10.4141/A98-031
Peric, V., Srebric, M., and Mladenovic-Drinic, S. (2013). Trypsin inhibitors in soybean. soybean: a dawn to the legume world: the future of soybean research is already here. J. Int. Legume Soc. 1, 23-24. Available online at: http://ils.nsseme. com/

Pojić, M., Mišan, A., Sakač, M., Dapčević Hadnadev, T., Šarić, B., Milovanović, I., et al. (2014). Characterization of byproducts originating from hemp oil processing. J. Agric. Food Chem. 62, 12436-12442. doi: 10.1021/jf5044426

Raboy, V., and Dickinson, D. B. (1984). Effect of phosphorus and zinc nutrition on soybean seed phytic acid and zinc. Plant Physiol. 75, 1094-1098.

Re, R., Pellegrini, N., Proteggente, A., Pannala, A., Yang, M., and Rice-Evans, C. (1999). Antioxidant activity applying an improved ABTS radical cation decolorization assay. Free Radic. Biol. Med. 26, 1231-1237. doi: 10.1016/S08915849(98)00315-3

Rodriguez-Leyva, D., and Pierce, N. G. (2010). The cardiac and haemostatic effects of dietary. hemp seed. Nutrit. Metabol. 7:32. doi: 10.1186/1743-7075-7-32

Russo, R., and Reggiani, R. (2013). Variability of antinutritional compounds in hemp seed meal of Italian and French varieties. Plant 1, 25-29. doi: 10.11648/j.plant.20130102

Russo, R., and Reggiani, R. (2015a). "Protein concentration and amino acid profile in hemp seed and flaxseed meal," in Eucarpia International Symposium on Protein Crops, ed A. De Ron (Pontevedra: Spanish Association for Legumes), 193-195.

Russo, R., and Reggiani, R. (2015b). Evaluation of protein concentration, amino acid profile and antinutritional compounds in hemp seed meal from dioecious and monoecious varieties. Am. J. Plant Sci. 6, 14-22. doi: 10.4236/ajps.2015.61003

Russo, R., and Reggiani, R. (2015c). Phenolics and antioxidant activity in flax varieties with different productive attitude. Int. Food Res. J. 22, 1736-1739. Available online at: http://www.ifrj.upm.edu.my

Sarwar, G. (1997). The protein digestibility-corrected amino acid score method overestimates quality of proteins containing antinutritional factors and of poorly digestible proteins supplemented with limiting amino acids in rats. $J$. Nutr. 127, 758-764.

Simopoulos, A. P. (2008). The importance of the omega-6/omega-3 fatty acid ratio in cardiovascular disease and other chronic diseases. Exp. Biol. Med. 233, 674-688. doi: 10.3181/0711-MR-311

Small, E., and Marcus, D. (2002). "Hemp: a new crop with new uses for North America," in Trends in New Crops and New Uses, eds J. Janick and A. Whipkey (Alexandria, VA: ASHS Press), 284-326.

Sosulki, F. W., Minja, L. A., and Christensen, D. A. (1988). Trypsin inhibitors and nutritive value in cereals. Plant Foods and Hum. Nutr. 38, 23-34. doi: 10.1007/BF01092307

Tang, C. H., Ten, Z., Wang, X. S., and Yang, X. Q. (2006). Physicochemical and functional properties of hemp (Cannabis sativa L.) protein isolate. J. Agric. Food Chem. 54, 8945-8950. doi: 10.1021/jf0619176

Tsukamoto, C., Shimada, S., Igita, K., Kudou, S., Kokubun, M., Okubo, K., et al. (1995). Factor affecting isoflavone content in soybean seeds: changes in isoflavones, saponins, and composition of fatty acids at different temperatures during seed development. J. Agric. Food Chem. 43, 1184-1192. doi: 10.1021/jf00053a012

Velioglu, Y. S., Mazza, G., Gao, L., and Oomah, B. D. (2006). Antioxidant activity and total phenolics in selected fruits, vegetables, and grain products. J. Agr. Food Chem. 46, 4113-4117. doi: 10.1021/jf9801973

Vonapartis, E., Aubin, M. P., Seguin, P., Mustafa, A. F., and Charron, J. B. (2015). Seed composition of ten industrial hemp cultivars approved for production in Canada. J. Food Comp. Anal. 39, 8-12. doi: 10.1016/j.jfca.2014.11.004

Conflict of Interest Statement: The authors declare that the research was conducted in the absence of any commercial or financial relationships that could be construed as a potential conflict of interest.

Copyright (C) 2016 Galasso, Russo, Mapelli, Ponzoni, Brambilla, Battelli and Reggiani. This is an open-access article distributed under the terms of the Creative Commons Attribution License (CC BY). The use, distribution or reproduction in other forums is permitted, provided the original author(s) or licensor are credited and that the original publication in this journal is cited, in accordance with accepted academic practice. No use, distribution or reproduction is permitted which does not comply with these terms. 


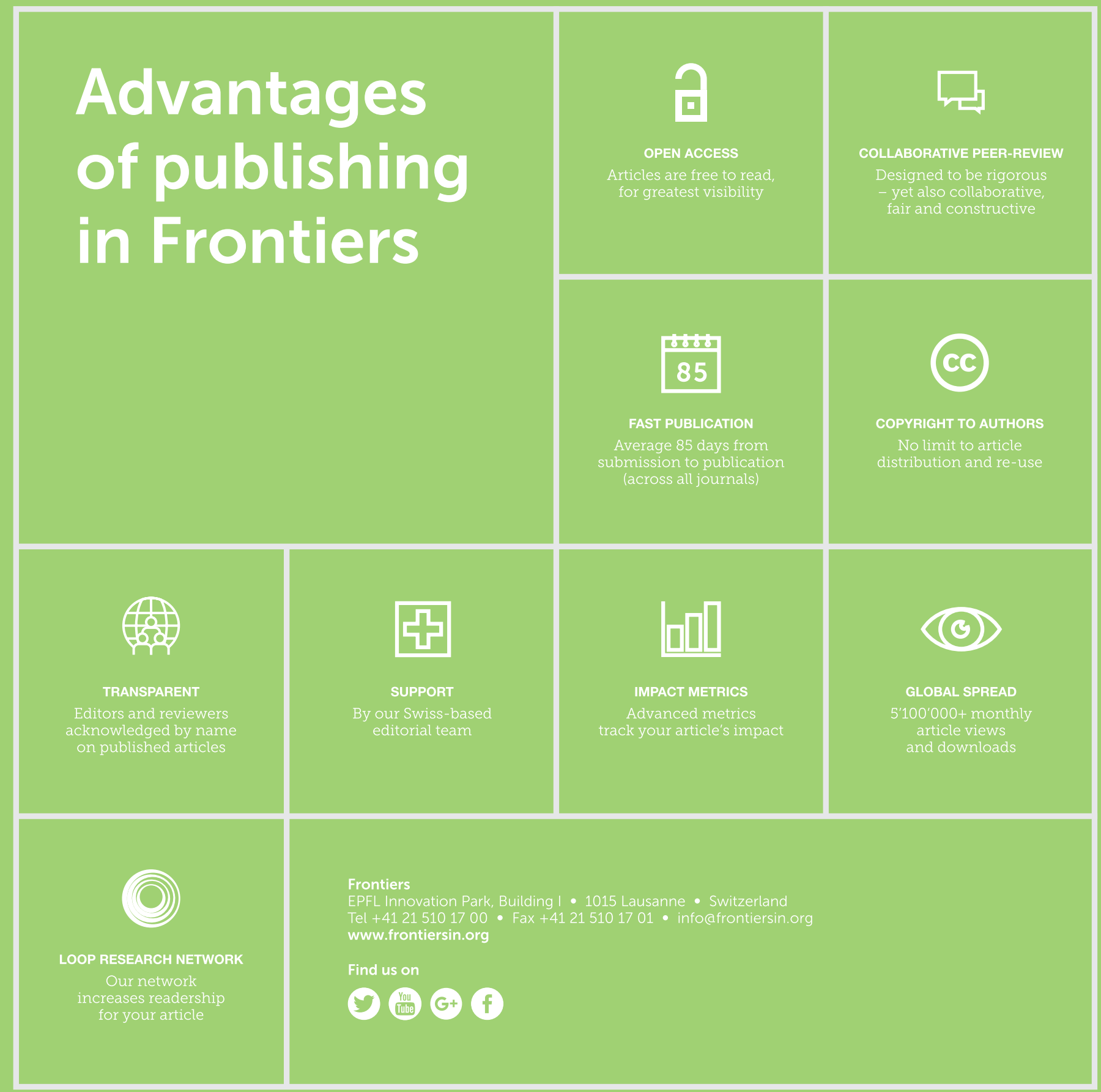

